DIPARTIMENTO DI SCIENZE DOCUMENTARIE, LINGUISTICO-FILOLOGICHE E GEOGRAFICHE

DOTTORATO DI RICERCA IN SCIENZE LIBRARIE E DOCUMENTARIE XXV CICLO

COORDINATORE: PROF. MARCO SANTORO

\title{
PROSPETTIVE DI SVILUPPO DEI CATALOGHI ELETTRONICI
}

DOTTORANDA

Dott.ssa ANTONELLA IACONO 



\section{CAPITOLO 1. IL CATALOGO ELETTRONICO TRA POSTMODERNITÀ E} RIVOLUZIONE DIGITALE.

1.1. La riflessione sulle funzioni del catalogo, il dibattito internazionale e le strategie bibliotecarie

1.2. Il catalogo come 'linguaggio' della biblioteca nel nuovo contesto informativo 40

1.3. L'utente 'postmoderno' e i 'nativi digitali' 48

1.4. La percezione delle funzioni del catalogo nelle indagini sull'utenza . 61

\section{CAPITOLO 2. OPAC 'DI NUOVA GENERAZIONE' E 'SISTEMI PER LA} SCOPERTA' 69

2.1. L'evoluzione dell'OPAC tradizionale e la nascita dei next generation catalogs.

2.2. Definire next generation catalogs e discovery systems. Una proposta 'operativa' 71

2.3. Perché nascono i cataloghi di nuova generazione. Le visioni del cambiamento. 77

2.3.1. Verso la ricerca globale: Dai next generation catalogs ai discovery systems

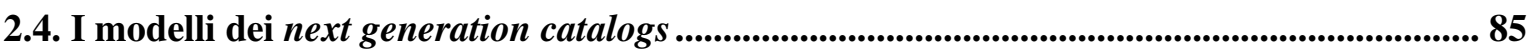

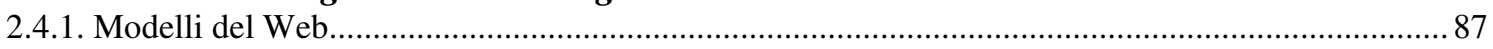

2.5. Le caratteristiche funzionali. Modelli di sviluppo e nuove funzionalità ................................ 94

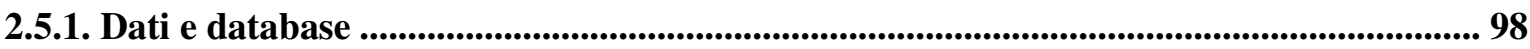

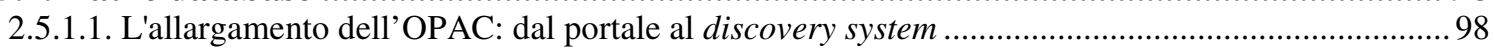

2.5.1.2 La ricerca globale: discovery systems e Web scale discovery services ............................................. 107

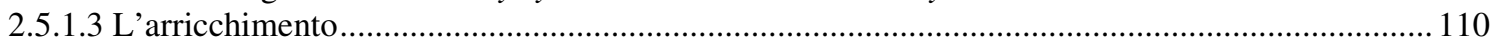

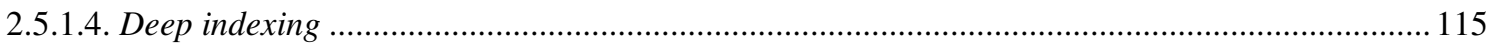

2.5.2. Ricerca, visualizzazione dei risultati e navigazione nel catalogo .................................... 116

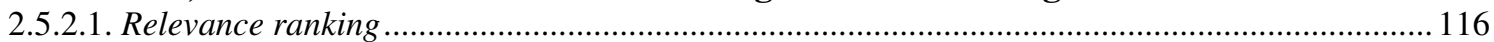

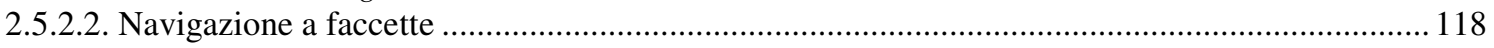

2.5.2.3. L'introduzione delle funzionalità dei motori di ricerca negli OPAC ............................................ 124

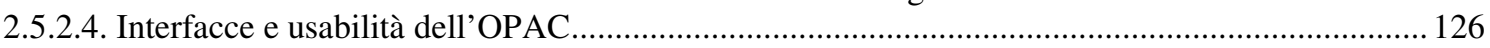

2.5.3. Nuove funzionalità per i servizi all'utenza......................................................................... 132

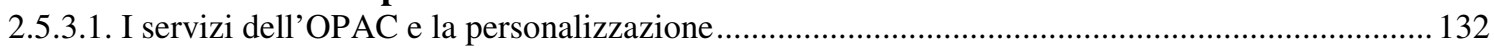

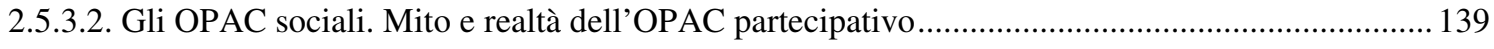

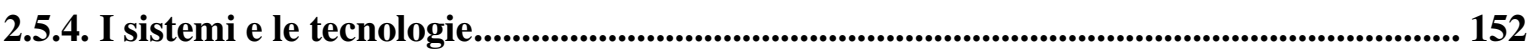

2.5.4.1. OPAC e ILS: la modularità come modello per la 'recombinant library' .................................. 152

2.5.4.2. L'implementazione dei discovery systems. Lavorare con i metadati ........................................ 157

2.5.4.3. L'implementazione dei discovery systems. Il workflow ................................................. 162 
2.6. I dati bibliografici negli OPAC di nuova generazione e nei discovery systems: arricchimento dei dati e impoverimento delle funzioni.

2.6.1. 'One size fits all'? dall'accesso unico alla moltiplicazione degli accessi....................................... 172

2.6.2. La ricerca semantica nei nuovi OPAC: ricerca per soggetto e ricerca per parola chiave ................... 173

2.7. Conclusioni. I Limiti del modello attuale e i futuri scenari. Le tematiche aperte............. 178

2.8. Appendice. Lavorare con i metadati. Extensible Catalog.................................................... 184

CAPITOLO 3. L'OPAC E IL MODELLO FRBR .......................................................189

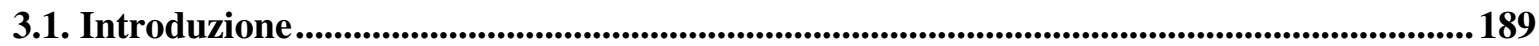

3.2. Il modello FRBR. Nascita e sviluppi della "famiglia FRBR". FRAD e FRSAD ...............190

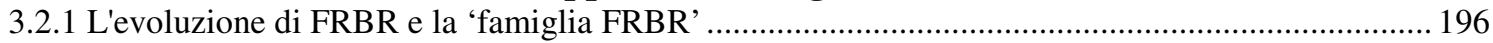

3.3. Da FRBR al rinnovamento dei principi e delle norme catalografiche...............................198

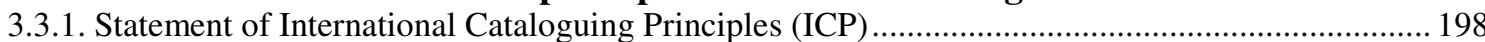

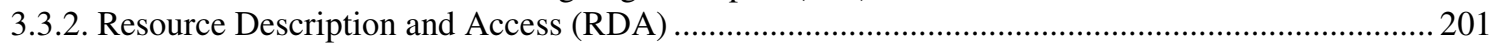

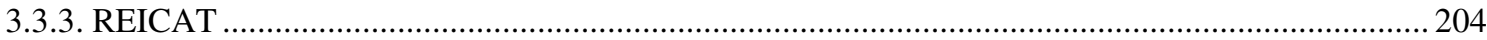

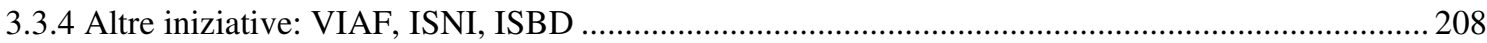

3.4. L'applicazione del modello FRBR ai cataloghi. Analisi delle entità FRBR .........................210

3.4.1. L'applicazione di FRBR alle diverse tipologie documentarie .................................................... 214

3.5. L'implementazione di FRBR negli OPAC: problematiche e opportunità ...........................219

3.5.1. Sistemi completi, prototipi, software ...................................................................................... 222

3.6. FRBR, Next Generation Catalogs e discovery systems: potenzialità di implementazione del modello nei sistemi per la scoperta ............................................................................................227

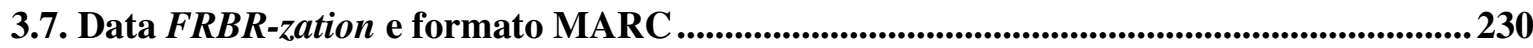

CAPITOLO 4: L'OPAC DAL WEB 2.0 AL WEB 3.0. LINKED DATA E WEB

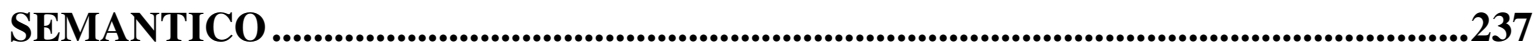

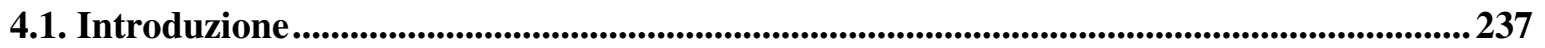

4.2. Dal Web 2.0 al Web 3.0. Open data e la Linked Open Data initiative (LOD) ....................238

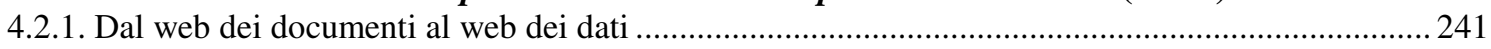

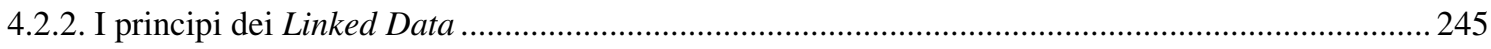

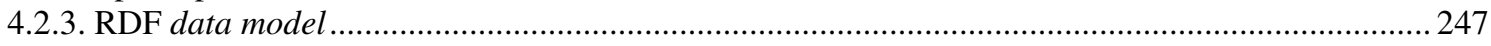

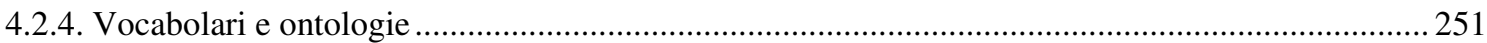

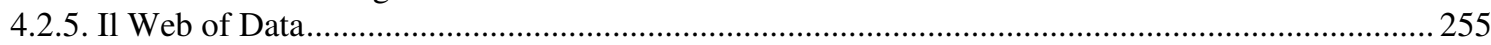

4.3. I dati delle biblioteche e il loro utilizzo nel Web Semantico .............................................264

4.4. Dal record al dato: atomizzazione e ristrutturazione del record bibliografico.................. 266

4.5. Pubblicare linked open data. Il workflow, le tecnologie, le licenze .........................................279

4.5.1. Le iniziative delle biblioteche nel campo dei linked data. Dataset, vocabolari, schemi di metadati

4.6. Web 3.0 per la ricerca semantica: arricchimento, impoverimento o evoluzione? .............301

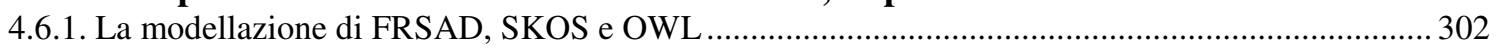

4.7. FRBR e Web Semantico: un incontro possibile? ..................................................................309 


\section{CAPITOLO 5. L'OPAC A SUPPORTO DEL PROCESSO DI RICERCA DELL'INFORMAZIONE. VERSO UN NUOVO MODELLO DI SVILUPPO PER IL CATALOGO ELETTRONICO

5.1. Verso una nuova identità del catalogo: dal recupero dell'informazione alla creazione di conoscenza.

5.2. Informazione e conoscenza nella Scienza dell'informazione.

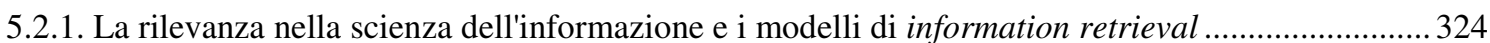

5.2.2. Un modello di information retrieval: Saracevic stratified model (1996) ........................................ 331

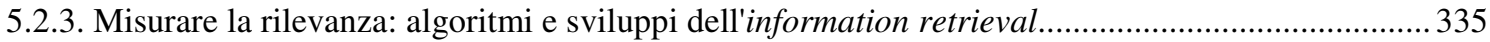

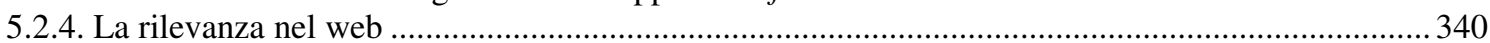

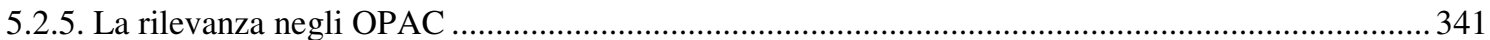

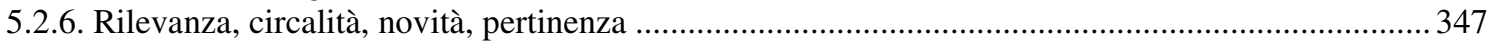

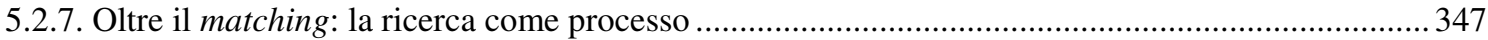

5.3. Dagli studi di information retrieval agli studi centrati sull'utente. I modelli del comportamento informativo e i modelli di ricerca dell'informazione

5.3.1. Teorie e metateorie socioculturali dell'informazione (Dervin, Belkin, Wilson) ................................352

5.3.1.1. Il sense-making model di Dervin e lo 'stato anomalo di conoscenza' di Belkin .............................354

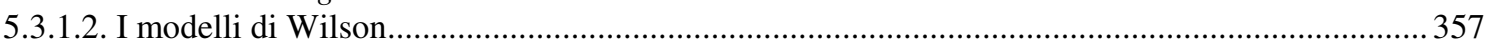

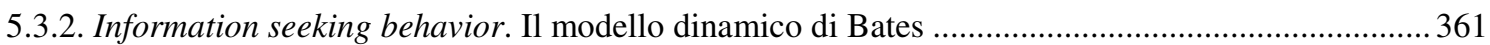

5.3.2.1. Ricerca attiva e acquisizione passiva di informazione: il modello integrato di Bates................... 367

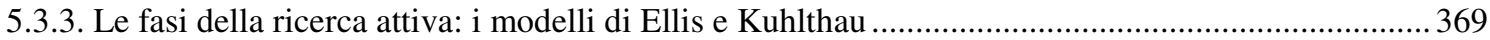

5.3.4. Il modello cognitivo di Ingwersen e le basi dell' information searching and retrieval (IS\&R) ....... 375

5.4. L'OPAC a supporto del processo di ricerca dell'informazione. Verso un nuovo modello

5.4.1. Verso un nuovo modello di sviluppo per l'OPAC

5.4.2. Analizzare il bisogno informativo. Variabili e barriere nel processo di recupero dell'informazione

5.4.3. Il rovesciamento della logica query oriented e l'attività di browsing. Le principali tattiche di ricerca:

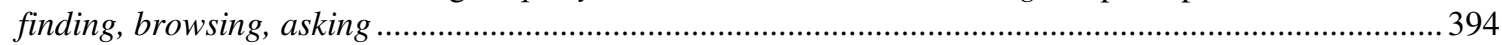

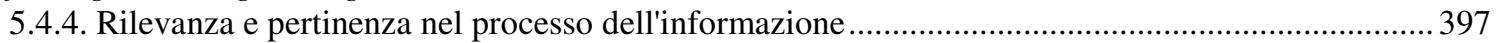

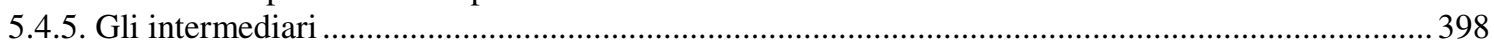

5.4.6. Le fasi del processo di ricerca dell'informazione. Funzioni-utente e funzionalità strategiche degli OPAC

5.4.6.1. Fase pre-focus. Attività dell'utente, funzionalità dell'OPAC e linee strategiche ............................ 404

5.4.6.2. La formulazione del focus. Attività dell'utente, funzionalità dell'OPAC e linee strategiche ...... 415

5.4.6.3. Fase post-focus. Attività dell'utente, funzionalità dell'OPAC e linee strategiche .......................... 424

5.5. Il ruolo dei linked data nel processo di ricerca dell'informazione

5.5.1. Il nuovo 'record bibliografico collegato' nella gestione del processo informativo ..............................434

5.5.2. La riduzione delle barriere cognitive nella ricerca dell'informazione...............................................435

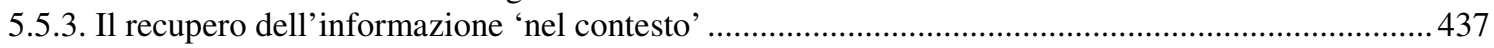

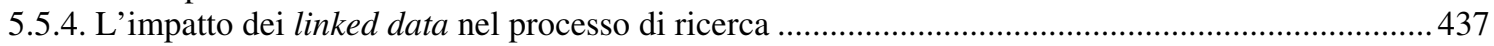

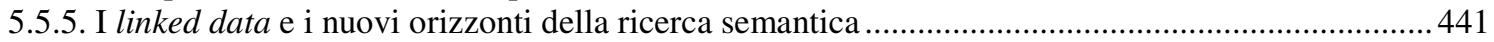

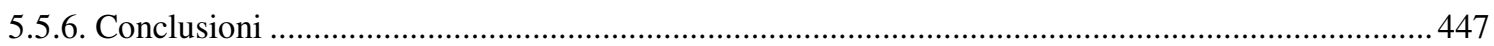

CONCLUSIONI. PROSPETTIVE E SVILUPPI FUTURI

1. Un solo OPAC, nessun OPAC? L'identità del catalogo di fronte alla sfida del Web Semantico. 


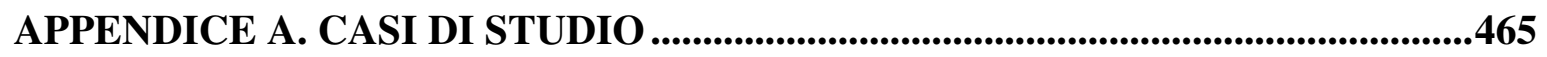

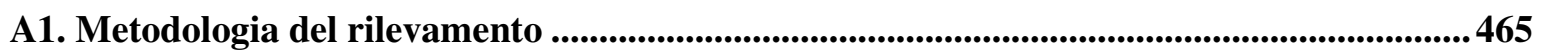

A2. Elementi di analisi e predisposizione della griglia di esame ..............................................465

Il nuovo OPAC del Servizio Bibliotecario Nazionale (SBN).............................................................. 471

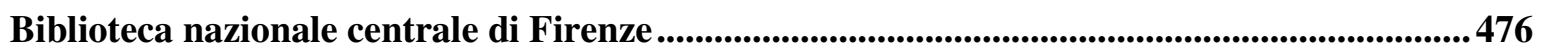

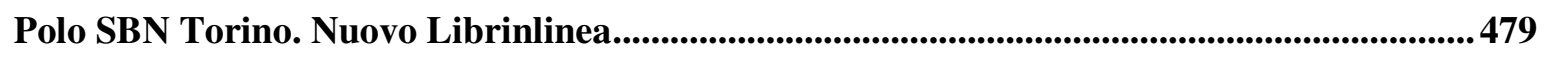

Sistema bibliotecario della Provincia di Verona...................................................................485

Sistema bibliotecario provinciale di Foggia .................................................................................................. 491

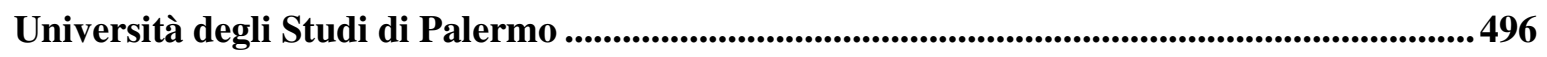

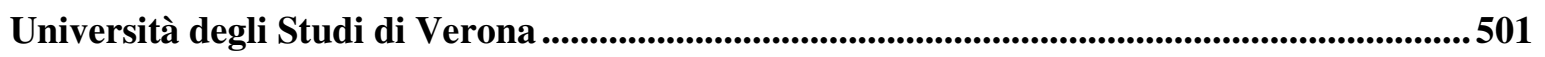

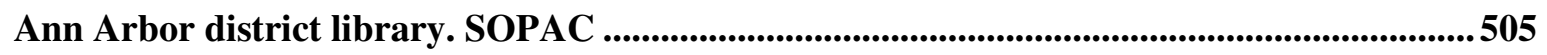

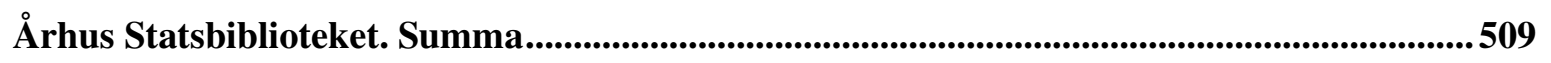

Université d'Artois. Visual Catalog ..............................................................................5514

Svenska forskningsbibliotekens gemensamma katalog. OPAC Libris .....................................519

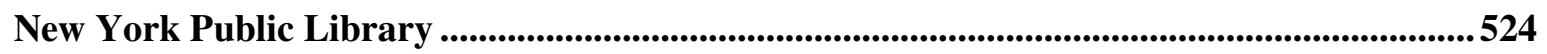

North Carolina State University. Endeca e Summon...............................................................529

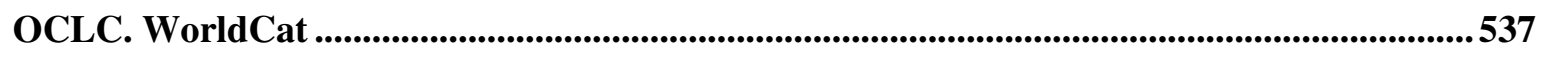

Harvard University. OPAC Hollis ................................................................................544

The Hong Kong University of Science and Technology library................................................549

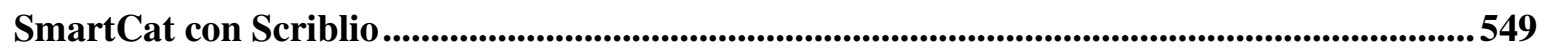

Universität Hamburg. Beluga.................................................................................................553

University of California Libraries. Melvyl................................................................................558

University of Virginia Library. Blacklite ..........................................................................562

Villanova University .........................................................................................................................569

APPENDICE B. SOFTWARE PER OPAC DI NUOVA GENERAZIONE E

SERVIZI DI WEB SCALE DISCOVERY .........................................................575

APPENDICE C. PROGETTI DI IMPLEMENTAZIONE DI FRBR NEGLI OPAC.

SISTEMI COMPLETI, PROTOTIPI, SOFTWARE ...................................................601 


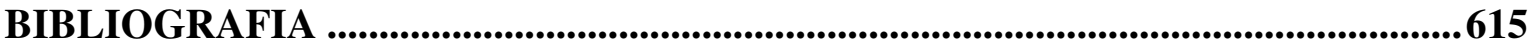





\section{RINGRAZIAMENTI}

I miei ringraziamenti vanno innanzitutto a Maria Teresa Biagetti e Gianfranco Crupi, che hanno seguito con costanza e dedizione il mio lavoro di ricerca in questi tre lunghi anni, guidandomi e sostenendomi con passione in questo cammino di crescita. Ringrazio inoltre il coordinatore e tutti i membri del Collegio di Dottorato per averci seguiti e incoraggiati fornendo spunti di riflessione sempre interessanti e stimolanti. Un doveroso riconoscimento va anche a tutti i docenti che nel corso delle lezioni hanno portato la loro esperienza e messo a disposizione di noi dottorandi la loro maturità professionale e umana.

Desidero ringraziare inoltre i miei cari amici dottorandi che hanno allietato questo percorso alternando ai proficui momenti di scambio culturale momenti di aggregazione sociale sinceri, divertenti e spensierati.

Vorrei ringraziare poi le tante persone che, a vario titolo, hanno contribuito alla realizzazione di questo lavoro e tutti gli amici, vicini e lontani, che in questi anni mi hanno sopportato. Un grazie e un tributo d'affetto, infine, alla mia famiglia e a Stefania per essermi stati vicini ed avermi aiutato costantemente, giorno per giorno, a superare le difficoltà di questo percorso. Dedico questo lavoro alla memoria di mia madre, di Davide e Sara e ai bei momenti trascorsi insieme. 



\section{INTRODUZIONE}

\begin{abstract}
«Nel lungo termine, le esperienze ottimali si sintetizzano in un senso di controllo, o meglio di partecipazione nella determinazione di ciò che si fa della propria vita: ed è questo tipo di esperienza che noi siamo in grado di immaginare pensando al concetto di felicità».
\end{abstract}

Mihaly Csikszentmihalyi

Oggetto di studio, presupposti e obiettivi della ricerca.

Nella più ampia riflessione sull'identità e i confini delle discipline bibliografiche e biblioteconomiche, quella sul catalogo come strumento attraverso il quale le biblioteche organizzano la conoscenza registrata nei documenti ai fini del suo recupero assume un significato fondante: il catalogo rappresenta, insieme ai servizi e agli spazi della biblioteca, il 'linguaggio' attraverso il quale, da secoli, essa svolge la primaria funzione della mediazione informativa.

Le tematiche del miglioramento e dell'evoluzione dei cataloghi elettronici nell'ultimo decennio hanno acquisito un rinnovato vigore nel dibattito biblioteconomico in relazione alla comparsa di quelli che vengono comunemente definiti 'OPAC di nuova generazione' e di 'sistemi per la scoperta', che oggi sostituiscono i cataloghi tradizionali o li affiancano, offrendo contenuti arricchiti e l'accesso a svariate risorse esterne.

L'evoluzione del catalogo elettronico in questa direzione si può considerare l'esito di un lungo dibattito che ha più volte rimarcato lo stato di 'crisi' del catalogo tradizionale di fronte ai grandi mutamenti che investono le forme di creazione, trasmissione e uso dell'informazione, dovuti alle profonde trasformazioni sociali, ad un mutato contesto tecnologico e ad un ambiente informativo sempre più complesso.

All'origine del ripensamento che ha condotto la biblioteconomia ad interrogarsi sulla 'tenuta' e la maturità del catalogo elettronico tradizionale vi sono due elementi di rilievo: la primaria esigenza di adeguare i cataloghi al ripensamento complessivo della teoria catalografica che si è espresso in un decennio ricco di iniziative di rilievo mondiale (che dalla pubblicazione di FRBR ha condotto alla riscrittura dei principi internazionali della catalogazione, della normativa angloamericana e italiana, fino agli importanti sviluppi sul versante del controllo bibliografico) e la necessità di rendere gli OPAC più vicini alle esigenze e ai desideri degli utenti e pienamente interoperabili con il web. 
Non si può non rilevare che in tale processo vi sia stata, infatti, la messa in discussione dei cataloghi elettronici in primis quali strumenti di comunicazione con gli utenti, capaci di rispondere ai loro bisogni informativi, alle loro aspettative e ai loro nuovi modi di approcciarsi all'informazione.

L'uso sempre più massiccio della rete ha imposto nuovi modelli di ricerca e di servizio che hanno cambiato radicalmente l'approccio dell'utenza con l'informazione, provocando una progressiva perdita di interesse per gli strumenti predisposti dalle biblioteche in favore di quelli apparentemente più duttili e amichevoli presenti nel web.

L'ingresso in campo di nuove tecnologie, come quelle del 'Web 2.0' e, oggi, dei linked data impongono al catalogo nuove trasformazioni e nuove sfide che necessitano di una riflessione, affinché gli OPAC nel loro processo evolutivo non perdano la coerenza e l'autorevolezza che da sempre li hanno contraddistinti.

La comparsa del modello dei linked data, nuovo modo di strutturare i dati bibliografici per integrarli nel Web Semantico, rappresenta una grande opportunità per le biblioteche di rendere i loro dati interoperabili con gli altri dati presenti nel web ed è in grado di modificare ulteriormente il modello attuale introducendo nel processo evolutivo dell'OPAC nuove e significative variabili. Il catalogo atomizzato e decostruito che la logica dei linked data richiede conserverà la sua identità e le sue caratteristiche, oppure subentreranno nuove modalità di ricerca dell'informazione? In definitiva, se l'utente potrà raggiungere un record catalografico tramite un motore di ricerca o altri servizi che il Web Semantico consentirà di sviluppare, vi sarà ancora spazio per il catalogo elettronico?

Di fronte ad uno scenario di grande cambiamento, ove è in atto una profonda trasformazione del più autorevole degli strumenti bibliotecari, la presente ricerca si è posta l'obiettivo di offrire una riflessione sulla natura, sull'evoluzione e sul futuro dei cataloghi elettronici.

La finalità principale della ricerca è, dunque, prima di tutto quella di approfondire e delineare le linee evolutive dei cataloghi elettronici, indagando il modello attuale di sviluppo rappresentato dagli OPAC 'di nuova generazione' e dai 'sistemi per la scoperta', rilevandone elementi innovativi e criticità e di proporre, infine, un modello per lo sviluppo dell'OPAC che si ponga come alternativa al modello corrente.

Le grandi trasformazioni dell'era digitale impongono una riflessione che si interroghi sull'identità e sulle funzioni primarie del catalogo elettronico, ossia su quegli elementi fondanti, su quelle qualità peculiari che lo rendono riconoscibile e lo distinguono dagli altri strumenti di recupero dell'informazione disponibili nel web. 
La ricerca si è posta, dunque, l'ambizioso obiettivo di offrire un nuovo modello di sviluppo per l'OPAC 'a supporto della ricerca dell'informazione' che sia fondato su un'analisi dei modelli comportamentali della ricerca informativa e centrato su un'analisi approfondita del processo di ricerca che si svolge nell'interazione con l'OPAC.

Il modello proposto nella ricerca delinea una linea di sviluppo che consente di mantenere un'identità 'forte' del catalogo, concentrandosi su una progettazione dell'OPAC che si basi su modelli nuovi, verso il superamento della logica del motore di ricerca, della rilevanza algoritmica e che possa fondarsi invece sull'utente, sui suoi bisogni informativi, i suoi comportamenti e sull'analisi delle componenti che entrano in gioco nel processo di ricerca dell'informazione. In questa prospettiva, 'cercare' l'informazione bibliografica vuol dire prima di tutto 'costruire' un percorso di ricerca e 'comprendere' l'informazione recuperata.

\section{Approccio concettuale}

L'approccio concettuale con il quale ho affrontato il lavoro di ricerca segue il metodo filosofico 'interpretativista', ormai consolidato specie in area anglosassone, che è centrato sull'utente, sui suoi bisogni informativi (information behaviour), sulle modalità e le procedure che gli utenti utilizzano nella ricerca dell'informazione (information seeking behaviour). Tale prospettiva teoretica ${ }^{1}$, basata su un'ottica costruttivista e cognitiva, si riflette nella presente ricerca finalizzata a esaminare il contesto nel quale si svolge la ricerca dell'informazione nel catalogo elettronico e nella proposta di un modello di OPAC nel quale riveste un ruolo centrale l'analisi del processo informativo in cui l'utente è coinvolto.

È in questa direzione che la presente ricerca accoglie un'interpretazione relativista e costruttivista della conoscenza che avviene nell'interazione con il catalogo, adottando

\footnotetext{
${ }^{1}$ Questa metodologia di ricerca si contrappone alla ricerca 'quantitativa', basata su un approccio filosofico positivista ai problemi della ricerca, che in passato ha condizionato la maggior parte degli studi biblioteconomici e che si è espressa nella bibliometria e negli studi quantitativi sull'utenza, quali le statistiche d'uso dei servizi bibliotecari. A differenza dell'approccio metodologico quantitativo, che rimane oggi pur sempre valido, la ricerca qualitativa e interpretativista si basa sull'assunto che non c'è mai una verità assoluta al di fuori dell'esperienza e del contesto nel quale si svolge l'esperienza stessa. Questo approccio tende ad indagare l'oggetto di ricerca in maniera olistica, ad interpretare i significati, i valori e l'esperienza per i membri di una comunità. Dunque una ricerca di questo tipo è essenzialmente descrittiva ed indirizzata a porre domande facendo emergere la teoria dal contesto, attraverso la metodologia della grounded theory, che non permette dunque formulazioni a priori o verificabili per via deduttiva o tramite l'adduzione. Si veda: Alison Pickard, La ricerca in biblioteca. Come migliorare $i$ servizi attraverso gli studi sull'utenza, introduzione, traduzione e cura di Elena Corradini; prefazione di Anna Maria Tammaro, Milano, Editrice Bibliografica, 2010, p. 13-35 e 53-79.
} 
come cornice teorica le principali metateorie della ricerca informativa e i principali modelli elaborati nel campo di studi dell' information behavior e dell' information seeking behavior e che si consolida nella proposta di un nuovo modello di OPAC mirato non solo al recupero dell'informazione, ma alla creazione di conoscenza.

La futura sopravvivenza del catalogo elettronico risiede dunque nella sua capacità di trasformarsi da strumento di recupero dell'informazione a strumento in grado di facilitare la creazione di conoscenza dell'universo bibliografico in esso rappresentato.

Per far ciò è necessario un ripensamento radicale del modello di sviluppo attuale, centrato sui meccanismi del matching e sulla rilevanza algoritmica e topica per concentrarsi sul processo di ricerca e sull'interazione che si svolge all'interno del catalogo e su una rinnovata struttura del record bibliografico che, collegando i dati del catalogo agli altri dati presenti nel web, sia in grado di sostenere la creazione di conoscenza.

\section{Tratti caratterizzanti e chiavi di lettura della ricerca}

Stiamo vivendo un passaggio d'epoca che si manifesta in una nuova rivoluzione scientifica, quella dell'informazione che, come le altre 'rivoluzioni', cambiano la percezione del mondo; la rivoluzione dell'informazione si può considerare la quarta dopo quelle copernicana, darwiniana e freudiana:

Tre rivoluzioni scientifiche hanno avuto un forte effetto da entrambi i punti di vista: nel modificare la nostra comprensione del mondo esterno, hanno mutato anche la concezione di chi siamo. Dopo Niccolò Copernico la cosmologia eliocentrica ha rimosso la Terra e quindi l'umanità dal centro dell'universo. Charles Darwin ha mostrato come ogni forma di vita si è evoluta nel tempo da progenitori comuni per mezzo della selezione naturale, rimuovendo in tal modo l'umanità dal centro del regno biologico. E, grazie a Sigmund Freud, riconosciamo oggi che la mente umana è anche inconscia e soggetta al meccanismo di difesa della repressione. Cosicché non siamo immobili, al centro dell'universo (la rivoluzione copernicana), non siamo innaturalmente separati e diversi dal resto del regno animale (la rivoluzione darwiniana), e siamo molto lontani dall'essere menti isolate interamente trasparenti a se stesse, come Cartesio, ad esempio, aveva ipotizzato (la rivoluzione freudiana) ${ }^{2}$.

La rivoluzione dell'informazione sta modificando non solo il nostro modo di interagire con il mondo, ma anche la comprensione di noi stessi: non siamo entità isolate, ma «organismi informazionali interconnessi, o inforg, che condividono con agenti

\footnotetext{
${ }^{2}$ Luciano Floridi, La rivoluzione dell'informazione, traduzione di Massimo Durante ; prefazione di Juan Carlos De Martin, Torino, Codice, 2012, p. 10.
} 
biologici e artefatti ingegnerizzati un ambiente globale costituito in ultima analisi dalle informazioni, l'infosfera» ${ }^{3}$.

L'impetuoso avvento delle tecnologie digitali testimonia l'ingresso in una nuova fase storica caratterizzata da una società complessa e instabile, che si esprime nella saturazione e nella pervasività dei media, nel sovraccarico informativo (information overload), cui si contrappone una povertà informativa senza precedenti e nei tratti dell'iperattività, transitorietà e incertezza che sono tipici dell'era postmoderna.

Una prima chiave di lettura della presente ricerca può, dunque, essere individuata nella natura storicamente determinata del catalogo, attraverso il quale la biblioteca conferisce una sistemazione culturale al sapere e nei cambiamenti che oggi investono le principali componenti dell'accesso bibliografico: l'utenza, ormai sempre più spesso remota e sconosciuta, le tipologie documentarie che esso è deputato a rappresentare, le modalità di fruizione dell'informazione, condizionate dalle nuove forme d'interazione che si manifestano nella rete e dagli strumenti di ricerca del web.

Il primo passo della ricerca è stato quindi quello di verificare, di fronte ai grandi mutamenti che contraddistinguono la nostra epoca, la maturità del catalogo elettronico e la coerenza del suo processo evolutivo; è stata compiuta dunque un'indagine approfondita del modello attuale di sviluppo e dei principali aspetti evolutivi che lo caratterizzano, delle urgenze e delle visioni che hanno condotto nell'ultimo decennio alla progettazione di OPAC di nuova generazione e discovery systems. L'indagine si è avvalsa dell'esame diretto di reali applicazioni o casi di studio (raccolti durante il primo anno di lavoro e allegati in appendice alla tesi) che ha consentito di rilevare i principali elementi di novità e di evidenziarne le criticità.

Sono state affrontate due tematiche giudicate particolarmente rilevanti per il compimento di un salto qualitativo nella progettazione degli OPAC: le problematiche dell'applicazione al catalogo del modello FRBR e le nuove opportunità che provengono dalla tecnologia emergente dei linked data, che offre una nuova modalità di pubblicare nel Web Semantico i dati autorevoli prodotti dalle biblioteche, consentendo una profonda ristrutturazione dei record bibliografici e la loro integrazione con gli altri dati del web.

Una seconda chiave di lettura della trattazione, non meno importante, è la centralità dell'utente attorno alle cui esigenze si costruiscono i servizi e gli strumenti bibliotecari; un'idea che, sebbene presente fin dagli esordi della disciplina, acquista un rinnovato vigore

\footnotetext{
${ }^{3}$ Ivi, p. 11.
} 
nei nuovi esiti della teoria catalografica e nelle nuove forme della valutazione qualitativa dei servizi bibliotecari.

Dalla constatazione della necessità di un rinnovamento del paradigma che sottende l'attuale progettazione dei cataloghi elettronici nasce la presente ricerca, il cui punto di arrivo risiede nell'elaborazione di un nuovo modello di sviluppo per l'OPAC.

Nella proposta del nuovo modello, l'attenzione è rivolta in primo luogo verso l'utente e i suoi comportamenti di ricerca, oggi influenzati dalle nuove tecnologie digitali.

Il quadro teorico che ha fornito la base per l'elaborazione del modello è costituito dagli studi sul comportamento informativo e sulla ricerca dell'informazione o 'metateorie socioculturali dell'informazione' elaborate nell'ambito della Scienza dell'Informazione a partire dagli anni '80, ovvero nel periodo in cui queste teorizzazioni cominciarono ad imprimere una nuova svolta negli studi della Library and Information science.

La centralità dell'utente alla quale si ispira la proposta non risiede nell'accettazione 'acritica' di funzionalità e tendenze provenienti dal web; infatti il funzionamento di un sistema di recupero dell'informazione non dipende dal gradimento o dal giudizio dell'utente, ma da un'adeguata progettazione del catalogo che deriva da un 'fondamento intellettuale di organizzazione dell'informazione' che sia riconoscibile e fondato sul raggiungimento degli obiettivi bibliografici ${ }^{4}$.

Tuttavia si avverte la necessità di dotare i cataloghi di nuove funzionalità che consentano agli utenti di svolgere al meglio il proprio processo informativo; per questo nel modello proposto l'attenzione è focalizzata sull'utente, sulle abitudini di ricerca, sul processo che svolge all'interno del catalogo, sulle motivazioni che attivano o ostacolano tale processo e il recupero dell'informazione bibliografica.

Nel corso del processo di ricerca la conoscenza dell'utente cambia grazie all'interazione con il catalogo; l'utente dunque non dovrebbe solo recuperare informazioni, ma acquisire la conoscenza dell'universo bibliografico in esso rappresentato e il catalogo dovrebbe aiutare l'utente in tutte le fasi della sua ricerca informativa. La nuova tecnologia dei linked data e il nuovo record de-costruito e 'collegato' agli altri dati sul web potrebbero essere la chiave di volta per un cambiamento del catalogo rivolto alla creazione di conoscenza.

\footnotetext{
${ }^{4}$ Cfr. Elaine Svenonius, Il fondamento intellettuale dell'organizzazione dell'informazione, traduzione di Maria Letizia Fabbrini; introduzione di Mauro Guerrini, Firenze, Le lettere, 2008.
} 
Al fine di strutturare organicamente i contenuti del lavoro la trattazione è stata articolata in cinque capitoli.

Il capitolo 1. si concentra sui presupposti che hanno determinato la recente evoluzione dei cataloghi elettronici, sulla natura e sulle funzioni del catalogo, riassume il dibattito internazionale sulle prospettive di miglioramento, indaga le recenti politiche bibliotecarie e, infine, traccia alcune riflessioni sulla fisionomia degli utenti giudicate utili a comprendere più a fondo le modalità di uso dei cataloghi elettronici.

Il catalogo riflette nella sua organicità e nelle sue funzioni il ruolo che, sin dai tempi più lontani, la biblioteca si è data all'interno della società come luogo dell'organizzazione, della selezione, della conservazione e preservazione della conoscenza registrata, ai fini della ricerca dell'informazione e del suo recupero per mezzo dei connotati semiotici e semantici dei documenti.

Il ripensamento delle modalità di fornire l'informazione bibliografica attraverso il catalogo, che ha condotto alle sue più recenti evoluzioni, deriva da un lungo dibattito all'interno della comunità bibliotecaria internazionale che ha più volte sottolineato la condizione di 'crisi' del catalogo tradizionale di fronte ai modelli imposti dai più popolari strumenti di ricerca del web.

Le riflessioni sulla natura, sulle funzioni e sulla futura sopravvivenza del catalogo come strumento qualificato di recupero dell'informazione non possono dunque essere scisse dalla più ampia riflessione epistemologica che investe natura, compiti e confini della Biblioteconomia del ventunesimo secolo, dal ripensamento del ruolo delle biblioteche nella società contemporanea, dalle problematiche della convivenza della biblioteca con la crisi dei modelli di socializzazione e dei nuovi canoni della comunicazione imposti dalle tecnologie digitali; un dibattito che in ultima analisi viene ricondotto alla frattura tra biblioteca e società.

La metafora ancor oggi pregnante del catalogo, come 'linguaggio' attraverso il quale la biblioteca esprime la sua funzione culturale di organizzare e sistematizzare il sapere, coinvolge una serie di aspetti che vengono ricondotti alla sua natura sistemica (il catalogo è un sistema necessariamente contingente per ordinare una raccolta di documenti ai fini del recupero) ${ }^{5}$ e a varie complicazioni come quella linguistica (i codici comunicativi della

\footnotetext{
${ }^{5}$ Elaine Svenonius cita tra gli antefatti filosofici rilevanti per il fondamento intellettuale dell'organizzazione dell'informazione la filosofia dei sistemi, introdotta nella biblioteconomia da Charles Ammi Cutter nella distinzione tra obiettivi, mezzi e principi per guidare la scelta dei mezzi, la filosofia della scienza, che ha dato
} 
biblioteca possono non essere compresi dall'utente), che si verifica nell'utilizzo degli indici per il recupero dell'informazione. È, dunque, necessario chiedersi, di fronte alle nuove modalità di creazione, ricerca e fruizione dell'informazione, in che modo riprogettare la comunicazione con l'utente, affinché il processo di ricerca che conduce al recupero dell'informazione catalografica venga svolto nella maniera più efficace.

Le biblioteche vivono, infatti, un momento di profonda trasformazione che le obbliga a riposizionarsi in un contesto informativo articolato e complesso e in tale compito gli OPAC rappresentano un punto importante di accesso al loro patrimonio documentario.

Non c'è dubbio che il catalogo sia ancora oggi strumento necessario e autorevole di recupero dell'informazione; tuttavia si avverte la profonda distanza del linguaggio del catalogo rispetto alle nuove aspettative dell'utente (che tende a trasferire i modelli mentali acquisiti nelle sue esperienze di ricerca nel web alla ricerca catalografica) e, in ultima analisi, rispetto ai 'nuovi linguaggi' che il web impone.

Questi interrogativi introducono la riflessione su quelle spinte 'esterne' che condizionano l'evoluzione del catalogo, cioè portano a considerare i condizionamenti della società contemporanea e l'impatto che le nuove tecnologie stanno avendo sulla vita umana, sui comportamenti sociali e sulle abitudini di ricerca degli utenti, indagando la fisionomia dell'utente 'postmoderno' e dei nuovi 'nativi digitali'.

Tali condizionamenti vengono rilevati nelle nuove modalità che l'utente manifesta nella fruizione del catalogo elettronico nei termini di 'consumo' e nei nuovi modi di interazione e uso dell'informazione fortemente condizionati dalle tecnologie digitali.

Segue dunque una panoramica delle iniziative recenti delle biblioteche nel campo del miglioramento degli OPAC, che si sono risolte in politiche catalografiche spesso controverse.

In chiusura un paragrafo è dedicato alle indagini che sono state compiute in ambito bibliotecario e hanno costituito la base per interpretare il rinnovamento dei cataloghi elettronici, culminato nel modello attuale che i nuovi software propongono e che viene analizzato nel capitolo successivo.

Nel capitolo 2. viene approfondito il modello attuale di OPAC derivante dai più recenti sviluppi che hanno evidenziato l'evoluzione dei cataloghi elettronici in OPAC 'di nuova generazione' (o next generation catalogs) e, successivamente, in quelli che sono più

origine alle teorie dell'information retrieval e della biliometria, e la filosofia del linguaggio che consente di introdurre e usare nell'organizzazione delle conoscenze, concetti come vocabolario, semantica e sintassi (ivi, p. 6-9). 
comunemente chiamati 'sistemi per la scoperta' (discovery systems, discovery tools). Il capitolo illustra la nascita e lo sviluppo dei next generation catalogs e degli strumenti di discovery, indagando i modelli che ne hanno determinato la nascita e le principali caratteristiche che li distinguono dai cataloghi tradizionali, che vengono illustrate in termini di dati, modalità di ricerca e di visualizzazione, servizi all'utenza, sistemi e tecnologie. Ci si interroga, infine, sul modello che queste nuove generazioni di cataloghi presentano in riferimento ai dati, al recupero semantico e alle funzionalità offerte, rilevando che la presenza di nuovi standard e norme catalografiche richiedono modelli sostenibili di sviluppo che devono orientarsi verso l'apertura, l'interoperabilità e una rinnovata progettazione del processo di ricerca.

Il quadro dello sviluppo degli OPAC delineato ha evidenziato nodi di riflessione ancora aperti:

* i cataloghi elettronici sono ancora lontani da un'adeguata rappresentazione delle entità che compongono l'universo bibliografico, poiché non si interviene sulla struttura dei record catalografici che vengono codificati in un formato non più adatto a realizzare la ricchezza e l'espressività offerta dal modello FRBR;

* i cataloghi si avvalgono di tecnologie non adatte all'apertura dei dati e all'interoperabilità. I dati sono costretti entro griglie di rappresentazione che favoriscono lo scambio esclusivamente all'interno della comunità bibliotecaria e non si integrano con il più ampio spazio delle risorse presenti sul web;

* la progettazione dei cataloghi ha imboccato la strada dell'imitazione del processo di ricerca tipico dell'ambiente web senza un'adeguata progettazione dei processi di conoscenza caratterizzanti la ricerca nel catalogo elettronico.

Ciò che si avverte è la distanza e l'arretratezza di questi strumenti rispetto all'evoluzione delle tecnologie attuali del web, dei modi di produzione, fruizione e scambio dell'informazione che oggi attraversano un profondo cambiamento. Mentre il web si avvia ad un mutamento che rivoluzionerà l'accesso ai dati, lo sviluppo dei cataloghi elettronici ha seguito vie diverse che hanno isolato le biblioteche e i dati prodotti e immagazzinati nei cataloghi elettronici dal resto del web.

La necessità di preservare la coerenza e l'autorevolezza del catalogo elettronico e di garantirne in definitiva la sua sopravvivenza dipendono da un'adeguata riprogettazione del catalogo che preveda un ripensamento dei modelli funzionali che oggi ispirano i next generation catalogs e gli strumenti di discovery verso: 
una rinnovata ed efficace rappresentazione al suo interno delle entità che compongono l'universo bibliografico e delle loro relazioni attraverso l'implementazione dei modelli elaborati dall'IFLA;

* l'apertura e il collegamento dei 'dati catalografici' agli altri dati presenti nel web, attraverso l'adozione di standard aperti e interoperabili e le emergenti tecnologie del Web Semantico come i linked data;

* un'adeguata riprogettazione del processo di ricerca nell'OPAC, che tenga conto di come gli utenti cercano l'informazione bibliografica, che sia capace di creare flussi di conoscenza all'interno del catalogo e, infine, di collegarsi con il più ampio spazio delle risorse informative presenti nel web.

Tali riflessioni hanno costituito le premesse per l'approfondimento degli argomenti che vengono affrontati nei capitoli seguenti.

Il capitolo 3. è dedicato all'approfondimento della tematica dell'applicazione del modello FRBR ai cataloghi elettronici e propone una riflessione sulle motivazioni della sua mancata applicazione. Il modello attuale è infatti basato su una progettazione del catalogo che favorisce la funzione del 'reperimento' a discapito di quella del 'raggruppamento' bibliografico delle entità, certamente più sofisticata, ma anche più difficile da ottenere per via algoritmica.

L'implementazione di FRBR ai cataloghi elettronici, infatti, ad oggi, non ha ancora portato a risultati apprezzabili; il modello è stato implementato in strumenti diversi (OPAC, banche dati, biblioteche digitali) a diversi livelli di completezza senza intervenire sulla struttura del record bibliografico, ma servendosi di algoritmi per lo schiacciamento di record e la loro visualizzazione per lo più in base all'entità opera.

Il capitolo ripercorre la nascita e gli sviluppi della 'famiglia' dei modelli FRBR ${ }^{6}$, il rinnovamento di principi e regole catalografiche e le altre iniziative che hanno avuto origine dallo studio dell' IFLA, indagando le problematiche di applicazione del modello a diverse tipologie documentarie e di implementazione nei cataloghi elettronici e fornendo una panoramica dei principali progetti avviati.

Nel capitolo, infine, si approfondiscono i motivi della mancata applicazione del modello FRBR ai discovery systems, ove confluiscono dati di diversa omogeneità; in questi

\footnotetext{
${ }^{6} \mathrm{Ci}$ si riferisce ai modelli che sono stati sviluppati a partire da FRBR: Functional requirements for authority data (FRAD) pubblicato nel 2009 e Functional Requirements for Subject Authority Data (FRSAD) pubblicato nel 2010.
} 
strumenti l'applicazione del relevance ranking per la visualizzazione dei risultati della ricerca dimostra tutta la sua intrinseca debolezza, mentre l'applicazione di FRBR manifesterebbe invece una straordinaria solidità.

Il capitolo 4. affronta uno degli argomenti maggiormente significativi del più recente dibattito biblioteconomico: il tema dell'interoperabilità e dell'apertura dei dati bibliografici.

I linked data rappresentano una tecnologia emergente del Web Semantico per rendere disponibili i dati nel web e una nuova opportunità per le biblioteche di produrre dati accessibili in rete, interoperabili e riutilizzabili, ma soprattutto costituiscono un nuovo linguaggio per la comunicazione globale che comporterà una modifica radicale nella creazione degli strumenti di mediazione tra universo bibliografico e utente.

Il capitolo introduce la nuova tecnologia dei linked data, illustra il data model RDF, il ruolo dei vocabolari e delle ontologie e la composizione del 'Web dei dati'.

I paragrafi successivi illustrano le modalità con cui i dati delle biblioteche possono divenire parte del web dei dati; affinché ciò avvenga, è necessario un radicale ripensamento del modo di offrire i dati bibliografici e una ristrutturazione del record bibliografico.

Vengono poi esposte le iniziative delle biblioteche nel campo del Web Semantico, affrontando le tematiche della pubblicazione di dati sotto forma di linked data, operazione che richiede notevoli sforzi alle biblioteche; sono illustrate le fasi del procedimento di pubblicazione e si affrontano le questioni riguardanti la scelta delle tecnologie più appropriate e delle licenze che regolano l'accesso e il libero riutilizzo dei dati nel web.

Nel capitolo ha un ruolo centrale l'impatto della tecnologia dei linked data sul cataloghi elettronici: l'adozione dei linked data comporterà una modifica radicale nella creazione dei record bibliografici composti di aggregazioni di dati provenienti da diverse fonti. La tecnologia dei linked data rappresenta una grande opportunità di cambiamento; offre infatti la possibilità di produrre dati aperti, interoperabili e riutilizzabili nel web; i dati autorevoli prodotti dalle biblioteche potranno essere riutilizzabili e liberamente fruibili nel web, cioè nell'ambiente oggi maggiormente frequentato e conosciuto dagli utenti con enormi vantaggi nella creazione, nella ricerca e nell'utilizzo dei dati bibliografici.

Gli ultimi paragrafi sono dedicati alla riflessione su due tematiche che interessano da vicino gli OPAC e che le tecnologie dei linked data consentono di sviluppare: le nuove 
possibilità della ricerca semantica e un'adeguata rappresentazione dell'universo bibliografico nei cataloghi elettronici.

Il capitolo 5. presenta l'elaborazione teorica di un nuovo modello di OPAC che viene proposto come alternativa al modello di sviluppo attuale.

Dal quadro emerso nei capitoli precedenti deriva la necessità di una revisione del paradigma corrente di sviluppo e la necessità di una progettazione dell'OPAC che si orienti verso il superamento della logica proposta dai motori di ricerca basata della rilevanza 'presunta' ottenuta per via algoritmica, e che ponga al centro dell' analisi l'utente e il suo comportamento informativo.

In questa prospettiva la ricerca dell'informazione bibliografica deve offrire la possibilità di costruire un percorso di ricerca e di comprendere l'informazione che viene recuperata tramite un' organizzazione intelligente dell' ambiente di ricerca.

Il capitolo espone in apertura le contraddizioni che caratterizzano il modello corrente, basato sul binomio relevance ranking/navigazione 'a faccette' come modalità di visualizzazione dei risultati e di navigazione nel catalogo, introducendo gli studi che nel campo della Scienza dell'informazione hanno indagato le molteplici dimensioni della rilevanza, che vanno ben oltre la rilevanza algoritmica e verso modalità di rilevanza 'soggettive'.

Successivamente si illustrano diversi modelli di ricerca ${ }^{7}$ che sono ricondotti all'interno delle due metateorie dell'informazione prima citate: quella costruttivista e quella cognitiva ${ }^{8}$ e che costituiscono il quadro teorico in base al quale è possibile proporre un modello di OPAC 'a supporto della ricerca dell'informazione' che ci consenta di osservare e rappresentare:

il contesto nel quale si svolge l'interazione nel catalogo elettronico;

* le diverse componenti coinvolte nel processo di ricerca e di uso dell'informazione bibliografica;

* il tipo di interazione tra le diverse componenti coinvolte;

\footnotetext{
${ }^{7}$ Vengono esposti i più noti modelli che si inseriscono in due principali metateorie: quella costruttivista e quella cognitiva che hanno tra gli esponenti di spicco Nicholas Belkin, Brenda Dervin, Marcia Bates, Carol Collier Kuhlthau e Peter Ingwersen; in particolare vengono esaminate due teorie generali del comportamento informativo (modelli di Wilson e Dervin); due modelli descrittivi della ricerca dell'informazione (Kuhlthau ed Ellis), il noto modello dinamico del berrypicking di Bates e il modello 'cognitivo' di Ingwersen.

${ }^{8}$ Il modello che si propone si inserisce entro le due principali linee teoriche sopra citate: quella costruttivista con particolare riferimento al modello di Kuhlthau e quella cognitiva, con riferimento alla teoria di Ingwersen. Le due teorie costituiscono l'orizzonte teorico del modello proposto: la prima induce a concepire la ricerca come una fase di costruzione, la seconda è utile a rappresentare le diverse componenti che interagiscono nel processo informativo che si svolge nell'OPAC.
} 
* le barriere e le variabili che si presentano all'utente nella fase di attivazione del processo di ricerca dell'informazione e che si frappongono nelle diverse fasi del processo informativo;

* le attività svolte, cioè il comportamento informativo (information seeking behavior) che l'utente manifesta nel processo di ricerca.

Nel modello di sviluppo proposto ho immaginato che l'utente sia coinvolto in un processo informativo che si svolge iterativamente attraverso tre fasi distinte (pre-focus, focus, post-focus) nelle quali l'utente è attivamente coinvolto in una situazione di problem solving che lo induce a svolgere varie attività che sono rivolte e vengono condizionate da tutte le componenti che entrano in gioco nel processo di ricerca: l'utente stesso, il sistema, i documenti e gli intermediari.

In base a questa analisi il modello individua così le funzionalità che gli OPAC dovrebbero attivare per sostenere tutte le fasi del processo e garantire che si compia una ricerca efficace e senza intoppi.

L'analisi mette in evidenza come l'attivazione del processo di ricerca non sia scontata, in quanto intervengono una serie di barriere che derivano dallo stato in cui l'utente si trova e nel quale sorge e si sviluppa il suo bisogno informativo. Tali barriere condizionano non solo i meccanismi di attivazione, ma sono presenti durante tutto il processo, poiché l'utente è iterativamente coinvolto nella comprensione e nel perfezionamento della sua ricerca; egli dunque modifica continuamente il suo bisogno, in quanto man mano che procede, costruisce e comprende l'oggetto della sua ricerca, modificando il suo stato cognitivo, affettivo e situazionale di partenza'

Questa visione 'costruttivista' e 'cognitiva' sulla quale si basa il modello elaborato nel presente lavoro ci consente di intendere il processo di ricerca come un processo di ‘creazione di senso' da parte dell'utente ovvero di comprensione dell'informazione nel suo contesto.

Ciò avvicina molto la visione che sottende il modello di OPAC da me proposto a quella recentemente prefigurata da Fattahi, laddove lo studioso afferma che il catalogo dovrebbe essere strutturato come un «sistema cognitivo integrato» nel quale l'utente è in grado di comprendere come viene rappresentata e strutturata la conoscenza e ne auspica il passaggio «da sistemi orientati al documento ai sistemi orientati alla conoscenza» ${ }^{10}$.

\footnotetext{
9 Il capitolo esamina alcuni concetti chiave della ricerca dell'informazione come quelli di bisogno informativo, rilevanza, pertinenza, browsing, finding e serendipità.

${ }^{10}$ Rahmatollah Fattahi, From information to knowledge: superworks and the challenges in the organization and representation of the bibliographic universe = Dall'informazione alla conoscenza: le super-opere e le sfide dell'organizzazione e rappresentazione dell'universo bibliografico: Lectio magistralis in
} 
I linked data rappresentano una nuova tecnologia in grado di fornire alle biblioteche un nuovo modo di strutturare i dati bibliografici rappresentati negli OPAC e negli altri strumenti di ricerca e servizi che vengono da esse predisposti.

Nei paragrafi conclusivi del capitolo si esplora la possibilità che proprio i linked data possano essere la tecnologia più appropriata per la costruzione di nuovi OPAC basati sulla creazione di conoscenza all'interno del processo informativo. In particolare, si esamina il ruolo che l'esposizione dei dati bibliografici sotto forma di linked data potrà avere nello sviluppo degli OPAC in relazione al modello da me proposto.

I linked data ci offrono oggi la possibilità di operare una profonda ristrutturazione o de-costruzione del record bibliografico nei 'dati' che lo costituiscono ovvero in una nuova struttura granulare e atomizzata; tale nuovo concetto di record bibliografico si esprime nel 'record collegato', costituito virtualmente dalla sintesi di varie triple che combinano dati creati dalle biblioteche e da altri enti.

Attraverso questa rinnovata struttura 'collegata', il nuovo record bibliografico potrà consentire all'utente di compiere non solo tutte le funzioni di base di un catalogo, ma anche un buon numero di altre funzioni individuate nel modello da me proposto.

Con la tecnologia dei linked data è, infatti, possibile generare automaticamente una struttura del record formato da tutte le entità previste dal modello FRBR, ma è possibile altresì dotare il record bibliografico di una rete di collegamenti che consentiranno all'utente di svolgere le funzioni delineate nel modello. Vengono messi in rilievo alcuni vantaggi che l'applicazione dei linked data può apportare al processo informativo delineato e approfondito nel modello proposto, in particolare:

la riduzione delle barriere cognitive che si presentano durante l'interazione utente-OPAC e con riferimento alla ricerca semantica. Tali barriere che intervengono soprattutto nella prima fase del processo di ricerca, possono essere sensibilmente ridotte. Applicando la tecnologia dei linked data è possibile migliorare l'accesso al catalogo (tramite il suggerimento di termini per la ricerca e l'interrogazione tramite il linguaggio naturale) e migliorare la ricerca semantica attraverso il collegamento di schemi e diversi sistemi di organizzazione delle conoscenze creati da comunità differenti. Ł̀ inoltre possibile sviluppare applicazioni visuali delle entità correlate, espandere la

Biblioteconomia, Firenze, Università degli Studi di Firenze, 2010, Firenze, Casalini Libri, 2010. Lo studioso iraniano affida al super-record quella funzione organizzativa necessaria alla costruzione di una tale visione di OPAC. 
ricerca con risultati recuperati automaticamente dal Web Semantico, dinamici e sempre aggiornati, di unire dimensioni di ricerca diverse per offrire modalità di esplorazione inedite, risolvere le barriere linguistiche;

* la possibilità di fornire un 'contesto' per la ricerca, che consente all'OPAC di trasformarsi da sistema di recupero dell'informazione a strumento di creazione di conoscenza e comprensione dell'informazione recuperata;

* la capacità di fornire al record bibliografico la struttura capace di sostenere ulteriori funzioni dell'utente, come quelle della valutazione della pertinenza e del riutilizzo dei dati.

I linked data potranno influire radicalmente nella progettazione complessiva di OPAC che possano consentire funzionalità migliorate 'a supporto della ricerca'. In questo paragrafo particolare spazio viene dato alle nuove e inedite prospettive che i linked data offrono alla ricerca semantica attraverso gli OPAC.

L'ultimo capitolo (Conclusioni) traccia le conclusioni della ricerca e propone una riflessione sul futuro dei cataloghi elettronici. Di fronte alle nuove tecnologie dei linked data è obbligatorio chiedersi quale sia il ruolo del catalogo elettronico nel futuro e se ci sia ancora spazio per un' 'idea' di catalogo. Ci si chiede, in definitiva, se il catalogo fondato su un record atomizzato e decostruito che la logica dei linked data richiede conserverà la sua identità e le sue caratteristiche, oppure subentreranno nuove modalità di ricerca dell'informazione. Se le linee di tendenza attuali vedono il catalogo ampliarsi per diventare uno strumento di recupero globale dell'informazione, la comparsa del modello dei linked data, come nuovo modo di strutturare i dati bibliografici per integrarli nel Web Semantico, pone nuovi interrogativi che coinvolgono la sopravvivenza delle modalità tradizionali di indicizzazione semantica e di organizzazione della conoscenza.

Con l'avvento delle tecnologie del Web Semantico l'OPAC si avvia verso una nuova tappa evolutiva che vedrà il verificarsi di una radicale trasformazione del catalogo e dei dati bibliografici, del rapporto tra utente e catalogo e tra il catalogo e l'universo globale dell'informazione. Le informazioni del catalogo potranno essere sfruttate in applicazioni del web e in modalità nuove per arricchire i percorsi a disposizione dell'utente e le modalità di accesso ai dati bibliografici, mentre si sposteranno nel web anche i meccanismi del controllo bibliografico e dell' organizzazione delle conoscenze.

La posizione intrapresa sostiene, dunque, che il catalogo potrà garantirsi un ruolo se manterrà un'identità forte e riconoscibile ovvero se, a differenza dei motori di ricerca del 
web, si porrà come uno strumento dove l'utente potrà non solo trovare, verificare o recuperare un'informazione bibliografica, ma come un ambiente in grado di sostenere il suo personale processo informativo, facilitando al contempo la creazione di conoscenza.

In appendice alla tesi (Appendici $A, B, C$ ) vengono elencati i casi di studio che hanno costituito la base per analizzare il modello corrente degli OPAC di nuova generazione e degli strumenti di discovery. Una prima parte (appendice A) è costituita dall'analisi di esempi applicativi di OPAC di nuova generazione e strumenti di discovery esaminati nel triennio che hanno costituito la base per l'interpretazione e lo studio del modello attuale di OPAC. Essi vengono analizzati secondo una griglia di rilevamento dei dati suddivisa in categorie e corredati di un breve commento, di immagini e riferimenti bibliografici.

La seconda e la terza appendice sono dedicate ai sistemi prototipali che hanno introdotto per via algoritmica il modello FRBR e ai più noti software per 'OPAC di nuova generazione' e 'sistemi per la scoperta'. 


\section{CAPITOLO 1. IL CATALOGO ELETTRONICO TRA POSTMODERNITÀ E RIVOLUZIONE DIGITALE}

«La 'società' è sempre stata un'entità immaginata, mai concessasi all'esperienza nella sua interezza»

Zygmunt Bauman

\subsection{La riflessione sulle funzioni del catalogo, il dibattito internazionale e le strategie bibliotecarie}

Da qualche anno Next generation catalogs e discovery systems sostituiscono i cataloghi tradizionali delle biblioteche.

Questi prodotti rappresentano l'esito di un lungo percorso di rinnovamento dei cataloghi elettronici che si è svolto a partire dalla metà del decennio scorso, quando si è avviato un lungo dibattito sulle funzionalità degli OPAC che ha visto autorevoli studiosi confrontarsi sulla possibilità di rinnovare i cataloghi arricchendoli e dotandoli di funzionalità più avanzate ${ }^{1}$.

Alle origini del dibattito che, a mio avviso, merita di essere ripercorso in queste pagine, si possono rilevare almeno due principali motivazioni: l'esigenza di adeguamento dei cataloghi alle iniziative che nell'ultimo decennio hanno rinnovato il panorama della catalogazione (dagli sviluppi della famiglia FRBR agli ICP, fino alle RDA ${ }^{2}$ ) e la necessità

\footnotetext{
${ }^{1}$ Tra le prime voci critiche vi furono quelle di Roy Tennant (Lipstick on a pig, «Library Journal» 130 (2005) n. 7, p. 34, <http://www.libraryjournal.com/article/CA516027.html>) che lamentava l'esigenza ormai irrinunciabile di cambiamenti effettivi e più profondi e di Timothy Burke nell'articolo Burn the catalog (20 January 2004, <http://www.swarthmore.edu/SocSci/tburke1/perma12004.html>). Altre voci critiche sono state quelle di Andrew Pace, Karen Markey, Karen G. Schneider che hanno scritto vari articoli polemici nel tentativo di segnalare gli aspetti maggiormente problematici dei cataloghi. Il dibattito in Italia sulla necessità di rivedere le caratteristiche degli OPAC è stato suscitato dalla traduzione italiana di un contributo di John Byrum (Recommendations for urgently needed improvement of OPAC and the role of the National Bibliographic Agency in achieving it, World Library and Information Congress: 71th IFLA General Conference and Council. Libraries - A voyage of discovery. August 14th - 18th 2005, Oslo. <http://www.ifla.org/IV/ifla71/papers/124e-Byrum.pdf>. Traduzione italiana: John D. jr Byrum, Raccomandazioni per miglioramenti urgenti dell'OPAC. Il ruolo delle agenzie bibliografiche nazionali, «Biblioteche oggi», 23 (2005), n. 10, p. 5-14) che ha maggiormente orientato le discussioni verso la tematica dell'arricchimento bibliografico. Alcuni importanti interventi si sono avuti in risposta a nuove politiche catalografiche che verranno riproposte e commentate in questo capitolo.

${ }^{2} \mathrm{Si}$ vedano rispettivamente: IFLA Study Group on the functional requirements for bibliographic records, Functional requirements for bibliographic records: final report, Münich, K. G. Saur, 1998. Disponibile anche online all'URL:<http://www.ifla.org/VII/s13/frbr/frbr.pdf>, <http://www.ifla.org/VII/s13/frbr/frbr.htm>. Trad. italiana: Requisiti funzionali per record bibliografici, ed. italiana a cura dell'Istituto centrale per il catalogo unico delle biblioteche italiane e per le informazioni
} 
di avvicinare gli OPAC ai desideri e alle aspettative degli utenti rendendoli al contempo 'integrati' in rete.

Tali urgenze sono state sollevate in coincidenza con alcuni fenomeni tecnologici di rilievo che hanno profondamente segnato la cifra del decennio appena trascorso, quali i grandi progetti di digitalizzazione di massa ${ }^{3}$, l'ascesa di Google e il suo ingresso nel mondo dei libri e delle biblioteche ${ }^{4}$ e, successivamente, l'emergere delle tecnologie del

bibliografiche, Roma, ICCU, 2000. Ultima versione: IFLA Study Group on the functional requirements for bibliographic records, Functional requirements for bibliographic records: final report. Approved by the Standing Committee of the IFLA Section on Cataloguing. September 1997. As amended and corrected through February 2009, <http://www.ifla.org/VII/s13/frbr/frbr_2008.pdf>; INTERNATIONAL FEDERATION OF LIBRARY ASSOCIATIONS AND INSTITUTIONS, IFLA Cataloguing Principles: Statement of International Cataloguing Principles (ICP) and its Glossary edited by Barbara Tillett and Ana Lupe Cristán, München: K.G. Saur, 2009 <http://www.ifla.org/VII/s13/icc/imeicc-statement_of_principles2008.pdf> [trad. it. Dichiarazione di principi internazionali di catalogazione, Roma: ICCU, 2009, <http://www.iccu.sbn.it/genera.jsp?id=94>,oppure <http://www.ifla.org/files/cataloguing/icp/icp_2009it.pdf $>$; American Library Association - Canadian Library Association, Chartered Institute of Library and Information Professionals (Great Britain)- Joint Steering Committee for Development of RDA, RDA: resource description \& access, Chicago, American Library Association, 2010.

${ }^{3}$ Tra il 1990 e il 2000, lo sviluppo delle reti e delle tecnologie accese un grande interesse per la possibilità di digitalizzare immensi patrimoni librari. La prima rilevante impresa, avviata all'inizio del decennio, fu quella del Million Book Project trasformatosi poi in Universal Digital Library che nel periodo 2000-2007 raggiunse la quota di 1 milione e 500 mila testi digitalizzati messi a disposizione in rete. Il progetto, partito dalla statunitense Carnegie Mellon University con l'obiettivo di creare una biblioteca digitale universale dal punto di vista linguistico, coinvolgeva biblioteche ed istituzioni culturali asiatiche, in particolare cinesi ed indiane come la cinese Zhejiang University, l'Indian Institute of Science e la biblioteca di Alessandria d'Egitto.

${ }^{4}$ Il 14 dicembre 2004 Google Inc., una delle più grandi aziende di internet, universalmente nota per il motore di ricerca Google, diede l'annuncio ufficiale del suo ingresso nel mondo delle biblioteche. Il Google Library Project prevedeva un accordo con le cinque più importanti biblioteche universitarie americane (l'University of Michigan, Harvard, Stanford, Oxford e la New York Public Library) per la digitalizzazione delle loro raccolte. Circa 15 milioni di libri e altri documenti diventavano così interrogabili tramite il nuovo servizio Google Print ${ }^{\mathrm{TM}}$ denominato successivamente Google Book Search, in Italia noto con il nome di Google Libri (<http://books.google.it/>). Google avrebbe incluso l'immenso patrimonio così digitalizzato in Google Book Search, l'innovativo servizio che avrebbe dovuto «al pari di un catalogo a schede, riportare le informazioni sul libro, il fulltext oltre che, in certi casi, alcuni frammenti, ossia alcune frasi contenenti il termine di ricerca nel contesto» (<http://books.google.it/googlebooks/library.html $>$ ). Nonostante l'entusiasmo delle biblioteche partecipanti, il progetto fu avviato e portato a compimento tra mille polemiche e fu più volte ostacolato da editori, librerie e degli stessi autori che si unirono lanciando l'accusa a Google di violare i diritti d'autore e che misero più volte a rischio la continuazione del progetto. Un anno più tardi, con il nome di Google ricerca libri, il servizio sbarcava anche in Italia, coinvolgendo alcune grandi case editrici italiane e appariva sul web in versione beta. L'elenco delle biblioteche partners del progetto è visibile in $<$ http://books.google.com/googlebooks/partners.html>. Accanto all'enorme progetto di digitalizzazione di massa, l'azienda lanciava quasi in contemporanea, nel novembre 2004, Google Scholar, un secondo motore dedicato appositamente ad organizzare la ricerca di materiale accademico e scientifico, dando accesso sia a risorse full text disponibili gratuitamente in rete o presenti nelle biblioteche aderenti al progetto tramite un servizio di distribuzione dei documenti. Nel giugno 2005, la Library of Congress annunciava l'avvio di un altro grande progetto di digitalizzazione di massa: il bibliotecario del Congresso James Billington propose la creazione di una World Digital Library che raccogliesse manoscritti e materiale multimediale allo scopo di riunire on line, materiali culturali rari e unici. Pochi mesi più tardi Billington e Sergey Brin, co-fondatore di Google, annunciavano che proprio Google avrebbe contribuito come primaria azienda del settore privato all'iniziativa con un finanziamento di tre milioni di dollari. L'ingresso di Google nel mondo dei libri e delle biblioteche scatenò un dibattito appassionato nella comunità internazionale, con echi anche in Italia, e cominciò a manifestarsi in molti il timore che internet potesse prendere il posto delle biblioteche e dei loro cataloghi. I risvolti più recenti della vicenda di Google Books sono analizzati da Alberto Petrucciani (Alberto Petrucciani, Ancora su Google e la giungla digitale: altri misteri, novità e tendenze, «AIB Studi», 52 (2012) n. 2, p. 197-204, <http://aibstudi.aib.it/article/view/6326/7213>. Tra queste la tendenza al copyfraud, ossia 
Web 2.0 e delle reti sociali; eventi che hanno profondamente cambiato le modalità di creazione, uso e condivisione delle conoscenze.

Di fronte al successo dei motori di ricerca e delle librerie online, ma anche all'emergere di nuove pratiche di comunicazione e diffusione del sapere, i bibliotecari si sono interrogati sul ruolo dei cataloghi e della catalogazione; nel confronto che ha animato la comunità bibliotecaria internazionale sono state sollevate varie argomentazioni in difesa dei cataloghi, quali la pericolosa associazione tra le biblioteche e le società commerciali, o la necessità di contrastare il fenomeno della 'disintermediazione', cioè quel processo per cui nel mondo dell'informazione elettronica l'utente tende ad approcciarsi all'informazione in modo diretto, senza ricercare l'aiuto professionale di bibliotecari o esperti.

Così nel nostro paese commentava Riccardo Ridi:

Le speranze di sopravvivenza sono comunque, a mio avviso, piuttosto elevate - almeno per i bibliotecari - perché parlare di disintermediazione coglie in realtà solo una parte del mutamento in atto. Se da una unilineare catena documentaria si passa una multilineare rete ipertestuale, i percorsi possono talvolta accorciarsi, certo, ma qualche volta possono anche correre il rischio di allungarsi o addirittura di girare nel vuoto o di finire nel nulla. Se da un canone del sapere si passa ad una serie di saperi intrecciati fra loro, ci sarà ancora più bisogno di prima di alfabetizzazione informatica e di altri ausilii per non perdersi, ovvero di quella disciplina e attività che «consiste nel mettere in contatto il lettore giusto con il libro giusto» (Ranghanathan 1992, p 376), comunque la si voglia chiamare. Il passaggio al modello (culturale e telematico) della rete non conduce in realtà alla disintermediazione, ma piuttosto verso la iper-intermediazione, (che include anche come caso limite particolare la mediazione di livello zero) perché aumentando i percorsi informativi, comunicativi, e documentari possibili, aumentano in proporzione anche le possibilità di scelta e il bisogno di una molteplicità di punti di vista da cui affrontare i problemi cognitivi e di criteri con cui ordinare il docuverso. «In un mondo di pressoché infinite scelte, l'informazione relativa alle scelte ha più valore delle scelte stesse ${ }^{5}$.

Il fascino dei motori di ricerca e delle librerie online, con le loro vincenti strategie di mercato, fece molta presa nel nascente dibattito, tanto che una tra le tante voci polemiche, quella di Roy Tennant nel lamentare l'esigenza ormai irrinunciabile di cambiamenti effettivi e più profondi negli OPAC ne auspicava gli sviluppi nella direzione di un avvicinamento ai modelli commerciali di Amazon e Google:

We need to focus more energy on important, systemic changes rather than cosmetic ones. If your system is more difficult to search and less effective than Amazon.com (and whose isn't?), then you have work to do. Stop asking for minor tweaks from vendors. After all, you can put lipstick on a pig, but it's still very much a pig ${ }^{6}$

quelle «manovre finalizzate a defraudare il pubblico della possibilità di utilizzare liberamente il materiale di dominio pubblico» (ivi, p. 197).

5 Riccardo Ridi, La Biblioteca come ipertesto. Verso l'integrazione dei servizi e dei documenti, Milano, Editrice Bibliografica, 2007, p. 79-81.

${ }^{6}$ Roy Tennant, Lipstick on a pig cit. 
Sì è avvertito nel dibattito, dunque, il senso di una profonda 'crisi' del catalogo; di fronte all'evoluzione tecnologica e alla comparsa di nuovi strumenti di ricerca disponibili in rete, che oggi condizionano profondamente i modelli mentali degli utenti che fanno ricerca, il catalogo è stato percepito come uno strumento poco amichevole e difficile da utilizzare.

Non c'è dubbio che la rete abbia imposto modelli di ricerca e di servizio nuovi e profondamente innovativi, tanto che si può affermare che l'approccio dell'utente della rete con l'informazione sia profondamente cambiato. Tuttavia, è stata proprio la concorrenza degli strumenti di ricerca commerciali come i motori di ricerca o le librerie online e lo sviluppo delle reti sociali (che hanno caratterizzato l'evoluzione del web tradizionale nel cosiddetto 'Web 2.0') ad imprimere una decisa accelerazione al processo di revisione e la ricerca di soluzioni plausibili per rilanciare il ruolo del catalogo; tali soluzioni hanno imboccato, almeno all'inizio, la via più rapida e agevole, ma non la più sicura, prendendo a riferimento tali esempi e correndo il rischio di orientare l'evoluzione del catalogo verso l'imitazione di modelli estranei al mondo delle biblioteche, non di certo basati su meditazioni approfondite. Senza una visione a tutto tondo delle problematiche inerenti ai cataloghi si è corso il rischio di guardare a questi strumenti in modo acritico e di imboccare la via dell'imitazione, come osservava Alberto Petrucciani ${ }^{7}$ :

Il confronto ossessivo, soprattutto nella letteratura americana, tra le biblioteche e i loro cataloghi da una parte e i servizi leader di Internet dall'altra, mentre testimoniano una disperata pulsione di "essere alla moda", comporta spesso un grosso abbaglio. È un classico non sequitur: dato che si sviluppano, crescono, si affermano cose come Google (Amazon ecc.), allora le biblioteche devono cercare di imitarle, di andare nella stessa direzione. È molto spesso ovvio il contrario [...] Il fatto che le persone possano già trovare "qualcosa" molto rapidamente con Google è un ottimo motivo per non cercare di fare la stessa cosa.

Il ripensamento delle funzionalità dei cataloghi, oltre ad essere al centro di un dibattito internazionale davvero intenso, è stato oggetto delle politiche bibliotecarie di importanti istituzioni che, a partire dalla seconda metà dello scorso decennio, hanno mutato le politiche catalografiche e ridefinito obiettivi e pratiche della catalogazione.

Può essere utile, dunque, riassumere in questa sede alcune tra le più importanti iniziative che hanno delineato fortemente il contesto nel quale si sono formate le visioni di sviluppo del catalogo elettronico e che hanno certamente orientato l'evoluzione dei cataloghi tradizionali verso quelli che oggi sono comunemente denominati next generation catalogs e discovery systems.

\footnotetext{
${ }^{7}$ Alberto Petrucciani, La catalogazione, il mercato e la fiera dei luoghi comuni, «Bollettino AIB», 43 (2006) n. 3 , p. 183.
} 
Ormai è ampiamente noto il dibattito svoltosi alla Library of Congress a partire dal 2006 sul futuro dei cataloghi e della catalogazione in seguito alle nuove politiche catalografiche avviate dall'istituto e che ebbe tra protagonisti Deanna Marcum, Karen Calhoun e Thomas Mann.

Deanna Marcum, bibliotecaria associata alla Library of Congress, propose una serie di riflessioni che esprimevano da parte della dirigenza l'urgenza di nuovi cambiamenti che investivano profondamente il ruolo dei cataloghi e della catalogazione ${ }^{8}$ e che furono oggetto di numerose critiche, sia all'interno della stessa istituzione, sia all'esterno con echi anche nella letteratura professionale italiana ${ }^{9}$. Marcum sosteneva che la maggior parte degli studenti nella ricerca di informazioni trascurasse le biblioteche in favore dei motori di ricerca e per rinforzare la sua tesi richiamava due recenti indagini sulle abitudini di ricerca degli studenti che dimostravano come questi, consapevoli dei difetti dei motori di ricerca (la pubblicità, l'abbondanza di informazione non pertinente che viene recuperata), trovassero comunque più agevole una ricerca con Google ${ }^{10}$. Partendo da queste premesse,

\footnotetext{
${ }^{8}$ Particolare clamore provocò l'intervento di Deanna Marcum in occasione dell'Ebsco Leadership Seminar che si tenne a Boston nel gennaio 2005. A seguito sia dei numerosi articoli polemici che di varie indagini sulle abitudini di ricerca degli utenti, Deanna Marcum presentò una relazione dal significativo titolo The Future of Cataloging. (Deanna B. Marcum, The future of cataloguing. Address to the Ebsco leadership seminar. Boston, $\quad$ Massachusetts, $\quad$ January 2005 , <http://www.loc.gov/library/reports/CatalogingSpeech.pdf>). La relazione segnò il principio di un lungo dibattito, all'interno della Library of Congress fra i numerosi bibliotecari addetti alla catalogazione molti dei quali riuniti nell'associazione professionale ASCME. Punto di partenza delle sue osservazioni era la constatazione dell'enorme somma spesa ogni anno dall'istituzione per le attività di catalogazione, ben 44 milioni di dollari, e la conseguente domanda se tale enorme impegno fosse realmente giustificabile nell'era di internet e dell'informazione digitale. Facendo riferimento ad alcuni studi recenti sulle abitudini di ricerca degli americani e degli studenti universitari, Marcum si dichiarava sconcertata nell'apprendere come un'alta percentuale di utenti cercasse le risposte di tutti i giorni in internet. Marcum prendeva in considerazione un recente studio (l'indagine del 2004 di Horrigan e Rainie ) sulla diffusione e l'uso di internet tra la popolazione americana che affermava come che il $60 \%$ per cento degli americani avesse un accesso internet e il $40 \%$ fosse online da più di tre anni e che la maggior parte di coloro che cercavano informazioni si aspettavano di trovarle in internet. Marcum intendeva dimostrare come sia più facile oggi per uno studente che intenda fare una ricerca, affidarsi alle funzioni di ricerca avanzata di un motore di ricerca come Google, piuttosto cliccare ripetute volte all'interno di un sito di una biblioteca per consultarne le basi dati e il catalogo: «So, is it any surprise that many students just go Googling instead of to the library, virtual or physical, and use whatever turns up first in the key-word search?» (ivi, p. 2-4).

${ }^{9}$ Il contributo più significativo di Thomas Mann fu pubblicato in Italia con una introduzione di Alberto Petrucciani (Alberto Petrucciani, La catalogazione cit.). Tra gli altri contributi che si occuparono di riassumere il dibattito internazionale è da citare anche Paul Gabriele Weston - Salvatore Vassallo ... "e il navigar m'è dolce in questo mare”. Linee di sviluppo e personalizzazione dei cataloghi, in Claudio Gamba Maria Laura Trapletti, La biblioteca su misura. Verso la personalizzazione del servizio, atti del convegno di "Biblioteche oggi", Milano, 9-10 marzo 2006, a cura di C. Gamba e M. L. Trapletti, Milano, Editrice Bibliografica, 2007, p. 131-167.

${ }^{10} \mathrm{Si}$ trattava di alcuni primi studi sulle abitudini di ricerca degli utenti, in particolare dell'indagine di Karl V. Fast e D. Grant Campbell, "I Still Like Google”: University Student Perceptions of Searching OPACs and the Web in Proceedings of the ASIS Annual Meeting, Medford, Information Today, 2004, p. 138-146, anche online in <http://www.asis.org/Conferences/AM04/abstracts/137.html>) e di una delle prime indagini sull'utenza compiute da OCLC (Online Computer Library Center (OCLC), OCLC White Paper on the Information Habits of College Students, published electronically by the OCLC Online Computer Library Center, Inc., June 2002, <http://www5.oclc.org/downloads/community/informationhabits.pdf>.
} 
Marcum manifestava apertamente il cambiamento di prospettiva e di priorità nella politica bibliotecaria della Library of Congress: tra le raccomandazioni vi era, infatti, quella di eliminare la pratica della soggettazione a beneficio di una maggiore velocità e di un risparmio sui costi delle operazioni di catalogazione ${ }^{11}$. In apertura al suo intervento Marcum poneva la seguente domanda:

But in the age of digital information, of Internet access, of electronic key-word searching, just how much do we need to continue to spend on carefully constructed catalogs? That is the question I have come here this evening to pose-how should we think about cataloging in the Age of Google? ${ }^{12}$

L'intervento di Deanna Marcum ricevette subito una risposta polemica nei vari articoli che Thomas Mann, bibliotecario di reference presso la stessa biblioteca, pubblicò qualche mese dopo ${ }^{13}$.

Il primo effetto del mutato corso della politica catalografica della biblioteca americana fu la comunicazione che annunciava la decisione della Library of Congress di terminare la creazione dei series authority records (SARs) and series title control ${ }^{14}$.

\footnotetext{
${ }^{11}$ Così affermava la studiosa: «My staff at the Library of Congress believes that, in providing access, there already has been a major shift. Cataloging now involves identifying metadata that already exist and taking advantage of existing description and access points. Different approaches are needed depending on whether resources are archived or linked and how long they will last. New hybrid systems take advantage of traditional library catalog information along with abstracting and indexing tools and online reference tools. [...] we are rethinking who does what in cataloging. For example, with the advent of ever more automated sophistication, the detailed attention that we have been paying to descriptive cataloging may no longer be justified. If the task of descriptive cataloging could be assumed by technicians, then retooled catalogers could give more time to authority control, subject analysis, resource identification and evaluation, and collaboration with information technology units on automated applications and digitization projects» (ivi, p. 10).

${ }^{12}$ Ivi, p. 2.

${ }^{13}$ Con l'articolo Will Google's keyword searching eliminate the need for LC cataloging and classification? Mann si adoperò per contrastare la visione eccessivamente ottimistica delle possibilità di ricerca offerte da Google Print (oggi Google Libri) e cioè la semplice ricerca per parola chiave. Come spiega Mann, se Google può fornire risposte su domande veloci, si dimostra totalmente inefficace nel fornire una panoramica coerente di ciò che è stato scritto intorno ad un argomento. Mann portava numerosi esempi per sostenere l'assoluta necessità di mantenere la catalogazione per soggetto, spiegandone le sostanziali differenze rispetto alla semplice ricerca per parola chiave. Nel saggio Survey of Library User Studies lo studioso riprendeva vari studi sulle abitudini di ricerca degli utenti con lo scopo di dimostrare che in realtà le biblioteche e i cataloghi vengono usati dagli utenti molto di più di quanto sembri, soprattutto quando la ricerca è orientata a fini di studio e di conoscenza approfondita su un certo argomento. (cfr. Thomas Mann, Will Google's keyword searching eliminate the need for LC cataloging and classification? August 152005. <http://www.guild2910.org/searching.htm> by Dr. Thomas Mann, Reference Librarian in the Library of Congress Main Reading Room, August 15 2005. <http://www.guild2910.org/searching.htm> e Survey of library user studies, October 2005, <http://www.guild2910.org/google.htm>). Sulla necessità della precoordinazione nell'indicizzazione per soggetto Mann si espresse anche nell'articolo Is precoordination unnecessary in LCSH? are Web sites more important to catalog than books? A reference librarian's thoughts on the future of bibliographic control, Library of Congress, Washington D.C., 15-17 November 2000. $<$ http://www.loc.gov/catdir/bibcontrol/mann_paper.pdf>. Il dibattito è proseguito con altri importanti contributi, cfr.: Id., The Peloponnesian war and the future of reference, cataloging, and scholarship in research libraries. Prepared for AFSCME 2910 The Library of Congress Professional Guild representing over 1,600 professional employees www.guild2910.org. Library of Congress, Washington D.C.: Library of Congress, 2007, <http://guild2910.org/Pelopponesian\%20War\%20June\%2013\%202007.pdf>; Id., Thomas Mann, "On the record" but off the track: a review of the report by The Library of Congress Working Group on The Future of Bibliographic Control, with a further examination of Library of Congress cataloging tendencies, 2008, <http://www.guild2910.org/WorkingGrpResponse2008.pdf>.
} 
Thomas Mann e Saul Schnidermann denunciarono più volte la dirigenza della Library of Congress di voler semplificare le procedure catalografiche a spese della catalogazione per soggetto e, in questo caso, anche del controllo bibliografico. Ma era ormai troppo tardi: nel 2006, il catalogo divenne oggetto di una nuova indagine che avrebbe mutato radicalmente la politica catalografica della più grande biblioteca americana di ricerca. Si tratta di un'inchiesta di ampio respiro sul futuro dei cataloghi che la biblioteca commissionò a Karen Calhoun della Cornell University Library. The changing nature of the catalog and its integration with other discovery tools, pubblicato nel 2006, può essere certamente annoverato tra gli scritti che hanno influenzato le politiche catalografiche e la visione evolutiva dei cataloghi elettronici ${ }^{15}$.

Nel report la studiosa ridisegnava le principali linee di sviluppo del catalogo, immaginando come tale strumento, giunto ormai al termine del suo ciclo vitale ${ }^{16}$, avrebbe potuto essere 'rivitalizzato' e proponeva delle direttive per il cambiamento dei processi di catalogazione.

Le direttive di Calhoun erano quelle di indirizzare le risorse dalla catalogazione dai formati di produzione di massa (quali, ad esempio, i libri) alla catalogazione di risorse uniche (archivi digitali, collezioni speciali, ecc.) e applicare alle politiche bibliotecarie un modello imprenditoriale che avrebbe consentito al catalogo di recuperare un rinnovato ruolo tra i servizi bibliotecari ${ }^{17}$.

\footnotetext{
${ }^{14}$ Series at the Library of Congress: June 1, 2006, <http://www.loc.gov/catdir/cpso/series.html>. A sostegno della decisione veniva evidenziato che le mutate condizioni e il potenziamento della ricerca per parola chiave fossero considerate oramai sufficienti per l'accesso e il recupero di questo materiale. La decisione provocò un'altra serie di proteste in quanto fu comunicata ed eseguita in soli dieci giorni. A riguardo si esprimeva così Alberto Petrucciani: «L'osservazione che le serie possono essere comunque ricercate per parole chiave è agghiacciante dal punto di vista biblioteconomico, perché chiunque abbia un po' di pratica di catalogazione sa quanto sia frequente che le collane abbiano titoli generici, che si ripetono spesso e volentieri, o sono costituiti più o meno dallo stesso repertorio di parole variamente combinate fra loro. Inoltre, come si sa o si dovrebbe sapere, molte serie sono numerate, e il controllo quindi non richiede soltanto l'identificazione a livello di titolo ma anche la gestione della numerazione progressiva. Ovvio dovrebbe essere, almeno per un bibliotecario, quanto sia importante il controllo delle serie per la politica delle acquisizioni o per la verifica del deposito legale» (Alberto Petrucciani, La catalogazione cit., p. 179).

${ }^{15}$ Karen Calhoun, The changing nature of the catalog and its integration with other discovery tools. Final report prepared for the Library of Congress, 17 March 2006, <http:www.loc.gov/catdir/calhoun-reportfinal.pdf>.

16 «Today, the online catalog is losing appeal for students and many scholars. Catalog usage, drifting downward compared to other discovery tools, may soon plummet. Fortunately, there are ways to use the knowledge that today's catalog has reached the end of its life cycle» (Ivi, p.10).

17 Il modello di sviluppo che Calhoun prende come riferimento è un modello di business e le sue argomentazioni si riferiscono ad un contesto (quello del mercato) cui le biblioteche sono state finora estranee. Le numerose interviste alla fine del rapporto miravano a fare emergere il tipo di informazione che ogni investitore vorrebbe avere su un prodotto la cui posizione di mercato venga erosa. Calhoun riprende le teorie economiche esposte in un contributo del 1965 Exploit the product life cycle da Theodore Lewitt, docente della Harward Business School Harvard Business Review (March 3, 2009).
} 
Le strategie proposte da Calhoun vengono disposte in un quadrante e si incrociano generando quattro livelli di profondità delle innovazioni: dalle meno innovative (promuovere un uso più frequente da parte degli utenti abituali) alle più profonde e impegnative (trovare nuovi servizi e nuovi utenti) che richiedono forti investimenti e specifiche competenze professionali ${ }^{18}$. La parte più controversa del rapporto ${ }^{19}$ è quella che

${ }^{18}$ Le quattro strategie previste sono (p. 11-12):

* promuovere un uso più frequente da parte degli utenti abituali: si tratta di apportare cambiamenti modesti al catalogo, quali un miglioramento delle modalità di ricerca e di visualizzazione dei risultati. Tale strategia non si rivela efficace a lungo termine ed è adatta a biblioteche che non dispongono di risorse economiche sufficienti o ai bibliotecari ancorati ad un atteggiamento conservatore, ma nel lungo termine porta all'indebolimento del catalogo a vantaggio di altri strumenti di ricerca;

* sviluppare nuovi servizi per gli utenti abituali promuovendo la catalogazione delle risorse elettroniche, fornendo servizi a valore aggiunto, es. l'esportazione di bibliografie in multiformato;

* trovare nuovi utenti per il prodotto esistente: si tratta di incrementare quei servizi di marketing rivolti a catturare l'interesse di nuove fasce di utenti. Prevede l'allestimento di più efficaci servizi all'utenza quali ricerche bibliografiche o brevettuali alle imprese, l'allestimento di servizi di document delivery con altre realtà territoriali etc.;

* trovare nuovi servizi e nuovi utenti, rappresentato nel quadrante in alto a destra raggruppa le trasformazioni più innovative che consentirebbero a lungo termine alle biblioteche di conservare il proprio ruolo in un contesto di forte innovazione tecnologica. Tra le raccomandazioni quella di intraprendere la valorizzazione della cultura locale favorendo il fenomeno del cosiddetto "turismo culturale", realizzare portali disciplinari, dedicarsi alla conservazione di siti web delle istituzioni locali, avviare progetti di digitalizzazione del patrimonio documentario, dedicarsi a progetti formativi nell'ambito del digitale, far leva sui cataloghi per supportare i progetti di digitalizzazione di massa e/o produrre effetti di long-tail per le collezioni librarie.

19 Anche il rapporto di Calhoun, come già avvenuto per le posizioni espresse da Marcum fu nuovamente contestato da bibliotecari interni all'istituzione (molti catalogatori dell'associazione professionale dell'AFSCME) riuniti intorno al bibliotecario Thomas Mann e da importanti personalità del panorama bibliotecomico; da molti il rapporto fu giudicato irrispettoso dell'identità stessa della biblioteca e aprì il dibattito anche in Italia dove alcuni studiosi sollevarono autorevoli critiche (Oltre all'intervento di Petrucciani si veda, ad esempio, il contributo di Paul Gabriele Weston, Caratteristiche degli opac e strategie delle biblioteche, «Bibliotime», XI (2008), n.1, <http://didattica.spbo.unibo.it/bibliotime/num-xi1/weston.htm>). Si vedano inoltre i due articoli polemici di Mann: What is going on at the Library of Congress?. Prepared for AFSCME 2910 by Thomas Mann, reference librarian in the Library of Congress Main reading room, June 19, 2006, <http://www.guild2910.org/AFSCMEWhatIsGoingOn.pdf>. ID, More on what is going on at the Library of Congress. Prepared for AFSCME 2910, 2007, <http://www.guild2910.org/AFSCMEMoreOnWhatIsGoing.pdf>.

Le posizioni espresse da Thomas Mann contro le politiche miopi della dirigenza della LC furono condivise anche da Michael Gorman, allora presidente dell'ALA, che non esitò a definire 'una catastrofe' i cambiamenti in atto nella politica catalografica della del Congresso. Così si esprimeva, nuovamente Thomas Mann nella sua 'recensione critica' al rapporto della studiosa: «According to the Calhoun report, library operations that are not digital, that do not result in resources that are remotely accessible, that involve professional human judgement or expertise, or that require conceptual categorization and standardization rather than relevance ranking of keywords, do not fit into its proposed "leadership" strategy. This strategy itself, however, is based on an inappropriate business model - and a misrepresentation of that business model to begin with. The Calhoun report draws unjustified conclusions about the digital age, inflates wishful thinking, fails to make critical distinctions, and disregards (as well as mischaracterizes) an alternative "niche" strategy for research libraries, to promote scholarship (rather than increase "market position"). Its recommendations to eliminate Library of Congress Subject Headings, and to use "fast turnaround" time as the "gold standard" in cataloging, are particularly unjustified, and would have serious negative consequences for the capacity of research libraries to promote scholarly research (Thomas Mann, The changing nature of the catalog and its integration with other discovery tools. Final report, March 17, 2006, prepared for the Library of Congress by Karen Calhoun. A critical review, 3 April 2006, <http://guild2910.org/AFSCMECalhounReviewREV.pdf>. Citazione a p. 1 ; trad. italiana: Thoman Mann, Il catalogo e gli altri strumenti di ricerca: un punto di vista dalla Library of Congress, «BollettinoAIB», 46 
illustra le alternative strategiche che possono essere praticate dalle biblioteche, che vanno dalla leadership cioè l'accentramento di tutti i servizi intorno alle strutture più "forti", al quick divestment che comporterebbe la rinuncia delle istituzioni bibliotecarie più deboli a mantenere un catalogo locale affidando la sua realizzazione e il mantenimento ad altre biblioteche "dominanti", liberando così risorse che avrebbero potuto così essere opportunamente sfruttate per altri servizi ${ }^{20}$. Ad ogni biblioteca o organizzazione era data la possibilità di scegliere, tra le varie alternative proposte per rivitalizzare il proprio catalogo, quella più idonea in base a criteri quali la situazione finanziaria, la posizione dominante $\mathrm{o}$ meno della propria biblioteca rispetto alle altre ${ }^{21}$.

Le due strategie intermedie consistevano nel trovare nicchie, cioè settori disciplinari o particolari utenze non sufficientemente servite dagli altri attori di mercato, oppure nel praticare l'harvesting importando dati e risorse da fonti esterne, limitandosi per i cataloghi alle procedure di gestione e manutenzione.

Il rapporto si soffermava inoltre sulle innovazioni necessarie alla riduzione dei costi

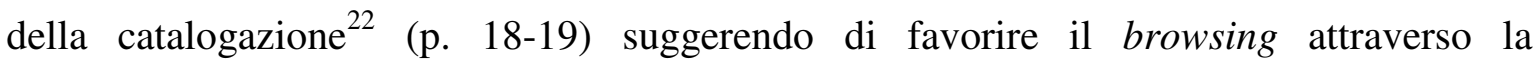
classificazione, studiare metodi per la classificazione automatica dei documenti, proponendo, dunque, di abbandonare la classificazione ottenuta per mezzo dall'attività umana $^{23}$.

(2006), 3, p. 186-205). Ancora una volta, Thomas Mann sottolineava come se, per ricerche rapide può essere sufficiente l'interrogazione degli strumenti della rete, per esigenze di studio e di ricerca il ruolo di questi strumenti è ridimensionato poiché ciò che fa la differenza è la costruzione e l'organizzazione delle raccolte. Sul sito web dell'AFSCME 2910 (<http://www.guild2910.org/>), associazione professionale che rappresenta circa 1600 impiegati della Library of Congress, sono ancora presenti i numerosi contributi di Mann nonché i contributi di Calhoun, Marcum e Saul Schniderman che animarono il dibattito (<http://www.guild2910.org/future.htm>).

${ }^{20}$ Le quattro strategie proposte sono: leadership, niche, harvest, or quick divestment.

${ }^{21}$ La valutazione della probabilità o meno di rivitalizzare il proprio catalogo dipende - secondo Calhoun strettamente dalla 'domanda': «In healthy businesses, the capacity to produce a product matches the demand for the product». Karen Calhoun, The changing nature of the catalog cit., p. 11

${ }^{22}$ Le misure che toccano profondamente le pratiche catalografiche della soggettazione e della classificazione sono esposte al punto 4 (ivi, p. 17-18) :

4.2.1 Use classification data to cluster catalog data for browsing by subject [13]

4.2.2 Explore automatic classification

4.2.3 Abandon the attempt to do comprehensive subject analysis manually with

LCSH in favor of subject keywords; urge LC to dismantle LCSH [14]

4.2.4 Encourage research and development in automatic subject analysis, including ways to reuse legacy data containing LCSH headings to support automatic subject analysis

${ }^{23} \mathrm{La}$ studiosa propone senza mezzi termini di terminare le procedure della catalogazione per soggetto («urge LC to dismantle LCSH», ivi, p. 18) favorendo invece la ricerca per parola chiave e incoraggiando studi e analisi sulla soggettazione automatica dei documenti. 
Infine, tra le altre raccomandazioni, alcune erano espressamente rivolte alle procedure per il miglioramento e l'arricchimento dei cataloghi e all'uso delle tecnologie per migliorare l'esperienza degli utenti (user experience) ${ }^{24}$.

Il rapporto Calhoun contiene in sé molti elementi di criticità che, a più di un quinquennio e alla luce dello sviluppo di strumenti per la scoperta, hanno un risvolto commerciale non poco significativo. Il rapporto - rilevava Mann- non solo era intriso di metafore, a cominciare da quella biologica del catalogo quale prodotto morente, ma anche viziato fin dall'inizio nel proporre alle biblioteche un modello imprenditoriale la cui finalità veniva individuata nel profitto e nella posizione di mercato. Sarebbe stato più opportuno collocare le biblioteche dentro il modello accademico finalizzato ad uno sviluppo del sapere e della cultura, poggiante su pratiche secolari e, per scelta, al di fuori delle richieste di mercato.

Semplificare le modalità di ricerca tenendo in conto solo le esigenze di chi cerca rapidamente un'informazione, avrebbe lasciato deliberatamente in ombra le esigenze degli studiosi, cioè di chi si aspetta di trovare nel catalogo un quadro esauriente di ciò che è stato scritto intorno ad un argomento: «È particolarmente preoccupante che il rapporto Calhoun - affermava ancora Mann - dimentichi totalmente che lo studio specialistico deve considerare anche libri in lingue straniere [...] Se si seguissero le proposte di Calhoun, queste risorse non sarebbero più visibili nelle ricerche per parole chiave perché non ci sarebbe più il controllo terminologico delle LCSH che riunisce opere in inglese e opere in altre lingue sotto voci di soggetto uniformi».

Accanto alla svolta nella politica bibliotecaria della Library of Congress va certamente citato il report Rethinking how we provide bibliographic services for the

\footnotetext{
${ }^{24} \mathrm{Nel}$ report di Calhoun ritroviamo molte delle caratteristiche implementate nei next generation catalogs e negli strumenti per la scoperta, che vengono proposte nel punto 5 (ivi p. 19), dedicato al miglioramento dell'esperienza dell'utente:

5. Improve the User Experience

5.1 Enrich the catalog with services (e.g., "more like this," "get it" options, new book lists, etc.), and data (cover art, reviews, TOCs)

5.2 Enable much better browsing and organization of large retrieval sets

5.3 Enable best-match retrieval (no search dead ends)

5.4 Provide relevancy ranking of search results

5.5 Continue working with available technologies (but look for better ones) to federate discovery and delivery of books, journals, and journal articles

5.6 Link the user to full text whenever possible

5.7 For items that cannot be delivered instantly, offer a range of unmediated, quick delivery options 5.8 Provide and market extended-hour hotlines, rush, and troubleshooting services to help users quickly solve delivery or connection problems

5.9 Push library metadata and links out to course Web pages and portals

5.10 Take advantage of e-commerce functions to serve non-members of the library community
} 
University of California ${ }^{25}$ che University of California pubblicava nel dicembre 2005 allo scopo di orientare le scelte catalografiche dell'ateneo.

Il proposito era di ridisegnare l'OPAC allo scopo di migliorare la ricerca e il recupero delle informazioni incoraggiando la promozione di nuove pratiche di catalogazione e potenziando in particolar modo l'accesso alle risorse digitali. Non è una novità che il punto di partenza delle proposte sia la constatazione dei cambiamenti in atto nella diffusione e nell'organizzazione delle risorse digitali offerte dai servizi commerciali del web quali Amazon, Google e i Tunes e un sempre più deciso orientamento degli utenti in favore di questi strumenti, dovuto principalmente alla percezione di immediatezza e alla facilità di uso che essi sembravano infondere nei loro utilizzatori ${ }^{26}$.

Il rapporto metteva in evidenza la questione dell'allargamento dell'OPAC: il trattamento delle fonti bibliografiche messo in atto dalle biblioteche, che solitamente offrono un accesso separato a tali fonti (catalogo, periodici elettronici, banche dati, archivi istituzionali, web) per la necessità di un diverso trattamento organizzativo e gestionale delle stesse, è in realtà oscuro all'utente in quale invece vorrebbe consultarle in un solo strumento $^{27}$. Nella visione del rapporto aggiungere valore al catalogo significa primariamente la messa in atto di tutti i collegamenti necessari tra le risorse digitali. Particolarmente significativo è il raggruppamento delle raccomandazioni nelle tre aree del potenziamento della ricerca, della ristrutturazione dell'OPAC e dell'adozione di nuove pratiche catalografiche ${ }^{28}$.

25 La biblioteca dell'università della California è la seconda biblioteca statunitense per importanza e ricchezza documentaria dopo la Library of Congress. Nella metà del 2005 all'interno del SOPAG (uno dei suoi comitati permanenti, incaricato di armonizzare i vari applicativi usati per supportare i servizi bibliografici nelle varie aree operative della biblioteca in modo da garantirne l'interoperabilità, venne istituito un apposito gruppo di lavoro incaricato di formulare una serie di raccomandazioni sul modo di riorganizzare $\mathrm{i}$ servizi bibliografici. Cfr. Bibliographic Services Task Force. University of California libraries, Rethinking how we provide bibliographic services for the University of California. Final report: December 2005, $<$ http://libraries.universityofcalifornia.edu/sopag/BSTF/Final.pdf>.

${ }^{26}$ Secondo il rapporto il catalogo non avrebbe saputo sfruttare a sufficienza i cambiamenti in atto nel modo di diffondere e organizzare le risorse digitali essendo percepito come uno strumento nel quale verificare dati ottenuti dai motori di ricerca e dalle librerie on- line, oppure come dispositivo per la localizzazione degli esemplari e per il prestito.

27 « The current Library catalog is poorly designed for the tasks of finding, discovering, and selecting the growing set of resources available in our libraries. It is best at locating and obtaining a known item. For librarians and for our users, the catalog is only one option for accessing our collections. We offer a fragmented set of systems to search for published information (catalogs, A\&I databases, full text journal sites, institutional repositories, etc) each with very different tools for identifying and obtaining materials. For the user, these distinctions are arbitrary» (ivi, p. 2). La conseguenza è che, «di fronte alla scelta fra più risorse, ciascuna munita di un proprio dispositivo di ricerca, sia sul web, sia all'interno della singola biblioteca, l'utente "che non ha tempo da perdere", si rivolge non alla singola risorsa, ma alla rete nel suo complesso, considerandola alla stregua di una risorsa unitaria e facendo uso di strumenti, come i motori, che tendono a conferire omogeneità a risposte provenienti per struttura, finalità, origine, stato di aggiornamento e così via» (Paul Gabriele Weston - Salvatore Vassallo ... “e il navigar m'è dolce in questo mare” cit., p. 163). ${ }^{28}$ Le tre categorie di intervento sono nel dettaglio: 
Anche in questo caso, come è avvenuto per la Library of Congress, emerge dal rapporto (per ridurre i costi della catalogazione manuale) la necessità di ripensare le procedure catalografiche, favorendo l'indicizzazione automatizzata dei contenuti dei documenti con un preciso richiamo alla possibilità di utilizzare metadati arricchiti (ad esempio, tables of contents o TOC) al posto dei soggetti e delle classificazioni, laddove si suggerisce l'abbandono di questi vocabolari:

Consider using controlled vocabularies only for name, uniform title, date, and place, and abandoning the use of controlled vocabularies [LCSH, MESH, etc] for topical subjects in bibliographic records. Consider whether automated enriched metadata such as TOC, indexes can become surrogates for subject headings and classification for retrieval ${ }^{29}$.

Infine, non vanno dimenticate le iniziative catalografiche che $\mathrm{OCLC}^{30}$, la più grande organizzazione non profit di servizi alle biblioteche, ha intrapreso per rinnovare il proprio catalogo collettivo Worldcat. Tra le iniziative va ricordato lo sviluppo di un software che ha consentito di ottenere per via algoritmica il raggruppamento bibliografico in base all'entità opera secondo il modello FRBR nella visualizzazione di WorldCat e quella di

1) Miglioramento della ricerca e dell'accesso all'informazione

* fornire agli utenti l'accesso diretto al documento

* suggerire la lettura di documenti che abbiano caratteristiche e contenuti simili a quelli ricercati

* favorire la personalizzazione permettendo all'utente di scegliere le risorse / basi dati da consultare, includendo un più vasto assortimento di risorse più ampio di quello supportato dagli odierni sistemi di metaricerca

* suggerire strategie di ricerca alternative, in special modo quando la ricerca non produce pochi risultati o risultato nullo. I suggerimenti dovrebbero riguardare termini alternativi, correlati, offrendo la possibilità di espandere la consultazione ad altri cataloghi quali Worldcat, offrendo un accesso a Google o dando all'utente la possibilità di chiedere informazioni al bibliotecario

* offrire una una migliore navigazione nel caso di risultati molto numerosi, implementando i concetti esposti in FRBR che permettono di distinguere l'opera, le sue espressioni (es le traduzioni in altre lingue) e differenti manifestazioni (edizioni o versioni di queste ultime)

* fornire i servizi bibliografici dove si trovano gli utenti

* introdurre criteri di ranking nei risultati

* agevolare la ricerca in ambienti multilingua

1) Ristrutturazione dell'OPAC

* creare una interfaccia unica per tutti i cataloghi

* unificare la ricerca per tutte le risorse informative e fornire i risultati raggruppati per formato, o in termini di granularità o altre faccette, con la possibilità per l'utente di scegliere i criteri di raggruppamento

2) Adozione di nuove pratiche catalografiche

* ripensare il flusso operativo, eliminando duplicazioni e massimizzando l'efficienza

* scegliere lo schema di metadati più idoneo a ciascuna tipologia di documento, evitando di applicare il Marc, le AACR2 o le LCSH ovunque.

* arricchire manualmente i metadati in particolar modo quelli di specifici elementi (quali nomi, titoli delle serie) o particolari ambiti (musica, letteratura ..)

* automatizzare la creazione di metadati.

29 Bibliographic Services Task Force. University of California libraries, Rethinking how we provide bibliographic services cit., p. 4 (§III.2c).

${ }^{30}$ OCLC (<http://www.oclc.org >) è la più vasta organizzazione non-profit di servizi informatici alle biblioteche che serve più 60.000 biblioteche in 112 paesi. OCLC e le biblioteche che vi aderiscono producono in cooperazione il noto catalogo collettivo WorldCat (<http://www.oclc.org/us/en/worldcat/default.htm>). 
rendere accessibile l'immenso contenuto del proprio catalogo sul web, permettendo di recuperare le descrizioni bibliografiche dai principali motori di ricerca Google e Yahoo ${ }^{31}$.

La strategia catalografica intrapresa da OCLC si è svolta nella direzione, già delineata nel rapporto Calhoun, di fornire strumenti centralizzati di ricerca e rappresenta, dunque, in chiusura di questa breve rassegna, uno dei primi esiti del dibattito sul rinnovamento dei cataloghi elettronici che si è concretizzato nella realizzazione del primo 'sistema per la scoperta' (discovery system), disponibile per le biblioteche partners OCLC e per il mercato mondiale.

Il progetto pilota Worldcat local, avviato nell'aprile $2007^{32}$, ha avuto come obiettivo quello di offrire una ricerca globale a partire da un unico strumento. WorldCat Local ${ }^{33} \mathrm{si}$ presenta come una versione 'su misura' di Worldcat.org che le biblioteche sostituiscono al proprio OPAC locale consentendo all'utente di interrogare contemporaneamente sia il catalogo locale sia Worldcat, in quanto vengono mostrati per primi i risultati della biblioteca e poi tutti quelli delle biblioteche aderenti al catalogo collettivo ${ }^{34}$.

A più di un quinquennio dal dibattito che ha visto la nascita e la diffusione di OPAC di nuova generazione e di sistemi per la scoperta, le cui caratteristiche e funzionalità verranno esposte nel capitolo seguente, lo scenario tecnologico oggi subisce una nuova e profonda evoluzione che aspira a rivoluzionare il mondo dell'informazione e a ricondurlo alla sua essenza primaria: il dato.

Le relazioni tra dati bibliografici e di altra natura rese possibili dall'adozione dei linked data, nuova tecnologia del Web Semantico, consentiranno di produrre cataloghi profondamente integrati con il resto del web, imprimendo una svolta decisiva nella struttura dei record catalografici e nelle funzioni che il catalogo potrà svolgere all'interno del più ampio spazio globale dell'informazione.

Il più recente confronto sul rinnovamento dei cataloghi elettronici si arricchisce oggi di nuove tematiche che emergono da questi sviluppi e che aprono nuove prospettive per i cataloghi elettronici nella direzione dell'integrazione dei dati catalografici nel web:

\footnotetext{
${ }^{31}$ Un accordo siglato nel 2004 con le due aziende e successivamente con altri partner ha previsto che i contenuti del catalogo collettivo Worldcat possano essere recuperati tramite una ricerca in internet, iniziativa che viene giustificata con il tentativo di rafforzare il ruolo delle biblioteche come fonte per la ricerca on-line. I risultati raccolti da WorldCat vengono mostrati tra i risultati di un'interrogazione nei motori con l'etichetta 'find in a library', collegando direttamente al posseduto delle biblioteche, ai loro OPAC o al full text.

${ }^{32} \mathrm{Al}$ progetto hanno aderito inizialmente l'Università di Washington ed una decina di biblioteche statunitensi. L'annuncio è ancora visibile in <http://www.oclc.org/news/releases/200659.htm>.

${ }^{33} \mathrm{Si}$ veda infra, Appendice B, Software per OPAC di nuova generazione e strumenti per la scoperta.

${ }^{34}$ Roy Tennant, non a caso, intitola Demise of local catalog ossia 'la morte del catalogo locale' un suo articolo dove annuncia, anche se con un certo ottimismo, questa nuova possibilità offerta da OCLC (Cfr. Roy Tennant, Demise of the local catalog, «Library Journal», 132 (2007), n. $12, \quad$ p.28. <http://www.libraryjournal.com/article/CA6457238.html>).
} 
Se in altre epoche la biblioteca si poteva considerare la fonte principale di informazione, oggi tutto ciò non è più vero. Ma questo, in sé, non è un problema. Dovremmo essere felici per la crescita dell'informazione e del suo uso, e per le conseguenti possibilità di creare una società sempre più informata, sempre più rappresentativa di un popolo attivo, che fa sentire la sua voce. Il problema, invece, è che le biblioteche sono lontane dalla principale fonte odierna di informazioni, cioè dal web. La spinta a muovere le biblioteche verso i linked data non risponde semplicemente al desiderio di modernizzare i cataloghi, ma rappresenta la necessità di trasformare i cataloghi delle biblioteche da database chiuso e separato in un sistema integrato con la tecnologia che la gente utilizza per la ricerca e per la creazione di nuove idee. Insomma, il catalogo della biblioteca, per essere visibile all'utente di oggi, deve cessare di essere un'entità staccata, un database separato e divenire un insieme di dati, milioni di dati tra gli innumerevoli dati del $w_{e b}{ }^{35}$.

Il quadro è arricchito anche dai nuovi approdi che provengono da un decennio ricco di iniziative catalografiche, originati dal ripensamento complessivo di principi e funzioni avviate dallo studio FRBR; lo studio rappresenta ad oggi la riflessione più matura sulla natura del catalogo e le sue funzioni, proponendo un modello di rappresentazione delle entità che compongono l'universo bibliografico che ancora le biblioteche non hanno applicato alla struttura del catalogo elettronico.

Di fronte alla maturazione della riflessione catalografica contemporanea ed un contesto informativo così profondamente cambiato rispetto al passato, all'entrata in scena di nuove tecnologie che cambieranno radicalmente i meccanismi di creazione, distribuzione e utilizzo dei dati bibliografici, ci si chiede quali saranno i futuri spazi per il catalogo elettronico nel nuovo contesto e quali le sue funzioni. Tali interrogativi ci portano focalizzare l'attenzione sulla natura del catalogo e delle sue funzioni.

\subsection{Il catalogo come 'linguaggio' della biblioteca nel nuovo contesto informativo}

Nell'affrontare le questioni dell'analisi della 'natura' del catalogo e delle sue funzioni non si può prescindere da un'indagine più profonda e dalla comprensione dei processi selettivi e comunicativi che attraverso di esso si manifestano nei confronti dell'universo documentario che il catalogo è chiamato a rappresentare e ordinare e dell'utente al quale esso è rivolto.

\footnotetext{
${ }^{35}$ Karen Coyle, Linked Data: an Evolution, Karen Coyle, Linked Data: an Evolution, «JLIS.it», 4 (2013), n. 1, p. 53-62, <http://leo.cilea.it/index.php/jlis/article/view/5443/7889>. Cit. a p. 3. Il contributo riprende la relazione presentata al Convegno Global interoperability and linked data in libraries, Firenze, 18-19 giugno, 2012.
} 
Il catalogo è lo strumento attraverso il quale la biblioteca opera un' 'interpretazione' dell'universo documentario servendosi del 'linguaggio bibliografico' che si esprime nell'evidenziare attraverso gli strumenti del controllo di autorità, della descrizione bibliografica, dell'indicizzazione nominale e semantica, i tratti semiotici e concettuali della produzione documentaria, cioè dell'universo bibliografico che vi è rappresentato.

La 'natura' e il 'valore' del catalogo vanno, dunque, ricercate nel suo porsi come apparato 'strumentale, ${ }^{36}$, interpretativo e di mediazione tra l'universo documentario e l'utente; in questo senso il catalogo rappresenta il 'linguaggio' della biblioteca.

Il 'linguaggio bibliografico' adoperato dal catalogo si pone come una soluzione coerente e 'necessaria', ma allo stesso tempo imperfetta e contingente, per consentire all'utente di esplorare il 'docuverso' tramite i canali e i percorsi in esso suggeriti ${ }^{37}$.

Esula dai contenuti della presente ricerca l'indagine teorica sulle implicazioni di tale operazione 'di controllo, sistematizzazione, osservazione e interpretazione che il catalogo impone 'per necessità', e che ci porterebbero ad affrontare i nodi, già ampiamente dibattuti, dei fondamenti teorici delle discipline bibliografiche e biblioteconomiche, sui quali già vari autori si sono espressi con autorevoli contributi ${ }^{38}$. Ci limiteremo, invece, in questa sede alla constatazione che il catalogo riflette il ruolo che la biblioteca ha rappresentato, sin dai tempi più lontani, all'interno della società, come luogo dell'organizzazione, della selezione e della conservazione della conoscenza registrata ai fini della ricerca dell'informazione e suo recupero per mezzo dei connotati semiotici e semantici dei documenti.

La riflessione sulla natura, sulle funzioni e in definitiva sul futuro del catalogo non può essere dunque separata della più ampia riflessione che investe natura, compiti e confini della Biblioteconomia del ventunesimo secolo. Si tratta di una riflessione epistemologica

\footnotetext{
${ }^{36}$ Ancora valida è l'affermazione di Diego Maltese (Diego Maltese, La biblioteca come linguaggio e come sistema, Milano, Editrice Bibliografica, 1985, p. 7) ove dichiara che il catalogo assolve ad una funzione vicaria nei confronti del patrimonio librario.

${ }^{37}$ Un'interessante analisi della 'natura' del catalogo e della sua funzione strumentale e vicaria dell'universo bibliografico ivi rappresentato è quella recentemente proposta da Roberto Ventura, (Il senso della biblioteca. Tra biblioteconomia, filosofia e sociologia, Milano, Bibliografica, 2011 p. 90) laddove afferma: «l'idea di catalogo in quanto mappa delle conoscenze può essere accettata nei termini di una contingente sistemazione documentaria che, non rivelando alcuna struttura sottostante dell'universo bibliografico, e facendo riferimento ad un determinato codice linguistico scelto tra le molteplici codificazioni possibili, si mostra, più o meno efficacemente, capace di consentire l'identificazione e il recupero di documenti presumibilmente e approssimativamente significativi per l'utente a partire da determinate chiavi di ricerca. Il catalogo, in altre parole, è una struttura necessaria e insufficiente che si costituisce successivamente e in modo indipendente dalla formazione dell'universo documentario stesso, nonostante i tentativi di aboutness bibliografica posti in essere dal bibliotecario [...] si tratta di instaurare, per lo meno nelle intenzioni, legami di carattere realistico, fondamentalmente dotati di stabilità, con l'universo documentario a partire da una scelta relativistica, cioè quella di effettuare un raggruppamento documentario in biblioteca».

${ }^{38}$ Tra i più recenti contributi che si interrogano sul ruolo delle biblioteche e sui fondamenti epistemologici della biblioteconomia sono da citare i saggi di Alberto Salarelli Biblioteca e identità: per una filosofia della biblioteconomia, Milano, Ed. Bibliografica, 2008 e di Roberto Ventura, Il senso della biblioteca cit.
} 
che oggi attraversa le discipline biblioteconomiche e bibliografiche e che mette in primo piano le questioni della natura della biblioteca e della Biblioteconomia, che possono essere affrontate - secondo Roberto Ventura- da almeno due diversi approcci:

1) dal punto di vista 'linguistico' in cui si misurano i codici comunicativi posti in essere dalle biblioteche (attraverso il catalogo, i servizi, gli spazi) e si confrontano con i nuovi modi di comunicazione del sapere;

2) oppure dal punto di vista sociologico (accogliendo così una visione 'sociologica' della Biblioteconomia e delle sue competenze), indagando sul ripensamento del ruolo delle biblioteche nella società contemporanea; in base a ciò Ventura afferma che «oggi il quadro problematico, tutto da districare, è dato dalla convivenza della biblioteca con la crisi dei modelli di socializzazione e i canoni comunicativi imposti dalle tecnologie digitali» e alla frattura tra biblioteche e società ${ }^{39}$.

Accogliendo quest'ultima visione, la più grande sfida del catalogo di fronte alla società postmoderna risiederebbe nella capacità di recepire il mutamento delle tecnologie in atto e le sue ricadute sulle modalità di rappresentazione, ricerca, recupero e uso dell'informazione bibliografica.

Nell'affrontare i temi della natura e delle funzioni del catalogo si fa spesso riferimento al catalogo quale 'linguaggio' della biblioteca e alla funzione di mediazione indiretta che si compie attraverso di esso tra $\mathrm{i}$ testi, rappresentati tramite surrogati ossia registrazioni bibliografiche, e l'utente quale destinatario del messaggio. È in questa cornice che possiamo affermare che il tema dell'evoluzione del catalogo elettronico si pone interamente nella sfera della comunicazione.

L'accesso ai documenti avviene, infatti, attraverso un meta-linguaggio ${ }^{40}$ che condiziona l'indeterminatezza del sistema bibliografico sotto il profilo della comunicazione. Come rilevava Ranganathan:

\footnotetext{
${ }^{39}$ Roberto Ventura, Il senso della biblioteca cit., p.43. Ventura rileva inoltre come tale frattura sia già stata evidenziata da Alberto Salarelli.

${ }^{40} \mathrm{Si}$ vedano su questi aspetti i fondamentali contributi di Alfredo Serrai Indici, logica e linguaggio : problemi di catalogazione semantica, Roma, Consiglio nazionale delle ricerche, Laboratorio di studi sulla ricerca e sulla documentazione scientifica, 1974 e Del catalogo alfabetico per soggetti: semantica del rapporto indicale, Roma, Bulzoni, 1979. La qualificazione del linguaggio bibliografico come metalinguaggio è ribadita anche in Alfredo Serrai - Marco Menato,.Biblioteche e bibliografia : vademecum disciplinare e professionale, Roma, Bulzoni, 1994, p. 155: «Poiché la bibliografia è un tipo di comunicazione che si svolge su messaggi, registrati, assume il carattere di un metalinguaggio, in quanto si riferisce, denotandoli, ad oggetti linguistici esterni che non fanno parte del discorso che li denota. Il livello metalinguistico delle formule linguistiche che li identificano, individuano, caratterizzano, qualificano, connotano, e definiscono i messaggi registrati è, pertanto, quello che spetta alle comunicazioni di natura bibliografica».
} 
Il catalogo della biblioteca è un intrico di convenzioni. È addirittura sleale, perché sembra scritto in un linguaggio familiare, naturale, mentre invece la lingua del catalogo è artificiale. La slealtà è data dal ricorso a parole ordinarie: le parole non sono morfologicamente o radicalmente artificiali, ma la sintassi sì. Anche la semantica è artificiale. L'uso dei segni di punteggiatura non è del tutto ortodosso. Gli elementi del nome di una persona sono invertiti. Tutto questo disorienta il principiante ${ }^{41}$.

I perni di questo metalinguaggio sono gli indici nominali e semantici assegnati al documento che consentono il reperimento delle notizie bibliografiche, operazione che è effettuata dal catalogatore, il quale poi dovrà fornire un ordine coerente alle registrazioni bibliografiche e si concretizzano nella costruzione di un 'sistema' in cui ogni utente potrà interagire con tali indici per recuperare l'informazione necessaria.

Possiamo, dunque, affermare che in questo senso i Principi della catalogazione, recentemente rinnovati, stabiliscano come questo metalinguaggio debba essere costruito; sebbene utilizzati per indicare i generali obiettivi del catalogo, i Principi guidano la costruzione dei linguaggi bibliografici ${ }^{42}$ e non vanno dunque confusi con le norme catalografiche.

${ }^{41}$ Shiyali Ramamrita Ranganathan, Reference service, Bangalore, Sarada Ranganathan Endowment for library science, 1961, §C25 citato in apertura del saggio di Mauro Guerrini, Catalogazione in Biblioteconomia: principi e questioni, a cura di Giovanni Solimine e Paul Gabriele Weston, Roma, Carocci, c2007, p. 185-220.

${ }^{42}$ I principi espressi nei nuovi International Cataloguing Principles sono: Interesse dell'utente, 2. Uso comune, 3. Presentazione, 4. Accuratezza, 5. Sufficienza e necessità, 6. Significatività, 7. Economia, 8. Coerenza e Standardizzazione, 9. Integrazione. (cfr.: International Federation of Library Associations and Institutions, IFLA Cataloguing Principles: Statement of International Cataloguing Principles (ICP) and its glossary edited by Barbara Tillett and Ana Lupe Cristán, München: K.G. Saur, 2009 $<$ http://www.ifla.org/VII/s13/icc/imeicc-statement_of_principles-2008.pdf> [trad. it. Dichiarazione di principi internazionali di catalogazione, Roma: ICCU, 2009, <http://www.iccu.sbn.it/genera.jsp?id=94>, oppure <http://www.ifla.org/files/cataloguing/icp/icp_2009-it.pdf>, p. 2).

In particolare a p. 2 (punto 2. Principi generali) vengono elencati i principi che guidano la costruzione dei codici di catalogazione, dopo aver affermato che il principio fondamentale è quello dell'interesse dell'utente. 2.1. Interesse dell'utente. Le decisioni adottate nel creare le descrizioni e le forme controllate dei nomi quali punti di accesso vanno prese tenendo presente l'utente;

2.2. Uso comune. Il lessico usato nelle descrizioni e nei punti di accesso deve concordare con quello della maggioranza degli utenti;

2.3. Presentazione. Le descrizioni e le forme controllate dei nomi delle entità per i punti di accesso devono essere basate sul modo in cui ciascuna entità descrive se stessa;

2.4. Accuratezza. L'entità descritta deve essere rappresentata fedelmente;

2.5. Sufficienza e necessità. Nelle descrizioni e nelle forme controllate dei nomi per i punti di accesso, devono essere inclusi solo gli elementi necessari per soddisfare l'utente e indispensabili per identificare in modo univoco un'entità;

2.6. Significatività. I dati devono essere bibliograficamente significativi;

2.7. Economia. Se per raggiungere uno scopo esistono modalità alternative, la preferenza deve essere data a quella che contribuisce maggiormente all'economia complessiva (vale a dire al costo minore o all'approccio più semplice);

2.8. Coerenza e Standardizzazione. Le descrizioni e la creazione dei punti di accesso devono essere il più possibile normalizzate. Ciò consente una maggiore coerenza, che a sua volta rende più facile la condivisione dei dati bibliografici e di autorità;

2.9. Integrazione. Le descrizioni per tutti i tipi di materiali e per le forme controllate dei nomi delle entità devono basarsi il più possibile su regole comuni. (ICP, §2, p. 2). 
I nuovi principi catalografici vanno affrontati in modo critico alla luce del nuovo contesto informativo; uno di questi, la 'convenienza dell'utente', può essere oggi variamente compreso e dare luogo ad interpretazioni errate o fuorvianti: se alcuni studi sottolineano che l'utente utilizza in definitiva solo un numero limitato di chiavi d'accesso ${ }^{43}$; tale circostanza non può risolversi nella semplificazione delle procedure di catalogazione, ma deve abilmente compensarsi con il 'principio dell'accuratezza' del trattamento bibliografico.

Il principio di comodità o convenienza dell'utente sembra oggi ispirare quelle politiche bibliotecarie e prassi catalografiche, che si riflettono negli 'OPAC di nuova generazione' e nei 'sistemi per la scoperta', tese a semplificare il numero di elementi della descrizione che vengono proposti all'utente nelle interfacce questi nuovi strumenti. Tale principio, invece, non dovrebbe essere accolto in maniera acritica come pretesto per la semplificazione dell' accesso bibliografico e per la riduzione dei costi della catalogazione ${ }^{44}$. Una compensazione tra i due principi dovrebbe risolversi, semmai, nella possibilità, a fronte della presentazione di una scheda con descrizione bibliografica ridotta, di ampliare $\mathrm{i}$ contenuti e visualizzare una descrizione più dettagliata per utenti esperti e i bibliotecari.

Tutti i principi vanno, dunque, tra loro adeguatamente conciliati in quanto ognuno di essi può far intraprendere strade diverse o talora divergenti alla strutturazione del catalogo ${ }^{45}$.

La catalogazione è influenzata fortemente dalla strategia e dalla politica bibliografica; a tale proposito Svenonius afferma come una fonte di problemi nell'organizzare l'informazione nasca dalla necessità di mantenere il passo con il progresso scientifico e tecnologico:

Un esempio di come il progresso tecnologico da adito a problemi è l'invenzione dei nuovi media, che ha costretto i sistemi bibliografici a rendere più generale il loro ambito, dai libri a qualsiasi mezzo che può comunicare informazioni. Un esempio di progresso politico che richiede adattamenti è la nascita dell'internazionalismo, che ha costretto questi sistemi ad estendere il loro raggio d'azione dal controllo bibliografico locale a quello universale [...]

Tali principi, frutto delle elaborazioni della teoria catalografica della Great Tradition sono stati rielaborati da Svenonius. Cfr. Elaine Svenonius, Il fondamento intellettuale dell'organizzazione dell'informazione cit., p. 96-117.

${ }^{43}$ Ivi, p. 99: Svenonius afferma che alcuni studi rivelano che l'utente si serve per la ricerca di un numero limitato di metadati che di regola corrispondono a quelli rilevabili nelle citazioni bibliografiche: autore, titolo, numero di edizione, editore, luogo di pubblicazione e data. Questo dato, rileva la studiosa, non deve stupire in quanto in generale la selezione di un numero di oggetti si svolge seguendo la regola individuata da Bradford, ovvero quella oggi rivisitata nella 'teoria della coda lunga' (cfr.: Chris Anderson, La coda lunga. Da un mercato di massa a una massa di mercati, trad. it. di Susanna Bourlot, Torino, Codice, 2007).

${ }^{44}$ Il riferimento è alle pratiche di abbandono dell'indicizzazione semantica, già evidenziate nel dibattito statunitense e alla semplificazione delle interfacce a discapito delle informazioni semantiche che oggi interessa la maggior parte di degli strumenti per la scoperta.

${ }^{45}$ Cfr. Roberto Ventura, Il senso della biblioteca cit., p. 149-152. 
$\mathrm{Ne}$ è un esempio il problema che nasce dal conflitto di due principi - quello della standardizzazione universale e quello della convenienza dell'utente. Culture e sotto-culture diverse classificano in modo differente, si servono di linguaggi di ricupero differenti, e sottostanno a convenzioni differenti relative ai nomi. Il problema tecnico da risolvere è come fornire la variante locale senza abrogare gli standard che facilitano il controllo bibliografico universale ${ }^{46}$

Alla luce delle nuove tecnologie del Web Semantico, possiamo affermare come tali principi oggi possano essere reinterpretati ed acquistare nuovo vigore nel contesto dei dati collegati: i linked data, come vedremo, consentono di rileggere le condizioni in cui avviene il controllo bibliografico, conciliando il principio di convenienza dell'utente con quello della standardizzazione e della coerenza.

Nello scenario tutto nuovo che si presenta oggi con lo sviluppo delle tecnologie del Web Semantico, è, dunque, possibile risolvere uno dei più antichi problemi che affliggono il controllo bibliografico:

La fonte più antica e più duratura di problemi che frustrano il lavoro del controllo bibliografico è il linguaggio usato nel tentativo di accedere all'informazione [...] pare che la fatica più colossale di tutte, concernente l'organizzazione dell'informazione, sia creare un linguaggio di descrizione non ambiguo, un linguaggio che conferisca sistematicità e metodo alla lingua naturale e allo stesso tempo permetta agli utenti di trovare ciò che vogliono con i nomi che conoscono ${ }^{47}$.

Si può riconoscere che gli obiettivi bibliografici che il catalogo persegue, ovvero le funzioni riconosciute al catalogo di una biblioteca, oggi riflettano ancora le linee essenziali stabilite da Charles Ammi Cutter nel 1876 nelle Rules for a printed dictionary catalogue $^{48}$; cioè richiedono al catalogo di:

1. mettere in grado una persona di trovare un libro di cui si conosca
a. l'autore;
b. il titolo;
c. il soggetto;

2. mostrare che cosa la biblioteca possiede

d. di un determinato autore;

e. su un determinato soggetto;

f. in un particolare genere letterario;

3. facilitare la scelta di un libro

g. attraverso le sue edizioni (in senso bibliografico);

\footnotetext{
${ }^{46}$ Elaine Svenonius, Il fondamento intellettuale dell'organizzazione dell'informazione cit., p. 17-18.

${ }^{47}$ Ivi p. $18-19$.

${ }^{48}$ Charles Ammi Cutter, Rules for a printed dictionary catalogue, in Department of Interior, Bureau of Education, Public libraries in the United States of America. Their history, condition, and management.Special report, Washington, Government Printing Office, 1876.
} 
h. attraverso la sua caratterizzazione (in senso letterario o topico $)^{49}$.

Gli obiettivi cutteriani definiscono le principali funzioni del catalogo: il primo obiettivo si riferisce a quella che viene definita funzione di 'reperimento' che consente all'utente di 'trovare' un documento partendo da un elemento noto (ad esempio, un autore, un titolo o un soggetto); la seconda fa riferimento alla funzione di 'raggruppamento', che consiste nel mostrare all'utente documenti che hanno un elemento in comune (ad esempio lo stesso autore, lo stesso soggetto o lo stesso genere letterario); il terzo obiettivo consiste nella possibilità di scelta tra documenti simili (es. due edizioni della stessa opera). Tali obiettivi, riveduti negli anni ' 60 da Lubetzky, sono stati riletti mettendo in evidenza le due principali funzioni di 'localizzazione' e 'raggruppamento'.

Oggi si ha l'impressione che tali obiettivi, delineati oltre un secolo fa da Cutter e tutto sommato arrivati fino a noi nelle elaborazioni dei Principi di Parigi e nei nuovi International cataloguing principles, non siano adeguatamente implementati nei cataloghi elettronici.

Nei nuovi Principi Internazionali di catalogazione (ICP), dei quali si parlerà approfonditamente più avanti, gli obiettivi sono stati riformulati in seguito allo studio FRBR e, successivamente, alle indicazioni provenienti dal saggio di Elaine Svenonius. I cinque obiettivi del catalogo sono oggi quelli di:

* trovare singole risorse documentarie o insiemi di risorse sulla base degli attributi e delle relazioni tra le entità;

* identificare una risorsa bibliografica o un agente;

* selezionare risorse rilevanti per l'utente in base alle chiavi di ricerca utilizzate;

* acquisire o ottenere l'accesso ai documenti e ai dati bibliografici;

* navigare, a livello globale, nei dati bibliografici per mezzo delle relazioni che sussistono tra le entità.

Nel corso di un secolo il catalogo ha visto mutare la sua forma: dai cataloghi in volume si è evoluto nel catalogo a schede e successivamente nel catalogo elettronico; tale percorso evolutivo ha comportato almeno due conseguenze:

1) una perdita di 'struttura bibliografica', in quanto la struttura a più livelli presente nel catalogo in volume e nei cataloghi a schede mobili non è stata adeguatamente riproposta nel catalogo elettronico, provocando la perdita della struttura sindetica, ovvero

\footnotetext{
${ }^{49}$ Mauro Guerrini, Catalogazione, Roma, AIB, 2005, p. 8.
} 
della rete di collegamenti tra le notizie bibliografiche che ha lo scopo di guidare gli utenti nella conduzione della propria ricerca;

2) un conflitto tra le due principali funzioni del catalogo, quella di 'reperimento' ovvero quella che risponde alle richieste di uno specifico documento, e quella di 'raggruppamento' ovvero quella che risponde alla richiesta di una classe di documenti che hanno una caratteristica in comune.

Lubetzky ha osservato «che utilizzare le potenzialità dell'elaboratore di ricuperare un certo libro per mezzo di alcuni elementi distintivi del frontespizio supera l'intera questione che riguarda l'uso del nome dell'autore. Si deve però notare che i cataloghi elettronici soddisfano solo la prima funzione del catalogo: aiutare il lettore a trovare il particolare libro che cerca ${ }^{50}$. La mancata applicazione dei modelli FRBR al catalogo, tematica che verrà approfondita nei capitoli successivi, penalizza fortemente l'utilizzo dei cataloghi elettronici.

Di fronte alle nuove sfide poste dalle tecnologie digitali, l'accesso catalografico oggi richiede un ripensamento che non solo recuperi l'approccio cutteriano ove catalogazione semantica e nominale confluiscono in un unico strumento, senza che l'una prevalga sull'altra e che si ispiri ai modelli dell'IFLA per rappresentare le entità di interesse dell'utente, ma che faccia convivere vecchi strumenti con nuove dimensioni della ricerca che oggi sono possibili attraverso le tecnologie del web.

Non si tratta dunque solamente di offrire all'utente la possibilità di compiere salti 'dimensionali' dalla dimensione nominale a quella semantica, ma di immaginare il catalogo come possibile punto di partenza per un'esplorazione personalizzata che oggi si arricchisce di nuove possibilità offerte dalle tecnologie del Web Semantico.

L'avvento delle tecnologie digitali, che investe direttamente le modalità di produzione, comunicazione e ricerca dell'informazione, provoca oggi un ripensamento teorico della disciplina, dei suoi ambiti di studio, dei suoi modelli di comunicazione, e porta la biblioteca ad interrogarsi sul suo 'ruolo' e sul suo posizionamento nella società. Di fronte ad un contesto profondamente mutato le biblioteche devono acquistare una consapevolezza critica e proporsi come spazio di crescita recependo le innovazioni che la contemporaneità impone, contando non solo su strumenti e pratiche acquisite in secoli di

\footnotetext{
${ }^{50}$ Carlo Bianchini - Mauro Guerrini, Quis custodiet ipsos custodes? : osservazioni sulle relazioni fra FRBR, $I C P, I S B D$ e RDA, «Il bibliotecario», $3^{\mathrm{a}}$ serie (2009), n. 1, p. 5. (L'articolo è la versione italiana rielaborata $\mathrm{e}$ ampliata del contributo From bibliographic models to cataloging rules: remarks on FRBR, ICP, ISBD, and RDA and the relationships between them. «Cataloging \& classification quarterly», 47 (2009), n. 2, p. 105124).
} 
storia, ma cercando la contaminazione con il mondo circostante, senza perdere di vista le proprie finalità.

Se ogni forma di sapere è necessariamente storica, così lo sono anche i meccanismi che regolano l'accesso bibliografico che si realizza nel catalogo. A cambiare sono, infatti, afferma Mauro Guerrini- le tre componenti dell'accesso bibliografico già individuate da Ranganathan $^{51}$ :

* l'universo dei fruitori ovvero gli utenti;

* l'universo della conoscenza registrata ovvero i documenti in esso rappresentati;

* l'universo degli strumenti della mediazione informativa.

Il catalogo elettronico, quale strumento con cui la biblioteca 'dialoga' con l'utenza di riferimento e, dunque, storicamente determinato dal contesto informativo e dalle esigenze dell'utente, deve adattarsi ai mutamenti di contesto e misurarsi costantemente con il mutare delle condizioni storiche in cui si realizza l'accesso alla conoscenza ${ }^{52}$.

Queste considerazioni introducono la riflessione su quelle spinte 'esterne' che condizionano l'evoluzione del catalogo, cioè portano a considerare i condizionamenti della società contemporanea e l'impatto che le nuove tecnologie stanno avendo sulla vita umana e sui comportamenti sociali e sulle abitudini di ricerca degli utenti, indagando la fisionomia dell'utente 'postmoderno' e dei nuovi 'nativi digitali'. Tali condizionamenti vengono rilevati nelle nuove modalità che l'utente manifesta nella fruizione del catalogo elettronico nei termini di 'consumo' e dalle nuove modalità di interazione e uso dell'informazione fortemente condizionate dalle tecnologie digitali.

\subsection{L'utente 'postmoderno' e i 'nativi digitali'}

Nel dibattito che ha visto vari studiosi interrogarsi sulle possibilità di miglioramento delle funzionalità offerte dagli OPAC si invoca sovente il ruolo dell'utente quale naturale

\footnotetext{
51 Mauro Guerrini, I Principi internazionali di catalogazione (ICP). Universo bibliografico e teoria catalografica all'inizio del XXI secolo, postfazione a cura di Attilio Mauro Caproni, Milano, Editrice Bibliografica, 2012, p. 297

${ }^{52} \mathrm{La}$ storicità del catalogo è stata invocata da Diego Maltese e da Luigi Crocetti, che avvertiva - in un contributo ormai classico e in momento storico che vedeva il lento passaggio dal catalogo cartaceo alla sua materializzazione elettronica - come il catalogo sia «uno strumento storicamente determinato: legato quindi ai bisogni di chi lo usa da una parte, ai modi in cui si organizza e si manifesta il processo informativo dall'altra» (Luigi Crocetti - Rossella Dini, ISBD (M). Introduzione ed esercizi, 3. ed., Milano, Editrice Bibliografica, 1995, p. 11).
} 
destinatario del catalogo, sia in termini di funzionalità delle registrazioni bibliografiche rispetto alle aspettative e necessità dell'utenza, sia in termini di rispondenza ai condizionamenti che le nuove modalità di ricerca del web impongono alla ricerca bibliografica.

Nella disamina del rapporto utente-catalogo rientrano non solo quegli aspetti soggettivi o psicologici che influiscono sul processo di ricerca, ma anche il condizionamento proveniente dal contesto sociale nel quale egli è inserito.

L’indagine così si apre ad approfondire meglio le influenze provenienti dai mutamenti sociali e tecnologici che condizionano il rapporto utente-OPAC e possono spiegare più a fondo le modalità d'uso dei cataloghi elettronici, ma allo stesso tempo anche le motivazioni del non uso dei cataloghi in favore di altri strumenti di ricerca del web.

Alla domanda 'Chi è l'utente del catalogo?' non si può rispondere se non tracciando il 'quadro della complessità' che caratterizza l'uomo contemporaneo nell'epoca attuale e che coinvolge i due concetti chiave di postmodernità e rivoluzione digitale.

L'epoca in cui stiamo vivendo è quella che $i$ filosofi definiscono della 'postmodernità ${ }^{53}$, ma anche nei termini di 'società post-industriale', 'società complessa',

\footnotetext{
${ }^{53}$ Il termine 'postmoderno' viene usato a partire dagli anni Sessanta in America e poi in Europa per denotare alcune pratiche culturali che si manifestarono in diverse discipline (letteratura, filosofia, teatro, architettura, arti figurative) o per riferirsi al mutamento della società postindustriale. Con il termine si indica una nuova comprensione della nostra era in opposizione al concetto di moderno. La nascita del postmoderno in filosofia si fa risalire alla pubblicazione del volume di François Lyotard La condition Postmoderne: rapport sur le savoir (Paris, Éditions de Minuit, 1979, in traduzione italiana: Jean Francois Lyotard, La condizione postmoderna, Milano, Feltrinelli, 1979), considerato unanimemente il 'manifesto' della postmodernità.

Il pensiero postmoderno è stato per lo più elaborato dai filosofi francesi, tra i quali oltre a Jean Francois Lyotard, sono da menzionare almeno Jean Baudrillard, Jacques Derrida, Paul Michel Foucault e in Italia, Gianni Vattimo.

Varie critiche sono state avanzate allo stesso termine 'postmoderno' che non viene inteso come fase tarda della modernità, bensì come superamento dell'epoca moderna e dunque all'insegna della rottura e della discontinuità con l'epoca moderna, come nuovo schema interpretativo rispetto ad esso.

Prima della postmodernità negli anni sessanta in america veniva usata la locuzione postindustriale a denotare la trasformazione delle tecnologie e il passaggio da un'economia fordista ad una società basata sul terziario e sui servizi (cfr. Alain Touraine, La società post-industriale, trad. it di Rolando Bussi, Bologna, Il Mulino, 1970 e id., Critica della modernità, trad. it. di Francesco Sircana, Milano, Il Saggiatore, 1997). Tale mutamento segnerà poi una profonda accelerazione con il passaggio dall'industria meccanica a quella elettronica, fino alle tecnologie delle comunicazioni e alle reti di dati. Il postmoderno afferma la forza del sapere come principale forza produttiva, concetto già delineato da Bell e ripreso da Lyotard e, più di recente da Jeremy Rifkin che, all'inizio del decennio scorso, ha teorizzato la nascita di una nuova economia, la net economy, dove il valore è generato dalla conoscenza e da un'economia priva di peso. Jeremy Rifkin, L'era dell'accesso. La rivoluzione della new economy, trad. it. di Paolo, Canton, Milano, Mondadori, 2000.

Tratti salienti del postmoderno sono «il rifiuto di ideologie totalizzanti, il pluralismo, il relativismo, il pensiero debole, l'olismo, l'incredulità, l'apparenza, lo spettacolo, il disincanto, l'ironia, il gioco, il sincretismo, l'ossimoro, il flusso, la superficie, la citazione, il collage, il recupero selettivo del passato, la quotidianità, l'assenza di regole, la parodia, il polimorfismo/camaleontismo, il meticciato, il multiculturalismo, la presentificazione, l'irriverenza, la pluralità degli stili e dei linguaggi, le coordinate plurime» (Giampaolo Fabris, Societing cit., p. 120).

Tratti comuni della postmodernità e del suo discorso teorico, letterario o artistico vengono ricondotti da Ihab Hassan ad alcuni punti:
} 
'post-materialistica', o 'post-capitalistica'; alcuni concetti chiave come differenziazione sociale e complessità, policentrismo e frammentazione, moltiplicazione delle realtà, delle logiche e dei contesti sono spesso citati per definire la realtà sociale contemporanea.

In questa sede ci preme considerare quali siano quei connotati della postmodernità che possono influenzare l'uso e le modalità di ricerca che gli utenti manifestano nell'interazione con il catalogo.

\section{L' Utente - consumatore}

Il tratto più squisitamente 'postmoderno' dell'utente, che oggi i nuovi cataloghi elettronici hanno tentato di interpretare nelle loro tensione al cambiamento, è certamente quella dell'utente visto nella dimensione di 'homo consumens ${ }^{54}$. Le azioni di consumo coprono nella società postmoderna una sfera significativa dell'agire umano; esse sono legate profondamente alla crescita del benessere economico e all'imporsi di un'ideologia del consumismo che ha profondamente degradato le relazioni sociali provocando una

* Indeterminazione, riferita alle rotture e alle ambiguità del linguaggi della conoscenza del postmoderno

* Frammentazione come luogo dell'indeterminazione. Il postmoderno sconnette e ripone la propria fiducia nei frammenti, contro ogni visione totalizzante e predilige il collage, il paradosso, gli objects trouves.

* Decanonizzazione o delegittimazione dei codici dominanti, delle convenzioni e delle autorità, verso la sovversione delle norme, la decostruzione, la demistificazione e il decentramento.

* Vacanza del sé, non ci sono profondità ma solo la superficie e l'uomo è in balia del gioco del linguaggio che dissemina significati e disperde il sé.

* L'impresentificabile si riflette nell'arte postmoderna che si confronta con stati subliminari (sublime, abietto, ineffabile) che sfidano la capacità di rappresentazione.

* Ironia, ibridazione e carnevalizzazione che si manifestano nel dialogo, nell'allegoria, nella trasmutazione dei generi, nella parodia e nel burlesco, nella logica del rovescio e del dietro-front.

* Performance il teatro postmoderno sociale o verbale invita alla performance. Nell'arte la performance trasgredisce i generi aprendosi allo sconosciuto.

* Costruzionismo. Il postmoderno è figurativo e costruisce la realtà attraverso le finzioni post-kantiane che implicano un intervento crescente della mente nella natura e nella storia ovvero un nuovo tipo di gnosticismo. Il costruzionismo si riflette nell'arte e nella scienza nei modi della costituzione del mondo di Goodman e nelle mosse pre-figurative di Hayden White.

* Immanenza. È la capacità di estendere se stesso nei linguaggi, nei media, nelle nuove tecnologie; si riflette nella diffusione dei nostri sensi percettivi presagito da Mc Luhan e nelle nelle teorie dell'essere del linguaggio di Foucault, nel pluralismo teorizzato da Hassan.

Si veda per approfondire: Giovanni Fornero, Postmoderno e filosofia in Nicola Abbagnano, Storia della filosofia, vol 4. La filosofia contemporanea, Milano, Tea, 1996, p. 3- 48.

Al distacco e alla razionalità, alla precisione, alla logica del metodo scientifico, il postmoderno oppone l'intuizione, la creatività e le emozioni.

${ }^{54}$ Zygmunt Baumann, Homo consumens. Lo sciame inquieto dei consumatori e la miseria degli esclusi. Trento, Erickson, 2007, p. 50-51: «La società dei consumi si fonda sull'insoddisfazione permanente, cioè sull'infelicità. Una strategia per ottenere una permanente insoddisfazione è quella di denigrare la merce che è appena stata messa sul mercato dopo averla promossa come la migliore possibile. Un altro modo, più efficace e più subdolo, è quello di soddisfare così completamente ogni desiderio che non possa nascere l'impulso a desiderare qualcosa di diverso: il desiderio si trasforma in bisogno e diventa un'esigenza compulsiva e una dipendenza. E funziona, come dimostra il diffuso bisogno di fare shopping per trovare sollievo contro l'angoscia e il dolore». 
diminuzione della cosiddetta felicità percepita ${ }^{55}$. Non a caso una delle definizioni della società contemporanea è quella di 'società dei consumi' che si fonda su un'economia basata sull'eccesso e sullo spreco.

La dimensione del consumo attraversa trasversalmente tutte le diverse attività della vita quotidiana e si configura come 'agire sociale dotato di senso' ${ }^{26}$; il consumatore riveste un ruolo più dialettico con il consumo e i mercati sfumano sempre più le loro caratteristiche fisiche per divenire «luoghi di conversazione» ${ }^{57}$. Ciò che emerge è la pervasività della dimensione del consumo nell'esperienza umana; l'utente consumatore abita «un universo semiotico il cui spazio è costantemente presidiato dal sistema delle icone commerciali» ${ }^{58}$ ove assume un ruolo sempre più determinante la dimensione 'esperienziale' del consumo in cui sono le esperienze ${ }^{59}$ a dare un senso agli acquisti dato che è ormai superata l'epoca in cui l'acquisto si configurava come soddisfazione dei bisogni e della fruizione. L'esperienza si identifica nell'immersione e nel coinvolgimento con cui le persone partecipano alle esperienze individuali e sociali. «I consumi sono ormai anche una forma di linguaggio con cui [il consumatore] comunica, a se stesso e agli altri, brani della propria identità, i propri stati d'animo o i mood del momento' ${ }^{60}$.

\footnotetext{
${ }^{55} \mathrm{Si}$ veda su queste tematiche anche Zygmunt Baumann, La società sotto assedio, trad. it. di Sergio Minucci, Roma [etc.], Laterza, 2007.

${ }^{56}$ Max Weber, Economia e società. 1. Teoria delle categorie sociologiche, trad. it. di Tullio Bagiotti, Franco Casabianca, Pietro Rossi, Edizioni di Comunità, Torino 1999.

${ }^{57}$ Lo slogan 'I mercati sono conversazioni' deriva dalla prima delle 95 tesi di un documento noto come Cluetrain Manifesto: the end of business as usual documento elaborato nel settore dell'economia e che ha mirato a ridefinire la natura del mercato nell'epoca del web immaginandolo come una comunicazione tra le persone (Rick Levine - Cristopher Locke - Doc Searle - David Weinberger, The Cluetrain Manifesto. The end of business as usual, Roma, Fazi, 2001). La metafora della conversazione ha ispirato alcune visioni del futuro della biblioteca, come quella proposta da David Lankes che ha lanciato l'idea delle 'le biblioteche sono conversazioni'.

${ }^{58}$ Giampaolo Fabris, Societing cit., p. 145.

${ }^{59}$ Questa dimensione esperienziale è stata sottolineata da Lella Mazzoli che distingue tre concetti chiave per comprendere il rapporto tra aspetto razionale ed emozionale che caratterizza la società postmoderna: l'immersione ovvero «il coinvolgimento, la nuova enfasi sensoriale con cui il soggetto partecipa dell'esperienza individuale e sociale [...] la contaminazione, intesa come miscellanea di paradigmi conoscitivi, di epistemologie, di modalità e modelli cognitivi $[\ldots]$ del nomadismo. Un continuo viaggiare attraverso le esperienze, vagabondaggio costante ... rifiuto della modalità di sedentarietà che contraddistinguono lo strutturarsi della modernità. Nomadismo come deterritorializzazione, come sottrazione, erosione di significati da contesti definiti, dai percorsi conoscitivi lineari a favore di un'erranza cognitiva che porta a costruire percorsi di senso oggettivo e interoggettivo mobili, mutevoli, multiformi». (Ivi, p. 147-148). Nella letteratura biblioteconomica la dimensione dell'esperienza nella progettazione del servizio bibliotecario quale approdo per un nuovo modello di biblioteca viene proposto da Anna Galluzzi che osserva come «non è più sufficiente limitarsi ad accumulare patrimonio bibliografico e a presentarne $i$ contenuti culturali a un utente passivo, bensì è indispensabile creare dei percorsi di ricerca mettendo in campo tutti quegli strumenti a disposizione della biblioteca che sappiano catturare l'attenzione e la partecipazione degli utenti, proporsi come luogo di sperimentazione delle nuove forme di socializzazione della conoscenza, rendere unico e originale il modo in cui fruire della biblioteca. Tutto ciò significa rendere la biblioteca un luogo realmente esperienziale» (Anna Galluzzi, Biblioteche per la città, Roma, Carocci, 2009, p. 90).

${ }^{60}$ Giampaolo Fabris, Societing cit., p. 143.
} 
Le biblioteche, investite da queste nuove pratiche di consumo culturale che si riflettono nei comportamenti degli utenti devono investire in nuovi modi di comunicazione con l'utente e di utilizzo dell'informazione in cui prevalgono nuovi paradigmi quali il movimento, la liquidità, il flusso la leggerezza e la diffidenza per ogni regola: l'ossimoro, il sincretismo, il melange, il pastiche, l'eclettismo, il pluralismo, il meticciato si riflettono nei comportamenti e nei valori dell'individuo postmoderno ${ }^{61}$.

Tra le caratteristiche dell'utente postmoderno vi sono certamente quelle che caratterizzano il consumatore o l'utente contemporaneo nei termini del flaneur, individuo d'elezione della postmodernità e che comprendono la dimensione del 'nomadismo' insita nei comportamenti umani e incarnano nuove modalità di approccio ai consumi culturali e all'informazione, che si manifestano oggi nei nuovi comportamenti di ricerca e di navigazione in rete con cui le biblioteche devono misurarsi:

La navigazione curiosa, random, talvolta un po' oziosa e voyeuristica, si adatta ancora meglio al termine flaneur. Internet diviene un gettonatissimo canale per conoscere nuovi prodotti e nuovi servizi, per imbattersi casualmente in nuove sollecitazioni o proposte di consumo che interessano, che intrigano, per ottenere informazioni - sovente personalizzate che orientino i propri acquisti, magari dopo aver visto le ultime news, dato un'occhiata al sito della squadra del cuore o partecipato a un forum di discussione. ${ }^{62}$

Nella navigazione in Internet, l'utente viaggia nella storia come in una banca dati, spostandosi da un punto all'altro della rete; questa esperienza è caratterizzata dalla perdita di punti di orientamento stabili, da un continuo attraversamento di confini, da un perenne migrare che non genera disorientamento, ma una senso di libertà ${ }^{63}$.

La dimensione del 'consumo' è ben presente sia nel dibattito professionale precedentemente citato, ove abbiamo visto come il catalogo venga varie volte immaginato

\footnotetext{
${ }^{61}$ Per Derrida il collage (o montaggio) è la forma principale del linguaggio della postmodernità così come la citazione che consente di saccheggiare la storia incorporandola nel presente. Il principale merito intellettuale del sociologo inglese di origine polacca Zygmunt Bauman risiede nella sostituzione del termine di 'postmodernita', con quello di 'modernita' liquida, per distinguere questa fase dall'epoca "solida" caratterizzata dalla formazione delle nazioni moderne, dall'uniformazione sociale, dall'espulsione di tutti gli elementi non assimilabili che caratterizzarono il periodo delle guerre e degli olocausti (cfr. Zygmunt Bauman, Modernità liquida, Modernita liquida, trad. it. di Sergio Minucci, Roma -Bari, Laterza, 2010 e Zygmunt Bauman, Modernità e olocausto, trad. it. Di Massimo Baldini, Bologna, Il mulino, 2010). Per Bauman la società postmoderna è liquida; come i fluidi che non hanno una coesione interna e un'integrazione o un'attrazione interna, essa non ha alcuna forma prestabilita, ma assume la forma sempre diversa del suo contenitore, e può inoltrarsi in molteplici direzioni. Come un liquido, può cambiare costantemente forma. Principali protagonisti della società liquida sono il flaneur e il vagabondo, il turista, il giocatore. Riprendendo il pensiero di Bauman si può affermare il flaneur si incarna oggi nell'utilizzatore della rete. Il 'flusso' viene preso a modello da Semprini per descrivere la società postmoderna in quanto la società del flusso contrappone alle posizioni prestabilite e alle griglie della modernità una logica della libera circolazione e del movimento perpetuo (cfr.: Andrea Semprini, La società di flusso: senso e identità nelle società contemporanee, Milano, Franco Angeli, 2003). Per Manuel Castells la nuova società è la «società in rete» che sta approdando ad una nuova forma di produzione ove la principale fonte di produttività risiede nella gestione del sapere (cfr. Manuel Castells, La nascita della società in rete, Milano, Università Bocconi, 2002). ${ }^{62}$ Giampaolo Fabris, Societing cit., p. 156.

${ }^{63}$ Ivi, p. 121.
} 
come un 'prodotto' da rivitalizzare, sia nei modelli che ispirano i sistemi per la scoperta che propongono questi strumenti come soluzioni di one-stop shopping di tutti i contenuti offerti dalla biblioteca, ove l'azione di cercare l'informazione viene paragonata all'esperienza del consumo. Questa dimensione si manifesta banalmente anche nelle nuove interfacce di questi strumenti con la comparsa nel catalogo elettronico del 'carrello della spesa' tipico delle librerie online e di altri siti di commercio elettronico, per indicare i contenitori di memoria temporanei ove possono essere salvati gli item che all'utente interessano nel corso di una ricerca.

Postmodernità e rivoluzione digitale sono strettamente collegate; c'è, infatti, una parte visibile del cambiamento tecnologico che si evidenzia negli strumenti odierni delle comunicazioni: internet, i telefoni cellulari, l'e-book e le altre tecnologie digitali.

Esiste una parte meno visibile di questo cambiamento che, invece, ha a che fare con il modo con cui oggi l'uomo rappresenta se stesso; in questo senso è in corso una vera e propria rivoluzione scientifica, la rivoluzione dell'informazione che, come le altre 'rivoluzioni', cambiano la percezione del mondo; quella dell'informazione è, dunque, la quarta rivoluzione dopo quelle copernicana, darwiniana e freudiana:

Tre rivoluzioni scientifiche hanno avuto un forte effetto da entrambi i punti di vista: nel modificare la nostra comprensione del mondo esterno, hanno mutato anche la concezione di chi siamo. Dopo Niccolò Copernico la cosmologia eliocentrica ha rimosso la Terra e quindi l'umanità dal centro dell'universo. Charles Darwin ha mostrato come ogni forma di vita si è evoluta nel tempo da progenitori comuni per mezzo della selezione naturale, rimuovendo in tal modo l'umanità dal centro del regno biologico. E, grazie a Sigmund Freud, riconosciamo oggi che la mente umana è anche inconscia e soggetta al meccanismo di difesa della repressione. Cosicché non siamo immobili, al centro dell'universo (la rivoluzione copernicana), non siamo innaturalmente separati e diversi dal resto del regno animale (la rivoluzione darwiniana), e siamo molto lontani dall'essere menti isolate interamente trasparenti a se stesse, come Cartesio, ad esempio, aveva ipotizzato (la rivoluzione freudiana) ${ }^{64}$.

Da parte di alcuni studiosi la nuova rivoluzione digitale è stata individuata nei nuovi media sociali e in quello che è comunemente conosciuto come Web 2.0, che è, in definitiva, il web attuale; i social network avrebbero cambiato i modi di comunicare, di creare e condividere informazioni:

Il bello delle reti è che sono strumenti che connettono persone e informazioni, con tutto quello che ne deriva: proprio perché connettono milioni di persone tra loro, i network finiscono per rispecchiare tutta la complessità di un sistema sociale. Quindi gestiscono relazioni, informazioni, attività delle persone. Ma anche cose meno visibili, come l'immaginario, i riferimenti culturali, i valori, gli affetti, le aspettative di noi tutti sul futuro immediato e su quello lontano. Sono tutte variabili che ci aiutano a determinare il modo in

\footnotetext{
${ }^{64}$ Luciano Floridi, La rivoluzione dell'informazione, trad. it. di Massimo Durante, prefazione di Juan Carlos
} De Martin, Torino, Codice, 2012, p. 10. 
cui operiamo delle scelte, il modo in cui immaginiamo noi stessi, troviamo i nostri equilibri ${ }^{65}$.

Non c'è dubbio che le nuove applicazioni sociali abbiano cambiato il modo con cui oggi le persone si relazionano all'informazione, introducendo variabili significative nel modo di produzione, ricerca e uso dell'informazione stessa, manifestatasi nella possibilità di contribuire attivamente al web, apportandovi contenuti in maniera semplice e veloce (i cosiddetti 'contenuti generati dagli utenti').

Tuttavia, la natura più autentica della rivoluzione digitale si manifesta nel complesso e rinnovato rapporto tra l'uomo e le tecnologie e tra l'uomo e l'informazione.

Il rapporto tra l'uomo e le tecnologie ha da sempre appassionato gli studiosi e non è una novità che si consideri l'uomo un essere 'culturalmente determinato' e che i modi con cui si rappresenta e le scelte che compie dipendano dall'ambiente circostante e dagli strumenti a disposizione.

C'è chi sostiene che con l'avvento dei social network l'uomo sia entrato 'dentro lo strumento', spostando una parte significativa della propria esistenza dentro lo spazio delle reti. Uno spazio non più condizionato dalla fisicità, ma immateriale, ove si compiono una serie di azioni che trascendono l'identità fisica e biologica. Molte delle azioni che possono essere compiute tramite le tecnologie digitali non contemplano la fisicità: possiamo intrattenere amicizie con persone che non abbiamo mai incontrato, partecipare ad eventi culturali e videoconferenze, acquistare nei negozi virtuali, ottenere un documento senza andare in biblioteca e persino vivere una seconda vita ${ }^{66}$.

Anche la contaminazione tra corpo e tecnologia, che tanto ha appassionato le fantasie degli scrittori ${ }^{67}$ oggi è realtà ${ }^{68}$, anche se non è certo nelle intenzioni dell'uomo, per quanto possa sembrare, quella di trasformare se stesso in cyborg, né quella di costruire robot che replicano le azioni quotidiane.

I media digitali rappresentano lo spazio entro il quale si compiono le nostre esperienze e l'uomo inizia a percepire gli strumenti digitali come un'estensione del suo essere corporeo: ogni giorno una fetta sempre più grande della 'memoria' collettiva viene

\footnotetext{
${ }^{65}$ Giuseppe Granieri, Umanità accresciuta. Come la tecnologia ci sta cambiando. Roma-Bari, Laterza, 2009.

${ }^{66}$ Si tratta della dimensione virtuale dell'esistenza ricreata in Second Life (<www.secondlife.com>).

${ }^{67} \mathrm{Si}$ pensi alle suggestioni letterarie di George Orwell (1984, trad. it. di Stefano Manferlotti, Milano, Mondadori, 2004) o di William Gibson, (Neuromante, trad. it. di Giampaolo Cossato e Sandro Sandrelli, Milano, Mondadori, 2003).

${ }^{68}$ Cfr. Giuseppe Granieri, Umanità accresciuta cit. p. 25-31. Il bio-technological make up è il risultato dell'applicazione sempre più vasta della cibernetica e delle tecnologie bioniche.
} 
immagazzinata in strumenti tecnologici, che conservano foto, ricordano eventi o le cose da fare.

I media digitali sono 'pervasivi' e oggi l'uomo non solo abita uno spazio costituito dall'informazione, ma lo diffonde nell'ambiente rendendolo pervasivo e garantendosi connessione ovunque egli si trovi ${ }^{69}$. La nuova realtà 'informazionale' dell'essere umano si manifesta non solo nella nuova pervasività dei media e nella mescolanza dell'analogico con il digitale definito nei termini di ubiquitous computing o 'internet delle cose'.

Uno dei problemi, frutto della rivoluzione digitale, con cui le biblioteche si devono misurare è, ad esempio, il cambiamento delle forme comunicative che vengono oggi influenzate dalla tecnologia informatica e dalle comunità della rete: una di queste nuove forme di creazione e condivisione delle conoscenze muove le persone verso la visualizzazione a discapito della lettura: la comunicazione avviene sempre più spesso attraverso media visivi (es. You Tube riceve circa sessanta ore al giorno di filmato) o più veloci (le chat, Twitter) rispetto al libro, che invece è un medium 'lento' e richiede uno sforzo cognitivo maggiore

In tal senso la tecnologia informatica, se non si provvederà a proporla come una opportunità da affiancare alle opportunità più tradizionali di fruizione dei messaggi, introdurrà modalità di fruizione comunicativa tipiche dell'era precedente l'invenzione della scrittura, con tutta una serie di conseguenze strutturali per quanto concerne la formazione e la trasmissione del sapere: tra le più evidenti e drammatiche possiamo annoverare la passività della visione rispetto al protagonismo critico della lettura; l'impossibilità di correggere le visioni subite rispetto al testo scritto duraturo e più volte percorribile; la mancanza di sistemazioni enciclopediche o di strutturazioni olistiche del sapere che per quanto imperfette e contestabili costituiscono un innegabile aiuto al mantenimento e all'alimentazione della memoria culturale; il basso grado di precisione delle citazioni ${ }^{70}$.

Le tecnologie della comunicazione non hanno solo cambiato significativamente i modi con cui oggi le persone si approcciano all'informazione e la utilizzano, ma hanno modificato il modo con cui l'uomo rappresenta se stesso.

Il passaggio alla società dell'informazione si manifesta in tutte le società più avanzate la cui economia, basata su beni intangibili, dipende oggi dalla corretta gestione dell'informazione.

Ciò avviene - riprendendo le suggestive considerazioni di Luciano Floridi - perché la società sta vivendo una nuova rivoluzione scientifica fondata sul riconoscimento che siamo

\footnotetext{
${ }^{69}$ Ivi, p. 24-25.

${ }^{70}$ Roberto Ventura, Il senso della biblioteca cit., p. 50-51. Si veda anche il provocatorio saggio di Nicholas G. Carr, Internet ci rende stupidi?: come la rete sta cambiando il nostro cervello, Milano, Raffaello Cortina, 2011 in cui l'autore sostiene come le persone attraverso l'uso della rete stiano modificando le abitudini di lettura a scapito della capacità di concentrarsi sui testi lunghi.
} 
inforg ovvero «organismi informazionali interconnessi che condividono con agenti biologici e costrutti tecnici un ambiente globale in ultima analisi costituito da informazione: l'infosfera» ${ }^{71}$.

La rivoluzione dell'informazione mostra la natura 'informazionale' degli esseri umani e non consiste semplicemente nel fatto che oggi molti individui possiedano un alter ego digitale o lascino dietro di sé un'ombra di dati nelle loro interazioni con le reti informatiche, ma nel più profondo cambiamento rappresentato dalla comprensione della realtà e di noi stessi.

Le ICT rappresentano, dunque, non solo applicazioni che apportano miglioramenti all'essere umano (come, ad esempio, gli arti artificiali o i robot) bensì dispositivi che comportano trasformazioni radicali poiché esse sono «ambienti in cui l'utente è in grado di entrare tramite porte di accesso (possibilmente amichevoli), sperimentando una sorta di iniziazione $\gg^{72}$. Tali tecnologie operano, dunque, una trasformazione che consiste non in una sorta di costruzione di un nuovo sistema sociale, ma nella trasformazione della sua ontologia; a tal fine Floridi utilizza il termine 'riontologizzare' per riferirsi al processo di «dislocazione e ridefinizione dell'essenza della nostra natura e del ruolo che rivestiamo nell'universo» secondo il quale «stiamo mutando la prospettiva consueta sulla natura ultima della realtà, vale a dire la nostra metafisica, da una materialistica, per la quale i processi e gli oggetti fisici giocano un ruolo chiave, a una informazionale» ${ }^{73}$.

Nell'infosfera disegnata da Floridi emergono questioni nuove come la proprietà di beni virtuali ${ }^{74}$, il nuovo ruolo dei consumi e della 'marca' come riappropriazione del sé che si manifesta nelle reti sociali, nella sostituzione dell'avatar, del nickname con informazioni reali sulla propria identità, sebbene nella nostra società sia radicato il diritto alla privacy ${ }^{75}$.

Bisogna sviluppare una nuova 'ecologia' tesa a preservare questo spazio informazionale come bene comune della collettività e a prevenire nuove forme di

\footnotetext{
${ }^{71}$ Luciano Floridi, La rivoluzione dell'informazione cit, p. IX.

${ }^{72}$ Ivi, p. 13.

${ }^{73}$ Ivi, p. 14.

${ }^{74}$ Una delle caratteristiche di questa trasformazione è la minore enfasi sulla natura fisica degli oggetti o dei processi, tale per cui i diritti di accesso e di uso vengono assimilati a quelli di proprietà. Nel futuro anche i beni virtuali diventeranno beni importanti e che gli utenti vorranno possedere. Pensiamo alle realtà virtuali come Second Life o altri ambienti virtuali dove le persone investono del tempo per costruire i propri avatar, creano oggetti e personaggi. Si veda in proposito il cap. 2 Identità senza corpo del saggio di Giuseppe Granieri, Umanità accresciuta cit., p. 89-117.

${ }^{75}$ In questo senso molti affermano che oggi con i nuovi social networks si assiste alla fine dell'idea gibsoniana di cyberspazio, con riferimento all'assenza di discontinuità tra identità fisica e identità digitale $\mathrm{e}$ al modo nuovo in cui l'uomo si approccia in rete ai problemi della privacy sostituendo la tendenza a nascondere la propria identità tipica delle chat e dei primi newsgroup con l'esibizione dei propri dati personale, (molti dei quali sono dati sensibili) e dei propri files privati in rete (es. video, foto etc).
} 
discriminazione che si identificano nel digital divide tra i nuovi ricchi e i nuovi poveri, tra quanti saranno cittadini digitali e chi invece, rimarrà escluso ${ }^{76}$.

Siamo dunque nel mezzo di una migrazione capace di ridisegnare la mappa della società mondiale, generando nuovi divari generazionali, socioeconomici e culturali:

Stiamo assistendo, dunque, a una migrazione epocale e senza precedenti dell'umanità dal suo habitat consueto, all'infosfera stessa, e ciò anche in ragione del fatto che quest'ultima sta assorbendo il primo. Il risultato è che gli esseri umani saranno inforg tra altri inforg e agenti (potenzialmente artificiali) che operano in un ambiente amichevole nei confronti delle creature informazionali. Quando gli immigranti digitali come noi saranno sostituiti da nativi digitali come i nostri figli, il corso dell'e-migrazione sarà completato e le future generazioni si sentiranno sempre più deprivate, escluse, svantaggiate o povere, ogni qualvolta si troveranno disconnesse dall'infosfera, come pesci fuor d'acqua ${ }^{77}$.

\section{2) La 'Google generation' e i nativi digitali}

Le tecnologie delle comunicazioni hanno cambiato significativamente i modi con cui oggi le persone si approcciano all'informazione e la utilizzano.

\footnotetext{
${ }^{76}$ Occuparsi oggi di ecologia dell'infosfera vuol dire anche - nella visione di Charlotte Hess ed Elinor Ostrom - salvaguardare i 'beni comuni della conoscenza' sottraendoli alla 'tragedia dei commons' ovvero garantendone la sostenibilità e l'accesso (cfr. Charlotte Hess - Elinor Ostrom, Introduzione. Panoramica sui beni comuni della conoscenza in La conoscenza come bene comune: dalla teoria alla pratica, a cura di Charlotte Hess - Elinor Ostrom, edizione italiana a cura di Paolo Ferri, premessa di Fiorello Cortiana, Milano, Mondadori, 2009). «La conoscenza, che nella sua forma digitale sembra possedere il dono dell'ubiquità, in realtà oggi è più vulnerabile che mai. Per esempio, quando biblioteche e singoli individui acquistavano una rivista cartacea, la dispersione territoriale di una molteplicità di copie garantiva la conservazione delle opere. Quando invece le riviste vengono pubblicate in forma digitale e concesse in licenza alla biblioteche o agli individui, le opere sono centralizzate e vulnerabili ai capricci dell'editore o del caso» (p. 11). Il 'divario digitale' può trasformarsi anche in 'divario digital-culturale', come spiega Fiorello Cortiana nella sua Premessa all'edizione italiana, ivi, p. XXXVI.: «La disintermediazione digitale, l'affermarsi di internet come sistema di creazione, diffusione e commercializzazione della conoscenza, combinato con il progressivo processo di digitalizzazione dei saperi e della comunicazione, rendono perciò obsoleti i tradizionali punti di snodo "pubblici" che garantivano l'apertura e l'accesso a tutti alla cultura alta del sapere scientifico: le librerie, le biblioteche, molti centri di alta formazione. [...] Nell'epoca di Gutenberg era lo stesso processo materiale di produzione e "pubblicazione" (editore, canale di vendita o di pubblica fruizione di prodotti cartacei materiali) che rappresentava la garanzia della "pubblicità" e apertura degli archivi della conoscenza. Oggi questa trasparenza si è persa, il circuito digitale di creazione dei saperi innovativi rende la conoscenza realmente innovativa e cutting edge, patrimonio esclusivo di ristrette "élite" di addetti ai lavori garantiti dal ruolo e dal censo». La tecnologia digitale che nelle intenzioni dei suoi creatori era immaginata come strumento di democratizzazione della conoscenza, traccia nuovi confini e nuovi enclosures i cui effetti sono ancora agli inizi»; ivi p. XXX-XXXI: «Si tratta [...] della nuova forma di segmentazione escludente della società. È il nuovo confine che separa coloro che hanno accesso a Internet, circa due miliardi di persone, da coloro che non lo hanno. Inoltre, all'interno dei connessi - i "salvati" della società informazionale - esiste un'ulteriore barriera. Si tratta della possibilità di accedere o meno alla conoscenza archiviata nelle basi dati di "editori scientifici privati" o di enti di ricerca pubblici e privati che garantiscono un accesso di "prima mano" ai saperi innovativi e alle ricerche originali [...] La rivoluzione digitale [...] rischia, cioè di aprire una nuova segmentazione di "censo intellettuale" - un cultural digital divide - tra connessi di serie A e connessi di serie $\mathrm{B}$, oltre che tra connessi e non connessi».

${ }^{77}$ Luciano Floridi, La rivoluzione dell'informazione cit., p. 14.
} 
Particolarmente rilevante nel nuovo contesto informativo è la presenza delle nuove generazioni di persone che sono nate con le nuove tecnologie quali computer, telefoni cellulari, e tecnologie digitali. Queste generazioni di utenti del web, definiti anche 'Google generation' o 'nativi digitali' sono stati oggetto di vari studi indirizzati a comprendere fino a che punto le nuove tecnologie dell'informazione abbiano modificato l'approccio all'informazione e l'apprendimento nelle nuove generazioni.

Uno studio del 2008, intitolato Information behaviour of the researcher of the future $^{78}$, frutto della collaborazione di British Library, University College London e del consorzio britannico JISC, si è proposto di sondare le modalità di accesso e di interazione con le risorse digitali della Google generation o dei cosiddetti digital native ${ }^{79}$, cioè coloro che hanno conosciuto internet sin dalla nascita, e quindi di disegnare l'identikit dei ricercatori del futuro che al momento dell'indagine si trovavano per lo più in età prescolare. L'indagine ha messo in evidenza alcuni dati meritevoli di riflessione.

Google è diventato uno strumento pervasivo confermandosi come il punto di partenza delle ricerche degli utenti e presente nell' $89 \%$ delle ricerche effettuate dalle nuove generazioni; solo un misero $2 \%$ degli intervistati inizia una ricerca dal sito web di una biblioteca. I giovani utenti richiedono l'informazione in modo semplice e veloce; dedicano poco tempo a valutare la pertinenza e la qualità di ciò che viene recuperato,

\footnotetext{
78 University College London - British Library - Joint Information Systems Committee, Information behaviour of the researcher of the future, [London]: UCL, 2008, $<$ http://www.jisc.ac.uk/media/documents/programmes/reppres/gg_final_keynote_11012008.pdf>. Sulla base di questa ricerca gli studiosi Gunther, Rowlands e Nicholas hanno avviato uno studio teso a verificare l'esistenza di una Google generation con caratteri distintivi e stili cognitivi propri. Gli studiosi hanno dimostrato che non vi è un'evidenza empirica che le nuove generazioni manifestino comportamenti totalmente diversi dispetto agli altri utilizzatori di internet (Cfr. Barrie Gunther - Ian Rowlands - David Nicholas. The Google generation: are ICT innovations changing information seeking behaviour?, Oxford, Chandos publishing, 2009). Dello stesso avviso sono anche gli studiosi Bennett, Marton e Kevin, che nel loro contributo hanno denunciato il 'moral panic' creatosi intorno all'argomento e dovuto ad una eccessiva esposizione mediatica del fenomeno, non supportata da evidenze empiriche (l'indagine, ad esempio rileva che non tutti i giovani 'millennials' hanno acquisito specifiche competenze tecnologiche e che sono ben presenti le differenze socio-economiche ed etniche). Cfr. Sue Bennett - Karl Marton - Lisa Kervin, The "digital native" debate: a critical review of the evidence, «British journal of educational technology», 39 (2008), n. 5, p. 775-786.

${ }^{79}$ Il termine, oggi per la verità un po' inflazionato, è stato coniato nel 2001 da Marc Prensky per distinguere le giovani generazioni, nate pressappoco negli anni 1980-94, rispetto agli immigranti digitali, ovvero coloro che hanno conosciuto internet solo nell'età adulta. (Cfr. Marc Prensky, Digital natives, digital immigrants «On the Horizon», 9 (2001), n. 5, p. 1-6, <http://www.scribd.com/doc/9799/Prensky-Digital-Natives-DigitalImmigrants-Part1>) e id., Digital natives, digital immigrants, part 2: Do they really think differently «On the Horizon», 9 (2001), n. 6, p. 1-6. Si vedano anche sull'argomento: John Palfrey - Urs Gasser, Nati con la rete, La prima generazione cresciuta su internet. Istruzioni per l'uso, Milano, Rizzoli, 2009 e Paolo Ferri, Nativi digitali, Milano, Mondadori 2011.
} 
accontentandosi anche di informazioni poco affidabili ${ }^{80}$. Il $93 \%$ si dichiara soddisfatto delle ricerche condotte tramite un motore di ricerca.

La lettura dell'indagine traccia dunque una realtà alquanto imbarazzante per le biblioteche: oggi milioni di giovani utenti si rivolgono a un 'motore' per trovare qualsiasi tipo di informazione, compresa quella bibliografica ${ }^{81}$. Il successivo studio di Gunther, Rowlands e Nicholas, che ha preso spunto dal report di JSC, ha avuto anche il merito di verificare l'esistenza o meno di una vera e propria Google generation ed ha evidenziato che sebbene le nuove generazioni abbiamo sviluppato una nuova forma di 'media literacy' manifestando nuove competenze e abilità cognitive che derivano da un uso intensivo delle tecnologie digitali ${ }^{82}$, per quanto riguarda il loro approccio con la ricerca dell'informazione non si può effettivamente parlare di una generazione che manifesti tratti distintivi o sostanzialmente diversi dalle precedenti: alcuni comportamenti di ricerca comuni alle nuove generazioni di utenti si ritrovano in tutte le fasce d'utenza interessate dall'uso delle tecnologie digitali ${ }^{83}$.

${ }^{80}$ Tale comportamento avvalora uno dei più importanti paradigmi della ricerca informativa, la teoria del minimo sforzo elaborata dallo psicologo e linguista George Kingsley Zipf nel saggio del 1949 Human behaviour and the principle of least effort: an introduction to human ecology, Cambridge, Addison-Wesley, 1949 studia il linguaggio verbale come attività regolata dal principio del minimo sforzo. Il 'principio del minimo sforzo', detto anche legge di Zipf è dunque una teoria generale del comportamento umano in base alla quale nello svolgimento di un compito un individuo adotterà con tutta probabilità l'azione che implicherà il minimo sforzo. La teoria trovò un immediato successo nella Linguistica, nell'Informatica e nella progettazione di sistemi di ricerca. Da questa semplice premessa discendono varie deduzioni; ad esempio, nel campo della ricerca dell'informazione si può affermare che una persona tende ad usare fonti informative che già ha usato in passato, o più facilmente raggiungibili, piuttosto che cercare nuove fonti di informazione. Per lo stesso motivo l'utente di internet si accontenta delle informazioni trovate in rete tramite un motore di ricerca e dunque più facili da ottenere anche se spesso non attendibili, piuttosto che intraprendere la ricerca in un OPAC, che invece richiede uno sforzo cognitivo maggiore.

Dello stesso parere è Fabio Metitieri. Riprendendo lo studio inglese (University College London - British Library - Joint Information Systems Committee, Information Behaviour of the Researcher of the Future cit.) che paragona il comportamento informativo dei nativi digitali a quello degli scoiattoli Metitieri ne mette in risalto l'incapacità di valutazione delle fonti: «La maggior parte del tempo è dedicata alla ricerca dei documenti e non al loro esame, con comportamenti che questo rapporto inglese definisce «da scoiattolo», perché tutto quanto viene trovato è salvato sul disco rigido, dove in molti casi rimarrà senza essere mai aperto», Fabio Metitieri, Il grande inganno del web 2.0, Roma-Bari, Laterza, 2009, p. 4.

${ }^{81}$ Ormai da qualche anno, e non è un caso, Google presenta in testa ai risultati le voci di Wikipedia, rafforzando così la tendenza ad affidarsi ad un motore di ricerca anche quando l'utente è in cerca di informazioni di base su un dato argomento. La ricerca dell'informazione rispetto al passato è divenuta più olistica, in quanto la maggior parte dell'informazione necessaria viene recuperata nel web. Cfr. Donald Owen Case, Looking for information : a survey of research on information seeking, needs, and behavior, 2. ed, Bingley, Emerald, c2008, p.4.

${ }_{82}$ L'utilizzo intensivo del computer e dei videogames ha fatto sì che queste nuove generazioni abbiano acquisito peculiari abilità cognitive quali, ad esempio, una migliore coordinazione visuale e motoria, la capacità di usare abilità visuali per identificare relazioni tra oggetti in uno spazio virtuale creando mentalmente configurazioni tridimensionali da visualizzazioni bidimensionali, la capacità di processare velocemente l'informazione ricevuta, di sviluppare strategie di problem solving, di ricordare immagini o intere sequenze di gioco. Molte di queste abilità sono distintive di una vera e propria 'Google generation' nonostante se ne rilevi la presenza anche negli adulti che usano intensivamente i computer.

${ }^{83}$ Le ipotesi che gli studiosi hanno voluto verificare erano ricavate da vari studi sui comportamenti di ricerca delle nuove generazioni di utenti. Alcune di queste supposizioni si sono dimostrate vere (la svolta verso le 
È da tener presente quanto scrive la ricercatrice statunitense Danah Boyd sui nativi digitali e sulla predilezione delle nuove generazioni per le reti sociali e le realtà virtuali; esse rappresentano un collante culturale, nuovi contesti condivisi nei quali i giovani consolidano i gruppi sociali ${ }^{84}$. Se dunque in un luogo pubblico, come in treno o in metropolitana, due giovani seduti uno accanto all'altro sono intenti a scrivere nei loro smartphone è molto probabile che stiano 'chattando' tra loro. La predilezione per le applicazioni sociali della rete nelle nuove generazioni inoltre non è sempre frutto di un interesse verso le nuove tecnologie; l'intimità che i giovani creano tra loro nel mondo virtuale restando costantemente connessi alla rete, seppur fisicamente distanti, non è incoraggiata solo dal fascino per gli strumenti tecnologici, ma dipende anche dalla mancanza di mobilità e di accesso a spazi non controllati dagli adulti ${ }^{85}$.

La più recente indagine italiana dell'Osservatorio permanente sui contenuti digitali evidenzia come il rapporto tra le nuove generazioni e le tecnologie sia, invece, essenzialmente esperienziale e rivolto all'attività ludica e all'entertainement ${ }^{86}$, manifestando al contempo che alla ricchezza di informazioni disponibili si contrappone una povertà informativa che oggi rappresenta un fenomeno difficile da contrastare.

forme di comunicazione digitali a discapito di quelle analogiche; la propensione al multitasking, la propensione ad essere agnostici rispetto al formato), altre sono comuni a tutte le fasce d'età o parzialmente vere (la necessità di essere costantemente connessi al web, mancanza di rispetto per la proprietà intellettuale, l'intolleranza per le attese durante la navigazione web, la preferenza per un apprendimento che si acquisisce con la pratica anziché con la lettura di libri o manuali); altre sono infondate (la preferenza dell'informazione visuale su quella testuale; la preferenza per una varietà di esperienze di apprendimento che contemplino l'intrattenimento; la preferenza per testi brevi a discapito dei fulltext, l'opinione che le realtà virtuali vengano concepite come reali, l'opinione che i nativi digitali giudichino attendibili le risorse provenienti dai loro pari rispetto alle fonti più autorevoli). Cfr. Barrie Gunther - Ian Rowlands - David Nicholas, The Google generation cit., p. 123-146.

84 Danah Boyd, ricercatrice statunitense, nella sua tesi di dottorato conseguita presso l'University of California, Berkeley ha indagato la cultura giovanile sui siti di social networking. Cfr.: Danah Boyd, Taken out of context: american teen sociality in networked publics. PhD Dissertation. University of CaliforniaBerkeley, School of Information, 2008.

${ }^{85}$ Secondo Boyd esistono tre categorie di spazio: pubblico, privato e controllato. Il primo è quello dove si incontrano persone, spesso sconosciute, ed estranei e dove la persona è portata ad esibire il suo lato migliore; lo spazio privato è identificato nella propria abitazione, dove ognuno può rilassarsi in famiglia e con gli amici; il luogo di lavoro è un esempio di spazio controllato, dove ci sono regole e si definiscono i comportamenti. Nei giovani - osserva Boyd - la scansione degli spazi è diversa rispetto a quella degli adulti: quasi tutto lo spazio occupato dai giovani è spazio controllato nel quale ai ragazzi si dice cosa fare e come comportarsi. Nella realtà virtuale i giovani ri-creano i loro spazi pubblici e privati nei quali hanno la possibilità di esprimersi, mentre si trovano fisicamente entro spazi controllati.

${ }^{86}$ Associazione Italiana per i Diritti di Riproduzione delle Opere dell'ingegno [et. Al], Osservatorio permanente sui contenuti digitali. Indagine 2009, Nielsen, 2009 <www.osservatoriocontenutidigitali.it> Principali partner del progetto sono Associazione Italiana per i Diritti di Riproduzione delle Opere dell'ingegno (AIDRO), Associazione Italiana Editori (AIE), Associazione Nazionale Industrie Cinematografiche Audiovisive e multimediali (ANICA), CINECITTÁ LUCE, - Federazione Industria Musicale Italiana (FIMI), Associazione dei Produttori Musicali Indipendenti (PMI) e Unione Italiana Editoria Audiovisiva (UNIVIDEO). 


\subsection{La percezione delle funzioni del catalogo nelle indagini sull'utenza}

Di fronte a questi grandi mutamenti di contesto, a partire dalla seconda metà del decennio scorso, sono state avviate varie indagini allo scopo di comprendere come le tecnologie dell'informazione stanno cambiando il modo in cui gli utenti cercano e usano l'informazione bibliografica e le risorse delle biblioteche. Particolarmente interessanti sono due recenti indagini di OCLC che si sono poste l'obiettivo di indagare la percezione che gli utenti hanno delle biblioteche e delle loro risorse e il modo con cui gli utenti utilizzano i cataloghi elettronici.

Perceptions of Libraries, 2010. Context and Community è la prosecuzione dell'inchiesta realizzata nel $2005^{87}$ allo scopo di indagare le nuove abitudini di ricerca dei 'consumatori di informazioni online'; l'indagine si era proposta di esplorare tre caratteristiche dell'ambiente informativo di internet: l'avanzamento verso l'autosufficienza, la soddisfazione e l'integrazione e lo studio diede inizio ad una serie di indagini che OCLC mise a punto per indagare in profondità le nuove caratteristiche degli 'information consumers ${ }^{88}$. A cinque anni di distanza dal precedente e a otto dal primo report di OCLC, questo nuovo rapporto indaga l'impatto dei cambiamenti sulla percezione e l'uso delle risorse informative delle biblioteche (In context, p. 10-49) nella popolazione americana. Le tecnologie informatiche, sempre più pervasive, hanno cambiato il modo di

\footnotetext{
${ }^{87}$ L'indagine ha voluto registrare i cambiamenti avvenuti nella società dai precedenti report 2003 OCLC Environmental Scan: Pattern Recognition e Perceptions of Libraries and Information Resources pubblicato nel 2005 come la recessione economica e i grandi mutamenti nelle tecnologie e valutarne l'impatto nella percezione e nell'uso delle risorse informative possedute dalle biblioteche. «While each study had a unique theme as we researched the hot topics of the time, the reports share a common goal-to provide a future frame for libraries by studying the perceptions, not just the practices, of the information consumer. Understanding beliefs cover behaviors has been our primary research objective. If it is true that perception is reality or, maybe more accurately, perception predicts tomorrow's reality, then our goal has been to provide hard data about the current perceptions of the library, Internet and information, and the ties among the three. We have explored the physical library, the online library, search engines, searching Internet privacy, trust, social networking, library funding and the concept of "library value." We have pushed hard to understand more about the information consumer's perception of the library brand» (OCLC, Perceptions of libraries, 2010: context and community cit., p. 2-3).

${ }^{88}$ Tra i report di OCLC sono da ricordare: ONLINE COMPUTER LIBRARY CENTER (OCLC), OCLC White paper on the information habits of college students, published electronically by the OCLC Online Computer Library Center, Inc., June 2002. <http://www5.oclc.org/downloads/community/informationhabits.pdf>, The 2003 OCLC environmental scan: pattern recognition. A report to the OCLC membership. Dublin, Online Computer Library Center, 2003, $<\mathrm{http}$ ///www.oclc.org/reports/escan/>; Perceptions of libraries and information resources. A report to the OCLC membership. Dublin, Online Computer Library Center, 2005 $<\mathrm{http} / / / \mathrm{www}$.oclc.org/reports/pdfs/Percept_all.pdf>; Sharing, privacy and trust in our networked world. A report to the OCLC membership. Dublin, Online Computer Library Center, 2007 $<$ http://www.oclc.org/reports/pdfs/sharing.pdf>; Online catalogs. What users and librarians want: an OCLC report, edited by Karen Calhoun and Diane Cellentani, Dublin Ohio, OCLC, 2009 $<$ http://www.oclc.org/reports/onlinecatalogs/fullreport.pdf $>$.
} 
accedere e di fruire l'informazione: solo cinque anni prima non era ancora esploso il fenomeno delle reti sociali e gli utenti accedevano ad Internet esclusivamente dal PC; Facebook era ancora un fenomeno limitato agli studenti universitari e You tube era appena nato; i dispositivi portatili come Iphone e ebook readers non erano ancora diffusi. In così poco tempo si è registrata una vertiginosa crescita di Internet (solo il 69\% degli americani aveva un accesso internet nel 2005 contro il 77\% del 2010, la popolazione in rete è aumentata del 12\%, la crescita delle tecnologie mobili del 100\%). Particolarmente rilevante è stata la crescita di motori di ricerca e delle reti sociali, che hanno registrato una crescita passando dal 37\% del 2007 al $66 \%$ nel $2010^{89}$ con la rete sociale Facebook (la cui popolazione è cresciuta del del $1.300 \%)$ che ha sorpassato per popolarità Google $(+84 \%) \mathrm{e}$ la crescita di altri strumenti per il collegamento internet come gli smartphones che hanno segnato un'impennata del $1,050 \%$ utilizzato nell' $80 \%$ dei casi per il collegamento ad internet tramite varie applicazioni (più note come 'apps').

Questa vera e propria rivoluzione digitale ha investito le biblioteche che si sono dotate di servizi mobili per i loro siti web (44\% delle biblioteche universitarie e il $34 \%$ di quelle pubbliche) e di reti wireless (7 biblioteche su 10 dichiarano di essere l'unica risorsa internet non a pagamento per le loro comunità) con un incremento delle reti wireless dal $37 \%$ all' $82 \%$ dal 2005 al 2009.

Particolarmente interessante è l'impatto della crisi economica sul servizio bibliotecario e sulla percezione dei servizi che le biblioteche offrono alla comunità. La recessione che a partire dal dicembre $2007^{90}$ ha sconvolto l'economia americana, fino alla sua chiusura ufficiale nel giugno $2009^{91}$ ha portato disoccupazione, impoverimento, significative perdite negli investimenti e una contrazione generalizzata dei consumi, manifestando complessivamente un impatto economico negativo sul $20 \%$ della popolazione nazionale, ovvero più di un terzo delle famiglie americane ${ }^{92}$.

\footnotetext{
${ }^{89}$ Attualmente due terzi della popolazione americana utilizza siti sociali. MySpace, YouTube e Facebook dominano per popolarità tutti gli altri siti sociali secondo un'indagine del 2007. Facebook, YouTube, MySpace, LinkedIn e Twitter (lanciato in rete nel luglio 2006) oggi conducono il mercato (Wireless and Mobile News, October 2010). In particolare si rileva l'ascesa di Twitter, che alla fine del 2010 ha registrato 24 milioni di visitatori statunitensi al mese, registrando una crescita del $1.100 \%$ rispetto al 2007 (i dati provengono da comScore).

${ }^{90}$ La manifestazione più evidente della crisi è stato un $358 \%$ di aumento di vendite delle case nel quadriennio 2005-2009 e con una diminuzione della costruzione delle nuove abitazioni del $215 \%$. L'indagine rileva anche che il $28 \%$ degli adulti tra i 25 e i 45 anni ha subito l'impatto della crisi nella vita lavorativa.

${ }^{91}$ I dati provengono dal National Bureau of Economic Research.

${ }^{92} \mathrm{Il}$ tasso di disoccupazione è salito al $9,4 \%$.
} 
L'utilizzo più intenso delle biblioteche in tempi di crisi è il dato più significativo: i cittadini toccati dalla recessione hanno cominciato a frequentare di più le biblioteche ${ }^{93}$ facendo registrare un aumento del 37\%; più di 7 milioni di americani colpiti dalla crisi ha significativamente aumentato l'uso di risorse online offerte dalle biblioteche. La popolarità di questa tipologia di risorse è cresciuta inglobando tutte fasce di età: dopo la posta elettronica e i motori di ricerca, le risorse internet più usate sono quelle sociali che si attestano al 78\% con un incremento del 106\% negli anni 2007-2010.

I dati confermano che le biblioteche non sono il punto d'inizio delle ricerche di informazione online e che i motori di ricerca sono la prima scelta ma anche, per contro, che i siti web delle biblioteche sono usati da circa un terzo degli americani; tuttavia nonostante i servizi di reference online siano molto diffusi, è cresciuta parimenti la percentuale di preferenza per i siti che offrono risposte online, che hanno triplicato l'uso dal $15 \%$ al $43 \%$ dal $2005^{94}$. Tra gli utenti delle biblioteche è cresciuta la percentuale dei prestiti con un picco per i DVD. Le principali ragioni d'uso dei servizi bibliotecari rilevati dall'indagine sono il risparmio di denaro $(75 \%)$, il piacere dei propri figli nell'andare in biblioteca (27\%); la necessità di compiere ricerche scolastiche (25\%) o la disponibilità di tempo libero $(25 \%)$. La presenza sempre più pervasiva di strumenti per l'accesso alla rete, nonostante la disponibilità di risorse online nelle biblioteche, non scalfisce il primato del libro, che è ancora considerato 'il marchio' distintivo della biblioteca; (questa identità si è rinsaldata con una crescità nei 5 anni dal $69 \%$ al 75\%) e la percezione del valore delle biblioteche rimane sostanzialmente immutato con una moderata crescita (p. 44-49).

Nella seconda parte del report (By community, p. 50-93) si indaga la percezione e l'uso delle risorse informative in base all'età e alle comunità di utenti (Studenti universitari, adolescenti e giovani), generazione x (25-45 anni), boomers (46-64), adulti $(\text { over } 65)^{95}$.

Più specificamente rivolta al catalogo è invece l'indagine che OCLC ha pubblicato nel $2009^{96}$, Online catalogs. What users and librarians want, che si è proposta di verificare

\footnotetext{
${ }^{93}$ Circa l' $81 \%$ delle persone colpite dalla crisi economica possiede la tessera di una biblioteca, contro il $68 \%$ di quelli non interessati dalla crisi, il 76\% dei consumatori ha ridotto la spesa in libri cd e dvd, il $75 \%$ degli utilizzatori delle biblioteche non spende denaro nell'acquisto di questi beni, mostrando come le biblioteche vengano usate per colmare il gap.

${ }^{94}$ Se nel 2005 l'82\% degli americani cominciava la ricerca di informazione in un motore di ricerca e solo l' $1 \%$ cominciava la ricerca nel sito web di una biblioteca, nel 2010 il primo dato è salito all' $84 \%$ e il secondo è sceso allo $0 \%$.

${ }^{95}$ Le categorie di utenti individuate dal rapporto sono: College students, teens and young aldults, Generation X, Boomers e seniors. (p. 52-89).

${ }^{96}$ OCLC, Online Catalogs What users and librarians want cit.
} 
quali siano i fattori di qualità dei cataloghi secondo le due principali categorie di utilizzatori degli OPAC: gli utenti e i bibliotecari.

L'indagine, che si presenta come un naturale prosieguo degli altri studi condotti negli ultimi anni dalla nota organizzazione ${ }^{97}$, ha avuto lo scopo di orientare la politica catalografica di OCLC che gestisce il catalogo collettivo WorldCat.

Quello di OCLC non è il primo report che negli ultimi anni si è occupato del futuro dei cataloghi elettronici ma si affianca ai già citati report dell'Università della California ${ }^{98}$ e al controverso studio di Karen Calhoun ${ }^{99}$, che ha firmato anche questo report. L'indagine offre pertanto vari spunti di riflessione rilevando un gap nella percezione e nelle modalità di utilizzo dei cataloghi da parte di utenti e bibliotecari:

I risultati mostrano due differenti tradizioni di organizzazione dell'informazione - una propria della biblioteconomia e una del web. Il valore dato dai bibliotecari alla qualità dei dati rimane fortemente influenzato dai principi classici di organizzazione dell'informazione, mentre le aspettative degli utenti sulla qualità dei dati derivano ampiamente dalla loro esperienza circa il modo in cui viene organizzata l'informazione nei siti web più conosciuti. Ciò che è necessario ora è, quindi, integrare il meglio di entrambi i due mondi in una nuova, più ampia definizione di ciò che si intende per qualità nei cataloghi elettronici ${ }^{100}$.

Così la qualità del catalogo, nelle intenzioni degli estensori del report, deve essere ricercata nella mediazione tra ciò che è conveniente per l'utente e ciò che è necessario per i bibliotecari.

Il rapporto rileva che i bibliotecari, i quali si rivolgono al catalogo per adempiere principalmente a mansioni lavorative, nell'elencare le priorità per migliorarne la qualità si mostrano più sensibili ad aspetti qualitativi quali la coerenza e l'accuratezza dei dati catalografici, ottenibili schiacciando i duplicati o correggendo eventuali errori nei record. $^{101}$

Per gli utenti, invece, gli elementi che maggiormente incidono sulla qualità del catalogo sono quelli che permettono di accorciare la distanza tra documenti e la loro rappresentazione nel catalogo: link diretti ai contenuti online, non solo di tipo testuale ma

\footnotetext{
${ }^{97}$ Un commento ai tre studi in: Andrea Marchitelli, La biblioteca nella percezione degli utenti: i risultati di tre indagini di OCLC, «AIB Notizie», 20 (2008), n. 4, p. 13-14 oppure $<$ http://www.aib.it/aib/editoria/n20/0413.htm3>.

${ }^{98}$ Bibliographic Services task force. University of California Libraries, Rethinking how we provide bibliographic services for the University of California cit.

${ }^{99}$ Karen Calhoun, The changing nature of the catalog cit.

${ }^{100}$ OCLC, Online Catalogs. What Users and Librarians Want cit, p. vi, la traduzione è mia.

${ }^{101}$ Nello specifico, ricordando che l'ordine di preferenza cambia a secondo del ruolo e della mansioni svolte, della tipologia di biblioteca e dell'area geografica, i bibliotecari giudicano quali priorità: schiacciamento dei record duplicati (52\%), aggiunta di tables of contents ai record (40\%), aggiunta di abstract ai record (28\%). Seguono correzione degli errori di battitura (27\%), aggiunta delle copertine alle liste di risultati $(25 \%)$, aggiunta di abstract alle liste di risultati (24\%), aumento dell'accuratezza delle informazioni sul posseduto (24\%) e dei record relativi alle risorse online (22\%); in fondo alla lista si posizionano richieste quali quella di aumentare l'esposizione del posseduto nel web (ivi, p. 25).
} 
anche di tipo multimediale, elementi che permettano di effettuare una valutazione più approfondita del contenuto semantico di un documento quali sommari e abstract, fino alla visualizzazione di brevi frammenti del documento (alla stregua di quanto avviene in Google ricerca libri o con la funzione look inside di Amazon).

Il rapporto suddivide i vari elementi presenti nell'elenco dei risultati nelle due macrocategorie della scoperta e della consegna; si ricava così che per gli utenti intervistati l'esperienza della consegna del materiale di cui hanno bisogno si rivela "molto importante, se non più importante dell'esperienza stessa della ricerca". In quella che il rapporto definisce una discovery-to-delivery experience, l'utente si aspetta un passaggio diretto dalla ricerca alla consegna, pretendendo informazioni immediate sulla reperibilità e disponibilità di un documento.

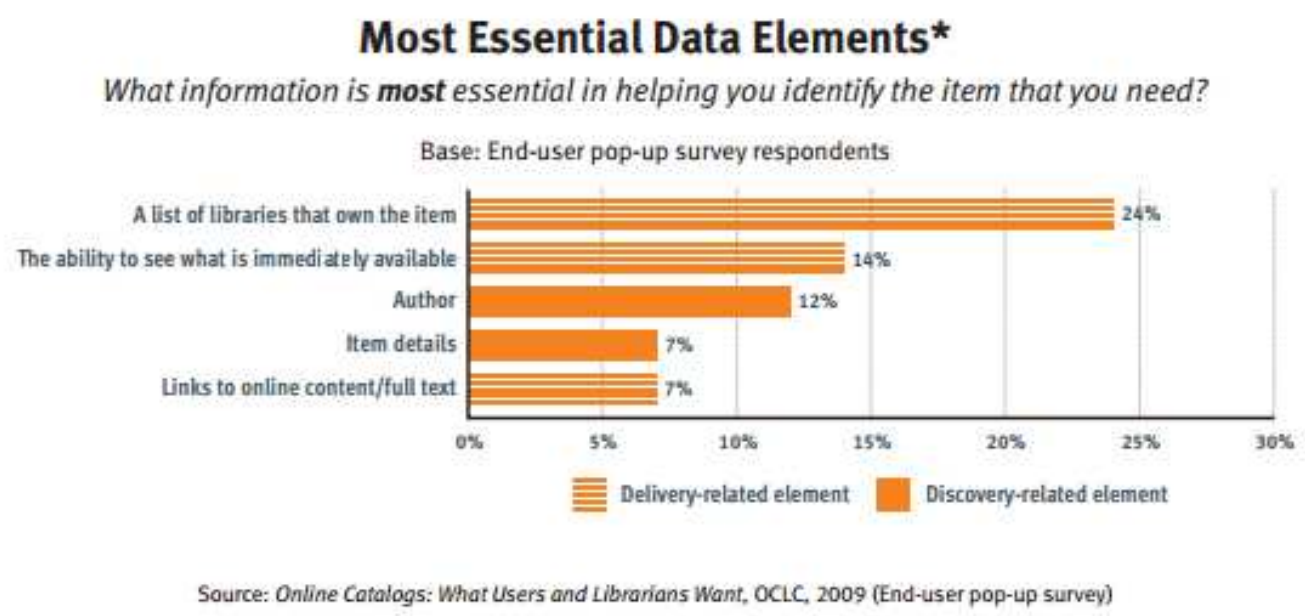

Fig. 1.1 - Gli elementi che gli utenti giudicano più importanti nel valutare l'informazione ${ }^{\mathbf{1 0 2}}$

La pertinenza, l'affidabilità e l'accuratezza dei dati vengono giudicati come elementi importanti che supportano tale passaggio, ma la scoperta di per sé viene considerata un inutile spreco di tempo ${ }^{103}$.

Il rapporto raccomanda così di considerare l'opportunità di avviare servizi innovativi di fornitura dei documenti, anche di tipo tradizionale, con consegne a domicilio in tempi brevi gestibili tramite consorzi bibliotecari o introducendo forme di print on demand alla stregua dei servizi di Amazon.

\footnotetext{
102 Ivi, p. 12.

${ }^{103}$ Ivi p. 20: «discovery alone is a waste of time». In alcuno software per OPAC è stata già introdotta la possibilità di cercare tra $\mathrm{i}$ soli item disponibili (un esempio in <http://www.aadl.org/catalog>). La caratteristica è oggi presente nella maggior parte degli strumenti di discovery.
} 
Un elemento ben radicato nelle richieste degli utenti sembra quello degli $\operatorname{arrichimenti}^{104}$; sommari, copertine e abstract vengono considerati più utili di altri arricchimenti di tipo sociale, quali commenti, rating delle risorse, tag, che incontrano il favore di specifiche fasce d'utenza.

\section{Top Five Desired Data Quality Enhancements}

What changes would be most helpful to you in identifying the item that you need?

Base: End-user pop-up survey respondents

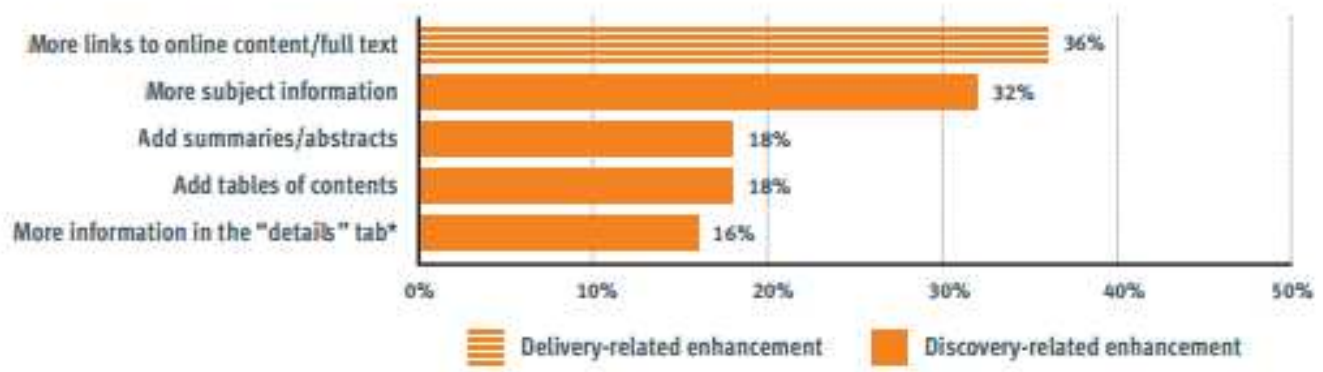

Source: Online Cotalogs: What Users and Libranans Want, OCLC, 2009 (End-user pop-up survey)

Fig. 1.2 - I principali elementi di qualità che gli utenti vorrebbero trovare nei cataloghi ${ }^{105}$

L'arricchimento del catalogo, volto a completare le descrizioni bibliografiche con elementi di tipo valutativo (abstract, sommari, copertine e fulltext) e di approfondimento (collegamento a risorse online, es. siti di biografie, etc.) appare un filone di sviluppo ormai consolidato di fronte alle crescenti esigenze dell'utente, che non si accontenta più della semplice rappresentazione del documento fornita dalla registrazione catalografica. Tali dati di arricchimento - secondo il report - richiedono modalità sostenibili di approvvigionamento, che si fondino sulla necessaria interoperabilità e sulla cooperazione. Essi possono essere ottenuti in automatico, sfruttando i dati già esistenti nei cataloghi, tramite l'accorpamento reso possibile dall'implementazione di FRBR, possono essere acquistati da fornitori commerciali o, infine, essere importati nei cataloghi utilizzando le API di servizi web o di partner commerciali ${ }^{106}$. Non si può però evitare di ricordare che l'arricchimento tramite tables of contents, raccomandato anche in altri studi quale opzione

\footnotetext{
${ }^{104} \mathrm{Si}$ segnala l'ovvia distanza tra utenti e bibliotecari su alcuni elementi quali ad esempio gli ISBN, importanti nel collegamento tra i record, nelle funzioni di schiacciamento dei duplicati e nell'interazione con risorse esterne per importare proprio i dati di arricchimento (importazione di copertine toc ecc..da fonti esterne) che essi stessi richiedono.

${ }^{105}$ Ivi, p. 12.

${ }^{106}$ Tra i vari fornitori di API vi sono community sociali dedicate ai libri (Librarything) e siti commerciali Amazon).
} 
sempre più richiesta dagli utenti, nasconde alcune insidie meno note, quali l'abbandono delle procedure manuali per la catalogazione semantica a favore di forme di indicizzazione automatizzata.

Secondo il rapporto, quando l'utente richiede più informazione 'di soggetto' non si riferisce alle forme controllate fornite dagli accessi per soggetti o per classi, ma a elementi per lo più esterni alle descrizioni catalografiche (sommari, abstract, link a risorse esterne); in questo modo si suggerisce che l'investimento sull'indicizzazione per soggetto debba essere reindirizzato verso forme di produzione più economiche ${ }^{107}$.

Per quanto riguarda, infine, le modalità di ricerca offerte dai cataloghi, il rapporto non riserva sorprese; la ricerca per parola chiave, introdotta nei nuovi Next generation catalogs come ricerca 'di base' si conferma la modalità preferita dagli utenti, i quali non disdegnano però anche le forme della ricerca avanzata per campi e le forme guidate con le opzioni di raffinamento offerte dalle interfacce a faccette che aiutano a filtrare, scorrere e gestire lunghi elenchi di risultati.

I fattori di contesto esaminati in questo capitolo costituiscono dunque il quadro nel quale si colloca lo sviluppo del catalogo elettronico che è oggi fortemente condizionato dai nuovi modi dell'uomo di interagire con l'informazione. Nel capitolo seguente ci si soffermerà sulle caratteristiche degli OPAC di nuova generazione e dei sistemi per la scoperta, rilevandone elementi innovativi e criticità.

107 «Given the growing concern that these traditional methods are not sustainable going forward, it may be necessary for libraries to find more economical means to achieve the benefits to end users that controlled subject vocabularies provide». (OCLC, Online catalogs. What users and librarians want cit., p. 52). 



\section{CAPITOLO 2. OPAC 'DI NUOVA GENERAZIONE' E 'SISTEMI PER LA SCOPERTA'}

«The catalogue has to tell you more than what you ask for».

Seymour Lubetzky

\subsection{L'evoluzione dell'OPAC tradizionale e la nascita dei next generation catalogs}

Il movimento verso una nuova generazione di interfacce per i cataloghi elettronici è stato una delle più importanti trasformazioni che le biblioteche hanno affrontato negli ultimi dieci anni. A partire dalla metà degli anni '90 con lo sviluppo dei software per cataloghi elettronici basati sul web (web based $^{1}$ ) una parte preponderante dell'interazione con l'utenza si svolge in rete, senza la mediazione di un bibliotecario (e solo di recente tale intermediazione si manifesta 'attivamente' all'interno del catalogo e nel processo di ricerca) attraverso l'interfaccia del catalogo elettronico.

Le nuove interfacce per la ricerca negli OPAC sono dunque frutto dell'adattamento ad un universo informativo sempre più complesso, al mutamento delle tecnologie e delle abitudini degli utenti. Di fronte all'emergere di nuove modalità di ricerca e di nuovi modelli di servizio provenienti dalla rete, gli OPAC tradizionali si sono dimostrati carenti di funzionalità e difficili da utilizzare per utenti non esperti.

Il quadro nel quale si inserisce lo sviluppo di interfacce di nuova generazione e di strumenti di discovery è influenzato da alcuni principali fattori:

1) Il nuovo contesto sociale nel quale avviene il recupero dell'informazione. I modelli forniti dalla rete hanno fatto emergere nuovi bisogni da parte dell'utenza e nuovi modi di approccio all'informazione, che certamente sono riconducibili al fenomeno della disintermediazione. L'utente vuole 'fare da sé' ed essere dunque il più possibile indipendente dalla mediazione del bibliotecario, vuole trovare un ambiente di ricerca user friendly, comprendere in maniera intuitiva gli strumenti messi a disposizione dalle biblioteche. È dunque importante che le interfacce e le modalità di interazione con l'OPAC

\footnotetext{
${ }^{1}$ I primi OPAC web-based furono sviluppati tra il 1995 e il 1997, tra questi: Ameritech WebPAC, Brodart LePacNET, DRAweb (WEB2), Endeavour, Innovative Interfaces, Library Corporation, SIRSI.
} 
e gli altri strumenti di ricerca della biblioteca siano il più possibile trasparenti per fornire un ambiente di ricerca unificato, come avviene per gli strumenti del web. L'uso sempre più massiccio dei motori di ricerca e di altre applicazioni commerciali, ma anche l'emergere delle applicazioni sociali e di tipo 2.0 hanno apportato modelli di ricerca e uso dell'informazione nuovi, basati sulla (spesso apparente) semplicità della ricerca (box unico per l'interrogazione, faccette, suggerimenti) e su un paradigma 'esplorativo'.

2) Il mutato contesto tecnologico. Le nuove possibilità offerte dalle tecnologie del web, ambiente nel quale l'OPAC opera, rendono possibili nuove soluzioni per lo sviluppo di OPAC che, sganciati dai gestionali, siano in grado di liberare i dati e renderli fruibili in altre applicazioni del web e, in prospettiva, pienamente utilizzabili nel Web Semantico. Le tecnologie sono ormai mature per progettare cataloghi pienamente integrati con le altre applicazioni del web e dunque interoperabili. Il nuovo sviluppo nella direzione dei linked data, che verrà approfondito nei capitoli seguenti, richiede che la progettazione di OPAC sia in grado di integrarsi con il resto del web.

3) un contesto informativo sempre più articolato dovuto all'incremento vertiginoso dell'informazione digitale e in fulltext e alla difficoltà per le biblioteche di gestire le risorse elettroniche in crescente aumento e a fronteggiare la concorrenza con i nuovi strumenti di ricerca dell'informazione del web, che impongono nuovi modelli di ricerca e di servizio. Le risorse informative sono abbondanti e l'attenzione dell'utente dedicata a ogni risorsa è scarsa $^{2}$. Le biblioteche si trovano a gestire una vera e propria 'emergenza informativa' che le obbliga a riposizionarsi nell'universo informativo, offrendo contenuti 'aperti', accessibili, ma di qualità e aggregando l'offerta.

In base a questi mutamenti di orizzonte gli studi sulla ricerca bibliografica e sui meccanismi di recupero dell'informazione si sono orientati verso nuovi modelli, abbracciando i paradigmi della 'ricerca esplorativa', che come vedremo, si rivelano utili per gestire le prime fasi del processo di ricerca, ma vanno affiancate da tecniche efficaci per il recupero mirato dell'informazione.

I nuovi OPAC sono, dunque, al centro di un profondo cambiamento che ne coinvolge profondamente le caratteristiche e le funzionalità; in questo quadro si situa lo sviluppo di next generation catalogs e degli strumenti di discovery che nell'ultimo decennio si sono imposti nel mercato come strumenti in grado di soppiantare i cataloghi tradizionali.

Il presente capitolo si occupa di indagare il modello di sviluppo che questi strumenti propongono, di approfondire le principali caratteristiche e, in definitiva, di valutare la

\footnotetext{
${ }^{2}$ Lorcan Dempsey, The (digital) library environment: ten years after, «Ariadne» 46 (2006), $<$ http://www.ariadne.ac.uk/issue46/dempsey/intro.html>.
} 
sostenibilità del modello attuale in termini di qualità e autorevolezza e alla luce dei nuovi sviluppi nel campo dei metadati e del Web Semantico.

\subsection{Definire next generation catalogs e discovery systems. Una proposta 'operativa'}

Nella letteratura professionale sull'evoluzione dei cataloghi elettronici ricorrono i termini next generation catalogs, discovery systems, discovery tools, OPAC arricchiti, interfacce per la scoperta ad indicare lo sviluppo di cataloghi nuovi, che presentano caratteristiche profondamente innovative rispetto a quelle presenti nei cataloghi tradizionali ${ }^{3}$.

La letteratura sull'argomento non ha mai chiarito la terminologia in maniera definitiva. Lo stratificarsi di termini diversi a connotare questi strumenti genera così una confusione quando si affronta l'argomento, poiché tali denominazioni vengono usate ad indicare talvolta prodotti diversi, talvolta prodotti sostanzialmente identici per caratteristiche e funzionalità ${ }^{4}$.

La prima questione da chiarire qualora si voglia tentare di definire i next generation catalogs è, dunque, di natura definitoria e ci porta ad indagare meglio la storia dello sviluppo di queste nuove interfacce per i cataloghi.

Due sono le principali spinte che hanno condotto alla nascita di next generation catalogs e di discovery systems e che, in un certo qual modo, segnano la differenza tra i due esiti di sviluppo delle interfacce e delle tecnologie per i cataloghi:

la necessità di offrire all'utente una ricerca semplificata che adotti paradigmi più in linea con la ricerca web e offra servizi aggiunti all'utente;

* la necessità di gestire le risorse elettroniche in crescente aumento.

\footnotetext{
${ }^{3}$ Per un inquadramento generale sulle tematiche dell'evoluzione dei cataloghi elettronici in OPAC di nuova generazione e strumenti per la scoperta i testi a cui fare riferimento sono: Marshall Breeding, Next-Gen Library Catalogs, New York, Neal-Schuman Publishers, 2010 e Jason Vaughan, Web Scale Discovery Services, Chicago, ALA TechSource, 2011. Nel nostro paese è a menzionare il recente volume di Marchitelli e Frigimelica (Andrea Marchitelli, Giovanna Frigimelica. OPAC. Roma, AIB, 2012). La vastissima letteratura sull'argomento è stata di recente condensata in: Mario Ceroti, Rassegna critica della letteratura scientifica italiana

«Biblioteche Oggi», 30 (2012), n. 11, p. 15-27. sugli

$O P A C$,

${ }^{4}$ «A discovery tool is often referred to as a stand-alone OPAC, a discovery layer, a discovery layer interface, an OPAC replacement, or the next generation catalog», Sharon Q. Yang - Kurt Wagner, Evaluating and comparing discovery tools: How close are we towards next generation catalog?, «Library Hi Tech», 28 (2010), n. 4, p. 691.
} 
Possiamo semplificare affermando che la prima necessità ha condotto allo sviluppo di quelli che oggi sono noti come next generation catalogs, mentre la seconda ha generato un'ulteriore evoluzione di questi strumenti nella direzione dei cosiddetti discovery systems o discovery tools.

Nel mio lavoro, per sgombrare il campo da equivoci, si propongo le definizioni che seguono, che saranno successivamente chiarite prendendo in considerazione la storia dell'evoluzione di questi strumenti:

1) Un 'OPAC di nuova generazione' (next generation catalog) è un'applicazione che, utilizzando ancora dati in formati tradizionali (MARC21, UNIMARC, Dublin Core), permette una ricerca facilitata per l'utente, migliorando la navigazione, l'utilizzo e la ricerca nei cataloghi rendendoli maggiormente usabili e simili alle interfacce di ricerca del web più note e conosciute. Tale applicazione può configurarsi come uno strumento d'arricchimento dell'OPAC, come un “involucro sociale” per i dati dell'OPAC o come un sostituto dell'OPAC tradizionale ${ }^{5}$.

Gli 'OPAC di nuova generazione' presentano numerose caratteristiche che li contraddistinguono dai cataloghi tradizionali e che riguardano i dati (contenuti estesi, integrazione di dati esterni, recupero dei fulltext, possibilità di utilizzo dei dati al di fuori dell'OPAC e nel web), la ricerca, il recupero e la visualizzazione dei risultati (navigazione a faccette, filtri, ordinamento per rilevanza, suggerimenti, sommari, tag e contenuti sociali), le funzioni attivabili dall'utente (riutilizzo e manipolazione delle informazioni ricevute) e le caratteristiche dei sistemi (sviluppo modulare dei software, utilizzo di nuove tecnologie open source etc.).

Molti cataloghi che presentano miglioramenti nell'interfaccia con l'introduzione di una o più di queste caratteristiche possono oggi essere definiti 'OPAC di nuova generazione' e per questo possono essere distinti dai tradizionali cataloghi e da prodotti più avanzati. La caratteristica principale è quella di essere strumenti che forniscono un'interfaccia amichevole per il catalogo tradizionale e che possono integrare risorse

\footnotetext{
5 «Cos'è un NGC? 1. Un'applicazione, separata o meno dall'ILS, che permette una ricerca facilitata per l'utente, pur continuando a utilizzare dati in formati tradizionali (MARC, UNIMARC, DC qualified...) 2. Uno strumento di arricchimento dell'OPAC 3. Un "involucro sociale" per i dati dell'OPAC; 4. Un sostituto dell'OPAC tradizionale; NGC ultimamente è diventato sinonimo di discovery interface o discovery tool. I discovery tool sono strumenti per il reperimento di risultati ritenuti pertinenti ponendo l'enfasi sulla scoperta (discovery) più che sul reperimento (retrieval)», Andrea Marchitelli, E pur si muove? Servizi integrati e di nuova generazione per la ricerca informativa, slides presentate al Seminario CENFOR "Le Biblioteche del 2011 scommettono sul futuro? Nuove tecnologie per l'accesso alle collezioni e servizi di telefonia mobile per gli utenti”, Milano, 4 Marzo 2011.
} 
esterne. Endeca, Aquabrowser, Encore, Vu Find e in area italiana Sebina You e Discovery NG rappresentano software per realizzare OPAC di nuova generazione ${ }^{6}$.

2) Un 'sistema per la scoperta' (discovery system o discovery tool, web scale discovery service) è un'interfaccia che dà più enfasi alla scoperta, rispetto al semplice reperimento. Oltre alle caratteristiche tipiche degli OPAC di nuova generazione, questi nuovi strumenti si caratterizzano per la loro copertura che non è più limitata alle sole risorse librarie. Un discovery system si propone come un punto unificato di ricerca e scoperta di tutte risorse documentarie e delle collezioni digitali possedute dalla biblioteca e da altre biblioteche. Per questo motivo sembra essere sempre meno appropriato il termine OPAC e più calzante l'appellativo di 'sistema per la scoperta'.

Possiamo intendere il discovery system non tanto come un catalogo arricchito e potenziato, ma come uno strumento che risponde all'esigenza dell'utente di avere tutto il contenuto disponibile a portata di mano tramite un'unica interfaccia, sostituendosi al portale bibliotecario. Il suo sviluppo non risponde solamente alla necessità di abbreviare il tempo dedicato alla ricerca ${ }^{7}$, ma - a mio parere - ad una vera e propria convergenza di vari strumenti informativi che solitamente le biblioteche approntano per propri utenti ${ }^{8}$.

Un discovery system si configura come uno strumento 'evoluto' nella direzione del paradigma orientato alla 'scoperta globale' dell'informazione disponibile e alla consegna, cioè al reperimento del fulltext. Questi nuovi strumenti hanno seguito un modello di sviluppo che in letteratura viene denominato 'discovery and delivery' ovvero 'scoperta e consegna' ${ }^{9}$. Oltre ad applicare il paradigma esplorativo tipico delle interfacce 'di nuova generazione', ad utilizzare le medesime tecnologie (harvesting dei metadati e uso di un motore di ricerca) e a fornire una serie di funzionalità avanzate, questi strumenti sono dedicati principalmente al recupero e all'aggregazione dell'informazione digitale da diverse fonti esterne.

La maggior parte dei software sviluppati attualmente, o in via di sviluppo, ricade in questa categoria; tra questi, alcuni software proprietari come WorldCat Local ${ }^{10}$, Primo ${ }^{11}$, Summon $^{12}$, Ebsco discovery and delivery ${ }^{13}$, sviluppati a partire dal 2009.

\footnotetext{
${ }^{6}$ Si vedano gli esempi applicativi nell' Appendice A. Casi di studio. Le descrizioni dei principali software sono fornite nell' Appendice B. Software per OPAC di nuova generazione e servizi di Web scale discovery ${ }^{7}$ Come sostiene Marchitelli (ivi, s.p.).

${ }^{8}$ Cfr. Henry Jenkins, Cultura convergente, trad. it. di Vincenzo Susca, Maddalena Pepacchioli, Virginio B. Sala, Milano, Apogeo, 2010.

${ }^{9}$ Carmel Denholm - Leto Kauler - Jan Lavelle - Lloyd Sokvitne, Making the new OPAC seamless: dealing with the transition from "finding" to "getting" «Library Hi Tech», 27 (2009), n.1, p. 13-29.

$10<$ www.oclc.org/worldcatlocal/default.htm>.

${ }^{11}<$ http://www.exlibrisgroup.com/category/PrimoOverview/>. Cfr. infra, Appendice B. Software per OPAC

di nuova generazione e servizi di Web scale discovery. Primo.
} 
I discovery systems, che sono utilizzati per lo più delle biblioteche accademiche o di ricerca, vengono integrati (o nel gergo tecnico 'popolati') con servizi commerciali che indicizzano notevoli quantità di contenuti servendosi di indici centralizzati. All'origine di questa ulteriore evoluzione vi è senz'altro l'aumento esponenziale dell'editoria elettronica, le opportunità commerciali di alcuni editori e distributori e l'esigenza delle biblioteche, specie quelle di ricerca, di rappresentare tramite un unico strumento le risorse possedute e di rendere più trasparente la ricerca per l'utente.

Il termine 'OPAC di nuova generazione' viene dunque utilizzato costantemente fino al 2010 in una forte fase sperimentale ${ }^{14}$ che vede la nascita di alcune interfacce nuove come Endeca $^{15}$, VuFind ${ }^{16}$, Encore ${ }^{17}$, Aquabrowser ${ }^{18}$, Blacklight ${ }^{19}$ : nella prima monografia interamente dedicata ai next generation catalogs ${ }^{20}$, Marshall Breeding, autorevole studioso di tecnologia dei sistemi di automazione per le biblioteche, utilizza questo termine per denotare questa nuova tipologia di strumenti. Ma Breeding avvertiva che il termine, benché ancora utilizzato nella sua monografia, si apprestava a divenire velocemente desueto in favore del più calzante appellativo di 'sistema per la scoperta'. Infatti, l'elemento più significativo, che aveva cominciato a delinearsi quale caratteristica distintiva dei discovery systems è quello della ricerca globale e onnicomprensiva di tutte le risorse cui la biblioteca ha accesso:

The word catalog isn't especially helpful in describing this category of products [...] generally they aim to modernize the interface and expand the scope beyond what was possible with the earlier products [...] This broader scope of search aims to help library users to find resources managed in multiple systems, including content to wich a library subscribes from external providers [...] this broader function has led to the term discovery interfaces ${ }^{21}$.

\footnotetext{
${ }^{12}<$ http://www.serialssolutions.com/en/services/summon/>. Cfr. infra, Appendice B. Software per OPAC di nuova generazione e servizi di Web scale discovery. Summon.

13 <http://www.ebscohost.com/discovery/>. Cfr. infra, Appendice B. Software per OPAC di nuova generazione e servizi di Web scale discovery. Ebsco discovery and delivery.

${ }^{14}$ In questa prima fase vi è una forte sperimentazione di soluzioni 'homegrown' ovvero di software 'fatti in casa' progettati da singole biblioteche o sistemi bibliotecari. Una mappa è stata approntata da Anne Christensen, responsabile del progetto Beluga all'Università di Amburgo ed è visibile all'url: $<$ http://www.communitywalk.com/next_generation_catalogs_in_europe/next_generation_catalogs_in_europe /map/363838>. (Cfr. Appendice A. Casi di studio. Universität Hamburg. Beluga). Sul progetto Beluga si veda anche Ann Christensen, Next generation catalogs: What do users think? Conclusions from the Beluga project. Convegno IFLA 75 pre-satellite Event. Emerging trends in technology: libraries between Web. 2.0 semantic web and search technology. Firenze 19-20 agosto 2009. $<$ http://www.ifla2009satelliteflorence.it/meeting3/program/assets/AnnChristensen.pdf>.

$15<$ http://www.lib.ncsu.edu/endeca/>.

${ }^{16}<$ http://vufind.org/>.

${ }^{17}<$ http://encoreforlibraries.com/>.

${ }^{18}<\mathrm{http}$ ://www.serialssolutions.com/en/services/aquabrowser/>.

${ }^{19}<$ http://projectblacklight.org/>.

${ }^{20}$ Marshall Breeding, Next-gen library catalogs cit.

${ }^{21}$ Ivi., p. 3.
} 
L'OPAC tradizionale, progettato per trattare le rappresentazioni catalografiche dei documenti contenuti, non è in grado di rappresentare in maniera appropriata al suo interno documenti in crescente aumento come i periodici elettronici e le banche dati on-line a testo pieno. Come verrà spiegato più approfonditamente, il discovery system viene sviluppato per fornire un unico contenitore nel quale interrogare basi dati separate e integrare contenuti digitali.

Per connotare questi strumenti per la scoperta, che richiedono normalmente alle biblioteche di sottoscrivere l'abbonamento ad un pacchetto commerciale e che si servono di grandi indici centralizzati, Vaughan propone di usare la definizione di Web scale discovery service, evidenziando la differenza dai precedenti strumenti:

Web scale discovery services are able to index a variety of content, whether hosted locally or remotely. Such content can include library ILS records, digital collections, institutional repository content, and content from locally developed and hosted databases. Such capabilities existed, to varying degrees, in "next generation library catalogs" which debuted beginning in the mid 2000s. In addition, Web scale discovery services preindex remotely hosted content, whether purchased or licensed by the library. This latter set of content hundreds of millions of items - can include items such as e-books, publisher or aggregator content for tens of thousands of full-text journals, content from abstracting and indexing databases, and materials housed in open access repositories. [...] Web scale discovery services are flexible services which provide quick and seamless discovery, delivery, and relevancy ranking capabilities across a huge repository of content. Commercial Web scale discovery vendors have brokered agreements with content providers (publishers, aggregators), allowing them to preindex item metadata and/or fulltext content (unlike the traditional federated search model). This approach lends itself to extremely rapid search and return of results ranked by relevancy ${ }^{22}$.

L'utilizzo del termine service, a denotare questo sviluppo, non è privo di senso in quanto questi prodotti non sono veri e propri cataloghi, ma si appoggiano anche a servizi commerciali e a pagamento che consentono di indicizzare una vastissima quantità di contenuti $^{23}$.

I discovery systems, che secondo alcuni studiosi rimpiazzeranno nel futuro gli OPAC di nuova generazione, oggi vengono mantenuti dalle biblioteche come accesso unificato alle risorse digitali o affiancati ad OPAC tradizionali o di nuova generazione anche se il loro utilizzo è in costante aumento. Essi non vanno confusi con gli strumenti per la ricerca federata: infatti i primi forniscono una singola interfaccia per la ricerca di risorse contenute in varie fonti (OPAC compreso) tramite la costituzione di indici centralizzati e sono

\footnotetext{
${ }^{22}$ Jason Vaughan, Investigations into library web scale discovery services, «Information Technology and Libraries», [preprint] <http://www.ala.org/ala/mgrps/divs/lita/ital/prepub/vaughan2011.pdf>, p. 3.

${ }^{23}$ Tra i contenuti vi sono basi dati di articoli in fulltext, collezioni digitali, repositories istituzionali e open access, indici, sommari e copertine, musica, video, ebook, e altri materiali.
} 
interfacce 'pubbliche', mentre la ricerca federata avviene in un ambiente protetto per via remota basandosi su appositi 'connettori'.

I discovery systems sono oggi in grado di gestire l'autenticazione per accedere anche a risorse remote riservate agli utenti di un'istituzione e si integrano dunque con strumenti per la ricerca federata e link resolvers, ma come vedremo molti di questi strumenti non riescono a gestire adeguatamente i servizi all'utenza che normalmente sono presenti in un OPAC, oltre a perdere alcune funzionalità importanti per la ricerca (ad esempio non consentono browsing per classi e soggetti), dunque non sono ritenuti in grado di rimpiazzare efficacemente i cataloghi tradizionali.

'Next generation catalogs' e 'strumenti di discovery' vengono dunque distinti in base all'uso che si fa di questi software, infatti essi possono essere utilizzati:

* per sostituire il catalogo, offrendo un punto unico di accesso a tutte le risorse della biblioteca, compreso il catalogo e assumendo tutti i servizi di ricerca di un portale (discovery system);

* come interfaccia “di nuova generazione' per l'OPAC (è il caso di molteplici usi del software VuFind);

* affiancando i due strumenti e presentando l'OPAC di nuova generazione per la ricerca nelle risorse della biblioteca e un discovery system per il recupero di una serie vasta di risorse esterne come articoli di riviste in full text e altro materiale che generalmente gli OPAC tradizionali e i NGC non recuperano.

Oggi generalmente gli studiosi denominano NGC quei prodotti che vengono utilizzati come interfaccia amichevole per l'OPAC recuperando sostanzialmente le risorse tradizionali o poco più ${ }^{24}$, per non confonderli con i sistemi per la scoperta che invece si propongono come punto unificato di ricerca di tutte le collezioni possedute o alle quali la biblioteca ha accesso ${ }^{25}$. Caratteristico di questi nuovi strumenti per la ricerca è proprio l'abbandono del termine catalogo, ormai comunemente giudicato inadatto a rappresentare le nuove tendenze evolutive di questi strumenti.

In questa sede si accoglie sostanzialmente questa visione - che sembra essere quella più consolidata nel nostro paese - distinguendo tra quelle realizzazioni che sono rivolte a

\footnotetext{
${ }^{24}$ VuFind è un esempio di discovery system che viene usato in molte biblioteche come interfaccia avanzata dell'OPAC per recuperare solo le risorse tradizionali; in altre situazioni è stato anche adottato come interfaccia amichevole per un discovery system commerciale. Si veda: Graham Seaman, Adapting VuFind as a front-end to a commercial discovery system, «Ariadne», $68 \quad$ (2012), $<$ http://www.ariadne.ac.uk/issue68/seaman/>.

${ }^{25}$ Per Andrew Nagy i cosiddetti NGC non hanno rappresentato davvero l'OPAC ideale e di nuova generazione che ci si aspettava: essi danno ancora accesso solo al posseduto locale a stampa e dunque costituiscono una fase intermedia verso gli strumenti per la scoperta a cui hanno aperto la strada: Andrew Nagy, Analyzing the Next-Generation catalog, «Library Technology Reports», ALA TechSource, 2011.
} 
migliorare la ricerca e che hanno apportato ai nuovi cataloghi caratteristiche avanzate e quelle che vi aggiungono un recupero globale dell'informazione/o che si agganciano a pacchetti commerciali.

Le definizioni adottate in questo lavoro, dunque, sono essenzialmente 'operative' e ci consentono di distinguere gli OPAC che hanno apportato nelle interfacce caratteristiche 'di nuova generazione' dai veri e propri strumenti di web scale discovery \& delivery, che le biblioteche sostituiscono o affiancano ai loro OPAC per la ricerca integrata di articoli o altre risorse esterne.

In base alle definizioni suggerite possiamo così elaborare una scansione temporale nell'evoluzione degli OPAC di nuova generazione e degli strumenti di discovery avvenuta nell'ultimo decennio.

\begin{tabular}{|l|l|}
\hline 2005-2007 & $\begin{array}{l}\text { Sviluppo di NGC, cataloghi con caratteristiche e interfaccia di nuova generazione: } \\
\text { (nuovo paradigma di ricerca esplorativo, caratteristiche nuove, servizi) Endeca, } \\
\text { Aquabrowser, Bibliocommons, VuFind etc. }\end{array}$ \\
\hline 2007-2010 & $\begin{array}{l}\text { nascita di veri e propri strumenti di discovery commerciali. Il primo è WorldCat } \\
\text { Local (2007), seguono a breve distanza Summon, Primo Central, Ebsco discovery } \\
\text { service. }\end{array}$ \\
\hline
\end{tabular}

Fig. 2.1 - Timeline dei Next Generation Catalogs e dei discovery systems

\subsection{Perché nascono i cataloghi di nuova generazione. Le visioni del cambiamento}

Come abbiamo accennato, il movimento verso i cataloghi di nuova generazione comincia a svilupparsi nella metà dal decennio scorso, in seguito ad alcuni contributi che mettevano in luce le criticità dei cataloghi tradizionali. Anche se il termine, coniato da Hildtreth $^{26}$, appare già alla fine degli anni ' 80 , si comincia più diffusamente a parlare nella letteratura professionale di 'next generation catalog' (sovente abbreviato in NGC) a partire dal 2005 quando i primi software con questo nome cominciano ad apparire sul mercato e il dibattito internazionale sul rinnovamento degli OPAC diventa più ricco.

La definizione è stata fornita 'per antitesi', come accade spesso quando ci si trova di fronte a fenomeni nuovi e imprevisti. Next generation catalogs è stata una denominazione generica usata più per segnare una discontinuità dagli strumenti di ricerca precedenti, che

${ }^{26}$ Charles Hildreth, Beyond boolean: designing the next generation of online catalogs, «Library Trends,» 35 (1987), n. 4, p. 647-667. 
per connotare significativamente le caratteristiche dei nuovi prodotti, ma nella definizione 'Next Generation Catalogs' vi erano elementi particolarmente interessanti:

1) il richiamo alle 'generazioni' di OPAC tracciate da Hildreth negli anni ' $80^{27}$;

2) l'uso del termine "catalog" per sottolineare il legame con il catalogo tradizionale e derivante dalla visione del miglioramento dell'OPAC preesistente con caratteristiche di nuova generazione, ma anche dal carattere prototipale e sperimentale che ha caratterizzato i nuovi prodotti almeno all'inizio del fenomeno.

I next generaton catalogs apportano un sostanziale mutamento rispetto al catalogo tradizionale, ma con modalità ed esiti differenti a seconda delle diverse interpretazioni del cambiamento, delle diverse urgenze che di volta in volta ne hanno ispirato la progettazione (OPAC più arricchito, OPAC più somigliante alle piattaforme commerciali, OPAC più 'sociale'; OPAC integrato con il web) o delle diverse tipologie bibliotecarie che li hanno adottati e alle loro esigenze $\mathrm{e}^{28}$.

$\mathrm{Ci}$ sono varie questioni oggi in merito all'uso dell'una o all'altra denominazione: alcuni studiosi preferiscono parlare di next generation catalog come una fase ormai conclusa di transizione tra il catalogo arricchito e il discovery system che è oggi il 'prodotto' o l'esito più diffuso di questa trasformazione in atto. Altri considerano ancora non concluso il processo di trasformazione in atto e parlano di NGC come di un catalogo elettronico del futuro, ancora da realizzare in maniera compiuta.

Il catalogo 'di nuova generazione' può considerarsi frutto di anni di ricerche e di tentativi di miglioramento dei cataloghi elettronici che si possono riassumere nelle tre generazioni di OPAC tracciate da Charles Hildreth, che tracciano la storia dello sviluppo tecnologico che ha caratterizzato la progettazione degli OPAC fino agli anni '90.

Hildreth $^{29}$ suggeriva l'esistenza nella storia degli OPAC di almeno tre generazioni:

1) la 'prima generazione', che si sviluppa dalla metà anni ' 70 agli inizi degli anni '80, ricalca le funzionalità del catalogo cartaceo, ma è persino peggiore. L'accesso al catalogo era ristretto all' autore o al titolo, la ricerca doveva contenere l'esatta parola o frase o almeno la prima parte di una frase o di una parola replicando quanto avveniva in un catalogo cartaceo; la risposta consisteva in visualizzazioni dei record povere di elementi, molto simili alle schede cartacee. L'accesso per soggetto non era contemplato, mancava la

\footnotetext{
${ }^{27}$ Charles Hildreth, Pursuing the ideal: generations of online catalogs, in Brian Aveney-Brett Butler, Online catalogs, Online Reference, Converging Trends, Chicago, American Library Association, 1984, p. 31-56.

${ }^{28}$ Le esigenze delle biblioteche di pubblica lettura hanno ispirato la progettazione di OPAC più amichevoli, con funzioni sociali, o arricchiti; dall'altra le biblioteche di ricerca hanno invece ispirato la progettazione di cataloghi in grado di fungere da punto unico per l'interrogazione delle diverse risorse possedute (banche dati, repositories, collezioni digitali, etc.).

${ }^{29}$ Charles Hildreth, Pursuing the ideal cit., p.31-56.
} 
possibilità di cercare nei record di autorità, non era implementata la struttura sindetica, con la conclusione che la possibilità del browsing nelle liste era praticamente impossibile.

2) La 'seconda generazione' (metà anni '80) introduce miglioramenti significativi ${ }^{30}$ provenienti dallo sviluppo degli studi sull'information retrieval.

Gli OPAC "di seconda generazione", secondo lo studioso, a partire dai loro esordi nella metà degli anni '80 furono progettati con l'intento di combinare due principali modelli di ricerca: da una parte quello dei cataloghi cartacei con i quali gli utenti avevano maggiore familiarità; dall'altra si cercò di emulare le potenzialità di ricerca offerte dai nuovi database di tipo commerciale.

Questa nuova generazione di cataloghi migliorava di molto le possibilità di ricerca poiché forniva una molteplicità di punti di accesso (ogni campo o parola o simbolo presente nei campi del record poteva costituire un punto d'accesso al documento), la possibilità di browsing negli indici, la ricerca per soggetto, per parola chiave e l'introduzione della ricerca booleana. Di seguito vengono elencati i punti di innovazione più significativi:

* la registrazione elettronica consente di avere più punti di accesso al record, in quanto ogni campo o parola del record può diventare un punto di accesso;

* vi sono più opzioni di browsing, indispensabili per chi non sa cosa sta cercando, introducendo anche un modello esplorativo;

* più modalità di visualizzare il catalogo che recupera un sapore "cutteriano", con la possibilità di visualizzare tutti i suoi possibili ordinamenti (per autore, per classi, per soggetti etc);

\footnotetext{
${ }^{30}$ L'elenco completo delle caratteristiche comprende:

- Card Catalog-Like Alphabetical Searching

- Subject Access Via Library-Assigned Headings

- Keyword Access

- Boolean Searching

- Index Term Browsing

- Shelf List Review/Scan

- Full Standard Bibliographic Records

- Multiple Display Formats

- Two or More Dialogue Modes

- Interactive Search Refinement/Modification

- Search Results Display/Print Manipulation

- Help Facility, Context-Sensitive

- Informative Error Messages

- Action and "How To" Option Prompts
}

Cfr: Charles R. Hildreth, Online catalog design models: are we moving in the right direction? A report submitted to the Council on library resources, August 1995, <http://eprints.rclis.org/archive/00000198/> oppure <http://myweb.cwpost.liu.edu/childret/clr-opac.html>. 
* miglioramento dei risultati di una ricerca, tramite il browsing dei 'cataloghi contenuti' o precisa tramite l'utilizzo degli operatori booleani;

- accesso remoto al catalogo. Il catalogo si appresta a divenire web based, consultabile da qualsiasi postazione;

* accesso ai dati sulla circolazione. L'OPAC è in grado di integrarsi con i servizi di prestito e fornire la disponibilità in tempo reale del documento;

accesso alle risorse possedute da altre biblioteche, tramite un database condiviso o collegamenti tra i sistemi;

* migliore utilizzo delle risorse librarie e soddisfazione nel processo di ricerca.

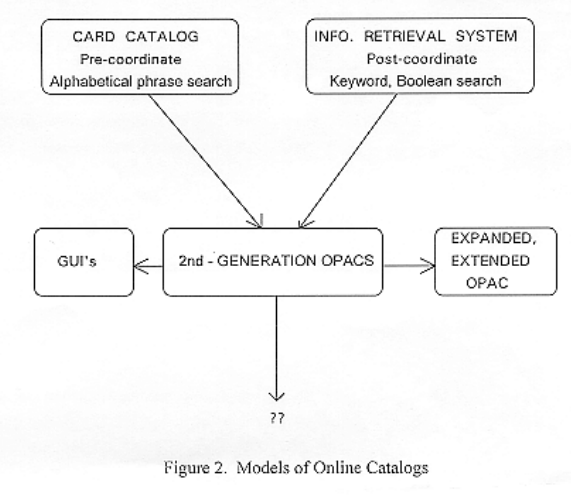

Fig. 2.2 - Modelli per i cataloghi elettronici ${ }^{31}$

3) la 'terza generazione', il cui nuovo sviluppo era previsto da Hildreth negli anni ' 80 e '90, rimane allo stadio 'sperimentale' e dunque non si traduce in prodotti consolidati; infatti per molto tempo si parlerà di 'fallimento della terza generazione di OPAC' .

Questa terza generazione avrebbe dovuto concretizzarsi nell'E3 OPAC, cioè un OPAC arricchito, esteso, ampliato ${ }^{32}$ :

La terza generazione di OPAC viene esposta introducendo il concetto di E3OPAC . L'OPAC di terza generazione si distingue per:

* funzionalità e usabilità migliorate (enhanced);

* indicizzazione del contenuto e copertura della collezione ampliate per essere un vero strumento di accesso a tutte le collezioni della biblioteca (expanded);

\footnotetext{
${ }^{31}$ Ivi, s.p.

${ }^{32}$ Charles R. Hildreth, Advancing toward the E3 OPAC: the imperative path, in Noelle Van Pulis, Think Tank on the Present and Future of the Online Catalog: Proceedings, American Library Association, Chicago, 1991, p. 39-48.
} 
accesso esteso (attraverso link e reti) ad includere tutte le collezioni e risorse di altre biblioteche (extended).

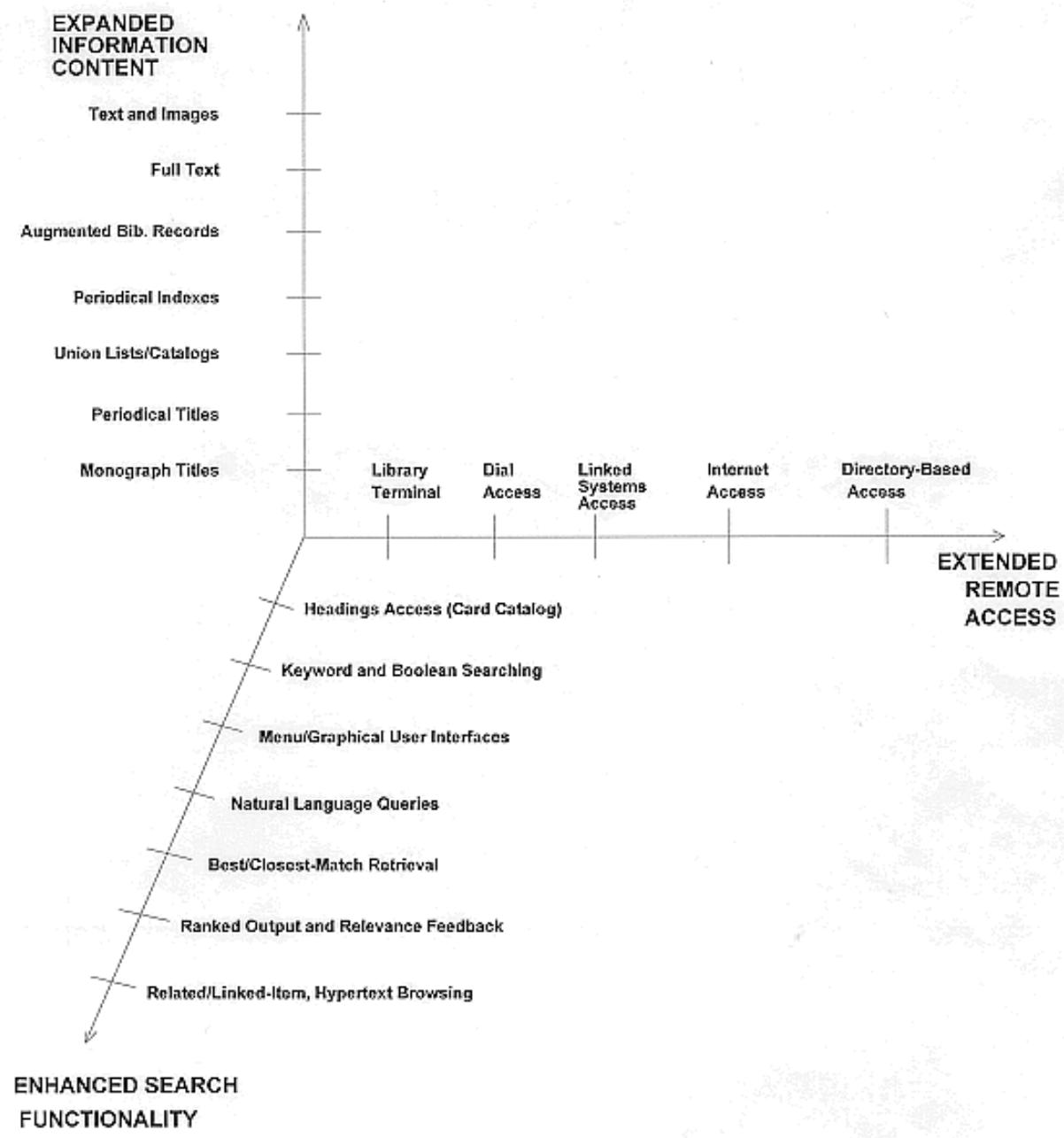

Figure 3. The E-3-OPAC

Fig. 2.3 - Modelli per i cataloghi elettronici. Il modello E-3 $3^{33}$

Per molto tempo questa terza generazione di OPAC è rimasta solo sulla carta; non vi erano, infatti, condizioni tecnologiche sufficientemente mature per compiere quel salto generazionale auspicato da Hildreth verso un OPAC realmente innovativo.

La visione di Hildreth contiene però in sé tutti i concetti che verranno ripresi all'inizio del decennio scorso da altri studiosi, quando si comincerà a parlare in maniera più diffusa di interfacce 'di nuova generazione': allargamento, integrazione, arricchimento. Tali concetti vengono ripresi e ampliati alla luce delle nuove possibilità offerte dalla tecnologia e la visione di next generation catalogs di Hildreth comincia a concretizzarsi.

${ }^{33}$ Charles Hildreth, Online Catalog Design Models cit. 
Possiamo prendere a riferimento le definizioni fornite da alcuni studiosi che si sono interrogati sul modo di migliorare i cataloghi elettronici, negli anni in cui tali prodotti cominciarono a fare la loro comparsa sul mercato, sul modello di sviluppo di questi nuovi software.

Eric Lease Morgan ci offre una visione dell'OPAC di nuova generazione mettendo l'accento sul nuovo modo di intendere il catalogo elettronico come "non catalogo" segnando una netta separazione cesura con l'OPAC tradizionale e con l'idea stessa di ‘catalogo'. La definizione di NGC fornita nel 2006 dallo studioso ${ }^{34}$ contiene in sé i più importanti elementi di innovazione di questi nuovi prodotti: l'integrazione, la centralizzazione, i servizi, l'apertura.

1. «The "next generation" library catalog is not a catalog, per se». Non si tratta dunque di un vero e proprio catalogo poiché non registra unicamente le risorse possedute dalle biblioteche, ma comprende tutte le risorse informative che possono essere utili agli utenti di una biblioteca. Nel catalogo non sono dunque contenute le sole risorse tradizionali, ma anche tutte le tipologie di risorse che oggi sono disponibili sul web. («data sets, images, full-text books and journal articles, archival finding aids, pre-prints \& other gray literature»).

2. «It avoids multiple databases». Esso consente di unificare la ricerca di tutte le risorse informative disponibili per agevolare la ricerca dell'utente e ridurre il problema della duplicazione dei dati. I database multipli sono infatti veri e propri silos non comunicanti ed aumentano la complessità della ricerca.

3. «It is bent on providing services against search results». È realizzato per offrire non solo i risultati di una ricerca, ma una serie di servizi a valore aggiunto, che vanno ben oltre i tradizionali servizi della verifica della disponibilità o di un prestito: si più scaricare un documento, acquistarlo in una libreria online, esportare la citazione etc. rendendo la ricerca più veloce e produttiva.

4. «It is built using things open». L'utilizzo di standard tecnici aperti e piattaforme software open source consente di favorire l'interoperabilità, la modularità, lo scambio e la condivisione. Molti contenuti open possono entrare a far parte dei cataloghi elettronici (banche dati fulltext, riviste open-access e repositories, preprints e altri contenuti che possono essere forniti tramite standard come OAI-PMH o XML).

\footnotetext{
${ }^{34}$ Eric Lease Morgan, A 'Next-Generation' library catalog - Executive summary (Part \#1 of 5) July 7, 2006 [blog post], «LITA Blog: Library Information Technology Association», <http://litablog.org/2006/07/07/anext-generation-library-catalog-executive-summary-part-1-of-5/>
} 
A partire dal 2005-2006 cominciano a svilupparsi progetti di cataloghi di nuova generazione, consistenti dapprima negli 'arricchimenti', poi in vere e proprie interfacce di nuovo tipo comprendenti tutte le caratteristiche sopra citate (e che verranno analizzate nel dettaglio) nel tentativo di porre rimedio alla scarsa usabilità dei cataloghi.

La nascita di cataloghi di nuova generazione si riconduce alla necessità di ovviare ad alcuni difetti dei cataloghi elettronici tradizionali e di avvicinarsi alla semplicità d'uso delle interfacce di ricerca del web.

Già nel 1987 Charles Hildreth descriveva in questi termini i limiti degli OPAC di seconda generazione:

1. do not facilitate open-ended, exploratory searching, by following preestablished trails and linkages between records in the database in order to retrieve materials related to those already found;

2. do not automatically assist the user with alternative formulations of the search statement or execute alternative search methods when the initial approach fails;

3. do not lead the searcher from successful free-text search terms (e.g., title words) to the corresponding subject headings or class numbers assigned to a broader range of related materials;

4. do not provide sufficient information in the retrieved bibliographic records (such as tables of contents, abstracts, and book reviews) to enable the user to judge the usefulness of the documents; and

5. do not rank the citations in large retrieval sets in decreasing order of probable relevance or closeness" to the user's search criteria ${ }^{35}$.

A distanza di un decennio, quando il confronto con il web provocava negli utenti un allontanamento dal catalogo in favore dei motori di ricerca e le tecnologie erano ormai mature per nuovi cambiamenti, molti difetti dei cataloghi tradizionali che erano ancora irrisolti divennero più evidenti, tra questi:

* la presenza di interfacce complesse e poco intuitive, la difficoltà di condurre ricerche esplorative;

* la mancata coerenza con le convenzioni rispettate dalle interfacce web più conosciute e usate dagli utenti (Google, Amazon, siti commerciali, banche dati);

35 Charles Hildreth, Beyond Boolean cit. 
* l'incapacità di rendere i risultati ordinati per ranking ovvero per rilevanza, modalità che i motori di ricerca avevano ormai imposto nei modelli mentali degli utenti;

* la limitatezza di estensione delle risorse recuperate e la necessità di espansione del catalogo per ricomprendere le risorse elettroniche in costante aumento ed evitare la ricerca frazionata su più strumenti;

* lo stretto legame con risorse tradizionali a stampa e la difficoltà a trattare le risorse elettroniche;

* l'incapacità di fornire i contenuti in fulltext agli utenti come invece accade nel web;

* la mancanza di caratteristiche sociali e di interazione con i dati catalografici;

* la mancanza di granularità, cioè l'incapacità di rendere conto degli articoli delle riviste che invece si trovano nelle banche dati possedute dalle biblioteche;

* la scarsa integrazione con le risorse elettroniche e, in definitiva, scarsa attitudine a trattare le risorse digitali. La difficoltà di inserimento delle risorse elettroniche nel catalogo, l'aumento delle stesse e la necessità di organizzare i contenuti digitali ed elettronici hanno spinto le biblioteche a progettare ambienti di ricerca e usare strumenti diversi per aggregare tali risorse. L'utente è spesso costretto a muoversi su più fronti (interrogando diversi contenitori che non comunicano tra di loro) e la ricerca non è integrata. Vi sono poi documenti per i quali i cataloghi elettronici sono stati carenti fin dall'inizio ad esempio la possibilità di rappresentare i periodici, trattati nel catalogo tradizionale come titoli;

* la scarsa usabilità e l'assenza delle caratteristiche più comuni dei motori e altri strumenti della ricerca che vengono approntati al di fuori delle biblioteche

* la chiusura dei dati dentro gli ILS, la mancata integrazione nel web e nel Web Semantico.

Questo elenco, seppur non esaustivo, consente di farsi un'idea delle principali questioni che sono state al centro dei tentativi di miglioramento dei cataloghi elettronici.

Al contrario, non bisogna dimenticare neanche i punti di forza che i cataloghi tradizionali presentano, consistenti in tutte quelle funzionalità complesse che consentono di recuperare l'informazione a stampa, in particolare il recupero mirato dell'informazione semantica. 


\subsubsection{Verso la ricerca globale: Dai next generation catalogs ai discovery systems}

L'OPAC preconizzato negli anni '90 da Hildreth è expanded ed extended, cioè non solo un punto unico di interrogazione di tutte le risorse della biblioteca, ma anche esteso a tutte le risorse esterne.

Si era parlato allora di 'allargamento' dell'OPAC, ma oggi si parla più opportunamente di centralizzazione delle interfacce di ricerca, in due principali accezioni:

1) la centralizzazione può riguardare la modalità di ricerca: le ricerche che gli utenti di una biblioteca solitamente svolgono in due o più strumenti, (es. più banche dati possedute dalla biblioteca, OPAC, biblioteca digitale, repository istituzionale etc.), possono essere svolte consultando una sola interfaccia, quella del discovery system, uno strumento ibrido tra il portale e il catalogo elettronico. Gli OPAC si presentano come un punto di interrogazione di varie fonti online e la linea di confine tra ciò che è posseduto/accessibile diventa più sottile, richiedendo una gestione più integrata da parte delle biblioteche per non disorientare l'utente, visto che sempre più spesso quest'ultimo è interessato all'accesso al documento e poco gli importa sapere dove questo si trovi.

2) la centralizzazione può riguardare il modo in cui la ricerca viene compiuta che si svolge ad un livello molto più ampio di quello della singola biblioteca o di un gruppo di biblioteche (consorzio di biblioteche pubbliche o universitarie) e viene estesa a livello se non planetario, comunque molto esteso (un esempio è WorldCat Local).

La nascita dei primi metaOPAC e, oggi, di cataloghi sempre più grandi porta alla centralizzazione della ricerca e alla scomparsa dei cataloghi locali in favore di OPAC collettivi e sistemi di discovery in grado di registrare il posseduto di molteplici istituzioni (comprendenti non solo l'OPAC ma tutte le risorse riservate appartenenti alle biblioteche collegate $)^{36}$.

\subsection{I modelli dei next generation catalogs}

La nascita dei nuovi prodotti che oggi si propongono come alternativa agli OPAC tradizionali è certamente frutto del ripensamento del modello classico di sviluppo che ha

\footnotetext{
${ }^{36}$ Roy Tennant, Demise of the local catalog cit.
} 
caratterizzato per molto tempo la progettazione dei cataloghi elettronici, specie per quanto riguarda la ricerca e l'accesso alle risorse. Le biblioteche, di fronte al problema di gestire un insieme di risorse sempre crescenti, si sono trovate di fronte a nuovi modelli organizzativi e di servizio che le applicazioni del web propongono agli utenti della rete.

La spinta maggiore è stata così quella di imitare i nuovi prodotti del web che oggi attraggono l'attenzione di milioni di utenti; una buona parte della letteratura sull'evoluzione dei cataloghi tradizionali sviluppatasi tra il 2005-2007 si è concentrata sull'influenza dei motori del web sulle abitudini di ricerca degli utenti ${ }^{37}$. Tale evidenza è stata confortata dalle indagini e dagli studi condotti da biblioteche e istituti di ricerca come OCLC, Digital Library Federation e JSC, dai quali è emerso un uso generalizzato di questi strumenti a discapito degli OPAC. Gli utenti, infatti, mostrano una preferenza per i motori di ricerca nonostante siano consapevoli dei loro limiti in quanto ad affidabilità, precisione, completezza o aggiornamento dell'informazione ricevuta ${ }^{38}$.

In seguito a queste indagini si è sviluppata una letteratura critica sull'argomento: abbiamo accennato ad alcuni articoli maggiormente polemici di alcuni studiosi (Pace, Tennant, Burke, Markey, Schneider) e a numerose liste di criticità stilate nel tentativo di segnalare gli aspetti maggiormente problematici dei cataloghi tradizionali.

Nella maggior parte di questi interventi nei quali, almeno all'inizio, fece molta presa anche la tendenza verso il nascente 'Web 2.0' con le sue caratteristiche tecnologiche e sociali, gli studiosi si confrontarono sul perché i cataloghi non riuscissero a trasmettere la semplicità d'uso che si ha, ad esempio consultando i motori di ricerca.

Tra le soluzioni prospettate per l'evoluzione dei cataloghi, ben presto si cominciò a parlare di googlizzazione, riferendosi, nel bene o nel male, a quei miglioramenti o arricchimenti (enhancements) che si orientavano in questa direzione. Amazoogle (neologismo coniato per l'occasione e derivante dall'abbreviazione in dei nomi di Amazon e Google) divenne agli inizi di questo mutamento, il modello dominante ${ }^{39}$.

Sul fatto che gli OPAC dovessero essere più amichevoli di quanto non lo fossero fino a quel momento le voci erano concordi, ma sulla possibilità che tali miglioramenti

\footnotetext{
${ }^{37}$ Tra i contributi sono da citare quelli di Karen Markey, The Online Library Catalog. Paradise lost and Paradise Regained?, «D-Lib Magazine», 13 (2007), n. 1/2 e di Holly Yu - Margo Young, The Impact of Web Search Engines on Subject Searching in OPAC, «Information Technology and Libraries», 23 (2004), n. 4, p. 168-180. <http://www.ala.org/ala/lita/litapublications/ital/volume23a/number4a/yu.pdf>.

${ }^{38}$ Il confronto tra i motori di ricerca e gli OPAC è stato oggetto dell'indagine condotta da Fast e Campbell (Cfr.: Karl V. Fast-D. Grant Campbell, "I Still Like Google": University Student Perceptions of Searching OPACs and the Web, in Proceedings of the ASIS Annual Meeting, Medford, Information Today, 2004, p. 138-146, anche online in <http://www.asis.org/Conferences/AM04/abstracts/137.html>.

${ }^{39}$ Neologismi come Amazoogle e Googlezone furono coniati da Lorcan Dempsey (cfr.: Lorcan Dempsey, The sound of words: Amazoogle and Googlezon, January 29, 2005, blog post $<$ http://orweblog.oclc.org/archives/000562.html>).
} 
potessero essere mutuati da questi strumenti il dibattito si è dimostrato piuttosto articolato e non privo di scontri di opinione $e^{40}$.

I motori di ricerca, le librerie online, i nuovi servizi come Librarything, Anobii, Goodreads certamente competono con gli OPAC in quanto a velocità e semplificazione della ricerca mostrandosi agli utenti più amichevoli; essi, infatti, predispongono alcuni aiuti alla ricerca che gli OPAC tradizionalmente non implementano ${ }^{41}$ : relevance ranking, uso delle radici delle parole, verifica della digitazione, uso facilitato degli operatori logici, suggerimenti (recommandations), filtri (faceting), ad es. per disciplina, genere della pubblicazione, formato, autore.

Nello sviluppo di nuovi cataloghi elettronici hanno dunque contato una serie di fattori che ne hanno condizionato il modello attuale e che derivano non solo dagli studi sulla ricerca dell'informazione, ma proprio dallo sviluppo di applicazioni web, dai motori alle librerie online, dal web sociale e in definitiva dai nuovi modi di comunicare, cercare e usare l'informazione.

Vale la pena, dunque, di soffermarsi su queste applicazioni, per indagare i modelli che hanno ispirato la progettazione delle nuove funzionalità dei cataloghi elettronici.

\subsubsection{Modelli del Web}

\section{a) Motori di ricerca}

Lo strumento più usato per interrogare i contenuti presenti sul web, il motore di ricerca (search engine) è un sistema automatico che raccoglie e analizza un insieme di dati e restituisce un elenco dei contenuti disponibili ordinandoli in base a formule statisticomatematiche o algoritmi che ne indicano il grado di rilevanza in relazione una determinata chiave di ricerca (query).

Tra i numerosi motori di ricerca attivi sul web, di gran lunga il più utilizzato è Google $^{42}$ (con un indice che supera gli 8 miliardi di pagine) seguito da Microsoft Live e

\footnotetext{
${ }^{40} \mathrm{Nel}$ nostro paese su questi temi si sono espressi Weston, Bergamin, Petrucciani.

${ }^{41}$ Vari studiosi appuntano le loro critiche sugli OPAC sul mancato recepimento di queste tecniche; tra gli altri anche i già citati contributi di Markey (The online library catalog cit.) e You - Young (The impact of Web search engines cit.).

${ }^{42}<$ http://www.google.com/>.
} 
Bing $^{43}$ e, infine, da Yahoo! e Ask ${ }^{44}$. La maggior parte dei motori di ricerca che opera sul web è gestito da compagnie private che utilizzano algoritmi proprietari e database tenuti segreti $^{45}$. Esistono comunque diversi tentativi di dar vita a motori di ricerca fondati sul software libero come Lucene, Nutch, Wikia Search, YaCy, OpenIndex ${ }^{46}$.

Non c'è dubbio che i motori di ricerca abbiano imposto un modello mentale forte negli utenti che li utilizzano, proponendo un nuovo modo di cercare le informazioni semplice e rapido che usa un semplice box di ricerca dove l'utente può immettere interrogazioni nel linguaggio naturale e ricevere in risposta documenti in fulltext e pagine web probabilmente rilevanti.

Il modello di sviluppo del motore di ricerca basato sulla corrispondenza algoritmica e sulla parola chiave oggi condiziona fortemente il modello mentale della ricerca ${ }^{47}$. Tuttavia i motori non offrono una ricerca mirata: il recupero si basa sui termini contenuti nelle pagine web e nei metadati e non sui concetti o sul contenuto delle pagine.

Recenti fattori di innovazione si sono registrati nel campo dei motori di ricerca intelligenti che sono in grado di fornire risposte ad interrogazioni formulate nel linguaggio naturale sfruttando le tecnologie sviluppate nel campo dell'intelligenza artificiale e della linguistica computazionale. Lexxe ${ }^{48}$, creato nel 2005, è un motore che cerca di fornire risposte basandosi sull'analisi del linguaggio naturale per ovviare ai difetti della ricerca semantica nei motori.

Lexxe consente di svolgere una ricerca specifica o generica attraverso l'utilizzo di chiavi semantiche (Lexxe's Semantic Keys) fornendosi di un database (knowledge database) integrato al motore e dunque tramite l'analisi linguistica dei termini immessi.

\footnotetext{
${ }^{43}$ Rispettivamente $<$ http://www.live.com $>,<$ http://www.bing.com $>$.

${ }^{44}<\mathrm{http}: / /$ www.yahoo.com/> e <http://www.ask.com/>.

$45 \mathrm{Si}$ veda The Dark side of Google, luci e ombre di Google, [risorsa online] $<$ http://eprints.rclis.org/bitstream/10760/12843/1/thedarksideofgoogle.pdf >

${ }^{46}$ Rispettivamente: <http://lucene.apache.org/core/>; <http://nutch.apache.org/>; <http://search.wikia.com> (ma non più attivo dal maggio 2009); <http://yacy.net/en/index.html>; <http://www.openindex.org/>.

${ }^{47}$ Cfr.: Jacob Nielsen, Mental models for search are getting firmer, «Jakob Nielsen's Alertbox», May 92005. $<$ http://www.useit.com/alertbox/20050509.html>.

${ }^{48}$ Lexxe <http://www.lexxe.com/> fu creato nel 2005 da Hong Liang Qiao, esperto australiano di tecnologia. Cfr.: Fugang $\mathrm{Xu}$, A search engine that answers questions, «Crienglish.com», 10 October 2005 <http://english.cri.cn/2238/2005-10-10/33@275908.htm>.
} 


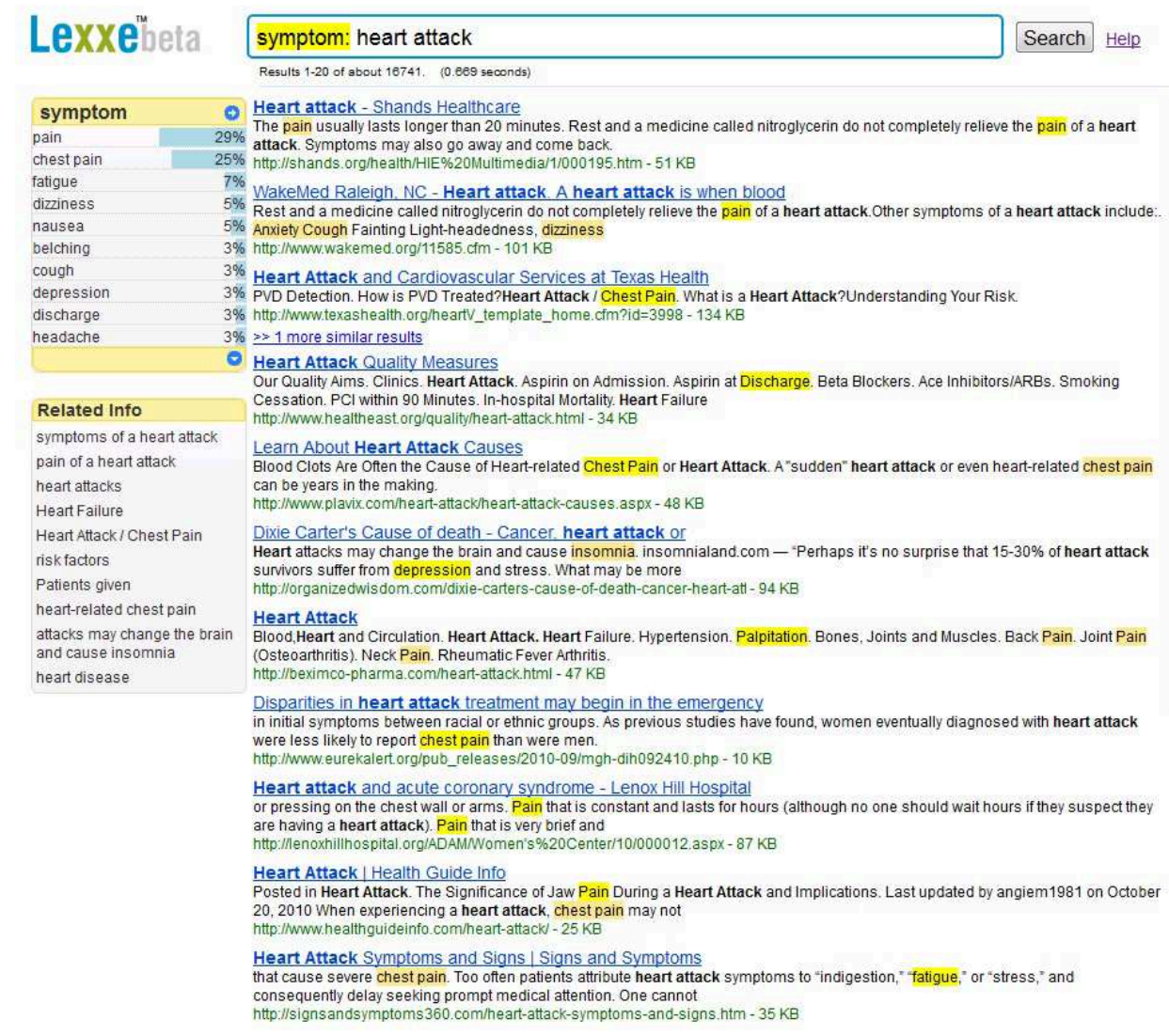

Fig. 2.4 - Lexxe, un motore di ricerca basato sull'elaborazione del linguaggio naturale (natural language processing $)^{49}$

Il tentativo di applicare tali calcoli linguistici ai motori di ricerca non ha dato però $\mathrm{i}$ frutti sperati, a causa dell'esasperata concorrenza dei motori di ricerca più conosciuti; tuttavia l'idea dei motori di ricerca di passare da 'catalogatori di siti' a 'fornitori di risposte' è stata ripresa con nuovo slancio dalle iniziative del Web Semantico.

In questo caso si attiva il processo inverso: se i dati vengono esposti nella forma di linked data o microdata, i motori di ricerca, utilizzando schemi prestabiliti, potranno utilizzarli per fornire risultati strutturati e consentire all'utente di visualizzare il contesto, trasformandosi davvero in fornitori di risposte.

Schema.org, progetto che anticipiamo in questa sede e siglato dai più importanti motori di ricerca del momento ${ }^{50}$, è un enorme passo di avvicinamento dei motori di ricerca al Web Semantico e consentirà in futuro il superamento della logica del matching e la realizzazione della ricerca semantica attraverso i motori di ricerca. Il progetto si basa sull'espressione del contenuto dei documenti in forma di microdata seguendo le specifiche

${ }^{49}<$ http://www.lexxe.com/>.
${ }^{50}$ L'accordo è stato siglato tra i motori Google, Microsoft, Yahoo! e Yandex!. 
del W3C per questo formato ${ }^{51}$ in base a schemi comuni che vengono raccolti nel sito www. schema.org ${ }^{52}$.
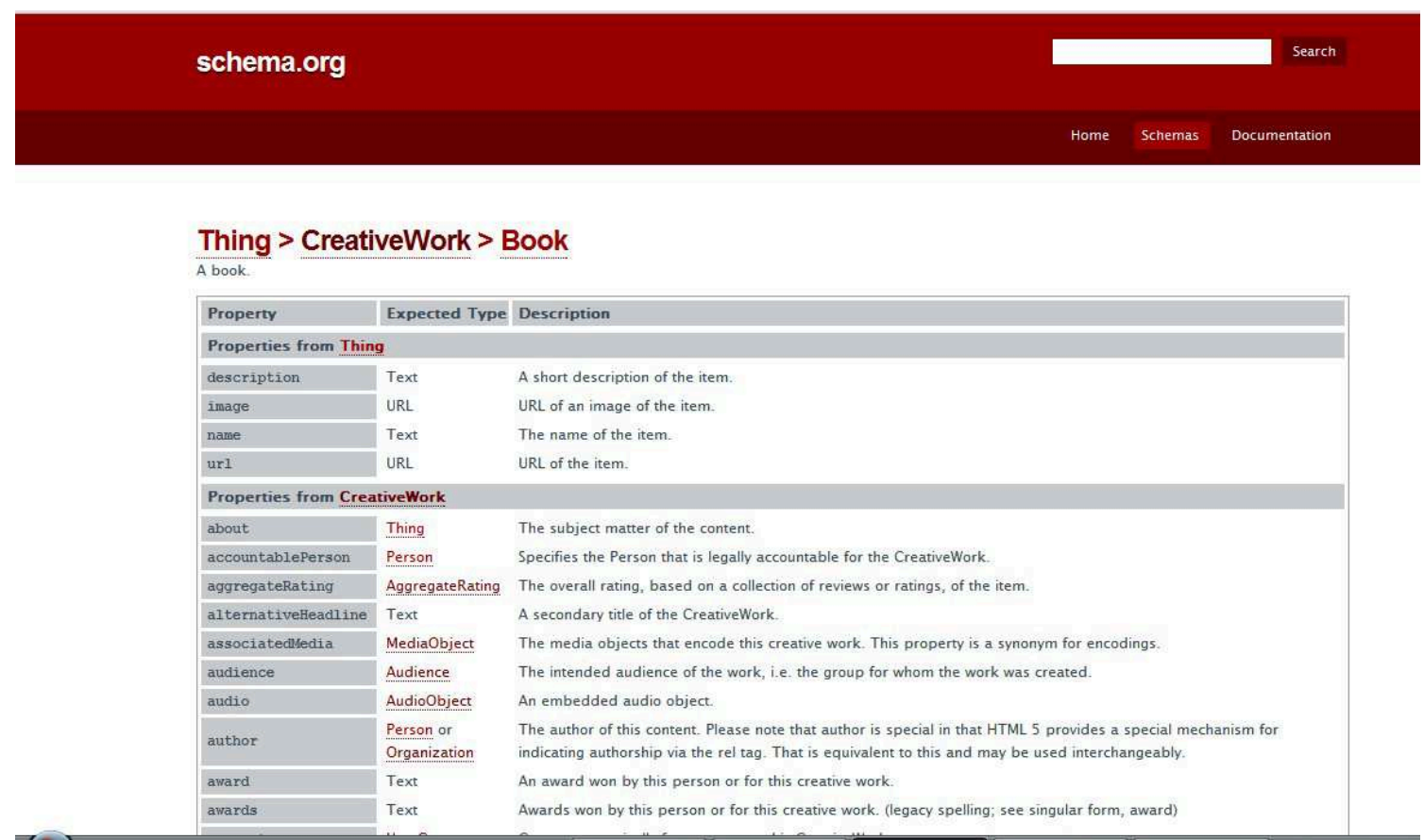

Fig. 2.5 - Schema.org consente di specificare i contenuti di una pagina web per renderli compatibili con il Web Semantico ${ }^{53}$

b) Librerie online

La popolarità delle librerie online è ormai indiscussa. Una su tutte, Amazon ${ }^{54}$, ha il merito di aver proposto alcune linee di tendenza particolarmente significative e che hanno incontrato un'elevata rispondenza da parte degli utenti. Le librerie online hanno apportato nella progettazione delle interfacce per il web numerosi elementi di innovazione tra i quali i suggerimenti imitativi e l'aggiunta di recensioni e commenti, proponendo il paradigma della ricerca 'esplorativa', basata sulla serendipità e rinforzata da una serie di funzioni sociali. Le persone che utilizzano le librerie online per i loro acquisti vi ritrovano anche il piacere di scoprire, di riceve suggerimenti personalizzati, di leggere i pareri degli altri utenti e di partecipare con le proprie opinioni. Particolarmente interessanti sono le

\footnotetext{
${ }^{51}$ Cfr: W3C, HTML Microdata, W3C Working Draft 24 May 2011, <http://dev.w3.org/html5/md-LC/>

${ }^{52}$ La maggior parte delle pagine web utilizza HTML. Il formato HTML istruisce il browser su come visualizzare ogni elemento racchiuso tra tag, ad esempio $<\mathrm{h} 1>\mathrm{Jaguar}</ \mathrm{h} 1>$ dice al browser di mostrare la stringa di testo 'Jaguar' in formato titolo1. L'HTML non dà alcuna informazione aggiuntiva sul significato della stringa, se Jaguar si riferisce all'animale o al noto marchio di autovetture. Schema.org fornisce un insieme di vocabolari controllati che i webmaster possono usare nelle loro pagine web e che possono essere compresi dai più diffusi motori di ricerca Google, Microsoft, Yandex and Yahoo! Utilizzando il formato HTML microdata insieme ai vocabolari di Schema.org aggiunge contenuto semantico alle pagine in HTML. L'obiettivo a lungo termine del progetto è di supportare oltre a HTML microdata anche altri formati.

${ }^{53}<$ http://www.schema.org/>.

${ }^{54}<\mathrm{http}: / /$ www.amazon.com/>.
} 
interfacce visuali che alcune librerie hanno approntato ${ }^{55}$ che replicano nel mondo virtuale l'azione fisica di cercare tra gli scaffali.

\section{c) Aggregatori}

Altrettanto diffuso in rete è l'utilizzo degli aggregatori. Particolarmente interessante è la possibilità di produrre mashups per sviluppare aggregatori in grado di riunire le informazioni presenti in più fonti. Molto diffusi a partire dal 2005-2006 gli aggregatori di feed, o lettori di feed, software o applicazioni Web che raccolgono contenuti web come titoli di notiziari, blog, podcast, e $\log ^{56}$ in un unico spazio per una consultazione facilitata. Gli aggregatori riducono il tempo e gli sforzi necessari per seguire regolarmente gli aggiornamenti di più siti web e permettono di creare uno spazio di informazione unico, in pratica una specie di 'notiziario personalizzato'. In termini aziendali, un 'aggregatore di servizio' utilizza la stessa logica: cioè, vengono offerti più servizi (online o offline), anche di diverso genere, in un unico 'contenitore', che sia una qualsiasi azienda o un qualsiasi operatore. Una particolare forma di aggregazione venne utilizzata nelle pagine personalizzate dei motori di ricerca, dove l'utente poteva mettere in rilievo feed provenienti da diversi siti sparsi nella rete: il più famoso fu Google Reader; oggi il più noto aggregatore è Google news ${ }^{57}$, dove si possono impostare aggiornamenti (feed) provenienti da diverse fonti, mentre $\mathrm{i}$ tra $\mathrm{i}$ più recenti prodotti in questo settore vi sono gli aggregatori di social network in grado di gestire la 'sfera sociale' di ogni utente del web, aggregando i contenuti provenienti dai siti sociali ai quali si è iscritti ${ }^{58}$.

\section{d) Google Scholar}

Tra gli strumenti del web che consentono l'accesso unificato alle risorse informative, Google Scholar è lo strumento che più si avvicina all'OPAC e alle funzionalità delle odierne banche dati. Nel frammentario universo della rete, Google Scholar rappresenta uno strumento di integrazione bibliografica molto potente; nonostante presenti ancora alcuni

\footnotetext{
${ }^{55}$ Tra queste si citano Zoomii books (<www.zoomii.com>), startup canadese per una libreria visuale creata nel 2008, ma oggi non più attiva, nata per presentare in forma visuale il contenuto di Amazon: all'utente veniva offerta la possibilità di esplorare gli scaffali, divisi per catagoria, replicando l'esperienza che avviene recandosi fisicamente in una libreria.

${ }^{56} \mathrm{Il}$ vlog (video blog) è una forma di blog nel quale la forma principale di comunicazione è costituita da comtenuti video.

$57<$ http://news.google.com/>.

${ }^{58}$ Hootsuite (<http://hootsuite.com/>) aggrega gli aggiornamenti provenienti dai social network ai quali si è iscritti riunendo in uno strumento i numerosi profili sociali.
} 
limiti, esso, oltre a fornire i fulltext di libri digitalizzati da Google e di articoli scientifici, svolge contemporaneamente le funzioni di metaricerca su fonti eterogenee, reference linking e ricerca delle citazioni. Google Scholar rappresenta nel web ciò che avviene all'interno del mondo delle biblioteche, ove lo sforzo di aggregare la massa di informazioni a disposizione degli utenti si concretizza negli strumenti di discovery o in strumenti di metainterrogazione e di reference linking ${ }^{59}$. In campo commerciale altri potenti strumenti di integrazione bibliografica sono invece gli indici citazionali ${ }^{60}$, usati per mostrare l'autorevolezza degli articoli scientifici (impact factor) e delle riviste di settore.

e) Siti di commercio elettronico e directories del web

I siti di commercio elettronico sono oggi tra le principali fonti di innovazione e di ispirazione per i nuovi OPAC, specie per quanto riguarda lo sviluppo e la costruzione di interfacce 'usabili'.

Tra le caratteristiche principali di questi strumenti troviamo alcuni elementi di organizzazione della ricerca (es. la possibilità di memorizzare, salvare oggetti fornite dai carrelli della spesa elettronici e dalle liste dei desideri) particolarmente interessanti. Questi siti forniscono anche elementi di progettazione (design elements) che oggi ritroviamo in molti cataloghi di nuova generazione come ad esempio tabs per separare contenuti di diversa tipologia, e altri oggetti grafici che semplificano la ricerca. Dato che l'utente di dei siti di commercio elettronico non è solito formulare interrogazioni, questi hanno fornito modelli di organizzazione dell'informazione (come quello delle faccette) esplorativi e basati sulla serendipità. Molti siti di e-commerce sfruttano la logica multidimensionale delle faccette per creare ambienti in cui l'approccio dell'utente è unicamente esplorativo. Tra questi <www.wine.com> <http://www.epicurious.com/> e l'italiano < www.zalando.it>. L'architettura dell'informazione per il web è una nuova disciplina che si è sviluppata attorno a queste tematiche e che applica $\mathrm{i}$ modelli e le teorie biblioteconomiche alla ricerca nel web ${ }^{61}$.

\footnotetext{
${ }^{59}$ Una panoramica di questi strumenti è fornita in Riccardo Ridi, La Biblioteca come ipertesto. Verso l'integrazione dei servizi e dei documenti, Milano, Editrice Bibliografica, 2007, p. 201-216.

${ }^{60}$ Ideati da Eugene Garfield, gli indici citazionali approdarono sul web con il nome di Web of Science (WOS). Ad essi si è affiancato poi il noto Scopus (<http://info.scopus.com/>) Cfr. La Biblioteca come ipertesto cit, p. 205. Una demo del funzionamento di Scopus è presente on-line all'url: $<$ http://www.info.scopus.com/setup/promo/demo/sc_DEMO_0407.html>.

${ }^{6} \mathrm{Si}$ vedano Peter Morville - Louis Rosenfeld, Information Architecture for the World Wide Web. Sebastopol, CA, O'Reilly, 2007 e Luca Rosati, Architettura dell'informazione: trovabilità: dagli oggetti quotidiani al Web, Milano, Apogeo, 2007.
} 
f) Reti sociali, strumenti e tecnologie del Web 2.0

Le nuove tecnologie del Web 2.0 hanno impresso una forte accelerazione allo sviluppo anche negli OPAC di caratteristiche 'sociali'. In molti OPAC di nuova generazione è stata introdotta tra le funzionalità offerte all'utente quella di costruire intorno al catalogo una comunità di lettori.

Fonte di ispirazione sono stati alcuni siti sociali dedicati ai libri e alla lettura come Anobii, Goodreads e Librarything ${ }^{62}$, dove gli utenti hanno modo di inserire i propri libri in uno spazio personale, di leggere suggerimenti e scambiare opinioni, di visualizzare gli altri utenti con gusti simili e che possiedono gli altri libri, di scoprire nuovi libri.

Librarything $^{63}$, che indicizza oggi più di 73.000 .000 di libri, ha sviluppato un' $\mathrm{API}^{64}$ per le biblioteche che consente di importare nei cataloghi elettronici alcuni arricchimenti come copertine, tag, recensioni, voti. L'API usa la corrispondenza dell'ISBN per offrire uno strumento di arricchimento per i cataloghi ${ }^{65}$.

Tra le novità, invece, si registrano le più recenti funzionalità di autenticazione integrate tra $\mathrm{i}$ vari strumenti sociali della rete, come ad esempio Facebook Connect ${ }^{66}$, che consente con l'account Facebook di accedere a varie altre reti sociali utilizzando i dati dell'account facebook, senza dover compilare nuovi form. In generale, le applicazioni del

\footnotetext{
$62 \quad$ Rispettivamente $\quad<h t t p: / / w w w . a n o b i i . c o m />, \quad<h t t p: / / w w w . g o o d r e a d s . c o m>\quad$ e $<$ http://www.librarything.com>.

${ }^{63}$ Librarything (<http://www.librarything.com/> e <http://www.librarything.it/>) con più di 1.500 .000 utenti consente di inserire i libri letti o un'intera biblioteca e si dichiara come "un catalogo semplice, a livello qualitativo di una biblioteca. I numeri di LibraryThing (dati aggiornati a maggio 2012) ci rivelano il successo di questa piattaforma: 1,542,115 utenti, 73,074,859 di libri 'catalogati' (di cui 6,915,224 sono opere uniche), $87,644,473$ di tag, 1,762,273 di recensioni, 11,746,618 di voti, 3,016,186 di copertine fornite dagli utenti, 42,548 immagini di autori. Notevole è anche il numero dei gruppi di lettori $(8,598)$.

${ }^{64}$ Un'API o Application Programming Interface è un insieme di procedure create per i programmatori allo scopo di interagire con una data applicazione; le API rappresentano programmi che altri software, librerie, o piattaforme possono usare per interagire con un programma e trasmettere informazioni. Attraverso le API è possibile creare mashups ovvero utilizzare parti di programmi preesistenti per creare nuove applicazioni. Un esempio di API utilizzata negli OPAC è quella di Google books che consente di importare le copertine dei libri nei cataloghi elettronici di nuova generazione.

$65<$ http://www.librarything.com/forlibraries $>$.

${ }^{66}$ Facebook Connect è uno strumento che può essere integrato nei siti web. Viene presentato attraverso un pulsante con il quale gli utenti possono collegare il proprio account Facebook al sito visitato. Lo scopo di questo sistema è quello di consentire agli utenti di creare una chiave di autenticazione sicura per tutti i siti web visitati, di mettersi in comunicazione con gli altri utenti visitatori di un sito, di condividere le informazioni e le azioni fatte su un sito con i propri amici Facebook. Questo sistema è analogo a Google Friend Connect con la differenza che appunto in questo caso si usa un'identità Google (si veda $<$ https://developers.facebook.com/docs/guides/web/>).
} 
web offrono un accesso ai contenuti senza barriere tra $\mathrm{i}$ vari servizi, mentre in biblioteca l'uso di servizi diversi richiede varie volte all'utente di autenticarsi.

\begin{tabular}{|c|c|}
\hline \multicolumn{2}{|l|}{ Ricerca } \\
\hline Motore di ricerca & $\begin{array}{l}\text { Interfaccia semplificata, relevance ranking, uso del linguaggio } \\
\text { naturale }\end{array}$ \\
\hline Libreria online & Ricerca esplorativa, suggerimenti imitativi. \\
\hline Aggregatore & Centralizzazione delle interfacce di ricerca in uno strumento unico \\
\hline $\begin{array}{l}\text { Google scholar/ Google } \\
\text { books }\end{array}$ & $\begin{array}{l}\text { Arricchimento, fulltext, strumenti } \\
\text { personalizzati (librerie personali) }\end{array}$ \\
\hline Reti sociali/Web 2.0 & $\begin{array}{l}\text { Ricerca Collaborativa (modello Yahoo Search), ricerca passiva } \\
\text { (feed RSS }\end{array}$ \\
\hline \multicolumn{2}{|l|}{ Interazione } \\
\hline Motore di ricerca & $\begin{array}{l}\text { Interazione semplificata tramite ricerca in box unico ed esposizione } \\
\text { dei risultati per ranking }\end{array}$ \\
\hline Libreria online & Suggerimenti imitativi e vocazione al browsing e alla scoperta \\
\hline Aggregatore & Informazione passiva con uso di metodologia push \\
\hline $\begin{array}{l}\text { Google scholar/ Google } \\
\text { books }\end{array}$ & I \\
\hline Reti sociali /Web 2.0 & $\begin{array}{l}\text { Grande interazione con immissione di contenuti generati dagli } \\
\text { utenti }\end{array}$ \\
\hline \multicolumn{2}{|r|}{ 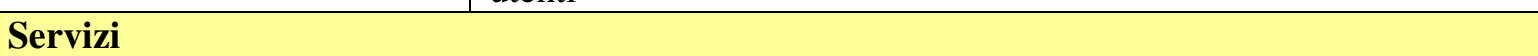 } \\
\hline Motore di ricerca & Recupero immediato di una risorsa in fulltext \\
\hline Libreria online & $\begin{array}{l}\text { Acquisto, visualizzazione del fulltext o preview della risorsa } \\
\text { (look inside) }\end{array}$ \\
\hline Aggregatore & I \\
\hline $\begin{array}{l}\text { Google scholar/ Google } \\
\text { books }\end{array}$ & $\begin{array}{l}\text { Recupero immediato di una risorsa in fulltext, preview di parti del } \\
\text { volume }\end{array}$ \\
\hline Reti sociali /Web 2.0 & Raccomandazioni, ratings, tags, comunità di pratica \\
\hline
\end{tabular}

Fig. 2.6 - La tabella mostra le caratteristiche dei modelli del web che hanno ispirato le funzionalità dei next generation catalogs in termini di ricerca, interazione, servizi.

\subsection{Le caratteristiche funzionali. Modelli di sviluppo e nuove funzionalità}

Le funzionalità offerte dai cataloghi tradizionali in confronto a quelle fornite dalle applicazioni della rete appena esaminate sono piuttosto povere in termini di accesso, ricerca, utilizzo dell'informazione ricevuta e integrazione con altre fonti. Così gli OPAC di nuova generazione hanno introdotto varie caratteristiche funzionali che li contraddistinguono dagli OPAC tradizionali ispirandosi a 'modelli funzionali' e tendenze tipiche del web. 
Weston e Vassallo ${ }^{67}$, prendendo come riferimento l'indagine elaborata nel 2006 dalla California Digital Library ${ }^{68}$, hanno individuato nelle attuali tendenze sei modelli funzionali per gli OPAC di nuova generazione:

- l'OPAC che si adatta: un OPAC fluido, che si adatta al comportamento di chi lo consulta, applicando tecniche di data mining a gruppi di utenti raggruppati in clusters di profili simili;

* l'OPAC che suggerisce: in grado, cioè di orientare l'utente nella ricerca specie quando questa giunge ad un punto morto. Applica la logica fuzzy e suggerisce all'utente percorsi e chiavi alternative per la ricerca;

* l'OPAC che apprende: sfruttando le tecniche del collaborative filtering per proporre all'utente nuovi percorsi, leggendo il comportamento dell'utente per adattarlo a profili simili;

* l'OPAC che integra: l'OPAC può limitarsi ad essere un semplice catalogo elettronico, o diventare punto di snodo degli altri servizi bibliotecari. Si arricchisce di contenuti esterni e si integra con altri servizi;

* l'OPAC che aggrega: presentando i risultati ordinati per rilevanza o scomposti in faccette o recependo la struttura logica di FRBR;

* l'OPAC che organizza il web: favorendo l'accesso alle risorse primarie, e 'portalizzandosi';

Tali modelli possono essere confrontati con le principali linee di tendenza tracciate da Ridi nel $2007^{69}$ :

* 'portalizzazione' o 'allargamento' degli OPAC, sia per integrare maggiormente le risorse offerte dalle biblioteche, sia per integrare il catalogo con le risorse offerte in rete;

* personalizzazione, da parte dell'utente, dell'interfaccia, dei percorsi della ricerca, dei risultati esportabili;

\footnotetext{
${ }^{67}$ Paul Gabriele Weston, - Salvatore Vassallo, "... e il navigar m'è dolce in questo mare". Linee di sviluppo e personalizzazione dei cataloghi, in La biblioteca su misura. Verso la personalizzazione del servizio, atti del convegno di "Biblioteche oggi", Milano, 9-10 marzo 2006, a cura di Claudio Gamba e Maria Laura Trapletti, Milano, Editrice Bibliografica, 2007, p. 130-167.

${ }^{68}$ Nella direzione di individuare come gli obiettivi dei nuovi OPAC potessero essere perseguiti tramite tecnologie più innovative come quelle presenti nei motori di ricerca e nelle librerie online California Digital library ha intrapreso nel 2006 il noto studio denominato Melvyl Recommender Project (California Digital Library, The Melvyl Recommender Project final report. 2006, $<$ http://www.cdlib.org/services/publishing/tools/xtf/melvyl_recommender/report_docs/Mellon_final.pdf>). Lo studio ha preso in considerazione cinque principali ambiti: sistemi di ricerca testuali, correzione automatica di errori di digitazione, strategie di presentazione dei risultati, ordinamento per rilevanza e suggerimenti per la ricerca.

${ }^{69}$ Riccardo Ridi, La Biblioteca come ipertesto cit., p. 122-124.
} 
* arricchimento dell'OPAC con l'aggiunta di ulteriori documenti e materiali (full text, immagini, recensioni, ecc..);

* integrazione o il dialogo con banche dati bibliografiche o testuali tramite le tecniche di reference linking o tramite la condivisione di authority files;

* adeguamento ad FRBR permettendo così di articolare in maniera più coerente le informazioni descritte nei cataloghi e consentendo all'utente di comprendere i rapporti tra le entità, di esplicitare la rete sindetica e di migliorare l'ipertestualità dei cataloghi,

* 'semantizzazione' ossia miglioramento delle tecniche di ricerca e dei metodi di visualizzazione finalizzati al recupero di informazioni di tipo semantico presenti nei cataloghi ma non ampiamente sfruttate ${ }^{70}$;

* adeguamento delle interfacce alle Guidelines for OPAC displays riducendo l'eterogeneità degli OPAC in quanto a terminologia, icone, funzioni di help e quantità di informazione presentata sullo schermo e per fornire all'utente delle capacità spendibili su OPAC diversi;

* rafforzamento della multilinearità tramite il potenziamento della tecnica dell'ipertesto. Si tratta sia dei legami che il catalogatore può creare in modo precoordinato in fase di catalogazione, sia di quelli che possono essere creati dall'utente nel momento dell'interrogazione. Ciò porterebbe alla creazione del 'catalogo post-coordinato' descritta da Weston ${ }^{71}$;

* sviluppo delle tecniche che suggeriscano percorsi di ricerca alternativi, nei casi in cui una ricerca compiuta dall'utente abbia esito negativo o giunga ad un punto morto;

* 'googlizzazione' rivolta alla semplificazione delle interfacce con la previsione della modalità di ricerca 'su tutti i campi' come modalità principale di ricerca e applicazione di tecniche caratteristiche dei motori (ranking, stemming etc.);

* estensione agli OPAC delle più avanzate tecniche di information retrieval e delle innovazioni riguardanti le interfacce umani/computer;

* estensione agli OPAC dei principi dell'usabilità e dell'accessibilità;

\footnotetext{
${ }^{70}$ L'indagine Opac semantici ha rappresentato in questo ambito un buon punto di partenza per esplorare le funzioni semantiche offerte dai cataloghi tradizionali nel nostro paese.

${ }^{71}$ Paul Gabriele Weston, Il catalogo elettronico. Dalla biblioteca cartacea alla biblioteca digitale, Roma, Carocci, 2002.
} 
Sulla base degli orientamenti e dei modelli su individuati, verranno esaminati, quindi, alcuni aspetti del dibattito biblioteconomico sull'evoluzione e il miglioramento complessivo dei cataloghi.

Negli ultimi 5-6 anni lo sviluppo di sistemi per la scoperta e di interfacce 'di nuova generazione' ha seguito tali orientamenti e mostrato una serie di caratteristiche funzionali che oggi sono comunemente riconosciute come distintive di prodotti 'di nuova generazione'. Yang e Wagner ${ }^{72}$, in un lavoro di valutazione di quelli che chiamano in modo generico next generation catalogs, distinguono le seguenti dodici funzionalità:

1. Unico punto di accesso per tutte le risorse.

2. Interfaccia adeguata alle interfacce commerciali per il web.

3. Contenuto arricchito

4. Navigazione a faccette

5. Box di ricerca 'google like'

6. Rilevanza, anche collegata alle statistiche sulla circolazione

7. Suggerimenti

8. Raccomandazioni/ materiali correlati

9. Contributi degli utenti (sommari, recensioni, voti, tag etc.)

10. $\quad$ Feed Rss

11. Integrazione con i social network

12. Url persistenti

In questo lavoro le funzionalità dei next generation catalogs e dei discovery systems verranno esposte nelle principali categorie che riguardano i dati, i sistemi, la ricerca e la visualizzazione dei risultati, le funzionalità e i servizi all'utenza, le interfacce.

\footnotetext{
${ }^{72}$ Sharon Q. Yang - Kurt Wagner, Evaluating and comparing discovery tools: how close are we towards next generation catalog? «Library Hi Tech», 28, (2010), n. 4, p. 690-709.
} 


\subsubsection{Dati e database}

\subsubsection{L'allargamento dell'OPAC: dal portale al discovery system}

Anche se le opportunità offerte dalle nuove tecnologie e il confronto con i concorrenti del web sono annoverati tra principali fattori che hanno indotto i progettisti a realizzare OPAC rinnovati, è stata soprattutto una maggiore complessità del sistema informativo in cui le biblioteche sono inserite a rendere necessario un cambiamento.

La crescita esponenziale di risorse online e delle collezioni digitali che le biblioteche si trovano a dover gestire, insieme ad alcuni fattori nuovi come la nascita dei numerosi repositories istituzionali favorita anche dallo sviluppo del movimento dell'Open Access (OA) e di software per i repositories compatibili con il web (es. Dspace), hanno messo le biblioteche di fronte alla necessità di progettare punti unici di ricerca per le svariate risorse elettroniche disponibili ${ }^{73}$.

Pagine web della biblioteca, catalogo, biblioteca digitale, repositories istituzionali, siti web, banche dati fulltext sono solo alcune delle risorse che possono essere ricercabili in biblioteca, con la conseguenza per l'utente di dover consultare un buon numero di interfacce diverse per ottenere un'informazione completa, così una delle caratteristiche degli strumenti di ricerca è la tendenza verso la creazione di un'interfaccia unica per l'esplorazione delle raccolte e delle risorse digitali.

La più importante funzione degli strumenti per la scoperta o discovery systems è fornire l'accesso da un unico punto alle risorse e collezioni della biblioteca e non ai soli dati bibliografici dell'OPAC.

Due sono le principali motivazioni che hanno spinto verso questo modello di sviluppo:

* l'enorme crescita delle risorse digitali e il mutamento del concetto di 'raccolta';

* la difficoltà di trattare le risorse digitali all'interno dell'OPAC con gli strumenti allora a disposizione.

Un argomento che per vari decenni ha alimentato il dibattito sugli OPAC è quello dell'inclusione delle risorse elettroniche remote al suo interno in modo da rendere il

\footnotetext{
${ }^{73}$ Un primo esempio di allargamento è stato OCLC WorldCat che nel 2009 ha inglobato OAISTER, l'enorme collettore di archivi aperti, sviluppato dall'Università del Michigan in collaborazione con Andrew Mellon Foundation a partire dal 2002. Oggi il catalogo collettivo indicizza anche i dati provenienti da JSTOR e HathiTrust.
} 
catalogo uno strumento veramente onnicomprensivo che consenta l'accesso a tutte le risorse sia possedute che selezionate dalla biblioteca per i propri utenti ${ }^{74}$. Nel 2005 Byrum esprimeva all'IFLA Conference di Oslo le preoccupazioni di molti studiosi americani sull'attuale generazione di OPAC ormai considerata vicina alla maturità proprio per l'inefficienza nel recuperare le risorse digitali in crescente aumento e chiedeva di apportare «miglioramenti urgenti» verso l'inclusione nell'OPAC di tali risorse esterne ${ }^{75}$.

Il concetto di OPAC 'esteso' o 'allargato' non è un concetto recente: l'argomento in passato era stato affrontato da Potter ${ }^{76}$ e Hildreth $^{77}$, ma l'enorme crescita di risorse digitali degli ultimi decenni lo ha riportato nuovamente al centro dell'attenzione, specie a partire dalla metà del decennio scorso, quando il movimento di rinnovamento degli OPAC ha ripreso nuovo slancio.

Hildreth prevedeva l'inserimento di queste risorse all'interno del catalogo, facendo assumere ad esso il ruolo di primario strumento di recupero dell'informazione e nel contempo fidelizzando gli utenti verso il catalogo che nelle intenzioni dello studioso doveva rimanere il principale strumento di ricerca in biblioteca. Ma è la stessa natura ipertestuale del catalogo elettronico a determinare questo nuovo sviluppo verso 1' 'allargamento': nel momento in cui il catalogo rende possibili, anche semplicemente attraverso i collegamenti ipertestuali, l'accesso diretto ai documenti primari, diventa una fonte di informazione primaria e di conseguenza uno strumento ibrido, il cui obiettivo va oltre la semplice rappresentazione dei documenti posseduti dalla biblioteca ${ }^{78}$.

Viene dunque a mutare profondamente il concetto di raccolta, che non si limita più ad essere costituita dalle sole risorse possedute ma anche da quelle selezionate e messe a disposizione dalla biblioteca per soddisfare le esigenze informative dei propri utenti.

Già nel 2001 Gorman affermava fortemente questo concetto, che avrebbe segnato a distanza di un decennio la nascita di OPAC di nuova generazione, dichiarando:

La raccolta di una biblioteca moderna consiste di quattro parti:

\footnotetext{
${ }^{74}$ Le risorse elettroniche ad accesso locale (REL) sono risorse per che necessitano per essere consultate di l'utente inserire un supporto fisico ( $\mathrm{cd}, \mathrm{dvd}$ ) in un computer o in una periferica collegata ad un computer. Le risorse ad accesso remoto (RER) possono essere utilizzate senza alcun supporto fisico ma attraverso un terminale o il collegamento ad una risorsa remota memorizzata in un altro sistema.

${ }^{75}$ John D. jr. Byrum, Recommendations for urgently needed improvement of OPAC and the role of the National Bibliographic Agency in achieving it, World Library and Information Congress: 71th IFLA General Conference and Council. Libraries - A voyage of discovery. August 14th - 18th 2005, Oslo. $<$ http://www.ifla.org/IV/ifla71/papers/124e-Byrum.pdf> ; trad. italiana: Byrum, John D. jr, Raccomandazioni per miglioramenti urgenti dell'OPAC. Il ruolo delle agenzie bibliografiche nazionali, «Biblioteche Oggi», 23 (2005), n. 10, p. 5-14.

${ }^{76}$ William Gray Potter, Expanding the online catalog, «Information Technology and Libraries», 8 (1989), n. 2, p. 99-104.

${ }^{77}$ Charles R. Hildreth, Online Catalog Design Model cit.

${ }^{78}$ Riccardo Ridi, La Biblioteca come ipertesto cit.
} 
- oggetti tangibili (libri, registrazioni,mappe, video, ecc.) posseduti dalla biblioteca e conservati in una determinata localizzazione fisica;

- risorse (elettroniche) intangibili (cd-rom, ecc.) possedute dalla biblioteca e disponibili per l'uso in biblioteca;

- oggetti tangibili (libri, ecc.) posseduti da altre biblioteche ma disponibili per la biblioteca attraverso sistemi interbibliotecari di condivisione delle risorse e cataloghi collettivi;

- risorse (elettroniche) remote, intangibili non possedute dalla biblioteca ma alle quali essa fornisce accesso. Quando facciamo nostra quest'ampia nozione, possiamo vedere la biblioteca come il centro di una serie di cerchi concentrici più estesi che alla fine comprendono la conoscenza registrata e l'informazione del mondo intero ${ }^{79}$.

Negli anni seguenti, con la crescita vertiginosa delle risorse elettroniche, si cominciò nel dibattito professionale a sostenere la catalogazione di tali risorse nell'OPAC, che ovviamente necessitavano di essere vagliate e selezionate in modo attento dalle biblioteche, sulla base delle caratteristiche delle risorse (accessibili liberamente o a pagamento) della mission della biblioteca e delle esigenze degli utenti. Purtroppo però l'enorme crescita dei documenti digitali produceva un impatto molto forte su chi doveva gestire le risorse, organizzarle e renderle disponibili. Le problematiche inerenti alla difficoltà di gestione delle risorse elettroniche, specie nelle biblioteche accademiche, sono state ampio oggetto di dibattito e hanno costituito anche l'occasione di confronto in numerosi incontri professionali e seminari ${ }^{80}$.

Le tecnologie non erano in grado di supportare questi cambiamenti e vi erano perplessità nella creazione di veri e propri OPAC allargati o estesi; alcune questioni riguardavano le modalità per l'accesso a banche dati e periodici elettronici per i quali le biblioteche pagavano una tariffazione, e che quindi avrebbero dovuto essere visibili solo agli utenti istituzionali e non a tutti gli utenti del web.

\footnotetext{
${ }^{79}$ Michael Gorman, Metadati o catalogazione? Una falsa alternativa, traduzione a cura di Stefano Gambari e Mauro Guerrini, «Biblioteche Oggi», 19 (2001), n. 6, p 8-18.

${ }^{80} \mathrm{Da}$ ricordare almeno il Convegno internazionale "Le risorse elettroniche: definizione, selezione $e$ catalogazione", Roma, 26-28 novembre 2001, <http://w3.uniroma1.it/ssab/er/it/home_nf.htm> promosso dall'Università di Roma La Sapienza, dal Ministero per i beni e le attività culturali e dall'Associazione italiana biblioteche. Al convegno furono presentati i contributi di molti studiosi italiani e stranieri quali: Mauro Guerrini, John Byrum, Marie-France Plassard, Riccardo Ridi, Juha Hakala, Igino Poggiali, Olivia Madison, Hildegard Schäffler, Michael Gorman. Per la gestione delle risorse elettroniche e l'analisi dei flussi di lavoro si veda anche Maria Cassella - Paola Gargiulo, Il workflow delle risorse elettroniche remote, «Biblioteche oggi», 24 (2006), n. 6, p. 46-58. <http://www.bibliotecheoggi.it/2006/20060604601.pdf>.
} 
La proposta suggerita da Ridi ${ }^{81}$ era quella di 'ristrutturare le modalità di accesso' all'OPAC permettendo all'utente di scegliere se consultare:

* l'OPAC tradizionale (o small opac) ristretto alle sole RER e al materiale documentario analogico o tradizionale;

* il VRD predisposto dalla biblioteca;

* un OPAC allargato, portalizzato o arricchito che include sia i documenti posseduti che le risorse elettroniche remote selezionate dalla biblioteca per i propri utenti.

e ipotizzava la creazione di un accesso riservato o 'dedicato' che avrebbe dato la possibilità agli utenti istituzionali e registrati di vedere le risorse in abbonamento.

In definitiva, prima dello sviluppo dei next generation catalogs, il problema della gestione delle rosorse elettroniche in crescente aumento creava non poche difficoltà alle biblioteche che, in base alle proprie risorse economiche, potevano decidere in che modo fornire l'accesso a tal risorse, cioè se tale accesso potesse essere costituito da un OPAC 'allargato', difficilmente realizzabile a basso costo, oppure dall'OPAC tradizionale affiancato da software per la gestione di portali bibliotecari ${ }^{82}$.

${ }^{81}$ Secondo Ridi (cfr. La Biblioteca come ipertesto cit. p. 117-118), uno dei primi studiosi che in Italia ha affrontato questa tematica, le principali alternative per la biblioteca che intende rendere disponibili le risorse elettroniche locali (REL) e le risorse elettroniche remote (REL) selezionate per i propri utenti erano le seguenti:

1) allargare le risorse dell'OPAC tradizionale, mediante l'inclusione dei link alle risorse elettroniche remote che la biblioteca seleziona per i propri utenti in base alla propria mission. Ciò equivarrebbe a trasformare però la natura essenzialmente catalografica dell'OPAC rendendolo un ibrido tra catalogo e bibliografia. Inoltre, una operazione del genere comporta sempre il pericolo di includere documenti che potrebbero in qualsiasi momento mutare, spostarsi richiedendo grandi carichi di lavoro ai bibliotecari per l'aggiornamento dei link e compromettendone la conservazione.

2) mantenere l'OPAC ristretto ai soli documenti posseduti dalla biblioteca (risorse "analogiche" e risorse elettroniche locali (REL) che sono fruibili direttamente recandosi presso la biblioteca riservando invece alle RER tre diversi possibili trattamenti:

- l'inserimento nell'OPAC dopo la loro trasformazione in REL, cioè dopo averle immagazzinate su scaffali virtuali della singola biblioteca, in modo da garantirne l'accesso e l'immutabilità nel tempo.

- oppure l'inserimento in liste o archivi separati da gestire con regole di catalogazione più snelle e che offrano modalità di interrogazione più semplici alimentati eventualmente con l'importazione di metadati forniti dai produttori delle RER o da terze parti, ovvero producendo quelli che vengono chiamati VDR, virtual reference desks, cioè raccolte di fonti informative e di strumenti di ricerca disponibili in rete predisposte dai bibliotecari e organizzate per disciplina o argomento contenenti spesso commenti, valutazioni e suggerimenti.

- $\quad$ oppure fornendo agli utenti la sola segnalazione di "liste di strumenti generali per la ricerca in internet presenti in rete (motori di ricerca, virtual reference desk presenti in rete ecc.. ).

${ }^{82}$ La necessità di usare strumenti diversi per offrire all'utente i contenuti digitali avrebbe poi portato allo sviluppo dei discovery systems per accedere a tutte le risorse bibliotecarie da un unico punto. 
L'idea di interrogare risorse diverse da un unico punto di interrogazione risale a molti anni fa, fin dai primi decenni dello sviluppo degli OPAC; lo standard Z39.50 ${ }^{83}$ consentì di interrogare fonti diverse e di presentarne i risultati (ovviamente non integrati tra di loro) in un'unica interfaccia; tecnologia che fu alla base dello sviluppo dei metaOPAC.

Per l'accesso alle risorse elettroniche locali e remote le biblioteche hanno scelto nel tempo diverse soluzioni: non potendo gestire tutte le risorse tramite l'accesso diretto dall'OPAC, la via più percorribile è sembrata quella di affiancare all'OPAC un portale attraverso il quale poter organizzare meglio le risorse offerte, raccogliendo nella home page gli accessi alle risorse possedute. Questi strumenti avevano il vantaggio di alleviare i bibliotecari dal compito di aggiornare i metadati delle risorse remote. A partire dagli anni '90 molte biblioteche, nella necessità di fronteggiare la concorrente espansione dell'informazione gratuita o a pagamento disponibile in rete, hanno cominciato a realizzare portali per permettere un migliore orientamento dei propri utenti nelle risorse offerte. I portali consentono ancora oggi alle biblioteche di organizzare e ordinare le risorse offerte fornendo il collegamento a basi dati commerciali o servizi di gestione dei periodici quando non si adotta un discovery system.

Lo sviluppo dei portali bibliotecari ha una sua storia, che è stata approfondita da un contributo di Maria Cassella ${ }^{84}$. Nella più recente generazione di portali in un primo tempo ci si è serviti dei metamotori (metasearcher) cioè software per la maggior parte open source, in grado di generare interrogazioni su diverse fonti (dai cataloghi ai database bibliografici alle risorse fulltext commerciali o locali a siti web selezionati o a servizi di indexing o abstracting). Tali strumenti permettevano, con una minima conoscenza tecnica di costruire portali ${ }^{85}$, intesi come piattaforme per organizzare l'accesso a servizi e contenuti

\footnotetext{
${ }^{83}$ Z39.50 è un protocollo di comunicazione informatica di tipo client-server che consente di ricercare attraverso una rete informatica informazioni in database differenti. La sua evoluzione è tuttora mantenuta dalla Library of Congress <http://www.loc.gov/z3950/agency/>. Il protocollo ha dato luogo alla norma ANSI/NISO Z39.50 e alle norme ISO 23950.

${ }^{84} \mathrm{La}$ studiosa distingue nella loro evoluzione due distinte generazioni. In una prima fase le biblioteche, grazie ad accordi di collaborazione e finanziamenti, costruirono in forma collaborativa grandi contenitori di informazioni digitali e che selezionavano strumenti rilevanti per la ricerca, costruendo i cosiddetti subject gateways o portali verticali o specialistici. Caratteristica di questa tipologia di portali era quella di focalizzare l'attenzione più sui contenuti che venivano messi a disposizione piuttosto che sulle esigenze degli utenti e sui servizi. Ciò si rivelò come un limite e portò ad un sostanziale insuccesso di questi strumenti. La seconda generazione di portali, che è quella oggi in atto, ha invece puntato sull' integrazione dei contenuti, sulla personalizzazione del servizio e sull'utilizzo di software open source. Cfr.: Maria Cassella, Le risorse elettroniche nei portali di biblioteche, «Biblioteche Oggi», 25 (2007), n. 10, p. 5-15.

${ }^{85}$ Tra i principali software per la costruzione di portali sono da citare: MetaLib (ExLibris), Millennium Access Plus (Innovative Interfaces), ENCompass (Endeavor Information Systems).
} 
in maniera personalizzata ${ }^{86}$. Purtroppo però questi primi strumenti di metaricerca, ancora oggi in uso nelle biblioteche, risultavano troppo poco integrati per essere effettivamente utili all'utente ${ }^{87}$. Essi infatti consentivano di lanciare una ricerca contemporaneamente su varie fonti diverse (e-journals, e books, banche dati o open archives), ma l'informazione recuperata non era integrata né integrabile. Rimanendo tali canali separati l'uno dall'altro, non era consentito attivare collegamenti reciproci né tra i macrodocumenti interrogati $(e$ journals, e-books, banche dati o open archives) né tra i microdocumenti (articoli e-printes, abstract, record bibliografici) che venivano recuperati generando risultati di ricerca duplicati e non integrabili o ordinabili, ad esempio, in un'unica schermata dei risultati.

Un relativo miglioramento della metaricerca è stato introdotto dalla cosiddetta 'ricerca federata' oggi in uso in molte biblioteche che consente l'interrogazione di diverse risorse organizzate in un unico contenitore. Gli strumenti per la ricerca federata migliorano di molto la presentazione dei risultati delle ricerche, presentandoli al lettore ordinati per ranking, aggregati e deduplicati e introducendo vari servizi (come il salvataggio delle ricerche o l'esportazione di citazioni o liste di riferimenti bibliografici) che non si trovavano nei precedenti strumenti.

Un ulteriore e più recente strumento, che consente di effettuare i collegamenti reciproci tra microdocumenti (il singolo articolo) e macrodocumenti (il titolo di una rivista), è invece dato dallo sviluppo della pratica del linking citazionale, o reference linking che si serve di software chiamati link resolvers ${ }^{88}$.

Gli strumenti che sfruttano il linking citazionale sono capaci, a partire dalle informazioni contenute in una citazione bibliografica su un qualunque servizio web, di arrivare all'oggetto informativo cui essa si riferisce e ad eventuali ulteriori servizi correlati, creando ambiente integrato per tutte le risorse digitali di un'istituzione ${ }^{89}$. Essi vengono usati per ottenere il fulltext di articoli o risorse riservate e si basano sullo standard OPEN URL, un formato standard di tipo open source per il trasferimento di metadati bibliografici

\footnotetext{
${ }^{86}$ Cinzia Bucchioni, - Serafina Spinelli, Teoria e pratica del linking citazionale «Biblioteche Oggi», 25 (2007), n. 8, p. 70-86. <http://www.bibliotecheoggi.it/2007/20070807001.pdf>.

${ }^{87}$ Riccardo Ridi, La Biblioteca come ipertesto cit., p. 202.

${ }^{88}$ Una Bibliografia selettiva sul "Link Resolver" è stata pubblicata rete dal CIBER/CASPUR nel maggio 2008 ed è visibile in rete all'indirizzo <http://www.uniciber.it/index.php?id=168>. Una lista aggiornata dei maggiori software e vendors è disponibile sul sito della Library of Congress alla url: $<$ http://www.loc.gov/catdir/lcpaig/openurl.html>.

${ }^{89}$ Per una panoramica più approfondita comprendente la storia del servizio di linking citazionale, i suoi sviluppi e le applicazioni pratiche si veda Cinzia Bucchioni, - Serafina Spinelli, Teoria e pratica del linking citazionale cit. Uno dei primi servizi di reference linking fu stato avviato nel 2007 nell'OPAC dell'Università di Pisa. I primi esperimenti su SFX e sullo standard Open URL condotti dallo studioso da Van de Sompel e da sui colleghi all'Università di Ghent in Belgio dal 1998 al 2000 sono stati tradotti in italiano e riassunti da Cinzia Bucchioni, SFX e OpenURL: gli esperimenti del team di Van de Sompel, «Bibliotime», V (2002), n. 2. $<$ http://didattica.spbo.unibo.it/bibliotime/num-v-2/bucchion.htm>.
} 
attraverso i servizi informativi. L'idea di partenza, dalla quale prese avvio la progettazione di questo strumento da parte dello studioso belga Van de Sompel, era data dalla possibilità di collegare una citazione al fulltext alla quale si riferisce.

In sostanza, una volta formulata l'interrogazione, all'utente viene fornito, insieme al risultato, un link (solitamente in forma grafica di pulsante) che gli consente di attivare, a partire da un documento selezionato, il resolver o 'risolutore di OpenURL' cioè una particolare URL (chiamata per l'appunto OpenURL) che viene generata al click del pulsante e che veicola i metadati.

Il resolver opera quindi una 'valutazione contestuale' in base ai dati ricevuti e al contesto della collezione di cui dispone l'istituzione e apre così una finestra con un elenco di servizi potenzialmente rilevanti per la citazione di partenza presentando all'utente i vari link che ne permettono l'esplorazione ${ }^{90}$.

I link resolvers trovano nell'OPAC l'utilizzo più appropriato. Essi producono infatti link 'caldi', che si rivelano così «massimamente efficaci, perché contestualizzati oggettivamente sulle reali caratteristiche (prima fra tutte la localizzazione), in un determinato istante e soggettivamente sui reali diritti di accesso, nel medesimo istante, dell'utente dato» ${ }^{91}$.

La tematica dell'allargamento dell'OPAC è dunque al centro del modello di sviluppo dei nuovi OPAC di nuova generazione e discovery systems. Gli strumenti di discovery si sono proposti nelle biblioteche come un rimedio all'uso di strumenti diversi; il loro ruolo è quello di sostituire, dove possibile, la ricerca federata e dunque i portali bibliotecari per consentire il recupero nell'interfaccia dell'OPAC di tutte le risorse disponibili; per questo all'inizio si parlò di next generation catalogs come 'OPAC portalizzati' e, usando una metafora commerciale, di ricerca one-stop shopping ${ }^{92}$. Ciò che risulta centrale è aggregare

\footnotetext{
${ }^{90}$ I vantaggi dell'adozione di questa tecnica sono stati così riassunti:

1) costruire un sistema di navigazione coerente che minimizza le possibilità di ricerche a risultato zero.

2) integrare e valorizzare nella collezione digitale messa a disposizione dalla biblioteche alcune tipologie di risorse poco conosciute, come ad esempio l' editoria free e archivi open access, i quali spesso non hanno visibilità nel web poiché non sostenuti da piattaforme commerciali forti.

3) valorizzare il ruolo nel catalogo nella sua doppia funzione di strumento di localizzazione di accesso alle risorse elettroniche e anche i servizi forniti (prestito interbibliotecario, document delivery, richiesta di informazioni al bibliotecario (Ask a ..) che "fungono da "integratori" tra la collezione digitale e cartacea.

4) consentire la possibilità di fidelizzare gli utenti alla biblioteca e al suo catalogo piuttosto che alle piattaforme commerciali o del web.

ma soprattutto l'importante vantaggio che si potrebbe ottenere dall'integrazione nell'opac di questi strumenti è quello di restituire alle biblioteche, per il tramite dei loro cataloghi il tradizionale ruolo di organizzazione, gestione e integrazione dei servizi in un contesto odierno caratterizzato, come abbiamo visto, da uno spostamento della ricerca fruizione dell'informazione al di fuori delle biblioteche. Cfr. Cinzia Bucchioni, Serafina Spinelli, Teoria e pratica del linking citazionale cit.

${ }^{91}$ Riccardo Ridi, La Biblioteca come ipertesto cit., p. 204.

${ }^{92}$ Roy Tennant, Cross-database search: one-stop shopping, «Library Journal»,126 (2001), n 17, p. 29-30.
} 
l'offerta per consentire all'utente una ricerca unificata e senza sforzo come avviene nella ricerca in rete ${ }^{93}$.

Per adempiere a questo compito le nuove interfacce si servono delle tecnologie dei motori di ricerca ${ }^{94}$ e si basano su un indice creato attraverso l'harvesting di contenuti da vari repositories che vengono così a creare un indice centralizzato. L'approccio che consente di interrogare risorse presenti in vari contenitori si basa sul protocollo OAI$\mathrm{PMH}^{95}$ : invece di richiedere in tempo reale $\mathrm{i}$ risultati di un'interrogazione, tecnica che rallenta notevolmente la ricerca, questo approccio si basa sulla preventiva indicizzazione dei documenti che vengono memorizzati in un indice centralizzato.

93 Cfr. Lorcan Dempsey, The (digital) library environment: ten years after, «Ariadne», 46 (2006) $<$ http://www.ariadne.ac.uk/issue46/dempsey/intro.html>.

${ }^{94}$ Lo stesso approccio è stato scelto da Google e Google Scholar. I loro indici contengono metadati e fulltext indicizzati da una varietà di risorse online e inclusi vari provider di contenuti usati dalle stesse biblioteche. Quando l'utente compie una ricerca Google Scholar non effettua una ricerca (cross-search) di ogni database, ma cerca nel proprio indice locale che raccoglie metadati e fulltext. Ciò ha reso Google Scholar più veloce degli strumenti per la ricerca federata e consente di evitare alcuni difetti, quali ad esempio quello di non poter includere nei risultati le risposte da un particolare server perché la ricerca è andata in timeout.

${ }^{95}$ OAI-PMH (Open Archives Initiative Protocol for Metadata Harvesting o Protocollo per la raccolta dei metadati dell'Open Archive Initiative) è un protocollo sviluppato dall'Open Archives Initiative come infrastruttura di comunicazione per il progetto l'Open Access. The Open Archives Initiative Protocol for Metadata Harvesting (OAI-PMH) un protocollo che consente di recuperare dati da sistemi diversi favorendo l'interoperabilità.

OAI-PMH fornisce un framework per l'interoperabilità che è indipendente da ogni applicazione e si basa sull'harvesting dei metadati. Vi sono due classi di partecipanti nel framework di OAI-PMH:

1) I fornitori di dati (data providers) sono repositories che espongono metadati strutturati attraverso l'OAI$\mathrm{PMH}$;

2) I fornitori di servizi (service providers) utilizzano i metadati raccolti attraverso l'OAI-PMH per costruire servizi. OAI-PMH « is a set of six verbs or services that are invoked within HTTP» utilizzando XML. Il set di metadati che correntemente viene usato dalla maggior parte dei repositories è Dublin Core. La versione corrente è la 2.0, aggiornata nel 2002: <http://www.openarchives.org/OAI/openarchivesprotocol.html> e http://www.openarchives.org/pmh/. Si veda anche OAI PMH Guidelines: $<$ http://www.openarchives.org/OAI/2.0/guidelines.htm>. 


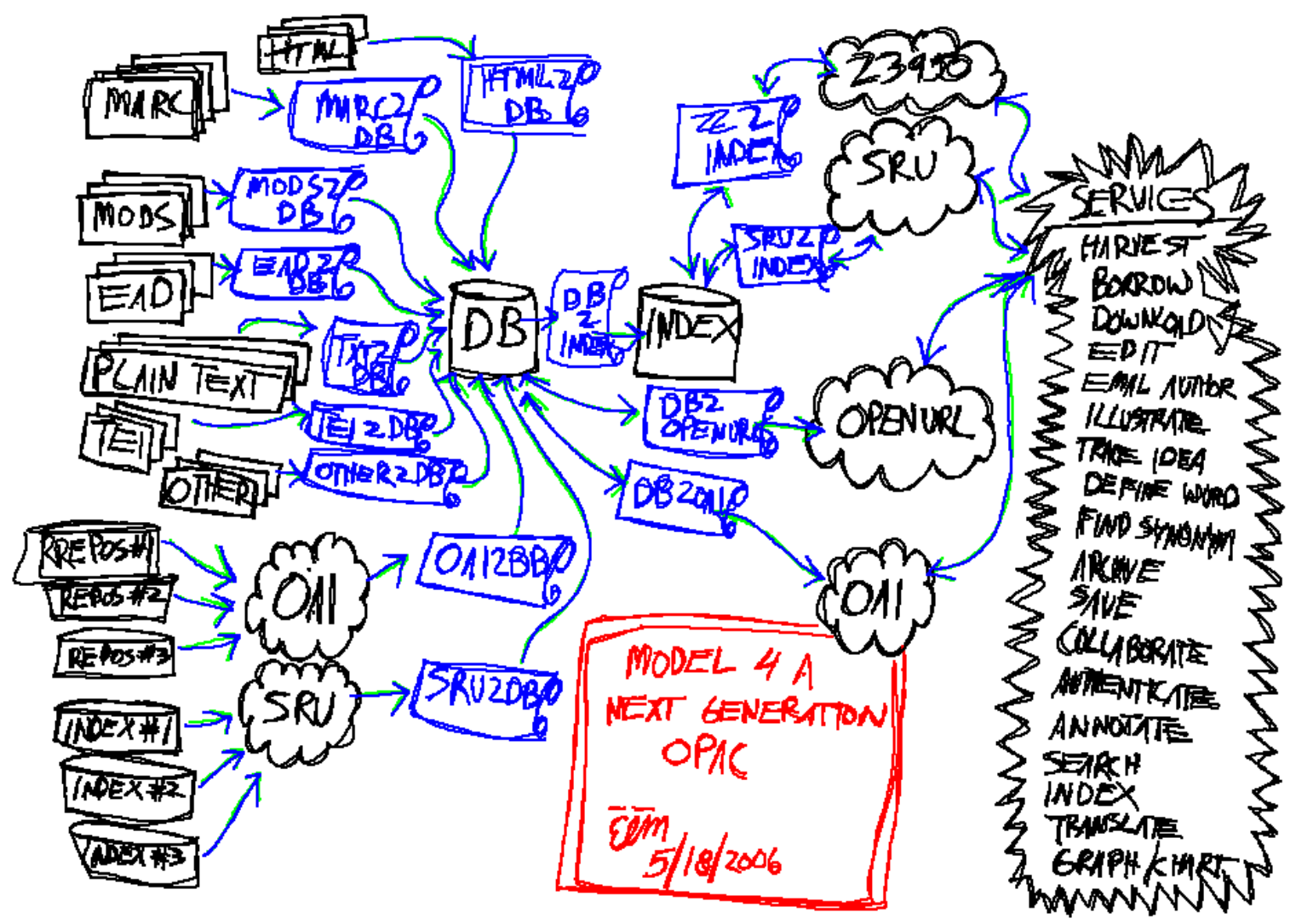

Fig. 2.7 - L'architettura dei next generation catalogs. Alla base della progettazione vi è la presenza dell'indice centrale ${ }^{96}$

L'harvesting di un intero database, che potrebbe oggi sembrare un'operazione lunga, in effetti è resa possibile dalla banda larga e dalle grandi capacità di calcolo dei computer attuali. Esso avviene prima della ricerca e si rivela una strategia più adatta per la ricerca in real-time, in quanto consente di velocizzare di molto il processo di ricerca, che oggi tocca i tempi raggiunti dai motori (qualche millesimo di secondo). L'interazione con la fonte (la ricerca remota) può avvenire durante la visualizzazione del dettaglio record, anche se la maggior parte dei dati MARC vengono indicizzati dal sistema ${ }^{97}$.

\footnotetext{
96 Eric Lease Morgan, Next generation library catalog, «Infomotions.com», 02-06- 2006, $<$ http://infomotions.com/musings/ngc/>.

${ }^{97} \mathrm{La}$ maggior parte dei dati dei record viene indicizzata per comparire nella pagina dei risultati in modo veloce. Gli altri dati non indicizzati vengono richiamati con interazione real-time al momento della visualizzazione del singolo record (dettaglio record). In questo modo si ottiene un miglioramento delle prestazioni al momento della ricerca.
} 


\subsubsection{La ricerca globale: discovery systems e Web scale discovery services}

Oggi la maggior parte dei next generation catalogs offre la possibilità di estrarre dati non solo dagli ILS, ma da una serie molto vasta di fonti: repositories, biblioteche digitali, banche dati di articoli fulltext.

L’integrazione degli articoli di riviste da fornitori diversi rende però l'utilizzo dei discovery system un'operazione non sempre risolutiva e richiede alla biblioteca un impegno notevole di risorse: non sempre è possibile recuperare tutto il materiale necessario (ad esempio abbonamenti di editori minori) e questa operazione sembra ancora piuttosto complessa, dunque si ricorre ancora alla ricerca federata per affiancare questi prodotti e gestire meglio il recupero degli articoli delle riviste in abbonamento che non sono contenute negli indici centralizzati o che non possono essere preventivamente indicizzati.

Oggi gli strumenti per la scoperta o Web Scale discovery services offrono indici già 'popolati' e pronti all'uso, comprendenti risorse free ma anche pacchetti a pagamento. Questi dati possono essere visualizzati velocemente grazie a questo sistema. Questi nuovi prodotti consentono di sfruttare indici già pronti e costituiscono uno sgravio di lavoro per le biblioteche.

Discovery tools should not be confused with federated search products. The former "promise to provide a single interface to multiple resources based on using a centralized consolidated index to provide faster and better search results", while the latter search remotely, rely on connectors, and provide "only partial and limited solutions" (Hane, 2009). In addition, a federated search tool usually requires user logon and works in a protected environment, while a discovery layer is open to the public. A federated search tool is dedicated to finding articles across a number of subscribed databases ${ }^{98}$

Nonostante ciò, se l'obiettivo dei Web Scale discovery services è arrivare a fornire una copertura globale delle risorse sottoscritte dalla biblioteca, ancora oggi la ricerca federata si propone come soluzione per affiancare questi strumenti per estendere il contenuto della ricerca a quei documenti che non è stato possibile indicizzare in precedenza. Molti discovery systems offrono il supporto della ricerca federata come componente integrabile con il software. L'uso della ricerca federata però rende un'interrogazione più lenta e l'integrazione dei risultati di un'interrogazione in un elenco ordinato per rilevanza non produce risultati soddisfacenti.

Nei primi anni di sviluppo dei discovery systems, per quanto riguarda le riviste, i contenuti recuperabili erano alquanto limitati. Tali prodotti incontravano infatti la

\footnotetext{
${ }^{98}$ Sharon Q. Yang - Kurt Wagner, Evaluating and comparing discovery tools cit., p. 691.
} 
riluttanza di editori a fornire i metadati degli articoli delle riviste. In seguito, l'idea di creare Web Scale discovery services in accordo con editori ha permesso di trovare soluzioni vantaggiose, consentendo così oggi a questi strumenti di discovery di espandere il loro mercato.

Attualmente i sistemi di discovery and delivery che si appoggiano a pacchetti commerciali come Primo non riescono a gestire in maniera unificata tutte le risorse in abbonamento, non consentono l'effettivo accesso senza barriere a contenuti che vengono prelevati tramite i link resolvers (gli utenti devo spesso autenticarsi varie volte per raggiungere i contenuti desiderati), e pongono alcuni problemi alle biblioteche in merito all'effettiva copertura delle risorse e alla qualità dei metadati.

Particolarmente interessante in tema di qualità è l'Open discovery Initiative (ODI), un progetto di $\mathrm{NISO}^{99}$ che si propone di definire standard e buone pratiche per una nuova generazione di servizi di scoperta per le biblioteche basati sulla ricerca negli indici ovvero i 'next generation library discovery services'. Usando indici che aggregano una vasta serie di risorse gratuite o a pagamento, da diversi providers, questi strumenti per la scoperta possono fornire servizi più sofisticati se paragonati alla ricerca federata o alle tecniche finora usate. Data la crescente sinergia tra i produttori di informazione (providers commerciali, istituzionali, open access) e i discovery services il gruppo intende stabilire buone pratiche per i contenuti che devono essere mostrati nei discovery systems, per l'interazione tra i creatori di questi servizi e gli information providers di queste risorse. Fondato alla fine del $2011^{100}$, il gruppo si è incontrato per la prima volta nel gennaio 2012 per dare avvio ai lavori ${ }^{101}$. Nel work item proposal ${ }^{102}$ vengono enunciati alcuni problemi

\footnotetext{
99 NISO (National Information Standards Organization) è un'organizzazione statunitense senza scopo di lucro che sviluppa, mantiene e pubblica standard tecnici relativi ad applicazioni per biblioteche. Gli standard approvati dal NISO sono pubblicati dall' ANSI, i più noti sono Z39.50 (protocollo per i dati bibliografici) e Z39.88 (OpenURL). Attraverso questi standard è possibile l'interoperabilità tra comunità diverse che producono informazione.

100 Comunicato stampa: NISO launches new Open Discovery Initiative to develop standards and recommended practices for library discovery services based on indexed search (October 25, 2011). <http://www.niso.org/news/pr/view?item_key=21d5364c586575fd5d4dd408f17c5dc062b1ef5f>.

101 Tra i membri del gruppo Marshall Breeding (Director for Innovative Technologies and Research, Vanderbilt University), e Jenny Walker (Consultant for Ex Libris). A proposito dei principali difetti dei DS Walker commenta (ivi): «They expect their uniquely licensed and purchased electronic content to be made available within the discovery service of their choice. But it is often not clear which resources are available, which are indexed in full text or by citations only, or both, and whether the metadata derives from aggregated databases or directly through the full-text.»e Breeding aggiunge: «The scope of discovery interfaces is broader than what is managed in the integrated library system [...] These services can include other local repositories and digital collections and the electronic resources in subscription information content products. We'd like to see a consistent vocabulary regarding all the elements involved, that there be clarity in the business rules that apply to the content once indexed, and that there be clear descriptors regarding the extent of indexing performed for each item or collection of content and the level of availability of the content».

${ }^{102}$ Standards and best practices for library discovery services based on indexed search: a proposed NISO work item voting member ballot, September 2 - October 2, 2011, Work item proposal:
} 
comuni che le biblioteche incontrano nell'uso di questi strumenti: i prodotti per la scoperta sono basati su accordi privati e metodologie di scambio spesso costruite ad hoc, è dunque importante che le biblioteche abbiano una chiara comprensione del grado di disponibilità di tali contenuti nel sistema di discovery scelto. Spesso non è chiaro se gli articoli o gli ebook sono disponibili, se sono indicizzati in full text, o solo per citazione, e se i metadati vengono ricavati da altri database aggregati o direttamente dal full text ${ }^{103}$. Inoltre le biblioteche si aspettano che tutte le risorse possedute siano indicizzate nell'indice centrale e chiarezza sulla copertura offerta dagli indici.

Libraries expect all resources to be indexed: increasingly difficult to deal with remnants of collection not represented in the discovery index. They also expect clarity on what is covered in the index: citations, abstracts, controlled vocabulary terms, full-text; and on the currency of discovery index, what is available in discovery system should closely match that of the native interface. The coverage of content by a particular discovery service is becoming a collection development tool ${ }^{104}$.

Le buone pratiche che il gruppo metterà a punto serviranno alle biblioteche per valutare la copertura e l'effettiva disponibilità e il grado di granularità dei contenuti e consentirà di ottenere una migliore intesa tra editori (information providers) e i venditori di questi sistemi sistemi. Tra le iniziative proposte vi è lo sviluppo di un vocabolario comune che consenta alle parti un'intesa migliore nello sviluppo di accordi reciproci riguardo a formato dei dati, metodi di scambio e di aggiornamento, diritti di accesso alle risorse. Lo scopo è di ottimizzare la comunicazione tra le parti in modo che le biblioteche possano identificare meglio i fornitori dell'informazione anche attraverso l'uso di identificatori persistenti ${ }^{105}$.

$<$ http://www.niso.org/apps/group_public/document.php?document_id=7175>.

${ }^{103}$ Ivi, p. 1.

${ }^{104}$ Ivi, p. 8.

${ }^{105}$ Nel dettaglio gli obiettivi sono (ivi, p. 3-4):

Specific Deliverables and Objectives:

Policy/business

a. Define levels of compliance

b. Content: Define the depth and breadth of content available to Open

Discovery indexes

c. Rights/authorization; Define level of access to indexed content (restricted to subscribers vs. open to all users)

d. Display: Define a minimum standard of what can be displayed from information provider content and metadata (and how to protect information provider intellectual property rights)

e. Linking: Define the incorporation of fair linking

ii. Technology

a. Rights: determination and sharing of authorizations when content discovery is restricted

b. Formats: content, and methods of updates

c. Usage statistics gathering and reporting 


\subsubsection{L'arricchimento}

Il circuito dell'informazione nel quale si inseriscono le biblioteche risulta oggi maggiormente complesso che in passato; accanto ai consueti protagonisti (biblioteche, editori, agenzie bibliografiche, centri di documentazione) entrano in scena nuovi attori, tra i quali anche le società commerciali che gestiscono i motori di ricerca e le librerie online.

Il web ha impresso un'accelerazione al processo di arricchimento degli OPAC: la diffusione di internet, l'impiego di tecnologie sempre più potenti e la crescita esponenziale dell'informazione in rete, specie in forma di fulltext, hanno portato gli utenti a non accontentarsi più della rappresentazione del documento fornita dalla descrizione catalografica, ma a pretendere di più dai cataloghi di biblioteca: il sommario, l'abstract o il full-text, la biografia dell'autore, la visualizzazione della copertina, i link alle risorse esterne, come una bibliografia sull'argomento cercato e, più di recente, i commenti e i pareri degli altri lettori.

Un catalogo elettronico che si qualifichi 'arricchito' è quindi sostanzialmente caratterizzato dalla presentazione, a fianco della tradizionale scheda catalografica, di una serie di materiali digitali e vari link a risorse informative.

L'arricchimento' degli OPAC è una pratica ormai comune ed è volta a completare la notizia del catalogo con elementi di tipo valutativo (abstract, sommari, copertine, link a fulltext) e di approfondimento (collegamento a risorse online, es. siti di biografie, di enciclopedie etc.). Tale filone di sviluppo sembra ormai pienamente consolidato di fronte alle crescenti esigenze dell'utente, che non si accontenta più della semplice rappresentazione del documento fornita dalla tradizionale registrazione catalografica.

L' 'arricchimento' ha condotto alla nascita dei cosiddetti 'OPAC arricchiti', una fase che è ormai comunemente considerata immediatamente precedente allo sviluppo dei Next generation catalogs e che, certamente, ne ha aperto la strada.

Come abbiamo appena visto, un particolare tipo di arricchimento dell'OPAC può sostanziarsi in una sua espansione, cioè nella capacità di dare l'accesso a risorse esterne. In questo senso abbiamo visto, quindi, come all'OPAC siano stati affiancati dapprima strumenti per la 'metaricerca' o per il 'linking' e come le soluzioni più avanzate spingano verso la creazione di sistemi per la scoperta che inglobano, oltre ai dati dell'OPAC, anche una serie molto vasta di risorse.

Anche il processo di arricchimento, come quello precedentemente descritto dell'estensione, ha avuto una fase sperimentale: molte biblioteche hanno dapprima 
arricchito i loro record catalografici incorporando risorse multimediali o altre risorse elettroniche locali presenti in biblioteca. In questo caso però l'arricchimento comportava un'onerosa attività in termini di tempo e di costi, oltre che l'appesantimento dei record catalografici.

In seguito il problema è stato affrontato in chiave più realistica ${ }^{106}$, cioè anziché arricchire il catalogo localmente, aggiungendovi quindi sommari, abstract ecc. predisposti dai bibliotecari, si è scelto di reperire le informazioni necessarie in altri sistemi informativi tramite opportuni collegamenti.

Questa modalità di arricchire il catalogo ha consentito ai record di conservare una struttura più leggera, in quanto la notizia catalografica viene arricchita dinamicamente nel momento dell'interrogazione, assemblando diversi elementi (toc, bibliografie, recensioni, abstract) prodotti in altre sedi.

Perché ciò possa avvenire occorre una collaborazione tra le biblioteche e le altre componenti del circuito bibliografico (editori, agenzie bibliografiche, centri di ricerca) e soprattutto, dal punto di vista tecnico, la necessità di disporre di metadati standard sia descrittivi che semantici, di favorire cioè l'interoperabilità ${ }^{107}$.

Tra le varie tipologie di arricchimento la meno recente è l'aggiunta di immagini. Già a partire dalla metà degli anni '80, lo sviluppo delle capacità di calcolo e di memoria degli elaboratori e l'introduzione di standard per la compressione delle immagini rese possibile affiancare alle notizie catalografiche anche immagini di parti del documento. Queste aggiunte si rivelano oggi preziose per la presentazione (e preservazione) del materiale antico o di pregio. Più recente è invece la presenza delle immagini delle copertine dei volumi dovuta alla diffusione delle librerie on-line sul web. Esse possono essere importate

\footnotetext{
${ }^{106}$ Così commentava Paul Gabriele Weston questi primi tentativi di arricchimento: «Ben più realistica e in linea con le potenzialità del Web è, invece, la scelta di reperire le informazioni necessarie altrove, cioè in altri sistemi informativi, bibliografici e non, e di stipulare gli opportuni collegamenti. Avendo individuato, all'interno di altre risorse (bibliografie, spogli, enciclopedie e dizionari, cataloghi di vendita e altri repertori più o meno specializzati) gli elementi che occorrerebbe integrare nella notizia bibliografica, si punta ad essi per mezzo di identificatori, senza essere obbligati a trascrivere tali dati all'interno della registrazione catalografica». Paul Gabriele Weston, La gestione elettronica delle biblioteche in Biblioteconomia: principi e questioni, a cura di Giovanni Solimine e Paul Gabriele Weston, Roma, Carocci, 2007, p. 221-256. La citazione si trova a p. 233-234.

${ }^{107}$ Sull'interoperabilità tra sistemi eterogenei si veda Paul Gabriele Weston, Dal controllo bibliografico alle reti documentarie. Il catalogo elettronico nella prospettiva dell'interoperabilità tra sistemi eterogenei. «Biblioteche Oggi», 20 (2002), n. 7, p. 44-56 e dello stesso autore Gli strumenti della cooperazione in rete : dal catalogo elettronico ai sistemi della ricerca interdisciplinare, Napoli, ClioPress, 2003. Versione elettronica in <http://www.storia.unina.it/cliopress/weston.html>, p. 7-14.
} 
automaticamente dai più conosciuti siti di servizi web o dalle librerie online (per mezzo di apposite $\mathrm{API}^{108}$ ) per completare la presentazione della notizia catalografica.

L'inserimento dei sommari o TOC (tables of content) negli OPAC è un altro tipo di arricchimento che si è diffuso da parecchi anni, specie in area anglosassone, dove i primi progetti risalgono a tempi non recenti ${ }^{109}$. L'utilizzo dei sommari a complemento delle descrizioni bibliografiche fu fortemente raccomandato da Byrum ${ }^{110}$ e nell'ultimo decennio anche in Italia si sono avuti i primi esperimenti in questo campo ${ }^{111}$ per poi diventare comuni in tutti i principali OPAC.

I sommari di volumi o riviste vengono passati allo scanner e convertiti in testo tramite appositi software di tipo OCR, dunque i TOC vengono opportunamente trattati con programmi software che consentono di eliminare le parti del discorso non significative ai fini della ricerca trasformando tali sommari in documenti costituiti interamente da parole chiave che possono quindi essere oggetto di una ricerca per parola chiave.

È evidente che l'inclusione dei sommari possa rappresentare per l'utente un ulteriore elemento di valutazione del contenuto dei documenti in aggiunta a quelli offerti dagli accessi semantici. Più discutibile è invece la possibilità che la ricerca per parola chiave su questi elementi interni al documento possa rendere superflua l'indicizzazione semantica ${ }^{112}$.

Recensioni e abstract, link a siti di biografie, a siti di risorse online quali, ad esempio enciclopedie $^{113}$, repertori ecc... costituiscono altre informazioni aggiuntive che è possibile

${ }^{108}$ Con lo sviluppo delle applicazioni 2.0, queste aggiunte sono sempre più spesso rilasciate sottoforma di API. È recente infatti il rilascio di un milione di copertine di volumi, da parte di Librarything, uno dei più fortunati siti di social tagging. La notizia (LibraryThing Releases One Million Free Covers) si trova all'url: $<$ http://www.libraryjournal.com/article/CA6586479.htm>. Tutte le API di Google si trovano su Google Code e su Google Developers (<https://developers.google.com>). Un'esperienza d'uso delle copertine di Librarything nell'OPAC della Clermont library è stata descritta in Jezmynne Westcott-Alexandra ChappellLebel Candace, LibraryThing for libraries at Claremont, «Library Hi Tech», 27 (2009), n.1, p. 78-81.

${ }^{109}$ Tra i primi progetti in area anglosassone si segnalano i progetti D-TOC e ONIX-TOC condotti dal gruppo Beat della Library of Congress citati da John D. jr. Byrum nelle Recommendations for urgently needed improvement of OPAC cit.

${ }^{110}$ Byrum ha portato a sostegno della sua idea gli studi compiuti da altri studiosi, tra i quali Ruth C. Morris. La studiosa ha rilevato, infatti, come vi sia stato un incremento dell'uso e della richiesta di quei volumi che presentavano i TOC.

${ }^{111}$ In Italia la prima esperienza significativa di utilizzo dei TOC è stata il progetto Indici e sommari in OPAC, realizzato dal CIB dell'Università di Bologna che ha arricchito il proprio OPAC di ateneo con la digitalizzazione dei sommari cfr. Raffaella Gaddoni, - Maria Laura Vignocchi, Nella selva oscura degli Opac, «Bibliotime», IX (2006), n.3 <http://www2.spbo.unibo.it/bibliotime/num-ix-3/gad-vign.htm>.

112 Anche qui il riferimento è alle posizioni espresse da Byrum (John D. jr. Byrum, Recommendations for urgently needed improvement of OPAC cit. p.6) laddove afferma: «le parole chiave dei TOC migliorano notevolmente l'efficacia della ricerca» e che «i TOC offrono il mezzo per superare le ristrettezze tradizionali della ricerca per soggetto. In effetti, uno studio durato undici anni ha stabilito che la ricerca per soggetto [è] stata rimpiazzata dalla ricerca per parole chiave, un punto decisamente a favore dei TOC, in quanto essi sono interamente costituiti da parole chiave».

${ }^{113}$ La tendenza verso questo tipo di collegamento è una caratteristica ormai adottata anche dei motori di ricerca. Google da qualche anno fa apparire tra i primi risultati proprio i link a Wikipedia, rafforzando così la 
importare nell'OPAC grazie alla collaborazione delle biblioteche con gli editori o all'utilizzo di API aperte. Il collegamento a Google Books e la funzione look inside è ormai caratteristica di molti OPAC, che sfruttano l'API di Google per visualizzare un'anteprima del contenuto di un documento.

Certamente l'arricchimento rappresenta un aiuto per l'utente e ormai una caratteristica irrinunciabile nei nuovi prodotti, che rafforza la multilinearità e l'ipertestualità dei cataloghi elettronici.

L'arricchimento bibliografico, collegando varie risorse elettroniche, dati e informazione referenziale e moltiplicando i percorsi possibili, ha portato alla realizzazione del catalogo post-coordinato preannunciato da Ayres dove la post-coordinazione risiede nella capacità per l'utente di scegliere i propri percorsi di ricerca ${ }^{114}$. Lo stesso concetto era stato espresso nella definizione del 'catalogo ipertestuale' da Weston e Galeffi, anche se il concetto di ipertestualità oggi sembra superato alla luce delle innovazioni del Web Semantico e dei linked data:

Combinando la ricerca per soggetti o descrittori con le indicazioni provenienti dalla guida bibliografica e mettendo in relazione risorse elettroniche, dati provenienti da scansione digitale e informazione referenziale, il catalogo finisce per diventare un vero e proprio sistema bibliografico complesso e nel dispiegarsi dell'intreccio di riferimenti incrociati tra descrizioni e bibliografie, documenti testuali e audiovisivi, rimandi interni ai documenti e tra documenti diversi, prende corpo il catalogo ipertestuale ${ }^{115}$.

In Italia i primi esempi di arricchimenti, che ormai si ritrovano in numerosissimi $\mathrm{OPAC}^{116}$, sono stati quelli dell'OPAC del polo bolognese, della Biblioteca Nazionale Centrale di Firenze ${ }^{117}$, del nuovo OPAC SBN, del Sistema bibliotecario provinciale di Verona $^{118}$.

tendenza degli utenti a rivolgersi ai motori anche per soddisfare la necessità di apprendere nozioni di base su un dato argomento.

114 Ayres (1999) citato in Paul Gabriele Weston, Il catalogo elettronico. Dalla biblioteca cartacea alla biblioteca digitale cit.

115 Weston-Galeffi 2004 citato in Paul Gabriele Weston, La gestione elettronica delle biblioteche in Biblioteconomia: principi e questioni, a cura di Giovanni Solimine e Paul Gabriele Weston. Roma, Carocci, 2007, p. 221-256. Cit. a pag. 235.

${ }^{116}$ Una prima panoramica degli OPAC 'arricchiti' in Italia fu fornita dal breve contributo di Frigimelica Giovanna Frigimelica, OPAC arricchiti: alcuni esempi italiani, «AIB Notizie», 19 (2007) n. 5, <http://www.aib.it/aib/editoria/n19/0507.htm3>.

${ }^{117}$ L'OPAC della BNCF fu tra i primi OPAC italiani a recepire alcune innovazioni presenti nelle interfacce 'di nuova generazione': lo strumento venne dotato di un 'navigatore Dewey' e di un'interfaccia completamente rinnovata che forniva le digitalizzazioni parziali di numerosi documenti con le immagini di copertina, occhietto, frontespizio e indici o sommari, ma anche copie digitali del volume. Le novità dell'OPAC fiorentino furono commentate da Bonaria Biancu, Opac 2.0: BNCF, «The Geek Librarian», [Blog post], 28 febbraio 2006, <http://bonariabiancu.wordpress.com/2006/02/28/opac-20-bncf>.

${ }^{118}$ L'OPAC oltre a presentare gli arricchimenti integra una serie di caratteristiche funzionalità tra le quali arricchimenti 'sociali' che gli utenti possono apportare alle notizie del catalogo. Cfr. infra Appendice A. Casi di studio. Nuovo OPAC SBN. 
C'è da considerare infine, se il solo arricchimento costituisca un reale potenziamento e arricchimento delle funzioni del catalogo.

Secondo Marchitelli e Piazzini ${ }^{119}$ l'OPAC arricchito rappresenta una fase transitoria tra l'OPAC tradizionale e l'OPAC di nuova generazione. L'arricchimento che abbiamo visto sin ora si sostanzia in un'aggiunta di ulteriori informazioni alle informazioni presenti nel catalogo ma, secondo lo studioso, le funzionalità di ricerca rimangono sempre le stesse, le interfacce sono poco usabili e l'interazione tra la biblioteca e l'utente resta sostanzialmente monodirezionale.

I dati di arricchimento inoltre - secondo il report di OCLC del $2009^{120}$ - richiedono modalità sostenibili di approvvigionamento, che si fondano sulla necessaria interoperabilità e sulla cooperazione. Essi possono essere ottenuti in automatico dal catalogo, possono essere acquistati da fornitori commerciali o, infine, essere importati nei cataloghi utilizzando le API di servizi web o di partner commerciali ${ }^{121}$.

Non si può però evitare di ricordare che l'arricchimento tramite tables of contents (toc), raccomandato anche in altri studi quale opzione sempre più richiesta dagli utenti, nasconde alcune insidie meno note, quali l'abbandono delle procedure manuali per la catalogazione semantica a favore di forme di indicizzazione automatizzata. Secondo il rapporto, quando l'utente richiede più informazione di soggetto non si riferisce alle forme controllate fornite dagli accessi per soggetti o per classi, ma a elementi per lo più esterni alle descrizioni catalografiche (toc, abstract, link a risorse esterne); in questo modo si suggerisce che l'investimento sull'indicizzazione per soggetto debba essere reindirizzato verso forme di produzione più economiche ${ }^{122}$.

Un ulteriore arricchimento è l'aggiunta di parole chiave o metadati 'qualificanti' al record, soluzione avanzata da Griffis e Ford ${ }^{123}$ e da Markey ${ }^{124}$.

\footnotetext{
${ }^{119}$ Andrea Marchitelli, - Tessa Piazzini, OPAC, SOPAC E Social networking: cataloghi di biblioteca 2.0?. «Biblioteche Oggi», $26 \quad$ (2008), n. $\quad 2, \quad$ p. $\quad 82-92 . \quad$ oppure: $<$ http://www.bibliotecheoggi.it/content/20080208201.pdf>.

${ }^{120}$ ONLINE COMPUTER LIBRARY CENTER (OCLC), Online catalogs. What users and librarians want cit.

${ }^{121}$ Tra i vari fornitori di API vi sono communities sociali dedicate ai libri (Librarything) e siti commerciali (Amazon).

122 «Given the growing concern that these traditional methods are not sustainable going forward, it may be necessary for libraries to find more economical means to achieve the benefits to end users that controlled subject vocabularies provide». (OCLC, Online catalogs cit., p. 52).

123 Patrick Griffis - Cyrus Ford, Enhancing OPAC records for discovery, «Information technology and libraries», 28 (2009), n. 4, p. 191-193.

${ }^{124}$ Karen Markey, The online library catalog cit.
} 
Tali tipologie di arricchimento possono essere vantaggiose per migliorare la rilevanza e la pertinenza di una risorsa, legando la valutazione alla dimensione soggettiva della ricerca: l'utente potrebbe dunque selezionare una risorsa

* in base alla conoscenza di un dato dominio disciplinare (esperti, principiante);

* secondo l'autorità dell' autore;

* secondo i destinatari della risorsa (adulti, ragazzi);

* secondo il soggetto e il genere letterario;

* secondo cluster di raggruppamenti cronologici cui si riferisce l'argomento;

* a seconda dell'uso che si può fare della risorsa (scaricabile, scaricabile ma protetta da scrittura, acquistabile, e altri tipologie di utilizzo (ascolta, guarda, leggi);

* a seconda della popolarità tra gli utenti;

* a seconda dell'esperienza che possono trarre dal documento ${ }^{125}$.

Infine un arricchimento particolare e che non richiede sforzo alle biblioteche è quello nel quale l'aggiunta di informazioni proviene dall'interazione dell'utente con il catalogo apportandovi la 'socialità'. Ciò avviene nel momento in cui al catalogo vengono applicate alcune tecniche tipiche del Web 2.0, i cosiddetti social software o gli strumenti propri del social networking di cui si parlerà più avanti. Si è parlato per qualche tempo di questa tipologia di arricchimenti come di OPAC 2.0 o social OPAC. Alcuni studiosi hanno studiato metodi per ricavare tali metadati 'qualificanti' dai social network importando i tag aggiunti volontariamente dagli utenti alle risorse nei numerosi siti sociali della rete.

Tuttavia, nonostante l'iniziale entusiasmo, l'impatto di questa massa critica non qualificata di metadati sul recupero dell'informazione non ha prodotto risultati soddisfacenti. Sebbene alcuni cataloghi oggi introducano questi tipi di arricchimenti presentando nuvole di tag, queste si riducono a metodi personali di ordinamento delle risorse senza beneficio alcuno per gli altri utenti del catalogo.

\subsubsection{Deep indexing}

Sempre nel campo del trattamento dei dati rientra la cosiddetta indicizzazione profonda o deep indexing, cioè la possibilità del sistema di indicizzare non solo i metadati di una risorsa, ma anche il fulltext. Se i cataloghi tradizionali infatti indicizzano i soli metadati costituiti dai record MARC, i nuovi strumenti invece consentono di indicizzare il fulltext ricavando ulteriori indici che possono essere sfruttati in fase di ricerca. I nuovi

${ }^{125}$ Ivi, s. p. 
discovery systems presentano la possibilità di indicizzare tramite l'harvesting i contenuti provenienti da diversi repositories, molti dei quali presentano informazioni testuali in fulltext, grazie ai progetti di digitalizzazione avviati da Google Book Search e OCA (Open Content Alliance). Tali indici centralizzati (si pensi ad esempio a Primo Central, o ad Ebsco) indicizzano una grande quantità di contenuti (es. articoli di riviste o e-book) in fulltext. La presenza di documenti di diversa omogeneità rende necessaria la presenza di metadati di alta qualità pena la difficoltà di creare le faccette necessarie, fornire un ranking sufficientemente efficace o supportare interrogazioni complesse ${ }^{126}$.

\subsubsection{Ricerca, visualizzazione dei risultati e navigazione nel catalogo}

La maggior parte delle innovazioni nella ricerca nel catalogo e nella visualizzazione dei risultati viene introdotta con la nascita dei primi next generation catalogs.

Due i principali modelli di riferimento:

* il motore di ricerca con il box unico di interrogazione, l'esposizione dei risultati per rilevanza, ed una serie di tecnologie avanzate di information retrieval;

* i siti commerciali, che hanno introdotto la modalità di navigazione a faccette per fornire filtri dinamici e alcune funzioni di memorizzazione e organizzazione dei dati.

In quest'area si contano le principali innovazioni di rilievo: relevance ranking, navigazione a faccette, raggruppamenti dinamici, correzione dello spelling, stemming, ricerca fuzzy, relevance feedback.

Alcune di queste tecniche, tipiche dei motori di ricerca, consentono di ridurre o ampliare la ricerca e di introdurre nella progettazione degli OPAC un approccio di tipo esplorativo.

\subsubsection{Relevance ranking}

Con lo sviluppo dei metaOPAC e dei cataloghi collettivi l'interrogazione dei cataloghi è divenuta più complessa; infatti, molto spesso un'interrogazione genera una lista

\footnotetext{
${ }^{126}$ Molto spesso i dati necessitano di un'operazione di 'pulizia' che va fatta da ogni singolo istituto, pena l'incoerenza dei risultati ottenuti.
} 
di occorrenze molto lunga e, in mancanza di meccanismi di raggruppamento, e della mancata implementazione del modello FRBR, ci si ritrova a scorrere molte schermate con evidente allungamento dei tempi della ricerca.

Il relevance ranking ovvero l'esposizione dei risultati per 'presunta rilevanza', com'è noto, proviene dagli algoritmi messi a punto dai motori di ricerca per ordinare i risultati di un'interrogazione, costituiti dalle pagine web, in ordine di rilevanza rispetto alla richiesta effettuata, presentando i risultati più pertinenti in cima alla lista.

L'applicazione di meccanismi di ranking negli OPAC, nonostante sia stata indicata come la prima e più urgente modifica da apportare ai cataloghi elettronici, presenta molti problemi complessi ${ }^{127}$.

Innanzitutto bisognerebbe chiedersi principalmente quali siano i metodi sui quali viene determinata la rilevanza, che come vedremo, si basa su un procedimento algoritmico di matching tra $\mathrm{i}$ termini presenti in un'interrogazione e i termini presenti nel documento o nei suoi metadati.

In secondo luogo, tali metodi dovrebbero essere sempre esplicitati all'utente, il quale dovrebbe avere in qualche modo una possibilità di scelta tra vari tipi di ordinamento.

Infatti non bisogna dimenticare che la rilevanza è un valore soggettivo e multidimensionale, non risolvibile nella semplice rilevanza algoritmica; dunque i metodi secondo i quali tale rilevanza viene oggettivata dovrebbero essere chiari ed implicare una possibilità di controllo da parte degli utenti:

Nella valutazione dei sistemi di ricerca la rilevanza è considerata un valore soggettivo, relativo all'utente, ora la troviamo oggettivata, secondo la frequenza dei termini richiesti, la loro posizione, i link ricevuti... Il vantaggio del relevance ranking allora risulta aleatorio per il singolo utente, che è inconsapevole e non interagisce. Quanto più pesa nell'algoritmo l'interazione collettiva (ciò che è più offerto e che più persone cercano è più rilevante), tanto più il criterio è funzionale all'omologazione di massa (ma ciò che vale in statistica o nella sociologia dei consumi, non necessariamente vale per me o per te, qui e ora). In ogni caso non è sostitutivo di un'impostazione meglio mirata della ricerca, di cui anzi segnala l'esigenza ${ }^{128}$.

\footnotetext{
127 Si vedano i contributi di Maria Teresa Biagetti, Pertinence perspective and OPAC enhancement, in Paradigms and conceptual systems in knowledge organization. Proceedings of the Eleventh International ISKO Conference 23-26 February 2010, Roma. Edited by Claudio Gnoli and Fulvio Mazzocchi. Würzburg, Ergon Verlag, 2010, p. 334-340 e Ead., Nuove funzionalità degli OPAC e relevance ranking, «Bollettino AIB», vol. 50 (2010) n. 4, p. 339-356.

128 Pino Buizza, Gli opac: funzionalità e limiti nel mondo del web, «Bibliotime», XI (2008), n.1 $<$ http://didattica.spbo.unibo.it/bibliotime/num-xi-1/buizza.htm>.
} 
La mancanza di controllo da parte dell'utente è la prima e più grave criticità della rilevanza applicata agli OPAC: molti studiosi, tra i quali Yee consigliano di evitare il relevance ranking perché toglierebbe all'utente il pieno controllo sulla propria ricerca ${ }^{129}$.

L'utilizzo del relevance ranking comporta, infatti, come vedremo quella procedura di pesatura dei termini (field weighting) che consente di dare maggior peso nell'algoritmo ai termini presenti in alcuni campi particolari (ad es. nel titolo o più volte nel testo); non viene, dunque, data all'utente la facoltà di scegliere a quale fattore dare più peso nella costruzione dell'algoritmo per il ranking. In ogni caso, il criterio usato per stabilire la rilevanza dovrebbe essere sempre esplicitato nelle pagine di aiuto ${ }^{130}$.

Sebbene deprecabile, tale tecnica oggi trova applicazione nella maggior parte degli OPAC di nuova generazione e in tutti gli strumenti di discovery. Come vedremo nel capitolo seguente, l'applicazione del modello FRBR agli OPAC può rendere superflua l'esposizione dei dati per rilevanza, mentre il concetto stesso di rilevanza coinvolge una serie di dimensioni che non possono essere ridotte alla sola rilevanza algoritmica e topica.

Ordinamento per rilevanza e navigazione a faccette sono due caratteristiche progettuali che vanno sempre insieme; la navigazione a faccette consente di filtrare infatti la lista dei risultati spesso lunga e priva di una logica immediatamente riconoscibile dall'utente ${ }^{131}$.

\subsubsection{Navigazione a faccette}

Nel campo della ricerca nell'OPAC, la cosiddetta navigazione a faccette (o faceted navigation) rappresenta la caratteristica di progettazione più adottata dalle nuove interfacce, insieme all'ordinamento per rilevanza. La creazione di cataloghi sempre più grandi e la crescita del numero di record contenuti negli OPAC hanno spinto i progettisti a trovare nuove soluzioni per presentare i risultati delle ricerche organizzati per una più

\footnotetext{
${ }^{129}$ Cfr.: Martha M. Yee, Beyond the Opac: future directions for Web-based catalogues, Relazione presentata al seminario Beyond the OPAC : future directions for Web-based catalogues. Perth, Western Australia, settembre $2006<$ http://www.nla.gov.au/lis/stndrds/grps/acoc/papers2006.html>.

${ }^{130}$ Giovanni Bergamin, OPAC: migliorare l'esperienza degli utenti, in Atti del seminario Il catalogo oggi: le norme catalografiche fra consolidamento e fluidità, Modena, 13 dicembre 2007, «Bibliotime», 11 (2008), n. $1,<$ http://didattica.spbo.unibo.it/bibliotime/num-xi-1/bergamin.htm>.

${ }^{131}$ Yang e Wagner etc. criticano fortemente il fatto che nel calcolo della rilevanza non vengano presi in considerazione i dati sulla circolazione; di recente alcuni nuovi strumenti per la scoperta come Bibliocommons hanno attivato questa funzionalità. Cfr. Sharon Q. Yang - Kurt Wagner, Evaluating and comparing discovery tools cit., p. 694.
} 
efficace fruizione. Naturalmente, dato che l'implementazione di FRBR in queste nuove interfacce tarda ad arrivare, i modelli sono stati principalmente quelli del web.

La rete ha abituato gli utenti all'interrogazione per parola chiave e a una presentazione dei risultati ordinati secondo la rilevanza. Di fronte alla vastità dei dati recuperati, gli utenti spesso esprimono l'esigenza di organizzare i risultati della propria ricerca in gruppi significativi allo scopo di comprendere e contestualizzare meglio i risultati ottenuti e proseguire nella propria ricerca. Alcuni studi citati da Hearst evidenziano come gli utenti cambino il proprio comportamento di ricerca in base alla disponibilità di questi meccanismi di raggruppamento ${ }^{132}$. Una tendenza recente sia da parte dei motori di ricerca sia di molti siti commerciali è però anche quella di raggruppare i risultati in modo più significativo per l'utente; ci si può riferire a tali meccanismi chiamandoli 'raggruppamenti dinamici'. Da qualche tempo si studiano, quindi, varie soluzioni per generare raggruppamenti efficaci e progettare delle interfacce che supportino l'esplorazione usando tali raggruppamenti.

Oggi sono due i metodi maggiormente indagati e usati nel web per creare tali raggruppamenti: il clustering e l'analisi a faccette.

La tecnica del clustering $^{133}$ sembra per il momento confinata all'uso nei motori di ricerca di nuova generazione ${ }^{134}$ come Clusty (oggi Yippy ${ }^{135}$ ), benché vi siano stati recenti tentativi di applicarla a metadati di soggetto. Come per il relevance ranking si tratta di una procedura che si applica ai risultati della ricerca libera cioè per parole chiave, che è ormai

\footnotetext{
${ }^{132}$ Marti A. Hearst, Clustering versus faceted categories for information exploration, «Communication of the ACM», 4 (2006), n. 4, p. 59-61, oppure <http://people.ischool.berkeley.edu/ hearst/papers/cacm06.pdf>

${ }^{133} \mathrm{Si}$ tratta di una modalità di disposizione dei risultati che vengono raggruppati in cluster. È una di una tecnica totalmente automatizzata: «Il clustering utilizza le medesime procedure statistiche della tassonomia numerica $[. .$.$] che cerca di raggruppare oggetti a seconda del numero di caratteri in comune. Nel caso dei$ documenti, i caratteri sono naturalmente le parole. Il clustering produce dendrogrammi, ossia alberi in cui i grappoli apparentemente più "simili" sono disposti su rami vicini. [...] Fondamentale per queste tecniche è quindi il concetto di distanza in base alla quale gli elementi vengono raggruppati e quindi l'appartenenza o meno ad un insieme dipende da quanto l'elemento preso in esame è distante dall'insieme. Le tecniche di clustering hanno il vantaggio di poter essere applicate in modo automatizzato ad una grande quantità di testi, ma lo svantaggio maggiore risulta l'impossibilità per il calcolatore di "sostituire la cognizione e l'esperienza di un operatore umano"» (Claudio Gnoli - Vittorio Marino - Luca Rosati, Organizzare la conoscenza. Dalle biblioteche all'architettura delle informazioni per il Web, Milano, Hops Tecniche nuove, 2006, p. 57). Tale tecnica è stata implementata a partire dal 2004 nel motore di ricerca Clusty (<www.clusty.com>) di Vivissimo oggi conosciuto come Yippy (<www.yippy.com $>$ ). Una volta lanciata una ricerca i risultati venivano dinamicamente raggruppati in categorie. Scarsi però sono i risultati: risulta evidente la difficoltà di generare raggruppamenti intuitivi e di generare etichette aderenti all'effettivo contenuto dei documenti. Dato che il clustering non è supervisionato e quindi non vi è una preliminare definizione di categorie di appartenenza ne risulta una totale impossibilità di prevedere $\mathrm{i}$ risultati che vengono generati. Ciò che a mio parere è penalizzante è il mescolamento di ambiti diversi che questa tecnica produce: una mescolanza tra elementi semiotici e semantici inedito in un contesto di recupero di informazione di qualità qual è quello dei cataloghi elettronici. Ancora oggi questi motori non riescono a fornire risultati soddisfacenti.

${ }^{134} \mathrm{Vi}$ sono stati alcuni recenti tentativi di applicare questa tecnica a metadati di soggetto LCSH e alle classificazioni.

${ }^{135}<$ http://yippy.com/>.
} 
caratteristica della ricerca nell'ambiente web. Tale tecnica è volta all'implementazione di uno strato 'semantico' a supporto della ricerca 'semiotica' ${ }^{136}$ : come conseguenza del continuo aumento dell'informazione in fulltext disponibile online: ci si propone di studiare procedure attraverso le quali ottenere per via algoritmica un risultato simile a quello che si potrebbe ottenere attraverso l'indicizzazione umana dei documenti e la loro comprensione da parte dell'indicizzatore. Tali tecniche però non hanno finora generato risultati soddisfacenti.

L'applicazione dell'analisi a faccette in ambiente digitale è, invece, una tecnica che oggi desta un particolare interesse fra gli esperti di architettura dell'informazione (AI o Information Architecture) e di gestione della conoscenza (Knowledge management).

Tale tipo di tecnica trova una più diffusa applicazione nei siti di commercio elettronico presenti sul web molto ricchi di contenuti, per i quali è maggiormente idonea un'architettura di tipo 'esplorativo' poiché l'utente non è solito formulare interrogazioni, anche se di faceted navigation si parla sempre di più anche nella letteratura biblioteconomica, in quanto questa tecnica è diventata la caratteristica progettuale più usata negli OPAC di nuova generazione e nei sistemi per la scoperta ${ }^{137}$.

Nella letteratura sull'argomento ci si riferisce a questo tipo di tecnica chiamandola anche 'classificazione a faccette', termine non del tutto corretto, che la mette in relazione con la classificazione analitico-sintetica ideata da Ranganathan, auspicandone una rivalutazione nel nuovo ambiente digitale ${ }^{138}$. Si tratta, invece, di una tecnica completamente diversa, tant'è che ci si riferisce più correttamente ad essa chiamandola faceted browsing, faceted navigation o ancor meglio, con il termine filtri ${ }^{139}$.

\footnotetext{
${ }^{136}$ D'altra parte scrivono Gnoli, Marino e Rosati il problema dei motori di ricerca più diffusi è che si limitano ad cercare l'occorrenza di una parola o di una stringa all'interno dei documenti, estraendo in tal modo o documenti spesso poco rilevanti. Una volta recuperato un item non c'è modo di comprenderne la relazione o la posizione rispetto ad altri documenti correlati. È quindi necessario adottare sistemi che funzionino da strato semantico di riferimento, cioè le ontologie, sistemi che permettano di mettere in relazione i concetti oppure di servirsi di queste tecniche automatizzate. Cfr: Claudio Gnoli - Vittorio Marino - Luca Rosati, Organizzare la conoscenza cit., p. 99-106.

${ }^{137}$ Un esempio sono i siti di commercio elettronico di un certo rilievo come Amazon.com.

138 Così si esprime Luca Rosati: «Benché riconosciuta come più potente dei sistemi tradizionali, la classificazione a faccette non si è mai imposta come standard in ambito bibliotecario, subendo anzi una sorta di oblio negli ultimi anni. È stato l'avvento dell'architettura dell'informazione per il web a rilanciare questa nozione, intuendone i grandi vantaggi derivanti dalla sua applicazione in ambiente digitale». Si veda: Luca Rosati, Per un accesso multidimensionale all'informazione o della classificazione a faccette, «The Information Architecture Institute Italia», [risorsa online], 2003 $<$ http://iainstitute.org/it/articoli/000204.html>.

139 Come facevano giustamente notare Gnoli, Marino e Rosati, per non incorrere in equivoci, bisogna innanzitutto chiarire che questo tipo di tecnica applicata ai contenuti del web è sostanzialmente diversa dalla sua formulazione originaria, cioè dallo schema di classificazione ideato da Ranganathan e poi rielaborato nuovamente dal Classification Research Group, gli studiosi sostengono che sia ormai una tendenza definire faceted qualsiasi schema che adotti una logica simile a quella della classificazione a faccette pur non
} 
Tale tecnica recepisce della teoria originaria di Ranganathan solo la logica multidimensionale di approccio all'informazione.

Il funzionamento della classificazione a faccette venne così esposto da Luca Rosati nel 2003:

La classificazione mediante faccette sfrutta un sistema di attributi (metadati) mutuamente esclusivi rappresentanti ciascuno un aspetto o proprietà persistente dell'oggetto e capaci - nel loro insieme - di descrivere esaustivamente l'oggetto stesso. Tali attributi sono detti faccette (termine introdotto in questa accezione dal biblioteconomista e matematico indiano Ranganathan), e sono contraddistinti da queste peculiarità:

* sono invariabili dal punto di vista semantico (ad es. la proprietà COLORE di un oggetto può variare in termini di valori che può assumere - giallo, rosso etc. ma è invariabile come concetto; cioè quell'oggetto avrà sempre un colore);

* costituiscono un insieme aperto, per cui è sempre possibile aggiungere nuove faccette a quelle già esistenti;

* sono utilizzabili come attributi di ricerca sia singolarmente sia in combinazione.

Tali caratteristiche rendono particolarmente efficace l'adozione di questo sistema in ambienti digitali, per un più veloce ed efficiente ritrovamento dell'informazione ${ }^{140}$.

Come avviene anche sul versante degli schemi enumerativi usati in numerosi portali e nei motori di ricerca per categorizzare l'immensa mole di informazioni contenuta e indicizzata in internet, nella maggior parte dei casi del web queste classificazioni a faccette vengono applicate in modo parziale e incoerente ${ }^{141}$.

condividendo in pieno tutte le caratteristiche della teoria originaria (Cfr. Claudio Gnoli - Vittorio Marino Luca Rosati, Organizzare la conoscenza cit. p. 71-95).

${ }^{140}$ Luca Rosati, Per un accesso multidimensionale all'informazione o della classificazione a faccette cit.

${ }^{141}$ L'incoerenza e la parzialità contraddistinguono anche i numerosi schemi enumerativi del web che vengono applicati in indici sistematici chiamati anche directories o indici ragionati o per categorie (tra i quali quali Yahoo! e Open directory). Questi servizi segnalano siti web raggruppandoli secondo schemi organizzativi a seconda del loro contenuto. Molto spesso però essi violano la regola dell'unico fundamentum divisionis per cui le classi e sottoclassi non producono categorie esclusive, ma spesso si sovrappongono, operando contemporaneamente diversi principi di divisione e producendo schemi incoerenti o ibridi. Una rassegna aggiornata di queste directories è presente curata da Riccardo Ridi all'URL: $<$ http://www.burioni.it/forum/ridi/home.htm>. Sull'utilità di usare nel schemi puri e coerenti anche nel web si sono espressi anche Chaim Zins (Models for classifying Internet resources, «Knowledge organization», 29 (2002), p. 20- 28), Rosenfeld e Morville (Information Architecture for the World Wide Web cit). La criticità delle classificazioni home-grown del web è stata rilevata anche da Michele Santoro (La disarmonia prestabilita: per un approccio ibrido alla conoscenza e ai suoi supporti, «Biblioteche oggi», 20 (2002), n.5, p. 46-57). Per approfondimenti si veda anche il capitolo 5 del volume di Gnoli, Marino e Rosati (Organizzare la conoscenza cit. p. 64-95). Differente e di altro spessore l'utilizzo delle faccette in altri contesti quali ad esempio le collezioni di oggetti digitali nel progetto Flamenco, mentre Gnoli faceva notare la compresenza di informazioni semantiche e descrittive nella faceted search di Jstor: «Una delle ultime mode sul web è la ricerca "a faccette", che trae origine proprio da una sofisticata tecnica biblioteconomica. Purtroppo, la sua applicazione sulla rete è spesso molto parziale, in quanto per faccette si intendono dei semplici canali di accesso alternativi, privi di notazione e di ordine di citazione e quindi, di nuovo, poco utili per lo scorrimento sistematico di grandi quantità di voci. In molti casi inoltre le informazioni semantiche, che sono la sede originaria dell'analisi a faccette, vengono mescolate con informazioni descrittive, come accade nella recente "faceted search" di Jstor» (Claudio Gnoli, Blopac semantici, «Bibliotime», XI (2008), n.1, $<$ http://didattica.spbo.unibo.it/bibliotime/num-xi-1/gnoli.htm>). 
Il più comune utilizzo delle faccette consiste nel creare delle categorie (cioè una serie di etichette che mostrano i concetti rilevanti di un determinato ambito disciplinare). Alle faccette così create, che corrispondono alle varie dimensioni o aspetti rilevanti della raccolta, si associa una gerarchia di termini. Una volta definite le categorie indipendenti (faccette) e le categorie ad esse collegate, anziché collocare gli item all'interno di categorie, le etichette delle faccette vengono assegnate agli oggetti, in modo che ogni oggetto possa essere cercato in molti modi, ovvero da più punti di vista. Al contempo viene progettata un'interfaccia che consente una navigazione flessibile organizzando i risultati in modo da supportare l'espansione e il raffinamento di una ricerca ${ }^{142}$. Venendo dunque, alla modalità di utilizzo nella faceted navigation negli OPAC il procedimento è simile a quello esposto in precedenza: il risultato di una ricerca ${ }^{143}$ viene presentato sia come lista breve, sia raggruppato a partire dagli elementi (campi) che compongono il record bibliografico: ogni campo - o combinazione di campi - «diventa così un punto di vista dal quale eventualmente partire per sfruttare meglio e progressivamente i risultati di una ricerca». ${ }^{144}$

\footnotetext{
${ }^{142}$ Nell' interfaccia classica (ad es. quella che il progetto Flamenco ha predisposto per Nobel Prize Winners $<\mathrm{http}$ ///orange.sims.berkeley.edu/cgi-bin/flamenco.cgi/nobel/Flamenco > o per la collezione del Museum of Fine Arts di San Francisco <http://orange.sims.berkeley.edu/cgi-bin/flamenco.cgi/famuseum/Flamenco >) all'inizio solitamente vengono mostrati all'utente tutti gli item e tutte le faccette in una disposizione simile a quella delle numerose directories del web. Si intuisce quindi che ogni ramo visualizzato rappresenta un percorso alternativo alla stessa collezione di oggetti. Le faccette principali rappresentano quindi non dei semplici contenitori ma dei descrittori semantici rappresentanti cioè una proprietà o un aspetto dell'oggetto. Le faccette possono quindi essere considerate come percorsi paralleli e alternativi allo stesso item. Non appena l'utente seleziona una etichetta all'interno di una faccetta, automaticamente si riduce il gruppo di item visualizzati, solitamente vengono mostrati solo quelli assegnati alla sottocategoria della faccetta e vengono eliminate alcune sottocategorie non più utili. Questa procedura è una sorta di filtraggio o raffinamento della ricerca. L'interfaccia di browsing viene appositamente predisposta per permettere al'utente di navigare nella raccolta costruendo implicitamente una query composta di tipo AND tra le faccette.

Il vantaggio delle faccette è di permettere all'utente di decidere il punto da quale partire nella propria ricerca e di decidere come esplorare e raggruppare gli oggetti. Tale decisione è favorita dalla presentazione della struttura logica delle faccette. Effettuata la selezione, le categorie non rilevanti per il raffinamento della ricerca in corso vengono escluse in modo automatico dalla visualizzazione, suggerendo così all'utente $i$ possibili raffinamenti e le possibili direzioni per procedere nella ricerca. Vengono inoltre mostrate le coordinate semantiche dell'insieme selezionato rispetto alle altre faccette del sistema. La ricerca per parola chiave può essere integrata in modo naturale con una struttura organizzativa a faccette; infatti le faccette consentono di effettuare interrogazioni complesse, evitare che la ricerca abbia risultato zero (quando si cerca per parola chiave) e rendere maggiormente efficace l'esplorazione di una raccolta. Esse consentono all'utente di dedurre aprima vista la tipologia degli oggetti presenti in una collezione ed evocano l'esperienza del browsing dei volumi in uno scaffale. Il maggiore svantaggio rispetto alle classificazioni è quello di non permettere a prima vista di catturare gli argomenti o i principali temi trattati e di non esplicitare le tipologie di relazioni che intercorrono tra le faccette. Inoltre i migliori risultati si hanno in ambienti disciplinari molto ristretti (es. nella descrizione di collezioni di oggetti d'arte, di fotografie e oggetti digitali).

${ }^{143} \mathrm{Vi}$ sono però anche casi in cui viene accettata anche una ricerca senza parametri che ha per risultato tutti $i$ record presenti nel catalogo raggruppati per faccette o punti di vista (es. nell'OPAC Endeca implementato nelle biblioteche della North Carolina State University (Cfr. Appendice A. Casi di studio. North Carolina State University).

${ }^{144}$ Giovanni Bergamin, OPAC: migliorare l'esperienza degli utenti cit.
} 
Secondo Gnoli et al ${ }^{145}$ l'uso delle faccette negli OPAC favorirebbe un approccio di tipo esplorativo all'informazione tale da garantire in ogni fase della ricerca approfondimenti, aggiustamenti del tiro o cambi di rotta secondo il modello del berrypicking elaborato da Marcia Bates ${ }^{146}$.

Tale tecnica ha trovato una prima applicazione nel software Endeca implementato nell'OPAC delle biblioteche della North Carolina State University (NCSU), ${ }^{147}$ giudicato da molti il primo catalogo di nuova generazione, per poi divenire caratteristica presente in tutti gli OPAC di nuova generazione e strumenti di discovery.

La finalità dell'implementazione di questa tecnica trova una giustificazione nel tentativo di rendere maggiormente amichevoli i cataloghi agli utenti favorendone un approccio esplorativo che può rivelarsi utile sia ad utenti che non hanno chiare le proprie esigenze di ricerca, sia a coloro che vogliono esplorare le modalità e i vari canali di ricerca forniti dai cataloghi, quindi in modo particolare per gli utenti inesperti ${ }^{148}$. In alcuni OPAC di nuova generazione come Blacklite ${ }^{149}$, le faccette sono diversificate per tipologia di

${ }^{145}$ Claudio Gnoli - Vittorio Marino - Luca Rosati, Organizzare la conoscenza cit., p. 110.

146 Marcia J. Bates, The design of browsing and berrypicking techniques for the online search interface, «Online Review», 13 (1989) n. 5, p. 407-424, <http://www.gseis.ucla.edu/faculty/bates/berrypicking.html>. Un'analisi sull'uso delle faccette da parte degli utenti nel catalogo della NCSU è Cory Lown, A transaction log analysis of NCSU's faceted navigation OPAC. A master's paper for the M.S. in L.S degree. April, 2008.< http://ils.unc.edu/MSpapers/3387.pdf>.

${ }^{147}$ L'opac è visibile all'URL: <http://www.lib.ncsu.edu/catalog/>, sull'applicazione di Endeca all'opac NCSU si veda anche Kristin Antelman, - Emily Lynema - Andrew K. Pace, Toward a twenty-first century library catalog, «Information Technology \& Libraries», $25 \quad$ (2006), n. 3 p. $128-139$ oppure: $<$ http://eprints.rclis.org/8177/1/antelman_lynema_pace.pdf $>$.

${ }^{148}$ Importanti studi sull'applicazione della teoria delle faccette nel web e negli opac sono quelli di Kathryn A. La Barre. In un suo studio comparativo su 75 siti di commercio elettronico ha rilevato come tale classificazione sia adottata in modo parziale, poco coerente e approssimativo. All'indirizzo $<$ http://courseweb.lis.uiuc.edu/ klabarre/facetstudy.html> sono visibili molti contributi della studiosa americana che fanno parte del suo progetto di ricerca Study: Facets on the web sull'applicazione dell'analisi a faccette nel web. A proposito degli OPAC invece commenta così: «To better leverage some legacy subject access systems ... more (not less) should be done to process these records and corresponding authority files (e.g. thesauri and other controlled vocabularies) in a manner that allows optimal access through the faceted navigation and browsing features of new search and discovery systems ... some of the systems currently implemented in libraries are heavily influenced by the commercial and business applications that preceded them ... Now it is the time to question the assumptions that are embedded in these commercial systems that were primarily designed to provide access to the concrete items through descriptions in order to enhance profit. ... Data assigned to cultural objects, especially as they make use of name and subject authority data, are more complex and powerful than most metadata routinely assigned to commercial products». Kathryn La Barre, Faceted navigation and browsing features in new OPACs: A more robust solution to problems of information seekers?, in Joseph T. Tennis, Proceedings North American Symposium on Knowledge Organization $2007 \quad 1, \quad$ p. 100-104, Toronto, 2007. Abstract in: $<$ http://arizona.openrepository.com/arizona/bitstream/10150/106157/1/71_La_Barre_(extended)2.pdf />.

149 Blacklite è l'OPAC di nuova generazione sviluppato nelle biblioteche dell'Università della Virginia a partire dal 2006 e reso pubblico nel 2008 in sostituzione del precedente OPAC. Le faccette vengono create utilizzando diversi schemi a seconda del tipo di materiale presentato (cfr. infra Appendice A. Casi di studio. University of Virginia libraries). Sul progetto si veda: Elizabeth Sadler, Project Blacklight: a next generation library catalog at a first generation university, «Library Hi Tech», 27 (2009), n.1, p. 57-67. 
materiale, offrendo all'utente un recupero più ottimale con differenti set di faccette per ogni tipo di oggetto (libri, dvd, musica).

I filtri si rivelano oggi indispensabili soprattutto per un motivo: l'ordinamento per presunta rilevanza non fornisce un quadro chiaro del contesto nel quale sono inseriti i risultati di una ricerca, cioè dell'universo bibliografico rappresentato nel catalogo, così i filtri soccorrono l'utente invitandolo a raffinare il risultato e produrre raggruppamenti utili.

\subsubsection{L'introduzione delle funzionalità dei motori di ricerca negli OPAC}

Negli OPAC di nuova generazione vengono implementate molte tecniche dei motori di ricerca del web, come quelle che consentono di correggere gli errori in fase di digitazione (correzione dello spelling) o di aggiungere al termine usato per un'interrogazione prefissi e suffissi per ampliare le possibilità di recupero (tecniche di stemming).

Nel primo caso si tratta della possibilità di correggere errori di digitazione compiute dall'utente al momento di immettere la stringa di ricerca (che nei motori ci appare con la nota frase «forse cercavi»). Alcuni OPAC hanno implementato questa funzionalità che, d'altra parte, può essere di effettivo aiuto all'utente; Bergamin, con un certo acume, ne suggeriva un uso semantico qualora fosse applicata nel contesto di una ricerca per soggetto, presentando le relazioni dei termini che risultino pertinenti al contesto della ricerca e che siano basate su un vocabolario controllato ${ }^{150}$.

La seconda tecnica è invece adottata nei motori di ricerca per ampliare quelle ricerche che producono scarsi risultati ed evitare le ricerche 'a risultato zero' tipiche degli OPAC tradizionali. Allo scopo si applica la ricerca post-booleana o probabilistica che si serve tipicamente della logica fuzzy ${ }^{151}$.

Sono state introdotte inoltre varie altre tecniche e soluzioni di progettazione della ricerca, che richiamano quelle presenti nei più comuni motori di ricerca e siti web. Tra queste sono da menzionare:

\footnotetext{
${ }^{150}$ Giovanni Bergamin, OPAC: migliorare l'esperienza degli utenti cit.

${ }^{151}$ La logica fuzzy (sfuocata, confusa o vaga) legata alla 'teoria degli insiemi sfuocati' teorizzata da Cartesio, Russell e Einstein assegna sia ai termini usati nella ricerca che agli operatori che li connettono valori percentuali e intermedi rispetto ai due casi estremi ( 1 e 0$)$ previsti dalla logica classica ottenendo risultati che si avvicinano di più alla realtà (Si veda: <http://it.wikipedia.org/wiki/Logica_fuzzy>).
} 
a. Suggerimenti imitativi

Nel web troviamo applicate diverse tipologie di suggerimenti: da quelli 'imitativi', basati sull'analisi del comportamento degli utenti per fornire suggerimenti di lettura ('chi legge xx legge anche yy') ai suggerimenti 'umani' cioè quelli creati manualmente che raccolgono i migliori risultati di una ricerca su un dato argomento ponendoli in cima alla lista; nei siti commerciali questa tecnica è nota come Best Bets.

I suggerimenti di lettura possono rivelarsi senz'altro utili per collegare le entità catalografiche e dar modo all'utente di esplorare il contenuto del catalogo; essi dovrebbero derivare:

1) dalla struttura sindetica di collegamento delle entità rappresentate nel catalogo derivanti dall'implementazione del modello FRBR: nel caso degli OPAC sarebbe più opportuno sfruttare le grandi potenzialità offerte dai soggetti e dalle classificazioni per proporre agli utenti volumi che trattano dello stesso argomento, oppure presentare gli altri volumi scritti dallo stesso autore, o appartenenti allo stesso genere letterario;

2) dai dati statistici sulla circolazione: la possibilità di sfruttare questi dati per proporre documenti che spesso vengono presi in prestito insieme, se è una caratteristica delle librerie online, oggi non è presente negli OPAC di nuova generazione né nei discovery systems.

A tutt'oggi la funzione "chi ha letto/preso in prestito $\mathrm{x}$ legge/prende in prestito anche y" non è attivata, in quanto quasi nessun OPAC utilizza i dati della circolazione per produrre questa tipologia di suggerimento. Troviamo invece la funzionalità di suggerimenti di 'item simili' che viene ottenuta tramite lo sfruttamento dei metadati delle risorse di volta in volta presentate.

\section{b. Visualizzazioni avanzate e word clouds}

Si tratta di mappe visuali che forniscono all'utente la visualizzazione di termini collegati al termine usato per la ricerca quali as es. varianti ortografiche, associazioni, traduzioni in altre lingue o termini ricavati dai tesauri. Sotto questo aspetto, alcuni prodotti come Aquabrowser hanno proposto interfacce visuali in aiuto alla ricerca, mentre altri OPAC presentano vetrine visuali per i nuovi arrivi o altre categorie. 


\section{c. Smart search e interfaccia 'alla Google'}

Per quanto riguarda invece le interfacce degli OPAC, una recente tendenza è quella di presentare almeno una finestra di tipo 'google like', cioè un box unico per la ricerca per parola chiave su tutti i campi. In definitiva, è sempre da tener presente che la maggior parte di queste tecniche hanno avuto fortuna nei motori di ricerca basati sulla ricerca libera in materiali full text (come le pagine web). L'applicazione di queste tecniche negli OPAC tende a dare forse troppo spazio a questa modalità di ricerca, che sembra, tendente ad omologare tutte le altre creando nell'utente una certa confusione tra il versante semiotico e quello semantico ${ }^{152}$. Le biblioteche invece dispongono di collaudate e potentissime tecniche per il recupero e l'indicizzazione dei documenti che negli OPAC potrebbero essere maggiormente sfruttate e portate a conoscenza degli utenti ${ }^{153}$.

\subsubsection{Interfacce e usabilità dell'OPAC}

Relevance ranking, navigazione a faccette e ricerca 'alla Google' rappresentano le principali innovazioni delle interfacce dei cataloghi 'di nuova generazione': questi espedienti derivano da una progettazione dell'OPAC che risponde alla volontà di adattarsi alle caratteristiche delle interfacce di risorse informative esterne al catalogo che oggi attraggono gli utenti imponendo loro nuovi modelli mentali e di ricerca. Tuttavia la progettazione non si avvale ancora delle opportunità che deriverebbero da una più profonda comprensione di meccanismi e strategie di ricerca che l'utente mette in atto nel momento in cui usa un OPAC e dei modelli comportamentali che lo guidano nei vari passi della ricerca, che insieme alla necessaria trasformazione della struttura dei dati catalografici verso una efficace rappresentazione del modello FRBR si rivelano si rivelano fondamentali per progettare interfacce che consentano di condurre con successo il dialogo tra utenti e OPAC.

Progettare interfacce per il catalogo che siano effettivamente funzionali e usabili si rivela, dunque, una questione molto complessa. Uno sforzo di analisi sull'impatto delle

\footnotetext{
${ }^{152}$ Pino Buizza, Gli opac: funzionalità e limiti nel mondo del web cit.

${ }^{153}$ Sulle innovazioni dell'interfaccia Endeca si vedano due contributi di Tito Sierra, Improving the catalogue interface using Endeca, Capitalize on access, 2006, <http://www.lib.ncsu.edu/endeca/presentations.html> e Id., A single search box interface to the NCSU libraries, two years later, Digital Library Federation Spring Forum 2007, Pasadena, California, April 24, 2007, <http://www.lib.ncsu.edu/dli/projects/quicksearch/>.
} 
tecnologie dell'informazione, delle nuove risorse informative sul comportamento degli utenti è fondamentale per progettare interfacce che sino realmente in grado di soddisfare $\mathrm{i}$ loro bisogni informativi.

In linea generale si può considerare l'interfaccia utente come ciò che si frappone tra la macchina e l'utente, ciò che fa dialogare l'uomo con la macchina, che consente all'utente di poter gestire in modo più o meno semplice le funzionalità di un sistema e in qualche modo di comprenderne il funzionamento ${ }^{154}$. È noto che il grado di usabilità di un qualsiasi prodotto, cioè il grado di soddisfazione e di facilità con cui si compie l'interazione tra l'utente e il prodotto stesso, dipende strettamente dall'incontro tra il modello concettuale del progettista (cioè dalle idee di questi riguardo il funzionamento del prodotto) e il modello concettuale dell'utente (cioè l'idea che l'utente concepisce del prodotto e del suo funzionamento) e si innalza proporzionalmente all'avvicinamento dei due modelli.

Affinché però l'utente riesca a dialogare in modo efficace con il sistema è necessario che in fase di progettazione dell'OPAC si tenga conto del modello concettuale che l'utente si è formato in base alle sue abitudini di ricerca e alla sua esperienza. Oggi più che mai $i$ modelli mentali degli utenti sono influenzati da modo di fare ricerca e dalle interazioni che si svolgono sul web.

Va però anche sottolineato che prodotti veramente innovativi hanno la capacità di creare nuovi modelli mentali e di influenzare i comportamenti degli utenti. Ciò poteva tradursi in un vantaggio al momento di progettare i primi OPAC. Invece, i modelli concettuali alla base dei primi cataloghi elettronici hanno ricalcato sostanzialmente le modalità di ricerca tipiche del catalogo cartaceo, finendo così per sprecare in qualche modo le potenzialità che potevano essere offerte dal nuovo mezzo elettronico. Si tratta di un'opportunità sprecata, che i cataloghi potevano cogliere in un periodo il web non esisteva ancora $^{155}$; invece i primi esperimenti furono alquanto deludenti, dando vita a prodotti persino inferiori ai cataloghi cartacei concepiti con lo scopo di guidare gli utenti che avevano familiarità con il catalogo cartaceo nel mondo della rete ${ }^{156}$. Con l'avvento del web le cose si complicarono ulteriormente; nel momento in cui l'OPAC, dapprima strumento per soli utenti esperti e bibliotecari, approda sul web aprendosi così all'utilizzo da parte di un'utenza generica e impreparata sotto certi aspetti (sia tecnici sia relativi alla struttura dei

\footnotetext{
${ }^{154}$ Una definizione ormai classica di interfaccia è quella data nel 2003 da Jef Raskin: «Interfaccia è il modo in cui si fa qualcosa con uno strumento: le azioni che dobbiamo eseguire e il modo in cui lo strumento risponde» Cfr. Jef Raskin, Interfacce a misura d'uomo. Milano, Apogeo, 2003. Citazione a p. 3.

${ }^{155}$ Tradizionalmente di fa risalire la nascita del Web al 6 agosto 1991, giorno in cui Berners-Lee mise on-line su Internet il primo sito web.

${ }^{156}$ Christine L. Borgman, Why are online catalogs still hard to use?, «Journal of the American Society for Information Science», 47 (1996), n. 7, p. 493-505.
} 
dati bibliografici), i problemi riguardanti la progettazione di interfacce amichevoli o user frendly hanno acquistato un'importanza che prima non avevano ${ }^{157}$.

Dall'avvento delle prime interfacce grafiche fino ai nuovi next generation catalogs l'evoluzione delle interfacce è stata sostanzialmente stagnante; le principali modalità di interrogazione tradizionalmente offerte all'utente erano limitate alle possibilità della ricerca semplice (per campi) e della ricerca avanzata (sfruttando gli operatori booleani). Con i nuovi OPAC si tende a preferire alle due modalità la ricerca per parola chiave su tutti i campi che sempre più spesso viene presentata come ricerca di base, e migliorare la user experience per mezzo di espedienti tecnologici e visualizzazioni avanzate ${ }^{158}$ L'utilizzo delle faccette o filtri nella ricerca comunque non ha ancora sfruttato appieno la possibilità che la ricerca si possa svolgere in più direzioni e con la possibilità per l'utente di ritornare sui propri passi, estendere o restringere a piacimento la propria ricerca o cambiare approccio in qualsiasi momento ${ }^{159}$.

\footnotetext{
${ }^{157}$ I primi OPAC presentavano un'interfaccia testuale o a linea di comando. L'utente interagiva con il sistema scrivendo righe di comando su un terminale testuale. Negli OPAC che usavano questa modalità, il sistema rimaneva inattivo fino a quando non riceveva un comando dall'utente. L'utente, dal suo canto, doveva comporre i comandi in forma di linee di testo rispettando una precisa sintassi. Dopo aver composto una riga, bisognava tipicamente battere il tasto Invio o Enter. A questo punto, il programma analizzava la riga di comando, e se questa era stata composta in modo formalmente corretto la eseguiva. Come effetto dell'esecuzione di un comando, si può avere la scrittura di testo sul terminale da parte del programma, in sostanza ciò che veniva restituito era un risultato in forma testuale che poteva contenere l'elencazione dei risultati o un messaggio di errore. Per poter interrogare il sistema l'utente doveva conoscere il linguaggio dal punto di vista sintattico e semantico. Un primo tentativo di semplificare le interfacce fu il successivo affiancamento alle interfacce a linea di comando di questi primi strumenti, di nuove interfacce a menù, dedicate ad utenti meno esperti. L'utente poteva scegliere quindi tra una serie di opzioni e immettere una scelta in una lista che gli veniva proposta. Un'interfaccia a menù ha il vantaggio di guidare l'utente nei passi della ricerca impedendogli di eseguire operazioni non valide. Con l'evoluzione della tecnologia e l'avvento delle prime interfacce grafiche (graphical user interface abbreviato GUI) nei primi microcomputer, fu possibile per l'utente interagire con il computer manipolando graficamente degli oggetti, svincolandolo quindi dalla necessità di imparare comandi da impartire con la tastiera. Grazie all'interfaccia grafica era possibile compiere vari compiti comuni e complessi senza il bisogno di un'approfondita conoscenza del funzionamento del computer. In un'interfaccia di questo tipo l'utente agisce direttamente sugli oggetti (rappresentati da icone o simboli) attraverso il mouse o altri dispositivi di puntamento e gli oggetti sono reattivi, cioè cambiano il loro aspetto o la loro posizione a seconda delle azioni impartite dell'utente. L'utente in queste interfacce riceve un feedback dagli oggetti, ma la sua ricerca non è guidata. Le interfacce grafiche hanno dunque cambiato volto degli OPAC senza introdurre reali innovazioni. Hildreth suggeriva già dai primi esperimenti, un approccio cauto all'uso delle interfacce grafiche negli opac avvertendo come molto spesso un'applicazione troppo disinvolta di questi strumenti potesse generare una maggiore e inutile complessità a danno dell'utente (Cfr.: Charles R. Hildreth, The GUI OPAC: approach with caution, «The public access computer systems review», 6 (1995), n. 5. p 1-6 oppure $<$ http://epress.lib.uh.edu/pr/v6/n5/hild6n5.html>). Sulla questione del complesso rapporto uomo-interfaccia si veda anche Marcia J. Bates, Where should the person stop and the information search interface start? «Information Processing \& Management», 26, (1990), p. 575-591.

${ }^{158}$ Ben nota l'interfaccia visiva di Aquabrowser che ebbe tra le prime implementazioni quella alla Queens Library.

${ }^{159}$ La una navigazione a faccette introdotta per la prima volta nel catalogo dall'interfaccia Endeca per l'OPAC della North Carolina State University presenta i possibili canali di ricerca che l'utente può esplorare. Tuttavia non sempre le faccette vengono utilizzate in maniera adeguata; le interfacce potrebbero essere notevolmente migliorate nelle capacità di raggruppamento qualora venisse implementato il modello FRBR.
} 
Le nuove interfacce studiate per OPAC 'di nuova generazione' si sovrappongono ad OPAC e sistemi di gestione tradizionali per estrapolarne i dati e presentarli in maniera più comprensibile; sfruttano la 'clusterizzazione' e la 'faccettazione' dei risultati delle ricerche e si affidano alle tecnologie per meglio strutturare i risultati e creare ambienti di ricerca veloci e più intelligenti ${ }^{160}$. Solitamente queste interfacce 'alla Google' presentano le caratteristiche proprie di Amazon e Google: tra queste abbiamo già citato relevance ranking, uso delle radici delle parole, verifica della digitazione, uso facilitato degli operatori logici, suggerimenti (recommandations) ${ }^{161}$ e filtri (faceting) ${ }^{162}$.

Nonostante ciò il problema delle interfacce utente è ancora lontano da una sua soluzione per l'approccio con cui oggi si cerca di migliorare il dialogo tra utenti e OPAC; ciò che ancora manca è soprattutto una resa migliore delle molteplici relazioni tra i dati. Ciò probabilmente deriva anche dal modo d'intendere l'interfaccia utente, spesso limitata alle sole funzioni dei formati per la visualizzazione dei dati o delle funzioni di aiuto in linea, senza focalizzare l'attenzione sull'importanza della struttura dei dati e delle loro relazioni, sul modo di raggruppare i risultati e sulle ricadute dei modelli dell'IFLA in termini di strutturazione e di presentazione dei dati bibliografici ${ }^{163}$.

\footnotetext{
${ }^{160}$ C'è da sottolineare che molto spesso in queste applicazione ciò che ne fa le spese è la presentazioni degli accessi semantici; sovente, infatti, va perduta la tenuta di strutture semantiche complesse, mentre i vantaggi ottenuti risiedono esclusivamente nella riduzione dei tempi della ricerca o nella sua semplificazione.

${ }^{161}$ Un primo prototipo di OPAC che forniva raccomandazioni di lettura fu Revyl, l'interfaccia messa a punto nell'ambito del Melvyl Recommeder Project della California Digital Library L'interfaccia restitiuva nella stessa videata tre categorie di record, Similar Records, Patrons who borrowed this item also borrowed, Amazon recommandations. (Cfr.: California Digital Library, The Melvyl Recommender Project final report cit.

${ }^{162}$ Vanno inoltre ricordati altri esperimenti di interfacce simili come Encore, che mostra all'utente un'interfaccia google like (è prevista anche la ricerca avanzata) restituisce i risultati ordinati per rilevanza, e tra i filtri mostra anche voci come Most relevant titles e Popular choices. ma anche filtri quali All Adult Materials, All Children's Materials, All Teen Materials (<http://ws4.starklibrary.org/iii/encore/app $>$ ) Tra i vari esempi di interfacce personalizzate anche quella fornita da Reading matters (<http://www.readingmatters.co.uk/bookchooser.php>), bookchooser indirizzato ai ragazzi. Gli utenti indicando le proprie preferenze nella schermata iniziale possono ottenere consigli di lettura adatti ai loro gusti. Indirettamente, il servizio aiuta le biblioteche ad orientare la loro politica degli acquisti.

${ }^{163}$ Già nel 1991 Yee richiedeva più attenzione, nella progettazione degli OPAC alla struttura dei dati sostenendo che l'inefficacia nella progettazione di OPAC dipendesse proprio da alcuni fondamentali equivoci che i progettisti dei sistemi avevano circa la natura dei record bibliografici e le reali esigenze degli utenti. La studiosa sottolineava come nella letteratura professionale ci fosse una certa confusione proprio sul modo di intendere il concetto di interfaccia. Il termine sarebbe stato originariamente applicato solo a quella parte del software con il quale l'utente entrava direttamente in contatto: metodi di ricerca, formati del display, assistenza all'utente, per cui l'interfaccia è stata tradizionalmente intesa come quella particolare componente del software, che permette di tradurre i risultati in forme comprensibili dagli utenti. Tali definizione che non comprende né l'hardware né la struttura dei dati, risulta incompleta in quanto è cruciale proprio il collegamento tra display e struttura dei dati: «[...] search method and display formats are mentioned as being examples of interface features. Search methods and display formats are both heavily dependent on data structures. [...] design of an effective user-computer interface requires effective design of the system as a whole, including relationships and structures inherent in the data itself. This is especially true in the case of OPAC's; made up 25 they are of cataloging records created, by means of a great deal of intellectual effort, largely for the purpose of demonstrating relationships to users; relationships such as those obtaining between 'all the works on a particular subject!' 'all the works of a particular author,' or 'the item retrieved and a newer
} 
Un approccio corretto dei progettisti di OPAC alla struttura dei record bibliografici e alle reali necessità degli utenti apporterebbe ai cataloghi indubbi benefici nella strutturazione dei dati e nell'articolazione delle interfacce ${ }^{164}$. Le soluzioni messe in atto per migliorare la presentazione dei risultati e le modalità di ricerca nei cataloghi 'di nuova generazione' si affidano alla tecnologia per ottenere un display dei dati migliore senza tuttavia ottenere una migliore resa della complessità delle molteplici relazioni tra i dati bibliografici. Anche la personalizzazione nel display dei dati è un argomento complesso, in quanto gli utenti devono poter sfruttare le conoscenze acquisite nella loro consultazione degli OPAC per poterle utilizzare in OPAC diversi. Uno degli obiettivi non ancora pienamente raggiunti nella progettazione di interfacce dei cataloghi elettronici, ma necessaria a garantire una omogeneità nella presentazione dei dati, è l'adeguamento delle visualizzazioni dei cataloghi alle Guidelines elaborate dall'IFLA ${ }^{165}$. Le Guidelines ormai da un decennio si propongono come un corpus di buone pratiche per assistere i bibliotecari

edition of the same work». Martha M. Yee, System design and cataloging meet the user: user interfaces to online public access catalogs, «Journal of American Society for Information Science», 42 (1991), n. 2, p. 7898, <http://escholarship.ucop.edu/uc/item/2rp099x6>. Citazione a p. 4.

${ }^{164}$ Uno tra i primi esempi di implementazione di FRBR in un OPAC (seppure in questo caso si tratti di un catalogo speciale) è l'interfaccia predisposta da OCLC, FictionFinder che diede una prima idea di come avrebbe potuto essese strutturato un catalogo 'di nuova generazione'. Fiction Finder è prototipo di interfaccia realizzato da OCLC per organizzare i dati bibliografici secondo il modello FRBR. Scopo del progetto è quello di usare il modello FRBR in un sistema prototipale che permetta la ricerca e il browsing dei record bibliografici che rappresentano la narrativa. Il sistema fornisce l'accesso a più di 3 milioni di record di libri, ebooks, e materiale audio classificato come narrativa nel catalogo di OCLC WorldCat (inclusi fumetti, opere teatrali, romanzi e racconti) L'interfaccia presenta una visualizzazione dei dati ispirata ad FRBR servendosi dell'algoritmo creato da OCLC (FRBR Workset Algorithm), che raggruppa i record in opere, facilita l'aggregazione dei dati dai singoli record, permette la creazione di descrizioni più ricche, facilitando la navigazione. L'interfaccia è visibile all'indirizzo: <http://fictionfinder.oclc.org/>. La pagina del progetto è invece: <http://www.oclc.org/research/projects/frbr/fictionfinder.htm>. Fiction Finder si serve di vocabolari controllati per approntare indici speciali, liste per il browsing e una nuvola di tag dei soggetti. Viene usato FAST per i soggetti, nonché altri vocabolari controllati (FAST (Faceted Application of Subject Terminology) è derivato dal LCSH e si serve di una sintassi semplificata (tra i vocabolari utilizzati Genre terms for fiction \& drama (GSAFD) Library of Congress Subject Headings (LCSH) Bilindex Répertoire de vedettes-matière). L'interfaccia produce una visualizzazione ordinata in base al numero delle localizzazioni. Sono pertanto previsti i consueti accessi per genere, personaggi, ambientazione ed argomento, ma sono presenti anche un breve riassunto della trama e un accesso per premio letterario. Il catalogo sfrutta infine i dati relativi alle localizzazioni, per valutare il tipo di pubblico a cui è destinata ogni risorsa (Kids, General, Special) e quindi fornire un suggerimento di lettura. (Cfr. Diane Vizine-Goetz, FictionFinder: imagining what a "Next generation" catalog might look like. Relazione presentata a OCLC Members Council Research and New Technologies Interest Group meeting, 6 February 2007, Québec, QC (Canada) and Dublin, Ohio (USA), 2007. <http://www.oclc.org/research/memberscouncil/2007-02/vizine-goetz.pdf> e Ead., FictionFinder: a FRBR works-based prototype, in Back to the future, ALCTS Preconference, ALA Annual Meeting, Orlando, June 25, $2004 \quad$ [on line], $\quad$ S. $\quad$ 1.], $\quad$ ALA, $\quad 2004$, $<$ http://www.ala.org/ala/alcts/alctsconted/presentations/VizineGoetz.pdf> )

${ }^{165}$ Le linee guida per la visualizzazione nell'OPAC si inseriscono nelle varie iniziative di standardizzazione perseguite dall'IFLA. Come già per altre iniziative dell'ente, l'obiettivo che il documento si propone è quello di stabilire dei requisiti minimi che gli OPAC dovrebbero avere in merito alla visualizzazione dei dati bibliografici. Il gruppo di lavoro è stato costituito nel 1997 in occasione dell'IFLA Conference di Copenaghen e una prima versione è stata pubblicata nel 1998. La quasi contemporanea pubblicazione di FRBR, però portò immediatamente alla necessità di una revisione sostanziale del documento e quindi alla pubblicazione, nel 2003, di un nuovo draft pubblicato nel sito dell'IFLA per essere sottoposto alla revisione da parte della comunità internazionale e del final report pubblicato nel 2005. 
nella progettazione delle interfacce dei propri OPAC prendendo in considerazione in primo luogo bisogni dell'utenza ${ }^{166}$. Oggetto del documento è esclusivamente la visualizzazione delle informazioni bibliografiche per cui tali raccomandazioni sono valide per qualsiasi tipologia di catalogo, sia che si basi su interfacce grafiche o web ${ }^{167}$. La coerenza del display nell'ottica Guidelines è dunque garanzia primaria per l'utente di acquisire abilità che possano essere sfruttate su qualsiasi tipo di OPAC consentendogli, una volta appresa la sintassi del catalogo, di interrogare agilmente OPAC diversi trovandosi sempre a proprio agio $^{168}$. Lo sforzo di produrre visualizzazioni coerenti, è però fortemente legato al formato dei record e alle regole di catalogazione e dunque allo stretto rapporto tra le interfacce, i formati e le regole catalografiche:

The content and structure of the records available for use in OPAC displays is determined by current cataloguing rules. The identification of the various types of data and records available is determined by the MARC formats. We take current cataloguing rules and current MARC formats as a given in this document, and try to suggest better ways to make use of existing records in OPAC displays. We do not try to suggest ways that actual changes in cataloguing rules might help to improve OPAC displays, although we recognize that some potential solutions to OPAC display problems lie in changes in cataloguing rules ${ }^{169}$.

Dunque ci si attende che il processo di rinnovamento, che a partire dall'elaborazione teorica offerta dal modello FRBR ha visto l'elaborazione di nuovi principi e norme catalografiche, si traduca in interfacce realmente innovative; in un contesto in cui il web impone modelli mentali e abitudini sempre più forti e difficili da scardinare ci si deve sforzare proprio nella progettazione delle interfacce più coerenti e usabili.

\footnotetext{
${ }^{166}$ Le Guidelines si presentano articolate in un'introduzione, in una serie di principi e di raccomandazioni e sono seguite da esempi, un'appendice, un glossario e una bibliografia sull'argomento. La parte più importante del documento è senz'altro costituita dai principi che ispirano il documento e dalle raccomandazioni. Queste ultime sono inserite nel testo all'interno di ogni principio e spiegano come realizzare il principio che viene espresso. Si veda: IFLA (International federation of library associations and institutions), Guidelines for online public access catalogue (OPAC) displays : final report, May 2005 / recommended by the task force on Guidelines for OPAC Displays, approved by a the Standing Committee of the IFLA Cataloguing Section, München, K. G. Saur, 2005. Draft del 2003 in <http://archive.ifla.org/VII/s13/guide/opacguide03.pdf>.

${ }^{167}$ Ivi, p. 7: «The focus of the guidelines is on the display of cataloguing information (as opposed to circulation, serials check-in, fund accounting, acquisitions, or bindery information). However, some general statements are made concerning the value of displaying to users information that is drawn from these other types of records. The guidelines do not attempt to cover HELP screens, searching commands, or command names and functions. Thus, the guidelines do not directly address the difference between menu-mode access and command-mode access».

${ }^{168}$ Ibidem: «An added benefit for such displays, if they are widely implemented, is that they could allow a user to transfer catalogue use skills acquired in one library to many other libraries. Now that so many library catalogues are available over the World Wide Web, this benefit is particularly attractive».

${ }^{169}$ Ivi, p. 8.
} 


\subsubsection{Nuove funzionalità per i servizi all'utenza}

\subsubsection{I servizi dell'OPAC e la personalizzazione}

Come in altri contesti, anche nell'ambito dei servizi bibliotecari la personalizzazione ha lo scopo ultimo di fidelizzare gli utenti ai servizi offerti dalle biblioteche. Essa attiene alla possibilità per le biblioteche di offrire servizi e contenuti maggiormente aderenti agli interessi degli utenti, consentendo di filtrare le risorse più idonee in base ai loro gusti, all'esperienza, agli interessi di ricerca e riducendo il rumore, cioè la presentazione di risorse relative a campi di interesse non espressamente richiesti.

Un approccio personalizzato consente all'utente di gestire servizi a distanza quali, ad esempio, la possibilità di salvare le ricerche fatte nell'OPAC, di richiedere servizi quali il prestito o la fornitura di documenti e di verificare in ogni momento la propria situazione utente.

Gli OPAC tradizionali offrono all'utenza la possibilità di avere un'area personale dove controllare la propria 'situazione utente' comprendente lo storico dei prestiti, i prestiti attivi le ricerche fatte. Oggi molti OPAC di nuova generazione offrono caratteristiche aggiuntive, come lo storico delle ricerche compiute.

Le caratteristiche di personalizzazione sono sempre più richieste e derivano dai nuovi modi di comunicazione tipici del web: la personalizzazione di un servizio, di una pagina web, di un portale è ormai una caratteristica alla quale la rete ci ha abituati, dato che proprio nel web si concentra la maggior parte dei servizi personalizzati e personalizzabili da parte degli utilizzatori di internet.

Tutti i portali si presentano con interfacce personalizzate o pagine iniziali appositamente predisposte che si 'aggiustano' cambiando modalità di presentazione e contenuti in relazione alle preferenze impostate dall'utente o che si servono, per la selezione dei dati da mostrare, delle tracce lasciate inconsapevolmente dall'utente nel corso della sua navigazione in rete per mostrare suggerimenti personalizzati, spesso consistenti in avvisi pubblicitari (si pensi alle varie pagine personalizzate dei motori di dicerca quali Igoogle, MyYahoo!, alle pubblicità personalizzate di Facebook, a Google+ che oggi crea un 'universo' di contenuti personali) ${ }^{170}$.

\footnotetext{
${ }^{170}$ Google Adsense è un programma gratuito che consente ai publisher online di guadagnare dalla visualizzazione di annunci 'pertinenti' in una vasta quantità di contenuti online.
} 
In realtà però molto spesso in rete ci si imbatte in una tipologia di personalizzazione dei servizi detta personalizzazione 'di massa' o 'standardizzazione personalizzata'. Si tratta di una procedura automatica, la quale essendo però facilmente riproducibile molto spesso si rivela di dubbia utilità e produce l'effetto opposto a quello voluto, giacché non è raro imbattersi in siti che utilizzano gli stessi strumenti e le stesse opzioni di personalizzazione $^{171}$. Il risultato è che tale tipologia di personalizzazione non apporta un vero valore aggiunto alla risorsa.

In linea generale, si distinguono due principali modalità di personalizzazione. La personalizzazione può definirsi 'esplicita' quando vi è il concorso diretto dell'utente, cioè viene chiesto a questi di esprimere particolari preferenze in base alle quali il servizio viene così personalizzato. È invece 'implicita' quando si basa su operazioni automatiche, che vengono compiute sulla base di dati registrati monitorando o analizzando le tracce lasciate dall'utente nella sua interazione con il sistema, senza che l'utente si accorga di essere osservato $^{172}$. Nell'ambito del web quest'ultima modalità è la più diffusa, specialmente nei siti di commercio elettronico, poiché non richiede nessun tipo di sforzo cognitivo da parte dell'utente e avviene in maniera del tutto silenziosa e inconsapevole. Le informazioni vengono raccolte attraverso l'uso di $\operatorname{cookies}^{173}$, oppure analizzando le 'tracce di navigazione' ovvero le interazioni dell'utente con il sistema durante la sua navigazione (onsite tracking).

Se la mole di dati raccolti è sufficientemente ampia come quella, ad esempio, di un libreria online come Amazon, possono essere definiti dei raggruppamenti o clusters cioè dei profili utente; ogni cluster rappresenta un insieme omogeneo di utenti che mostrano gli stessi comportamenti, e quindi hanno esigenze o interessi simili. Maggiore è la quantità di informazione raccolta, più tali clusters possono essere specifici, per consentire un servizio maggiormente personalizzato.

\footnotetext{
${ }^{171}$ Cfr.: Laura Ballestra, Servizi on line personalizzati: luci e ombre in La biblioteca su misura. Verso la personalizzazione del servizio, atti del convegno di "Biblioteche oggi", Milano, 9-10 marzo 2006, a cura di Claudio Gamba - Maria Laura Trapletti, Milano, Editrice Bibliografica, 2007, p. 243-267.

${ }^{172}$ Cfr. Nicky Ferguson - Seb Schmoller - Neil Smith, Personalisation in presentation services. A report commissioned by JISC (Joint Information Systems Committee-UK), 15 July 2004, [risorsa online] $<$ http://www.therightplace.plus.com/jp/jp-study-15.pdf > .

${ }^{173}$ I cookies (parola che letteralmente vuol dire biscottini) sono file di testo che vengono inviati dal sito web al pc dell'utente per essere in esso memorizzati (nei files temporanei del browser) ed essere reinviati in seguito al sito nelle visite successive. Essi contengono varie informazioni codificate, relative all'indirizzo IP dell'utente, alle abilitazioni dell'utente e alla tracciatura dei movimenti, che permettono al sito di 'riconoscerlo' nelle visite successive, attivando così una serie di comportamenti tra i quali ad es. la presentazione di una pagina personalizzata.
} 
La tecnica maggiormente conosciuta che si basa su tali raggruppamenti di profili utente, è nota con il nome di collaborative filtering ${ }^{174}$; essa permette di fare delle previsioni a partire, ad esempio, da un prodotto e proporre suggerimenti di acquisto sulla base dei comportamenti di altri utenti.

I 'filtri collettivi' si sono rivelati una scelta vincente in contesti nei quali ci si rivolge ad una utenza non omogenea e diversificata, ad esempio gli utenti di siti commerciali quali Amazon $^{175}$. I suggerimenti delle librerie online «chi ha acquistato $\mathrm{xx}$ ha acquistato anche yy» costituiscono la più nota applicazione di questa tecnica e oggi difficilmente vengono replicati nelle interfacce degli OPAC.

La rilevazione delle regolarità di comportamento degli utenti viene affidata a tecniche quali il data mining, che permettono di estrarre informazioni significative dai dati, cogliere analogie e regolarità, di riconoscere patterns cioè schemi di comportamento ${ }^{176}$. In base a tali schemi oggi è possibile indirizzare il comportamento di acquisto degli utenti.

Purtroppo, però, tali suggerimenti sono spesso invadenti e non tengono conto del fatto che i gusti, le esigenze e gli interessi cambiano nel tempo, o possono avere natura contingente.

Amazon è un ottimo esempio: se oggi cerco un libro che corrisponde ai miei interessi di lettura, domani mi verranno proposti libri simili, se però oggi ho cercato per conto di un amico un libro che non mi interessa direttamente, domani troverò suggerimenti che non mi interessano. Tali tecniche, inoltre, per giungere a risultati soddisfacenti, devono potersi applicare ad grandi quantità di dati e pongono dunque vari problemi ${ }^{177}$.

La stessa riflessione si può estendere ai comportamenti di lettura, dunque l'utente deve comunque avere la possibilità di esplicitare un bisogno, o all'opposto, il rifiuto di un'informazione.

La modalità 'esplicita' di personalizzazione prevede una partecipazione attiva dell'utente; egli può manifestare attivamente un giudizio di gradimento, di non gradimento,

\footnotetext{
${ }^{174}$ Altre tecniche simili sono il content-base filtering o filtraggio cognitivo, che si basa sulle prefenze attuali e passate di un utente per proporgli prodotti coerenti con il suo profilo e il rule-based filtering che invece stabilisce dei profili utente statici, in base al quale estrapolare delle regole per la distribuzione di contenuti web potenzialmente rilevanti. Per un approfondimento su queste tematiche si vedano i contributi di Ventura, Ballestra e Barlach in La biblioteca su misura cit.

${ }^{175} \mathrm{La}$ personalizzazione automatica in vari casi non si rivela la scelta migliore perché per funzionare necessità di molti dati sull'utenza.

${ }^{176}$ È vero anche che, come fa notare Ballestra, in genere i comportamenti d'acquisto sono guidati dai bisogni, non dai precedenti comportamenti d'acquisto, in quanto i bisogni cambiano nel tempo.

${ }^{177}$ Tra i risvolti negativi della personalizzazione, ad esempio, bisogna fare attenzione ai probabili rischi di violazione della privacy, a volte effettuata dai gestori dei servizi tramite la vendita a terzi o la diffusione di dati.
} 
oppure a quest'ultimo vengono richiesti dati in base ai quali egli ha la possibilità di crearsi un portale o una pagina personalizzata.

Le due tipologie di personalizzazione sono state impiegate con maggiore o minore fortuna a partire dagli anni '90 dalla maggior parte dei siti e dei portali commerciali. Dapprima la personalizzazione esplicita è stata la regola, poi con la possibilità di disporre di una massa di dati ormai immagazzinata, la personalizzazione implicita ha finito per prevalere. Oggi i siti commerciali sfruttano modalità di personalizzazione 'esplicite' che, in base alla nota teoria di Zipf ${ }^{178}$, richiedono il minimo sforzo (es. mi piace /non mi piace in Facebook o il rating, giudizio da esprimere tramite le 'stelle').

Accanto a questi due grandi paradigmi, una terza via, di recente utilizzata dalle aziende, è quella di affiancare communities di utenti/clienti attorno ad un prodotto o un servizio e di sfruttare l'analisi dei $\log$ cioè le registrazioni delle loro interazioni con il sistema per trarre spunti utili al miglioramento del servizio stesso. L'offerta di pagine personalizzate sta pian piano cedendo il posto a questo tipo di interazione, dove l'apporto dell'utente non rimane confinato alla propria pagina personale, ma si integra e viene convogliato nel sapere di una comunità. Librerie e applicazioni commerciali come Amazon, Ebay, etc. si basano su questi saperi condivisi.

Scegliere la tipologia giusta di personalizzazione per un OPAC o un sito web, non è semplice; la personalizzazione comporta dei costi e dei rischi e il rapporto tra personalizzazione e soddisfazione dell'utente non è privo di complessità ${ }^{179}$.

Dalle esperienze raccolte emerge che la tipologia 'esplicita' di personalizzazione risulta in genere più gradita all'utente che, nonostante non abbia tempo da perdere, preferisce comunque compilare i dati per creare il proprio profilo e avere così un maggior controllo, potendo modificarli a piacere nel caso i propri interessi dovessero per qualche motivo mutare. Un'altra motivazione che spinge gli utenti in questa direzione è che non temono così che vengano loro precluse risorse potenzialmente rilevanti. Quest'ultima motivazione è però la stessa che, in molti casi, spinge gli utenti a rifiutare un servizio personalizzato $^{180}$. Oggi l'utente utilizza nel web una gran quantità di servizi e trova sempre meno il tempo per compilare form per avere un servizio su misura, dunque oggi è necessario sfruttare nuove forme di personalizzazione. Avere un' 'identità digitale' in

\footnotetext{
${ }^{178}$ George Kingsley Zipf, Human behaviour and the principle of least-effort cit.

${ }^{179}$ Roberto Ventura, User satisfaction e personalizzazione: quale rapporto, in La biblioteca su misura. Verso la personalizzazione del servizio, atti del convegno di "Biblioteche oggi", Milano, 9-10 marzo 2006, a cura di Claudio Gamba e Maria Laura Trapletti. Milano, Editrice Bibliografica, 2007, pubblicato anche in «Biblioteche Oggi», 24 (2006), n. 6, p. 29-44.

${ }^{180}$ Laura Ballestra, Servizi on line personalizzati: luci e ombre cit.
} 
Facebook o in Google, probabilmente diventerà una nuova forma per avere contenuti personalizzati, senza bisogno di immettere dati e preferenze per ogni nuovo sito visitato.

Nel campo delle biblioteche, la personalizzazione è stata applicata dapprima nei portali bibliotecari e successivamente anche negli OPAC. Tra i primi sistemi innovativi di gestione altamente personalizzabili, si può citare l'esperienza di My library all’Università della California. MyLibrary@NCSU è stato per anni il miglior esempio di portale personalizzabile ${ }^{181}$. Oggi però si preferisce quindi creare semplici pagine 'ad accesso personale' dalle quali gli utenti accedono a vari servizi bibliotecari (es. verifica dei prestiti, rinnovo o prenotazione di volumi, salvataggio delle ricerche fatte negli OPAC alle quali si affiancano servizi innovativi, quali la possibilità di avere un blog personale o di creare una community) e che quindi non si propongono come unico punto di accesso all'informazione.

La possibilità di partire dall'OPAC come punto di partenza personalizzato per l'esplorazione di tutti i servizi bibliotecari si ricollega direttamente al dibattito OPAC / portale che ha visto più volte vari studiosi confrontarsi su questa tematica ${ }^{182}$ e che si è

\footnotetext{
${ }^{181}$ Sviluppato e creato da Eric Lease Morgan per le biblioteche della NCSU, MyLibrary rappresentava una soluzione software per la creazione di 'miniportali' personalizzabili da parte degli utenti, dove i vari contenuti erano integrati. Allo scopo di filtrare i contenuti limitandoli alle proprie esigenze il software permetteva di creare un proprio spazio personalizzato di accesso alla biblioteca. Il software, con le parole di Antonella De Robbio, che fornì una sintesi delle caratteristiche del prodotto permetteva all'utente di «disporre di "una specie di "banco di lavoro virtuale" per l'utente finale,comprendente tra i vari attrezzi di lavoro: il motore di ricerca preferito, le banche dati più utilizzate (cui l'ente ha accesso), la selezione dei siti preferiti subito pronti sotto mano, la selezione delle pagine Web della biblioteca o della struttura in cui la biblioteca è incardinata, maggiormente utilizzate e sistemate nell'ordine che si preferisce, i periodici elettronici in uno scaffalino virtuale per una rapida e costante consultazione, i numeri di "pronto soccorso" help-desk per ottenere informazioni dai bibliotecari specialisti in quel settore cui l'utente afferisce. Oltre a queste opzioni [il software permetteva] ... l'accesso all'OPAC che consente il recupero delle informazioni bibliografiche dall'OPAC per argomenti selezionati o dalla lista delle nuove accessioni». (Antonella De Robbio, My library. Note informative e tecniche stilate sulla base della documentazione disponibile e sulla base di una prima sperimentazione tecnica del software. A cura di Antonella De Robbio. Gruppo di Lavoro per Integrazione delle Risorse Elettroniche IRE, Sistema Bibliotecario di Ateneo. Università degli Studi di Padova, (Unpublished report, 28-02-2002, ultimo aggiornamento 30-03-2002), <http://eprints.rclis.org/4031/>). L'idea di un portale 'su misura' così strutturato è stata però successivamente messa in discussione. Dopo vari anni di utilizzo alla NCSU e in varie altre biblioteche americane, tali 'portalini' sono stati abbandonati in favore di un accesso più integrato con gli altri servizi e nella maggior parte dei casi non si presentano più come uno spazio separato (silo). La pagina web del progetto $(<\mathrm{http} / / /$ my.lib.ncsu.edu/ $>)$ infatti non è più attiva. Per l'evoluzione del progetto MyLibrary@NCSU si veda il cit. contributo di Ballestra. MyLibrary è stato utilizzato anche in Italia a partire dal 2001, nella biblioteca Mario Rostoni della LIUC. Tra le possibilità offerte anche quella, in fase di interrogazione dell'opac e dopo essersi autenticati, di salvare le proprie ricerche nel proprio archivio libri, al quale si accede anche al di fuori dell'OPAC.

${ }^{182}$ La dicotomia OPAC/portale è stata oggetto di discussione nell'ultimo decennio tra professionisti all'interno di liste di discussione quali ad es. Web4lib in conseguenza soprattutto del crescente fenomeno delle librerie on-line quali Amazon. Mi piace riportare in questa sede una testimonianza che ho avuto modo di citare in un mio precedente contributo: già nel 1999 nella lista si era originata la discussione in un thread dal titolo Opac as Portal. A titolo esemplificativo, si riporta un estratto di un post di uno dei partecipanti alla discussione, Tony Barry (n.d.r. la discussione è ancora visibile negli archivi della lista all'indirizzo <http://web4lib.org/archive/1999-October/029015.html>). La personalizzazione dell'OPAC è in questo esempio molto spinta, anche se alcune caratteristiche esposte, sembrano quanto mai attuali (n.d.r. La traduzione è mia):
} 
evoluta verso l'integrazione dei due strumenti. Sicuramente prevale oggi una tendenza verso l'integrazione dell'OPAC con tutti gli altri servizi offerti dalle biblioteche (es. accesso a banche dati, risorse e servizi web). Nella maggior parte delle più recenti applicazioni software è prevalente infatti la logica dell'accesso unico (una sola autenticazione per tutti i servizi ${ }^{183}$ ), in modo che l'identificazione segua l'utente per tutto il suo percorso sia nell'OPAC sia nel portale di una biblioteca. Per ottenere tale risultato è indispensabile adottare sistemi aperti e modulari (meglio se open source).

Alcune interessanti esperienze si sono sviluppare a partire dal modello MyLibrary e giungendo ad esiti originali, verso lo sviluppo di sistemi ove l'OPAC ha un ruolo più centrale nell'ottica della personalizzazione. Gli OPAC interagiscono quindi con scaffali virtuali dei singoli utenti oppure a partire da essi, vengono sviluppati nuovi servizi all'utenza $^{184}$.

«una prima chiave per un ruolo più esteso dell'opac è l'autenticazione. L'opac può personalizzare il servizio a misura dell'utente se è in grado di riconoscerlo. Le biblioteche hanno fallito questo importante passaggio poiché pensano all'opac come ad un servizio pubblico di tipo generico come avveniva per il catalogo cartaceo [...] Accoglienza: una volta fatto il log vorrei un opac che mi fornisse informazioni immediate utili nel caso io volessi recarmi in biblioteca, del tipo "ciao Tony, oggi è mercoledì 10 ottobre e sono le $10 \mathrm{am}$. La sezione $\mathrm{A}$ è aperta e chiude alle $7 \mathrm{pm}$. La sezione $\mathrm{b}$ è aperta e chiude alle $7 \mathrm{pm}$. La sezione $\mathrm{C}$ è chiusa fino alle 9am di Lunedì. È prevista pioggia. Hai 8 libri in prestito. Dirac P., Quantum mechanics devi restituirlo venerdì, vuoi rinnovare il prestito? Ci sono 15 nuovi libri che possono interessarti. Alcune riviste che hai richiesto sono arrivate." [...] Raffinamento della ricerca: vorrei un opac che mi permettesse di raffinare la ricerca secondo i libri che ho preso in prestito in passato, le intestazioni di soggetto e le classificazioni che ho usato nelle precedenti sessioni in base alla data del prestito [...]Recensioni e valutazioni: se prendo in prestito un volume vorrei che il sistema mi permettesse di scrivere un commento e dare una valutazione. Nella ricerca dovrebbero essere disponibili le recensioni e una media dei giudizi degli altri lettori [...]Risorse e aiuti in fase di ricerca: nella ricerca di un documento, vorrei che mi venissero mostrati dei link ai documenti collegati agli stessi indici, con la formula "more like this", che mi permetta di selezionare simultaneamente i documenti che mi piacciono tralasciando quelli che non mi interessano. L'algoritmo dovrebbe basarsi non solo sugli indici in comune, ma anche sull'analisi incrociata dei prestiti. Materiale non posseduto: L'opac non dovrebbe limitarsi al materiale posseduto dalla biblioteca o alle risorse alle quali la biblioteca accede a pagamento ma una ricerca di una monografia che non ha successo dovrebbe portare ad un link ad una libreria online, nel caso l'utente volesse acquistare il libro. L'opac dovrebbe prevedere accesso anche ai periodici non posseduti con un collegamento al servizio di Document delivery o al prestito interbibliotecario. [...] Sulla base delle risposte raccolte automaticamente dal sistema, la biblioteca potrebbe decidere quali libri acquistare o avere una chiara indicazione della domanda dell'utenza. Libri in arrivo: le notizie all'utente sui libri arrivati in biblioteca potrebbero essere personalizzate tenendo conto dei soggetti e delle classificazioni maggiormente usate dall'utente, dall'indicazione dei volumi presi in prestito dalle persone che hanno gli stessi interessi che scaturiscono da un "borrowing pattern" simile, dai libri che l'utente aveva specificato di voler prendere in prestito».

${ }_{183}$ Questo argomento è al centro dell'attenzione nel dibattito più recente. Una sola autenticazione per diversi servizi offerti dalla biblioteca consente all'utente di passare da uno strumento all'altro (es. dall'OPAC ad una banca dati), o accedere a diversi servizi della biblioteca senza doversi nuovamente autenticare.

${ }^{184}$ Tra le esperienze più significative è da menzionare il progetto My Library, condotto da sette importanti biblioteche danesi Gitte Barlach, "My library": un accesso integrato alle risorse della biblioteca basato sui servizi web. in La biblioteca su misura. Verso la personalizzazione del servizio, atti del convegno di "Biblioteche oggi", Milano, 9-10 marzo 2006, a cura di Claudio Gamba - Maria Laura Trapletti, Milano, Editrice Bibliografica, 2007, p. 119-128. A partire dall'OPAC esse permettevano di accedere una volta effettuata un' interrogazione su un titolo, a vari servizi tra i quali un portale di biografie e un servizio di raccomandazioni basato sui prestiti effettuati dagli altri utenti, alla maniera di Amazon. Il sistema di raccomandazioni usato per la personalizzazione dei suggerimenti di lettura ha previsto l'uso dei filtri collettivi unitamente alla possibilità di scelta/rifiuto del consiglio di lettura, quale modalità per la 
Sull'uso della 'personalizzazione automatizzata' o 'implicita' nei cataloghi, il dibattito è ancora aperto. I dati d'uso relativi, ad esempio, ai libri presi in prestito, alle proposte di acquisto dell'utente e degli altri utenti non vengono ancora usati per fornire un servizio 'su misura'. Weston e Vassallo hanno suggerito per questo tipo di personalizzazione il modello funzionale dell' «OPAC che si adatta» riferendosi alle operazioni di data mining effettuate su clusters di utenti ${ }^{185}$.

Tale modello funzionale è strettamente legato anche a quello, suggerito dagli stessi studiosi, dell' 'OPAC che apprende'. L'OPAC può apprendere dai comportamenti dell'utente e in modo dinamico e di conseguenza adattarsi ai gusti e alle preferenze dell'utente, fornendogli un ambiente personalizzato o proponendogli nuovi percorsi di ricerca.

Tale visione porterebbe a strutturare i cataloghi stessi come agenti intelligenti e a dare un maggior peso a fattori quali l'uso dei documenti; significativi in questo senso sono gli interrogativi posti da Ventura ${ }^{186}$ :

È possibile realizzare un OPAC in grado di "riconoscere" i propri utenti, di "apprendere" le loro abitudini di ricerca e di lettura, di presentarsi come un sistema "proattivo" nei confronti dell'utenza, che non si limiti a forme di recupero strettamente rispondenti alle chiavi di recupero imputate dall'utente? Può tutto ciò far pensare a nuove forme di indicizzazione temporanea dei documenti basate su schemi comportamentali, legate cioè all'uso dei documenti piuttosto che all'interpretazione del significato in essi racchiuso? L'OPAC, in definitiva, come 'agente intelligente'?

Oggi funzionalità di personalizzazione vengono sempre più spesso offerte dai cataloghi che si proclamano 'di nuova generazione', purtroppo però l'evoluzione verso sistemi di discovery non si è orientata verso lo sviluppo di caratteristiche personalizzate, anzi ha comportato la perdita di alcune funzionalità importanti per l'utente. Un sistema di discovery in genere non gestisce l'area personale che solitamente si ritrova nei nuovi OPAC: se vi è la possibilità di ricavare dall'ILS informazioni sulla disponibilità in tempo reale del documento, questi sistemi non consentono di gestire un'area personale, che viene ancora delegata all'OPAC tradizionale.

personalizzazione e per l'adattamento dinamico dell'opac alle esigenze degli utenti. Anche l'OPAC del Servizio bibliotecario provinciale di Verona prende spunto dal modello fornito dal progetto MyLibrary@NCSU. La soluzione adottata dal SBPVr ha introdotto tra le novità anche strumenti tipici del 2.0 come le folksonomie e i commenti alle risorse dell'OPAC. Cfr. infra, Appendice A. Casi di studio. Sistema bibliotecario della Provincia di Verona.

${ }^{185}$ Paul Gabriele Weston, - Salvatore Vassallo, "... e il navigar m'è dolce in questo mare". Linee di sviluppo e personalizzazione dei cataloghi cit.

${ }^{186}$ Roberto Ventura, User satisfaction e personalizzazione: quale rapporto cit. 


\subsubsection{Gli OPAC sociali. Mito e realtà dell'OPAC partecipativo}

Per qualche anno il tema dell'OPAC sociale e collaborativo ha animato il dibattito bibliotecario sullo sviluppo degli cataloghi. Nel giro di pochi anni nella letteratura professionale si cominciò a parlare di Web 2.0, 'reti sociali', 'software sociale', 'network collaborativo'.

Sull'onda di quella che sembrò essere una vera rivoluzione del web, anche in campo bibliotecario, in poco tempo, è stato teorizzato il modello della 'Library 2.0' ispirato alla filosofia di questo nuovo corso del web, così anche nello sviluppo dei cataloghi ha preso vita una vera e propria 'visione" ${ }^{187}$ : l'OPAC 2.0 o OPAC sociale e partecipativo ${ }^{188}$.

L'applicazione dei principi del Web 2.0 ai cataloghi comporta sostanzialmente l'ingresso negli OPAC degli attributi di socialità e interattività ormai considerati i tratti più distintivi della recente evoluzione della rete.

Chi si batte a favore dell'ingresso delle caratteristiche 2.0 nei cataloghi di biblioteche è spinto dalla considerazione che biblioteche che offrono all'utenza cataloghi di tipo tradizionale possano perdere utenti in favore dei motori di ricerca del web o dei siti di social cataloguing. Il rischio, così, è quello di rimanere fuori da nuovi canali comunicativi e da nuovi modi di produrre informazione che contraddistinguono le applicazioni del web.

Ciò è vero, ma solo in parte: come vedremo, la trasformazione del catalogo nella direzione del Web 2.0 non è avvenuta pienamente: anche se i software sociali sono entrati lentamente nelle vite di milioni di persone e hanno effettivamente cambiato il modo di

\footnotetext{
${ }^{187}$ Marchitelli e Piazzini videro negli gli opac sociali la 'fase tre' di una evoluzione che dal catalogo tradizionale si era svolta fino al catalogo arricchito: «Possiamo definire i SOPAC - affermava Marchitellicome degli OPAC arricchiti da particolari strumenti di socializzazione e di condivisione di contenuti creati dagli utenti, o viceversa, con le parole degli sviluppatori dell'opac della Ann Arbor Disctrict Library, come un «set di strumenti tipici delle reti sociali integrati nel catalogo della biblioteca. Questo offre agli utenti la possibilità di votare, recensire, commentare le risorse e applicare ad esse le sue etichette (Blyberg 2007). In questi sistemi l'utente può generalmente lasciare commenti, inserire tag, votare le risorse e, soprattutto, trovarsi a far parte di un network che gli permette di incontrare altri utenti con un profilo simile, dalle cui letture potrà trarre ulteriore ispirazione» Andrea Marchitelli, - Tessa Piazzini, OPAC, SOPAC E Social networking cit. Si vedano anche altri due brevi contrbituti di Andrea Marchitelli: OPAC, bloOPAC e social networking: gestione della conoscenza 2.0?. slides presentate al 3' Incontro ISKO Italia - UniMIB, Milano, 2007, <http://eprints.rclis.org/archive/00010667/01/opac-blopac-e-social-networking1722.pdf> e ID, Il buono, il brutto, il cattivo: esistono davvero gli OPAC 2.0? [Slides presentate all'incontro CILEA "Oltre l'OPAC". Jesi, Biblioteca Planettiana, 9 giugno 2008], $<$ http://eprints.rclis.org/archive/00013795/01/Il_buono\%2C_il_brutto\%2C_il_cattivo.pdf>. Si veda sullo stesso tema ancheil contributo critico di Fabio Metitieri, L'OPAC collaborativo tra socialità $e$ folksonomia, «Biblioteche Oggi», 27 (2009), n.2, p. 7-14.

${ }^{188}$ Il primo OPAC sociale (SOPAC) venne creato da John Blyberg e mantenuto da Darien Library derivava il suo nome dall'unione dei termini Social e OPAC. Oggi il software è alla versione 2 e tra le implementazioni visibili oltre a quella della Darien Library (<http://www.darienlibrary.org/catalog $>$ ) vi sono quelle di Ann Arbor district library (Cfr. infra, Appendice A. Casi di studio. Ann Arbor District llibraries. SOPAC) e Palos Verdes Library District <http://www.pvld.org/>.
} 
comunicare e di utilizzare l'informazione, nel catalogo le tecnologie del Web 2.0 vengono ampiamente utilizzate, ma i contenuti degli utenti sono scarsi e sempre meno richiesti, sia dagli stessi utenti, sia dai bibliotecari.

È ormai comunemente accettato che il Web 2.0 sia stato un processo di rinnovamento tecnologico, e una nuova filosofia di sviluppo del web; il meme è diventato subito sinonimo di innovazione, vitalità e cambiamento ${ }^{189}$ rispetto ad un web statico e monodirezionale.

Hanno suscitato grande interesse alcune tematiche ad esso collegate - per alcuni forse ingiustamente ricomprese sotto tale etichetta - come le teorie economiche della lunga coda e della 'saggezza delle folle" ${ }^{190}$, che hanno contribuito a renderlo una vera e propria filosofia del web, un'attitudine ${ }^{191}$.

In realtà la natura e la portata del fenomeno sono state oggetto discussione e le ipotesi interpretative hanno visto l'alternarsi di una visione rivoluzionaria, destinata a creare una rete migliore, e di quella evolutiva che oggi è maggiormente accolta.

Non mancano neppure le critiche da parte di chi ha visto nel fenomeno un vero e proprio bluff, una vasta operazione di mercato compiuta da Tim O'Reilly e soci per rilanciare le discussioni intorno ai loro prodotti editoriali ${ }^{192}$.

All'inizio, la caratteristica più significativa del Web 2.0 era stata la volontà di avvicinare l'esperienza degli utenti nella rete alle applicazioni desktop; tendenza che si è manifestata nel modello produttivo del Software as Service (Saas). Sono nate così

\footnotetext{
${ }^{189}$ Il termine Web 2.0 indica comunemente uno stato di evoluzione di quella parte di internet che conosciamo come World Wide Web sia a livello tecnologico sia a livello sociale: da più parti si ritiene che il web 2.0 non sia stato un semplice processo di rinnovamento tecnologico, ma, piuttosto, una nuova filosofia di sviluppo del web, dove al centro vi è in primo luogo la socialità. Una prima definizione di web 2.0 venne data dal fondatore del meme Tim O'Reilly nel celebre articolo What is Web 2.0. Design patterns and business models for the next generation of software ove il mutamento tra tecnologie venne spiegato con una semplice tabella comparativa Tim O'Reilly e i suoi collaboratori cercarono di spiegare il nuovo corso del web con una tabella elaborata nel loro primo brainstorming, paragonando siti di quello che retroattivamente definirono web 1.0 con le nuove applicazioni. Successivamente O' Reilly fornì una prima definizione del fenomeno elaborando una mappa concettuale (meme map) e definendo il web 2.0 come una serie di principi e pratiche. Ne articolò quindi l'essenza in sette principi: il web come piattaforma, sfruttare l'intelligenza della folla, i dati sono il prossimo 'intel inside', la fine del ciclo delle release dei software, modelli di programmazione leggeri, il software supera il livello del singolo dispositivo, esperienze dell'utente ricche. Cfr. Tim O'Reilly, What is Web 2.0. Design patterns and business models for the next generation of software, «O'Rellynet.com», 30 september 2005. <http://www.oreillynet.com/pub/a/oreilly/tim/news/2005/09/30/what-is-web-20.html>.

${ }_{190} \mathrm{Si}$ vedano rispettivamente: Chris Anderson, The long tail, «Wired», 12 (2004) n. 10. $<$ http://www.wired.com/wired/archive/12.10/tail.html> e James Surowiecki, The wisdom of crowds. Why the many are smarter than the few and how collective wisdom shapes business, economies, societies, and nations, New York, Doubleday, 2004. In traduzione italiana: Id., La saggezza della folla, Milano, Internazionale, 2007.

${ }^{191}$ Ian Davis, Talis, Web 2.0 and all that, «Internet Alchemy blog», [Blog post], 4 July 2005, $<$ http://iandavis.com/blog/2005/07/talis-web-20-and-all-that $>$.

${ }^{192}$ Si vedano: Geert Lovink, Zero comments. Teoria critica di Internet, trad. it. di Alessandro Delfanti, Milano, Mondadori, 2008 (in particolare le p. 3-37) e Fabio Metitieri, Il grande inganno del web 2.0., Bari, Laterza, 2009.
} 
tecnologie nuove come Ajax, Ria, i widgets e mashups che hanno permesso, utilizzando linguaggi come XML, di creare applicazioni dinamiche e interattive ${ }^{193}$.

Il Web 2.0 ha però avuto il merito di portare, accanto alla tecnologia, una certa dose di novità nel modo di usare e interagire nel web; le nuove tecnologie hanno permesso a chiunque di creare gruppi in modi nuovi e con grande facilità, così il web in poco tempo è diventato "sociale" 194 .

Tim O’ Reilly fornì una definizione compatta del Web 2.0 in una pagina del suo $\operatorname{blog}^{195}$ :

Web 2.0 is the network as platform, spanning all connected devices; Web 2.0 applications are those that make the most of the intrinsic advantages of that platform: delivering software as a continually-updated service that gets better the more people use it, consuming and remixing data from multiple sources, including individual users, while providing their own data and services in a form that allows remixing by others, creating network effects through an "architecture of participation," and going beyond the page metaphor of Web 1.0 to deliver rich user experiences ${ }^{196}$.

Tale definizione metteva in rilievo quelli che oggi sono riconosciuti come i due principali aspetti del fenomeno:

1) Un primo aspetto è di tipo tecnologico e viene identificato da molti con la semplicità d'uso delle interfacce dei siti 2.0, che risultano simili a vere e proprie applicazioni come quelle che si istallano sul proprio computer. Internet diviene quindi una piattaforma $^{197}$ di servizi. Oggi in rete tale aspetto ha realmente modificato l'interazione degli utenti con internet: le immagini, i documenti, possono essere conservate nella rete in appositi spazi, molte applicazioni che prima erano stand alone si trovano oggi su internet:

\footnotetext{
${ }^{193}$ Nicole C. Engard, Library mashups: exploring new ways to deliver library data, Medford, Information Today, Inc., c2009. Anche in <http://mashups.web2learning.net/> (in particolare la parte III: Mashing up Catalog Data). Inoltre si veda: Bonaria Biancu, Aggregare le informazioni sul web: esperienze a confronto. in Biblioteche come conversazioni: 2.0 due punto zero intorno e oltre l'architettura: Giornata di studio del CNBA 2007, Roma, 18 maggio 2007 / a cura di Serena Sangiorgi e Sandra Astrella. - [S.1.]: Coordinamento nazionale biblioteche di architettura, c2008. - (I quaderni del CNBA ; 9) oppure $<$ http://digital.casalini.it/17240611>.

${ }^{194}$ Sugli aspetti sociali dell'organizzazione delle conoscenze nel Web 2.0 si veda Clay Shirky, Here comes everybody. The power of organizing without organizations, New York: Penguin Press, c2008. In traduzione italiana: Uno per uno, tutti per tutti. Il potere di organizzare senza organizzazione, Torino, Codice, 2009.

${ }^{195}$ La definizione è stata poi ulteriormente arricchita nel 2006 (cfr. Tim O'Reilly, 2.0 compact definition: trying again «O'Reilly Radar», blog post, december 10, 2006, $<$ http://radar.oreilly.com/archives/2006/12/web_20_compact.html>).

196 Tim O'Reilly, Web 2.0: compact definition?, «O'Reilly Radar», blog post, October 12005. $<$ http://radar.oreilly.com/archives/2005/10/web_20_compact_definition.html>.

${ }^{197}$ Questo aspetto è sottolineato anche nella definizione che ne danno Ken Chad \& Paul Miller della Talis (Ken Chad - Paul Miller, Do libraries matter? The rise of Library 2.0. A white paper. Version 1.0 [Risorsa online] November 2005, <http://www.talis.com/applications/downloads/white_papers/DoLibrariesMatter.pdf>): «What the next generation of applications enable is the creation of a participative 'platform' that empowers both users and the builders of user-facing applications. In the web world, growing interest in this idea is being dubbed Web 2.0.».
} 
è possibile editare testi, modificare immagini o creare effetti speciali, creare grafici, usare un antivirus online senza istallare alcun software su proprio pc.

2) Un secondo aspetto del Web 2.0 risiede nella la sua natura collaborativa e 'sociale'. Molti ritengono che la vera rivoluzione consista nell'approccio con il quale gli utenti si rivolgono al web, che passa fondamentalmente dalla semplice consultazione (seppure supportata da efficienti strumenti di ricerca, selezione e aggregazione) alla possibilità di contribuire popolando e alimentando il web con i propri contenuti ${ }^{198}$.

\begin{tabular}{|c|c|c|}
\hline Web 1.0 & & Web 2.0 \\
\hline DoubleClick & $-->$ & Google AdSense \\
\hline Ofoto & $-->$ & Flickr \\
\hline Akamai & $-->$ & BitTorrent \\
\hline mp3.com & $-->$ & Napster \\
\hline Britannica Online & $-->$ & Wikipedia \\
\hline personal websites & $-->$ & blogging \\
\hline evite & $-->$ & upcoming.org and EVDB \\
\hline domain name speculation & $-->$ & search engine optimization \\
\hline page views & $-->$ & cost per click \\
\hline screen scraping & $-->$ & web services \\
\hline publishing & $-->$ & participation \\
\hline content management systems & $-->$ & wikis \\
\hline directories (taxonomy) & $-->$ & tagging ("folksonomy") \\
\hline stickiness & $-->$ & syndication \\
\hline
\end{tabular}

Fig. 2.8 - La tabella di Tim O’ Reilly paragona applicazioni 2.0 a quelle del web $1.0^{199}$.

\footnotetext{
${ }^{198}$ Una delle definizioni è: «Web 1.0: è una lezione universitaria, in cui un professore spiega e gli alunni ascoltano. Web 2.0: è una lezione autogestita in cui tutti parlano e partecipano».

${ }^{199}$ Tim O'Reilly, What is Web 2.0 Design patterns and business models for the next generation of software, «O'Rellynet.com», 30 september 2005. <http://oreilly.com/web2/archive/what-is-web-20.html>.
} 


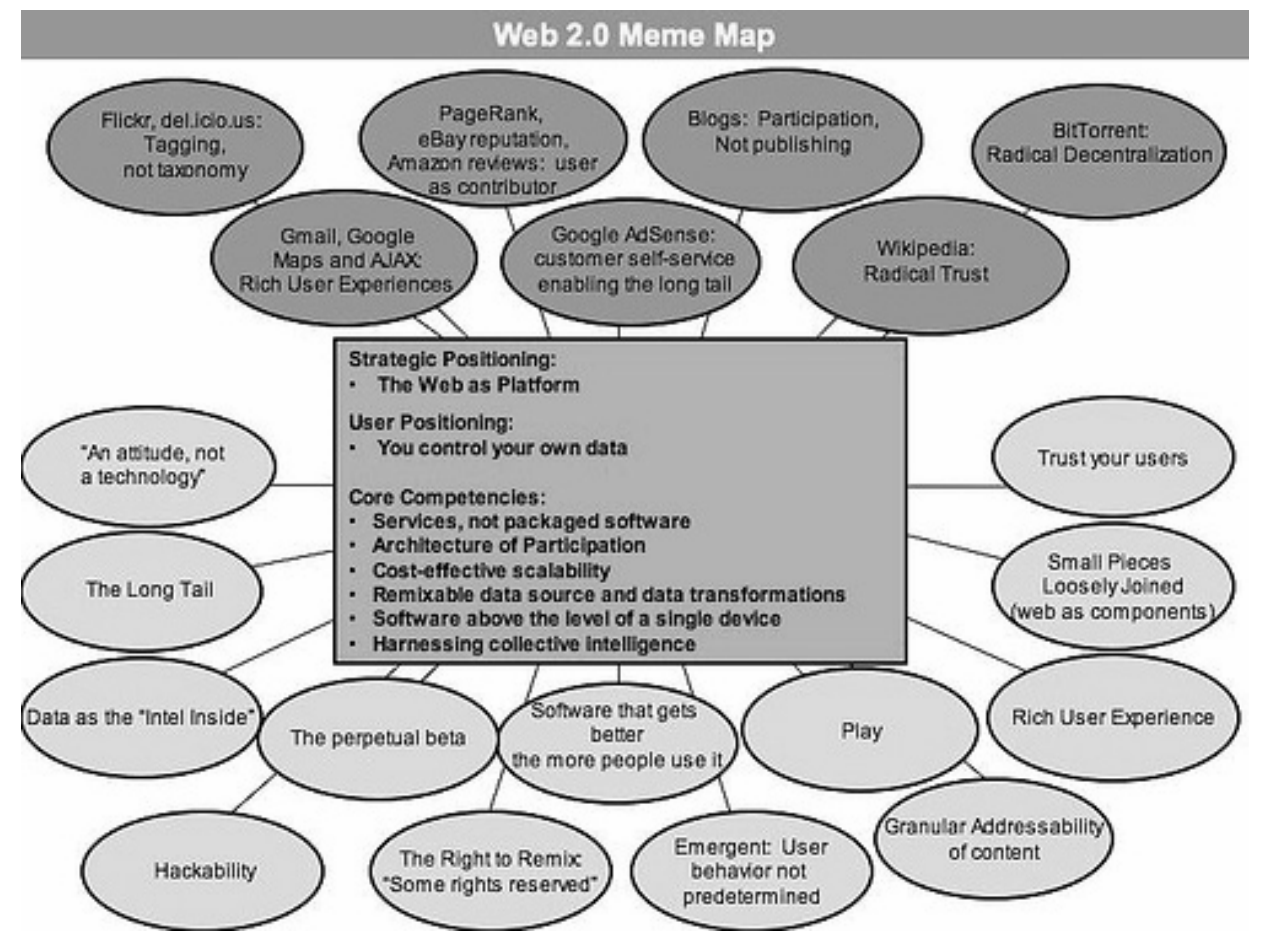

Fig. 2.9 - Web 2.0 meme map ${ }^{200}$

Le applicazioni tipiche del Web $2.0^{201}$ sono quelle di social networking ${ }^{202}$ che consentono agli utenti di collaborare (wiki), di condividere interessi e contenuti (sharing).

${ }^{200}$ Ivi, s.p.

${ }^{201}$ Si deve a Paul Anderson un'analisi approfondita delle applicazioni del web 2.0 (i blog, wiki, tagging e social bookmarking, folksonomy, multimedia sharing, audio blogging e podcasting, rss e syndication), dei differenti tipi di servizi che si possono costruire tramite tali applicazioni e un'analisi dei concetti fondamentali alla base nuovo indirizzo intrapreso dal web dettati da Tim Berners Lee (Cfr.: Paul Anderson, What is Web 2.0? Ideas, technologies and implications for education, JISC tech watch report (Joint Information Systems Committee-UK), february 2007 , <http://www.jisc.ac.uk/media/documents/techwatch/tsw0701b.pdf $>$ ). Per quanto riguarda la caratteristica che Anderson cita come 'produzione individuale e contenuti generati dagli utenti', in un articolo Nicola Benvenuti, riferendosi alla tabella elaborata da di Tim O'Reilly e soci, notava come la parte più interessante di quella rappresentazione del nuovo ambiente consista nella sostituzione della 'pubblicazione' con la 'partecipazione'. (Nicola Benvenuti, Social tagging e biblioteche: implicazioni e suggestioni di una "classificazione generata dagli utenti che emerge attraverso un consenso dal basso", «Biblioteche oggi», 25, (2007), n. 3, p. 35-42). Il web 2.0 ha permesso con facilità agli utenti di passare dalla condizione passiva di consumatori dei contenuti del web a quella di prosumer, cioè di produttori e consumatori di contenuti.

Con le nuove applicazioni 'sociali' gli utenti possono pubblicare nel web in modo molto semplice immettendovi i propri filmati, foto, commenti esprimendo le proprie idee, condividendo esperienze e progetti nelle reti sociali; possono classificare le risorse nei siti di social tagging, possono scambiarsi e condividere i propri interessi di ricerca nei siti di social bookmarking. Dalle prime piattaforme per blogging si è passati così velocemente allo sviluppo di quello che viene definito software sociale, o software collaborativo cioè applicazioni che consentono agli individui di incontrarsi, interagire e collaborare in rete e, in particolare, di creare comunità on-line per condividere testi, immagini, link e foto fino a condividere ogni aspetto della propria esistenza come pensieri, immagini, link, foto e altri contenuti nella piattaforma Web 2.0 per eccellenza, Facebook. Una nuova realtà democratica si è incarnata nei wiki, spazi che consentono agli utenti di aggiungere contenuti e di modificare contenuti creati dagli altri. La più grande impresa collaborativa in forma di wiki è Wikipedia, enciclopedia on line libera e aperta alla collaborazione di chiunque, che utilizza un "sistema di modifica e pubblicazione aperto" e viene pubblicata in 250 lingue differenti .

Una delle implicazioni di questa nuova realtà, rafforzata dell'architettura della partecipazione, sembra essere proprio la modificazione del concetto di autorità cioè di chi è autorizzato a scrivere o pubblicare, alla quale si sostituisce il criterio della trovabilità (findability) come Peter Morville, esperto di usabilità e architettura 
Queste nuove tecnologie hanno consentito agli utenti del web di diventare prosumers, cioè allo stesso tempo consumatori (producers) e fruitori (consumers) dei contenuti che immettono sul web.

Tale genere di apporto, o contenuto generato dagli utenti (user generated content), in realtà non è del tutto inedito (basti pensare ai vecchi newsgroups o ai forum); l'elemento di novità tra i vecchi ambienti collaborativi e quelli tipicamente 2.0 è l'estrema facilità nella produzione di tali contenuti e nell'immissione di questi nel web.

Così da un web 'read only', dove l'atteggiamento dei fruitori del web era passivo e il flusso dell'informazione unidirezionale, si è passati ad un web 'read/write', che può essere in ogni momento riscritto grazie al contributo collaborativo degli utenti che lo popolano in un flusso 'molti a molti'.

dell'informazione per il web ha sottolineato in un articolo pubblicato sul suo blog (Cfr.: Peter Morville, Authority, «Semantic studios», blog post, 11 october 2005 , <http://semanticstudios.com/publications/semantics/000057.php>.Come accade in Wikipedia, anche nell'intera internet l'autorità non deriva da filtri o selezioni preventive di contenuti ma da un'attribuzione di valore che viene sancita dalla popolarità e dal consenso, concetto che si traduce, in applicazioni quali Google, nella reperibilità dell'informazione. Profondamente legato alla produzione di contenuti da parte degli utenti è il concetto di sfruttare il potere della folla, che deriva dal fortunato saggio di James Surowiecki Wisdom of the crouds cit.; è forse il concetto più innovativo ma soprattutto il più conosciuto del 2.0 ed è noto anche come sfruttare la saggezza collettiva. Ciò che è fondamentale è la produzione collaborativa di conoscenza. Legati a questo concetto sono le folksonomies o social tagging.

Un altro aspetto significativo del web è l'enorme quantità di dati che vengono raccolti e sfruttati dalla rete. $\mathrm{O}^{\prime}$ Reilly ammette che il core business delle aziende 2.0 è quello di raccogliere e incanalare queste grandi quantità di dati, raccolti monitorando le azioni degli utenti e sfruttandoli per migliorare e innovare i servizi di aziende come Google, Amazon o Ebay. L'altro aspetto legato ai dati è la possibilità di aggregare dati da fonti diverse per creare nuovi servizi web attraverso la pratica dei mash-up che è resa possibile attraverso l'uso delle API facilmente abbinabili, cioè programmi che vengono usati per fare comunicare delle applicazioni. Esse permettono di incorporare nei software 'pezzetti di codice' non direttamente manipolabili .

Strettamente legata alla possibilità di far viaggiare nella la rete i dati è la tecnologia dei RSS feed. Si tratta della syndication dei dati, una particolare applicazione pull: anzichè pensare a trattenere gli utenti, chi realizza contenuti fa in modo che questi possano essere fruiti non solo sul sito, ma anche attraverso canali diversi, senza la necessità di visitare il sito stesso.

Importanti sono i concetti di architettura della partecipazione e di effetto di rete.

Il primo concetto si riferisce alla possibilità di migliorare un servizio web attraverso l'uso del servizio stesso. Più il servizio viene usato più il software migliora. Quindi i servizi acquistano valore solo se vengono utilizzati dagli utenti. Allo scopo i servizi vengono progettati proprio per sfruttare le interazioni degli utenti aggiungendo quindi valore al servizio. Il secondo concetto, mutuato dall'economia è la grande importanza delle interconnessioni della rete e dell'uso nell'aumentare il valore di un servizio. Cosicchè molte applicazioni del web 2.0 sono progettate per godere dei benefici degli effetti di rete, e della possibilità quindi di beneficiare di aspetti comportamentali e di tendenze e di mode che la rete contribuisce a diffondere. Il concetto della lunga coda è uno di quelli che hanno avuto maggior risonanza anche tra i bibliotecari (Chris Anderson, La coda lunga cit.)

Un'altra caratteristica del web 2.0, riguardante il modo di creare applicazioni, è il 'sempre beta' cioè il prendere coscienza che nessun software è mai veramente completo finchè una comunità di utenti lo sta commentando. Esso è inoltre una strategia di progettazione. Molte grandi applicazioni vengono rilasciate in piccoli pezzettini in versione beta che vengono così commentate e manipolate separatamente da sviluppatori diversi e commentati da comunità diverse.

Infine, il concetto di apertura, rafforzatosi con il 2.0 è quello che porta a creare ambienti aperti e interoperabili, che si servono di standard non proprietari e che utilizzano software open source.

${ }^{202}$ Basta guardare la più recente Web trend map per accorgersi del successo delle aziende che hanno puntato sulle reti sociali $<$ http://informationarchitects.jp/wtm4/>. 
Nel variegato mondo del Web 2.0 fatto di reti sociali, di blog e wiki, dove si creano comunità professionali, di pratica, occasionali o dove (come nel caso della nota Wikipedia) si crea nuova conoscenza in modo collaborativo, si assiste così a una continua rielaborazione del web e dei suoi contenuti.

Anche il successo di piattaforme commerciali come Amazon dipende anche da vari fattori 'di tipo 2.0', tra i quali l'effetto prosumer e i noti 'effetti di rete'. Amazon non solo suggerisce, aggrega, mostra intere parti dei documenti, ma costruisce anche conversazioni intorno agli oggetti in vendita; gli utenti possono lasciare i propri commenti o dare il proprio voto, possono aprire thread cioè creare vere e proprie discussioni (come veri forum) intorno ad ogni oggetto presentato ${ }^{203}$. Ciò avviene anche nei numerosi siti di social cataloguing, dove si creano gruppi attorno all'interesse comune per i libri, per la fotografia, per la musica, si intrecciano e si svolgono conversazioni in cui tutti scrivono, tutti pubblicano, tutti partecipano.

Nel nuovo web anche le applicazioni, secondo uno dei principi del Web 2.0 ${ }^{204}$, sono perennemente in beta version, cioè in fase di sviluppo e modifica. Le applicazioni migliorano con l'uso; la fortuna o l'insuccesso di un'applicazione vengono sancite da un processo decisionale che si origina dal basso e deriva dal consenso implicito dei gruppi che la adoperano.

Negli ultimi anni del decennio scorso i contributi sulla tematica '2.0' sono notevolmente cresciuti, attirando l'attenzione da parte del mondo bibliotecario. Non c'è dubbio che grazie alle nuove tecnologie gli utenti hanno cambiato il loro approccio con la rete, trasformandosi da consumatori passivi di contenuti a produttori di informazione e l'informazione stessa è dinamica e può essere ricombinata e riutilizzata.

Nonostante i creatori si siano soffermati al principio sulle caratteristiche tecnologiche del fenomeno possiamo oggi affermare che la vera essenza del Web 2.0 è dunque costituita dai concetti di centralità dell'utente e di partecipazione: 'le persone sono il contenuto dei siti $^{205}$. Ciò significa una nuova concezione del web, dove i siti non sono fatti, come accadeva in passato, di informazioni messe a disposizione degli utenti, ma degli utenti stessi, che mettono a disposizione informazioni per il loro stesso consumo, per creare reti

\footnotetext{
${ }^{203}$ Un esempio di conversazione su un libro in Amazon: <http://www.amazon.com/Dewey-Small-TownLibrary-Touched-World/forum/Fx3KD3RILMA7DU9/-

/1/ref=cm_cd_dp_rtr_po?_encoding $=$ UTF8\&asin=0446407410 $>$.

${ }^{204}$ I principi del web 2.0 sono stati illustrati dall'inventore del meme: Tim O'Reilly, What is Web 2.0. Design patterns and business models for the next generation of software cit.

${ }^{205}$ «Here's my take on it: Web 2.0 is an attitude not a technology. It's about enabling and encouraging participation through open applications and services. By open I mean technically open with appropriate APIs but also, more importantly, socially open, with rights granted to use the content in new and exciting contexts.» (Ian Davis, Talis, Web 2.0 and all that cit.).
} 
di relazioni, dove l'utente è al centro della rete ${ }^{206}$. Oggi You Tube, Facebook e Wikipedia rappresentano gli esempi più popolari di questo nuovo modo di usare la rete: il primo, che accoglie 60 ore di filmato al minuto, costituisce un'immensa vetrina di filmati, la maggior parte amatoriali e una gigantesca comunità di pratica; Facebook è la più grande rete sociale al mondo con circa 845 milioni di identità digitali.

Le tematiche del Web 2.0 sono entrate presto a far parte del dibattito biblioteconomico. Si è originato così il movimento della 'Library 2.0"207 incentrato sulle tematiche della partecipazione e della conversazione che ha avuto per qualche tempo largo spazio nella letteratura professionale $\mathrm{e}^{208}$.

Sono nati in rete numerosi blog, liste di discussione e wiki dedicati al fenomeno, sono stati organizzati vari convegni sul tema, i bibliotecari sono stati incoraggiati a sondare le nuove possibilità offerte da queste tecnologie e a familiarizzare con questi nuovi tools .

Il movimento della Library 2.0 si fa portavoce della necessità per le biblioteche di andare incontro agli utenti, di valutare costantemente l'offerta di servizi e di orientarsi maggiormente al cambiamento; tematiche che tutto sommato non sono nuove nelle teorie

\footnotetext{
${ }^{206}$ Non a caso nel 2006, anno dell'esplosione delle reti sociali, il prestigioso settimanale americano TIME, noto per assegnare il titolo di personaggio dell'anno a personalità che si sono messe in luce per importanti iniziative che hanno coinvolto le persone a livello internazionale, scriveva che quell'anno in copertina non ci sarebbe stata l' immagine di un personaggio famoso ma solo uno schermo di un computer che rifletteva l'immagine di chi lo guardava, perché - titolava l'articolo- «Il personaggio dell' anno 2006 sei TU, utente di Internet»e proseguiva «Lev Grossman of Time Magazine says you are the Person of the Year for "Seizing the reins of the global media, for founding and framing the new digital democracy, for working for nothing and beating the pros at their own game, Time's Person of the Year for 2006 is you».

${ }^{207}$ Secondo quanto riferito da Wikipedia il termine sembra sia stato coniato e utilizzato per la prima volta da Michael Casey nel suo blog LibraryCrunch (<www.librarycrunch.com $>$ ) come espressione derivata dai termini Business 2.0 e Web 2.0. Ma anche in questo caso, come già avvenuto per il web 2.0 sul significato del termine non è stato raggiunto un unanime consenso. Walt Crawford in un suo contributo (Library 2.0 and “Library 2.0”, «Cities \& Insights», 6 (2006), n. 2, <http://citesandinsights.info/civ6i2.pdf $>$ )elenca 62 punti di vista e sette diverse definizioni di Library 2.0 a volte anche in contrasto tra loro. Secondo David Lankes l'uso del termine sta a indicare semplicemente il tentativo di applicare alcuni concetti del web 2.0 agli obiettivi della biblioteca. Una meme map dei concetti collegati alla library 2.0 fu elaborata in Italia da Bonaria Biancu. (Library 2.0 meme map - version 2.0. Immagine. <http://www.flickr.com/photos/bonaria/113222147/>).

Le definizioni fornite da Crawford, si possono confrontare con i principi chiave del movimento Library 2.0 inseriti nella relativa voce di Wikipedia: I principi elencati in Wikipedia sono:

1. Browser + Web 2.0 Applications + Connectivity $=$ Full-featured OPAC

2. Harness the library user in both design and implementation of services

3. Library users should be able to craft and modify library provided services

4. Harvest and integrate ideas and products from peripheral fields into library service models

5. Continue to examine and improve services and be willing to replace them at any time with newer and better services.

Leggendo le varie definizioni si potrebbe affermare che Library 2.0 si proponga quindi come un modo nuovo di fornire servizi bibliotecari sfruttando le nuove tecnologie di internet, ma con una particolare importanza per la partecipazione degli utenti e la loro interazione con i servizi bibliotecari.

${ }^{208}$ Non è possibile citare in questa sede i numerosissimi contributi su questa tematica, si rimanda duque all'ottima Bibliografia curata dalla Biblioteca Luigi Crocetti: Bibliografia di scritti di biblioteconomia, in italiano, sul web 2.0. A cura della Biblioteca Luigi Crocetti, <http://www.cultura.toscana.it/biblioteche/bsb/bibliografie/webduepuntozeroinitaliano-2009.shtml> aggiornata al 2009, un'altra bibliografia su biblioteca e web 2.0 curata da Paola Gargiulo e Maria Pia Colapenna e aggiornata al 2012 è presente in <http://www.uniciber.it/index.php?id=489>.
} 
biblioteconomiche, poiché da sempre le biblioteche sono orientate alla soddisfazione dell'utente, anche prima dell'avvento del 2.0. Già da vari anni in ambito bibliotecario si è avvertita la necessità di portare il catalogo incontro agli utenti, suggerendone una possibile evoluzione (tracciata da Ayres) nel catalogo ipertestuale o post-coordinato, nel quale l'utente sceglie i propri percorsi e mette in atto le proprie strategie di ricerca. D'altro canto, l'emergere di nuovi modelli concettuali come FRBR e di nuovi principi e regole di catalogazione, testimoniano la volontà di mettere al centro del rinnovamento l'utente. Ciò che risultava nuovo era dunque il 'ruolo attivo' dell'utente nei servizi bibliotecari e, di riflesso, negli OPAC.

Chad e Miller ricondussero a tre i principi della Library 2.0:

* La biblioteca è ovunque

* La biblioteca non ha barriere

* La biblioteca invita alla partecipazione ${ }^{209}$

Dal movimento della Library 2.0 scaturì una letteratura ricchissima di contributi che si ispiravano al nuovo fenomeno per chiedersi come i cataloghi potessero beneficiare delle nuove tecnologie e accogliere gli stimoli provenienti da tali teorie. ${ }^{210}$

È proprio a partire dall'idea di partecipazione e di conversazione che Lankes, Silverstein e Nicholson, in uno dei contributi a mio avviso più originali dell'intero dibattito, nel documento Participatory networks: The library as conversation lanciarono l'idea di una Biblioteconomia partecipativa, vista come paradigma per progettare un futuro sostenibile per le biblioteche e i loro OPAC ${ }^{211}$. Questi ultimi non dovrebbero essere un mero contenitore di surrogati di informazione digitale che viene gestito separatamente dai documenti stessi - sostengono gli autori, ma un vero e proprio catalogo potenziato dovrà abbattere la barriera artificiale tra catalogo (inteso come sistema di controllo inventariale) e

\footnotetext{
${ }^{209}$ Ken Chad - Paul Miller, Do libraries matter? The rise of Library 2.0. A white paper cit.

210 Nella Library 2.0, movimento che ha trovato un'ampia partecipazione nell'ambito della cosiddetta 'blogosfera', una particolare attenzione venne rivolta proprio al catalogo, e proprio da un blog proviene il Catalog manifesto elaborato da Christopher Harris che allo scopo di «gettare una nuova luce sul rapporto tra bibliotecari, utenti, e cataloghi» recitava al primo punto, rivolgendosi ai bibliotecari: «Il catalogo della biblioteca non è nostro» intendendo il nuovo ruolo attivo e di partecipazione degli utenti. (Christopher G. Harris, Catalog Manifesto, «Infomancy», 14.6.2007, [Blog post], <http://schoolof.info/infomancy/?p=388>. La possibilità di creare cataloghi più partecipati e collaborativi cominciò a farsi strada e si originò il concetto il concetto di OPAC 2.0: secondo gli esponenti di Library 2.0 l'OPAC funziona meglio quanti più utenti sono coinvolti nel processo di interazione con il catalogo immettendo e condividendo con gli altri utenti i contenuti e favorendo la partecipazione. Immaginando il catalogo nei termini di una 'conversazione' ci si rendeva conto di come essa fosse posta in termini molto formali unidirezionali: ciò che mancava ai cataloghi era proprio l'interazione e l'incontro con gli utenti e, in definitiva, quella socialità che si riscontrava nella nuova tendenza del web.

211 R. David Lankes - Joanne Silverstein - Scott Nicholson, Participatory networks: the library as conversation, «Information Research», 12 (2007), n. 4, <http://informationr.net/ir/12-4/colis/colis05.html>. La traduzione italiana del documento è disponibile all'url: <http://www.aib.it/aib/cg/gbdigd07.htm3> .
} 
contenuti (cioè i contenuti digitali che costituiscono il 'deposito di comunità'). Lankes sosteneva, inoltre, che dal momento che le biblioteche sono nel business della conversazione, il ruolo dei cataloghi debba passare dalla descrizione dei manufatti al collegamento delle conversazioni che si svolgono attorno agli oggetti rappresentati. Secondo lo studioso le conversazioni avrebbero dovuto influenzare le procedure di ranking degli OPAC, restituendo quelle più interessanti. ${ }^{212}$

Altro punto caldo del dibattito biblioteconomico fu l'applicazione delle folksonomies, cioè di una semantica di tipo 2.0 al catalogo, che generò un confronto ampio e qualificato $^{213}$.

Possiamo ormai parlare pacificamente di fallimento della 'semantica 2.0' nei cataloghi elettronici: è palese che se le classificazioni tradizionali presentano evidenti limiti (quali la difficoltà di applicazione a differenti tipi di risorse o la lentezza nel recepire il cambiamento), le folksonomies, veloci e facili da applicare, generano però metodi di recupero dell'informazione incoerenti e poco affidabili. Ciò è dovuto, oltre che all'intrinseca debolezza di questi strumenti, al fatto che gli utenti non comprendendone la funzione sociale ne fanno spesso un uso per fini personali e in contesti informali.

L'ipotesi di affiancare agli accessi semantici controllati, basati sull'approccio tradizionale della codificazione, questa nuova modalità piatta di organizzazione delle conoscenze capace di rappresentare le nicchie ${ }^{214}$ e bottom-up (che emerge da un consenso dal basso degli utenti) necessita così di una lettura diversa: non si tratta quindi, com'è ovvio, di cedere a una nuova citizen librarianship ${ }^{215}$ e delegare funzioni di organizzazione della conoscenza agli utenti, né di creare un improbabile catalogo 'folksonomico', ma di affiancare a strumenti più esperti di recupero dell'informazione nuovi canali, allo scopo di apportare alla ricerca, come avviene già per il browsing, una certa dose di serendipità e multidimensionalità di approccio alla ricerca dell'informazione.

212 David Lankes, Fighting for the soul of libraries, June 12th, 2009. $<$ http://quartz.syr.edu/rdlankes/Presentations/2009/NYSLAA.pdf>.

${ }^{213}$ Una rassegna degli studi sulle folksonomies, in cui vengono citati più di 180 contributi, si trova in Jennifer Trant, Studying social tagging and folksonomy: a review and framework, «Journal of Digital Information» 10 (2009) n.1. oppure <http://dlist.sir.arizona.edu/2595/>; si veda anche: Tom Steele, The new cooperative cataloging, «Library Hi Tech», 27 (2009), n.1, p. 68-77. Nel nostro paese sono da menzionare oltre al già citato intervento di Nicola Benvenuti anche quelli di Quintarelli e Santoro (Emanuele Quintarelli, Folksonomies: power to the people. [Relazione presentata al convegno ISKO Italia - UniMIB, Milano, 24 giugno 2005, <http.://www.iskoi.org/doc/folksonomies>; Michele Santoro, Questa sera si cataloga a soggetto. Breve analisi delle folksonomies in prospettiva bibliotecaria, «Bibliotime», X (2007), n. 2, $<$ http://www2.spbo.unibo.it/bibliotime/num-x-2/santoro.htm>).

${ }^{214}$ Sul rapporto tra folksonomie e lunga coda si veda: Rossana Morriello, La coda lunga e le biblioteche, «Bollettino AIB », 49 (2009) 1, p. 69-82.

${ }^{215}$ Il richiamo è al citizen journalism il giornalismo collaborativo dei blog, accusato da più parti di essere la causa del declino dei quotidiani. 
Sono state molte le iniziative nella direzione della creazione di nuova generazione con caratteristiche sociali, sia in ambito internazionale che nel nostro paese. Alcuni produttori hanno lanciato software per OPAC o specifici moduli che integrano gli strumenti tipici dei social software. Tra questi si segnalano AFI-OPAC, Scriblio ${ }^{216}$, VuFind, Encore, Primo e Aquabrowser MyDiscoveries ${ }^{217}$. In Italia invece meritano una menzione l'OPAC del Sistema bibliotecario di Verona, primo tentativo di social OPAC italiano $^{218}$ basato sul software Discovery NG di Comperio e quello della Biblioteca provinciale di Foggia, basato sull'implementazione di Sebina You ${ }^{219}$.

Alcuni OPAC importano, attraverso la pratica del mashup, le recensioni degli utenti di Amazon e Librarything. Più sensato - a mio parere - che ad arricchire il catalogo sia il bacino degli utenti registrati, piuttosto che una community generica di utenti di un social network o di una libreria online. In definitiva l'eventualità di accogliere i contenuti generati dagli utenti, introducendo arricchimenti di tipo 'sociale' (voti, tag, commenti) al catalogo, richiede che ogni singola biblioteca valuti il 'valore biblioteconomico' complessivo di tali arricchimenti in termini qualitativi e di soddisfazione dell'utenza. L'invito a sperimentare che venne proposto dai teorici della Library 2.0 oggi va accolto in maniera cauta e nella misura in cui tali aggiunte possano essere di effettivo aiuto nel fornire all'utenza servizi realmente personalizzati.

È condivisibile invece la necessità di progettare OPAC presenti e ricercabili sul web o di aggiungervi funzioni che consentano di comunicare con le applicazioni più diffuse della rete. Il concetto, espresso già da Lankes, Silverstein e Nicholson, è quello di «essere dove la conversazione ha luogo». Il maggiore sforzo nel dialogare con i propri utenti e nell'essere maggiormente presenti nei luoghi da loro frequentati e conosciuti si traduce così nella nozione di una biblioteca 'aperta' e 'pervasiva',220, dotata di un catalogo perfettamente integrato con il resto del web ${ }^{221}$.

\footnotetext{
$216<$ http://about.scriblio.net/about/>. L'OPAC è basato sulla piattaforma per blogging Wordpress. Una realizzazione è visibile alla Plymouth State University. Si riporta a titolo esemplificativo un record per dare l'idea dell'abbondanza dei dati di arricchimento e delle funzionalità aggiuntive di tipo 2.0: $<$ http://library.plymouth.edu/read/338504\#bsuite_share_bookmark>. Si confronti lo stesso libro in Amazon $<$ http://www.amazon.com/Dewey-Small-Town-Library-TouchedWorld/dp/0446407410/ref=sr_1_1?ie=UTF8\&s=books\&qid=1245704724\&sr=8-1\#capbody $>$.

217 Per una panoramica più approfondita di questi strumenti si veda anche: Andrea Marchitelli - Tessa Piazzini, OPAC, SOPAC e Social networking cit.

${ }^{218}$ L'OPAC è consultabile all'url: <http://sbp.provincia.verona.it/> ed è stato prodotto da E-portaltech, oggi Comperio (<http://www.comperio.it/sscms/>). Cfr. infra, Appendice A. Casi di studio. Sistema bibliotecario della Provincia di Verona.

${ }^{219}$ Cfr. infra, Appendice A. Casi di studio. Sistema bibliotecario provinciale di Foggia.

${ }^{220}$ Ken Chad - Paul Miller, Do libraries matter? cit.

221 In proposito Coyle fa notare come non sia tanto l'avvento del documento digitale ad orientare l'evoluzione del catalogo, quanto invece la spinta verso una profonda integrazione con il Web: «Some even
} 
Sono state sperimentate varie soluzioni per 'aprire' i cataloghi e renderli maggiormente visibili nella rete reindirizzando contenuti da e verso il web.

Vengono sfruttate forme di syndication dei dati che consentono di far "viaggiare nel web' i dati dei cataloghi tramite i feed RSS (es. dando la possibilità agli utenti di impostare alert che ripetono nell'OPAC le ricerche preferite, di abbonarsi a bollettini delle novità o a liste tematiche proposte dai bibliotecari) ${ }^{222}$.

Si favorisce il riuso dei dati bibliografici. L'OPAC Worldcat permette, ad esempio, agli utenti di creare bibliografie pubbliche e private, di esportare i dati del catalogo in altri software (EndNote, Refworks) e nei più noti social network, di linkare ad una scheda di catalogo da qualsiasi punto della rete tramite il permalink. OCLC, per un certo periodo e in via sperimentale, ha reso il contenuto di WorldCat interrogabile tramite i motori di ricerca $^{223}$.

Si cerca di reindirizzare gli utenti dalla rete verso il catalogo. Vari cataloghi sono accessibili da Facebook nelle pagine delle biblioteche che si rendono visibili tramite il noto social network. Alcune biblioteche progettano widgets e componenti aggiuntivi per i browser che permettono, da qualsiasi punto del web, di convogliare gli utenti verso il catalogo.

Sono ormai diffuse, infine, soluzioni per adattare gli OPAC alla consultazione su telefoni cellulari e altri dispositivi mobili.

Tuttavia oggi il concetto di 'essere nella rete' si carica di nuovi significati, che vanno ben oltre la socialità e attengono al concetto di Web Semantico e dell'apertura dei dati sotto forma di linked data.

La necessità non è più quella di essere nel web, ma di essere parte del web dei dati, cioè del Web Semantico. Attraverso questo suo 'essere del web' dal catalogo possono essere sviluppate applicazioni che consentano un migliore e più diffuso utilizzo dei dati delle biblioteche e l'integrazione con la rete.

predicted the eventual dissolution of the physical library, with all documents and all services being digital. But none of them envisioned the rise of a vibrant information environment entirely outside of libraries: the World Wide Web. Our users have shifted their attention from the library to other sources of information. The question today is not how do we get users into the library, but how can we take the library to the users. The answer will necessarily involve a transformation of the library catalog» Karen Coyle, The library catalog in a 2.0 world, «Journal of Academic Librarianship», 33 (2007), n. 2, p. 289-291, anche in <http://www.kcoyle.net/jal_33_2.html>.

${ }^{222}$ Cfr.: Antonella De Robbio, Servizi bibliotecari personalizzati basati su rss feeds : $i$ diversi volti di un nuovo canale di comunicazione. In La Biblioteca su misura cit., p. 184-206. Si veda anche: Gino Roncaglia, Blogosfera e feed rss : una palestra per il semantic web?, «Networks», 2003, n. 2, $<$ http://www.merzweb.com/testi/saggi/blogosfera.pdf $>$ oppure $<$ http://eprints.rclis.org/5742/>.

${ }^{223}$ In questa direzione si muove anche il progetto eXtensible Catalog (XC) $<$ http://www.extensiblecatalog.org/> analizzato in appendice al presente capitolo. 
Possiamo in definitiva chiederci quanto le caratteristiche sociali siano oggi effettivamente presenti nei cataloghi bibliotecari.

A distanza ormai di circa un quinquennio dalle prime esperienze di social OPAC possiamo affermare che gli OPAC hanno adottato la maggior parte delle tecnologie che sono ricomprese sotto l'etichetta di 'Web 2.0' che lentamente hanno pervaso tutti gli strumenti di ricerca del web, si è ben lontani dall'aver recepito nei cataloghi la 'filosofia della partecipazione': la capacità di inglobare contenuti generati dagli utenti, elemento che rappresenta la vera essenza del Web 2.0, non ha mai avuto grande successo nei cataloghi elettronici.

Se le caratteristiche 'sociali' sono citate tra le caratteristiche distintive di quelli che definiamo 'next generation catalogs', in realtà le indagini ci mostrano che gli utenti non le cercano, né gli OPAC delle biblioteche li implementano.

Ciò è testimoniato da alcuni recenti studi; l'indagine comparativa di Yang e Hoffmann mostra che tra gli arricchimenti, quelli 'sociali' sono i meno diffusi e implementati:

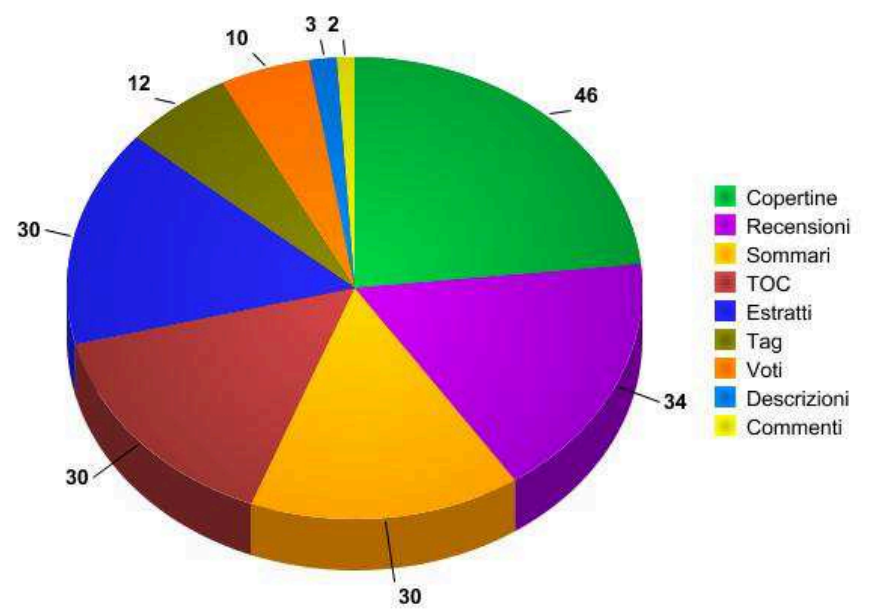

Fig. 2.10 - Arricchimenti negli OPAC di nuova generazione: i meno diffusi sono quelli 'sociali, ${ }^{224}$

Tra gli arricchimenti 'sociali' gli OPAC che implementano $\operatorname{tag}^{225}$ sono una bassissima percentuale (che si attesta all'11\%) mentre le altre caratteristiche (voti, recensioni) scendono sotto il $7 \%$ e vengono implementate da prodotti commerciali come Encore, Aquabrowser, Vu Find.

\footnotetext{
${ }^{224}$ Rielaborazione grafica dei dati forniti da Sharon Q. Yang - Melissa A. Hofmann, Next generation or current generation?: A study of the OPACs of 260 academic libraries in the USA and Canada, «Library Hi Tech», 29 (2011), n. 2, p. 266 - 300, La tabella dei dati rielaborati è a p. 280.

${ }^{225}$ Tag 11\%, Recensioni 7\%; Voti 4; Commenti 1\%; Descrizioni 0; Annotazioni 0.
} 


\subsubsection{I sistemi e le tecnologie}

\subsubsection{OPAC e ILS: la modularità come modello per la 'recombinant library'}

Una delle principali novità dei NGC e dei DS è squisitamente tecnologica e comporta la messa in discussione della architettura tradizionale dei sistemi di automazione per le biblioteche. Integrated Library System o ILS è il termine tradizionalmente usato per indicare i sistemi integrati adoperati dalle biblioteche. Il grande vantaggio degli ILS è stato per molto tempo quello di consentire la condivisione degli archivi e il colloquio dei vari moduli che gestiscono i principali servizi bibliotecari (acquisti, prestito, circolazione, catalogazione ecc...) in modo da evitare ridondanze e ripetizioni di dati. Ciò ha consentito per molti anni una notevole riduzione dei costi e un miglioramento complessivo della qualità dei cataloghi. L'evoluzione degli ILS ha visto questi sistemi incentrarsi in principio sullo snellimento delle procedure interne alla biblioteca, per poi concentrarsi sulla valorizzazione delle risorse bibliotecarie mediante strumenti di consultazione e di accesso ai documenti quali il modulo OPAC, interlibrary loan (ILL), document delivery (DD) e la condivisione delle risorse elettroniche locali.

Gli ILS hanno sviluppato alcune funzionalità di servizio all'utenza e di interazione: poter accedere da un'unica interfaccia a funzioni di ricerca e gestionali ha enormi vantaggi, tra i quali, ad esempio, la possibilità di cercare nell' OPAC e di richiedere contestualmente un prestito, di ottenere informazioni in tempo reale sullo stato di disponibilità di un documento e sulla propria situazione utente.

Da circa 20 anni il maggiore sforzo di chi progetta gli ILS è rivolto ad integrare le risorse esterne e a potenziare l'accesso al patrimonio documentario delle biblioteche. L'integrazione delle risorse esterne, che come abbiamo visto è particolarmente problematica, è strettamente connessa con le questioni dell'interoperabilità tra sistemi eterogenei e delle iniziative nel campo dello sviluppo e della standardizzazione dei metadati e dei protocolli di scambio. Secondo alcuni studiosi, alla fine degli anni '90 lo sviluppo degli ILS, considerati prodotti ormai maturi, avrebbe subito un rallentamento poiché le società produttrici cominciarono a dedicarsi ad altri prodotti a beneficio del web e del digitale. 
È stata soprattutto l'accelerazione delle tecnologie a frenare un pacifico e pianificato sviluppo di questi sistemi; essa avrebbe infatti imposto agli ILS un adeguamento continuo consistente in migrazioni di dati, cambiamento dei sistemi operativi, sviluppo dell'architettura client-server, riorganizzazione delle procedure operative in conseguenza dello sviluppo delle applicazioni del web. I gestionali hanno iniziato ad aggregare prodotti diversi, che svolgono specifiche procedure ${ }^{226}$.

Si è determinato così quel fenomeno di trasformazione degli ILS (Integrated library systems) in Dis-integrated library sistems, cioè la scomposizione di questi sistemi in una serie di componenti separate. I gestionali si sono orientati quindi verso un'architettura modulare di progettazione del software. A partire dal 2006, non esistendo un sistema integrato in grado di far fronte alle necessità di tutte le biblioteche nella gestione delle risorse elettroniche, gli ILS tradizionali hanno cominciato a gestire una parte sempre minore delle operazioni che avvengono in biblioteca:

The integrated library system? The integrated library system (ILS) remains a central library investment, although one wonders how long this will continue in its current form. The financial and operational burden of periodically transitioning such a monolithic system seems too high to sustain and is one incentive for disintegration of the ILS into more manageable components. Another is that the library is acquiring additional systems: the ILS is managing a progressively smaller part of the overall library operation ${ }^{227}$.

Come abbiamo visto, l'ILS (con la sua interfaccia OPAC) sempre più spesso si ritrova in questo contesto affiancato dal portale ${ }^{228}$ che raggruppa tutte le altre risorse che costituiscono contenitori diversi e si collega con altri applicativi che consentono l'interrogazione di svariate risorse:

The case of the catalogue is especially interesting, where there is a growing desire to provide a discovery experience in another environment (a standalone catalogue application with more features, from other databases, from Amazon, from reading lists or social bookmarking systems, and so on) [...] there is a need to connect the discovery experience back to the library system for further action [...] All these factors point to a reduced interest in the ILS per se, as it becomes more of a back-office application. And the library vendors are certainly competing around other components, which include a resolver, a metasearch application, an electronic resource management system, maybe an institutional repository, and other digital asset management systems. The existing library system is very much a silo application with

\footnotetext{
${ }^{226}$ Tra queste Weston elenca: architettura di rete e protocollo TCP-IP; adozione di un database relazionale di tipo commerciale (RDBMS), architettura object-oriented; OPAC consultabile sul web; interfaccia grafica per tutti i moduli; conformità allo standard Z39.50; adozione del protocollo ILL, compatibilità con lo stndard EDI; (Cfr. Paul Gabriele Weston, La gestione elettronica delle biblioteche cit.) ; nei sistemi più evoluti sono da citare anche lo standard UNICODE, le API (Application programming interfaces) e i nuovi moduli OAIPMH.

${ }^{227}$ Lorcan Dempsey, The (Digital) Library Environment: Ten Years After cit.

${ }^{228}$ Come avviene ad esempio nell'opac della NCSU, (cfr. infra Appendice A. Casi di studio. North Carolina State University Libraries).
} 
limited data flow and interconnection. It sits as an inhospitable peak in the flat Web environment ${ }^{229}$.

Gli enormi progressi tecnologici ormai raggiunti hanno consentito di progettare OPAC 'sganciati' dai tradizionali ILS per poter meglio integrare le risorse e servizi offerti in rete:

The growth of modern technology and high speed networks now mean that it is possible to separate the OPAC from the bibliographic database used by the library to manage its holdings. The capacity to extract, move, and import large amounts of data in modern computer systems is well developed; and this had been coupled with the capacity of new search-engine type applications to efficiently index large volumes of data to provide powerful real time searching and results handling. In this new context, an OPAC need not necessarily be part of the ILMS, and as a consequence, need not be limited by traditional ILMS based OPAC functionality ${ }^{230}$.

Il concetto-chiave nelle tecnologie degli OPAC di nuova generazione e dei sistemi di discovery è quello di decouplement cioè di separazione dei software di gestione (ILS) dai software per la ricerca. Questo processo è stato anche chiamato dis-integrazione degli ILS, o sviluppo modulare del software ${ }^{231}$.

La nuova possibilità di estrarre dati dai sistemi di gestione di una biblioteca, spesso basati su tecnologie proprietarie, e farli elaborare da un software esterno per restituirli arricchiti, migliorati, integrati con altri dati, in nuove interfacce più in linea con i gusti e le esigenze degli utenti finali, ha rappresentato una vera e propria tecnologia dirompente nel mercato dei software per biblioteche ${ }^{232}$.

Un'impostazione di questo tipo consente di introdurre per mezzo dell'OPAC anche miglioramenti rivolti allo sviluppo di nuovi servizi per gli utenti, come la possibilità di accedere all'OPAC e ai servizi bibliotecari tramite il cellulare o il palmare, la segnalazione delle novità tramite feed $^{233}$ e altro sia di introdurre arricchimenti - sostiene Sokvitne - che consentono di costruire un sistema maggiormente tagliato su misura delle specifiche esigenze degli utenti. Tra queste l'arricchimento dell'OPAC con informazioni aggiuntive utili all'utente per scegliere in modo più preciso i documenti di proprio interesse che si dimostrava carente nei record bibliografici gestiti entro gli ILS tradizionali.

\footnotetext{
${ }^{229}$ Ivi, s. p.

${ }^{230}$ Lloyd Sokvitne, Redesigning the OPAC: moving outside of the ILMS, «Australian Academic \& Research

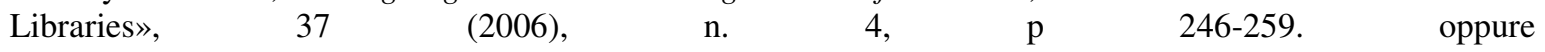
<http://www.nla.gov.au/lis/stndrds/grps/acoc/documents/Sokvitne.doc>.

${ }^{231}$ Sul conseguente ridimensionamento del ruolo degli ILS si veda: Art Rhyno, The end of ILS, «Inside OLITA», 5 (2001).

${ }^{232}$ Il termine disruptive innovation o disruptive technology, coniato nel 1995 da C. Christensen è usato in letteratura e nella tecnologia di business per descrivere le innovazioni che consentono di migliorare un prodotto o servizio in modo che il mercato non si aspetta (cfr. la relativa voce di Wikipedia $<$ http://en.wikipedia.org/wiki/Disruptive_innovation>).

${ }^{233}$ Antonella De Robbio, Servizi bibliotecari personalizzati basati su rss feeds cit.
} 
Utilizzando la tecnica del mashup e le API diventa così possibile collegarsi ad altri servizi web per importare dati da altre applicazioni web (ad esempio dalle librerie online come Amazon) e in tempo reale. Il fenomeno della 'disintegrazione degli ILS' e della loro ricomposizione in elementi modulari è profondamente legata anche all'uso di software non proprietari o open source (OSS) ${ }^{234}$.

I produttori di sistemi di automazione, infatti, già da qualche anno si orientano verso questa soluzione, aprendo così questi sistemi alla possibilità di essere compatibili e integrabili con altre applicazioni e quindi, in definitiva, maggiormente interoperabili. L'obiettivo è anche quello di rendere maggiormente integrabili i formati usati per la descrizione bibliografica con la piattaforma web; in questo senso i formati MARC rendono difficile l'integrazione dei dati bibliografici nelle interfacce web. La migrazione verso $\mathrm{XML}^{235}$ e l'uscita in formati basati su XML consentono esportare dati 'compatibili con il web; un ruolo centrale è svolto dal protocollo OAI $-\mathrm{PMH}^{236}$.

La nuova architettura modulare consente anche di integrare l'OPAC con altri software che, come abbiamo visto, gestiscono l'accesso agli articoli delle riviste (link resolver) e recuperano risorse esterne.

I produttori hanno incrementato lo sviluppo di queste nuove interfacce "sganciate" in grado di operare con un gran numero di software di gestione proprietari e di restituire i dati con modalità di visualizzazioni e funzioni più innovative $\mathrm{e}^{237}$.

C'è stata una grande varietà di "soluzioni interpretative" che hanno portato allo sviluppo di una gran quantità di prodotti molto diversi tra loro, in relazione sia alle necessità delle biblioteche che li hanno predisposti, sia alle tecnologie usate (aperte o proprietarie), ai costi per svilupparli e non ultimo alle visioni dei progettisti stessi. La sperimentazione, almeno in un primo periodo, è stata molto forte e non sempre le soluzioni sperimentate hanno aumentato la qualità dell'informazione recuperata.

Da queste preoccupazioni è nata l'iniziativa della Digital library Federation (DLF) che nel 2008 ha condotto all'accordo di Berkeley. L'accordo è stato promosso per garantire

\footnotetext{
${ }^{234}$ Su questo argomento uno dei primi contributi fu quello di Robi Sturman, Il software open source per la gestione integrata delle biblioteche: una nuova risorsa?, «Bollettino AIB», 44 (2004), n. 3 p. 257-270.

${ }^{235} \mathrm{La}$ migrazione verso altri formati è già iniziata alla Library of Congress che detiene lo sviluppo del MARC, (vedi Deanna Marcum, A bibliographic framework for the digital age, October 31, 2011 $<$ http://www.loc.gov/marc/transition/pdf/bibframework-10312011.pdf>) Yee raccomandava già da tempo di migrare verso linguaggi XML e di recente sono state studiate nuove soluzioni, consistenti in linguaggi intermedi o crosswalks, che permettono di fare dialogare più schemi di metadati e linguaggi diversi (Cfr.: Martha M. Yee, Beyond the Opac: future directions for Web-based catalogues, cit.

${ }^{236}$ Marshall Breeding, Next-Gen Library Catalogs cit., p. 35 e p. 72-73.

${ }^{237}$ Nell'esame dei software per biblioteche rimane un punto importantissimo di riferimento il sito web Library technology guide <http://www.librarytechnology.org/> curato dallo studioso americano Marshall Breeding.
} 
l'interoperabilità tra ILS e nuovi strumenti di discovery. I nuovi trend di sviluppo impongono che gli ILS diventino piattaforme in grado di supportare gli strumenti per la scoperta che si basano sui loro dati ${ }^{238}$. Scopo dell'accordo è produrre API per l'accesso ai dati dell'ILS maggiormente standardizzate e condivisibili.

Il documento ha individuato tre principali funzioni che vanno supportate per garantire l'interoperabilità a livello di tecnologie adottando protocolli aperti e standard condivisi:

a. Harvesting: cioè le funzioni che consentono la raccolta dei dati delle collezioni librarie sia per intero, sia dei record aggiornati. Le opzioni di harvesting devono includere sia il record stesso sia i dati che vengono completati da altre informazioni supplementari come le localizzazioni e i dati sulla circolazione). Entrambe le operazioni devono essere supportate tramite un'interfaccia OAI-PMH;

b. Real-time Availability: cioè le informazioni sullo stato di disponibilità devono essere disponibili in tempo reale tramite l'adozione di una modalità standard attraverso un'API dedicata. Questa funzionalitò dovrà essere implementata tramite un'interfaccia REST specificata dal ILS-DI task group;

c. Linking: cioè la capacità del sistema di produrre URL stabili che rendono possibile il deep linking ai singoli records. Ad esempio un link stabile alla pagina che contiene il record e un link che consenta di ottenere informazioni su quell'item. Questa funzionalità viene implementata tramite un modello di URL che viene specificato dal ILS-DI task group.

Il testo dell'accordo contiene in appendice l'elenco dei 10 più grandi fornitori stranieri di gestionali per biblioteche che hanno sottoscritto il documento ${ }^{239}$.

Purtroppo nessuno dei produttori italiani di software per biblioteche ha sottoscritto l'accordo, così le biblioteche italiane si trovano penalizzate nella scelta di software di

\footnotetext{
238 «These trends imply that the ILS needs to become a platform that supports appropriate interfaces for discovery applications living on top of it instead of trying to do everything on its own [...]At present, a number of ILS vendors provide proprietary methods for accessing their underlying data stores. These may consist of command-line API tools accessible only for a special fee to trained users, or direct SQL queries against the ILS's database tables. While these methods provide much needed hooks into the ILS, making library resources more widely usable requires a larger, standards-based API. To enable outward integration, organizations will require that ILS's adopt a more standardized method for providing API access to the data store, moving away from traditional library-centric protocols like Z39.50 toward an XML-based web services API model. Such a model will enable developers outside of the library community to more easily access the information stored within the ILS, creating opportunities for greater integration with non-library applications like course management tools», Ivi p. 4.

${ }^{239}$ Digital Library Federation. ILS Discovery Interface Task Group (ILS-DI). Technical Recommendation. An API for effective interoperation between integrated library systems and external discovery applications. Revision 1.1. December 8, 2008, <http://www.diglib.org/architectures/ilsdi/DLF_ILS_Discovery_1.1.pdf>. Hanno sottoscritto il Berkeley Accord il April 4, 2008: Talis, Ex Libris, LibLime, BiblioCommons, SirsiDynix, Polaris Library Systems, VTLS, California Digital Library, OCLC, AquaBrowser.
} 
nuova generazione applicabili ai loro ILS o rischiano di non poter adottare alcuni sofware per next generation catalogs o i nuovi strumenti di discovery.

\subsubsection{L'implementazione dei discovery systems. Lavorare con i metadati}

La parte più complessa della preparazione e implementazione di uno strumento di discovery riguarda un intenso lavoro sui metadati: il contesto dei metadati è profondamente cambiato e si trova tuttora in un momento di forte transizione. A partire dal 1998 con l'elaborazione di FRBR le biblioteche si sono dotate di un nuovo modello concettuale per la rappresentazione delle entità che compongono l'universo bibliografico; nel 2009 sono stati elaborati i nuovi Principi internazionali di catalogazione (ICP) e nel 2010 nuovi modelli di dati come RDA e tutto ciò è stato influenzato dal dibattito sui modelli di gestione dell'informazione bibliografica e sui bisogni informativi dell'utente in un contesto profondamente influenzato dal web.

Le biblioteche si sono dotate dunque di nuovi standard che prescrivono quale debba essere il contenuto dei dati bibliografici.

Allo stesso tempo il panorama dei metadati è profondamente cambiato: le biblioteche non hanno più a che fare con i soli metadati bibliografici, ma parlare di metadati significa entrare in relazione con un ecosistema costituito da schemi, strutture, modelli, protocolli etc. Il bibliotecario deve dunque acquisire una serie di conoscenze sulle tipologie di metadati che consentiranno loro di sviluppare nuovi servizi ${ }^{240}$.

Gli OPAC operano un contesto sfaccettato in cui le dimensioni sono costituite da vari elementi che adottano standard specifici per i formati dei dati, per le funzioni dei metadati (descrittivi, organizzativi etc.), e le tecnologie (protocolli, codifiche etc).

\footnotetext{
${ }^{240}$ Si veda: Steven J. Miller, Metadata for digital collections. A how-to-do-it manual, New York, NealSchuman Publishers, 2011, Sito web con esempi: <http://www.neal-schuman.com/metadata-digitalcollections $>$. Sulla tematica della compatibilità tra metadati di diversa provenienza si veda anche Zorana ERCEGOVAC, Toward a global access to bibliographic information: converging patterns, new paradigms: [presented as a keynote presentation at the COBISS/SICRIS User Conference in Maribor, Slovenia, 29 November 2000], [risorsa on line], [Los Angeles]: [University of California], [s.d.], $<$ http://www.cs.ucla.edu/Leap/zer/maribor.htm>. Disponibile anche in lingua slovena in «Obvestila», 5 (2000), n. 4, p. 4-28.
} 


\begin{tabular}{|l|l|l|}
\hline Standard catalografici & $\begin{array}{l}\text { Definiscono il contenuto dei } \\
\text { metadati }\end{array}$ & $\begin{array}{l}\text { RDA, FRBR, FRAD, FRSAD, } \\
\text { REICAT etc. }\end{array}$ \\
\hline \hline Schemi di metadati & $\begin{array}{l}\text { Definiscono la struttura dei } \\
\text { metadati in una registrazione } \\
\text { catalografica o "record" }\end{array}$ & $\begin{array}{l}\text { UNIMARC element sets } \\
\text { come Dublin Core, MODS, } \\
\text { CDWA, etc. }\end{array}$ \\
\hline \hline Codifiche di metadati & $\begin{array}{l}\text { Definiscono le modalità in cui } \\
\text { devono essere registrati i } \\
\text { metadati per essere elaborati }\end{array}$ & ISO2709, XML, etc. \\
\hline \hline Protocolli di comunicazione & $\begin{array}{l}\text { Definiscono le modalità di } \\
\text { registrazione dei dati ai fini } \\
\text { della loro trasmissione e } \\
\text { utilizzo }\end{array}$ & $\begin{array}{l}\text { FTP, OAI-PMH, OpenURL, } \\
\text { etc. }\end{array}$ \\
\hline
\end{tabular}

Fig. 2.11 - Standard catalografici

Ciò comporta il dover trattare vari tipi di standard che non sono strettamente bibliotecari

* standard per il contenuto dei dati (regole catalografiche, guide, come RDA, REICAT;

* standard per la struttura dei dati o element sets come MARC Dublin Core, MODS, CDWA, RDA elements, EAD elements etc.);

* standard per i valori dei dati come LCSH, e thesauri come TNG e AAT;

* standard o formati per lo scambio dei dati (MARC XML, ISO2709, EAD XML etc);

* standard per la visualizzazione dei dati (es. negli OPAC gli ISBD, CSS, etc. ).

Si tratta di standard concettuali (modelli e norme) e tecnologici (codifiche, protocolli). Gli standard concettuali cui si rapportano gli strumenti per la ricerca nascono nel mondo delle biblioteche, mentre gli altri provengono da altri ambiti, tuttavia la dimensione concettuale e quella operativa o tecnologica sono strettamente collegate ${ }^{241}$.

\footnotetext{
241 Ad esempio l'uso di OAI-PMH richiede un'esportazione dei dati da SBN in UNIMARCXML, trasformando la rete dei record collegati in un record piatto. Alcuni record collegati come ad esempio i titoli per i collegamenti di tipo a $\mathrm{A}$ e $\mathrm{B}$ vengono perduti così bisogna adattare lo schema risultante per accogliere queste informazioni.
} 


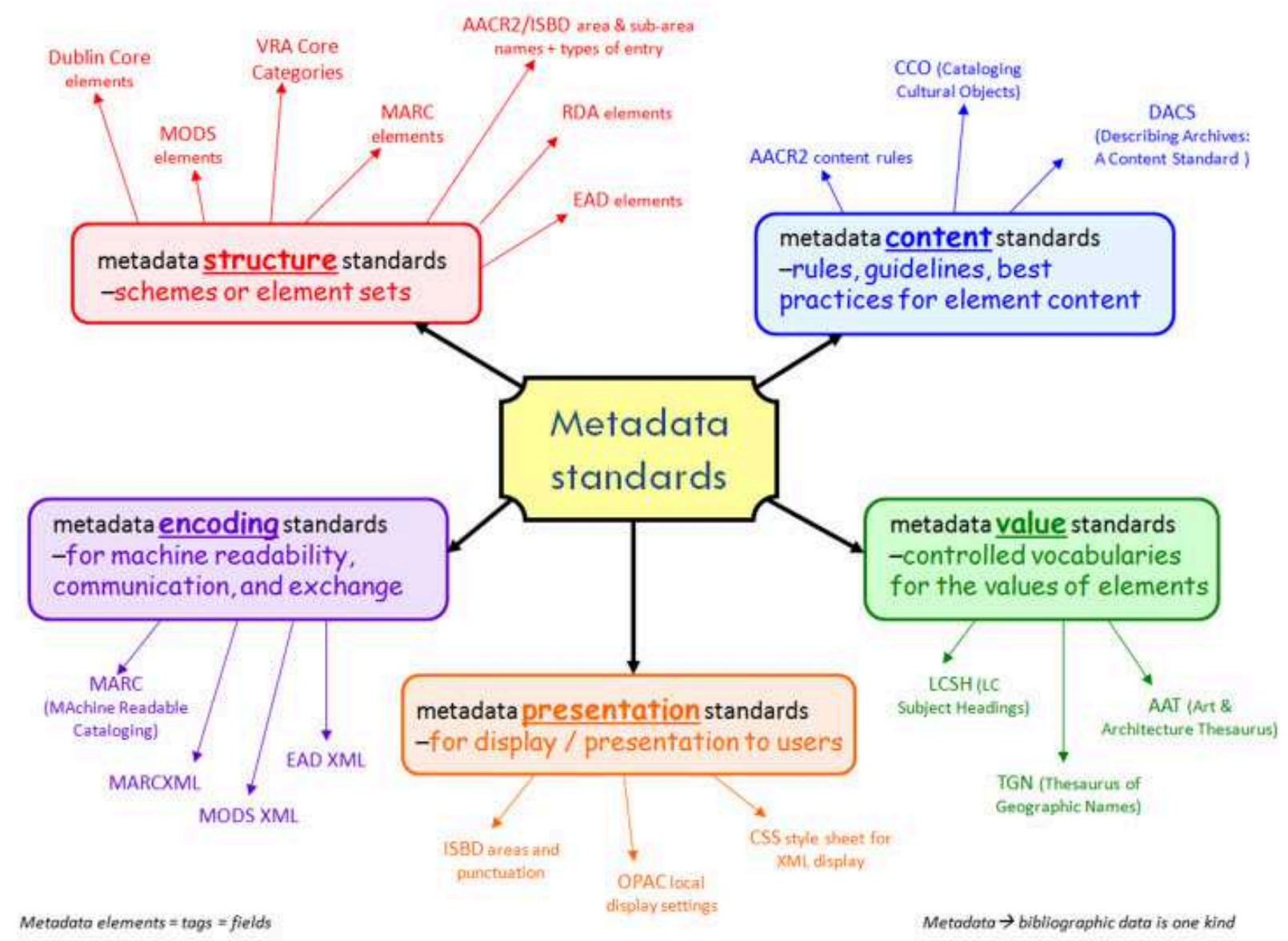

Fig. 2.12 - Tipologie dei metadati. Lo scenario attuale ${ }^{242}$

Il contesto fornito dai nuovi standard catalografici (FRBR, FRAD, FRSAD, RDA) enfatizza la granularità dei cataloghi, tramite la definizione nuove entità per i dati bibliografici che non erano presenti nei record creati con i precedenti standard. I dati contenuti negli OPAC fanno parte di un modello relazionale che ha per lungo tempo influenzato la modalità di strutturazione dei database bibliografici.

La struttura dei dati bibliografici è molto importante ed è profondamente legata alla visualizzazione del record nelle interfacce e, in definitiva all'usabilità dei cataloghi stessi. Marta $\mathrm{Yee}^{243}$ sostiene infatti che un approccio più granulare al record necessiti di un attento riequilibrio per aggregare i dati nelle entità di FRBR, che sono in definitiva quelle entità cercate dagli utenti. Gli utenti del catalogo infatti non necessitano di dati sparsi, ma di entità immediatamente riconoscibili. La struttura del MARC, il formato che ha definito

\footnotetext{
${ }^{242}$ Steven Miller, Metadata and cataloging online resources: selected reference documents, web sites and articles. Compiled by Steven J. Miller, Updated June 1, 2011, University of Wisconsin-Milwaukee School of Information Studies, <http://www.uwm.edu/ mll/resource.html>.

${ }^{243}$ Martha M. Yee, The single shared catalog revisited, in Conversations with catalogers in the 21st century, edited by Elaine Sanchez, Santa Barbara, Libraries Unlimited, 2011, p. 121-136.
} 
la struttura del record bibliografico e l'architettura dei database bibliografici, non è in grado di supportare questa accresciuta granularità.

Per Barbara Tillet gli OPAC dovranno riflettere la struttura relazionale dei database bibliografici per consentire all'utente di navigare tra famiglie di opere che compongono l'universo bibliografico visualizzando le relazioni gerarchiche e associative, le famiglie di espressioni distinte per formato, le famiglie di espressioni distinte per lingua ${ }^{244}$.

Finora i tentativi di applicare FRBR nei next generation catalogs e nei discovery tools si sono fermati alla superficie; non si è intervenuti sulla 'struttura' dei record, ma sulla semplice visualizzazione dei record tradizionali nell'interfaccia di questi nuovi strumenti, attraverso 'algoritmi per la frbrzation' dei dati, dunque i cataloghi elettronici attuali non sono in grado di presentare visualizzazioni gerarchiche delle famiglie di opere.

Di fronte all'evoluzione degli standard catalografici il modello dei discovery system appare fortemente inadeguato a rappresentare l'evoluzione dei modelli concettuali espressi dalla teoria catalografica e dalle nuove possibilità offerte dal Web Semantico e dai linked data.

Chi interroga un motore di ricerca per trovare un'opera, ottiene in risposta le pagine web che parlano di quell'opera, l'eventuale scansione digitale in Google Books e i dati dei cataloghi di vendita di libri in commercio e di antiquariato, ma non dagli opac, i cataloghi delle biblioteche. Alcuni opac sono visibili, come per esempio WorldCat o la National Library of Australia e, a livello nazionale, le Biblioteche Civiche Torinesi e la Braidense, ma rimangono eccezioni. Paradossalmente i discovery tools, l'ultimo ritrovato delle aziende fornitrici di software per il mondo bibliotecario, garantiscono l'esatto contrario di ciò di cui le biblioteche hanno bisogno: gli opac sono aperti verso il web e quando un utente non trova nel catalogo ciò che cerca, viene indirizzato sul web aperto. Inseguiamo il web imitando i motori di ricerca con i next generation catalogues e i discovery tools, ma questo approccio continua a tenere diviso il mondo dei dati bibliografici dal mondo dei dati del web. Se i cataloghi non diventano parte del web, e i dati bibliografici non si integrano nel web, i lettori continueranno a non trovarli nei loro primi approcci alla ricerca e, con tutta probabilità, continueranno a ignorarli ${ }^{245}$.

I maggiori limiti degli strumenti di discovery possono essere così definiti:

1. non consentono di applicare il modello FRBR creando nuove entità;

2. sfruttano l'approccio della "FRBRization" che è basato sul ricorso a un algoritmo invece che sulla creazione di nuove entità al momento della conversione dei metadati (approccio del progetto eXtensible Catalog);

\footnotetext{
244 Barbara B. Tillet RDA (Resource Description and Access): lo sviluppo di un nuovo codice di catalogazione internazionale, «Bibliotime», XI (2008), n. $1<$ http://didattica.spbo.unibo.it/bibliotime/num-xi1/tillett.htm>.

${ }^{245}$ Carlo Bianchini, Library Linked Data e il futuro delle biblioteche, 2012, (risorsa online - in corso di pubblicazione, attualmente disponibile in: $<$ https://sites.google.com/site/homepagecarlobianchini/Ricerca/library-linked-data-e-il-futuro-dellebiblioteche $>$ ).
} 
3. Nella visualizzazione, la struttura relazionale si basa ancora sul meccanismo tradizionale ipertestuale, dunque non collega opere, espressioni, manifestazioni;

4. L'approccio è centrato sui record, invece che sui dati, è incapace di creare visualizzazioni gerarchicamente strutturate e ispirate al modello FRBR, e si basa invece sulla navigazione guidata tra i record tradizionali

Gli strumenti di discovery adottano dunque un modello di sviluppo diametralmente opposto a quello di RDA e FRBR e si basa su due principali elementi di progettazione:

* le faccette;

* la FRBRization', gli algoritmi di ranking e di aggregazione automatica per le edizioni (manifestazioni).

Gli strumenti di discovery che si dichiarano 'Frbrzzati', come Vu Find, WorldCat, Primo, basandosi sui record tradizionali in formato MARC non sono in grado di applicare il modello: essi lavorano su clusters di manifestazioni che vengono ottenuti sfruttando con algoritmi i record sottostanti per generare tali raggruppamenti ${ }^{246}$.

La debolezza di questo approccio era nota sin dagli inizi: quando i primi next generation catalogs fecero la comparsa nel mercato tale elemento fu sottolineato dagli stessi creatori $^{247}$. L'approccio usato dai next generation catalogs e dai discovery systems non consente di applicare il modello non tanto per una questione di dati, ma per l'approccio complessivo alla progettazione adottato da questi strumenti: essi sfruttano i record provenienti dalle descrizioni per l'importazione dei record e la loro visualizzazione, e non lavorano sui record di autorità che invece rappresentano le entità richieste dagli utenti $^{248}$. Le faccette vengono offerte come mezzo alternativo di recupero di quelle informazioni che potrebbero essere ottenute tramite l'implementazione del modello.

Tuttavia tali raggruppamenti si dimostrano incoerenti: ad esempio le faccette tipologia di materiale o lingua non consentono di scegliere realmente tra tutte le diverse espressioni: tali raggruppamenti sono incoerenti e imprecisi oltre a presentare il difetto di non creare una lista navigabile o esplorabile dei raggruppamenti creati con le faccette.

\footnotetext{
246 Breve, ma efficace la riflessione di Rockind sul termine Frbrzation che in realità si traduce nell'applicazione di algoritmi per il raggruppamento in base all'opera. Jonathan Rochkind, "FRBRization" is not FRBRization, «Bibliographic Wilderness», Blog post. 31/07/2008 $<$ http://bibwild.wordpress.com/2008/07/31/frbrization-is-not-frbrization/>.

${ }^{247}$ Per l'OPAC di nuova generazione Endeca si vedano le osservazioni di Antelman, Lynema, Pace Toward a twenty-first century library catalog cit.

${ }^{248}$ Un esempio di progettazione opposta è invece Wordlcat Identities che lavora solo sulle voci di autorità presenta tutte le funzionalità che dovrebbero essere fornite da un catalogo elettronico.
} 
Solitamente gli strumenti di discovery hanno un modulo che consente la conversione dei metadati, che potrebbe essere sfruttato per introdurre nuovi campi, tuttavia non è possibile rappresentare nuove entità in quanto non si è poi in grado di stabilire le relazioni appropriate, che sono alla base della visualizzazione secondo il modello FRBR.

Tuttavia oggi l'unico progetto che trasforma i metadati per ottenere le entità di FRBR è Extensible Catalog, vero modello per un catalogo di nuova generazione.

L'elemento di debolezza dei discovery system è il loro 'approccio algoritmico' alla progettazione degli OPAC per quanto riguarda:

* la struttura del record;

* la progettazione della ricerca.

L'approccio di sviluppo dei discovery systems e dei next generation catalogs è lontano da una progettazione che si basa sulla trasformazione e rielaborazione della struttura dei record: ecco perché tali strumenti rivelano tutta la loro debolezza in fase di ricerca: essi si basano su algoritmi invece di creare nuove entità, dunque non sono in grado di esprimere entità e relazioni previste dal modello.

\subsubsection{L'implementazione dei discovery systems. Il workflow}

Proponendo una metafora per le varie questioni da affrontare al momento dell'implementazione di questi nuovi strumenti, Eric Lease Morgan affermava che «there is more than one way to skin a cat» come utile metafora per intendere che ogni implementazione può presentare risultati differenti anche per lo stesso prodotto, a seconda delle necessità e della "visione" che ogni singola biblioteca ha dello strumento di discovery ${ }^{249}$.

In generale prima di tutto, ogni biblioteca avrà cura di preparare un'indagine degli strumenti di discovery presenti per valutare sistema da scegliere e contestualmente verrà svolta un'analisi degli strumenti che dovranno confluire nel sistema di discovery per preparare uno schema di mappatura ${ }^{250}$.

\footnotetext{
249 Eric Lease Morgan, Next generation library catalog, «Infomotions.com», 02-06- 2006, $<$ http://infomotions.com/musings/ngc/>.

${ }^{250}$ Un'analisi valutativa di questo tipo visibile online è quella del CUWL Resource Discovery Exploratory Task Force del sistema delle biblioteche universitarie del Wisconsin-Madison che ha preparato un rapporto sugli strumenti di discovery presenti sul mercato per valutarne l'adottabilità nel proprio sistema bibliotecario. Resource Discovery Exploratory Task Force Final Report. University of Wisconsin - Madison Libraries
} 
Una volta scelto lo strumento, seguendo lo schema suggerito da Breeding ${ }^{251}$, si prevedono tre fondamentali fasi di implementazione:

\footnotetext{
* integrazione del discovery system con l'ILS, estrazione dei dati, importazione dei dati nel discovery system, sincronizzazione e aggiornamento;

* caricamento di contenuti da altri repositories;

fase di test, di lancio e monitoraggio del sistema.
}

a) Integrazione del Discovery system con l'ILS, estrazione dei dati, importazione dei dati nel discovery system, sincronizzazione e aggiornamento

Il concetto principale è che i Discovery System non sono progettati per gestire i dati separatamente, ma li importano in un indice centralizzato e questa architettura di progettazione li distingue dalla ricerca federata e li rende al contempo più veloci. È dunque necessaria una fase preliminare di importazione e di indicizzazione dei dati all'interno del sistema nell'indice dello strumento di discovery.

La fondamentale nozione di decouplement, prevede che al sistema di discovery venga delegata la ricerca, mentre il mantenimento delle funzioni e dei dati gestionali resti affidato all'ILS sottostante, che continua ad mantenere le funzioni di catalogazione, acquisto, prestiti e circolazione. Se i moduli OPAC integrati erano avvantaggiati dalla loro completa integrazione nell'ILS potendo accedere a tutti i dati sempre aggiornati, i nuovi discovery system, invece, lavorano all'esterno, dunque sono fondamentali le attività di estrazione dei dati, di trasferimento, di sincronizzazione continua di questi dati che cambiano velocemente e l'interazione deve avvenire in tempo reale. Ciò impone un'attività di costante aggiornamento e sincronizzazione del discovery system.

La più importante fase è quella dell'importazione dei dati catalografici presenti all'interno dell'ILS, operazione delicata e critica, in quanto comporta un trasferimento massivo di dati, che devono essere continuamente sincronizzati per mantenerne l'aggiornamento. Lo stesso vale per tutte le operazioni che gli utenti compiono con i dati, (quali, ad esempio, prestiti, rinnovi, visualizzazione in tempo reale della disponibilità) dove risulta necessario che questi sistemi possano recuperare le informazioni aggiornate e

Submitted to the Library Services Council, 6/2/2008 <http://staff.library.wisc.edu/rdetf/RDETF-finalreport.pdf>.

${ }^{251}$ Le fasi dell'implementazione sono state descritte anche dalla Library of Congress e da Morgan (cfr. Terry Ballard - Anna Blaine, User search-limiting behavior in online catalogs. Comparing classic catalog use to search behavior in next-generation catalogs. New Library World 112 (2011) n. 5-6, p 261-273, $<$ http://search.proquest.com/docview/889388524?accountid=13698>). 
consentire all'utente di autenticarsi una sola volta nel discovery system, (e non in entrambi i sistemi), dal quale poi accedere a tutti i servizi.

L'operazione di estrazione dei dati presenta alcune grosse criticità; non sempre tutti i dati presenti nell'ILS sono importabili nello strumento di discovery e l'importazione può avvenire anche per tutti i dati relativi agli item ${ }^{252}$. Ciò dipende dalla 'visione' che ogni singola biblioteca avrà dello strumento di discovery, se esso dovrà costituire una semplice interfaccia semplificata per le risorse della biblioteca e sostituire del tutto l'OPAC, oppure se viene mantenuto l'OPAC e lo strumento di discovery recupererà nella sua interfaccia molte altre risorse. Nel caso della sostituzione, dovranno essere importati tutti i dati, nel primo caso invece potrebbero essere importati solo alcuni dati, lasciando al sistema la possibilità di collegarsi sul momento all'OPAC per recuperare quelli mancanti.

Prima dell'importazione bisogna però compiere una lunga serie di operazioni preliminari che riguardano l'analisi dei sistemi che verranno ricercati nel discovery system (sistemi sorgente), cioè verificare i protocolli di comunicazione dei dati, i formati di metadati che tali sistemi (OPAC, repository, biblioteca digitale) utilizzano nel formato di export verso lo strumento di discovery. L'obiettivo di questa preliminare analisi è quello di poter ottenere tutte le informazioni che serviranno per preparare la mappatura. L'analisi va condotta sui metadati esportati via OAI-PMH. Verranno esaminati anche i protocolli di comunicazione tra il catalogo; gli archivi istituzionali; SFX, ecc. e il sistema di destinazione cioè l'indice del Discovery system.

È importante sottolineare che il DS lavora in maniera del tutto automatizzata, dunque rispetto ad un OPAC non dispone di funzioni di editing dei metadati (es. modifica, cancellazione, etc) che dovranno essere effettuate sul sistema di partenza: l'indice del discovery system importerà al suo interno tutti i metadati presenti nelle risorse importate che dovranno essere normalizzati e trasformati in un unico formato, quello presente nello strumento di discovery.

Il protocollo maggiormente usato per l'importazione massiva di dati è OAI-PMH che è un protocollo pubblico creato appositamente per automatizzare il trasferimento di metadati bibliografici tra due server la cui versione attuale risale al 2002. Il modello utilizzato dai discovery systems segue le specifiche di OAI-PMH utilizzando questo protocollo per il trasferimento dei dati ${ }^{253}$.

\footnotetext{
${ }^{252}$ Queste operazioni sono sovente a carico delle singole biblioteche, ma i maggiori vendors hanno predisposto guide tecniche.

${ }^{253}$ Il modello dell'harvesting, che ha sostituito quello precedente della 'ricerca federata' che aveva dominato la scena fino poco tempo fa, è ormai il metodo più usato per il trasferimento di grandi quantitativi di
} 
Attraverso questo protocollo multiformato si ottengono file $\mathrm{XML}^{254}$. Il protocollo si limita a richiedere dati strutturati in XML, mentre altri standard ne definiranno strutture e contenuti. Il lavoro dei discovery systems richiede un'attività che si concentrerà sulla 'struttura' dei dati, in quanto il trasferimento tramite OAI-PMH richiede una mappatura e una normalizzazione dei dati. Il procedimento comporta la conversione di tutti i formati provenienti dai sistemi di partenza in un formato intermedio (basato su XML) e, infine, in un formato di destinazione seguendo un processo di normalizzazione dei metadati che vengono convertiti in un formato unico attraverso schemi di mappatura o 'crosswalks'.

La conversione di diversi schemi di metadati in un formato basato su XML (export XML) consente all'harvester del discovery system di importare i dati tramite il protocollo OAI-PMH, che dovranno in seguito di essere riconvertiti nel formato proprietario del discovery system; operazione che consentirà l'indicizzazione dei dati importati che possono così essere ricercati nell'indice centralizzato.

Le biblioteche devono dunque dotare i loro gestionali di un modulo (Modulo OAI $\mathrm{PMH})$ che consente l'export dei dati in DC o in MARCXML, o UNIMARC XML a seconda dei casi.

La conversione dei dati dai formati MARC verso i formati XML non è priva di difficoltà, anzi si dimostra la fase più critica del processo. È infatti indispensabile un'analisi del record in MARCXML o UNIMARCXML per individuare errori o incoerenze che potrebbero portare a problemi nell'importazione e visualizzazione dei dati nello strumento di discovery ${ }^{255}$ e ovviare ai problemi che possono insorgere ${ }^{256}$.

informazione da qualunque tipo di repository che offre questo tipo di 'uscita' dei dati (Marshall Breeding, Next-gen library catalogs cit., p. 73).

${ }^{254}$ L'adattamento dell'XML ai formati catalografici è iniziato nel 2003 con la creazione di MARC21 XML: la codifica XML del MARC (febbraio 2003: primi tests da parte della Library of Congress di records in formato MARC21 XML). Il formato UNIMARC XML usato da Sebina Open Library è stato creato da Giovanni Bergamin: lo schema si trova a: <http://www.bncf.firenze.sbn.it/progetti/unimarc/slim/documentation/unimarcslim.xsd> e anche http://www.bncf.firenze.sbn.it/progetti/unimarc/slim/documentation/unimarcslim.html?rigamenu=UNIMAR CXML>

${ }^{255}$ Ad esempio nel caso di Sebina può essere operata una conversione da SBN in UNIMARC e poi a UNIMARCXML che comporta il passaggio da una struttura reticolare del record ad una struttura piatta (basata su MARC). Gli errori di tag costituiscono un problema notevole nel caso di un discovery system che visualizza $\mathrm{i}$ dati in campi etichettati. I campi etichettati richiedono etichette corrette che corrispondano al contenuto del campo e può accadere che il contenuto sia erroneo. È dunque indispensabile un'analisi del record in MARCXML o UNIMARCXML per individuare errori o incoerenze che potrebbero portare a problemi nella visualizzazione dei dati nello strumento di discovery. Un esempio di record in UNIMARC nell'OPAC di

Sebina: $<$ http://opac.biblio.polimi.it/SebinaOpac/OAIHandler?verb=GetRecord\&metadataPrefix=oai_unimarc\&ident ifier=oai:SebinaOL.PMI:0741569>.

${ }^{256}$ Ad esempio nel formato dei dati di Primo (PNX) mancano importanti elementi:

* altri titoli (titolo variante; titolo originale; titolo uniforme)

* classificazione;

* legame del tipo comprende (per le opere multivolume); 
Il processo di normalizzazione dei dati recuperati si rivela insostituibile per gestire sia dati che provengono da diversi ILS (ad esempio ILS differenti in un DS di un consorzio, come nel caso delle biblioteche che utilizzano WorldCat Local) sia di diversi repositories (oggi tutti i più importanti repositories es. DSpace, Fedora etc. supportano OAI-PMH) che dovranno essere ricercabili tramite l'interfaccia del discovery system.

La stessa procedura deve essere seguita dunque per tutti gli strumenti che devono integrarsi con il discovery system ad esempio quando bisogna importare dati da un repository istituzionale come Dspace, dati archivistici o una collezione digitale. Questi strumenti hanno sovente la funzionalità dei export dei dati in DublinCore. Visto che OAI$\mathrm{PMH}$ è un protocollo multiformato che accetta qualsiasi file strutturato tramite XML bisogna decidere di adottare formati intermedi che siano flessibili e multiuso come MODS e Dublin Core Application Profile.

L'operazione di importazione dei dati estratti dall'ILS nel discovery system, varia da prodotto a prodotto, e generalmente presenta anche le seguenti questioni:

* arricchimento: di solito i dati vengono integrati con altri dati esterni, o arricchimenti, che sfruttano le potenzialità dei motori di ricerca integrato nel DS. Gli arricchimenti possono derivare da:

* importazione di toc, copertine e sommari da servizi esterni tramite API gratuite ${ }^{257}$ (es. Google Book Search, Amazon, Librarything, oppure commerciali (Syndetic Solutions, Baker and Taylor Content Café);

* ricerca del fulltext;

* ricerca tramite gli authority files di autori e soggetti per recuperarne forme varianti;

* utilizzo dei dati recuperati nell'interfaccia: alcune questioni che sorgono sono relative all'adattamento dei dati all'interfaccia:

1. come verranno presentati i dati nelle nuove interfacce;

2. come verranno usati i dati nel sistema negli algoritmi che determinano la rilevanza;

\footnotetext{
* legami specifici dei periodici;

* informazioni sulla storia del periodico (Area 3 delle ISBD);

* informazioni specifiche per il materiale cartografico (Area 3 delle ISBD)

* note (area della note delle ISBD in tag 300 UNIMARC);

* informazioni inserite nella semiarea di responsabilità che chiariscono i diversi contributi.

${ }^{257}$ L'arricchimento del record può avvenire importando elementi aggiuntivi che vengono mostrati insieme alle descrizioni bibliografiche. I più comuni arricchimenti sono rappresentati dalle copertine, facilmente importabili tramite apposite API, molte delle quali sono gratuite, e da altri arricchimenti testuali, rappresentati da sommari, recensioni, biografie degli autori etc. La maggior parte degli strumenti di discovery consente di gestire direttamente e configurare gli arricchimenti, che richiedono comunque una certa conoscenza e dimestichezza con le API.
} 
3. quali faccette, etichette o dati dovranno essere presentati nelle interfacce (es. nella visualizzazione breve dei record o nel dettaglio record).

Queste questioni dipenderanno da ogni singola biblioteca, che deciderà cosa mostrare e come mostrare i propri record nell'interfaccia del discovery system. Generalmente tali sistemi sono in grado di fornire all'utente in risposta alla sua interrogazione una visualizzazione del record che contiene tutti gli elementi identificativi che consentano all'utente di identificare i documenti necessari, selezionarli a schermo e lanciare l'esplorazione del dettaglio del record. Ma il modo con cui avviene la visualizzazione del record può variare dal deep linking dell'URL persistente attribuito alla risorsa, o in tempo reale tramite una richiesta all'ILS tramite un'API. Tali modalità dipendono dal discovery system utilizzato e richiedono, nel secondo caso, un lavoro preliminare di assegnazione di un URL persistente ad ogni singola risorsa ${ }^{258}$.

Le modalità di visualizzazione possono essere personalizzate in base alle preferenze di ogni singola biblioteca, grazie ad una serie di funzionalità che questi software generalmente presentano.

- Interazioni in tempo reale. Una volta svolte queste operazioni è necessario che vi sia la possibilità di stabilire tra il discovery system e l'OPAC interazioni in tempo reale necessarie per tenere continuamente aggiornate le informazioni relative alla disponibilità dei documenti, e dare conto dei nuovi acquisti e della catalogazione di nuovo materiale. Se dunque l'harvesting richiede trasferimenti massivi di dati all'interno dell'indice del discovery system, varie operazioni di sincronizzazione devono poi essere programmate per mantenere i dati continuamente aggiornati ${ }^{259}$. Le operazioni di sincronizzazione dei dati per il loro costante aggiornamento sono rese necessarie dal fatto che i dati gestiti dentro gli ILS cambiano costantemente. La frequenza di aggiornamento dipende dall'esigenza di ogni singola biblioteca e può variare da due o tre aggiornamenti l'ora ad uno due aggiornamento al giorno e dipende anche dal tempo necessario per l'aggiornamento. In particolare la disponibilità del documento richiede l'aggiornamento costante dei dati altrimenti verranno mostrati all'utente le disponibilità relative all'ultimo aggiornamento effettuato.

- Integrazione con $i$ servizi all'utente, autenticazione. Si tratta di gestire dallo strumento di discovery le autenticazioni richieste per accedere a servizi personalizzati di prestito, scaffale personale, liste personalizzate, accesso a proxy per le risorse (banche dati

\footnotetext{
${ }^{258}$ Cfr. Marshall Breeding, Next-Gen Library Catalogs cit., p. 76-77.

${ }^{259}$ Il modulo OAI-PMH dovrà richiedere al gestionale solo i record che risultano modificati.
} 
etc) riservate agli utenti della biblioteca, richieste di prestito interbibliotecario etc. Generalmente tali software gestiscono l'autenticazione e offrono servizi che non vengono offerti dall'OPAC tradizionale: salvataggio di risultati, riutilizzo di queries, liste personalizzate. Nelle biblioteche universitarie i discovery system devono integrarsi con altri software che gestiscono, calendari, scadenze, email, iscrizione ai corsi per il suggerimento dei materiali di studio etc. La necessità di svolgere una sola autenticazione per accedere a tutti i servizi disponibili ha portato allo sviluppo di applicativi dedicati a tale importante funzionalità. Una delle più delicate e complesse operazioni di configurazione del discovery system consiste nell'integrazione con gli altri applicativi esistenti per fornire un'unica autenticazione (single sign-on ) per tutti i servizi.

b) Caricamento di dati da repositories esterni

Generalmente i discovery systems presentano oltre ai dati provenienti dall'OPAC, altri documenti recuperati da fonti esterne. In particolar modo alcuni software per 'discovery systems' che si agganciano a indici 'prepopolati' di dati di svariata provenienza (es. e-journals, databases citazionali etc.) e di copertura globale sono ormai particolarmente diffusi: Summon, Primo ed Ebsco Discovery Service sono i più conosciuti Web Scale discovery services.

L'integrazione di questi dati a differenza di quelli che vengono estratti dagli ILS non presenta particolari difficoltà, e segue un iter simile che prevede l'estrazione dei dati, il trasferimento, la configurazione della visualizzazione e delle autenticazioni, la sincronizzazione. Il 'popolamento' dei sistemi di discovery con questi dati generalmente non crea particolari problemi, in quanto queste fonti spesso presentano i metadati in XML $\mathrm{o}$ in altri standard altamente compatibili come Dublin Core; il fattore più critico è la rappresentazione adeguata di tali dati insieme agli altri già contenuti nel discovery system, in modo che le visualizzazioni siano omogenee, e dunque l'aspetto più critico si focalizza nella scelta dei campi da mostrare.

I nuovi strumenti per la scoperta fanno un uso più vasto dei metadati rispetto agli OPAC tradizionali ${ }^{260}$. Essi non servono solamente per implementare il modello FRBR (es. per il raggruppamento in base all'entità opera) ma il loro approccio ai metadati è completamente diverso: alcune incorerenze prima nascoste possono essere messe in rilievo proprio nell'interfaccia di un discovery system, ad esempio nella visualizzazione delle

\footnotetext{
${ }^{260}$ Marshall Breeding, Next-Gen Library Catalogs cit., p. 79.
} 
faccette. Questa motivazione induce spesso varie biblioteche che usano lo stesso prodotto ad adottare faccette diverse, non tanto per una preferenza estetica, quanto per necessità di mascherare incoerenze altrimenti visibili. Un'altra complicazione sorge dal fatto che $\mathrm{i}$ discovery systems incorporano dati che prima venivano sfruttati su interfacce diverse e separate: una volta recuperati in un'interfaccia unica questi possono presentare carenze, disomogeneità e duplicazioni che vanno accuratamente eliminate. In particolar modo si rende necessaria un'attenzione più costante all'attività di catalogazione e di creazione di metadati.

\section{b.1) E-journals}

Una delle caratteristiche più interessanti dei discovey systems è senz'altro l'importazione di articoli di riviste. È un tema molto discusso in passato, quando l'impossibilità di importare direttamente negli OPAC tale tipologia di documento aveva portato i produttori di software a introdurre sul mercato appositi applicativi chiamati ERMS e poi ad affidarsi alla ricerca federata. È nota la difficoltà di gestire all'interno degli OPAC questo materiale. Negli OPAC tradizionali le riviste sono state trattate 'a livello titolo', senza fornire l'accesso al full-text dei singoli articoli. La possibilità per i discovery systems di fornire l'accesso a tutti i documenti posseduti dalle biblioteche riguarda anche l'accesso ai fulltext degli articoli delle riviste online sottoscritte da una biblioteca. Generalmente le due principali tecniche prevedono la cosiddetta indicizzazione diretta e la ricerca federata.

Di solito la prima funzione è quella maggiormente utilizzata e consiste nell'indicizzazione dei documenti direttamente all'interno del discovery system.

Essa consente di recuperare nella stessa interrogazione tutto il contenuto accessibile da quella biblioteca, ottenendo una esperienza di ricerca integrata e unificata, di migliorare la rilevanza e di usare meglio le faccette. Il workflow da seguire è lo stesso dei dati dell'OPAC e consiste nell'harvesting dei metadati e nella loro indicizzazione dentro il discovery system, ma l'investimento in termini di risorse e personale è notevole. Ormai i cosiddetti servizi di Web scale discovery forniscono indici già pronti che si trovano all'interno del discovery system e che si possono acquistare con pacchetti commerciali ad hoc. Questi contengono un enorme quantitativo di articoli in full-text già indicizzati sollevando le biblioteche che vogliono trattare gli articoli, da un compito gravoso e oneroso. In questo caso la biblioteca si limita ad effettuare delle attività meno impegnative, 
come il controllo delle riviste sottoscritte, un'operazione di profiling cioè di settaggio delle impostazioni iniziali (riscontro delle riviste inserite nel discovery system con quelle effettivamente sottoscritte) e pochi altri lavori di manutenzione.

Affidarsi alla ricerca federata è ancora possibile, benché oggi sconsigliato; si preferisce a questa metodologia l'harvesting. Però non sempre le biblioteche si trovano nella condizione di praticare questa ultima tecnica: ad esempio quando il contenuto offerto nell'indice centralizzato non comprende alcune riviste specializzate che invece la biblioteca vuole recuperare, o quando si trova già in possesso di strumenti per la ricerca federata.

Solitamente l'appesantimento dovuto alla simultanea ricerca federata, in combinazione con quella svolta dal discovery system, rende sconsigliabile questa pratica, ma nella realtà è molto comune. Pertanto la maggior parte di questi strumenti ha nel tempo incorporato applicativi che riescono ad integrare questi sistemi ottimizzandone le prestazioni. Infine anche i link resolvers vengono integrati in questi sistemi, per l'accesso ai documenti riservati agli utenti iscritti. Non è raro vedere insieme i tre strumenti discovery system, link resolver e ricerca federata, usati contemporaneamente da una stessa istituzione. Cosi la scelta di uno strumento di discovery dipende sovente dagli strumenti già usati dalla biblioteca, con i quali le interfacce per la scoperta devono integrarsi.

\section{c ) Monitoraggio del sistema ed altri aspetti dell'implementazione}

Le ultime fasi sono quelle dell'implementazione e il monitoraggio del sistema che richiedono alcuni aspetti decisionali e comportano una serie di operazioni pratiche.

L'interfaccia verrà infatti sottoposta ad una fase di test, e fornita in beta version prima di assumere la sua veste definitiva e numerosi input verranno dal feedback che utenti e bibliotecari manifesteranno in queste fasi e poi durante il prosieguo. Solitamente viene previsto un periodo di coesistenza del nuovo discovery system con l'OPAC preesistente prima della definitiva sostituzione.

Successivamente il nuovo discovery system diverrà l'interfaccia di default per la ricerca e verrà integrato nelle pagine web del sito della biblioteca. Questa fase è molto delicata e richiede un attento monitoraggio, per capire come gli utenti usano queste interfacce, se ad esempio, usano di meno l'OPAC probabilmente riscontreranno problemi con la nuova interfaccia. 
Va, infine, sottolineato che il modo in cui ogni singola biblioteca implementa un OPAC di nuova generazione o un discovery system può essere molto diverso, a seconda delle competenze tecniche di chi implementa tali interfacce, e che tale implementazione può influenzare le modalità di recupero dell'informazione di questi sistemi e in definitiva la qualità dell'informazione recuperata ${ }^{261}$.

Da queste preoccupazioni ${ }^{262}$, su iniziativa della Digital Library Federation è nato un gruppo di studio allo scopo di indagare il rapporto tra ILS e interfacce per la scoperta e di pervenire allo studio di un'API (Application programming interface) che risponde alla necessità di assicurare che nei collegamenti tra sistemi di gestione bibliotecaria (ILS) e discovery systems siano mantenute alcune caratteristiche essenziali che permettono di cercare, identificare, selezionare, ottenere una risorsa e navigare adeguatamente nei nuovi sistemi.

Il punto di arrivo di tale progetto è stato l'accordo di Berkeley siglato nel 2008 tra $\mathrm{i}$ più grandi produttori di software per biblioteche che si sogno impegnati a mantenere nello sviluppo di nuovi sistemi le funzionalità più importanti ${ }^{263}$.

\subsection{I dati bibliografici negli OPAC di nuova generazione e nei discovery systems: arricchimento dei dati e impoverimento delle funzioni}

In definitiva, possiamo chiederci come si presentano i dati bibliografici negli OPAC di nuova generazione e soprattutto se la ricerca in questi strumenti fornisce agli utenti risultati di qualità: negli OPAC che si definiscono di nuova generazione e negli strumenti di discovery la ricerca offre un miglioramento complessivo della qualità dei risultati recuperati?

\footnotetext{
${ }^{261}$ Ivi p. 74.

${ }^{262} \mathrm{Ma}$ anche da quella di preservare lo sviluppo dei cataloghi da logiche puramente commerciali.

${ }^{263}$ La funzionalità sono raggruppate in tre categorie: harvesting e recupero dei dati bibliografici compatibili con OAI-PMH, availability per il recupero dei dati sulla circolazione in tempo reale e linking che si riferisce alla possibilità di stabilire link permanenti ad ogni item presente a catalogo. Per ogni gruppo vengono definite delle funzioni astratte, mentre si rimanda a successive linee guida che avranno il compito di stabilire le soluzioni tecnologiche più adatte alla realizzazione nel concreto di tali funzioni.
} 


\subsection{1. 'One size fits all'? dall'accesso unico alla moltiplicazione degli accessi}

Come abbiamo avuto modo di accennare, next generation catalogs e discovery systems non offrono un accesso unico alle risorse possedute, sottoscritte o raggiungibili da una biblioteca.

Se per istituzioni come le biblioteche pubbliche spesso basta un next generation catalog per offrire agli utenti un accesso semplificato alle risorse librarie, le biblioteche accademiche si trovano a sperimentare vari strumenti di ricerca per gestire le proprie risorse in fulltext. In molti casi un solo strumento di discovery non basta e ci si ritrova a dover integrare più strumenti. L'obiettivo dell'accesso unico non viene quasi mai raggiunto con l'aggravio di disorientare l'utente.

Un esempio emblematico della situazione della moltiplicazione degli accessi si ritrova nel caso delle biblioteche della Grand Valley State University (GVSU libraries). Le biblioteche nel 2010 fornivano ben tre accessi ai dati dell'OPAC:

1) l'OPAC tradizionale per la ricerca di materiale tradizionale;

2) l'OPAC con interfaccia di nuova generazione Encore per una ricerca semplificata nell'OPAC;

3) il discovery system Summon per la ricerca di articoli (e altro materiale).

Oggi l'accesso Encore è stato rimosso e la biblioteca offre due accessi: Summon, che recupera anche i dati dell'OPAC per la ricerca degli articoli e il catalogo classico per il recupero delle risorse tradizionali a stampa. L'OPAC della biblioteca consente funzionalità semantiche che nell'OPAC di nuova generazione non erano applicabili e che riguardano la ricerca semantica, come lo scorrimento delle liste e degli indici ${ }^{264}$.

\footnotetext{
${ }^{264}$ Le vicende relative all'adozione in contemporanea dei software Encore e Summon alla Grand Valley State University sono analizzate nel contributo di Sharon Q. Yang -Melissa A. Hofmann, Next generation or current generation? cit.
} 


\subsubsection{La ricerca semantica nei nuovi OPAC: ricerca per soggetto e ricerca per parola chiave}

Il particolare mutamento del contesto nel quale i nuovi cataloghi elettronici si sono sviluppati nell'ultimo quinquennio, da più parti è stato visto come il principale fattore per un vero e proprio un mutamento di paradigma nella ricerca. Un paradigma nuovo, ricalcante la ricerca nel web, è quello che vede due principali tipologie di approccio alla ricerca per soggetto:

* la 'ricerca esplorativa', che si serve di filtri e canali già stabiliti per aiutare l'utente che non sa cosa sta cercando;

* la ricerca per parola chiave, che viene introdotta come ricerca di default a scapito della ricerca per soggetto.

Se la prima modalità come vedremo nei prossimi capitoli risulta una caratteristica funzionale indispensabile che l'OPAC deve attivare a vantaggio dell'utente, la ricerca per parola chiave nasconde varie insidie.

Il dibattito sulla ricerca 'per parola chiave' contrapposta alla ricerca 'per soggetto' avviato dagli anni '80, quando tale tecnica venne introdotta nei cosiddetti cataloghi di seconda generazione, oggi acquista nuova enfasi ${ }^{265}$.

${ }^{265}$ La questione si riduce alla fondamentale differenza tra indicizzazione pre-coordinata nella quale la correlazione tra i termini per costruire la stringa di soggetto viene effettuata dal catalogatore tramite l'uso di vocabolari (es. LCSH, Soggettario di Firenze) e l'indicizzazione post-coordinata che viene effettuata al momento del recupero tramite la coordinazione di più termini con l'uso di operatori booleani.

I vantaggi della precoordinazione si riflettono nelle modalità di recupero e di visualizzazione:

1. la struttura sindetica consente di aumentare il richiamo e compiere ricerche mirate ed esaustive;

2. lo sforzo cognitivo è a carico del catalogatore che ha tutti gli strumenti per garantire il controllo bibliografico e disambiguare i termini usando una specifica sintassi;

3. la precoordinazione supporta il browsing delle liste, raggruppando sotto un termine tutti i termini correlati e consentendo lo scorrimento degli indici di classi o di soggetti;

4. la precoordinazione supporta il multilinguismo, poiché una stessa stringa può essere attribuita a pubblicazioni in lingue diverse.

Dunque l'uso in contemporanea di indicizzazione pre e post-coordinata è risultato indispensabile per produrre maggior richiamo e precisione nella ricerca. Si ricorderà in questo senso un contributo di Svenonius (cfr.: Elaine Svenonius, Unanswered questions in the design of controlled vocabularies, «Journal of the American Society for Information Science», 37 (1986) n. 5, p. 331-340). L'alternativa tra le due modalità di ricerca nel catalogo elettronico è stata definitivamente superata negli anni ' 80 quando si comprese che i cataloghi elettronici potevano sfruttare entrambi i vantaggi dei due approcci utilizzando stringhe coordinate dal catalogatore e creando in base ad esse indici di termini singoli che potevano essere indicizzati dal sistema. (Marcia J. Bates, Subject access in online catalogs: a design model, «Journal of the American Society for Information Science», 37 (1986), n. 6, p. 357-376. Solo di recente lo scontro tra i due approcci si è riacceso in seguito alla fortuna che l'indicizzazione fulltext dei motori ha riscosso nella ricerca web: si è ritornati su posizioni antagoniste che hanno visto contrapporsi le motivazioni di chi ritiene che l'indicizzazione manuale sia necessaria per la precisione della ricerca (es. Mann) a chi invece ritiene questo approccio ormai largamente superato, economicamente insostenibile $\mathrm{e}$, in definitiva, non più necessario (è il caso di Calhoun). 
Gli OPAC di seconda generazione avevano introdotto miglioramenti significativi alla ricerca per soggetto: anziché utilizzare per la ricerca le stringhe coordinate assegnate al documento, l'utente poteva coordinare più parole chiave. Rispetto alla ricerca per soggetto ottenibile nel catalogo cartaceo vi erano alcuni vantaggi: la ricerca non doveva più iniziare obbligatoriamente dal primo termine della voce di soggetto, ma era possibile usare qualsiasi termine contenuto nella stringa; e inoltre possibile utilizzare i collegamenti ipertestuali per collegare i termini e le voci di soggetto.

Nel tempo sono emersi anche altri vantaggi: ad esempio, la possibilità di includere nella ricerca per soggetto anche altre parti informative (come i sommari o persino il fulltext dei documenti) e di creare vocabolari di accesso (entry vocabulary) di termini per mappare il linguaggio naturale utilizzato dall'utente nell'interrogazione al linguaggio controllato presente negli indici per soggetto e per classi. Il modello che influenza la nascita dei nuovi OPAC mette, dunque, al centro la ricerca per parola chiave ispirandosi al web e offrendo la ricerca 'google like' ossia su tutti i campi come ricerca predefinita; la ricerca per parola chiave è, infatti, il modello di ricerca che tutti gli utenti della rete si aspettano e fornirla in un OPAC risponde ad esigenze di usabilità dell'interfaccia.

Tale scelta ha sollevato il dibattito sull'efficacia della ricerca nei cataloghi elettronici: la parola chiave, infatti, ha sostituito definitivamente l'uso degli operatori booleani, per lo più sconosciuti agli utenti di oggi e vuole sostituirsi alla ricerca per sogetto che utilizza il linguaggio controllato delle stringhe di soggetto e di classificazione. Come abbiamo visto, il dibattito è stato particolarmente acceso alla Library of Congress e all'Università della California dove le linee di politica bibliotecaria si sono orientate verso l'abbandono dell'indicizzazione manuale.

Il punto è se nella ricerca per soggetto ci si possa basare sul valore informativo di termini collocati in aree del record che hanno una qualche funzione informativa: ad esempio il titolo e l'area delle note e i sommari e gli indici che si trovano nelle risorse in fulltext. Questa ipotesi è uscita notevolmente rafforzata dalle indagini sugli utenti compiute nell'ultimo decennio.

Certamente la ricerca per parola chiave consente di recuperare termini all'interno di una stringa di soggetto, migliorando le possibilità di recupero rispetto ai primi OPAC, ma sfortunatamente tale modalità ha numerosi svantaggi ed è stato dimostrato come negli OPAC si recuperino con questa tecnica risultati insoddisfacenti, dunque non si presenta come un valido sostituto alla ricerca per soggetto. 
Infatti non è raro recuperare all'interno dei risultati documenti non pertinenti: ciò è dovuto ai casi di falsa coordinazione che vengono recuperati dal motore di ricerca e dai suoi algoritmi.

La ricerca per parola chiave su box singolo (Google like) e su tutti i campi rispetto alla ricerca nel campo soggetto produce risultati imprevedibili in quanto il sistema non effettua il matching sulle stringhe di soggetto.

Di solito la query viene interpretata dal sistema collegando implicitamente i termini con l'operatore AND ed eseguendo la ricerca su tutti i campi ${ }^{266}$. La maggior parte delle volte il sistema non riesce a riconoscere se i termini contenuti in una query fanno tutti parte della stessa stringa di soggetto, di un titolo, o di un punto d'accesso controllato (es. autore).

Negli OPAC tradizionali, dato che il sistema era incapace di 'comprendere' la query, un'utile possibilità di ricerca era data dal browsing dei soggetti e delle classi. Il browsing dei soggetti consentiva di partire da un termine per delimitare il campo e focalizzare la ricerca su un determinato aspetto. Tuttavia il browsing delle liste non veniva sfruttato appieno: un OPAC dovrebbe fornire infatti non solo la lista in cui il termine compare in prima posizione, ma l'elenco di tutte le stringhe di soggetto che contengono il termine selezionato con i loro collegamenti. Ciò non è quasi mai avvenuto nella ricerca negli OPAC tradizionali ${ }^{267}$. Bisogna inoltre ricordare che non tutti gli OPAC tradizionali erano in grado di sfruttare adeguatamente gli accessi per soggetto per produrre liste navigabili di soggetti, o alberi classificatori esplorabili, e tale inefficienza viene replicata negli OPAC di nuova generazione e nei discovery systems ${ }^{268}$.

Nei nuovi OPAC e nei principali strumenti di discovery la questione non è stata risolta: mentre in OPAC arricchiti è ancora possibile trovare browsing di soggetti e classi, tutti gli strumenti di discovery commerciali hanno abbandonato del tutto il browsing dei soggetti e delle classi e tutte le modalità tradizionali di scorrimento degli indici, per alcuni principali motivi:

\footnotetext{
${ }^{266}$ Nella maggior parte degli OPAC di nuova generazione (un esempio è il software VuFind, cfr. Appendice B. VuFind) questa opzione deve essere impostata dalla biblioteca, infatti l'operatore booleano di collegamento di default è OR. (cfr. Birong Ho - Keith Kelley -Scott Garrison, Implementing VuFind as an alternative to Voyager's WebVoyage interface: one library's experience, «Library Hi Tech», 27 (2009), n. 1, p. 82-92).

${ }^{267}$ Solo alcuni OPAC come Millennium e Voyager consentono questa funzionalità. Millennium è implementato all'Università cattolica del sacro cuore.

${ }^{268}$ Molti OPAC consentono una ricerca sulle sugli accessi per soggetto e delle liste solo tramite una ricerca per termine iniziale (tramite il troncamento automatico a destra), dunque non implementano la ricerca 'per termine e stringa' che dovrebbe invece fornire un elenco navigabile di tutte le stringhe contenenti il termine richiesto. In questo modo l'utenet avrebbe modo di selezionare la stringa corretta in base alla sua query e di proseguire la ricerca in modo mirato e aumentandone la precisione.
} 
1. offrendo un'interfaccia unica per il recupero di libri, articoli, ebook e altro materiale che la biblioteca possiede o che ha in abbonamento, più una serie di risorse esterne, il sistema riceve metadati di diversa omegeneità e completezza specie per quanto riguarda le informazioni semantiche. Non solo i metadati di queste risorse sono spesso privi di informazioni semantiche, ma quando essere vengono recuperate possono essere disomogenee per la diversità degli strumenti di organizzazione delle conoscenze utilizzati (es. non tutte usano gli stessi vocabolari o la stessa classificazione), dunque lo strumento di discovery non riesce a gestire nelle faccette questi metadati;

2. nei nuovi OPAC si preferisce fornire il browsing di canali opportunamente selezionati dalla biblioteca: tra questi i nuovi arrivi, scaffali tematici, categorie opportunamente distinte per audience.

Non è raro il caso di OPAC di nuova generazione che presentano in prima pagina proprio le categorie attraverso le quali effetturare il browsing, preferendo dunque come primo approccio al catalogo quello esplorativo. Il catalogo online di Ann Arbor District Libraries rappresenta un bell'esempio di approccio esplorativo ricco di canali tematici ${ }^{269}$.

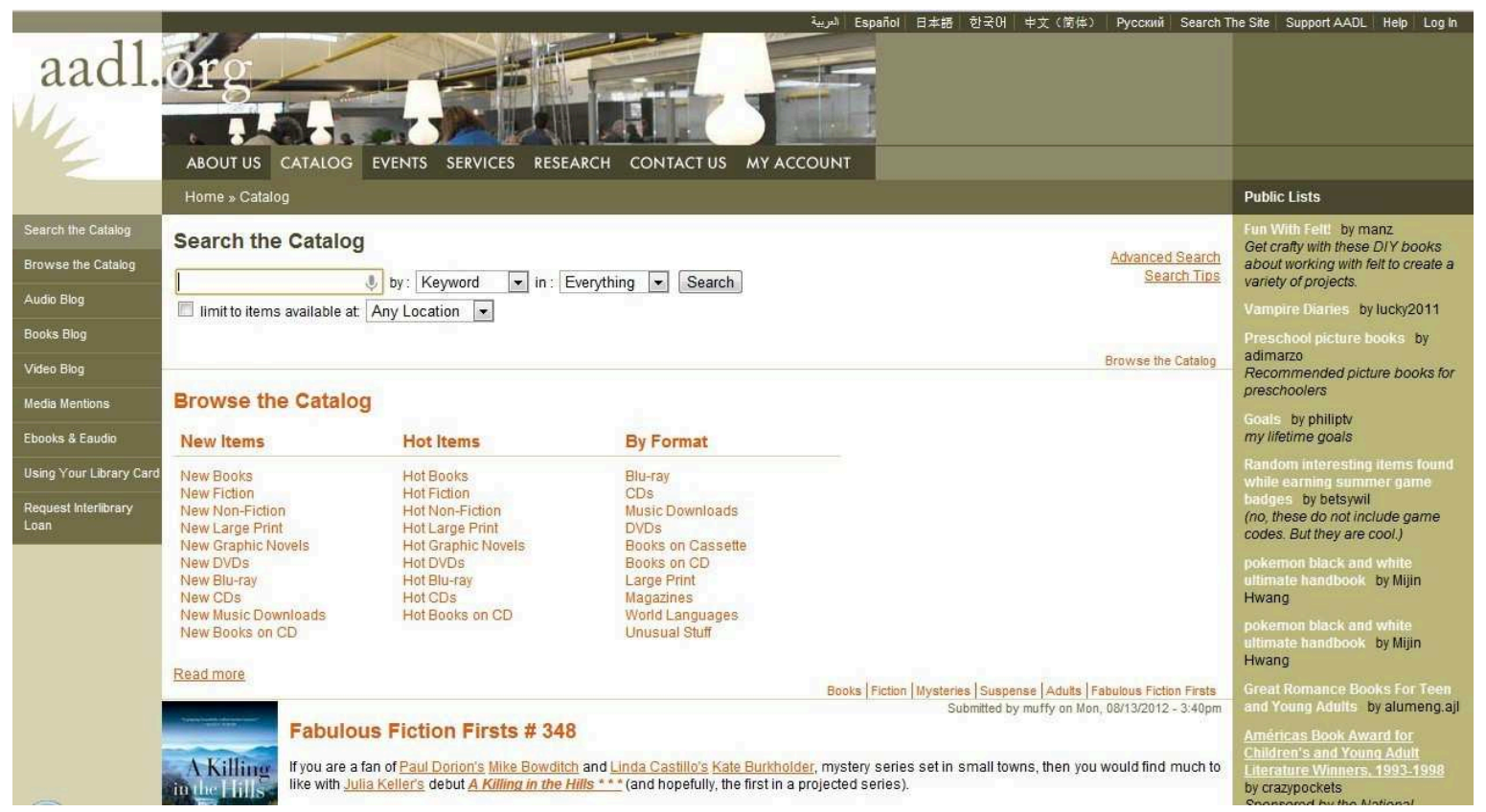

Fig. 2.13 - L'OPAC delle biblioteche di Ann Arbor: un esempio di ricchezza di canali d'accesso al catalogo 270 .

\footnotetext{
${ }^{269}$ Cfr. infra, Appendice A. Casi di studio. Ann Arbor District Libraries.

${ }^{270}<$ http://www.aadl.org/catalog/>.
} 
Come si vedrà più avanti questo approccio non è sbagliato ma, per essere efficace, va affiancato alla presenza di altre tecniche di ricerca che consentano di aumentare la precisione.

Dunque oggi ci si affida ad alcune soluzioni:

1. l'arricchimento dei record con informazioni supplementari sull'argomento. L'arricchimento con elementi ridondanti o non controllati provenienti dai sommari e da altre parti del record aumenta il richiamo a discapito della precisione: non consente di disambiguare i termini, né di creare raggruppamenti efficaci dei record da mostrare in liste navigabili. È così necessario un ordinamento per rilevanza che e necessiterebbe del supporto di thesauri;

2. relevance ranking e relevance feedback diventano soluzioni obbligate per rimediare ai difetti di una ricerca per parola chiave;

3. la creazione di un 'vocabolario di accesso', come proposto da Bates ${ }^{271}$, diventa una necessità sebbene questa soluzione non sia oggi praticata. Un vocabolario di accesso è una mappatura del linguaggio naturale con i termini controllati del sistema. Purtroppo l'implementazione di tali vocabolari è una scelta che si rivelerebbe utile, ma che non trova ancora applicazione.

La possibilità di mantenere la ricerca 'google like' come accesso preferito alle risorse di un catalogo, presuppone, per avere una mimima efficacia, che nei nuovi OPAC sia adottata una soluzione di questo tipo: una ricerca per parola chiave su tutti i campi deve effettuare il matching sulle voci di autorità: soggetti, classi, autori, titoli e consentire il relevance feedback attraverso la funzione di autocompletamento intelligente.

Next generation catalogs e discovery systems hanno seguito le linee di tendenza emerse dal dibattito internazionale e offrono per la ricerca per soggetto le seguenti soluzioni:

1. indicizzazione full text su termini del linguaggio controllato e del linguaggio naturale (parole chiave estratte da parti del record come il titolo, toc) tramite l'indicizzazione full text di tutto il record bibliografico quale alternativa alla ricerca per termini controllati sfruttando l'uso del linguaggio naturale utilizzato nei titoli, nei sommari, negli indici. Questo consente di avere una indicizzazione ridondante che aiuta l'utente in fase di ricerca con una maggiore varietà di termini che funzionano da punto di accesso;

2. arricchimento dei record tramite sommari e indici per aumentare il recupero e avere più termini per l'indicizzazione;

${ }^{271}$ Marcia J. Bates, Subject access in online catalogs: a design model cit. 
3. uso della 'faceted navigation' per estrarre alcune faccette di soggetto da mostrare all'utente, che indicano i termini controllati assegnati al set di risultati che viene proposto $^{272}$. Tuttavia questa presunta 'mappatura' del linguaggio naturale estrae soggetti attributi ad un elenco di risultati già imperfetto perché derivante dall'applicazione di algoritimi di ranking, dunque la scelta dell'utente avviene a posteriori e senza possibilità preventiva di scorrimento di liste, o di classi. Queste voci possono funzionare da filtri per il raffinamento quando delimitano un set dei risultati, ma non forniscono elenchi navigabili;

4. Suggerimenti per estendere la ricerca utilizzando le voci di soggetto non come lista navigabile ma in forma di link per lanciare una nuova ricerca.

In definitiva viene a mancare la possibilità di sfruttare il browsing in soggetti e classi; tale funzione viene spostata su nuovi 'canali' predisposti da ogni singola biblioteca e sulle potenzialità alquanto ridotte delle faccette.

L'arricchimento dei dati, dunque non sempre corrisponde ad un arricchimento delle funzioni di ricerca, anzi si traduce sovente in un impoverimento generale in termini di precisione. Ecco perché le interfacce di nuova generazione sono generalmente utili per offrire all'utente una ricerca semplificata ma spesso penalizzano la ricerca per soggetto, mentre gli strumenti di discovery sono del tutto carenti sotto questo importante aspetto.

\subsection{Conclusioni. I Limiti del modello attuale e i futuri scenari. Le tematiche aperte}

In definitiva, possiamo concludere questa lunga panoramica con una riflessione riguardante l'attuale stato dell' arte degli OPAC ed alcune brevi considerazioni sul modello che tali prodotti propongono, per capire fino a che punto il modello attuale di sviluppo degli OPAC di nuova generazione e di sistemi per la scoperta sia un modello sostenibile per la ricerca negli OPAC.

Nell'ultimo decennio si è assistito ad un'evoluzione degli OPAC che possiamo sintetizzare nel passaggio dall'OPAC tradizionale agli OPAC di nuova generazione che successivamente si sono evoluti nei cosiddetti discovery systems.

\footnotetext{
272 Secondo alcuni studiosi questa funzione consente una 'mappatura' dei termini utilizzati per l'interrogazione con quelli controllati che può sostituire la presentazione di una lista navigabile dei soggetti.
} 
I primi tentativi di miglioramento degli OPAC hanno visto, infatti, una fase di arricchimento del record catalografico grazie alla possibilità di inserimento di elementi provenienti dall'esterno; successivamente sono state colte le opportunità tecnologiche offerte dal Web 2.0 trasformando tali strumenti in OPAC 'di nuova generazione' e in alcuni casi enfatizzando le caratteristiche sociali del fenomeno (generando per qualche tempo i cosiddetti 'Social OPAC'); infine gli OPAC si sono ulteriormente evoluti in quelli che più correttamente vengono oggi definiti discovery systems o discovery interfaces ${ }^{273}$, strumenti dedicati più alla scoperta che al semplice reperimento, in quanto in grado di recuperare oltre ai record catalografici, anche risorse esterne.

Il lavoro di analisi ha rilevato che la sostituzione degli OPAC tradizionali con questi nuovi strumenti presenta ancora varie problematiche aperte.

Innanzitutto il panorama di questa lenta trasformazione è molto diversificato. In molti casi le biblioteche hanno mantenuto la possibilità di visualizzare l'OPAC tradizionale ritenuto lo strumento principale di accesso alle raccolte e riservato alle sole risorse possedute dalle biblioteche (ad esempio, l'Università di Harvard mantiene l'accesso al nuovo catalogo Hollis e al precedente Hollis classic ${ }^{274}$, l'Università della Virginia, oltre al nuovo VirgoBeta, presenta il tradizionale catalogo Virgo $^{275}$ ) e tante altre biblioteche sperimentano l'utilizzo di interfacce di nuova generazione affiancandoli a strumenti di discovery.

Molte altre istituzioni, specie accademiche e di ricerca hanno, invece, abbandonato il loro OPAC tradizionale affidandosi ad un nuovo sistema per la scoperta, in base all'idea che per l'utente che si approccia ad un sito web di una biblioteca, frazionare la ricerca in più strumenti diversi e che non comunicano tra loro come OPAC, banche dati, repositories, periodici elettronici, biblioteche digitali, può essere frustrante per gli utenti che non sanno dove cercare le informazioni e non sono a conoscenza di tutte le risorse a disposizione ${ }^{276}$. In questo modo tutto ciò che interessa viene recuperato tramite il discovery system. È un dato di fatto inoltre che gli utenti, abituati alla ricerca nel web preferiscono cercare l'informazione in un solo punto e trovarvi documenti di fruizione immediata e a livello più approfondito rispetto a quanto tradizionalmente offerto da un OPAC, ad esempio, vogliono

\footnotetext{
${ }^{273}$ Marshall Breeding dichiara che oggi al posto della denominazione $O P A C$ di nuova generazione è più corretto usare il termine discovery interfaces o discovery system. (Cfr.: Marshall Breeding, State of the Art in Library Discovery 2010, «Computers in libraries», 30 (2010), n. 1 p. 31-35, anche in <http://www.librarytechnology.org/ltg-displaytext.pl?RC=14574>).

${ }^{274}$ Cfr. infra, Appendice A. Casi di studio. Harvard University.

${ }^{275}$ Cfr. infra, Appendice A. Casi di studio. University of Virginia Library. Blacklite.

${ }^{276}$ In molte indagini tra gli utenti emerge spesso la scarsa conoscenza degli strumenti di ricerca di cui le biblioteche dispongono, in particolare delle risorse elettroniche (banche dati, periodici elettronici).
} 
recuperare un determinato capitolo di un libro o un articolo, richiedendo funzionalità che un OPAC tradizionale non riesce a gestire in maniera adeguata.

Questi nuovi prodotti sono variamente personalizzabili e possono essere usati come versione avanzata dell'OPAC (veri OPAC potenziati o di nuova generazione) o come sistemi per la scoperta, quando recuperano molti dati esterni al catalogo, oggetti digitali, dati di repositories e banche dati, etc.

Le biblioteche possono, ad esempio, adottare un discovery system open-source come VuFind decidendo come e quali debbano essere i dati da recuperare, se usarlo principalmente come interfaccia per l'OPAC, scegliendo così un'implementazione di "basso profilo"277, oppure usarlo per cercare a più ampio raggio in tutte le risorse della biblioteca, nell'OPAC e nelle banche dati, nella biblioteca digitale, etc. Tra i software disponibili, come già sottolineato, alcuni prodotti hanno portato al limite estremo questa capacità di recuperare più risorse possibili. Ad esempio Primo, Ebsco discovery service e Summon $^{278}$ sono integrati con pacchetti commerciali, basi dati, indici e risorse web e consentono di recuperare centinaia di milioni di registrazioni configurandosi come veri Web-Scale discovery services ${ }^{279}$.

Lo stato dell'arte oggi è quello di tante biblioteche che si sforzano di gestire tale 'sovrabbondanza informativa' ricorrendo, sperimentando, implementando questi nuovi strumenti.

Molte biblioteche che si servono di questi enormi contenitori di informazioni preferiscono affiancarli ai loro OPAC, come hanno fatto le biblioteche della North Carolina State University (NCSU). L'OPAC Endeca, ad esempio, cerca tra il posseduto della biblioteca; a tutto il resto provvede il discovery system Summon ${ }^{280}$.

Non tutte le biblioteche sono disposte a rinunciare al proprio OPAC per affidarsi completamente ad un discovery system anche perché l'implementazione di tali interfacce di scoperta pone alcuni limiti evidenti non ancora risolti relativi proprio alla loro funzione di strato aggiuntivo, come ad esempio quelli di personalizzazione, aggiornamento in tempo

\footnotetext{
${ }^{277}$ Un'implementazione di questo tipo in Italia è visibile nel nuovo OPAC del Polo bibliotecario biellese. Nonostante le funzionalità avanzate di VuFind rendano questo prodotto un sistema di discovery, il software è stato implementato ad un livello base di interfaccia amichevole per l'OPAC recuperando le sole risorse tradizionali a stampa.

${ }^{278} \mathrm{Si}$ vedano gli approfondimenti dei tre software (cfr. infra, Appendice B. Software per OPAC di nuova generazione e servizi di Web scale discovery).

${ }^{279}$ Ad esempio, il software Primo recupera le informazioni provenienti dai repositories locali (OPAC, archivi digitali, ambienti di e-learning,altro della biblioteca/biblioteche collegate e da Primo Central che contiene centinaia di milioni di record, risorse in licenza d'uso e ad accesso libero, e-books, e-journals, banche dati bibliografiche, risorse remote (di cui oltre 600 ad accesso gratuito), milioni di e-journals ed $e$-books.

${ }^{280}$ Cfr. infra, Appendice A. Casi di studio. North Carolina State University. Endeca.
} 
reale dei dati dall'ILS e di integrazione con altri strumenti dell'ILS o dei portali che richiedono come abbiamo visto ulteriori autenticazioni ${ }^{281}$.

Alcune istituzioni preferiscono mantenere l'OPAC preesistente magari rinnovando l'interfaccia con un next generation catalog, dando la possibilità di visualizzare persino l'OPAC tradizionale, altre si affidano con fiducia ai nuovi sistemi di scoperta.

Di fatto però la sostituzione di OPAC con sistemi e interfacce per la scoperta sta lentamente avvenendo, così presto la maggior parte dei cataloghi verrà sostituita con questi nuovi strumenti. Attualmente le biblioteche che adottano sistemi per la scoperta e next generation catalogs sono in aumento; sono poche però le biblioteche che sostituiscono del tutto l'OPAC con un sistema per la scoperta. Si tratta per la maggior parte di biblioteche accademiche, che ricorrono allo strumento di discovery per ovviare alla metaricerca. Tuttavia il dato è in aumento; lo dimostrano le recenti implementazioni di Primo all'Università Ca' Foscari e di Summon nell'ateneo di Palermo ${ }^{282}$ : in entrambe le realtà questi strumenti per la scoperta, che operano in maniera lievemente differente, sostituiscono l'OPAC tradizionale e vengono presentati come unica interfaccia di ricerca.

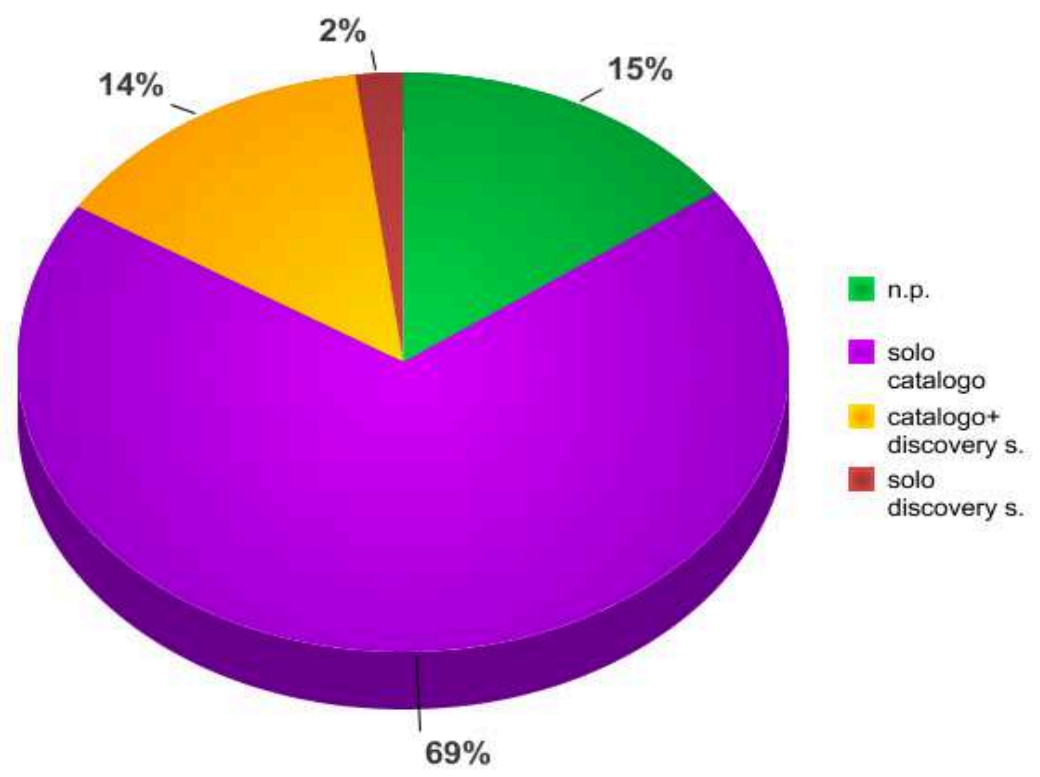

Fig. 2.14 - Le biblioteche che hanno sostituito il loro OPAC con un sistema di discovery sono ancora una piccola percentuale. La maggior parte delle biblioteche che ha adottato un sistema per la scoperta preferisce affiancarlo all’OPAC già esistente ${ }^{283}$

\footnotetext{
${ }^{281}$ Solo in questi ultimi anni si sta provvedendo a studiare metodi per l'autenticazione federata in grado di garantire il 'single sign on istituzionale' autenticando gli utenti localmente e autorizzandoli ad accedere a più risorse (Shibboleth <http://shibboleth.net/> è un software open source che offre quest'opportunità).

${ }^{282}$ Cfr. infra, Appendice A. Casi di studio, Università di Palermo.

${ }^{283}$ Rielaborazione grafica dei dati forniti in Sharon Q. Yang, Melissa A. Hofmann, Next generation or current generation?: A study of the OPACs of 260 academic libraries in the USA and Canada, «Library Hi Tech», 29 (2011), n. 2 p. $266-300$.
} 
Cosa ne sarà dell'OPAC al termine di questo processo di evoluzione è ancora presto per dirlo. Si possono però ipotizzare alcuni scenari plausibili:

l'OPAC potrebbe assolvere una funzione meramente inventariale, di localizzazione dei documenti presso una tal biblioteca. Le funzioni di ricerca verrebbero svolte da sistemi di scoperta (discovery system) dalla copertura sempre più ampia. L'OPAC tradizionale verrebbe assorbito totalmente in questi sistemi. In questo scenario le biblioteche sacrificano l'OPAC tradizionale, ma è conveniente adottare alcuni accorgimenti: le interfacce devono essere estremamente chiare all'utente che deve essere consapevole in ogni momento di dove sta cercando (nell'OPAC locale, nel posseduto di altre biblioteche? in una banca dati libera o a pagamento? in internet? nel repository istituzionale?) Questo scenario è quello più plausibile e verso il quale puntano i produttori di software;

l'OPAC potrebbe rimanere uno strumento autonomo, con la propria interfaccia di ricerca di nuova generazione (quindi più amichevole per l'utente) affiancato da uno strumento di discovery per la ricerca a più ampio raggio (come nel caso, già illustrato, di NCSU libraries). Le biblioteche in questa seconda ipotesi preferiscono mantenere più punti di accesso alle loro collezioni mantenendo l'OPAC uno strumento separato di accesso alle collezioni locali che la biblioteca dispone. Questo secondo scenario è quello attuale e, al momento, il più desiderabile, purché vengano mantenute alcune funzionalità complesse di recupero semantico che si ritrovano negli OPAC tradizionali.

Rimangono dunque ancora delle aree aperte di riflessione che, specie alla luce della nuova evoluzione in direzione dei linked data, meritano una riflessione quando si progettano questi nuovi strumenti.

Innanzitutto l'ingresso nell'OPAC di risorse esterne rende più complessa un'esposizione ordinata dei risultati; la soluzione adottata è quella di proporre la ricerca per parola chiave come ricerca predefinita, di adottare l'ordinamento per rilevanza (relevance 
ranking) come modalità di visualizzazione dei risultati e di offrire una serie di filtri per il raffinamento in forma di faccette ${ }^{284}$.

Questa modalità di navigazione del catalogo è certamente una novità rispetto ai cataloghi tradizionali ma, chiaramente, è ben al di sotto delle reali potenzialità degli OPAC. L'utente può, infatti, scegliere di navigare tra raggruppamenti di dati che condividono una proprietà particolare (ad esempio una certa data, un certo soggetto, etc. ) per filtrare l'immenso numero di risultati che riceve da una ricerca per parola chiave. Si tratta, però, più che di una possibilità, di una vera e propria necessità, quella di filtrare una ricerca che ha un alto richiamo (e di 'rumore') e un grado bassissimo di precisione, a causa dell' assenza di altri meccanismi logici di ordinamento.

$\mathrm{Al}$ di là degli arricchimenti dei cataloghi e di alcuni utili ausili alla ricerca, come, ad esempio, la correzione dello spelling, la funzione 'forse cercavi', ed altre tecniche sofisticate di information retrieval (la logica fuzzy e lo stemming), i nuovi cataloghi non apportano ancora quelle modifiche sostanziali che dovrebbero consentire agli utenti di navigare in maniera ordinata nell'universo bibliografico ivi rappresentato. Alcune prime indagini sull'uso dei cataloghi di nuova generazione e discovery systems hanno rilevato alcuni elementi interessanti:

1) alcuni arricchimenti (come ad esempio i sommari) si rivelano utili ai fini della valutazione della pertinenza dei documenti;

3) gli utenti gradiscono la possibilità di filtrare i dati usando le faccette (raggruppamenti) e considerano questa caratteristica più importante del relevance ranking;

2) le funzioni sociali non sono in genere richieste. Gli utenti non sono interessati a contribuire con recensioni, né a visualizzare le raccomandazioni degli altri utenti, né ad usare le folksonomie, ma, stranamente, vorrebbero avere la possibilità di visualizzare una nuvola di tag ${ }^{285}$.

\footnotetext{
${ }^{284}$ Tutti questi dati, se importati nell'OPAC, vengono forniti in un'interfaccia unica di ricerca, deduplicati e visualizzati ordinati per ranking, con la tipica faccettazione dei risultati. In caso contrario, vengono utilizzati link resolvers per collegarsi con le risorse esterne (in cui di norma è richiesta l'autenticazione).

${ }^{285}$ Gli studi sull'utenza cui si fa riferimento sono quelli di WinnieTam - Andrew M. Cox- Andy Bussey, Student user preferences for features of next-generation OPACs: A case study of University of Sheffield international students. «Program: Electronic Library \& Information Systems», 43 (2009) n. 4, p. 349-374 e Jeppe Lomholt Akselbo - Lise Arnfred, Sten Barfort [et al.], The hybrid library: from the users' perspective: a report for the DEFF Project "The loaners" expectations and demands for the hybrid library. February 2006, National Library and Copenhagen University Library, Aarhus School of Business Library, State and University Library, and University Library of Southern Denmark, 21 September 2006, $<$ http://www.statsbiblioteket.dk/summa/fieldstudies.pdf/view $>$.
} 
Questi risultati all'apparenza contraddittori, tradiscono probabilmente un bisogno di ordine, di logica e di coerenza. Come ha recentemente sottolineato Fattahi ${ }^{286}$ infatti:

i cataloghi in linea non sono in grado di presentare un quadro chiaro dell'universo bibliografico. Risultati di ricerca disseminati disordinatamente, moltiplicati in recuperi di grandi dimensioni, danno luogo a liste di record recuperati che devono essere riorganizzati, se vogliamo che siano di qualche utilità.

Queste riflessioni ci inducono a concludere che oggi il processo di rinnovamento degli OPAC debba passare per altre strade, e non imboccare quelle 'scorciatoie' poco praticabili rappresentate dall'approccio algoritmico, dalla chiusura dei dati, dalla riproduzione automatizzata dei processi della conoscenza.

La coerenza e l'autorevolezza del catalogo elettronico dovranno manifestarsi in più direzioni che si possono così sintetizzare:

* un'appropriata rappresentazione nel catalogo delle entità che compongono l'universo bibliografico e delle loro relazioni attraverso l'implementazione del modello FRBR nei cataloghi;

* l'apertura e il collegamento dei 'dati catalografici' nel web, attraverso l'adozione di standard aperti e interoperabili;

* un'adeguata riprogettazione del processo di ricerca nell'OPAC, che tenga conto di come gli utenti cercano l'informazione bibliografica che sia capace di creare flussi di conoscenze all'interno del catalogo elettronico e di collegarsi con il più ampio spazio di tutte le risorse presenti nel web.

\subsection{Appendice. Lavorare con i metadati. Extensible Catalog}

EXtensible Catalog sviluppato all'Università di Rochester è, al momento, tra i progetti più innovativi nella creazione di un OPAC 'di nuova generazione' in grado di esprimere le entità previste dal modello FRBR. Partner del progetto sono varie biblioteche e istituzioni ${ }^{287}$ tra le quali OCLC, Andrew W. Mellon Foundation e le Università di Cornell e Yale.

\footnotetext{
${ }^{286}$ Rahmatollah Fattahi, From information to knowledge: superworks and the challenges in the organization and representation of the bibliographic universe =Dall'informazione alla conoscenza: le super-opere e le sfide dell'organizzazione e rappresentazione dell'universo bibliografico: Lectio magistralis in Biblioteconomia, Firenze, Università degli Studi di Firenze, 2010, Firenze, Casalini Libri, 2010.

287 Partner del progetto: University of Rochester, The Andrew W. Mellon Foundation, Consortum of Academic and Research Libraries in Illinois (CARLI); University of Notre Dame; Rochester Institute of
} 
$\mathrm{XC}$ può essere definito un modello di next generation catalog che interviene sulla struttura dei dati e implementa il modello FRBR. Il software mette in atto procedure di conversione dei record studiate per poter ottenere le entità previste dal modello. L'architettura di progettazione è basata su un approccio completamente diverso da quello visto nei NGC e discovery systems, che lavora sulla struttura dei dati ed è noto come "Dublin Core Application Profile"288.

XC Schema combina elementi di metadati da due diversi schemi, RDA and Dublin Core (DC), cui aggiunge nuovi XC schema elements ${ }^{289}$. Agli elementi DC aggiunge 22 elementi RDA tra cui: elementi con funzione descrittiva che mancano a DC; elementi con funzione di espressione delle entità FRBR.

I records MARC vengono dunque convertiti in XC e gli elementi del record originale vengono suddivisi in diversi record che rappresentano le 4 entità FRBR.

In sostanza, nell'attuale situazione di transizione, la soluzione dell'eXtensible Catalog basata sulla conversione dal tradizionale formato MARC a un formato misto appare la scelta più innovativa e più compatibile con la necessità di convertire i record dal vecchio al nuovo formato. A differenza di discovery systems come Primo, XC interviene sulla struttura dei dati incorporando gli elementi previsti da RDA e suddividendo il record tradizionale nelle entità previste dal modello.

I discovery systems non fanno questa operazione: WorldCat e VuFind non prevedono schemi estendibili di metadati ${ }^{290}$. La possibilità di estendere un set di metadati in XML è la chiave di volta per l'implementazione di FRBR nei sistemi. La 'struttura del record' può essere modificata in una nuova struttura in grado di accogliere nuove entità FRBR, anche tramite link esterni (es un rimando ad un'entità persona in WordlCat Identities).

Technology; Kyushu University, University of North Carolina - Charlotte; Serials Solutions; OCLC, University of Buffalo, Cornell University, Yale University, Ohio State University, Nylink.

${ }^{288}<$ http://dublincore.org/usage/documents/profile-guidelines/>.

289 Lo schema ì visibile $<$ http://www.extensiblecatalog.org/sites/default/files/fulltext/XCSchemaProperties20090107.pdf>.

${ }^{290}$ Il software Primo (cfr.: infra, Appendice B. Software per OPAC di nuova generazione e servizi di Web scale discovery. Primo.) contiene un set estendibile di metadati in formato XML che consente l'inserimento di nuovi campi, ma non consente di esprimere nuove relazioni, dunqie non consente di esprimere nuove entità e relazioni. Può solo creare raggruppamenti di manifestazioni. 


\begin{tabular}{|c|c|c|}
\hline Property Name in Schema & $\begin{array}{l}\text { URI (note that URIs for XC } \\
\text { properties are provisional) }\end{array}$ & $\begin{array}{l}\text { Description (if XC } \\
\text { property) }\end{array}$ \\
\hline dcterms:abstract & http://purl.org/dc/terms/abstract & \\
\hline rdarole:artist & http://rdvocab.info/roles/artist & \\
\hline dcterms:audience & http://purl.org/dc/terms/audience & \\
\hline rdarole:author & http://rdvocab.info/roles/author & \\
\hline rdarole:compiler & http://rdvocab.info/roles/compiler & \\
\hline rdarole:composer & http://rdvocab.info/roles/composer & \\
\hline $\begin{array}{l}\text { rdvocab:coordinatesOfCartograp } \\
\text { hicContent }\end{array}$ & $\begin{array}{l}\text { http://rdvocab.info/Elements/coordinat } \\
\text { esOfCartographicContent }\end{array}$ & \\
\hline dcterms:coverage & http://purl.org/dc/terms/coverage & \\
\hline xc:coverage & $\begin{array}{l}\text { chttp://www.extensiblecatalog.info/Ele } \\
\text { ments/coverage }\end{array}$ & $\begin{array}{l}\text { A parallel property } \\
\text { to the dcterms } \\
\text { property of the } \\
\text { same name, } \\
\text { defined to enable } \\
\text { use of link to } \\
\text { Library of } \\
\text { Congress authority } \\
\text { files (lcnaf) }\end{array}$ \\
\hline dcterms:creator & http://purl.org/dc/terms/creator & \\
\hline xc:creator & $\begin{array}{l}\text { http://www.extensiblecatalog.info/Ele } \\
\text { ments/Creator }\end{array}$ & $\begin{array}{l}\text { A parallel property } \\
\text { to the dcterms } \\
\text { property of the } \\
\text { same name, } \\
\text { defined to enable } \\
\text { use of an attribute } \\
\text { to link }\end{array}$ \\
\hline
\end{tabular}

Fig. 2.15 - Un estratto dello schema XC con alcuni elementi relativi all'opera. Gli elementi del set provenienti dagli schemi DC e RDA vengono integrati con nuovi elementi (xc: $)^{291}$

Lo schema deriva da una suddivisione del record 'piatto' di MARCXML in record collegati e basati sulle entità previste dal modello FRBR, cui viene aggiunto un record relativo alle localizzazioni (XC Holdings); tutti i record vengono forniti di un identificatore unico.

Il workflow dei metadati che le diverse componenti del software gestiscono prevede le 5 fasi di conversione, normalizzazione, trasformazione, aggregazione, indicizzazione.

La conversione viene effettuata dal modulo XC OAI Toolkit, compatibile con la maggior parte degli ILS, espone i metadati dell'ILS nell'interfaccia di XC, sincronizza i cambiamenti nei record dell'ILS automaticamente, converte il MARC in MARC XML, risolve il problema degli identificatori.

I seguenti tre passaggi (normalizzazione, trasformazione e aggregazione) vengono gestiti dal modulo XC Metadata Services Toolkit è uno strumento per processare e aggregare i metadati che può elaborare una mole ingente di metadati da diverse fonti

\footnotetext{
${ }^{291}<$ http://www.extensiblecatalog.org/sites/default/files/fulltext/XCSchemaProperties20090107.pdf>.
} 
(silos), risolvere i problemi dei formato e gestire la qualità dei metadati. Il modulo aggrega, de-duplica i metadati, e sincronizza i cambiamenti nell'interfaccia di discovery.

Nel procedimento di normalizzazione (MARCXML Normalization Service) si risolvono problemi di spelling, si normalizzano le forme degli identificativi OCLC, si sostituiscono alcuni codici che possono essere meglio visualizzati nelle faccette, si scompongono i soggetti di LCSH in modo che le parti che li costituiscono possano essere rappresentati nelle apposite faccette (di genere, geografiche, argomento, etc).

Successivamente si interviene con la trasformazione di MARCXML in XC. Si suddividono i record MARC in record basati su FRBR in base a XC Schema in modo che ad un record in input corrispondano in output vari record, si gestiscono le relazioni tra $\mathrm{i}$ diversi record prodotti, vengono creati i record opera e espressione, si gestiscono i casi di libri legati insieme,

Il modulo gestisce l'aggregazione dei record che rappresentano la stessa risorsa a livello di manifestazione, e opera, gestisce le relazioni tra i record (che rappresentano diverse entità FRBR) pone le basi per l'implementazione di RDA al di fuori del MARC. Il modulo consente la normalizzazione e la trasformazione di altri schemi e la trasformazione dei dati XC in RDF, cioè in triple dei linked data.

L'indicizzazione viene gestita dal modulo XC Drupal Toolkit, un CMS che si preoccupa di importare i metadati in Drupal. Apache Solr, motore di ricerca opensource, viene utilizzato per consentire un risultato tramite faccette, e cercare contemporaneamente nel catalogo, in tutti i repositories collegati, e nelle risorse del sito web. L'integrazione con l'ILS per i dati sulla circolazione viene gestito da un modulo separato NCIP Toolkit, che ottiene dati aggiornati. 


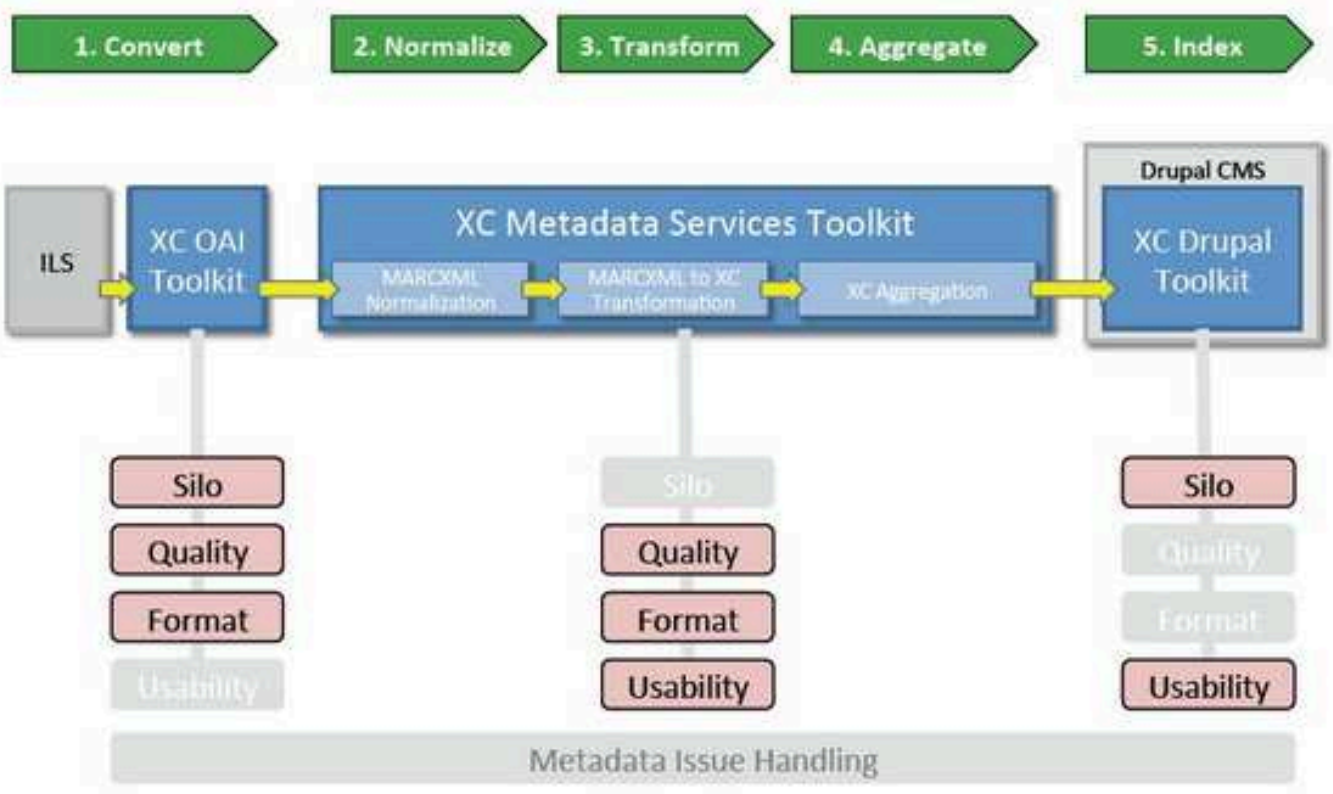

Fig. 2.16 - Il processo di conversione e trasformazione dei metadati compiuto dalle componenti del software $\mathrm{XC}^{292}$

Paragonato all'approccio di XC il modello di sviluppo dei discovery systems e dei next generation catalogs è dunque lontano da una progettazione che si basa sulla trasformazione e rielaborazione della struttura dei record: ecco perché tali strumenti rivelano tutta la loro debolezza in fase di ricerca: essi si basano su algoritmi invece di creare nuove entità, dunque non sono in grado di esprimere entità e relazioni previste dal modello.

\footnotetext{
${ }^{292}$ David Lindahl, EXtensible Catalog: software portfolio, presentazione tenuta a ALA Midwinter 2011 LITA Next Generation Catalog IG, 9 gennaio 2011, $<$ http://docushare.lib.rochester.edu/docushare/dsweb/Get/Document-47024>.
} 


\section{CAPITOLO 3. L'OPAC E IL MODELLO FRBR} «L'efficacia di un sistema per accedere all'informazione
è una funzione diretta dell'intelligenza adoperata per
organizzarlo»

Elaine Svenonius

\subsection{Introduzione}

Come abbiamo avuto modo di rilevare nei precedenti capitoli, il modello di sviluppo in uso nei nuovi cataloghi elettronici denominati next generation catalogs e nei discovery systems presenta una serie di caratteristiche nuove che consentono di avvicinare maggiormente gli utenti ai cataloghi, offrendo loro una ricerca più simile a quella del web e introducendo caratteristiche tipiche degli ambienti da loro più frequentati e conosciuti, come le reti sociali o i siti di commercio elettronico.

La principale carenza degli attuali OPAC, tuttavia, non è quella di essere strumenti poco personalizzati, arricchiti o 'sociali', ma l'incapacità di rappresentare ordinatamente l'universo bibliografico e di consentire ai suoi utilizzatori una navigazione logica e significativa.

La navigazione nel catalogo si svolge spesso per tentativi e gli aiuti rappresentati dai filtri per la ricerca offerti dalla cosiddetta 'navigazione a faccette' soccorrono l'utente in un momento successivo rispetto alla presentazione del set dei risultati, esposti per rilevanza.

L'esposizione dei risultati per ranking di presunta rilevanza per l'utente, come abbiamo avuto modo di osservare, non rende conto della complessità e della ricchezza delle relazioni tra le entità rappresentate nel catalogo e finisce dunque per impoverire la navigazione e comprometterne l'efficacia.

A maggior ragione oggi, le interfacce rinnovate degli OPAC di nuova generazione e dei discovery systems sembrano confermare la tendenza a utilizzare queste soluzioni di riordino e presentazione dei risultati anziché focalizzare gli studi sulle modalità di realizzare ed evidenziare quella rete di relazioni che consentirebbe una presentazione ordinata, secondo un «principio direttivo costante» e immediatamente riconoscibile, di tutte le entità dell'universo bibliografico ${ }^{1}$.

\footnotetext{
${ }^{1}$ Cfr Carlo Bianchini, FRBR prima di FRBR. Il numero di libro nella Colon Classification, «JLIS.it», 1 (2010), n. 1, p. 11-39, <http://leo.cilea.it/index.php/jlis/article/view/31>. Così lo studioso si esprime in merito all'utilizzo dell'ordinamento per rilevanza nei cataloghi elettronici e nel catalogo del consorzio OCLC
} 
La maggior parte degli OPAC di nuova generazione e dei discovery systems (nei casi in cui questi strumenti vengano a soppiantare l'OPAC tradizionale) non consente all'utente di navigare dall'opera e dalle sue espressioni alle manifestazioni fino ad arrivare alle copie e di visualizzarne le relazioni con le altre entità, di manifestare il reticolo di relazioni tra le entità, ovvero la struttura sindetica del catalogo.

È proprio la funzione di 'navigare', introdotta nei nuovi ICP tra le funzioni del catalogo, a mancare nelle nuove interfacce, che non sono in grado di offrire un'appropriata organizzazione dei dati bibliografici.

In definitiva, ormai da più di un decennio il modello FRBR si propone come valida alternativa all'attuale modello di sviluppo degli $\mathrm{OPAC}^{2}$. Quali sono dunque le principali problematiche di implementazione e perché ancora oggi lo sviluppo degli OPAC non prende in considerazione l'esposizione dei dati secondo il modello? Per rispondere a queste domande nel presente capitolo si esamina la nascita, l'evoluzione del modello FRBR e le problematiche di applicazione e implementazione ai cataloghi elettronici.

\subsection{Il modello FRBR. Nascita e sviluppi della "famiglia FRBR". FRAD e FRSAD}

Nell'ambito degli studi compiuti nell'ultimo ventennio, Functional requirements for bibliographic records $(F R B R)$ rappresenta senza dubbio la riflessione teorica più rilevante. Oggi principi e norme catalografiche si ispirano a questo importante studio elaborato nel

\footnotetext{
WorldCat, (p. 16): «i cataloghi elettronici, anche quelli cosiddetti FRBR-zzati, non prevedono una struttura adeguata per evidenziare la maggior parte delle relazioni che sussistono tra tutte le risorse bibliografiche e per navigare realmente nell'intero spazio bibliografico rappresentato dal catalogo. Anzi, in WorldCat proprio il riconoscimento dell'impossibilità di fornire ordinamenti diversi, ha suggerito di inserire la funzione di "ordinamento per rilevanza", che si basa probabilmente su un algoritmo simile a quello presente in Google e in altri motori di ricerca. Eppure ciò che distingue un catalogo realizzato da professionisti dell'informazione da uno 'stupido' motore di ricerca è proprio la rete di relazioni che consentono una presentazione ordinata, secondo un "principio direttivo costante" immediatamente riconoscibile, di tutte le entità dell'universo bibliografico».

${ }^{2}$ IFLA. Study Group on the functional requirements for bibliographic records, Functional requirements for bibliographic records: final report, Münich, K. G. Saur, 1998. Disponibile anche online all'URL: <http://www.ifla.org/VII/s13/frbr/frbr.pdf>, o: <http://www.ifla.org/VII/s13/frbr/frbr.htm>. Trad. italiana: Requisiti funzionali per record bibliografici, ed. italiana a cura dell'Istituto centrale per il catalogo unico delle biblioteche italiane e per le informazioni bibliografiche, Roma, ICCU, 2000. Ultima versione: IFLA Study Group on the functional requirements for bibliographic records. Functional requirements for bibliographic records: final report. Approved by the Standing Committee of the IFLA Section on Cataloguing. September 1997. As amended and corrected through February 2009, <http://www.ifla.org/VII/s13/frbr/frbr_2008.pdf>.
} 
1998 dall'IFLA. Il merito dello studio è stato quello di intraprendere, a quarant'anni dai Principi di Parigi ${ }^{3}$, una profonda riflessione sugli oggetti della catalogazione e sulle funzioni dei record bibliografici alla luce dei nuovi cambiamenti emersi nell'ultimo cinquantennio, tra i quali:

* l'evoluzione delle tecnologie, che ha permesso la creazione e lo scambio di record bibliografici a livello mondiale;

* la moltiplicazione di nuove tipologie di materiali da gestire quali le risorse elettroniche;

* la necessità di ridurre i costi della catalogazione e di favorire l'interoperabilità;

* la comparsa di nuove modalità di trasmissione e accesso all'informazione (dalle riviste elettroniche al più recente movimento Open Access);

* l'esigenza di produrre interfacce migliori per la ricerca, che riproducano in maniera adeguata l'universo bibliografico che vi è rappresentato allo scopo di migliorare l'esperienza di ricerca dell'utente.

Lo studio dell'IFLA, inoltre, ha dato immediato impulso al rinnovamento dei principi e delle norme catalografiche a livello internazionale ${ }^{4}$. Merito di FRBR è stato soprattutto quello di rielaborare i principi già esistenti in una luce funzionale fornendo una solida base per la rielaborazione di nuovi principi di catalogazione e risolvendo alcune questioni che erano rimaste irrisolte nella precedente elaborazione:

La triade di elementi che costituiscono la natura dell'oggetto bibliografico (opera, edizione, copia) che hanno rappresentato e sono i perni attorno ai quali si è sviluppata la prassi e la

${ }^{3}$ Cfr. Pino Buizza, Dai Principi di Parigi a FRBR, «Bibliotime», V, (2002) n. 1, $<$ http://www.spbo.unibo.it/bibliotime/num-v-1/buizza.htm>.

${ }^{4} \mathrm{La}$ differenza tra principi, funzioni e requisiti viene così espressa da Mauro Guerrini, Le funzioni del catalogo dall'ICCP a FRBR, AIB. SEZIONE TOSCANA, <http://www.aib.it/aib/sezioni/toscana/> [2000], [risorsa online], <http://www.aib.it/aib/sezioni/toscana/conf/frbr/guerrini.htm>:

«Possiamo tracciare lo sviluppo della teoria catalografica in tre fasi:

1. la formulazione di principi;

2. l'individuazione di funzioni;

3. la definizione di requisiti.

I principi sono teorici, le funzioni sono pragmatiche, i requisiti sono funzionali.

1. I principi sono orientati alla costruzione di norme catalografiche. La loro formulazione ha consentito di porre le basi della catalografia moderna, come i principi elaborati da Lubetzky per la costruzione degli accessi, che hanno costituito la base delle decisioni assunte dall'ICCP.

2. Le funzioni - ad esempio, quelle stabilite da Charles A. Cutter nelle sue Rules for a dictionary catalogue del 1876, da Shiyali Ramamrita Ranganathan in Classified catalogue code del 1934, da Seymour Lubetzky in Code of cataloging rules del 1960 o da S. Michael Malinconico alla fine degli anni Settanta - hanno lo scopo di connotare il catalogo come un insieme coerente, logico e sistematico di registrazioni di documenti. La funzione di localizzazione (finding or location function) facilita il reperimento "di una particolare pubblicazione, ovvero di una particolare edizione di un'opera che si trova in biblioteca", e la funzione di raggruppamento (gathering or collocation function) persegue l'obiettivo di riunire, collegare ed organizzare i record.

3. I requisiti sono gli elementi di funzionalità che permettono al catalogo di essere efficace; FRBR li definisce in relazione alle operazioni che gli utenti compiono quando ricercano informazioni in bibliografie nazionali e in cataloghi». 
teoria catalografica moderna sono stati analizzati in passato soprattutto come enunciati di principio e non quali oggetti della catalogazione e quali oggetti ricercati dagli utenti. Le definizioni e le derivazioni normative scaturite da questa impostazione sono andate invariabilmente a cozzare con la realtà strumentale del catalogo. Ciò che è mancato finora è stata la chiara definizione strutturale, formale e funzionale della registrazione e del catalogo. FRBR non trasforma i principi catalografici, bensì li rielabora in una luce funzionale con l'applicazione all'analisi del record degli strumenti tipici della modellazione delle basi dati relazionali ${ }^{5}$.

Di fatto, come vedremo, l'elaborazione teorica fornita da FRBR ha dato modo di sanare quella dicotomia tra le funzioni del catalogo che all'epoca dei principi parigini era stata sollevata $^{6}$ e di fatto si era imposta; i principi di Parigi avevano lasciato fondamentalmente in sospeso l'annosa questione sull'oggetto della catalogazione, che aveva visto contrapporsi le teorie di Seymour Lubetzki, che infine avevano prevalso nella stesura dei Principi, alle argomentazioni di Eva Verona ${ }^{7}$ :

\footnotetext{
${ }^{5}$ Carlo Ghilli - Mauro Guerrini, Introduzione a FRBR : Functional requirements for bibliographic records $=$ Requisiti funzionali per record bibliografici, Milano, Bibliografica, 2001, p.27-28.

${ }^{6}$ Anche se in realtà, tale dicotomia- secondo il parere di Teresa Grimaldi - sembra avere non tanto un fondamento teoretico né sembra dipendere da fattori strettamente tecnologici, piuttosto andrebbe ricondotta al mancato recepimento, nella pratica catalografica delle teorie sul concetto di opera già elaborate da Lubetzky: «In realtà quella dicotomia non aveva alcun fondamento teoretico: si trattava, piuttosto, di recepire i risultati più maturi e consapevoli della recente riflessione sui principi catalografici, in particolare quelli raggiunti da Lubetzky, che, sulla scia delle profonde intuizioni catalografiche di Panizzi e Cutter, puntualizzava che l'aspetto più elusivo ma intrinsecamente più importante del libro è l'identità dell'opera in esso contenuta; e di calibrare, di conseguenza, la metodologia descrittiva e i meccanismi catalografici al fine di caratterizzare e correlare opportunamente tutti i molteplici aspetti della complessa realtà ideale e materiale dei documenti. Tuttavia la dicotomia non solo fu posta, ma fu risolta in maniera ambigua e contraddittoria: infatti, l'obbligato ossequio del mondo catalografico occidentale alla tradizione letteraria induceva al privilegiamento dell'opera; ma, in mancanza di una definizione esplicita e puntuale, e sulla base di un ingiustificato capovolgimento categoriale, l'essenza dell'opera veniva individuata in riferimento, più che alla identità di una sostanza intellettuale, alla sua concretizzazione in un prodotto bibliografico-editoriale, coincidente il più delle volte con il documento, e si giungeva addirittura, attraverso un rimando tautologico tra opera e pubblicazione, alla assunzione che ogni pubblicazione è una nuova opera a sé stante: rimando fondato sul disconoscimento della creatività come il tratto essenziale dell'opera. Di qui derivano insanabili contraddizioni all'interno della politica di indicizzazione, come quella determinata - nei Principi di Parigi e nei codici di diretta derivazione - dalla duplice pretesa di 1) riflettere adeguatamente in un'unica registrazione catalografica entrambi gli aspetti della complessa entità bibliografica, la creazione intellettuale di un autore e il medium usato per trasmetterla; 2) di adottare due distinte procedure, incaricata l'una di descrivere l'oggetto fisico, l'altra, tramite la formulazione dell'intestazione, l'entità intellettuale. Ciò spiega perché l'indicizzazione delle opere non è ritenuta una funzione descrittiva autonoma e primaria, ma risulta un'attività informativa residuale rispetto al compito principale di descrivere i documenti in cui quelle sono contenute. La contraddittorietà di queste assunzioni non è determinata da difficoltà di tipo tecnologico - la necessità di assumere, nei cataloghi manuali, un'unità di registrazione bibliografica di base - ma dalla mancata chiarificazione teoretica dell'oggetto della catalogazione descrittiva: infatti, ancora oggi, nonostante la struttura multidimensionale e relazionale del catalogo informatizzato, permane come oggetto principale del catalogo un'unica classe di oggetti, l'entità discreta del documento, in assenza di principi e procedure logicoconcettuali adeguate all'analisi e indicizzazione delle multiformi e stratificate realtà del documento» (Teresa Grimaldi, L'oggetto della descrizione bibliografica, Seminario su FRBR (Functional requirements for $\begin{array}{lllll}\text { bibliographic } \quad \text { records), } & \text { Firenze, } & \text { 27-28 }\end{array}$ <http://www.aib.it/aib/sezioni/toscana/conf/frbr/grimaldi.htm>.

${ }^{7}$ Sulla questione si veda Paul Gabriele Weston, Tra controllo bibliografico e controllo intellettuale: qualche riflessione sui sistemi bibliografici elettronici. Relazione presentata all'International Conference Electronic Resources, Roma, 2001. <http://eprints.rclis.org/4121/1/weston_ita.pdf/>.
} 
Un catalogo elettronico così strutturato può finalmente consentire il superamento di quella dicotomia funzionale "lista di reperimento" (il reperimento di specifici documenti all'interno di una raccolta libraria) - "strumento bibliografico" (il raggruppamento di tutte le opere di un determinato autore e di tutte le edizioni e le manifestazioni di una specifica opera) che $\mathrm{i}$ Principi di Parigi non avevano provveduto a chiarire ${ }^{8}$

FRBR, dunque, approfondisce prima di tutto la questione ontologica ${ }^{9}$, cioè la comprensione di quali siano gli oggetti della descrizione bibliografica servendosi di un modello ${ }^{10}$, cioè per mezzo della concettualizzazione.

Dato che l'oggetto della concettualizzazione è una base di dati bibliografica - il catalogo- sono gli obiettivi del catalogo a determinare l'ontologia e la sua costruzione ${ }^{11}$.

Pertanto si può quindi definire lo studio elaborato dall'IFLA come un modello concettuale finalizzato all'adempimento delle funzioni del catalogo che, dunque, sposta il focus dai principi teorici verso gli obiettivi e le funzioni di questo strumento, definendo gli oggetti di interesse di chi utilizza i record bibliografici ${ }^{12}$ :

Definire quali sono le entità di interesse del lettore, stabilire quali devono essere i nuclei attorno ai quali la struttura catalografica deve riuscire a convogliare informazioni ed elaborarne le applicazioni, presuppone che il processo di catalogazione sia concepito in termini di funzioni e di obiettivi e che proprio l'aspetto funzionale della catalogazione sia considerato prioritario ${ }^{13}$.

Lo studio definisce le finalità dei record bibliografici e le modalità di strutturazione degli stessi in relazione alle diverse tipologie di documenti e alle necessità degli utenti e, allo stesso tempo, analizza, anche nell'ottica di una riduzione dei costi, la quantità minima di informazione che debba possedere un record bibliografico per essere funzionale, cioè per soddisfare le esigenze delle diverse componenti del circuito bibliografico costituito da tutti coloro che scambiano, ricercano e utilizzano record bibliografici.

\footnotetext{
${ }^{8}$ Paul Gabriele Weston, Gli strumenti della cooperazione in rete : dal catalogo elettronico ai sistemi della ricerca interdisciplinare, Napoli, ClioPress, 2003. Versione elettronica in $<$ http://www.storia.unina.it/cliopress/weston.html>. Citazione a p. 28.

${ }^{9} \grave{\mathrm{E}}$ opportuno sottolineare come la distinzione tra le varie nature del libro (opera, testo, edizione ed esemplare) sia stata proposta molto tempo prima dell'elaborazione del modello FRBR da Alfredo Serrai in numerosi contributi tra i quali: Biblioteche e cataloghi, Firenze, Sansoni, 1983, p. 83-97; Ricerche di biblioteconomia e bibliografia, Firenze, Giunta regionale toscana, La Nuova Italia, 1983, p. 49-50; Il cimento della bibliografia, Milano, Sylvestre Bonnard, 2001 p. 13-32.

${ }^{10}$ Intendiamo per modello uno strumento che tenta di comprendere e analizzare un fenomeno ricostruendo relazioni, sequenze e processi. I modelli sono indispensabili per formulare vere e proprie teorie.

${ }^{11}$ Cfr. Elaine Svenonius, Il fondamento intellettuale dell'organizzazione dell'informazione cit., p. 46: «Là dove gli obiettivi determinano l'ontologia, una volta specificata un'ontologia, si possono sviluppare modelli alternativi. Per esempio, un modello potrebbe descrivere una variabile stabilita, quale l'autore, o il soggetto, come entità, un altro modello potrebbe descriverla come attributo, un altro ancora ambedue le cose. La scelta di un modello fra i diversi modelli alternativi dipende [...] dall'uso al quale è destinato».

${ }^{12}$ Per una panoramica sulle teorie catalografiche che, a partire dalle teorie di Panizzi fino ai tempi più recenti, hanno precorso l'elaborazione del modello FRBR si veda William Denton, FRBR and the history of cataloging in Understanding FRBR: what it is and how it will affect our retrieval tools, edited by Arlene Taylor, Westport, Libraries Unlimited, 2007, p. 35-57 e il contributo di Biagetti (Maria Teresa Biagetti, Nuovi modelli catalografici, «Bibliotheca», 2 (2002), p. 38-59).

${ }^{13}$ Ivi, p. 40.
} 
FRBR è, dunque, un modello che rappresenta l'universo bibliografico attraverso la definizione delle entità che lo compongono e delle loro relazioni. A tale scopo FRBR si è servito di un modello E-R (entità-relazione) per individuare gli oggetti di interesse primario per gli utenti che utilizzano record bibliografici, cioè le Entità, determinare le caratteristiche di tali Entità (attributi) e stabilire le relazioni che intercorrono fra di esse.

L'analisi entità-relazione consente di scoprire la natura logica dei dati bibliografici che vengono espressi come:

* Entità: oggetti (cioè opere, persone e concetti) di interesse primario per gli utenti che usano l'informazione bibliografica. Questi oggetti, suddivisi in tre principali gruppi, contengono dati bibliografici che aiutano l'utente a trovare, identificare e ottenere l'informazione desiderata;

* Attributi: dati bibliografici associati alle entità. Essi sono specifici per ogni entità; il modello elenca gli attributi associati ad ogni entità (es. attributi dell'opera sono il titolo, la forma, la data);

* Relazioni: rappresentano le associazioni tra le entità (ad esempio l'entità opera e l'entità persona possono essere correlate tramite una relazione "creato da" che esprime la paternità intellettuale oppure "ha come soggetto" che esprime una relazione semantica).

I requisiti funzionali dei record vengono individuati alla luce delle funzioni-utente cioè delle funzioni che svolgono gli utenti che utilizzano le registrazioni bibliografiche: questa è una caratteristica fondamentale del modello, che si sforza appunto di definire logicamente quale informazione l'utente si aspetta di trovare in un record bibliografico e come l'informazione possa essere utilizzata.

L'analisi di ciò che gli utenti chiedono a un record bibliografico nel processo di recupero dell'informazione si traduce nelle seguenti tipologie di azioni o 'funzioniutente ${ }^{14}$ :

\footnotetext{
${ }^{14}$ IFLA. Functional requirements for bibliographic records cit., p. 82: «6. User Tasks [...]

- to find entities that correspond to the user's stated search criteria (i.e., to locate either a single entity or a set of entities in a file or database as the result of a search using an attribute or relationship of the entity);

- to identify an entity (i.e., to confirm that the entity described corresponds to the entity sought, or to distinguish between two or more entities with similar characteristics);

- to select an entity that is appropriate to the user's needs (i.e., to choose an entity that meets the user's requirements with respect to content, physical format, etc., or to reject an entity as being inappropriate to the user's needs);

- to acquire or obtain access to the entity described (i.e., to acquire an entity through purchase, loan, etc., or to access an entity electronically through an online connection to a remote computer)».
} 
trovare cioè reperire i materiali che corrispondano a determinati criteri (ad esempio le opere di un autore, su un dato soggetto, o pubblicate in un dato anno, ecc.) tramite gli attributi o le relazioni;

* identificare cioè verificare l'identità di un'entità e distinguerla da altre entità che hanno con essa caratteristiche in comune;

selezionare cioè scegliere, tra le entità recuperate, l'entità che ha le caratteristiche desiderate, ad esempio il cui testo sia scritto in una data lingua;

* ottenere l'accesso, cioè accedere all'entità descritta (ad esempio, ottenere una copia in prestito, o accedere al fulltext).

Alle prime quattro funzioni-utente stabilite dal testo del 1998, si potrebbe aggiungere la funzione di navigare nel catalogo ${ }^{15}$, introdotta tra le funzioni utente ${ }^{16}$ già nella prima bozza della Dichiarazione di principi di catalogazione internazionali elaborata dall' IME ICC nel 2003 e poi nel testo definitivo del $2009^{17}$.

L'elaborazione del modello FRBR ha riscosso immediato successo, in quanto portatrice di elementi innovativi che sono stati sottolineati e riconosciuti da vari studiosi anche nel nostro paese ${ }^{18}$ : l'originalità del modello - secondo il parere di Weston ${ }^{19}$ - risiede nel riconoscimento e nell'esaltazione di quella dimensione relazionale e organizzativa del catalogo che risulta da una sua articolazione secondo il modello ${ }^{20}$.

\footnotetext{
${ }^{15}$ La funzione fun introdotta in seguito allo studio di Elain Svenonius, The intellectual foundation of information organization cit.

${ }^{16}$ Barbara Tillet non esclude che in futuro tali funzioni possano essere ulteriormente ampliate. Cfr.: Barbara B. Tillet, What is FRBR? a conceptual model for the bibliographic universe, revised february 2004, Library of Congress Cataloging Distribution Service, <http://www.loc.gov/cds/downloads/FRBR.PDF> pubblicato anche in «Technicalities», 25 (2003), n. 5.

${ }^{17} \mathrm{Si}$ veda in merito anche Paul Gabriele Weston, FRBR e utente: considerazioni sulla ricerca, AIB. Sezione Toscana, relazione presentata al Seminario su FRBR (Functional requirements for bibliographic records), Firenze, 27-28 gennaio 2000, risorsa online, <http://www.aib.it/aib/sezioni/toscana/conf/frbr/weston.htm>.

${ }^{18}$ Subito dopo la pubblicazione dello studio dell'IFLA si tenne a Firenze nei primi mesi del 2000 il Seminario su FRBR organizzato dalla Sezione Toscana che fu un primo e importante incontro per discutere l'applicazione del nuovo modello nella realtà catalografica italiana. Si veda: AIB. Sezione Toscana, Seminario su FRBR (Functional requirements for bibliographic records) - Firenze, 27-28 gennaio 2000 [risorsa on line], [Firenze], Associazione italiana biblioteche, Sezione Toscana. <http://www.aib.it/aib/sezioni/toscana/conf/cfrbr.htm>. Disponibile anche a stampa: Seminario FRBR : Functional requirements for bibliographic records $=$ Requisiti funzionali per record bibliografici, Firenze, 27-28 gennaio 2000 : atti a cura di Mauro Guerrini = FRBR Seminar : Functional requirements for bibliographic records $=$ Requisiti funzionali per record bibliografici, Florence, 27th-28th January 2000 : proceedings / edited by Mauro Guerrini, Roma, AIB, 2000.

${ }^{19}$ Paul Gabriele Weston, Gli strumenti della cooperazione in rete cit.

${ }^{20}$ Un'altra considerazione sui livelli del catalogo strutturato secondo FRBR è quella di Carlyle e Fusco che riassumono nel loro contributi i vantaggi di economicità della visualizzazione quando venga strutturata secondo i livelli previsti dal modello (Allyson Carlyle - Lisa M. Fusco, Functional requirements for bibliographic records: Understanding FRBR as a conceptual model: FRBR and the bibliographic universe, «Bulletin of the American Society for Information Science and Technology», 33 (2007), n. 6, p. 12-16).
} 
L'OPAC strutturato secondo il modello FRBR - a parere dello studioso richiederebbe un'articolazione su almeno due livelli: al di sopra la rete delle relazioni tra le varie entità e, ad un livello inferiore, l'archivio delle descrizioni delle singole manifestazioni a cui verrebbero collegate le localizzazioni e le informazioni sugli esemplari che permettono alle biblioteche di espletare le attività amministrative e gestionali $^{21}$. Il modello ha dunque il pregio di distinguere e definire gli elementi ideali (opera ed espressione) e fisici (manifestazioni/item) che compongono l'universo bibliografico.

\subsubsection{L'evoluzione di FRBR e la 'famiglia FRBR'}

Il modello concettuale elaborato dall'IFLA nel 1998 ha sollevato, fin dalla pubblicazione, varie questioni in seguito alle diverse interpretazioni che sono emerse dal dibattito internazionale e dai primi tentativi di implementazione nei cataloghi.

Lo studio dell'IFLA, che già nel Rapporto finale era stato preannunciato come un lavoro non definitivo, ha dato il via a numerosi gruppi di studio che hanno approfondito alcuni aspetti particolari e certe tematiche affrontate nel report in modo incompleto.

Nel 2002 si è formato l'FRBR Review Group $^{22}$ incaricato di esaminare alcune problematiche in vista di una revisione del modello: una di queste è stata una migliore formulazione per l'entità Espressione, oggetto di perplessità da parte di vari studiosi e oggetto di ricerca di un apposito gruppo di studio (IFLA Working Group in The Expression Entity) formatosi nel $2007^{23}$.

Altrettanto importanti furono i due gruppi costituiti per approfondire e rielaborare il modello in base ai suggerimenti provenienti dalla comunità internazionale.

Nell'aprile del 1999 su iniziativa di IFLA Division on Bibliographic Control e IFLA Universal Bibliographic Control si formò il gruppo di lavoro FRANAR (Functional Requirements of Authority Numbering and Records) per stabilire i requisiti per i record di

\footnotetext{
${ }^{21}$ Ben prima della nascita degli OPAC di nuova generazione e degli strumenti di discovery Weston intravedeva le possibilità applicative del modello per la creazione di un livello superiore costituito dai collegamenti tra le entità (indispensabile ai fini della ricerca) e da livelli inferiori destinati alla gestione degli esemplari.

${ }^{22}$ Il Working Group nel 2003 assume la denominazione di FRBR Review Group; dopo lo scioglimento del gruppo lo sviluppo fu affidato al Working Group on Aggregates formatosi nel 2005.

${ }^{23} \mathrm{Fu}$ introdotta una modifica nel testo che evitava di considerare ogni cambiamento minimo (es. correzioni dello spelling, o punteggiatura) come nuova Espressione.
} 
autorità che dopo un decennio di lavori ha elaborato un primo draft, sottoposto alla revisione internazionale nel $2005^{24}$ e convertito in testo definitivo nel $2009^{25}$.

FRANAR ha sviluppato il gruppo 2 del modello introducendo novità significative come il concetto di famiglia (family) tra le entità del gruppo, soluzione sollecitata dalla comunità archivistica.

Il gruppo di lavoro sui soggetti, FRSAD (Functional Requirement for Subject Authority Data), si è costituito nel $2005^{26}$ con l'intento di sviluppare e approfondire il modello concettuale per quanto riguarda i soggetti. FRSAD, pubblicato nel giugno del $2010^{27}$, ha introdotto le entità thema e nomen. La prima è una superclasse di tutte le entità che possono costituire il soggetto di un'opera ${ }^{28}$ cioè delle 10 entità definite da FRBR. Il nomen viene introdotto come entità, anziché come attributo. Tra le funzioni utente viene inserita la funzione di esplorare (explore), basata sugli studi dell'utenza condotti dal gruppo.

Nel contempo, la necessità di armonizzare e rendere compatibile FRBR con le descrizioni della comunità museografica ha portato al nuovo studio FRBR-OO (objectoriented $)^{29}$ basato sul modello orientato agli oggetti e frutto del lavoro del Working Group on FRBR/CIDOC CRM Harmonization.

Nel 2008 è stata pubblicata, infine, la seconda versione del modello ${ }^{30}$ dopo un decennio che ha visto un intenso lavoro di ricerca e dibattito, testimoniato dalla ricchezza della letteratura professionale sull'argomento, della quale il gruppo di studio dà tempestivamente notizia mantenendo online un'utile e aggiornata bibliografia ${ }^{31}$.

\footnotetext{
${ }^{24}$ IFLA UBCIM, Working Group on Functional Requirements and Numbering of Authority Records (FRANAR), Functional requirements for authority records: a conceptual model Draft 2005-06-15 $<$ http://www.cidoc-crm.org/docs/frbr_oo/frbr_docs/FRANAR-Conceptual-M-Draft-e.pdf>.

${ }^{25}$ Glenn E. Patton - IFLA Working Group on Functional Requirements and Numbering of Authority Records (FRANAR) - International Federation of Library Associations and Institutions, Functional requirements for authority data: A conceptual model, München, K.G. Saur, 2009.

${ }^{26}<$ http://archive.ifla.org/VII/s29/pubs/wgfrsar-roster.pdf>.

${ }^{27}$ IFLA Working Group on Functional Requirements for Subject Authority Records (FRSAR), Functional Requirements for Subject Authority Data, A Conceptual Model (FRSAD), Berlin -München, De Gruyter Saur, 2011, anche online: IFLA. Functional Requirements for Subject Authority Data (FRSAD) Final Report Approved by the Standing Committee of the Classification and Indexing Section, June 2010. $<$ http://www.ifla.org/files/classification-and-indexing/functional-requirements-for-subject-authority-

data/frsad-final-report.pdf $>$.

${ }^{28}$ Ivi, p. 45.

${ }^{29}$ The FRBRoo è un'ontologia formale creata allo scopo di rappresentare le informazioni semantiche dei record bibliografici per facilitarne l'integrazione e lo scambio con i metadata provenienti dai musei, il sito di riferimento è: <http://www.cidoc-crm.org/frbr_inro.html>.

${ }^{30}$ Functional Requirements for Bibliographic Records: Final report cit. Online sono presenti le versioni aggiornate: <http://www.ifla.org/VII/s13/frbr/> (Sept. 1997, as amended and corrected through February 2008). Al momento la versione on-line più aggiornata è del Febbraio 2009.

${ }^{31}$ La bibliografia è consultabile all'indirizzo: <http://www.ifla.org/VII/s13/wgfrbr/FRBR_bibliography.pdf>.
} 


\subsection{Da FRBR al rinnovamento dei principi e delle norme catalografiche}

La possibilità che FRBR consenta lo scambio di metadati non solo tra biblioteche, ma anche tra tutti gli altri soggetti che producono e usano informazione bibliografica (agenzie bibliografiche, musei, archivi, biblioteche digitali) richiede la creazione di standard per produrre metadati che siano interoperabili e compatibili tra di loro. FRBR costituisce una buona base per garantire l'interoperabilità, ma si rende necessaria una completa ristrutturazione dei record bibliografici e di autorità possibile solo con la creazione di nuove regole di descrizione e, dunque, di nuovi Principi di catalogazione.

\subsubsection{Statement of International Cataloguing Principles (ICP)}

Lo studio dell'IFLA ha dato l'avvio a importanti riflessioni sui principi della catalogazione, sugli standard sulle norme di catalogazione, destinate a cambiare profondamente la struttura dei cataloghi elettronici.

Scopo dei nuovi International Cataloguing Principles (ICP) ${ }^{32}$, che hanno sostituito i vecchi principi di catalogazione, è stato quello di fornire, sulla base del modello IFLA, linee guida per la redazione di norme catalografiche nazionali e internazionali orientate verso il recepimento del modello FRBR.

La straordinaria diffusione di OPAC, metaOPAC e, oggi, di OPAC di nuova generazione e discovery systems, ha reso sempre più stringente e improcrastinabile la necessità di regole di catalogazione condivise a livello internazionale e quindi, ancora prima, di principi di catalogazione internazionali. Tale esigenza ha dato il via ai cinque incontri IME ICC (IFLA Meeting of Experts on an International Cataloguing Code o Incontro IFLA di esperti su un codice di catalogazione internazionale) tenuti dal 2003 al 2007 che hanno avuto l'obiettivo di verificare i punti in comune e le divergenze tra i diversi codici nazionali in uso fino ad allora e di formulare nuovi principi di catalogazione più aderenti alla realtà del catalogo elettronico e delle risorse digitali.

\footnotetext{
${ }^{32}$ IFLA Cataloguing Principles: Statement of International Cataloguing Principles (ICP) and its Glossary edited by Barbara Tillett and Ana Lupe Cristán, München, K.G. Saur, 2009, in italiano all'URL $<$ http://www.ifla.org/files/cataloguing/icp/icp_2009-it.pdf>.
} 
D'altra parte a più di quarantacinque anni dai Principi di Parigi del 1961, che avevano costituito la base teorica dei codici nazionali di molti paesi, già da tempo si avvertiva nella professione la necessità di un loro ripensamento, della verifica della loro validità in un contesto attuale ormai molto diverso da quello nel quale tali principi erano stati elaborati.

Tra i principali fattori di cambiamento vi sono proprio le caratteristiche tecnologiche dei nuovi cataloghi elettronici, i nuovi modi di accedere alle risorse bibliografiche, che sono sostanzialmente diversi rispetto a quelle dei cataloghi cartacei e, in contemporanea, la progressiva trasformazione e l'ampliamento delle risorse offerte dalle biblioteche.

Dal punto di vista del rapporto tra i cataloghi e l'informatica, all'epoca della redazione dei principi parigini, poiché le tecnologie informatiche erano agli albori, non era stato possibile prevederne gli sviluppi e le influenze future sulle notizie bibliografiche nei record dei cataloghi elettronici. Più volte negli anni successivi emersero critiche nei confronti dei codici di catalogazione sviluppatisi senza trarre un reale beneficio dalle potenzialità dell'automazione dei cataloghi ${ }^{33}$.

Un'altra questione, già accennata in precedenza, è che a differenza del contesto nel quale nacquero i principi, date le caratteristiche del catalogo cartaceo, fu necessario operare una scelta tra unità letteraria e bibliografica, scelta che venne vista allora come un compromesso irrinunciabile: FRBR ha invece fornito un modello nel quale, con chiarezza, è oggi possibile fornire accesso agli oggetti materiali e a quelli ideali che compongono l'universo bibliografico riuscendo così a sanare tale dicotomia.

The Statement of International Cataloguing Principles (Dichiarazione di principi di catalogazione internazionali) si propone come punto d'arrivo dei cinque convegni IME $\mathrm{ICC}^{34}$, ed è stata pubblicata sul sito web dell'IFLA in una prima bozza nel $2003^{35}$ per essere sottoposta alla revisione internazionale, poi nella versione definitiva The Statement of International Cataloguing Principles (ICP 2009), pubblicata nel febbraio del 2009 e disponibile in numerose lingue. La dichiarazione viene proposta come base per la redazione di nuovi codici di catalogazione e di un codice di catalogazione internazionale. ${ }^{36}$

\footnotetext{
${ }^{33}$ In realtà queste osservazioni furono sollevate anche durante la conferenza. Per approfondimenti si veda Paul Gabriele Weston, Gli strumenti della cooperazione in rete cit., p. 15-17 e Id., Tra controllo bibliografico e controllo intellettuale cit.

${ }^{34}$ Per la genesi del testo si veda il recente volume di Mauro Guerrini, I Principi internazionali di catalogazione (ICP). Universo bibliografico e teoria catalografica all'inizio del XXI secolo, postfazione a cura di Attilio Mauro Caproni, Milano, Editrice Bibliografica, 2012.

35 Il primo frutto della conferenza IME-ICC del 2003 fu Principles for library catalogues and other bibliographic files (cfr: Mauro Guerrini, I Principi internazionali di catalogazione (ICP) cit. p. 87).

${ }^{36}$ La sfida per il nuovo codice era conciliare le diversità di linguaggio, scrittura di pratiche citazionali di diverse comunità come biblioteche, archivi, musei, e di proporre un insieme di regole di facile utilizzo basate
} 
La nuova dichiarazione fornisce principi, obiettivi per la costruzione di codici di catalogazione, norme per la catalogazione, disposizioni per il reperimento dell'informazione e un glossario.

Particolarmente importante è la dichiarazione delle funzioni del catalogo dove ritroviamo vari elementi di novità; le funzioni del catalogo sono riprese dalle funzioniutente previste nel modello FRBR, e includono la funzione di navigare proveniente dall'elaborazione di Svenonius ; vi si ritrovano nuovi termini come 'risorsa', 'creatore', 'agente' e 'dato', tipici del linguaggio delle comunità che si occupano di metadati e che rimandano ai più recenti sviluppi nell'ambito della catalogazione e al mutato ambiente informativo ${ }^{37}$.

4. Obiettivi e funzioni del catalogo

Il catalogo deve essere uno strumento efficiente ed efficace che consente all'utente:

4.1. di trovare risorse bibliografiche in una raccolta come risultato di una ricerca basata sugli attributi o sulle relazioni delle risorse:

\subsection{1. per trovare una singola risorsa}

\subsection{2. per trovare insiemi di risorse che rappresentino:}

tutte le risorse che appartengono alla stessa opera

tutte le risorse che incorporano la stessa espressione

tutte le risorse che esemplificano la stessa manifestazione

tutte le risorse associate a una determinata persona, famiglia o ente

tutte le risorse su un determinato soggetto

tutte le risorse definite secondo altri criteri (come lingua, luogo di pubblicazione, data di pubblicazione, tipo di contenuto, tipo di supporto, ecc.), di solito utilizzati per selezionare ulteriormente i risultati della ricerca

4.2. di identificare una risorsa bibliografica o un agente (cioè, avere conferma che l'entità descritta in una registrazione corrisponda a quella cercata o distinguere fra due o più entità con caratteristiche simili);

4.3. di selezionare una risorsa bibliografica appropriata alle necessità dell'utente (cioè, scegliere una risorsa che risponda ai requisiti posti dall'utente per quanto riguarda formato, contenuto, supporto, ecc. oppure rifiutare una risorsa in quanto inadeguata alle proprie esigenze);

sul web,orientate a qualsiasi tipo di risorsa (libri, periodici, musica, siti web) e compatibili con altre regole di descrizione e accesso alle risorse documentarie.

${ }^{37}$ Particolarmente significativa è la sostituzione del termine libro con risorsa. La terminologia ci riporta al Web Semantico, dove qualsiasi cosa descritta da RDF è detta risorsa ed è identificata da un URI (Universal Resource Identifier). Il modello di dati RDF è formato infatti da risorse, proprietà e valori. 
4.4. di acquisire o ottenere accesso a un esemplare descritto (cioè, fornire informazioni che consentano all'utente di ottenere un esemplare mediante acquisto, prestito, ecc. o di accedere a un esemplare in modalità elettronica, tramite una connessione in linea a una fonte remota); oppure di accedere, acquisire o ottenere dati di autorità o bibliografici);

4.5. di navigare in un catalogo e al di fuori (cioè tramite la disposizione logica dei dati bibliografici e di autorità e la presentazione di chiare modalità per muoversi, compresa la presentazione delle relazioni fra opere, espressioni, manifestazioni, esemplari, persone, famiglie, enti, concetti, oggetti, eventi e luoghi) ${ }^{38}$.

\subsubsection{Resource Description and Access (RDA)}

In campo normativo la natura dello studio dell'IFLA, cioè quella di essere una rappresentazione ex novo dell'universo bibliografico indipendente da ogni codice di catalogazione precedente e da ogni reale implementazione, ha avuto un impatto considerevole sul rinnovamento dei codici di catalogazione nazionali, sollecitato anche dalle modificazioni intervenute nell'ambiente informativo.

Le nuove regole di catalogazione angloamericane RDA (Resource Description and Access) pubblicate nel 2010 rispondono alla necessità di fornire un codice di catalogazione innovativo basato sulle elaborazioni fornite dal modello FRBR e, data la straordinaria diffusione a livello mondiale del codice angloamericano, si sono proposte come norma di riferimento a livello internazionale ${ }^{39}$.

L'elaborazione del codice nella sua forma definitiva è stata lunga e non priva di ripensamenti, anche radicali.

L'ipotesi di sostituire le AACR2, che già da tempo venivano percepite come inadeguate a rispondere alle nuove trasformazioni in atto nella tecnologia dei cataloghi e nella descrizione delle nuove risorse documentarie, aveva portato già dal $1997^{40}$ alla volontà di revisionare totalmente il codice di catalogazione americano che, grazie alle iniziative nel campo della catalogazione derivata avviate a partire dagli anni settanta, aveva

\footnotetext{
38 IFLA, Cataloguing Principles: Statement of International Cataloguing Principles (ICP), traduzione italiana cit., p. 3-4.

39 American Library Association - Canadian Library Association, Chartered Institute of Library and Information Professionals (Great Britain)- Joint Steering Committee for Development of RDA. RDA: resource description \& access. Chicago, American Library Association, 2010. Si veda anche la pagina web RDA Toolkit: Resource Description \& Access, [2010],<http://rdatoolkit.org/>.

40 Il Comitato per lo sviluppo di RDA (Joint Steering Committee, JSC) convocò una conferenza internazionale sui Principi e lo sviluppo futuro delle AACR per autori di regole di catalogazione ed esperti di tutto il mondo, che si incontrarono a Toronto nel 1997.
} 
conosciuto una straordinaria diffusione. Particolari problemi sorgevano per i continui adeguamenti del codice alle nuove risorse digitali; l'articolazione delle norme era farraginosa e un'ulteriore criticità riguardava l'inadeguato trattamento delle relazioni bibliografiche ${ }^{41}$. Veniva anche ampiamente riconosciuto che l'uso del formato MARC non era adatto a favorire l'interoperabilità con la più vasta comunità dell'informazione.

A fronte della necessità di trovare soluzioni innovative ma definitive, il codice che dapprima assunse il nome di AACR3 fu oggetto di numerose critiche, che portarono ad un rallentamento dei lavori in attesa di una verifica della validità del codice ${ }^{42}$. Tra i difetti imputati l'insufficiente adozione del modello FRBR e FRAD ${ }^{43}$.

Particolarmente significativo però fu il cambiamento di rotta nella direzione di una possibile integrazione con il Web Semantico ${ }^{44}$.

$\mathrm{Ci}$ fu anche un invito a passare a un approccio ai metadati basato su dati singoli, piuttosto che costruire stringhe descrittive, per essere più compatibili con i servizi di metadati per il web nella più ampia comunità dell'informazione. Ciò era più coerente con l'approccio entitàrelazioni dei modelli concettuali dell' IFLA ${ }^{45}$

Nel 2003 a Londra gli esponenti del gruppo di sviluppo RDA (JSC) e i rappresentanti delle comunità Dublin Core, IEEE/LOM e del Web Semantico avviarono un proficuo confronto che avrebbe portato all'apertura di RDA verso i metadati. Nacque il gruppo DCMI/RDA Task Group allo scopo di sviluppare i registri RDA e di rendere disponibili sul web i vocabolari controllati e l'insieme degli elementi di RDA al fine di una loro integrazione con il Web Semantico ${ }^{46}$.

\footnotetext{
${ }^{41}$ L'iter che portò allo sviluppo del codice è stato recentemente ripercorso da Barbara Tillet, cfr.: Barbara Tillett, Keeping libraries relevant in the Semantic Web with RDA: Resource Description and Access, «JLIS.It», 2, (2011) n. 2, <10.4403/jlis.it-4760> e in Ead., Barbara B. TILLET, RDA (Resource Description and Access): lo sviluppo di un nuovo codice di catalogazione internazionale, «Bibliotime», XI (2008), n. 1 $<$ http://didattica.spbo.unibo.it/bibliotime/num-xi-1/tillett.htm>.

${ }^{42}$ Nell'aprile 2005 la prima bozza delle AACR3 ebbe un riscontro negativo da parte delle comunità bibliotecarie di tutto il mondo. Tra le critiche vi furono quelle avanzate da Karen Coyle e Diane Hillmann che consideravano RDA ancora troppo ancorato al passato e inadatto al nuovo ambiente informativo; le studiose sostenevano che il codice si fosse allontanato dal modello FRBR. Cfr. Karen Coyle - Diane Hillmann, Resource Description and Access (RDA). Cataloging rules for the 20th century, «D-Lib Magazine», 13 (2007), n. 1/2. <http://www.dlib.org/dlib/january07/coyle/01 coyle.html>.

43 «Quei modelli concettuali davano una nuova prospettiva alla descrizione delle risorse, spostando l'attenzione sul supporto e sul formato, e considerando persone, famiglie ed enti associati a quelle risorse in termini di loro caratteristiche identificanti. Le entità e le relazioni di FRBR e il vocabolario usato per descriverle erano importanti per la comunità internazionale di riferimento. Probabilmente un aspetto importante che derivava dai modelli concettuali era l'attenzione all'uso di caratteristiche identificanti nel descrivere le risorse per soddisfare le attività di base dell'utente: trovare, identificare, selezionare e ottenere» (Barbara B. Tillet, Keeping Libraries Relevant in the Semantic Web with RDA cit., p. 4).

${ }^{44}$ Tale cambio di rotta avrebbe portato da una concezione del nuovo standard catalografico statico, codificato, chiuso ad uno standard costruito su RDF e all'apertura verso il Web Semantico, un linguaggio che avrebbe consentito alle regole di essere modificate, aggiornate in tempo reale e integrate con schemi diversi

${ }^{45}$ Ivi, p. 4.

${ }^{46}$ La portata di questa decisione venne subito recepita dagli studiosi come un cambiamento destinato a generare grandi benefici nella strutturazione e integrazione dell'OPAC con il web. Scriveva Nicola Benvenuti
} 
Nel frattempo, anche i cinque incontri IME-ICC furono occasione di riflessione e riorganizzazione costante delle norme in base ai nuovi spunti che avrebbero generato il testo dei nuovi principi ICP. L'idea di catalogo proposta da RDA è davvero innovativa e oggi particolarmente attuale: non solo si basa sulla possibilità di integrazione nel Web Semantico, ma si fonda sull'idea di creare descrizioni utilizzabili da tutti gli attori del circuito bibliografico e in generale dell'informazione.

Lo stesso titolo dato al codice Resource Description and Access è sinonimo di una forte discontinuità con il passato e di un codice totalmente nuovo, basato su una nuova ristrutturazione logica del record bibliografico e di un nuovo modo di intendere la catalogazione, che è sottolineato dallo stesso abbandono del termine 'catalogazione' nel titolo del codice: si parla ormai infatti di 'descrizione' e 'accesso' alle risorse.

Dalla metà del 2010 RDA Resource Description and Access ${ }^{47}$ offre un'alternativa rispetto alla tradizionale pratica catalografica proponendo un nuovo codice che consente alle biblioteche che lo adottano di produrre metadati strutturati pienamente interconnessi con l'ambiente digitale e del Web Semantico.

Ciò che è fondamentalmente nuovo nel codice è la sua strutturazione: esso è composto infatti di due sole parti principali dedicate agli attributi delle entità e alle relazioni tra le entità, mentre è assente la parte della descrizione, proprio perché intenzione degli estensori è quella di separare nettamente i contenuti dalla forma di presentazione degli stessi. RDA è un codice che si basa solo sui contenuti, indipendentemente dalla forma di presentazione degli stessi e dalla visualizzazione dei dati, in ciò risiede la principale novità.

Ciò significa che siamo di fronte da un codice nuovo poiché:

* è dotato di flessibilità che gli consente di coprire potenzialmente tutte le tipologie di materiale;

(Dal MARC ai metadati: comincia una nuova stagione per le biblioteche, Bibelot Blog, [Blog post], 21 Maggio 2007, <http://www.bibelot.toscana.it/?p=130>): «L'adeguamento delle regole catalografiche ai mutamenti di formati sarà immediato; i metadati creati dal mondo delle biblioteche potranno costituire un forte impulso al semantic web e diventare utili a tutti gli utenti della rete; ma soprattutto il catalogo potrà uscire dal deep web ed essere ricercabile e gestibile in Internet. Si aprirà così la possibilità di arricchire il catalogo con servizi di valore aggiunto [...] di comporre (mashup) i dati delle biblioteche con altri servizi di rete, arricchendo enormemente la ricerca dell'informazione condotta con i motori di ricerca (ad esempio con opzioni che rimandino alla disponibilità di libri e articoli su determinati argomenti oggetto della ricerca, indicando il posseduto presso la biblioteca locale). E si intravede così la fine di quei mostri di gestionali bibliotecari, carissimi, chiusissimi, non comunicanti e invece vincolanti della libertà di fare informazione delle biblioteche che oggi dominano il mercato, in favore della utilizzazione di software specializzati per i singoli servizi, aggiornabili e sostituibili senza dover ricomprare tutto il sistema».

${ }^{47}$ Sul sito dello Joint Steering Committee <http://www.rda-jsc.org/> vengono pubblicati gli aggiornamenti. L'attuale chair del gruppo è Barbara Tillett, rappresentante per la Library of Congress. 
* è dotato di modularità che consente di prevedere il trattamento di risorse nuove;

* è dotato di una certa adattabilità che consente la creazione di dati utilizzabili in differenti contesti tecnologici, da quelli tradizionali agli OPAC del futuro $^{48}$.

RDA dunque è al momento l'elaborazione normativa più innovativa nel panorama internazionale; particolarmente interessante sarà l'adozione del codice nei nuovi cataloghi elettronici; la Library of Congress ha annunciato che implementerà la catalogazione dei suoi dati bibliografici in RDA a partire dal 31 Marzo $2013^{49}$.

\subsubsection{REICAT}

Anche in Italia il moto di rinnovamento si è manifestato in due grandi iniziative: da una parte, nell'ambito della catalogazione semantica il rinnovamento del Soggettario ${ }^{50}$, dall'altra in campo normativo, nei lavori per la revisione delle RICA che hanno condotto alle nuove REICAT ${ }^{51}$.

Immediatamente dopo la diffusione dello studio dell'IFLA ${ }^{52}$ e a vent'anni dalla stesura del codice, elaborato nel 1979, si è costituita per iniziativa dell' ICCU (Istituto Centrale per il Catalogo Unico delle biblioteche italiane e per le informazioni bibliografiche) una Commissione per la revisione e l'aggiornamento delle regole. La nuova Commissione ha riflettuto sull'opportunità di prendere come riferimento il modello FRBR

\footnotetext{
${ }^{48}$ Le tre caratteristiche elencate sono riprese dal contributo Carlo Bianchini, FRBR prima di FRBR cit.

${ }^{49}$ Per l'annuncio si veda <http://www.loc.gov/catdir/cpso/news_rda_implementation_date.html>.

${ }^{50}$ Le regole si sono concretizzate nel Nuovo Soggettario. Cfr. Biblioteca nazionale centrale di Firenze, Nuovo soggettario: guida al sistema italiano di indicizzazione per soggetto. Prototipo del Thesaurus, Milano, Bibliografica, (C2006.

51 ICCU - Istituto Centrale per il Catalogo Unico delle biblioteche italiane e per le informazioni bibliografiche, Regole italiane di catalogazione: REICAT, Roma, ICCU, 2009.

${ }^{52}$ A proposito commentava Alberto Petrucciani: «Le nuove Regole italiane si inseriscono in maniera chiara ed esplicita nella fase attuale di evoluzione delle normative catalografiche, una fase che si può far datare dalla diffusione del rapporto finale dello studio FRBR (1998). L'importanza di questo studio, come ho notato in altre occasioni, non sta tanto nell'originalità dei suoi contenuti, tutto sommato relativa, quanto nel ruolo di catalizzatore che ha avuto, un po' in tutto il mondo, dopo un periodo di eclisse dell'interesse per il campo della catalogazione e dell'indicizzazione. Lo studio FRBR ha il merito decisivo di aver riportato in primo piano la riflessione sui temi della catalogazione (ossia dell'organizzazione bibliografica) costituendo nel contempo una base comune ed autorevole per la comunità bibliotecaria a livello internazionale e nei singoli paesi, capace di suscitare interesse anche in altri campi più o meno affini o connessi» (cfr. Alberto Petrucciani, Le nuove RICA nel contesto dell'evoluzione delle normative catalografiche, «Bibliotime», XI (2008), n.1, <http://didattica.spbo.unibo.it/bibliotime/num-xi-1/petrucci.htm>.
} 
per la nuova revisione del codice ${ }^{53}$. A partire dal 2003 sono state pubblicate sul web, ogni sei mesi, le parti del testo delle nuove regole e la prima bozza complessiva è stata diffusa nel febbraio $2008^{54}$ (REICA 2008). Le nuove Regole italiane di catalogazione (REICAT) vengono pubblicate nel $2009^{55}$ risultando essere così il primo codice di catalogazione ad adottare il modello FRBR.

Le nuove norme vengono definite un testo «fondamentalmente nuovo» ${ }^{56}$, sia nell'articolazione che nell'esposizione della materia, ma entro il quale vengono recuperate molte indicazioni presenti nelle vecchie RICA $^{57}$.

Particolarmente importanti sono i motivi che hanno spinto alla creazione di un codice nuovo, ispirato allo studio FRBR:

* il cambiamento nelle forme di produzione e di consultazione del catalogo;

* l'innovazione apportata dall'uso di sistemi automatizzati;

* l'esigenza di trattare in maniera omogenea le nuove e diverse tipologie documentarie;

* l'emergere di una nuova concezione di catalogo che non è più isolato ma è profondamente integrato con altri cataloghi ${ }^{58}$.

In particolare quest'ultimo punto è in linea con le recenti tendenze della creazione di cataloghi sempre più grandi, frutto della realizzazione di cataloghi collettivi e di vaste reti bibliotecarie e supportato dallo sviluppo di nuove tecnologie.

Queste ultime hanno consentito la creazione di software in grado di gestire grandi quantitativi di dati provenienti da più biblioteche e come abbiamo visto, anche da risorse

\footnotetext{
${ }^{53}$ La riflessione sulle novità del modello FRBR e sulle problematiche per l'impiego nelle norme RICA è stata riassunta in un documento pubblicato sul sito dell'ICCU (ICCU - Istituto Centrale per il Catalogo Unico delle biblioteche italiane e per le informazioni bibliografiche. Commissione RICA, L'applicazione del modello FRBR ai cataloghi: problemi generali e di impiego normativo. 22 ottobre 2001, [risorsa online pubblicata sul sito dell'ICCU nell'ottobre 2003], <http://www.iccu.sbn.it/upload/documenti/rica-frbr.pdf>). Tappe importanti del lavoro di revisione del codice sono state anche le occasioni di incontro con i bibliotecari organizzate dalla Commissione stessa con il supporto dell'ICCU. Da menzionare i seminari pubblici organizzati a Roma nel 1998 (La catalogazione verso il futuro), nel 2002 (Catalogazione e controllo di autorità) e nel 2006 (Le nuove regole italiane di catalogazione: opere ed espressioni, responsabilità) i cui atti sono disponibili sul sito dell'ICCU (<http://www.iccu.sbn.it $>$ ), ad eccezione del primo, pubblicato dall'ICCU.

${ }_{55}<$ http://www.iccu.sbn.it/upload/documenti/ReicaFeb2008.pdf?l=it>.

55 ICCU - Istituto Centrale per il Catalogo Unico delle biblioteche italiane e per le informazioni bibliografiche, Regole italiane di catalogazione: REICAT. Roma, ICCU, 2009.

56 Alberto Petrucciani, Ragioni e principi della revisione delle RICA: per un nuovo codice italiano di catalogazione, «Bollettino AIB», 45 (2005), n. 2, p. 149-184.

57 Al di là del ripensamento complessivo dell'impostazione del catalogo, gli estensori delle nuove norme hanno evitato di introdurre modelli e terminologie proprie del modello FRBR che, al momento della preparazione del nuovo testo, erano ancora da sviluppare e testare, in base ad un suggerimento fornito dall'FRBR Review Group dell'IFLA.

${ }^{58}$ Ivi, p. 149.
} 
esterne, ed a una nuova concezione di catalogo, ove l'integrazione di dati prodotti in differenti istituti devono convivere:

Nel catalogo collettivo [...] il mantenimento della coerenza interna richiede un lavoro incessante e impegnativo di controllo e correzione, che si svolge per forza di cose a posteriori piuttosto che al momento della creazione della registrazione. É bene, perciò, che le norme indichino le soluzioni da raggiungere in maniera più precisa e meno aperta a interpretazioni soggettive (che pure rimangono, com'è ovvio, in qualche misura inevitabili) [...]. In questo contesto [...] viene esaltata la possibilità di confrontare i dati forniti dal libro che si ha in mano con quelli delle altre pubblicazioni già registrate nei grandi cataloghi collettivi, mentre risulta poco praticabile e poco indicativo il confronto con strumenti repertoriali di tipo tradizionale ${ }^{59}$.

La necessità di elaborare ex novo un testo fruibile da un gran numero di istituti ha condotto ad una nuova strutturazione logica delle norme, in grado di facilitare l'apprendimento per mezzo di spiegazioni chiare e il più possibile complete di tutte le indicazioni, un linguaggio puntuale in grado di farne uno strumento professionale di lavoro per i catalogatori.

Rispetto all'articolazione dei codici più tradizionali REICAT apporta alcune significative novità; ad esempio, le norme specifiche sono precedute per quanto possibile da una norma generale con funzione anche riassuntiva e il codice risulta più facilmente consultabile, in quanto ogni norma è inserita nel contesto che la riguarda.

Particolare rilievo viene dato alla parte riguardante la forma delle intestazioni ${ }^{60}$, oggi particolarmente importante per le cresciute esigenze del controllo sui punti d'accesso per garantirne l'univocità specie in cataloghi come quelli di oggi sempre più vasti, che possono arrivare a milioni di registrazioni e che presentano spesso intestazioni duplicate e sovrapposizioni. Qui si è avvertita l'esigenza di costituire, fin da subito, una guida utile ai catalogatori, chiarendo e superando alcuni equivoci riguardanti le regole di forma per le intestazioni, i problemi dell'uso linguistico delle forme dei nomi e dei titoli, e il caso spinoso delle identità bibliografiche.

\footnotetext{
${ }^{59}$ Ivi, p. 150.

${ }^{60}$ Diversamente dai nuovi Principi internazionali di Catalogazione (ICP) nelle REICAT, permane il termine 'intestazione'. Petrucciani, ne spiega così la motivazione, ivi, p. 160: «Per inciso, il termine "intestazione" ci pare ancora idoneo a indicare con esattezza quella formulazione, a volte piuttosto complessa, che il catalogatore stabilisce, seguendo norme precise, per rappresentare nel catalogo una determinata entità (una persona o un ente, un'opera, un tema, ecc.). "Punti di accesso" a una registrazione bibliografica, invece, sono tutti gli elementi utilizzabili per una ricerca, che nei cataloghi elettronici vengono in pratica a coincidere con qualsiasi elemento della registrazione stessa, utilizzabile da solo o in combinazione con altri. Il termine «intestazione» si può considerare sinonimo di "punto d'accesso controllato", ma esprime in maniera più chiara e non ambigua il fatto che si tratta di un elemento formulato dal catalogatore, non semplicemente "controllato" (o magari, all'inverso, non controllato abbastanza...). "Intestazione" fa capire chiaramente che si tratta di una sequenza di simboli, in genere linguistici, rispetto all'evanescenza del termine "punto di accesso"».
} 
Più interessante, in questa sede, è la parte relativa alle funzioni del catalogo ${ }^{61}$ che richiama i Principi di Parigi, mentre tutta la strutturazione del codice è basata sul recepimento del modello FRBR. Alle nuove entità opera ed espressione è dedicata una parte del codice.

Rispetto alle nuove norme angloamericane RDA, le neonate REICAT sono parse ad alcuni studiosi, anche alla luce dei nuovi sviluppi della catalogazione e del Web Semantico, già invecchiate. Soprattutto sembra che ad aver penalizzato l'attualità del codice sia stata la sua pubblicazione in un momento storico particolare: quasi in contemporanea con i nuovi ICP e all'inizio del dibattito sui linked data.

A dimostrazione di questo ritardo, le REICAT presentano un problema terminologico con l'adozione di termini ormai desueti (come, ad esempio, 'intestazione' ormai sostituito nei nuovi ICP dal più appropriato 'punto d'accesso' o 'titolo uniforme' utilizzato in luogo del più corretto 'punto di accesso controllato') che rimandano alla teoria e alla pratica della catalogazione tradizionale e cartacea ${ }^{62}$, un problema di compatibilità con l'edizione ISBD consolidata $^{63}$ e un problema di implementazione del codice nel software SBN e in altri software che vengono oggi commercializzati nel nostro paese ${ }^{64}$.

61 ICCU - Istituto Centrale per il Catalogo Unico delle biblioteche italiane e per le informazioni bibliografiche, Regole italiane di catalogazione: REICAT. p. 14-15:

$\ll 0.1 .3$. Funzioni del catalogo

0.1.3 A. Il catalogo ha lo scopo di:

1) permettere di trovare una particolare pubblicazione, attraverso una o più caratteristiche sufficienti a individuarla;

2) permettere di trovare tutte le pubblicazioni che hanno in comune una o più caratteristiche rilevanti (p.es. le edizioni di una stessa opera, quelle delle opere di uno stesso autore, quelle delle opere su uno stesso argomento o materia, quelle edite in una stessa collana, in una stessa località o in uno stesso anno, etc.);

3) fornire le informazioni utili per identificare o per selezionare le pubblicazioni (o altre entità) reperite, cioè per verificare che una determinata registrazione si riferisca effettivamente all'entità desiderata, e non ad altra simile o omonima, o per scegliere tra più entità quelle più confacenti alle proprie esigenze, escludendo quelle con caratteristiche non desiderate (p.es. di contenuto, lingua, formato, data, etc.);

4) fornire le informazioni utili per l'accesso a un esemplare della pubblicazione cercata o selezionata (p.es. la sua collocazione e disponibilità in una biblioteca o la sua accessibilità a distanza tramite una rete informatica); 5) fornire le indicazioni e i mezzi utili a svolgere efficacemente una ricerca (p.es. a reindirizzarla, a restringerla o ampliarla, o a visualizzare registrazioni connesse)

1.0.1.3 B. Le norme sono finalizzate in particolare ad assicurare le seguenti funzioni, stabilite nella Definizione di principi approvata dalla Conferenza internazionale sui principi di catalogazione (Parigi 1961):

1) permettere di trovare una particolare pubblicazione;

2) permettere di trovare tutte le edizioni di una particolare opera;

3) permettere di trovare tutte le opere di un particolare autore».

${ }^{62}$ I termini non più usati nei nuovi ICP sono: Unità bibliografica (Manifestazione), Intestazione (Punto di accesso autorizzato, punto di accesso controllato), Rinvio (Forma variante del nome); Titolo uniforme (Punto di accesso autorizzato, Forma autorizzata del nome, Nome).

${ }^{63}$ Tali problemi sono elencati in Carlo Bianchini, FRBR prima di FRBR cit. p. 233.

${ }^{64}$ I vari problemi riscontrati sono stati discussi a Roma nel seminario REICAT: contenuti, applicazione, elementi di confronto. Roma, Biblioteca Nazionale Centrale di Roma, 18 febbraio 2010 i cui interventi sono visibili in rete all'indirizzo: <http://www.iccu.sbn.it/opencms/opencms/it/main/attivita/gruppilav_commissioni/pagina_369.html>. 


\subsubsection{Altre iniziative: VIAF, ISNI, ISBD}

Si segnalano, per completezza, le altre iniziative a livello internazionale di importante rilievo; tra queste va certamente citato il progetto VIAF (Virtual International Authority File $)^{65}$, implementato e mantenuto da OCLC, che si propone di creare un database internazionale di forme di nomi ${ }^{66}$.

Il tema della qualità dei dati nei cataloghi è sempre più spesso al centro del dibattito bibliotecario; essa è il prerequisito per garantire al catalogo autorevolezza e coerenza. Il nuovo autority file di VIAF:

* collega i record di autorità prodotti a livello nazionale e regionale, creando raggruppamenti di record collegati;

* espande il concetto di controllo bibliografico universale (UBC) ${ }^{67}$ consentendo alle varianti nazionali e regionali espresse nella forma accettata di coesistere e supportando le esigenze di varianti nel linguaggio preferito e nella forma e grafia preferita;

* Gioca un ruolo importante nel Web Semantico esponendo le registrazioni sotto forma di linked data.

VIAF fornisce gli identificativi accessibili sul web delle entità di interesse dei bibliotecari e degli utenti e consente la localizzazione dei dati bibliografici delle entità a partire dalle varianti locali dei nomi. Gli utenti che accedono a VIAF sono in grado di visualizzare i nomi nella forma autorizzata nel loro paese e di visualizzare le varianti dello stesso nome e le forme autorizzate stabilite dalle agenzie bibliografiche degli altri paesi aderenti al progetto, facilitando la ricerca dei dati bibliografici in diverse lingue. ${ }^{6} \mathrm{Si}$ tratta,

\footnotetext{
$65<$ http://viaf.org $>$.

${ }^{66} \mathrm{Al}$ progetto di OCLC parteciparono inizialmente Library of Congress, Deutsche Nationalbibliothek, e Bibliothèque nationale de France. Nel sito web (<http://www.oclc.org/research/projects/viaf/>) relativo al progetto esso è definito «A joint project [...] VIAF explores virtually combining the name authority files of all three institutions into a single name authority service». È stato realizzato un sistema prototipo che è visibile all'indirizzo <http://viaf.org/>. Oggi partecipano 20 agenzie di 16 paesi. L'ICCU partecipa a Viaf dal 2009 con i record di autorità SBN autori personali secondo il formato UNIMARC/A.

Si veda anche l'annuncio: Library leaders to extend Virtual International Authority File. OCLC News Release, 14 November 2007. Si veda anche: Thomas B. Hickey, The Virtual International Authority File: expanding the concept of universal bibliographic control, «NextSpace», 13 (2009), $<$ https://www.oclc.org/content/dam/oclc/publications/newsletters/nextspace/nextspace_013.pdf>.

67 Thomas B. Hickey, The Virtual International Authority File: Expanding the concept of universal bibliographic control, «NextSpace», 13 (2009).

${ }^{68}$ OCLC ha gestito la parte tecnologica del progetto e ha predisposto un apposito algoritmo al fine di collegare gli archivi dei nomi di autorità delle biblioteche nazionali partecipanti. L'algoritmo controlla in modo automatico le relazioni tra i nomi delle persone nella forma in cui si trovano nelle registrazioni
} 
dunque, di un progetto che potrà in futuro essere la base di un nuovo modo di intendere il controllo bibliografico che è così decentrato e distribuito, a vantaggio delle comunità del web. A questo proposito Guerrini recentemente ha sottolineato come sia oggi attuale la proposta di Ayres e - in Italia, - di Weston, di abbandonare il principio di uniformità e indicizzare un attributo nella forma in cui compare nella risorsa:

Collegare la scelta della forma di un nome alla sua effettiva manifestazione significa renderla, in qualche modo, indipendente dalle normative nazionali e dalle interpretazioni locali. Ne dovrebbe derivare un vantaggio ai fini della ricerca all'interno dei cataloghi collettivi o nell'ambito delle reti internazionali di biblioteche [...] la brillante proposta di Weston prefigura un nuovo principio di multiformità e permette di eliminare la ripetizione di alcuni elementi attualmente registrati, in forme diverse, due volte all'interno della registrazione (per esempio, la responsabilità); offrirebbe inoltre l'opportunità di scavalcare la questione della scelta della forma ${ }^{69}$.

Per concludere vanno segnalate altre due iniziative di particolare rilievo. Nel 2009 completa il progetto VIAF l'ideazione da parte di ISO dell'International Standard Name Identifier (ISNI), di un identificatore numerico che viene associato alle identità di persone ed enti coinvolti nella creazione di risorse allo scopo di garantire quel principio di uniformità numerica che aveva già contraddistinto il progetto ISADN ${ }^{70}$.

Infine, sono state di recente innovate anche le norme descrittive $\operatorname{ISBD}^{71}$ con la redazione delle nuove International Standard Bibliographic Description (ISBD), Preliminary consolided edition, che si sono proposte di armonizzare in un testo unico le otto ISBD fino ad oggi pubblicate ${ }^{72}$; la nuova versione però è stata oggetto di critica in quanto non avrebbe recepito il modello FRBR e da più parti si è levata la necessità di una nuova rielaborazione degli standard descrittivi.

In conclusione, l'analisi degli sviluppi del modello FRBR e delle iniziative catalografiche ad esso collegate ci mostrano uno scenario di grande trasformazione, ma

bibliografiche e di autorità e le registrazioni bibliografiche dove i nomi appaiono come punti di accesso permettendo di individuare automaticamente le corrispondenze e di creare i legami tra le registrazioni di autorità della stessa entità negli archivi di istituzioni diverse e presentare il ventaglio di forme varianti in VIAF. Si veda anche Richard Bennet - Christel Hengel - Thomas B. Hickey- Edward T. O'Neill - Barbara B. Tillett, Virtual International authority file [Presentazione ad ALA Annual Conference, New Orleans, LA, June 22-28, 2006, <http://www.oclc.org/research/projects/viaf/ala2006c.ppt>.

${ }^{69}$ Mauro Guerrini, I Principi internazionali di catalogazione (ICP) cit., p. 158.

${ }^{70}$ Il progetto riguarda - come ha sottolineato ancora Guerrini - solo le identità pubbliche attive (persone ed enti con ruolo attivo) e non quelle passive (es. di soggetto).

${ }^{71}$ IFLA. ISBD Review Group e International Federation of Library Associations and Institutions. Cataloguing Section. Standing Committee, ISBD International Standard Bibliographic Description, Berlin, De Gruyter Saur, 2011.

72 IFLA, International Standard Bibliographic Description (ISBD), Preliminary consolided edition, recommended by the ISBD Review Group, approved by the Standing Committee of the IFLA Cataloguing Section, München, Saur, 2007. In traduzione italiana: IFLA, International Standard Bibliographic Description (ISBD), edizione consolidata preliminare raccomandata dall'ISBD Review Group; approvata dallo Standard Committee dell'IFLA Cataloguing Section. Edizione italiana / a cura dell'Istituto centrale per il catalogo unico delle biblioteche italiane e per le informazioni bibliografiche (ICCU), Roma, ICCU, 2010. 
frammentato, in cui caratteristiche e funzionalità del catalogo elettronico sono messe a dura prova dalla coesistenza e dallo sviluppo in parallelo delle diverse iniziative. Non sorprende quanto Guerrini affermava qualche tempo fa quando, interrogandosi sul rapporto tra nuovi FRBR, ICP, RDA e ISBD, osservava:

Ignorando lo stato instabile ed eterogeneo che caratterizzava il panorama della riflessione biblioteconomica a livello internazionale prima della pubblicazione di FRBR, il passaggio dal passato al futuro in questi domini è estremamente complicato per molte altre ragioni. Ciascun processo è complesso, ma i processi in corso sono molti e riguardano i diversi livelli logici della catalogazione, sicché nessun livello può trovare appoggio sugli altri, perché tutto è in movimento. La complessità da affrontare per gestire il cambiamento generale ha pertanto un livello superiore della somma delle complessità di ciascun singolo processo e livello logico. Non basta neppure rilevare che ogni singolo passaggio e ogni decisione è studiata e valutata attentamente da ciascuna entità coinvolta: manca infatti qualcuno (qualche ente o agenzia bibliografica) che si occupi della gestione dell'intero processo e non è chiaro a quale principio ci si potrebbe ispirare [...] Attualmente molti organismi stanno lavorando a ciascuna fase del processo e ciascuno di essi dimostra la volontà di cooperare e di integrare il proprio lavoro con gli altri; l'impressione è tuttavia che i lavori procedano in modo autonomo e parallelo. La questione diviene allora: quis custodiet ipsos custodies $?^{73}$.

\subsection{L'applicazione del modello FRBR ai cataloghi. Analisi delle entità FRBR}

Nel dibattito professionale sul modello FRBR vanno distinte le problematiche di applicazione del modello, che si riferiscono alla discussione teorica sull'applicabilità di FRBR a vari contesti (biblioteche digitali, specifiche collezioni, archivi, musei) da quelle che riguardano l'implementazione nei sistemi, termine con il quale si indica la creazione di sistemi basati su FRBR che di solito avviene tramite la realizzazione di appositi algoritmi e software $^{74}$.

Lo studio dell'applicabilità del modello FRBR ai cataloghi elettronici e le prime implementazioni del modello agli OPAC hanno portato alla luce criticità e punti di forza del modello. In particolare le entità del primo gruppo che sono state oggetto

\footnotetext{
${ }^{73}$ Carlo Bianchini - Mauro Guerrini, Quis custodiet ipsos custodes?: osservazioni sulle relazioni fra FRBR, $I C P, I S B D$ e RDA/, «Il bibliotecario. Rivista di studi bibliografici», 3. serie (2009), n. 1 p. 59-85 (Versione italiana, rielaborata e ampliata, di: From bibliographic models to cataloging rules: remarks on FRBR, ICP, $I S B D$, and RDA and the relationships between them. «Cataloging \& classification quarterly», vol. 47 (2009), n. 2, p. 105-124. La citazione è a p. 22-23.

${ }^{74}$ Yin Zhang - Athena Salaba, Implementing FRBR in libraries: key issues and future directions, New York, Neal-Schuman Publishers, 2009.
} 
dell'implementazione in alcuni prototipi hanno sollevato alcune questioni che vengono qui riassunte.

\section{L'opera}

L'opera viene definita in FRBR come una «creazione intellettuale o artistica distinta» o come un' «entità astratta». Si sottolineano nella definizione di opera due diverse valenze che ne contraddistinguono rispettivamente i punti di forza e debolezza: se dunque da un punto di vista ontologico l'opera come pura astrazione si apre a diverse interpretazioni e ad una molteplicità di realizzazioni, a livello catalografico essa manifesta la sua valenza operativa e organizzativa:

A livello catalografico, l'astrattezza dell'Opera si traduce in una grande flessibilità, che è alla base della sua forza collocativa: è grazie all'astrattezza che l'Opera di FRBR rappresenta un espediente tecnico che permette di regolare il livello di raggruppamento delle realtà bibliografiche ${ }^{75}$.

Lo stesso concetto è ribadito nel fondamentale testo di Svenonius, che osserva l'opera dal punto di vista 'concettuale' come una porzione di un contenuto intellettuale e artistico e dal punto di vista 'operativo', che consente al catalogo di svolgere la sua funzione aggregante: «Dal punto di vista operativo - afferma la studiosa- possiamo definire un'opera in termini di procedure da eseguire per creare l'insieme di tutti i documenti che hanno in comune il fatto di contenere essenzialmente le stesse informazioni» ${ }^{76}$.

La possibilità di formare 'insiemi opera' ovvero raggruppamenti in base all'opera consente dunque di raggruppare documenti contenenti le stesse informazioni e costituisce l'azione fondamentale dell'organizzazione dell'informazione. Tali insiemi svolgono due principali funzioni di organizzare le visualizzazioni e di collegare le entità bibliografiche correlate $^{77}$.

\section{L'espressione}

L'espressione è l'elemento più innovativo del modello, ma anche quello che ha generato le principali critiche e certamente il più dibattuto nella letteratura professionale su FRBR.

\footnotetext{
${ }^{75}$ Mauro Guerrini, I Principi internazionali di catalogazione (ICP) cit., p. 114

${ }^{76}$ Elaine Svenonius, Il fondamento intellettuale dell'organizzazione dell'informazione cit., p. 48.

${ }^{77}$ Ivi, p. 52.
} 
Definita dal modello come la realizzazione artistica o intellettuale di un'opera, l'espressione è la «specifica forma artistica o intellettuale che un'opera assume ogni volta che viene realizzata ${ }^{78}$.

Anche l'espressione è ritenuta importante nel catalogo per la sua valenza organizzativa, poiché risolve alcuni problematiche come la questione delle versioni e dei supporti; vi sono tuttavia alcuni motivi di critica che riguardano:

1) gli aspetti rivolti alla definizione dell'entità (identità e confini) e alla sua applicabilità ai cataloghi elettronici specie nelle relazioni con le altre entità. In alcuni casi risulta particolarmente difficile stabilire se l'entità collegata a un'opera è un'altra opera o una sua espressione;

2) la questione che tale entità viene compresa con difficoltà dagli utenti. Il concetto di espressione non viene naturalmente compreso dagli utenti;

3) alcuni particolari problemi che sorgono nell'applicabilità dell'entità ad alcune particolari tipologie di materiali a stampa, ad esempio nel libro antico, nelle arti visive, nelle rappresentazioni dal vivo etc. Un particolare problema, oggi risolto, è stato quello della "variante testuale minima" in quanto l'espressione in FRBR era basata sul criterio della completa identità testuale: ogni variante anche minima del testo era considerata una nuova espressione. Il principio così formulato ostava all'applicazione corretta di FRBR per il materiale a stampa antico, dove avrebbe portato ad una pericolosa e controproducente proliferazione di espressioni. Le numerose critiche in merito hanno poi portato alla necessità di rivedere la definizione di espressione e la parte riguardante il criterio della variante testuale minima è stata stralciata nel nuovo draft del $2008^{79}$.

\section{La manifestazione e l'item}

La manifestazione e l'item, cioè rispettivamente l'edizione / pubblicazione e la copia fisica, sono le entità concrete del modello FRBR. Se l'item è l'entità che crea meno problemi, in quanto ben individuabile e caratterizzata per via della sua fisicità e concretezza, la manifestazione impone qualche chiarificazione. L'edizione, com'è noto, è una particolare manifestazione di un'opera e ha in comune con le altre manifestazioni lo

\footnotetext{
${ }^{78}$ Carlo Ghilli - Mauro Guerrini, Introduzione a FRBR: Functional requirements for bibliographic records $=$ Requisiti funzionali per record bibliografici, Milano, Bibliografica, 2001, p. 49.

${ }^{79} \mathrm{Si}$ veda anche Mauro Guerrini, I Principi internazionali di catalogazione (ICP) cit., p. 115-120
} 
stesso contenuto informativo ${ }^{80}$, ma non sempre identificare un'edizione è un'operazione semplice, poiché il concetto di edizione ha dei contorni sfumati ${ }^{81}$.

In FRBR l'entità manifestazione è definita come entità concreta e di natura materiale, ma la sua definizione apre alcuni spunti interessanti di riflessione - recentemente riproposti e sintetizzati in Italia da Mauro Guerrini - relativi alla natura stessa dell'entità così come viene descritta nel modello (se l'espressione è un'entità materiale, altrettanto può dirsi per alcuni aspetti editoriali o questi possono considerarsi come contributo intellettuale?) e al rapporto con altre entità (nel caso di manifestazioni in rapporto 'molti a molti' con le espressioni).

\section{Super-opera}

Il concetto introdotto da Domanovszky ${ }^{82}$ di discendenza da un'origine comune è alla base del concetto di Super-Work (super-opera). Sebbene assente dal modello FRBR e dai nuovi Principi internazionali di catalogazione (ICP), tale concetto ha trovato numerosi sostenitori $^{83}$. Più opere vengono ricondotte ad una stessa super-opera quando contengono contenuti simili in virtù del fatto di emanare dallo stesso archetipo. L'entità è stata ideata a scopi chiaramente organizzativi, per migliorare la funzione di raggruppamento. Raggruppare $\mathrm{i}$ record in base alla super-opera mette dunque insieme opere, film, registrazioni sonore, registrazioni di spettacoli dal vivo, parodie e opere di derivazione, commentari e bibliografie ampliando i confini tradizionali del recupero a questi importanti legami tra le diverse opere. Tale concetto, nel nuovo scenario che vede la comparsa di metaOPAC e dei recenti discovery systems, andrebbe dunque rivalutato per la sua dimensione operativa. Svenonius ne rileva la sua funzione di supporto allo studio letterario

\footnotetext{
${ }^{80}$ Cfr. Elaine Svenonius, Il fondamento intellettuale dell'organizzazione dell'informazione cit., p. 55-56.

${ }^{81}$ Ivi, p. 55: «Il concetto di edizione, come quello di opera, ha confini sfumati; il problema (ancora una volta) consiste nell'identificare le differenze e valutarne il grado di rilevanza - distinguere fra avere sostanzialmente lo stesso contenuto (l'essenza dell'opera) e avere in comune lo stesso contenuto (essenza dell'edizione). Il modo in cui viene risolto questo problema ha conseguenze pratiche. Spesso, nel loro lavoro quotidiano, i catalogatori sono chiamati a decidere se due documenti rappresentano la stessa edizione oppure edizioni differenti di un'opera».

82 Ákos Domanovszky, Functions and objects of author and title cataloguing, München, Verlag Dokumentation, 1975.

${ }^{83}$ Tra questi il più noto è Rahmatollah Fattahi. Nella sua tesi di dottorato il concetto è applicato a quello di 'Super-record'. Cfr. Rahmatollah Fattahi, The relevance of cataloguing principles to the online environment: an historical and analytical study: a thesis submitted in fulfillment of the requirements for the degree of doctor of philosophy in the School of information, library and archive studies, University of South Wales [Sidney, Australia], 1997 Anche online all'URL: <http://wilma.silas.nsw.edu.au/students/rfattahi/ chappref.htm>.
} 
e di mezzo efficace per raggiungere l'obiettivo del catalogo - da lei introdotto - di navigare.

È proprio nel raggiungimento di questo obiettivo, oltre che nella capacità operativa di raggruppamento, che la super-opera troverebbe oggi un'appropriata applicazione nei nuovi OPAC che reperiscono una gran quantità di informazioni correlate provenienti da diverse fonti, su supporti e formati diversi, ove ci si potrebbe avvantaggiare oltre che del raggruppamento in base all'opera, anche di quello per famiglie di opere. Numerosi, infine, sono gli studi dedicati al concetto di super-opera o super-registrazione. ${ }^{84}$

In particolare Fattahi ha rilevato come tale concetto consenta di «comprendere, differenziare, correlare, organizzare e sintetizzare le informazioni sulle famiglie bibliografiche» e quindi sia un fondamentale strumento di conoscenza nell'OPAC:

Esso infatti illustra a) quanto è grande una famiglia bibliografica (la consistenza), b) quali tipi di entità sono correlate a un'opera (le categorie), c) quali tipi di relazioni esistono fra due o più opere (l'associazione), d) come sono ordinate le opere correlate (organizzazione). Questi elementi aiutano l'utente a trarre maggiore conoscenza sull'universo bibliografico. Se ripensiamo i fondamenti teorici delle funzioni del catalogo di biblioteca possiamo sviluppare cataloghi che rappresentino la conoscenza invece che l'informazione ${ }^{85}$.

\subsubsection{L'applicazione di FRBR alle diverse tipologie documentarie}

Un importante campo di indagine negli studi sul modello FRBR è quello relativo all'applicazione del modello a diverse tipologie di risorse documentarie. Il modello logico fornito dallo studio FRBR può essere applicato in vari contesti; a beneficiare della struttura fornita dal modello non sono dunque i soli cataloghi elettronici, ma anche vari insiemi e tipologie di documenti come, ad esempio, collezioni di oggetti digitali, di oggetti d'arte e

\footnotetext{
${ }^{84}$ I concetti di 'super-record' e 'super-opera sono stati elaborati rispettivamente da Carlyle e Fattahi. Cfr: Allyson Carlyle, Ordering author and work records: an evaluation of collocation in online catalog displays, «Journal of the American Society for Information Science», 47 (1996), n.7, p. 538-554 e Rahmatollah Fattahi, The relevance of cataloguing principles to the online environment cit.

${ }^{85}$ Rahmatollah Fattahi, From information to knowledge cit. p. 28.
} 
manufatti, particolari tipologie di pubblicazioni come le arti visive e dello spettacolo, collezioni di archivi e musei etc.

L'applicabilità del modello a diverse tipologie di raccolte è stato argomento di numerosi studi ed è oggi particolarmente attuale: OPAC di nuova generazione e strumenti di discovery hanno ampliato la capacità di questi strumenti nell'accogliere una gran quantità di risorse non solo di diversa provenienza, ma anche di diversa tipologia: oggetti e collezioni digitali, collezioni particolari (musicali, cinematografiche o di arti visive, risorse elettroniche ed e-book) possono entrare a far parte dei nuovi cataloghi elettronici ed essere recuperati insieme ai materiali tradizionali (libri, periodici, materiale a stampa antico, materiale cartografico etc.) così è indispensabile che il modello possa essere applicato con coerenza a tali materiali.

In generale, si può affermare che il modello esprime appieno la sua potenzialità quando è applicato ad alcune tipologie di risorse, quali la narrativa, le collezioni musicali, $\mathrm{i}$ periodici; in altri contesti l'applicabilità del modello solleva varie questioni, specie in contesti nei quali gli oggetti da descrivere sono oggetti unici, come nel caso delle opere d'arte. In linea di massima, il modello esprime la sua efficacia quando le opere hanno più espressioni e manifestazioni collegate. I primi prototipi sono stati testati proprio su raccolte di questo tipo, ad esempio, sulle opere di narrativa; esse infatti rappresentano un campo ottimale di applicazione del modello; tali collezioni, infatti, includono opere con diverse espressioni e manifestazioni, come le numerose traduzioni in altre lingue e un numero ampio di opere correlate rappresentate da adattamenti, parodie ed altre modificazioni sostanziali.

Le collezioni di narrativa sono state, insieme alla musica, tra i primi materiali a costituire la base di prototipi per lo studio dell'applicazione del modello, tra i quali il più noto è FictionFinder di OCLC ${ }^{86}$.

La possibilità di usare i record bibliografici già esistenti per testare l'applicabilità del modello è stata oggetto anche di ricerche su opere particolarmente diffuse e problematiche ${ }^{87}$ come The Expedition of Humphry Clinker o Flatlandia ${ }^{88}$.

Il primo studio ha sollevato le difficoltà di applicazione dell'espressione ai record catalografici, rilevando che i record non hanno elementi sufficienti per riconoscere le

\footnotetext{
${ }^{86}$ Si veda infra, Appendice C. Progetti di implementazione di FRBR negli OPAC.Prototipi. FictionFinder.

${ }^{87}$ Gli studi di OCLC Research sul modello FRBR sono riassunti nella pagina del progetto (FRBR projetc) $<$ http://www.oclc.org/research/activities/past/orprojects/frbr/default.htm>.

${ }^{88}$ Edward T. O'Neill, FRBR: application of the entity-relationship model to Humphry Clinker, «Library Resources and Technical Services», 46 (2002) n. 4, p. 150-159. $<\mathrm{http}: / /$ www.oclc.org/research/publications/archive/2002/oneill_frbr22.pdf >. Si veda anche la pagina web dedicata: <http://www.oclc.org/research/activities/frbr/clinker.html>.
} 
espressioni e collegarle correttamente alle manifestazioni, mettendo in rilevo come il modello alla prova di situazioni bibliografiche complesse mostri alcune debolezze, ove invece dovrebbe mostrare tutta la sua forza. Le conclusioni dei due studi sono state di una certa importanza per comprendere l'applicabilità del modello ai record bibliografici esistenti e hanno confermato che alcune tipologie di collegamenti sono essenziali nella pratica catalografica agli scopi del modello, ad esempio l'utilizzo dei titoli uniformi, o i ruoli di autori o responsabilità secondarie.

Particolarmente interessanti si rivelano inoltre le strategie messe in atto da FictionFinder per il catalogo dedicato alle opere di narrativa di WorldCat.

Il catalogo che, ricordiamo, non è un OPAC, include libri, e-books e audiolibri provenienti dalle opere di narrativa presenti in WorldCat, in particolare contenenti romanzi e racconti.

Basato sull'algoritmo sviluppato da OCLC (OCLC Work-set Algorithm ${ }^{89}$ ), il catalogo ricorre ad alcuni espedienti tecnici per presentare un'implementazione parziale di FRBR particolarmente rivolta al raggruppamento delle opere. Il secondo livello di raggruppamento è invece frutto di un compromesso; essendo un mix di espressionimanifestazioni esso mostra tutte le difficoltà insite nel raggruppamento delle espressioni quando si opera sui record bibliografici tradizionali codificati nel formato MARC e basati sulle attuali regole di catalogazione.

Particolarmente interessanti si rivelano le problematiche relative all'applicazione del modello ad altre tipologie documentarie, che qui si riassumono brevemente ${ }^{90}$.

1. Opere d'arte e manufatti artistici.

Le opere d'arte rappresentano una tipologia documentaria nella quale un'attenzione particolare deve essere riservata al concetto di opera, che per questi materiali non coincide esattamente con la definizione fornita da FRBR, essendo le opere d'arte oggetti unici. La scansione delle entità del gruppo 1 non è chiaramente applicabile, in quanto le norme catalografiche relative a questi materiali (CCO) applicano una catalogazione objectoriented, che non si sposa bene con il modello. Se tutti i prodotti artistici sono rappresentati da una realizzazione fisica (manifestazione) sorgono problemi per la definizione delle

\footnotetext{
${ }^{89}$ Si veda: infra, Appendice C. Progetti di implementazione del modello FRBR. Algoritmi.

${ }^{90} \mathrm{Si}$ rimanda per un approfondimento alla lettura dei saggi contenuti nel volume curato da Arlene Taylor Understanding FRBR: what it is and how it will affect our retrieval tools, Westport, Libraries Unlimited, 2007.
} 
espressioni. Due diverse versioni di un dipinto sono considerate espressioni diverse per FRBR, ma opere diverse nelle regole catalografiche $(\mathrm{CCO})^{91}$.

\section{Materiale a stampa antico}

Una tipologia di materiale molto presente negli OPAC è il materiale antico a stampa. I libri antichi rappresentano un caso problematico di applicazione del modello, dove l'applicazione di FRBR è apparsa fin da subito limitante e persino controproducente.

Nella descrizione del libro antico, la definizione dell'entità espressione crea notevoli problemi: pensiamo ad esempio alle varianti di edizione, diverse emissioni o alle varianti di stato, concetti che vanno meglio precisati se si vogliono ottenere descrizioni coerenti.

In particolare, dalla pubblicazione del modello fu chiaro da subito che le entità previste dal modello FRBR non si adattavano al mondo della produzione artigianale del libro.

Secondo il modello FRBR tutte le copie (item), sono identiche, cosa che non si verifica nelle edizioni antiche a stampa, dove la produzione manuale introduce una serie di variabili che non sono previste nel modello.

Le piccolissime differenze presenti nelle diverse edizioni secondo il modello avrebbero dato luogo ad espressioni diverse introducendo una complessità non facilmente gestibile, e una semplificazione non attentamente studiata avrebbe potuto generare confusione ed essere estremamente pericolosa. Con il nuovo draft del 2008 è stato risolto problema della 'variante minima', ma restano ancora alcuni passi da fare.

\section{Collezioni musicali}

Le collezioni musicali sono un materiale su cui si sono avviati vari progetti di realizzazione del modello ${ }^{92}$. Le principali questioni sono relative alla difficoltà in alcuni casi di stabilire le opportune differenze tra le espressioni; molto complesse si presentano per questa tipologia di materiale anche le relazioni opera-opera frequenti in quanto un'opera può contenere frammenti di altre opere, aggregazioni di opere etc. e alcune

\footnotetext{
${ }^{91}$ L'esempio è fornito da Yin Zhang - Athena Salaba, Implementing FRBR in libraries cit.

${ }^{92}$ Uno di questi è il progetto Variation avviato all'Indiana University. Il software Variations, in uso dal 1996 è un sistema open source progettato su un modello entità-relazione, che si ispira al modello FRBR. Cfr.: infra, Appendice C. Progetti di implementazione di FRBR negli OPAC. Sistemi completi, prototipi, software.
} 
difficoltà nascono quando si deve stabilire l'opera. (es. in una poesia espressa in forma musicale qual è l'opera? la musica o il testo?), i titoli uniformi spesso non sono espressivi.

A testimonianza della difficoltà di trattamento vi è la recente implementazione di FRBR nel catalogo dell'Australian Music Center (<australianmusiccentre.com.au>) dove ritroviamo solo il raggruppamento in base all'opera.

4. Spettacoli dal vivo, esibizioni, balletti

Le registrazioni di spettacoli dal vivo e concerti, balletti e performance teatrali rappresentano problematiche più complesse e in comune con il trattamento di questi materiali in biblioteca, ove di solito vengono trattati in maniera differente, a causa dell'l'assenza di normative catalografiche specifiche. FRBR in questo campo deve risolvere numerosi interrogativi che riguardano nuovamente le entità di opera e di espressione: ad esempio la coreografia di un balletto che a sua volta si basa su un'opera scritta è un'espressione o un'opera in quanto proveniente dalla creazione intellettuale del coreografo? Particolari problemi sorgono nelle relazioni dall'opera alle opere correlate (es. il testo della coreografia, le foto scattate durante la performance).

\section{Film e TV}

Raccolte riguardanti il cinema e la tv sono tra le tipologie di materiale cui l'applicazione del modello apporta grandi benefici. Tuttavia anche in questa tipologia di materiale presenta alcune problematiche quando bisogna applicare le entità previste nel modello. Tra gli studiosi che si sono occupati delle problematiche del trattamento in FRBR di questa tipologia di materiale vi è la statunitense Martha M. Yee, che in alcuni suoi scritti ha rilevato varie problematiche che tali materiali sollevano ${ }^{93}$.

\footnotetext{
${ }^{93} \mathrm{Si}$ veda in particolare il contributo di Martha M. Yee, FRBR and moving image materials: content (Work and Expression) versus carrier (Manifestation) in Understanding FRBR: what it is and how it will affect our retrieval tools, edited by Arlene Taylor, Westport, Libraries Unlimited, 2007, p. 117-130. In particolare Yee compie una disamina accurata dei concetti di opera ed espressione nel materiale audiovisivo e conclude (p. 118-119): «Suffice it to say here that any intentional change in content of a moving image work (as opposed to changes in carrier) creates a new expression of the work, except in the rare cases where the change in content is so substantial as to create a new work. [...] a film of Mozart's Magic flute would also be a new work. I have argued elsewhere that moving image works are essentially visual works, not textual or musical works, and that the transformation of a textual or musical work into a visual work necessarily creates a new work. It would appear that FRBR agrees with this analysis. FRBR examples of different expressions of the same work include a moving image example (Jules et Jim), which indicates that any change in the sound, text, music, or image of a moving image work creates a new expression of that work».
} 


\subsection{L'implementazione di FRBR negli OPAC: problematiche e opportunità}

L'applicazione di FRBR ai cataloghi elettronici è oggi un argomento di particolare importanza e rilievo nel dibattito internazionale. In particolare la scelta degli OPAC di nuova generazione e dei discovery systems di affidarsi ciecamente al recupero dell'informazione bibliografica tramite gli algorimi di relevance ranking acuisce il senso di disagio di fronte ad un'occasione mancata, almeno finora, di rendere l'esplorazione nel catalogo davvero comprensibile e significativa per l'utente.

L'applicazione del modello FRBR consentirebbe di risolvere molti dei difetti imputati ai cataloghi odierni; tra le carenze che gli OPAC presentano infatti vi è l'incapacità di rendere pienamente conto:

* dell'identità di un'opera sotto i suoi diversi titoli, e le differenze tra le edizioni /manifestazioni;

* delle relazioni fra opere, ad esempio, tra le opere derivate e opere che hanno per oggetto altre opere;

* dell'identificazione degli autori, utili in particolar modo in casi di omonimia, che potrebbero essere risolti fornendo i dati bibliografici identificativi dei record di autorità;

* dei diversi ruoli di responsabilità degli autori, per cui non è possibile visualizzare, a partire da un autore le opere ed espressioni nelle quali l'autore abbia responsabilità di differente tipo (es. tutte le opere nelle quali ha una responsabilità principale, tutte quelle in cui invece ha una responsabilità secondaria);

* delle relazioni tra enti collegati, per quanto concerne i cambiamenti di denominazione e la discriminazione tra enti omonimi;

* le sequenze dei diversi titoli di un periodico;

FRBR si presenta quindi indispensabile per risolvere almeno due importanti questioni:

la capacità di raggruppamento di tutte le espressioni e manifestazioni di un'opera e quindi la capacità del catalogo di svolgere al meglio la funzione 'aggregante'; 
un maggiore controllo bibliografico dato dalla possibilità di dar conto di tutte le varianti dei nomi e dei titoli;

Risulta indispensabile applicare ai record esistenti la struttura del modello FRBR in quanto essi risultano ancorati alla pratica della pubblicazione mettendo al primo posto la conoscenza dell'opera:

Qualcosa in più, quindi, dovrebbe essere fatto per migliorare la struttura dei record bibliografici esistenti; l'attuale struttura del record è basata sull'attuale pratica della pubblicazione: gli utenti vogliono le opere, ma gli editori pubblicano manifestazioni. Così, in verità, è la manifestazione che comanda e non l'opera. ${ }^{94}$

Come osserva Fattahi «gli utenti degli OPAC vorrebbero vedere i record recuperati presentati e ordinati in un contesto significativo in modo da poter comprendere il tipo, la natura e il valore di ciascuna opera (cioè acquisire conoscenza relativa all'opera)» ${ }^{95}$.

Particolarmente importante è conservare la possibilità di eseguire una ricerca contemporaneamente per autore e titolo, restituendo la massima precisione nella ricerca di quelle opere note delle quali si conosca qualche parola del titolo e il nome dell'autore ${ }^{96}$.

La strutturazione dei record secondo il modello FRBR apporterebbe ai cataloghi una maggior semplicità nelle interfacce rendendo la presentazione dei risultati maggiormente comprensibile all'utente; per far ciò è però necessario che gli OPAC implementino tutte le funzionalità necessarie e che gli algoritmi vengano studiati per fornire all'utente visualizzazioni significative in base alle entità del modello.

Restano ancora valide le indicazioni di $\mathrm{Yee}^{97}$ sulle funzionalità che gli OPAC dovrebbero presentare all'utente, basandosi sull'implementazione del modello:

consentire all'utente di compiere una ricerca simultaneamente per nome e titolo;

consentire all'utente che conosce un'opera e il suo autore di identificare quali parole chiave della sua ricerca sono parole chiave di un autore e quali sono parole chiave del titolo (entrambi i due espedienti consentono di aumentare la precisione della ricerca);

\footnotetext{
${ }^{94}$ Martha M. Yee, Beyond the Opac cit.

${ }^{95}$ Rahmatollah Fattahi, From information to knowledge cit., p.34.

${ }^{96} \mathrm{La}$ necessità è sottolineata da Weston che cita Serrai: «Occorre preservare, più che mai all'interno di un sistema bibliografico di tipo relazionale, quello che Serrai definisce "l'inscindibile sinolo autore-titolo"». Cfr. Paul Gabriele Weston, Gli strumenti della cooperazione in rete cit, p. 31. Si veda anche il contributo di Martha M. Yee., FRBRization: a method for turning online public finding lists into online public catalogs, «Information Technology and Libraries», 24 (2005), n. 3, pag. 77-95, anche in <http://repositories.cdlib.org/postprints/715> ove l'autrice afferma a p. 77: «Probably one of the most common OPAC searches is for a work of which the author and title are known».

${ }^{97}$ Martha M. Yee, Applying FRBR to library catalogues : a review of existing FRBRization projects. Relazione presentata al seminario Beyond the OPAC : future directions for Web-based catalogues. Perth, Western Australia, settembre 2006, <http://www.nla.gov.au/lis/stndrds/grps/acoc/papers2006.html>.
} 
* il matching di una ricerca per autore dovrebbe avvenire sui record di autorità, inclusi rinvii e richiami e sulle intestazioni dei record bibliografici; quello di una ricerca per titolo invece sui campi titolo e sui record di autorità dei titoli. Preferibilmente il matching di parole chiave multiple per la stessa entità dovrebbe essere effettuato sui raggruppamenti di record. Ad esempio la corrispondenza di termini dello stesso autore dovrebbe riguardare tutti i record di autorità collegati a quell'autore (inclusi pseudonimi ed enti con le loro suddivisioni); quella di termini appartenenti alle stessa opera dovrebbe essere verificata su tutti i record di autorità relativi all'opera e alle sue parti. Bisogna recuperare dunque ogni identificatore dell'opera che ricorre o in un record bibliografico o in un record di autorità che presenti la corrispondenza simultanea di un autore e di un titolo;

* se, in risposta a un'interrogazione, vengono trovati diversi identificatori per l'opera, bisogna mostrarli all'utente consentendogli di identificare l'opera desiderata. Bisognerebbe inoltre dare in risposta non solo i record bibliografici che hanno fornito la corrispondenza, ma anche gli autori e i titoli uniformi;

* una volta che un'opera viene selezionata, mostrare le sue espressioni, le manifestazioni, le opere correlate, in una visualizzazione sintetica e per raggruppamenti logici, in modo che l'utente possa facilmente visualizzare le espressioni e le manifestazioni o le opere correlate e scegliere se visualizzare o meno le altre opere correlate, le opere sulle opere, le espressioni etc. in maniera indipendente.

* offrire all'utente varie opzioni di ordinamento del set dei risultati, cioè delle espressioni/manifestazioni, in base agli attributi (data / luogo di pubblicazione, editore, illustratore, traduttore) più idonei;

* una volta selezionata una manifestazione, fornire all'utente la visualizzazione dell'intero record della manifestazione, che includa tutti gli elementi discriminatori che differenziano l'espressione/manifestazione da altre espressioni / manifestazioni ${ }^{98}$

* consentire a chi cerca per soggetto o per parola chiave di ottenere i massimi risultati dal processo dalla FRBR-zation. Ad esempio, mostrare all'utente che

\footnotetext{
${ }^{98}$ Yee elenca i seguenti elementi «statements of subsidiary authorship (illustrator, translator, editor, and the like), edition statements, series statements, publisher and date, extent statements (paging, playing time, etc.), presence or absence of illustrations in the physical description, edition history notes and notes about version, and the like».
} 
cerca per soggetto, tutte le opere afferenti a quel dato soggetto, o alla parola chiave.

Per quanto riguarda, infine, l'impatto sulle strutture bibliografiche uno studio di OCLC su più di 40 milioni di registrazioni presenti in Worldcat dimostra che più dell'80\% delle opere ha una sola manifestazione. Ciò si traduce in un grande vantaggio, nella catalogazione derivata e partecipata in vista dell'adeguamento dei cataloghi a FRBR, in quanto i maggiori sforzi si concentrerebbero su un $20 \%$ rappresentato da quelle opere che hanno più espressioni/manifestazioni. Le classificazioni e i soggetti potrebbero essere dati una volta sola a livello dell'opera, piuttosto che essere più volte ripetuti, come nella corrente prassi, per ogni manifestazione, eliminando così ridondanze e riducendo i costi della catalogazione ${ }^{99}$.

\subsubsection{Sistemi completi, prototipi, software}

Con il termine 'implementazione' si indica la creazione di sistemi basati su FRBR, tramite la realizzazione di appositi algoritmi e software.

Le implementazioni di FRBR trovano applicazione sia nei cataloghi elettronici sia in collezioni speciali, archivi, musei, biblioteche digitali. In questo paragrafo ci si soffermerà sulle problematiche di implementazione negli OPAC.

Non c'è dubbio che l'implementazione di FRBR negli OPAC offra grandi opportunità per migliorare le funzionalità di ricerca. Riprendendo le funzioni utente previste dal modello, la possibilità di visualizzare nell'OPAC i dati secondo il modello entità-relazione di FRBR, consentirebbe all'utente un'esplorazione significativa dei contenuti, potendo l'utente navigare tra le entità che formano l'universo bibliografico rappresentato nel catalogo.

Numerosi sforzi sono stati compiuti per implementare il modello negli OPAC, ma bisogna ricordare che FRBR è un modello concettuale e dunque non offre raccomandazioni pratiche di implementazione del modello nei cataloghi, né in altre tipologie di strumenti come collezioni digitali, archivi etc., dunque gli approcci all'implementazione sono tutti di tipo sperimentale.

Molti progetti avviati sono rimasti allo stato prototipale, creati allo scopo di condurre valutazioni pratiche sull'effettiva possibilità di implementazione del modello, per stimolare

\footnotetext{
${ }^{99}$ Barbara B. Tillet, What is FRBR? cit.
} 
la riflessione sull'argomento, sviluppare software e algoritmi per costruire sistemi basati sul modello.

Seguendo la ripartizione proposta da Zhang e Salaba, i progetti di implementazione possono essere distinti in tre grandi categorie ${ }^{100}$ :

* sistemi completi. Si tratta di sistemi che supportano tutti i servizi e le funzioni dei cataloghi elettronici. Tra le implementazioni sono da menzionare WorldCat, Film and Television Archive sviluppato dall'UCLA;

* sistemi sperimentali e prototipi. Si tratta di sistemi creati per valutare le possibilità di implementazione del modello e che non supportano i servizi bibliotecari (prestito, disponibilità, etc). Tra questi è noto il prototipo sviluppato dai Library Labs per la Biblioteca nazionale australiana (NLA);

* algoritmi e software particolarmente studiati per facilitare l'implementazione del modello, ad esempio per la "FRBR-zation" i record nel formato tradizionale MARC e renderli compatibili con le entità e le relazioni previste nel modello. Tra questi vari tools che consentono di creare raggruppamenti (cluster) in base all'opera realizzati dalla Library of Congress (FRBR display tool) e da OCLC (OCLC FRBR Workset Algorithm).

Va rilevato come uno dei problemi più grandi sia convertire il formato MARC e i miliardi di record prodotti con questo formato in dati FRBR-compliant, cioè compatibili con il modello.

Sviluppare nuovi sistemi in grado di usare FRBR sarebbe infatti un compito poco gravoso se si pensasse di costruire sistemi completamente nuovi. Ciò che crea maggiori problemi non è studiare una struttura bibliografica nuova, ma piuttosto convertire i record esistenti per poterli utilizzare in un formato dei dati in grado di riflettere le entità, gli attributi e le relazioni previste dal modello FRBR. Dunque gli sforzi finora sostenuti sono rivolti ai record strutturati nel formato MARC, universalmente adottato per rappresentare i dati bibliografici, in modo da rendere possibile la loro conversione in una struttura compatibile con il modello.

\footnotetext{
${ }^{100}$ Yin Zhang - Athena Salaba, Implementing FRBR in libraries cit.. Si veda anche: Athena Salaba - Yin Zhang, From a conceptual model to application and system development, «Bulletin of the American Society for Information Science and Technology», 33 (2007), n. 6, p. 17-23. Disponibile anche online all'URL: <http://www.asist.org/Bulletin/Aug-07/Bulletin_AugSep07.pdf>.
} 
Particolarmente rivolti a questo scopo sono gli algoritmi che vengono sviluppati per fare in modo che i metadati tradizionali possano essere 'mappati' con gli attributi delle entità previste dal modello.

Tra i sistemi completi WorldCat e UCLA Film and Television Archive OPAC costituiscono i più importanti esempi di sistemi in attività.

Nel campo dei sistemi sperimentali ritroviamo i più interessanti esempi, come i noti FictionFinder e AustLit, progetti rivolti alle opere di narrativa, FRBR prototype di Library Labs e Kent State University FRBR Project. Altri progetti sperimentali non più attivi sono quelli di RedLightGreen (sostituito da WorldCat) e Bibsys (non più disponibile). Particolarmente interessanti sono stati anche i tentativi di sviluppo di nuovi software per i cataloghi elettronici che di recente sono stati affiancati anche dallo studio delle modalità di conversione dei record bibliografici in nuovi formati basati su XML e su RDF. Tra questi Extensible Catalog (XC), VIRTUA ILS, Innovative interfaces, Visual Cat, OPENFRBR, IFPA sono gli esempi più rappresentativi.

Allo scopo di mappare i record esistenti per estrarne gli attributi delle entità FRBR sono rivolti gli algoritmi e i software noti come 'convertitori', tra i quali si distinguono OCLC FRBR Work-Set Algorithm, Converting tool for bibliographic records e altri software per la visualizzazione come FRBR Display tool, FRBR Floater.

In generale, dalla panoramica delle implementazioni, si possono osservare alcune caratteristiche distintive:

1) I progetti correlati a FRBR sono stati applicati a cataloghi collettivi, cataloghi di consorzi internazionali come WorldCat e cataloghi speciali come FictionFinder. In particolare si nota l'applicazione a cataloghi speciali riguardanti collezioni specifiche come la letteratura e la musica: due esempi sono UCLA Film and television archive e OCLC Fictionfinder.

2) Quasi sempre ci troviamo di fronte ad implementazioni parziali del modello, di solito limitata alle entità del gruppo 1 , con qualche compromesso, ad esempio quasi sempre viene eliminata l'entità espressione. Dunque il modello viene sovente 'reinterpretato' per favorire un raggruppamento dei risultati che comprende solo alcune entità dando rilievo ad alcuni attributi, ad esempio, la lingua nel caso dell'espressione. Il prototipo di Libraries Australia introduce il raggruppamento per super-opera per le entità rappresentate da più di un'opera. RedLightgreen, una delle prime implementazioni poi sostituita da WorldCat, schiacciava i quattro livelli corrispondenti alle entità in due: opere e manifestazioni. Worldcat.org che lo ha sostituito utilizza un appoccio FRBR-like, 
implementando il modello con i tre livelli opera, espressione, manifestazione. Per ogni record è possibile visualizzare le altre manifestazioni. FictionFinder introduce l'entità espressione, usata principalmente per il raggruppamento delle manifestazioni in base alla lingua. In questo campo, gli algoritmi per la frbr-zation dei dati, che sono i principali mezzi per ottenere visualizzazioni significative, non forniscono risultati soddisfacenti: Yee ha sottolineato come ad esempio FRBR display tool, lo strumento per la FRBRzation dei dati creato dalla Library of Congress, presenti alcuni importanti difetti: forza l'utente alla ricerca o sul nome o sul titolo (non entrambi), non effettua il matching sui record di autorità, produce display non compatti e difficilmente scansionabili, crea confusione nel mostrare le opere sulle opere e le opere correlate, non raggruppa tutte le opere (a volte sottili differenze nel sottotitolo generano opere diverse), raggruppa espressioni e manifestazioni nell'unico livello delle "edizioni”.

3) FRBR viene implementato come alternativa al relevance ranking. La visualizzazione in FRBR dei risultati è uno dei grandi vantaggi dell'applicazione di FRBR ai cataloghi. In particolare l'applicazione di FRBR produce al posto di un elenco di risultati ordinati per presunta rilevanza un insieme strutturato in maniera logica (es. una lista di opere) che si presta a una navigazione gerarchica che dall'opera arriva all'item e reticolare (opere collegate, soggetti correlati etc.).

Particolari problemi sorgono nel raggruppamento di opere quando gli autori manifestano diverse identità bibliografiche ${ }^{101}$ (es. Mark Twain e Samuel Clemens) o nel caso di pseudonimi. Ciò avviene quando gli algoritmi che gestiscono il raggruppamento non funzionano come dovrebbero (non prendono in considerazione i titoli uniformi oppure, le registrazioni per errore non li possiedono). Il problema nel trattamento di pseudonimi è stato rilevato da Vizin-Goetz nell'implementazione di FictionFinder e da Yee che ha riscontrato il problema in alcune note implementazioni di FRBR tra cui FictionFinder, RedLightGreen, Virtua e nel software FRBR display tool.

Purtroppo a ormai più di dieci anni dalla prima versione di FRBR sono ancora pochi gli sforzi compiuti per dispiegare appieno il potenziale del modello nonostante la letteratura sviluppatasi intorno al modello dell'IFLA sia molto consistente, rivelando che gli studi IFLA rappresentano un argomento al centro dell'interesse.

La spiegazione di questo ritardo va riferita, a mio parere, ad una serie di circostanze:

\footnotetext{
${ }^{101}$ Il caso delle identità bibliografiche è un particolare caso di pseudonimia e derivante dall'uso di pubblicare opere o scritti sotto un nome diverso da quello reale per opere di generi letterari differenti. Questa problematica non è solo catalografica, ma solleva anche interrogativi teorici.
} 
un'evoluzione lenta di FRBR e dei modelli correlati che vanno a formare la famiglia dei modelli FRBR (FRBR, FRAD e FRSAR), elaborati in tempi diversi e solo di recente giunti a termine;

- il mancato coordinamento delle iniziative di studio. Un esempio è quello di FRSAD. Pubblicato nel 2010 a soli due anni dalla nuova revisione del modello FRBR, introduce rispetto a quest'ultimo alcune novità significative come le nuove entità nomen e thema (da considerarsi come superclasse delle dieci entità previste da FRBR) non presenti nella revisione del 2008. In più i recenti anni hanno visto numerose iniziative parallele: i nuovi principi di catalogazione (ICP), nuove norme catalografiche nazionali (RDA, REICAT) nuovi standard per la descrizione (ISBD consolidated edition) e gli esiti prima accennati di FRAD e FRSAD. In tutto ciò è mancato forse un coordinamento in grado di armonizzare le diverse iniziative, come sottolineato già da Guerrini ${ }^{102}$;

* l'implementazione di modelli di sviluppo per gli OPAC che hanno contemplato soluzioni alternative per le interfacce di ricerca, come quelle proposte dai next generation catalogs e dai discovery systems basati sulla visualizzazione dei dati ordinati per ranking e sulla navigazione a faccette quale mezzo per l'esplorazione e il raffinamento del set dei risultati;

* la recentissima tematica dei linked data, che ha spostato l'attenzione verso l'integrazione dei dati bibliografici nel Web Semantico;

* la scarsità di test sull'utenza specifici per le funzioni previste dal modello. Solo di recente alcune importanti indagini sono state rivolte ad accertare le difficoltà dell'utenza nella ricerca con le entità FRBR. Un importante studio è quello di Zhang e Salaba ${ }^{103}$ su tre sistemi prototipi FictionFinder, Libraries Australia demonstrator, e WorldCat. In particolare gli studiosi hanno rilevato che alcune funzioni vengono svolte senza problemi (trovare un'opera, trovare opere correlate), mentre in altre gli utenti incontrano dubbi e perplessità (trovare una manifestazione, trovare un'espressione). Anche la funzione di ottenere l'accesso viene svolta con notevole difficoltà. Sempre secondo questo studio, gli utenti gradiscono miglioramenti che riguardano prima di tutto l'interfaccia, l'indicazione di documenti correlati, le opzioni di raffinamento, le opzioni di ordinamento del set dei risultati e le informazioni sulla disponibilità. Significative si rivelano anche le preferenze

${ }^{102}$ Carlo Bianchini - Mauro Guerrini. Quis custodiet ipsos custodes? Osservazioni sulle relazioni fra FRBR, ICP, ISBD e RDA «Il Bibliotecario», III S. (2009), n. 1, p. 59-85., <http://eprints.unifi.it/archive/00001743/>. ${ }^{103}$ Yin Zhang - Athena Salaba, Implementing FRBR in Libraries cit. 
richieste per il miglioramento dei sistemi: maggiori link ad opere / item correlati, migliori funzioni di raggruppamento, maggiori possibilità di riordino del set di risultati, miglioramento della grafica dell'interfaccia, uso di una terminologia più chiara per le etichette, possibilità della ricerca avanzata nella pagina principale dell'OPAC.

\subsection{FRBR, Next Generation Catalogs e discovery systems: potenzialità di implementazione del modello nei sistemi per la scoperta}

Gli OPAC di nuova generazione e i discovery systems hanno introdotto una serie di caratteristiche che si propongono come alternative alla visualizzazione dei dati secondo il modello FRBR; tra queste il relevance ranking e la navigazione a faccette vengono proposte come modalità di visualizzazione e navigazione nei dati bibliografici.

Un'altra caratteristica significativa di questi sistemi è però anche tecnologica: la capacità di sovrapporsi agli ILS preesistenti per formare uno livello aggiuntivo di ricerca, che consente a queste applicazioni di lavorare completamente sganciate dall'applicativo sottostante.

Se fino a qualche tempo fa non era possibile implementare FRBR senza ricorrere ad un dispendio notevole di energie e risorse finanziarie, sistemi di questo tipo offrono oggi grandi opportunità per sviluppare delle visualizzazioni dei dati in FRBR. In particolare alcuni software per la visualizzazione come FRBR Display tool scaricabili gratuitamente offrirebbero buone opportunità per sviluppare interfacce migliori per la ricerca. Si potrebbe dunque investire nello sviluppo di interfacce che sfruttino il modello FRBR, implementando la sua logica e le sue architetture e utilizzando tecnologie open source, adattando gli algoritmi per la visualizzazione in FRBR ai nuovi sistemi.

Se fino a poco tempo fa non erano ancora stati sviluppati algoritmi per la data frbrzation, oggi invece varie soluzioni sono disponibili in forma gratuita e spesso open source per implementare il modello nelle nuove interfacce, anche se, chiaramente, una vera e propria implementazione completa del modello prevederebbe l'intervento sulla struttura dei record.

Prendendo a riferimento il modello attuale di sviluppo degli OPAC di nuova generazione e degli strumenti di discovery, analizzeremo dunque alcune caratteristiche 
distintive di questo modello e vedremo come l'applicazione del modello FRBR si possa integrare in maniera fruttuosa e vantaggiosa con il modello esistente per fornire all'utente interfacce di ricerca che consentano un'esplorazione significativa dell'universo bibliografico rappresentato nell'OPAC.

\section{Relevance ranking e navigazione a faccette}

Abbiamo visto, nel capitolo precedente, come relevance ranking e navigazione a faccette siano due caratteristiche strettamente collegate tra loro: l'utente infatti ottiene dalla sua ricerca una lista di record potenzialmente rilevanti in base ad un procedimento algoritmico di matching tra i termini contenuti nell'interrogazione e i metadati delle risorse bibliografiche del catalogo.

Le faccette risultano dunque necessarie nei nuovi OPAC per filtrare una lista di record per presunta rilevanza che non dice all'utente quasi nulla sulla struttura dell'universo bibliografico rappresentato. Si può inoltre affermare che le faccette abbiano finora fornito un mezzo per esplicitare gli attributi delle entità, quando invece ciò che manca nel catalogo, ed è fondamentale per la comprensione dei dati bibliografici in esso contenuti, è l'esplicitazione delle relazioni.

L'implementazione del modello FRBR nel catalogo elettronico implica uno scardinamento del modello attuale di sviluppo, in quanto essa si propone come alternativa all'ordinamento per rilevanza.

Applicando il modello ai cataloghi, FRBR diventa la modalità principale di interrogazione e navigazione lungo tutto il percorso di ricerca. Ciò significa che un'interrogazione di un catalogo strutturato secondo il modello potrebbe da subito mostrare le relazioni tra le entità, fornendo una lista di opere e tutti i necessari collegamenti con le entità correlate e consentendo all'utente una navigazione logica nelle porzioni dell'universo bibliografico che di volta in volta visualizzerà nel corso della sua ricerca.

Le faccette, che oggi sono spesso celebrate quale mezzo per consentire un utile raffinamento dei dati potrebbero essere utilizzate come ulteriori filtri, stavolta davvero utili, cioè come modalità alternativa di presentazione degli attributi collegati alle entità che l'utente recupera durante il suo percorso di ricerca.

In questo caso l'utente oltre a muoversi nel reticolo di opere, espressioni, manifestazioni e altre entità correlate che all'utente vengono visualizzate come risultato di un'interrogazione, potrà ricorrere alle faccette per utilizzare gli attributi che vengono 
estratti dalle entità che compongono il set dei risultati per compiere 'salti dimensionali', e lanciare una nuova ricerca a partire da attributi o entità (es. soggetti correlati, classi) che le faccette mostrano. L'utente può, infatti, tramite le faccette mostrate dal sistema, saltare velocemente dalla dimensione semantica a quella semiotica, saltare dall'entità agli attributi $\mathrm{o}$ alle altre entità correlate per lanciare nuova ricerca.

Un discorso diverso si apre per quegli strumenti per la scoperta denominati discovery systems o web scale discovery services che invece si propongono come strumento unificato di accesso a diverse fonti e dove viene recuperata, insieme ai record dell'OPAC, una grande quantità di materiali di diversa provenienza e di diversa tipologia.

Oltre ai metadati dell'OPAC, questi strumenti recuperano varie risorse esterne (oggetti digitali, collezioni di vario tipo, dati provenienti da banche dati, repositories etc.) e di varia tipologia (libri, e-book, immagini, video, musica, oggetti museali, risorse elettroniche etc.)

L'eterogeneità delle risorse recuperabili rende più complessa l'implementazione di FRBR, che come abbiamo visto trova alcune difficoltà di applicazione in alcune particolari tipologie di materiali. In questi strumenti però il modello rivelerebbe alcuni punti di forza nel raggruppamento per opere e super-opere, riunendo cioè tutte le opere discendenti dallo stesso archetipo.

2. Trasformazione dell'interfaccia con introduzione di caratteristiche proprie dei motori di ricerca. Enfasi sulla ricerca per parola chiave per la ricerca semantica

La trasformazione delle interfacce degli OPAC di nuova generazione ha come elemento distintivo l'esibizione del box unico di ricerca come principale modalità di ricerca. Come abbiamo già detto, l'interrogazione secondo questa modalità genera un set di registrazioni bibliografiche che dicono molto poco della complessità dell'universo bibliografico che stiamo esplorando. L'applicazione di FRBR ai cataloghi può rendere questa modalità di interrogazione più significativa attivando in fase di interrogazione la visualizzazione delle relazioni tra le entità.

L'applicazione di FRBR può in qualche modo anche rivalutare la funzione della 'ricerca avanzata', oggi particolarmente trascurata nei cataloghi elettronici che, come abbiamo visto, sono indirizzati verso una ricerca semplificata 'Google like'. Come sottolinea Martha M. Yee ${ }^{104}$, l'applicazione di FRBR consentirebbe l'interrogazione

${ }^{104}$ Martha M. Yee, Applying FRBR to library catalogues cit. 
simultanea per autore e per titolo, funzione oggi negata nella maggior parte dei cataloghi elettronici e in grado di fornire una grande precisione qualora l'utente sia a conoscenza di qualche parola del titolo e del nome dell'autore.

\subsection{Data FRBR-zation e formato MARC}

Nella letteratura professionale si usa il termine data FRBR-zation per riferirsi al processo di trasformazione dei dati MARC in un formato compatibile con FRBR.

Negli anni più recenti la possibilità di adattare i formati MARC ad una strutturazione con FRBR è stata oggetto di vari studi che hanno cercato di individuare nei record MARC gli elementi e le relazioni FRBR che permetterebbero di strutturare le interfacce in modo da mostrare le relazioni tra le entità. Tuttavia vari studiosi hanno costatato le difficoltà dei record tradizionali codificati in questo formato di adattarsi all'espressione delle entità previste dal modello. Il passaggio è reso ancora più difficile poiché anche il formato MARC è stato sottoposto nel tempo a numerosi aggiustamenti derivanti dal cambiamento delle norme catalografiche.

Come è noto, la maggior parte dei record bibliografici contenuti nei cataloghi elettronici di tutto il mondo è basata su MARC (MAchine-Readable Cataloging) ${ }^{105}$, che molto tempo ha fornito alle biblioteche non solo un formato per lo scambio dei dati bibliografici, ma anche un'architettura logica per strutturare i record bibliografici. Il suo scopo principale è stato quello di codificare i record bibliografici contenuti nelle schede catalografiche in un formato leggibile dalle macchine e consentire alla Library of Congress di trasmettere le schede in forma elettronica alle altre biblioteche.

In definitiva, il formato MARC ha rappresentato un'infrastruttura molto potente che ha consentito, sin dagli anni 70 un notevole risparmio dei costi della catalogazione. Inizialmente sviluppato per la Library of Congress è stato poi adottato da istituzioni di livello internazionale come OCLC.

Dopo più di 40 anni di utilizzo, il MARC nella sua evoluzione MARC 21 (e più di recente MARCXML) è uno standard universalmente diffuso e intorno ad esso è stata creata un'infrastruttura (reti, software) fortemente dipendente dal formato di scambio dei dati bibliografici.

\footnotetext{
$105<$ http://www.loc.gov/marc/>.
} 
Se da anni ormai si parla di una dismissione del formato di codifica, in realtà tale evenienza genera grandi difficoltà. Il formato è strettamente legato alle norme catalografiche utilizzate. Ogni cambiamento nelle regole comporta una modifica nel formato e genera ridondanze, in quanto MARC deve continuamente adattarsi alle novità ma, allo stesso tempo, garantire la sopravvivenza di dati bibliografici obsoleti, dunque, ad esempio non si possono facilmente riusare gli stessi tag. Tentativi di semplificazione si dimostrano impossibili in quanto il formato deve garantire la compatibilità con i dati più vecchi o prodotti con precedenti regole di catalogazione.

Altri formati, come ad esempio Dublin Core, non sono stati in grado di sostituire adeguatamente il formato MARC, in quanto non garantiscono la ricchezza e la profondità bibliografica che esso è in grado di offrire.

Allo stesso tempo le nuove regole di catalogazione, come RDA richiedono una vasta rete di link tra i dati bibliografici che MARC 21 con la sua struttura 'piatta' non riesce a soddisfare.

L'implementazione di XML come formato di codifica e scambio ha dimostrato di possedere una buona flessibilità; esso, infatti, è collegato a una serie di iniziative di sviluppo molto solide che compongono l'ambiente XML, come ad esempio XLTS (EXtensible Stylesheet Language Trasformations) che trasforma XML in altri schemi basati su XML e XMLQuery che consente un'interrogazione di dati in XML. Il formato MARCXML ${ }^{106}$ è oggi un formato diffuso in quanto si basa su una trasformazione della struttura ISO2709 del MARC21 in XML conservando il tagging e la codifica di MARC21. Un altro formato di codifica è MODS ${ }^{107}$.

Questi nuovi formati basati su XML consentono di sfruttare la ricchezza dei dati MARC nell'ambiente tecnologico attuale e basato sul web; essi consentono infatti di utilizzare i dati del MARC nei nuovi protocolli come SRU, OAI, METS che richiedono dati codificati in XML.

Nonostante la Library of Congress mantenga tutti questi standard, oggi si fa avanti una volontà di cambiamento del formato di codifica dei dati bibliografici verso l'abbandono del MARC21:

Recognizing that Z39.2/MARC are no longer fit for the purpose, work with the library and other interested communities to specify and implement a carrier for bibliographic

\footnotetext{
$106<$ http://www.loc.gov/standards/marcxml/>.

107 Metadata Object Description Schema (MODS) è uno schema per insiemi di dati bibliografici che può essere impiegato per una varietà di scopi, in particolare per le biblioteche . Lo standard, come per MARC è mantenuto dal Network Development and MARC Standards Office della Library of Congress $<$ http://www.loc.gov/standards/mods/>.
} 
information that is capable of representing the full range of data of interest to libraries, and of facilitating the exchange of such data both within the library community and with related communities. ${ }^{108}$

In risposta al nuovo modello FRBR sono emerse varie questioni relative all'adeguatezza del formato MARC. $\mathrm{Ci}$ si è chiesti se il formato sia in grado di rappresentare tutti gli elementi e le strutture presenti nel modello o se sia il caso di adottare un nuovo schema. In particolare è apparsa la difficoltà nel rappresentare nel record MARC tutte le informazioni richieste dal modello (es. gli attributi dell'opera, dell'espressione, delle manifestazioni etc.).

Lo studio più noto su questa tematica è quello di Tom Delsey ${ }^{109}$ che ha verificato la possibile mappatura del record MARC con FRBR, rilevando che dei 30 attributi relativi all'espressione la metà non ha un corrispettivo tag in un record formattato in MARC. È emerso anche che molti degli attributi richiesti sono sepolti indistintamente nelle note e dunque difficilmente estraibili per le operazioni di mappatura.

I vari progetti di implementazione di FRBR in OPAC e in altri strumenti e contesti hanno fatto emergere varie problematiche, tanto da far concludere agli sviluppatori che MARC non è idoneo a supportare FRBR (o meglio un'implementazione completa del modello) in quanto la struttura piatta di MARC non sarebbe in grado di rappresentare le complesse relazioni richieste dal modello FRBR.

Uno di questi studi è stato condotto su BIBSYS sistema sperimentale in Norvegia ${ }^{110}$. MARC è incapace di sostenere la complessità del modello, e si dimostra compatibile solo in alcuni scenari elementare (opere con una sola espressione, una sola manifestazione) e incontra notevole difficoltà nell'esprimere le relazioni con gli altri gruppi del modello.

In definitiva, si può sostenere che MARC si dimostra necessario al mantenimento di dati tradizionali, ma negli scenari tecnologici che si stanno prefigurando prevale la necessità di strutture basate su XML.

Anche RDA ha reso necessario un confronto di compatibilità con MARC, ma in questo caso il formato riesce a incorporare la maggior parte dei dati richiesti in RDA. Gli

\footnotetext{
${ }^{108}$ Library of Congress Working Group on the Future of Bibliographic Control, On the record: report of the Library of Congress Working Group on the future of bibliographic control, January 9, 2008, <http://www.loc.gov/bibliographic-future/news/lcwg-ontherecord-jan08-final.pdf > p. 25 .

109 Tom Delsey, Functional analysis of the MARC 21 bibliographic and holdings formats [on line]. Washington, Library of Congress, January 4, 2002, <http://www.loc.gov/marc/marc-functionalanalysis/home.html> poi in versione aggiornata e rivista: Library of Congress. Network Development and MARC Standards Office, Functional analysis of the MARC 21 bibliographic and holdings formats, updated and revised [on line], Library of Congress, April 6, $2006<\mathrm{http} / / / \mathrm{www} . l o c . g o v / m a r c / m a r c-f u n c t i o n a l-$ analysis/functional-analysis.html>.

${ }^{110}$ Cfr. infra, Appendice C. Progetti di implementazione di FRBR negli OPAC. Sistemi completi, prototipi, software.
} 
studiosi si sono divisi su posizioni contrastanti: alcuni credono si debba sostituire MARC pena l'incompatibilità con FRBR; altri sostengono che anche RDA sia inadatto a FRBR.

Tuttavia il cambiamento di formato è già in atto; infatti, in seno alla Library of Congress ormai da anni si sta valutando l'abbandono del MARC in favore di nuovi formati, come dimostra la costituzione di un gruppo di studio per la transizione dal MARC21 ad un formato più idoneo agli sviluppi più recenti ${ }^{111}$. La Bibliographic Framework Transition Initiative ${ }^{112}$ avviata nell'ottobre $2011^{113}$ avrà come principale attività lo sviluppo di nuove modalità di cattura e condivisione dei record bibliografici e la ricerca di una soluzione per la sostituzione del formato MARC per lo scambio dei dati bibliografici:

A major focus of the initiative will be to determine a transition path for the MARC 21 exchange format in order to reap the benefits of newer technology while preserving a robust data exchange that has supported resource sharing and cataloging cost savings in recent decades

Il nuovo Bibliographic framework indicherà lo sviluppo di un nuovo ambiente piuttosto che un "formato"; in questo senso il Web Semantico e il modello linked data sono stati individuati come una possibilità interessante di sviluppo per le biblioteche e le istituzioni culturali.

Il progetto di un nuovo bibliographic framework si centra dunque sull'ambiente web, e adotterà il modello dei dati fornito da RDF, i principi e le specifiche dei linked data. Le idee che sottostanno ai linked data e ai suoi protocolli di scambio hanno trovato accoglimento nel settore delle biblioteche e degli altri componenti del circuito dell'informazione e l'uso di RDF viene individuato quale mezzo di integrazione dei dati bibliografici sul web e una modalità importante di accesso all'informazione.

Il gruppo individua una serie di punti che dovranno essere tenuti in considerazione nello sviluppo del nuovo ambiente, che qui si riassumono ${ }^{114}$ :

1. Ampio adattamento alle regole per i contenuti e ai modelli di dati. Si sottolinea che il nuovo ambiente dovrà essere 'agnostico' alle regole catalografiche utilizzate, in considerazione del fatto che ogni comunità adotta le proprie specifiche regole di descrizione, create in momenti storici diversi e che in altri casi alcuni metadati non sono

\footnotetext{
${ }^{111}$ Collaborano all'iniziativa come partner ufficiali Library and Archives Canada e British Library ma anche altri patners come Deutsche Nationalbibliothek e altre agenzie bibliografiche e istituzioni che utilizzano il formato MARC, varie commissioni come MARBI Committee di ALA, Canadian Committee on MARC, e il BIC Bibliographic Standards Group britannico.

$112<$ http://www.loc.gov/marc/transition/>

113 Deanna Marcum, A bibliographic framework for the digital age, October 31, 2011 <http://www.loc.gov/marc/transition/pdf/bibframework-10312011.pdf>

${ }^{114}$ Ivi, § Requirements for a New Bibliographic Framework Environment.
} 
basati su regole. L'accoglimento in RDA delle precedenti AACR2 viene visto come un elemento importante al pari dell'accoglimento delle norme descrittive adottate da altre comunità $^{115}$;

2. Previsione delle tipologie di dati che sono logicamente collegati o supportano la descrizione bibliografica. Si tratta dei metadati delle localizzazioni, le classificazioni, dati sulla preservazione, tecnici, sui diritti e archivistici. Questi dati devono essere opportunamente integrati tramite link e altre soluzioni tecnologiche;

3. Adattamento dei dati testuali e di linked data con URI al posto dei testi. Studiando le modalità di trasmissione dei dati testuali e dei link e tenendo conto che esiteranno ambienti e sistemi con diverse capacità di trasmettere e ricevere dati testuali e link;

4. Considerazione delle relazioni tra i formati di comunicazione, le convezioni di input dei record e la conservazione/manipolazione dei sistemi. L'immagazzinamento, la visualizzazione dei dati e le schermate di input non saranno più in stretta relazione con $\mathrm{i}$ formati di comunicazione come è avvenuto in passato ${ }^{116}$;

5. Considerazione delle necessita delle biblioteche di diversa tipologia e grandezza. Il nuovo ambiente dovrà permettere alle biblioteche di sviluppare i servizi secondo le proprie possibilità e servire gli interessi delle piccole biblioteche di pubblica lettura quanto delle grandi biblioteche di ricerca;

6. Dismissione dello sviluppo del formato MARC in quanto non più necessario. Il gruppo riconosce che i sistemi e i servizi basati su MARC21 saranno un'importante parte dell'infrastruttura ancora per molti anni e che un cambio dei sistemi su larga scala implica per molte realtà un impegno economico notevole. Con la migrazione di AACR a RDA sarà necessario avere la piena compatibilità di RDA con MARC, pertanto si prevede di non apportare altri sviluppi al formato che non siano strettamente collegati a RDA;

7. Compatibilità con i record basati su MARC. Dato che il nuovo schema per la comunicazione dei dati bibliografici sarà uno schema completamente nuovo, esso dovrà prevedere la piena integrazione con i dati già prodotti in MARC. Dato che descrivere nuovamente queste risorse non è un'opzione praticabile, è necessario che vengano preservati il più possibile tutti i dati presenti nei record MARC;

\footnotetext{
115 DACS (Describing Archives, a Content Standard), DACS), VRA (Visual Resources Association) Core, CCO (Cataloging Cultural Objects).

116 A questo proposito Martha M. Yee si è espressa più volte, sottolineando il profondo legame tra la visualizzazione delle interfacce dei cataloghi e la struttura del record.
} 
8. Misure per la trasformazione dal MARC 21 al nuovo ambiente bibliografico. Un requisito necessario sarà la creazione di software che convertano i dati dal MARC al nuovo ambiente bibliografico e la loro sperimentazione.

Gli sviluppi nella modellazione web sono oggi incentrati su RDF, che è il metodo raccomandato da W3C per le descrizioni concettuali e per la strutturazione dell'informazione. Visto che i dati in RDF possono essere 'serializzati' in diversi formati tra i quali XML (eXtensible Markup Language), essi possono essere utilizzati nei database relazionali che compongono i cataloghi.

I Triplestores, database specificamente progettati per immagazzinare e interrogare dati RDF sono oggi ampiamente disponibili e forniranno alla comunità bibliotecaria più opportunità per conservare e scambiare i suoi dati, una riduzione dei costi e la possibilità di contare su un'infrastruttura tecnologica capace di migliorare le conoscenze e le abilità dei professionisti dell'informazione.

Embracing common exchange techniques (the Web and Linked Data) and broadly adopted data models (RDF) will move the current library-technological environment away from being a niche market unto itself to one more readily understandable by present and future data creators, data modelers, and software developers ${ }^{117}$.

L'applicazione di FRBR ai cataloghi trova dunque un ulteriore campo di indagine nell'applicazione di RDF ai dati catalografici e già vari studiosi si sono interrogati sulla possibilità di implementare il modello FRBR basandosi su dati compatibili con il Web Semantico ${ }^{118}$.

Queste ultime osservazioni introducono la tematica che verrà trattata nel capitolo seguente cioè il passaggio dai 'record' ai 'dati' e il nuovi approdi del catalogo nell' ambito del Semantic Web.

\footnotetext{
${ }^{117}$ Deanna Marcum, A bibliographic framework for the digital age cit.

${ }^{118}$ Tra questi, Martha M. Yee ha condotto un esperimento per progettare regole che consentano di mappare le entità del gruppo 1 di FRBR con RDF in modo da ottenere record in grado di fornire visualizzazioni raggruppate in base a tali entità. Cfr. Martha $\mathrm{M}$. Yee, Can bibliographic data be put directly onto the Semantic Web?, «Information Technology and Libraries», 28 (2009), n. 2, p. 55-80, $<$ http://escholarship.org/uc/item/91b1830k $>$. Si veda anche Ian Davis, - Richard Newman, Expression of core FRBR concepts in RDF, [risorsa on line], [S. 1.], Ian Davis, c2005, <http://vocab.org/frbr/core>.
} 



\title{
CAPITOLO 4: L'OPAC DAL WEB 2.0 AL WEB 3.0. LINKED DATA E WEB SEMANTICO
}

\begin{abstract}
«Propongo di considerare questa domanda: "Le macchine sono in grado di pensare?"»

Alan Turing
\end{abstract}

\subsection{Introduzione}

Il quadro dello sviluppo degli OPAC delineato nei precedenti paragrafi ci ha offerto una panoramica delle principali problematiche che sono state affrontate nell'evoluzione dei cataloghi elettronici e ci consente di presentare alcuni nodi di riflessione ancora aperti:

1) i cataloghi elettronici sono ancora lontani da un'adeguata rappresentazione delle entità che compongono l'universo bibliografico poiché non si interviene sulla struttura dei record catalografici che vengono codificati in un formato non più adatto a realizzare la ricchezza e l'espressività offerta dal modello FRBR;

2) i cataloghi si avvalgono di tecnologie non adatte all'apertura dei dati e all'interoperabilità. I dati sono costretti entro griglie di rappresentazione che favoriscono lo scambio esclusivamente all'interno della comunità bibliotecaria e non si integrano con il più ampio spazio delle risorse presenti sul web;

3) la progettazione dei cataloghi ha imboccato la strada dell'imitazione del processo di ricerca tipico dell'ambiente web senza un'adeguata progettazione dei processi di conoscenza tipici della ricerca nel catalogo elettronico;

Ciò che si avverte è la distanza e l'arretratezza di questi strumenti rispetto all'evoluzione delle tecnologie attuali del web, dei modi di produzione, fruizione e scambio dell'informazione che oggi attraversano un profondo cambiamento. Mentre il web si avvia ad un mutamento che rivoluzionerà l'accesso ai dati, lo sviluppo dei cataloghi elettronici ha seguito vie diverse che hanno isolato le biblioteche e i dati prodotti e immagazzinati nei cataloghi elettronici dal resto del web.

Un'opportunità di cambiamento viene oggi offerta dal Web Semantico ed in particolare dalla tecnologia dei linked data che consente la produzione di dati aperti, interoperabili e riutilizzabili nel web. I dati autorevoli prodotti dalle biblioteche potranno essere riutilizzabili e liberamente fruibili nel web, cioè nell'ambiente oggi maggiormente 
frequentato e conosciuto dagli utenti con enormi vantaggi nella creazione, nella ricerca e nell'utilizzo dei dati bibliografici.

I linked open data offrono un enorme potenziale per le istituzioni del patrimonio culturale come biblioteche, archivi, musei. I dati bibliografici possono avere un uso più ampio ed essere collegati a dati prodotti da altre istituzioni accrescendo il valore dei dati stessi, formando la creazione di un grafo globale in grado di collegare le risorse culturali tra loro e alle altre risorse del web ${ }^{1}$.

Nel quadro cosi delineato i dati bibliografici non rimarranno chiusi negli OPAC, ma saranno disponibili per l'utilizzo in altre applicazioni del Web Semantico: «Library data about our resources is no longer just meant to be stored in catalog drawers as the inventory tool to access an individual library's collections. It can now be made available to anyone, anywhere in the world, at any time» ${ }^{2}$.

I dati delle biblioteche potranno essere accessibili alle altre applicazioni del web e riutilizzabili per creare nuovi servizi profondamente integrati con le applicazioni che sfruttano le potenzialità del Web Semantico.

Appoggiare questa nuova modalità di produzione dei dati bibliografici può offrire grandi vantaggi alle biblioteche e ai loro utenti: le biblioteche potranno collegare i loro dati fra di loro, condividere strumenti bibliografici, schemi, ontologie e sistemi di organizzazione delle conoscenze, migliorare il controllo bibliografico e costruire sui dati nuovi servizi per i propri utenti.

\subsection{Dal Web 2.0 al Web 3.0. Open data e la Linked Open Data initiative (LOD)}

Oggi viviamo in un ecosistema informativo basato sui dati; non solo siamo continuamente circondati dai dati, ma essi giocano un ruolo sempre più importante nella

\footnotetext{
1 Jan Hannemann - Jürgen Kett, Linked data for libraries, relazione presentata World Library and Information Congress: 76th IFLA General Conference and Assembly Open access to knowledge - promoting sustainable progress, Gothenburg, $\quad$ Sweden $\quad 10-15 \quad$ August 2010, $<\mathrm{http}$ //www.ifla.org/files/hq/papers/ifla76/149-hannemann-en.pdf>.

${ }^{2}$ Barbara B. Tillett, RDA and the Semantic Web, Linked Data Environment. Barbara B. Tillett, RDA and the Semantic Web, Linked data environment, «JLIS.it», 4 (2013), n. 1, p. 139-146, $<$ http://leo.cilea.it/index.php/jlis/article/view/6303/7876>. Citazione a p. 140.
} 
vita delle persone: potremmo dire di essere entrati nell'era della data economy ${ }^{3}$. Sui dati si costruiscono le fortune di colossi come Amazon, Google e Yahoo! che offrono e utilizzano dati per i propri scopi. I dati acquistano un valore di scambio sempre maggiore facendo sorgere nuove micro-economie che al contempo accelerano il progresso scientifico e democratico.

Oggi si parla sempre più diffusamente di Open data poiché è indubbio che l'accesso alle conoscenze prodotte dalle comunità costituisca il terreno sul quale costruire e sviluppare non solo le attività economiche, ma anche quelle intellettuali. I dati così acquistano valore per il fatto di essere accessibili e aperti cioè utilizzabili senza restrizioni ${ }^{4}$.

In particolare è importante che siano resi pubblici e aperti i dati di interesse ambientale, economico-sociale, medico e genetico i quali si costituiscono attraverso la ricerca finanziata con denaro pubblico, dunque sono da considerarsi al pari dei beni strumentali e tangibili, beni comuni che si formano grazie alla collettività e che devono dunque essere fruiti dalla collettività che li ha prodotti ${ }^{5}$. Le numerose iniziative che sono state avviate nel campo degli Open data si inseriscono nella scia del più famoso movimento dell'Open access $(\mathrm{OA})^{6}$ e di tutte le altre iniziative volte ad aumentare la consapevolezza della necessità dell'accesso aperto per i dati prodotti nell'ambito scientifico e della pubblica amministrazione ${ }^{7}$.

Al centro di tutte le comunità open $^{8}$, il movimento Open data ha l'obiettivo di rendere accessibili i dati della ricerca senza restrizioni e limitazioni.

\footnotetext{
${ }^{3}$ Tom Heath - Christian Bizer, Linked data: evolving the Web into a global data space, Morgan \& Claypool, 2011, <http://linkeddatabook.com/editions/1.0/>.

4 Thomas Baker, Designing data for the open world of the Web, «JLIS.it», 4 (2013), n. 1, p. 63-66, $<$ http://leo.cilea.it/index.php/jlis/article/view/6308/7865>.

${ }^{5}$ Questa spinta anima $\mathrm{i}$ numerosi progetti di pubblicazione di Open Data da parte di amministrazioni pubbliche che rilasciano sempre più spesso dataset aperti contribuendo così in maniera significativa al movimento Open data. Particolarmente rilevanti anche le iniziative che hanno seguito la dichiarazione di Ghent relative all'uso di dati pubblici, software e risorse nel campo educativo. Per una panoramica sui progetti nel campo degli Open data si veda il recentissimo contributo di Antonella De Robbio, Antonella De Robbio, Forme e gradi di apertura dei dati. I nuovi alfabeti dell'Open Biblio tra scienza e società, «Biblioteche Oggi», 30 (2012), n. 6, p. 11-24.

${ }^{6}$ L'Open Access (OA) ha mirato all'accesso aperto dei risultati delle ricerche accademiche finanziate con denaro pubblico e si è consolidato con le dichiarazioni di Budapest (2002), Bethesda (2003) e Berlino (2003).

${ }^{7} \mathrm{Si}$ tratta di iniziative che coinvolgono soprattutto i dati scientifici, ove vi sono varie aree di resistenza all'uso pubblico dei dati e in cui il dibattito sui dati aperti si rivela cruciale. Uno di questi campi, citato da De Robbio è quello dei dati che si riferiscono a virus ed epidemie, ove l'apertura dei dati si rivela critica per le conseguenze che potrebbero derivarne, tra le quali un esempio è il bio terrorismo. Altri studiosi fanno risalire le origini dell'Open Data al movimento dell'Open goverment, che prevede la trasparenza dei dati delle pubbliche amministrazioni.

${ }^{8}$ Il punto di riferimento per tutte le comunità open è l'Open Knowledge Foundation (OKF), organizzazione non-profit nata nel 2004 per promuovere la conoscenza aperta e la condivisione dei dati e delle conoscenze a fini benefici. Tra le comunità open più note si segnalano: l'Open source per le licenze dei software, l'Open Content dedicato alle opere della creatività, l'Open Knowledge che si prefigge la diffusione delle conoscenze senza barriere linguistiche, di accessibilità, di comprensione. L'Open Access riguarda il contenuto della
} 
La domanda di dati è crescente, dunque è indispensabile trovare un modo per fornire l'accesso ad essi in modo che possano essere facilmente riutilizzati da tutta la comunità del web, per facilitare la scoperta dei dati e per integrare dati provenienti da diverse fonti.

L'esposizione dei dati nella forma di linked open data ${ }^{9}$, cioè di dati tra loro correlati, aperti e interoperabili risponde a queste nuove esigenze, consentendo la scoperta, l'accesso, l'uso e l'integrazione dei dati e offrendo un potente strumento per condividere e riutilizzare i dati su scala mondiale.

Rispetto al movimento Open data che promuove le iniziative volte ad eliminare le barriere economiche sociali e culturali alla libera condivisione dei dati, linked data mira ad abbattere le barriere tecnologiche che impediscono la libera condivisione dei dati per creare uno spazio unico e libero di condivisione e di riutilizzo dei dati nel web.

Il termine linked data, nella definizione classica fornita da Tim Berners Lee, fondatore del web e principale esponente del movimento del Web Semantico, si riferisce ad una serie di buone pratiche per pubblicare e collegare dati strutturati sul web, ma linked data significa soprattutto un mutamento di paradigma: vuol dire creare dati che siano 'del web' e non solo 'sul web, 10 .

Attraverso i linked data è possibile pubblicare dati sul web in una modalità leggibile e interpretabile da una macchina, il cui significato viene definito da una stringa di parole e marcatori (tripla) per costituire un reticolo di dati collegati appartenenti ad un dominio e collegabili ad altri dataset relativi ad altri domini presenti nel web. Si costruisce così una rete di dati globale, i cui contenuti possono essere scambiati e interpretati dalle macchine, ovvero la base per il Web Semantico ${ }^{11}$.

La prospettiva del Web Semantico coinvolge direttamente lo sviluppo di sistemi di information retrieval come i cataloghi e le modalità ricerca consentendo il superamento dell'information retrieval tradizionale e facilitando il ritrovamento dei contenuti dei documenti stessi e l'integrazione con altri documenti presenti nella rete creando una rete diffusa di conoscenze. A differenza del recupero classico basato sulla corrispondenza dei

\footnotetext{
ricerca scientifica e accademica, l'Open Learning riguarda l'accesibilità della piataforme per la didattica a distanza e infine l'Open Linked data che è l'oggetto di questo capitolo, che riguarda la disponibilità di dati in rete aperti, accessibili e interoperabili. Tra le iniziative merita una menzione l'Open Library project, iniziativa di Internet Archive che nella piattaforma Open library crea una pagina web per ogni libro pubblicato fornendo l'accesso libero, la condivisione e la diffusione di ogni opera presente.

${ }^{9}<$ www.linkeddata.org > . L'iniziativa, come si spiegherà anche più avanti. nasce dall'interesse di Tim Bernes Lee fondatore del Web, e si riferisce ai dati pubblicati in base a principi progettati per facilitare i collegamenti tra dataset, set di elementi e vocabolari nel Web Semantico.

${ }^{10}$ Karen Coyle, Linked data: an evolution, «JLIS.it», 4 (2013), n. 1, p. 53-62, $<$ http://leo.cilea.it/index.php/jlis/article/view/5443/7889>.

${ }^{11}$ Tim Berners-Lee - James Hendler - Ora Lassila, The Semantic Web, «Scientific American», 284, 5, (2001) p. 34-43.
} 
termini dell'interrogazione con quelli presenti nel documenti, il Web Semantico propone un recupero basato sui concetti e la possibilità di ampliare le conoscenze a risorse che vengono automaticamente collegate tra loro, superando i limiti del tradizionale collegamento ipertestuale.

Se il web 1.0 collegava i documenti e il recente web 2.0 ha collegato le persone e i contenuti da loro generati, oggi il web 3.0 o meglio il 'Web dei dati' si propone di mettere in relazione i dati per produrre e organizzare la conoscenza.

\subsubsection{Dal web dei documenti al web dei dati}

Per comprendere il cambio di prospettiva che sottende la logica dei linked data è necessario focalizzare l'attenzione sul modo che fino ad oggi è stato adottato per rappresentare e condividere dati strutturati sul web: HTML, i microformati e le API.

HTML $^{12}$ è il principale mezzo di pubblicazione dei dati sul web, ma è un linguaggio orientato alla rappresentazione in un browser di documenti testuali (e non di dati) e dunque non consente di estrarre significato dai testi, né l'utilizzo di dati semanticamente strutturati. Oggi la maggior parte dell'informazione presente sul web si trova nella forma di pagine HTML, cioè di documenti testuali che vengono collegati l'uno con l'altro attraverso i collegamenti ipertestuali o hyperlink. Tali documenti possono essere letti e compresi dall'uomo, ma i loro significati restano oscuri alle macchine in quanto i dati in essi contenuti non sono adeguatamente strutturati.

La struttura dei dati è fondamentale per la condivisione e il riutilizzo dei dati stessi; estrarre dati strutturati dalle pagine web è un problema con cui il web si è confrontato sin dagli albori e che è stato solo parzialmente risolto tramite l'invenzione dei microformati ${ }^{13}$.

I microformati, tecnologia più recente dell'HTML, consentono di inglobare dati strutturati dentro le pagine web e sono stati usati in numerosi progetti ${ }^{14}$, ma hanno un limite: tali dati sono relativi a set molto piccoli ove vengono definite le entità, le loro proprietà e relazioni e, al momento dell'attuale sviluppo tecnologico, non sono in grado di esprimere relazioni complesse. Tuttavia di recente questa tecnica trova applicazione nei

\footnotetext{
$12<\mathrm{http}$ //www.w3.org/html/>

$13<\mathrm{http}: / /$ microformats.org/>.

${ }^{14}$ Tra il 2005 e il 2012 il sito segnala i seguenti progetti, alcuni dei quali sono poi stati abbandonati: 20052009: StructuredBlogging; 2005-2011: Google Base schema; 2007-2011: Google Data API/Elements 20092009: Yahoo et al CommonTag.org; 2010-2012+ Facebook OGP meta tags; 2011-2012+ Google/MS/Y! Schema.org; 2012-2012+ Twitter Cards meta tags; 2012-2012+ OpenMetadata.org.
} 
microdata, un'evoluzione dei microformati e una specifica di HTML5, di cui l'esempio più noto è Schema.org che ha lo scopo di esporre dati comprensibili dai motori di ricerca ottenendo risultati più intelligenti, tramite l'utilizzo di dati strutturati all'interno delle pagine web.

Infine, un altro approccio largamente usato per condividere dati, introdotto dallo sviluppo delle tecniche del Web 2.0, è l'utilizzo delle API (Application Programming interfaces $^{15}$ ).

Le Web API oggi sono utilizzate quasi dappertutto per creare mashups di vario tipo ovvero ricombinazioni di dati provenienti da applicazioni diverse ${ }^{16}$. A differenza dei collegamenti ipertestuali delle pagine HTML, le API creano astrazioni di dati che possono essere riutilizzate per una particolare applicazione; lo svantaggio è che i dati restituiti restano in frammenti isolati e non consentono di risalire alle pagine web dalle quali sono estratti, dunque non consentono di scoprire altri dati.

Inoltre le API non assegnano identificatori unici alle risorse, così gli identificatori valgono solo per la particolare applicazione che li utilizza e restano confinati a quella particolare applicazione.

Se i microformati e le API sono tecnologie che consentono di trasmettere dati tra applicazioni del web, la struttura delle pagine web è in larga parte espressa in HTML.

Attraverso HTML nel web si crea una rete di documenti (web of documents), cioè una rete di documenti e di oggetti connessi tramite link non classificati.

Collegare i dati presenti sul web richiede invece una modalità di collegamento che consenta di descrivere le cose reali (i dati) e di specificare perché due o più dati sono collegati: si tratta di passare da un web dei documenti al web dei dati (web of data) cioè ad uno spazio globale di dati collegati.

Il concetto di linked data è strettamente legato al concetto di Semantic Web, nel senso che i linked data si possono considerare una metodologia per la realizzazione del Web Semantico proposto da Tim Berners Lee, fondatore del Web: l'utilizzo dei linked data rende possibile il passaggio da una rete di link ad una rete di concetti tra loro collegati, cioè di dati.

\footnotetext{
${ }^{15}$ Un’ Application programming interface (API) è un insieme di procedure disponibili per i programmmatori, di solito raggruppate a formare un set di strumenti specifici per l'espletamento di un determinato compito all'interno di un certo programma. La finalità è ottenere un'astrazione di dati semplificando così il lavoro di programmazione. $\mathrm{Si}$ veda la relativa voce in Wikipedia <http://it.wikipedia.org/wiki/Application_programming_interface>.

${ }^{16}$ Tra queste, le API di Google (es. Google Maps e Google Books) e di Amazon sono certamente le più conosciute. Un elenco completo di API è mantenuto dal sito Programmable Web $<\mathrm{http}: / /$ www.programmableweb.com/>.
} 


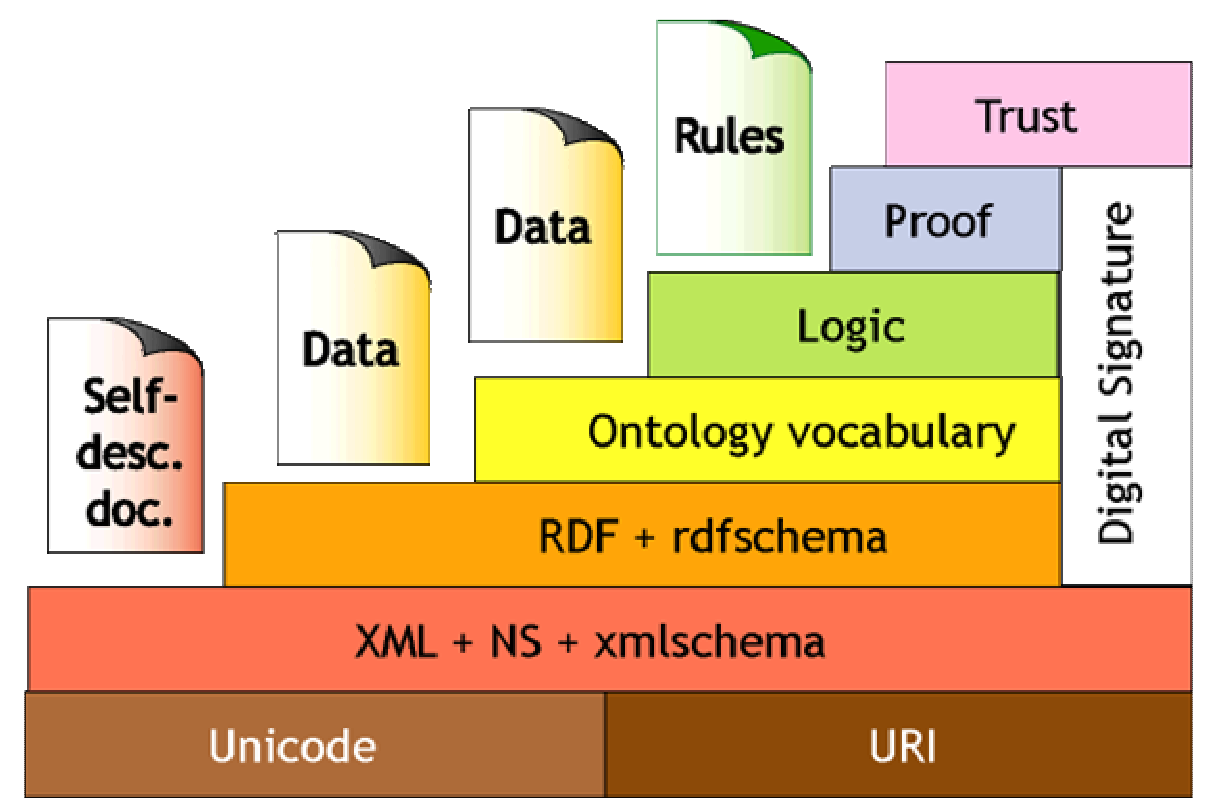

Fig. 4.1 - Gli strati del Web Semantico ${ }^{17}$

A partire dal 2006 il progetto di realizzazione del Web Semantico è stato revisionato in favore degli emergenti linked data, che sono stati individuati come la tecnologia in grado di fornire la struttura portante per la costruzione del Web Semantico ${ }^{18}$.

Il raggiungimento del Semantic Web richiede la creazione di uno strato di contenuti accessibili e rielaborabili dalle macchine e dunque l'essenza dei linked data è che segnano un cambiamento nel modo di pubblicare dati sul web; dai dati HTML comprensibili all'uomo si passa a dati interpretabili e comprensibili alle macchine realizzando il passaggio dal web dei documenti al web dei dati.

La differenza tra i due modi di concepire il web è stata esposta nel 2008 da Tim Berners Lee Christian Bizer e Tom Heath ${ }^{19}$ :

Il web ipertestuale, un web 'di documenti' è fatto di dati sconnessi tra loro ed è:

17 Eric Miller, Digital Libraries and the Semantic Web, <http://www.w3.org/2001/09/06-ecdl/slide170.html>.

${ }^{18}$ In un articolo pubblicato nel 2006 dal titolo The Semantic Web revisited Tim Berners-Lee, Nigel Shadbolt e Wendy Hall hanno ridefinito alcuni aspetti del Web Semantico in funzione della dinamicità del Web. L'idea era quella di sviluppare e migliorare le ontologie in maniera collaborativa, grazie all'intervento di comunità di pratica. In seguito, questo nuovo approccio si è orientato sempre più a far sì che i dati vengano strutturati sotto forma di Linked Data che non sotto forma di ontologie. Nell'articolo del 2009 i Linked Data vengono considerati come la tecnologia per la costruzione del Web Semantico. (Cfr. rispettivamente: Tim BernersLee, Nigel Shadbolt e Wendy Hall, The Semantic Web Revisited, «Intelligent systems», 21 (2006), n. 3, p. 96-101, <http://eprints.soton.ac.uk/262614/1/real/OLD_Semantic_Web_Revisted.pdf> e Christian Bizer Tom Heath - Tim Berners-Lee Linked data - The story so far, «IJSWIS», 5 (2009) n. 3, p. 1-22, $<$ doi:10.4018/jswis.2009081901>).

${ }^{19}$ Christian Bizer - Tom Heath - Tim Berners-Lee, Linked Data: Linked data: principles and state of the art, [relazione presentata a] 17th International World Wide Web Conference, W3C Track @ WWW2008, Beijing, China, 23-24 April 2008, <http://www.w3.org/2008/Talks/WWW2008-W3CTrack-LOD.pdf>. 
* simile ad un file system globale, ricco ma monolitico;

* una descrizione piatta di oggetti e di documenti;

* una rete di relazioni tra oggetti costituita da relazioni tra gli oggetti che non sono connaturate negli oggetti stessi, dunque la semantica del contenuto e dei legami tra gli oggetti non dipende dal contenuto ma vi è apportata da operatori umani;

* il grado di struttura degli oggetti è troppo basso;

* gli oggetti sono utilizzabili dagli esseri umani e non dalle macchine.

Il Web Semantico o 'Web dei dati' è invece:

* un database globale simile ai database relazionali costituito non da documenti ma da singoli oggetti (cose) che hanno specifiche relazioni tra loro;

una descrizione degli oggetti che diventa oggetto del web e riutilizzabile;

* una rete di relazioni tra oggetti correlati in base alla natura intrinseca degli oggetti stessi e con un grado alto di struttura;

* una rete di relazioni tra 'cose' studiata principalmente per essere compresa dalle macchine e secondariamente dall'uomo ${ }^{20}$.

Linked data è un modo di pubblicare, condividere e connettere i dati sul web; allo scopo di costruire questa rete di relazioni tra i dati, linked data si serve di una struttura standard che è fornita dal linguaggio RDF.

Anche il paragone con i database relazionali, utilizzati per immagazzinare i dati può essere utile per comprendere il cambio di prospettiva proposta dai Linked data e dal Web Semantico:

* RDF collega dati o 'cose' e non documenti testuali;

* i collegamenti sono 'qualificati' (typed) cioè esprimono la natura della connessione e consentono di scoprire altri dati.

\footnotetext{
${ }^{20}$ Mauro Guerrini - Tiziana Possemato, Linked data: a new alphabet for the semantic web, «JLIS.it», 4 (2013), n. 1, p. 67-90, <http://leo.cilea.it/index.php/jlis/article/view/6305>.
} 


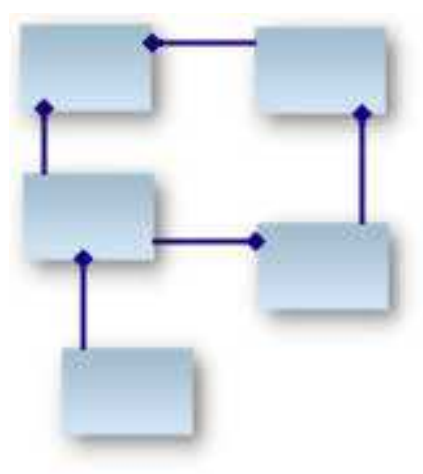

Relational DB

Tables Related By Primary Key

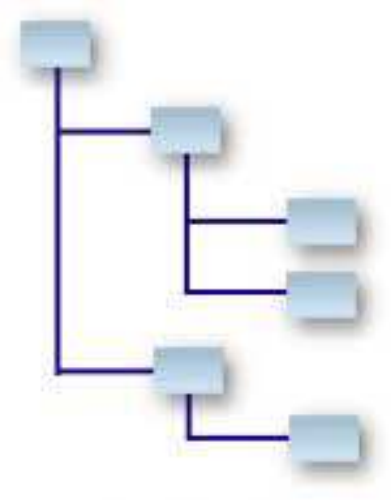

Hierarchical DB

Parent Nodes Have More Intrinsic Importance

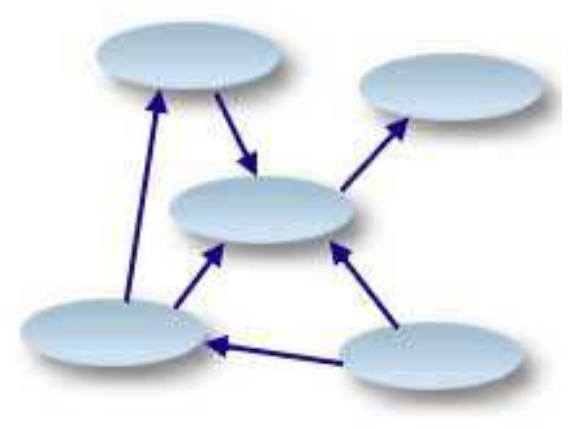

Graph DB

Arbitrary Object Relations No Intrinsic Importance

Fig. 4.2. - Differenza tra database relazionale, gerarchico e a grafor $^{21}$

\subsubsection{I principi dei Linked Data}

Con il termine linked Data Tim Berners Lee, fondatore del World Wide Web, si riferisce ad una serie di buone pratiche per pubblicare dati strutturati nel web che vengono esposte in quattro principi comunemente noti come 'Linked data principles' ${ }^{22}$ :

* usare gli URI per i nomi degli oggetti;

* usare HTTP URIs per dare modo agli utenti di trovare questi nomi;

* una volta trovato un URI, fornire informazioni utili utilizzando standard (RDF e SPARQL);

* includere links ad altri URIs in modo che possano scoprire più cose.

L'idea è quella di utilizzare l'architettura del web per condividere dati su scala globale. Il web, come è noto, si serve di tre principali standard: gli URI (Uniform Resource Identifiers) per l'identificazione, l'HTML (HyperText Markup Language) per i contenuti e HTTP (HyperText Transfer Protocol) come protocollo di scambio e accesso ai dati. Il Web Semantico si basa sulla stessa architettura per condividere i dati su scala globale.

Il primo principio afferma che bisogna usare HTTP URI ${ }^{23}$ per identificare gli oggetti e i concetti. Per pubblicare dati sul web tutti gli oggetti di interesse e le loro relazioni

\footnotetext{
${ }^{21}<$ http://www.linkeddatatools.com/semantic-web-basics $>$.

22 Berners-Lee, Tim. Linked data: Design issues, 27 luglio 2006, $<$ http://www.w3.org/DesignIssues/LinkedData.html>.

${ }^{23}$ La sintassi per la costruzione degli URI è definita in Tim BERNERS-LEE [et al.] Uniform Resource Identifier (URI): generic syntax. Request for Comments: 3986, 2005, <http://tools.ietf.org/html/rfc3986> .
} 
devono essere identificati da HTTP URI evitando altri schemi conosciuti come URN $^{24}$ o $\mathrm{DOI}^{25}$. Gli URI fungono da nomi e da accessi all'informazione.

Il secondo principio afferma che gli URI devono essere 'dereferenziabili' intendendo per 'dereferenziazione' la possibilità di accedere alle descrizioni delle risorse identificate dall' ${ }^{\prime} I^{26}$.

In tal modo un client HTTP può cercare l'URI e recuperare la descrizione della risorsa identificata dall'URI. Tutte le descrizioni di oggetti destinate alle macchine devono essere rappresentate da dati in RDF. In tal modo HTTP verrà usato per rendere le informazioni comprensibili agli essere umani e RDF alle macchine.

Il terzo prescrive l'utilizzo di un singolo modelli di dati (data model) per pubblicare dati strutturati sul Web e cioè RDF, modello di dati a grafo particolarmente progettato per il Web Semantico.

Il quarto prescrive l'utilizzo dei link (link RDF) per collegare non più i documenti, ma le $\operatorname{cose}^{27}$. In uno spazio globale $\mathrm{i}$ link RDF collegano dati provenienti da dataset diversi, così linked data si basa sull'idea classica di web, ma la trasforma in un web di dati correlati.

Ci soffermeremo, dunque, sugli ultimi due punti introducendo il modello RDF ed esaminando i principali tipi di collegamento tra i dati.

\footnotetext{
${ }^{24}$ Un Uniform Resource Name (URN) è uno standard che identifica una risorsa all'interno di un namespace, ma, a differenza di un URL, non permette l'identificazione della locazione della risorsa stessa. Un esempio di URN è i campo bibliotecario il codice ISBN.

${ }^{25}$ Digital Object Identifier (DOI) è uno standard che consente l'identificazione duratura, all'interno di una rete digitale, di qualsiasi entità che sia oggetto di proprietà intellettuale e di associarvi i relativi metadati.

${ }^{26}$ Ciò significa che un client http che trova un URI può usare il protocollo HTTP per recuperare una descrizione della risorsa. Due sono le principali strategie per rendere glu URI dereferenziabili: 303 URI e Hash URI. Sulle due modalità di accesso si veda Heath- Bizer, Linked data: evolving the Web into a global data space cit. In generale il primo metodo è più indicato per dataset di grandi dimensioni, mentre il secondo per i vocabolari.

${ }^{27}$ Sono chiamati semplicemente links per distinguerli dai collegamenti ipertestuali o hyperlinks.
} 


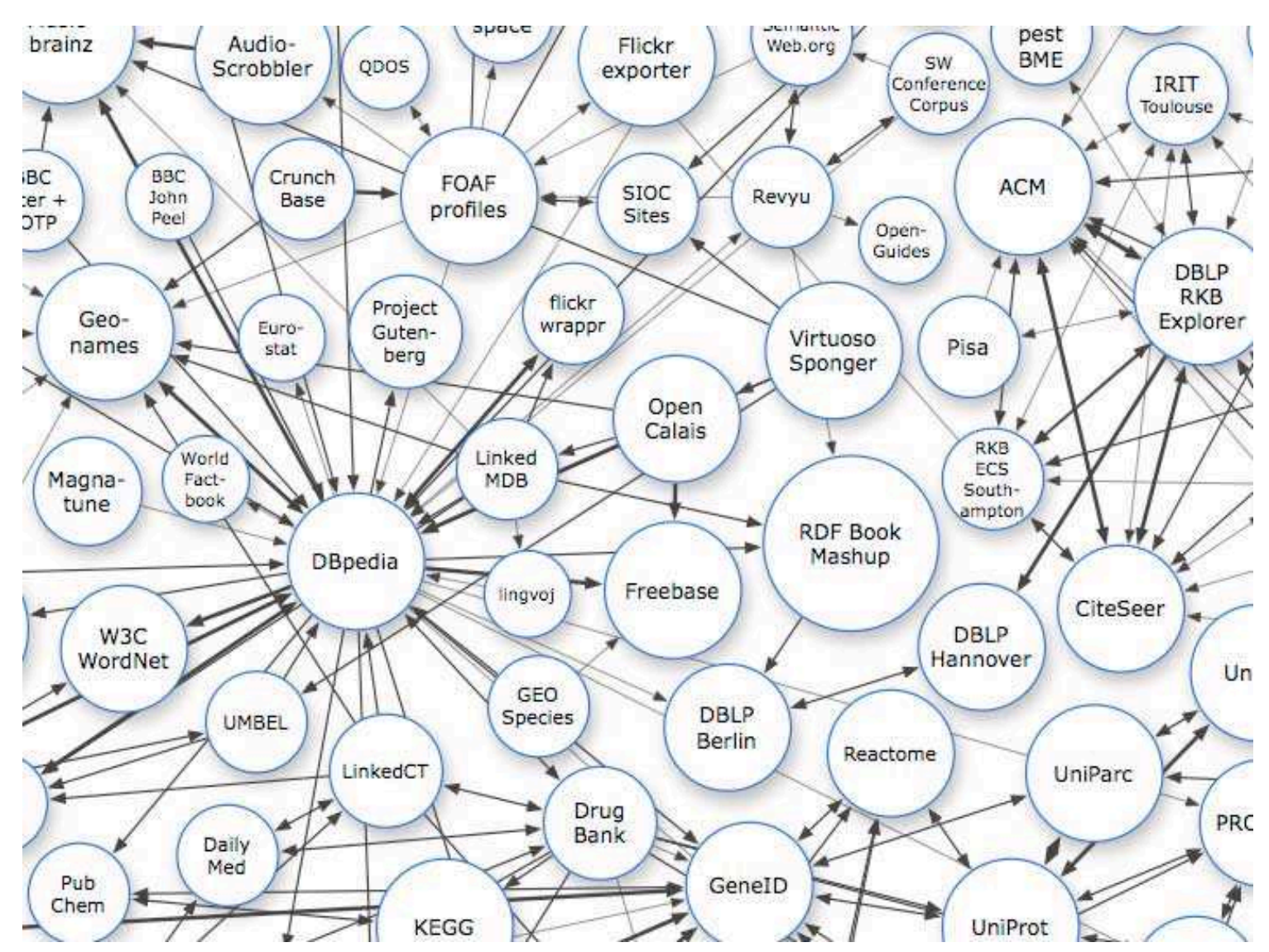

Fig. 4.3 - Una porzione della 'nuvola dei linked data'28

\subsubsection{RDF data model}

Produrre linked data significa esprimere il contenuto delle informazioni, ovvero il loro 'significato', rendendole condivisibili e riutilizzabili nel web.

Il modello dei dati di riferimento è Resource Description Framework (RDF) ${ }^{29}$, uno standard sviluppato e mantenuto dal consorzio $\mathrm{W}^{3} \mathrm{C}^{30}$ per descrivere semanticamente le risorse e le loro relazioni.

Il modello RDF codifica i dati nella forma di asserzioni (statements) formate da tre parti:

1. soggetto: la parte della frase che identifica la cosa descritta ovvero la risorsa

2. predicato: la proprietà della cosa

3. oggetto: il valore della proprietà della cosa

\footnotetext{
${ }^{28}<$ http://www.linkeddata.org $>$.

${ }^{29}<$ http://www.w3.org/RDF/>.

${ }^{30}$ Una rappresentazione visiva della timeline degli standard creati da W3C si trova in: $<$ http://www.dblab.ntua.gr/ bikakis/XML\%20and\%20Semantic\%20Web\%20W3C\%20Standards\%20Timeli ne-History.pdf $>$.
} 
Le asserzioni sono dette 'triple' e sono concetti atomici ovvero unità minime di significato; esse vengono espresse in RDF in forma di grafi cioè di nodi e archi

Un'asserzione è ad esempio:

Alberto Moravia -- è autore di -- La noia .

\section{Soggetto -- Predicato -- Oggetto}

Una tripla può essere rappresentata come un grafo e codificata tramite una sintassi basata su XML, come in questo esempio:

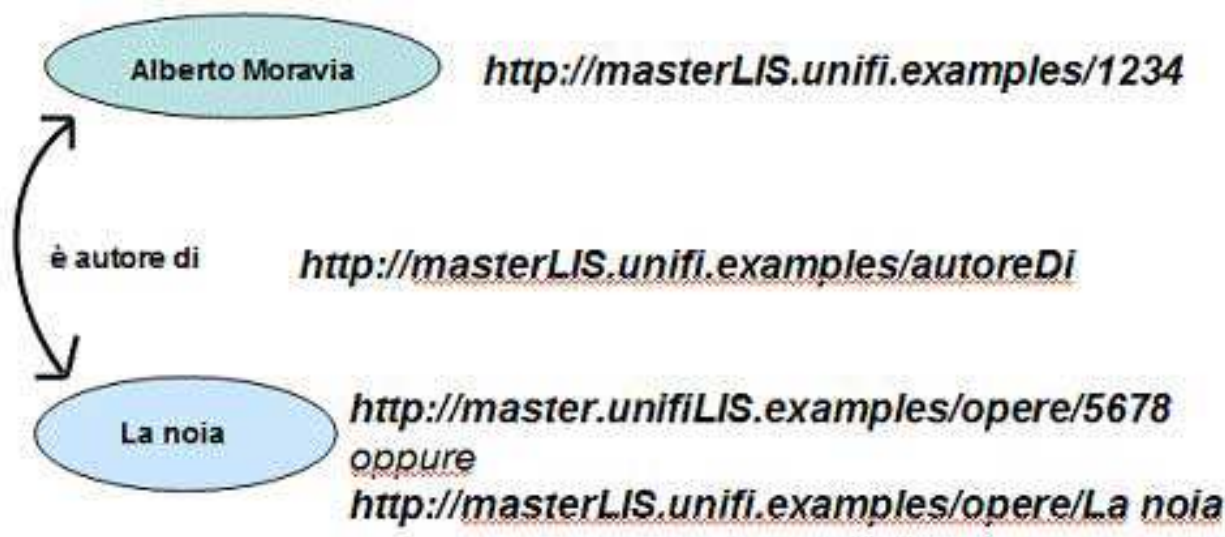

Fig. 4.4a - Esempio di tripla rappresentata in forma di grafo e fornita di URI ${ }^{31}$

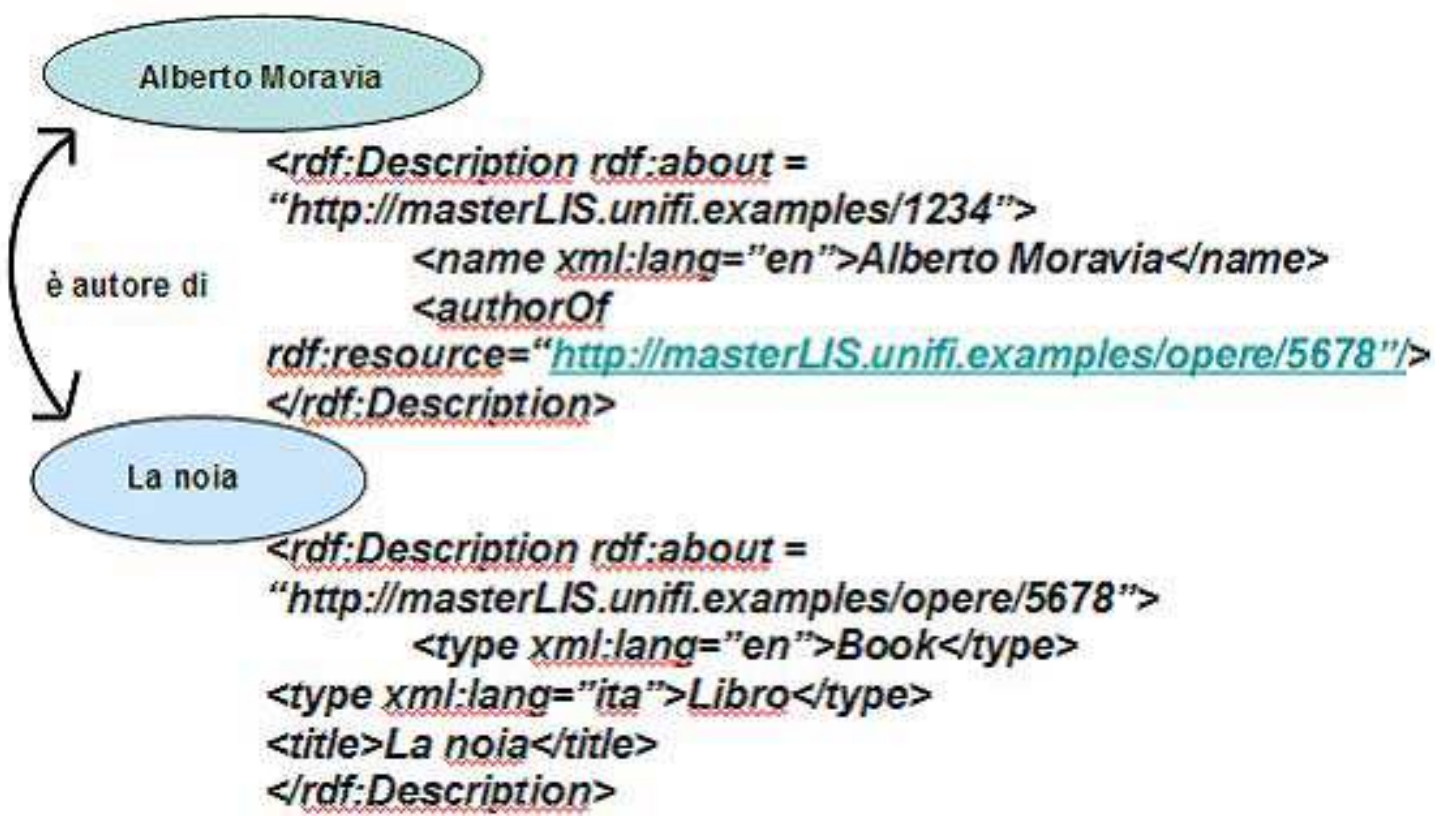

Fig. 4.4b - Esempio di tripla espressa in RDF/XML ${ }^{32}$

Una volta espresso un certo numero di asserzioni le macchine sono in grado di dedurre conoscenza tramite un meccanismo inferenziale ${ }^{33}$. Ogni asserzione aggiunge

\footnotetext{
${ }^{31}$ Mauro Guerrini - Tiziana Possemato, Linked data: a new alphabet for the semantic web cit, p. 81 .

${ }^{32}$ Ivi, p. 82.
} 
nuova informazione, generando nuove inferenze e producendo nel web una quantità sempre maggiore di informazione strutturata e classificata.

Le triple sono codificate tramite una sintassi basata su XML; l'uso di una sintassi XML è l'elemento più importante ai fini della loro apertura e del loro riutilizzo: i dati possono così essere letti non solo nell'ambiente nel quale vengono prodotti (es. il catalogo di una biblioteca), ma nell'intero sistema informativo globale.

Tutti gli elementi della tripla per poter essere riutilizzabili devono essere espressi tramite URI dereferenziabili anche se è possibile, ma non consigliato, esprimere il soggetto/l'oggetto in modalità testuale. Il predicato viene espresso tramite l'uso di vocabolari, tra i quali i più diffusi in ambito bibliotecario sono Dublin Core, Bibliontology, Foaf e SKOS.

Al fine di pubblicare linked data sul web è necessario adottare formati per la 'serializzazione' dei dati, ovvero usare una sintassi basata su RDF; questo è infatti un 'modello per i dati' che consente di esprimere dati utilizzando un soggetto, un predicato e un oggetto.

Il consorzio W3C mantiene vari formati per la 'serializzazione', tra i quali i principali standard RDF/XML ${ }^{34}$ e $\mathrm{RDFa}^{35}$.

La sintassi RDF/XML consente di pubblicare linked data sul web, mentre RDFa consente di incorporare delle triple in un documento HTML. Altri formati di serializzazione utilizzati sono anche Turtle ${ }^{36} \mathrm{JSON}^{37}$.

Anche i link utilizzati per collegare le risorse devono essere 'link RDF'; il funzionamento del Web Semantico si basa proprio sull'attivazione di questa nuova tipologia di link, i quali sono profondamente diversi dai link ipertestuali: se questi collegano documenti, i link RDF invece collegano 'cose' e il tipo di collegamento, ovvero la natura della relazione tra due cose, è specificata nel link stesso.

\footnotetext{
${ }^{33}$ L'inferenza è il processo deduttivo con il quale date due proposizioni si deduce la verità di una proposizione da un'altra giudicata vera, ovvero vi è la possibilità di dedurre conoscenza tramite percorsi. Il calcolo inferenziale è conosciuto in matematica e nella logica ed è largamente usato nell'informatica. La caratteristica del modello RDF è proprio quella di produrre asserzioni che intersecandosi producono per inferenza nuove asserzioni. Sul meccanismo dell'inferenza si veda anche la definizione di W3C all'URL: $<$ http://www.w3.org/standards/semanticweb/inference>.

34 Dave Beckett - Brian McBride. RDF/XML Syntax Specification (Revised)W3C Recommendation 10 February 2004, <http://www.w3.org/TR/REC-rdf-syntax/>. RDF/XML è il principale formato di serializzazione di RDF.

${ }^{35}$ RDFa 1.1 Primer Rich Structured Data Markup for Web Documents W3C Working Group Note 07 June 2012, <http://www.w3.org/TR/rdfa-primer/>RDFa consente di esprimere in RDF dati contenuti nelle pagine web.

${ }^{36}$ Terse RDF Triple Language (<http://www.w3.org/TeamSubmission/turtle/>) è un formato tabellare che consente di esprimere triple in RDF.

37 JavaScript Object Notation (<http://www.json.org/>) è un formato adatto per lo scambio dei dati in applicazioni client-server basato sul linguaggio JavaScript.
} 
Nel Web Semantico devono essere attivati tre importanti tipi di link che sono necessari al suo funzionamento:

- i link relazionali esprimono un collegamento tra diversi dataset puntando a cose, persone luoghi presenti in un altro dataset o insieme di dati collegati;

- i link di identità collegano tra loro URI di dataset diversi che si riferiscono alla stessa cosa o allo stesso concetto (sono anche denominati 'alias URI');

- i link di vocabolario puntano dal dato alle definizioni dei termini dei vocabolari usati per descrivere il dato stesso. In tal modo è possibile un'integrazione tra vocabolari diversi ${ }^{38}$.

I link RDF consentono il collegamento tra descrizioni che ri riferiscono alla stessa 'cosa' ovvero allo stesso URI e la fusione di grafi che hanno in comune degli elementi per formare grafi sempre più vasti e complessi.
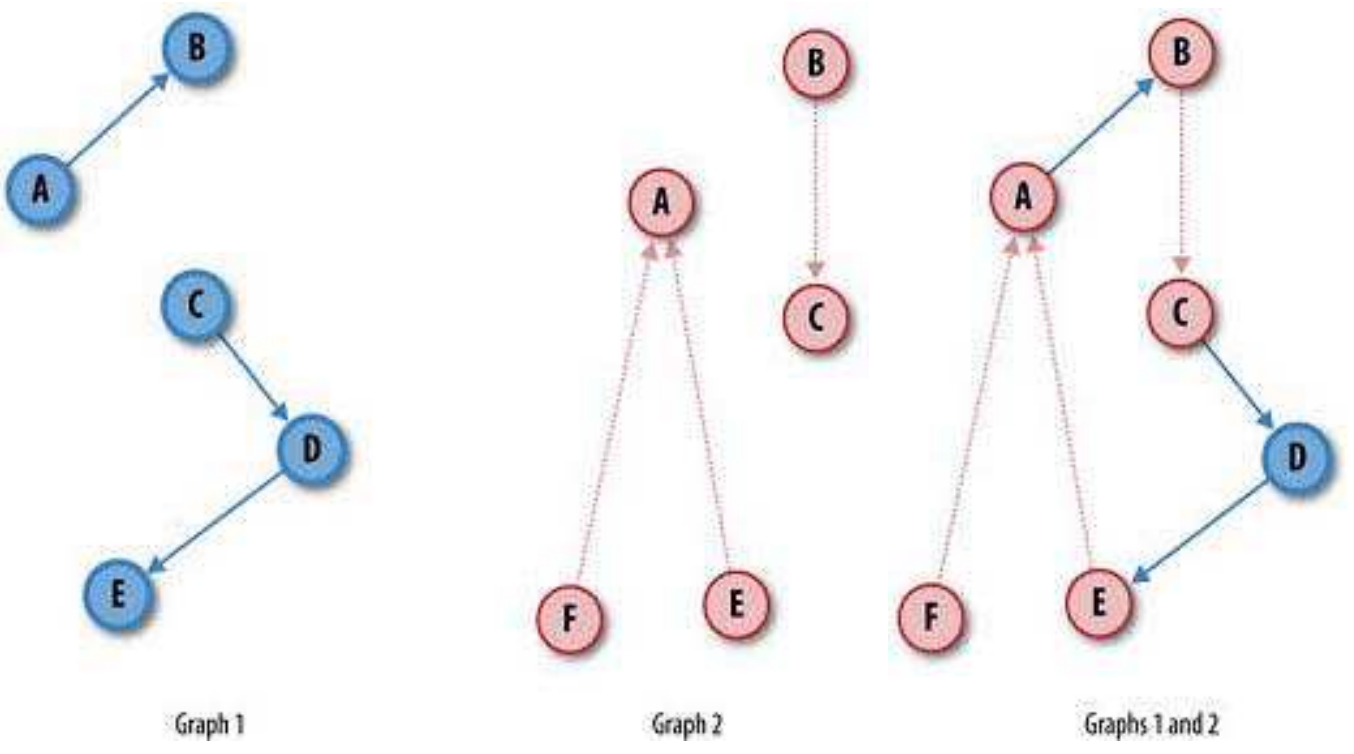

Fig. 4.5 - Unione di grafi aventi identificatori in comune ${ }^{39}$

\footnotetext{
${ }^{38}$ Cfr. Tom Heath - Christian Bizer, Linked Data: evolving the Web into a global data space cit.

${ }^{39}$ Toby Segaran - Colin Evans - Jamie Taylor, Programming the Semantic Web. Build flexible applications with graph data, Beijing, O'Reilly, 2009, p. 27.
} 


\subsubsection{Vocabolari e ontologie}

Affinché il meccanismo delle connessioni tra i dati collegati funzioni è necessario disporre di un'infrastruttura tecnologica in cui ogni concetto sia identificato univocamente e possa essere così riconosciuto da una macchina, ma è anche necessario che oltre ad esprimere asserzioni circa una particolare risorsa vi sia la possibilità di definire i vocabolari di riferimento che consentiranno di stabilire quali termini usare per descrivere una risorsa e le sue proprieta; è necessario dunque il ricorso a vocabolari e ontologie.

Nel Web Semantico i vocabolari e le ontologie definiscono concetti e termini usati per descrivere e rappresentare una particolare area di interesse. Lo scopo dei vocabolari è classificare i termini che vengono usati in un particolare settore disciplinare ed indicare le relazioni e gli utilizzi del termine in un particolare contesto; dunque i vocabolari e le ontologie rappresentano il cuore del Web Semantico.

Nel Web Semantico non c’è una vera e propria distinzione tra vocabolari e ontologie. Generalmente si usa il termine 'ontologia' quando è presente un formalismo stretto, che per i vocabolari non è invece necessario.

La necessità di creare descrizioni a diversi livelli formali è implicita nel processo della modellazione semantica. I modelli consentono la comunicazione umana, la comprensione degli eventi e la loro previsione, permettono di mediare punti di vista differenti e, in definitiva, di condividere e creare conoscenza.

Generalmente i modelli utilizzati per la comunicazione umana possono possedere un grado di formalismo basso ed essere dunque genericamente 'informali', in quanto possono essere corretti, riscritti e interpretati dall'uomo; i modelli informali sono spesso validi in un determinato contesto e possono esistere modelli con vari gradi di formalita ${ }^{40}$. Tuttavia la possibilità di organizzare la conoscenza, ovvero di spiegare fenomeni e effettuare previsioni dipende dall'uso di 'formalismi'. I modelli formali costituiscono la base dei modelli matematici e di quelli destinati alle macchine.

Nel Web Semantico è necessario adottare un modello formale che consenta a tutti i partecipanti di condividere i significati e di esprimere le differenze, che riguardano i diversi modi di comprendere e organizzare il sapere. Il Web Semantico offre una piccola quantità di modelli formali, tramite i quali è possibile produrre conoscenza tramite le

\footnotetext{
${ }^{40}$ Un esempio sono le norme giuridiche e tutti i modelli scritti nel linguaggio naturale, ma anche quelli che si basano sulla comunicazione visiva mediante immagini. Informali sono anche i meccanismi di tagging del web. Gradi di formalità si possono trovare nelle norme giuririche valide in contesti diversi, spesso sovrapposti (es. norme comunitare, legislazione nazionale, regionale, comunale).
} 
inferenze, inoltre fornisce un'infrastruttura nella quale ogni comunità può esprimere punti di vista differenti su uno stesso concetto organizzando i punti in comune e le divergenze. Ad esempio diverse comunità potranno descrivere lo stesso ambito di conoscenza utilizzando propri linguaggi e strumenti o diversi livelli di specificità. La modellazione semantica deve quindi prevedere diversi livelli di 'espressività', che sono necessari per trattare lo stesso argomento a livelli diversi e con strumenti diversi da comunità differenti. Vocabolari e ontologie sono dunque necessari per offrire a tutte le comunità che operano nel Web Semantico la possibilità di esprimersi in merito a qualsiasi cosa utilizzando le entità, le relazioni e la terminologia più appropriata ${ }^{41}$.

I linked data, come vedremo, offrono una potente tecnologia per collegare ontologie prodotte da comunità diverse.

Nel campo dell'organizzazione delle conoscenze un'ontologia ${ }^{42}$ è uno schema strutturato e condiviso di concetti e delle relazioni che descrivono un' area della conoscenza utilizzando linguaggi non ambigui e dunque processabili da una macchina ${ }^{43}$.

Le ontologie sono da tempo considerate lo strumento attraverso il quale è possibile superare i limiti posti dall'information retrieval tradizionale ai fini del recupero dell'informazione semantica nel web. Esse possono essere utilizzate, insieme ai tesauri documentari, per un recupero mirato dell'informazione e, dunque, nella fase di ricerca, istruendo i sistemi ad effettuare il matching tra i termini contenuti in una query e i termini presenti nell'ontologia. Attraverso l'uso di un'ontologia a supporto della ricerca in un catalogo, ad esempio, è possibile non solo aumentare la precisione, ma fornire all'utente una rete di concetti che consentono di espandere la ricerca ${ }^{44}$.

A differenza dei thesauri documentari che offrono un'espressività limitata delle relazioni tra i concetti, che sono riconducibili alle sole relazioni gerarchiche e associative, le ontologie possiedono un'espressività semantica di gran lunga superiore, dettagliando più

\footnotetext{
${ }^{41}$ Sul ruolo della modellazione semantica nel Web Semantico si veda: Dean Allemang- James Hendler, Semantic web for the working ontologist, Morgan Kaufmann Publishers - Elsevier, 2008, p. 13-23.

${ }^{42}$ Molto nota è la definizione di Thomas Robert Gruber: «An ontology is an explicit specification of a conceptualization» fornita nel contributo A translation approach to portable ontology specifications, «Knowledge Acquisition», 5 (1993) n. 2, p. 199-220.

${ }^{43}$ La definizione è tratta dal contributo di Biagetti, cui si rimanda per un'analisi delle principali ontologie presenti in rete e del loro utilizzo a supporto della ricerca (Maria Teresa Biagetti, Le ontologie come strumenti per l'organizzazione della conoscenza in rete, in Numero speciale monografico su le ontologie a cura di Maria Teresa Biagetti, «AIDA informazioni», 28 (2010) n. 1-2, p. 9-31, $<$ http://www.aidainformazioni.it/2010/122010.html>).

${ }^{44}$ Le ontologie, infine, possono essere utilizzate anche nel processo di indicizzazione semiautomatica dei documenti, facilitando le operazioni di assegnazione dei termini più appropriati.
} 
approfonditamente la tipologia di relazione tra i concetti e offrendo una molteplicità di relazioni logiche che consentono ai sistemi (agenti intelligenti) di produrre inferenze ${ }^{45}$.

Le ontologie consentono, in definitiva, di rappresentare le entità e di descriverne le caratteristiche, di esplicitare le relazioni tra i concetti e di consentire categorizzazioni e ragionamenti di tipo deduttivo.

Nel Web Semantico le ontologie e i vocabolari sono indispensabili per creare dataset, ovvero set di dati che rappresentano una collezione di risorse o grafi che appartengono ad uno stesso dominio disciplinare o che sono accomunati da una qualche caratteristica e identificati tramite URI dereferenziabili.

L'insieme dei dataset forma oggi un enorme grafo comunemente denominato Web of data. Essi rappresentano attraverso milioni di triple ogni sorta di 'cosa': dalle persone, ai luoghi geografici, a dati statistici e governativi, fino ai film, libri, musica e cinema.

Il Web dei dati è dunque un grafo di dati che può contenere qualunque tipologia di dato: chiunque può pubblicare dati, persino tra loro contradditori senza vincoli nella scelta dei vocabolari; le entità vengono connesse tramite link RDF creando una gigantesco grafo nel quale è possibile scoprire altre informazioni. Nel Web dei dati, inoltre, ogni dato si autodescrive: un'applicazione può ottenere una descrizione del significato di ogni dato; i dati sono autoparlanti.

Rispetto al semplice collegamento tra dati di provenienza eterogenea ottenibile attraverso l'utilizzo di API, il Web dei dati si manifesta integrato: l'accesso ai dati è più semplice, in quanto i dati utilizzano lo stesso modello (data model) per rappresentare i dati, ovvero RDF.

Il Web Semantico consente di esprimere vocabolari e ontologie utilizzando linguaggi come RDF Schema ${ }^{46}$, Simple Knowledge Organization System (SKOS) ${ }^{47}$ e Web Ontology Language $(\mathrm{OWL})^{48} 49$.

Mentre il primo consente di creare vocabolari di ogni tipo, gli ultimi due sono linguaggi per la creazione di vere e proprie ontologie di concetti, in grado dunque di esprimere gerarchie e tassonomie di concetti e dotate di un alto grado di espressività.

${ }^{45}$ Alcuni thesauri documentari sono stati reingegnerizzati sottoforma di ontologie formali, tra questi il thesaurus Agrovoc; altri si presentano come ontologie-thesauri (es. WorldNet). Oggi queste ontologie vengono fornite sottoforma di linked data. AGROVOC, ad esempio, è stato pubblicato come linked data ed espresso in Simple Knowledge Organization System (SKOS).

${ }^{46}<$ http://www.w3.org/TR/rdf-schema/>.

${ }^{47}<$ http://www.w3.org/2009/08/skos-reference/skos.html\#ConceptScheme>.

${ }^{48}<$ http://www.w3.org/TR/\#tr_OWL_Web_Ontology_Language $>$.

49 Nel sito di W3 $<$ http://www.w3.org/standards/techs/rdf\#w3c_all $>$. Una guida per creare i vocabolari è quella del W3C: Best Practice Recipes for Publishing RDF Vocabularies W3C Working Group Note 28 August 2008. $<$ http://www.w3.org/TR/swbp-vocab-pub/>. 
RDF Schema (RDFS) è un linguaggio per descrivere ontologie leggere in RDF ovvero vocabolari consistenti in una elencazione di elementi e di proprietà ${ }^{50}$.

Web Ontology Language (OWL) estende l'espressività di RDFS ad esempio stabilendo altri concetti per modellazione come owl:equivalentClass e owl:equivalentProperty che consentono di definire mappature tra vocabolari diversi oppure owl:inverseOf che consente di stabilire che una proprietà è l'inverso di un' altra.

Mentre RDFS e OWL consentono di esprimere modelli concettuali in termini di classi e proprietà. $\operatorname{SKOS}^{51}$ è un vocabolario per esprimere gerarchie di concetti più complesse e progettato per supportare l'uso di sistemi di organizzazione delle conoscenze (KOS) come thesauri, schemi di classificazione, liste di soggetti e tassonomie nell'ambito del Web Semantico.

I vocabolari e le ontologie più diffuse che possono essere usate nella creazione di linked data sono reperibili anche nella directory Linked Open Vocabularies (LOV) ${ }^{52}$; tra questi si segnalano in questa sede i più utilizzati, che forniscono anche il contesto nel quale i dati bibliografici prodotti dalle biblioteche possono essere collegati ad altri dati presenti nel Web Semantico:

- FOAF (Friend of a Friend) ${ }^{53}$ è un'ontologia che descrive le persone, le loro attività, le relazioni con altre persone o cose. L'ontologia può essere utilizzata per costruire liste di autorità;

- Dublin Core Metadata Initiative (DCMI) ${ }^{54}$ definisce attributi di metadati come titolo, creatore, data, soggetto etc.;

- The Music Ontology ${ }^{55}$ definisce termini per descrivere vari aspetti che si riferiscono alla musica: artisti, album, tracce, etc. e le relazioni (es. 'ha iniziato l'attività', 'homepage');

- The Event Ontology ${ }^{56}$ definisce termini per descrivere eventi;

\footnotetext{
${ }^{50}$ Le due classi principali sono rdfs:Class rdf:Property consentono di definire classi di risorse e proprietà. le annotazioni semantiche vengono espresse tramite le due proprietà rdfs:label e rdfs:comment, infine vi sono altre proprietà che stabiliscono relazioni tra classi e tra proprietà come rdfs:subClassOf, rdfs:subPropertyOf, rdfs:domain e rdfs:range.

${ }^{51}$ SKOS (Simple Knowledge Organisation System) è il data model sviluppato dal W3C Semantic Web Deployment Working Group(SWDWG) per favorire l'uso, la condivisione e l'interoperabilità di sistemi di organizzazione della conoscenza (come thesauri, classificazioni, tassonomie, folksonomie etc.) nel contesto del Web Semantico. Oggi i principali thesauri gestiti da Biblioteche nazionali stanno progressivamente adottando questo standard per i propri vocabolari controllati. Tra questi LCSH, LCC, CDDe il Thesaurus del Nuovo Soggettario.

$52<$ http://labs.mondeca.com/dataset/lov/>. Progetto di Bernard Vatant e Pierre-Yves Vandenbussche mantiene una reppresentazione dell'ecosistema dei linked open vocabularies (in RDFS o ontologie in OWL) presenti nella Linked Data Cloud e collegate tra loro attraverso il vocabolario VOAF.

$53<$ http://xmlns.com/foaf/spec/>.

${ }^{54}<$ http://dublincore.org/documents/dcmi-terms/>.

$55<\mathrm{http}: / /$ musicontology.com/>.
} 
- The Programmes Ontology definisce termini per descrivere programmi TV e radio;

- Creative Commons Schema ${ }^{57}$ definisce i termini per descrivere le licenze in RDF;

- Bibliographic Ontology ${ }^{58}$ o Bibliontology è un'ontologia per decrivere concetti e proprietà in citazioni e riferimenti bibliografici.

- SIOC (Semantically-Interlinked Online Community) ${ }^{59}$ è un vocabolario creato per descrivere aspetti delle comunità online, come forum, utenti, post, etc.;

- The Good relations Ontology ${ }^{60}$ definisce termini per descrivere prodotti e servizi commerciali online.

\subsubsection{Il Web of Data}

Il Web dei dati (Web of Data) è, dunque, un gigantesco grafo che collega i diversi dataset presenti nella rete. Varie comunità ad oggi hanno reso disponibili i propri dati nella forma di linked data, ma pubblicare linked data sul web significa rendere i dati non solo accessibili, ma 'aperti' e dunque liberamente fruibili e riutilizzabili nel web.

Le origini del Web of Data risalgono al 2007 e al Linked Open Data Project ${ }^{\mathbf{6 1}}$, iniziativa del consorzio $\mathrm{W} 3 \mathrm{C}^{62}$, cui hanno partecipato dapprima piccoli enti e

${ }^{56}<$ http://motools.sourceforge.net/event/event.html\#product $>$.

$57<\mathrm{http}: / /$ creativecommons.org/schema.rdfA $>$.

${ }^{58}<$ http://bibliontology.com/>. L'ontologia è utilizzata anche nell'OPAC delle biblioteche svedesi Libris ed visualizzabile all'URL: <http://bibotools.googlecode.com/svn/bibo-ontology/tags/1.3/bibo.xml.owl\#>; si forniscono di seguito a scopo esemplificativo le definizioni di soggetto e titolo:

$<!--$ http://purl.org/dc/terms/subject -->

<owl:ObjectProperty rdf:about="http://purl.org/dc/terms/subject">

<skos:scopeNote xml:lang="en">Used to describe the subject of a bibliographic resource.

$<$ /skos:scopeNote $>$

$</$ owl:ObjectProperty $>$

$<!--$ http://purl.org/dc/terms/title -->

<owl:AnnotationProperty rdf:about="http://purl.org/dc/terms/title">

<rdf:type rdf:resource="http://www.w3.org/2002/07/owl\#ObjectProperty"/>

<skos:scopeNote xml:lang="en">Used to describe the title of a bibliographic resource</skos:scopeNote>

$</$ owl:AnnotationProperty $>$

$59<\mathrm{http}: / /$ rdfs.org/sioc/spec/>

${ }^{60}<\mathrm{http}: / /$ purl.org/goodrelations/>.

61 L'iniziativa è del gruppo Semantic Web Education and Outreach Interest Group (SWEO IG) <http://www.w3.org/wiki/SweoIG/TaskForces/CommunityProjects/LinkingOpenData>. L'obiettivo di W3C SWEO Linking Open Data community project è «to extend the Web with a data commons by publishing various open data sets as RDF on the Web and by setting RDF links between data items from different data sources».

62 Il World Wide Web Consortium (W3C) è un'organizzazione non governativa internazionale fondata nel 2004 al Massachusetts Institute of Technology (MIT) da Tim Berners-Lee in collaborazione con il CERN e che ha come scopo lo sviluppo del World Wide Web. La principale attività svolta dal W3C consiste nello stabilire standard tecnici per il World Wide Web inerenti sia i linguaggi di marcatura che i protocolli di 
successivamente alcuni importanti enti e istituti quali BBC, Reuters e Library of Congress. Il progetto ha avuto lo scopo di convertire i dataset già esistenti in strumenti aperti e di stabilire dei principi per pubblicare dataset in RDF aperti ovvero non condizionati da licenze commerciali o da restrizioni.

Da allora il numero di dataset liberamente disponibili è cresciuto e tale crescita è graficamente visibile nella Linked open data cloud $^{63}$, che dal 2007 registra la crescita del progetto e viene continuamente aggiornata con i nuovi dataset che vengono resi disponibili sul web e nel registro $\mathrm{CKAN}^{64}$.

I principali dataset spaziano nei campi e nelle più diverse discipline. Di seguito vengono elencati i più noti, suddivisi per categorie

-Interdisciplinari: Dbpedia ${ }^{65}$ e Freebase ${ }^{66}$ sono i principali dataset generali. Il primo estrae gli articoli di Wikipedia, assegnando URI alle cose e ai concetti presenti nell'enciclopedia ed ha costituito la base per lo sviluppo e il collegamento di altri dataset. Posto al centro della cloud è un vero hub ovvero uno nodo centrale della nuvola. Quasi tutti i dataset linkano a Dbpedia. Freebase fornisce descrizioni degli oggetti prelevando dati da Dbpedia e Geonames.

-Geografici: Geonames ${ }^{67}$ è il primo e più autorevole dataset di luoghi geografici con circa 8 milioni di luoghi censiti. Dati geografici sono presenti anche in dataset statistici come EuroStat, WorldFactbook e US Census.

-Governativi: i dati prodotti da organismi governativi rappresentano una parte determinante della LOD cloud. La presenza in rete di questi dati sotto forma di dati aperti rappresenta motivo di trasparenza per i governi e impulso alla realizzazione dell'Open government. L'amministrazione Obama negli USA e il governo britannico hanno disposto

\footnotetext{
comunicazione. Il W3C ha stabilito e ufficializzato più di 50 standard tra i quali HTML, XML, URL, URI, CSS. RDF, OWL e PNG.

${ }^{63}<$ http://richard.cyganiak.de/2007/10/lod/>. L'immagine mostra i dataset che sono stati pubblicati nel formato Linked data dalle comunità che aderiscono al progetto Linking Open Data Project e di altri organismi ed è basato sui metadati raccolti nella directory CKAN. Il diagramma mantenuto da Richard Cyganiak (DERI, NUI Galway) e da Anja Jentzsch (Freie Universität Berlin). L'ultimo aggiornamento del 19 settembre 2011 conta 295 dataset.

${ }^{64}<$ http://www.ckan.net/> oggi The Data Hub <http://thedatahub.org/> è un repository mantenuto da Open Knowledge Foundation. Ogni dataset registrato in CKAN viene fornito di una descrizione dei dati e di altre informazioni utili. Un altro repository italiano che segnala progetti relativi agli Open Data è Spaghetti Open Data.

$65<$ http://dbpedia.org/>. DBpedia rappresenta lo sforzo condiviso per estrarre informazione strutturata da Wikipedia. La versione inglese della DBpedia knowledge base attualmente descrive 3.77 milioni di cose, di cui 2.35 milioni sono classificate in una vasta ontologia che include 764,000 persone, 573,000 luoghi (di cui 387,000 luoghi abitati), 333,000 opere dell'ingegno (tra cui 112,000 album di musica, 72,000 film and 18,000 video games), 192,000 organizzazioni e enti (di cui 45,000 aziende e 42,000 istituti di formazione scuole e università), 202,000 specie and 5,500 malattie.

${ }^{66}<\mathrm{http}: / /$ www.freebase.com>.

${ }^{67}<$ http://www.geonames.org/>.
} 
la creazione dei portali integrati <www.data.gov> e <www.data.gov.uk> che forniscono un'ingente mole di dati in triple $\mathrm{RDF}^{68}$. Altre importanti iniziative sono quelle dei dei governi di Svezia ${ }^{69}$ e Austria ${ }^{70}$ e delle amministrazioni di San Francisco, Toronto, Londra, Saragozza. Nel nostro paese dati.piemonte.it è tra i primi progetti italiani nella direzione dei linked data.

-Media: nel campo dei media una delle prime organizzazioni a riconoscere le potenzialità dei linked data è stata la British Broadcasting Corporation (BBC) che ha rilasciato a partire dal 2008 due siti web che hanno esposto i dati dei programmi televisivi e di quelli musicali forniti via radio in forma di linked data e di pagine web convenzionali. Successivamente $\mathrm{BBC}$ ha rilasciato Wildlife Finder ${ }^{71}$ che si presenta come un sito web dedicato ad ogni specie animale. Ogni singola risorsa viene fornita in HTML ed esposta anche in forma di linked data. Successivamente anche altri colossi dell'informazione hanno seguito l'esempio: il New York Times ha rilasciato il vocabolario dei suoi soggetti sotto una licenza $\mathrm{CCA}^{72}$.

Nel luglio del 2012 l'ultimo arrivato è Nature Publishing Group ${ }^{73}$, il gruppo editoriale della prestigiosa rivista Nature che espone i suoi dati offrendo nella forma di linked data circa 270 milioni di triple relative agli argomenti trattati nella rivista sotto licenza CC0 che consente il massimo riutilizzo dei dati.

-Istituzioni culturali: le biblioteche sono fortemente impegnate nella produzione di dataset, vocabolari, e set di elementi in linked data. I vocabolari della Library of Congress, Libris, gli element set di RDA e British national bibliography sono solo alcune delle numerose iniziative che le biblioteche stanno mettendo in atto nel campo dei linked data, cui si riserva un approfondimento nei paragrafi successivi. Particolarmente importante in questo settore è stata la creazione in seno al W3C del Library linked Data Incubator Group cui si affianca la presenza di Open Biblio, comunità che registra le novità nel campo degli Open Data nel settore biblioteconomico. Particolarmente attivo è anche il settore educativo ove si registrano interessanti sviluppi quali DBLP. RBKexplorer e Talis Aspire ${ }^{74}$.

\footnotetext{
$68<$ http://www.data.gov/semantic $>$.

$69<$ http://www.opengov.se/>.

${ }^{70}<$ http://gov.opendata.at/site/blog $>$.

$71<$ http://www.bbc.co.uk/wildlifefinder $>$.

$72<$ http://data.nytimes.com/>. I dati del soggettario mantenuto da oltre 150 dalla testata americana conta circa 10.000 descrittori di soggetti, persone, organizzazioni luoghi. I dati sono stati rilasciati nel gennaio 2010 con licenza CCBY.

$73<$ http://data.nature.com/>.

$74<$ http://www.talis.com/aspire $>$.
} 
- Scienze della vita: i dati relativi a questo settore disciplinare sono particolarmente importanti per il progresso scientifico e tecnologico. In questo settore Bio2 $\mathrm{RDF}^{75}$ rappresenta il principale progetto di riunione di importanti archivi relativi alla genetica, alla chimica e alla medicina che mantengono singolarmente propri dataset come UniProt, KEGG e Pubmed. Ulteriori iniziative si hanno nel campo farmaceutico. Linking Open Drug Data è un progetto di W3C che coinvolge note case farmaceutiche che scambiano cooperativamente dati e analisi di laboratorio per supportare la ricerca sui farmaci.

- Commercio e industria: nel settore dell'e-commerce vi sono alcuni progetti interessanti; tra le ontologie si segnala Good relations: the Web vocabolary for the ecommerce, un'ontologia che aggrega aziende, prodotti, servizi, offerte, orari di apertura e prezzi provenienti da diversi siti. Dal 2008 mantiene un vocabolario che consente di promette di rendere recuperabili attraverso i prodotti e i servizi delle aziende attraverso motori di ricerca e applicazioni mobili ${ }^{76}$.

RDF Book Mashup ${ }^{77}$ è stato il primo servizio a pubblicare i dati sui prodotti recuperati da Amazon e Google. ProductDB ${ }^{78}$ aggrega link provenienti da diversi dataset commerciali.

- Reti sociali e contenuti collaborativi: 112.0 ha generato nel web una mole molto vasta di quelli che vengono chiamati UGC ovvero contenuti generati dagli utenti, dei quali Wikipedia, Flickr e Facebook rappresentano oggi i più importanti esempi. Così anche in questo settore si registrano importanti iniziative. Oltre al già citato DBPedia, sono sorti alcuni progetti intorno a reti sociali come Flickr e Revyu. FlickrWrapp espone linked data relativi alla rete collaborativa di condivisione di immagini, Revyu.com si incentra sulle recensioni degli utenti, Semantic MediaWiki e Ontowiki ${ }^{79}$.

Di recente Facebook ha adottato l'Open Graph Protocol ${ }^{80}$ che consente di inserire nelle pagine web contenuto semantico utilizzando il formato di serializzazione RDFa.

Il quantitativo di dataset dal 2007 è notevolmente cresciuto come dimostra la seguente immagine tratta dal registro CKAN relativa al settembre $2011^{81}$.

\footnotetext{
${ }^{75}$ <http://bio2rdf.org/>. Tra i dataset presenti: UniProt, KEGG, Pubmed. Si veda anche: François BELLEAU Marc-Alexandre NOLIN -Nicole TOURIGNY- Philippe RIGAULT- Jean MORISSETTE, Bio2RDF: towards a mashup to build bioinformatics knowledge systems «Journal of Biomedical Informatics», 41 (2008) n.5, p. 706-716.

$76<$ http://www.heppnetz.de/projects/goodrelations/>. Aggiungendo delle righe di codice al contenuto delle pagine i dati possono essere compresi ed estratti dai motori di ricerca. Il software utilizza i microformati di Schema.org. Il software è sviluppato dal prof. Dr. Martin Hepp dell'E-Business and Web Science Research Group dell'Universität der Bundeswehr München. La licenza utilizzata è Creative Commons Attribution 3.0.

$77<$ http://wifo5-03.informatik.uni-mannheim.de/bizer/bookmashup/>.

${ }^{78}<$ http://productdb.org/>.

${ }^{79}$ Rispettivamente <http://semantic-mediawiki.org/> e <http://ontowiki.net/Projects/OntoWiki $>$.

${ }^{80}<$ http://opengraphprotocol.org/>.
} 


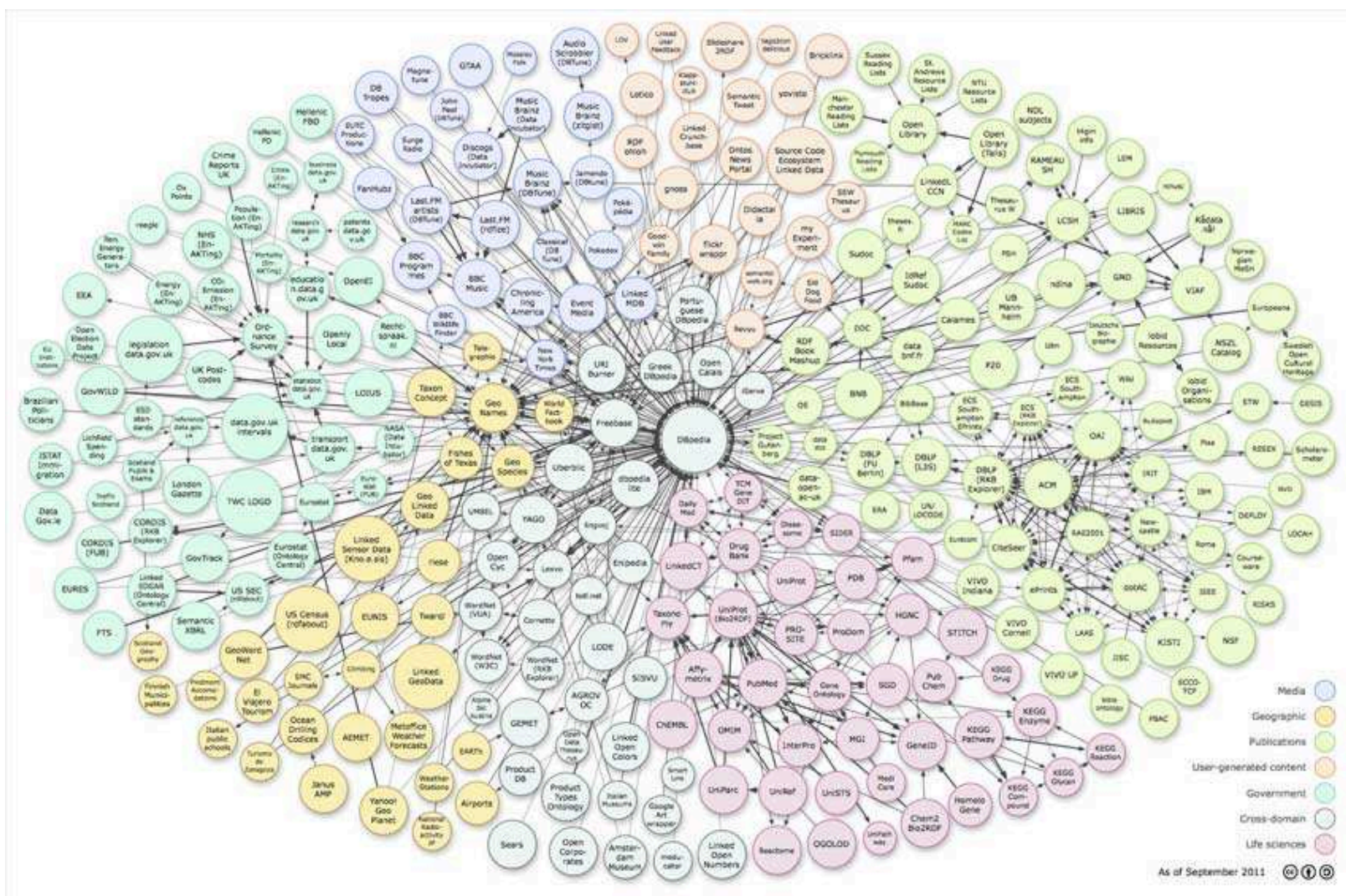

Fig. 4.6 - Stato della Linked data cloud nel Settembre $2011^{82}$

La pubblicazione sempre più ampia di dataset sul web rende oggi possibile lo sviluppo di applicazioni che consentono il miglioramento della ricerca e una migliore fruizione delle informazioni contenute nel web, che diventano così accessibili ai motori di ricerca.

La pubblicazione di dataset in forma di linked data, come vedremo nel dettaglio più avanti, prevede che si possa pubblicare un data dump in RDF ovvero un set di dati interpretabile da una macchina, ma prevede anche modalità più semplici adatte alla pubblicazione di dati contenuti in database relazionali oppure contenuti all'interno delle pagine web.

La via di sviluppo è duplice: da una parte si assiste alla creazione di nuovi motori di ricerca più sofisticati e intelligenti, che sfruttano le informazioni semantiche contenute nei dataset del web come Sig.ma ${ }^{83}$ e SWSE ${ }^{84}$; questi 'motori di ricerca semantici' raccolgono

\footnotetext{
${ }^{81}$ Le statistiche relative al numero e alla composizione dei dataset nel registro CKAN si trovano all'URL: $<$ http://www4.wiwiss.fu-berlin.de/lodcloud/state/>.

$82<$ http://richard.cyganiak.de/2007/10/lod/lod-datasets_2011-09-19_colored.html>.

83 <http://sig.ma> Sig.ma produce 'al volo' 'Live views on Web(s) of Data' ossia visualizzazioni del web dei dati fornendo informazioni interattive e unendo dati provenienti da migliaia di fonti. Le pagine di Sig.ma sono dotate di permalinks e possono essere incorporate nelle pagine web. Si veda anche: Giovanni Tummarello - Richard Cyganiak - Michele Catasta - Szymon Danielczyk - Renaud Delbru - Stefan Decker,
} 
e indicizzano le informazioni presenti nella linked open data cloud per fornire risposte intelligenti a domande poste nel linguaggio naturale, come già avviene in Google e negli altri motori tradizionali. Linksailor ${ }^{85}$ è un linked data browser che interpreta $\mathrm{i}$ dati recuperati a partire da un URI nel Web Semantico e fornisce in risposta una pagina web composta dai dati estratti da diversi dataset disponibili nel web.

Dall'altra parte si favorisce il recupero di informazioni semantiche ottenibili tramite una migliore strutturazione dei dati contenuti nelle pagine web, sfruttando i microformati e i nuovi microdata.

Schema.org ${ }^{86}$ ha segnato un passo importante per l'utilizzo di dati strutturati nel web e in prospettiva, data la recente adozione dei microformati anche da parte di OCLC Worldcat $^{87}$, anche nel mondo delle biblioteche. Il progetto è stato siglato il 2 giugno 2012 dai principali motori di ricerca Google Bing, Yahoo! e nel novembre dello stesso anno dal motore di ricerca russo $\operatorname{Yandex}^{88}$ e il suo scopo è quello di migliorare il recupero semantico da parte dei motori di ricerca tradizionali.

Si tratta di una raccolta di schemi di elementi per descrivere le risorse o 'cose' presenti nelle pagine HTML del web come persone, luoghi, prodotti dell'ingegno (libri, film, musica), prodotti, eventi etc. L'idea alla base del progetto è quella di utilizzare i microdata per incorporare dati semantici strutturati nelle pagine web e di incoraggiare gli sviluppatori di siti web di qualsiasi tipologia ad utilizzare $i$ vocabolari presenti in Schema.org per aggiungere alle pagine html il codice necessario a connotare semanticamente le 'cose' descritte nel web. Molti siti web dinamici infatti vengono generati tramite dati strutturati che vengono immagazzinati in database. Al momento della loro strutturazione in HTML diventa difficile recuperare i dati strutturati originali; utilizzando questi schemi i motori di ricerca potranno beneficiare di un facile accesso a questi dati strutturati e le aziende del web potranno ottenere una maggiore visibilità delle proprie pagine nel web. Proprio per la sua ricaduta in termini di $\mathrm{SEO}^{89}$ questa tecnologia oggi riscuote un certo successo.

Sig.ma: live views on the Web of data, «Journal of Web Semantics: Science, Services and Agents on the World Wide Web», 8 (2010) n. 4, p. 355-364.

${ }^{84}<$ http://www.swse.org/>.

${ }^{85}<\mathrm{http}: / /$ linksailor.com/>. I dati vengono arricchiti con quelli recuperati nella piattaforma Talis.

${ }^{86}<$ http://www.schema.org/>.

${ }^{87}<\mathrm{http}: / /$ www.worldcat.org/>.

${ }^{88}$ Rispettivamente: <http://www.bing.com/>, <http://www.google.com/>, <http://www.yahoo.com>; $<$ www.yandex.ru>.

${ }^{89}$ Search Engine Optimization (SEO) è una tecnica, studiata e utilizzata principalmente per le aziende commerciali, finalizzata a migliorare il recupero delle pagine web nei motori di ricerca per ottenerne un buon posizionamento nelle pagine dei risultati (SERP). 
Schema.org si basa sui microdata, una nuova e recente tecnologia del web tuttora in via di sviluppo.

I microdata $^{90}$ si presentano come un'evoluzione dei microformati ${ }^{91}$ creati per consentire a chi pubblica pagine web di aggiungere specifici attributi ad HTML o XHTML per specifici ambiti definiti dal microformato, fornendo informazioni comprensibili alle macchine che descrivono 'cose' come eventi, ricette, prodotti. Microdata è una specifica di HTML $^{92}$ che consente di incorporare informazioni semantiche nel codice delle pagine web già esistenti ed è compatibile con RDF e JSON. Come i microformati anche i microdati si appoggiano a vocabolari, ma, a differenza dei microformati, i microdati consentono di estendere i vocabolari e risolvono i conflitti tecnici come quelli relativi agli attributi dei $\mathrm{CSS}^{93}$.

Schema.org definisce uno schema di marcatura per dati strutturati che si appoggia ad un vocabolario condiviso e che consente ai webmaster di incorporare informazioni leggibili dalle macchine nel codice scritto in $\mathrm{HTML}^{94}$.

Gli schemi comprendono vari oggetti, concetti, eventi, luoghi. Ad esempio, un libro fa parte della categoria opere della creatività che a sua volta fa parte della categoria cose ed eredita le proprietà delle classi di appartenenza. Le immagini che seguono mostrano come le informazioni contenute in una pagina web e relative ad un libro possono essere codificate utlilizzando lo schema previsto in Schema.org

\footnotetext{
${ }^{90}<$ http://www.w3.org/TR/2011/WD-microdata-20110525/>.

$91<$ http://microformats.org/> è il sito di riferimento che raccoglie i progetti in corso.

92 HTML5 è attualmente in via di sviluppo: L'Editor's Draft 26 June 2012 si trova alla URL: <http://dev.w3.org/html5/spec/single-page.html> . Il World Wide Web Consortium ha annunciato che la prima versione dello standard sarà pronta per il luglio 2014.

${ }_{93}$ Cascading Style Sheets, ovvero i fogli di stile, utilizzati per formattare le pagine HTML, XML, XHTML allo scopo di separare contenuti e formattazione.

${ }^{94}$ Per la leggibilità dell'esempio qui riportato si indicano gli attributi principali definiti dallo standard Microdata:

itemscope - Crea un oggetto e indica che i discendenti di questo elemento contengono informazioni su di esso.

itemtype - Un URL valido di un vocabolario che descrive l'oggetto e le sue proprietà.

itemid -Indica un identificatore univoco dell'oggetto.

itemprop - indica che il tag che lo contiene esprime il valore di una specifica proprietà dell'item. Le proprietà e i valori sono descritte nel vocabolario relativo all'item, i valori delle proprietà possono consistere in stringhe di testo, ma possono usare anche URL usando l'elemento 'a' e l'attributo 'href', oppure l'elemento 'img' e l'attributo 'src', o altri elementi che linkano oppure includono risorse esterne.

itemref - Le proprietà che non discendono dall'elemento con l'attributo itemscope possono essere associate all'oggetto utilizzando questo attributo. Esso fornisce una lista di identificativi degli elementi com proprietà aggiuntive in altre parti del documento.
} 
$<$ a href="category/books.html" $>$ Books $</$ a $>>$

$<$ a href="category/books-literature.html" $>$ Literature \& Fiction $</ a>>$

$<$ a href="category/books-classics" $>$ Classics $</ a>$

$<$ img src="catcher-in-the-rye-book-cover.jpg" />

The Catcher in the Rye - Mass Market Paperback

by <a href="/author/jd_salinger.html" $>$ J.D. Salinger $</ a>$

4 stars - 3077 reviews

Price: $\$ 6.99$

In Stock

Product details

224 pages

Publisher: Little, Brown, and Company - May 1,1991

Language: English

ISBN-10: 0316769487

Reviews:

5 stars - $<$ b $>$ "A masterpiece of literature" $</$ b $>$

by John Doe. Written on May 4, 2006

I really enjoyed this book. It captures the essential challenge people face as they try make sense of their lives and grow to adulthood.

4 stars - <b>"love it LOLOL111!" $<$ /b $>$

by Bob Smith, Written on June 15, 2006

Catcher in the Rye is a fun book. It's a good book to read.

Fig. 4.7a - Pagina web in HTML contenente informazione non strutturata 
<body itemscope itemtype="http://schema.org/WebPage">

$\ldots$

$<$ div itemprop="breadcrumb" >

$<$ a href="category/books.html" $>$ Books $</$ a $>>$

$<$ a href="category/books-literature.html" $>$ Literature \& Fiction $</ a>>$

$</ \operatorname{div}>$

$<$ a href="category/books-classics" $>$ Classics $</ a>$

<div itemscope itemtype="http://schema.org/Book">

img itemprop="image" src="catcher-in-the-rye-book-cover.jpg" />

$<$ span itemprop="name" $>$ The Catcher in the Rye $</$ span $>$ -

$<$ link itemprop="bookFormat" href="http://schema.org/Paperback">Mass Market

Paperback by <a itemprop="author" href="/author/jd_salinger.html">J.D. Salinger $</ a>$

<div itemprop="aggregateRating" itemscope itemtype="http://schema.org/AggregateRating" > $<$ span itemprop="ratingValue" $>4</$ span $>$ stars -

$</$ div $>$

$<$ span itemprop="reviewCount" $>3077</$ span $>$ reviews

$<$ div itemprop="offers" itemscope itemtype="http://schema.org/Offer">

Price: <span itemprop="price" $>\$ 6.99</$ span $>$

$<$ meta itemprop="priceCurrency" content="USD" />

$</ \operatorname{div}>$

$<$ link itemprop="availability" href="http://schema.org/InStock">In Stock

Product details

$<$ span itemprop="numberOfPages" $>224<$ /span $>$ pages

Publisher: < span itemprop="publisher">Little, Brown, and Company</span> -

$<$ meta itemprop="datePublished" content="1991-05-01">May 1, 1991

Language: $<$ span itemprop="inLanguage" $>$ English $<$ /span $>$

ISBN-10: <span itemprop="isbn">0316769487</span>

Reviews:

$<$ div itemprop="review" itemscope itemtype="http://schema.org/Review"> $<$ span itemprop="reviewRating" $>5</$ span $>$ stars -

$<$ b $>$ "<span itemprop="name" $>$ A masterpiece of literature $</$ span $>$ " $<$ /b $>$

by $<$ span itemprop="author" $>$ John Doe</span $>$,

Written on $<$ meta itemprop="datePublished" content="2006-05-04">May 4, 2006

$<$ span itemprop="reviewBody">I really enjoyed this book. It captures the essential

$</$ div $>$

challenge people face as they try make sense of their lives and grow to adulthood. $<$ /span $>$

$<$ div itemprop="review" itemscope itemtype="http://schema.org/Review">

$<$ span itemprop="reviewRating" $>4</$ span $>$ stars -

$<$ b $>$ "<span itemprop="name" $>$ A good read. $</$ span $>$ " $<$ /b $>$

by $<$ span itemprop="author" $>$ Bob Smith $</$ span $>$,

Written on $<$ meta itemprop="datePublished" content="2006-06-15">June 15, 2006

$<$ span itemprop="reviewBody">Catcher in the Rye is a fun book. It's a good book to read.</span $>$

$</$ div $>$

$</$ div $>$

$</$ body $>$

Fig. 4.7b - Pagina web in HTML con informazione strutturata in base a Schema.org ${ }^{95}$

$95<$ http://www.schema.org>. 


\subsection{I dati delle biblioteche e il loro utilizzo nel Web Semantico}

Da sempre le biblioteche producono dati autorevoli e di qualità. L'interesse a pubblicare dati di biblioteche nel Web Semantico è duplice. Da una parte le biblioteche hanno interesse ad essere presenti nel Web Semantico, altrimenti i dati dei loro cataloghi saranno irraggiungibili dagli utenti del web, dall'altra il Web Semantico ha interesse verso i dati autorevoli prodotti dalle biblioteche per creare reticoli di dati qualificati e controllati.

I cataloghi elettronici sono stati progettati per gestire le collezioni delle biblioteche, localizzare risorse e facilitare l'accesso ai documenti. Tuttavia oggi gli OPAC falliscono questo importante obiettivo: gli utenti generalmente preferiscono affidarsi a motori di ricerca quale punto privilegiato per iniziare le proprie ricerche.

Attraverso la ricerca in un motore un utente può trovare ciò che interessa, un'informazione, una pagina web di un autore e sempre più spesso anche i riferimenti bibliografici, mentre i cataloghi elettronici vengono sempre meno utilizzati in quanto i dati contenuti non possono essere recuperati nel web.

Le biblioteche si trovano così in una fase di profondo squilibrio che minaccia la sopravvivenza dei cataloghi quali strumenti autorevoli di recupero dell'informazione bibliografica.

I dati prodotti dalle biblioteche (come anche quelli di archivi e banche dati bibliografiche) sono sempre meno accessibili e, a parte qualche autorevole eccezione, sono racchiusi entro formati inaccessibili al web e i nuovi next generation catalogs e strumenti di discovery esaminati nei precedenti capitoli ci offrono un quadro di sviluppo nettamente in controtendenza se paragonato agli sviluppi del Web Semantico e al movimento Open data $^{96}$.

\footnotetext{
${ }^{96}$ Nonostante vi sia lo sforzo di questi nuovi prodotti verso la trasformazione dei record dai formati MARC verso XML per ottenere raggruppamenti in base al modello FRBR. È il caso del software Extensible Catalog (XC), cfr: David Lindahl, eXtensible catalog: transforming legacy metadata towards FRBR, RDA, and Linked Data. ALA Midwinter $2011<$ http://www.extensiblecatalog.org/learnmore/publications/alamidwinter-2011-lita-next-generation-catalog-ig-extensible-catalog-transfo> e Id., EXtensibleCatalog's Library, 2010, <http://www.screencast.com/users/eXtensibleCatalog >. Per una presentazione del software si veda anche id., EXtensible Catalog: software portfolio, presentazione tenuta a ALA Midwinter 2011 - LITA $\begin{array}{llllll}\text { Next Generation } & \text { Catalog } & \text { IG, } & 9 & \text { gennaio }\end{array}$ $<\mathrm{http}: / /$ docushare.lib.rochester.edu/docushare/dsweb/Get/Document-47024>.

$\mathrm{Si}$ vedano in proposito anche: Jennifer Bowen, Moving library metadata toward linked data: opportunities provided by the eXtensible Catalog. In Diane I. Hillmann - Michael Lauruhm, Making metadata work harder: celebrating 15 years of Dublin Core. Proceedings of the International Conference on Dublin Core and Metadata Applications, Pittsburgh 20-22 October 2010, Dublin Core Metadata Initiative, 2010, p. 44-59, $<$ http://dcpapers.dublincore.org/ojs/pubs/article/view/1010/979>; Ead., Metadata to support next-generation library resource discovery: Lessons from the eXtensible Catalog, Phase 1., «Information Technology and Libraries», 27 (2008), n. 2, p. 6-19, <https://urresearch.rochester.edu/handle/1802/5757>.
} 
Milioni di dati bibliografici contenuti negli OPAC rimangono così racchiusi entro silos non comunicanti con l'esterno e invisibili ai motori di ricerca.

Lo sviluppo di software per gli OPAC che non tiene conto di questo mutato ambiente informativo è destinato ad allontanare sempre di più $\mathrm{i}$ dati bibliografici dagli altri dati presenti nel web: se i dati delle biblioteche non sono interoperabili le biblioteche rischiano di rimanere escluse dal circuito dell'informazione in rete ${ }^{97}$.

Il tema dell'interoperabilità è stato da sempre al centro delle questioni biblioteconomiche: le biblioteche e l'IFLA da tempo si occupano di garantire l'interoperabilità tra archivi, biblioteche, e altri componenti del circuito bibliografico.

Oggi la questione diventa cruciale e soprattutto si estende al web: non si tratta di produrre dati interoperabili tra gli attori del settore culturale, ma di produrre dai interoperabili con il web; se gli utenti non troveranno i dati delle biblioteche liberamente accessibili nel web tenderanno ad ignorare le biblioteche e i servizi da esse offerti.

Proprio per questo motivo il MARC è stato abbandonato in favore di formati e strutture dei dati compatibili con il web. È stato proprio il formato utilizzato per immagazzinare e scambiare dati il principale problema che ha causato il rallentamento dell'evoluzione degli OPAC e lo ha indirizzato verso soluzioni che impediscono di fatto l'utilizzo, lo scambio e il ritrovamento dell'informazione bibliografica nel web ${ }^{98}$.

Oggi le biblioteche cominciano a fare passi importanti nel campo del Web Semantico pubblicando i loro dati nella forma di linked data e confrontandosi su queste nuove tematiche.

Nel nostro paese il recente Convegno internazionale Global interoperability and linked data in libraries, tenutosi a Firenze il 18 e 19 Giugno 2012 è stato il più importante e autorevole momento di confronto tra esperti internazionali e rappresentanti delle istituzioni del settore culturale per riflettere sulle modalità di intervento, sulle problematiche e le opportunità che lo scenario dei linked data apre alla fruizione del patrimonio delle biblioteche ${ }^{99}$.

\footnotetext{
${ }^{97}$ Cfr.: Axel Kaschte, Linked open data on its way into next generation library management and discovery solutions, «JLIS.it», 4 (2013), n. 1, p. p. 305-312, <http://leo.cilea.it/index.php/jlis/article/view/5492/7913>.

${ }^{98} \mathrm{Si}$ vedano in proposito le riflessioni di Martha M. Yee sulle difficoltà di utilizzo dei dati bibliografici formattati in MARC nel Web Semantico: Martha M. Yee, Can bibliographic data be put directly onto the Semantic Web? cit.

$99<$ http://www.linkedheritage.org/linkeddataseminar/>. Il Seminario è stato promosso da: Università degli studi di Firenze, Istituto centrale per il catalogo unico delle biblioteche italiane (ICCU), Biblioteca nazionale centrale di Firenze (BNCF), Casalini Libri, Comune di Firenze, Conferenza dei rettori delle università italiane (CRUI), Associazione italiana biblioteche (AIB), Istituto di teoria e tecniche dell'informazione giuridica del Consiglio nazionale delle ricerche (ITTIG-CNR), Fondazione Rinascimento digitale. Tra le tematiche affrontate elencate: Web dei dati; Linked data, Dati bibliografici aperti; Gestione e distribuzione dei dati bibliografici; Scambio di dati aperti; Tecnologie per il Web Semantico; Condivisione della
} 
Abbracciare il modello dei linked data vuol dire prima di tutto strutturare i record bibliografici secondo il modello RDF, creare i vocabolari indispensabili per rappresentare entità e proprietà e le relazioni e pubblicare sul web le risorse bibliografiche, ovvero rendere disponibili dataset contenenti i dati catalografici degli OPAC in modo che siano aperti, interoperabili e riutilizzabili nel web.

\subsection{Dal record al dato: atomizzazione e ristrutturazione del record bibliografico}

Nell'ottobre 2011 in seno al consorzio W3C si è creato un gruppo dedicato ai dati di natura bibliografica : il W3C Library Linked Data Incubator Group (LLD XG) ${ }^{100}$.

Il gruppo ha avuto come obiettivo lo studio di fattibilità dei Library linked data (LLD), cioè ha stabilito quali requisiti debbano possedere i dati bibliografici per poter essere interconnessi e pienamente utilizzabili nel Web Semantico. Gli obiettivi del gruppo richiamano alla mente quelli dell'IFLA e del rapporto FRBR ma, a differenza del modello FRBR, nato allo scopo di valutare i requisiti dei record bibliografici per lo scambio tra tutti gli attori dell'universo bibliografico e di ridurre i costi della catalogazione, il nuovo gruppo, nato in un ambiente non strettamente bibliotecario, si è occupato di definire i requisiti dei dati bibliografici nel Web Semantico, senza riferimento alle norme, alle regole di catalogazione o alle strutture catalografiche che le biblioteche utilizzano.

Ciò non solo ha ampliato gli orizzonti della disciplina, attraverso un progetto non strettamente destinato alla comunità bibliotecaria, favorendo la collaborazione tra esperti del settore delle biblioteche ed esperti della comunità del Web Semantico, ma ha posto su nuove basi il modo di pensare ai metadati prodotti dalle biblioteche come dati fruibili non solo all'interno della ristretta cerchia di chi opera nel settore culturale, ma nell'intero web.

conoscenza e connessione dei dati; Sviluppo di standard tecnici aperti; Buone pratiche per la pubblicazione e la connessione di dati strutturati sul web; Archivi aperti; Accesso aperto; Modelli concettuali; Sistemi di organizzazione della conoscenza (Knowledge Organisation Systems - KOS); Riutilizzo dei dati bibliografici; Schema di metadati condivisi; Open Library data.

$100<$ http://www.w3.org/2005/Incubator/lld/wiki/Main_Page>. Obiettivi del gruppo: «to help increase global interoperability of library data on the Web, by bringing together people involved in Semantic Web activities - focusing on Linked Data - in the library community and beyond, building on existing initiatives, and identifying collaboration tracks for the future». Si veda il report del gruppo: Library linked data incubator group, Library linked data incubator group final report, Unpublished draft, 6 marzo 2012, <http://www.w3.org/2005/Incubator/lld/XGR-lld-20111025/>. 
Le risorse che le biblioteche producono, cioè i dati bibliografici, di autorità e gli schemi concettuali possono così essere pienamente riutilizzabili e fruibili nel Web Semantico a vantaggio di tutti gli utenti della rete e possono dunque divenire parte del Web Semantico.

Allo scopo di garantire un efficace utilizzo dei dati bibliografici nel Web Semantico è indispensabile che avvenga una trasformazione nel modo di concepire i metadati bibliografici, che si orienti verso:

* una maggiore granularità, ovvero una più ampia gamma di elementi o dati da rappresentare, collegare e registrare;

* una maggiore complessità del data model utilizzato. Il record tradizionale non è sufficiente ad esprimere la complessità delle entità e delle relazioni tra i dati costituenti un record bibliografico;

* nuove tecnologie e nuovi formati più adatti alla rappresentazione dei dati e alla loro rielaborazione da parte delle macchine attraverso la sostituzione del formato MARC con formati nuovi basati sul data model RDF. ${ }^{101}$

Creare dati bibliografici 'collegati' in forma di linked data significa in primo luogo adottare RDF come nuovo data model cioè un nuovo modello logico per l'espressione dei dati bibliografici e abbandonare il concetto di record come è stato tradizionalmente concepito.

Come è accaduto per il web, dove da una rete di documenti in html collegati tramite link e concepiti come blocchi unici di informazione si passa ad un web di dati collegati, anche nel campo delle biblioteche abbracciare la logica dei linked data vuol dire costruire relazioni semplici tra gli elementi di una registrazione bibliografica e non più tra i singoli record.

L'uso dei dati bibliografici nel Web Semantico richiede che i dati bibliografici adottino forme di registrazione caratterizzate da una granularità maggiore rispetto al passato.

Finora la registrazione bibliografica è stata costituita da un blocco indivisibile di dati bibliografici, ovvero il record tradizionale testuale e monolitico; ciò ha rallentato notevolmente lo sviluppo di software per i cataloghi.

La struttura della registrazione bibliografica codificata nel formato MARC ha rallentato il processo di evoluzione dei cataloghi elettronici in quanto il record bibliografico è stato progettato come un blocco unico di testo (così come è avvenuto per le

101 Carlo Bianchini, Library Linked Data e il futuro delle biblioteche, 2012, il contributo, in corso di pubblicazione e attualmente disponibile in <http://eprints.rclis.org/16886/>. 
pagine html del web) e i software si sono basati su tecnologie proprietarie inadatte all'integrazione dei dati stessi con il web.

Nella progettazione di OPAC di nuova generazione non si è intervenuti sulla struttura dei record e ci si è concentrati invece sulle nuove funzionalità di ricerca, lasciando in secondo piano lo sviluppo di sistemi aperti e interoperabili e proseguendo nella progettazione di sistemi chiusi e inaccessibili al web.

Per contro, le nuove norme catalografiche come RDA hanno compiuto un passo in avanti e seguendo la logica dei linked data hanno introdotto nella catalogazione un nuovo scenario ove ogni registrazione bibliografica è formata dall' estrazione e ricomposizione ordinata di 'dati' provenienti da diverse fonti, costituite da archivi di nomi, di opere, espressioni, manifestazioni, luoghi etc ${ }^{102}$.

Le regole catalografiche RDA si sono dimostrate pronte a recepire il modello dei dati di RDF e oggi il codice è compatibile con il Web Semantico: le RDA descrivono le entità da rappresentare nei record bibliografici in appositi element sets e definiscono gli attributi in appositi vocabolari. Tutti set di elementi e vocabolari di RDA sono stati depositati nell'Open metadata registry ${ }^{103}$ all'indirizzo <http://rdvocab.info/>.

Le entità previste da RDA sono le 14 entità già provenienti dalla famiglia dei modelli FRBR. Altri schemi di elementi (element sets) vengono stabiliti per il gruppo 1 (466) il gruppo 2 (59) e il gruppo 3 (16) che stabiliscono gli attributi (es. titolo, forma dell'opera, data dell'opera, data dell'espressione, etc.), mentre un altro element set RDA Relationships for Works, Expressions, Manifestations, Items ${ }^{104}$ stabilisce le relazioni espresse nella parte centrale di ogni tripla, ad esempio, creato da, è parte di, è continuazione di, etc. secondo il seguente modello

\footnotetext{
${ }^{102} \mathrm{Si}$ vedano i contributi di Alan Danskin, Linked and open data: RDA and bibliographic control, «JLIS.it», 4 (2013), n. 1, p. 147-159, <http://leo.cilea.it/index.php/jlis/article/view/5463/7897> ([relazione presentata al Convegno Global interoperability and linked data in libraries, Firenze, 18-19 giugno 2012), Barbara Tillett, Keeping Libraries Relevant in the Semantic Web with RDA: Resource Description and Access. «JLIS.it» [Online], 2(2011) n. 2 e Diane Hillmann - Karen Coyle - Jon Phipps - Gordon Dunsire, RDA Vocabularies. «D-Lib Magazine», 16 (2010), n. 1-2, <http://www.dlib.org/dlib/january10/hillmann/01hillmann.html.> e Karen Coyle, RDA vocabularies for a twenty-first-century data environment, «Library Technology Reports», 46 (2010) n. 2 (numero monografico).

103 The Open Metadata Registry (<http://metadataregistry.org >) è considerato una infrastruttura tecnica fondamentale per la realizzazione del Web Semantico. Nato originariamente per supportare la National Science Digital Library (NSDL), il Registro è liberamente accessibile a chiunque voglia usare i suoi servizi.Il registro è ispirato a quello tenuto e sviluppato in open-source dalla comunità Dublincore: Dublin Core Metadata Initiative (DCMI) Registry. Il registro esente gli obiettivi del registro DCMI : consentendo 1) la creazione automatizzata e il mantenimento di schemi e application profiles 2) la submission di schemi al registro per la revisione e la pubblicazione.

${ }^{104}$ RDA Relationships for Works, Expressions, Manifestations, Items,

$<\mathrm{http}$ ://metadataregistry.org/schema/show/id/13.html>.
} 


$\begin{array}{lll}\text { entità } & \text { relazione } & \text { entità } \\ \text { soggetto } & \text { predicato } & \text { oggetto } \\ \text { valore } & \text { valore } & \text { valore }\end{array}$

In ogni tripla verranno esplicitate le tipologie delle relazioni tra le entità, ovvero i tipi di responsabilità che intercorrono tra un'opera e una persona oppure la tipologia di relazione che collega due opere (es. Based on (Work), Abridgement of (Work) Abstract of (Work) Adaptation of (Work)

così nella tripla

persona è autore di opera

le relazioni vengono espresse dal set di elementi $R D A$ roles ove vengono esplicitati i ruoli di autore, regista, attore, fotografo e ogni altro tipo di responsabilità principale o secondaria etc.

Oltre agli element sets, ovvero elencazioni di entità i cui valori sono liberi (un'opera può avere qualsiasi titolo), RDA ha previsto una serie di 76 vocabolari (vocabularies) che contengono valori prestabiliti. Essi vengono usati per creare insiemi chiusi, ovvero definire i valori di alcuni attributi definiti negli altri dataset.

Ad esempio il vocabolario book format ${ }^{105}$ contiene gli elementi prescritti dal codice per la descrizione del formato nel libro antico e cioè gli elementi (12mo, 16mo, 24mo, $32 \mathrm{mo}$, folio etc. ) ovvero i valori che possono assunti dall'attributo 'formato della manifestazione'.

Nell'ottica dei linked data un record bibliografico sarà sostituito da un grafo, costituito da diverse triple o dichiarazioni che collegheranno diversi dataset e utilizzeranno diversi vocabolari creati dalla comunità bibliotecaria e da altre comunità e presenti nel web. La creazione dei dati che si riferiscono ad una descrizione bibliografica sarà più granulare in quanto ogni record sarà costituito dalla sintesi di un numero molto vasto di triple che combineranno dati creati dalle biblioteche e da altri enti di natura non bibliotecaria.

Immaginiamo la seguente descrizione bibliografica:

The difference engine / William Gibson and Bruce Sterling. - London :Vista, 1996. - 383 p. ; 20 $\mathrm{cm}$.

${ }^{105}<$ http://metadataregistry.org/vocabulary/show/id/76.html >. 
e la sua rappresentazione nel formato MARC21:

$00001647 \mathrm{cam}$ a22003737 4500

0015060570

003 SE-LIBR

00520100303170021.0

008011211 s1996 xxk 0000 eng c

020 a 0-575-60029-2 (pbk);c £5.99 : CIP entry (May.)

03590575600292

040 a Li

082 a 813.54220

082 a 813.54220

084 a Heq. $012 \mathrm{kssb} / 6$

1001 a Gibson, William,d 1948-0 220040

2450 4a The difference engine /c William Gibson and Bruce Sterling

260 a London :b Vista,c 1996

300 a 383 p. ;c $20 \mathrm{~cm}$

500 a Originally published: London: Gollancz, 1990

6507 a American fictiony 20th century2 BNB

650 4a Steampunk9 TEST0 308074

7001 a Sterling, Bruce,d 1954-0 307779

950 k 6 650i Svenska ämnesord (sao):a Fantastisk litteraturw i0 144795u Steampunk 950 k6 650i Svenska ämnesord (sao):a Science fiction0 164863u Steampunk

950 k6 650i Svenska ämnesord (sao):a Cyberpunk (litteratur)0 142481u Steampunk 955 k6 650i Genreämnesord för skönlitteratur (sao):a Fantasyw i0 243892u Steampunk 955 k6 650i Genreämnesord för barn- och ungdomslitteratur (barn):a Fantasyw i0 245336u Steampunk 955 k6 650i Genreämnesord för skönlitteratur (sao):a Science fictionw i0 245382u Steampunk 955 k6 650i Genreämnesord för barn- och ungdomslitteratur (barn):a Science fictionw i0 244025u Steampunk 955 k6 650a Cyberpunku Steampunk

976 2a Heq.01b Skönlitteratur

8415 Lda xa ab 0201070u 0 4000uu |000000e 1

8525 Ldb Ldz Skönlitteratur. Annan uppl. kan finnas vid biblioteket

Fig. 4.8 - Una descrizione bibliografica e la sua rappresentazione nel formato MARC2 $1^{106}$

\footnotetext{
${ }^{106}$ OPAC Libris <http://libris.kb.se/bib/5060570>.
} 
Infine, esaminiamo il grafo risultante dalla rappresentazione in RDF delle triple che si riferiscono ad un reticolo bibliografico di cui la manifestazione fa parte:

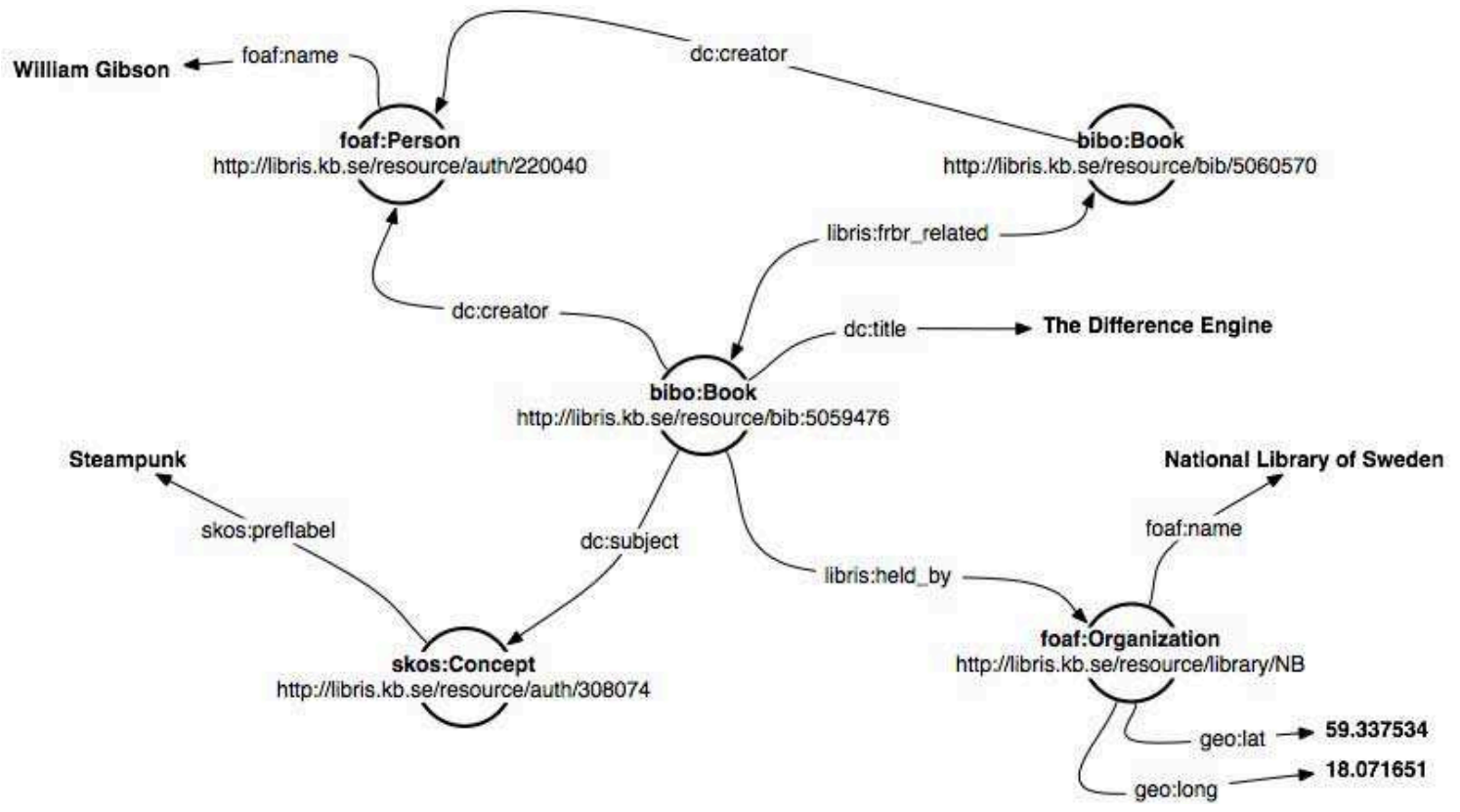

Fig. 4.9 - Un reticolo bibliografico espresso con i Linked data ${ }^{107}$

L'esempio, tratto dall'OPAC Libris ${ }^{108}$, primo catalogo ad esporre i dati nella forma di linked data, mostra come può essere espresso un reticolo bibliografico in un OPAC servendosi del data model RDF.

Come vediamo, il record bibliografico è un grafo costituito da un certo numero di triple che asseriscono che vi sono due risorse correlate il cui autore è William Gibson, che hanno per soggetto 'Steampunk' e che sono possedute dalla Biblioteca nazionale svedese $^{109}$.

L'esempio mostra che il record bibliografico viene ottenuto assemblando un insieme di triple quali ad esempio:

* Una persona il cui nome è William Gibson

\footnotetext{
107 Martin Malmsten, LIBRIS available as Linked Data, 312 2008. Blog post, «Librisbloggen.kb.se», LIBRIS nationella bibliotekssystem. <http://librisbloggen.kb.se/2008/12/03/libris-available-as-linked-data/>. $108<$ http://libris.kb.se/>. Cfr.: Cfr. infra, Appendice A. Casi di studio. Svenska forskningsbibliotekens gemensamma katalog. OPAC Libris. Si vedano anche sull'OPAC Libris i seguenti contributi di Martin Malmsten, Cataloguing in the open - the disintegration and distribution of the record, «JLIS.it», 4 (2013), n. 1, p. 417-424, <http://leo.cilea.it/index.php/jlis/article/view/5512>, Id., Making a library catalogue part of the Semantic Web, in J Greenberg - W. Klas, Metadata for semantic and social applications. Proceedings of the International Conference on Dublin Core and Metadata Applications. Berlin, 22-26 September 2008, pp. 146-150. Singapore and Universitätsverlag Göttingen, Dublin Core Metadata Initiative, 2008 <http://webdoc.sub.gwdg.de/univerlag/2008/DC_proceedings.pdf>; Id., Exposing library data as Linked data, relazione tenuta al Satellite Meetings IFLA 2009, Emerging trends in technology: libraries between Web 2.0, semantic web and search technology, Florence, 19-20 Agosto 2009, $<$ http://www.ifla2009satelliteflorence.it/meeting3/program/assets/MartinMalmsten.pdf>.

${ }^{109}$ Da una finestra tipo terminale si può usare la stringa: curl --include --location --header accept:text/rdf $+\mathrm{n} 3$ $<$ http://libris.kb.se/resource/bib/5060570> per vedere in atto la negoziazione dei contenuti.
} 
* Un concetto la cui forma preferita è Steampunk

* Un libro il cui titolo è The different Engine

* Un’organizzazione denominata Biblioteca Nazionale Svedese

L'esempio mostra come tutte le entità siano identificate attraverso URI (es. <http://libris.kb.se/bib/5059476>; <http://libris.kb.se/bib/5060570> etc.) e siano usate diverse ontologie presenti in rete: per esprimere le entità e i collegamenti tra le entità sono utilizzate ontologie e vocabolari noti come Foaf, Bibliontology, Geonames e vengono costruite diverse triple che sono tra loro collegate:

* William Gibson è autore di The different engine

- William Gibson è una persona

* The different engine è una risorsa di tipo libro

* The different engine ha per soggetto Steampunk

La prima tripla risulta dalla sintesi di tre triple che fanno riferimento alle ontologie Foaf, Bibliontology, DublinCore e usa questi vocabolari per esprimere le entità: libro, concetto, persona e le relazioni soggetto e creatore (dc:subject e dc:creator). Ogni parte della tripla viene associata ad un identificatore unico, ovvero un URI (ad esempio: $<$ http://libris.kb.se/bib/5059476>).

La Biblioteca nazionale svedese viene georeferenziata utilizzando l'ontologia Geonames per esprimere la posizione della biblioteca e fornendo al Web Semantico un collegamento utile per utilizzare quel dato per altre applicazioni e viceversa alle biblioteche per costruire una particolare applicazione basata su quei dati (ad esempio, ricavare il parcheggio pubblico più vicino alla biblioteca, etc.)

Questo esempio ci mostra come dati bibliografici possano essere esposti in forma di dati collegati e ci offre anche un primo quadro dei vantaggi che si possono ottenere utilizzando il modello RDF per esprimere un record bibliografico:

1) Controllo bibliografico e di autorità

Il controllo di autorità, ovvero l'uso di forme normalizzate degli accessi nominali e semantici e il mantenimento delle relazioni tra i documenti correlati, garantisce la coerenza della struttura sindetica del catalogo, migliora la navigazione e si rivela fondamentale nel collegamento dei dati bibliografici con altri basi dati esterne e con il web. 
Un concetto fondamentale del data model RDF è che ogni parte della tripla viene identificata attraverso un URI ovvero un identificatore unico e persistente. Anche i predicati, ovvero le relazioni che intercorrono tra le entità (e che nel caso specifico sono collegate ad alcuni vocabolari come Dublin Core, Bibliontology, Foaf o Skos) vengono provviste di URI in modo che possano essere riutilizzate.

Ciò significa, facendo un esempio pratico, che William Gibson ${ }^{110}$, noto fondatore del movimento Cyberpunk è identificato da un URI che lo distingue dagli altri omonimi presenti nel web, dunque dal calciatore e allenatore scozzese William Gibson ${ }^{111}$ che sarà invece identificato da un URI diverso. La relazione di identità tra due entità del Web Semantico viene stabilita dunque dall'URI.

Allo stesso modo vengono risolte le questioni relative agli pseudonimi: Samuel Clemens e Mark Twain avranno due URI diversi, ma saranno collegati da una relazione di equivalenza che consentirà alla macchina di riconoscere che si tratta della stessa persona. Questa tipologia di relazione, che collega due alias URI può essere esplicitata facendo ricorso a linguaggi formali e ontologie come OWL e SKOS, stabilendo una relazione di equivalenza (come, ad esempio, owl:sameAs).

Ciò si traduce in un vantaggio notevole in fase di ricerca e di creazione dei metadati bibliografici e risolve un annoso problema che ha afflitto da sempre la ricerca sia nei motori di ricerca, sia negli OPAC.

La stessa logica si applica per i titoli e i soggetti; due o più soggetti potranno essere considerati simili se si riferiscono allo stesso concetto e due diversi titoli varianti, anche in lingue diverse potranno essere ricondotti allo stesso titolo originale identificato da un preciso URI.

L'adozione da parte delle biblioteche del modello dei dati RDF consente dunque di risolvere le questioni del controllo bibliografico che da sempre hanno afflitto i cataloghi elettronici penalizzando la qualità del recupero dell'informazione.

2) Riutilizzo di dataset esistenti e approccio distribuito alla creazione dei dati bibliografici

Nello scenario previsto dai linked data i dati bibliografici potranno provenire da dataset di natura bibliografica e non bibliografica. Ad esempio, il valore relativo al nome dell'autore William Gibson potrà essere collegato alla forma prevista dal dataset Foaf $^{12} \mathrm{e}$

\footnotetext{
$110<$ http://it.wikipedia.org/wiki/William_Gibson>.

$111<$ http://it.wikipedia.org/wiki/William_Gibson_\%28calciatore\%29>.

112 http://www.foaf-project.org/ ; FOAF Vocabulary Specification 0.98 Namespace Document 9 August 2010
} 
alla forma presente in $\operatorname{VIAF}^{113}$. Nel Web dei dati è indispensabile il riutilizzo, ove possibile, di vocabolari e dataset già esistenti; ciò consente numerosi vantaggi, che divengono immediati: il collegamento dei dati delle biblioteche ad altri dati presenti nel web assicurandone la visibilità, un risparmio notevole delle risorse in fase di catalogazione e, infine, la possibilità di collegare direttamente una descrizione all'archivio di autorità che contiene tutte le forme varianti.

Oggi i cataloghi mantengono archivi di autorità non condivisi e inaccessibili dall'esterno; i catalogatori creano le forme degli autori secondo le regole previste nelle norme catalografiche. La possibilità di collegare il dato alla forma in VIAF, che contiene tutte le forme (REICAT, RAK, RDA etc.) porterebbe alla semplificazione del lavoro di catalogazione e ad un vantaggio nella ricerca. L'utente potrebbe trovare una descrizione catalografica partendo dalla forma desiderata e trovarvi il collegamento a tutte le forme varianti.

Allo stesso modo nel mondo dei linked data qualsiasi dato può essere collegato ad un archivio diverso: ad esempio, i luoghi possono essere derivati da un dataset geografico come Geonames. Dato che esistono nel web dataset autorevoli, linked data prescrive l'utilizzo di questi archivi di autorità. Dunque in futuro insieme a dati provenienti da archivi bibliografici troveranno luogo in una descrizione bibliografica anche dati provenienti da archivi statistici, da anagrafi e banche dati di istituzioni pubbliche, di enti territoriali, governativi, etc.

3) Interoperabilità tecnologica

L'utilizzo di formati di serializzazione di RDF come RDF/XML RDFS, SKOS basati su XML consente di produrre dati interoperabili con gli altri dati prodotti nel Web. L'interoperabilità diventa dunque cruciale per evitare l'isolamento dei dati bibliografici e di conseguenza delle biblioteche stesse dal luogo ove oggi si trova l'informazione, ovvero il Web.

La Bibliographic Framework Transition Initiative avviata alla Library of Congress ${ }^{114}$ (Marcum 2011) ha decretato l'abbandono del formato MARC in favore di formati basati su XML, che possano dunque garantire la necessaria interoperabilità e introdurre nel record

$<$ http://xmlns.com/foaf/spec/ >.

${ }^{113}$ The Virtual International Authority File, <http://viaf.org/>.

114 Deanna Marcum, A bibliographic framework for the digital age (October 31, 2011): Bibliographic Framework Transition Initiative (Library of Congress), 31 october 2011, <http://www.loc.gov/marc/transition/news/framework-103111.html>. 
bibliografico quella flessibilità necessaria nella struttura dei dati bibliografici. È necessario che le biblioteche adottino nuovi formati più strutturati e granulari, e di conseguenza interoperabili con il web ${ }^{115}$.

La formazione del gruppo di lavoro sui Library Linked Data, costituito da esperti nel campo dei metadati e del settore delle biblioteche garantisce oggi una maggiore interoperabilità dei dati bibliografici, mettendo in evidenza le problematiche connesse alla conversione e adattamento dei dati bibliografici tradizionali al nuovo ambiente informativo $^{116}$.

\section{4) Riduzione dei costi}

Uno degli obiettivi perseguiti dalle biblioteche e dal modello FRBR dell' IFLA è la riduzione dei costi della catalogazione. L'approccio fornito dai linked data offre grandi opportunità per la condivisione della catalogazione tra più enti e per la riduzione dei costi della pratica catalografica. Se il record bibliografico viene sostituito da un insieme di dati collegati, provenienti da diverse fonti produttrici di informazioni, il carico di lavoro anziché gravare su pochi enti può automaticamente essere ridistribuito tra le comunità che operano nel web. Il record bibliografico non sarà creato da una sola istituzione, ma le sue componenti deriveranno da molti archivi di autorità (luoghi, soggetti, nomi etc) creati da diversi componenti del Web Semantico.

5) Superamento delle barriere linguistiche

Il perseguimento di questo obiettivo è al centro del dibattito sul controllo bibliografico da diversi anni. Le nuove possibilità offerte dalla tecnologia dei cataloghi elettronici basati sul web consentono oggi di abbattere le barriere linguistiche e il progetto

\footnotetext{
${ }^{115}$ Sono ormai tanti i contributi di studiosi che si sono espressi in favore dell'abbandono del MARC, si veda: Karen Coyle, Murdering MARC, September 1, 2006,. «Coyle's InFormation», $<$ http://kcoyle.blogspot.com/2006/09/murdering-marc.html>; Roy Tennant, MARC must die, «Library Journal», 127 (2004), n17, p. 26-27. http://www.libraryjournal.com/article/CA250046.html e MARC exit $\begin{array}{lllllll}\text { strategies, } \quad \text { LLibrary Journal», } 127 \text { (2004), n. } & \text { 19, } & \text { p. }\end{array}$ $<$ http://www.libraryjournal.com/article/CA256611.html?q=tennant+exit>.

${ }^{116}$ Sulla possibilità di creare metadata compatibili con RDF si veda: Iryna Solodovnik, Comunicazione $e$ ricerca semantica di contenuti informativi tra metadati, Linked Open Data e ontologie, «Bibliotime», XIV (2011), n. 2, <http://didattica.spbo.unibo.it/bibliotime/num-xiv-2/solodovnik.htm> e Karen Coyle, Understanding the Semantic Web: bibliographic data and metadata. «Library Technology Reports», 46 (2010) n. 1 (numero monografico).
} 
VIAF è stato il primo tentativo ad andare in questa direzione, riunendo le forme dei nomi usate dalle agenzie nazionali di diversi paesi. Linked Data offre un supporto tecnologico che consente il superamento delle barriere linguistiche; un URI, infatti, è totalmente indipendente dal linguaggio usato per esprimere un concetto, così ogni comunità sarà libera di esprimere un concetto con il proprio linguaggio e di collegare tale descrizione allo stesso URI.

L'uso di identificatori univoci consente di collegare diverse descrizioni allo stesso concetto: utilizzando identificatori univoci e globali per indicare nomi, opere, eventi, luoghi, soggetti e concetti le biblioteche sono in grado di immettere nel web un quantitativo enorme di dati che potranno essere riutilizzati e tramite il collegamento ad altri dataset rendere le descrizioni bibliografiche più ricche ed accessibili.

6) Produzione di dati modulari, condivisibili e riutilizzabili nel web

La tecnologia dei linked data è immediata, infatti i dati, una volta immessi e collegati con altri dati, divengono immediatamente disponibili e utilizzabili da tutte le comunità che operano nel Web Semantico.

Così un utente che cerca informazioni può ottenere immediatamente risultati che provengono da diverse agenzie che hanno fornito informazioni sul medesimo oggetto, tramite il reticolo di grafi collegati che il Web Semantico mette a disposizione.

Nel momento in cui un sistema di recupero dell'informazione estrae dati dal Web Semantico è possibile il recupero di tutti i collegamenti che interessano un determinato URI, evidenziando una rete di collegamenti ben più vasta di quella che può essere prodotta da un singolo ente. I dati bibliografici escono così dai silos non comunicanti per integrarsi con dati di altra natura.

Due sono, infine, le principali risorse cui le biblioteche possono fare riferimento nella creazione e diffusione dei propri dati: il Final report del Library linked data incubator group e OPEN BIBLIO.

Il primo consiste in linee guida per l'applicazione dell'approccio linked data alle biblioteche, mentre il secondo è un sito web e un repository di esperienze di applicazione dell'accesso aperto e del più vasto approccio Open data al mondo delle biblioteche e verrà approfondito più avanti in questo capitolo, quando si esaminerà il tema delle licenze per i dati. 
Il rapporto del Library linked data incubator group (LLD XC) ${ }^{117}$ costituisce una valida guida per la creazione di linked data relativi ai dati bibliografici. Il Final Report definisce i benefici dell'approccio linked data ai dati bibliografici, analizza alcune problematiche che sorgono nell'utilizzo dei dati bibliografici nel Web Semantico, fornisce una serie di raccomandazioni e raccoglie in appendice un censimento e un'analisi delle diverse iniziative nel campo dei linked data in ambito biblioteconomico ${ }^{118}$.

Il rapporto rappresenta dunque un buon punto di partenza e un'utile guida nella creazione di linked data di tipo bibliografico.

Innanzitutto il rapporto sottolinea i benefici dell'approccio linked data per i dati creati dalle biblioteche e i vantaggi che esse possono ottenere rispetto alle attuali pratiche catalografiche e di condivisione dei dati.

I linked data e, in particolare, i linked open data (LOD) sono condivisibili, estendibili e riutilizzabili non solo dalle stesse istituzioni bibliotecarie ma dall'intero web, infatti supportano il multilinguismo in quanto gli URI sono 'language-agnostic'. L'utilizzo di URI per la descrizione dei dati bibliografici rende i linked data una tecnologia che avvantaggia notevolmente le biblioteche nella creazione collaborativa, nella condivisione dei dati e nel controllo bibliografico.

Anche gli utenti delle biblioteche possono trarre vantaggi dalla navigazione nei linked data: essi potranno sfruttare nuove forme di scoperta e di utilizzo dei dati bibliografici navigando tra informazioni librarie e non librarie e scoprendo attraverso i dati nuove risorse migliorando così complessivamente le possibilità di ricerca e scoperta. Gli OPAC che utlilizzeranno la tecnologia dei linked data saranno perfettamente integrati nel web, e a partire dalle informazioni bibliografiche contenute nei cataloghi l'utente avrà la possibilità di scoprire senza soluzione di continuità altri dati presenti nello stesso spazio informativo, ovvero nel Web dei dati.

Le biblioteche non sono in grado di produrre dati bibliografici ad alto livello di granularità producendo descrizioni per loro stesse: la produzione di un record attraverso i linked data avverrà dal basso e sarà decentrata e distribuita tra vari attori che fanno parte

117 <http://www.w3.org/2005/Incubator/lld/XGR-lld-20111025/>. La nascita del gruppo è frutto della collaborazione del gruppo costituito dagli studiosi Thomas Baker, (Dublin Core Metadata Initiative); Emmanuelle Bermès (Centre Pompidou - Francia) Karen Coyle (Consulente, US), Gordon Dunsire, (Consulente UK); Antoine Isaac (Europeana and Vrije Universiteit Amsterdam, Netherlands); Peter Murray (LYRASIS, US); Michael Panzer (OCLC Online Computer Library Center, Inc., US); Jodi Schneider, (DERI Galway at the National University of Ireland, Galway, Ireland); Ross Singer (Talis Group Ltd, UK); Ed Summers (Library of Congress, US); William Waites (University of Edinburgh); Jeff Young (OCLC Online Computer Library Center, Inc., US); Marcia Zeng, (Kent State University, US).

${ }^{118}$ Library linked data incubator group: Use Cases, W3C Incubator group report, Daniel Vila Suero Editor, 25 October 2011, <http://www.w3.org/2005/Incubator/lld/XGR-lld-usecase-20111025/>. Ultima versione disponibile all'URL: <http://www.w3.org/2005/Incubator/lld/XGR-lld-usecase/>. 
non più del solo circuito bibliografico, ma dell'intero web e l'informazione verrà collegata allo spazio informativo del web in un gigantesco grafo.

Usando i linked data le biblioteche creeranno insiemi di dati autorevoli, aperti e riutilizzabili per descrivere altre risorse, incrementando la propria visibilità nel web.

Infine gli sviluppatori di software possono facilmente produrre applicazioni che sfruttino i linked data con un doppio vantaggio: la possibilità di utilizzare protocolli e standard non più 'bibliocentrici' ma comuni a tutti il web e facilmente interoperabili e riutilizzabili ${ }^{119}$ e la possibilità di riutilizzare dati e applicazioni prodotte nel web per migliorare i software per le biblioteche. Essi non dovranno più lavorare con standard bibliotecari come ISO $2709^{120}$ e MAchine-Readable Cataloging (MARC) ${ }^{121}$, che richiedono software appositamente progettati, ma produrrano dati comprensibili da tutte le applicazioni del web e potranno lavorare in contatto con le altre comunità di sviluppo del web.

Il rapporto si sofferma sulle problematiche e sui limiti che i dati tradizionali prodotti dalle biblioteche presentano:

* i dati delle biblioteche non sono integrati con le risorse web (§ 3.1.1);

* i dati delle biblioteche sono progettati solo per la comunità bibliotecaria (§ 3.1.2);

* i dati sono espressi principalmente in forma testuale e nel linguaggio naturale ( $§$ 3.1.3);

* La comunità bibliotecaria e quella del Web Semantico utilizzano terminologie differenti per gli stessi concetti espressi dai metadati (§ 3.1.4);

* lo sviluppo e il mutamento delle tecnologie delle biblioteche dipende strettamente dai creatori dei sistemi software $(\S 3.1 .5)$;

Particolarmente significative sono le raccomandazioni che il Library Linked data Incubator Group fornisce nel report: attraverso i linked data le biblioteche possono recuperare il ruolo di leadership nei servizi di gestione delle risorse informative e di conservazione a lungo termine e di accesso a tali risorse. Il rapporto raccomanda alle biblioteche di focalizzare l'attenzione sulle questioni di apertura dei dati, agli organismi che si occupano della standardizzazione di contribuire alla standardizzazione dei linguaggi del Web Semantico, di sviluppare dati e standard compatibili con i Linked data attraverso

\footnotetext{
${ }^{119}$ Pensiamo ad esempio ai protocolli di accesso ai dati strettamente bibliotecari come Z39.50 Information Retrieval Protocol (<http://www.niso.org/standards/resources/Z39.50_Resources>) i linked data utilizzano il protocollo più diffuso nel web ovvero Hypertext Transport Protocol (HTTP).

${ }^{120}<\mathrm{http}: / /$ www.iso.org/iso/iso_catalogue/catalogue_tc/catalogue_detail.htm?csnumber=41319>.

$121<$ http://loc.gov/marc/>.
} 
la diffusione di best practices e di collaborare con le comunità che operano nel Web Semantico. Agli sviluppatori e ai gestori dei dati bibliografici (es. le agenzie nazionali) il report raccomanda la progettazione di sistemi compatibili con i linked data, l'assegnazione di URI alle risorse create.

\subsection{Pubblicare linked open data. Il workflow, le tecnologie, le licenze}

Affinché si costituisca il Web dei dati le aziende e le persone che operano sul web devono esporre i propri dati sottoforma di dati collegati o Linked data. La pubblicazione di dataset di Linked data è un passo fondamentale che porta alla costituzione di un gigantesco grafo in cui ogni oggetto o concetto rappresentato in un dato potrà essere identificato e collegato ad altri dati presenti nel web.

E dunque necessario considerare $\mathrm{i}$ diversi aspetti che ogni ente deve tenere in considerazione nel processo di pubblicazione dei Linked data nel web ovvero il procedimento della pubblicazione e le sue fasi, le soluzioni tecnologiche a disposizione e le licenze.

a. Il workflow

Pubblicare i propri dati come linked data sul web richiede un certo numero di operazioni, che vanno dall'identificazione della fonte dei dati fino alla predisposizione dei dati per il loro riutilizzo ${ }^{122}$.

${ }^{122}$ Le fasi del processo di pubblicazione sono state efficacemente sintetizzate nella guida Methodological guidelines fot publishing linked data da Boris Villazon Terrazas e Oscar Corcho (<http://delicias.dia.fi.upm.es/wiki/images/7/7a/07_MGLD.pdf $>$ ) che sintetizzano in sette passi il processo di pubblicazione:

* identificazione della fonte dei dati

* modellizzazione del vocabolario

* generazione dei dati in RDF

- bonifica dei dati prodotti

* creazione di collegamenti tra dataset differenti identificando linking target e validando le relazioni individuate

* pubblicazione del dataset nel registro CKAN

Altre autorevoli guide sono quelle di Chris Bizer - Richard Cyganiak - Tom Heath How to Publish Linked Data on the Web <http://sites.wiwiss.fu-berlin.de/suhl/bizer/pub/LinkedDataTutorial/20070727/> e di Leigh Dodds - Ian Davis, Linked data patterns. A pattern catalogue for modelling, publishing, and consuming Linked data, 2011, [ebook], <http://patterns.dataincubator.org/book/>. Altre utili guide sono rivolte ai dati governativi sono: Linking Government Data, David Wood editor, New York, Springer, 2011; Bernadette Hyland - Boris Villazón-Terrazas, Cookbook for Open Government Linked Data, $<$ http://www.w3.org/2011/gld/wiki/Linked_Data_Cookbook>. 
1) Il primo passaggio è l'identificazione delle fonti dalle quali estrarre i dati; questa fase richiede che si valutino attentamente le fonti (se tali fonti sono già pubbliche o se bisogna intervenire sui dati originali), che si analizzino in prima battuta le principali entità e le relazioni e che si affrontino in via preliminare le questioni relative alla costruzione degli URI alla scelta della licenza più appropriata.

La scelta degli URI è un'operazione particolarmente delicata, in quanto gli URI scelti, a differenza degli URL del web, devono risultare significativi e non 'opachi' ${ }^{123}$.

2) Il secondo passaggio riguarda la modellizzazione del vocabolario che induce a considerare la possibilità del riutilizzo di vocabolari diversi già esistenti (soluzione consigliata ove possibile) o la creazione di nuove ontologie (operazione più complessa, ma in alcuni casi necessaria).

3) Segue la generazione di file di dati RDF, passaggio che si compie tramite i diversi linguaggi di mappatura e che dipende dal formato di origine. In base ai dati di provenienza si deciderà se sono necessarie operazioni di trasformazione e pulizia dei dati e a seconda del formato di origine saranno disponibili vari strumenti e software per la trasformazione dei dati in base ai vocabolari creati nella fase di modellazione. Verranno dunque effettuate delle verifiche dei dati prodotti per eliminare eventuali errori prodotti nella conversione.

4) Un successivo e importante passo è quello della creazione dei collegamenti con altri dataset. Verranno identificati nel registro CKAN i dataset che contengono dati equivalenti a quelli presenti nel dataset creato e si stabiliranno le opportune equivalenze che verranno poi validate ${ }^{124}$. Si potrà ad esempio collegare l'URI relativo alla città di Madrid nel dataset di partenza, ad esempio miodataset.it/... Madrid con gli URI relativi ad una stessa città in due dataset differenti come Dbpedia e Geonames: http://dbpedia.org/resource/Madrid e http://sws.geonames.org/6355233/. Per le equivalenze si usano ontologie come SKOS e OWL.

5) Il passaggio finale è quello della pubblicazione del dataset nel Web Semantico per renderlo disponibile al riutilizzo. I dati immessi nel web potranno essere interrogati tramite SPARQL endpoints ovvero applicazioni che utilizzano il linguaggio di interrogazione SPARQL.

Prima di pubblicare i dati è importante porsi delle domande che costituiranno una sorta di checklist ovvero misureranno la rispondenza del proprio dataset ad una lista di

\footnotetext{
${ }^{123} \mathrm{Si}$ veda il capitolo 4 Linked data design consideration e 4.1.2 Guidelines for creating cool URIs, in Heath - Bizer, Linked data: evolving the Web into a global data space cit.

${ }^{124}$ Un validatore dei collegamenti di tipo 'Same As' è SameAs Validator, $<$ http://oegdev.dia.fi.upm.es:8080/sameAs/>.
} 
qualità necessarie per la pubblicazione: qualità dei dati di provenienza, link ad altri dataset; uso di licenze aperte; utilizzo di vocabolari, dereferenziabilità degli URI; mappatura dei vocabolari; dati relativi al dataset; altri metodi di accesso al dataset.

In questa fase finale bisogna affrontare alcuni passi importanti come ad esempio pubblicare i metadati relativi al dataset che si sta pubblicando e preparare il dataset in modo che possa essere trovato a partire da altri dataset presenti nel web ${ }^{125}$. Una caratteristica fondamentale del Web dei dati è che tutti i dati di diversi dataset siano collegati tra loro in modo che - nelle parole di Berners Lee- 'si possano scoprire più cose'. Dunque il passo successivo è la registrazione del dataset nel registro $\mathrm{CKAN}^{126}$, la generazione di un Sitemap ${ }^{127}$ del proprio dataset e la segnalazione ai motori come Google e Sindice $^{128}$.

b. Le soluzioni tecnologiche

Le biblioteche oggi pubblicano e rendono disponibili i dati bibliografici nei loro OPAC o in piattaforme più ampie come i sistemi per la scoperta, in grado di offrire all'utente attraverso un'unica interfaccia anche descrizioni di oggetti digitali e altre risorse provenienti da banche dati libere o a pagamento, repositories del web e istituzionali e, infine, risorse web. Molti dati bibliografici possono essere reperiti anche nei portali e nelle grandi biblioteche digitali che sono oggi sempre più diffuse.

I dati bibliografici, come abbiamo visto, restano però ancorati a formati e protocolli strettamente bibliotecari, interpretabili e comprensibili dai software per le biblioteche e solo di rado vengono esportati in formati interoperabili con il web come XML o in $\mathrm{RDF}^{129}$. La maggior parte dei formati utilizzati per i dati bibliografici è proprietaria e ciò rallenta sia lo sviluppo dei software sia il processo di armonizzazione e scambio dei dati con il

\footnotetext{
${ }^{125}$ VoID e Open Prov sono due strumenti che consentono di esprimere i metadati relativi al dataset che si vuole pubblicare.

${ }^{126}$ Per raccogliere la meta informazione sui dataset presenti nel web è stato creato CKAN, un registro di open data fornito dalla Open Knowledge Foundation. Una pagina fornisce le indicazioni per esprimere i metadati del dataset in CKAN. Cfr.: Guidelines for Collecting Metadata on Linked Datasets in CKAN. $<$ http://www.w3.org/wiki/TaskForces/CommunityProjects/LinkingOpenData/DataSets/CKANmetainformati on>.

${ }^{127}$ Sitemap4rdf è uno strumento che utilizza Sitemap Protocol, per indicizzare sui motori pagine disponibli per il crawling. È supportato dai principali motori di ricerca come Google, e da motori basati sui dati come Sindice. Sitemap4rdf uno strumento a linea di comando che genera una mappa del sito in XML per i siti che espongono linked data tramite un' applicazione SPARQL.

${ }_{128}<$ http://sindice.com/> Sindice è un motore di ricerca che indicizza dati strutturati compatibili con RDF che usano tecnologie come Microformati, Microdata, Schema.org, RDFa e Opengraph.

${ }^{129} \mathrm{Al}$ momento l'unico software che consente la trasformazione dei dati bibliografici in XML e in RDF è Extensible Catalog (XC), la procedura per la conversione è però ancora in via di sviluppo.
} 
resto del web. Le biblioteche che utilizzano questi software sono legate nelle scelte di sviluppo dei propri strumenti agli sviluppatori (vendors) che impongono i propri formati di codifica dei dati.

In generale, il record bibliografico è stato progettato per lo scambio e la visualizzazione negli OPAC, mantiene una forma testuale e una bassa granularità. La granularità nella struttura dei dati bibliografici è una caratteristica che implica dei costi alti che le biblioteche non sono in grado da sole di sostenere.

La pubblicazione di dati bibliografici sotto forma di linked data apre alle biblioteche la strada dell'integrazione con gli altri dati prodotti nel web e ad una nuova struttura del record bibliografico atomizzata e modulare oltre che a nuove forme di produzione dei dati bibliografici, distribuite e collaborative.

Produrre descrizioni nella forma di dati collegati non vuol dire rinunciare a quanto è stato fatto finora: i dati che oggi vengono pubblicati nelle pagine html o che sono contenuti nelle basi dati bibliografiche delle biblioteche possono essere strutturati in una modalità compatibile con i linked data, mediante un lavoro di conversione dei dati esistenti e con l'adozione di nuove modalità di produzione dei dati bibliografici, che verrà distribuita tra biblioteche e altre comunità che operano nel web.

Le biblioteche nella creazione di linked data si trovano coinvolte in varie problematiche come la scelta degli identificatori persistenti, la modellizzazione dei dati, il collegamento ad altri dataset, l'allineamento semantico, la creazione, quando necessario, di nuovi vocabolari e ontologie, l'esposizione dei dati affinché siano reperibili nei motori di ricerca tradizionali.

\section{Identificatori univoci e scelta degli URI}

Esporre i dati bibliografici come linked data comporta l'assegnazione di identificatori univoci alle risorse bibliografiche. Di recente le biblioteche, spinte dal fenomeno Web 2.0 e dalle sue tecnologie hanno cominciato ad assegnare identificatori univoci alle risorse bibliografiche per poter richiamare le stesse in applicazioni esterne attraverso le API e la tecnologia del Mashup ${ }^{130}$.

\footnotetext{
${ }^{130}$ Il tema degli identificativi persistenti è stato oggetto di approfondimento anche nel nostro paese, cfr.: Oriana Bozzarelli - Maria Cassella, Gli identificativi persistenti. A Torino un seminario di presentazione, «Biblioteche Oggi», 29 (2011) n. 8 p. 66-70. Anche in: <http://eprints.rclis.org/handle/10760/16344>.
} 
Tuttavia la maggior parte dei software per biblioteche non utilizza identificatori univoci per le risorse bibliografiche e quando li utilizza questi non sono compatibili con il Web Semantico. Utilizzare URI http consente di collegare i dati bibliografici agli altri dataset esistenti, di separare il contenitore dal contenuto e, a lungo termine, di preservare il contenuto dell'informazione e risolvere il problema dell'obsolescenza dei formati. La scelta degli URI per le classi e per i soggetti è una scelta particolarmente complessa.

\section{Creazione del data model}

Creare il data model significa scegliere i vocabolari da utilizzare per esprimere le entità e le loro relazioni. Una volta scelto di utilizzare linked data come modello logico per la struttura dei dati, le biblioteche dovranno compiere delle scelte relative alla modellazione del dataset ovvero scegliere quali dataset di elementi utilizzare e quali vocabolari di valori per esprimere le entità bibliografiche, gli attributi e le loro relazioni. Il modello utilizzato per rappresentare l'universo bibliografico è FRBR e dunque si farà riferimento alle entità, agli attributi e alle relazioni previste dai modelli della famiglia FRBR, che sono stati pubblicati nel Web Semantico.

3. Collegamento ad altri dataset, creazione di nuovi vocabolari e ontologie, allineamenti semantici

Il collegamento tra i dataset diversi è il cuore della tecnologia del Web Semantico e dei linked data e l'allineamento semantico è quella operazione che consiste nel produrre link RDF tra entità semanticamente equivalenti, similari o correlate presenti in altri dataset.

VIAF e AGROVOC rappresentano esempi di strumenti che forniscono l'allineamento semantico tra forme di autorità presenti creati da diverse agenzie bibliografiche nazionali rendendole disponibili in un unico authority file virtuale. AGROVOC è il dataset relativo all'omonimo thesauro di FAO per i soggetti e nomi relativi all'agricoltura e alla nutrizione, che oggi è collegato a 13 importanti dataset.

Come abbiamo accennato, le ontologie forniscono le modalità per il collegamento dei dati di diversi dataset attraverso linguaggi semplici come RDFS oppure più complessi come SKOS e OWL. 
Nella fase di costruzione del proprio modello di dati, le biblioteche dovranno individuare le principali fonti interne con le quali produrre gli allineamenti semantici e fornire i collegamenti semantici con le principali e più note fonti esterne, utilizzando quando possibile questi vocabolari per descrivere entità, e relazioni e occorrenze e definire le relazioni tra termini del mondo bibliotecario e quelli prodotti da altre comunità.

Gli allineamenti semantici sono necessari per i set di elementi dei metadati per la pratica di riutilizzo di set di metadati già esistenti o per la costruzione di application profiles che riutilizzano elementi di metadati provenienti da diversi dataset.

Ad esempio, le entità di FRBR sono espresse in vocabolari diversi, dunque il compito importante è quello di allineare le definizioni provenienti dai diversi vocabolari già esistenti.

Allo stesso modo è importante e cruciale collegare i diversi dataset prodotti dalle biblioteche, per evitare che i dati delle biblioteche continuino ad essere prodotti entro silos non comunicanti: tutti i dati contenuti nei dataset che rappresentano le risorse di una biblioteca devono essere collegati con gli altri dataset per evitare la duplicazione dei dati bibliografici, che fino ad oggi ha caratterizzato il lavoro di produzione dei record bibliografici nei cataloghi di tutto il mondo. In questo modo si avvia un processo distribuito e virtuoso di deduplicazione dei dati bibliografici che alla fine del processo risulteranno collegati nel vasto Web Semantico. Il collegamento dovrà comprendere altri dataset bibliografi e non bibliografici. Ad esempio la voce per l'autore William Gibson verrà collegata alla sua forma in VIAF e Dbpedia, che a sua volta è un'astrazione di Wikipedia. 


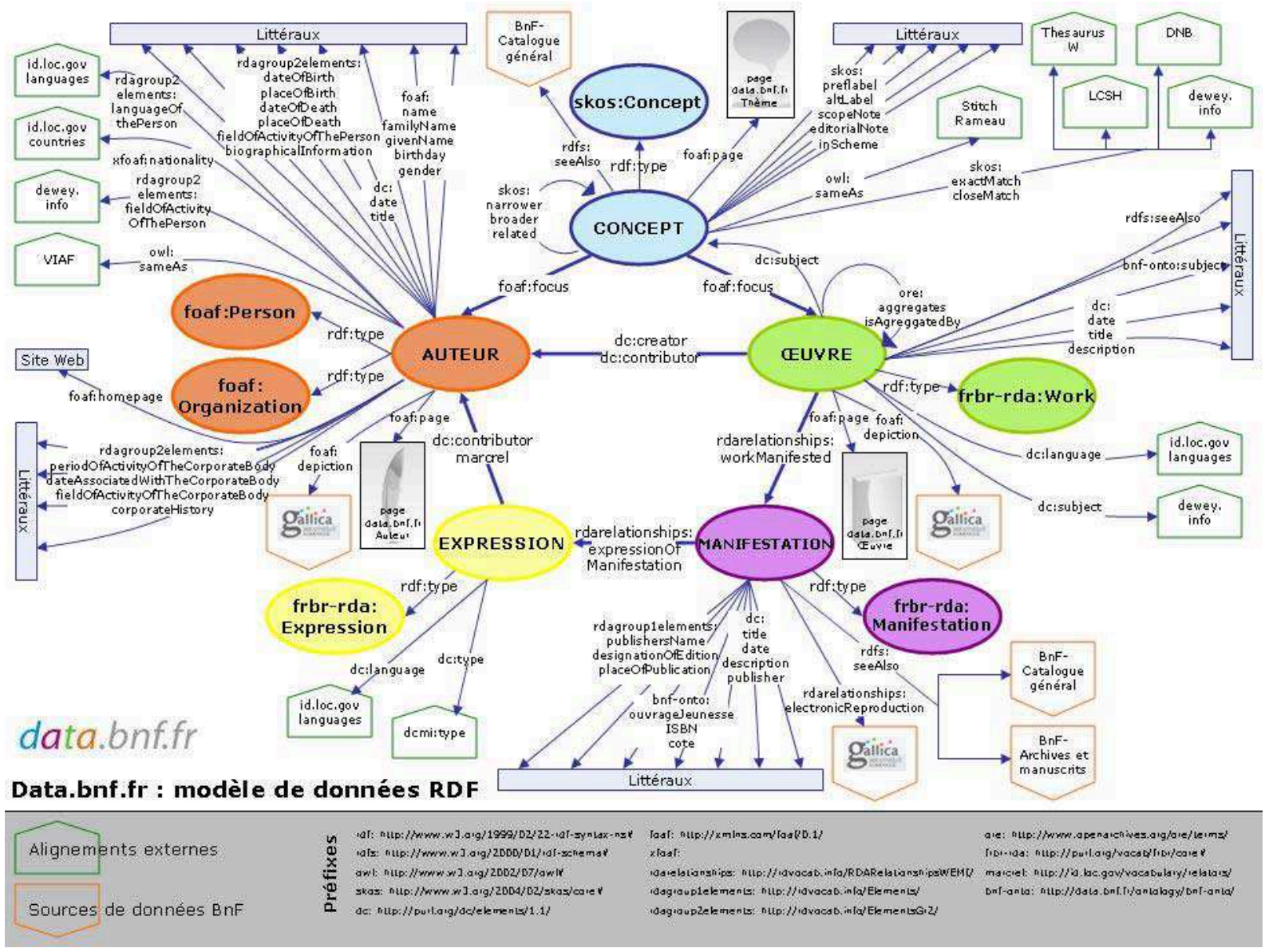

Fig. 4.10 - Il data model di data.bnf.fr nella versione completa ${ }^{131}$

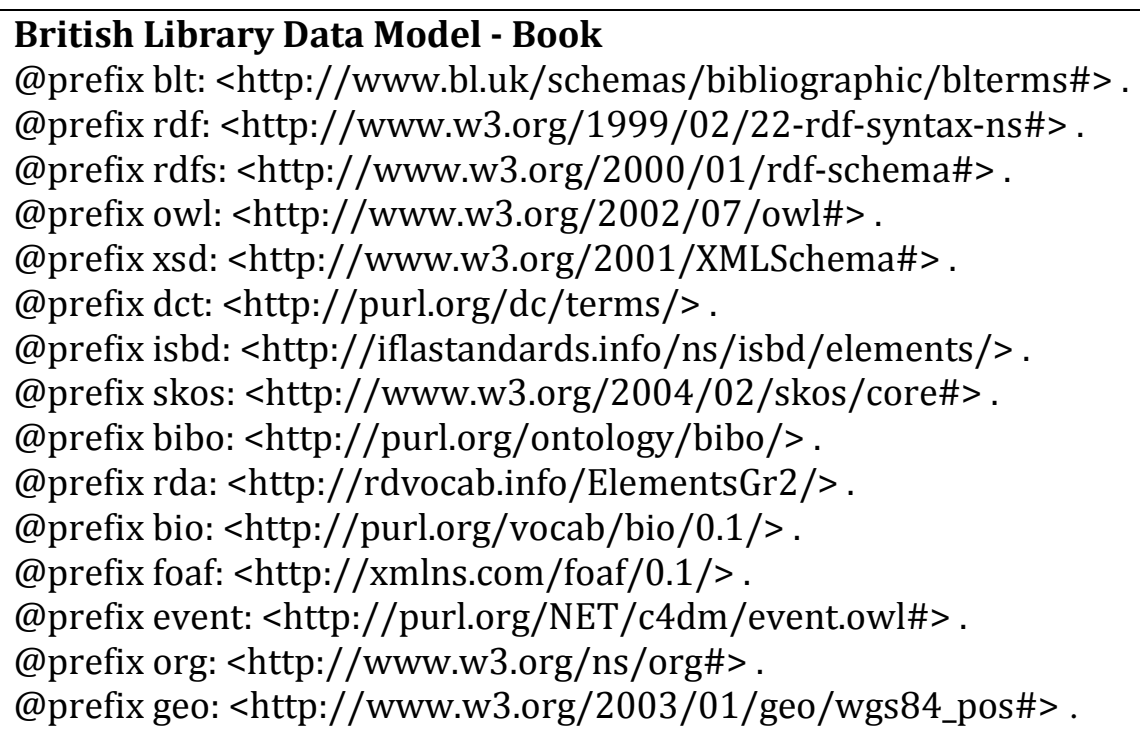

Fig. 4.11- I prefissi utilizzati nel data model di British Library Bibliography rivelano l'uso di proprietà provenienti da diverse ontologie e vocabolari ${ }^{132}$

\footnotetext{
$131<$ http://data.bnf.fr/semanticweb-en>.

$132<$ http://www.bl.uk/bibliographic/pdfs/bldatamodelbook.pdf>.
} 
4. Creazione di nuove ontologie

Sebbene sia buona norma il riutilizzo di ontologie e vocabolari già esistenti, quando si rivela necessario, le biblioteche potranno creare nuove ontologie per definire classi e proprietà non previste in altri schemi. Ad esempio per la British Library Bibliography è stata creata una nuova ontologia ${ }^{133}$ che ha lo scopo di definire classi e proprietà per la descrizione delle risorse bibliografiche e per il collegamento ad altre entità che completano quelle fornite da altri schemi.

Tra queste vengono definite, ad esempio, la classe Famiglia come sottoclasse della classe Gruppi definita dall'ontologia Foaf:

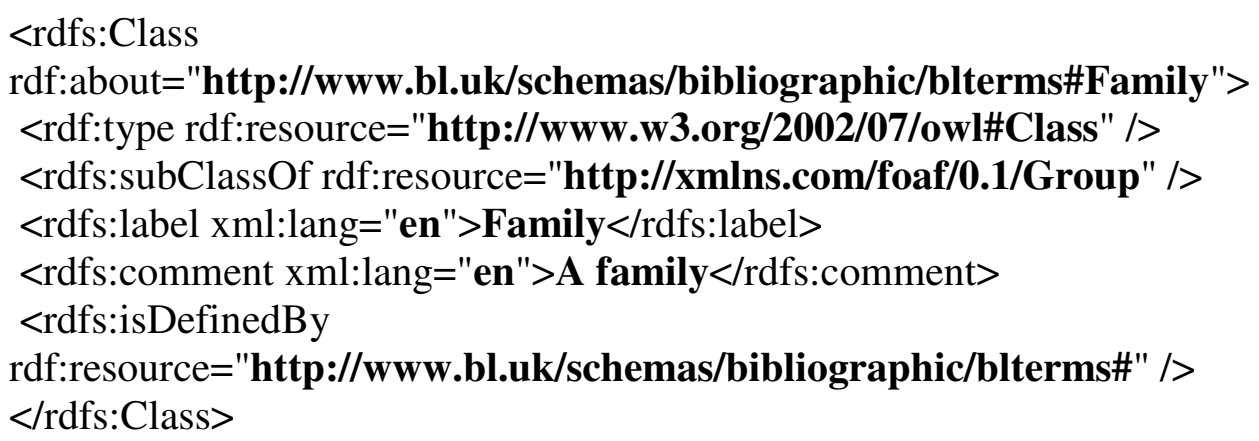

ed una proprietà per associare un numero BNB ad una risorsa:

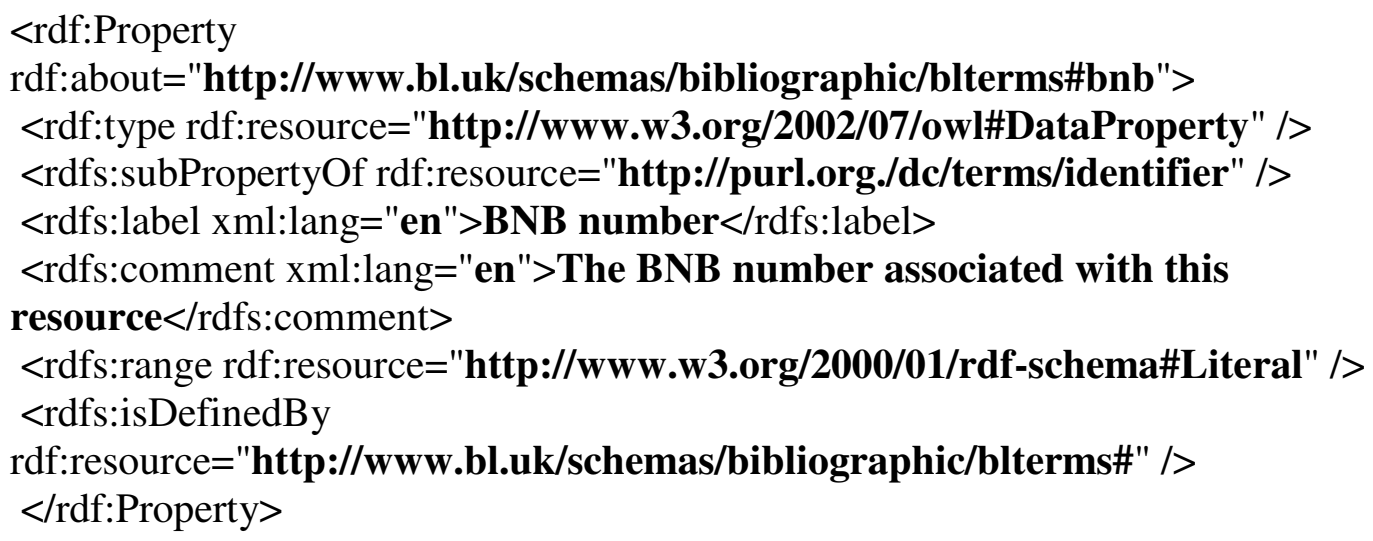

5. recupero dei dati bibliografici da parte dei motori di ricerca

Una volta che i dati sono stati esposti e pubblicati come linked data potranno essere recuperati e tramite i collegamenti ad altri schemi potranno essere utilizzati dalle altre applicazioni del Web Semantico. È importante che le biblioteche predispongano diverse serializzazioni di RDF che consentono il recupero e la comprensione dei dati anche da parte dei motori di ricerca o di altri applicazioni del Web Semantico.

\footnotetext{
${ }^{133}$ L'ontologia è visibile all'indirizzo: <http://www.bl.uk/schemas/bibliographic/blterms\#>.
} 
Produrre linked data vuol dire non solo applicare il modello di dati RDF, ma anche rendere pubblicamente 'aperti' e disponibili i propri dati; per questo si utilizza più propriamente la denominazione Linked Open Data ${ }^{134}$.

Ma cosa significa produrre dati 'aperti'? Quale deve essere il grado più opportuno di apertura per i dati bibliografici e quali sono le possibilità che vengono offerte alle biblioteche e agli altri enti che vogliono pubblicare dati collegati sul web?

I concetti di 'dato' e di 'apertura' sono strettamente connessi ai linked data e al più vasto movimento Open Data (OD) e meritano in questa sede una breve digressione.

Il concetto di 'dato' non va confuso con quello di 'informazione' che, come vederemo anche nel capitolo successivo, è generalmente considerata come 'una differenza percepita ${ }^{135}$. Il dato grezzo da solo non è capace di produrre conoscenza, ma deve essere organizzato, rielaborato e strutturato per divenire 'informazione': in definitiva esso costituisce la materia prima sulla quale l'informazione viene generata.

Le biblioteche fanno uso di dati, ma sono specializzate nella creazione di 'metadati' (i 'dati sui dati'), rappresentativi delle risorse informative da descrivere ai fini del loro recupero in un sistema di IR o in un catalogo elettronico o utilizzati allo scopo di trattare le risorse digitali a fini amministrativi, gestionali o conservativi.

Il concetto di 'apertura' ci riporta direttamente alle definizioni di Open Data e in un contesto più ampio di Open Knowledge ovvero di tutte quelle iniziative volte all'eliminazione delle barriere all'utilizzo dei prodotti della scienza e dell'intelligenza umana intendendo per 'Open knowledge' «qualsiasi contenuto, informazione o dato che le persone sono libere di utilizzare, riutilizzare e ridistribuire senza alcuna restrizione legale, tecnologica o sociale» e intendendo per 'conoscenza' i contenuti come musica, film, libri, dati scientifici, storici, geografici, informazione governativa o amministrativa.

$\mathrm{L}^{\prime}$ Open Knowledge Foundation $(\mathrm{OKF})^{136}$ ha fornito alcune importanti definizioni di Open Data, Open Content ed Open information ${ }^{137}$.

\footnotetext{
${ }^{134}$ Si veda in proposito: Antonella De Robbio, - Silvia Giacomazzi, Dati aperti con LODe, «Bibliotime», XIV (2011), n. $2<$ http://didattica.spbo.unibo.it/bibliotime/num-xiv-2/derobbio.htm>.

${ }^{135}$ Cfr.: Gregory Bateson, Verso un'ecologia della mente, trad. it. di Giuseppe Longo, Milano, Adelphi, 2004.

136 Open Knowledge Foundation è una fondazione senza scopo di lucro creata nel 2004 allo scopo di promuovere la conoscenza aperta dei dati provenienti da diversi ambiti disciplinari (geografici, statistici, biomedici etc.) per renderli fruibili, riutilizzabili senza barriere sociali, culturali o tecnologiche. La comunità italiana mantiene il sito web <http://it.okfn.org/>.
} 
Negli ambiti di azione di $\mathrm{OKF}^{138}$ si situano le iniziative volte a garantire l'apertura dei dati bibliografici, ovvero l'Open Bibliographic data (OPEN BIBLIO) ${ }^{139}$ relativo all'apertura dei dati e dei metadati provenienti dal settore bibliotecario, in particolare i dati provenienti dai cataloghi e i relativi metadati.

In quest'ambito OKF ha stabilito dei principi (Open Bibliographic data Principles POBD $)^{140}$, ha creato un gruppo di studio (Open Bibliographic Data Working Group) che pubblica discussioni e progetti nel sito OpenBiblio.net e attivato una mailing list (Open Bibliographic Data mailing list) allo scopo di discutere progetti e iniziative.

I Principles on Open Bibliographic Data ${ }^{141}$, ovvero i principi da adottare per produrre dati bibliografici 'aperti', sono quattro:

137 Open Knowledge Foundation, Open Definition, Version $1.1<$ http://opendefinition.org/okd/> (n.d.r la traduzione e rielaborazione del testo è di chi scrive). In particolare un'opera per essere 'aperta' deve soddisfare i seguenti requisiti:

1. Accesso. L'opera deve essere disponibile per intero e senza costi di riproduzione e preferibilmente scaricabile via internet e deve essere offerta in una forma aperta alle modifiche. Si tratta di una apertura 'sociale'.

2. Ridistribuzione. La licenza non deve escludere che le parti possano vendere o donare l'opera in se o come parte in una pacchetto piàù ampio derivato da opere provenienti da diverse fonti. La licenza non deve prevedere royalty o tariffe per tale vendita o distribuzione.

3. Riutilizzo. La licenza deve consentire le modifiche e le opere derivate e permettere che esse siano ridistribuite nei termini dell'opera originale. Questa clausola non impedisce l'uso di licenze share-alike che richiedono la ridistribuzione delle modifiche negli stessi termini dell'opera originale.

4. Assenza di restrizioni tecnologiche. L'opera deve essere fornita in una forma tale che non vi siano ostacoli tecnologici all'esecuzione delle precedenti attività. Ciò può essere raggiunto prevedendo la distribuzione dell'opera in un formato di dati aperto, le cui specifiche siano pubblicamente e apertamente disponibili e che non ponga restrizioni finanziarie sul suo utilizzo.

5. Attribuzione. La licenza può richiedere come condizione per la ridistribuzione e il riutilizzo l'attribuzione ai creatori o ad altre persone che hanno contribuito all'opera. Se questa condizione viene imposta non deve comportare oneri $[\ldots]$.

6. Integrità. La licenza può porre come condizione per l'opera che viene distribuita in una forma modificata che l'opera risultante dalla modifica porti un nome diverso o un diverso numero di versione rispetto all'opera originale.

7. Nessuna discriminazione a danno di persone o gruppi. La licenza non deve discriminare alcuna persona, né gruppi di persone.

8. Nessuna discriminazione a danno di particolari campi applicazione. La licenza non deve essere restrittiva per chi utilizza l'opera in uno specifico campo di studio o di applicazione. Ad esempio essa non può limitare l'utilizzo nel settore commerciale o nel campo della ricerca genetica.

9. Distribuzione della licenza. I diritti sull'opera devono applicarsi a tutti coloro ai quali l'opera è ridistribuita senzala necessità di applicazione di una licenza aggiuntiva per le parti.

10. Licenze non specifiche per un pacchetto. I diritti sull'opera non devono dipendere dal fatto che l'opera sia parte di un particolare pacchetto. Se l'opera è estratta da quel pacchetto e usata e distribuita entro i termini della licenza dell'opera, tutte le parti alle quali l'opera è distribuita dovranno avere gli stessi diritti che sono garantiti nel pacchetto originale.

11. La licenza non deve porre restrizioni alla distribuzione di altre opere. La licenza non deve porre restrizioni su altre opere che vengono distribuite insieme all'opera licenziata. Ad esempio, la licenza non deve richiedere che altre opere distribuite sullo stesso medium siano aperte.

${ }^{138}$ I principali ambiti di intervento sono quelli dell'Open Government, dell'Open bibliographic data e dell'Open data in Science, riferiti rispettivamente ai dati governativi, bibliografici e scientifici.

$139<$ http://opendefinition.org/bibliographic/>.

$140<$ http://openbiblio.net/principles/> scaricabili <http://openbiblio.net/files/2011/01/POBD.pdf>.

${ }^{141}$ I principi sono stati elaborati con la collaborazione di: Karen Coyle, Mark MacGillivray, Peter MurrayRust, Ben O' Steen, Jim Pitman, Adrian Pohl, Rufus Pollock e William Waites. 
1. utilizzare per la pubblicazione dei dati bibliografici una licenza esplicita e robusta, ovvero precisa irrevocabile e basata su una dichiarazione legale in termini di deroga o licenza;

2. utilizzare una licenza o deroga che sia appropriata per i dati bibliografici. Una varietà di deroghe e licenze utilizzabili sono descritte nel documento $<$ http://opendefinition.org/licenses/\#Data>. L'uso delle comuni licenze Creative Commons $^{142}$ (tranne che per la $\mathrm{CC} 0$ ) è fortemente scoraggiato;

3. utilizzare i dati nelle modalità definite dalla Open definition che prevedono l'assenza di clausole restrittive per garantire la massima fruizione e il riutilizzo dei dati;

4. utilizzare per i dati bibliografici licenze come PDDL (Public Domain Data Licence) o CC0 (Creative Commons Zero).

Tali importanti principi introducono alla complessa problematica delle licenze per i dati che vengono pubblicati nel web.

Secondo i principi dell'Open Biblio i dati bibliografici contenuti in OPAC, database bibliografici, archivi aperti e basi di conoscenza come schemi di classificazione e thesauri dovranno essere forniti in modalità compatibili con i linked data e con licenze adeguate alla loro fruizione. Generalmente gli OPAC offrono l'accesso libero ai dati bibliografici che vengono visualizzati nelle interfacce per la ricerca, ma la fruizione e il riutilizzo dei dati non sono sempre concessi o garantiti, a causa della scarsa familiarità con l'uso di licenze compatibili con il web e probabilmente anche alla mancanza di consapevolezza sulle potenzialità dei dati aperti che finora ha caratterizzato il nostro settore.

Le biblioteche che vogliono pubblicare dati di dominio pubblico hanno a disposizione una varietà di licenze aperte come Open data commons ${ }^{143}, \mathrm{CC}^{144} \mathrm{e} \mathrm{PDDL}^{145}$

\footnotetext{
${ }^{142}$ Oltre a quelle elencate è sconsigliato anche l'uso di altre diffuse licenze come GFDL, GPL, BSD.

$143<$ http://opendatacommons.org/> sono le licenze per gli Open data fornite da OKF e sono rispettivamente: Public Domain Dedication and License (PDDL) "Public Domain for data/databases"; Attribution License (ODC-By) — "Attribution for data/databases"; Open Database License (ODC-ODbL) — "Attribution ShareAlike for data/databases" (<http://opendatacommons.org/licenses/>).

$144<\mathrm{http}$ ///creativecommons.org/publicdomain/zero/1.0/>. La licenza consente di attribuire un'opera al pubblico dominio. Una traduzione provvisoria in lingua italiana è presente in (<http://wiki.creativecommons.org/Publicdomain/zero/1.0/LegalText_\%28Italian\%29>). La persona che associa un'opera a questa tipologia di licenza dedica l'opera al pubblico dominio rinunciando a tutti i diritti su di essa. Secondo CC0: "You can copy, modify, distribute and perform the work, even for commercial purposes, all without asking permission.» I titolari rinunciano definitivamente ai diritti sull'opera al fine di contribuire ad un patrimonio comune di opere creative, culturali e scientifiche ('Commons') che il pubblico può sviluppare ulteriormente, modificare, inserire in altre opere, riutilizzare e ridistribuire del tutto liberamente, in qualsiasi forma e per ogni finalità. Sono inclusi senza alcun limite anche gli scopi commerciali, potendo fare affidamento sul fatto che non sarà intrapresa alcuna azione legale per violazione
} 
che non richiedono attribuzione e comportano la rinuncia a tutti i diritti d'autore sui dati bibliografici creati.

Molte istituzioni che hanno pubblicato i dati sottoforma di linked data tuttavia adottano licenze restrittive. È il caso di OCLC che per i dati catalografici ha adottato una licenza Open Data Commons Attribution (ODC-BY) ${ }^{146}$ e di software proprietari che generalmente pongono problemi quanto al libero utilizzo dei dati contenuti. Vi sono invece iniziative come quelle della Bibliothèque nationale de France e delle biblioteche nazionali di Germania, Regno Unito e Spagna che rendono disponibili i loro dati con licenze aperte che ne favoriscono il libero riutilizzo.

Nel nostro paese l'OPAC SBN del Servizio bibliotecario Nazionale in quanto principale produttore di dati bibliografici sul territorio e finanziato pubblicamente dovrebbe vagliare la disponibilità all'apertura dei dati tramite licenze di pubblico dominio. Alcune criticità sono state recentemente rilevate per i cataloghi di istituzioni universitarie che essendo strumenti decentrati riservano alcune problematiche relative alla gestione dei dati e dei diritti da parte delle biblioteche che li utilizzano ${ }^{147}$. Sempre più spesso le biblioteche di questa tipologia si affidano a soluzioni di discovery che sostituiscono i cataloghi elettronici con discovery system commerciali, che contengono oltre ai dati dei cataloghi anche dati provenienti da banche dati a pagamento. La questione dell'apertura dei dati in questi casi diventa più spinosa e di difficile soluzione.

\subsubsection{Le iniziative delle biblioteche nel campo dei linked data. Dataset, vocabolari, schemi di metadati}

Le biblioteche e le grandi agenzie nazionali hanno cominciato a produrre alcuni archivi bibliografici come linked data, cui si aggiunge la pubblicazione in questo formato

\footnotetext{
dei diritti da parte dei titolari. Le persone che adottano CC0 possono contribuire ai Commons per promuovere l'ideale di una cultura libera e la produzione ulteriore di opere creative, culturali e scientifiche, o per costruirsi una reputazione, o per una maggiore distribuzione delle proprie opere in parte attraverso l'utilizzo e l'attività di altre persone.

${ }^{145}$ Open Data Commons Public Domain Dedication and License (PDDL), $<$ http://opendatacommons.org/licenses/pddl/>.

146 Open Data Commons Attribution License, <http://opendatacommons.org/licenses/by/>.

${ }^{147} \mathrm{Si}$ veda il recente contributo di Antonella De Robbio, Forme e gradi di apertura dei dati cit. Su questa tematica si sono svolte alcune riunioni di persone interessate al movimento OpenBiblio in Italia: $<$ http://it.okfn.org/category/openbiblio/> e <http://openbiblio.net/>.
} 
di alcuni schemi di metadati come DCMI e FRBR e dei principali vocabolari controllati come Dewey e LCSH.

Le iniziative si muovono verso la creazione di tre categorie di strumenti:

1. Datasets che descrivono risorse bibliografiche come la British National Bibliography, i cataloghi delle biblioteche nazionali di Svezia e Ungheria e Spagna, Open libray, CrossRef ed Europeana e il progetto della Biblioteca Nazionale francese. Un dataset è un insieme di dati strutturati, (descrizioni di libri o altri oggetti). Sono insieme di triple che descrivono cose, elementi (attributi, relazioni) di entità e valori degli elementi. Gli elementi possono essere forniti da un metadata element set come Dublin Core e i valori possono provenire da vocabolari controllati come LCSH, VIAF, oppure essere stringhe di testo (es. il titolo di un'opera);

2. Vocabolari che descrivono i valori come Library of Congress Subject Headings, il thesaurus AGROVOC, il Virtual International Authority File (VIAF), Dewey Decimal Classification, e GeoNames. Possono essere assimilati ai thesauri, agli schemi di classificazione, alle tassonomie, ai vocabolari controllati, alle liste di autorità e più generalmente agli schemi di organizzazione delle conoscenze. Un vocabolario di valori definisce argomenti, autori etc. che possono essere usati come valori degli elementi dei record. Un vocabolario di valori rappresenta una lista chiusa di valori autorizzati per un dato elemento;

3. Metadata element sets ovvero insiemi di termini che definiscono entità, relazioni e attributi come i termini DCMI Metadata e gli elementi di RDA: Resource Description and Access, Simple Knowledge Organization System (SKOS), e il vocabolario Friend of a Friend (FOAF). Un metadata element set definisce classi di entità e attributi (o elementi) di un'entità. Generalmente un metadata element set non definisce entità bibliografiche, ma fornisce elementi che possono essere usati per descrivere queste entità. Ad esempio Dublin Core è uno schema che definisce elementi come Creatore e Data, ma non definisce i record bibliografici che usano questi elementi. FRBR definisce entità come Opera e Manifestazione, Espressione etc egli elementi che le collegano e le descrivono. 
Di seguito vengono riassunti i più significativi contributi delle biblioteche ai linked open data $^{148}$.

1. Dataset di risorse bibliografiche

In campo bibliotecario i principali dataset esistenti sono quelli che mettono a disposizione i dati di cataloghi e bibliografie nazionali che sono stati pubblicati in RDF.

\section{British National Bibliography}

La pionieristica impresa della British Library è stata la pubblicazione della British National Bibliography (BNB), <http://bnb.bl.uk/> da parte del British Library Metadata Services $^{149}$. Il dataset è collegato ai dataset VIAF, LCSH, Lexvo, GeoNames, MARC country e language, Dewey.info, RDF Book Mashup secondo le specifiche contenute nel modello dei dati (data model $)^{150}$. Lo scopo è di pubblicare le descrizioni di tutti i libri e le riviste ricevute dalla British Library a partire dal 1950. Oggi vengono rilasciati circa 2.6 milioni di descrizioni di libri (84,961,180 triple) pubblicati nel regno unito negli ultimi 60 anni. I dati bibliografici sono rilasciati con licenza CCO.

Data.bnf. fr

La maggior parte dei dati bibliografici dei cataloghi e delle biblioteche digitali non è accessibile nel web né ricercabile tramite i motori di ricerca. Ciò ha spinto la Bibliothèque nationale de France a pubblicare i suoi dati nel web, a renderli accessibili ai motori e riutilizzabili nel Web Semantico ${ }^{151}$.

La Bibliothèque nationale de France è entrata a far parte degli enti che forniscono Linked open data con il progetto Data.bnf. fr per la creazione di una piattaforma di ricerca che unisce dati dei cataloghi (MARC), degli archivi (EAD) e di oggetti digitali (DC) ${ }^{152}$.

\footnotetext{
${ }^{148}$ L'elenco completo dei soli dataset bibliografici si trova in nel registro CKAN in un'apposita pagina $<$ http://thedatahub.org/group/bibliographic>; una lista di vocabolari e schemi di metadati si trova in $<$ http://www.w3.org/2005/Incubator/lld/XGR-lld-vocabdataset-20111025/>.

$149<$ http://bnb.data.bl.uk/id/>.

$150<$ http://www.bl.uk/bibliographic/pdfs/bldatamodelbook.pdf> [version 1.3, March 2012]. Un esempio di descrizione in RDF è <http://thedatahub.org/dataset/bluk-bnb/resource/a814828a-0ea9-43aa-9628cd6b999e14cb>.

${ }^{151}$ Si veda: Romain Wenz, Linked open data for new library services: the example of data.bnf.fr, «JLIS.it», 4 (2013), n. 1, p. 403-416, <http://leo.cilea.it/index.php/jlis/article/view/5509/7919>.

${ }^{152}$ Una presentazione del progetto si trova in: <http://data.bnf.fr/docs/databnf-presentation-en.pdf>.
} 
Data.bnf. $\mathrm{fr}^{153}$ pubblica pagine web che contengono circa 2,5 milioni di dati espressi in forma di triple e raggruppa tutte informazioni provenienti dai diversi cataloghi e dalla biblioteca digitale Gallica.

La piattaforma di ricerca data.bnf.fr pubblica pagine web relative ad opere, autori e soggetti. La pagina relativa a un autore, ad esempio Dante Alighieri (<http://data.bnf.fr/11898585/dante_alighieri/>) mostra l'elenco delle sue opere e delle diverse edizioni con link ai cataloghi, alla biblioteca digitale, e ad altre tipologie di documenti. Sono presenti testi di cui è autore, commentatore e i collegamenti ai traduttori, commentatori delle sue opere o scrittori a lui associati, sono elencate le opere derivate, gli adattamenti e altre opere correlate.

Allo stesso modo, si possono scorrere o ricercare soggetti e la pagina di un soggetto (<http://data.bnf.fr/11979282/abus_de_pouvoir/>), oltre alle forme varianti e collegate, ha collegamenti agli autori che hanno trattato quel soggetto e l'indicazione delle opere estratte dai cataloghi.

Il data model per il dataset del progetto data.bnf.fr utilizza dati creati in vari formati come InterMarc per il catalogo, XML-EAD per gli inventari di archivi e Dublin Core per la biblioteca digitale. I dati vengono raggruppati automaticamente, modellati, arricchiti e pibblicati nella semantica di RDF. Il risultato è serializzato in tre differenti formati di sintassi RDF: RDF-XML, RDF-N3, and RDF-NT. Parte dei dati provengono da fonti esterne come vocabolari di valori; id.loc.gov per le lingue e le nazioni, dewey.info per i soggetti DCMI type per le tipologie documentarie e infine altri dataset esterni presenti in CKAN come Dbpedia e VIAF. La Bibliothèque nationale de France fornisce dunque un ricco dataset dove ogni risorsa è identificata da un URI e la visualizzazione dei dati in RDF.

Il software utilizzato è CubicWeb, una piattaforma open source per applicazioni rilasciata sotto licenza LGPL. Il data model è visibile all'indirizzo $<$ http://data.bnf.fr/semanticweb-en>.

Il progetto prevede l'implementazione di FRBR per la visualizzazione ${ }^{154}$ dei dati bibliografici con singole pagine per le opere, gli autori e i soggetti.

Le pagine web vengono rese disponibili ai motori di ricerca tramite l'utilizzo di Schema.org e riutilizzabili nei social network tramite Opengraph Protocol (OG). I dati sono forniti sotto una licenza aperta Licence Ouverte ${ }^{155}$.

\footnotetext{
$153<$ http://data.bnf.fr>

${ }^{154} \mathrm{Si}$ veda per maggiori dettagli $<$ http://data.bnf.fr/semanticweb-en>.

${ }^{155}$ Licence Ouverte Open licence; il testo in: <http://data.bnf.fr/docs/Licence-Ouverte-Open-Licence.pdf>.
} 
La più recente iniziativa è quella della biblioteca nazionale spagnola ${ }^{156}$ che espone come linked data circa 4 milioni di record di autorità relativi a persone, enti, conferenze, titoli uniformi e soggetti e 2.4 milioni di record bibliografici relativi a volumi antichi, moderni, risorse elettroniche, manoscritti, periodici, mappe, stampe, fotografie, registrazioni video e sonore in un dataset formato da più di 50 milioni di triple collegando il suo dataset ${ }^{157}$ con altre risorse come VIAF, SUDOC, GND German national library, Libris, Dbpedia) ${ }^{158}$. Tutti i dati sono forniti con licenza CC0.

Deutsche Nationalbibliografie (DNB) e HBZ Union Catalog

Il Linked Data Service della biblioteca nazionale tedesca Deutsche Nationalbibliothek ha rilasciato dati bibliografici a partire dal $2012^{159}$. Sono disponibili i dati bibliografici delle collezioni librarie della DNB e i seriali del catalogo unico dei periodici $(\mathrm{ZDB})^{160}$. La licenza scelta per il rilascio è $\mathrm{CC} 0$.

Il contenuto del catalogo in forma di raw open data viene offerto in forma di linked data in <http://thedatahub.org/dataset/lobid-resources> aggiornato giornalmente con i metadati bibliografici del HBZ Union Catalog per quelle biblioteche che hanno acconsentito al rilascio dei loro dati bibliografici ${ }^{161}$. Più di 10 milioni di record bibliografici vengono così forniti sotto una licenza CCO.

\footnotetext{
$156<$ http://datos.bne.es $>$.

157 Il dataset è stato creato dall' Ontology Engineering Group, Facultad de Informática, Universidad Politécnica de Madrid ed è manetnuto da Daniel Vila-Suero and Boris Villazón-Terrazas.

${ }^{158}$ Per la modellazione dei dati si veda: <http://www.bne.es/es/Catalogos/DatosEnlazados/FuentesDatos/>. Per la migrazione dei dati e il loro collegamento è stato utilizzato Marimba un apposito strumento per convertire e collegare dati bibliografici.

${ }^{159}$ Il primo servizio per la pubblicazione di Linked open data (LOD) della Biblioteca nazionale tedesca è stato la pubblicazione, nel 2010, dell'authority file dei nomi personali e dei soggetti. Attualmente i dati resi disponibili riguardano: record relativi alla collezione principale (tranne record per la musica a stampa), il catalogo unico dei periodici tedeschi (Zeitschriftendatenbank, ZDB) e l'authority file tedesco (Gemeinsame Normdatei, GND). Si veda: Gabriele Meßmer, Linking library metadata to the Web: the German experiences, «JLIS.it», 4 (2013), n. 1, p. 391-402, <http://leo.cilea.it/index.php/jlis/article/view/5507/7878>. $160<\mathrm{http}: / /$ www.dnb.de/EN/datendienste/linkedData $>$.

$161 \mathrm{Al}$ momento le biblioteche interessate sono USB Cologne; Cologne University of Applied Sciences Library;Library Centre of Rhineland-Palatinate (catalog of RLB Koblenz); Rhineland-Palatinate (catalog of PLB Speyer); Library Centre of Rhineland-Palatinate (catalog of BB Zweibrücken); Central Library of Sports Science; RWTH Aachen University Library catalog; German National Library of Medicine, Specialist; Library of Nutritional, the Environmental, and Agricultural Sciences; German National Library of Medicine (ZB MED); Dortmund University Library; Duisburg-Essen University Library,Campus Essen; Duisburg-Essen University Library, Campus Duisburg; Bochum Ruhr University Library and all Institutions; Rhein-Waal University Library; Rhein-Waal University Library section Kamp-Lintfort; Dortmund College Library; FHB Dortmund; Hochschule Ostwestfalen-Lippe; Fachhochschule Aachen; Fachhochschule Gelsenkirchen; FHB Bielefeld; Hochschule Niederrhein; Fachhochschule Südwestfalen, Fachbibliothek
} 


\section{LIBRIS}

Il catalogo collettivo delle biblioteche svedesi ${ }^{162}$ è il primo catalogo di biblioteca ad essere fornito interamente nella forma di linked data. Il catalogo comprende la Swedish National Bibliography e gli authority data. Nel settembre 2011 è stata rilasciata la Swedish National Bibliography e gli authority data di persone, soggetti ed enti con una licenza CC0. I dati contengono links a Wikipedia, DBPedia, LC Authorities (per i nomi e i soggetti) e VIAF. L'obiettivo è quello di rilasciare con una licenza aperta tutti i dati contenuti nel catalogo.

OCLC WordlCat

Il 20 giugno 2012 OCLC ha annunciato il rilascio dei dati di WorldCat, il catalogo più grande del mondo, aderendo a Schema.org, il progetto siglato nel 2011 tra i principali motori di ricerca ${ }^{163}$.

WorldCat.org offre così il più grande data set di dati bibliografici come linked data sul web e attraverso l'aggiunta della marcatura prevista da Schema.org tutti i libri, le riviste ed altre risorse bibliografiche saranno rese disponibili ai motori di ricerca del web come Bing e Google che possono riusare questi metadati per costruire i propri indici ed altre applicazioni.

\section{CERN}

La biblioteca del CERN ha rilasciato i dati del catalogo della sua biblioteca per il download $^{164}$. Attualmente il file ${ }^{165}$ è aggiornato all'export del 6.12.2009 ed è fornito in un file MARCXML compresso ma si prevede il rilascio in RDF. e i dati sono disponibili sotto licenze CC0 and PDDL versione $1.0^{166}$.

\footnotetext{
Hagen, Zentralbibliothek; Universitäts- und Landesbibliothek Münster (160 biblioteche)Fachhochschule Münster, Hochschulbibliothek; Hochschulbibliothek der Fachhochschule Düsseldorf; Universitäts- und Landesbibliothek Bonn (fonte: Open Data from the hbz Union catalog, http://thedatahub.org/dataset/hbz_unioncatalog). $162<$ http://libris.kb.se>

163 Per l'annuncio si veda: OCLC, OCLC adds Linked Data to WorldCat.org, 20 Giugno 2012. <http://www.oclc.org/uk/en/news/releases/2012/201238.htm>.

$164<$ http://library.web.cern.ch/library/Library/bookdata.html>. Responsabile del progetto è Patrick Danowski

${ }^{165}<$ http://library.web.cern.ch/library/files/books_export.xml.zip>. L'annuncio in: <http://gs-servicebookdata.web.cern.ch/gs-service-bookdata/announcement.html>.

${ }^{166}$ Public Domain Dedication and License version 1.0, <http://www.opendatacommons.org/licenses/pddl/>.
} 
Si segnalano infine altre iniziative nate allo scopo di riunire dati provenienti da diverse istituzioni e progetti relativi al patrimonio culturale:

Open Library $^{167}$ è l'iniziativa di Internet Archive rivolta alla costruzione di una pagina web per ogni libro pubblicato contiene dati estratti da milioni di record bibliografici, un'infrastruttura dinamica che consente di recuperare informazione da migliaia di fonti, un'interfaccia wiki, un supporto multilingua e la possibilità per tutti di contribuire immettendo i dati dei libri. Attualmente indicizza 30 milioni di record (di cui 13.4 milioni sono disponibili attraverso il sito web). L'interfaccia di ricerca ha adottato la navigazione a faccette e consente di visualizzare il full text di oltre 230,000 libri.

Project Gutenberg ${ }^{168}$ pubblica i metadati relativi alle opere di pubblico dominio presenti nell'omonimo progetto.

Europeana $^{169}$ è la biblioteca digitale europea che riunisce contenuti digitalizzati dalle istituzioni culturali dei 27 Paesi dell'Unione Europea. Europeana è promotrice dell'iniziativa Linked Open Data per incentivare lo sviluppo, in ambito culturale, di applicazioni innovative per la produzione di contenuti digitali e la creazione di nuovi servizi sul web. Dal $1^{\circ}$ luglio 2012 Europeana espone i metadati sotto forma di Linked open data dati aperti relativi a 2,4 milioni di oggetti digitali del patrimonio culturale europeo. L'accordo sullo scambio dei dati (Data Exchange Agreement) sottoscritto dai partner culturali europei per inserire i propri metadati in Europeana è basato sulla licenza aperta CC0 per consentire il libero riutilizzo dei dati.

\section{Vocabolari e ontologie}

Vocabolari della Library of Congress

La Library of Congress ha pubblicato i suoi vocabolari sotto forma di linked data creando un Linked Data Service ${ }^{170}$, ovvero una piattaforma nella quale sono ospitati e resi disponibili i principali standard e vocabolari mantenuti dall'istituzione Tra questi i vocabolari controllati Library of Congress Subject headings (LCSH), LC Children's Subject Headings, la Library of Congress Classification (LCC), e le liste di autorità LC Name Authority File, thesauri e liste di termini LC Genre/Form Terms; Thesaurus for

\footnotetext{
$167<$ http://openlibrary.org/>.

$168<$ http://www4.wiwiss.fu-berlin.de/gutendata/ $>$.

$169<$ http://www.europeana.eu/portal/>.

${ }^{170}<$ http://id.loc.gov/>.
} 
Graphic Materials, e Vocabolari per la preservazione (Preservation Events, Preservation Level Role, Cryptographic Hash Functions) più altre liste di termini controllati e di identificatori $^{171}$.

Tra i vocabolari, i più noti e utilizzati sono senz'altro Library of Congress subject headings (LCSH) e Library of Congress Classification (LCC), utilizzati per fornire alle descrizioni bibliografiche gli accessi semantici per i soggetti e le classi: LCSH è il soggettario mantenuto dalla Library of Congress sin dal 1898 per l'indicizzazione dei materiali posseduti alla Library of Congress. Il soggettario, utilizzato pressoché da tutte le biblioteche dell'area statunitense ha raggiunto una diffusione universale grazie alla catalogazione derivata e partecipata ed è usato in diverse aree geografiche nella traduzione locale.

LCC è lo schema di classificazione della Library of Congress che ha pubblicato una selezione di classi come linked data nel servizio Id.loc.gov. La classificazione è stata pubblicata servendosi di SKOS. Sono stati estratti i primi tre livelli della classificazione: ogni numero di classe è un concetto $\operatorname{SKOS}^{172}$.

\section{RAMEAU}

È l'esposizione in SKOS del soggettario RAMEAU, mantenuto dalla Bibliothèque nationale de France nell' ambito del progetto data.bnf.fr. È allineato al vocabolario LCSH tramite link e mappatura SKOS. Pubblicato in SKOS/RDF nel progetto STITCH è attivo dal 2012; tutti gli URI dei concetti RAMEAU reindirizzano alle descrizioni di data.bnf.fr.

\section{Dewey.info}

Dewey.info è uno spazio sperimentale per i linked data relativi alla Classificazione Decimale Dewey (DDC) che si propone come piattaforma sperimentale per pubblicare dati Dewey sul web.

Il servizio, fornito da $\mathrm{OCLC}^{173}$ ha avuto come principali obiettivi pratici la creazione di URI dereferenziabili per ogni classe, la codifica della classificazione in RDF/SKOS e la

\footnotetext{
${ }^{171}$ MARC Relators, MARC Countries; MARC Geographic Areas; MARC Languages; ISO639-1 Languages; ISO639-2 Languages; ISO639-5 Languages; Extended Date/Time Format.

${ }^{172} \mathrm{Si}$ veda: Kevin Ford, LC Classification as linked data, «JLIS.it», 4 (2013), n. 1, p. 161-176, $<$ http://leo.cilea.it/index.php/jlis/article/view/5467/7899>.

${ }^{173}$ Il resoconto delle operazioni per la conversione del sistema di classificazione Dewey in Linked data è stato fornito dall'intervento di Joan S. Mitchell e Michael Panzer al recente convegno di Firenze Global
} 
messa a disposizione dei dati secondo una licenza che consentisse il libero riutilizzo dei dati così prodotti nel Web Semantico.

Particolare attenzione è stata rivolta alla creazione degli URI: essi non solo devono consentire il recupero delle descrizioni, ma consentire attraverso l'URI di riconoscere e identificare lo schema, parti di esso, l'edizione utilizzata, la lingua e tener conto dell'evoluzione dello schema ${ }^{174}$.

Dewey.info è inoltre progettato per contenere le rappresentazioni dello schema utilizzabili dalle macchine e dagli esseri umani nella comunità vasta del Web Semantico. Per questo sono stati scelti in output tre diverse serializzazioni di RDF: il classico RDF/XML, Turtle e JSON. Dapprima sono stati pubblicati i sommari (primi tre livelli della CDD) in undici lingue, successivamente sono stati aggiunti numeri CDD dell'edizione ridotta 14 in inglese, italiano e vietnamita. È prevista l'integrazione con i numeri derivati dall'ultima edizione integrale CDD23. La licenza scelta è Creative Commons BY-NC-ND.

\section{VIAF}

VIAF (Virtual International Autority File) ${ }^{175}$ è un servizio implementato e mantenuto da OCLC - in cooperazione con 20 biblioteche nazionali che raggruppa virtualmente gli autority files dei nomi ricorrenti nei cataloghi in un solo servizio di authority. Scopo del progetto è l'abbattimento dei costi e delle barriere linguistiche e la messa a disposizione sul web dell'authority file. Dopo un breve periodo di incertezza, i dati sono rilasciati sotto la licenza Open Data Commons Attribution license ${ }^{176}$.

\footnotetext{
interoperability and linked data in libraries, Firenze, 18-19 giugno, 2012, cui si rimanda per le problematiche incontrate dal gruppo che ne ha curato la conversione. Cfr Joan S. Mitchell - Michael Panzer, Dewey linked data: Making connections with old friends and new acquaintances, «JLIS.it», 4 (2013), n. 1, p. 177-200, <http://leo.cilea.it/index.php/jlis/article/view/5467>.

174 Al momento della creazione degli URI la situazione riguardante lo sviluppo e la diffusione della classificazione risultava piuttosto complessa: la DDC22, pubblicata originariamente nel 2003, risultava tradotta in diverse lingue quali tedesco, francese, italiano. Inoltre erano presenti le versioni ridotte (edizione 14 ridotta) in francese, italiano, vietnamita, ebraico e spagnolo. I sommari basati sull'edizione 22 erano pubblicati in inglese e in altre dieci lingue; infine erano state pubblicate altre opere derivate delle schema riguardanti la classe Religione e altre due traduzioni della DDC 22 in francese e in spagnolo utilizzate per l'ordinamento delle rispettive bibliografie nazionali. La coesistenza di queste diverse traduzioni delle edizioni completa e ridotta hanno posto le più importanti criticità e la necessità di una programmazione accurata del processo di creazione degli URI.

$175<$ http://viaf.org/viaf/data/>.

${ }^{176} \mathrm{Nel}$ sito del progetto sono esplicitate le modalità della licenza.
} 


\section{AGROVOC}

AGROVOC Linked Open Data (LOD) ${ }^{177}$ è un progetto che ha lo scopo di convertire il noto thesauro mantenuto da Food and Agriculture Organization of the UN (FAO). Il thesauro contiene più di 40.000 concetti in 22 lingue sugli argomenti relativi al cibo, alla nutrizione, all'agricoltura e ad altre discipline affini. La knowledge base AGROVOC descrive attualmente oltre 4 milioni di risorse e circa 21.000 sono i link in uscita verso altri vocabolari tra i quali LCSH, RAMEAU, Dbpedia, DC, Geopolitical Ontology e 3.000 i collegamenti in entrata ${ }^{178}$.

Rådata nå!

È la rappresentazione in RDF dell'authority file di BIBSYS, il catalogo delle biblioteche norvegesi creato con il sostegno di Norwegian Archive, Library and Museum Authority (ABM-utvikling) da BIBSYS e NTNU University Library ed è pubblicato con licenza Open Data Commons Public Domain Dedication and Licence (PDDL) ${ }^{179}$.

3. Schemi di metadati

Gli schemi di metadati sono essenziali per descrivere insiemi di elementi come entità e attributi di entità (autore, creatore, opera, data, titolo, etc.): tra i principali set di metadati sono da menzionare RDA, FRBR e Dublin Core ${ }^{180}$.

RDA

RDA Resource description and Access è uno standard per la descrizione dei contenuti che come molti altri standard (REICAT, RAK etc.) risponde alle esigenze di

\footnotetext{
177 <http://aims.fao.org/standards/agrovoc/about>. Il thesaurus è in hosting presso MIMOS, partner scientifico di FAO.

$178<$ http://aims.fao.org/news/agrovoc-linked-10-new-datasets>.

$179<$ http://brinxmat.posterous.com/bibsys-personal-name-linked-open-data-publish>.

${ }^{180}$ A questi si aggiunge anche l'adattamento delle ISBD al Web Semantico con la finalità di «migliorare la portabilità dei dati bibliografici nell'ambiente del Web Semantico e l'interoperabilità dello standard ISBD con altri standard di contenuto» per opera dell'ISBD Review Group e International Federation of Library Associations and Institutions. Cataloguing Section. Cfr.: Elena Escolano Rodrìguez, ISBD adaptation to semantic web of bibliographic data in linked data, «JLIS.it», 4 (2013), n. 1, p. 119-138, $<$ http://leo.cilea.it/index.php/jlis/article/view/5484/7895>.
} 
descrizione delle risorse bibliografiche ${ }^{181}$. Oltre ad essere il primo standard per i contenuti creato da quando i cataloghi sono passati alla forma elettronica, è anche uno standard aperto ed è esplicitamente strutturato attorno al modello FRBR. Di recente il comitato di sviluppo di RDA ha deciso di rendere disponibili sia i vocabolari sia gli element set in RDF per il libero riutilizzo nel web pubblicandoli sull'Open Metadata Registry ${ }^{182}$. Il percorso di creazione dei vocabolari è stato riassunto da Alan Daskin al recente convegno fiorentino ${ }^{183}$ e tutti i vocabolari sono disponibili all'url <http://rdvocab.info/>.

\section{Dublin Core}

Si tratta del più noto schema di metadati ${ }^{184}$ sviluppato in ambito OCLC dalla Dublin Core metadata community, che unisce produttori autori e detentori dei diritti sulle risorse pubblicate in rete per fornire elementi di descrizione e accesso alle risorse digitali. Il set minimo (core) è costituito di 15 elementi. Ciascun elemento è definito usando un set di 10 attributi ricavati dalla norma ISO 11179.

FRBR e il lavoro di FRBR Review group

Il principale lavoro dell'FRBR review Group è recentemente rivolto alle iniziative nel campo dei linked data, volte alla pubblicazione dei modelli e degli standard in una forma autorevole per il riutilizzo dei dati ${ }^{185}$. Le recenti iniziative hanno riguardato la scelta e la pubblicazione dei namespaces per il modello nell'Open Metadata Registry e la scelta di un dominio permanente e stabile per i namespaces che è stato individuato in iflastandards.info, e il modello per la costruzione dei namespaces ${ }^{186}$ che rappresenta la base per la creazione di tutti i namespaces, tra i quali ad esempio quello per la famiglia

\footnotetext{
${ }^{181}$ Gli standard per i contenuti stabiliscono come descrivere una risorsa, quanto descrivere, come trattare le responsabilità relative alla risorsa, da quali fonti trarre le informazioni, etc.

${ }^{182}<$ http://metadataregistry.org>. I set di elementi e i vocabolari sono visibili all'URL: <http://rdvocab.info/>

${ }^{183}$ Alan Danskin, Linked ad open data: RDA and bibliographic control cit.

$184<\mathrm{http}$ ://dublincore.org/documents/dcmi-terms/>.

${ }^{185}$ FRBR Review group è il gruppo costituito in seno alla Sezione di Catalogazione dell'IFLA che si occupa di assicurare il mantenimento e lo sviluppo del modello FRBR pubblicato per la prima volta nel 1998 e della famiglia dei modelli FRBR sviluppati inseguito quali FRAD e FRSAD, pubblicati rispettivamente nel 2009 e nel 2010 e del loro consolidamento. Sul recente lavoro del gruppo si veda la relazione di Pat Riva: FRBR Review Group initiatives and the world of linked data, «JLIS.it», 4 (2013), n. 1, p. 105-118, $<$ http://leo.cilea.it/index.php/jlis/article/view/5482/7893>.

${ }^{186}$ La creazione dei namespaces è stata oggetto di un progetto apposito affidato a Gordon Dunsire a partire dal 2007, <http://www.ifla.org/about-the-frbr-review-group\#projects>.
} 
FRBR $^{187}$. Nel maggio 2011 è seguita la pubblicazione del dataset di elementi FRBR, e nel febbraio 2012 sono stati publicati i set di elementi di FRAD e FRSAD.

Lo sviluppo dei namespaces ha reso evidenti alcune problematiche che riguardano l'armonizzazione e l'attività di consolidamento dei modelli facenti parte della famiglia FRBR, che sono stati sviluppati a partire dal 1998, in tempi diversi e in un periodo di forte cambiamento che ha caratterizzato principi, norme catalografiche e ha visto l'affacciarsi di nuove tecnologie del Web Semantico.

\subsection{Web 3.0 per la ricerca semantica: arricchimento, impoverimento o evoluzione?}

La ricerca semantica ha sempre rappresentato un punto critico nella progettazione dei cataloghi elettronici. Quando si approccia al catalogo l'utente che sceglie questa modalità di interrogazione procede spesso per tentativi per vari motivi: nelle prime fasi del processo di ricerca, come vedremo nel prossimo capitolo, l'utente è maggiormente coinvolto in attività cognitive che consistono in un processo di creazione di senso dell'ambiente circostante, in questo caso dell'universo bibliografico con il quale l'utente entra in contatto attraverso il catalogo, degli strumenti di recupero dell'informazione, della terminologia utilizzata e non ultimo del suo stesso bisogno informativo.

Per questo è importante offrire all'utente oltre a modalità di ricerca attive anche modalità di browsing, principalmente per soggetti e per classi. La maggior parte degli OPAC tradizionali e anche di quelli che si dichiarano di nuova generazione non implementa visualizzazioni gerarchiche o interfacce visuali per soggetti e classi, con una perdita di efficacia nel recupero delle ricerche per soggetto. Stringhe di soggetto e numeri di classe vengono spesso mostrati come elementi 'inerti' e non attivabili, e nel caso delle classi, sovente privi degli equivalenti verbali.

Ci soffermeremo dunque dapprima sulle tematiche di compatibilità della modellazione di FRSAD con i modelli offerti dal Web Semantico per esprimere le ontologie e sulle nuove modalità per esprimere i soggetti e le classi in forma di linked data; successivamente analizzeremo i vantaggi che ne possono derivare per l'utente nella ricerca per soggetto.

\footnotetext{
$187<$ iflastandards.info/ns/fr $>$.
} 


\subsubsection{La modellazione di FRSAD, SKOS e OWL}

Il data model fornito da RDF e illustrato nei paragrafi precedenti è molto simile a quello delineato da FRSAD, lo schema dei modelli FRBR per esprimere i soggetti.

Functional Requirements for Subject Authority Data(FRSAD) ${ }^{188}$, lo schema concettuale di IFLA per modellare la circalità (aboutness) delle opere, introduce un modello fondato sulle entità thema e nomen: thema è il concetto, ovvero il soggetto di un'opera e nel modello ogni entità stabilita dai modelli FRBR e FRAD può essere il thema di un'opera. Nomen si riferisce ad ogni segno o sequenza di segni (caratteri alfanumerici, simboli o suoni) utilizzati per riferirsi al Thema.

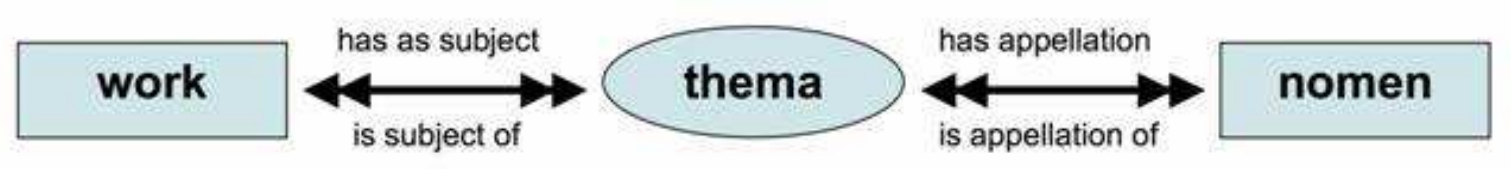

Fig. 4.12 - Il modello FRSAD per modellare la circalità

Oltre alla relazione con l'entità opera e con le altre entità dei modelli della 'famiglia FRBR', di cui thema è una sorta di superclasse, FRSAD introduce varie relazioni tra le due entità definite nel modello: relazioni thema - thema gerarchiche o associative e relazioni nomen - nomen come quelle partitive (nomi composti) o di equivalenza (sostituisce/è sostituito da; ha per forma variante/è la forma variante di; ha per derivazione/è derivato da; ha per acronimo/è l'acronimo di; ha per abbreviazione/è l'abbreviazione di; ha per traslitterazione / è la traslitterazione di).

\footnotetext{
188 IFLA. Functional Requirements for Subject Authority Data (FRSAD) Final Report Approved by the Standing Committee of the Classification and Indexing Section, June 2010. $<$ http://www.ifla.org/files/classification-and-indexing/functional-requirements-for-subject-authoritydata/frsad-final-report.pdf>In particolare si veda FRSAD Final Report: Appendix C: FRSAD Model and Other Model, p. 48-50.
} 


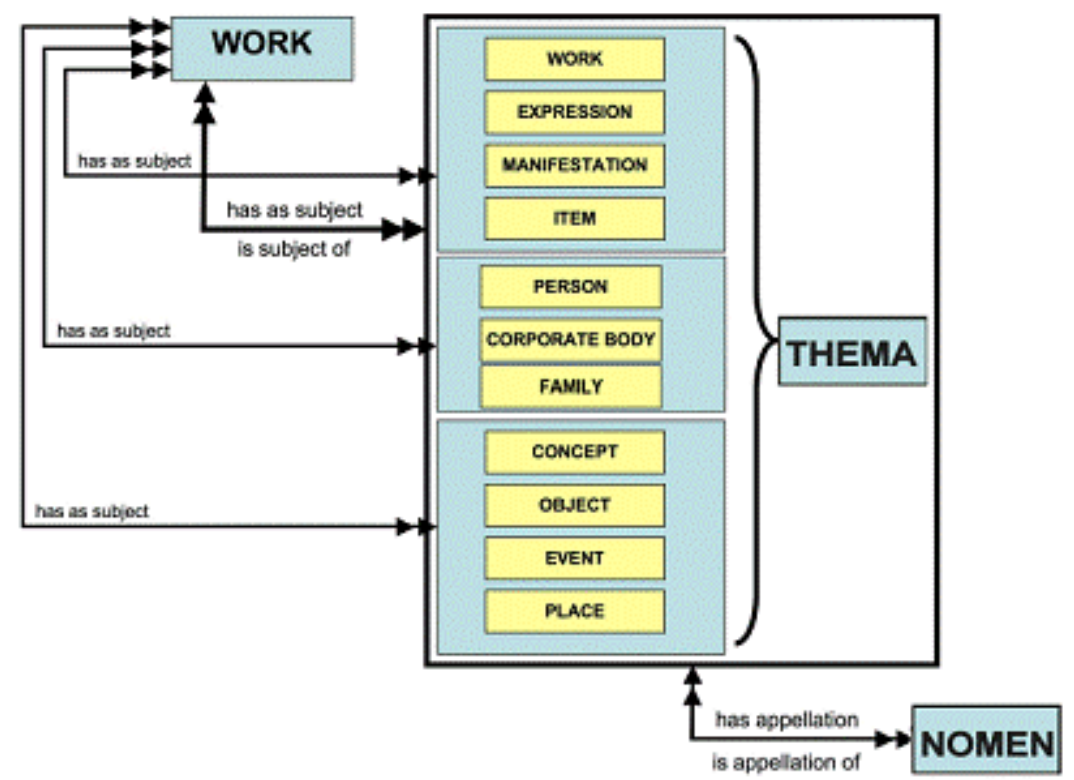

Fig. 4.13- L'entità thema nel modello FRSAD è una superclasse delle entità definite negli altri modelli dell' IFLA

Il modello concettuale thema - nomen stabilito da FRSAD trova adeguate corrispondenze in schemi di codifica per il Web Semantico come Simple Knowledge Organization System (SKOS) and Web Ontology Language (OWL), che forniscono modelli e per esprimere le strutture di sistemi di oganizzazione delle conoscenze ${ }^{189}$. In particolare, $\operatorname{SKOS}^{190}$ definisce classi e proprietà per esprimere la struttura di base e il contenuto di schemi concettuali come thesauri, schemi di classificazione, tassonomie, soggetti, folksonomies e altri vocabolari controllati; applicando il data model fornito da RDF consente la composizione dei concetti, la loro pubblicazione nel web e la possibilità di integrazione dello schema con altri schemi concettuali presenti nel Web Semantico. Attraverso SKOS i concetti possono essere organizzati in gerarchie e reti di associazioni, aggregati in schemi concettuali, ordinati in collezioni ordinate e mappati ai concetti presenti in altri altri schemi.

Il modello SKOS è un modello basato sul 'concetto' e definisce la separazione tra concetti e etichette (ovvero i termini per esprimerli) già espressa in FRSAD: tutto il vocabolario è modellato sui concetti che a loro volta sono rappresentati da etichette, dunque essi possono essere confrontati con le entità definite in FRAD ovvero thema, nomen e i loro attributi.

${ }^{189}$ Sulla tematica si veda: Maja Žumer - Marcia Lei Zeng, Modelling Knowledge Organization Systems and Structures: A discussion in the context of conceptual and data models. Slides presentate a: ISKO UK Biennial Conference, London, 4-5 luglio $2011<\mathrm{http}: / /$ www.iskouk.org/conf2011/ppt/zumer.pdf>.

$190<$ http://www.w3.org/TR/2009/REC-skos-reference-20090818/>. 
Nella costruzione di una tripla un concetto viene associato - tramite il predicato rdf:type alla classe specifica skos:Concept ed identificato in modo univoco da un URI. Con l'etichetta skos:prefLabel, che può essere espressa in diverse lingue ${ }^{191}$, si identificano i termini che all'interno dello schema concettuale sono accettati e vengono usati come descrittori nel sistema d'indicizzazione. Lo standard consente di descrivere anche un gruppo di termini che sono di corredo al concetto, perché legati a questo stesso, ma che non hanno un'identità semantica e quindi non verranno identificati con un URI ma solo con delle etichette: con skos:altLabel vengono determinati i sinonimi o i quasi sinonimi, e con skos:hiddenLabel le varianti ortografiche, le abbreviazioni e gli acronimi ${ }^{192}$, secondo una modellazione che nella versione core di SKOS non consente di esprimere tutte le relazioni nomen-nomen previste da FRSAD in quanto i termini vengono trattati come attributi e non come entità, ma che può essere attivata tramite l'uso di un'apposita estensione di SKOS ${ }^{193}$.

Il modello definito da FRSAD trova mappature appropriate nelle classi, negli attributi e nelle relazioni semantiche stabilite dallo standard del W3C SKOS: frsad:thema equivale a skos:Concept; frsad:nomen equivale a skos:label (oppure skosxl:Label) ${ }^{194}$ e altre proprietà come skos:broader, skos:narrower skos:related consentono di esprimere le relazioni gerarchiche e associative tra i concetti tipiche di un'organizzazione thesaurale.

Web Ontology Language (OWL) è un linguaggio per esprimere ontologie di concetti nel Web Semantico con un significato formalmente definito. Esso consente di esprimere le

$191<$ MyResource>

skos:prefLabel "animals"@en ;

skos:altLabel "fauna"@en ;

skos:hiddenLabel "aminals"@en ;

skos:prefLabel "animaux"@fr ;

skos:altLabel "faune"@fr.

${ }^{192}$ Le hidden labels possono essere usate per reindirizzare per rimediare agli errori di battitura in fase di interrogazione e contenere forme errate dei termini. Se la query errata corrisponde con un hidden label, l'utente sarà in grado di trovare il concetto rilevante.

${ }^{193}$ Alcune relazioni tra etichette possono essere risolte solo non ricorrendo ad un'estensione di SKOS, SKOS-XL. Vi possono essere ad esempio problemi qualora sia necessario collegare tra loro le etichette per particolari fini come testimoniare le relazioni di variante storica (generalmente espresse con HSF, Historical see for) che collegano termini preferiti con termini preferiti in passato e non più accettati oppure relazioni di scomposizione che creano (espressa con USE+/UF+): creano un collegamento reciproco tra termini composti e i singoli termini derivabili dalla scomposizione. Su queste e altre problematiche affrontate nella conversione in RDF del thesaurus del nuovo Soggettario si veda: Giovanni Bergamin - Anna Lucarelli, The Nuovo soggettario as a service for the linked data world, «JLIS.it», 4 (2013), n. 1, p. 214-226, $<$ http://leo.cilea.it/index.php/jlis/article/view/5474/7903>.

194 SKOS-XL è un'estensione di SKOS che fornisce un suppporto per descrivere entità lessicali (termini) attribuiti ai concetti. Lo scopo è 'reificare' le etichette di skos:Concepts, trattandole come risorse RDF effettive. Ciò consente la possibilità per esse di essere ulteriormente annotate utilizzando ad esempio una proprietà "È una traduzione di". Lo schema è visibile in : <xmlns:xl="http://www.w3.org/2008/05/skos-xl\#>. Si veda anche Alistair Miles - Sean Bechhofer, SKOS Simple Knowledge Organization System eXtension for Labels (SKOS-XL) Namespace Document - HTML variant 18th August 2009 recommendation edition, $<$ http://www.w3.org/TR/skos-reference/skos-xl.html>. 
relazioni tra vari concetti (thema) previsti da FRSAD in maniera più completa rispetto a SKOS in quanto consente di esprimere ulteriori relazioni tra le classi come SubClassOf, EquivalentClasses, DisjointClasses, e DisjointUnion.

I modelli SKOS e OWL consentono dunque di esprimere 'concetti', ovvero accessi per soggetti e per classi e le loro relazioni, ma anche di mappare concetti presenti in diversi schemi.

L'assegnazione di un valore univoco ai concetti facilita l'interoperabilità tra KOS differenti, cioè la possibilità di mappare entità semantiche facenti parte di schemi concettuali profondamente diversi come ad esempio un soggettario e una folksonomia. Per stabilire questi collegamenti lo standard SKOS definisce alcune particolari proprietà che stabiliscono differenti livelli di equivalenza: skos:closeMatch; skos:exactMatch; skos:broaderMatch e skos:narrowerMatch; skos:relatedMatch.

Di recente i più importanti e diffusi soggettari e schemi di classificazione sono stati tradotti nella forma di linked data, tra questi i vocabolari della Library of Congress (LCSH, LCC) e la Classificazione Decimale Dewey, RAMEAU e il Thesaurus del Nuovo Soggettario.

Come si vede nella figura seguente, che rappresenta un accesso per soggetto di LCSH, una stringa di soggetto (Italy--History--1492-1559--Fiction) viene identificata da un particolare URI, come istanza di skos:concept ed è formata da varie componenti che a loro volta sono presenti in altri schemi.

Il componente della stringa Italy è presente infatti nel dataset dei nomi Library of Congress Name Authority File, ha a sua volta un proprio URI (<http://id.loc.gov/authorities/names/n79021783>) è collegato alle forme varianti (nel caso

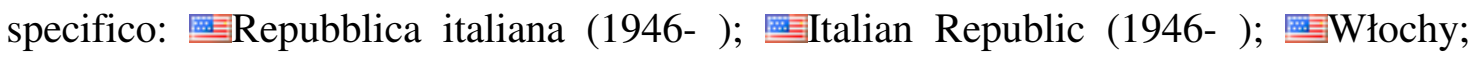

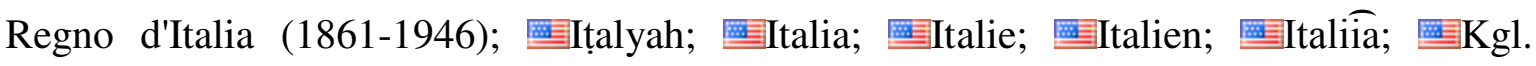

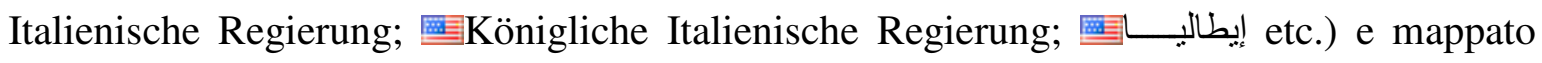
con altri schemi come VIAF (<http://viaf.org/viaf/152361066/\#skos:Concept >). 


\begin{tabular}{|c|c|}
\hline \multicolumn{2}{|c|}{$\begin{array}{l}\text { 潧 Italy--History--1492-1559--Fiction } \\
\text { URI(s) }\end{array}$} \\
\hline$\circ$ & http://id.loc.gov/authorities/subjects/sh2008115565 \\
\hline ○ & info:Ic/authorities/sh2008115565 \\
\hline o & http://id.loc.gov/authorities/sh2008115565\#concept \\
\hline \multicolumn{2}{|c|}{ - Instance Of } \\
\hline ○ & MADS/RDF ComplexSubject \\
\hline$\circ$ & MADS/RDF Authority \\
\hline$\circ$ & SKOS Concept $\square$ \\
\hline \multicolumn{2}{|c|}{ - Components } \\
\hline ○ & 澴Italy 6 \\
\hline$\circ$ & 潧History \\
\hline$\circ$ & 㲘1492-1559 \\
\hline ○ & 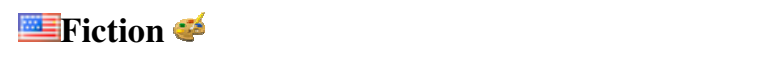 \\
\hline \multicolumn{2}{|c|}{ - Scheme Membership(s) } \\
\hline$\circ$ & Library of Congress Subject Headings \\
\hline \multicolumn{2}{|c|}{ - Collection Membership(s) } \\
\hline ○ & LCSH Collection - Authorized Headings \\
\hline$\circ$ & LCSH Collection - General Collection \\
\hline \multicolumn{2}{|l|}{ - Sources } \\
\hline$\circ$ & found: Work cat.: The family, 2001 \\
\hline \multicolumn{2}{|c|}{ - Editorial Notes } \\
\hline$\circ$ & [Record generated for validation purposes.] \\
\hline \multicolumn{2}{|c|}{ - Change Notes } \\
\hline$\circ$ & 2008-03-14: new \\
\hline \multicolumn{2}{|c|}{ - Alternate Formats } \\
\hline$\circ$ & RDF/XML (MADS and SKOS) \\
\hline$\circ$ & N-Triples (MADS and SKOS) \\
\hline$\circ$ & JSON (MADS/RDF and SKOS/RDF) \\
\hline$\circ$ & MADS - RDF/XML \\
\hline$\circ$ & MADS - N-Triples \\
\hline$\circ$ & MADS/RDF - JSON \\
\hline$\circ$ & SKOS - RDF/XML \\
\hline$\circ$ & SKOS - N-Triples \\
\hline$\circ$ & SKOS - JSON \\
\hline$\circ$ & MADS/XML \\
\hline$\circ$ & MARC/XML \\
\hline
\end{tabular}

Fig. 4.14- Esempio di accesso per soggetto $\mathrm{LCSH}^{195}$

$\overline{195}<$ http://id.loc.gov/authorities/subjects/sh2008115565.html>. 
La conversione di soggettari, thesauri, ontologie e altri sistemi di organizzazione delle conoscenze nei linguaggi del Web Semantico come SKOS e OWL richiede un'attenta valutazione e un lavoro concettuale particolarmente impegnativo per risolvere i problemi che si presentano.

SKOS e OWL consentono di esprimere nel linguaggio del Web Semantico oltre ai soggettari anche gli accessi per classe.

Generalmente per rendere fruibili i dati di uno schema di classificazione in forma di dati collegati bisogna codificare la semantica dello schema di classificazione in $\mathrm{RDF} / \mathrm{SKOS}$, fornire ad ogni classe un URI dereferenziabile e processabile, fornire le rappresentazioni in un doppio formato, per gli esseri umani e per le macchine; usare licenze che consentano il libero riutilizzo dei dati nella comunità del Web Semantico.

La Classificazione Decimale Dewey, recentemente pubblicata in RDF per la parte relativa ai sommari non ha presentato particolari problemi nella conversione in SKOS, in quanto ha riguardato per ora una la parte enumerativa dello schema, che viene riassunta nei sommari e nelle edizioni ridotte dello schema. Tuttavia insorgono alcuni problemi dovuti alla complessita insita nallo schema di classificazione. Sappiamo infatti che la CDD sebbene alle origini fosse uno schema enumerativo, nella sua evoluzione si è lentamente trasformato facendo spazio a modalità di costruzione del numero, ovvero alla sintesi notazionale.

Recenti studi hanno mostrato la difficoltà nella traduzione in SKOS dei numeri costruiti e di particolari intestazioni come le voci centrate che ad esempio consentono di mettere in relazione un insieme di numeri ad un particolare concetto ${ }^{196}$.

Particolarmente interessante si rivela la possibilità di scomposizione di notazioni complesse nei suoi numeri costitutivi, che consentirebbe di collegare parti del numero di classificazione, come quelle che rappresentano le suddivisioni geografiche con altri schemi, come ad esempio Geonames.

Ad esempio il collegamento di una faccetta geografica con l'equivalente entità fornita da Geonames consente di creare approcci alternativi alla ricerca e favorire la mappatura automatica di schemi totalmente diversi per tipologia delle entità descritte;

\footnotetext{
${ }^{196}$ Michael Panzer - Marcia Lei Zeng, Modeling classification systems in SKOS: some challenges and bestpractice recommendations, in Semantic interoperability of linked data : Proceedings of the international Conference in Dublin Core and Metadata Applications, Seoul, October 12-16, 2009. Edited by S. Oh. Sugimoto - S. A. Sutton, Seoul, Dublin Core Metadata Initiative and Nation Library of Korea, 2009, p. 3-14. Disponibile anche all'URL: <http://dcpapers.dublincore.org/index.php/pubs/article/view/974/944>.
} 
Geonames, infatti, oltre a fornire le coordinate geografiche nel Web Semantico fornisce URI autorevoli per moltissime entità geografiche ${ }^{197}$.

Il collegamento con queste URI di classificazioni o soggettari crea una mappatura tra due schemi diversi che forniscono in una notazione o in un soggetto il richiamo ad uno stesso URI creato da Geonames, accomunando due intestazione che si riferiscono, per esempio, ad una stessa città, tramite un nesso di 'circalità', che può costituire un mezzo inedito per espandere la ricerca.

Ad esempio se Dewey e LCSH collegano un elemento geografico della notazione o della stringa all'URI creato da Geonames i tre dataset verranno automaticamente collegati con la possibilità in fase di ricerca di passare da uno schema all'altro ampliando le possibilità di ricerca e fornendo nuove modalità di visualizzazione.

Dato che concetti geografici (luoghi fisici, che sono istanze di classi come Places) non possono essere collegati direttamente a soggetti della classe skos:concept, per collegare due diverse tipologie di entità si utilizza la proprietà dell'ontologia Foaf, foaf:focus, che consente di correlare la concettualizzazione di una cosa alla cosa stessa ${ }^{198}$. Attraverso questo espediente si compie un salto dimensionale dalla dimensione concettuale a quella fisica che può ampliare le modalità di ricerca per soggetto e per classe. Ad esempio, sarà possibile vedere il risultato di una query visualizzato anche su una mappa geografica, oppure a partire da determinati luoghi su una mappa è possibile visualizzare tutte le stringhe di soggetto e le classi che contengono il riferimenti a un determinato luogo e estendere la ricerca a quelle dei luoghi fisicamente vicini (es. due stati confinanti) o attraverso le nuove tecnologie di geolocalizzazione, più vicine all'utente che compie la ricerca in un dato momento.

Alcuni data model, come ad esempio quello utilizzato per la British national Bibliograhy introducono questa tipologia di collegamento tra entità di diversa tipologia (soggetti geografici, persone, organizzazioni) arricchendo così le possibilità di ricerca semantica $^{199}$.

Nel nostro paese particolarmente significativo in questo senso è l'avvio della collaborazione tra la Biblioteca Nazionale Centrale di Firenze e il Comune di Firenze

\footnotetext{
${ }^{197}$ Questa possibilità è tuttora al vaglio del gruppo che in OCLC si sta occupando dello sviluppo dello schema completo in Dewey.info. I lavori sono stati recentemente riassunti nel convegno di Firenze da Joan S. Mitchel e Michael Panzer (Dewey linked data: Making connections with old friends and new acquaintances, «JLIS.it», 4 (2013), n. 1, p. 177-200, <http://leo.cilea.it/index.php/jlis/article/view/5467>.

$198<$ http://xmlns.com/foaf/spec/>. La proprietà focus è stata inserita nello nell'ontologia di foaf per l'uso con il vocabolario SKOS al fine di indicare specifiche 'cose' (solitamente persone, luoghi, o oggetti) che sono menzionati in schemi differenti che utilizzano SKOS (ad esempio i thesauri).

$199<$ http://www.bl.uk/bibliographic/pdfs/bldatamodelbook.pdf>.
} 
$<\mathrm{http} / / /$ opendata.comune.fi.it/> per collegare attraverso le applicazioni dei linked data dati bibliografici relativi al nuovo Soggettario espresso in SKOS e dati amministrativi (ad esempio lo stradario di Firenze).

Attraverso il collegamento di dataset di diversa natura e attraverso la mappatura di concetti presenti in differenti schemi, la ricerca per soggetto può essere enormente potenziata, sia in fase di interrogazione, in quanto è ormai possibile utilizzare i dataset creati per aiutare l'utente a formulare interrogazioni, sia in fase di navigazione in quanto è possibile produrre visualizzazioni avanzate che sfruttano il collegamento tra schemi diversi (es. soggetti e classificazioni), utilizzati sia nel settore bibliotecario che in altri settori, per espandere ulteriormente la ricerca.

\subsection{FRBR e Web Semantico: un incontro possibile?}

Nel precedente capitolo ci siamo soffermati sul modello FRBR e sulla sua applicabilità alle differenti tipologie di risorse bibliografiche e infine sulle questioni che sorgono nell'implementazione di FRBR ai record bibliografici prodotti dalle biblioteche.

Gli OPAC attuali più avanzati nella forma di next generation catalogs e di strumenti di discovery non applicano ancora pienamente il modello FRBR nella presentazione dei dati bibliografici. Ciò è dovuto al fatto che essi utilizzano per l'espressione dei dati bibliografici formati proprietari e non compatibili con il web e che realizzano raggruppamenti in base ad algoritmi senza intervenire sulla struttura dei record.

La modellazione RDF può contribuire significativamente ad esprimere i dati bibliografici nelle entità previste dal modello FRBR e fornire visualizzazioni avanzate in grado di esplicitare il modello.

Data.bnf.fr rappresenta un buon esempio di piattaforma basata sui linked data, che sfrutta i dati bibliografici e le descrizioni provenienti da OPAC, archivi e biblioteche digitali come Gallica, per produrre visualizzazioni basate su FRBR e, dunque, nel nostro caso, anche un ottimo spunto per la visualizzazione dei dati bibliografici negli OPAC e un esempio interessante per la progettazione di nuove interfacce per i cataloghi ${ }^{200}$.

\footnotetext{
${ }^{200}$ Romain Wenz, Linked open data for new library services: the example of data.bnf.fr, «JLIS.it», 4 (2013), n. 1, p. 403-416, <http://leo.cilea.it/index.php/jlis/article/view/5509/7919>.
} 
Sebbene ci troviamo di fronte ad uno strumento di ricerca che consente di integrare dati provenienti da diverse fonti e che certamente non prevede le raffinate possibilità di ricerca generalmente offerte da un OPAC, la piattaforma data.bnf.fr si caratterizza per le capacità di trasformazione e arricchimento e per il collegamento di fonti interne ed esterne (vari dataset) per la pubblicazione di pagine HTML che possano essere comprese ed elaborate dai motori di ricerca attraverso varie serializzazioni, mentre tutti i dati grezzi sono disponibili per lo scaricamento in RDF.

Nel data model rappresentato nella figura vengono espresse e modellate le entità di FRBR che corrispondono alle principali modalità di ricerca che questo strumento offre, ovvero gli autori, i titoli, gli argomenti.

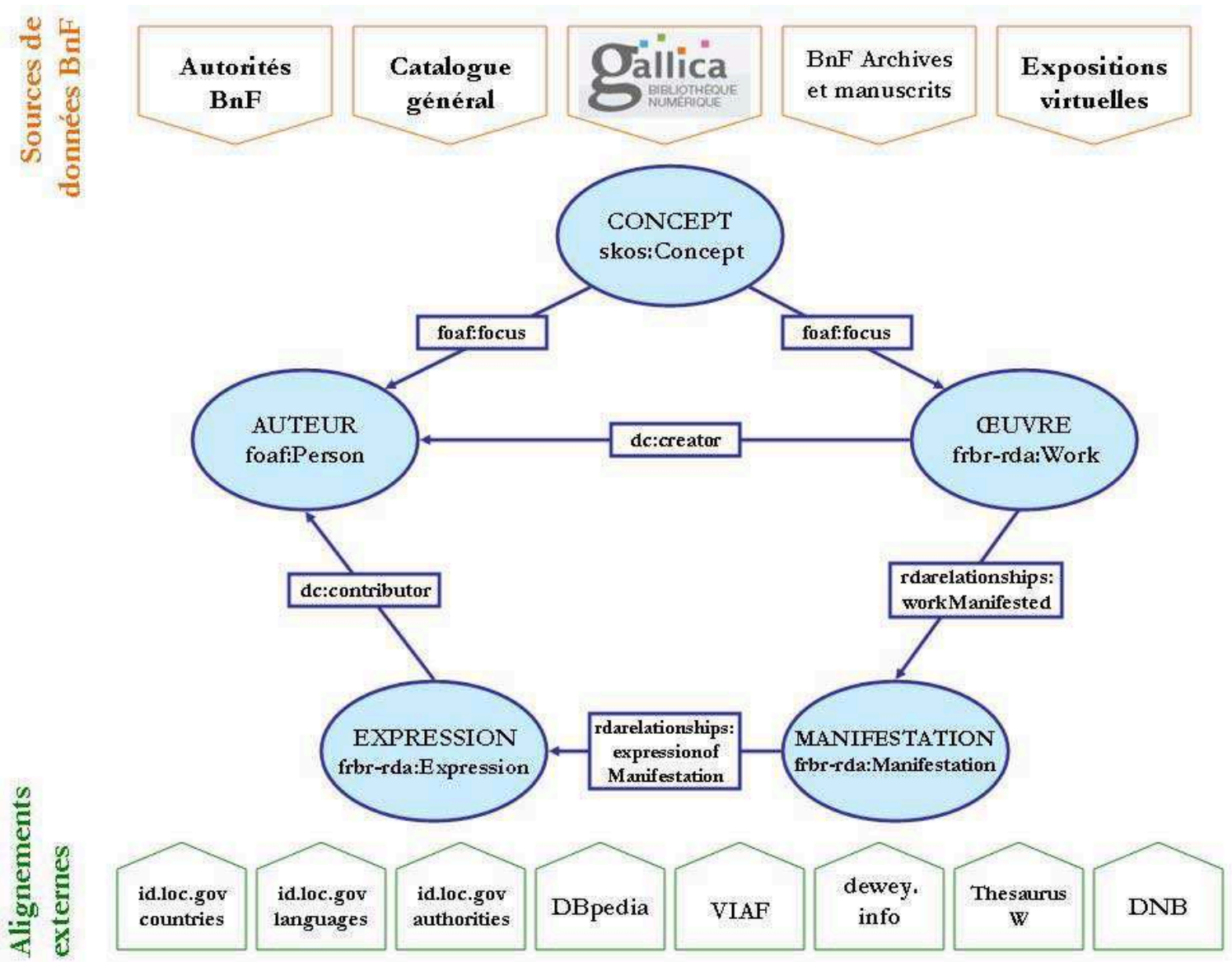

Fig. 4.15 - Il data model di data.bnf.fr. Lo schema mostra la modellazione di base delle entità FRBR e gli allineamenti semantici interni ed esterni ${ }^{201}$

La costruzione delle tre principali modalità di accesso ai dati bibliografici: l'opera, l'autore, l'argomento prevede la creazione di pagine distinte per le tre entità che possono essere oggetto del recupero da parte di un utente che vengono generate dinamicamente in base ai linked data.

\footnotetext{
$201<$ http://data.bnf.fr/semanticweb-en>.
} 
La pagina dell'opera viene creata in base ai record di autorità per l'opera fornite dal catalogo della BnF ed è strutturata secondo livelli previsti da FRBR. Vengono mostrati i dettagli del contenuto, l'insieme dei livelli di opere, espressioni (in apposite pagine RDF), manifestazioni e item.

Vengono mostrate le opere correlate e le relazioni intero parte tra opere e tra manifestazioni.

La pagina web di un autore oltre a contenere i dati completi mostra l'elenco delle sue opere e dei differenti ruoli dell'autore nelle opere correlate.

Tutte le pagine vengono arricchite da dati provenienti da diversi dataset esterni, come Wikipedia, dal quale vengono estratte informazioni supplementari su opere, titoli e soggetti.

Le pagine vengono pubblicate in HTML e per renderle visibili ai motori di ricerca vengono estratti i dati descrittivi più rilevanti e descritti nelle risultanti pagine web applicando le marcature previste dallo standard RDFa cui si aggiunge l'uso di vocabolari condivisi come Schema.org utilizzato per codificare le pagine con i microdata, oggi riconosciuti dai principali motori di ricerca e lo schema di marcatura Opengraph Protocol (OG).

Questi strumenti rappresentano modi nuovi di utilizzare i dati presenti nel web per creare interfacce che sfruttano in maniera appropriata la presenza di dati provenienti dalle fonti più diverse, ma soprattutto creano visualizzazioni in grado di manifestare naturalmente la scansione delle entità previste in FRBR.

\subsection{Conclusioni. Linked data e nuove prospettive dell'accesso bibliografico}

Come è emerso in queste pagine, la nuova tecnologia dei linked data rappresenta una grande opportunità per le biblioteche di produrre dati bibliografici aperti, interoperabili e riutilizzabili nel web con enormi vantaggi nell'accesso ai dati, nella loro creazione e nel loro riutilizzo.

In definitiva, i linked data consentono di aprire nuove prospettive alle modalità di accesso all'informazione bibliografica allo scopo di garantirne la fruibilità e l'accesso gettando nuova luce sui temi dell'interoperabilità che da decenni è oggetto di riflessione $\mathrm{e}$ 
sui concetti di pluralità e convergenza che sono alla base di un'adeguata progettazione di ambienti digitali: «Per interoperabilità si intende [infatti] la capacità di assicurare che sistemi, procedure e cultura di una specifica organizzazione siano gestiti in modo tale da massimizzare le opportunità di scambiare e riusare informazioni all'interno e all'esterno» ${ }^{202}$. Oggi le tecnologie dei linked data possono garantire le quattro principali tipologie di interoperabilità, già tracciate da Leombroni, per la costruzione di ambienti digitali integrati e cioè quelle dell'interoperabilità tecnologica, semantica, organizzativa e delle risorse umane:

interoperabilità tecnica: la garanzia dell'interoperabilità fra sistemi e applicazioni. Essa è resa possibile dall'adozione di una tecnologia unificante come quella fornita dal data model RDF;

* interoperabilità semantica: la tecnologia dei linked data consente alle varie comunità coinvolte di definire concetti il cui significato è condiviso tramite il meccanismo degli URI che consente di collegare ai concetti i termini con i quali le diverse comunità li identificano e utilizzano per la ricerca;

* interoperabilità delle risorse umane: i linked data consentono una convergenza dei dati e dei servizi che attorno ad essi si sviluppano; a tal fine consentono l'interoperabilità delle competenze distribuite nel Web Semantico che vengono collegate insieme per lo sviluppo di nuovi servizi basati sui dati

* interoperabilità organizzativa: i linked data consentono di sviluppare una convergenza dei servizi delle istituzioni che operano nell'ambito del cultural heritage, della ricerca scientifica, dei servizi culturali, che cooperano insieme nel web affinché venga garantita la qualità e l'integrità e dei dati immessi attraverso idonee procedure di certificazione della provenienza dei dati. Quest'ultima tipologia comprende anche una nuova interoperabilità 'giuridica' relativa alle licenze con le quali i dati vengono esposti nel Web Semantico, raggiungibile attraverso l'armonizzazione delle licenze d'uso presenti a livello internazionale tramite l'adozione di licenze di utilizzo dei dati più appropriate, e possibilmente, aperte.

202 Cfr.: Claudio Leombroni, Appunti per un'ontologia delle biblioteche digitali: considerazioni sulla Biblioteca digitale italiana, «Bollettino AIB», 44 (2004), n. 2, p. 115-131; Leombroni individuava quattro diverse diverse tipologie di interoperabilità:

1. interoperabilità tecnica: la garanzia dell'interoperabilità fra sistemi e applicazioni;

2. interoperabilità semantica: le varie componenti del sistema devono essere definite da concetti il cui significato è condiviso;

3. interoperabilità delle risorse umane: nell' ambito della convergenza dei servizi è richiesto un discreto tasso di interoperabilità delle competenze;

4. interoperabilità organizzativa: nell'ambito della convergenza dei servizi i confini fra gli istituti culturali sono più sfumati. 


\title{
CAPITOLO 5. L'OPAC A SUPPORTO DEL PROCESSO DI RICERCA DELL'INFORMAZIONE. VERSO UN NUOVO MODELLO DI SVILUPPO PER IL CATALOGO ELETTRONICO
}

\begin{abstract}
«Quale struttura connette il granchio con l'aragosta, l'orchidea con la primula e tutti e quattro con me? E me con tutti voi? E tutti e sei noi con l'ameba da una parte e con lo schizofrenico dall'altra?»
\end{abstract}

Gregory Bateson

\subsection{Verso una nuova identità del catalogo: dal recupero dell'informazione alla creazione di conoscenza}

Le nuove funzioni che gli attuali OPAC presentano derivano da un processo di ripensamento complessivo delle funzionalità del catalogo dettato dall'esigenza di tenere il passo con le nuove tecnologie informatiche e delle abitudini di ricerca degli utenti, i quali si rivolgono sempre più spesso per cercare l'informazione a strumenti alternativi approntati al di fuori delle biblioteche. Con l'avvento dei motori di ricerca e la diffusione di Internet lo scenario della ricerca è profondamente mutato rispetto al passato: motori di ricerca, librerie online, basi di dati commerciali danno certamente all'utente una sensazione di immediatezza del risultato e di facilità d'uso che tradizionalmente mancavano nella consultazione del catalogo elettronico. A paragone con la ricerca che l'utente svolge nel web, nella quale esprime il bisogno informativo usando il linguaggio naturale e servendosi di norma di un solo campo di ricerca, i cataloghi tradizionali sono stati percepiti quali strumenti poco amichevoli.

Gli utenti degli OPAC tendono a trasferire i loro modelli mentali, consolidati nella ricerca in rete, nell'uso dei cataloghi elettronici, aspettandosi le funzionalità che trovano nei motori di ricerca, nelle librerie online e nelle altre applicazioni del web.

Man mano che i cataloghi si sono evoluti verso quelli che definiamo next generation catalogs o discovery systems, una gran quantità di risorse esterne, prima ricercabili attraverso singoli strumenti (basi dati, portali, etc), è divenuta recuperabile tramite $i$ cataloghi; la grande quantità di risorse digitali disponibile e la proliferazione dei supporti 
ha imposto un modello di sviluppo improntato al recupero tramite un'unica interfaccia di risorse prima recuperabili tramite strumenti diversi (consultazione di banche dati, risorse web, collezioni digitali, repositories) allargando a dismisura i documenti recuperabili.

Oggi next generation catalogs e 'sistemi per la scoperta' integrano nella ricerca catalografica una gran quantità di dati provenienti da fonti esterne quali oggetti digitali, dati di repositories e banche dati, articoli di periodici in fulltext e, infine, pacchetti commerciali di e-journals, immettendo così nel catalogo una massa critica di dati impensabile fino a qualche tempo fa.

La ricerca e la navigazione nel catalogo in una massa sempre più imponente di documenti, sono divenute certamente più complesse, in quanto un'interrogazione genera sovente una lunga lista di documenti, che devono essere ordinati in maniera significativa se il catalogo vuole essere di qualche aiuto all'utente.

Se nei cataloghi elettronici 'tradizionali' sin dalle origini la ricerca è stata incentrata sulla formulazione di un'interrogazione, presupponendo che l'utente fosse in grado sia di descrivere il suo bisogno informativo, sia di usare o prevedere il linguaggio usato dal sistema, di misurarsi dunque con il 'linguaggio bibliografico', oggi le difficoltà insite nella ricerca catalografica vengono solo apparentemente risolte dando la preferenza alla modalità di ricerca per parola chiave e offrendo un monocampo 'alla Google' per la ricerca su tutti i campi e proponendo una lista di risultati ordinati per presunta rilevanza.

In generale, lo sviluppo dei cataloghi elettronici nell'ultimo decennio, oltre al fenomeno dell'allargamento delle risorse in esso rappresentate, è stato caratterizzato dall'introduzione di nuove funzionalità per gestire più efficacemente la gran mole di risorse recuperabili; innovazioni che tuttavia non hanno migliorato la ricerca e la comprensione dell'informazione ricevuta poiché basate ancora su un approccio algoritmico della progettazione della ricerca, sulla ricerca per parola chiave e sull'arricchimento dei record.

La progettazione dei cosiddetti OPAC di nuova generazione, per ovviare alla mole di documenti che riescono a recuperare, ha seguito un modello di sviluppo basato su:

* presentazione della modalità di ricerca per parola chiave su tutti i campi come ricerca $d i$ default (le parole immesse vengono automaticamente e implicitamente concatenate dal sistema con l'operatore AND) e relegazione delle modalità della ricerca tradizionali in secondo piano, nella cosiddetta ricerca avanzata o esperta;

* ordinamento dei risultati per rilevanza (relevance ranking); 
* adozione delle faccette per circoscrivere i risultati della ricerca (faceted browsing) e come principale mezzo per navigare nel catalogo.

L'approfondimento del modello attuale degli OPAC di nuova generazione o sistemi per la scoperta mostra dunque alcuni elementi di novità, ma anche varie criticità in quanto risulta imperniato sulla teoria classica della ricerca dell'informazione e sui recenti sviluppi sull'information retrieval; in particolare si rileva:

* una progettazione della ricerca legata alle teorie dell'IR classico e imperniata sulla coppia ordinamento per rilevanza / navigazione a faccette: le due caratteristiche sono complementari perché l'ordinamento per rilevanza produce un elenco di risultati poco significativi e che vanno poi filtrati;

* un meccanismo di recupero dell'informazione 'ingenuo' basato sul matching cioè sulla corrispondenza algoritmica tra l'interrogazione e le parole presenti nel documento o nei suoi metadati;

* una pericolosa deriva verso lo strumento di discovery e la centralizzazione delle interfacce di ricerca, con l'annegamento dei dati dei cataloghi in questi nuovi strumenti unificati basati su una presentazione scarsamente strutturata dell'informazione contenuta e dunque incapaci di supportare l'apprendimento dell'informazione nel contesto;

* un processo di arricchimento del record bibliografico che da solo, è incapace di aiutare l'utente a comprendere e valutare l'informazione nel contesto rappresentato nel catalogo;

* un 'approccio esplorativo' basato sull'estrazione automatica di dati dai record bibliografici e non sull'esposizione della struttura delle relazioni bibliografiche tra le entità.

In definitiva, dunque, se esaminiamo i più recenti sviluppi, la progettazione dei cataloghi elettronici ha emulato gli strumenti del web con una conseguente perdita di 'identità' del catalogo elettronico.

Ciò è ancora più evidente se immaginiamo che l'atto di cercare in un catalogo implica non solamente la messa in atto di algoritmi per la ricerca, ma una profonda interazione dell'utente con l'informazione ricevuta affinché essa sia inserita nell'insieme di conoscenze già acquisite, ovvero l'informazione modifichi lo stato cognitivo dell'utente che fa ricerca.

Si avverte dunque la necessità di creare un ambiente informativo che risponda alla necessità di trovare non solo l'informazione su un argomento noto o conosciuto, ma di 
gestire in maniera più efficace quelle situazioni di ricerca nelle quali l'utente non sa cosa sta cercando e di organizzare in maniera efficace i risultati delle interrogazioni in modo che l'utente possa comprenderne il 'contesto'.

In questa prospettiva il catalogo quindi non dovrebbe limitarsi semplicemente a fornire risultati a partire da un'interrogazione di cui si conosca in anticipo l'oggetto (know item search), ma dovrebbe anche fornire all'utente un ambiente di ricerca che favorisca una esplorazione migliore dei contenuti.

L'esposizione significativa dei risultati costituisce un elemento importante per l'efficacia della ricerca, che è stato ampiamente sottovalutato nella progettazione degli OPAC fin dalle prime applicazioni e che oggi non trova una soluzione adeguata.

In passato, infatti, in assenza di meccanismi logici di ordinamento, il catalogo elettronico presentava i risultati secondo un ordinamento che corrispondeva solitamente alla data di immissione del singolo record nel catalogo, limitando le possibilità di browsing alla sola ricerca per liste, cioè allo scorrimento di liste ordinate di termini (es. nomi di autori, stringhe di soggetto, più raramente classi) a partire da un dato termine immesso. Negli OPAC tradizionali le interfacce di ricerca sono state costruite in modo da circoscrivere la ricerca in canali ben precisi (autore, titolo, soggetti, classi, etc.), corrispondenti ai dati indicizzati dal sistema tra i quali l'accesso per parola chiave era solo uno dei tanti canali possibili.

L'ingresso della modalità di ordinamento per 'presunta rilevanza' (relevance ranking) nella progettazione degli OPAC ha allineato le interfacce dei cataloghi a quelle dei motori di ricerca, in modo che gli utenti possono esprimere il proprio bisogno informativo utilizzando il linguaggio naturale a loro più congeniale, senza dunque dover imparare linguaggi per l'interrogazione e senza curarsi della terminologia usata dal sistema; ma il processo non è pacifico e nasconde, come vedremo alcune insidie, tra le quali la perdita di controllo della propria ricerca e una contestualizzazione non ottimale dei risultati ottenuti.

La ricerca per soggetto, l'esplorazione e l'esposizione significativa dei risultati di una ricerca sono ancora tutt'oggi le principali aree di criticità nell'uso dei moderni OPAC di nuova generazione e negli strumenti di discovery. Il sottosviluppo di queste aree è il risultato di una visione 'debole' del catalogo che prende come riferimento i modelli inadatti a produrre conoscenza come quelli che derivano dai motori di ricerca del web e che si manifestato nella sostituzione della ricerca per soggetto con la parola chiave e nella 
messa in atto di un paradigma esplorativo basato sulla coppia relevance ranking/navigazione a faccette estratte dai documenti.

Progettare cataloghi che siano davvero di nuova generazione vuol dire dunque prima di tutto rovesciare questa visione del catalogo per progettare OPAC nuovi basati sulla creazione di conoscenza.

Questo capitolo dunque prenderà in considerazione i recenti studi sulla natura e le diverse manifestazioni della rilevanza, in modo che ci si possa chiedere se l'efficacia di un OPAC può essere misurata in termini di 'rilevanza presunta'.

La domanda, quando si saranno compresi la natura, le manifestazioni e i criteri in base ai quali gli utenti forniscono valutazioni in merito alla rilevanza potrà così essere riformulata nei seguenti termini: quale tipologia di rilevanza viene fornita negli OPAC? Quali sono i criteri in base ai quali vengono formulati gli algoritmi che misurano la rilevanza? Quale ‘tipologia’ di rilevanza gli OPAC dovrebbero applicare per migliorare il recupero dell'informazione?

L'esame delle modalità con cui gli OPAC oggi presentano i risultati delle interrogazioni dimostra che il concetto di rilevanza è modellato sul concetto di circalità (rilevanza topica o aboutness) ottenuta mediante procedimenti di tipo algoritmico: vedremo invece che l'analisi più approfondita del concetto di rilevanza dovrebbe orientare la progettazione degli OPAC anche verso un tipo di rilevanza soggettiva e dinamica che prenda in considerazione anche la dimensione della pertinenza, cioè la corrispondenza del risultato alle necessità informative e soggettive dell'utente.

Affinché ciò avvenga non è necessario progettare algoritmi più sofisticati, ma riprogettare la ricerca in modo che l'utente riesca a valutare la pertinenza dell'informazione recuperata attraverso una riprogettazione del processo informativo.

In questa prospettiva la ricerca di informazione nell'OPAC va ben oltre il recupero dell'informazione (IR) e coinvolge vari aspetti che possono essere ricompresi nel modello più generale di 'comportamento informativo' che comprende il bisogno informativo, la ricerca, lo scambio e l'uso dell'informazione.

Lo studio dei comportamenti di ricerca è da decenni il campo d'indagine delle teorie dell'information behavior ossia di quel filone di indagine che nell'ambito della Scienza dell'informazione propone un approccio qualitativo del recupero dell'informazione, mettendo in evidenza il contesto sociale nel quale si compie il processo informativo. Nell'ambito di questo importante filone di studio, che ha impresso una svolta 'qualitativa' nella disciplina, si sono originate le 'metateorie sociali del comportamento informativo' e 
gli studi noti come Information seeking behavior; queste teorie, che saranno approfondite nel corso di questo capitolo, permettono di esaminare più a fondo i meccanismi del recupero dell'informazione mettendo l'accento su come le persone cercano e usano l'informazione e possono costituire la base per progettare i sistemi non solo più intelligenti, ma più 'realistici'.

L'ingresso di queste teorie nel campo della Scienza dell'Informazione risale alla metà degli anni '80 quando si assiste a un nuovo orientamento degli studi sull'Information retrieval verso una dimensione sociale e cognitiva che considera nel recupero dell'informazione anche il contesto nel quale avviene, i processi mentali e i comportamenti messi in atto dagli utenti nella ricerca dell'informazione. Nonostante si tratti di un campo di studi che conta una vasta mole di scritti e di contributi e che ha condotto all'elaborazione di modelli e di importanti teorie che trovano applicazione anche in altre discipline e contesti, nella teoria biblioteconomica esse non trovano lo spazio che meriterebbero.

La progettazione di sistemi di recupero dell'informazione e dunque di cataloghi elettronici 'di nuova generazione' dovrebbe dunque avvantaggiarsi degli esiti prodotti da questo filone studi che ha indagato le modalità reali di ricerca dell'informazione, nelle quali l'immissione di un'interrogazione (searching) è solo uno dei comportamenti possibili nel più generale processo di ricerca dell'informazione.

Accogliere queste teorie come base per la progettazione degli OPAC consente di superare l'attuale visione squisitamente algoritmica della ricerca concentrata sui sistemi e in cui si presuppone che l'utente debba recuperare un record o un'informazione e di concentrarsi sulla ricerca come processo, poiché l'utente che compie una ricerca nell'OPAC è coinvolto prima di tutto in un processo informativo che lo conduce dalla definizione del suo bisogno informativo fino alla scoperta e al recupero dell'informazione pertinente. Sulla ricerca dell'informazione si esprimeva così Brian Vickery già nel 1965:

[la ricerca dell'informazione è] una sorta di processo graduale che evolve per fasi successive attraverso l'interazione tra l'utente e il sistema informativo; è un vero e proprio processo di apprendimento: mano a mano che la conoscenza aumenta anche lo spettro di informazioni che giudichiamo pertinenti e utili ${ }^{1}$.

Un riconoscimento implicito di questa funzione di 'apprendimento' si ritrova a mio parere nella funzione di 'navigare' di FRBR introdotta tra le funzioni-utente previste nei nuovi principi internazionali di catalogazione, che consente, appunto, attraverso

\footnotetext{
${ }^{1}$ Brian Vickery, On retrieval system theory, London, Butterworths, 1965, p. 10 (ndr. la traduzione è mia).
} 
l'implementazione del modello nelle interfacce, di offrire una visualizzazione delle entità oggetto di interesse del lettore e di poter navigare nella rete delle entità correlate, offrendo un'informazione contestualizzata e dunque capace di creare processi di conoscenza.

I nuovi next generation catalogs e gli strumenti di discovery si dimostrano dunque oggi inadeguati a gestire i processi di conoscenza ed essenzialmente rivolti verso il recupero di singoli record, o verso una funzione di metaricerca che consente il recupero di una grandissima quantità di record a discapito della qualità del recupero.

Il futuro del catalogo elettronico risiede quindi nella capacità di trasformarsi da strumento di recupero dell'informazione a strumento in grado di facilitare la creazione di conoscenza dell'universo bibliografico in esso rappresentato.

Per far ciò è necessario abbandonare il modello di sviluppo attuale, centrato sui meccanismi del matching e sulla rilevanza algoritmica e topica per concentrarsi sul processo di ricerca e sull'interazione che si svolge all'interno del catalogo.

Nella parte centrale del capitolo viene dunque proposto un nuovo modello di OPAC che si basa sulle principali metateorie della ricerca informativa e sui principali modelli elaborati nel campo dell'information seeking behavior che mettono al centro i concetti di bisogno informativo, di strutture della conoscenza, di uso dei sistemi informativi e di recupero e di uso dell'informazione.

Si dimostrerà come un nuovo modello di OPAC che produca flussi di conoscenza debba essere fondato sulle teorie del comportamento nella ricerca informativa allontanandosi così dal modello evolutivo attuale basato unicamente sugli sviluppi dell'information retrieval e sulla rilevanza algoritmica. La tematica si arricchisce con gli approfondimenti che derivano dalle tecnologie oggi a disposizione.

Le opportunità oggi offerte dalla tecnologia dei linked data consentono di collegare $\mathrm{i}$ record catalografici, scomposti nei dati costituenti, ad altri dati presenti sul web consentendo l'attivazione di servizi nuovi a vantaggio della ricerca e dell'intero processo informativo.

Vedremo dunque come la nuova tecnologia dei linked data e un modo nuovo di riprogettare il record bibliografico possano divenire un valido aiuto verso la costruzione di OPAC nuovi basati sulla produzione di conoscenza durante il processo di ricerca. 


\subsection{Informazione e conoscenza nella Scienza dell'informazione}

Nell'affrontare il concetto di rilevanza nel recupero dell'informazione e, dunque, quale ambito di interesse della Scienza dell'informazione, merita almeno un breve accenno il concetto di 'informazione' che costituisce il suo principale oggetto di studio ${ }^{2}$, essendo la Scienza dell'informazione «la disciplina che studia, sul piano teorico e applicativo, i processi e gli strumenti con cui l'informazione viene prodotta, registrata, distribuita, immagazzinata e recuperata, ovvero l'intero ciclo di vita dell'informazione stessa» ${ }^{3}$.

Definire l'informazione non è un compito agevole in quanto - ha osservato Floridi essa è un labirinto concettuale difficilmente districabile: «l'analisi del concetto di informazione - afferma il filosofo - versa ancora in quello stadio deplorevole in cui il disaccordo investe il modo stesso in cui i problemi sono provvisoriamente formulati e contestualizzati entro cornici teoriche» ${ }^{4}$.

Probabilmente il più conosciuto tentativo nella storia della disciplina di definire l'informazione è quello della tanto influente quanto restrittiva 'teoria matematica della comunicazione' (MTC), successivamente nota come 'teoria dell'informazione', elaborata da Shannon e Weaver ${ }^{5}$.

Questa teoria ha condotto ad una concezione dell'informazione legata alla comunicazione e alla codifica dei messaggi secondo un modello che studia la trasmissione dell'informazione da una sorgente ad una destinazione ignorando il contenuto semantico del concetto: l'informazione è quella misurabile e nella misura dell'informazione risiede lo straordinario successo della teoria nel campo della scienza, dell'ingegneria, dell'informatica e delle telecomunicazioni.

\footnotetext{
${ }^{2}$ Per una panoramica degli oggetti di studio si veda: Tefko Saracevic, Information Science in Encyclopedia of Library and Information sciences, 3 ed., edited by Marcia J. Bates and Mary Niles Maack, New York, CRC Press, 2010, p. 2381-2391: «Information science is the science and practice dealing with the effective collection, storage, retrieval and use of information» (citazione a p. 2381). Secondo Saracevic la Scienza dell'informazione tenta di rispondere a tre principali domande (le cosiddette 'Big Questions' già individuate da Marcia Bates): 1. la domanda fisica: quali sono le caratteristiche e le leggi dell'informazione registrata?; 2. la domanda sociale: come le persone si relazionano all'informazione, come la cercano e come la usano?; 3. la domanda progettuale: come migliorare e rendere più rapido l'accesso all'informazione registrata?.

${ }^{3}$ Alberto Salarelli, Introduzione alla scienza dell'informazione, Milano, Bibliografica, 2012, p. 31.

${ }^{4}$ Luciano Floridi, La rivoluzione dell'informazione, trad.it. di Massimo Durante; pref. di Juan Carlos De Martin, Torino, Codice, 2012, p. XV.

${ }^{5}$ Elaborata da Shannon e Weaver nel noto saggio del 1948, questa teoria che propose un approccio quantitativo alla Scienza dell'Informazione, si concentrava sui segnali e sui canali di comunicazione trascurando la sfera semantica e il contesto sociale nel quale si compie la comunicazione dell'informazione. Cfr. Claude Elwood Shannon, A mathematical theory of communication, «Bell System Technical Journal», 27, (1948), p. 379-423 e 623-656, anche in <http://circuit.ucsd.edu/ yhk/ece287a-win08/shannon1948.pdf> poi ripreso e ampliato in Warren Weaver - Claude Elwood Shannon. Shannon, The Mathematical Theory of Communication, Urbana, Illinois, University of Illinois Press, 1949.
} 


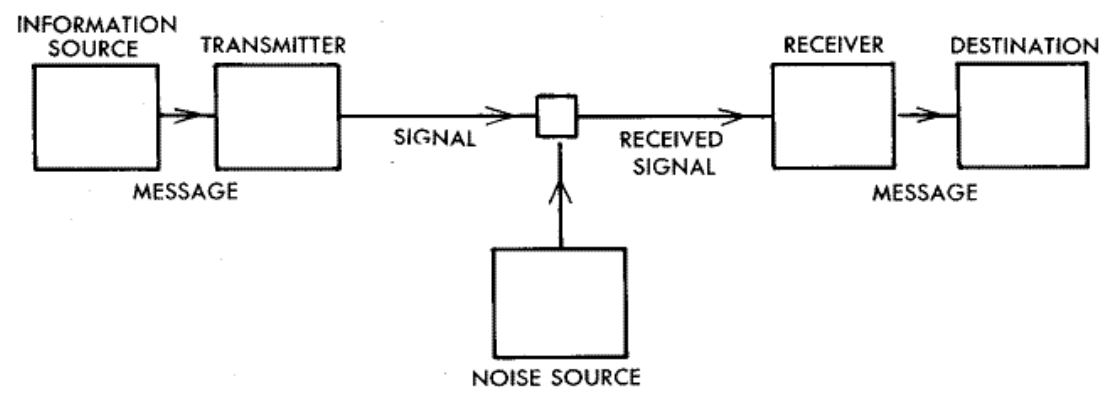

Fig. 5.1 - Diagramma di un sistema di comunicazione nella teoria matematica dell'informazione di Shannon e Weaver ${ }^{6}$

Fu proprio la limitatezza del modello d'informazione proposta dai due studiosi, ove il concetto informazione è completamente indipendente dal suo significato, ad aver condotto allo sviluppo di altre teorie sull'informazione; la teoria matematica della comunicazione non era minimamente interessata al significato, né ai concetti di rilevanza, di utilità o di interpretazione dell'informazione ignorando tutto ciò che non può essere misurato; come osserva Donald Case, la maggior parte delle problematiche che sorgono quando analizza il concetto dell'informazione nei termini proposti da Shannon e Weaver non sono ricomprese nella spiegazione del concetto fornita dai due studiosi: si tratta dei concetti emersi in altre teorie, sviluppatesi anche come risposte polemiche alla MTC che nel definire l' 'informazione' fanno riferimento ai concetti di utilità (l'informazione deve essere utile per produrre un effetto?), di fisicità (deve avere una forma fisica, deve essere osservabile?); di struttura (deve essere strutturata in qualche modo? deve implicare un processo?), di intenzionalità (deve essere intenzionale e mirata alla comunicazione di qualcosa?) e di verità (l'informazione per essere definita informazione deve essere vera?) ${ }^{7}$. Tutte le teorie che si distinguono dalla $\mathrm{MTC}^{8}$ e che caratterizzano il filone 'cognitivo' del

\footnotetext{
${ }^{6}$ Claude Elwood Shannon, A mathematical theory of communication cit., p. 7.

${ }^{7}$ Per un approfondimento del concetto di 'informazione' e dei principali studi che hanno esplorato questo fenomeno si vedano Alfredo Serrai, Dalla informazione alla bibliografia: la professione bibliotecaria. Milano, Bibliografica, 1984; Luciano Floridi, La rivoluzione dell'informazione cit.; Donald Owen Case, Looking for information : a survey of research on information seeking, needs, and behavior, 2. ed. - Bingley : Emerald, c2008, p. 39-67, Alberto Salarelli, Introduzione alla scienza dell'informazione cit, p. 11-30, Marcia J. Bates, Information, in Encyclopedia of Library and Information sciences, 3 ed., edited by di Marcia J. Bates and Mary Niles Maack, New York, CRC Press, 2010 p. 2347-2360 (Disponibile anche online all'URL: <http://pages.gseis.ucla.edu/faculty/bates/articles/information.html>) e Rafael Capurro - Birger Hjørland, The concept of information, «Annual Review of Information Science and Technology», 37 (2003), n. 1, p. 343-411 (Draft version in: <http://www.capurro.de/infoconcept.html>).

${ }^{8}$ Le più note definizioni del concetto di informazione vengono ripercorse nella voce Information da Marcia $\mathrm{J}$. Bates che, al termine del contributo osserva (ivi, p. 2358): «The understanding of the core of "information" in information science remains a highly contested area. Information is seen as:

* a proposition, a structure, a message, or an event;

* as requiring truth or indifferent to truth;

* as socially embedded ad under perpetual re-interpretation, or as measurable in bits;

* as a worn-out idea deserving of dispatch, or an exciting conception in terms of evolutionary forces.
} 
recupero dell'informazione presentano come tratto comune quello del 'soggetto interpretante' analizzando così non solo gli aspetti sintattici, ma anche gli aspetti semantici e pragmatici della comunicazione dell'informazione ${ }^{9}$.

Un diagramma delle dimensioni e dei significati che ruotano intorno al concetto di informazione è quello fornito da Luciano Floridi che ha tracciato una mappa del concetto, le cui basi si possono ricondurre al concetto di dato + significato $(\text { data }+ \text { meaning })^{10}$; una spiegazione quanto mai attuale che conduce alla riflessione dei due principali aspetti che il concetto di informazione veicola: quello oggettivo, costituito dai dati, e quello soggettivo, legato alla capacità di interpretazione da parte di un soggetto interpretante ${ }^{11}$. Dunque le aggregazioni di dati diverrebbero pienamente significative quando sono in grado di trasmettere un messaggio comprensibile in chi le osserva.

\footnotetext{
The much-debated concept of information remains at the lively heart of information science».

${ }_{9}^{9}$ Queste tre dimensioni dell'informazione sono evidenziate da Tefko Saracevic in un contributo che definisce le competenze della Scienza dell'informazione ove riconduce i concetti di informazione a tre categorie: 1) l'informazione in termini di segnali e messaggi secondo un approccio quantitativo e 'acontestuale' che considera solo la sintassi, trascurando semantica e pragmatica 2) un approccio cognitivo all'informazione che considera i processi cognitivi che intercorrono tra l'interprete e il messaggio veicolato ove si assite ad un rapporto tra le dimensioni soggettiva dell'informazione (messaggio) e oggettiva (supporto); l'approccio pragmatico, che colloca l'atto dell'informazione e i supporti nel contesto ('in context') storico, economico, sociale nel quale avviene l'uso dell'informazione e che si pone delle domande sul perché viene prodotta o utilizzata l'informazione (cfr: Tefko Saracevic, Information Science cit.).

${ }^{10}$ Luciano Floridi, La rivoluzione dell'informazione cit. p. 25. La definizione generale di informazione (DGI) che Floridi propone è fondata su tre caratteristiche fondanti dell'informazione: che ogni in formazione possa essere sconponibile in una quantità di dati, che i dati siano 'ben strutturati' ossia organizzati secondo un costrutto logico e che i dati siano dotati di significato.

11 Nella definizione proposta da Floridi, Salarelli rileva che la caratteristica strutturale individuata dal filosofo, ossia che i dati siano 'ben formati', trascende la semantica sottolineando che l'informazione debba essere considerata anche sotto l'aspetto semiotico ovvero si debba anche considerare quello che nella linguistica strutturale viene denominato il 'significante'. In questo senso la teoria della semiotica di Charles Sanders Peirce - secondo lo studioso - definendo l'informazione come «ciò che sta per qualcosa, in un determinato contesto, per un determinato interprete» - ha fornito un quadro efficace nel quale inserire il concetto di informazione. Cfr.: Alberto Salarelli, Introduzione alla scienza dell'informazione cit. p. 14-16.
} 


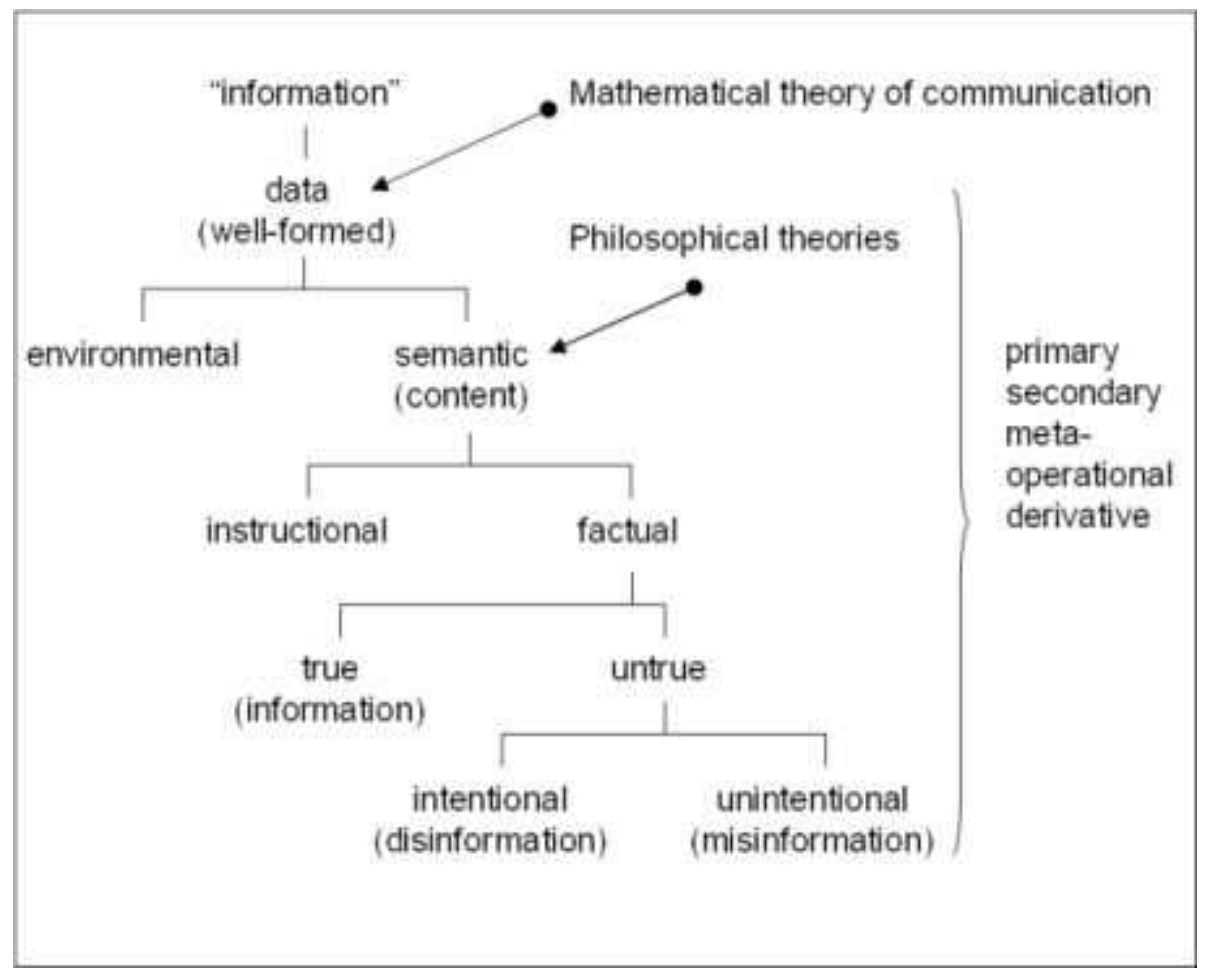

Fig. 5.2 - Una mappa concettuale dell'informazione ${ }^{12}$

Tale definizione avvicina il concetto di informazione a quello delineato da Gregory Bateson ove l'informazione può essere considerata come una frattura che produce una differenza nella struttura cognitiva dell'utente, e nel quale, dunque, l'informazione viene definita come una «differenza che crea una differenza» ${ }^{13}$.

Questa definizione ci induce, infine, a chiarire la differenza tra informazione e conoscenza, ove per conoscenza si intende il processo di modifica della propria struttura cognitiva, un atto dunque 'soggettivo', legato alle esperienze e all'interpretazione da parte di ogni singolo individuo, che dunque, a differenza dell'informazione che può essere comunicata, trasferita, consumata, venduta, non è mai oggettiva o misurabile. La conoscenza attiene ad una sfera più alta di valori, che è ottenibile attraverso un processo di rielaborazione dell'informazione ottenuta per inserirla nel proprio bagaglio conoscitivo. Questa motivazione spinge oggi le società più avanzate a passare da società dell'informazione a società della conoscenza, ovvero a studiare metodi per filtrare

\footnotetext{
${ }^{12}$ Luciano Floridi, Semantic Conceptions of Information, First published Wed Oct 5, 2005; substantive revision Fri Jan 28, 2011, online, <http://plato.stanford.edu/entries/information-semantic/>.

${ }^{13}$ Ivi, p. 16-17. Il concetto della «differenza che produce una differenza» viene fatto risalire agli studi di filosofia e cibernetica di Bateson (cfr. Gregory Bateson, Verso un'ecologia della mente, trad. it di Giuseppe Longo, Milano, Adelphi, 1976, p. 470). Il concetto venne anche espresso in termini matematici da Bertram C. Brookes con la formula $K[\mathrm{~S}]+\Delta I=\mathrm{K}[\mathrm{S}+\Delta \mathrm{S}]$, dallo studioso definita come equazione fondamentale della Scienza dell'informazione Cfr.: Bertram C. Brookes, The foundations of information science. Part 1: Philosophical aspects. «Journal of Information Science», 2 (1980), p. 125-133.
} 
l'informazione disponibile e trasformarla in conoscenza, ovvero informazione 'collocata', integrata e organizzata, che è trasformata e inserita in una struttura cognitiva.

Attraverso la conoscenza le persone sono in grado di giudicare la realtà e compiere delle scelte più vantaggiose, ovvero di migliorarsi. Questo processo viene amplificato dalla rivoluzione digitale e dall'emergenza informativa costituita dall'enorme crescita della quantità delle conoscenze prodotte, degli individui che la producono e dalle nuove forme assunte dalla comunicazione in rete, dunque, dal fenomeno dell'information overload, di fronte al quale le biblioteche svolgono un'utile funzione di filtro e di mediazione.

È la società della conoscenza - digitale, interattiva, convergente e pervasiva - che consente [...] un ecosistema cognitivo a carattere virale nel quale gli sguardi, i paradigmi, le modalità di produzione e di scambio di contenuti sono di natura informazionale ${ }^{14}$.

Nelle pagine che seguono ci riferiremo al concetto di informazione in un senso ampio intendendo per 'informazione' quel concetto già individuato da Bateson, ovvero di differenza percepita o di riconoscimento di schemi nel mondo che ci circonda, che si può trasmettere, organizzare distinguendola dalla conoscenza che, invece, in quanto profondamente soggettiva, non si è in grado di creare, ma semmai si può facilitare tramite un'organizzazione appropriata dell'informazione.

Questi concetti ci consentono di comprendere meglio perché oggi la progettazione degli OPAC basata sul concetto di rilevanza presunta dell'informazione ricevuta, debba essere ricorretta in favore dell'accoglimento delle teorie che sono emerse dal filone cognitivo dell'information retrieval che, a partire dalla fine del secolo scorso, hanno portato al centro dell'attenzione della Scienza dell'informazione nuovi argomenti di ricerca e approcci qualitativi al recupero dell'informazione.

\subsubsection{La rilevanza nella scienza dell'informazione e i modelli di information retrieval}

La rilevanza è generalmente riconosciuta come il concetto fondante degli studi sui sistemi di information retrieval (IR) a partire dall'International Conference for Scientific Information del $1958^{15}$.

\footnotetext{
${ }^{14}$ Fiorello Cortiana, Premessa all'edizione italiana in La conoscenza come bene comune cit., p. XV.

${ }^{15}$ La prima Conference on Scientific Information (ICSI) si tenne a Washington dal 16 al 21 novembre del 1958 e fu sponsorizzata da National Science Foundation, National Academy of Sciences e American Documentation Institute (ente predecessore dell' American Society for Information Science). L'avvio del
} 
Gli studi intrapresi a partire dagli anni '60 per valutare l'efficacia dei sistemi di information retrieval, fondano la loro analisi sul concetto di rilevanza e le misure più importanti usate nell'IR, richiamo e precisione, sono basate su un concetto dicotomico o binario della rilevanza che considera un documento rilevante o non rilevante. Tale concezione dualistica del concetto di rilevanza, come vedremo, non descrive adeguatamente i processi coinvolti nella ricerca e nel recupero dell'informazione.

Gli studi più recenti hanno infatti dimostrato che la rilevanza è soggettiva, dinamica e multidimensionale. È necessario dunque esaminare più a fondo il concetto di rilevanza per comprenderne natura, manifestazioni e caratteristiche.

Una delle più note e recenti definizioni di rilevanza che potremmo considerare come il punto d'arrivo dei più recenti studi, è quella fornita dagli scritti di Saracevic ${ }^{16}$ :

We consider relevance as having a number of dimensions or attributes: Relevance is a relation. Relevance is a property. Relevance is a measure. Relevance has a context, external and internal. Relevance may change. Relevance has a number of manifestations or kinds. Relevance is not given. Relevance is inferred. Relevance is created or derived. Relevance involves selection. Relevance involves interaction. Relevance follows some intentionality ${ }^{17}$.

La definizione di rilevanza proposta induce in primo luogo ad affrontare il tema della natura della rilevanza, cioè dei modi in cui viene compresa la rilevanza e in cui essa si manifesta.

Saracevic ${ }^{18}$ afferma che la rilevanza è un concetto intuitivo e primitivo, nel senso che è universalmente compresa. Le persone comprendono la rilevanza ${ }^{19}$ per istinto, come

programma spaziale sovietico culminato nel lancio dello Sputnik provocò per reazione una forte crescita degli studi sull'informazione fino ad allora poco indagati (tra le aree di studio: Organization of information for storage and search: comparative characteristics of existing systems, Organization of information for storage and retrospective search: intellectual problems and equipment considerations; Organization of information for storage and retrospective search: possibility for a general theory; Responsibilities of Government, Societies, Universities, and industry for improved information services and research). Per approfondimenti si veda: International Conference on Scientific Information, Proceedings of the International Conference on Scientific Information. Washington, National Academy of Sciences, 1959.

${ }^{16}$ Cfr.: Tefko Saracevic, Relevance: a review of and a framework for the thinking on the notion in information science, «Journal of the American Society for Information Science», 26 (1975) n. 6, p. 321-343; Id., Relevance reconsidered, in Peter Ingwersen - Niels Ole Pors, Proceedings Colis 2: Second international conference on conceptions of Library and information science: integration in perspective, October 13-16, 1996 Copenhagen, Royal School of Librarianship, 1996, p. 201-218; Id., Relevance: a review of the literature and a framework for thinking on the notion in information science. Part II: nature and manifestations of relevance, «Journal of the American Society for Information Science and Technology», 58 (2007), n. 3, p. 1915-33; Tefko Saracevic, "Relevance: a review of the literature and a framework for thinking on the notion in information science. Part III: Behavior and effects of relevance", "Journal of the American Society for Information Science and Technology», 58 (2007) n. 13, p. 2126-44.

${ }^{17}$ Tefko Saracevic, Relevance: a review of the literature [...] Part II cit., p. 1920.

${ }^{18}$ Ibidem.

19 «Nobody has to explain to users of IR systems what relevance is, even if they struggle (sometimes in vain) to find relevant stuff. People understand relevance intuitively» (Tefko Saracevic, Relevance reconsidered cit., p. 215). 
comprendono gli altri concetti primitivi di tempo, spazio, cultura e, intuitivamente, la intendono come correlata o riferita implicitamente a qualcosa, che è rappresentato dal contesto.

Più in profondità, invece, la rilevanza è un concetto che consiste in un numero di parti che interagiscono e sono correlate tra loro da proprietà o criteri, ovvero si può comprendere la rilevanza prendendo in considerazione il numero di 'dimensioni' attraverso le quali le parti possono essere correlate e interpretate. Queste ultime non sono statiche, ma cambiano in base alle circostanze.

La rilevanza può essere dunque considerata come una 'proprietà' in base alla quale le parti sono tra loro correlate, ma anche come una 'misura' dell'efficacia di queste relazioni ${ }^{20}$ Nella scienza dell'informazione la rilevanza viene considerata come una 'relazione' tra gli oggetti informativi e il contesto (che è costituito da stati cognitivi e affettivi) in base ad una determinata proprietà che riflette la manifestazione della rilevanza che si vuole ottenere (es. utilità, circalità, corrispondenza cognitiva etc.); gli oggetti informativi e il contesto possono essere rappresentati da oggetti tangibili o intangibili.

Volendo astrarre e individuare uno schema generale per le numerose definizioni di rilevanza che sono state date, potremmo dire che la rilevanza è, dunque, la misura della corrispondenza (utilità o aderenza) tra l'informazione fornita e la richiesta d'informazione determinata da chi giudica, utente o specialista dell'informazione:

Relevance is the A of a B existing between a C and a D as determined by an E," where A may be "measure, degree, estimate ...;" B may be "correspondence, utility, fit, ... ;" C may be "document, information provided, fact ...;" D may be "query, request, information requirement ... ;" and E may be "user, judge, information specialist.",21

La rilevanza nella Scienza dell'informazione è considerata un'inferenza; viene infatti creata per inferenza dai sistemi e dagli automatismi, e viene derivata per inferenza dagli gli esseri umani $^{22}$. Insieme al concetto di creazione - derivazione, necessario per comprendere appieno il concetto di rilevanza, altri due elementi sono molto importanti: il primo è che il processo di creazione o derivazione della rilevanza implica necessariamente un processo di 'selezione' tra documenti o informazioni rilevanti e non rilevanti, processo che è orientato alla massimizzazione del risultato e alla minimizzazione dello sforzo; in secondo luogo che il processo di selezione coinvolge una serie di interazioni di vario tipo. Dunque anche i processi di selezione e interazione sono connaturati nel concetto di rilevanza.

\footnotetext{
${ }^{20}$ Tefko Saracevic, Relevance: a review of the literature [...] Part II cit., p. 1918.

${ }^{21}$ Tefko Saracevic, Relevance: a review of the literature ... cit., p. 328.

${ }^{22}$ Tefko Saracevic, Relevance: a review of the literature ... Part II cit., p. 1919
} 
Gli 'attributi' o le 'dimensioni della rilevanza' sono stati ben indagati da Cosijn e Ingwersen $^{23}$; gli studiosi considerano uno dei possibili modi di modellare il concetto di rilevanza della proposta di Saracevic in un sistema di attributi della rilevanza che comprende:

Relazione: la rilevanza nasce quando si esprime una relazione in base ad una certa proprietà, di solito attraverso degli scambi comunicativi che coinvolgono le persone e l'informazione o gli oggetti informativi.

Scopo: la relazione che esprime la rilevanza coinvolge le intenzioni - obiettivi, ruoli aspettative e motivazione.

Contesto: lo scopo nell'espressione della rilevanza deriva sempre dal contesto ed è diretto verso un contesto. La rilevanza non può dunque essere considerata al di fuori del contesto. A sua volta il contesto può essere ‘interno' laddove la rilevanza coinvolga stati affettivi e cognitivi oppure 'esterno', se la rilevanza è diretta verso una situazione, un compito, il problema in esame. Possono essere coinvolte nel contesto anche componenti sociali e culturali.

Inferenza: la rilevanza implica la valutazione di una relazione e su questa base è creata o derivata.

Selezione: l'inferenza può implicare una selezione di fonti concorrenti allo scopo di massimizzare i risultati o della minimizzare dello sforzo impiegato per ottenerli

Interazione: l'inferenza è ottenuta come processo dinamico e interattivo, nel quale un'interpretazione degli altri attributi può variare, quando varia il contesto.

Misura: la rilevanza coinvolge una misura della efficacia o del grado di massimizzazione della relazione data, come la valutazione dell'informazione recuperata, in base allo scopo orientato verso un contesto.

Considerando la 'manifestazione' come la prova dell'esistenza o della presenza di qualcosa, si comprende che, come molti altri fenomeni, anche la rilevanza ha un certo numero di manifestazioni ${ }^{24}$.

Gli studi di Vickery $^{25}$ riconobbero per la prima volta l'esistenza di diverse manifestazioni della rilevanza, definendo user relevance il criterio consistente nella

\footnotetext{
${ }^{23}$ Gli studiosi riprendono e arricchiscono le definizioni fornite da Saracevic (Tefko Saracevic, Relevance reconsidered cit p. 5) cfr. Erica Cosijn - Peter Ingwersen, Dimensions of relevance, «Information Processing and Management: an International Journal archive», 36 (2000), n. 4, p. 533-550.

${ }^{24}$ Dal momento che la rilevanza implica una relazione, le varie definizioni di manifestazioni della rilevanza sono state espresse facendo riferimento a quella particolare relazione e aggettivando dunque il termine come in 'topical relevance' o usando un singolo termine che denota una particolare manifestazione es. 'pertinence'
} 
verifica del livello ottimale di discriminazione contrapponendolo alla subject relevance o anche topic o system relevance. Da allora queste manifestazioni sono state trattate come opposte e concorrenti. Swanson nel $1986^{26}$ le definì 'rilevanza oggettiva' e 'rilevanza soggettiva', equiparando i due tipi di rilevanza alla teoria dei tre mondi di Popper ${ }^{27}$.

Dagli anni '60 la rilevanza, principale oggetto di studio della scienza dell'informazione, è stato oggetto di ricerca di varie altre discipline ${ }^{28}$ e di studi che hanno considerato il comportamento e gli effetti della rilevanza in diversi contesti parlando così di rilevanza di sistema, comunicativa, situazionale e psicologica, ma nessuno di questi schemi è stato universalmente accettato. Nel campo della Scienza dell'informazione sostiene Saracevic - gli studiosi non sono ancora pervenuti ad una 'teoria della rilevanza' condivisa, consistente in principi e proposizioni testate per spiegare come la rilevanza sia applicabile alla pratica della disciplina specie nel campo del recupero dell'informazione (IR), per comprenderne le manifestazioni e prevederne comportamenti ed effetti ${ }^{29}$. A livello teorico invece vari modelli sono stati messi a punto per spiegare gli elementi e le diverse variabili coinvolte nei giudizi di rilevanza. Secondo Mizzaro tali modelli hanno seguito due diversi orientamenti: dal 1959 al 1976 gli studi si sono orientati prevalentemente sul documento e l'interrogazione; dal 1977 in poi, invece, la ricerca ha cercato di comprendere e formalizzare una rilevanza più soggettiva, dinamica e multidimensionale ${ }^{30}$. Un dualismo che come vedremo si manifesta tutt'oggi in modelli di IR opposti: quelli orientati ai sistemi e orientati agli utenti formulati da comunità di studiosi che tra loro hanno comunicato poco ${ }^{31}$ :

${ }^{25}$ Brian Vickery, The structure of information retrieval systems. In Proceedings of the International Conference on Scientific Information, vol. 2, Washington, National Academy of Sciences, 1959, p. 12751290.

${ }^{26}$ Don R. Swanson, Subjective versus objective relevance in bibliographic retrieval systems, «The Library Quarterly», 56 (1986), n. 4, p. 389-398.

27 «World One, is the phenomenal world, or the world of direct experience. World Two is the world of mind, or mental states, ideas, and perceptions. World Three is the body of human knowledge expressed in its manifold forms, or the products of the second world made manifest in the materials of the first world (e.g.books, papers, symphonies, and all the products of the human mind). World Three, he argued, was the product of individual human beings. The influence of World Three, in his view, on the individual human mind (World Two) is at least as strong as the influence of World One», (Karl Popper citato in Tefko Saracevic, Relevance: a review of the literature [...] Part II cit., p. 1929).

${ }^{28}$ Nella logica, orientata agli studi sull'intelligenza artificiale, in filosofia, nel campo dell'epistemologia, nel campo della comunicazione che studia l'informazione come fenomeno e processo e dove la rilevanza è stata indagata nel campo degli studi sulla comunicazione verbale

${ }^{29}$ Tefko Saracevic, Relevance: a review of the literature [...] Part III cit., p. 1923.

30 Stefano Mizzaro, Relevance: the whole history, «Journal of the American Society for Information Science», 48 (1997) n. 9, p. 827.

31 «In a massive study of cocitation patterns in information science for the period 1972-1995, White and McCain (1998), among others, mapped the structure of the field showing two broad clusters or subdisciplines calling them domain analysis and information retrieval: Specialties can be aggregated upward in two large subdisciplines: (1) The analytical study of learned literatures and their social contexts, comprising citation 
Some researchers have been able to cross between this divide, but in the words of Saracevic: "we have two camps, two islands, with, unfortunately, relatively little traffic in between" 32

La definizione delle manifestazioni della rilevanza è stata oggetto di studio di numerose ricerche: nel 1998 Mizzaro nell'articolo How many relevances in information retrieval? si interrogava sulle principali manifestazioni della rilevanza, mentre Saracevic ammette alla radice della rilevanza una ripartizione dualistica dalla quale scaturisce un sistema pluralistico di rilevanze ${ }^{33}$.

Prendendo a riferimento le elaborazioni teoriche di Cosijn e Ingwersen, di Borlund ${ }^{34}$ e di Saracevic si possono distinguere le seguenti manifestazioni della rilevanza:

* rilevanza di sistema o algoritmica data dalla relazione tra una query e l'informazione o gli oggetti informativi in un file o in un sistema sia recuperati sia non recuperati, da una data procedura o algoritmo. Ogni sistema possiede specifiche modalità attraverso le quali gli oggetti sono rappresentati, organizzati e confrontati con la query. Essi comprendono l'assunzione di rilevanza, visto che lo scopo è quello di recuperare una serie di oggetti che il sistema ha dedotto essere rilevanti per una interrogazione. L'efficacia comparativa nella deduzione della rilevanza è il criterio della rilevanza di sistema.

* rilevanza topica o di soggetto: la relazione tra il soggetto espresso nella query e il soggetto coperto dall'informazione o dagli oggetti informativi recuperati. Si presuppone che sia la query che gli oggetti informativi possano essere identificati come facenti parte di un particolare argomento o soggetto. La circalità (aboutness) è il criterio attraverso il quale la rilevanza topica (topicality) è dedotta;

* rilevanza cognitiva o pertinenza: è la relazione tra lo stato di conoscenza dell'utente e l'informazione o gli oggetti informativi recuperati. La corrispondenza cognitiva, la capacità informativa, la novità, la qualità

analysis and citation theory, bibliometrics, and communication in science and R\&D; and (2) the study of the human-computer-literature interface, comprising experimental and practical retrieval, general library systems theory, user theory, OPACs, and indexing theory. . . . [Authors] are essentially "'literatures people", or "'retrieval people.', (p. 337) Their conclusion: "Two subdisciplines of information science are not yet well integrated” (p. 337)». Tefko Saracevic, Relevance: a review of the literature [...] Part II cit., p. 1925.

${ }^{32}$ Tefko Saracevic, Information science, «Journal of the American Society for Information Science», 50 (1999), n. 12, p. 1057. Sull'argomento si veda anche Jan Nolin, "Relevance” as a boundary concept: Reconsidering early information retrieval, «Journal of Documentation», 65 (2009), n. 5, 745-767.

33 Stefano Mizzaro, How many relevances in information retrieval? «Interacting with Computers», 10 (1998), n. 3, 303-320.

${ }^{34}$ Erica Cosijn - Peter Ingwersen, Dimensions of relevance cit.; Pia Borlund, The concept of relevance in IR. «Journal of the American Society for Information Science and Technology», 54 (2003), n. 10, p. 913-925. 
dell'informazione e simili sono criteri attraverso i quali viene dedotta la rilevanza cognitiva o pertinenza;

* rilevanza situazionale o utilità: è la relazione tra la situazione, il compito o il problema in esame e gli oggetti informativi recuperati. L'utilità nel prendere decisioni, l'appropriatezza dell'informazione nella risoluzione di un problema, la riduzione dell'incertezza sono $\mathrm{i}$ criteri in base ai quali la rilevanza situazionale è dedotta. Essa può essere estesa e ricomprendere anche fattori sociali e culturali;

* rilevanza affettiva: è la relazione tra gli scopi, gli obiettivi, le emozioni o le motivazioni dell'utente e l'informazione recuperata. La soddisfazione, il successo, il raggiungimento di un obiettivo sono i criteri per dedurre la rilevanza motivazionale o affettiva. Possiamo dire che la rilevanza affettiva è alla base di altre manifestazioni della rilevanza, in particolare della rilevanza situazionale.

Oltre ad analizzare le diverse manifestazioni della rilevanza, vari studiosi si sono chiesti quali siano i più importanti indizi della rilevanza per le persone, cioè cosa rende un'informazione o un oggetto informativo rilevante. I risultati di queste ricerche si sono concretizzati nello stabilire categorie o criteri di rilevanza; in generale le ricerche hanno dimostrato che utenti diversi usano criteri simili per dedurre la rilevanza, anche se l'importanza che essi danno allo stesso criterio differisce di molto a seconda dei compiti, del progresso nel tempo di un determinato compito o obiettivo, e della tipologia di utenza. I criteri (relevance clues) sui quali gli utenti si basano per produrre deduzioni della rilevanza si possono raggruppare nelle seguenti categorie che possono riguardare il contenuto (argomento, qualità, ampiezza della trattazione, aggiornamento trattamento, chiarezza), l'oggetto (le caratteristiche dell'oggetto informativo tipo, organizzazione, rappresentazione, formato, disponibilità, accessibilità, costi), la validità (accuratezza dell'informazione fornita, autorità, affidabilità delle fonti, verificabilità), l'uso o corrispondenza alla situazione (appropriatezza rispetto a una situazione, a un compito di ricerca, usabilità, urgenza, valore nell'uso), la corrispondenza cognitiva (comprensione, novità, sforzo mentale), la corrispondenza affettiva (risposta emotiva all'informazione, gioia, frustrazione, incertezza), la corrispondenza a un'opinione (opinione personale che si dà all'informazione, fiducia) $)^{35}$.

\footnotetext{
${ }^{35}$ Tefko Saracevic, Relevance: a review of the literature [...] Part III cit., p. 2130.
} 
Gli utenti non usano un solo criterio per volta, ma li combinano, usandoli in modo interattivo, come accade nel modello disegnato da Saracevic ${ }^{36}$ nel quale avviene l'interazione tra le caratteristiche degli oggetti informativi, (le prime tre nell'elenco) e quelle dell'utente.

Tra le caratteristiche, i criteri orientati al contenuto sono quelli che prevalgono, ma in interazione con altri criteri.

Una delle prime considerazioni che vanno fatte nella considerazione del concetto di rilevanza è che il giudizio di rilevanza non dipende solo dalla corrispondenza del risultato con un particolare argomento espresso nella query quindi non esiste esclusivamente la rilevanza topica, ma vari altri attributi giocano un ruolo fondamentale.

\subsubsection{Un modello di information retrieval: Saracevic stratified model (1996)}

La rilevanza è dunque un fenomeno complesso che coinvolge una serie di aspetti e di variabili che interagiscono tra loro e in cui sono coinvolti processi, strutture, sistemi, fenomeni e concetti. Il concetto di rilevanza si è modificato nel tempo grazie all'evoluzione dei modelli di information retrieval verso paradigmi più interattivi capaci di rappresentare le diverse variabili legate non solamente al giudizio di rilevanza ma all'intero processo di recupero dell'informazione.

Uno dei modelli che rappresentano la complessa interazione che avviene tra l'utente e il sistema è quello elaborato da Saracevic ${ }^{37}$ che propone un modello 'stratificato' nel quale i diversi elementi vengono rappresentati come un numero di strati o livelli interconnessi e le inferenze sulla rilevanza sono create o derivate nel processo di interazione tra diversi livelli o strati.

I presupposti alla base del modello proposto da Saracevic sono che l'utente interagisce con sistemi di IR per trovare le informazioni e che l'uso dell'informazione è connesso alla conoscenza, alle applicazioni e al contesto che, a sua volta, è connesso con la rilevanza. I principali elementi del modello proposto da Saracevic sono dunque l'utente e il computer, ognuno con una serie di variabili proprie, che dialogano o vengono in contatto in

\footnotetext{
${ }^{36}$ Tefko Saracevic, The stratified model of information retrieval interaction: extension and applications, «Proceedings of the American Society for Information Science», 34 (1997), p. 313-327.

${ }^{37}$ Tefko Saracevic, The stratified model of information retrieval interaction cit.
} 
uno strato superficiale, rappresentato dall'interfaccia (surface level). Gli strati, i quali rappresentano gli elementi variabili e i processi coinvolti nell'interazione, non sono inclusivi l'uno dell'altro e non formano al loro interno gerarchie ma sono governati da relazioni molto più complesse.

Figure 1. Elements in the stratified model of IR interaction

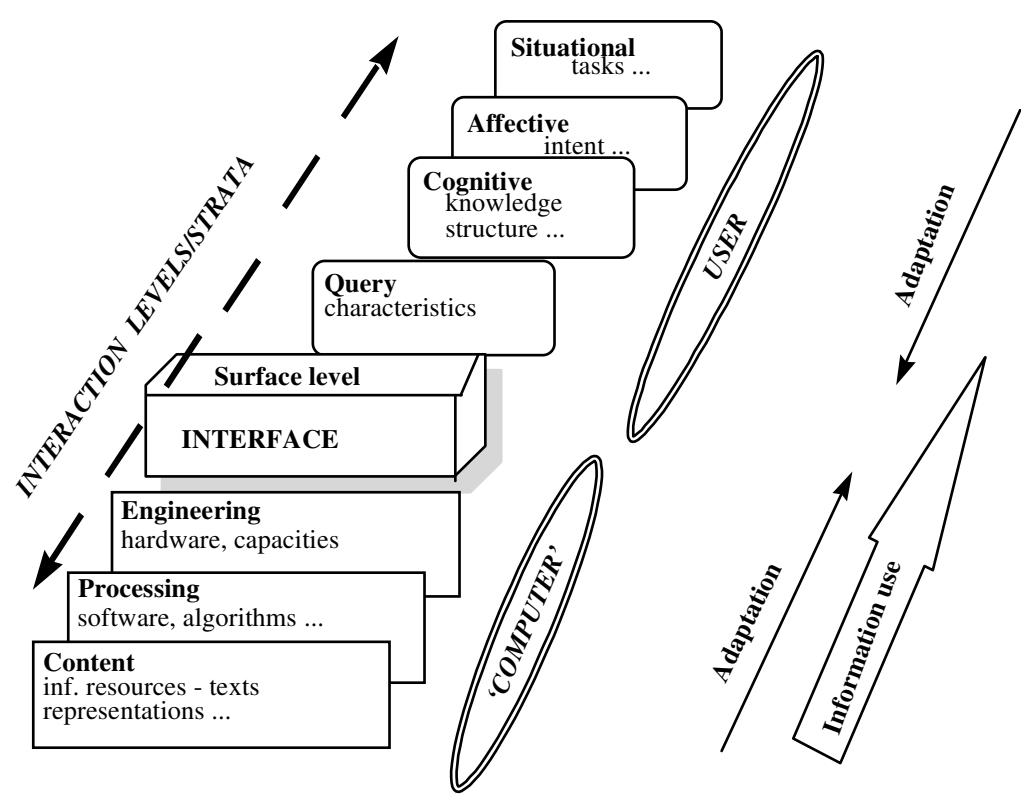

Fig. 5.3 - Il 'modello stratificato della rilevanza' di Saracevic ${ }^{38}$

Dal lato dell'utente troviamo i livelli cognitivo, affettivo e situazionale; dal lato computer gli strati sono individuati nell'ingegneria (hardware), nell'elaborazione (software utilizzato) e nel contenuto cioè nelle risorse informative contenute nel sistema. Il modello è aperto ad adattamenti che possono dipendere dal variare del contesto o da aspetti che riguardano i computer, come l'interfaccia, i software e gli algoritmi utilizzati e le risorse informative rappresentate. In particolare, il contesto nel modello può consistere in un aspetto sociale (organizzativo, istituzionale, riguardante una data comunità) o culturale (es. storico). Ciò che è importante rilevare è che il contesto determina il problema che si vuole esaminare o risolvere.

\footnotetext{
${ }^{38}$ Thomas D. Wilson, Models in information behaviour research, «Journal of Documentation», 55 (1999), n. 3, p. 249-270, disponibile anche all'URL: <http://informationr.net/tdw/publ/papers/1999JDoc.html>.
} 
Nel modello stratificato di Saracevic dunque l'interazione in un sistema di IR può essere immaginata come un dialogo tra vari partecipanti - gli elementi associati all'utente e quelli associati al computer- attraverso l'interfaccia, allo scopo primario di modificare lo stato cognitivo dell'utente tramite l'uso effettivo dell'informazione rilevante recuperata, in relazione al problema che si vuole esaminare e che deriva dal contesto. Tale dialogo può essere reiterato, incorporare vari tipi di feedback e mostrare al suo interno schemi che possono essere oggetto di studio. Ogni strato implica diversi e specifici processi che dal lato umano possono essere affettivi, psicologici e cognitivi, e dal lato-macchina fisici o simbolici.

L'interfaccia fornisce un livello di superficie nel quale si svolge tale interazione: gli utenti istaurano il dialogo tramite delle espressioni (i comandi impartiti al sistema) e ricevono risposte (espresse dai computer) attraverso l'interfaccia nella quale non avviene solo la ricerca e il matching (come nel modello tradizionale di IR), ma si istaurano una serie di processi o "cose" che avvengono oltre il semplice 'cerca-e-confronta', come la comprensione e la deduzione di un dato componente del computer o di una risorsa informativa, il browsing, la navigazione, la visualizzazione dei risultati, la ricezione di vari tipi di feedback, la creazione di giudizi e valutazioni etc. I computer a loro volta dialogano con l'utente attraverso processi e meccanismi logici propri e forniscono risposte al dialogo, con deduzioni o richieste alle risposte dell'utente.

Ulteriori considerazioni sulla rilevanza vengono tratti da altri studi: è stato dimostrato che valutare un documento in termini di rilevante/non rilevante non fornisce una misura reale della valutazione dell'utente provando che il giudizio di rilevanza non è un valore binario. Rees e Schultz alla fine degli anni '60 hanno elaborato una scala a 11 punti rappresentata graficamente da ben 11 intervalli di rilevanza che vanno dal documento completamente irrilevante a quello estremamente rilevante ${ }^{39}$.

Indagare a fondo i criteri in base ai quali gli utenti stabiliscono la rilevanza fornisce, dunque, un quadro più completo del processo complessivo di attribuzione della rilevanza; e consente anche di cogliere utili suggerimenti su come migliorare i sistemi.

Un secondo importante dato è che il giudizio di rilevanza non è 'indipendente'; spesso la valutazione della rilevanza di un documento dipende da cosa si è esaminato in precedenza.

\footnotetext{
${ }^{39}$ Alan M. Rees - Douglas O. Schultz, A field experiment approach to the study of relevance assessments in relation to document searching: Final report to the National Science Foundation, Cleveland, Case Western Reserve University, Center for Documentation and Communication Research, 1967, p. 87-91.
} 
Un ulteriore elemento di grande importanza è che la rilevanza è 'dinamica'. Più si va avanti nel progresso di una ricerca, più si focalizza un argomento, tanto più cambiano i fattori in base ai quali viene dedotta la rilevanza. Più l'argomento è chiaro, più la discriminazione migliora ed è possibile compiere deduzioni più stringenti.

La consapevolezza della dimensione dinamica della rilevanza non è recente. Già nel 1967, Cuadra e Katter ${ }^{40}$ individuavano la difficoltà di controllare tutte le variabili che potevano influenzare il giudizio di rilevanza da parte dell'utente perché esse potevano cambiare nel tempo. La questione viene sollevata anche da Kuhlthau in una critica alle misurazioni classiche della rilevanza. Troppo spesso l'informazione era stata concepita come 'un prodotto da fornire', come 'la risorsa giusta' o 'il risultato esatto' e la valutazione affidata alla singola risposta a una specifica domanda misurata in termini di accuratezza.

Le valutazioni misurate in termini di richiamo e precisione sono state riconosciute come inadeguate a valutare il processo di apprendimento nel quale gli utenti sono coinvolti quando cercano l'informazione; i giudizi di rilevanza basati su queste misure possono essere adeguati per valutare una singola risposta ad una domanda, ma quando l'utente è coinvolto nel processo dinamico di informarsi la rilevanza non rimane statica. Ciò che è rilevante al principio della ricerca, può diventare irrilevante nel prosieguo, e viceversa. Così il concetto di rilevanza come entità statica, limita profondamente il processo dinamico della formulazione di un problema di ricerca e il processo di apprendimento su un particolare argomento ${ }^{41}$.

La dimensione dinamica della rilevanza è messa in rilievo anche dai recenti studi di Taylor, Zhang e Amadio ${ }^{42}$; gli studiosi esaminano i cambiamenti nella valutazione della rilevanza e durante il processo di ricerca dell'informazione ${ }^{43}$. I dati raccolti dai loro test

\footnotetext{
${ }^{40}$ Carlos Cuadra, Experimental studies of relevance judgments: final report. Vol. 1: Project summary, Santa Monica, System Development Corp., 1967.

41 Carol Collier Kuhlthau, Seeking meaning: a process approach to library and information services, Westport, Libraries Unlimited, 2004, p 3.

42 cfr.: Arthur Taylor - Xiangmin Zhang - William J. Amadio, Examination of relevance criteria choices and the information search process". «Journal of Documentation», 65 (2009), n. 5, p. 719 - 744 .

43 Cfr. anche Arthur Taylor, User relevance criteria choices and the information search process, «Information Processing and Management», 48 (2012), n. 1, p. 136-153. L'autore prende in considerazione vari elementi tra i quali: accuracy, advertisement, affectiveness, authority, bias, breadth of subject area, definitions, history, novelty, recency, source, structure, time, tips, topic, understandability, etc.Si tratta di aspetti che gli utenti considerano importanti nel giudizio di rilevanza. Essi possono riguardare il contenuto di un documento ( $\mathrm{ad}$ esempio, il documento appartiene alla mia area di indagine, fornisce un buon approfondimento sull'argomento contiene linee guida o direttive, contiene notizie storiche o di base sull'argomento, contiene consigli e istruzioni, contiene informazioni recenti e aggiornate; il documento copre molti argomenti nell'area indagata; contiene definizioni avanzate/di base, è un avviso o una pubblicità) la sua validità (il documento contiene un'informazione accurata, l'autore è un esperto in materia, proviene da una fonte - es. una rivista, sito web - che ha una buona reputazione nell'area di indagine; l'autore prende una posizione o ha una specifica opinione) l'oggetto (la struttura che rende più facile da leggere e comprendere) la 'corrispondenza cognitiva' (è di facile comprensione, aggiunge nuove informazioni a quelle già in mio
} 
confermano la relazione tra i criteri di rilevanza scelti dagli utenti e il processo di ricerca dell'informazione, cioè la fase della ricerca nella quale essi si trovano; man mano che gli utenti avanzano nella ricerca, cambiano i criteri in base ai quali determinano la rilevanza e alcuni criteri si confermano più importanti in alcune fasi piuttosto che in altre. Gli utenti dimostrano una preferenza per alcuni criteri come la capacità di comprensione, la chiarezza, l'ampiezza/profondità dell'argomento trattato, la precisione e la specificità; tali criteri crescono di importanza nel corso della ricerca.

\subsubsection{Misurare la rilevanza: algoritmi e sviluppi dell'information retrieval}

Il principale obiettivo degli studi sull'information retrieval è la misurazione della rilevanza nel recupero dell'informazione al fine di migliorare i sistemi. Dato che la maggior parte delle interfacce di ricerca prevede la formulazione di un'interrogazione in forma testuale, questi studi si concentrano sulle modalità di rappresentazione dei documenti ai fini del loro recupero e affrontano le tematiche dell'indicizzazione manuale e automatica ovvero dello sviluppo di algoritmi e di tecniche che consentono di convertire i documento in insiemi di termini di indice utilizzabili per il recupero dell'informazione. Si tratta dunque di un filone di studio che prende a riferimento principalmente i sistemi e si basa sullo studio dei termini di ricerca e sulla valutazione dei risultati in termini di rilevanza. Nell'ambito di questo filone di studi sono stati elaborati modelli di accesso o di interrogazione che esplorano cosa avviene all'interno dei sistemi di recupero e che vengono comunemente chiamati modelli di information retrieval.

Per recuperare l'informazione, i sistemi di IR usano vari modelli di interrogazione che si sono evoluti nel tempo ${ }^{44}$. Tali modelli di retrieval assegnano una misura di similarità tra una query e un documento. I tre modelli 'classici' sono quello booleano, quello a spazio vettoriale e il modello probabilistico ${ }^{45}$.

I primi sistemi utilizzavano il modello booleano, che permetteva agli utenti di specificare il proprio bisogno informativo immettendo interrogazioni i cui termini dovevano essere collegate attraverso gli operatori booleani AND, OR, NOT. Tali sistemi

possesso), affettiva (è piacevole) o situazionale (il documento è utile in base al tempo disponibile per la ricerca).

${ }^{44}$ Per una panoramica dei modelli di accesso all'informazione si veda: Edie Rasmussen, Access models in Ian Interactive information retrieval edited by Ian Ruthven and Diane Kelly, London, Facet, 2010, p. 95-111.

${ }^{45}$ Si veda: Christopher D. Manning - Prabhakar Raghavan -Hinrich Schütze, Introduction to Information Retrieval, Cambridge University Press, 2008. Anche online all’URL: <http://nlp.stanford.edu/IR-book/>. 
avevano numerosi difetti: oltre a non permettere l'ordinamento dei risultati per rilevanza, richiedevano all'utente la conoscenza degli operatori, necessaria a formulare correttamente una query. Con questi sistemi era possibile un ordinamento dei risultati per data, o in base qualche altro dato del documento, ma non era possibile quantificare o limitare il numero di documenti recuperati.

Successivamente, la necessità di ordinare i documenti secondo una misura di utilità per l'utente, corrispondente alla rilevanza del documento, ha condotto allo sviluppo di sistemi più sofisticati, in base all'idea che era possibile assegnare un punteggio al documenti in base alla presunta rilevanza per l'utente. I due principali modelli in grado di misurare la rilevanza che si affermarono furono il modello a spazio vettoriale e il modello probabilistico.

Nel modello a spazio vettoriale elaborato alla fine degli anni '80 da Gerard Salton e dai suoi studenti ${ }^{46} \mathrm{i}$ testi vengono rappresentati come vettori di termini (parole o frasi). Se vengono scelte come termini le parole, ogni parola sarà una dimensione indipendente nello spazio vettoriale. Seguendo l'idea di assegnare un punteggio numerico ad un documento in relazione alla query effettuata, il modello misura la somiglianza tra il vettore generato per la query (convertendo dunque la query in un vettore) e il vettore calcolato per il documento. La similarità tra vettori viene tipicamente calcolata misurando la distanza (in termini di convergenza/divergenza) tra i due vettori cioè l'angolo formato usando le misura numerica del coseno ${ }^{47}$.

L'idea del recupero probabilistico venne originariamente proposta da Maron e Kuhns nel $1960^{48}$. L'insieme dei modelli probabilistici è basato sul principio generale che $\mathrm{i}$ documenti di una base dati possano essere ordinati in ordine decrescente in base alla probabilità della loro rilevanza per una determinata query. Il probability ranking principle $(P R P)$ venne proposto qualche anno dopo da Robertson ${ }^{49}$ :

If a reference retrieval system's response to each request is a ranking of the documents in the collection in order of decreasing probability of relevance to the user who submitted the request, where the probabilities are estimated as accurately as possible on the basis of whatever data have been made available to the system for this purpose, the overall

\footnotetext{
${ }^{46}$ Gerard Salton, Automatic text processing: the transformation, analysis, and retrieval of information by computer, Reading, Addison-Wesley, 1988.

${ }^{47}$ La funzione trigonometrica del coseno si rivela una misura efficace dato che è 1.0 per i vettori identici e and 0.0 per quelli ortogonali.

48 Melvin E. Maron - John L. Kuhns, On relevance, probabilistic indexing and information retrieval, «Journal of the ACM», 7 (1960) n, 3, p. 216-244.

${ }^{49}$ Stephen E. Robertson, The probability ranking principle in IR, «Journal of Documentation», 33 (1977) p. 294-304.
} 
effectiveness of the system to its user will be the best that is obtainable on the basis of those data $^{50}$.

I modelli probabilistici stimano la probabilità di rilevanza dei documenti in base alla query e differiscono l'un l'altro in base alle tecniche di stima che vengono utilizzate.

La valutazione dell'efficacia di un sistema di information retrieval gioca un ruolo primario nelle ricerche sul recupero dell'informazione. I test di Cranfield condotti a partire dal 1960stabilirono una serie di caratteristiche dei sistemi di recupero dell'informazione e proposero le misure che più tardi vennero comunemente accettate dalla comunità di studiosi come le più adatte a valutare la qualità dei sistemi: il richiamo (recall) e la precisione (precision) ${ }^{51}$. Il richiamo è la proporzione dei documenti rilevanti recuperata dal sistema, la precisione è la proporzione dei documenti recuperati che risultano rilevanti. Un sistema efficace dovrebbe avere gradi alti di richiamo e precisione, recuperando più documenti possibile riducendo la presenza di documenti non rilevanti. Le due misure del richiamo e della precisione però, come è stato poi dimostrato, si contraddicono, ad un alto grado di richiamo corrisponde un indebolimento della precisione e viceversa, dunque i progettisti bilanciano le due misure per ottenere il grado di precisione o di richiamo desiderati. Tra le misure utilizzate la precisione media valuta il grado di precisione in un certo intervallo di documenti recuperati.

Le principali tecniche che consentono di implementare un sistema di recupero dell'informazione fanno riferimento alla cosiddetta pesatura dei termini del documento e alla modifica della query tramite il relevance feedback. La maggior parte dei sistemi di information retrieval fa uso di uno schema per la pesatura dei termini e di specifiche per l'espansione della query. In entrambi i modelli vettoriale e probabilistico le tre principali misure si basano 1) sul computo della frequenza del termine (TF), cioè delle volte in cui un termine appare nel documento, considerando rilevanti quei documenti nei quali la parola appare più spesso, misura però non sufficiente da sola ad indicare la rilevanza del documenti; 2) sulla frequenza inversa (inverse document frequency o IDF), proposta negli anni '70 da Stephen Robertson e Karen Spärck Jones ${ }^{52}$ tesa a controbilanciare la frequenza del termine secondo l'idea che le parole molto comuni e che compaiono in molti documenti non sono realmente indicative del contenuto del documento e che viene misurata

\footnotetext{
${ }^{50}$ Ivi, p. 295.

${ }^{51}$ I 'test di Cranfield' condotti dal Cranfield Institute of Technology erano volti a stabilire criteri e misure per valutare i sistemi di recupero dell'informazione. Sotto l'influenza di Cyril Cleverdon, a capo del progetto, la rilevanza divenne il principale criterio per la valutazione dei sistemi, misurata nei termini di recall e precision.

52 Stephen E. Robertson- Karen Spärck Jones, Relevance weighting of search terms, «Journal of the American Society for Information Science», 27 (1976), n. 3, p. 129-146.
} 
calcolando il rapporto tra il numero dei documenti presenti nella base dati e il numero dei documenti nei quali è stata rilevata la presenza del termine cercato; 3) sulla lunghezza del documento, ulteriore computo teso a perfezionare la pesatura evitando che i documenti più lunghi poiché contengono numerose ripetizioni del termine siano considerati e possano inficiare la misura della rilevanza.

Con l'avvio delle conferenze TREC $^{53}$ i modelli di recupero vettoriale e probabilistico hanno avuto la possibilità di essere testati nel recupero di grandi quantità di documenti, consentendo lo sviluppo di tecniche di pesatura dei termini (term weighting) e l'elaborazione di schemi più sofisticati. Alla City University di Londra, un gruppo di studiosi diretti da Robertson perfezionò uno schema di pesatura nell'ambito del modello probabilistico (Okapi weighting ${ }^{54}$ ) e alla Cornell University fu elaborata una particolare funzione logaritmica per la pesatura dei termini (TF) e, successivamente, vari schemi per migliorare le tecniche di pesatura dei termini tenendo conto della lunghezza del documento (Pivoted normalization weighting $)^{55}$. Comunemente oggi si usa la frase pesatura tf-idf (tfidf weighting) per riferirsi a qualsiasi tecnica utilizzi queste misure, dimostrando che il computo basato sul rapporto TF -IDF introdotto da Robertson e Spärck Jones ha mantenuto una straordinaria solidità.

${ }^{53}$ Le conferenze TREC (Text REtrieval Conference), nascono su iniziativa del National Institute of Standards and Technology (NIST) e del Dipartimento della Difesa degli Stati Uniti (U.S. Department of Defense) nel 1992 come parte del TIPSTER Text program, un progetto finanziato dal Defense Advanced Research Projects Agency (DARPA), l'agenzia governativa del sopracitato dipartimento che si occupa dello sviluppo delle tecnologie ad uso militare, per sostenere l'avanzamento delle correnti tecnologie per la manipolazione dei testi, il recupero testuale, la 'sommarizzazione' e l'estrazione di informazione dai documenti. Lo scopo di TREC è di sostenere la ricerca delle comunità di studiosi che si occupano di information retrieval fornendo l'infrastruttura necessaria per la valutazione su larga scala delle metodologie di information retrieval. Gli obiettivi dei convegni TREC sono più specificamente: il supporto alla ricerca su ampie collezioni di testi, l'incremento della comunicazione tra settori diversi (industria, università, organi governativi) tramite la creazione di un ambiente di discussione aperto allo scambio di idee sulla ricerca, l'incoraggiamento al trasferimento delle tecnologie dai laboratori di ricerca ai prodotti commerciali per il miglioramento delle metodologie di recupero dell'informazione applicate a problemi reali e, infine, la messa a disposizione di tecniche di valutazione e di nuove tecniche applicabili agli attuali sistemi a vantaggio dell'industria e delle università, (cfr: <http://trec.nist.gov/overview.html>). Le conferenze si articolano seguendo vari tracks cioè vari percorsi corrispondenti alle aree di interesse sulle quali si concentrano le ricerche. Nel 2003 erano 93 i gruppi che hanno partecipato all'iniziativa in rappresentanza di 22 nazioni.

${ }^{54}$ Sugli esperimenti di Robertson si veda anche: Stephen E. Robertson - Micheline Hancock-Beaulieu, On the evaluation of IR systems, «Information Processing \& Management», 28 (1992) n. 4, p. 457-466. Nell'articolo gli studiosi, di fronte ai nuovi filoni cognitivi della Scienza dell'informazione, discutono quanto sia difficoltosa una reale valutazione dell'efficacia dei sistemi di information retrieval.

${ }^{55}$ Entrambe le misurazioni prendono in considerazione i seguenti elementi:

* If frequenza del termine nel documento

* qtf frequenza del termine nella query

* $\quad N$ numero totale dei documenti presenti nella collezione

* df numero dei documenti che contengono il termine

* dl lunghezza del documento (espressa in bytes),

* avdl lunghezza media del documento 
La seconda tecnica introdotta per migliorare il recupero dell'informazione e ovviare alle difficoltà degli utenti di inserire i termini corretti per l'interrogazione è il relevance feedback.

Ideato da Rocchio nel $1971^{56}$, si basava sull'idea di modificare la query di partenza in base al giudizio di rilevanza /non rilevanza fornito dall'utente stesso nel corso della sua ricerca. Usando il feedback ricevuto il sistema poteva automaticamente generare una nuova query collegando termini correlati per lanciare una nuova ricerca. Invitando l'utente a giudicare i primi risultati proposti dal sistema, esso genera automaticamente una nuova query (query expansion) per trovare documenti maggiormente rilevanti, calcolando una rilevanza su quei documenti o servendosi, ad esempio nel caso di cataloghi elettronici dei metadati di soggetto/classe assegnati al documento. La necessità di non caricare l'utente dello sforzo aggiuntivo di fornire manualmente il feedback richiesto ha poi condotto alla sperimentazione tecniche di query expansion che agiscono anche in assenza di feedback specificati da parte dell'utente. Le tecniche di pseudo-feedback, efficaci soprattutto per le query molto brevi, sono automatiche e agiscono presupponendo che i primi risultati che vengono generati in risposta all'interrogazione siano rilevanti per l'utente; usano dunque le informazioni contenute nei documenti stessi per generare una nuova query, che viene impiegata per ordinare $\mathrm{i}$ documenti che vengono presentati all'utente in risposta all'interrogazione.

Le più recenti evoluzioni del relevance feedback riguardano l'utilizzo dei cluster, cioè di raggruppamenti generati automaticamente dai sistemi in base alle caratteristiche comuni dei documenti contenuti in una base dati, tecnica che però ha avuto più successo nelle interfacce (in particolare nei siti web e negli OPAC di nuova generazione) per sviluppare nuove modalità di navigazione e di browsing. Un uso interessante di questa tecnica è quello di utilizzare per l'espansione della query i raggruppamenti generati automaticamente da dizionari o ontologie sviluppate al di fuori delle basi dati, ad esempio, in ontologie lessicali come WordNet ${ }^{57}$, e che consentirebbero all'utente di allargare la ricerca ai termini sinonimi e collegati al termine/ai termini prescelti, espandendo la ricerca

\footnotetext{
${ }^{56}$ Joseph J. Jr. Rocchio, Relevance feedback in information retrieval, in Gerard Salton, The SMART retrieval system: experiments in automatic document processing, Prentice Hall, 1971, p. 313-323.

$57<$ http://wordnet.princeton.edu/>WordNet ${ }^{\circledR}$ è un database lessicale per la lingua inglese. Nomi, verbi, aggettivi e avverbi sono raggruppati in insiemi di sinonimi (cognitive synonyms o synsets), ognuno dei quali esprime un concetto distinto. Tali synsets sono collegati tra di loro per mezzo di relazioni concettualisemantiche e lessicali. Si crea così una rete di termini e concetti correlati semanticamente tra loro che può essere esplorata tramite la navigazione in un browser.
} 
ai concetti correlati al termine di partenza, ad esempio tutti i termini dei concetti che fanno parte di una determinata classe ${ }^{58}$.

\subsubsection{La rilevanza nel web}

I motori di ricerca determinano la rilevanza secondo alcuni fattori che possono essere suddivisi nelle due grandi categorie dei fattori dipendenti e indipendenti dalla query immessa nel sistema. I primi dipendono strettamente dall'interrogazione immessa, gli altri riguardano fattori esterni o associati esternamente al documento ${ }^{\mathbf{5 9}}$.

Tra i primi ritroviamo alcuni calcoli statistici propri dei sistemi di information retrieval quali la frequenza della parola cercata nel documento, la distanza e l'ordine dei termini di ricerca immessi. La posizione dei termini di ricerca nel documento porta a preferire quei documenti nei quali il termine è posto in punti chiave del documento es. il titolo, i titoli dei paragrafi (rilevati attraverso i marcatori di formattazione per il grassetto o il corsivo) e i nei metatag o l'IDF (inverse document frequency). Altri criteri presi in considerazione sono la preferenza dei testi che appaiono nelle ancore o criteri che ricorrono a fattori linguistici (verranno privilegiati nel recupero ad esempio i documenti che sono scritti nella lingua usata nell'interfaccia) o a forme di geo-localizzazione, dando un peso maggiore alle pagine web che sono più vicine al luogo dell'utente ${ }^{60}$.

I criteri indipendenti dal documento vengono usati allo scopo di determinare la qualità di un documento. La considerazione di questi elementi nella formazione degli algoritmi segnò il passaggio dalla prima generazione di motori di ricerca, i cui algoritmi erano deboli e facilmente aggirabili, ad una nuova generazione di motori, volti a migliorare la qualità delle risorse recuperate.

Tra questi il più noto è l'algoritmo di PageRank, brevettato alla Stanford University ${ }^{61}$ dai fondatori di Google per l'omonimo motore di ricerca e rivolto a misurare la popolarità

\footnotetext{
${ }^{58}$ Maria Teresa Biagetti, Pertinence perspective and OPAC enhancement cit.

${ }^{59}$ Dirk Lewandowski, Web searching, search engines and information retrieval, «Information Service and Use» 25 (2005), n. 3/4 p. 137-147.

${ }^{60} \mathrm{~L}$ 'operazione è resa possibile identificando l'indirizzo IP della macchina da cui proviene l'interrogazione.

${ }^{61}$ La formula dell'algoritmo per il calcolo del PageRank, derivata dalla teoria dei processi di Markov, fu sviluppata dai fondatori di Google, Sergey Brin e Larry Page e fa parte degli algoritmi di Link Analysis Ranking. Cfr.: Sergey Brin - Lawrence Page, The anatomy of a large-scale hypertextual web search engine, Seventh International World-Wide Web Conference (WWW 1998), April 14-18, 1998 Brisbane, Australia, [risorsa online], <http://infolab.stanford.edu/ backrub/google.html>.
} 
di una pagina $w^{6} b^{62}$. Il peso che l'algoritmo assegna ad una risorsa web dipende dal numero di pagine web che puntano al documento ed è volta a misurare la popolarità di un link all'interno di un insieme di pagine rilevanti per una determinata query. L'algoritmo di Google è stato perfezionato nel tempo e oggi il motore prende in considerazione nella determinazione della rilevanza oltre 200 variabili incluso il Pagerank.

Altri fattori indipendenti dalla query sono il numero di click che un documento riceve nella pagina dei risultati, o il posizionamento nella gerarchia delle directories web, la provenienza, la lunghezza e il formato del documento e l'aggiornamento.

Miglioramenti recenti introducono misurazioni basate sulla pertinenza prendendo in considerazione alcuni fattori come le pagine precedentemente visitate dall'utente.

Anche le tecniche di relevance feedback vengono oggi comunemente usate nel web per fornire all'utente suggerimenti d'acquisto, proponendo articoli simili. Tra i primi siti web che ne hanno sfruttato le potenzialità la libreria on-line Amazon, che ricorre alle tecniche di pseudo-feedback o feedback implicito per suggerire all'utente nuovi prodotti in base a quelli precedentemente acquistati dall'utente stesso o dagli altri utenti che usano la piattaforma di e-commerce.

\subsubsection{La rilevanza negli OPAC}

Tra gli strumenti utilizzati per apportare un significativo miglioramento alle possibilità di ricerca negli OPAC il relevance ranking, cioè l'ordinamento dei risultati del catalogo in ordine di rilevanza, è una delle principali caratteristiche introdotte di recente nei cataloghi che si definiscono di nuova generazione.

Il relevance ranking, proveniente dagli algoritmi messi a punto dai motori di ricerca per ordinare le pagine web in ordine di rilevanza rispetto alla richiesta effettuata presentando i risultati più rilevanti in cima alla lista, è una modalità di ricerca ormai familiare agli utenti del web e desiderata in tutte le interfacce per la ricerca.

Come è accaduto nel web, dove l'ordinamento per ranking ha consentito di filtrare una massa critica di pagine web rendendole facilmente recuperabili tramite i motori di ricerca, anche nel campo degli OPAC, lo sviluppo di grandi cataloghi collettivi e di metaOPAC reso possibile dallo sviluppo delle tecnologie, ha notevolmente complicato l'interrogazione dei cataloghi rendendola più complessa rispetto al passato.

${ }^{62}<\mathrm{http}: / /$ www.google.com/about/corporate/company/tech.html . 
Infatti, molto spesso un'interrogazione genera una lista di occorrenze molto lunga e, in mancanza di meccanismi di raggruppamento, che potrebbero essere risolti dall'implementazione del modello FRBR, ci si ritrova a scorrere molte schermate con il risultato che l'utente allunga notevolmente i tempi della ricerca e spesso non trova ciò che sta cercando o si accontenta di ciò che viene proposto nelle prime pagine dei risultati.

Non a caso la letteratura annovera vari contributi ${ }^{63}$, confortati da indagini sull'utenza, che hanno considerato l'ordinamento per rilevanza tra le modifiche più urgenti da introdurre nei cataloghi elettronici ${ }^{64}$.

L'applicazione di meccanismi di ranking, nonostante l'apparente semplicità che essa manifesta, presenta molti problemi complessi. Prima di tutto bisogna chiedersi in che modo gli OPAC oggi determinano la rilevanza, poi bisogna comprendere se la rilevanza offerta, alla luce delle teorie sopra esposte, sia un mezzo realmente efficace per esporre i risultati di un'interrogazione, o se gli OPAC debbano, invece, orientarsi verso altre dimensioni della rilevanza, al di là di quella topica e algoritmica.

La rilevanza viene comunemente misurata attraverso algoritmi che prendono in considerazione le due principali misure precedentemente illustrate di Term frequency (TF) e Inverse document frequency (IDF).

La prima si riferisce alla frequenza dei termini nel fulltext di un documento oppure nel record catalografico; mentre la seconda prende in considerazione la frequenza dei termini nella raccolta di documenti rappresentata nell'OPAC, assegnando un peso più alto nel calcolo complessivo ai termini che ricorrono con meno frequenza,

Rispetto all' informazione fulltext, il recupero dei dati bibliografici negli OPAC introduce alcune complicazioni: non essendo fondata sull'analisi dei testi ma dovendo

\footnotetext{
${ }^{63}$ Cfr. supra, cap. 1.

${ }^{64}$ L'applicazione del relevance ranking agli OPAC è stata consigliata da John D. jr. Byrum, (Recommendations for urgently needed improvement of OPAC cit.), Karen Markey (Karen Markey, The online library catalog. Paradise lost and paradise regained?cit.), You e Young (cfr. Holly Yu-Margo Young, The impact of Web search engines on subject searching in OPAC, «Information Technology And Libraries», 23 (2004), $\quad$ n. $4, \quad$ p. $168-180, \quad$ anche in <http://www.ala.org/ala/lita/litapublications/ital/volume23a/number4a/yu.pdf>) è invece vivamente sconsigliato da Martha M. Yee (Martha M. Yee, Beyond the Opac: future directions for Web-based catalogues, [Relazione presentata al seminario Beyond the Opac: future directions for Web-based catalogues. Perth, Western Australia, settembre 2006] <http://www.nla.gov.au/lis/stndrds/grps/acoc/papers2006.html>che in merito si esprime così : «Avoid relevancy ranking. Computers cannot understand what a human being is looking for. Computers cannot understand which item in the collection is most likely to help a given user-even another human being can't necessarily do this; the user must be in control!». C'è persino chi definisce il relevance ranking il pilastro della ricerca: «relevance ranking - the building block of search, found in any search engine, from Google to Amazon to Internet Movie Database to little old Librarians' Internet Index» (cfr. Karen G. Schneider, How OPACs suck, Part 1: relevance rank (or the lack of it), 13 March 2006 [blog post] $<$ http://www.techsource.ala.org/blog/2006/03/how-opacs-suck-part-1-relevance-rank-or-the-lack-ofit.html>).
} 
considerare i surrogati dei testi stessi costituiti dalle registrazioni bibliografiche, vi è la necessità di adattare gli schemi per la pesatura in modo che alcune parti del record siano considerate più significative rispetto ad altre.

Il primo e più noto OPAC di nuova generazione ad aver introdotto l'ordinamento per rilevanza dei risultati è l'OPAC della North Carolina State University ${ }^{65}$. Il motore di ricerca di Endeca (Endeca MDEX) può essere programmato per effettuare il recupero con varie tecniche (e.g., matchall, matchany, matchboolean, matchallpartial). L'implementazione scelta per NCSU usa la tecnica di recupero match-all per la ricerca per parola chiave, correlando le parole immesse con l'operatore AND e recuperando i risultati che contengono tutte le parole chiave immesse o la loro forma corretta (spell-corrected) o troncata. Dunque non è necessario introdurre operatori booleani; essi vengono compresi tra le stopword cioè tra i termini ignorati dal sistema, lasciando all'utente la possibilità di utilizzare gli operatori nella ricerca avanzata. Anche se i classici modelli di recupero tendono ad associare il ranking alla ricerca probabilistica o basata su vettori, Endeca include una gamma di opzioni che possono essere applicate alla ricerca e personalizzate in base alle esigenze della singola biblioteca per ottenere algoritmi personalizzati. Ad ogni indice di ricerca creato in Endeca può essere assegnato un diverso peso nell'algoritmo di ranking. Nella ricerca l'algoritmo dà priorità ai termini esattamente come vengono immessi (non vengono considerati la correzione dello spelling, la corrispondenza a termini di un tesauro, o il troncamento). Per le ricerche contenenti più di un termine i risultati che contengono la frase esatta vengono considerati più rilevanti. L'algoritmo creato da NCSU considera la frequenza dei termini e la frequenza inversa e ha definito una priorità basata sui campi del record, considerando più rilevanti quei risultati nei quali la corrispondenza avviene nel campo del titolo rispetto ad altri campi del record, ad esempio quello delle note.

È allo studio l'applicazione di tecniche più avanzate come il matchallpartial retrieval che non sono vere e proprie tecniche probabilistiche o vettoriali, ma permettono di ampliare una ricerca proponendo termini affini, in caso di risultato zero. Altre tecniche associate alla ricerca per campi come la correzione dello spelling vengono usate dal catalogo Endeca per migliorare il recupero. L'autocorrezione dello spelling utilizza un approccio basato sugli indici e non sulla presenza di un vocabolario. Il relevance ranking però è stato configurato per visualizzare il risultato in base alla query introdotta, e anche in

\footnotetext{
${ }^{65}$ Cfr. infra, Appendice A. Casi di studio. L'implementazione del software nelle biblioteche della North Carolina State University è stata esposta in Kristin Antelman, - Emily Lynema - Andrew K. Pace, Toward a Twenty-first Century Library Catalog, «Information Technology \& Libraries», 25 (2006), n. 3 p. 128-139 oppure: <http://eprints.rclis.org/archive/00007332/01/antelman_lynema_pace.pdf>.
} 
presenza di errori, viene restituito il risultato, dando in un secondo momento la possibilità di re-immettere la query in base al suggerimento fornito dal sistema. Tra le opzioni di ordinamento anche quella per item più popolari.

È ormai una caratteristica distintiva dei nuovi OPAC quella di offrire l'ordinamento per ranking di default, ma non sempre i meccanismi che regolano la formazione degli algoritmi vengono esposti in maniera chiara all'utente.

Alcuni esempi virtuosi in questo senso sono quelli dell'OPAC delle biblioteche dell'Università Cattolica ${ }^{66}$ e dell'OPAC sperimentale australiano ${ }^{67}$ dove troviamo presentazioni chiare e ricche di dettagli tecnici.

Nell'OPAC WebpacPro dell'Università Cattolica (fig. 5.4), il sistema suddivide i risultati della ricerca in 5 gruppi e li presenta all'utente mostrando ai primi posti quelli che hanno ottenuto il grado di rilevanza più alto in base ai seguenti criteri

\begin{tabular}{|l|l|}
\hline & $\begin{array}{l}\text { I PIÙ RILEVANTI } \\
\text { il titolo principale contiene tutte le parole cercate come un'unica } \\
\text { frase }\end{array}$ \\
\hline & $\begin{array}{l}\text { ALTAMENTE RILEVANTI } \\
\text { il complemento del titolo contiene tutte le parole cercate come } \\
\text { un'unica frase }\end{array}$ \\
\hline$-\square \square \square$ & $\begin{array}{l}\text { MOLTO RILEVANTI } \\
\text { il titolo della collana, una nota di contenuto o un soggetto } \\
\text { contengono tutte le parole cercate come un'unica frase }\end{array}$ \\
\hline $\begin{array}{l}\text { RILEVANTI } \\
\text { le singole parole cercate sono presenti nel titolo }\end{array}$ \\
\hline $\begin{array}{l}\text { POCO RILEVANTI } \\
\text { le parole cercate sono presenti in qualche campo della } \\
\text { registrazione bibliografica }\end{array}$ \\
\hline$-\square$
\end{tabular}

Fig. 5.4 - Parametri per il calcolo della rilevanza nell'OPAC dell'Università Cattolica del Sacro Cuore $^{68}$

In SOPAC, l'OPAC sperimentale implementato nelle biblioteche pubbliche di Ann Arbor oltre ai tradizionali campi del record inclusi nel computo della rilevanza si trovano

\footnotetext{
${ }^{66}<\mathrm{http}: / /$ millennium.unicatt.it/screens/help_index_ita.html $>$.

${ }^{67}<$ http://1101.nla.gov.au/> e <http://1101.nla.gov.au/docs/LuceneRRNotes.html>.

${ }^{68}<$ http://millennium.unicatt.it/screens/tipi_ricerca_ita.html\#split=05>.
} 
anche tag e recensioni. Nel prototipo di OPAC della National Library Australia (NLA) si forniscono $\mathrm{i}$ dettagli tecnici descrivendo tutti $\mathrm{i}$ parametri che vengono presi in considerazione per formare l'algoritmo di ranking ${ }^{69}$. Come è possibile rilevare dalla documentazione fornita, l'algoritmo assegna al record un peso complessivo in base alla corrispondenza del termine/dei termini / della frase esatta nei diversi campi del record ${ }^{70}$.

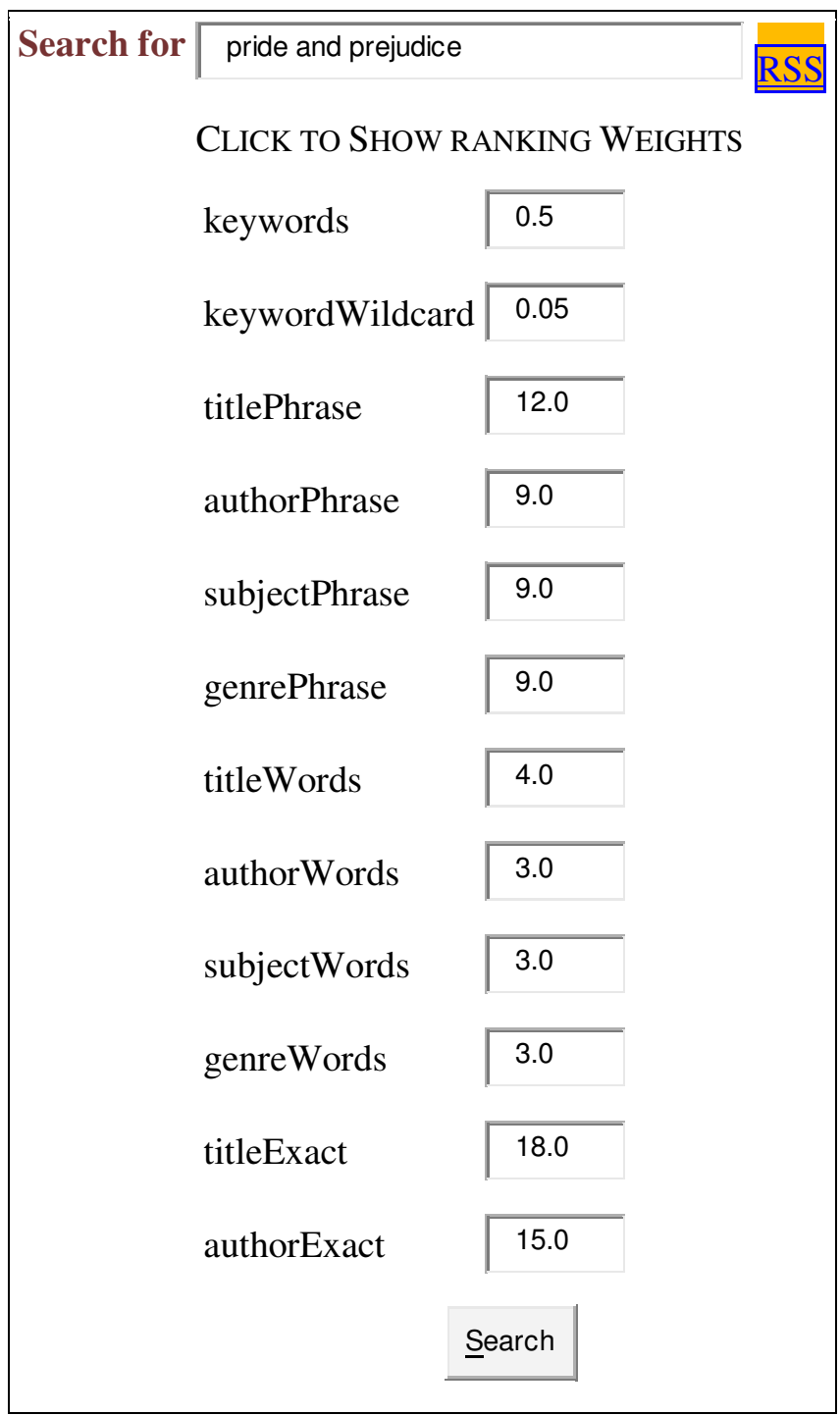

Fig. 5.5 - Parametri per il calcolo della rilevanza nell'OPAC sperimentale della National Library of Australia $^{71}$

\footnotetext{
${ }^{69}$ L'OPAC consente anche di visionare il calcolo algoritmico che viene effettuato per i primi 15 record della lista dei risultati (aggiungendo a ricerca effettuata il comando \&debug=y alla fine dell'url corrispondente alla richiesta visualizzata sullo schermo).

${ }^{70}$ Cfr. Alison Dellit - Tony Boston, Relevance ranking of results from MARC-based catalogues: from guidelines to implementation exploiting structured metadata, National Library Of Australia Staff Papers, 2009, <http://www.nla.gov.au/openpublish/index.php/nlasp/article/view/1052/1321>.

${ }^{71}<$ http://1101.nla.gov.au/>.
} 
Nel formulare il peso complessivo assegnato al record ai fini del suo ordinamento nel set dei risultati entrano in gioco anche le misurazioni classiche della frequenza del termine (TF) e della frequenza inversa (IDF) come è visibile nell'esempio in basso che riporta una sezione dell'algoritmo:

$67.65536=(\mathrm{MATCH})$ product of:

$109.93996=(\mathrm{MATCH})$ sum of:

$0.1874402=(\mathrm{MATCH})$ sum of:

0.18019457 = weight(keywords:"pride and prejudice" 999^0.5 in 7951247), product of:

0.014872668 = queryWeight(keywords:"pride and prejudice" 999^0.5), product of:

$0.5=$ boost

$19.57588=\mathbf{i d f}($ keywords: pride $=7879$ and $=10236404$ prejudice $=4806$ )

$0.0015194891=$ queryNorm

12.11582 = fieldWeight(keywords:"pride and prejudice" in 7951247), product of:

$1.4146645=\mathbf{t f}($ phraseFreq $=2.0012755)$

$19.57588=\operatorname{idf}($ keywords: pride $=7879$ and $=10236404$ prejudice $=4806$ )

$0.4375=$ fieldNorm(field=keywords, doc=7951247)

Fig. 5.6 - Una sezione dell'algoritmo del prototipo di OPAC di National Library of Australia ${ }^{72}$

Inoltre l'algoritmo prende in considerazione anche elementi esterni al record attribuendo un peso anche fattori che non dipendono dalla query, preferendo i record che sono localizzati in molte biblioteche, che sono posseduti / disponibili in una delle biblioteche scelte dall'utente, sono disponibili online, mentre altri criteri di ranking che verranno implementati in futuro prevedono un più stretto collegamento a siti commerciali dando preferenza ai volumi che hanno molte valutazioni/più venduti su LibraryThing /Amazon, che sono citati da pagine web autorevoli, oppure che sono disponibili per l'acquisto a prezzi convenienti o di seconda mano.

\footnotetext{
${ }^{72}$ Ibidem.
} 


\subsubsection{Rilevanza, circalità, novità, pertinenza}

Prendendo dunque in considerazione le modalità con cui gli OPAC calcolano la rilevanza, si può affermare che tali strumenti riducano la rilevanza misurata ai concetti della rilevanza algoritmica e topica. Una volta appurato che la circalità (topic relevance) può essere considerata come una delle tante dimensioni che caratterizzano la rilevanza, gli OPAC dovrebbero prendere a modello una rilevanza più sfaccettata considerando anche le altre dimensioni, finora sottoutilizzate.

Una recente ricerca ha preso in considerazione cinque criteri di rilevanza: circalità, novità, affidabilità, comprensibilità, ampiezza e ha rilevato che la circalità e la novità sono i criteri più significativi che vengono associati alla rilevanza, mentre ad un grado più basso, ritroviamo l'affidabilità, la comprensibilità e l'appropriatezza ${ }^{73}$. La novità, intesa come novità per l'utente, è dunque uno degli altri aspetti della 'rilevanza cognitiva', una rilevanza soggettiva in cui entra in gioco il bagaglio di conoscenze dell'utente.

\subsubsection{Oltre il matching: la ricerca come processo}

Gli OPAC di nuova generazione hanno introdotto il relevance ranking come metodo di ordinamento dei risultati della ricerca; nonostante però l'ordinamento per rilevanza sia generalmente avvertito come una delle innovazioni più importanti nella costruzione di interfacce di nuova generazione, gli algoritmi restano ancorati ad una misurazione che si identifica nella dimensione topica della rilevanza costruita sulla corrispondenza algoritmica ovvero sulla presenza del termine in determinate parti del record.

Il recupero si basa dunque su modelli che rimangono ancorati ad una definizione classica di information retrieval nella quale i sistemi forniscono risposte alle interrogazioni formulate dagli utenti.

Online catalogs must serve a population of information seekers that is heterogeneous in terms of age, language, culture, subject knowledge, and computing expertise, most of whom will be perpetual novices at information retrieval. To design systems that can support question answering rather than simply query matching, we need to learn more about the search process. One way to identify the features and functions to incorporate into the next

73 Yunjie (Calvin) Xu-Zhiwei Chen, Relevance judgment: what do information users consider beyond topicality?, «Journal of the American Society for Information Science and Technology», 57 (2006), n. 7, p. 961-973. 
generation of systems is to examine why systems based on query design models are hard to use $^{74}$.

L'applicazioni di metodi e tecniche dell'information retrieval agli OPAC da solo non basta ad ottenere un miglioramento significativo dell'efficacia dei cataloghi, per questo motivo è importante prendere a riferimento il campo di studi del comportamento informativo.

Come abbiamo visto, gli studi più recenti introducono nel concetto di information retrieval la componente dinamica, la componente cognitiva e quella situazionale della ricerca dell'informazione e il concetto del 'contesto', che è centrale nell'approccio qualitativo alla ricerca dell'informazione.

Ciò è frutto di riorientamento degli studi sulla ricerca dell'informazione che a partire dagli anni '80 si manifesta nella Scienza dell'informazione verso lo studio del comportamento informativo (information behavior) inteso come lo studio dei processi mentali e delle strategie coinvolte nel recupero dell'informazione. Gli studiosi cominciano a considerare gli aspetti cognitivi e sociali coinvolti nel processo di ricerca.

Negli anni '90 questo nuovo filone comincia a consolidarsi e il termine information behavior diventa il termine preferito per descrivere i molti modi in cui gli esseri umani interagiscono con l'informazione ed in particolare i modi con cui essi cercano e usano l'informazione ${ }^{75}$.

L'orientamento di studio rivolto alla comprensione del bisogno informativo e dell'uso dell'informazione diviene dunque uno dei filoni di studio della Scienza dell'Informazione ${ }^{76}$ e l'area di studio è sostenuta e alimentata dalle numerose iniziative che vedono confrontarsi studiosi di tutto il mondo ${ }^{77}$.

Negli studi sulla ricerca dell'informazione si assiste dunque ad una biforcazione che vede due filoni di studio ben distinti che si identificano in due diverse discipline: gli studi

\footnotetext{
${ }^{74}$ Christine L. Borgman, Why are online catalogs still hard to use?, «Journal of the American Society for Information Science», 47 (1996), n. 7, p. 494.

${ }^{75}$ Marcia J. Bates, Information behavior in Encyclopedia of Library and Information sciences, 3 ed., edited by Marcia J. Bates and Mary Niles Maack, New York, CRC Press, 2010 p. 2381-2391 (Disponibile anche online all'URL: <http://www.gseis.ucla.edu/faculty/bates/articles/information-behavior.html>).

${ }^{76}$ Saracevic, Tefko, Information Science in Encyclopedia of Library and Information sciences cit.

77 Tra queste meritano di essere citate per l'ampiezza degli studi prodotti le conferenze Information Seeking in Context (ISIC) che si tengono ogni due anni a partire dal 1996. La prima conferenza tenutasi a Tampere vide la partecipazione, tra gli altri,di Brenda Dervin, Sanda Erdelez, Carol C. Kuhlthau, Kimmo Tuominen, Reijo Savolainen, Pertti Vakkari e Thomas D. Wilson. Dato che il campo della ricerca informativa è multidisciplinare le conferenze vedono confrontarsi ricercatori di diverse discipline quali la scienza dell'informazione, la gestione dell'informazione, la psicologia, la psicologia sociale, l'informatica, e altre discipline. L'ultima conferenza ISIC si è tenuta nel settembre 2012 a Tokyo (vedi $<$ http://informationr.net/isic/index.html>). Altre iniziative di rilievo sono i simposî dello Special Interest Group on Information Seeking and Use (SIG USE) che si tengono nell'ambito delle conferenze dell' American Society of Information Science and Technology (ASIST), <http://siguse.wordpress.com/>.
} 
orientati all'utente e al suo comportamento informativo sono oggetto di studio della scienza dell'informazione, mentre quelli più propriamente rivolti ai sistemi e alle misurazioni della rilevanza, allo studio di algoritmi e modalità di rappresentazione dei testi vengono coltivati nell'ambito dell'informatica. L'avvicinamento dei due filoni di studio, da molto tempo auspicato, e inaugurato dai contributi di Ingwersen e Jarvelin ${ }^{78}$, è oggetto di una nuova area di ricerca che oggi viene definita interactive information retrieval ${ }^{79}$ che prevede la possibilità di utilizzare i risultati provenienti dalle due aree di studio per migliorare i sistemi di ricerca dell'informazione. Secondo questa nuova visione il miglioramento complessivo della ricerca non può provenire solamente dallo sviluppo di algoritmi in grado sfruttare le risorse informative, ma dalla capacità tener conto del comportamento informativo. Tuttavia il collegamento tra le due discipline non ha ancora generato quella sinergia che ci si aspettava.

Il modello di information searching proposto Saracevic, preso in considerazione per spiegare gli elementi coinvolti nel giudizio di rilevanza è finalizzato ad illustrare l'interazione tra l'utente e un sistema e a mettere in evidenza le diverse variabili coinvolte nell'interazione tra un utente e un sistema. Questo modello e gli altri modelli di information retrieval sviluppati secondo un riorientamento cognitivo del recupero dell'informazione (tra i quali il modello cognitivo di Ingwersen, l'Episode model di Belkin rappresentano le più alte elaborazioni) hanno una grande importanza nella comprensione delle diverse dimensioni coinvolte nel recupero dell'informazione, ma il principale limite è quello di non associare i fattori identificati alle strategie di ricerca dell'informazione e ai comportamenti che l'utente manifesta nel processo di recupero dell'informazione; alcuni dei concetti chiave che influenzano il recupero dell'informazione rimangono fuori dal campo di indagine di questi modelli ${ }^{80}$; essi sono oggetto di studio di un filone ben distinto di ricerca, quello dell'information seeking behavior che annovera una vastissima letteratura e che viene considerato, insieme a quello dedicato all'information retrieval, un filone fondamentale di ricerca nel campo delle scienze dell'informazione.

Nel tentativo di migliorare le funzionalità offerte all'utenza, ci si può avvalere per la progettazione degli OPAC degli studi sui modelli comportamentali di ricerca dell'informazione; per molto tempo i cataloghi elettronici si sono basati su modelli che privilegiavano una ricerca orientata al risultato, trascurando il processo dinamico nel quale

\footnotetext{
${ }^{78}$ Peter Ingwersen - Kalervo Järvelin, The turn: integration of information seeking and retrieval in context, Heidelberg, Springer, 2005.

${ }^{79}$ Per un approfondimento degli studi che hanno condotto a questo nuovo filone di studio si veda il recente volume curato da Ian Ruthven e Diane Kelly, Interactive information seeking, behaviour and retrieval cit.

${ }^{80}$ Iris Xie, Interactive information retrieval in digital environments, New York, IGI Global, 2008, p. 196.
} 
l'utente viene coinvolto nel corso della ricerca, oggi invece i cataloghi dovrebbero affiancare ad un efficace recupero dell'informazione anche una gestione ottimale del processo di ricerca basandosi sui modelli che rappresentano la ricerca come processo e studiano i comportamenti dell'utente.

Questi studi si basano su un approccio 'centrato sull'utente' identificando modelli comportamentali e process-based, che indagano i modi in cui gli utenti cercano e usano l'informazione. A differenza dei modelli di information retrieval che rimangono sostanzialmente ancorati a paradigmi basati sul matching, questi modelli si sono orientati verso la scoperta dei processi che regolano l'accesso, lo scambio e l'uso dell'informazione.

Questo approccio qualitativo alla ricerca dell'informazione è basato sulla necessità di comprendere i modi in cui l'informazione si manifesta in relazione al contesto e i processi di comprensione e apprendimento, ovvero la sua possibilità di trasformarsi in conoscenza.

Nel presente lavoro si prenderanno a riferimento i più significativi sviluppi in questo settore di studio, che costituiranno il quadro teorico dal quale, a mio avviso, si possono trarre le premesse per la costruzione di cataloghi rinnovati che possano facilitare la creazione di conoscenza.

È necessario guardare al contesto sociale nel quale si compie la ricerca e l'uso dell'informazione e non solo alla semplice conoscenza delle componenti di un sistema di comunicazione (canale, emittente, ricevente, codice) o di recupero dell'informazione (sistemi, algoritmi, rappresentazione dei testi).

Per questo motivo gli studi sull'utenza e i modelli elaborati all'interno del filone dell'information behavior costituiscono una grande risorsa per lo studio, la modellazione e la progettazione di cataloghi innovativi.

\subsection{Dagli studi di information retrieval agli studi centrati sull'utente. I modelli del comportamento informativo e i modelli di ricerca dell'informazione}

Come abbiamo già accennato, vi sono due principali orientamenti nelle ricerche sul recupero dell'informazione: un approccio centrato sull'utente e uno centrato sui sistemi. L'approccio 'di sistema' ha svolto un ruolo predominante nel passato nella progettazione dei sistemi di information retrieval. 
In seguito ha prevalso il riconoscimento che approcci centrati sui sistemi non riescono a soddisfare le esigenze degli utenti e che il modello classico di recupero dell'informazione basato sulla corrispondenza (matching) tra la rappresentazione dei documenti e la rappresentazione dei bisogni degli utenti in una query risulta un modello inadeguato per progettare sistemi di ricerca. È emerso dunque un approccio cognitivo all'information retrieval, che dalla rappresentazione del documento si sposta verso il riconoscimento e l'indagine delle strutture cognitive dell'utente.

In questi studi, l'utente è riconosciuto la componente essenziale dell'interazione con i sistemi e punto centrale dell'osservazione. Si è dunque sviluppato un nutrito filone di ricerca (che si richiamava ai cosiddetti user studies ${ }^{81}$ ) volto ad indagare i comportamenti di ricerca degli utenti e il loro approccio alla ricerca dell'informazione.

Purtroppo nonostante l'enorme quantità degli studi prodotti, non c'è ancora stata un'influenza decisiva di queste teorie nella progettazione dei sistemi e in particolar modo dei cataloghi elettronici e questi ultimi vengono ancor sviluppati presupponendo che l'utente abbia obiettivi fissi nel tempo e che sia in grado di formulare richieste al sistema.

Una progettazione centrata sull'utente (o user centered design) dovrebbe permettere ai sistemi di determinare quale azione è più opportuna in un determinato momento, di rendere visibile il modello concettuale sul quale è stato progettato il sistema, di assicurare che in ogni momento l'utente sappia cosa può fare e che sia in grado di comprendere cosa sta accadendo ovvero abbia il controllo della propria ricerca ${ }^{82}$.

Una delle più recenti definizioni riguardanti i principali campi di indagine dell'Information science riconosce lo studio dei modi con cui le persone si relazionano all'informazione come uno dei principali ambiti di ricerca della disciplina ma il richiamo a questo particolare filone di indagine negli studi sulle strategie di miglioramento dei cataloghi elettronici fa riferimento ad alcuni contributi apparsi già partire dagli anni ' $90^{83}$. Christine Borgman attribuiva la difficoltà d'uso dei cataloghi elettronici ad una scarsa comprensione dei comportamenti di ricerca messi in atto dagli utenti:

Despite numerous improvements to the user interface of online catalogs in recent years, searchers still find them hard to use. Most of the improvements are in surface features rather

\footnotetext{
${ }^{81}$ Le origini degli 'studi sull'utenza' sono stati rilevati in due reports presentati alla Royal Society Scientific Information Conference nel 1948. Si tratta dello studio di Urquhart (un'indagine sulle preferenze le motivazioni della ricerca e la soddisfazione degli utenti della London Science Museum Library) e di Bernal (un'inchiesta sull'uso di periodici scientifici da parte degli scienziati britannici). Cfr.: Tefko Saracevic, Foreword in Interactive information seeking, behaviour and retrieval cit., p.xxv-xxxii.

${ }^{82}$ Sul tema si veda: Donald A. Norman-Stephen W. Draper, User centered system design: new perspectives on human-computer interaction, Hillsdale, Lawrence Erlbaum Associates, 1986.

${ }^{83} \mathrm{Cfr}$. Tefko Saracevic, Information Science in Encyclopedia of Library and Information sciences cit.
} 
than in the core functionality. We see little evidence that our research on searching behavior studies has influenced online catalog design ${ }^{84}$.

Secondo Bates ${ }^{85}$ lo sviluppo di cataloghi elettronici deve prendere in considerazione non solo gli studi sull'uso dei cataloghi o dei sistemi che hanno certamente rappresentato un ricco ambito di ricerca negli ultimi 25 anni, ma prima di tutto gli studi generali sulla ricerca dell'informazione ${ }^{86}$.

\subsubsection{Teorie e metateorie socioculturali dell'informazione (Dervin, Belkin, Wilson)}

Tutta la letteratura sulla ricerca dell'informazione che ha dato origine a quelle che vengono definite 'metateorie socioculturali dell'informazione' ${ }^{87}$ si basa sui modelli generali del comportamento umano nei confronti dell'informazione.

\footnotetext{
${ }^{84}$ Christine L. Borgman, Why are online catalogs still hard to use? cit., p. 501.

${ }^{85}$ Marcia J. Bates, Task Force Recommendation 2.3 Research and design review, improving user access to library catalog and portal information, final report, (version 2.3), 2003, [risorsa online], <http://www.loc.gov/catdir/bibcontrol/2.3BatesReport6-03.doc.pdf>

${ }^{86}$ Lo sviluppo delle interfacce dei sistemi di recupero dell'informazione è frutto di decenni di studi che si sono svolti almeno in due direzioni: 1) Gli studi sull'utenza rivolti ad analizzare l'interazioni tra utenti e sistemi. Non sempre questi studi sono tra loro comparabili poiché si servono di almeno due metodologie tra loro in contrasto:

* i focus groups, dove gli utenti vengono opportunamente selezionati e invitati a svolgere determinati compiti (es. trovare un dato termine, cercare per soggetto etc.) e/o a rispondere a certe domande.

* l'analisi dei log (TLA, Transaction log analysis) dove si osservano le interazioni che avvengono spontaneamente tra gli utenti e i sistemi, si raccolgono i dati e si traggono conclusioni per via induttiva.

Entrambe le metodologie mostrano dei limiti; la prima perché dipende dalle valutazioni soggettive di chi predispone l'indagine e per la scarsa comparabilità dei risultati di ricerche che adottano la stessa metodologia; la seconda perché per funzionare deve scendere a compromessi (non è possibile identificare con certezza gli utenti, si ignora il motivo iniziale di una ricerca e gli obiettivi, lasciando forse troppo spazio all'interpretazione).

In questo campo di studi lo sviluppo degli OPAC a partire dagli anni '80 ha avuto un'influenza decisiva per la possibilità di registrare i comportamenti degli utenti nelle sessioni di ricerca.

2) L'elaborazione di modelli nell'ambito dell'information seeking behavior e lo studio dei comportamenti di ricerca dell'informazione ha avuto uno sviluppo straordinario a partire dagli anni '80, tuttavia come emerge dall'analisi delle modalità di ricerca e di visualizzazione degli OPAC, è evidente che lo sviluppo di questi strumenti non ha ancora saputo beneficiare appieno dei risultati di questi studi.

Sull'argomento si vedano anche le osservazioni di Karen Markey (cfr.: Karen Markey,Twenty-five years of end-user searching, Part 1: research findings, «Journal of the American Society for Information Science and Technology», 58 (2007) n. 8, p. 1071-1081, <http://hdl.handle.net/2027.42/56093> e Ead., Twenty-five years of end-user searching, Part 2: future research directions. «Journal of the American Society for Information Science and Technology», 58 (2007) n. 8, 1123-1130, <http://hdl.handle.net/2027.42/56094>).

${ }^{87}$ Recentemente ripercorse in Alberto Salarelli, Introduzione alla scienza dell'informazione cit. p. 79-91. Una panoramica delle teorie e dei più noti modelli del comportamento informativo si trova in Theories of information behaviour, edited by Karen E. Fisher - Sanda Erdelez - Lynne Mckechnie, Medford, published for the American Society for Information Science and Technology by Information Today, 2005.
} 
Il concetto di information behavior è un concetto ampio, usato per descrivere i molti modi in cui gli esseri umani interagiscono con l'informazione, in particolare e le modalità con cui le persone cercano e usano l'informazione ${ }^{88}$.

Si deve a Thomas D. Wilson ${ }^{89}$ la sistematizzazione dei campi di studio che vengono ricondotti al filone di studio del comportamento informativo; lo studioso identifica tre campi principali di ricerca che sono nidificati l'uno nell'altro:

* l'information behavior è «la totalità del comportamento umano in relazione alle fonti e ai canali di informazione, comprendente la ricerca attiva e passiva dell'informazione e l'uso dell'informazione stessa»;

* l'information seeking behavior è un sottocampo di indagine particolarmente rivolto alla varietà dei metodi che le persone sviluppano per cercare l'informazione;

* l'information searching behavior restringe ulteriormente l'indagine alle interazioni che avvengono (con o senza intermediari) tra gli utenti dell'informazione e i sistemi automatizzati, dei quali i sistemi di recupero dell'informazione per i dati testuali rappresentano una particolare tipologia.

La ricerca attiva dell'informazione (information seeking behavior) può essere considerata una parte del più ampio scenario del comportamento informativo (information behavior), volto a indagare i comportamenti generali che le persone mettono in campo nel loro uso dell'informazione.

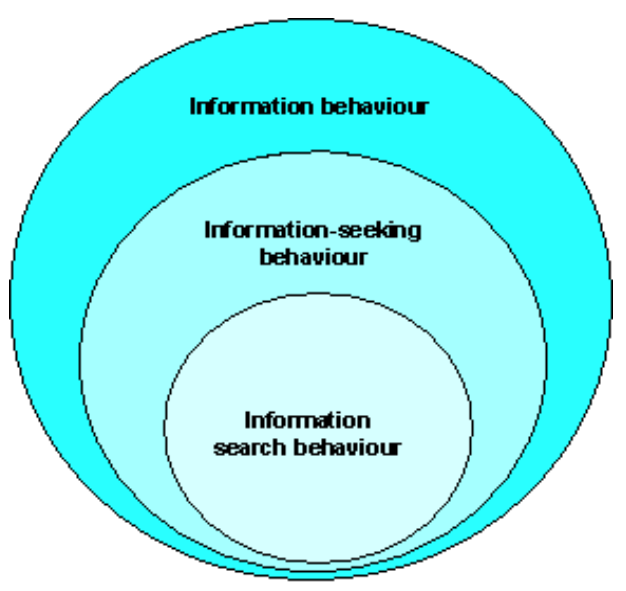

Fig. 5.7 - I tre campi di indagine negli studi sulla ricerca dell'informazione ${ }^{90}$

\footnotetext{
${ }^{88}$ Marcia J. Bates, Information behavior in Encyclopedia of Library and Information sciences cit.

${ }^{89}$ La tripartizione dei campi di studio è teorizzata nel saggio di Thomas D. Wilson, Models in information behaviour research, «Journal of Documentation», 55 (1999), n. 3, p. 249-270, disponibile anche all'URL: $<$ http://informationr.net/tdw/publ/papers/1999JDoc.html>.

${ }^{90}$ Ivi p. 263.
} 
Nello spazio di indagine ricoperto dall'information behavior vi sono dunque quelle teorie di ampio respiro o metateorie ${ }^{91}$ e teorie che inseriscono la ricerca dell'informazione in modelli generali di comportamento, che riassumeremo brevemente, per esplorare in seguito i modelli che hanno cercato di descrivere il comportamento nella ricerca attiva dell'informazione (Ellis, Kuhlthau, Bates), che rientrano nel campo più specialistico dell'information seeking behavior e costituiscono il quadro teorico di riferimento quando si voglia indagare più a fondo le modalità di ricerca attiva degli utenti. La maggior parte di questi approcci, come vedremo, sono interrelati ${ }^{92}$.

\subsubsection{Il sense-making model di Dervin e lo 'stato anomalo di conoscenza' di Belkin}

La necessità di spiegare come e perché si origina la ricerca dell'informazione è oggetto dei modelli elaborati da Dervin e Belkin. Il modello proposto da Brenda Dervin ${ }^{93}$ è comunemente considerato una metateoria, essendo una vera e propria filosofia che sta alla base delle numerose teorie che sono state sviluppate. La sense-making theory, sviluppata in un numero considerevole di anni a partire dal 1972, è considerata molto più di un semplice modello di comportamento di ricerca dell'informazione; è piuttosto una serie di principi e

\footnotetext{
${ }^{91}$ Per un'introduzione ai concetti di metateorie, teorie e modelli si veda anche l'introduzione di Bates: Bates. Marcia J. An introduction to metatheories, theories and models in Theories of information behavior cit., p. 124. Una metateoria indica un approccio diverso rispetto a quello teorico, potendosi considerare come una 'filosofia della teoria' che alza lo sguardo sulle idee fondamentali che le sottendono.

${ }^{92}$ A differenza dei modelli query centric, basati sulla rappresentazione e l'analisi dei testi, i modelli centrati sull'utente comprendono fattori cognitivi e situazionali; questi possono essere raggruppati nelle categorie dei modelli:

* cognitivi: basati sui processi cognitivi che si svolgono nella ricerca dell'informazione (es. Dervin e Nilan, e Ingwersen);

* strategici: basati sulle strategie che l'utente utilizza nella ricerca dell'informazione (Bates, Xie); * process-based: sviluppano rappresentazioni di stadi del processo di ricerca (Kulthau, Marchionini);

* episodici: rappresentano episodi di ricerca e non stadi del processo informativo (es. Belkin);

* stratificati: rappresentano l'interazione di diversi componenti in forma di strati del sistema, in cui ogni strato influenza l'interazione.

Cfr.: Ryen White, Interactive tecniques in Interactive information seeking, behaviour and retrieval, edited by Ian Ruthven and Diane Kelly, London, Facet, 2011, p. 171-188. La categorizzazione dei modelli è a p. 172. Per un'ampia panoramica dei principali modelli si veda:Theories of information behaviour cit.

${ }^{93}$ Brenda Dervin è professore di comunicazione all'Ohio State University e ricerca nell'ambito della comunicazione e della Library and information science. Le sue ricerche sul comportamento di ricerca e l'uso dell'informazione hanno condotto allo sviluppo del Sense-Making, una vera e propria metateoria del comportamento informativo che ha influenzato numerosi altri studi nel campo dell'information behavior.
} 
un approccio metodologico, un insieme di metodi di ricerca e una 'pratica' per spiegare come le persone si relazionano all'informazione ${ }^{94}$ :

a set of assumptions, a theoretic perspective, a methodological approach, a set of research methods, and a practice' ... designed to cope with information perceived as, 'a human tool designed for making sense of a reality assumed to be both chaotic and orderly ${ }^{95}$

Il sense making è un comportamento sia interno che esterno che coinvolge sia gli aspetti cognitivi che le procedure e che permette all'individuo di costruire i suoi movimenti nel tempo e nello spazio. L'uso e la ricerca dell'informazione sono considerate in un processo di costruzione di senso. Secondo un approccio costruttivista e situazionale Dervin costruisce il modello situazione-gap-utilizzo dell'informazione. La teoria è fondata su quattro elementi costitutivi: una situazione nel tempo e nello spazio che definisce il contesto in cui si origina il bisogno o il problema informativo, un gap cioè la carenza o il bisogno di informazione che è da intendersi come la differenza tra la situazione fornita dal contesto e la situazione desiderata, un risultato che è la conseguenza del processo di sensemaking (creazione di senso). Nel modello gli elementi sono posti ai vertici in un triangolo: situazione, gap/bridge e risultato, ma il modello può essere rappresentato più efficacemente usando la metafora del ponte, infatti la definizione del gap e il suo superamento sono essenziali per l'approccio basato sul sense making.
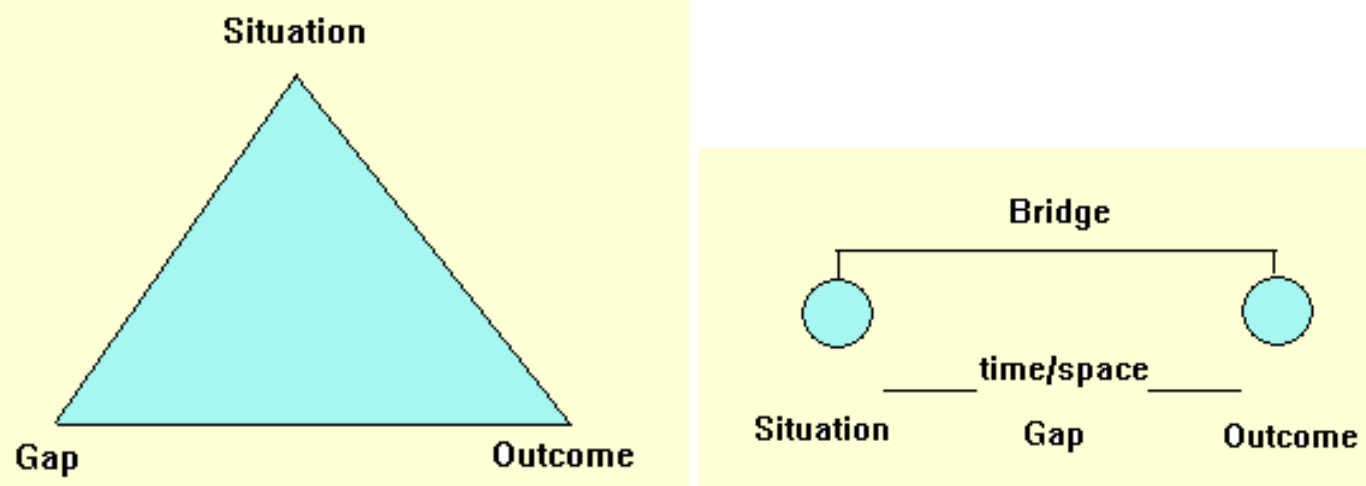

Fig. 5.8 - Il 'triangolo del sense-making' e la metafora del ponte ${ }^{96}$

Il triangolo della sense making theory comprende una situazione circoscritta nel tempo e nello spazio, un gap inteso come una carenza informativa da superare e usa l'informazione come un ponte superarla. Molti studiosi concordano nel ritenere che la forza

\footnotetext{
${ }^{94}$ Sebbene la teoria venga esposta compiutamente solo nel 1992: Brenda Dervin, From the mind's eye of the user: the sense-making qualitative-quantitative methodology. In Jack D. Glazier-Ronald R. Powell, Qualitative research in information management, Englewood, Libraries Unlimited Inc., 1992.

${ }_{95}$ Thomas D. Wilson, Models in information behaviour research cit., p. 253.

${ }^{96}$ Ivi, p. 253-254.
} 
del modello di Dervin risieda nelle sue conseguenze metodologiche: nel comportamento informativo, può indurre a un tipo di ragionamento che può rivelare la natura di una situazione problematica, il grado in cui l'informazione funziona da ponte per superare il gap dell'incertezza e della confusione e la natura dei risultati che provengono dall'uso dell'informazione. Alcuni studiosi hanno usato il modello per analizzare i bisogni informativi di particolari tipologie di utenza o analizzare nuovi ambienti di ricerca come ad esempio la ricerca nel web. Utilizzando questo particolare tipo di approccio Savolainen e Kari $^{97}$ hanno individuato tecniche tipiche della ricerca nel web, identificando i principali gap e le tattiche di ricerca utilizzate dagli utenti per superare il gap (gap-bridging).

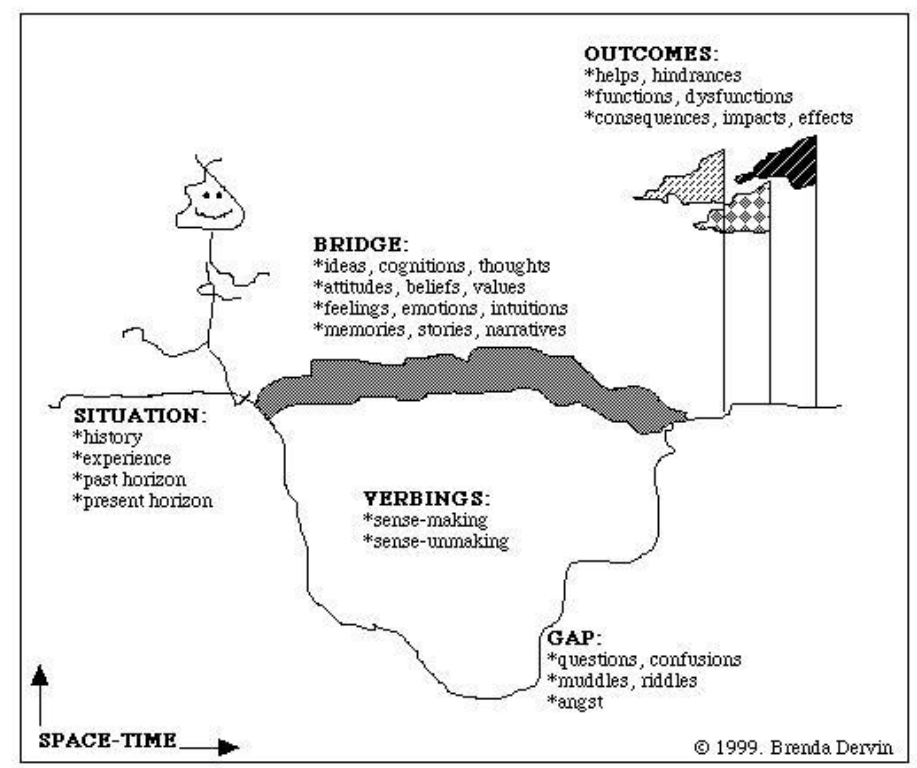

Fig. 5.9 - Un'altra rappresentazione concettuale della teoria di Dervin ${ }^{98}$

Con la sua teoria Dervin si allontana anche dai precedenti modelli di ricerca che rappresentavano il processo di ricerca con il tradizionale modello della trasmissione dell'informazione dalla fonte al ricevente. Nella visione della studiosa l'informazione è socialmente costruita, dunque non può essere mai separata dal contesto dal quale si origina.

Le teorie di Dervin hanno costituito una svolta negli studi verso metodi e teorie centrate sull'utente e sulla persona, mettendo in evidenza come alcune delle assunzioni su cui si basavano gli studi orientati ai sistemi fossero infondate, ad esempio che solo l'informazione oggettiva fosse osservabile o che l'informazione possa essere fornita al di fuori di un contesto.

\footnotetext{
${ }^{97}$ Reijo Savolainen-Jarkko Kari, Facing and bridging gaps in Web searching. «Information Processing Management», 42 (2006), n. 2, p. 519-537.

${ }^{98}<$ http://informationr.net/ir/11-4/paper269.html>.
} 
La necessità di spiegare la forza dalla quale si origina la ricerca dell'informazione portò Nicholas Belkin a formulare il concetto di Anomalous State of Knowledge (ASK) ${ }^{99}$. La teoria di Belkin prende le mosse dalla teoria dei livelli di bisogni informativi analizzati da Taylor nel $1968^{100}$ e dalle teorie della situazione problematica di Weirsig ${ }^{101}$ può essere considerata un'analisi più approfondita del gap analizzato da Dervin. Secondo Belkin quando l'utente, imbattendosi in una situazione problematica, non può risolvere il problema applicando la conoscenza acquisita, il suo stato anomalo crea un' 'incertezza cognitiva' che gli impedisce di esprimere adeguatamente il suo bisogno informativo. Ha dunque bisogno di maggiori informazioni per chiarire i propri pensieri, forza che lo guida a rivolgersi ad un sistema di recupero dell'informazione.

\subsubsection{I modelli di Wilson}

Tra le teorie e i modelli riguardanti la complessità del comportamento informativo sono da citare senz'altro i vari modelli che Thomas D. Wilson mette a punto in circa un ventennio di ricerche.

A partire dal 1971 Wilson sviluppa un primo modello generale nel quale si propone di analizzare i diversi fattori che attivano la ricerca di informazione e le barriere che impediscono alle persone di intraprendere l'azione di cercare l'informazione.

Il primo modello, nella versione perfezionata e proposta nel 1981, suggerisce una visione tripartita della ricerca dell'informazione che include il contesto del ricercatore, il sistema utilizzato e le risorse informative che esistono entro un universo delle conoscenze. Centrale nel modello di Wilson è l'idea che il contesto fornito dal ruolo sociale, personale e ambientale può generare il bisogno di informazione e che dunque i bisogni che originano l'information seeking behavior (comportamento di ricerca dell'informazione) possono essere distinti in fisiologici, affettivi e cognitivi. Da tali premesse Wilson afferma che l'idea stessa di bisogno informativo si rivela un concetto ormai superato e inefficace agli scopi della ricerca, e propone la sua sostituzione con il termine "information behavior" in quanto

\footnotetext{
${ }^{99}$ Nicholas J. Belkin, Anomalous states of knowledge as a basis for information retrieval, «Canadian Journal of Information Science», 5, (1980), p. 133-143.

100 Robert S. Taylor, Question-negotiation and information seeking in libraries, «College and research libraries», 29 (1968), p. 178-194.

${ }^{101}$ Gernot Wersig, The problematic situation as basic concept of information science in the framework of the social sciences. In Theoretical problems for Informatics: new trends in informatics and its terminology, Moscow, International Federation for Documentation, 1979, p. 48-57.
} 
non è il bisogno ma il comportamento, corrispondente allo stato mentale interno, l'oggetto direttamente osservabile.

Dopo il primo tentativo di sviluppare un modello generale di information seeking behavior, nel modello del 1981 viene dunque approfondito il concetto del contesto che origina il bisogno informativo.

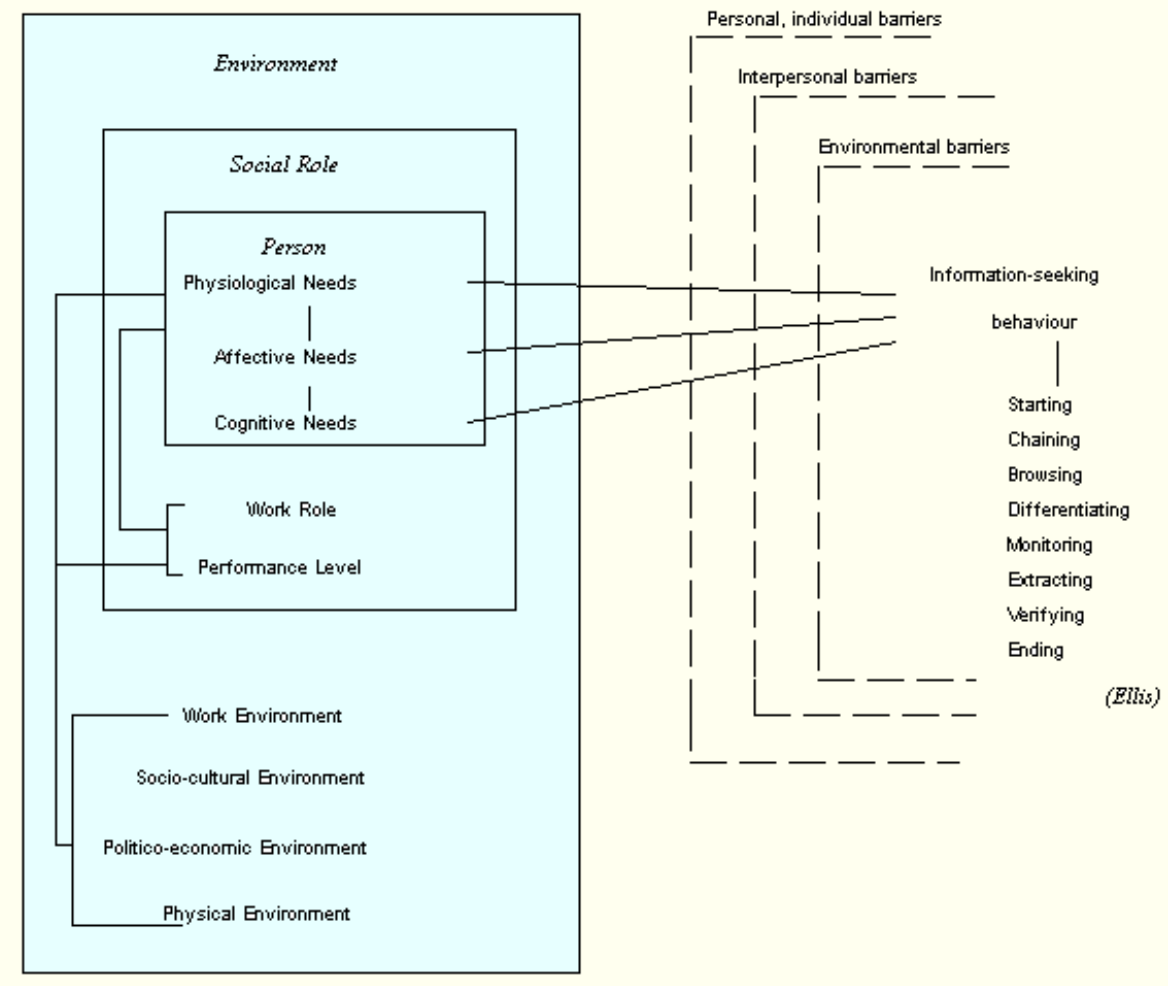

Fig. 5.10 - Il modello di Wilson del $1981^{102}$

Nel modello (fig. 5.10) viene analizzato il contesto nel quale sorge il bisogno informativo, le barriere eventuali che possono impedire alla persona di intraprendere la ricerca dell'informazione e l'attivazione del processo di ricerca che dà luogo a una serie di comportamenti che, nel frattempo, sono stati esaminati più approfonditamente da modelli più specifici, come quello di Ellis.

L'ultima versione del modello, elaborata nel 1996, è certamente la più completa e interessante poiché nel processo di ricerca dell'informazione l'autore prende in esame le diverse teorie che possono essere usate per esplorare $\mathrm{i}$ vari aspetti, alla luce di alcune teorie e modelli che nel frattempo erano emersi.

\footnotetext{
102 Thomas D. Wilson, On user studies and information needs, «Journal of Documentation», 37 (1981), n. 1, p. 3-15, ripubblicato in «Journal of Documentation», 62 (2006), n. 6, pp. 658-670, $<$ http://informationr.net/tdw/publ/papers/1981infoneeds.html>. Figura a p. 663.
} 
Il modello delineato del $1996^{103}$ (fig. 5.11) è dunque un modello molto ampio, in grado di ospitare al suo interno altre teorie che possono aiutare a spiegare più nel dettaglio non solo alcuni aspetti fondamentali del comportamento umano, ma anche i vari approcci al comportamento di ricerca dell'informazione. Nel modello la decisione di intraprendere un'azione per soddisfare un desiderio informativo è legata ad alcune variabili o barriere che possono essere illustrate da alcune note teorie ${ }^{104}$.

Anche il modello di sense making di Dervin, ad esempio, può essere associato al modello disegnato da Wilson poiché ha a che fare con la percezione di un bisogno informativo e con la necessità di superare un ostacolo, cioè di colmare un gap informativo. Il gap può essere un modo per rappresentare quelle barriere o variabili che nel modello di Wilson del 1981 sono frapposte tra il bisogno informativo (e il contesto in cui si genera tale bisogno) e la decisione di intraprendere un'attività di ricerca. ${ }^{105}$ Rientrano nel modello di Wilson anche teorie elaborate successivamente quali l'information encountering di Erdelez $^{106}$ (che analizza quello che potremmo definire l'atto di imbattersi nell'informazione o quella che oggi viene comunemente chiamata serendipità) che può essere una elaborazione della modalità di attenzione passiva ${ }^{107}$ e il modello Kuhlthau delle fasi del processo di ricerca dell'informazione, che invece, come si vedrà più avanti, è una analisi dettagliata delle fasi della ricerca attiva.

Il modello di Wilson deve dunque la sua forza alla generalità che lo contraddistingue prendendo a riferimento la totalità del comportamento umano nei confronti dell'informazione e mostrando come lo sviluppo di una parte specifica può contribuire a comprendere l'insieme ${ }^{108}$.

103 Il modello venne esposto in Thomas D. Wilson - Christina Walsh, Information behaviour: an interdisciplinary perspective, Sheffield, University of Sheffield, Department of Information Studies, 1996, $<$ http://informationr.net/tdw/publ/infbehav/cont.html>.

${ }^{104}$ Wilson identifica variabili e le barriere che si rifanno alle seguenti teorie, che verranno approfondire più avanti: la Stress/coping theory (capacità di fronteggiare l'ansia) di Folkman, Self efficacy theory di Bandura, la scelta di attivare la ricerca è associata alla Risk-reward theory di Settle \& Alreck, infine collega le varie teorie di apprendimento alla fase information processing and use, area di ricerca in cui l'approccio più noto è fornito dalla Teoria costruttivista dell'apprendimento (Steffe \&Gale 1995, citato in Thomas D. Wilson Christina Walsh, Information behaviour cit.).

${ }^{105}$ Queste considerazioni sono proposte da Natalia Goldbold in un suo recente contributo anche se, come vedremo, il gap attiva un comportamento informativo ma non attiva necessariamente l'attività di cercare l'informazione, né la ricerca è necessariamente originata da un gap. (Natalia Godbold, Beyond information seeking: towards a general model of information behaviour, «Information Research», 11 (2006) n. 4, p. 269 $<$ http://InformationR.net/ir/11-4/paper269.html>).

106 Sanda Erdelez, Information encountering: a conceptual framework for accidental information discovery in Pertti Vakkari-Reijo Savolainen-Brenda Dervin, Information seeking in context: proceedings of international conference on research in information needs, seeking and use in different context, London, Taylor Graham, 1997, p. 412-421.

${ }^{107}$ È una modalità di acquisizione dell'informazione che trova spazio in altri modelli successivi come quello che Marcia Bates propone nel 2002.

108 Thomas D. Wilson, Models in information behaviour research, cit. 


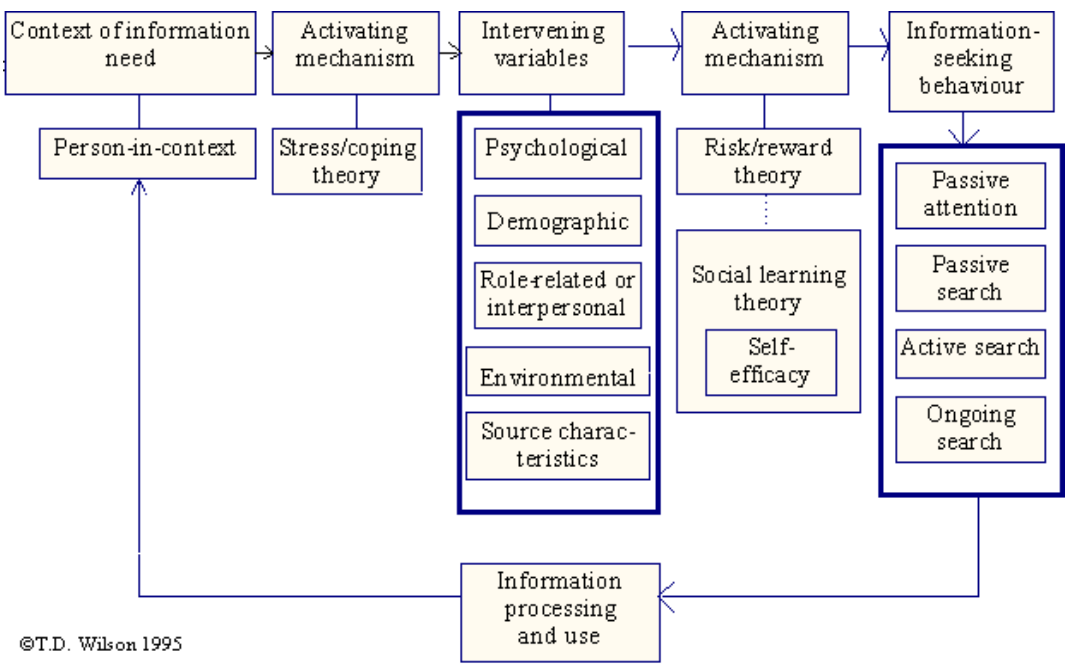

Fig. 5.11 - Il modello di Wilson del $1996^{109}$

Più di recente Wilson ha proposto un modello che interpreta l'attività di ricerca dell'informazione l'information seeking behavior come un comportamento determinato dagli obiettivi e condizionato dall'incertezza (uncertainty) in cui è centrale il concetto di problem solving ${ }^{110}$; nel quale chi cerca l'informazione si muove attraverso diverse fasi di soluzione del problema come problem recognition, problem identification, problem resolution e solution statement ${ }^{111}$.

I modelli elaborati da Wilson possono dunque essere considerati modelli di base che consentono di avere un quadro complessivo dei problemi di ricerca dell'informazione; lui stesso li definisce information behaviour models in quanto di tratta di modelli generali che riguardano l'attività di ricerca dell'informazione con una prospettiva più ampia rispetto al semplice uso di sistemi di information retrieval.

\footnotetext{
${ }^{109}$ Thomas D. Wilson, Models in information behaviour research cit., p. 257.

${ }^{110}$ Questo nuovo modello è in grado di spiegare i cosiddetti 'episodi multipli di ricerca' esaminati nei modelli elaborati da Belkin e Spink. In base al modello l'utente, infatti, procede in base al feedback ricevuto. A Belkin si deve un noto modello (l'episode model) che considera l'interazione tra l'utente ed un sistema di recupero dell'informazione come una sequenza di interazioni di diverso tipo in un 'episodio di ricerca' dell'informazione (Cfr. Nicholas J. Belkin - Colleen Cool - Adelheit Stein -Ulrich Thiel, Cases, scripts and information seeking strategies: on the design of interactive information retrieval systems, «Expert Systems with Applications», 9, (1995) p. 379-395. Un profondo studio dei meccanismi di feedback negli studi di information seeking behavior si deve ad Amanda Spink che ha elaborato un modello del processo di ricerca formato da più cicli (o episodi), nel quale in ogni ciclo di ricerca l'utente utilizza varie tattiche, valuta i risultati e attiva un altro ciclo di ricerca in base al feedback ricevuto nell'interazione con il sistema. Cfr.: Amanda Spink, Multiple search sessions model of end-user behavior: an exploratory study, «Journal of the American Society for Information Science», 47 (1996), p. 603-609; Amanda Spink - Robert M. Losee, Feedback in information retrieval, "Annual Review of Information Science and Technology», 31 (1996), p. 33-78; Amanda Spink, Study of interactive feedback during mediated information retrieval, «Journal of the American Society for Information Science», 48 (1997) n. 5, p. 382-394.

111 Una prospettiva che lo avvicina ai modelli elaborati da Ellis e Kulthau che verranno esposti successivamente. Cfr. Thomas D. Wilson, Exploring models of information behaviour: the 'Uncertainty' Project, «Information Processing and Management», 35(1999), p. 839-849.
} 
È importante sottolineare che secondo Wilson gli studi teorici che provengono dall'area generale dell'information behavior sono ritenuti capaci di condizionare i principi di progettazione di questi sistemi e permettere agli sviluppatori di specificare meglio $\mathrm{i}$ percorsi della navigazione, quale tipo di informazione o tipologia di dato deve essere contenuto nel record, ma la specificazione delle modalità concrete di progettazione di sistemi interattivi sulla base di tali modelli non è realizzabile e rimane un compito dei progettisti dei sistemi ${ }^{112}$.

Il modello di Ellis suggerisce che i sistemi di IR dovrebbero fornire più percorsi di navigazione, rispetto alla semplice ricerca booleana o alle strategie di best-matching, come la capacità di collegare le citazioni e i testi e di navigare tra le citazioni, l'opportunità di agenti intelligenti che monitorino le aggiunte ai database a seconda dell'ultima ricerca dell'utente o di un profilo stabilito.

Inoltre, dato che teoricamente tutti gli studi sull'information seeking behaviour mostrano l'importanza di reti personali i sistemi dovrebbero incorporare meccanismi che possano consentire agli utenti di essere messi in contatto con persone interessate alla stessa area di ricerca.

\subsubsection{Information seeking behavior. Il modello dinamico di Bates}

Secondo Marcia Bates ${ }^{113}$ il termine information-seeking si riferisce ad un insieme di processi e di strategie messe in atto dalle persone nella loro richiesta e nel loro uso dell'informazione ${ }^{114}$.

Rispetto ai modelli visti in precedenza, nati allo scopo di comprendere aspetti generali del comportamento informativo, gli studi sull'information seeking si sono sviluppati nel tentativo di cogliere la natura dinamica del processo di ricerca dell'informazione. Nella maggior parte delle ricerche questi processi si intendono come

\footnotetext{
${ }^{112}$ Ad esempio, nel modello di Wilson del 1996 lo studioso ipotizza che un sistema di IR dovrebbe essere progettato per ridurre il rischio di fallimento, aumentare il senso di autoefficacia (self efficacy) ma i mezzi con cui il rischio deve essere ridotto - afferma Wilson - rientrano nei compiti dei progettisti. I sistemi potranno essere ideati meglio se chi li progetta comprenderà le idee di risk/reward e di self efficacy.

${ }_{113}$ Marcia J. Bates è tra i più influenti studiosi nell'area di ricerca del comportamento informativo. Ha pubblicato numerosi contributi nell'area delle strategie dei sistemi di information retrieval, dell'organizzazione della conoscenza e del comportamento di ricerca dell'informazione. Dopo aver ricevuto numerosi incarichi in università americane si è ritirata dall'insegnamento con la carica di Professor Emerita conferitole dall'Università della California. Ha svolto il ruolo di consulente per numerosi enti tra i quali Library of Congress, Getty Research Institute (part of the Getty Trust), Council on Library and Information Resources, U.S. National Commission on Libraries and Information Science. Attualmente è responsabile editoriale dell'Encyclopedia of Library and Information Sciences.

${ }^{114}$ Marcia J. Bates, Information behavior in Encyclopedia of Library and Information sciences cit.
} 
riferiti ad un obiettivo (goal-directed). Donald Case, ad esempio, definisce la ricerca dell'informazione come «uno sforzo conscio di acquisire informazione in risposta ad una necessità o un gap (tradurre come bisogno informativo, lacuna informativa) nella propria conoscenza» ${ }^{115}$. La definizione si estende anche alle fasi di questo processo.

Marchionini e Kuhlthau mettono in evidenza la ricerca dell'informazione come processo definendola rispettivamente come «[a] process in which humans purposefully engage in order to change their state of knowledge» ${ }^{116} \mathrm{e}$ «the process of construction within information seeking involves fitting information in with what one already knows and extending this knowledge to create new perspectives» ${ }^{117}$. L'information seeking essendo parte del più generale information behavior cioè del comportamento umano verso l'informazione è strettamente dipendente dal contesto.

Il modello elaborato nel 1989 da Marcia Bates nel suo saggio The design of browsing and berrypicking techniques for the online search interface ${ }^{118}$ riguarda la ricerca attiva e diretta dell'informazione ed è noto per aver messo profondamente in discussione il modello classico che aveva dominato le teorie dell'information retrieval e della ricerca on-line per almeno venticinque anni.

Il modello classico era infatti basato sulla semplice equazione che vedeva da una parte la collezione di documenti e la rappresentazione dei contenuti del sistema e dall'altra il bisogno informativo la sua rappresentazione in una query. Al centro del modello vi è il risultato, basato sulla corrispondenza (matching) tra la query che l'utente ha costruito e la rappresentazione del documento.

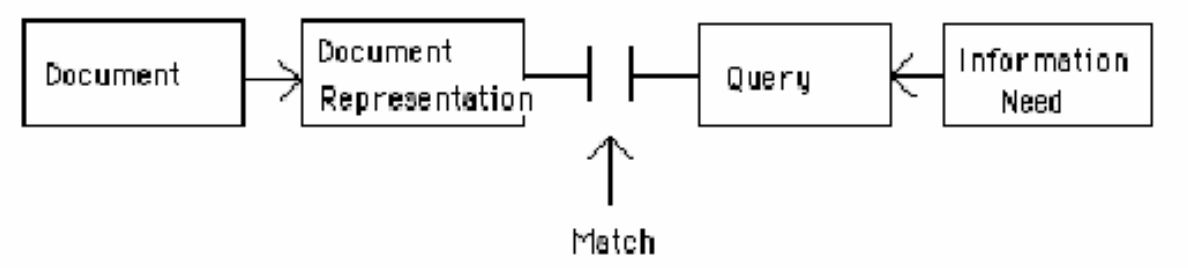

FIGURE 1: The Classic Information Retrieyal Model

Fig. 5.12 - Il modello 'classico' di Information retrieval ${ }^{119}$

\footnotetext{
115 Gary Marchionini, Information seeking in electronic environments, Cambridge, Cambridge University Press, 1995, p. 6.

116 Ivi, p 5.

${ }^{117}$ Carol Collier Kuhlthau, Seeking meaning cit.

118 Marcia J. Bates, The design of browsing and berrypicking techniques for the online search interface, «Online Review», 13 (1989) n. 5, p. 407-424.

${ }^{119}$ Ivi, p. 408.
} 
Un modello semplice, statico e 'ingenuo' di IR, in quanto incapace di cogliere la natura interattiva e dinamica del processo di recupero dell'informazione.

A questo paradigma Bates contrappone un modello innovativo più vicino al reale comportamento di chi fa ricerca e di conseguenza più adatto alla progettazione di interfacce per la ricerca online.

Secondo la studiosa man mano che si incrementano le tipologie di documenti e di fonti disponibili in rete (banche dati fulltext etc., enciclopedie online e altre fonti di reference), l'utente si trova di fronte un ambiente complesso sia in termini di risorse disponibili sia in termini di tecniche di ricerca da utilizzare con queste fonti ${ }^{120}$ :

We need to expand our understanding of these resources and the search techniques to use in them, and at the same time, expand our view of how the search interface should be designed to assist searchers with their new, complex tasks ${ }^{121}$.

Già prima di Bates il modello classico della ricerca era stato messo in discussione da alcuni studi che avevano preso considerazione una parte del modello (quella lato-utente); Belkin, Oddy e Brookes ${ }^{122}$ si erano chiesti se fosse necessario per chi cerca trovare un modo per rappresentare il bisogno informativo in una query comprensibile dai sistemi, o se invece i sistemi avrebbero dovuto aiutare l'utente ad esprimere il bisogno informativo nel linguaggio naturale, invece di tradurlo nel linguaggio artificiale del sistema. Dall'altra (quella dei sistemi) si era più volte sottolineato che lo sviluppo tecnologico e la possibilità della ricerca fulltext facevano sì che la rappresentazione tradizionale dei documenti (vocabolario controllato) giocasse un ruolo minore nella pratica del recupero dei documenti.

Ciò che è particolarmente rilevante nella riflessione proposta da Bates è che la studiosa mette in discussione l'intero modello classico e non semplicemente una sua parte (quella dei sistemi o quella dell'utente), in quanto considera quel modello nella sua totalità come inadeguato a rappresentare compiutamente la ricerca dell'informazione.

Il modello proposto da Bates è innovativo rispetto ai precedenti per quattro principali elementi: la natura della query, la natura dell'intero processo di ricerca, la varietà delle

\footnotetext{
${ }^{120}$ Le tecniche o tattiche di ricerca sono state approfondite dalla studiosa in un precedente contributo ove venivano identificate 29 tattiche e varie strategie di ricerca. Lo studio di questi aspetti è oggetto di un particolare filone di indagine che Wilson ha denominato information searching behavior. Cfr.: Marcia J. Bates, Information search tactics, «Journal of the American Society for Information Science», 30 (1979), p. 205-214.

${ }^{121}$ Ivi p. 407.

${ }^{122}$ Nicholas J. Belkin, R. N. Oddy, H. M. Brooks, ASK for Information Retrieval: Part II. Results of a Design Study, «Journal of Documentation», 38 (1982), n. 3, p. 145-164.
} 
tecniche di ricerca usate, information domain o ambito disciplinare in cui la ricerca è condotta $^{123}$.

Nel modello classico la domanda di ricerca è statica e rimane sempre la stessa durante tutto il corso della ricerca; ciò semplifica eccessivamente il modello in quanto nella realtà le cose si svolgono diversamente e la ricerca più che a un evento singolo assomiglia all'azione della 'raccolta delle bacche' in una foresta:

In real-life searches in manual sources, end users may begin with just one feature of a broader topic, or just one relevant reference, and move through a variety of sources. Each new piece of information they encounter gives them new ideas and directions to follow and, consequently, a new conception of the query. At each stage they are not just modifying the search terms used in order to get a better match for a single query. Rather the query itself (as well as the search terms used) is continually shifting, in part or whole. This type of search is here called an evolving search. Furthermore, at each stage, with each different conception of the query, the user may identify useful information and references. In other words, the query is satisfied not by a single final retrieved set, but by a series of selections of individual references and bits of information at each stage of the ever-modifying search. A bit-at-a-time retrieval of this sort is here called berrypicking. This term is used by analogy to picking huckleberries or blueberries in the forest. The berries are scattered on the bushes; they do not come in bunches. One must pick them one at a time. One could do berrypicking of information without the search need itself changing (evolving), but in this article the attention is given to searches that combine both of these features ${ }^{124}$.

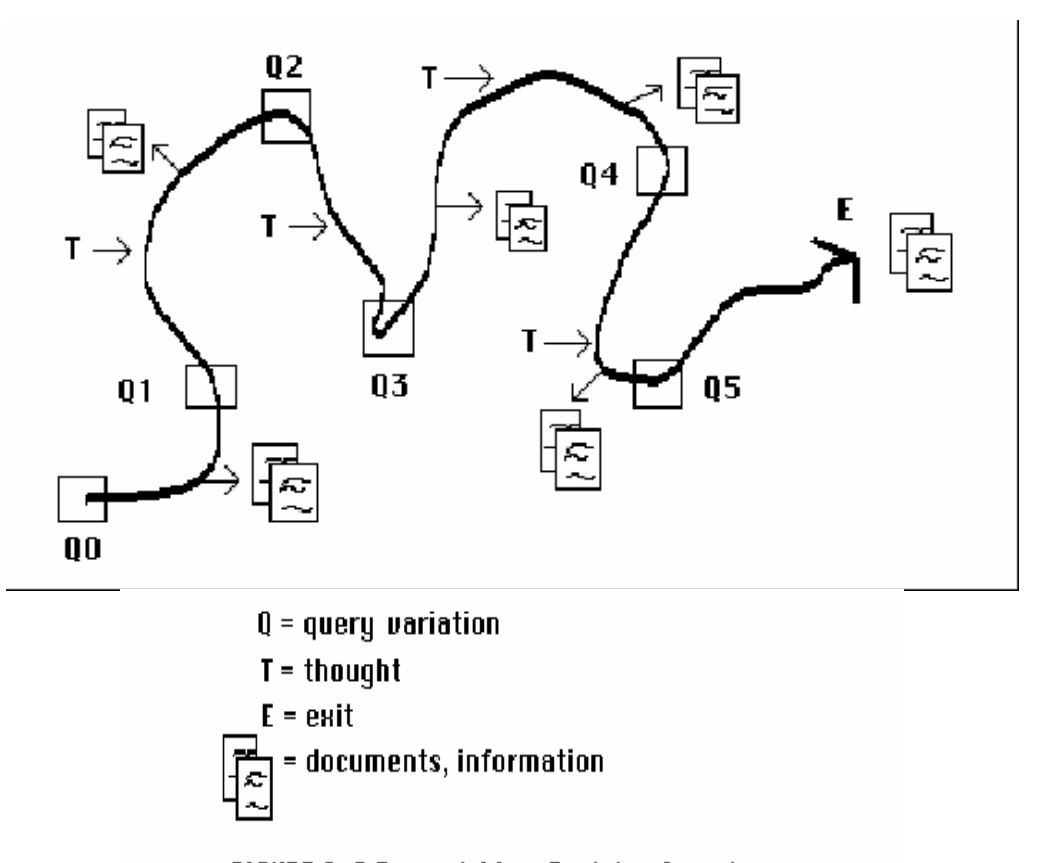

FIGURE 2: A Berrypicking, Euoluing Search

Fig. 5.13 - Il modello del berrypicking elaborato da Bates ${ }^{125}$

\footnotetext{
${ }^{123}$ Si veda: Marcia J. Bates, Berrypicking in Theories of information behaviour edited by Karen E. Fisher Sanda Erdelez - Lynne Mckechnie, Medford, published for the American Society for Information Science and Technology by Information Today, 2005, p. 58 -62.

${ }^{124}$ Marcia J. Bates, The Design of Browsing and Berrypicking Techniques cit., p. 409-410.

${ }^{125}$ Ivi, p. 410.
} 
Il modello del berrypicking, a differenza del modello classico di recupero dell'informazione, usando le parole di Bates, suggerisce che:

* le ricerche non sono statiche ma spesso si evolvono;

* la ricerca non è costituita da una semplice elencazione di risultati, bensì da una sequenza di briciole di informazione;

* chi ricerca informazioni usa una varietà di tecniche di ricerca che si estendono al di là di quelle che comunemente vengono associate alle basi dati bibliografiche;

* [che gli utenti] usano una varietà di risorse che si estendono ben oltre le basi dati bibliografiche.

Mentre il modello classico trova il suo focus nel matching cioè nella corrispondenza tra le rappresentazioni del documento e del bisogno informativo, nel modello di Bates il focus si sposta ad una sequenza di comportamenti. L'utente si muove verso l'obiettivo attivando una serie di azioni. I cambi di direzione rappresentano i cambiamenti di una ricerca che si evolve poiché l'utente opera una serie di cambi di rotta e di pensiero. Nel caso di una ricerca basata sul matching (o single match search) il processo è diretto e lineare e comporta una sola domanda e una solo set di risultati in risposta all'interrogazione.

Rispetto a tale modello emergono dunque alcune importanti differenze: la natura della query è in evoluzione e cambia nel corso della ricerca, la natura del processo di ricerca segue un percorso simile a quello della raccolta delle bacche, invece di condurre al singolo set di risultati. Man mano che l'utente aggiunge informazione all'informazione già acquisita, riconcettualizza la precedente query come risultato di un processo di comprensione più profonda del dominio che viene indagato o dell'argomento cercato.

Non è dunque scontato che l'approdo di una ricerca sia un set di risultati; durante il corso della ricerca infine l'utente può usare diverse fonti e mettere in atto diverse strategie di ricerca (search tactics).

Accanto al modello di Bates altri studiosi hanno dimostrato che la ricerca ha un andamento evolutivo; tra questi Ellis e Kuhlthau hanno dimostrato nei loro modelli che c'è un grande quantità di ricerca esplorativa nel processo di ricerca.

Il modello di Bates suggerisce anche che nella ricerca reale ci sono molti modi con cui l'utente si approccia all'informazione seguendo e alternando alcune principali strategie, tra le quali quella di seguire le note a piè pagina trovate in volumi e articoli di interesse per scoprire nuove informazioni (footnote chasing); la ricerca delle citazioni, servendosi di 
indici citazionali e risalendo dalla citazione trovata ai documenti che citano il documento (forward chaining); la strategia di visualizzare, una volta identificata una rivista in un'area di interesse, l'elenco dei numeri pubblicati per individuare le annate o i fascicoli che interessano (journal run); quella di fare browsing nel materiale che è collocato fisicamente in prossimità del materiale esaminato in precedenza per recuperare documenti simili (una tecnica che dalle biblioteche a scaffale aperto viene oggi replicata in alcuni OPAC tramite interfacce e ricerche visuali); e, infine, quella di cercare per soggetto in bibliografie e servizi di abstracting e indexing (A\&I services) e per autore.

La ricerca per soggetto e la ricerca per autore, che chiudono il quadro delle tecniche descritte da Bates, sono spesso viste come due modalità di ricerca contrastanti nella letteratura professionale sull'uso dei cataloghi dove si parla di known item search opposta alla subject search. Ma la ricerca per autore - secondo Bates - può essere parte di una ricerca per soggetto, ad esempio quando un utente usa il nome dell'autore per vedere se l'autore ha scritto opere sullo stesso soggetto.

I suggerimenti di Bates per progettare interfacce di ricerca 'di tipo berrypicking', comprendono dunque l'implementazione delle funzionalità in grado di soddisfare le principali funzioni sopra descritte.

* browsing. La visione della ricerca come un processo in evoluzione, simile alla raccolta delle bacche e che usa una varietà di fonti d'informazione e di tecniche di ricerca cambia il modo con cui si intendono il browsing e la navigazione;

* riferimenti bibliografici: implica la ricerca a partire dalle note a pié pagina, dalle citazioni e la possibilità di muoversi tra le sezioni di un documento;

* ricerca delle citazioni: a partire da un riferimento o una citazione si deve essere in grado di vedere un set di riferimenti che citano il riferimento di partenza. L'utente dovrebbe poter visualizzare gli spogli delle riviste, blocchi di testo, abstract e sommari;

* spoglio delle riviste: comporta la possibilità di visualizzare le annate, i fascicoli delle riviste, e il loro contenuto;

* area scanning: è la possibilità di visualizzare lo scaffale relativo ad un'area di interesse.

Le funzioni descritte sono attivabili non solo nella ricerca attiva dei documenti cartacei, ma anche negli ambienti digitali.

Le tecniche descritte da Bates nel 1989 sono oggi estremamente attuali e utili nello sviluppo di nuove funzionalità per i cataloghi elettronici per alcune ragioni: 
il modello supera la tradizionale impostazione del matching e introduce nuove dimensioni nella ricerca dell'informazione aprendo alle modalità esplorative e di browsing e considerando la navigazione nel catalogo, a lungo trascurata nei cataloghi elettronici, un mezzo naturale di acquisizione dell'informazione;

* alcune funzionalità descritte (es. ricerca delle citazioni, area scanning, spoglio delle riviste) sono ormai applicabili agli OPAC di nuova generazione e nei discovery system che hanno introdotto al loro interno una gran quantità di documenti in fulltext e di articoli di periodici.

\subsubsection{Ricerca attiva e acquisizione passiva di informazione: il modello integrato di Bates}

A partire dalla prima conferenza internazionale riguardante la ricerca dell'informazione nel contesto (International Conference on Information Seeking in Context) svoltasi nel 1996 in Finlandia apparvero un gran numero di contributi che indagarono la ricerca dell'informazione sotto vari aspetti; essi riguardavano approcci di tipo diverso e indagini in particolari contesti come quello lavorativo ma anche tipologie di ricerca non solamente attive, ma anche non intenzionali e passive, come il fenomeno di imbattersi accidentalmente nell'informazione, teorizzato da Sanda Erdelez ${ }^{126}$ o di evitare l'informazione (information avoidance) ${ }^{127}$.

Nel 2002 Marcia Bates nel saggio Toward an integrated model of information seeking and searching ${ }^{128}$ offre una nuova sintesi dei modelli comportamentali di ricerca delle informazioni, ricomponendo in un unico modello "integrato" le diverse prospettive

\footnotetext{
${ }^{126}$ Sanda Erdelez, Information encountering: a conceptual framework for accidental information discovery in Pertti Vakkari-Reijo Savolainen-Brenda Dervin, Information seeking in context: proceedings of international conference on research in information needs, seeking and use in different context, London, Taylor Graham, 1997, p. 412-421.

${ }^{127}$ Il comportamente di evitare l'informazione viene studiato in special modo nel contesto dell'informazione medica, ma può dipendere anche dalla povertà informativa, cioè dalla mancanza di strumenti adatti a comprendere l'informazione ricevuta, dovuto anche da una scarsa alfabetizzazione informativa o alle condizioni sociali; si vedano in proposito i vari saggi di Elfreda Chatman, in particolare: Elfreda Chatman, The impoverished life world of outsiders, «Journal of the American society of Information science», 47 (1996), p. 193-206. Su concetto di 'information poverty' nella Library and information science si veda anche: Jutta Haider and David Bawden, Conceptions of 'information poverty' in library and information science: a discourse analysis, «Journal of Documentation» 63, (2007), n. 4, p. 534-557.

${ }^{128}$ Marcia J. Bates, Toward an integrated model of information seeking and searching, Keynote Address, Fourth international Conference on Information Needs, Seeking and Use in Different Contexts, Lisbon, Portugal, September 11, 2002 <http://www.gseis.ucla.edu/faculty/bates/articles/info_SeekSearch-i030329.html>.
} 
con cui gli studiosi hanno indagato negli anni più recenti i comportamenti di ricerca dell'informazione nei vari contesti. Nel modello, unendo gli aspetti sociali e culturali con quelli biologici e antropologici, Bates ricostruisce un quadro unitario delle diverse strategie di raccolta delle informazioni.

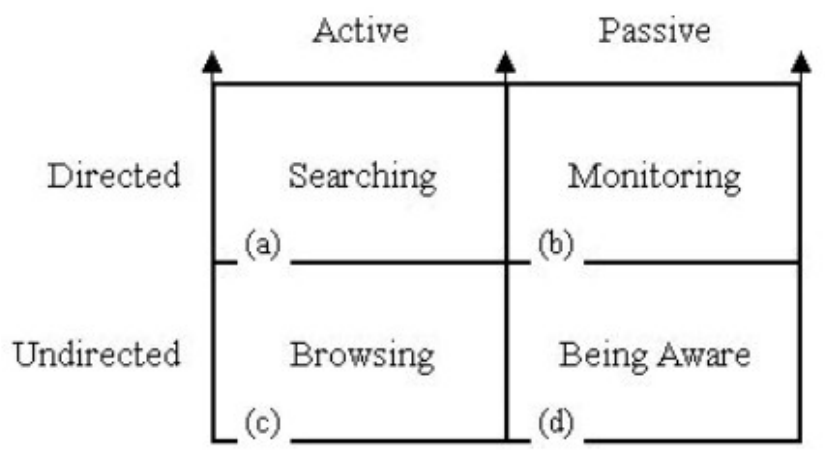

Fig. 5.14 - Il modello integrato di Bates ${ }^{129}$

I risultati sono stati riassunti da Luca Rosati ${ }^{130}$ :

Bates distingue quattro strategie fondamentali di raccolta o ricerca dell'informazione, disposte in una matrice bidimensionale. I due assi della matrice indicano il grado di consapevolezza dell'individuo circa l'informazione ricercata (asse verticale) e il grado di volontarietà del processo di ricerca stesso (asse orizzontale). Per ciascuno di questi assi vengono distinte due modalità opposte:

* asse verticale (grado di consapevolezza dell'oggetto ricercato; grado in cui sappiamo di aver bisogno di conoscere una data informazione)

- diretto: posso specificare cosa sto cercando, se pure con minore o maggiore precisione

0 indiretto: non so cosa sto cercando

asse orizzontale (grado di volontarietà)

○ attivo: ricerca attiva di una informazione

○ passiva: acquisizione passiva di una informazione (dal contesto, da altri etc.)

Dalle diverse combinazioni di tali modalità scaturiscono le quattro strategie fondamentali di ricerca:

* ricerca attiva e consapevole (searching) di una informazione specificabile

* monitoraggio (monitoring): capacità di assorbire dall'esterno informazioni di specifico interesse senza però cercarle direttamente; si tratta di una sorta di propensione alla serendipità

* esplorazione (browsing): non abbiamo uno specifico interesse o bisogno conoscitivo, ma ci esponiamo attivamente alla possibilità di acquisire nuove informazioni

\footnotetext{
${ }^{129}$ Ivi, s.p.

130 Luca Rosati, Un modello integrato di interazione uomo-informazione, Trovabile, 2007, $<$ http://trovabile.org/articoli/un-modello-integrato-di-interazione-uomo-informazione>.
} 
acquisizione/consapevolezza (awareness): acquisizione passiva di informazioni (sono le informazioni che ci vengono incontro, non siamo noi a cercarle) e indiretta (in assenza di esigenze conoscitive specifiche)".

La sintesi di Bates offre degli spunti interessanti specie nel momento in cui analizza la ricorrenza delle strategie di ricerca delineate nel modello, nella vita dell'uomo. Le strategie attive di raccolta delle informazioni, cioè quelle che richiedono uno sforzo mentale (searching e browsing) sono le meno usate e circa il $94 \%$ delle informazioni che acquisiamo nel corso della vita avvengono tramite una modalità passiva di acquisizione delle informazioni ${ }^{131}$.

\subsubsection{Le fasi della ricerca attiva: i modelli di Ellis e Kuhlthau}

Tra i modelli orientati all'utente, alcuni studi hanno cercato di descrivere sequenze di comportamenti e fasi della ricerca attiva dell'informazione. Questi modelli possono offrire nuovi spunti a chi progetta sistemi di recupero dell'informazione.

1. Ellis Model of information seeking behavior: un modello comportamentale di recupero dell'informazione

L'intenzione di Ellis, quando propose il suo lavoro, era di fornire un modello di information seeking behavior fondato empiricamente, che potesse dunque influenzare significativamente la progettazione di sistemi di IR concentrandosi sui principali comportamenti che gli utenti manifestano nel processo di ricerca dell'informazione ${ }^{132}$. Il modello di Ellis rispecchia dunque non un modello cognitivo, ma piuttosto un approccio comportamentale alla ricerca dell'informazione. Il presupposto di partenza è che dietro l'attività di ricerca dell'informazione vi sia un certo numero di tipologie diverse di comportamenti che Ellis individua nelle seguenti attività:

\footnotetext{
${ }^{131}$ Così commenta Luca Rosati, ivi s.p.: «Circa l'80\% delle informazioni che acquisiamo nel corso della nostra vita ci vengono attraverso la modalità di awareness; circa il 14\% attraverso il monitoring; solo il $5 \%$ è acquisito mediante browsing e un esiguo $1 \%$ mediante searching. La quasi totalità $(94 \%)$ del nostro bagaglio conoscitivo deriva quindi da una modalità passiva di acquisizione delle informazioni».

${ }^{132}$ David Ellis, A behavioural approach to information retrieval system design. «Journal of Documentation», 45 (1989), p. 171-212.
} 
Starting: riguarda le strategie utilizzate per iniziare la ricerca d'informazione, quali, ad esempio consultarsi con i mediatori dell'informazione (amici, studiosi, insegnanti, bibliotecari).

Chaining: seguire i collegamenti tra le citazioni e le note a pie di pagina dei documenti trovati per scoprire altro materiale; comprende anche l'attività di seguire a ritroso le citazioni, consultando indici citazionali.

Browsing: 'semi-directed or semi-structured searching' ricerca semidiretta o semistrutturata (Ellis, 1989, p. 187) in cui si dà una veloce occhiata ad un'area di potenziale interesse.

Differentiating: differenziare le risorse ottenute, ma anche gli approcci adottati, la qualità e il livello dell'informazione, la qualità, il livello, il tipo di trattamento come mezzo per filtrare il quantitativo di informazione ottenuta.

Monitoring: mantenersi aggiornati sugli sviluppi di un determinato settore di studi selezionando specifiche fonti di informazione.

Extracting: identificare selettivamente il materiale rilevante da una fonte informativa

Verifying: controllare l'accuratezza dell'informazione ricevuta

Ending: tirare le conclusioni e giungere alla conclusione della ricerca.

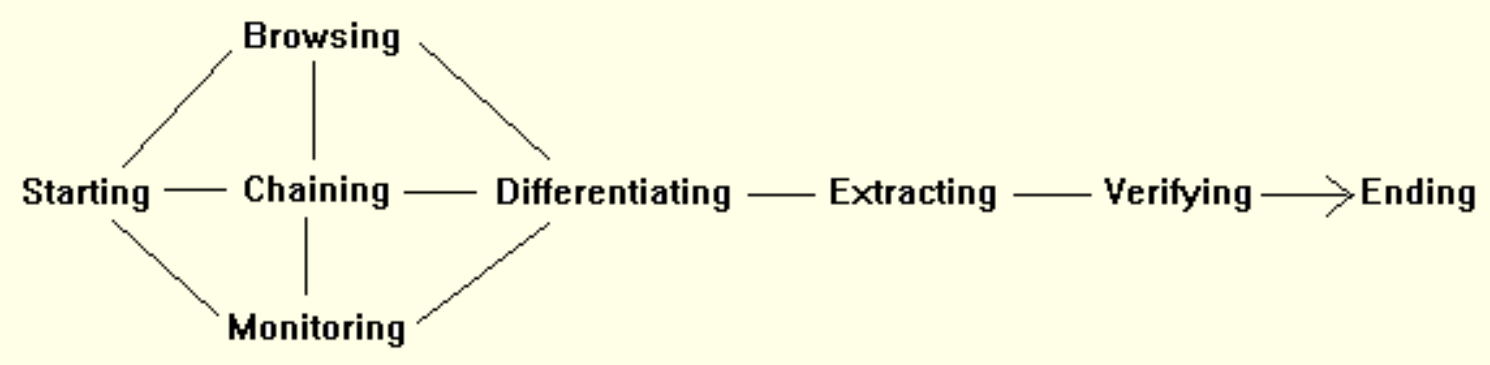

Fig. 5.15 - Il modello 'comportamentale' di David Ellis ${ }^{133}$

Nel modello di Ellis i vari comportamenti non sono definiti in termini di fasi della ricerca, ma di caratteristiche comportamentali e strategie, dunque il processo descritto non è da intendersi come lineare; i comportamenti individuati interagiscono e si combinano tra loro in vari modi e possono formare schemi diversi di ricerca dell'informazione a seconda delle circostanze nelle quali avviene la ricerca, della persona coinvolta e del particolare punto temporale nel quale la ricerca avviene. Nel modello vi sono fasi (di inizio e di fine), una serie di comportamenti (differentiating, extracting, verifying) e una serie di strategie (browsing, chaining, monitoring). Gli studi che Ellis ha condotto in collaborazione con

\footnotetext{
${ }^{133}$ Thomas D. Wilson, Models in information behaviour research, cit., p. 255.
} 
altri studiosi (tra i quali Ford, Spink, Wilson) hanno indagato gli aspetti dell'incertezza, degli stili cognitivi e dell'interazione tra utenti e intermediari, aprendo nuove strade per esplorare il comportamento nella ricerca dell'informazione.

2. Kuhlthau Information Search Process (ISP): le fasi del processo di ricerca dell'informazione

Lo studio di Carol Kuhlthau ${ }^{134}$ sul processo di ricerca ha avuto come esito un modello comunemente noto come Information Seeking Process (ISP) ${ }^{135}$.

Il modello si basa sulla teoria costruttivista di Kelly ${ }^{136}$ e rappresenta la ricerca dell'informazione come un processo di 'costruzione'. Secondo Kuhlthau la ricerca dell'informazione è prima di tutto un processo intellettuale ove i bisogni informativi si evolvono da una vaga consapevolezza di qualcosa che manca fino al recupero dell'informazione che contribuisce alla comprensione e al significato.

Il modello focalizza l'attenzione sull'utente e sulle sue percezioni durante il processo, identificando la parte più significativa nella formulazione del focus della ricerca, prima della quale l'utente permane in una situazione di incertezza (uncertainty) che ne può provocare il fallimento. La ricerca si svolge secondo una serie di passaggi che si snodano in un processo lineare che conduce l'utente dall'incertezza alla confidenza e dalla rilevanza alla pertinenza; seguendo il modello, si aprono spazi di intervento da parte dei sistemi nel processo della ricerca in quei punti critici in cui l'utente si trova in difficoltà a formulare, esprimere o manifestare il bisogno informativo.

Nello studio della ricerca dell'informazione dal punto di vista cognitivo è centrale il modello che la persona che cerca ha del mondo, determinato dall'educazione e dalle esperienze pregresse. Ciò che Ingwersen chiama knowledge structures ovvero strutture della conoscenza, o che altri definiscono modelli cognitivi.

Lo studio propone un modello del processo di ricerca dell'informazione per fasi che comprende lo sviluppo di considerazioni su un argomento (topic), le sensazioni che accompagnano lo sviluppo di questo pensiero, l'azione di cercare e usare le risorse.

\footnotetext{
${ }^{134}$ Professor II Emerita del Department of Library and Information Science alla Rutgers University, è direttore del Center for International Scholarship in School Libraries (CISSL). È conosciuta a livello internazionale per la sua ricerca innovativa sul processo di ricerca dell'informazione (ISP) e per il modello ISP degli aspetti emotivi, cognitivi e fisici articolata in sei fasi della ricerca e dell'uso dell'informazione. Si veda: Carol Collier Kuhlthau, Seeking meaning cit.

${ }^{135}$ Già prima di Kuhlthau, altri studiosi avevano tentato di descrivere le fasi del processo informativo; tra questi Krikelas aveva schematicamente descritto le fasi della ricerca dellinformazione in un modello costruito su tre fasi : information seeking, information gathering, information giving. Cfr.: James Krikelas, Information-seeking behavior: patterns and concepts, «Drexel library quarterly», 19 (1984), p. 5-20

${ }^{136}$ George Kelly, A theory of personality: the psychology of personal constructs, New York, W. W. Norton \& Co, 1963.
} 
Il compito intrapreso in ogni fase della ricerca viene categorizzato in base alle tre classi del pensiero, delle azioni, delle sensazioni e, come corollario, delle strategie utili o delle modalità che producono i risultati più soddisfacenti.

Nel corso delle fasi dell'ISP i pensieri evolvono, dapprima sono vaghi e non chiari, diventano poi precisati, o nel linguaggio di Belkin si passa da uno stato anomalo di conoscenza (ASK) alla cosiddetta specificità.

I passaggi centrali del modello sono costituiti dalla formulazione del focus, fase fondamentale del processo che costituisce l'idea che guida l'utente nel processo, dà una direzione da seguire affinché la ricerca dell'informazione desiderata abbia successo.

Il processo di ricerca (ISP) si articola in sei fasi che dall' inizio del compito di ricerca portano l'utente al completamento della ricerca; all'interno di ogni stadio vengono prese in considerazione tre categorie principali: la sfera affettiva (sensazioni); quella cognitiva (pensieri) e quella fisica (azioni intraprese).

1. Inizio del compito di ricerca. In questa fase la persona comprende il compito assegnato e va alla ricerca dei possibili argomenti. Prevale un senso di incertezza e di solito l'azione intrapresa è la discussione con amici e colleghi sui possibili argomenti e l'azione pratica di fare browsing in biblioteca.

2. Selezione dell'argomento. In questa fase l'utente sceglie un argomento generale, e dà avvio alla ricerca. Nella scelta sono coinvolte una serie di analisi che coinvolgono l'interesse personale, la quantità di informazione disponibile, i requisiti del progetto e il tempo assegnato. In questa fase l'utente tende a confrontarsi con i mediatori (es. l'insegnante o il bibliotecario), a fare un'attività di scanning, per avere un quadro d'insieme dell'argomento e delle possibilità di sviluppo della sua ricerca, come consultare una collezione di reference.

3. Esplorazione precedente alla formulazione dell'oggetto di ricerca (pre-focus exploration). In questa fase l'utente non ha ancora determinato il focus della ricerca e dunque il compito principale è quello di indagare l'informazione sull'argomento generale da estendere alla comprensione personale dell'argomento al fine di formulare un focus.

4. Formulazione dell'oggetto di ricerca. È il punto di svolta del processo di ricerca, che fa diminuire l'incertezza e aumentare la fiducia . Il compito è quello di formare un focus, dunque selezionare e identificare le idee che per mettono di mettere a fuoco l'argomento. Fattori che condizionano il risultato sono l'interesse personale, il compito assegnato, l'informazione disponibile e il tempo a disposizione. 
5. Raccolta dell'informazione. È in questa fase che l'interazione tra l'utente e il sistema di recupero dell'informazione funziona in modo efficace, infatti il compito è di raccogliere l'informazione su un focus ormai formato.

6. Conclusione della ricerca. Nella fase di chiusura della ricerca, l'utente esibisce un senso di sollievo. Le persone finiscono la ricerca per motivi diversi: sentono di aver messo abbastanza impegno nella ricerca, diminuisce la rilevanza e cresce la ridondanza dei documenti trovati, oppure ad un certo punto smettono di cercare l'informazione perché devono prepararsi ad affrontare la stesura di una ricerca. Prevalgono strategie di organizzazione in quanto ci si prepara a presentare la ricerca.

Nel modello Kuhlthau prende in considerazione anche il concetto di rilevanza: secondo la studiosa nelle prime fasi del processo l'utente cerca l'informazione rilevante correlata all'argomento generale; dopo aver formulato il focus della ricerca procede alla ricerca dell'informazione pertinente correlata all'argomento che ha messo a fuoco ${ }^{137}$.

I concetti di 'rilevanza' e 'pertinenza' fanno riferimento alle elaborazioni di Saracevic ${ }^{138}$, cioè la rilevanza è la determinazione che l'informazione è correlata o si può applicare all'argomento o oggetto di ricerca che si sta esaminando, dunque è utile nella ricerca che si sta conducendo, mentre l'irrilevanza è considerata la non appartenenza ad un concetto, e l'inutilità ai fini della ricerca. La pertinenza è correlata invece al proprio bisogno informativo.

Bisogna precisare che il modello non riguarda propriamente la ricerca nei cataloghi elettronici o in sistemi di information retrieval, ma schematizza un generale compito di ricerca, che può concretizzarsi in più azioni diverse, tra le quali anche quella di cercare in un catalogo o in un sistema. La studiosa però non esclude che il modello delineato possa servire da spunto della progettazione di sistemi di recupero dell'informazione ${ }^{139}$.

\footnotetext{
${ }^{137}$ Carol Collier Kuhlthau, Seeking meaning cit. p. 41.

${ }^{138}$ Tefko Saracevic, Relevance: a review of and a framework for the thinking on the notion in information science cit.

${ }^{139}$ Ivi, p. 208-209.
} 
Model of the Information Search Process

\begin{tabular}{|c|c|c|c|c|c|c|c|}
\hline & Initiation & Selection & Exploration & Formulation & Collection & Presentation & Assessment \\
\hline $\begin{array}{l}\text { Feelings } \\
\text { (Affective) }\end{array}$ & Uncertainty & Optimism & $\begin{array}{l}\text { Confusion } \\
\text { Frustration } \\
\text { Doubt }\end{array}$ & Clarity & $\begin{array}{l}\text { Sense of direction / } \\
\text { Confidence }\end{array}$ & $\begin{array}{l}\text { Satisfaction or } \\
\text { Disappointment }\end{array}$ & $\begin{array}{l}\text { Sense of } \\
\text { accomplish- } \\
\text { ment }\end{array}$ \\
\hline $\begin{array}{l}\text { Thoughts } \\
\text { (Cognitive) }\end{array}$ & vague & 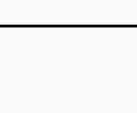 & $\longrightarrow$ & focused & increased & interest & $\begin{array}{l}\text { Increased } \\
\text { self- } \\
\text { awareness }\end{array}$ \\
\hline $\begin{array}{l}\text { Actions } \\
\text { (Physical) }\end{array}$ & seeking & $\begin{array}{l}\text { relevant } \\
\text { Exploring }\end{array}$ & information & seeking & $\begin{array}{c}\text { pertinent } \\
\text { Documenting }\end{array}$ & information & \\
\hline
\end{tabular}

Fig. 5.16 - Il processo di ricerca dell'informazione (ISP) nel modello di Kuhlthau ${ }^{140}$

I modelli di Ellis e Kuhlthau sono stati spesso citati insieme nella letteratura sulla ricerca dell'informazione in quanto entrambi prendono in considerazione le fasi in cui si articola il processo di ricerca; Wilson ha rappresentato le due elaborazioni in un modello unico che ingloba le fasi analizzate da Kuhlthau e i comportamenti individuati da Ellis dimostrando la complementarità dei due modelli.

\section{S7ge: Initiation Selection/exploration Formulation Collection Presentation}

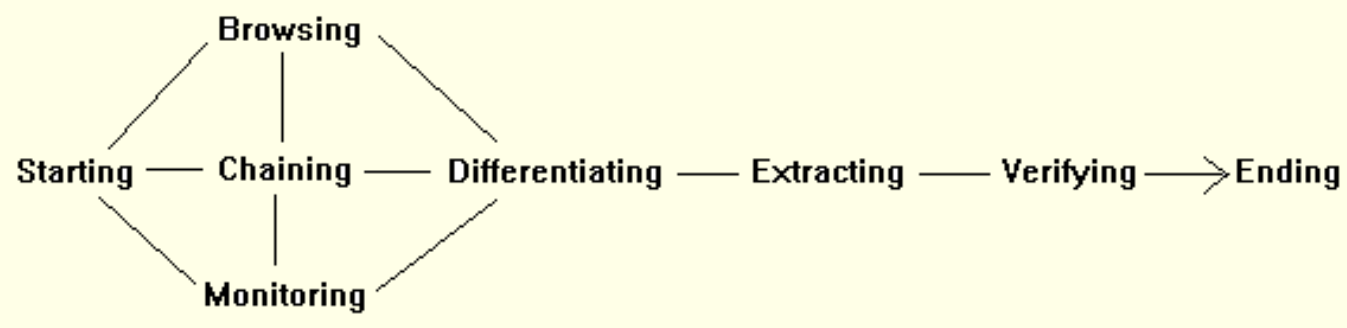
Action:
Recognize
Identify/formulate
Gather
Complete

Fig. 5.17 - Sovrapposizione di modelli: la complementarità dei modelli di Kuhlthau e Ellis ${ }^{141}$

Il modello di Kuhlthau ha fornito infine la struttura per lo sviluppo della teoria del processo basato sulle funzioni (Task-based IR process) elaborato da Vakkari $(2001)^{142}$ in base allidea che le fasi dello svolgimento di un compito di ricerca possono influenzare le tattiche di ricerca dell'informazione e i tipi di informazione ricercata, i cambiamenti delle

\footnotetext{
School of information, $<$ http://comminfo.rutgers.edu/ kuhlthau/information_search_process.htm>.

${ }^{141}$ Thomas D. Wilson, Models in information behaviour research, cit.

${ }^{142}$ Pertti Vakkari, A theory of the task-based information retrieval process: A summary and generalization of a longitudinal study, «Journal of Documentation», 57 (2001), n.1, p. 44-60.
} 
tattiche utilizzate nel corso della ricerca, dei termini di ricerca, dei giudizi di rilevanza e, in definitiva, il tipo di informazione ottenuta.

\subsubsection{Il modello cognitivo di Ingwersen e le basi dell' information searching and retrieval (IS\&R)}

A chiusura dei modelli esposti non può mancare il modello cognitivo che Ingwersen sviluppò in un decennio di studi e che ha dato origine alla teoria cognitiva della ricerca dell'informazione (Cognitive theory for information retrieval interaction) ${ }^{143}$.

Il modello, che si basa su una rappresentazione dell'interazione nel processo di recupero dell'informazione, ha dimostrato una straordinaria forza grazie al suo livello di completezza; esso considera nel recupero dell'informazione tutte le componenti coinvolte e le loro 'strutture cognitive', «rappresentando l'utente, il sistema, le fonti informative, i meccanismi di intermediazione in un contesto globale, utilizzando una prospettiva cognitiva per comprendere i principali concetti e fenomeni del recupero dell'informazione ${ }^{144}$, come la natura dei bisogni informativi, l'incongruenza cognitiva e la sovrapposizione del recupero, l'incertezza logica, il concetto di documento e le misure della rilevanza.

La rappresentazione fornita da Ingwersen è frutto dall'elaborazione di una teoria cognitiva dell'interazione che avviene nel recupero dell'informazione e considera il processo di recupero dell'informazione come un processo di apprendimento; secondo il punto di vista cognitivo ogni atto dell'elaborazione dell'informazione è mediato da un sistema di categorie e concetti che per il sistema di recupero dell'informazione costituiscono un modello della realtà.

Un punto centrale della teoria è che sia la ricezione che la generazione dell'informazione costituiscono atti dell'elaborazione dell'informazione; il modo in cui tale elaborazione viene espletata dipende dal modello della realtà dell'attore coinvolto, che può essere una persona o un elaboratore. Secondo Ingwersen dunque, a differenza delle precedenti teorie cognitive in cui la persona era il destinatario di segni, dati e informazioni generate da un sistema, nella teoria proposta anche il sistema ha un proprio modello della realtà.

143 Peter Ingwersen, Cognitive perspectives of information retrieval interaction, «Journal of Documentation», 52 (1996), n. 1, p. 3-50.

${ }^{144}$ Ivi, p. 3. 
Nell'elaborazione dell'informazione da parte dell'uomo, il modello della realtà rappresenta lo spazio cognitivo individuale, costituito dalle cosiddette 'strutture cognitive', che corrispondono agli stati cognitivi/emotivi determinati dalle esperienze pregresse. Nell'elaborazione da parte delle macchine (o 'simbolica') invece il modello della realtà consiste nelle strutture cognitive che sono state incorporate nel sistema prima dell'elaborazione. Tali strutture cognitive interagiscono con quelle delle persone, quando queste cercano l'informazione. Tale elaborazione però rimane a livello linguistico e segnico e solo quando il destinatario è una persona potrà avvenire a livello cognitivo, generando una trasformazione degli stati cognitivi dell'utente in nuovi stati, dunque trasformando l'informazione recuperata in conoscenza ${ }^{145}$. Secondo la teoria cognitiva esposta, nella scienza dell'informazione il concetto di informazione deve soddisfare due condizioni: essere il risultato della trasformazione delle strutture cognitive di chi ha generato l'informazione, e dall'altro essere qualcosa che se percepito condiziona e trasforma gli stati di conoscenza del ricevente. L'informazione va dunque - secondo lo studioso - oltre il significato: una frase o un'immagine possono avere diversi significati e produrre diversi tipi di elaborazione o diverse trasformazioni dello stato di conoscenza a seconda della situazione del ricevente. La conseguenza più evidente nell'interazione tra uomo e macchina è che i messaggi diretti all'uomo possono trasformarsi in conoscenza, ma i segni comunicati alle macchine, rimarranno segni, e dunque a livello linguistico di superficie come informazione potenziale ${ }^{146}$.

Dunque il processo di recupero dell'informazione avrà due fondamentali caratteristiche: sarà caratterizzato da incertezza e imprevedibilità in quanto ogni presupposizione e significato che si dà ai messaggi viene perso: l'interpretazione umana non è legata ad un processo di codifica-decodifica di un messaggio ma alla presenza di una semantica comune.

Il 'modello cognitivo' proposto da Ingwersen (fig. 5.18) rappresenta dunque l'interazione che avviene nel recupero dell'informazione dove le strutture cognitive che rappresentano le idee e la conoscenza umana prendono la forma di trasformazioni generate da diversi 'attori' umani, dunque provenienti da diverse origini cognitive (es. il produttore, il progettista, l'indicizzatore, l'autore dei testi) e gli utenti (ad esempio gli algoritmi rappresentano le strutture cognitive di chi li ha ideati etc.), la trasformazione della richiesta dell'utente in una query rappresenta una struttura cognitiva aggiunta a livello di interfaccia, mentre a destra il modello rappresenta lo spazio cognitivo dell'utente.

\footnotetext{
${ }^{145}$ Ivi, p. 5-6.

${ }^{146}$ Ivi, p. 8.
} 


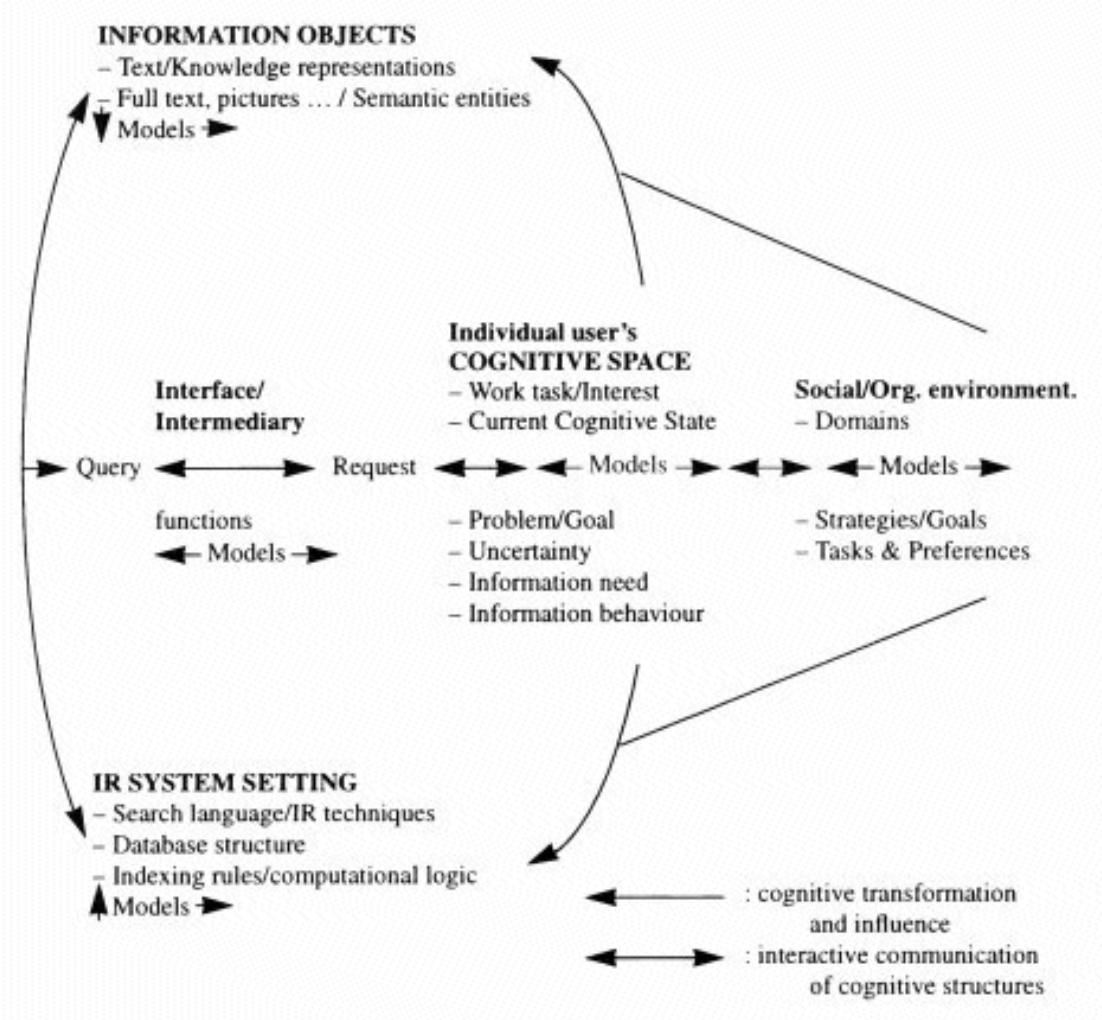

Fig. 5.18 - Il modello 'cognitivo' di Ingwersen ${ }^{147}$

Il modello proposto da Ingwersen nel 1996 ha aperto nuovi scenari nella ricerca sull'information-seeking and retrieval (IS\&R) un campo di indagine nuovo che è stato proposto in un nuovo contributo di Ingwersen and Järvelin ${ }^{148}$ e in un modello (General model of cognitive information seeking and retrieval) elaborato allo scopo di riunire i due filoni dell' information retrieval e dell' information seeking che dà origine a quel nuovo filone di studio che gli studiosi definiscono Information seeking and retrieval (IS\&R) ${ }^{149}$, derivante dall'unione del punto di vista cognitivo (Belkin e Ingwersen) con gli sviluppi dell'information retrieval o Interactive information retrieval.

Il modello rappresenta l'interazione tra le componenti coinvolte nel processo ed è focalizzato sugli 'attori cognitivi': gli autori degli oggetti informativi, i progettisti delle interfacce, i progettisti del sistema di indicizzazione delle risorse informative, i membri del progetto che giocano il ruolo di referenti nella selezione degli oggetti informativi, gli studiosi che interagiscono con gli oggetti informativi e/o li producono e infine coloro che utilizzano le interfacce dei sistemi per le loro ricerche. Ognuno di questi attori viene

\footnotetext{
${ }^{147}$ Ivi, p. 9.

${ }^{148}$ Peter Ingwersen-Kalervo Järvelin, The turn cit. La svolta verso una teoria cognitiva dell'information retrieval è descritta anche in Peter Ingwersen, Information retrieval interaction, London, Taylor Graham, 1992, p. 123-156.

${ }^{149} \mathrm{Si}$ veda il contributo di Peter Ingwersen, Interactive Framework for Information Seeking and Interactive Information Retrieval in Theories of information behaviour cit., p. 215-220.
} 
considerato nel proprio contesto culturale, sociale, lavorativo evidenziando i fattori che possono influenzarne il comportamento informativo.

Il modello suggerisce che anche la componente algoritmica, ambito dell'indagine dell'IR algoritmico, dovrebbe essere integrata negli studi sull'information seeking auspicando la collaborazione di due tradizioni di ricerca che per molto tempo hanno operato in autonomia e isolamento.

\subsection{L'OPAC a supporto del processo di ricerca dell'informazione. Verso un nuovo modello}

Scopo della presente elaborazione è delineare alcune nuove funzionalità che gli OPAC potrebbero implementare per aiutare l'utente nel processo informativo e tracciare un modello che tenga conto dei modi con cui gli utenti cercano e usano le informazioni ${ }^{150}$.

La scelta metodologica del 'modello' pone la presente ricerca in un orizzonte più ristretto rispetto a quello della teoria: i modelli si rivelano utili allo sviluppo di teorie, in quanto propongono 'letture' e 'interpretazioni' della realtà che possono essere testate al fine di produrre delle vere e proprie teorie. Essi rappresentano il primo gradino dello sviluppo delle teorie, ovvero descrivono fenomeni, affinché si possano sviluppare spiegazioni dei fenomeni stessi e prevederli ${ }^{151}$.

Il modello di OPAC che si propone nel presente lavoro di ricerca deriva dall'approfondimento delle 'metateorie socioculturali dell'informazione'152 elaborate a partire dagli anni '70 come reazione al noto modello della teoria matematica della comunicazione (MTC) e che ebbero tra i primi e più grandi protagonisti gli studiosi Jesse

\footnotetext{
150 Intendendo per 'processo informativo', seppur prendendo a riferimento i modelli generali di ricerca dell'informazione, quello di ricerca di una particolare tipologia di informazione, cioè l'informazione bibliografica.

${ }^{151}$ La maggior parte della riflessione nel campo dell'information behavior e della Library and information science si trova nello stadio della modellazione.

${ }^{152}$ Il concetto di metateoria ha affinità e sovrapposizioni con quello di paradigma individuato da Thomas Kuhn, che rappresenta tuttavia un concetto ancora più ampio in quanto racchiude, oltre alle metateorie, anche teorie e la metodologia di un campo disciplinare. Le principali metateorie o approcci sono ripercorsi nel saggio introduttivo di Marcia Bates al volume curato da Karen E. Fisher, Sanda Erdelez e Lynne Mckechnie, Theories of information behavior cit., p. 10-14 sono quelle che possono essere ricondotte ad alcuni principali approcci: 1. storico, 2. costruttivista, 3. costruzionista o discorsivo analitico 4. filosofico-analitico, 5. della teoria critica, 6. etnografico, 7. socio-cognitivo, 8. cognitivo, 9. bibliometrico, 10. fisico, 11. ingegneristico, 12. della progettazione centrata sull'utente. 13. evoluzionista.
} 
Shera ${ }^{153}$, Birger Hjørland ${ }^{154}$ e Brian Vickery ${ }^{155}$ ed in particolare considera due importanti teorie che da questi studi traggono origine:

1) la teoria cognitiva dell'informazione ${ }^{156}$, che considera nel processo dell'informazione il ruolo chiave del soggetto interpretante e che mette in primo piano le strutture cognitiva dell'utente. Secondo questa metateoria l'informazione può essere considerata come una frattura che produce una differenza nella struttura cognitiva dell'utente, concetto teorizzato da Bateson ed espresso in termini matematici da Bertram C. Brookes, e nel quale l'informazione viene definita come una «differenza che crea una differenza ${ }^{157}$. Secondo questa teoria la comprensione avviene quando creatore e interprete utilizzano e comprendono lo stesso sistema di segni, concetto da cui discende la teoria socio-cognitiva.

2) la teoria costruttivista dell'informazione, che considera il processo di ricerca come un processo di costruzione. Secondo tale teoria, che trova le origini nelle discipline della pedagogia e della filosofia, ogni individuo crea la propria realtà in base a modelli mentali che non sono innati, ma derivano dall'esperienza e si trasformano in base ad essa. Ambiti di indagine della teoria sono i concetti di bisogni informativo, di strutture della conoscenza, di uso dei sistemi informativi e di recupero dell'informazione per migliorarne l'usabilità e le funzioni ${ }^{158}$.

Le 'metateorie sociali dell'informazione' e i principali modelli di ricerca formulati entro quei paradigmi e analizzati nel presente capitolo ${ }^{159}$ costituiscono l'orizzonte teorico del modello che si propone nel presente lavoro; esse hanno dimostrato che nel recupero dell'informazione sono coinvolti diversi fattori che sono stati oggetto di analisi sia dei

\footnotetext{
${ }^{153}$ Jesse Hauk Shera, Knowing books and men; knowing computers too, Littleton, Libraries Unlimited, 1973. Nel noto saggio, uno dei più importanti della letteratura biblioteconomica, Shera criticava il concetto di informazione fornito da Shannon e Weaver e il loro approccio quantitativo, proponendo di allargare gli orizzonti epistemologici della disciplina e proponendo un approccio cognitivo e sociale.

${ }^{154}$ Birger Hjørland è il più noto esponente della metateoria socio-cognitiva, denominata anche collettivismo, che ha le sue radici nell'elaborazione teorica di Vijgotskij. Secondo la teoria le caratteristiche del gruppo sociale nel quale l'individuo opera hanno una influenza decisiva nel modo di gestire l'informazione. All'interno di questa disciplina si situa l'analisi di dominio (domain analyisis) elaborata da Birger Hjørland e Hanne Albrechtsen, teoria secondo la quale i sistemi di organizzazione delle conoscenze (es. gli schemi di classificazione) sono profondamente legati alla comunità di riferimento.

155 Il volume di Brian e Alina Vickery è un testo classico per l'approccio teorico alla Scienza dell'informazione. Si veda: Brian C. Vickery- Alina Vickery, Information science in theory and practice, London, Bowker-Saur, 1992.

${ }^{156}$ Da non confondere con il cognitivismo che invece è un filone di studio della psicologia che assimila il funzionamento della mente umana a quello del computer.

${ }^{157}$ Gregory Bateson, Verso un'ecologia della mente cit, p. 470.

${ }^{158}$ A questo filone si ascrivono i principali studi sul miglioramento dei sistemi di recupero dell'informazione con un'attenzione alle caratteristiche di usabilità e di personalizzazione delle funzionalità di ricerca.

${ }^{159}$ In particolare le principali metateorie come quelle di Dervin, Belkin, Ingwersen, Wilson e i modelli di ricerca come quelli di Kuhlthau, Bates, Goldbold, Saracevic.
} 
principali modelli di information retrieval, sia dei più ampi modelli di information behavior, sia infine dei modelli di information seeking behavior finora esaminati.

Tali teorie ci inducono a considerare nel processo di ricerca alcuni concetti chiave:

* il contesto sociale e organizzativo in cui il bisogno informativo nasce e si sviluppa;

* gli obiettivi e i compiti concreti che svolgono gli utenti;

* la struttura cognitiva dell'utente e le conoscenze pregresse;

* le strategie di ricerca dell'informazione che gli utenti mettono in campo;

* gli oggetti informativi / i testi / i documenti rappresentati nei sistemi;

* i requisiti hardware e software dei sistemi e la tipologia dei sistemi;

* le interfacce dei sistemi di recupero dell'informazione;

* gli altri intermediari coinvolti nel processo di recupero dell'informazione;

Tali modelli hanno anche dimostrato che i concetti di interattività ${ }^{160}$ e di incertezza ${ }^{161}$ hanno un grande rilievo nel processo di recupero dell'informazione: l'utente è impegnato in uno sforzo continuo di riduzione dell'incertezza, di comprensione (o di 'creazione di senso') e di 'contestualizzazione' dell'informazione trovata nel quadro delle sue conoscenze pregresse, in un processo dinamico e iterativo.

I modelli di Ellis e Kuhlthau sopra analizzati e alcuni modelli più generali visti in precedenza, come quelli di Bates, Wilson e Ingwersen offrono interessanti spunti per costruire un modello ideale di OPAC in grado di delineare alcune nuove funzionalità che gli OPAC di nuova generazione potrebbero implementare per offrire all'utente funzioni di ricerca più efficaci. Tali rappresentazioni, infatti, analizzando in profondità la ricerca attiva diretta e indiretta dell'informazione offrono un'indagine più completa del processo di ricerca e ci inducono a prendere in considerazione alcuni concetti che finora sono stati

\footnotetext{
${ }^{160} \mathrm{Si}$ fa riferimento alla dimensione interattiva della ricerca che proviene da alcune principali elaborazioni sulla natura dinamica del processo di ricerca dell'informazione come quella di Bates.

${ }^{161} \mathrm{Ci}$ si riferisce all'incertezza cognitiva proposta da Belkin e Dervin e che Kuhlthau prende a riferimento come principio di base dell'Information Science. Il 'Principio dell'incertezza' formulato da Kuhlthau individua nell'incertezza cognitiva l'origine del processo di ricerca: «Uncertainty is a cognitive state that commonly causes symptoms of anxiety and lack of confidence. Uncertainty and anxiety can be expected in the early stages of information search process. The affective symptoms of uncertainty, confusion, and frustration are associated with vague, unclear thoughts about a topic or question. as knowledge states shifts to more clearly focused thoughts, a parallel shift occurs in feelings of increased confidence. uncertainty due to a lack of understanding, a gap in meaning, o a limited construction initiates the process of information seeking». (Carol Collier Kuhlthau, Seeking meaning cit., p. 92). Il concetto di incertezza come concetto fondante della Scienza dell'Informazione non è nuovo e deriva dalla teoria matematica della comunicazione (MTC) proposta da Shannon e Weaver sebbene nella teoria dei due studiosi l'incertezza assuma una connotazione diversa: la possibilità per un messaggio di acquisire un significato deriva dalle possibilità che esso ha di manifestarsi nel codice di cui fa parte, dunque l'informazione deriva dalla riduzione dell'incertezza. In questo caso però siamo di fronte semplicemente ad una teoria quantitativa e statistica che non prende affatto in considerazione gli aspetti semantici.
} 
trascurati nella progettazione delle interfacce e delle funzionalità di ricerca degli OPAC. Esse consentono di focalizzare l'attenzione non solo sulle rappresentazioni dei testi e sugli algoritmi per il recupero, ma sulle reali necessità dell'utente e sul contesto nel quale si svolte l'attività di ricerca.

In particolare i due modelli descrittivi elaborati da Ellis e Kuhlthau possono costituire una base per l'analisi del processo informativo in un catalogo elettronico: mentre i passaggi individuati da Ellis possono essere intesi come situazioni tipiche o vere e proprie tecniche di ricerca che l'utente adotta nel passaggio dalla fase iniziale (starting) alla fase conclusiva (ending) di una ricerca ${ }^{162}$ e che possiamo paragonare alle 'tattiche' di ricerca proposte da Bates, i passaggi individuati da Kuhlthau rappresentano le fasi del processo in cui si articola la ricerca dell'informazione.

I modelli forniti da Ellis e Kuhlthau non sono però da intendersi come rappresentazioni lineari del processo informativo perché l'utente non cerca l'informazione in maniera semplicistica o compiendo un percorso che dall'inizio conduce alla conclusione seguendo fasi ben definite, ma compie percorsi irregolari: può tornare indietro, come suggerito dal modello del berrypicking di Bates o ripetere iterativamente le fasi della ricerca che sono state individuate nei due modelli.

Prendere a modello questi schemi non significa dunque tentare di progettare un OPAC che sia in grado di seguire l'utente nel suo percorso di ricerca, da una parte perché è impossibile prevedere in quale stadio del processo di ricerca (ISP) si trovi l'utente nel momento in cui si rivolge al catalogo per le sue ricerche, dall'altra perché i percorsi di ricerca non sono mai lineari.

Tuttavia una più efficace comprensione delle diverse dimensioni della ricerca è utile in quanto:

* è possibile studiare il comportamento dell'utente nell'intero processo della ricerca. Ciò consente di focalizzare la propria attenzione non solo sullo strumento informativo (nel nostro caso l'OPAC) con cui la persona entra in relazione, ma di prendere in considerazione modelli generali di comportamento, abitudini e stili cognitivi, caratteristiche che accomunano tutti gli esseri umani nel momento in cui si mettono alla ricerca dell'informazione o vengono a contatto con strumenti informativi;

\footnotetext{
${ }^{162} \mathrm{Nel}$ modello di Ellis solo le fasi di inizio e fine della ricerca sono da intendersi come vere e proprie fasi, gli altri sono meglio definiti come comportamenti di ricerca.
} 
è possibile fare delle previsioni e agire per influenzarne il comportamento modificando i sistemi, analizzando meglio i processi che avvengono nell'uso dei cataloghi elettronici.

I modelli analizzati costituiscono dunque la base teorica per delineare alcune funzionalità da implementare negli OPAC per aiutare l'utente nel processo informativo e tentare di progettare un modello che tenga conto dei modi con cui gli utenti cercano e usano le informazioni nella loro esperienza di ricerca.

\subsubsection{Verso un nuovo modello di sviluppo per l'OPAC}

Nel modello di OPAC che viene proposto lo scopo principale è analizzare le diverse componenti che entrano in gioco nell'interazione dell'utente con l'OPAC e di studiare nel dettaglio il processo di ricerca delineando le attività che vengono svolte durante il processo di ricerca dell'informazione.

Il modello si basa su alcuni assunti di partenza:

l'utente si rivolge al catalogo per colmare un bisogno informativo (gap). Alla fine della ricerca l'utente può giungere ad una conclusione fruttuosa della ricerca e colmando così la sua lacuna oppure concludere la ricerca in maniera insoddisfacente. L'utente dunque compie un percorso di ricerca che non è lineare; si può supporre che in vari momenti della ricerca l'utente analizzi il proprio bisogno informativo e decida se continuare la ricerca, cambiare strategia o percorso, reiterare i passi precedentemente fatti, o abbandonare la ricerca;

* intendendo la ricerca come un processo di 'costruzione' della conoscenza, il catalogo non deve solo fornire l'informazione cercata, ma aiutare l'utente a costruire il proprio personale percorso di ricerca, mettendo a disposizione e attivando tutte quelle funzionalità che gli consentano di compiere il proprio processo di 'informarsi', cioè di raggiungere, comprendere e usare l'informazione bibliografica;

* un OPAC che voglia davvero essere centrato sull'utente deve offrire le funzionalità che aiutino l'utente nelle diverse fasi della ricerca in cui si può trovare: supportare le diverse attività compiute da chi si trova allo stadio iniziale, dall'utente che ha già definito l'oggetto della sua ricerca, o da chi 
invece si trova alla conclusione dell'attività, attivando le funzionalità più appropriate;

* le cinque funzioni del catalogo individuate da FRBR e dai ICP (trovare, identificare, selezionare, acquisire o ottenere l'accesso e navigare ${ }^{163}$ ) costituiscono i principali obiettivi che il catalogo soddisfa nelle diverse fasi individuate e le principali attività che l'utente svolge nella sua interazione con i dati bibliografici. Queste funzioni si integrano con ulteriori funzioni che sono tipiche dei sistemi di information retrieval e che derivano da una definizione più dettagliata dei compiti che gli utenti svolgono nelle diverse fasi del processo e dalle componenti coinvolte;

* una progettazione efficace dell'OPAC dovrà tenere conto del modo in cui gli utenti oggi cercano le informazioni, delle profonde trasformazioni dei modelli mentali, delle abitudini di ricerca e degli stili cognitivi dovute alla grande influenza della ricerca web nella vita quotidiana di chi cerca le informazioni.

Una migliore organizzazione logica dei dati bibliografici, una più efficace strutturazione del processo di ricerca e una più profonda comprensione dei meccanismi di recupero dell'informazione, possono essere considerate le tre principali aree di evoluzione dei cataloghi elettronici.

Muovendo da queste preliminari considerazioni è stato elaborato un nuovo modello di sviluppo per l'OPAC che sia in grado di analizzare e rappresentare:

* il contesto nel quale si svolge l'interazione nel catalogo elettronico;

* le diverse componenti coinvolte nel processo di ricerca e di uso dell'informazione bibliografica;

* il tipo di interazione tra le diverse componenti coinvolte; e in particolare:

* le barriere e le variabili che si presentano all'utente nella fase di attivazione del processo di ricerca dell'informazione e che si frappongono nelle diverse fasi del processo informativo;

* le attività svolte, cioè il comportamento informativo (information seeking behavior) che l'utente manifesta nel processo di ricerca.

163 La funzione di navigare, non presente originariamente nelle funzioni-utente di FRBR, venne introdotta tra le funzioni del catalogo nei nuovi International Cataloguing Principles del 2009 in seguito all'importante studio di Elaine Svenonius The Intellectual Foundation of Information Organization. (Cambridge, MIT Press, 2000). 
Il modello che si propone nelle pagine seguenti può dunque essere considerato una rielaborazione originale dei modelli precedenti e si inserisce entro le due principali linee teoriche sopra citate: quella costruttivista con particolare riferimento al modello di Kuhlthau e quella cognitiva, con riferimento alla teoria di Ingwersen.

Le due teorie si sono dimostrate efficaci nell'elaborazione del modello: il primo punto di vista, che induce a concepire la ricerca come una fase di costruzione, viene usato in particolare per spiegare il comportamento di ricerca dell'informazione che avviene nel catalogo tramite l'interfaccia, il secondo è preso a riferimento per rappresentare le diverse componenti che interagiscono in un sistema di IR e, in questo caso, all'interno di un OPAC.

Il modello fornisce una rappresentazione il più possibile completa dell'interazione che si svolge in un catalogo elettronico, in grado cioè di prendere in considerazione tutte le componenti coinvolte: l'utente con il suo stato cognitivo, le barriere e le variabili che attivano/ostacolano l'informazione; l'universo bibliografico formato dai documenti e le loro rappresentazioni, dai sistemi di organizzazione delle conoscenze in uso; il sistema con le sue caratteristiche hardware e software e, infine, gli intermediari, bibliotecari o communities di lettori, che sono coinvolti nel processo di ricerca e uso dell'informazione bibliografica.

Prendendo a riferimento i modelli precedentemente esposti (Kuhlthau 2004 ${ }^{164}$, Saracevic $1996^{165}$, Ingwersen $1996^{166}$, Wilson $1999^{167}$ ) il nuovo modello esamina e rappresenta l'interazione che avviene nell'OPAC in quanto sistema di information retrieval e che si svolge tra diverse componenti che entrano in gioco nel processo di ricerca:

* gli oggetti informativi (es. testi e la loro rappresentazione nei record bibliografici e le entità bibliografiche rappresentate in FRBR, FRSAR, FRAD). Questi sono oggetto delle funzioni-utente delineate in FRBR e riprese nei Principi internazionali di catalogazione (ICP);

* lo spazio cognitivo dell'utente formato dal particolare compito di ricerca, dal suo interesse personale, dal problema che intende risolvere, dall'incertezza e dal suo bisogno informativo, dal suo comportamento informativo e oggetto di interesse nei modelli sopra esposti (in particolare Wilson, Saracevic, Ingwersen);

\footnotetext{
${ }^{164}$ Carol Collier Kuhlthau, Seeking meaning cit.

${ }^{165}$ Saracevic, Tefko, Relevance reconsidered, cit

${ }^{166}$ Peter Ingwersen, Cognitive perspectives of information retrieval interaction cit.

167 Thomas D. Wilson - Christina Walsh, Information behaviour cit.
} 
- le caratteristiche del sistema: ad esempio le funzionalità di ricerca offerte, le particolari caratteristiche del software in uso;

* gli intermediari coinvolti nel processo di recupero dell'informazione, ad esempio i bibliotecari, e, nei nuovi OPAC sociali, delle communities di utenti che si creano attorno al catalogo.

Nell'elaborazione teorica si ritrovano dunque alcuni concetti già evidenziati dai modelli precedenti analizzati, che vengono ripresi e rielaborati:

le 'strutture cognitive' del modello di Ingwersen. Esse vengono introdotte nel modello per spiegare la tipologia di interazione che intercorre tra le diverse componenti del modello e per analizzare la nascita del bisogno informativo nel contesto;

* le 'barriere' e le 'variabili' del modello di Wilson. Vengono riprese e introdotte nel modello le variabili o barriere che si presentano all'attivazione del processo (rischio, costi-benefici, auto-efficacia) e durante il processo di ricerca (ISP ${ }^{168}$ );

* il gap (incertezza cognitiva o bisogno informativo) dei modelli di Dervin e di Belkin. Il concetto di gap viene ripreso e approfondito per spiegare il processo dinamico e non lineare del processo informativo;

* le 'fasi' e i 'comportamenti' del processo informativo dei modelli Kuhlthau ed Ellis. Scopo dei due modelli, in particolare del modello di Kuhlthau, è quello di intendere la ricerca come un processo di costruzione che si svolge in diverse fasi. Una versione semplificata del modello di Kuhlthau si è dimostrata efficace per schematizzare il processo di ricerca nell'OPAC ed individuare le attività dell'utente collegate.

Particolare rilievo assume l'analisi del processo informativo. La ricerca che si svolge nell'interfaccia, che è rappresentata nella parte centrale del modello, viene articolata in varie fasi, riprendendo l'elaborazione teorica di Kuhlthau, che viene utilizzata come schema per rappresentare il processo di ricerca dell'informazione.

In base al modello teorico proposto, tra le diverse fasi del processo l'utente compie un'analisi del proprio bisogno informativo (gap) che gli consente di decidere se proseguire

${ }_{168}$ Acronimo di Information Search Process è usato nel presente lavoro quando si vuole indicare in forma abbreviata il processo di ricerca dell'informazione. 
nel proprio percorso o se fermarsi, se ripetere la fase precedente; la ricerca è rappresentata dunque come un processo di costruzione non lineare, ma iterativo.

L'articolazione del processo in 'fasi' distinte ci offre l'opportunità di delineare stati mentali (cognitivi, affettivi, situazionali) nei quali l'utente si può trovare al momento dell'interazione con l'OPAC. Nelle tre fasi dell'ISP evidenziate, l'utente compie una serie di azioni che gli consentono di trovare/identificare/valutare/correlare /usare l'informazione bibliografica contenuta nell'OPAC.

In base all'interazione delle componenti coinvolte nel processo e alle azioni corrispondenti vengono così delineate le funzionalità che un OPAC dovrebbe offrire per supportare il processo di ricerca dell'informazione.

Il modello presentato raccoglie gli spunti provenienti dagli studi del comportamento informativo introducendo nella progettazione degli OPAC alcuni elementi di originalità che possono essere così sintetizzati:

* la raffigurazione del processo di ricerca viene considerato all'interno del 'contesto', rappresentato dalle componenti che interagiscono nell'OPAC: l'utente condizionato dal suo 'stato' (cognitivo, affettivo e situazionali); il sistema, i documenti, gli intermediari;

* la raffigurazione del processo informativo non consiste nella rappresentazione lineare di un 'iter ideale di ricerca' che si compie all'interno di un catalogo elettronico (rappresentato ad esempio dal modello di Kuhlthau), ma rappresenta le fasi di una ricerca dinamica e iterativa influenzata da più fattori, variabili e barriere che si manifestano nel corso del processo e che derivano dall'interazione delle componenti prima citate;

- l'individuazione delle attività legate alle diverse fasi del processo informativo si traduce nell'individuazione di funzioni-utente più ampie che possono essere soddisfatte dalle registrazioni bibliografiche e che ampliano le funzioni già previste da FRBR e che dipendono dal processo e dall'interazione delle componenti in esso coinvolte;

* tali funzioni - utente a loro volta sono alla base dell'analisi delle funzionalità che gli OPAC dovrebbero offrire per il soddisfacimento delle funzioni stesse e dunque per un più efficace recupero dell'informazione. 


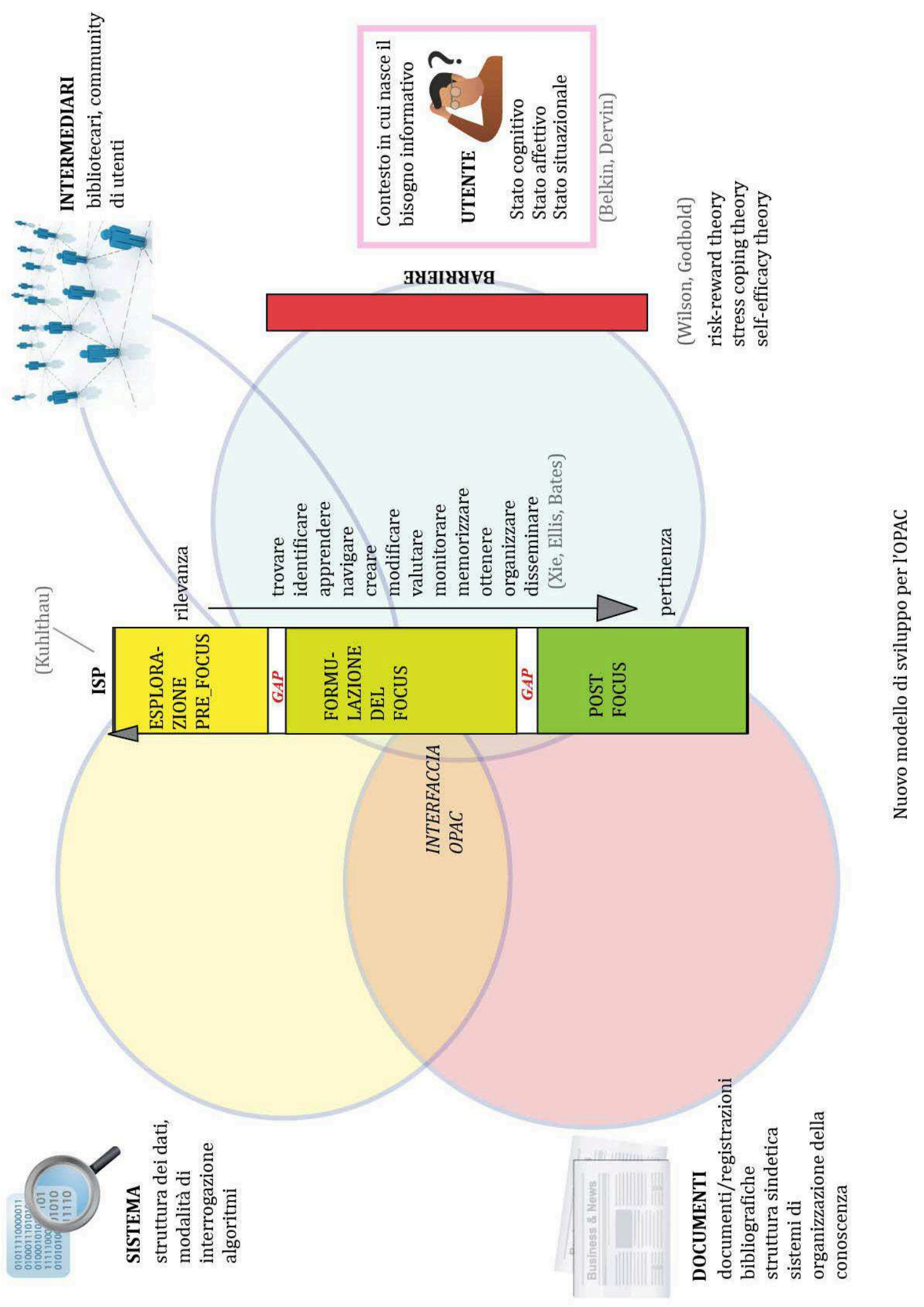

Fig. 5.19 - Un nuovo modello di sviluppo per L'OPAC. Rappresentazione delle componenti che entrano in gioco nell'interazione in un OPAC: utente, sistema, documenti, intermediari. Al centro del modello è rappresentato il processo informativo e le principali funzioni che l'utente compie durante il processo. Sono anche presenti le principali barriere che intervengono all'attivazione e nel corso del processo di ricerca. 
I paragrafi che seguono sono dedicati ad approfondire nel dettaglio i principali aspetti del modello, alcuni concetti fondamentali e, infine, il processo informativo.

Verranno dunque analizzati:

il concetto di bisogno informativo nella ricerca, il gap e le barriere;

* searching, browsing, asking: le tre modalità principali di recupero dell'informazione;

* gli intermediari e il loro ruolo nel processo di recupero dell'informazione ${ }^{169}$;

* le fasi del processo di ricerca dell'informazione bibliografica;

* le attività che l'utente svolge nelle diverse fasi della ricerca.

\subsubsection{Analizzare il bisogno informativo. Variabili e barriere nel processo di recupero dell'informazione}

Una delle più semplici domande che sorgono nell'analisi del processo di ricerca dell'informazione è quella della sua attivazione, ovvero perché gli utenti iniziano una ricerca.

L'utente può intraprendere una ricerca di informazione per colmare una lacuna informativa (gap) e adottare quelle strategie che gli permettono di 'riacquisire il senso' in un mondo disordinato e senza leggi ${ }^{170}$. Possiamo immaginare che nellinterazione con l'OPAC l'utente sia coinvolto - riutilizzando il concetto espresso da Dervin - in una continua acquisizione di senso rivolta a comprendere e interpretare l'universo bibliografico ivi rappresentato.

Secondo Robert S. Taylor, cui si deve la più celebre analisi della capacità di espressione del bisogno informativo e delle sue dimensioni, un bisogno informativo può essere presente ma inespresso (visceral need), descritto a livello conscio (conscious need),

\footnotetext{
${ }^{169}$ La presenza degli intermediari nel modello tra le componenti dell'interazione in un catalogo elettronico è un elemento originale del modello presentato, che si ricollega alle sempre più presenti componenti sociali, alle esperienze di chat nel catalogo con esperti bibliotecari (es. quanto avviene nell'OPAC Summa. Cfr. Appendice A. Casi di studio) e che apre a realizzazioni innovative, specie se si pensa alle nuove possibilità offerte dalle recenti tecnologie, che consentiranno nell'immediato futuro ad un utente di sapere chi, nel momento in cui compie una ricerca, sta effettuando la stessa ricerca nel web e di attivare un contatto, che a sua volta influirà sugli esiti della ricerca.

${ }^{170}$ Cfr. Brenda Dervin, Chaos, order, and Sense-Making Brenda Dervin, Chaos, order, and Sense-Making: a proposed theory for information design, in Robert Jacobson, Information design, Cambridge, MIT Press, 1999, p. 35-57.
} 
formalizzato nel linguaggio naturale (formalised need), espresso ad un sistema di recupero dell'informazione (compromised need $)^{171}$.

Inoltre il bisogno informativo può essere categorizzato in molti modi: ad esempio l'utente può cercare nuova informazione, può aver bisogno di chiarire un'informazione già posseduta, o di verificare e confermare l'informazione trovata ${ }^{172}$.

Non sempre però un bisogno informativo attiva la ricerca dellinformazione; ad esempio gli individui potrebbero non cercare l'informazione quando percepiscono il bisogno troppo grande da superare, o quando il compito o la ricerca da svolgere coinvolgono il concetto di rischio cioè, ad esempio, quando percepiscono che l'informazione ricevuta potrebbe essere dannosa (rischio emozionale, politico o fisico), portare cattive notizie, o quando temono il fallimento.

Allo stesso tempo possono non attivare la ricerca dell'informazione quando percepiscono di non avere abbastanza tempo da dedicare all'attività o non sanno come muoversi o cosa fare. Nel modello di Wilson, queste considerazioni fanno parte delle barriere che si frappongono tra il bisogno dell'utente e l'attività di ricerca dell'informazione.

Similmente anche la percezione di un bisogno informativo troppo piccolo può portare a non attivare la ricerca dell'informazione, in particolare quando gli utenti tendono a minimizzare il problema dopo aver misurato il rapporto costi-benefici dell'operazione ${ }^{173}$.

Questa valutazione può da una parte non attivare la ricerca dell'informazione o dall'altra portare al compimento l'azione della ricerca. Natalia Godbold suggerisce che il bisogno informativo può essere risolto in molti altri modi che non contemplano la ricerca dell'informazione (ad esempio creare l'informazione, metterla in discussione, distruggerla, ignorarla e prendere un altro percorso, prendere mentalmente nota).

Nonostante sia da più parti affermato che il vero oggetto di studio della Scienza dell'informazione è la ricerca 'attiva' dell'informazione, cioè quella che viene attivata con una certa dose di consapevolezza, le nuove modalità di utilizzo delle risorse della rete, ove sovente strumenti di ricerca sono integrati con strumenti di intrattenimento, quali ad

\footnotetext{
${ }^{171}$ Robert S. Taylor, Question-negotiation and information seeking in libraries cit. Per una spiegazione generale del modello e del suo impatto nelle teorie successive si veda anche Phillip M. Edwards, Taylor Question-Negotiation, in Theories of information behavior cit., p. 358-362. Un'analisi del bisogno informativo si trova anche nel volume di Alfredo Serrai Guida alla biblioteconomia, Firenze, Sansoni, 1981, a p. 34-35 dove l'autore distingue tra i concetti di esigenza, necessità, desiderio, richiesta e uso dell'informazione.

${ }^{172}$ Weights \& al. citato in Thomas D. Wilson - Christina Walsh, Information behaviour: an interdisciplinary perspective, Sheffield, University of Sheffield, Department of Information Studies, 1996. $<\mathrm{http}$ ///informationr.net/tdw/publ/infbehav/cont.html>. Tali categorie concordano con le fasi dell'ISP di Kulthau.

${ }^{173}$ Natalia Godbold, Beyond information seeking cit., p. 269.
} 
esempio le librerie online, ci mettono di fronte a comportamenti inediti e meritevoli oggi di studi più approfonditi.

L'utente può dunque approcciarsi all'informazione senza l'esistenza di un vero e proprio bisogno informativo. Come è stato notato da vari studiosi ${ }^{174}$ a volte le persone attivano la ricerca di informazione per un piacere in se, per scoprire nuove cose, o per il desiderio di soddisfare piccoli bisogni informativi.

L'utente potrebbe così scegliere di utilizzare un OPAC senza una specifica richiesta attivando modalità di ricerca esplorative. Tale comportamento, descritto da Bates ${ }^{175}$, fa sì che nel corso di una ricerca attiva non ci sia uno specifico interesse o bisogno conoscitivo, ma ci si esponga attivamente alla possibilità di acquisire nuove informazioni. Pensiamo oggi a chi utilizza le librerie on-line come Amazon o gli strumenti sociali come Flickr, Anobii o Goodreads ai quali spesso gli utenti si rivolgono non tanto per trovare ma per il piacere di scoprire nuovi libri in base ai suggerimenti di lettura offerti dal sistema o dagli altri utenti attivando un' esplorazione dei contenuti basata sulla serendipità. Si tratta dunque di modalità nuove e inedite con le quali la Scienza dell'Informazione oggi si deve misurare.

Profondamente legate al concetto di bisogno informativo vi sono le 'variabili' o 'barriere' che possono condizionare il processo di ricerca dell'informazione, facendo decidere all'utente se attivare/ non attivare il processo, se interromperlo e abbandonare la ricerca, se tornare indietro sui propri passi.

Possiamo infatti ipotizzare che l'utente incontri una serie di barriere (o variabili) che possono provenire sia dal suo contesto (personale, sociale, ambientale) sia da altri fattori, quali ad esempio la scarsa conoscenza dello strumento di ricerca, o dalla scarsa comprensione del suo compito di ricerca.

Dunque avremo barriere che fanno riferimento a caratteristiche della personalità, fisiologiche, cognitive ed emozionali; che si riferiscono al ruolo-sociale e ai suoi condizionamenti economici (es. accesso all'informazione, costo dell'informazione anche riferito al valore del tempo); barriere ambientali e situazionali (tempo a disposizione, fattori geografici e linguistici) ${ }^{176}$.

\footnotetext{
${ }^{174}$ Si tratta degli studi di Krikelas (1983), Savolanien (1995), Bates (2003), Rioux (2005) citati in Natalia Godbold, Beyond information seeking cit.

${ }^{175}$ Marcia J. Bates, Toward an integrated model of information seeking and searching cit.

${ }^{176}$ In un contesto interdisciplinare tali barriere vengono indagate nel campo della psicologia: rientrano in questa tipologia di barriere i concetti di dissonanza cognitiva (necessità di risolvere il conflitto tra nozioni in disaccordo), esposizione selettiva (tendenza degli individui ad esporsi a idee che sono in accordo con i loro interessi, necessità o attitudini), degli stili cognitivi intesi come modalità di elaborazione dell'informazione che il ricercatore mette in atto nelle fasi del processo di apprendimento e che sono legate alla scelta concreta
} 
Tra le 'barriere' coinvolte nel processo di ricerca il modello prende in considerazione tre teorie già proposte nel modello di Wilson del 1996: la teoria della riduzione dello stress, l'analisi costi-benefici, e il concetto di autoefficacia ${ }^{177}$.

Il concetto di stress nella ricerca dell'informazione, introdotto in psicologia da Richard Lazarus e Susan Folkman, viene utilizzato in altri contesti disciplinari (es. nella ricerca dell'informazione medica) per spiegare alcuni comportamenti di ricerca. L'idea di stress/coping ${ }^{178}$ cioè della capacità di attivare effetti comportamentali (coping) per affrontare, ridurre o tollerare situazioni di stress è stata una chiave per interpretare i processi di ricerca in alcuni contesti e meriterebbe ulteriori indagini e applicazioni nello studio dei sistemi di recupero dell'informazione ${ }^{179}$.

Analogamente anche la teoria della valutazione del rischio (legata alla risk/reward theory di Robert Settle e Pamela Alreck ${ }^{180}$ ) può illuminare su alcuni aspetti della ricerca dell'informazione anche nei cataloghi elettronici, supponendo che l'utente nell'analisi del suo gap includa anche una valutazione dello sforzo da sostenere commisurato alla ricompensa, dunque un'analisi del rischio potenziale della sua ricerca.

Infine, anche il concetto di autoefficacia introdotto da Albert Bandura e inteso come l'idea di competenza rispetto al compito prefissato (sense of personal mastery) è una

delle strategie cognitive utilizzate per risolvere un compito (non vanno confusi con le abilità), delle conoscenze pregresse e livello di conoscenza di un campo disciplinare, di fattori demografici: età, sesso, e altri fattori indagati anche nel settore del marketing per valutare il comportamento dei consumatori.

${ }^{177} \mathrm{Si}$ fa riferimento nel modello alle barriere presenti in ingresso e durante il processo informativo già individuate da Wilson e in particolare alla stress coping theory, alla risk/reward theory e alla self efficacy theory. Cfr. Thomas Wilson - Chistina Walsh, Information behaviour: an interdisciplinary perspective cit., capitolo 4, <http://informationr.net/tdw/publ/infbehav/chap4.html>.

${ }^{178}$ Esiste una letteratura molto vasta sul coping inteso come quel meccanismo che consente alle persone di fronteggiare situazioni di stress e li porta a mettere in atto strategie per gestire i problemi e le conseguenze che essi generano. Tra le teorie più rilevanti quella cognitivo-transazionale proposta da Lazarus e Folkman Si vedano: Richard Lazarus - Susan Folkman, Stress, appraisal, and coping, New York, Springer Pub. Co, 1984 e Susan Folkman, Personal control and stress and coping processes: a theoretical analysis. «Journal of Personality and Social Psychology», 46 (1984), p. 839-852.

${ }^{179}$ Un modello che rappresenta la relazione tra la ricerca dell'informazione e il coping indagando i comportamenti di rifiuto dell'informazione specie nel campo della ricerca medica è stato elaborato da Krohne nel contributo Vigilance and cognitive avoidance as concepts in coping research citato in Cfr. Thomas Wilson - Chistina Walsh, Information behaviour: an interdisciplinary perspective cit., cap 3. $<$ http://informationr.net/tdw/publ/infbehav/chap3.html>.

${ }^{180}$ Elaborata nell'ambito della ricerca sui consumi, la teoria prevede quali fattori di rischio: performance risk (probabilità che il prodotto corrisponda alle aspettative desiderate, un financial risk (posso permetteri questo prodotto o posso trovarne uno meno caro sul mercato?) physical risk (è un azzardo per le mie disponibilità finanziarie?), social risk (impressionerò amici e colleghi?) e ego risk (il prodotto migliorerà il mio status sociale o la mia felicità? (concepito anche in termini di autostima). È molto interessante notare che più il fattore di rischio è alto, più l'utente attiverà modalità di ricerca attive. Una teoria nata dunque per analizzare comportamenti di acquisto come questa, può spiegare alcuni aspetti della ricerca nei sistemi di IR. È stato accertato che gli utenti diventano voraci ricercatori (utilizzando modalità attive dell'informazione), quando il fattore di rischio connesso al compito che stanno svolgendo è alto, dunque quando vi è un alto grado di incertezza. Dunque il principio dell'incertezza, alla base di molte teorie e modelli (es. Wilson 2002, Kulthau 2004, Belkin) è confermato anche in ricerche compiute in altri campi disciplinari. Cfr.: Robert B. Settle Pamela L. Alreck, Reducing buyers' sense of risk. «Marketing Communications», 14 (1989) p. 34-40. 
variabile coinvolta: consente all'utente di farsi una idea delle possibilità di raggiungere un obiettivo prefissato rispetto alle proprie capacità e di condizionare dunque i suoi comportamenti sia al momento dell'attivazione (intraprendere o meno una ricerca), sia nel prosieguo (abbandonare, cambiare direzione, tornare indietro) ${ }^{181}$.

Seguendo queste teorie e prendendo in considerazione il modello elaborato dal Wilson è dunque importante analizzare a fondo quelle barriere che si frappongono fra l'utente e l'utilizzo dell'informazione contenuta in un OPAC e le variabili che possono condizionare il processo di ricerca. Tali barriere, a mio avviso, sono presenti non solo al momento dell'attivazione della ricerca dell'informazione bibliografica, ma anche lungo l'intero processo e le sue fasi, in quanto l'utente rianalizza e riconcettualizza varie volte il suo bisogno informativo che, come evidenziato da vari studiosi (Bates, Godbold), varia dinamicamente nel corso della ricerca dell'informazione.

Christine Borgman identifica vari livelli di conoscenze richieste per interagire con un $\mathrm{OPAC}^{182}$ :

1) una conoscenza concettuale dei processi di IR cioè la capacità di convertire l'informazione richiesta in una query;

2) una conoscenza semantica relativa alla costruzione della query in un particolare sistema;

3) una serie di abilità tecniche che consentano all'utente di costruire una query; A ciò possiamo aggiungere

4) la necessaria conoscenza del settore disciplinare di una ricerca (domain knowledge);

5) le barriere provenienti dai modelli mentali che gli utenti hanno costruito nell'utilizzo di altri strumenti di ricerca ${ }^{183}$.

L'analisi delle barriere nel recupero dell'informazione bibliografica rappresenta oggi un'esigenza irrinunciabile per la progettazione efficace della ricerca.

\footnotetext{
${ }^{181}$ Albert Bandura, Self efficacy: towards a unifying theory of behavioural change. «Psychological Review», 84 (1977), p. 191-215.

182 Christine L. Borgman, Why are online catalogs still hard to use? «Journal of the American Society for Information Science», 47 (1996), n. 7, p. 493-503.

183 Holly Yu-Margo Young, The Impact of Web Search Engines on Subject Searching in OPAC, «Information Technology And Libraries», 23 (2004), n. 4, p. 168-180, anche in <http://www.ala.org/ala/lita/litapublications/ital/volume23a/number4a/yu.pdf>.
} 


\subsubsection{Il rovesciamento della logica query oriented e l'attività di browsing. Le principali tattiche di ricerca: finding, browsing, asking}

All'inizio degli anni '90 nella letteratura professionale apparvero alcuni contributi che lamentavano la scarsa funzionalità degli OPAC nel recupero dell'informazione. Non si trattava solamente di introdurre funzionalità più avanzate, ma si mettevano in discussione i modelli di ricerca che fino ad allora erano stati alla base della progettazione dei sistemi.

In particolare Hildreth ${ }^{184}$ facendo riferimento ai recenti studi di Bates, sottolineava che il passaggio ai nuovi OPAC 'di terza generazione' avrebbe dovuto implicare una ridefinizione dei modelli di ricerca sottostanti poiché la maggior parte degli OPAC rifletteva nella progettazione e nelle funzionalità offerte, un modello concettuale inadeguato dell'attività di recupero dell'informazione che faceva riferimento alle teorie sviluppate nel campo dell'information retrieval nei decenni precedenti.

I modelli di interrogazione alla base dell'information retrieval, dal modello booleano a quelli più avanzati come quello probabilistico, rientrano in quella modalità di approccio all'informazione che possiamo definire query oriented ${ }^{185}$. Essi infatti presuppongono che il punto di partenza della ricerca sia la formulazione di una domanda e la sua espressione verbale e che si ottenga un risultato in caso di corrispondenza (esatta o migliore) tra il termine/termini ricercato/i e i termini dei documenti che sono stati indicizzati nel sistema. Molta letteratura sulla progettazione dei cataloghi elettronici negli anni ' 80 ha così posto al centro dell'attenzione le modalità di immissione/modifica/previsione delle interrogazioni che l'utente immetteva nel corso della ricerca nei cataloghi elettronici.

Lo sviluppo degli studi sull'utenza e dei modelli comportamentali ha invece rilevato che accanto all'attività di finding, un altro modo di ricercare le informazioni che ci servono e che ha immediato riscontro nella vita di tutti i giorni, è il browsing, attività che in ambito

\footnotetext{
${ }^{184}$ cfr. Charles R. Hildreth, Advancing toward the E3 OPAC: the imperative path, in Noelle Van Pulis, Think Tank on the Present and Future of the Online Catalog: Proceedings, Chicago, American Library Association, 1991, p. 39-48 e Id., Online catalog design models: are we moving in the right direction? A report submitted to the Council on library resources, August, 1995, <http://eprints.rclis.org/archive/00000198/> oppure <http://myweb.cwpost.liu.edu/childret/clr-opac.html>.

${ }^{185}$ Ivi, cap. 5, s. p.: «most operational OPACs and retrieval systems are based on a design model that is not fully descriptive of much actual information seeking activity. This query-based model assumes the user comes to the system with a known, specified or specifiable information need. A second assumption of this design model is that for any given query there exists a single best output set that should be targeted for retrieval. Bates has called this "The Fallacy of the Perfect Thirty-Item Online Search».
} 
bibliotecario trova un'applicazione pratica nel più immediato comportamento di ricerca dello scorrere i volumi presenti negli scaffali ${ }^{186}$.

Nella letteratura professionale il browsing, cui ci si è riferiti usando denominazioni diverse (come ricerca non lineare, esplorativa, navigazione o berrypicking), è stato oggetto crescente di attenzione sin dagli anni '70, anche se è nell'uso del web che tale modalità di ricerca è divenuta familiare agli utenti, suscitando un nuovo interesse per tale tecnica.

Già nel lontano 1971 Apted individuava una tipologia di browsing che definiva "general purposive browsing" consistente nell'esame, pianificato o no, di diverse fonti (giornali, libri o altri media) alla ricerca di un'informazione utile", tale tecnica fu descritta poco dopo da Morse come lo scorrere tra i volumi finché "qualcosa non cattura il proprio interesse", mentre altri studiosi identificarono il comportamento generale della ricerca in un'attività generica di browsing ${ }^{187}$. Successivamente gli studi sull'argomento dimostrarono che gli utenti consideravano tale modalità di ricerca la più agile e intuitiva e la preferivano come modalità di ricerca nei sistemi elettronici.

I motivi per i quali gli utenti preferiscono navigare piuttosto che formulare interrogazioni sono stati ricondotti da Gary Marchionini ad alcune principali motivazioni quali, ad esempio, non avere chiare le proprie esigenze informative, avere difficoltà nell'esprimerle o nel tradurle nel linguaggio usato dal sistema ${ }^{188}$.

Un'ulteriore motivazione consiste nella comodità insita in questa modalità di ricerca, poiché se il processo di browsing è orientato naturalmente all'azione, quello di finding $o$ searching impone invece all'utente una preliminare riflessione e uno sforzo mentale poiché prevede, appunto, la formulazione di una query; tale ipotesi conferma gli enunciati della legge del minimo sforzo individuata da $\mathrm{Zipf}^{189}$ in base alla quale gli utenti preferiscono l'informazione più facile da trovare, anche se di bassa qualità rispetto a quella di qualità ma difficilmente accessibile.

Secondo l'analisi di Hildreth, l'auspicato passaggio ad una nuova generazione di OPAC implica una ridefinizione dei modelli sottostanti tale per cui l'attività di browsing,

\footnotetext{
${ }^{186}$ Alcuni studiosi ravvisano fra le differenze tra i due filoni di studio dell'information seeking e information retrieval il fondamentale passaggio dalla modalità di searching a quella di browsing come elemento comportamentale che segna un cambio di paradigma verso lo studio dell'interazione tra sistema e utente.

${ }^{187}$ Gli studi di Apted (1971) e Morse (1973) sono citati in Charles Hildreth, Online catalog design models cit.

${ }^{188}$ Gary Marchionini, An invitation to browse: designing full-text systems for novice users, «The Canadian Journal of Information Science», 12 (1987), n. 3/4, p. 69-79. Inoltre, alcuni studi citati dallo studioso confermerebbero come gli utenti preferiscano scorrere i materiali organizzati sugli scaffali piuttosto che cercarli nel catalogo cartaceo della biblioteca.

${ }^{189}$ Le varie tipologie di browsing sono analizzate anche nel recente contributo di Marcia J. Bates, What is browsing—really? A model drawing from behavioural science research, «Information Research», 12 (2007), n. 4 p. 330, anche in <http://InformationR.net/ir/12-4/paper330.html>.
} 
finora nella maggior parte degli casi relegata nel ruolo di supporto a quella di searching, acquisti una sua autonomia.

In questo processo, Bates rimarca come vi sia un'errata tendenza a vedere l'attività di browsing in contrasto rispetto alla ricerca normale o tradizionale, quando invece i due modelli di ricerca dovrebbero essere complementari; le due strategie non sono dunque in contrasto, ma si completano. Le teorizzazioni di Bates ${ }^{190}$ hanno portato alla conclusione che per giungere a formulare modelli di ricerca più realistici, occorra non solo rovesciare il paradigma query oriented («this "known-subject need, best-match, end product-oriented" information retrieval paradigm» ${ }^{191}$ ) ma soprattutto intendere la ricerca come una serie di passaggi tra loro correlati, nei quali entrambe le attività di ricerca e navigazione (searching e browsing) si completano e si integrano: il recupero dell' informazione così si evolve dall'essere output-orientated ad un'attività process-orientated ${ }^{192}$.

Accogliendo gli spunti provenienti dai modelli di Kuhlthau e Vakkari, possiamo proporre che l'uso dell'una o dell'altra strategia di ricerca siano influenzate dalla fase dell'ISP in cui l'utente si trova supponendo che alcune fasi (quelle che Vakkari definisce come pre-focus) richiedano maggiormente un approccio di tipo esplorativo che finora la progettazione dell'OPAC ha ampiamente trascurato.

In alcune fasi della ricerca l'utente si predispone naturalmente all'attività browsing e alla navigazione approcciandosi al catalogo in maniera attiva e indiretta, esponendosi alla possibilità di acquisire nuova informazione (ad esempio, quando un utente di una biblioteca pubblica scorre gli ultimi arrivi disposti per categorie in cerca delle novità).

Il passaggio dall'interrogazione al browsing e viceversa è inoltre necessario quando l'utente deve effettuare un cambio di dimensione nella propria ricerca, cioè saltare dal livello semiotico (degli autori, dei titoli e dei tratti semiotici delle pubblicazioni) a quello semantico (dei contenuti e dei concetti) ${ }^{193}$.

Le due funzioni di base dell' information retrieval cioè finding e browsing non sono però sufficienti da sole a risolvere tutti i casi di ricerca dell'informazione, in quanto nel processo entrano in gioco anche fattori umani, rappresentati nei sistemi dalle funzioni che consentano, durante il processo di chiedere aiuto, e costituite non solo dagli aiuti in linea

\footnotetext{
${ }^{190}$ In particolare ci si riferisce alle elaborazioni del modello berrypicking del 1998 e del modello integrato del 2002.

${ }^{191}$ Ivi, cap. 5

${ }^{192}$ Aida Slavić, Interface to classification: some objectives and options. «Extension \& Corrections to the UDC», 28 (2006), <http://dlist.sir.arizona.edu/1621/>

${ }^{193}$ Gli OPAC oggi consentono questo passaggio tramite l'esposizione dei dati estratti dalle notizie nelle faccette, tuttavia si sottolinea come le faccette semantiche siano sovente incomplete e gli accessi semantici penalizzati
} 
che tutti gli OPAC forniscono e che pure si rivelano importanti, ma anche da quelle funzioni di reference online che possono consistere nell'attivare una chat con il bibliotecario, o in percorsi prestabiliti (frequently asked questions), oppure nel contatto con persone che si occupano dello stesso argomento (tramite le reti sociali, come quelle del web 2.0).

Questo tipo di tattica entra a far parte oggi con i nuovi OPAC, dei processi che si svolgono all'interno del catalogo. L'ingresso delle funzioni di asking (richiesta di aiuto) rappresenta un completamento delle funzionalità di ricerca offerte dal catalogo, che prima erano limitate alle sole funzioni di ricerca e browsing, ed avvicina la ricerca in OPAC a quella che avviene in un ambiente fisico, cioè in biblioteca.

\subsubsection{Rilevanza e pertinenza nel processo dell'informazione}

Nel modello proposto entrano a far parte i concetti della 'rilevanza' e della 'pertinenza' che fanno parte di un processo di valutazione che caratterizza la ricerca dell'informazione.

Considerando le fasi di ricerca descritte da Kuhlthau, i concetti della rilevanza topica e della pertinenza sono due dimensioni della rilevanza necessarie: durante le prime fasi l'utente cerca l'informazione generica rilevante, mentre nel prosieguo della ricerca prevale maggiormente la ricerca dell'informazione specifica e pertinente. L'OPAC dovrebbe dunque supportare entrambe le attività fornendo all'utente la possibilità di valutare se l'informazione ottenuta sia rilevante, e introducendo alcuni elementi di personalizzazione che consentano al sistema di monitorare le precedenti ricerche dell'utente o acquisire conoscenza relativa alle sue preferenze e alle sue conoscenze (ad esempio il grado di conoscenza o expertise dell'area di soggetto che è oggetto di indagine dell'utente, o delle sue preferenze tramite qualche forma di relevance feedback) per consentire all'utente di ricevere l'informazione pertinente. Ciò consente anche di prevenire un'altra delle barriere che si frappongono tra l'utente e l'informazione ossia quell'information overload, inteso come sovraccarico informativo che investirebbe l'utente, qualora i risultati non siano adeguatamente filtrati e risultino eccessivi o fuorvianti. In questo senso una navigazione tra risultati strutturati significativamente per l'utente può ridurre il senso di impreparazione o lo sforzo cognitivo nel corso della ricerca consentendogli di avere il pieno controllo del suo 
percorso informativo, e producendo al contempo apprendimento e la comprensione dei processi messi in atto dal sistema ${ }^{194}$.

\subsubsection{Gli intermediari}

Una delle principali aree di sviluppo degli OPAC rappresentate nel modello è costituita dagli intermediari che operano nel processo di ricerca dellinformazione. Nell'ambiente fisico infatti un utente che cerca informazioni nel catalogo può avvalersi della mediazione del bibliotecario, che può soccorrerlo nella scelta degli strumenti più idonei, nella consultazione del catalogo o nello scorrimento degli scaffali.

La collaborazione nel processo di ricerca in un ambiente fisico consente di superare quel senso di isolamento che invece caratterizza la ricerca in un catalogo elettronico, ove l'utente si trova ad interagire in solitudine con l'interfaccia, spesso soccorso dalle sole funzioni di aiuto in linea che gli OPAC predispongono.

Nei precedenti modelli che hanno rappresentato l'interazione nei sistemi di IR, il principale intermediario coinvolto nell'interazione utente-catalogo è rappresentato dall'interfaccia, luogo nel quale si svolge l'interazione tra le diverse componenti.

Oltre all'interfaccia, quale strato superficiale nel quale si incontrano e interagiscono le strutture cognitive dell'utente, quelle rappresentate nei documenti e nei sistemi, oggi entrano anche nell'OPAC altri intermediari, la cui presenza può condizionare la ricerca e lo svolgimento del processo.

Un esempio è rappresentato dall'introduzione della funzione di chat con il bibliotecario, che sempre più spesso è presente negli OPAC di ultima generazione e che è in grado di connettere l'utente a un aiuto umano più concreto e immediato rispetto alle semplici funzioni di aiuto in linea che tradizionalmente sono state incluse nell'OPAC, o dalle nuove funzioni di recupero, insieme ai risultati di una ricerca, degli 'esperti' nell' area di soggetto oggetto dell'interrogazione come ad esempio avviene nell'OPAC danese Summa ${ }^{195}$.

\footnotetext{
${ }^{194}$ Sugli effetti dell'information overload nel processo di ricerca si vedano gli scritti di David Bawden e in particolare: David Bawden, Information and digital literacies; a review of concepts, «Journal of Documentation», 57 (2001), n. 2, p. 218-259, Id., Smoother pebbles and the shoulders of giants: the developing foundations of information science, «Journal of Information Science», 34 (2008), n 4, p. 415-426, e David Bawden - Lyn Robinson, The dark side of information: overload, anxiety and other paradoxes and pathologies, «Journal of Information Science», 35 (2009) n. 2, p. 180-191. Disponibile anche all'URL: $<$ http://intranet.welch.jhmi.edu/intranet/journalclub/reference/journalclub32010dblr.pdf>.

${ }^{195}$ Cfr. Appendice A. Casi di studio. OPAC Summa.
} 
Altrettanto interessanti sono le funzionalità che gli OPAC sociali introducono a vantaggio degli utenti. In questi OPAC, dove si crea una rete sociale intorno alle registrazioni del catalogo, si formano communities di utenti che entrano in contatto tra loro costituendo una rete di scambio di opinioni, commenti e suggerimenti.

Ulteriori possibilità ci riservano le nuove funzionalità del web e suoi recentissimi sviluppi ispirati da Facebook e da altri siti di ricerca collaborativa nel web ${ }^{196}$. Si assiste alla nascita di motori di ricerca sociali come Volunia ${ }^{197}$, che collegano alla ricerca la componente sociale secondo il nuovo paradigma «seek\&meet» (cerca e incontra).

In base a queste tecnologie, qualora venissero applicate all'OPAC, l'utente potrebbe scoprire nel corso della sua ricerca quali altri utenti stanno visualizzando lo stesso record, ed entrare in contatto con loro, sfruttando una rete sociale fortemente interattiva, in grado di condizionare in tempo reale le scelte e gli esiti della propria ricerca.

\subsubsection{Le fasi del processo di ricerca dell'informazione. Funzioni-utente e funzionalità strategiche degli OPAC}

Sebbene, come abbiamo accennato in precedenza, si possa supporre che esista una certa percentuale di utenti che si approccia ai cataloghi elettronici per soddisfare bisogni discreti oppure senza un vero e proprio bisogno informativo attivando modalità di ricerca indirette, o compiendo operazioni di routine o affidate alla serendipità, generalmente l'interazione che l'utente svolge in un OPAC rientra nella cosiddetta "ricerca attiva" dell'informazione ovvero intenzionale, rivolta al soddisfacimento di uno scopo o alla soluzione di un problema di ricerca.

Per illustrare dunque il processo di ricerca in un catalogo prenderemo come riferimento un modello generale in grado di illustrare le diverse attività che l'utente svolge nel corso di una ricerca.

\footnotetext{
196 Un esempio molto noto nel web è Yahoo Answers (<http://answers.yahoo.com/>), dove gli utenti pongono domande e risolvono quesiti in maniera collaborativa. Recenti indagini (cfr. OCLC, Perceptions of libraries, 2010: context and community, edited by Cathy De Rosa et al. OCLC, 2011 $<$ http://www.oclc.org/reports/2010perceptions.htm> e anche cfr. supra cap. 1, § 1.4 hanno dimostrato un netto incremento nell'utilizzo di questa tipologia di siti web.

$197<$ http://www.volunia.com>. Il nuovo motore di ricerca, sviluppato in Italia dal team del prof. Marchiori all'Università di Padova e lanciato all'inizio del 2012 consente all'utente di commentare le pagine visitate e visualizzare chi le ha visionate.
} 
Allo scopo di analizzare più approfonditamente la ricerca che si svolge in un OPAC ci serviremo dello schema del processo di ricerca (ISP) fornito da Kuhlthau che presenta un modello in grado di illustrare le fasi che compongono la ricerca attiva dell'informazione ${ }^{198}$.

La struttura delle 'fasi' del modello di Kuhlthau che vengono qui proposte nella versione semplificata offerta da Vakkari ${ }^{199}$ verrà utilizzata nel modello da me proposto come schema dei tre principali stadi in cui l'utente si trova quando utilizza l'OPAC. Tali fasi determinano il modo con cui l'utente cerca e usa l'informazione; in queste fasi gli utenti utilizzano strategie diverse in base alla tipologia del compito da svolgere, del tempo a disposizione, dell'interesse personale e delle conoscenze di cui dispongono.

Possiamo dunque prendere come riferimento tre principali fasi in cui si articola il processo di ricerca:

la fase precedente alla formulazione del focus, nella quale l'utente è impegnato nell'attività di trovare un argomento specifico per la sua ricerca;

* la fase della formulazione e del perfezionamento del focus;

* la fase successiva alla formulazione del focus ove l'utente compie l'attività di raccolta delle informazioni per avviarsi alla conclusione della ricerca.

\begin{tabular}{|l|l|}
\hline Kuhlthau (1991, 2004) & Vakkari (2001) \\
\hline Inizio della ricerca & \multirow{2}{*}{ Fase pre-focus } \\
\cline { 1 - 1 } selezione dell'argomento & \\
\cline { 1 - 1 } esplorazione & Formulazione del focus \\
\hline Formulazione del focus & Fase post-focus \\
\cline { 1 - 1 } Raccolta dell'informazione & \\
\cline { 1 - 1 } Conclusione della ricerca & \\
\hline
\end{tabular}

Fig. 5.20 - Tabella di confronto delle fasi di Kuhlthau $(1991,2004)$ rielaborate e semplificate in Vakkari (2001)

In queste fasi si compie un processo informativo che può essere schematizzato nelle seguenti attività 'cognitive':

* riconoscere un problema (ovvero un bisogno informativo, un gap nella conoscenza);

* identificare l'informazione necessaria;

\footnotetext{
${ }^{198}$ Carol Collier Kuhlthau, Seeking meaning cit.

${ }^{199}$ Cfr. Pertti Vakkari, A theory of the task-based information retrieval process cit.
} 
* esplorare / formulare una richiesta;

* analizzare e valutare i risultati;

- aggiungere nuova conoscenza alla propria 'struttura cognitiva'.

Nel modello proposto, nel processo di ricerca rappresentato al centro del modello avviene l'interazione tra le diverse componenti coinvolte: gli oggetti informativi, lo spazio cognitivo dell'utente, il sistema, gli intermediari coinvolti nel processo di recupero dell'informazione.

Nelle tre fasi del processo di ricerca dell'informazione nell'OPAC delineate avremo dunque funzioni rivolte alle 'entità' che si riferiscono agli oggetti informativi contenuti nell'OPAC cioè all'universo bibliografico rappresentato nel catalogo, ovvero le funzioni 'bibliografiche' (es. trovare un determinato documento) e altre funzioni o attività che l'utente svolge e che derivano dalle componenti che entrano in contatto nell'interazione che avviene nel catalogo elettronico e nel recupero dell'informazione bibliografica (ad esempio trovare una strategia di ricerca, identificare il proprio bisogno informativo).

Tali attività richiedono di essere considerate e valutate in quanto derivano dell'interazione tra l'utente e il sistema e i documenti e gli eventuali intermediari coinvolti nel processo dell'informazione. Dalle componenti dell'interazione discendono alcuni fattori che si riferiscono ad esempio, dal lato utente, alla conoscenza dell'utente del sistema, di un particolare campo disciplinare, al proprio processo di ricerca, dal lato dei sistemi ai dati trattati, al software utilizzato, alle funzionalità proposte e sono ampiamente rappresentate nei modelli di ricerca precedentemente analizzati.

Le funzioni - utente identificate nel modello FRBR dunque rappresentano l'interazione tra l'utente e gli oggetti informativi presenti nel catalogo costituiti dalle entità che compongono l'universo bibliografico e che sono oggetto dell'interesse degli utenti, dai loro attributi e dalle loro relazioni. reciproche. In particolare tali funzioni servono a verificare la validità stessa del modello FRBR in relazione agli scopi che si vogliono ottenere.

Nel modello le funzioni-utente rappresentano infatti l'uso che si fa dei dati bibliografici; secondo il modello l'utente deve essere in grado di:

* utilizzare i dati di cui dispone per trovare le risorse che corrispondono ai criteri stabiliti nella ricerca;

* utilizzare i dati ottenuti per identificare_una entità;

* utilizzare i dati per selezionare una entità che corrisponda alle proprie necessità; 
* utilizzare i dati per ottenere l' accesso all'entità descritta ${ }^{200}$.

Possiamo dunque proporre che per migliorare le funzionalità offerte dai cataloghi elettronici si debba tener conto non solo di quelle 'funzioni-utente' che si riferiscono agli oggetti informativi rappresentati nel catalogo, ma anche di quelle funzioni che scaturiscono dalle altre principali componenti dell'interazione: l'utente stesso e il suo stato cognitivo e il sistema e gli eventuali intermediari.

Dando per scontato che le funzioni-utente, ovvero le funzioni bibliografiche individuate da FRBR siano le funzioni primarie e indispensabili all'utilizzo dei dati bibliografici indipendentemente dallo stato cognitivo in cui l'utente si trovi, ipotizzeremo in questa sede che vi siano altre sotto-funzioni che invece dipendono dalle altre componenti: l'utente e il sistema e che tali funzioni pratiche possano variare al variare della fase del processo di ricerca nella quale si trova l'utente nella sua interazione con l'OPAC

Per rappresentare le funzioni pratiche, attività o le sotto-funzioni che l'utente svolge nel corso delle diverse fasi del processo di ricerca in un sistema di recupero dell'informazione Xie ha elaborato le seguenti 'interactive intentions': identificare, apprendere, esplorare, creare, modificare, valutare, monitorare, tenere a memoria, accedere, organizzare, ottenere, disseminare ${ }^{201}$.

Rielaborando e sovrapponendo tali funzionalità con le funzioni-utente previste dal modello FRBR, ricaviamo dunque le seguenti dodici funzionalità:

1. trovare (un'entità tramite i suoi attributi);

2. identificare (un'entità tramite gli attributi / il proprio bisogno informativo/una strategia di ricerca);

200 IFLA Study Group on the functional requirements for bibliographic records. Functional requirements for bibliographic records: final report. Approved by the Standing Committee of the IFLA Section on Cataloguing. September 1997. As amended and corrected through February 2009, <http://www.ifla.org/VII/s13/frbr/frbr_2008.pdf>. A p. 79: «four generic user tasks have been defined for the purposes of this study. The tasks are defined in relation to the elementary uses that are made of the data by the user:

- to find entities that correspond to the user's stated search criteria (i.e., to locate either a single entity or a set of entities in a file or database as the result of a search using an attribute or relationship of the entity);

- to identify an entity (i.e., to confirm that the entity described corresponds to the entity sought, or to distinguish between two or more entities with similar characteristics);

- $\quad$ to select an entity that is appropriate to the user's needs (i.e., to choose an entity that meets the user's requirements with respect to content, physical format, etc., or to reject an entity as being inappropriate to the user's needs);

- to acquire or obtain access to the entity described (i.e., to acquire an entity through purchase, loan, etc., or to access an entity electronically through an online connection to a remote computer)».

${ }^{201}$ Iris Xie, Interactive Information Retrieval in Digital Environments, cit. p. 233-234. 
3. apprendere (le caratteristiche di un sistema /una strategia di ricerca/i confini semantici di un'area di soggetto/ la conoscenza dei entità correlate all'entità esaminata);

4. esplorare /navigare/correlare/differenziare (le relazioni tra le entità);

5. creare (una query per dare inizio/proseguire la propria ricerca);

6. modificare (una query per dare inizio/proseguire la propria ricerca);

7. valutare (la rilevanza / la pertinenza di una risorsa/ una strategia di ricerca);

8. organizzare (i risultati ricevuti/ la propria ricerca/ integrare le nuove conoscenze);

9. monitorare (la propria ricerca/ una determinata area di soggetto/ un gruppo di documenti che hanno caratteristiche in comune ad esempio le novità);

10. tenere a memoria (un record / lo svolgimento delle attività durante l'interazione);

11. accedere / ottenere (ottenere l'accesso a una risorsa /ottenere una copia fisica);

12. disseminare (l'informazione trovata per riutilizzarla al di fuori dell'OPAC).

Nella tabella che segue vengono dunque associate le diverse interazioni in relazione al processo di ricerca che si svolge nel catalogo:

\begin{tabular}{|c|c|c|c|}
\hline Attività & Pre-focus & Focus & Post-focus \\
\hline *Trovare & $\square$ & $\square$ & $\square$ \\
\hline *Identificare & $\mathbf{\square}$ & $\mathbf{\square}$ & $\square$ \\
\hline Apprendere/correlare & $\mathbf{\square}$ & $\square$ & $\square$ \\
\hline *Navigare & $\boldsymbol{\square}$ & $\square$ & $\square$ \\
\hline Creare & $\square$ & $\mathbf{\square}$ & $\square$ \\
\hline Modificare & $\square$ & $\bar{\square}$ & $\square$ \\
\hline \multicolumn{4}{|c|}{ Valutare / (Selezionare*) } \\
\hline -la rilevanza & $\mathbf{a}$ & $\square$ & $\mathrm{O}$ \\
\hline -la pertinenza & O & $\boldsymbol{\square}$ & $\square$ \\
\hline \multicolumn{4}{|l|}{ Monitorare } \\
\hline il processo di ricerca & $\mathbf{a}$ & $\boldsymbol{\square}$ & $\square$ \\
\hline Memorizzare & O & $\square$ & $\mathbf{\square}$ \\
\hline *Accedere /Ottenere & $\mathbf{\square}$ & $\square$ & $\bar{\square}$ \\
\hline Organizzare & $\square$ & $\boldsymbol{\square}$ & $\boldsymbol{\square}$ \\
\hline Disseminare & $\mathrm{O}$ & $\bar{O}$ & $\bar{\square}$ \\
\hline
\end{tabular}

a valore alto $\square$ valore medio $\bigcirc$ valore scarso *Funzioni-utente in FRBR

Fig. 5.21 - Le attività dell'utente in relazione alle fasi del processo di ricerca dell'informazione (ISP) 


\subsubsection{Fase pre-focus. Attività dell'utente, funzionalità dell'OPAC e linee strategiche}

L'utente che si approccia all'OPAC nella fase che definiamo pre-focus è impegnato nel processo di comprensione di un compito assegnato o della sua stessa esigenza di ricerca, dunque in una primissima analisi del suo bisogno informativo, ed è alla ricerca dei possibili argomenti che potranno far parte della propria ricerca. Come già indicato da Kuhlthau in questa fase del processo prevale un forte senso di incertezza che caratterizza tutte le azioni che l'utente compie.

Nella fase di 'inizio di una ricerca' l'utente non è ancora in grado di esprimere il proprio bisogno informativo perché non è ancora in grado di concettualizzarlo; deve compiere un enorme sforzo cognitivo per comprendere gli ambiti e i confini del suo compito di ricerca e mettere a fuoco un argomento. In un ambiente fisico la principale azione intrapresa dall'utente è la richiesta di aiuto, che si può concretizzare in una discussione sui possibili argomenti, nel dialogo e nella richiesta di un consulto con gli insegnanti, i bibliotecari, i compagni di corso o con qualche persona informata. Tale comportamento è confermato anche dai comportamenti previsti dal modello di Ellis nella fase di inizio (starting). Un'altra probabile azione che Kuhlthau cita come caratteristica di questa fase consiste nel 'fare browsing' negli scaffali della biblioteca.

In questa prima fase del processo rientra anche la selezione dell'argomento di ricerca $^{202}$, fase in cui l'utente sceglie un argomento generale per dare avvio alla ricerca. $\grave{E}$ stato dimostrato che in questa fase prevale la necessità di partire da argomenti o idee generali per riconoscere e ricollegare nuove idee alle idee già esistenti, ovvero di farsi 'un'idea generale dell'argomento'. I motivi che giustificano la scelta dell'argomento dipendono da molti fattori diversi dell'utente, che possono essere cognitivi (l'interesse personale) oggettivi (la quantità di informazione disponibile, i requisiti del progetto e situazionali (il tempo assegnato). Anche nella scelta di un argomento l'utente tende a confrontarsi con i mediatori (es. l'insegnante o il bibliotecario), a fare un'attività di scanning, per avere un quadro d'insieme sull'argomento e sulle possibilità di sviluppo della sua ricerca; in un ambiente fisico esempio può consultare una collezione di reference.

Si avvia così una vera e propria 'fase esplorativa' caratterizzata da sensazioni di incertezza e dubbio crescenti nel tempo nella quale il compito è quello di indagare

${ }^{202}$ Carol Collier Kuhlthau, Seeking meaning cit., p. 29-53. 
l'informazione sull' argomento generale che verrà poi esteso alla comprensione personale dell'argomento al fine di formulare un focus. È il punto più difficile della ricerca in quanto la persona cerca di trovare un orientamento nell'argomento e di informarsi a sufficienza per formulare un focus e un punto di vista personale. Non sempre l'informazione raccolta fornisce all'utente un quadro chiaro e spesso fonti informative contrastanti lo portano a situazioni di scoraggiamento, a volte in vere e proprie tentazioni ad abbandonare il compito di ricerca.

Nel dialogo con un sistema di recupero dell'informazione si rileva la difficoltà di formulare l'oggetto di ricerca nei sistemi, ovvero nel riconoscere e verbalizzare l'argomento in termini da inserire nell'interrogazione.

Nella fase pre-focus (che comprende dunque le tre fasi descritte nell'ISP da Kuhlthau) dunque l'utente svolge all'inizio della ricerca una serie di attività che gli consentono di formulare con precisione l'oggetto della sua ricerca.

In questa fase l'utente è coinvolto in un processo intenso di riduzione dell'incertezza cognitiva che caratterizza le fasi iniziali del processo di ricerca ${ }^{203}$.

Le funzionalità che l'OPAC appronta devono essere dunque dirette a supportare la fase di costruzione che l'utente compie per definire e perfezionare l'oggetto della sua ricerca. In questa fase nell'OPAC devono essere attivate tutte quelle funzionalità che consentano all'utente di trovare l'informazione rilevante, sfruttando modalità esplorative e di supporto alla formulazione di un'interrogazione ed effettuando in risposta una serie di raggruppamenti significativi per l'utente.

Le principali azioni pratiche intraprese dall'utente in questa prima fase sono quelle di

* identificare lo strumento più appropriato al quale rivolgersi o una particolare entità / le entità correlate per dare avvio alla ricerca, oppure una particolare strategia di ricerca;

* apprendere le caratteristiche del un sistema /database, la struttura dei dati contenuti nell'OPAC;

* la conoscenza relativa ad un'area di soggetto che vuole indagare;

* esplorare: attivare un comportamento esplorativo consenta di trovare un'entità, di visualizzare le entità collegate, ad esempio in una certa area di soggetto;

* creare una query per dare avvio alla ricerca;

\footnotetext{
${ }^{203}$ Sulla riduzione dell'incertezza durante il processo informativo si veda anche: Jesper Kallehauge, Stagedriven information seeking process: value and uncertainty of work tasks from initiation to resolution, «Journal of Information Science», 36 (2010), n. 2, p 242-262.
} 
valutare l'informazione rilevante cioè avere la possibilità di stabilire tramite le caratteristiche degli oggetti informativi se un determinato documento corrisponde alla query immessa o rientra nell'argomento oggetto della query.

Sebbene il comportamento dell'utente descritto da Kuhlthau faccia riferimento alla sola ricerca 'attiva' dell'informazione, si può anche verificare che l'utente si approcci all'OPAC senza una specifica richiesta, attivando un comportamento esplorativo, attivo e indiretto $^{204}$ nel quale senza un interesse specifico si espone attivamente alla possibilità di acquisire nuove informazioni. In questo caso, ancora poco considerato dagli studi sull'information seeking behavior che sono maggiormente orientati alla ricerca 'intenzionale' in cui l'utente ha un problema da risolvere, possiamo supporre che l'incertezza cognitiva abbia un peso minore nelle attività che l'utente svolge ${ }^{205}$.

\section{Funzionalità strategiche che gli OPAC dovrebbero offrire}

In base alle funzioni pratiche che l'utente svolge in questa fase, possiamo delineare alcune funzionalità che dovrebbero essere sviluppate nella progettazione dei cataloghi elettronici per consentire all'utente di interagire più efficacemente con il catalogo, di specificare il bisogno informativo e recuperare l'informazione rilevante in relazione alla sua ricerca.

* offrire all'utente funzioni di orientamento (es. ricerca nell'OPAC, espansione ai contenuti esterni);

* facilitare la ricerca esplorativa tramite il browsing;

* offrire suggerimenti di lettura in forma visuale (es. vetrine) e canali di accesso al catalogo;

* offrire l'informazione generica (background information) e di riferimento su un particolare soggetto;

consentire all'utente di esplorare il contesto semantico di un concetto;

\footnotetext{
${ }^{204}$ Marcia J. Bates, Toward an integrated model of information seeking and searching cit.

${ }^{205}$ Ciò vale anche nel caso di utenti inesperti. Un approccio esplorativo si rivela più efficace della formulazione di un'interrogazione. Cfr.: Shelda Debowski, Wrong way: Go back! An exploration of novice search behaviours while conducting an information search, «The Electronic Library», 19 (2001), p. 371-382 e Karen Markey Drabenstott, Do non-domain experts enlist the strategies of domain experts?, "Journal of the American Society for Information Science and Technology», 54 (2003), n. 9, p. 836-854. Anche online in : $<$ http://deepblue.lib.umich.edu/bitstream/handle/2027.42/35291/10281_ftp.pdf?sequence=1>.
} 
* aiutare l'utente a formulare una query individuando i termini sinonimi e correlati;

* raggruppare i documenti che hanno caratteristiche comuni;

* suggerire le modalità di ricerca più adatte al risultato che si vuole ottenere.

a. Offrire all'utente funzioni di orientamento, integrare gli strumenti bibliografici

Le funzionalità che i nuovi OPAC dovrebbero mettere in campo riguardano quindi alcune funzioni che potremmo definire 'di orientamento' per l'utente e di guida agli strumenti più idonei da utilizzare.

Queste funzionalità sono state tradizionalmente fornite dai portali bibliotecari e sono state svolte all'esterno del catalogo, anche se di recente si notano alcune tendenze innovative. I servizi di reference a distanza asincroni (Ask a librarian) che trovano solitamente posto nella homepage della biblioteca hanno ceduto il posto a forme di reference a distanza sincrone ove l'utente può interagire direttamente con il bibliotecario in fase di ricerca. Tali funzionalità trovano oggi spazio anche all'interno degli OPAC, nella forma delle chat sperimentate all'interno dei cataloghi ${ }^{206}$.

Una funzione più utile per l'utente potrebbe essere quella di fornire un aiuto iniziale riguardo agli strumenti di ricerca messi a disposizione delle biblioteche. In questo campo si concentrano le principali innovazioni e le maggiori criticità: tra le caratteristiche degli OPAC di nuova generazione vi è quella di offrire all'utente una ricerca "one stop shopping" che consente di interrogare per mezzo di una sola interfaccia una varietà molto estesa di risorse provenienti dal catalogo tradizionale e da varie fonti esterne. I discovery systems mettono l'utente di fronte ad una complessità dovuta alla massa critica dell'informazione recuperata; affiancandosi all'OPAC consentono un recupero su vasta scala che ricomprende le risorse dell'OPAC, ma molto spesso la presenza di questo doppio canale di ricerca non è compresa dall'utente.

Un approccio più praticabile potrebbe essere quello di un'integrazione tra gli strumenti dell'OPAC e del discovery system, dando all'utente la possibilità di cominciare la sua ricerca consultando un OPAC e una volta visualizzato il set di risultati, estendere la ricerca ai contenuti provenienti da fonti esterne generando un'interrogazione che ampli il

\footnotetext{
${ }^{206}$ L'OPAC della Topeka and Shawnee County Public Library è stato il primo ad introdurre in via sperimentale la chat all'interno dell'OPAC, <http://www.tscpl.org/>. Oggi la funzione trova anche posto nella home page del sito web.
} 
set di risultati ricomprendendo le risorse provenienti dal discovery system. In questo modo si otterrebbero alcuni vantaggi:

* dare una maggiore centralità all'OPAC che diventerebbe il punto di accesso iniziale di tutte le ricerche;

* apportare alla ricerca bibliografica una maggiore chiarezza e trasparenza per l'utente;

nel caso di ordinamento per ranking (solo qualora tale ordinamento sia fornito come modalità alternativa al raggruppamento delle entità/famiglie bibliografiche e non in sostituzione della visualizzazione secondo il modello FRBR) un miglioramento della qualità dei risultati ottenuti. L'ordinamento per ranking nei discovery system è infatti impreciso poiché vengono presi in considerazione ai fini dell'ordinamento documenti disomogenei: sia i record bibliografici quali surrogati dei documenti, sia i fulltext degli articoli di riviste e dei documenti presenti in rete $\mathrm{i}$ quali nell'ordinamento ricevono un grado più alto di rilevanza in base al fatto che si indicizzano per l'ordinamento anche le parole contenute nei testi.

b. Facilitare la ricerca tramite il browsing

L'OPAC dovrebbe fornire all'utente la possibilità iniziare il proprio percorso di ricerca attraverso il browsing cioè di avviare una 'ricerca esplorativa' nel catalogo. Il catalogo dovrebbe offrire la possibilità fin dalla prima schermata di accedere al contenuto del catalogo attraverso il browsing dei soggetti, delle classi, cioè di accedere ai contenuti dell'OPAC senza dover formulare un'interrogazione, oppure di formulare un'interrogazione e di ricevere in risposta dal sistema:

* i raggruppamenti semantici che contengono il termine o i termini immessi;

raggruppamenti di opere contenenti nel titolo il termine o i termini immessi.

L'OPAC dovrebbe dunque presentare fin da subito la possibilità per l'utente di scegliere se formulare un'interrogazione oppure affidarsi a canali di ricerca predisposti. Per molto tempo gli OPAC hanno presentato all'utente la sola possibilità di immettere un'interrogazione. Il browsing dovrebbe avvantaggiarsi di vocabolari controllati che consentano di effettuare raffinamenti / espansioni a partire da un termine in modo semplice e rapido e senza la necessità per l'utente di conoscere la struttura sottostante dei 
collegamenti semantici, che dovrebbero comunque essere esplicitati. L'utente dovrebbe poter espandere/restringere la sua ricerca tramite un semplice comando espandi/riduci, e dunque con il minor sforzo possibile ma tale funzione deve essere svolta in modo chiaro e comprensibile per l'utente: dovrebbe essere manifestata all'utente la struttura sindetica cioè la rete di collegamenti tra le entità, offrendo il raggruppamento per famiglie bibliografiche.

c. La formulazione della query. Vocabolari d'accesso e uso delle ontologie

Anche nelle fasi iniziali di una ricerca l'utente immette interrogazioni o perché è in possesso di elementi noti (es. il nome di un autore, un titolo) o perché ha in mente un soggetto generico dai quali partire per iniziare la ricerca.

L'utente dovrebbe essere messo in grado di creare agevolmente una query e di modificarla in base al set di risultati ottenuti, o a varie strategie di relevance feedback che possono essere attivate. Numerosi studi hanno dimostrato che gli utenti hanno difficoltà a formulare interrogazioni ai sistemi, ciò risulta evidente se gli utenti si trovano nelle fasi iniziali del processo di ricerca ${ }^{207}$.

Secondo Marchionini e White ${ }^{208}$ la formulazione di una interrogazione richiede almeno due tipologie di corrispondenza (mapping):

a) una corrispondenza semantica (semantic mapping), tra il vocabolario dell'utente e quello del sistema, necessario per articolare l'interrogazione in un vocabolario comprensibile dal sistema;

b) una corrispondenza di azioni (action mapping) cioè delle tattiche di ricerca dell'utente rispetto a quelle consentite dai sistemi.

L'utente in questa fase del processo dovrebbe essere messo in grado di

* identificare l'entità più appropriata per dare inizio alla ricerca;

* creare la query di partenza per la ricerca dell'entità di interesse;

* modificare la query in base ai risultati ottenuti (o automaticamente sfruttando le funzionalità di relevance feedback);

${ }^{207}$ Tefko Saracevic - P. Kantor, Studying the value of library and information services. I. Establishing a theoretical framework. «Journal of the American Society for Information Science», 48 (1997), n. 6, p. $527-$ 542 .

${ }^{208}$ Gary Marchionini - Ryen White, Find what you need, understand what you find, «International Journal of Human-Computer Interaction», 23 (2007) n. $3, \quad$ p. 205-237, $<$ http://www.tandfonline.com/doi/abs/10.1080/10447310701702352\#preview>. 
valutare la rilevanza dell'informazione recuperata (TOC, etc. arricchimenti, livello di approfondimento di un argomento) attraverso un'esposizione significativa dei risultati della ricerca.

Una caratteristica che gli OPAC hanno introdotto per ovviare alle difficoltà della ricerca in questa fase è la ricerca google like, cioè la ricerca per parola chiave su tutti i campi del record, che produce una massa critica di risultati che vengono ordinati per rilevanza.

Come è stato già rilevato, l'ordinamento dei risultati in base a una presunta rilevanza per l'utente e basato esclusivamente sul matching dei termini in un documento non è in grado di fornire risultati soddisfacenti in quanto produce una carenza di struttura bibliografica nella visualizzazione dei risultati e una mancanza di controllo sui risultati di un'interrogazione. L'utente potrebbe infatti fermarsi ad esaminare solo la prima pagina dei risultati, ritenendo che le pagine seguenti siano meno significative e fidandosi dell'ordinamento proposto. L'utilizzo negli algoritmi di modalità fuzzy amplia a dismisura la quantità dei record recuperati, rendendo quasi impossibile per l'utente comprendere l'estensione del set dei risultati davvero rilevanti. La misura dei confini dell'universo rappresentato nei cataloghi è sempre più sfuggente, e l'ordinamento per rilevanza non è in grado di fornire all'utente un aiuto in questo senso ${ }^{209}$.

Allo stesso modo l'utente, abituato a compiere le ricerche in rete, si rivolge sempre più spesso al catalogo usando il linguaggio naturale; trovando nei nuovi OPAC la ricerca google like come ricerca di default, si sforza sempre di meno a tradurre la sua query in un linguaggio comprensibile dai sistemi e vi cerca caratteristiche proprie dei motori, come la correzione degli errori in fase di digitazione che, infatti, in molti nuovi OPAC è stata introdotta.

L'utilizzo di vocabolari controllati a supporto della ricerca è necessario per aiutare l'utente a formulare, riformulare ed espandere la query di partenza, dunque la presenza di un vocabolario controllato che consenta all'utente di accedere con precisione ai contenuti dell'OPAC è una funzionalità critica che nella progettazione degli OPAC dovrebbe essere implementata se si vuole migliorare il processo di ricerca dell'utente.

Vari studiosi hanno proposto la creazione di un vocabolario di accesso, inteso come vocabolario da sovrapporre alla rete dei collegamenti semantici presenti nel catalogo, per

\footnotetext{
${ }^{209} \mathrm{Su}$ questo argomento si vedano anche le riflessioni di Bates (Marcia J. Bates, Where should the person stop and the information search interface start? «Information Processing \& Management», 26, (1990), p. $575-591$.
} 
consentire all'utente di raggiungere da una query di partenza il termine / i termini più adatti a formulare un'interrogazione nel linguaggio di indicizzazione usato nel catalogo ${ }^{210}$.

I più recenti sviluppi del Web Semantico, che, come abbiamo visto, cominciano ad avere alcune prime applicazioni nello sviluppo dei cataloghi elettronici potrebbero avere in questo campo alcune applicazioni significative ${ }^{211}$.

L'OPAC potrebbe servirsi dei nuovi strumenti del Semantic Web per appoggiarsi a ontologie lessicali o a ontologie costruite $a d$ hoc a partire dagli accessi semantici assegnati alle registrazioni bibliografiche, ad esempio le stringhe di soggetto $\mathrm{LCSH}^{212}$.

Nuove funzionalità in grado di appoggiarsi alle ontologie rappresentano, nello scenario tecnologico che si sta preannunciando, una nuova possibilità di costruire interfacce amichevoli per l'espressione delle interrogazione ai sistemi. La possibilità di reingegnerizzare i vocabolari utilizzati negli OPAC in ontologie formali può rappresentare una valida alternativa alla semplice ricerca per parola chiave in un box di ricerca 'alla Google', soluzione che al momento nelle interfacce degli OPAC di nuova generazione viene privilegiata come accesso più amichevole alle risorse del catalogo ${ }^{213}$.

Attraverso l'uso delle ontologie come strato di accesso ai contenuti semantici/semiotici dell'OPAC l'utente potrebbe inserire le sue interrogazioni nel sistema usando il linguaggio naturale e avere in risposta una serie di 'indicazioni dinamiche' sui termini preferiti per dare avvio a una ricerca più mirata.

Una volta immessi uno o più termini nel box, l'OPAC dovrebbe presentare all'utente le corrispondenze dei termini immessi con cluster di soggetti o classi corrispondenti e indirizzare l'utente alla modalità di ricerca più appropriata in base al termine o ai termini usati per formulare l'interrogazione.

Alcuni studiosi hanno ipotizzato la possibilità di costruire ontologie basate sulle stringhe di soggetto $\mathrm{LCSH}$, in grado di strutturare semanticamente le relazioni implicite e esplicite tra le stringhe di soggetto LCSH ai fini di un miglioramento del recupero per soggetto dei documenti nelle biblioteche digitali. Le relazioni esplicite si riferiscono alla struttura sindetica esplicita tra le stringhe di soggetto, e cioè i collegamenti tesaurali

\footnotetext{
${ }^{210}$ Marcia J. Bates, Task Force Recommendation 2.3 Research and design review, Improving User Access to Library Catalog and Portal Information, final report (Version3), 2003 [risorsa online], <http://www.loc.gov/catdir/bibcontrol/2.3BatesReport6-03.doc.pdf>.

${ }^{211}$ Cfr. infra Appendice A. Casi di studio. OPAC Libris.

${ }^{212}$ Ioannis Papadakis - Konstantinos Kyprianos - Rosa Mavropodi - Michalis Stefanidakis, Subject-based information retrieval within digital libraries employing LCSHs, «D-Lib Magazine», 15 (2009) n. 9/10 < http://www.dlib.org/dlib/september09/papadakis/09papadakis.html>.

${ }^{213}$ Maria Teresa Biagetti, Le ontologie come strumenti per l'organizzazione della conoscenza in rete, in Numero speciale monografico su le ontologie a cura di Maria Teresa Biagetti, «AIDA informazioni», 28 (2010) n. 1-2, p. 9-31, <http://www.aidainformazioni.it/2010/122010.html>.
} 
gerarchici e relazionali, mentre le relazioni implicite collegano le stringhe di soggetto che condividono suddivisioni comuni (topiche, geografiche, formali, e cronologiche) e che costituiscono quella che gli autori chiamano 'struttura sindetica estesa' ${ }^{214}$. Il vantaggio di costruire un'ontologia di questo tipo, secondo gli autori è quello di sfruttare le potenzialità semantiche già presenti nei cataloghi elettronici, dotati di descrittori di qualità, per migliorare l'efficacia del recupero dell'informazione nella ricerca per soggetto. LCSH, come i più comuni soggettari in uso dalle biblioteche, impongono il controllo della sinonimia ed è indubbio che la ricchezza semantica e l'espressività offerte dalle stringhe di soggetto sono indubbiamente più ampie di quelle offerte dal singolo termine di ricerca. L'impopolarità della ricerca per soggetto nei cataloghi deriva dall'incompatibilità delle LCSH con l'ambiente elettronico e con la difficoltà nel recupero usando gli operatori booleani. Gli utenti infatti usano termini a loro familiari corrispondenti al soggetto cercato e li combinano con gli operatori, ma una ricerca di questo tipo raramente fornisce risultati apprezzabili, in quanto di rado i termini usati corrispondono con il linguaggio di indicizzazione utilizzato dal sistema. In questo campo l'applicazione dei thesauri potrebbe già avere un effetto positivo nel recupero dell'informazione, anche se questi strumenti sono poco utilizzati.

Il proposito di estendere la struttura sindetica e di mapparla con uno schema di ontologia potrebbe - secondo gli studiosi - fornire la struttura per sviluppare interfacce che aiutano l'utente a scegliere il soggetto più appropriato per la propria ricerca.

In base allo schema dell'ontologia proposta dagli studiosi, anche nel caso di corrispondenza parziale dei termini, la struttura sindetica estesa potrebbe aiutare l'utente in maniera dinamica indirizzandolo al soggetto desiderato. Un recupero di questo tipo potrebbe di gran lunga migliorare le prestazioni della ricerca per soggetto nei moderni cataloghi. Gli studiosi propongono un'interfaccia in grado di sfruttare l'autocompletamento della query in modo intelligente e significativo per l'utente.

Di recente in alcuni cataloghi è stata introdotta tra le nuove caratteristiche proprio la funzione di autocompletamento della query, funzionalità già presente in Google, che offre suggerimenti in base alle ricerche compiute dagli utenti nelle loro interrogazioni nel motore di ricerca ${ }^{215}$.

\footnotetext{
${ }^{214}$ Ioannis Papadakis et. al., Subject-based Information Retrieval cit.

215 Il funzionamento del completamento automatico offerto da Google è spiegato in <http://support.google.com/websearch/bin/answer.py?hl=it\&answer=106230> «Mentre digiti, l'algoritmo di Google prevede e visualizza le query di ricerca in base alle attività di ricerca degli altri utenti. Queste query vengono determinate in modo algoritmico in base a una serie di fattori assolutamente oggettivi (come la popolarità dei termini di ricerca), senza alcun intervento umano. Tutte le query previste visualizzate sono
} 
Tale funzione, come avviene già in Google, viene usata per suggerire termini già cercati in precedenza nel catalogo e sarebbe uno strumento più utile se si appoggiasse ad un vocabolario controllato di termini sinonimi in grado di aiutare l'utente nella formulazione dinamica delle sue interrogazioni oppure ad un'ontologia creata ad hoc da soggettari o classificazioni. L'autocompletamento in fase di digitazione avrebbe la funzione, in questo caso, non tanto di presentare i termini maggiormente cercati dagli utenti che hanno già visitato il catalogo, ma di presentarsi come una vera e propria guida dinamica ai termini più idonei per formulare un'interrogazione.

Anche nel caso della ricerca per parola chiave più interessante risulterebbe la possibilità nella fase di interrogazione di appoggiarsi a vocabolari controllati, thesauri, vocabolari d'accesso o ontologie che siano in grado di rilevare quali tra i termini immessi siano contenuti in stringhe di soggetto, classi, titoli etc. e di presentare all'utente un suggerimento dinamico con le relative occorrenze e in grado di guidare l'utente alla modalità più corretta di interrogazione del catalogo.

In molti moderni OPAC la funzione di autocompletamento viene usata in maniera banale e non vengono sfruttate le potenzialità insite in questa modalità di ricerca.

È il caso di alcuni OPAC rinnovati di recente come, ad esempio, la versione del nuovo OPAC SBN. Avvertendo il lettore che si tratta, comunque di una versione beta del software, dunque lanciata in rete in veste provvisoria e sperimentale, l'OPAC presenta in questa funzionalità di recente introdotta alcune inefficienze evidenti, in quanto l'autocompletamento proposto non si appoggia, come dovrebbe ad un vocabolario controllato $^{216}$.

\footnotetext{
state digitate in precedenza da utenti Google. Il set di dati della funzione di completamento automatico viene aggiornato spesso in modo da offrire query di ricerca nuove e in aumento. Inoltre, se hai eseguito l'accesso al tuo account Google e hai attivato la Cronologia web, potrebbero essere visualizzate query di ricerca basate su ricerche pertinenti che hai eseguito in passato». I vantaggi della tecnologia secondo Google sono riposare le dita, accorgersi di errori di digitazione, ripetere ricerche già fatte, e infine nella 'scoperta' dell'informazione.

${ }^{216}$ Un esempio di questo tipo di autocompletamento è visibile inserendo nel box di ricerca una query nel linguaggio naturale come "Argentina storia" i cui termini sono contenuti nella stringa Argentina - storia; l'OPAC dovrebbe completare la query segnalando nel menu a discesa gli accessi che contengono il termine/i termini immessi. Il suggerimento fornito è invece un elenco dei termini cercati precedentemente dagli utenti, senza alcun computo che possa rivelarsi utile all'utente. In vari casi $\mathrm{i}$ termini cercati in fase di autocompletamento vengono automaticamente sostituiti con un solo termine (se presente tra i termini cercati nel catalogo).
} 


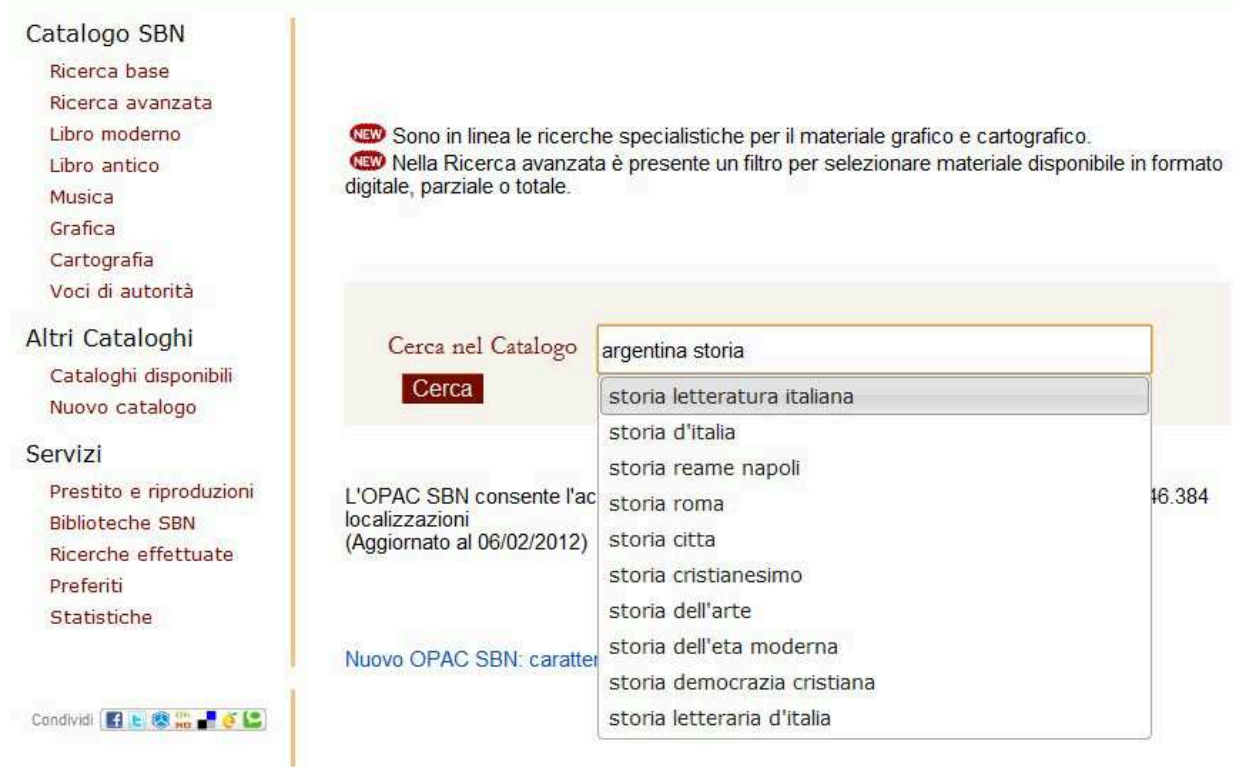

Copyright $82010 \mathrm{ICCU}$ |Istituto Centrale per il Catalogo Unico delle biblioteche italiane e per le informazioni bibliografiche - Realizzato da Inera s.r.l.

WBC : RHTML1.0V WBS CSS -

Fig. 5.22 - L'autocompletamento in fase di digitazione della query dell'OPAC del Servizio bibliotecario nazionale non si appoggia ad una lista di termini controllati ${ }^{217}$

d. Offrire l'informazione generica/specifica su un argomento

La descrizione del processo di ricerca descritto da Kuhlthau prevede che prima della formulazione del focus della propria ricerca, l'utente cercherà un argomento generale, dal quale far partire la propria ricerca; il comportamento è confermato da numerosi studi che attestano come l'utente formuli nella maggior parte dei casi una query su un argomento ampio, sia perché non è a conoscenza della terminologia usata dal sistema, sia perché molto spesso non è pratico del campo disciplinare che sta indagando.

Vari studi hanno notato che l'utente nell'esprimere la query al sistema in un'interrogazione per soggetto molto spesso la formula ad un livello di specificità non corretto, più ampio o più ristretto rispetto all'argomento oggetto del focus. È dunque importante che l'utente sia in grado di formarsi una conoscenza dell'ambito semantico che sta indagando, funzionalità che è espressa tramite la struttura sindetica del catalogo e attraverso la rete dei collegamenti tra le entità.

Un aiuto che l'OPAC potrebbe offrire all'utente è quello di specificare il grado di profondità o di specificità e di completezza con cui viene trattato un argomento in un dato

\footnotetext{
${ }^{217}<$ http://www.sbn.it/opacsbn/opac/iccu/free.jsp>. Cfr. infra, Appendice A. Casi di studio. OPAC SBN.
} 
documento $^{218}$. Ciò comporterebbe livelli di indicizzazione più approfonditi e sfaccettati, che richiederebbero un potenziamento notevole dell'espressività semantica del contenuto di un documento, ma allo stesso tempo un notevole incremento dei costi della catalogazione.

Pre-focus. Linee strategiche di sviluppo.

L'analisi approfondita delle funzioni che gli utenti svolgono in questa fase ci offre lo spunto per evidenziare alcune criticità e suggerire alcuni miglioramenti che si potrebbero apportare nella progettazione degli OPAC.

Tra i miglioramenti più significativi:

* strutturazione significativa dell'accesso alle risorse dell'OPAC/del discovery system;

* sviluppo delle funzionalità che consentano all'utente di esplorare in maniera significativa il catalogo tramite il browsing (di soggetti, di classi, di altri canali di accesso alle risorse contenute nel catalogo, es liste delle novità, cluster di soggetti, etc.);

* introduzione di funzionalità che consentano di visualizzare l'informazione generica su un dato argomento;

* sviluppo delle funzionalità di aiuto nella formulazione della query tramite l'appoggio a vocabolari d'accesso, thesauri, ontologie costruite sugli accessi semantici presenti nel catalogo.

\subsubsection{La formulazione del focus. Attività dell'utente, funzionalità dell'OPAC e linee strategiche}

Il punto di svolta del processo di ricerca, già individuato da Kuhlthau e ripreso nel modello da me presentato, è la formulazione di un focus.

Una volta che l'oggetto della ricerca è compreso dall'utente, egli è in grado di muoversi con fiducia verso le fasi successive di raccolta e utilizzo dell'informazione

218 Maria Teresa Biagetti, Nuove funzionalità degli OPAC e relevance ranking. «Bollettino AIB», vol. 50 (2010) n. 4, p. 339-356. 
ricevuta. L'utente che si approccia all'OPAC quando ha già trovato un focus specifico ha la possibilità di svolgere l'interazione con il sistema in maniera efficace perché ormai ha un senso chiaro di direzione della ricerca. Il suo compito è di raccogliere l'informazione su un focus già formato, dunque l'utente è in grado di specificare non solo qual è l'informazione rilevante, ma anche di valutare l'informazione pertinente ${ }^{219}$.

Una volta recuperata l'informazione, l'utente è impegnato in una serie di attività che gli consentono di:

* esaminare i risultati ottenuti, riformulare e dunque creare una nuova query;

* modificare la query in base ai risultati ottenuti (o automaticamente sfruttando le funzionalità di relevance feedback);

* valutare la 'rilevanza' dell'informazione recuperata rispetto ad un focus ormai formato (un argomento specifico) e selezionare le informazioni rilevanti ottenute;

* valutare la 'pertinenza' dell'informazione recuperata, in base alle conoscenze pregresse all'interesse personale, al tempo a disposizione e, dunque, ad una serie di fattori soggettivi che sono stati evidenziati nei modelli analizzati

Funzionalità strategiche che gli OPAC dovrebbero offrire

Secondo Kuhlthau nel processo informativo vi è un incremento della ricerca dell'informazione pertinente. Una volta formulato e compreso l'argomento della ricerca, si cercherà l'informazione 'rilevante' rispetto all'argomento specifico che si è messo a fuoco, per poi procedere alla selezione dell'informazione 'pertinente'.

La progettazione dell'OPAC dovrebbe dunque offrire all'utente funzionalità che consentano di:

formulare e modificare la query di partenza in maniera dinamica;

* valutare la rilevanza e la pertinenza dell'informazione recuperata

\footnotetext{
${ }^{219}$ Carol Collier Kuhlthau, Seeking meaning cit.
} 
a. Riformulazione della query, espansione e modifica dinamica della query di partenza.

Una volta formulato l'oggetto di ricerca l'utente può accorgersi di aver creato la query in modo impreciso, dunque potrà creare nuovamente la query per avvicinarsi maggiormente all'oggetto di ricerca, o apprendendo informazioni dalla navigazione nell'OPAC, potrà creare nuove query. Tale comportamento è esposto nel modello proposto da Bates $^{220}$ che suggerisce che la ricerca non è statica, ma si evolve e che quindi la prima query che l'utente inserisce, è destinata a cambiare nel corso della ricerca. Il catalogo, dunque, non deve fermarsi a dare una risposta, ma consentire all'utente di navigare tra le entità, di tornare indietro, di poter raffinare o riformulare la query nel corso della ricerca in base a spunti e suggerimenti.

Lo strumento che gli OPAC di nuova generazione hanno introdotto per ovviare alla massa di risultati che si ottengono tramite una ricerca per parola chiave in un box 'Google like' è l'ordinamento per rilevanza (o rilevanza presunta) affiancato dai filtri, che consentono di restringere, ampliare il set dei risultati dinamicamente e senza formulare una nuova interrogazione. La faceted navigation, introdotta per la prima volta dalle biblioteche della North Carolina State University per l'OPAC basato sulla piattaforma Endeca, consente di filtrare dinamicamente il set di risultati ottenuti tramite una serie di filtri, che possono essere aggiunti o eliminati, dando luogo ad una ristrutturazione dinamica del set dei risultati in base all'operazione eseguita. Le faccette in realtà sono raggruppamenti dinamici generati a partire dalle registrazioni presenti nel catalogo ${ }^{221}$. Differente dalla funzionalità dell'autocompletamento è la funzione di query espansion: mentre la prima è un aiuto dinamico che avviene in fase di digitazione e dunque aiuta nell'indirizzare l'utente al termine più idoneo a iniziare la sua ricerca, l'espansione della query avviene successivamente al recupero dei risultati ${ }^{222}$, e ha una funzione di indirizzo per l'utente per il prosieguo della ricerca, configurandosi come un vero e proprio 'suggerimento'. Anche la funzione di query espansion rientra tra le funzionalità introdotte negli OPAC di nuova generazione. Funzionalità di questo tipo vengono evocate a partire dal dettaglio del record, per ampliare la ricerca a documenti simili, dello stesso autore, o che trattino lo stesso argomento. Interessante funzione potrebbe essere quella di espandere la query in base

\footnotetext{
${ }^{220}$ Marcia Bates, The Design of Browsing and Berrypicking Techniques for the Online Search Interface cit. ${ }^{221}$ Cfr. supra, cap. 2. $\$$ 2.5.2.2. Navigazione a faccette.

${ }^{222}$ Efthimiadis (1999) citato in Gary Marchionini - Ryen White, Find what you need, understand what you find cit.
} 
all'argomento trattato, con la possibilità di espandere e restringere la query in un solo clic a documenti che trattano lo stesso argomento in modo più generico/specifico.

b. Valutazione del documento: dalla 'rilevanza' alla 'pertinenza'

Quando l'utente utilizza l'OPAC con un focus ben formato può valutare i documenti recuperati ai fini della rilevanza e della pertinenza e dunque svolgere un processo di selezione dei documenti che giudica utili per la propria ricerca. Come abbiamo visto, nell'OPAC un documento è considerato rilevante e dunque viene recuperato, quando vi è corrispondenza algoritmica tra i termini presenti nell'interrogazione e i termini presenti in alcuni metadati o nell'intero documento (ad esempio la presenza dell'autore cercato nel punto di accesso associato alla registrazione nella ricerca per autore). Nel caso di interrogazione per parola chiave, il sistema tramite un algoritmo provvede alla pesatura dei termini, indispensabile ad ottenere i documenti in ordine di "presunta rilevanza".

Come già anticipato, la valutazione della rilevanza nel processo di ricerca è un processo dinamico perché documenti rilevanti all'inizio possono poi risultare non rilevanti nel momento della formulazione di un focus, inoltre, secondo gli studi citati l'utente nel suo percorso di ricerca, cercherà nelle prime fasi i documenti rilevanti, mentre nelle ultime fasi darà più importanza all'informazione pertinente, valutando il documento in base a criteri soggettivi.

Un documento recuperato potrà essere rilevante ma poi rilevarsi non pertinente in base ad una serie di valutazioni soggettive dell'utente. Nella valutazione della rilevanza di un documento entrano dunque in gioco i fattori già elencati da Saracevic ${ }^{223}$ :

Contenuto. L'utente può valutare un documento in base al contenuto esaminando la corrispondenza dell'argomento (aboutness o 'circalità') o guardando ad altri fattori come la qualità dell'informazione, l'ampiezza della trattazione nel documento, il suo grado di aggiornamento, il trattamento di un argomento nel documento e la chiarezza dei contenuti esposti. Le funzionalità offerte dagli OPAC di nuova generazione si sono concentrate su questo aspetto, infatti l'arricchimento degli OPAC ha rappresentato un notevole passo avanti rispetto agli OPAC tradizionali; la presenza di sommari, recensioni, valutazioni, l'aggiunta del fulltext, rappresentano un valido aiuto per l'utente, rispetto alla visualizzazione della semplice registrazione bibliografica.

\footnotetext{
${ }^{223}$ Tefko Saracevic, Relevance: a review of the literature and a framework for thinking on the notion in information science. Part II cit.
} 
Come già accennato in precedenza, andrebbero maggiormente sviluppati quegli strumenti semantici che consentano di rilevare

* il grado di autorevolezza di un documento;

* il livello della trattazione di un particolare soggetto o argomento nel documento;

* il tipo di trattamento (di discorso) che si fa intorno ad un particolare soggetto.

Nel primo caso, la ricerca per citazioni, tattica descritta da Bates e da Ellis ${ }^{224}$, che consente di sapere quali e quanti documenti citano un documento, potrebbe rivelarsi un aiuto nella valutazione. Nel secondo caso, si tratta di informare l'utente se l'argomento del documento è trattato in maniera completa nel documento e quali altri argomenti sono presenti. Nel terzo caso si tratta di informare l'utente sul tipo di discorso che si fa intorno all' argomento stesso, cioè il punto di vista.

Oggetto. L'utente può valutare la rilevanza esaminando le caratteristiche dell'oggetto informativo, ad esempio la tipologia o il formato di documento (libro, risorsa elettronica etc. ) la disponibilità del documento, l'accessibilità e i costi per ottenerlo. Generalmente gli OPAC forniscono questi dati, in molti OPAC di nuova generazione è possibile scegliere fin da subito tra i documenti immediatamente disponibili, ad esempio quelli non in prestito e in qualche caso anche tra quelli accessibili (come la possibilità di selezionare le sole risorse online in full text).

Validità. L'accuratezza dell'informazione fornita, l'autorità e l'affidabilità dell'autore, la verificabilità, sono in genere alcune caratteristiche che l'utente ricerca per valutare la validità e l'autorevolezza di un documento.

Gli OPAC di nuova generazione hanno introdotto vari perfezionamenti atti a migliorare le possibilità di valutazione di un documento da parte dell'utente. L'arricchimento del record bibliografico con sommari e abstract rappresenta un miglioramento senz'altro importante per valutare la rilevanza dell'informazione ricevuta.

Worldcat Identities, che si presenta come un authority file arricchito delle voci di autorità presenti nell'OPAC Worldcat, è un esempio di come l'OPAC può fornire elementi valutativi sulle entità bibliografiche, in questo caso le entità del primo tipo, autori ed enti delle opere presenti nel catalogo. Per ogni voce d'autorità presente nell'OPAC viene creata una pagina web, consultabile a partire dalla registrazione bibliografica in OPAC oppure dall'esterno, ricercandola nella homepage del progetto. Per ogni autore sono visibili, oltre a tutte le forme varianti del nome, il numero totale delle edizioni, e la distribuzione nel

\footnotetext{
${ }^{224}$ Nella teoria del berrypicking di Marcia J. Bates questa funzionalità viene introdotta tra le tattiche 0 strategie di ricerca; Ellis la considera invece un comportamento di ricerca (chaining).
} 
tempo in forma di grafico, il genere principale, i soggetti e le classificazioni collegate, $\mathrm{i}$ ruoli (paternità intellettuale) principali e secondari, le lingue in cui compaiono le edizioni delle sue opere, le opere più conosciute e maggiormente possedute dalle biblioteche con il numero complessivo delle edizioni di ogni opera e una breve recensione, la tipologia di audience, le identità (voci di autorità) correlate, una serie di link esterni (es. Wikipedia). Alcuni dati sono anche espressi in forma visuale tramite nuvole di tag (soggetti, livello di audience).

Worldcat Identities si presenta come uno strumento avanzato, utile all'utente per la funzione di valutazione del documento ai fini della determinazione della rilevanza e, soprattutto, della pertinenza. Valutare infatti l'attendibilità, la notorietà o farsi un'idea delle tematiche trattate da un autore nel corso della sua attività, fornisce all'utente elementi utili a giudicare la validità di un documento.

\section{5ु. WorldCat" Identities Beta}

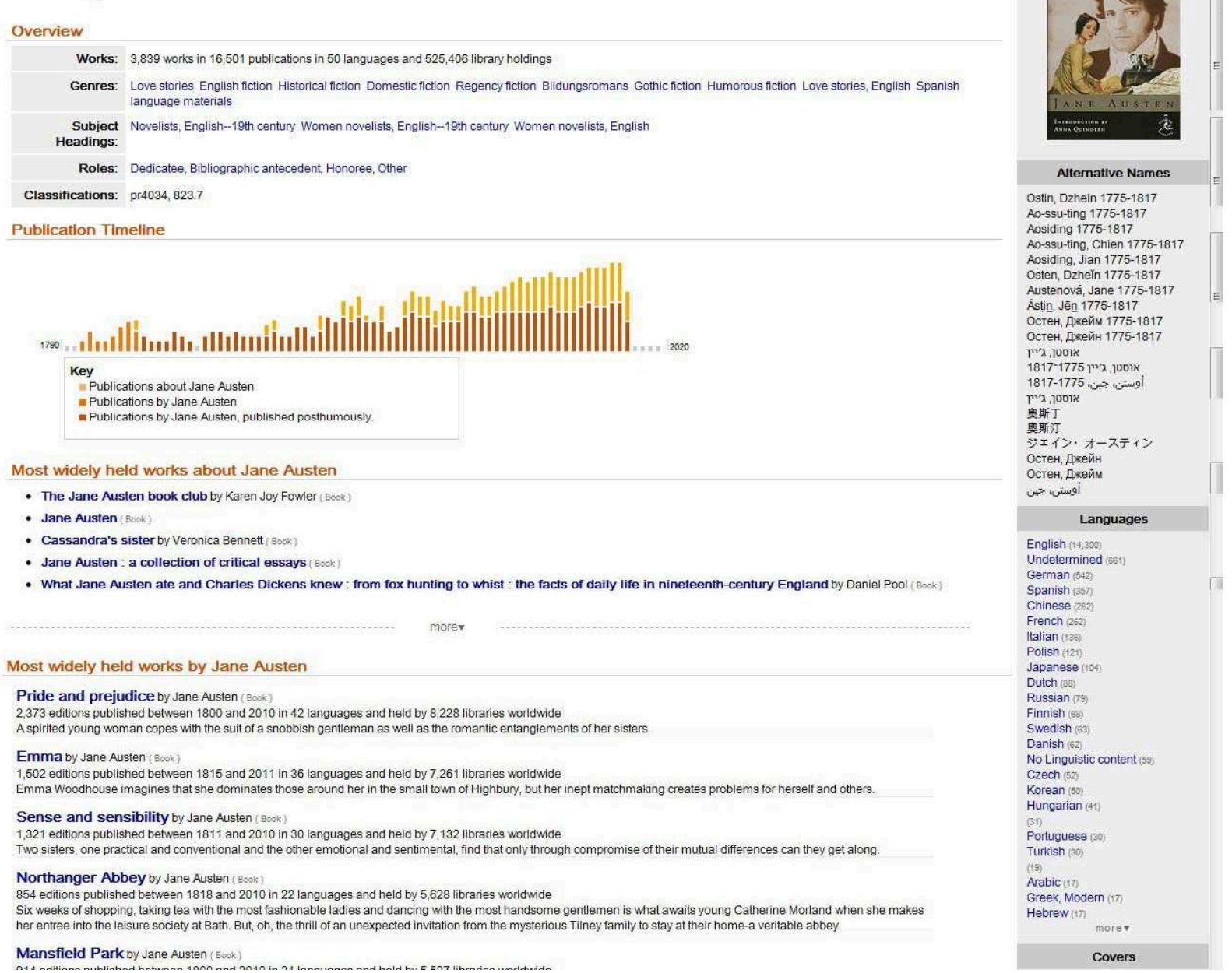

Fig. 5.23 - WorldCat Identities offre un'ampia quantità di dati che consentono una valutazione della produzione letteraria di un autore: le opere del'autore e sull'autore, i generi trattati, i soggetti collegati, i tag assegnati dagli utenti, una timeline che consente di verificare 
l'autorevolezza dell'autore, la tipologia dei destinatari, le varianti del nome, un riassunto del contenuto delle opere ${ }^{225}$

Infine, specie per la letteratura professionale, la ricerca per citazioni cioè la possibilità di verificare, ad esempio, quali altre opere hanno citato un documento è una funzione che potrebbe essere introdotta, in particolar modo per gli articoli dei periodici che vengono oggi recuperati tramite un discovery system ${ }^{226}$.

Dare all'utente la possibilità di valutare la 'pertinenza' di un documento prevede l'attivazione di nuove funzionalità che permettano di stabilire la 'rilevanza soggettiva' di un documento rispetto alle proprie esigenze informative.

Nella valutazione della pertinenza entra in gioco quella che Ingwersen chiama la struttura cognitiva dell'utente; l'utente valuta infatti la pertinenza in base alle conoscenze che già possiede, alle sue esperienze pregresse, e ad altri fattori soggettivi che riguardano la propria situazione ad esempio il tempo a disposizione.

Per dedurre la pertinenza l'utente prenderà in considerazione quelle corrispondenze che si relazionano al suo bisogno informativo, al suo stato mentale, alla sua struttura cognitiva, ad una serie di condizionamenti che provengono dal contesto, nel quale il suo bisogno informativo si forma e si perfeziona.

Le valutazioni che egli compie possono riferirsi ad esempio:

* all'uso o corrispondenza alla situazione. L'utente valuta l'appropriatezza rispetto alla situazione in cui si trova, al proprio compito di ricerca e al tempo disponibile per svolgere il compito, valutando alcune caratteristiche quali l'usabilità, urgenza, valore nell'uso;

* alla corrispondenza cognitiva. L'utente valuta la capacità di comprensione e lo sforzo mentale che dovrà sostenere per usare la risorsa oppure ne valuta l'importanza in termini di novità per l'utente (un documento rilevante che non è stato esaminato in precedenza). Non è escluso che anche nella ricerca dell'informazione bibliografica rientrino alcune valutazioni che l'utente compie quando cerca l'informazione in generale dunque nella valutazione del documento possa rientrare anche la generalità/specificità dell'argomento trattato, da valutare in base alla propria conoscenza pregressa;

\footnotetext{
$225<$ http://www.worldcat.org/wcidentities/lccn-n79-32879>.

${ }^{226}$ Cfr. supra, cap. $2, \S 1,2$.
} 
* alla corrispondenza affettiva consistente nella risposta emotiva all'informazione ricevuta e condizionata da fattori come gioia, frustrazione, incertezza (ben presenti nei modelli di ricerca indagati);

* alla corrispondenza ad una opinione consistente in una valutazione che si relaziona all'opinione personale che si da all'informazione ricevuta (ad es opinione politica), alla fiducia che l'utente dà all'informazione recuperata.

L'OPAC dunque potrebbe essere progettato per favorire l'individuazione di quelle caratteristiche del documenti che consentano all'utente di stabilire la pertinenza del documento che è necessaria fina dal momento della formulazione del focus.

Più complessa è invece l'eventualità che sia l'OPAC in base a determinare la pertinenza in base ai feedback forniti dall'utente stesso. Si entra infatti nel discorso, più articolato, della personalizzazione ${ }^{227}$. Non sempre l'utente è disposto ad fornire al sistema indicazioni sulle sue preferenze compilando un form, esprimendo un giudizio, caricando la sua ricerca di uno sforzo aggiuntivo ${ }^{228}$.

In questo campo, l'introduzione di funzioni sociali nell'OPAC, consistenti in recensioni, tag e commenti etc. sono state annunciate come una forma immediata e spontanea di feedback; tuttavia tali strumenti non sono idonei a svolgere questa delicata funzione per varie ragioni, che sono state analizzate da vari studiosi ${ }^{229}$. Va inoltre precisato che gli OPAC di nuova generazione che si definiscono sociali per aver introdotto tali funzionalità, spesso importano le recensioni e le valutazioni da fonti esterne.

\section{c. Esame dei risultati}

Uno dei problemi che affligge pesantemente la ricerca negli attuali OPAC consiste nella modalità di esposizione agli utenti dei risultati di un'interrogazione.

L'introduzione dell'ordinamento 'per presunta rilevanza', come abbiamo già rilevato, presenta gravi limiti e non offre una visualizzazione significativa per l'utente, poiché non rende conto della complessità e della ricchezza delle relazioni tra le entità rappresentate provocando così un generale impoverimento della navigazione e compromettendo l'efficacia complessiva del processo di ricerca.

\footnotetext{
${ }^{227}$ Cfr. supra, cap. $2, \S 2.5 .3 .1$. I servizi dell’OPAC e la personalizzazione.

$228 \mathrm{Si}$ tratta della personalizzazione 'esplicita' indirizzata a raccogliere indicazioni tramite espressa indicazione dell'utente.

${ }^{229}$ Cfr. Nicola Benvenuti, Social tagging e biblioteche cit. e Laura Ballestra, Servizi on line personalizzati: luci e ombre. cit.
} 
Nella modalità di visualizzazione di un'interrogazione basata sulla rilevanza viene a mancare una visualizzazione sistematica dei record recuperati in grado di rendere possibile una conoscenza approfondita dell'universo bibliografico rappresentato nel catalogo.

Sebbene da ormai un decennio il modello FRBR si proponga come valida alternativa all'attuale modello di sviluppo degli OPAC ancora oggi la mancata applicazione del modello si presenta come uno dei problemi ancora da risolvere nella progettazione dei cataloghi elettronici e che potrebbe di gran lunga migliorare il processo di valutazione dei risultati di una ricerca.

Abbiamo già affrontato nei precedenti capitoli le questioni relative all'importanza dell'applicazione e dell'implementazione di FRBR nei cataloghi elettronici, rilevandone i vantaggi, tra questi uno dei più importanti è costituito dall'efficacia delle entità previste dal modello in termini operativi ovvero organizzativi; pensiamo, ad esempio, all'efficacia del raggruppamento all'opera e delle funzioni non solo 'concettuali' ma 'operative' delle entità opera e super-opera come modalità organizzative del catalogo.

L'utente può, infatti, sfruttare i risultati visualizzati in base alle entità di FRBR per compiere una valutazione dei documenti rilevanti per una data ricerca. La visualizzazione secondo il modello FRBR dei risultati di una ricerca migliora la comprensione dei risultati recuperati facilitando il processo di conoscenza che deriva dal recupero dell'informazione contestualizzata nella porzione di universo bibliografico di riferimento, grazie al collegamento tra entità e alla funzione 'operativa' dei raggruppamenti generati tramite il modello.

Formulazione del focus. Linee di sviluppo.

L'esame approfondito di questa fase intermedia ma cruciale della ricerca, suggerisce che gli OPAC presentano ancora delle importanti criticità nelle funzionalità dell'esame dei risultati, nella creazione e modifica della query, nelle funzioni della valutazione della rilevanza e della pertinenza e che lo sviluppo di questa area potrebbe apportare grandi vantaggi per l'utente nell'interrogazione dei cataloghi elettronici. In particolare sembra opportuno offrire all'utente la possibilità di:

* navigare nel catalogo elettronico in base ad una disposizione dei risultati secondo il modello FRBR;

* valutare appropriatamente un documento perché si verifichi più pienamente la funzione del catalogo di 'selezionare' prevista da FRBR e ICP. 


\subsubsection{Fase post-focus. Attività dell'utente, funzionalità dell'OPAC e linee strategiche}

Le fasi successive del processo di ricerca vedono l'utente coinvolto in tutte quelle operazioni che gli consentono di organizzare i risultati ottenuti, tenere a memoria dati per riutilizzi futuri, accedere e ottenere l'informazione bibliografica, disseminare e riutilizzare i dati all'esterno dell'OPAC.

L'utente è dunque impegnato nella raccolta dell'informazione pertinente e l'interazione tra l'utente e il sistema di recupero dell'informazione si svolge in modo efficace, infatti il compito è di raccogliere l'informazione su un focus ormai ben definito; tutte le energie sono volte supportare il focus e l'utente che ha ormai risolto l'incertezza cognitiva che tanto peso aveva nei comportamenti della prima fase del processo, acquista una certa sicurezza dovuta all'acquisizione della 'direzione' da dare alla propria ricerca; egli è dunque in grado di specificare qual è l'informazione rilevante ad un sistema o a un bibliotecario, di valutare l'informazione pertinente raccogliendo tutte le risorse disponibili e di avviarsi così verso la conclusione della ricerca.

Nella fase di chiusura del processo, l'utente generalmente esibisce un senso di sollievo.

I motivi che spingono l'utente a concludere la ricerca hanno una grande importanza nella valutazione dell'efficacia di un sistema quasi quanto quelli che la attivano ${ }^{230}$.

Secondo Kuhlthau ${ }^{231}$ le persone concludono la ricerca per motivi diversi: perché sentono di aver messo abbastanza impegno, perché diminuisce la rilevanza e cresce la ridondanza dei documenti trovati, oppure perché ad un certo punto smettono di cercare semplicemente l'informazione perché devono prepararsi ad affrontare la stesura di una ricerca. Nell' ambiente fisico le energie e le attività sono volte a definire strategie di organizzazione dell'informazione recuperata.

Nella fase conclusiva di una ricerca l'utente è impegnato nelle attività di raccolta e di verifica dell'informazione ricevuta per avviarsi alla conclusione. L'utente svolge dunque una serie di attività che potremmo così definire:

* organizzare i risultati ottenuti per una navigazione più efficace;

\footnotetext{
${ }^{230}$ I motivi che spingono un utente ad abbandonare il processo di ricerca acquistano un certo rilievo in negli studi di 'architettura dell'informazione', un campo di interesse che, attingendo dalle teorie biblioteconomiche studia il modo di organizzare l'informazione nel web. Si vedano: Peter Morville - Louis Rosenfeld, Information Architecture for the World Wide Web, Sebastopol, CA, O'Reilly, 2007 e Peter Morville - Jeffery Callender, Search patterns, Sebastopol, CA, O'Reilly Media, 2010.

${ }^{231}$ Carol Collier Kuhlthau, Seeking meaning cit.
} 
- monitorare (la ricerca in corso/ un determinato soggetto/ le novità);

* tenere a memoria (un particolare record, i record visualizzati nelle precedenti ricerche);

* accedere (ottenere l'accesso al documento);

* ottenere (ottenere copia fisica/digitale del documento);

* disseminare (riutilizzare l'informazione ottenuta esportando liste di record, citazioni bibliografiche, linkare al documento da fonti esterne il permalink di un record etc.);

Alcune delle attività citate sono importanti anche nelle prime fasi della ricerca: le funzioni di ottenere e accedere (in FRBR 'ottenere l'accesso') possono essere importanti fin da subito: l'utente dovrebbe poter selezionare i documenti immediatamente disponibili (quelli ammessi al prestito, quelli che sono disponibili per il prestito in un dato momento, $\mathrm{i}$ documenti in formato digitale scaricabili etc.) per filtrare la sua ricerca.

Nella progettazione degli OPAC di recente si è manifestata una certa attenzione alle attività che l'utente svolge in questa fase finale della ricerca; gli OPAC tradizionali fornivano all'utente un set di risultati senza offrire le opportunità di riutilizzo delle informazioni ottenute, che tradizionalmente erano limitate alla eventualità di richiedere il documento in prestito, o di prenotarlo; raramente l'utente aveva la possibilità di salvare il proprio lavoro o sfruttare i dati raccolti nell'OPAC per interagire con strumenti esterni. Nello sviluppo recente si è provveduto a dotare i nuovi prodotti di funzionalità tese a migliorare questa fase conclusiva della ricerca.

Funzionalità strategiche che gli OPAC dovrebbero offrire

Le tre principali funzionalità che gli OPAC dovrebbero offrire in questa fase sono le seguenti

* aiutare l'utente ad organizzare l'informazione ricevuta, di memorizzare i dati e i risultati relativi;

* attivare funzionalità di monitoraggio;

* attivare funzionalità di riutilizzo dell'informazione all'esterno dell'OPAC. 
Nella fase finale della ricerca prevale la necessità di organizzare l'informazione ricevuta, in modo da renderla fruibile una volta abbandonato il catalogo.

Gli OPAC di nuova generazione hanno introdotto varie caratteristiche che aiutano l'utente in questa fase della ricerca. In particolare sono state sviluppate alcune nuove funzionalità che si sono avvalse dello sviluppo della tecnologia del web.

La possibilità di riordino del set dei risultati in diversi modi è una funzionalità ormai presente in molti cataloghi elettronici, essa consente all'utente di riorganizzare i risultati in base alle proprie preferenze.

Tra le altre funzionalità che gli OPAC oggi introducono a vantaggio dell'utente in questa fase della ricerca vi sono

Possibilità di avere spazi e servizi personalizzati all'interno dei cataloghi

* Possibilità di creare bibliografie pubbliche o private, con la possibilità di "taggare", cioè aggiungere etichette ai documenti inseriti in tali liste

* Possibilità di creare profili utente; cioè di avere la propria pagina di inizio o disporre di tipi di ricerca predefiniti e display personalizzati ecc.

Alcune caratteristiche di personalizzazione consentono agli utenti di avere un'area personale, tramite autenticazione, consentendo di organizzare meglio le proprie ricerche nel catalogo. Gli OPAC di nuova generazione hanno introdotto la possibilità di creare liste personali private o pubbliche di documenti visualizzati nell'OPAC. Queste dovrebbero essere dotate di tag, cioè di etichette che l'utente può apporre alle risorse per organizzare in maniera personalizzata i propri documenti.

L'utilizzo del social tagging, che nel catalogo ha dimostrato la propria debolezza come mezzo di organizzazione e recupero dell'informazione, potrebbe in questo caso, essere di qualche aiuto per l'utente in questi spazi privati, quando le risorse da organizzare e recuperare sono quelle che l'utente ha selezionato.

Gli spazi personali possono essere di aiuto anche nel semplificare le operazioni di settaggio della ricerca; l'utente può impostare una sola volta alcuni parametri di default per personalizzare la propria schermata di ricerca: numero dei record da visualizzare, grandezza dei caratteri, lingua dell'interfaccia etc.

Le librerie online e i siti commerciali hanno suggerito altre funzionalità che riguardano l'interazione e il design: ad esempio il "carrello della spesa" è una memoria virtuale che segue l'utente durante il percorso di ricerca. Le liste di oggetti, i carrelli, le 
liste di acquisto, o liste dei desideri nei siti di commercio elettronico fungono da organizzatori di memoria o da scomparti temporanei di memoria (Amazon ad esempio, permette di tenere in memoria oggetti per un utilizzo futuro). Tali funzionalità vengono sempre più spesso introdotte negli OPAC, rispondendo anche a quelle situazioni di information encountering, ove l'utente imbattendosi in un'informazione non richiesta, ma che giudica utile utilizzi futuri, può tenere a memoria l'oggetto, per visionarlo successivamente.

b. Attivare funzionalità di monitoraggio

Le attività di monitoraggio che l'utente svolge nella sua interazione con l'OPAC sono principalmente di due tipi

\footnotetext{
* monitoraggio del percorso della propria ricerca;

* monitoraggio di un'area di interesse (ad esempio le novità o un'area di soggetto);
}

Nel primo caso l'OPAC deve predisporre una serie di funzionalità che consentono all'utente di tenere a memoria il percorso della propria ricerca. Dunque l'interfaccia dovrebbe fornire un aiuto visuale che consenta all'utente di ripercorrere il percorso fatto, tornare indietro, individuare le ricerche svolte in una sessione di ricerca, salvare una sessione. Alcuni OPAC hanno introdotto la cosiddetta history della ricerca svolta, visualizzando le ricerche compiute durante la sessione. Utile anche una funzione che tenga traccia dei filtri usati durante la ricerca, rintracciabile in alcuni OPAC che hanno introdotto la faceted navigation come modalità di visualizzazione/ filtraggio della ricerca.

Nel secondo caso l'OPAC dovrebbe disporre di funzionalità che consentano all'utente di rimanere informato su un'area di interesse (es. un'area di soggetto), oppure sulle nuove aggiunte al catalogo.

In quest'ambito gli OPAC di nuova generazione hanno introdotto alcune interessanti novità. Vengono introdotti servizi di notifica cioè la possibilità di impostare avvisi (alerts) nel catalogo per ricevere nella forma preferita (email, RSS) notizie circa la disponibilità di documenti riguardanti una particolare area di interesse, di abbonarsi a bollettini delle novità o a liste tematiche proposte dai bibliotecari. Tali alerts ripetono a intervalli nell'OPAC le ricerche impostate dall'utente e sfruttano forme di syndication che consentono di far viaggiare nel web i dati dei cataloghi tramite i feed RSS, dando la possibilità agli utenti di ricevere le informazioni senza visitare l'OPAC. 
c. Attivare funzionalità di riutilizzo dell'informazione bibliografica

Gli OPAC di nuova generazione hanno puntato molto sulle caratteristiche di riutilizzo dell'informazione ricevuta all'esterno dell'OPAC.

Oggi si sperimentano varie soluzioni per aprire i cataloghi e renderli maggiormente visibili nella rete. Tra le principali funzionalità introdotte vi sono quelle che consentono di disseminare l'informazione bibliografica e riutilizzarla una volta usciti dall'OPAC.

L'OPAC Worldcat permette, ad esempio, agli utenti di creare bibliografie pubbliche e private, di esportare i dati del catalogo in altri software (EndNote, Refworks) e nei più noti social network, di linkare ad una scheda di catalogo da qualsiasi punto della rete tramite il permalink. OCLC, per un certo periodo e in via sperimentale, ha reso il contenuto di WorldCat interrogabile tramite i motori di ricerca ${ }^{232}$.

Alcune funzionalità di riutilizzo si collegano alle funzionalità che permettono un'interazione dell'utente con il catalogo o con altri utenti del catalogo es. le caratteristiche sociali: la possibilità di esprimere un giudizio sulle risorse presenti nel catalogo (con l'aggiunta di tag, recensioni, commenti) è collegata alla conseguente possibilità di recuperare anche le discussioni intorno all'oggetto di interesse; la possibilità di creare all'interno dell'OPAC communities o gruppi di lettura favorisce una rete di collegamenti ad oggetti del catalogo anche da applicazioni esterne.

Altre funzionalità consentono di utilizzare il catalogo anche da siti web esterni; un esempio sono i Widget di WorldCat per Facebook e per altre applicazioni 'sociali'; con essere si cerca di indirizzare gli utenti dalla rete verso il catalogo. Vari cataloghi sono già accessibili da Facebook nelle pagine delle biblioteche che si rendono visibili tramite il noto social network. Alcune biblioteche progettano widgets e componenti aggiuntivi per $\mathrm{i}$ browser che permettono, da qualsiasi punto del web, di convogliare gli utenti verso il catalogo. Sono disponibili inoltre varie soluzioni per adattare gli OPAC alla consultazione su telefoni cellulari e altri dispositivi mobili ${ }^{233}$.

Fase post-focus . Linee di sviluppo

Gli OPAC di nuova generazione hanno introdotto numerose funzionalità che aiutano l'utente in questa fase della ricerca. Permangono ancora alcune aree aperte di riflessione

\footnotetext{
${ }^{232}$ In questa direzione si muove anche il progetto eXtensible Catalog (XC). $<$ http://www.extensiblecatalog.org/>.

${ }^{233}$ Tra le interfacce per dispositivi mobili: WorldCat <http://www.worldcat.org/mobile/default.jsp > e l'OPAC Airpac dell'Università di Sydney <http://opac.library.usyd.edu.au/airpac/> .
} 
intorno ad alcune funzionalità ancora poco sviluppate come quelle che consentono di organizzare meglio e monitorare il proprio percorso di ricerca.

\begin{tabular}{|c|c|}
\hline EFOCUS & \\
\hline Attività dell'utente & Funzionalità dell'OPAC \\
\hline $\begin{array}{l}\text { Identificare lo strumento più appropriato al quale } \\
\text { rivolgersi o una particolare entità / le entità correlate } \\
\text { per dare avvio alla ricerca, oppure una particolare } \\
\text { strategia di ricerca }\end{array}$ & $\begin{array}{l}\text { Offrire all'utente funzioni di } \\
\text { orientamento integrare gli strumenti } \\
\text { informativi (es. ricerca nell'OPAC, } \\
\text { espansione ai contenuti esterni) } \\
\text { Offrire canali già pronti di accesso alle } \\
\text { risorse offerte dalla biblioteca }\end{array}$ \\
\hline $\begin{array}{l}\text { Apprendere le caratteristiche del un sistema } \\
\text { /database, la struttura dei dati contenuti nell'OPAC / } \\
\text { la conoscenza relativa ad un'area di soggetto che si } \\
\text { vuole indagare } \\
\text { Correlare le conoscenze nuove a quelle già } \\
\text { acquisite }\end{array}$ & $\begin{array}{l}\text { Offrire l'informazione generica } \\
\text { (background information) e di } \\
\text { riferimento su un particolare soggetto } \\
\text { Facilitare la ricerca esplorativa tramite il } \\
\text { browsing } \\
\text { Offrire suggerimenti di lettura in forma } \\
\text { visuale (es. vetrine delle novità) e canali } \\
\text { di accesso al catalogo } \\
\text { Offrire un collegamento tra diversi } \\
\text { schemi di organizzazione delle } \\
\text { conoscenze }\end{array}$ \\
\hline $\begin{array}{l}\text { Esplorare/navigare: attivare un comportamento } \\
\text { esplorativo consenta di trovare un'entità, di } \\
\text { visualizzare le entità collegate, ad esempio in una } \\
\text { certa area di soggetto. } \\
\text { Interagire con gli item che mappano i confini del } \\
\text { compito (meta-informazione) }\end{array}$ & $\begin{array}{l}\text { Facilitare la ricerca esplorativa tramite il } \\
\text { browsing } \\
\text { Raggruppare i documenti che hanno } \\
\text { caratteristiche comuni } \\
\text { Offrire una visualizzazione dei dati } \\
\text { bibliografici basata sulle entità primarie } \\
\text { di interesse dell'utente nella ricerca nel } \\
\text { catalogo }\end{array}$ \\
\hline
\end{tabular}




\begin{tabular}{|c|c|}
\hline Creare una query per dare avvio alla ricerca. & $\begin{array}{l}\text { Aiutare l'utente a formulare una query } \\
\text { individuando termini sinonimi/correlati }\end{array}$ \\
\hline Valutare l'informazione rilevante & $\begin{array}{l}\text { Offrire arricchimenti del record e } \\
\text { contenuti esterni (es. Worldcat Identities) }\end{array}$ \\
\hline \multicolumn{2}{|l|}{ FASE 2. FORMULAZIONE DEL FOCUS } \\
\hline Attività dell'utente & Funzionalità dell'OPAC \\
\hline Creare una query sull'argomento messo a fuoco & $\begin{array}{l}\text { Aiutare l'utente a formulare una query } \\
\text { individuando termini sinonimi/correlati }\end{array}$ \\
\hline $\begin{array}{l}\text { Modificare la query sull'argomento messo a fuoco } \\
\text { per ottenere una ricerca più precisa }\end{array}$ & $\begin{array}{l}\text { Fornire funzionalità di modifica } \\
\text { dinamica della query in base ai termini } \\
\text { correlati e a strategie di relevance } \\
\text { feedback }\end{array}$ \\
\hline $\begin{array}{l}\text { Valutare l'informazione pertinente rispetto a ciò che } \\
\text { già si conosce su un dato argomento o allo stato } \\
\text { situazionale in cui ci si trova (obiettivo a lungo } \\
\text { termine, poco tempo a disposizione) }\end{array}$ & $\begin{array}{l}\text { Offrire arricchimenti del record e } \\
\text { contenuti esterni (es. Worldcat Identities) }\end{array}$ \\
\hline \multicolumn{2}{|l|}{ FASE 3. POST-FOCUS } \\
\hline Attività dell'utente & Funzionalità dell'OPAC \\
\hline $\begin{array}{l}\text { Organizzare i risultati ottenuti per una navigazione } \\
\text { più efficace }\end{array}$ & $\begin{array}{l}\text { Offrire modalità di visualizzazione che } \\
\text { consentano di organizzare } \\
\text { l'informazione selezionata dall'utente } \\
\text { come pertinente alla propria ricerca }\end{array}$ \\
\hline $\begin{array}{l}\text { Monitorare la ricerca in corso/ un determinato } \\
\text { soggetto/ le novità /tenersi aggiornato su un } \\
\text { argomento seguendo regolarmente le risorse } \\
\text { attinenti. }\end{array}$ & $\begin{array}{l}\text { Fornire elementi che consentano di } \\
\text { visualizzare: la ricerca corrente e le } \\
\text { ricerche effettuate in precedenza } \\
\text { Offrire il monitoraggio delle novità e di } \\
\text { aree di soggetto. } \\
\text { Offrire modalità di syndication dei dati } \\
\text { relativi ad una particolare ricerca } \\
\text { Offrire la possibilità di impostare alert su } \\
\text { una determinata ricerca } \\
\text { Offrire la possibilità di creare community }\end{array}$ \\
\hline
\end{tabular}




\begin{tabular}{|c|c|}
\hline & $\begin{array}{l}\text { e gruppi di interesse su particolari } \\
\text { argomenti /aree di soggetto }\end{array}$ \\
\hline $\begin{array}{l}\text { Tenere a memoria (un particolare record, i record } \\
\text { visualizzati nelle precedenti ricerche) }\end{array}$ & $\begin{array}{l}\text { Offrire contenitori virtuali di memoria. } \\
\text { Ricordare la cronologia delle ricerche } \\
\text { compiute, sia durante la sessione di } \\
\text { ricerca, sia successivamente con } \\
\text { l'attivazione di spazi personali per } \\
\text { l'utente. } \\
\text { Consentire all'utente di ripetere } \\
\text { automaticamente una ricerca condotta in } \\
\text { precedenza. }\end{array}$ \\
\hline Accedere (ottenere l'accesso al documento) & $\begin{array}{l}\text { Offrire quando possibile l'accesso al } \\
\text { fulltext del documento } \\
\text { Progettare modalità di ricerca orientate al } \\
\text { contenuto (es. modalità di ricerca che } \\
\text { consentano di cercare solo tra i } \\
\text { documenti digitali) }\end{array}$ \\
\hline $\begin{array}{l}\text { Ottenere (ottenere copia fisica/digitale del } \\
\text { documento) }\end{array}$ & $\begin{array}{l}\text { Offrire il fulltext oppure modalità } \\
\text { intuitive di collegamento a ai servizi di } \\
\text { prestito /document delivery o a fonti } \\
\text { esterne per l'acquisto } \\
\text { Offrire modalità di ricerca che } \\
\text { consentano di cercare solo tra i } \\
\text { documenti immediatamente disponibili }\end{array}$ \\
\hline $\begin{array}{l}\text { Disseminare (riutilizzare l'informazione ottenuta } \\
\text { esportando liste di record, citazioni bibliografiche, } \\
\text { linkare al documento da fonti esterne il permalink di } \\
\text { un record etc.) }\end{array}$ & $\begin{array}{l}\text { Offrire modalità di riutilizzo dei dati } \\
\text { bibliografici all'esterno del catalogo: } \\
\text { esportazioni di liste di documenti e } \\
\text { bibliografie } \\
\text { collegamento a gestori di bibliografie, } \\
\text { salvataggio, stampa, condivisione del } \\
\text { documento o di una lista di documenti. }\end{array}$ \\
\hline
\end{tabular}

Fig. 5.24 - Le fasi del processo informativo, le attività dell'utente e le funzionalità dell'OPAC che dovrebbero essere offerte all'utente per supportare il processo informativo 


\subsection{Il ruolo dei linked data nel processo di ricerca dell'informazione}

Nel modello di sviluppo delineato abbiamo immaginato che l'utente sia coinvolto in un processo informativo che si svolge iterativamente attraverso tre fasi distinte (pre-focus, formulazione del focus, post-focus) nelle quali l'utente è attivamente coinvolto in una situazione di problem solving che lo induce a svolgere varie attività che, come abbiamo visto, sono rivolte e vengono condizionate da tutte le componenti che entrano in gioco nel processo di ricerca: l'utente stesso, il sistema, i documenti e gli intermediari.

In base a questa analisi il modello individua così le funzionalità che gli OPAC dovrebbero attivare per sostenere tutte le fasi del processo e garantire che si compia una ricerca efficace e senza intoppi.

Abbiamo visto anche che l'attivazione del processo di ricerca non è scontata in quanto intervengono una serie di barriere che derivano dallo stato in cui l'utente si trova e nel quale sorge e si sviluppa il suo bisogno informativo. Tali barriere condizionano non solo i meccanismi di attivazione, ma sono presenti durante tutto il processo, poiché l'utente è iterativamente coinvolto nella comprensione e nel perfezionamento della sua ricerca; egli dunque modifica continuamente il suo bisogno, in quanto man mano che procede, costruisce e comprende l'oggetto della ricerca, modificando il suo stato cognitivo, affettivo e situazionale di partenza.

Questa visione costruttivista e cognitiva sulla quale si basa il modello elaborato nel presente lavoro ci consente di intendere il processo di ricerca come un processo di 'creazione di senso' da parte dell'utente, ovvero di comprensione dell'informazione nel suo contesto.

Ciò avvicina la visione che sottende il modello di OPAC da me proposto a quella recentemente prefigurata da Fattahi laddove lo studioso afferma:

il catalogo di biblioteca non è un sistema con il quale l'utente deve acquisire conoscenza a forza di sperimentare, per tentativi ed errori: dovrebbe essere un sistema cognitivo integrato che si autodefinisce e in grado di armonizzare l'ambiente e il comportamento di ricerca informativa dell'utente, al di là e oltre la ricerca d'informazione. Dovrebbe mostrare come è rappresentata, strutturata e visualizzata la conoscenza nel catalogo

e auspica il passaggio dei cataloghi «da sistemi orientati al documento ai sistemi orientati alla conoscenza» ${ }^{234}$.

\footnotetext{
${ }^{234}$ Fattahi affida al super-record quella funzione organizzativa necessaria alla costruzione di una tale visione di OPAC. Rahmatollah FATTAHI, From information to knowledge: superworks and the challenges in the organization and representation of the bibliographic universe = Dall'informazione alla conoscenza: le
} 
I linked data, come abbiamo avuto modo di illustrare nel precedente capitolo, rappresentano una recente tecnologia in grado di fornire alle biblioteche un nuovo modo di strutturare i dati bibliografici rappresentati negli OPAC e negli altri strumenti di ricerca e servizi che vengono da esse predisposti.

Nelle pagine conclusive di questo capitolo si esplorerà la possibilità che i linked data possano essere la tecnologia più appropriata per la costruzione di nuovi OPAC basati sulla creazione di conoscenza all'interno del processo informativo.

In particolare si esaminerà il ruolo che l'esposizione dei dati bibliografici sotto forma di linked data potrà avere negli sviluppo degli OPAC in relazione al modello da me proposto. Ritengo, infatti, che la tecnologia dei linked data possa avere un ruolo significativo:

* nel ridurre le barriere cognitive che si presentano nell'interazione dell'utente con l'OPAC;

* nel creare un contesto per la ricerca, che consente all'OPAC di trasformarsi da strumento di recupero dell'informazione a strumento di creazione di conoscenza;

* nel fornire al record bibliografico la struttura capace di sostenere ulteriori funzioni dell'utente;

e, quindi, nella progettazione di OPAC che possano consentire funzionalità migliorate a supporto della ricerca.

Nei paragrafi che seguono verrà valutato l'impatto della tecnologia dei linked data nel modello da me presentato in relazione alle attività che l'utente svolge nel processo informativo, al fine della progettazione di nuove funzionalità che consentano di migliorare il processo di ricerca.

super-opere e le sfide dell'organizzazione e rappresentazione dell'universo bibliografico : Lectio magistralis in Biblioteconomia, Firenze, Università degli Studi di Firenze, 2010, Firenze, Casalini Libri, 2010, p. 42. 


\subsubsection{Il nuovo 'record bibliografico collegato' nella gestione del processo informativo}

Il principale strumento per rappresentare l'informazione bibliografica nel catalogo, il record bibliografico, è un insieme di metadati che rappresentano le entità e gli attributi che caratterizzano le dimensioni semiotiche e semantiche di un 'oggetto bibliografico' (una monografia, un documento sonoro, etc.) e le relazioni con le altre entità collegate.

Come abbiamo avuto modo di sottolineare nel capitolo precedente, per molto tempo la produzione di record bibliografici si è basata su una struttura piatta e lineare del record, incapace di rappresentare compiutamente sia le diverse entità (opera, espressione, manifestazione, item, soggetti ${ }^{235}$, autori, enti, famiglie), sia i legami con le altre entità.

Più volte si è lamentata la mancanza di struttura del record bibliografico come principale ostacolo all'espressione adeguata delle entità rappresentate nel catalogo. Un record bibliografico che manca di struttura adeguata e di granularità, non consente infatti di assolvere alle basilari funzioni del catalogo (funzioni -utente) previste in FRBR.

I linked data ci offrono oggi la possibilità di operare una profonda ristrutturazione del record bibliografico che si presenta con una struttura granulare e atomizzata; tale nuovo concetto di record bibliografico si esprime nel 'record collegato', costituito virtualmente dalla sintesi di varie triple che combinano dati creati dalle biblioteche e da altri enti.

Attraverso tale struttura 'collegata' il nuovo record bibliografico sarà capace di consentire all'utente di compiere non solo tutte le funzioni di base di un catalogo, ma anche un buon numero di altre funzioni individuate nel modello da me proposto.

Con la tecnologia dei linked data è dunque è possibile generare automaticamente una struttura del record formata da tutte le entità previste dai modelli FRBR, ma è possibile altresì dotare il record bibliografico di una rete di collegamenti che consentiranno all'utente di svolgere le nuove funzioni delineate nel processo informativo.

Possiamo dunque immaginare il nuovo 'record collegato' come prosecuzione dell'idea di 'super-record', più volte auspicata da vari studiosi, tra i quali, Fattahi (1996) e del modello FRBR (1998) e di meta-record recentemente riproposto da Fattahi in grado di creare una struttura che possa essere agevolmente navigata in modo da fornire all'utente «un ambiente di apprendimento per mezzo del quale il record catalografico facilita

\footnotetext{
${ }^{235} \mathrm{Si}$ fa riferimento all'entità thema che in FRSAD ha inglobato le entità soggetto, evento e luogo precedentemente stabilite da FRBR che invece sono definite in FRSAD come attributi del thema.
} 
l'organizzazione e la rappresentazione della conoscenza nel catalogo, come pure la capacità di acquisire, elaborare e interiorizzare la conoscenza da parte di chi utilizza il catalogo» $^{236}$.

Il nuovo 'record collegato' che si viene a creare quando i dati del catalogo vengono esposti come linked data è formato da un collegamento di triple in grado di connettere dati provenienti da diverse fonti sia bibliografiche sia di altra natura creando un reticolo illimitato di collegamenti tra i dati, il Web of data.

L'elemento che consente di realizzare questo collegamento è il concetto di URI ovvero un identificatore unico e persistente associato al dato.

Nel nuovo 'record collegato' ogni parte della tripla viene identificata attraverso un URI, avremo dunque URI che identificano le opere, le espressioni, gli autori, i soggetti e le classi, e i predicati, ovvero le relazioni che intercorrono tra le entità che provviste così di URI possano essere riutilizzate. Si crea così un reticolo di dati in cui tutte le entità e le relazioni previste dai modelli FRBR possono essere espresse. Ciò è reso possibile dallo sviluppo di set di elementi, di vocabolari e ontologie che provengono dal settore bibliotecario (es. Dublin Core, RDA) o da altre discipline (Foaf, Skos)

Questo collegamento dei dati, sebbene possa a prima vista, far immaginare un'immensa mole di informazione difficile da gestire, in realtà viene creato automaticamente tramite il collegamento di diversi dataset e con uno sforzo che non ricade unicamente sulle biblioteche, ma che viene condiviso tra le biblioteche e le altre comunità del Web Semantico.

I linked data, oltre a collegare dati di varia natura offrono una tecnologia in grado di migliorare il controllo bibliografico, di implementare il modello FRBR nei cataloghi e di migliorare complessivamente il processo di ricerca.

\subsubsection{La riduzione delle barriere cognitive nella ricerca dell'informazione}

Il modello di sviluppo da me presentato in questa ricerca analizza la ricerca dell'informazione bibliografica nel contesto, ovvero prende in considerazione non

${ }^{236}$ Rahmatollah Fattahi, From information to knowledge cit., p. 37 
solamente il sistema, ma tutte le componenti che interagiscono nel processo di ricerca all'interno di un OPAC.

Abbiamo anche visto che il modello prende in considerazione il processo di ricerca che è dinamico e non lineare, in cui è centrale il concetto di 'gap', quale situazione problematica o carenza cognitiva che l'utente vuole colmare attivando e proseguendo nelle fasi del processo informativo e quello delle 'barriere' che si frappongono sia al momento dell'attivazione sia lungo il processo.

Tra le barriere maggiormente significative vi sono quelle cognitive, che possono consistere

* in una scarsa comprensione dell'oggetto della ricerca, il focus, presente qualora l'utente si trovi in una fase iniziale della sua ricerca;

* in una difficoltà di espressione dell'oggetto di ricerca, derivante dalla scarsa conoscenza dell' area disciplinare che si sta indagando;

* nella scarsa conoscenza di un sistema e dei suoi meccanismi di recupero dell'informazione (barriere tecnologiche);

* nella scarsa conoscenza dell'universo bibliografico rappresentato e delle modalità di rappresentazioni dei testi;

* nelle barriere linguistiche.

L'applicazione del relevance ranking ai cataloghi elettronici, di cui sono state esaminate le evidenti criticità all'inizio di questo capitolo, è la conseguenza dell'attuale difetto nella struttura dei record bibliografici espressi nel formato MARC.

I linked data consentono di adottare una struttura del catalogo che consenta una riduzione dell'incidenza di questa tipologia di barriere.

In particolare consentono di creare un processo di apprendimento che è fondamentale per il successo di una ricerca nel catalogo e che oggi viene ostacolato dagli sviluppi recenti degli OPAC di nuova generazione e degli strumenti di discovery.

L'impatto dei linked data nella struttura dei record bibliografici può migliorare la ricerca nell'OPAC aumentando l'efficacia delle seguenti funzionalità e consentendo all'utente di superare le barriere cognitive in quanto consente di svolgere adeguatamente le seguenti funzioni individuate nel modello:

* Navigazione e browsing nel catalogo

* Interrogazione tramite formulazione di una query

Valutazione dell'informazione ricevuta 
Accesso alle risorse bibliografiche

Potenziamento della ricerca semantica

\subsubsection{Il recupero dell'informazione 'nel contesto'}

Il principale ruolo dei linked data nell'innovazione dei cataloghi elettronici risiede nella possibilità che il Web dei dati venga utilizzato come 'contesto esteso' per l'esplorazione dei contenuti del catalogo che si affianca a quello rappresentato in un catalogo ossia quello delle entità e dei loro collegamenti, per espandere e migliorare il processo di ricerca dell'informazione.

Il concetto di 'contesto' è profondamente connaturato nella definizione di conoscenza, in quanto la conoscenza stessa viene creata e appunto collocata in un contesto. Una progettazione efficace del catalogo, dovrebbe creare dunque un contesto nel quale l'utente può collocare ogni singola informazione per mezzo della relazione tra le entità costitutive. In tal modo il catalogo si muove dall'essere un sistema di recupero dell'informazione verso uno strumento teso a facilitare la creazione di nuove conoscenze. La visione costruttivista dell'apprendimento, che viene assunta ad orizzonte teorico della presente trattazione e una visione più chiara del processo informativo che si svolge nel catalogo, che è stato approfondito in queste pagine, ci consentono di immaginare che la conoscenza venga costruita attraverso un procedimento di 'costruzione di senso' che l'utente svolge nelle sue esplorazioni del catalogo.

I linked data consentono di appoggiare il catalogo ad una infrastruttura cognitiva costituita dalle conoscenze presenti nel web dei dati, che collega le conoscenze formatesi in contesti diversi; queste collegate alle entità bibliografiche presenti nel catalogo diventano ulteriori elementi di un contesto esteso, che si va così ad aggiungere all'universo bibliografico rappresentato nel catalogo.

\subsubsection{L'impatto dei linked data nel processo di ricerca}

E' da considerare, infine, l'impatto che la tecnologia dei linked data può avere sullo svolgimento del processo informativo e sulle funzioni che l'utente compie nella sua interazione con l'OPAC: 
a. Navigazione e browsing nel catalogo

Come abbiamo avuto modo di osservare, nel modello da me proposto si presuppone che l'utente nelle prime fasi della ricerca (quelle che abbiamo denominato come pre-focus) sia maggiormente predisposto alla ricerca 'esplorativa', in quanto, non avendo ancora chiaro l'oggetto della ricerca, preferisce (come accade nella realtà fisica, ad esempio, di una biblioteca) modalità attive ma 'indirette' di esplorazione. Alcuni OPAC oggi propongono canali, percorsi, o suggeriscono le novità. La tecnologia dei linked data offre varie opportunità, prima fra tutte quella di migliorare i percorsi di navigazione nel catalogo.

Attraverso i linked data è infatti possibile applicare il modello FRBR in modo tale da rappresentare tutte le entità previste e consentire una navigazione gerarchica che dal raggruppamento di opere simili mostri le singole opere, le espressioni e tutte le manifestazioni e gli item ad esse collegati. In tal modo si compiono le funzioni bibliografiche che l'utente svolge nel catalogo e si realizza una navigazione 'significativa' per l'utente ove si svolge, al contempo, un processo di apprendimento.

La navigazione non avviene solo nel contesto del catalogo, ma attraverso il collegamento ad altri dataset può estendersi al web, inglobando dinamicamente informazioni provenienti da fonti esterne. Questi collegamenti possono attivare nuovi percorsi di esplorazione, che partendo da dati bibliografici, raggiungono informazioni di altra natura.

b. Interrogazione tramite formulazione di una query

Nelle fasi successive del processo di ricerca, in cui l'utente è in grado di formulare interrogazioni al sistema, abbiamo visto quanto sia importante offrire un aiuto nel formulare l'interrogazione. Sebbene gli OPAC oggi ricorrano all'autocompletamento della query, sovente questa tecnica non si appoggia a vocabolari controllati, rivelandosi inefficace. I più recenti sviluppi del Web Semantico, che, come abbiamo visto, cominciano ad avere alcune prime applicazioni nello sviluppo dei cataloghi elettronici ${ }^{237}$, potrebbero avere in questo campo alcune ricadute particolarmente significative.

${ }^{237}$ Un esempio è l'OPAC Libris. Cfr. infra, Casi di studio. OPAC Libris. 
I linked data consentono di poter usufruire di ontologie già create in maniera dinamica e automatica all'interno del Web Semantico e dunque utilizzabili a vantaggio della ricerca nei cataloghi. Come alcuni studiosi hanno già sottolineato qualche tempo fa ${ }^{238}$ ciò consente di ottenere una struttura sindetica estesa che serve per aiutare l'utente a formulare interrogazioni basata su ontologie lessicali. Oggi i linked data consentono di sfruttare i legami che si creano automaticamente tra i diversi dataset, offrendo all'OPAC una struttura sindetica potenzialmente illimitata.

Ad esempio, gli accessi semantici di un catalogo (es. le stringhe LCSH utilizzate o le notazioni delle classi) vengono automaticamente mappati ai concetti correlati e dunque sono recuperabili non solo a partire dai termini che compongono il soggetto, ma anche a partire da termini semanticamente simili, perché collegati allo stesso concetto. La rete dei dati presente nel Web dunque potrebbe utilmente essere sfruttata dalle biblioteche per sviluppare interfacce che aiutano l'utente a scegliere il soggetto più appropriato per la propria ricerca.

Come abbiamo avuto modo di accennare, la tecnologia dei linked data consente anche di abbattere le barriere linguistiche all'utilizzo di un catalogo, in quanto tutte le forme varianti di un nome o di un titolo o di un soggetto possono essere collegate alla forma accettata secondo il meccanismo già noto dell'authority file con un vantaggio in più per la ricerca semantica: la possibilità di esprimere un concetto in una lingua diversa da quella per cui il catalogo è progettato: i termini dell'interrogazione sebbene in una lingua diversa da quella espressa in un catalogo possono venire ugualmente collegati automaticamente tramite i linked data con un indubbio vantaggio per chi ricerca.

Un enorme vantaggio è quello di poter interrogare il catalogo servendosi del linguaggio naturale per essere indirizzati ai termini più adatti per la ricerca nel catalogo o lanciando una ricerca esplorativa a partire dai termini suggeriti dal sistema.

\section{c. Valutazione dell'informazione ricevuta}

La valutazione dei risultati di un'interrogazione o dei record recuperati tramite un'attività esplorativa consiste, per l'utente che si trova nelle prime fasi del processo delineate nel modello, essenzialmente nel valutare la rispondenza all'argomento ricercato (rilevanza topica), mentre per chi si trova già nelle fasi successive anche alla corrispondenza soggettiva, ovvero un ultima analisi alla pertinenza e all' 'utilità' .

\footnotetext{
${ }^{238}$ Papadakis e i sui colleghi proponevano il ricorso ad ontologie lessicali da creare ad hoc.
} 
L'opportunità offerta dalla tecnologia dei linked data tramite il record bibliografico ‘collegato' risiede nella possibilità di ampliare il contesto utile all'utente per effettuare la valutazione della rilevanza e della pertinenza, ampliandolo a quello delle migliaia di risorse presenti nel Web Semantico.

La quantità di dati che dinamicamente possono essere recuperati attraverso un record collegato al Web of data è non solo potenzialmente illimitata, ma consente di arricchire il risultato di informazioni supplementari sempre aggiornate, che consentano che la valutazione sia arricchita di elementi esterni: ad esempio la possibilità di visualizzare un raggruppamento di tutti gli argomenti trattati da un autore nelle sue opere, di visualizzare la fortuna editoriale di un autore in un certo periodo di tempo o l'autorevolezza, sono funzionalità tutto sommato non particolarmente complesse, ma di grande aiuto all'utente, che possono essere implementate per mezzo del collegamento del record a dati presenti nel web $^{239}$.

d. Accesso alle risorse bibliografiche

La nuova modalità di esporre i dati delle biblioteche sottoforma di linked data costituisce un modo per migliorare l'accesso alle risorse bibliografiche del catalogo. Ciò non si risolve solo nelle nuove modalità di interrogazione che sono rese possibili attraverso l'uso di questa tecnologia, ma anche e soprattutto nella possibilità di accedere al catalogo da fonti esterne; una volta che i dati bibliografici entrano a far parte del Web of data essi saranno oggetto del recupero dell'informazione da qualsiasi altro dato collegato al Web Semantico.

e. Visualizzazioni avanzate

Una delle novità introdotte dagli OPAC di nuova generazione è certamente la componente visuale del recupero dell'informazione, manifestatasi nella comparsa non solo delle copertine dei volumi quale arricchimento del record, ma soprattutto nella predisposizione di nuove modalità di browsing delle collezioni e costruzione di canali di esplorazione delle risorse. Alcuni di questi espedienti replicano le azioni reali della ricerca e dello scorrimento di uno scaffale in una biblioteca fisica (ad esempio le visualizzazioni degli scaffali in base ad uno schema di classificazione); altri creano raggruppamenti che si

\footnotetext{
${ }^{239}$ Funzionalità di questo tipo sono state recentemente introdotte per via algoritmica nel Web. Amazon ha lanciato una nuova tecnica per il ranking degli autori che ha provocato non poche critiche.
} 
reputano utili a valutare la pertinenza dei documenti (ad esempio le 'vetrine delle novità) richiamandosi al modello della biblioteca-libreria; altri ancora sono diretti a creare mappe semantiche visuali di associazioni e termini simili e a presentare in una rappresentazione visuale (come nel caso del noto software per OPAC di nuova generazione Aquabrowser ${ }^{240}$ ). Non c'è dubbio che oggi molte applicazioni del web si distinguano anche per la loro veste accattivante che si esprime, sovente, nella creazione di mappe visive ${ }^{241}$. Il record bibliografico 'collegato' che la logica dei linked data consente di costruire getta le basi per la costruzione di «ambienti arricchiti di conoscenza» ${ }^{242}$ nei quali sarà possibile per l'utente attivare nuovi percorsi di esplorazione del catalogo che saranno possibili a partire dalle entità oggetto di interesse.

Non dobbiamo infatti dimenticare che le nuove possibilità di implementazione del modello FRBR ai cataloghi renderanno possibile in futuro organizzare i risultati delle ricerche tramite raggruppamenti logici che dal livello delle super-opere (ovvero dei raggruppamenti di famiglie di opere che hanno caratteristiche in comune) potrà svolgersi fino al livello dell'item.

Il record collegato compie un ulteriore passo in avanti: attraverso il collegamento dei dataset delle biblioteche con quelli presenti nel web dei dati può dare luogo alla realizzazione di modellizzazioni ontologiche dei risultati delle ricerche che gli utenti mettono in atto, capaci di aprire orizzonti inaspettati per la ricerca semantica.

\subsubsection{I linked data e i nuovi orizzonti della ricerca semantica}

Il data model fornito da RDF che è alla base dell'architettura dei linked data consente di strutturare l'informazione bibliografica in modalità nuove che possono potenziare le funzionalità della ricerca semantica. Ciò è dovuto al fatto che nel Web Semantico tutti gli URI che si riferiscono allo stesso 'concetto' vengono correlati creando una vasta rete semantica di relazioni che viene automaticamente collegata al catalogo.

Come abbiamo avuto modo di affermare nel capitolo 1. i cataloghi soffrono della contingenza e della difficoltà per l'utente dovuta all'utilizzo dei dispositivi indicali. Gli

\footnotetext{
${ }^{240}$ Cfr. infra, Appendice B. Software per OPAC di nuova generazione e servizi di Web scale discovery. Aquabrowser.

${ }^{241}$ Esempi del Web di interfacce visive sono Literature map <http://www.literature-map.com/> e Gnod (<http://www.gnod.net $>$ ) per la musica, i libri e i film.

${ }^{242}$ Rahmatollah Fattahi, From information to knowledge cit., p. 36.
} 
indici rappresentano una necessità, ma pongono all'utente il problema di misurarsi con il 'linguaggio bibliografico, 243 .

In particolare, gli accessi semantici oggi sono sempre meno utilizzati dagli utenti, che preferiscono immettere parole chiave ed effettuare una ricerca su tutti i campi, che però spesso fornisce risultati imprecisi, ma, soprattutto, gli accessi semantici che le biblioteche utilizzano ai fini dell'organizzazione delle registrazioni catalografiche e del recupero riflettono le modalità di organizzazione delle conoscenze, ossia il modo in cui le biblioteche organizzano il sapere.

Inoltre - come ha giustamente evidenziato Biagetti - le biblioteche hanno seguito strategie di indicizzazione content oriented ovvero tese ad individuare con precisione nei termini indicali il contenuto di un documento, sacrificando così le potenzialità di utilizzazione dei libri stessi in quanto le molteplici dimensioni e interpretazioni che possono provenire da studiosi di formazione e provenienza scientifica diversa vengono ridotte alla creazione di un'unica stringa 'coestesa' ${ }^{244}$.

Oggi i linked data ci offrono l'opportunità di collegare le sistemazioni concettuali operate dalle biblioteche alle altre modalità di organizzare e rappresentare le conoscenze create da altre comunità che operano nel web:

An essential process is the joining together of subcultures when a wider common language is needed. Often two groups independently develop very similar concepts, and describing the relation between them brings great benefits... The Semantic Web, in naming every concept simply by a URI, lets anyone express new concepts that they invent with minimal effort. Its unifying logical language will enable these concepts to be progressively linked into a universal $\mathrm{Web}^{245}$.

L'affermazione di Tim Berners Lee esprime l'enorme potenzialità dell'applicazione del Web Semantico ai cataloghi: il fatto di essere una logica unificante che consente di

${ }^{243}$ Cfr. Alfredo Serrai, Del catalogo alfabetico per soggetti, cit.

${ }^{244} \mathrm{Si}$ veda Maria Teresa Biagetti, Indici semantici e ricerca scientifica, in L'organizzazione del sapere. Studi in onore di Alfredo Serrai, a cura di Maria Teresa Biagetti, Milano, Sylvestre Bonnard, 2004, p. 15-29. Biagetti analizza il ruolo degli indici semantici per la ricerca scientifica in un articolo che offre interessanti spunti di riflessione specie alla luce delle nuove tecnologie del Web Semantico. La studiosa rileva come rispetto agli aspetti sintattici del processo di indicizzazione sia stata tradizionalmente riservata una minore attenzione all'analisi dei contenuti semantici e alla scelta delle strategie di indicizzazione da adottare. Ciò è dovuto - afferma la studiosa - al prevalere di metodologie che si sono richiamate al procedimento della 'coestensione' ovvero alla necessità di condensare in una sola stringa i molteplici contenuti semantici di un libro. A tale strategia orientata al contenuto (content oriented) si contrappone, specie nell'indicizzazione di documenti che ricadono nelle Scienze umane, la possibilità di studiare strategie need-oriented, ovvero indirizzate alle necessità e alle possibili richieste di ricercatori formatisi in contesti scientifici diversi (ivi, $p$. 16): «I contenuti dei libri non solo possono offrire molteplici possibilità d'interpretazione, ma possono risultare significativi in modo diverso, sia in relazione a discipline differenti, sia rispetto ai vari scenari di ricerca nei quali essi possono essere di volta in volta utilizzati».

${ }^{245}$ Si tratta delle conclusioni di un noto articolo di Tim Berners-Lee - James Hendler - Ora Lassila, The Semantic Web, cit, citato anche in Corey A. Harper - Barbara B. Tillett, Library of Congress controlled vocabularies and their application to the Semantic Web, «Cataloging \& Classification Quarterly», 43 (2007), n. $3 / 4$, p. 1 . 
collegare i diversi linguaggi che nel web vengono utilizzati per denominare cose, eventi, luoghi, concetti, persone.

La possibilità di portare il catalogo 'dentro il web' e in particolare 'dentro' il Web Semantico riveste dunque una particolare importanza per la ricerca semantica e apre al contempo modalità inedite per la ricerca.

Le opportunità che i linked data offrono alla ricerca semantica sono ravvisabili in almeno due principali punti

* mappatura di concetti presenti in schemi e sistemi di organizzazione delle conoscenze diversi ai fini dell'interrogazione e della visualizzazione dei risultati

* sviluppo di modalità multidimensionali per la ricerca.

Il Web Semantico fornisce un'infrastruttura tecnologica che consente di collegare allo stesso concetto identificato da un URI i termini o le espressioni utilizzate da diverse comunità del web; ciò significa che è possibile attraverso il collegamento dei dataset di una biblioteca agli altri dataset presenti ottenere una struttura sindetica estesa già pronta che le biblioteche possono utilizzare per i propri scopi.

Attraverso il collegamento di dataset di diversa natura e la mappatura di concetti presenti in differenti schemi, la ricerca per soggetto può essere enormemente potenziata, sia nella fase di interrogazione, in quanto è ormai possibile utilizzare la rete di collegamenti del record per aiutare l'utente a formulare interrogazioni, sia in fase di navigazione in quanto è possibile produrre visualizzazioni avanzate che sfruttano il collegamento tra schemi diversi (es. soggetti e classificazioni), utilizzati sia nel settore bibliotecario che in altri settori scientifici, per espandere ulteriormente la ricerca.

Una delle più immediate possibilità offerte dalla tecnologia dei linked data è quella di collegare schemi diversi di organizzazione delle conoscenze (KOS) come soggetti e classi.

Questo tipo di collegamento, che non viene ancora implementato negli OPAC, è un espediente che favorisce la navigazione e l'apprendimento che si può realizzare durante il processo informativo. L'utente può infatti partire da un soggetto per visualizzare tutte le classi che utilizzano quel soggetto e navigare nell'albero classificatorio correlato, o al contrario visualizzare tutti i soggetti che sono legati alle opere che fanno parte di una determinata classe.

Un'altra possibilità è di collegare schemi completamente diversi per tipologia e profondità, ad esempio un soggettario e una folksonomia: una conseguenza 
dell'applicazione dei linked data agli OPAC è dunque il collegamento automatico dei record di autorità ovvero di quelle forme che si presume possano essere cercate dalla maggior parte degli utenti, a varie altre forme presenti sul web che possono diventare, se collegate ai dati catalografici, punti d'accesso aggiuntivi al catalogo.

Un'ulteriore possibilità è quella di formulare un'interrogazione secondo una logica 'sfumata': la possibilità di collegare concetti non identici ma simili è prevista nel Web Semantico, così sarà possibile recuperare un concetto partendo da concetti ad esso correlati.

In questo modo è possibile migliorare la ricerca semantica e la fruizione dei documenti stessi, che potranno essere più facilmente recuperati e utilizzati.

Queste nuove possibilità aprono prospettive inedite per la ricerca semantica sia in ambito generalista che in ambito scientifico e rivelano un'area di sovrapposizione di competenze tra Biblioteconomia e Scienza dell'informazione: se le biblioteche in base alla conoscenza della propria utenza di riferimento hanno per lungo tempo adottato strategie di indicizzazione semantica rivolte alla 'sommarizzazione' e cioè ad estrarre i contenuti del documento per ricavarne una 'circalità' (aboutness) adatta ai lettori di riferimento, oggi i cataloghi elettronici, rivolti ad un'utenza che non è prevedibile a priori, registrano un intervento sempre meno intensivo del catalogatore al quale è richiesta una mediazione minima, volta ad evidenziare esclusivamente i contenuti che l'autore presuppone che l'utente conosca ${ }^{246}$. Si tratta, dunque di quella tipologia di indicizzazione che Biagetti definisce anche entity oriented indexing che nei casi più spinti può essere totalmente automatizzata attraverso l'analisi linguistica di un documento fulltext, ricavandone i termini rilevanti da tradurre nel linguaggio d'indicizzazione. Questa soluzione, ovviamente, contrasta con la visione 'pragmatista' espressa da Hjørland nella teoria della domain analysis (analisi di dominio) secondo la quale le proprietà che distinguono semanticamente un documento possono avere significati diversi all'interno di diversi

\footnotetext{
${ }^{246}$ Le due prospettive metodologiche richiamate nel contributo di Biagetti sono quelle contrapposte della 'sommarizzazione' e della 'presupposizione' che hanno condizionato le strategie di indicizzazione semantica. «La prima risulta più adatta nel caso di biblioteche specializzate e di centri di documentazione, nei quali gli indicizzatori siano a conoscenza delle esigenze dei loro lettori, e stabiliscano dunque indici non in modo neutrale, ma sulla basedell'individuazione delle necessità del lettore "ideale", della conoscenza di quali aspetti egli coglierà di ciascun argomento, mentre la seconda è tipica delle biblioteche pubbliche generali e dei servizi bibliografici, nei quali gli indicizzatori non conoscono con sufficiente certezza i profili culturali dei possibili lettori "ideali", e dinque non devono compiere alcun tentativo di assumere una posizione circa il livello di conoscenza posseduto dal lettore, ma devono invece prendere in considerazione, in termini di aboutness, e registrare negli indici, qual è la "presupposizione" dell'autore, quali argomenti l'autore del libro presuppone che il lettore debba conoscere: in questo caso, l'interferenza dell'indicizzatore, tra il lettore e il libro, è minima».
}

Cfr. Maria Teresa Biagetti, Indici semantici e ricerca scientifica cit., p. 20 
ambiti disciplinari $^{247}$. Lo studioso danese elabora una teoria del soggetto come «potenziale epistemologico dei documenti» ${ }^{248}$, in quanto le proprietà significative del soggetto non sono stabilite a priori, ma dipendono dal contesto nel quale vengono elaborate, dunque è la società stessa a produrre e determinare $\mathrm{i}$ soggetti e in definitiva lo sviluppo 'epistemologico' di un soggetto è il frutto dell' avanzamento scientifico e sociale. In questo senso emergono gli elementi di novità e le nuove opportunità che la tecnologia dei linked data offre alla rielaborazione di una rinnovata teoria del soggetto che vede congiuntamente impegnate la Biblioteconomia e la Scienza dell'informazione ${ }^{249}$. Il passaggio da una rete di 'dati' ad una rete di 'significati' consente di allargare il raggio di analisi alle modalità di rappresentazioni della conoscenza in diverse comunità scientifiche ed equivale alla possibilità per i nuovi sistemi di recupero dell'informazione di usufruire di quella struttura di significati che costituisce il 'portato epistemologico' di un soggetto, creando le condizioni per proficui scambi disciplinari e per un recupero dell'informazione qualitativamente differente.

Particolarmente interessanti si rivelano, in secondo luogo, i 'salti dimensionali' che si possono compiere durante una ricerca, che possono introdurre nella ricerca semantica funzionalità decisamente innovative: ciò è reso possibile attraverso il collegamento di dataset bibliografici a dataset di altra natura, ad esempio, quelli di persone, quelli geografici, quelli tematici (es. dataset di film, di opere teatrali) o di altre istituzioni del patrimonio culturale come musei e archivi.

Si forniscono di seguito alcuni esempi significativi di nuove funzionalità che potrebbero essere sviluppate ricorrendo alla tecnologie dei linked data per migliorare la ricerca semantica.

\footnotetext{
${ }^{247}$ Ivi, p. 23.

${ }^{248}$ Ibidem.

${ }^{249}$ Così si esprime Biagetti (ivi p. 25): «La Biblioteconomia e la Scienza dell'informazione, secondo l'impostazione offerta da Birger Hjørland, devono elaborare una "Teoria del soggetto" che permetta di produrre rappresentazioni corrette della conoscenza espressa nei libri, anche prendendo in considerazione l'ampio orizzonte costituito dai diversi campi scientifici. L'orientamento "pragmatista" trova quindi un fondamento nella concezione, definita Domain analysis, che considera gli utenti dei sistemi d'informazione e delle biblioteche come individui che fanno parte di organizzazioni sociali, all'interno delle quali si mettono in evidenza i diversi campi scientifici, con le loro peculiarità. Secondo questo orientamento, oltre a tener conto dei processi cognitivi e psicologici, dell'attività linguistica e comunicativa, ad analizzare i contenuti informativi sulla scorta di principi universali e ad individuare caratteristiche permanenti, la Scienza dell'informazione dovrà elaborare i contenuti che si presentano all'interno dei diversi campi di conoscenza, cioè nell'ambito delle diverse comunità di pensiero o do produzione scientifica che, nella trattazione di Hjørland, vengono avvicinati ai "paradigmi" scientifici emergenti e dominanti in un dato periodo storico, che Thomas Kuhn ha posto a fondamento della sua teoria sulle rivoluzioni scientifiche».
} 
a. Collegamento tra entità bibliografiche e di altra natura

Nel recupero dell'informazione, un'entità di interesse (es. un'opera) può essere visualizzata in collegamento con le altre entità (es. opere derivate, riduzioni, adattamenti), ma sarà anche possibile visualizzare le influenze di autori sull'opera di altri autori attraverso il collegamento tra entità secondole relazioni previste da FRBR; tramite la struttura dei dati collegati sarà possibile visualizzare il collegamento tra un'opera e tutte le opere che in qualche modo sono poste in relazione con essa, tra tutti gli autori e soggetti correlati. Sarà possibile anche saltare da un soggetto ad un oggetto museale che lo rappresenta, es. un quadro, una scultura, tramite il collegamento con i dataset del patrimonio culturale.

b. Collegamento tra sistemi di organizzazione delle conoscenze

La scomposizione dei soggetti e delle classi nei concetti che li compongono potrà unire diversi sistemi di organizzazione delle conoscenze (KOS) creati dalle biblioteche e sistemi creati da altre comunità del Web Semantico, offrendo modalità avanzate di recupero dell'informazione che si possono avvalere di una rete sindetica di rimandi tra termini già pronta all'uso. Sarà possibile raggiungere un soggetto o una classe a partire da termini provenienti da KOS di diverso tipo. Le tecnologie del Web Semantico consentono di collegare tra loro anche termini affini e concetti simili ampliando il raggio della ricerca semantica.

c. Collegamento tra dati bibliografici e 'cose' reali

La possibilità di collegare $\mathrm{i}$ dati bibliografici alle cose descritte e collegate nel Web Semantico ha indubbi vantaggi nella costruzione di modalità innovative di ricerca. Sarà possibile, ad esempio, attraverso il collegamento a database geografici, visualizzare a partire da una mappa geografica i soggetti correlati o gli autori che pubblicano in un determinato paese o che hanno avuto più influenza in una certa area geografica. 
d. Possibilità di costruire pagine dinamiche a partire dalle entità di interesse

La visualizzazione dei dati bibliografici potrà avvalersi delle tecnologie del Web Semantico che consentono, ad esempio, la visualizzazione di pagine dinamiche per ogni entità di interesse del catalogo, ad esempio un'opera, un soggetto, un autore. In tal modo viene ampliata la possibilità di recupero di un'entità nel suo contesto rappresentando la sua collocazione nell'universo bibliografico del catalogo e usufruendo di collegamenti con gli altri dati nel web. Un esempio di come potrebbe apparire la pagina di un soggetto è quella fornita da data.bnf.fr: a partire da un determinato soggetto viene creata dinamicamente una pagina che offre collegamenti a documenti, film, immagini, risorse multimediali e collegamenti esterni.

Visualizzazioni avanzate create a partire dalle entità di interesse del catalogo possono realizzare un recupero dell'informazione bibliografica in un contesto arricchito da altre risorse autorevoli recuperate nel web. Si passa così da un recupero ispirato all'information retrieval ad un recupero in base al modello entità-relazione, che si rivela particolarmente efficace nel raggruppamento dei concetti nella ricerca semantica.

\subsubsection{Conclusioni}

L'analisi di teorie e modelli di information seeking behavior elaborati nella Scienza dell'informazione ha rappresentato un buon punto di partenza per analizzare e comprendere più a fondo le modalità con cui gli utenti si approcciano alla ricerca di informazione e i compiti che svolgono nel processo di ricerca; un'analisi più approfondita dei comportamenti e delle azioni che gli utenti svolgono durante il processo ci ha consentito di individuare alcune nuove funzionalità che consentono un più efficace utilizzo dell'OPAC.

L'indagine si è posta l'obiettivo di analizzare la progettazione degli OPAC alla luce dell'analisi del processo di ricerca per appurare se tutte le funzionalità necessarie all'utente nelle fasi di ricerca in cui l'utente si può trovare al momento in cui si rivolge all'OPAC siano state previste.

Le funzionalità che oggi i nuovi OPAC hanno attivato per migliorare la ricerca non hanno ancora migliorato in maniera consistente il recupero dell'informazione. 
Per contro, il modello elaborato in queste pagine fornisce uno strumento di analisi delle componenti e delle variabili coinvolte nel recupero dell'informazione al fine di sviluppare funzionalità che possano tenere conto delle modalità con cui gli utenti cercano e usano l'informazione bibliografica, facilitando la creazione di conoscenza nel recupero dell'informazione bibliografica.

L'analisi porta, dunque, a concepire la ricerca non più in termini di corrispondenza tra una query e il risultato, ma ad ampliare la visuale all'intero processo di ricerca. Immaginando la ricerca dell'informazione come un processo di costruzione emergono senz'altro alcune nuove funzionalità che dovrebbero essere tenute in considerazione nell'evoluzione futura di questi strumenti.

Dall'analisi emergono alcuni elementi utili a riprogettare le interfacce e le funzioni dei cataloghi elettronici. In particolare dovrebbero essere attivate quelle funzionalità che consentano all'utente, specie a chi si trova nelle prime fasi del processo, di esplorare in maniera significativa i contenuti del catalogo per trovare l'informazione rilevante e di proseguire nel percorso della propria ricerca raccogliendo l'informazione pertinente alla propria ricerca.

Per riassumere, si sottolineano alcune criticità rilevate nell'analisi che potranno essere oggetto di riflessione in una progettazione efficace delle funzionalità dei nuovi OPAC:

* sviluppo delle funzioni di orientamento, navigazione e browsing nel catalogo come supporto alla fase iniziale della ricerca;

* sviluppo delle funzioni di valutazione della rilevanza e della pertinenza per le fasi centrali;

* sviluppo di funzionalità di monitoraggio, organizzazione e riutilizzo dell'informazione per supportare le fasi conclusive del processo.

Le funzionalità proposte nel modello espandono le principali funzioni-utente delineate nel modello FRBR e nei nuovi Principi internazionali di catalogazione per consentire all'utente un più efficace utilizzo dei cataloghi elettronici e introducono nuove funzionalità che derivano dall'analisi del comportamento informativo.

Si esplora infine la possibilità che i linked data possano essere la tecnologia più appropriata per la costruzione di nuovi OPAC, basati sulla creazione di conoscenza all'interno del processo informativo esaminando le possibilità offerte da questa nuova tecnologia nello sviluppo degli OPAC in relazione al modello proposto. L'analisi rileva 
che la profonda ristrutturazione o de-costruzione del record bibliografico nei 'dati' che lo costituiscono, resa possibile dall'adozione del modello RDF, e dunque la creazione di un nuovo modello di record, il 'record collegato' può offrire una struttura in grado di rinnovare profondamente le funzionalità di ricerca del catalogo, facilitando l'attivazione e lo svolgimento delle funzionalità proposte nel modello.

Il modello logico RDF consente infatti di innovare profondamente la struttura il record per ricavare le entità previste nel modello FRBR e consentire un recupero dell'informazione in grado di sfruttare il modello entità-relazione con visualizzazioni dei risultati che evidenzino le entità e le loro relazioni. Inoltre, la rete di collegamenti che il 'record collegato' consente di attivare con il più vasto 'web dei dati' consentirà all'utente di svolgere le funzioni previste nel processo informativo da me analizzato. L'adozione delle tecnologie dei linked data può avere, infine, un ruolo significativo nel processo informativo analizzato nel mio modello, nella riduzione delle barriere cognitive, nella formulazione di un'interrogazione, nella navigazione tra i record bibliografici ed altre informazioni provenienti dal web, nelle valutazioni di rilevanza e pertinenza e, infine, nella ricerca semantica. 



\title{
CONCLUSIONI. PROSPETTIVE E SVILUPPI FUTURI
}

\begin{abstract}
«Nessuno scrive davvero del futuro. Tutto quello che ci rimane quando fingiamo di scrivere del futuro è il momento in cui stiamo scrivendo. Ecco perché ogni futuro immaginato diventa obsoleto come un gelato che si scioglie mentre uscite dalla gelateria all'angolo»
\end{abstract}

William Gibson

\section{Un solo OPAC, nessun OPAC? L'identità del catalogo di fronte alla} sfida del Web Semantico

Lo scenario nel quale si svolge l'attuale evoluzione del catalogo elettronico delineato in queste pagine è quello di un mutamento radicale delle forme di creazione, condivisione e utilizzo dell'informazione profondamente segnate dalla grande trasformazione del digitale.

Se fin dai tempi più remoti possiamo affermare che viviamo in una società dell'informazione, in quanto dotata di sistemi di registrazione delle conoscenze, è solo in tempi più recenti che il progresso e il benessere umano dipendono strettamente da una gestione consapevole ed efficace del ciclo di vita dell'informazione che comprende la creazione, la trasmissione, il processo, la gestione e l'uso dell'informazione stessa attraverso la condivisione, la modificazione, l'indicizzazione ai fini del suo recupero, la conservazione e l'immagazzinamento, l'apprendimento e l'istruzione ${ }^{1}$.

Nella rivoluzione digitale il progresso delle tecnologie della comunicazione gioca un ruolo centrale; esse sono in grado di portare indubbi vantaggi economici e di progresso scientifico, ma, al contempo, di produrre significative trasformazioni sulla natura della realtà e della conoscenza e di incidere profondamente sulla società, fino a ridisegnare le mappe della società mondiale in cui il digital divide rischia di creare inedite forme di discriminazione e divario generazionale, geografico, economico e culturale.

In questo articolato contesto il principale ruolo delle biblioteche, quali strutture che si occupano di organizzare il sapere, è quello di filtrare, mediare e organizzare la mole d'informazione disponibile.

\footnotetext{
${ }^{1}$ Luciano Floridi, La rivoluzione dell'informazione cit.
} 
Assume dunque un'importanza strategica la creazione di strumenti di recupero dell'informazione che consentano, al pari degli altri servizi bibliotecari, di mettere il lettore nelle migliori condizioni affinché l'informazione possa trasformarsi in conoscenza.

La riflessione sulla natura e sulle funzioni dei cataloghi elettronici svolta nella presente ricerca ha posto al centro il catalogo elettronico inteso come 'linguaggio' attraverso il quale la biblioteca esprime la sua funzione culturale di organizzazione del sapere. In questo senso le riflessioni hanno indagato la natura del catalogo quale sistema di organizzazione delle conoscenze e di recupero dell'informazione tramite un codice comunicativo che si esprime negli strumenti della descrizione bibliografica, degli indici nominali e semantici e del controllo bibliografico che le biblioteche hanno raffinato in secoli di pratica bibliotecaria.

Di fronte alle nuove modalità di creazione, ricerca e fruizione dell'informazione in atto, ci si è chiesti, dunque, in che modo riprogettare la comunicazione con l'utente, affinché il processo di ricerca che conduce al recupero dell'informazione bibliografica venga svolto nella maniera più efficace.

Il presente lavoro si è posto anche un importante interrogativo; quello di indagare se i cataloghi sfruttino appieno le potenzialità che possono provenire da una progettazione intelligente del recupero dell'informazione.

La creazione di strumenti di recupero dell'informazione poggia infatti su quel fondamento intellettuale già individuato da Svenonius nella sua riflessione seminale sui fondamenti teorici che soggiacciono all'organizzazione e al recupero dell'informazione. In merito agli strumenti per l'organizzazione dell'informazione Svenonius affermava:

Un sistema per organizzare l'informazione, per essere efficace, deve basarsi su un fondamento intellettuale; questo fondamento intellettuale consiste di diverse parti:

* un'ideologia, formulata in termini di scopi (gli obiettivi che un sistema per organizzare l'informazione deve conseguire) e di principi (le direttive che informano la progettazione);

* le formalizzazioni dei processi implicati nell'organizzazione dell'informazione, come quelli forniti dalle concettualizzazioni linguistiche e dai modelli relazionali entità/attributi;

* la conoscenza acquisita tramite la ricerca, in particolare quella espressa in forma di generalizzazioni di livello alto sulla progettazione e l'uso di sistemi organizzativi; e

* nella misura in cui la disciplina è definita dai punti focali della sua ricerca, i problemi chiave che occorre risolvere per organizzare in modo intelligente l'informazione e far progredire la scienza dell'informazione ${ }^{2}$.

\footnotetext{
${ }^{2}$ Elaine Svenonius, Il fondamento intellettuale dell'organizzazione dell'informazione cit., p. 3.
} 
Non c'è dubbio che i primi due elementi sono stati oggetto di rielaborazione nella teoria catalografica dell'ultimo decennio che si è concretizzata nei nuovi Principi internazionali di catalogazione e nel modello FRBR.

I primi hanno ridefinito obiettivi e funzioni del catalogo, l'altro rappresenta la più alta elaborazione teorica per descrivere l'ontologia dell'universo bibliografico e al contempo uno strumento operativo per strutturare un record che possa risultare funzionale alle diverse comunità che lo utilizzano.

Sugli altri due elementi fondamentali manca una riflessione organica e aggiornata di livello internazionale che, sulla base delle nuove tecnologie, dei nuovi sviluppi della teoria catalografica e dello scenario informativo attuale, guidi ad una progettazione dell'OPAC basata su modelli nuovi ${ }^{3}$.

Il presente lavoro si è addentrato nell'analisi di questi aspetti tentando di esplorare le pratiche e le teorie che si sono sviluppate all'interno della Biblioteconomia e della Scienza dell'informazione per quanto riguarda la progettazione della ricerca nei cataloghi.

I nuovi strumenti che oggi vengono proposti per rimpiazzare le interfacce tradizionali dei cataloghi elettronici, OPAC 'di nuova generazione' e 'strumenti per la ricerca', sebbene mirino a creare interfacce semplificate e ad unificare la ricerca che altrimenti l'utente svolgerebbe in più strumenti, si orientano verso modelli di sviluppo che tendono all'imitazione della ricerca che avviene nel web invece di proporre funzionalità realmente innovative.

Essi dovrebbero puntare, invece, sulla predisposizione di una nuova struttura dei dati catalografici che sia in grado di accogliere i più recenti sviluppi delle tecnologie informatiche e di informare una progettazione più intelligente del catalogo elettronico che conduca l'utente verso la conoscenza e l'apprendimento dell'universo bibliografico ivi rappresentato.

Il risultato è uno scivolamento verso una rischiosa 'perdita d'identità' del catalogo in favore di strumenti che vengono approntati all'interno delle biblioteche (sistemi per la scoperta e web scale discovery services) e al di fuori (altri strumenti di ricerca presenti nel web).

Nel momento in cui i dati bibliografici, che prima restavano registrati in OPAC chiusi e non interoperabili, diventano accessibili, collegati e integrati con gli altri dati e compatibili con le tecnologie del web, si assiste ad una crescita dell'interesse per i dati bibliografici, ma allo stesso tempo sembra diminuire l'interesse per il catalogo come

\footnotetext{
${ }^{3}$ Oltre alle riflessioni di Svenonius (Il fondamento intellettuale dell'organizzazione dell'informazione cit.) e alle Guidelines for OPAC displays che oggi appaiono certamente da revisionare.
} 
servizio della biblioteca, come strumento identificabile insieme agli altri servizi che vengono offerti all'utente. Ciò deriva da una sovrapposizione di fattori che sono stati indagati nelle pagine di questo lavoro.

In primo luogo, lo sviluppo del catalogo elettronico è profondamente legato al passaggio dalla gestione fisica dei documenti a quella digitale; ciò ha comportato il declino della concezione del catalogo come principale strumento informativo della biblioteca, poiché di fronte alla crescita delle risorse informative il segmento di informazione tradizionalmente rappresentato nel catalogo elettronico e limitato alle sole risorse di un'istituzione perde inevitabilmente di interesse. In passato il catalogo elettronico rappresentava lo strumento e descriveva ciò che era disponibile localmente e le ricerche avevano come punto focale la biblioteca, oggi si assiste al contrario ad una predisposizione di un ambiente di ricerca nel quale il catalogo è solo una delle componenti. Le biblioteche, infatti, offrono all'utente, oltre alle collezioni a stampa, una vasta serie di risorse che vanno dalle banche dati, alle collezioni digitali, ai repositories istituzionali, alle collezioni di $e$ journals che necessitano di essere recuperate per essere visibili al'utenza.

Il catalogo si trova così nel delicato passaggio che lo vede trasformarsi da uno strumento istituzionale a un dispositivo che opera nel più ampio contesto del web, ovvero in grado di inserirsi nei flussi informativi che hanno luogo nel web.

In secondo luogo, la ricerca e l'uso dell'informazione si sono spostati nella rete, così le biblioteche vedono il verificarsi di una sorta di rivoluzione copernicana che da centro di interesse o punto di partenza privilegiato della ricerca, le obbliga a riconfigurarsi intorno all'utente creando servizi e strumenti che si integrino nell'ambiente di lavoro dell'utente.

La rete cambia i modi in cui le conoscenze vengono create, registrate e distribuite coinvolgendo in ultima analisi il modo in cui avviene la comunicazione tra gli individui; nella rete essa assume una forma reticolare, cioè i flussi della comunicazione si ridistribuiscono passando da un modello 'uno a molti' ad uno 'molti a molti' e modificando le tradizionali modalità di creazione, accesso e uso dell'informazione.

Lo spostamento della ricerca a livello della rete impone che l'accesso e la scoperta dell'informazione avvengano principalmente attraverso altri servizi di internet e che le biblioteche debbano sforzarsi di costruire sempre di più servizi che ruotano intorno all'utente e che si inseriscono nei nuovi flussi di lavoro e di attività che l'utente svolge nella rete; ciò significa che l'informazione bibliografica viene fornita in una molteplicità di formati, di modalità di visualizzazione e di possibilità di utilizzo che vanno oltre la ricerca in un catalogo elettronico. Le informazioni del catalogo possono essere sfruttate in 
applicazioni del web e in modalità nuove per arricchire i percorsi a disposizione dell'utente e le modalità di accesso ai dati bibliografici. Inoltre in rete la ricerca dell'informazione è legata a forti e ben progettate user experiences; l'utente nella ricerca dell'informazione nel web vive un'esperienza di ricerca integrata, a differenza dell'ambiente di ricerca tradizionale predisposto dalle biblioteche.

Se i flussi dell'informazione sono radicalmente cambiati, emerge allora con forza la necessità delle biblioteche di 'riposizionarsi' «attraverso un lavoro di continuo e progressivo adeguamento, a mano a mano che si modificano i bisogni del pubblico, che si evolvono le forme di produzione e circolazione del sapere e dei documenti in cui esso è registrato, che si presenta l'esigenza di elaborare nuovi strumenti di lavoro» ${ }^{4}$.

Dapprima questa tendenza si è manifestata:

* con l'accoglimento di nuove fonti di informazione per arricchire i record bibliografici inglobando risorse provenienti dall'esterno e creando strumenti in grado di espandere il raggio della ricerca;

* tramite la creazione di strumenti atti a creare intorno al catalogo uno spazio informativo integrato delle risorse presenti in biblioteca manifestatosi nella nascita di discovery systems.

Le linee di tendenza attuali, esaminate ed approfondite in questo lavoro, vedono il catalogo ampliarsi e trasformarsi per diventare uno strumento di recupero globale dell'informazione; oggi si assiste alla creazione di 'strumenti per la ricerca' che tendono alla creazione di super-OPAC in grado di recuperare dati provenienti da varie fonti esterne, ma anche a rendere disponibili all'esterno le risorse digitali che le biblioteche producono. Ciò risponde alla tendenza che Lorcan Dempsey ha denominato 'inside-out ${ }^{5}$, ovvero alla necessità per le biblioteche di fornire un accesso unificato non solo alle risorse che le biblioteche acquistano o dispongono in licenza d'uso (periodici elettronici, banche dati etc), ma anche a quelle risorse prodotte in forma sempre più massiccia dalle istituzioni stesse (repositories e collezioni digitali) cui le biblioteche vogliono offrire una visibilità a tutti gli utenti del $\mathrm{web}^{6}$. La nascita degli strumenti di discovery che oggi si sostituiscono o

\footnotetext{
${ }^{4}$ Giovanni Solimine, La biblioteca e la rete nella società dell'apprendimento cit. p. 410.

${ }^{5}$ Lorcan Dempsey, Outside in and inside out, «Lorcan Dempsey’s Weblog», blog post, 11 .01. 2010, $<$ http://orweblog.oclc.org/archives/002047.html>.

${ }^{6}$ Lorcan Dempsey, Thirteen ways of looking at libraries, discovery, and the catalog: scale, workflow, attention, «EDUCAUSE Review Online», 10 December 2012, <http://www.educause.edu/ero/article/thirteenways-looking-libraries-discovery-and-catalog-scale-workflow-attention $>$.
} 
si affiancano agli OPAC tradizionali costituiscono la più diretta conseguenza di questa tendenza.

In questa prospettiva sono emersi alcuni elementi di rilievo che affliggono la progettazione dei cataloghi attuali, che seguono strategie ispirate all'information retrieval classico o che si ispirano a tendenze imposte da altri strumenti informativi, piuttosto che seguire, come si dovrebbe, la via della riflessione matura sulla natura e le funzioni del catalogo elettronico nel nuovo contesto informativo.

Oggi lo scenario tecnologico subisce una nuova e importante evoluzione che rivoluzionerà il mondo dell'informazione riconducendolo alla sua essenza primaria: il dato. Tale innovazione provocherà effetti di enorme portata sulla struttura del catalogo elettronico. Così ai metadati prodotti in ambito bibliotecario si aggiungono oggi, con la tecnologia dei linked data, anche i dati provenienti dal web; essi potranno essere utilizzati per proporre nuovi servizi e ampliare le potenzialità di ricerca.

La comparsa del modello dei linked data come nuovo modo di esporre i dati bibliografici per integrarli nel Web Semantico pone vari interrogativi che coinvolgono la sopravvivenza delle modalità tradizionali di indicizzazione semantica e di organizzazione della conoscenza e di accesso alle risorse bibliografiche. Di fronte alle nuove tecnologie dei linked data è obbligatorio chiedersi quale sia il ruolo del catalogo elettronico nel futuro e se ci sia ancora spazio per un' 'idea' di catalogo. Le condizioni di convergenza e interoperabilità che l'apporto del Web Semantico è in grado di offrire ai dati bibliografici e del patrimonio culturale creerà un web senza barriere, uno spazio globale ove i dati bibliografici saranno collegati agli altri presenti web. Questo processo di collegamento potrebbe far immaginare ai più scettici uno scenario in cui la possibilità di recuperare dati bibliografici attraverso altri strumenti del web renda ormai superfluo il ruolo del catalogo e far così intravedere la fine di questo importante strumento.

Ciò che è certo è, invece, che le tecnologie dei linked data consentiranno alle biblioteche di immettere nel Web Semantico i dati autorevoli prodotti da una lunga tradizione teorica e pratica che le biblioteche hanno accumulato nell'organizzazione, nella conservazione e preservazione del sapere ai fini della ricerca dell'informazione e suo recupero esercitata tramite gli strumenti del controllo di autorità, della descrizione bibliografica, dell'indicizzazione nominale e semantica, negli strumenti che consentono di compiere associazioni tra i documenti. 


\section{Scenari futuri e prospettive di sviluppo}

Se non possiamo ancora sapere quali sarà l'esito definitivo del processo di evoluzione dei cataloghi elettronici, tuttavia è chiaro che con l'avvento delle nuove tecnologie del Web Semantico ci si avvia verso una nuova importante tappa del loro percorso evolutivo che vedrà il verificarsi di una radicale trasformazione del catalogo e dei dati bibliografici:

La qualità di una biblioteca non si misura tanto dalla quantità di documenti posseduti, quanto dalla capacità di strutturare e di modellizzare i dati, di renderli accessibili, conservando al contempo la stratificazione dei contesti, la relazione tra quello nuovo che si crea e il contesto d'origine, oltre che con tutti gli altri documenti con cui essi stringono relazioni semantiche tacite o palesi. Essa cioè deve saper ricostruire le relazioni logiche e genetiche tra $\mathrm{i}$ documenti, rendendoli tuttavia disponibili a nuove ricomposizioni semantiche, lasciate dinamicamente a totale giudizio e scelta dell'utente; deve, insomma, saper esibire i contesti multipli a cui i documenti fanno riferimento. Di qui la necessità di uno sforzo - già in atto per rendere identificabili in modo univoco i propri dati nel contesto del web per renderli disponibili a essere leggibili, interpretabili e usabili dalle macchine ${ }^{7}$.

Il catalogo dunque si avvia a diventare uno strumento in grado di collegare le conoscenze prodotte dalle biblioteche agli altri dati presenti nel web.

La posizione intrapresa in questo lavoro sostiene che il catalogo potrà garantirsi un ruolo in futuro se manterrà un'identità 'forte' e riconoscibile ovvero se, a differenza dei motori di ricerca del web, si porrà come uno strumento dove l'utente potrà non solo trovare, verificare o recuperare un'informazione bibliografica, ma come un ambiente in grado di sostenere il suo personale processo informativo producendo al contempo conoscenza.

Da questa premessa emergono alcuni significativi elementi di riflessione che potranno essere oggetto di approfondimento in futuro e che si ritiene siano stati sollevati in questo lavoro di ricerca:

* la possibilità di una rinnovata progettazione del processo informativo che avviene nel catalogo elettronico ai fini di un miglior recupero dell'informazione bibliografica;

* il nuovo ruolo del record bibliografico nel processo informativo;

* un'adeguata riprogettazione degli accessi al catalogo;

un nuovo rapporto tra utente e informazione bibliografica e tra universo bibliografico e universo globale dell'informazione.

\footnotetext{
${ }^{7}$ Gianfranco Crupi, Beyond the Pillars of Hercules: Linked data and Cultural heritage, «JLIS.it», 4 (2013), n. 1, p. 25-49, <http://leo.cilea.it/index.php/jlis/article/view/8587/7887>.
} 
Sul primo punto il presente lavoro ha sollevato la necessità di guardare più da vicino come si svolge il processo di ricerca dell'informazione nel catalogo. Lo svolgimento della mediazione informativa attraverso lo strumento del catalogo richiede infatti l'acquisizione della consapevolezza di tutti quei fattori che influenzano i comportamenti di ricerca e dunque l'orientamento del lavoro si è focalizzato sull'esame del processo informativo, che necessariamente coinvolge i concetti di informazione, di bisogno informativo, di ricerca dell'informazione e di comportamento informativo.

Ciò che però segnerà profondamente il processo di evoluzione del catalogo elettronico sarà lo spostamento a livello della web dei meccanismi del controllo bibliografico e dell'organizzazione della conoscenza, ovvero delle principali 'vie d'accesso' al catalogo.

Ogni 'dato' può servire da punto d'accesso per il raggiungimento dell'entità bibliografica presente in un catalogo, attivando proficui collegamenti con le altre entità presenti nell'universo bibliografico rappresentato nel catalogo; in questo senso cambieranno profondamente le caratteristiche dell' accesso bibliografico.

Il controllo bibliografico, come è noto, rappresenta un elemento indispensabile per garantire la qualità del catalogo; esso supera di gran lunga per importanza la creazione delle descrizioni bibliografiche, in quanto è deputato a assicurare il corretto recupero dell'informazione e a facilitare il ritrovamento delle entità correlate, garantendo un'efficace struttura sindetica del catalogo.

I record di autorità consentono di correlare le notizie bibliografiche ad elementi esterni, come basi di dati, descrizioni prodotte da altre istituzioni del patrimonio culturale, oppure da editori e distributori, per consentire un'effettiva interoperabilità, in quanto consentono a partire dalle registrazioni catalografiche l'accesso e il collegamento a descrizioni prodotte in diverse lingue, in paesi diversi, o caratterizzate da un diverso trattamento catalografico dell'informazione bibliografica. Nelle nuove applicazioni possibili tramite i linked data gli authority files consentono di raggiungere facilmente dati di altra natura per ampliare il potenziale informativo dei cataloghi.

Riguardo agli accessi semantici, l'evoluzione dei linked data potrebbe portare ad una nuova strutturazione di questi accessi con notevoli benefici nella ricerca e nella scoperta dell'informazione. Se dalla nascita dei cataloghi elettronici uno dei più frustranti compiti per l'utente è stato quello di misurarsi nella ricerca con il linguaggio bibliografico del catalogo, in futuro i linked data consentiranno un più facile accesso: la rete dei dati permetterà di raggiungere una notizia partendo dai termini che gli utenti 
conoscono e di arrivare agli stessi concetti utilizzando la terminologia più congeniale alle diverse comunità. In questo senso, si può riproporre in questa sede il pensiero di Ventura, che riprendendo un passo di Jesse Shera, qualche anno fa auspicava un cambio di paradigma e rifletteva sulla classificazione e la soggettazione in questi termini:

La classificazione, sebbene sia stata utilizzata come schema per radunare libri sullo stesso soggetto e ordinare soggetti in una biblioteca, si è mostrata inadeguata ad affrontare l'esplosione documentaria verificatasi nell'ambito della comunicazione grafica: una delle conseguenze è che gli stessi bibliotecari hanno finito per usare la notazione classificatoria per localizzare libri piuttosto che come guida semantica agli argomenti o alle relazioni interdisciplinari. L'organizzazione bibliografica dovrebbe assumere altre forme e sarebbe opportuno studiare i modi in cui le registrazioni bibliografiche sono usate da differenti gruppi di utenza, al fine di massimizzare l'utilità sociale delle informazioni bibliografiche stesse: l'ordinamento bibliografico dovrebbe così riflettere l'organizzazione effettiva del pensiero piuttosto che un ordine universale e astratto della natura, e gli schemi classificatori potrebbero essere congegnati di conseguenza. L'accesso alla conoscenza dovrebbe avvalersi degli odierni strumenti bibliografici e tecnologici, dato che questi strumenti non appiattiscono il contenuto multidimensionale del libro all'ordine lineare e monodimensionale stabilito dalla classificazione gerarchica [...] La classificazione resta un'attività fondamentale, ma grazie all'apporto dei nuovi strumenti essa può ridefinirsi in modo tale da consentire ai ricercatori la scoperta di nuove relazioni tra conoscenze o nuove interpretazioni delle conoscenze registrate ${ }^{8}$.

Perciò - continuava Ventura - la classificazione dovrebbe divenire più pragmatica, «legata a usi della letteratura scientifica da parte di comunità definite e specializzate, offrendo un approccio multiplo agli oggetti correlati e giocando sulla selezione delle proprietà rilevanti degli oggetti di studio».

I linked data ci offrono oggi una tecnologia in grado di riprogettare il controllo bibliografico e impostare su nuove basi la ricerca semantica, in cui è centrale il passaggio da una rete di dati ad una rete di significati. Il collegamento tra dataset di diversa natura e provenienza consentono infatti di passare da una rete di dati ad una rete di saperi e di conoscenze intrecciate tra loro. In questo senso i nuovi cataloghi elettronici abbandonano le 'metanarrazioni' dei cataloghi moderni per trasformarsi in 'luoghi' nei quali si intersecano tanti discorsi tenuti insieme da un linguaggio comune.

La ridefinizione dell'accesso catalografico cambierà infine profondamente il rapporto tra utente e universo bibliografico e tra l'universo bibliografico e quello globale dell'informazione in quanto la più profonda integrazione tra $\mathrm{i}$ dati catalografici e $\mathrm{i}$ dati prodotti da altri soggetti renderà i dati delle biblioteche più completi. Ciò comporterà la possibilità di fruire l'informazione bibliografica anche in contesti diversi da quello delle biblioteche tramite i nuovi servizi che potranno essere approntati grazie alla struttura del Web Semantico.

\footnotetext{
${ }^{8}$ Roberto Ventura, Il senso della biblioteca cit., p. 241-242.
} 
L'utente potrà avvalersi di un linguaggio comune al web per interrogare il catalogo accorciando così le distanze tra il linguaggio del web e quello delle biblioteche; nel record bibliografico ‘collegato' ogni dato disporrà di un 'contesto allargato' che potrà essere navigato dall'utente per raggiungere ulteriori informazioni apportando così un valore aggiunto alle risorse presenti nel catalogo.

\title{
3. Biblioteconomia e Scienza dell'informazione: due «naturali alleati»"
}

\begin{abstract}
Nella società contemporanea, con la sempre più ampia diffusione di sistemi in grado di veicolare un quantitativo impressionante di dati che si riversano sul corpo sociale, emerge con forza la volontà di comprendere il ruolo dell'informazione in rapporto al suo manifestarsi come processo e dunque alla sua capacità di essere assimilata per potersi trasformare in conoscenza ${ }^{10}$.
\end{abstract}

Abbiamo visto che il passaggio fondamentale che distinguerà gli odierni cataloghi da quelli del futuro sarà la possibilità di ottenere tramite questi strumenti un'esperienza di ricerca che dal recupero dell'informazione si orienti verso la creazione di conoscenza. I nuovi orizzonti che i linked data aprono nel campo della ricerca semantica risiedono nell'uso di ontologie create dinamicamente con cui si riuscirà a collegare ogni specifico dominio del sapere e ad esplicitarne i collegamenti reciproci: tutto ciò avverrà automaticamente attraverso i processi inferenziali e deduttivi che consentono la fusione di grafi provenienti da diversi dataset.

È fondamentale, nel percorso informativo che l'utente compie nel catalogo, la possibilità di avvalersi di questa nuova struttura di navigazione per trovare l'informazione pertinente che consiste, in via definitiva, per le biblioteche, nel garantire i percorsi di ricerca che l'utente vorrà intraprendere attivando i collegamenti tra dati bibliografici e dati di altra natura, che consentono di scoprire nuove associazioni semantiche tra l'universo bibliografico e l'universo informativo.

Nuovamente, dunque, il futuro del catalogo elettronico passa attraverso il binomio 'informazione - conoscenza'. In questo contesto un esame più approfondito del concetto di 'informazione' e degli approcci che la Scienza dell'informazione e le altre discipline hanno adottato per esplorare $\mathrm{i}$ modi in cui avviene la ricerca dell'informazione si è

\footnotetext{
${ }^{9}$ Cfr. Alberto Salarelli Biblioteconomia e information science: diversi linguaggi per due naturali alleati?, relazione presentata al convegno I nuovi alfabeti della biblioteca, Milano, Fondazione Stelline, 15-16 marzo 2012 cui si rimanda per un approfondimento dei rapporti tra le discipline.

${ }^{10}$ Alberto Salarelli, Introduzione alla scienza dell'informazione cit. p. 41.
} 
rivelato un'utile traccia per approfondire le caratteristiche del processo informativo che si svolge nel catalogo.

Le teorie prese in considerazione in questo studio, che rappresentano la cornice teorica nella quale si inserisce la presente riflessione e il modello proposto, si interrogano sul concetto di informazione nelle dimensioni cognitiva e pragmatica e, pertanto, pongono la riflessione sulle funzioni del catalogo non tanto sotto la dimensione dell'oggetto, ma sull'informazione come 'atto di comunicazione', che avviene nel catalogo attraverso il linguaggio bibliografico, tra il 'sistema bibliografico' e l'utente.

In questo senso le nuove e più recenti elaborazioni teoriche intervenute nella disciplina della Scienza dell'informazione contribuiscono a fornire un apporto teorico e intellettuale alla Biblioteconomia e costituiscono la base per un fecondo incrocio di competenze tra le due discipline, che Jesse Sherà definì appunto "naturali alleati'11.

Anche se la questione delle connessioni tra i due ambiti di studio nel dibattito professionale è tutt'altro che pacifica ${ }^{12}$, non dobbiamo infatti sottovalutare l'apporto teorico che la Scienza dell'informazione fornisce alla Biblioteconomia, sottolineato anche da Saracevic, che riconosce ai due settori di studio forti rapporti interdisciplinari sebbene li consideri separati da particolari peculiarità, ma anche da Shera e Vakkari, secondo i quali invece la Scienza dell'informazione troverebbe una base teorica proprio nella Biblioteconomia.

Ciò che è certo è che la Scienza dell'informazione ha mutato i suoi paradigmi di riferimento nel corso della storia della disciplina che sono passati dallo studio delle biblioteche nel loro contesto sociale, a quello dei sistemi di recupero dell'informazione, al paradigma 'fisico' basato sulla teoria matematica della comunicazione, fino

${ }^{11}$ Jesse Hauk Shera, Knowing books and men; knowing computers too, Littleton, Libraries Unlimited, 1973.

12 Alberto Salarelli, Biblioteca e identità: per una filosofia della biblioteconomia, Milano, Ed. Bibliografica, 2008 p. 203-204. Lo stesso Salarelli, infatti, qualche anno fa commentava «la questione acquista una posizione centrale nel dibattito biblioteconomico allorché si confrontino due possibili esiti futuri della disciplina ovvero, riprendendo una riflessione di Michele Santoro, si ponga in atto una contrapposizione tra coloro che rimangono fedeli all'idea del servizio bibliotecario e alle tradizionali forme di gestione e disseminazione dei documenti, e coloro che invece si rifanno al «paradigma dell'informazione» e conclude: «Rifarsi al paradigma dell'informazione e dunque prevedere l'incorporazione della biblioteconomia nella scienza dell'informazione significa perdere di vista il fondamento di ogni biblioteconomia, cioè la biblioteca. Qualora ogni singolo atto di mediazione informativa venga considerato nella sua unicità, dimentichi di una struttura generale di riferimento chiamata biblioteca, preoccupandosi unicamente delle prometeiche caratteristiche di quella cosa chiamata informazione, significa perdere di vista il linguaggio comune (comune a tutti gli utilizzatori) della biblioteca e dunque avviarsi lungo una strada lastricata di offerte individuali e personalizzate. Lo spostamento dell'orizzonte d'indagine da un livello strategico a un piano tattico - partire dai problemi per fornire soluzioni - è del tutto sintomatico della frammentazione del mondo del sapere tipico della realtà contemporanea: il case study diventa l'emblema di un approccio alla realtà basato spesso e unicamente sul problem solving: ebbene, se per alcuni esponenti della scienza dell'informazione questo modo di operare può rappresentare una specificità del suo statuto epistemologico, per la biblioteconomia il vincolo sociale impone di procedere verso sintesi teoriche che siano in grado di essere condivise - o messe a confronto - in un orizzonte di più ampia portata». 
all'approccio cognitivo, oggi poco indagato e conosciuto, almeno nel nostro paese, che considera i processi informativi nel contesto storico e sociale.

La Scienza dell'informazione si è allontanata così dalla Computer science alla quale è rimasto il compito di indagare i processi di elaborazione dei dati attraverso i computer, avvicinandosi ad altre discipline come la Biblioteconomia ${ }^{13}$.

Ne è testimonianza il diverso approccio con cui il concetto di informazione è stato trattato nel tempo sia nella Scienza dell'informazione sia in altre discipline; Saracevic ${ }^{14}$ distingue tre livelli di crescente complessità:

- l'approccio «quantitativo, atomistico e aconcettuale» che considera l'informazione in termini di segnali e messaggi, ignorando tutto ciò che non può essere misurabile. Tale approccio considera solo gli aspetti sintattici, tralasciando quelli semantici e si pone come obiettivo lo studio delle condizioni ideali della trasmissione, dell'immagazzinamento e dell'elaborazione dell'informazione attraverso le tecnologie informatiche e si esprime nella teoria matematica della comunicazione;

* l'approccio che considera i processi cognitivi dell'informazione ovvero la comunicazione tra il messaggio e il soggetto interpretante ove si assiste a un processo dialettico tra la dimensione oggettiva dell'informazione, espressa nel dato, e quella soggettiva di chi la produce o la utilizza;

* l'approccio pragmatico, che considera il contesto specifico nel quale avviene il processo informativo considerando quegli aspetti storici, economici e sociali che influenzano il ciclo di vita dell'informazione e che si interroga sulle modalità di uso dell'informazione da parte di un individuo o di un gruppo sociale.

Risiede certamente nella tecnologia il principale punto di connessione tra le due scienze $^{15}$. Già nel lontano 1968 Alfredo Serrai aveva intuito che la 'rivoluzione dell'informazione' avrebbe portato la Biblioteconomia a riflettere su se stessa:

L'elaboratore darà un poderoso calcio intellettuale alla biblioteconomia e la lancerà in un mondo di altre dimensioni. Il calcolatore costringerà i bibliotecari se non altro a tentare di respingerlo. Per prender posizione nei suoi confronti, essi dovranno esaminare le basi filosofiche e operative della propria pratica professionale, e quindi cercare di darsi un corpo

\footnotetext{
${ }^{13}$ Vakkari citato in Vilma Alberani - Elisabetta Poltronieri, Documentazione e scienza dell'informazione: interazioni con la biblioteconomia, «Bollettino AIB», 43 (2003), n. 2, p. 189-211.

${ }^{14}$ Tefko Saracevic citato in Salarelli, Introduzione alla scienza dell'informazione, p. 58-59.

${ }^{15}$ Come si desume peraltro anche dalla norma UNI/ISO 5127/1 (Vilma Alberani - Elisabetta Poltronieri, Documentazione e scienza dell'informazione: interazioni con la biblioteconomia cit, p. 190) che definisce la Biblioteconomia come «la scienza dell'informazione applicata all'organizzazione, all'amministrazione e alle operazioni proprie delle biblioteche».
} 
di solide conoscenze teoretiche colle quali vagliare l'apporto dello strumento tanto nuovo e rivoluzionario da esse chiamato "cervello" elettronico ${ }^{16}$.

Serrai inoltre sottolineava alcuni benefici di carattere concettuale che la Scienza dell'informazione avrebbe potuto apportare alla Biblioteconomia ${ }^{17}$ :

La professione bibliotecaria viene a trovarsi al centro di una tale disciplina non più con mansioni di collegamento fra dati registrati e individuo, bensì in funzione di coordinamento e di integrazione fra la dinamica sociale e il correlativo flusso di informazione [...]. Le biblioteche hanno la possibilità - e se non lo faranno verranno sostituite da altri organismi di assumere il ruolo di catalizzatore delle conoscenze della società [...]. Per raggiungere questo, il bibliotecario è obbligato ad allargare al massimo il raggio dei propri interessi e delle proprie ricerche, a collegarsi e a beneficiare delle impostazioni, delle metodologie e dei risultati relativi a discipline apparentemente lontane dalla propria, quali sono la neurologia, la linguistica, la semeiotica, la logica simbolica, la cibernetica, la matematica applicata, la teoria delle comunicazioni, la teoria dei sistemi, la psicologia ${ }^{18}$.

In base all'idea che la principale funzione della Biblioteconomia sia quella di offrire una mediazione informativa tra utenti e documenti di qualità differente rispetto alle alternative offerte da altri strumenti di ricerca non possiamo che accogliere questa visione, sottolineando l'apporto delle prospettive provenienti dal filone cognitivo che si è sviluppato in seno alla Scienza dell'informazione e che in questa trattazione vengono ripercorse e che forniscono l'approccio teorico sul quale si basa la presente trattazione.

La scienza dell'informazione potrebbe fornire un valido apporto se si avesse il coraggio di utilizzarla non come una mera appendice [...] ma per quello che essa effettivamente può offrire, vale a dire - innanzitutto - quegli strumenti teorici in grado di tagliare

${ }^{16}$ Alfredo Serrai, La biblioteca di fronte alla rivoluzione concettuale e tecnologica della scienza moderna, in I congressi 1965-1975 dell'Associazione Italiana Biblioteche, a cura di Diana La Gioia, Roma: AIB, 1977, p. 105-122. Citazione a p. 111. Il passo di Serrai è citato così come appare nel contributo di Alberani e Poltronieri, cui si rimanda per la profonda analisi dei rapporti interdisciplinari tra Biblioteconomia, Documentazione e Scienza dell'informazione. Si veda anche il volume di Alfredo Serrai Alfredo Serrai, Biblioteconomia come scienza: introduzione ai problemi e alla metodologia, Firenze, Olschki, 1973.

${ }^{17}$ Alberani e Poltronieri evidenziano la riflessione di Robert S. Taylor circa le connessioni della Scienza dell'informazione con la Biblioteconomia, che si manifesterebbe in almeno cinque aree di indagine:

«-Analisi dei sistemi: la scienza dell'informazione è interessata allo sviluppo di modelli e di tecniche di simulazione per lo studio della biblioteca, di sue parti o di configurazioni più grandi come le reti di biblioteche. A loro volta le biblioteche, i loro servizi e le loro procedure possono ottenere notevoli risultati da sistemi più sofisticati che permetteranno loro di essere meglio comprese in un contesto di comunicazione più ampio come istituzione sociale nel processo educativo, culturale e innovativo.

- Contesto ambientale: quadro sociale nel cui ambito si colloca la biblioteca (le relazioni fra le risorse e i servizi informativi e lo sviluppo tecnologico e le attitudini culturali dell'ambiente nei riguardi dell'informazione, della conoscenza e dell'istruzione).

- Canali d'informazione: nel passato la biblioteca ha offerto un unico canale, costituito dalla letteratura pubblicata. La maggior parte della comunicazione scientifica avviene al di fuori della biblioteca (in biblioteche speciali o in centri d'informazione non dipendenti dalla biblioteca) e il bibliotecario sembra essere un partecipante passivo piuttosto che innovativo, che si è voluto escludere da quel dinamismo proprio della comunicazione. La molteplicità di canali richiede profondi cambiamenti.

- Processo di classificazione, cioè l'organizzazione e l'analisi bibliografica: anche in questo caso il compito sembra sfuggire dalle mani dei bibliotecari (con le debite eccezioni) per passare ai linguisti, matematici ecc. - Interazione uomo-sistema: l'insieme delle interazioni che hanno luogo fra l'utente e qualsiasi punto del sistema (servizi, strumenti bibliografici ecc.)», Vilma Alberani - Elisabetta Poltronieri, Documentazione e scienza dell'informazione: interazioni con la biblioteconomia cit, p. 197.

${ }^{18}$ Alfredo Serrai, Dalla informazione alla bibliografia cit., p. 137-138. 
trasversalmente l'esistente, di porre questioni globali e strategiche come sono quelle che coinvolgono il concetto di informazione ${ }^{19}$.

In questo senso, le riflessioni svolte in queste pagine sottolineano il proficuo rapporto tra $\mathrm{i}$ due ambiti disciplinari che si auspica possa in futuro ampliare ulteriormente le competenze della Biblioteconomia contemporanea ${ }^{20}$.

Le dinamiche che regolano l'accesso all'informazione e alla conoscenza, alla luce dei recenti sviluppi del Web Semantico, aprono così nuovi orizzonti di interazione interdisciplinare che la Biblioteconomia dovrà cogliere affinché le biblioteche rimangano garanti nel futuro del libero accesso alla conoscenza registrata.

${ }^{19}$ Alberto Salarelli, Biblioteconomia e Information science: diversi linguaggi per due naturali alleati? cit.

${ }^{20}$ È che solo a partire dagli anni ' 90 , infatti, che questo approccio interdisciplinare si concretizza nel nostro paese a livello accademico in percorsi formativi nei quali rientrano appieno materie attinenti all'ambito disciplinare della Scienza dell'informazione e ove si realizza una più stretta collaborazione tra le due discipline. 


\section{APPENDICE A. CASI DI STUDIO}

\section{A1. Metodologia del rilevamento}

La presente appendice raccoglie l'analisi di un campione di OPAC analizzati nel corso della ricerca che hanno costituito la base per lo studio più approfondito delle caratteristiche di OPAC di nuova generazione e sistemi per la scoperta.

Nella prima fase della ricerca è stata compiuta un'analisi preliminare di un ampio numero di casi di applicazione di sistemi per la scoperta e OPAC di nuova generazione implementati in biblioteche pubbliche e accademiche e di soluzioni sperimentali allo scopo di scegliere i casi più rappresentativi che sono qui presentati. In tutte le biblioteche e sistemi bibliotecari presi in considerazione i cataloghi sono stati oggetto di modifiche migliorative: sono stati sostituiti da nuovi next generation catalogs che si sono sovrapposti ai tradizionali cataloghi per offrire interfacce più amichevoli, sono stati affiancati o sostituiti da discovery systems. Per disporre di una solida base di analisi, gli OPAC scelti sono stati predisposti da biblioteche diverse per dimensioni, tipologia e destinazione.

\section{A2. Elementi di analisi e predisposizione della griglia di esame}

I casi di studio sono esaminati in base ad una griglia di elementi d'indagine suddivisa in varie categorie che vanno dalla tipologia di dati inclusi nel sistema, alle modalità di ricerca e di visualizzazione dei risultati, fino alle funzionalità offerte all'utente per il riutilizzo dell'informazione ricevuta.

Nella realizzazione della griglia di analisi, la difficoltà iniziale è stata quella di incasellare $\mathrm{i}$ casi di studio in categorie che potessero essere funzionali all'indagine. L'individuazione ad esempio della tecnologia adottata, come criterio di suddivisione dei casi di studio, è apparsa da subito un fattore debole e insufficiente per distinguere categorie ben definite, in quanto gli strumenti di cui si parla sono stati utilizzati, almeno agli inizi, in maniera modulare nei modi più svariati e con un altissimo grado di sperimentazione. Ciò mi ha portato ad optare per una diversa strategia di analisi; sono stati dunque esaminati i 
casi di studio individuati tra le realizzazioni che hanno avuto più ampia eco nella letteratura professionale e è stata sviluppata un'indagine per 'faccette', individuando gli elementi meritevoli di approfondimento e traendo le opportune conclusioni.

La griglia si compone di varie partizioni logiche corrispondenti a cinque principali aree di analisi:

\section{Dati e database.}

Quest'area d'indagine riguarda il modo in cui l'OPAC viene ampliato per aggiungere oltre alle risorse possedute anche contenuti esterni, come articoli di riviste provenienti dalle basi dati e arricchimenti delle notizie bibliografiche attraverso il collegamento ad archivi di autorità, approfondimenti e altre risorse esterne. Questo aspetto rivela la recente evoluzione del catalogo elettronico verso la centralizzazione delle interfacce di ricerca, cioè la tendenza verso un punto unico di interrogazione che dai portali bibliotecari ha condotto ai discovery tools. Viene preso dunque in considerazione il modo di organizzare la metaricerca o ricerca estesa ossia una ricerca che deve potenzialmente includere il maggior numero di risorse disponibili, analizzando la tipologia dei dati recuperabili dal sistema.

\section{Ricerca nel catalogo}

In quest'area viene preso in considerazione il modo in cui è progettata la ricerca nell'OPAC. Uno degli aspetti più interessanti dei cataloghi elettronici di nuova generazione è proprio la realizzazione di interfacce per la ricerca che hanno caratteristiche molto diverse da quelle dei cataloghi elettronici tradizionali e che si ispirano a quelle presenti nel web. In rete la progettazione delle interfacce ha conosciuto nell'ultimo decennio un immenso sviluppo volto a riprogettare la ricerca per offrire un' 'esperienza utente' il più possibile soddisfacente. Negli OPAC si tende ad utilizzare quei modelli del web che si sono rivelati più efficaci e al contempo hanno trasmesso all'utente modelli mentali solidi influenzandone i comportamenti.

Si iscrivono in quest'ambito i seguenti campi di indagine:

* ricerca nel catalogo: vengono indagate le modalità di ricerca possibili (browsing, navigazione a faccette, uso dei filtri) e, nel caso della navigazione a faccette, le categorie utilizzate per il raffinamento (ambito disciplinare, livello 
della trattazione, audience, genere letterario, la data di creazione $\mathrm{o}$ pubblicazione, modalità di accesso al documento). La navigazione a faccette si richiama ad un modello 'esplorativo' che rovescia il paradigma query oriented degli OPAC tradizionali orientato alla domanda e rende la ricerca più simile alla scoperta (process oriented);

* funzionalità di aiuto per la creazione di interrogazioni al sistema: si tratta di alcune funzionalità correlate alla ricerca per parola chiave e strettamente dipendenti dal linguaggio del catalogo come la correzione dello spelling, lo stemming, e di altre tecniche come logica fuzzy che serve ad ampliare i risultati per evitare ricerche 'a risultato zero', l'autocompletamento.

\section{Visualizzazione dei risultati, del singolo record e navigazione nel catalogo}

La visualizzazione dei dati nel catalogo è forse l'aspetto più innovativo rispetto al passato, ma anche quello nel quale si concentrano le maggiori criticità; dei cataloghi esaminati verranno presi in considerazione:

* ordinamento per rilevanza (relevance ranking) dei risultati di un'interrogazione o esposizione dei risultati secondo il modello FRBR;

* possibilità di esaminare il full text del documento (funzione 'look inside' alla di Amazon e Google Books),

* visualizzazione delle immagini delle copertine, sommari e visualizzazioni intermedie;

* visualizzazione breve della notizia con l'indicazione delle opere correlate, delle varianti della stessa opera attraverso l'implementazione di FRBR o tramite clustering, cioè per mezzo di raggruppamenti automatici;

* visualizzazione di suggerimenti di lettura. Essi rispondono a domande del tipo: 'cosa posso leggere oltre sull'argomento?', 'qual è il miglior libro di partenza su questo dato argomento?' e assumono la forma: 'se hai letto xx potrebbe piacerti anche yy') o di suggerimenti anche in forma di mappe visive;

* ricerca orientata al contenuto ovvero al fulltext se esiste oppure, se non c'è, alla biblioteca che lo possiede o, nel caso il volume non sia posseduto da una biblioteca, alla libreria per l'acquisto; 
tecniche che permettono una certa serendipità nella ricerca e nella visualizzazione dei risultati della ricerca; uso delle interfacce visive e di modalità aggiuntive di esplorazione dei risultati.

\section{Funzioni- utente}

Oltre alle funzioni-utente previste in FRBR vengono rilevate le altre funzionalità offerte all'utente nella sua consultazione di un catalogo, come quelle di riutilizzo dell'informazione ricevuta:

* possibilità di riordino del set dei risultati;

* possibilità di avere spazi e servizi personalizzati all'interno dei cataloghi;

* possibilità di creare bibliografie pubbliche o private, con la possibilità di 'taggare', cioè aggiungere etichette ai documenti del catalogo;

* possibilità di creare profili utente; cioè di avere la propria pagina di inizio o disporre di tipi di ricerca predefiniti e display personalizzati;

* funzionalità che riguardano l'interazione e il design come il 'carrello della spesa', una memoria virtuale che segue l'utente durante il percorso di ricerca;

- servizi di notifica; possibilità di impostare avvisi (alert) nel catalogo per ricevere nella forma preferita (email, RSS) notizie circa la disponibilità di documenti o dei nuovi arrivi;

* liste di oggetti. I carrelli, le liste di acquisto, o liste dei desideri nei siti di commercio elettronico fungono da organizzatori di memoria o da scomparti temporanei di memoria;

* possibilità di cercare nel catalogo anche da siti esterni (un esempio sono i widget di WorldCat per Facebook);

* modalità che consentono una maggiore interazione dell'utente con il catalogo o con altri utenti, come ad esempio, le caratteristiche 'sociali', la possibilità di esprimere un giudizio sulle risorse presenti nel catalogo con tag, recensioni, commenti, con la conseguente possibilità di recuperare anche le discussioni intorno all'oggetto recuperato; la possibilità di creare communities o gruppi di lettura. 


\section{Funzioni e caratteristiche del sistema}

Si approfondiscono gli aspetti tecnologici. Tra questi:

* approccio service-layer. Ciò comporta la progettazione di OPAC non monolitici, ma modulari; lo sganciamento del catalogo dall'ILS (sistema di gestione sottostante) e un approccio 'a livelli' dei sevizi aggiuntivi;

* esposizione del contenuto attraverso open API in modo che il catalogo possa interagire con sistemi esterni e permettere a questi sistemi di formulare interrogazioni verso il catalogo o di fare harvesting dei dati del catalogo per i propri scopi;

* esposizione dei dati come linked data;

* uso del set di caratteri Unicode (consente di visualizzare senza errori alfabeti diversi);

* permalink. L'uso ormai generalizzato del permalink permette di utilizzare i dati all'esterno per vari scopi quali, ad esempio, aggiungere facilmente i dati a bibliografie, liste di letture, bollettini di novità e simili, o riutilizzarli in altre applicazioni del web.

Verrà inoltre analizzato il contesto nel quale l'OPAC è inserito, come è progettato l'ambiente di ricerca e quali sono le modalità di interazione dell'OPAC con gli altri strumenti predisposti dalle biblioteche, proponendo infine una breve discussione del caso, le immagini più rappresentative e i riferimenti bibliografici. 
Si fornisce di seguito l'elenco dei casi di studio esaminati

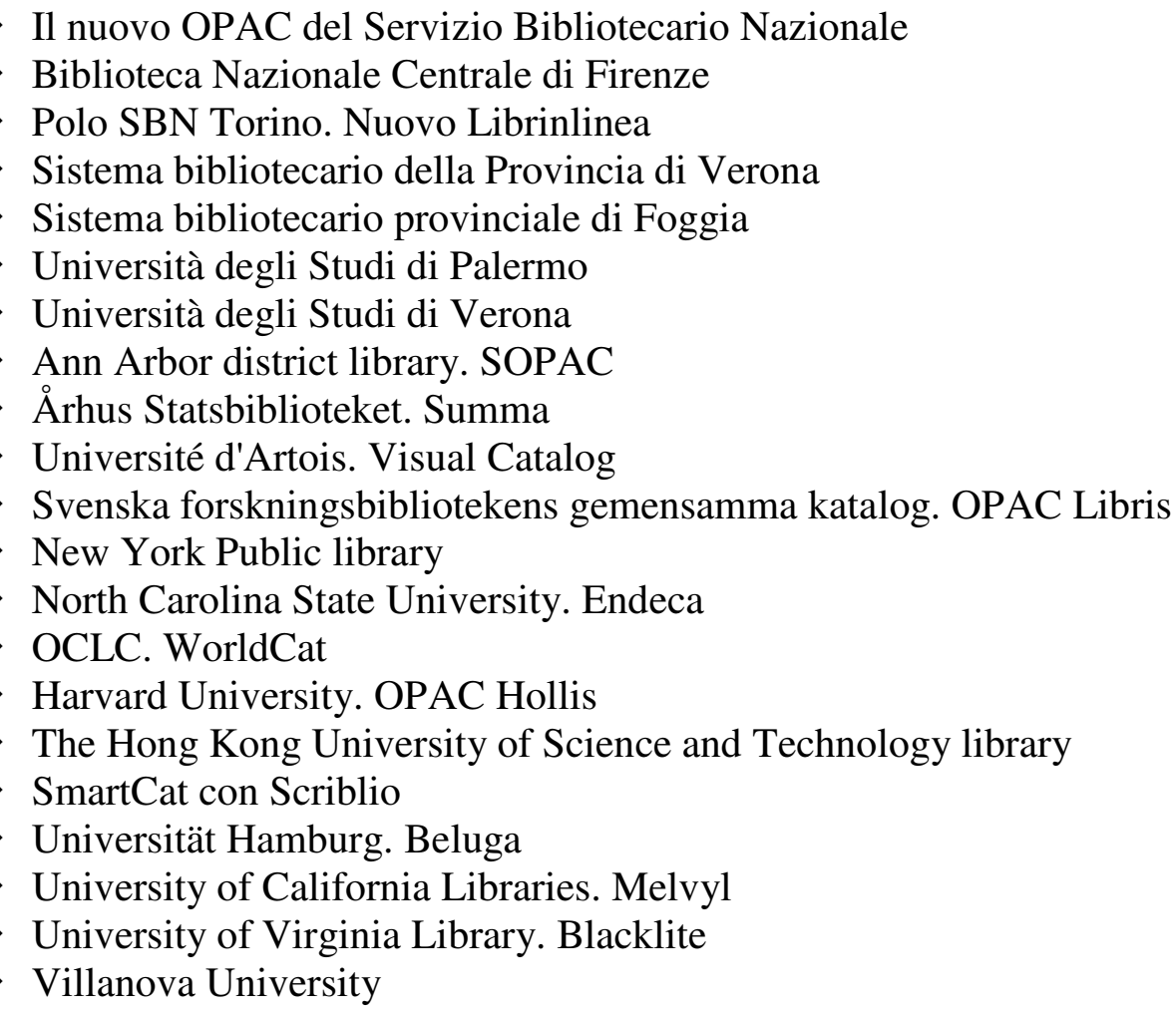




\section{Il nuovo OPAC del Servizio Bibliotecario Nazionale (SBN)}

URL: <http://opac.sbn.it/opacsbn/opac/iccu/free.jsp>

L'OPAC SBN è il catalogo collettivo delle biblioteche partecipanti al Servizio Bibliotecario Nazionale. Oggi SBN conta 81 poli per un totale di 4875 biblioteche ${ }^{1}$.

Il catalogo contiene le descrizioni e le localizzazioni di materiale librario e altre tipologie di documenti: materiale antico (pubblicazioni monografiche a stampa dal XV secolo fino al 1830), materiale moderno (pubblicazioni monografiche, comprese registrazioni sonore e video, archivi elettronici etc., a partire dal 1831 e pubblicazioni periodiche senza limiti di data), materiale scritto di interesse musicale (musica manoscritta, musica a stampa e libretti per musica), materiale grafico e materiale cartografico. Una novità è proprio la ristrutturazione della base dati relativa alla musica, che consente la ricerca di questo materiale.

Nel gennaio 2011 è stata lanciata in rete la versione 'beta', che è stata sostituita alla precedente versione dell'OPAC nel settembre 2011.

L'OPAC SBN consente l'accesso a 11.777 .646 notizie bibliografiche, corredate da 58.393 .052 localizzazioni $^{2}$.

Tra le particolarità della nuova interfaccia di ricerca l'adozione di software open source; in particolare l'OPAC utilizza il motore di indicizzazione e ricerca Lucene/SOLR che permette la navigazione 'a faccette' nei risultati e migliori prestazioni in fase di ricerca allineando il processo di ricerca alle interfacce dei next generation catalogs.

Tra le nuove funzionalità introdotte per la ricerca e la visualizzazione dei dati si segnalano la ricerca monocampo 'google like' nella home page con suggerimenti in fase di digitazione della query sulla base delle ricerche già effettuate dagli utenti.

Nella modalità Ricerca libera (con un singolo campo di testo) i termini inseriti vengono ricercati nei canali autore, titolo, soggetto, descrizione Dewey e concatenati mediante l'operatore logico "AND". I suggerimenti che compaiono durante la digitazione nel campo sono dovuti al meccanismo del "completamento automatico" basato sulle statistiche legate alla frequenza delle ricerche effettuate.

Con la Ricerca Base è possibile interrogare contemporaneamente tutte le notizie presenti nel Catalogo dell'Indice SBN. L'interrogazione viene effettuata attraverso i canali di ricerca comuni a tutta la base dati. Non richiede da parte dell'utenza nessuna conoscenza dell'uso degli operatori logici e di struttura. I canali di ricerca disponibili Autore, Titolo, Soggetto, Numero di Classificazione Dewey, Descrizione di Classificazione Dewey, Tutti i campi.

La ricerca avanzata prevede la possibilità di cercare notizie che soddisfino contemporaneamente fino a tre criteri di ricerca differenti. Per ciascun criterio sono disponibili: un menù a tendina dal quale selezionare un canale di ricerca; un campo di testo in cui inserire un termine da cercare nel canale selezionato; un menù a tendina dal quale selezionare la struttura dei termini inseriti ("parole in AND", "parole in OR", "frase"); un menù a tendina dal quale selezionare un operatore logico con cui combinare un criterio col canale successivo (solo per i primi due criteri, "AND", "OR", "AND NOT"). È possibile aggiungere dei filtri.

Le ricerche libera, di base e avanzata non permettono di rintracciare notizie che hanno campi non comuni come, ad esempio, la marca tipografica o l'impronta, che sono caratteristiche del libro antico. Per interrogare queste notizie si utilizzano le ricerche specializzate, che mettono a disposizione dell'utente quei canali non disponibili nelle maschere generiche. Attualmente sono disponibili le seguenti ricerche specializzate:libro antico, libro moderno, musica.

Tramite la Ricerca nelle voci di autorità è possibile visualizzare la scheda di un record di autorità.

Accesso all'OPAC /autenticazione

A tutti gli utenti del web / non vi sono aree che richiedono autenticazione

Possibilità di visualizzare l'interfaccia in più lingue

\footnotetext{
${ }^{1}$ Dato aggiornato ad agosto 2012.

${ }^{2}$ Dati aggiornati al 13/11/2011
} 


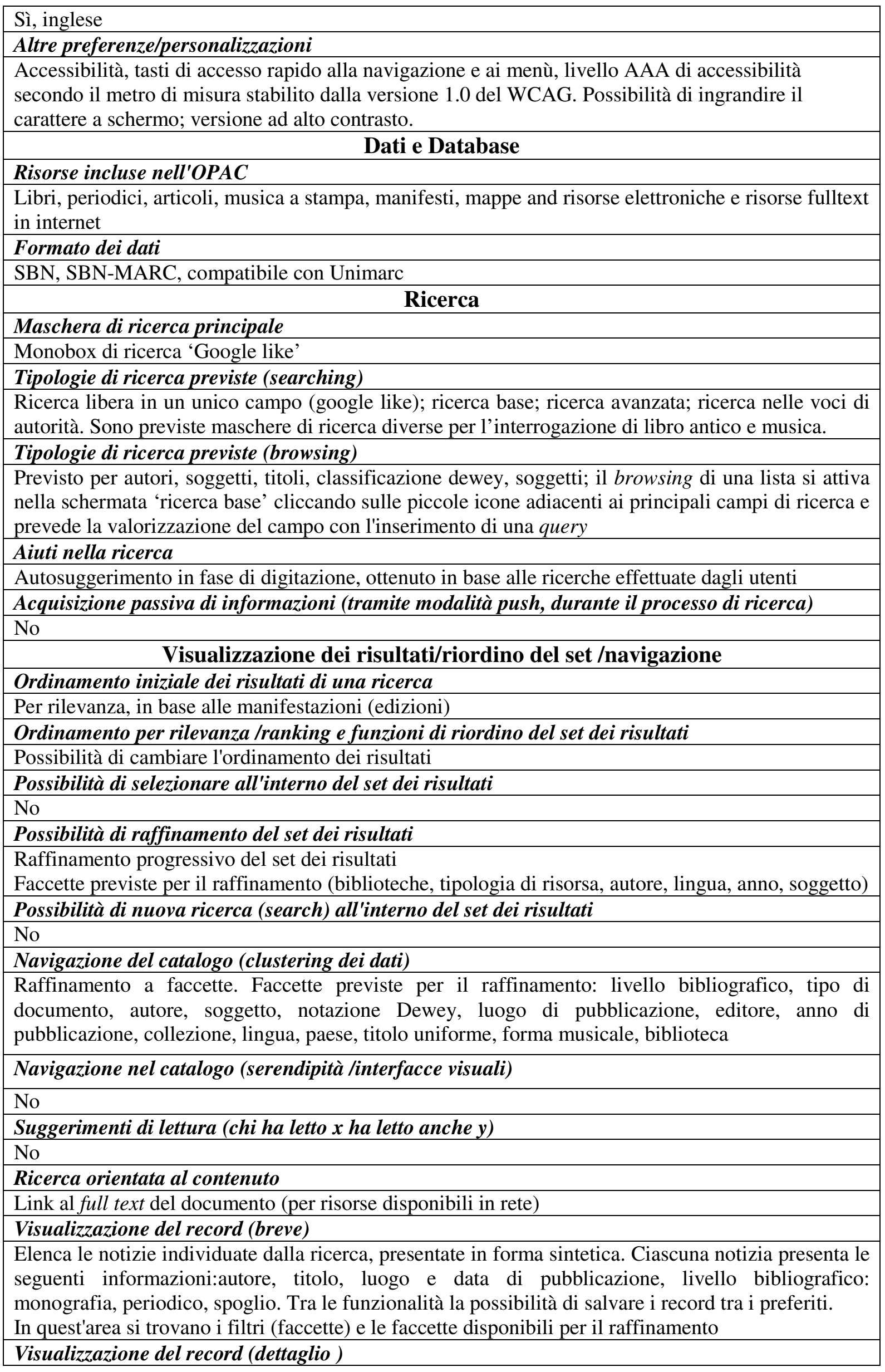


Descrizione bibliografica della notizia (campi attivabili lanciano una nuova ricerca: autori, soggetti, classi); link al record di autorità; Scelta della visualizzazione del formato: etichette, ISBD; link a scarico scheda in formato Unimarc, esportazione in Endnote e Refworks, esportazione della citazione (APA e MLA), aggiunta ai preferiti, stampa, email, prestito /richiesta del documento; elenco delle localizzazioni

\section{Contenitori temporanei /permanenti di memoria}

Funzione 'preferiti' (dal menu preferiti è poi possibile attivare funzioni per la stampa e l'esportazione). La cronologia dell ricerche effettuate viene memorizzata per l'intera durata della sessione di lavoro

\section{Funzioni di esportazione /riutilizzo/sociali}

\section{Esportazione della citazione del documento}

Sì, esportazione anche verso software di gestione bibliografie (RefWorks and EndNote)

Invio dei risultati per email / Stampa dei risultati

Sì, dal dettaglio record, dai preferiti

Altre modalità di riutilizzo dei dati

Sì, dal dettaglio record, dai preferiti

Condivisione nei social network

Sì

Permalink

Sì

Creazione di una lista personale di documenti

Lista dei preferiti, viene salvata solo nella sessione

Funzioni sociali (aggiunta di tag, recensione, valutazione)

No

RSS (syndication dei dati)

No

Interfacce/applicazioni per dispositivi mobili

No

Software utilizzato

\section{Caratteristiche del Sistema}

Copyright ICCU Istituto Centrale per il Catalogo Unico delle biblioteche italiane e per le informazioni bibliografiche - Realizzato da Inera s.r.l.

Tecnologie utilizzate

Open source, basato su Lucene e SOLR

Particolari implementazioni

I

\section{Ambiente di ricerca}

\section{l \\ Discussione}

Con la nuova versione del software SBN, basata sul motore di ricerca Lucene, l'OPAC del Servizio Bibliotecario Nazionale si è rinnovato nella grafica e nelle funzionalità offerte all'utenza.

La pagina dell'OPAC ha una presentazione pulita e semplificata ove spicca al centro della pagina con evidenza il monocampo di ricerca 'Google like'.

L'offerta documentaria recuperabile ricalca sostanzialmente quella offerta dal precedente OPAC con la possibilità di ricerca nelle basi dati libro antico, moderno, musica, grafica, cartografia e nell' authority file delle voci di autorità.

L'OPAC presenta ben tre modalità di interrogazione che consentono un buon approccio all'utente inesperto e mediamente esperto, ma allo stesso tempo accontentano i più esperti ricercatori che nella ricerca avanzata possono trovare un'ampia scelta di possibilità nei numerosi campi da combinare con i tradizionali operatori booleani. Nelle ricerche speciali (musica, cartografia, libro antico) l'utente può trovare campi di ricerca specifici per la particolare tipologia di materiale e non presenti nella maschera della ricerca base.

L'OPAC ha mantenuto una buona dotazione di possibilità per effettuare il browsing nelle liste. Èpossibile navigare agevolmente negli indici, anche se alcuni accorgimenti grafici migliorerebbero 
ulteriormente la comprensione dei meccanismi di interrogazione.

La formulazione dell'interrogazione viene facilitata grazie alla possibilità di sfruttare l'autocompletamento in fase di digitazione della query scegliendo tra i termini suggeriti dal sistema. La navigazione complessiva nel catalogo risulta, grazie alla navigazione 'a faccette', decisamente migliorata, grazie alla possibilità di utilizzare questa particolare tipologia di navigazione per orientare il proprio processo di ricerca.

L'esposizione dei risultati presenta l'ordinamento per ranking come opzione di default con la possibilità di riordinare il set e modificarlo dinamicamente in base alle faccette selezionate.

Le funzioni di aiuto sono esaustive e ben fatte. L'utente ha a disposizione una ricca pagina di spiegazioni suddivise per argomenti che può visualizzare in una finestra separata; particolarmente utile è il glossario dei termini attivabile dalle etichette durante l'esplorazione del catalogo

Le funzionalità di riutilizzo dei dati sono poche ma essenziali, come la possibilità di esportare liste $\mathrm{e}$ citazioni, nulle invece le funzionalità sociali.

Non vi è un'area personalizzata per l'utente, ma solo la possibilità di salvare le ricerche per la durata della sessione di ricerca.

Non c'è dubbio che il nuovo OPAC SBN rappresenti nel panorama italiano un avanzamento nella modalità di concepire le funzionalità dei cataloghi. Seppur progettato ad hoc il sistema coglie l'opportunità offerta dalle tecnologie open source già utilizzate in altri progetti (ad esempio nel progetto Blacklite ${ }^{3}$.

\section{Punti di forza / novità}

L'utilizzo di tecnologie open source, le funzioni di aiuto ben strutturate ed esaustive.

\section{Criticità}

Alcune carenze nell'espressività delle etichette relative alle funzioni di navigazione degli indici nella ricerca base (scansione liste titoli, autori e soggetti) ove sono stati mantenuti alcuni elementi grafici poco espressivi che potrebbero essere sostituiti più efficacemente da etichette esplicative.

L'autocompletamento in fase di digitazione potrebbe effettuare il matching sugli accessi nominali e semantici invece che sui termini derivati dalle ricerche già compiute a catalogo da altri utenti.

\section{Riferimenti bibliografici}

Daniela Gigli, Bibliografia sul Servizio Bibliotecario Nazionale 1979-2011, Roma, ICCU, Gennaio 2012, <http://www.iccu.sbn.it/opencms/export/sites/iccu/documenti/2012/Bibliografia_SBN_19792011.pdf >.

\section{Immagini}

\footnotetext{
${ }^{3}$ Cfr. infra, University of Virginia Library. Blacklite.
} 


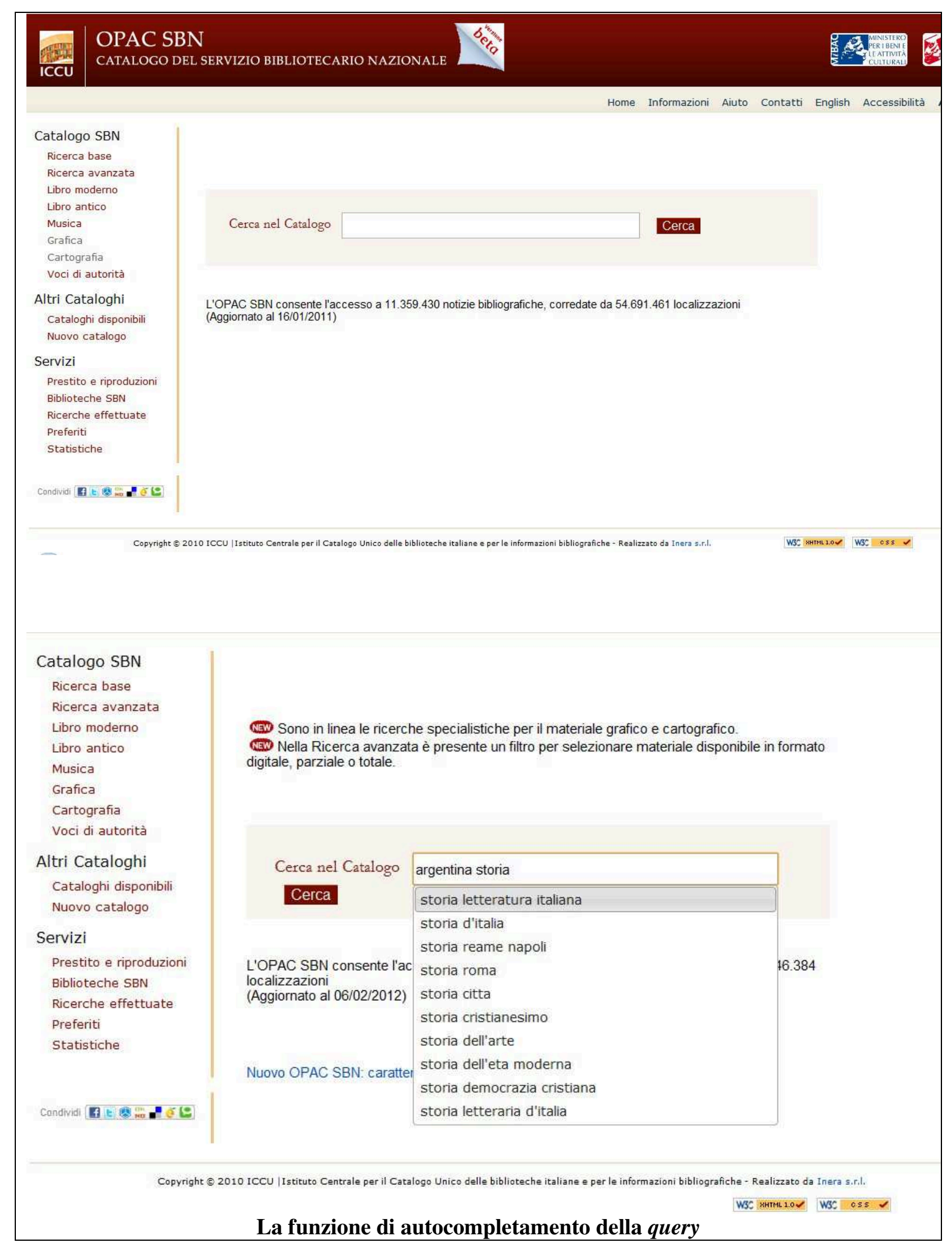




\section{Biblioteca nazionale centrale di Firenze}

URL: <http://opac.bncf.firenze.sbn.it/opac/controller.jsp>

Il catalogo on-line della BNCF attivo online dal 1985 consente le ricerche nella base dati del Polo BNCF della Biblioteca nazionale e di altre sette biblioteche del polo.

Nell'OPAC della Biblioteca nazionale centrale di Firenze è stata sperimentata a partire dal 2006 un'interfaccia utente nuova che si distingue per un' interpretazione più innovativa delle tipologie della ricerca (ricerca libera in evidenza, ricerca base e avanzata) e per l'adozione un navigatore Dewey progettato sulla prima versione di OCLC Dewey browser che consente di navigare agevolmente nel catalogo visualizzando l'albero classificatorio (primi tre livelli di profondità).

\section{Accesso all'OPAC /autenticazione}

Box unico di ricerca nella pagina web della biblioteca; pagina dell'OPAC

Possibilità di visualizzare l'interfaccia in più lingue

Sì, inglese

Altre preferenze/personalizzazioni

No

\section{Dati e Database}

Risorse incluse nell'OPAC

Libri, periodici, articoli, musica a stampa, manifesti, mappe and risorse elettroniche e risorse

digitalizzate

Formato dei dati

SBN

Maschera di ricerca principale

Box di ricerca per parola chiave

Tipologie di ricerca previste (searching)

Ricerca in un box unico (google like) + ricerca base+ ricerca avanzata; la ricerca base permette di combinare fino a quattro campi di ricerca $i$ cui elementi sono forniti in un menu a discesa (i campi previsti sono: Autore, Titolo, Soggetto, Collana, Classe (numero CDD) Classe (parola chiave CDD); Luogo; Editore; Identificatori standard (numero ISBN, BNI, CUBI, impronta ecc.); Marca; Collocazione; Parole chiave; Identificativo titolo; Identificativo autore; Identificativo soggetto; Inventario

Ricerca avanzata (per linea di comando) o ricerca esperta sono disponibili dal menu principale; la ricerca permettere di combinare i dati provenienti da più campi con gli operatori booleani e operatori fuzzy. Se in ricerca libera, ricerca base (con l'accortezza di riempire sempre il primo campo), ricerca avanzata, il sistema non riesce a trovare nessun documento con i termini digitati, viene presentata un'ulteriore opzione di ricerca: la ricerca per termini simili.

Tipologie di ricerca previste (browsing)

Esame della lista dei titoli, degli autori e dei soggetti (deve essere attivata dall'utente tramite l'inserimento di un termine iniziale in un box di ricerca); navigatore Dewey

Aiuti nella ricerca

Se in ricerca libera, ricerca base, ricerca avanzata, il sistema non riesce a trovare nessun documento con i termini digitati, viene presentata un'ulteriore opzione di ricerca: la ricerca per termini simili che può essere attivata dall'utente tramite un pulsante. La funzione non propone dei termini ma lancia l'interrogazione che propone un nuovo set di risultati.

Acquisizione passiva di informazioni (tramite modalità push, durante il processo di ricerca) No

\section{Visualizzazione dei risultati/riordino del set /navigazione}

Ordinamento iniziale dei risultati di una ricerca

Per rilevanza, in base alle manifestazioni (edizioni)

Ordinamento per rilevanza/ranking e funzioni di riordino del set dei risultati

Programmabile a partire dal set di risultati / si, per rilevanza, anno decrescente, autore titolo 
Possibilità di selezionare all'interno del set dei risultati

Tutti i documenti, digitalizzazioni, libri, tesi di dottorato, periodici, carte geografiche, editoria digitale, manoscritti, musica a stampa, grafica

Possibilità di raffinamento del set dei risultati

No

Possibilità di nuova ricerca (search) all'interno del set dei risultati

No

Navigazione del catalogo (clustering dei dati)

No

Navigazione nel catalogo (serendipità /interfacce visuali)

No

Suggerimenti di lettura (chi ha letto $x$ ha letto anche $y$ )

No

Ricerca orientata al contenuto

Link al full text del documento (per edizioni digitalizzate)

Visualizzazione del record (breve)

Comprende: autore, titolo, data, tipo di risorsa

Visualizzazione del record (dettaglio )

Comprende: Autore, Titolo /autore principale e secondario (area 1), isbn, luogo, editore, data, collana, soggetti e classi (navigabili), tipologia di materiale, lingua, elenco delle localizzazioni,

link al record in formato unimarcxml

Contenitori temporanei /permanenti di memoria

No

\section{Funzioni di esportazione /riutilizzo/sociali}

Esportazione della citazione del documento

Sì, esportazione apre una finestra del browser con un file testuale

Invio dei risultati per email / Stampa dei risultati

$\mathrm{No} / \mathrm{No}$

Altre modalità di riutilizzo dei dati

No

Condivisione nei social network

No

Permalink

No

Creazione di una lista personale di documenti

No

Funzioni sociali (aggiunta di tag, recensione, valutazione)

No

RSS (syndication dei dati)

No

Interfacce/applicazioni per dispositivi mobili

No

\section{Caratteristiche del Sistema}

Software utilizzato

Opac Versione: 1.0 - 5/2/2006 - Servizi informatici, Biblioteca nazionale centrale Firenze. Sviluppo:

Webdev

Tecnologie utilizzate

Proprietaria

Particolari implementazioni

I

Ambiente di ricerca 


\begin{tabular}{|c|}
$\begin{array}{l}\text { La biblioteca digitale e i repertori elettronici, i cataloghi storici hanno accessi separati nel sito web } \\
\text { della biblioteca. }\end{array}$ \\
\hline Discussione \\
\hline La Bibliseca nazo
\end{tabular}

La Biblioteca nazionale centrale di Firenze è stata una delle prime istituzioni nel nostro paese a rimodellare il proprio OPAC in base alle tendenze dei nuovi next generation catalogs. Già nel 2006 introdusse nell'OPAC il box unico, rimodulando le modalità di ricerca offerte all'utente. Di recente ha incorporato il box unico di ricerca nella home page della biblioteca. Resta ancora un buon esempio di sviluppo l'originale navigatore Dewey, che consente il browsing delle classi consentendo all'utente di familiarizzare con il sistema di organizzazione delle conoscenza adottato.

Dal maggio 2006 tutte le digitalizzazioni della BNCF sono consultabili dall'OPAC e dalla sezione Le collezioni digitali con un nuovo visualizzatore Internet Archive BookReader, reader open source sviluppato da Internet Archive per Open Library. L'OPAC negli ultimi anni, tuttavia, non ha conosciuto altre evoluzioni e lo sviluppo è fermo alla versione 1.0 pubblicata nel 2006.

\section{Punti di forza / novità}

La possibilità di utilizzare uno strumento di browsing delle classi favorisce il recupero dell'informazione facilitando al contempo l'apprendimento del sistema di organizzazione delle conoscenze utilizzato (Classificazione Decimale Dewey). Questa modalità di navigazione facilita la scoperta di nuove informazioni.

\section{Criticità}

Nella progettazione dell'OPAC non sono state implementate le più comuni funzionalità presenti in interfacce di nuova generazione.

\section{Riferimenti bibliografici}

Bonaria BIANCU, Opac 2.0: BNCF, «The Geek Librarian», Blog post, 28 febbraio 2006. $<$ http://bonariabiancu.wordpress.com/2006/02/28/opac-20-bncf $>$.

\section{Immagini}

$\begin{array}{lll}\text { A. dettaglio documento } & \times \\ 3 & \text { BIBLIOTECA NAZIONALE CENTRALE DI FIRENZE }\end{array}$

ricerca libera | ricerca base | ricerca avanzata | lista titoli | lista autori | lista soggetti | navigatore dewey | (2) aiuto | crediti

Documento 6 di 1104

Bosso, Bianca

Casa di bambola / Bianca Bosso

Bologna : Calderini, [1996].

$x, 116 \mathrm{p}$. : ill. ; $23 \mathrm{~cm}$

ISBN 88-7019-996-7. BN 97-8066.

1. Case di bambola
745.5923 (ed. 20) - ARTIGIANATO. CASA E MOBILI PER BAMBOLE

Monografia - Materiale a stampa - Pubbl. in: Italia - Lingua: italiano

Bibl. Nazionale Centrale di Firenze

Gli utenti registrati possono accedere ai servizi della biblioteca scegliendo il mumero di inventario del documento desiderato. Controllare modalità e orario dei servizi

Collocazione: GEN B10 03356

Inventario: $\quad$ CF005285957 $1 \mathrm{v}$

Digitalizzazione parziale

$$
\begin{aligned}
& \text { record in formato unimarcxml } \\
& \text { Aggiornato al: } 14 / 08 / 2008(2.066 .040 \text { monografie e } 151.581 \text { periodici) }
\end{aligned}
$$




\section{Polo SBN Torino. Nuovo Librinlinea}

URL: <http://www.librinlinea.it >

Librinlinea è lo strumento di ricerca delle biblioteche che aderiscono al Polo piemontese TO0 del Servizio Bibliotecario Nazionale e consente di interrogare il catalogo delle biblioteche del polo ed accedere ai servizi bibliografici.

Il catalogo contiene i documenti (libri, periodici, manoscritti, spogli) pervenuti nelle biblioteche dalla data della loro adesione a SBN.

La nuova versione dell'OPAC è stata lanciata il 23 dicembre 2011 in versione beta per sostituire l'interfaccia precedente <http://sbnweb.csi.it:8092/semplice.html > che è rimasta visibile per qualche mese.

L'OPAC consente di effettuare ricerche bibliografiche per singola biblioteca o per gruppi, di accedere alle informazioni contenute nella base dati nazionale, di proporre ad una biblioteca l'acquisto di nuovi libri e di verificare la disponibilità di un volume presso una biblioteca per chi desiderasse prenderlo in prestito.

Tra le innovazioni rispetto al precedente OPAC vi sono la ricerca 'monobox', i contenitori temporanei di memoria, alcuni arricchimenti (link esterni a Wikipedia, immagini delle copertine e una grafica decisamente migliorata.

Di recente, la nascita nell'area piemontese di nuovi sistemi bibliotecari e l'arrivo sulla scena di software italiani per interfacce di nuova generazione compatibili con SBN ha ridotto il numero delle biblioteche aderenti. Tra le biblioteche che non sono più rappresentate spiccano le Biblioteche Civiche Torinesi, passate nel 2012 al software Comperio.

Tuttavia, il processo di rinnovamento del sistema è stato premiato dall'utenza: gli accessi a Librinlinea nel 2012 sono aumentati in maniera considerevole: da 22.676 accessi di utenti unici nel mese di Gennaio 2012, a più di 46.300 visite nel mese di Giugno per un totale di 213.000 visite nel primo semestre con oltre un milione e settecento mila pagine visualizzate (fonte CSI Piemonte).

Tra le novità recenti un'applicazione per dispositivi mobili, che consente di interrogare i cataloghi e accedere ai servizi da cellulari, smartphone e tablet ${ }^{4}$.

L'applicazione consente di cercare i documenti attraverso il titolo, l'autore, il codice ISBN o cercare le biblioteche più vicine. Ogni scheda del catalogo contiene oltre alle informazioni sul documento, la localizzazione e il percorso per raggiungere la biblioteca che lo possiede.

Lo sviluppo dell'OPAC è in continua evoluzione; nel 2013 il software è stato nuovamente aggiornato, aggiungendo la possibilità di utilizzare i filtri per circoscrivere la ricerca, una nuova versione è stata annunciata per febbraio 2013.

Accesso all'OPAC /autenticazione

Dalla pagina dedicata /no

Possibilità di visualizzare l'interfaccia in più lingue

No

Altre preferenze/personalizzazioni

No

Risorse incluse nell'OPAC

Libri, periodici, manoscritti, spogli

Formato dei dati

SBN

Maschera di ricerca principale

Monobox (di tipo Google) con ricerca su tutti i campi

Tipologie di ricerca previste (searching)

Ricerca su tutti campi, ricerca avanzata

${ }^{4}<$ http://www.csipiemonte.it/app/gallery/librilinea.php $>$ 


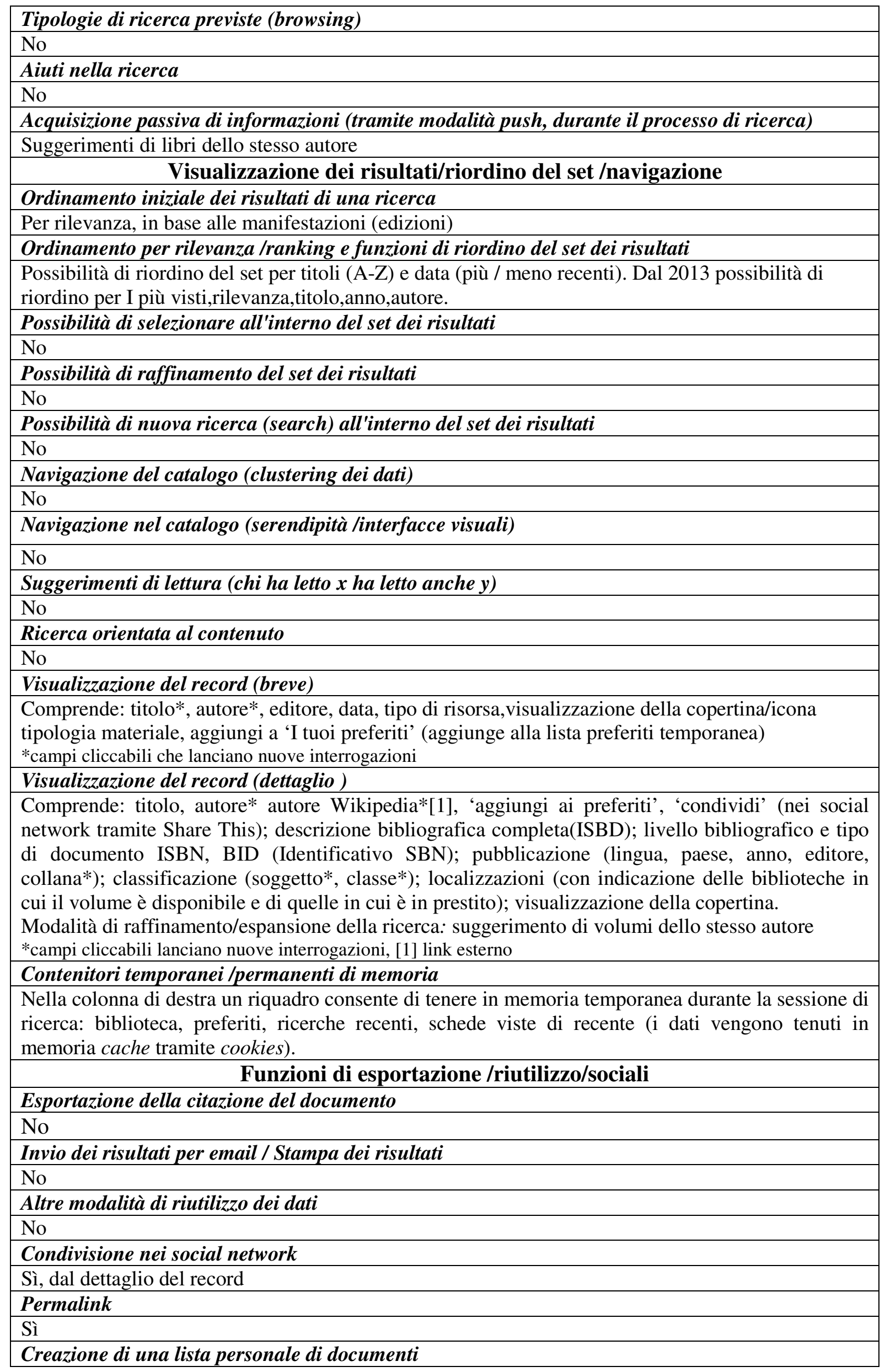




\begin{tabular}{|c|}
\hline $\begin{array}{l}\text { No } \\
\text { Funzioni sociali (aggiunta di tag, recensione, valutazione) }\end{array}$ \\
\hline No \\
\hline RSS (syndication dei dati) \\
\hline No \\
\hline Interfacce/applicazioni per dispositivi mobili \\
\hline Sì, è stata creata un'apposita App per dispositivi Apple e Android \\
\hline Caratteristiche del Sistema \\
\hline Software utilizzato \\
\hline Librinlinea, sviluppo CSI Piemonte CSI Piemonte per Regione Piemonte \\
\hline Tecnologie utilizzate \\
\hline Proprietarie, sviluppate da CSI Piemonte \\
\hline Particolari implementazioni \\
\hline I \\
\hline Ambiente di ricerca \\
\hline I \\
\hline Discussione \\
\hline $\begin{array}{l}\text { Librinlinea rappresenta il tentativo della Regione Piemonte di offrire agli utenti delle biblioteche } \\
\text { piemontesi del polo TO0 uno strumento di ricerca unificato allineato ai più recenti sviluppi dei } \\
\text { software bibliotecari per quanto riguarda l'interfaccia e le funzionalità. } \\
\text { Nonostante gli elementi di novità che il sistema presenta l'interfaccia rimane alquanto statica e la } \\
\text { rappresentatività delle risorse incluse e delle funzionalità lo rendono simile ad un OPAC } \\
\text { tradizionale. } \\
\text { I contenuti sono gli stessi offerti dal precedente OPAC, limitati alle risorse tradizionali a stampa } \\
\text { delle biblioteche rappresentate. } \\
\text { Mancano nel software alcune funzionalità ormai diffuse nelle più comuni interfacce di nuova } \\
\text { generazione: funzioni sociali, la possibilità di navigare adeguatamente nella lista dei risultati, la } \\
\text { presenza di veri e propri filtri, l'applicazione di raggruppamenti in base alle entità del modello } \\
\text { FRBR che rendono la navigabilità dell'OPAC piuttosto limitata. Una volta effettuata la ricerca, vi è } \\
\text { la possibilità di applicare filtri per restringere la ricerca, ma non è possibile correggere il tiro o } \\
\text { tornare indietro; assenti anche aiuti automatici in fase di ricerca. Risultano, infine, del tutto assenti } \\
\text { funzionalità di browsing come la navigazione in liste di soggetti o classi. } \\
\text { Altrettanto carente si presenta la disponibilità di funzioni di esportazione e di riutilizzo dei dati } \\
\text { bibliografici contenuti nel catalogo, che è limitata alla sola esportazione nei social network e all'e- } \\
\text { mail (fornite dal noto servizio gratuito Share This }{ }^{5} \text { ). }\end{array}$ \\
\hline $\begin{array}{l}\text { Significativa è anche la perdita di funzionalità che l'OPAC offriva nella sua versione precedente } \\
\text { come quelle di personalizzazione: agli strumenti tradizionali di ricerca; Librinlinea affiancava la } \\
\text { possibilità di interrogazioni mediante profili di interesse definibili e modificabili dall'utente: tale } \\
\text { funzione di disseminazione selettiva delle informazioni (DSI) consentiva inoltre l'invio periodico } \\
\text { all'utente, tramite casella di posta elettronica o SMS, delle novità bibliografiche previste dal suo } \\
\text { profilo. } \\
\text { Ancor più penalizzante a mio avviso la perdita di funzionalità di ricerca semantica e di browsing } \\
\text { che il vecchio sistema offriva all'utente, come la possibilità dello scorrimento degli indici dei } \\
\text { soggetti che viene del tutto eliminata nel nuovo sistema (mentre nel precedente sistema era } \\
\text { possibile, sebbene dalla ricerca avanzata e con funzionalità limitate). } \\
\text { Infine il miglioramento complessivo della grafica e della leggibilità generale ha reso l'interfaccia } \\
\text { più leggera rispetto al precedente sistema, tuttavia tali miglioramenti non sono sufficienti a farne } \\
\text { uno strumento evoluto. }\end{array}$ \\
\hline $\begin{array}{l}\text { Punti di forza / novità } \\
\text { Il miglioramento dell'interfaccia grafica. L'OPAC piemontese è tra i primi nel nostro paese a }\end{array}$ \\
\hline
\end{tabular}

${ }^{5}<$ http://www.sharethis.com>. 


\begin{tabular}{|l|}
\hline $\begin{array}{l}\text { disporre di un'interfaccia per la consultazione su dispositivi mobili. } \\
\text { Criticità } \\
\text { La limitatezza delle funzionalità di ricerca e navigazione e l'assenza di funzioni di browsing. }\end{array}$ \\
\hline Riferimenti bibliografici \\
\hline Immagini \\
\hline
\end{tabular}




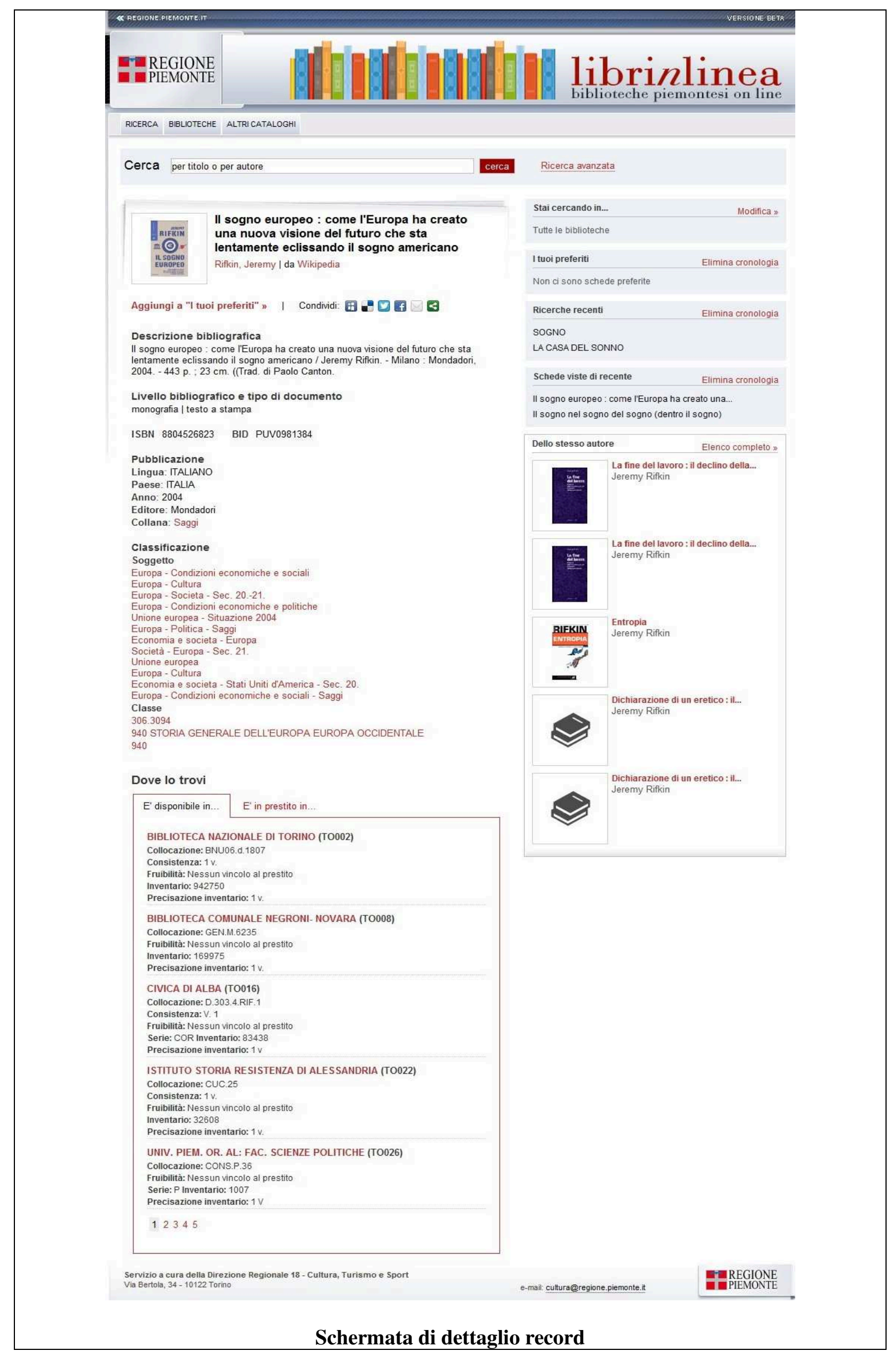




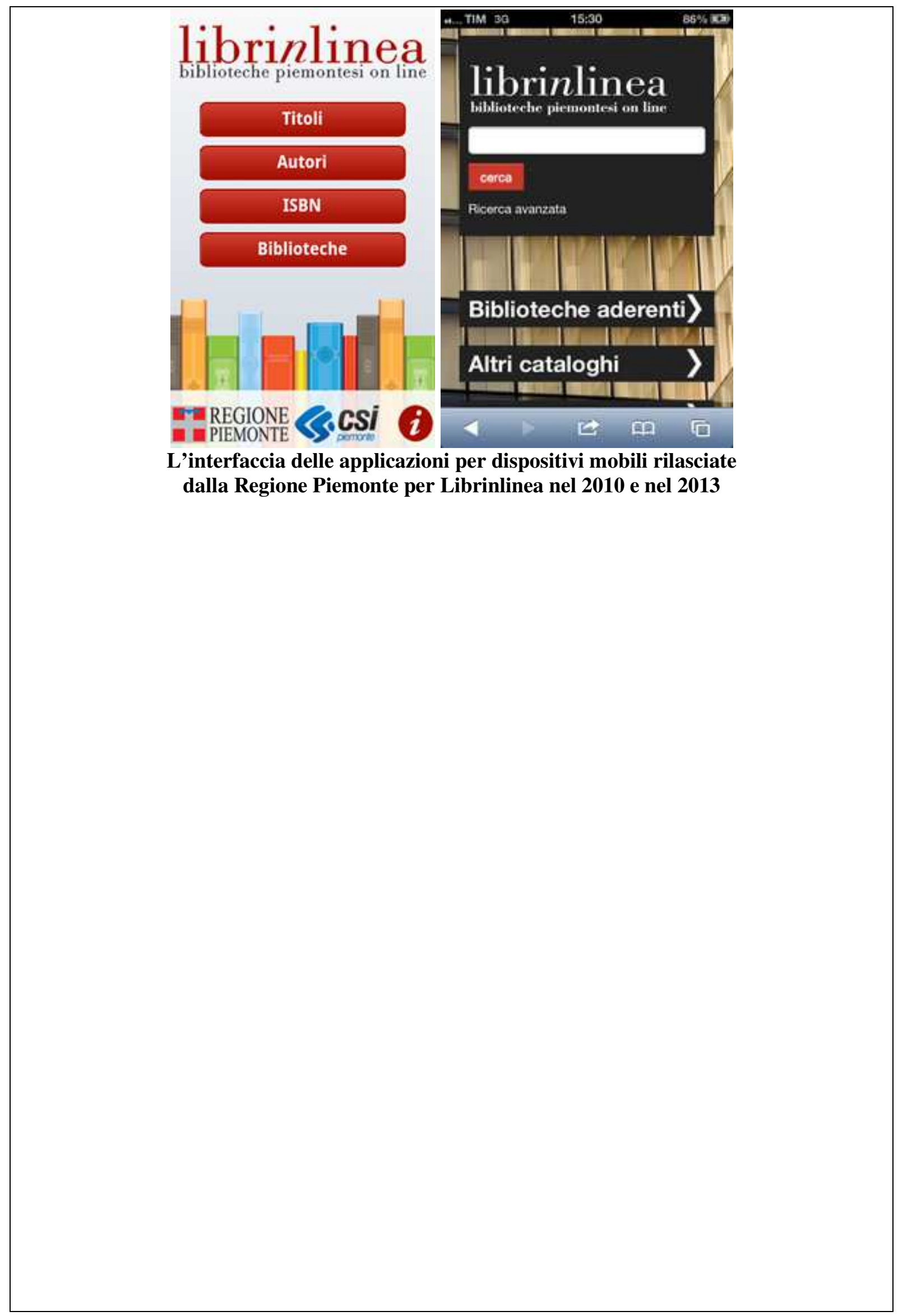




\section{Sistema bibliotecario della Provincia di Verona}

URL: <http://sbp.provincia.verona.it/>

L'OPAC del Sistema bibliotecario di Verona (SBPVr) rappresenta un valido esempio di OPAC 'sociale' o '2.0', denominazione in uso a partire dal 2006 per indicare quei cataloghi che hanno privilegiato le funzionalità tipiche dei social network.

Comperio, la ditta produttrice del software, è stata la prima azienda italiana ad ottenere dall'ICCU la certificazione di compatibilità con il protocollo SBNMarc, pertanto una delle prime aziende in grado di sviluppare un OPAC innovativo nello stagnante panorama italiano.

Il sistema veronese è stato uno dei primi ad utilizzare le soluzioni software di Comperio adottando il gestionale Clavis e, successivamente, il sistema di ricerca Discovery NG per l'OPAC di nuova generazione sostituendo il precedente software Sebina. Il progetto viene avviato già nel 2004 quando è messa in opera la prima versione del gestionale Clavis presso il Sistema Bibliotecario della Provincia di Verona. Il sofware è basato sul Content management system Silverstripe.

Accesso all'OPAC /autenticazione

Accesso integrato nella home page del portale della biblioteca

Possibilità di visualizzare l'interfaccia in più lingue

No

Altre preferenze/personalizzazioni

/

Risorse incluse nell'OPAC

Dati e Database

Risorse tradizionali

Formato dei dati

SBN-MARC, con possibilità di 'uscita' nel formato MARCXML

Maschera di ricerca principale

Box di ricerca 'Google like'

Tipologie di ricerca previste (searching)

Box 'Google like' con modalità di ricerca su tutti i campi e avanzata

Tipologie di ricerca previste (browsing)

Browsing dei nuovi titoli (vetrina), della nuvola dei tag, dei titoli più letti, più prestati, consigliati (dalla home page)

Aiuti nella ricerca

No

Acquisizione passiva di informazioni (tramite modalità push, durante il processo di ricerca) No

Ordinamento iniziale dei risultati di una ricerca

Per rilevanza, in base alle manifestazioni (edizioni)

Ordinamento per rilevanza /ranking e funzioni di riordino del set dei risultati

Possibilità di aggiungere o eliminare una faccetta ricomponendo il set dei risultati

Possibilità di selezionare all'interno del set dei risultati

Tra i documenti di una specifica biblioteca aderente al sistema

Possibilità di raffinamento del set dei risultati

Raffinamento progressivo del set dei risultati

Faccette previste per il raffinamento (livello, materiale, nomi, editore, lingua, data, paese soggetto)

altre faccette sono chiuse per default

Possibilità di nuova ricerca (search) all'interno del set dei risultati

No

Navigazione del catalogo (clustering dei dati) 


\begin{tabular}{|c|}
\hline Uso dei filtri per navigare tra i risultati \\
\hline Navigazione nel catalogo (serendipità /interfacce visuali) \\
\hline Vetrina delle novità \\
\hline Suggerimenti di lettura (chi ha letto $x$ ha letto anche y) \\
\hline Nella home page vengono mostrati elenchi dei libri più letti, più prestati, consigliati \\
\hline Ricerca orientata al contenuto \\
\hline Link al full text del documento (per risorse disponibili in rete) \\
\hline Visualizzazione del record (breve) \\
\hline $\begin{array}{l}\text { Autore (cliccabile), titolo(cliccabile), titolo/autore luogo editore, data, tipo di risorsa, link al record } \\
\text { (dettaglio), visualizzazione della copertina, pulsanti per commenti, scaffali, prenota, numero di } \\
\text { copie totali, in prestito, prenotate, abstract. Per visualizzare gli scaffali e prenotare è necessario } \\
\text { autenticarsi }\end{array}$ \\
\hline Visualizzazione del record (dettaglio ) \\
\hline $\begin{array}{l}\text { Titolo e contributi, pubblicazione, descrizione fisica, serie, numero standard, data, lingua, paese, } \\
\text { altri titoli, note (estratte dalla descrizione), nomi (è segnalata la responsabilità principale), soggetti, } \\
\text { genere, classi (gli ultimi } 3 \text { sono cliccabili e lanciano una nuova ricerca) } \\
4 \text { tabs separano le pagine: Localizzazioni con indicazione in tempo reale dello stato del volume e } \\
\text { delle condizioni di prestabilità (vista di default), sommario, dettagli, allegati, commenti } \\
\text { Sono presenti le funzioni sociali vota, commento, scaffali, prenota }\end{array}$ \\
\hline Contenitori temporanei /permanenti di memoria \\
\hline $\begin{array}{l}\text { Area personale per salvataggio richieste, creazioni di liste private e pubbliche. Dall'area personale è } \\
\text { possibile visualizzare i commenti e i post inseriti nel forum, gestire un proprio profilo pubblico per } \\
\text { la community, visualizzare gli scaffali pubblici, il proprio nome, avatar e minibiografia. Dall'area } \\
\text { personale è possibile gestire i tradizionali servizi (prestiti, rinnovi, salvataggio di sessioni etc.) }\end{array}$ \\
\hline Funzioni di esportazione /riutilizzo/sociali \\
\hline Esportazione della citazione del documento \\
\hline No \\
\hline Invio dei risultati per email / Stampa dei risultati \\
\hline Sì, dall'area personale \\
\hline Altre modalità di riutilizzo dei dati \\
\hline I \\
\hline Condivisione nei social network \\
\hline No \\
\hline Permalink \\
\hline No \\
\hline Creazione di una lista personale di documenti \\
\hline Sì, nell'area personale vi sono 3 scaffali predefiniti e si possono creare altri scaffali \\
\hline Funzioni sociali (aggiunta di tag, recensione, valutazione) \\
\hline Tag, recensioni, valutazioni \\
\hline RSS (syndication dei dati) \\
\hline No \\
\hline Interfacce/applicazioni per dispositivi mobili \\
\hline No \\
\hline Caratteristiche del Sistema \\
\hline Software utilizzato \\
\hline Comperio Discovey NG 3.0 \\
\hline Tecnologie utilizzate \\
\hline Open source \\
\hline Particolari implementazioni \\
\hline l \\
\hline Ambiente di ricerca \\
\hline $\begin{array}{l}\text { L'OPAC è affiancato da MOV un sistema di ricerca multicatalogo (metaopac), che interroga in } \\
\text { modo cumulativo le reti bibliotecarie del territorio veronese. MOV integra il posseduto presente in }\end{array}$ \\
\hline
\end{tabular}


tre cataloghi collettivi: Catalogo collettivo dell'Università di Verona (Università di Verona); Archivio bibliografico veronese (Comune di Verona); Sistema bibliotecario della Provincia di Verona (SBPVr). Oltre alle reti bibliotecarie veronesi, MOV interroga anche altre fonti bibliografiche:

* OAIster (un catalogo di risorse digitali di carattere accademico come libri elettronici, $e$ journals, file audio, immagini, film, testi di consultazione raccolti da più di 700 archivi istituzionali, usando il protocollo Open Archives Initiative per la raccolta dei metadati. Normalmente ogni notizia bibliografica presenta un link al full text o all'oggetto digitale liberamente disponibile: tuttavia la collezione include anche materiale ad accesso riservato.

* Open Directory Project ovvero una delle più grandi directory del Web costruita e mantenuta da una vasta comunità globale di editori volontari.

* Progetto Gutenberg: una vasta collezione di versioni elettroniche liberamente riproducibili di libri stampati.

\section{Discussione}

Nell'economia del portale del sistema veronese (<http://sbp.provincia.verona.it/>) l'OPAC riveste un ruolo primario, con l'accesso al centro della home page rappresentato dal box di ricerca libera su tutti i campi di tipo 'google like'. Attorno all'OPAC ruota tutta la costruzione della homepage del sito, che presenta vari spazi che accolgono dati provenienti dal catalogo. Èil caso di 'Ultimi arrivi' e 'Classifiche' mentre 'Ultimi blogpost' riporta le recenti discussioni del blog, vera novità intesa appunto a rafforzare la comunità di utenti.

Per quanto riguarda i contenuti recuperati, il catalogo del SBPVr può considerarsi a tutti gli effetti un OPAC di nuova generazione. Non è quindi un sistema per la scoperta in quanto le risorse ivi rappresentate sono quelle tradizionali formate dalle raccolte presenti in biblioteca.

Riguardo alle funzioni di ricerca, il catalogo della biblioteca è fruibile già dalla homepage del sito, dove è possibile attivare la ricerca su tutti i campi o andare alla schermata della ricerca avanzata. Già nella homepage uno spazio è dedicato al browsing del catalogo in base a tematiche o "scaffali virtuali" di volta in volta predisposti dai bibliotecari (proposte di viaggio, lo scaffale dei classici, donne e lavoro etc.) o iniziando dalla nuvola dei tag più usati.

Il catalogo non incorpora caratteristiche avanzate come correzione dello spelling, stemming o logica fuzzy. Se se non vi è corrispondenza tra la parola cercata e il termine presente a catalogo la ricerca ha risultato zero.

Esaminando la disposizione dei risultati e le funzionalità di navigazione, i risultati vengono presentati ordinati per titolo o per data, con un elenco di manifestazioni affiancato dalle faccette per raffinare i risultati stessi; non è ancora prevista la possibilità di esaminare il full text del documento (funzione Search/look inside alla Amazon o Google Books). Ogni record bibliografico è arricchito con TOC e copertine; tra le particolarità l'indicazione 'Destinatari' riferito alla tipologia di utenza alla quale il documento è destinato. Interessante anche l'indicazione delle 'Proposte' in calce al record: si tratta dei suggerimenti di lettura alla Amazon 'se hai letto xx potrebbe piacerti yy'; altri arricchimenti presenti sono allegati, esemplari e recensioni. Le responsabilità principale e secondaria nel record non sono distinguibili poiché sono accorpate sotto un'unica etichetta "nomi". La ricerca può essere impostata solo sui record disponibili per il prestito.

La ricerca semantica lascia un po' a desiderare; dalla visualizzazione per record è possibile attivare la navigazione sui titoli che hanno lo stesso soggetto; la classificazione contiene gli equivalenti verbali ma non permette di lanciare una navigazione per classi. Allo stesso modo le classi non figurano tra le faccette per il raffinamento.

Tale le funzioni utente previste, il catalogo indubbiamente privilegia quelle 'sociali' tese a creare una comunità di lettori e fidelizzare gli utenti delle biblioteche del sistema. Nell'OPAC ogni lettore può creare il proprio profilo, interagire nel blog e commentare le risorse.

Il catalogo contiene i commenti e i post del blog degli utenti e dei bibliotecari, ma anche i voti (ratings) e i tag inseriti dagli utenti alle risorse catalografiche.

Anche l'area personale My Discovery, accessibile tramite un link nella homepage, conserva questa impostazione 'sociale'; si può a gestire il proprio profilo (scegliere un avatar, password, inserire informazioni personali), consultare i prestiti attuali e lo storico dei prestiti, visionare e modificare lo stato delle prenotazioni attuali, inserire nuovi messaggi del blog e molto altro. 


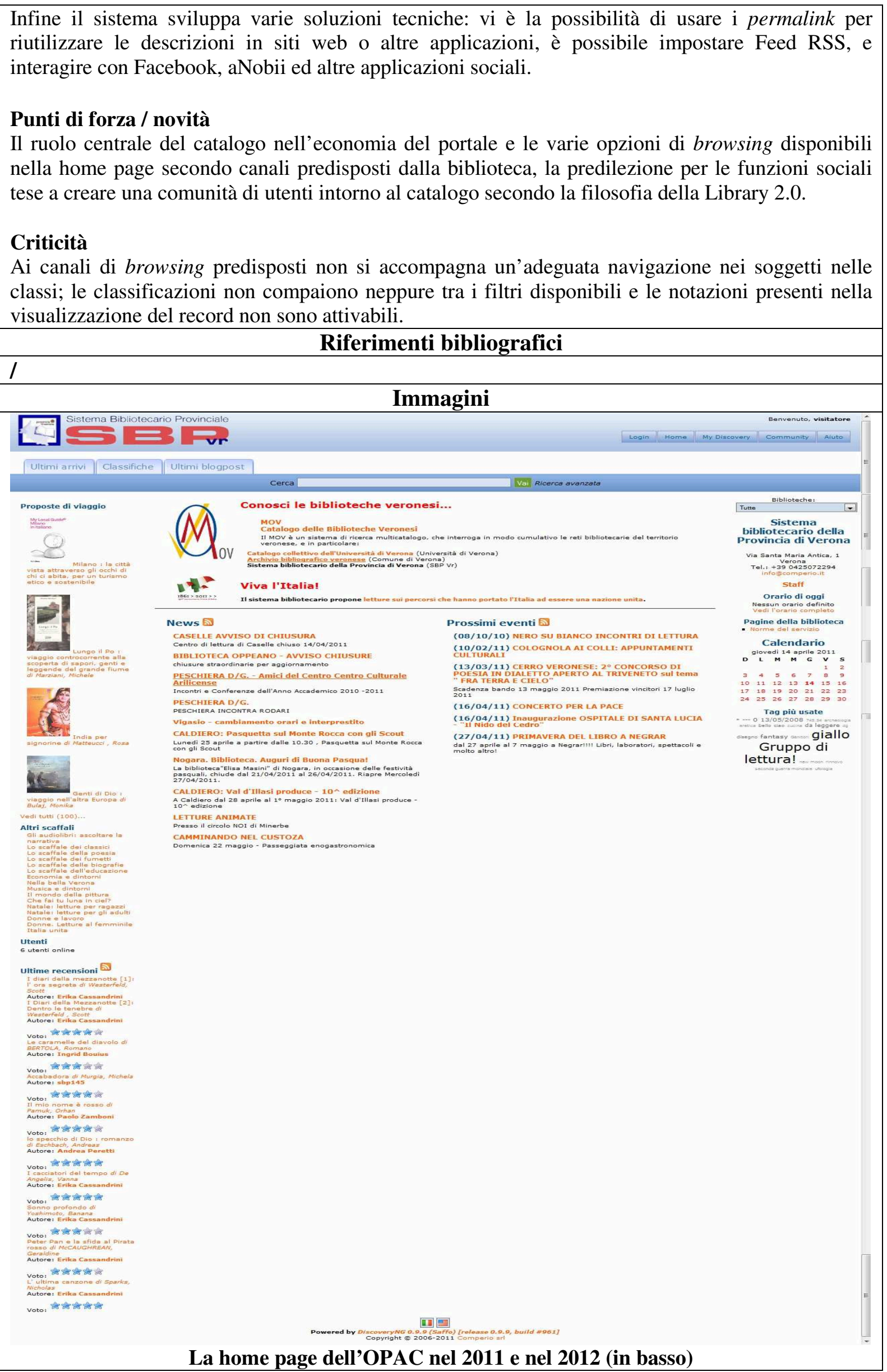




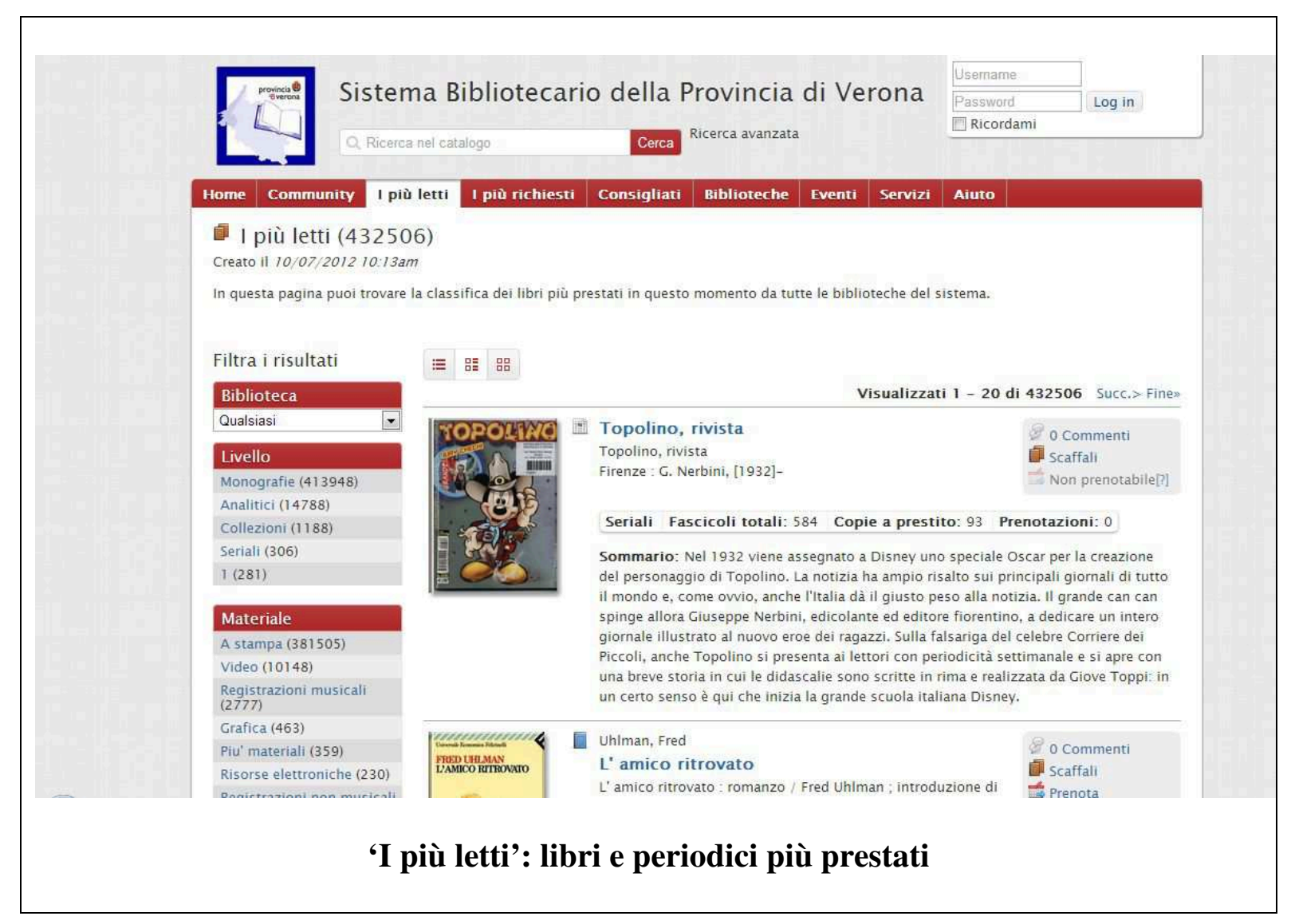




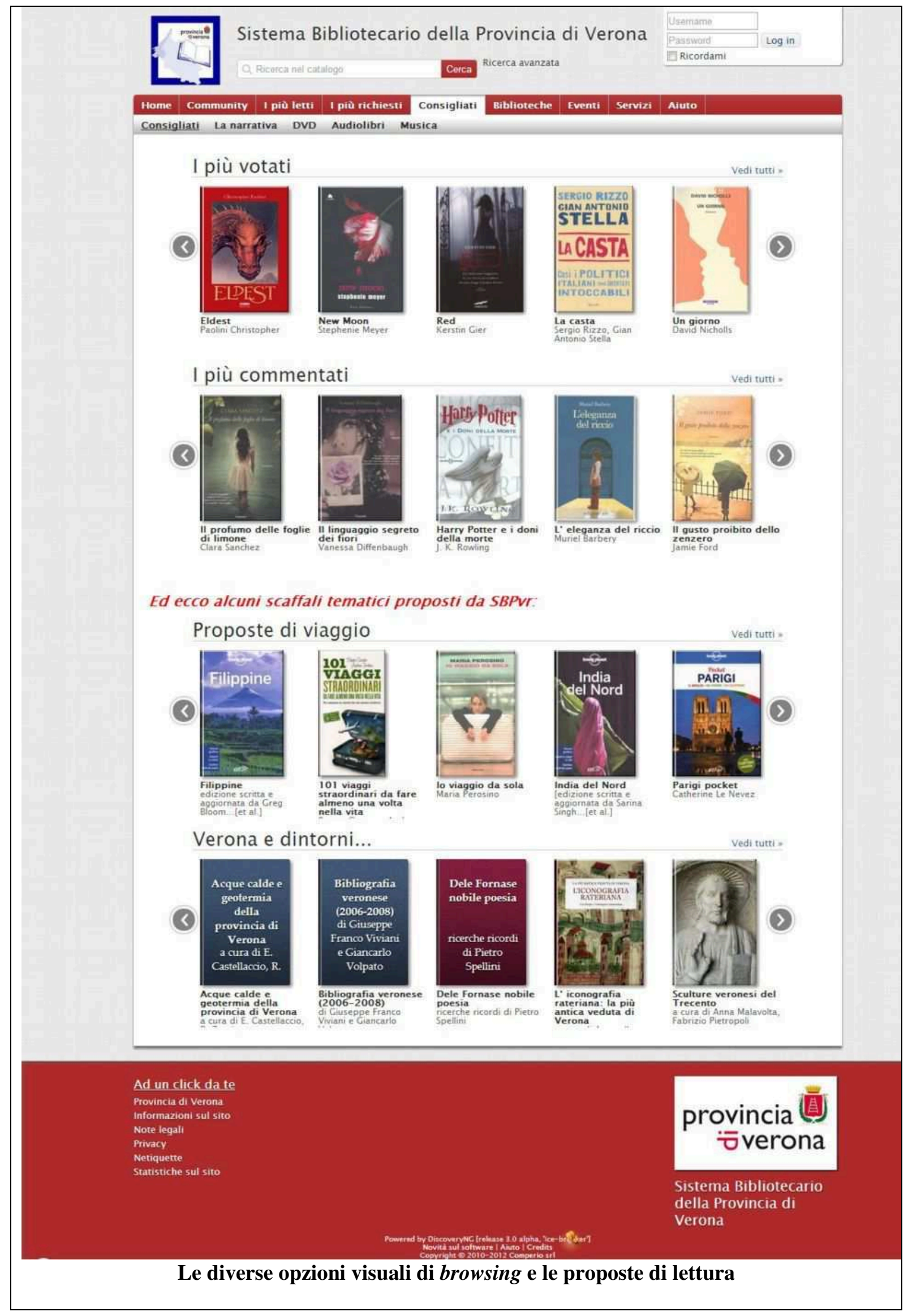




\title{
Sistema bibliotecario provinciale di Foggia
}

\author{
URL: <http://opac.bibliotecaprovinciale.foggia.it/SebinaOpacyou/Catalogo20.do>
}

Il sistema bibliotecario provinciale di Foggia ha implementato a partire dal 2011 Sebina You, il modulo per 'OPAC di nuova generazione' di Sebina Open library.

Il sistema bibliotecario provinciale La Magna Capitanata (www.bibliotecaprovincialefoggia.it) è l'ente guida del polo Bibliotecario SBN di Foggia che comprende 45 biblioteche di varia tipologia (tra queste la Biblioteca multimediale del Consiglio regionale pugliese Teca del Mediterraneo).

Il nuovo OPAC delle biblioteche pubbliche è stato lanciato con il nome di Catalogo 2.0.

Gabriella Berardi ha descritto il processo decisionale che ha portato ad affidarsi a questo tipo di catalogo. La decisione di affidarsi a un nuovo OPAC rientra nella strategia di miglioramento che ha portato al rinnovamento generale dei servizi all'utenza che sviluppato su tre fronti: il rinnovamento dell'OPAC, la biblioteca digitale e il progetto Meravigliosa Capitanata, un archivio di informazioni sugli autori locali e le tematiche legate al territorio della Capitanata.

Nell'implementazione dell'OPAC è stato scelto di mantenere un 'profilo basso', nel senso che non si è voluto un OPAC che si sovrapponesse al portale della biblioteca, che rimane il punto dal quale è possibile accedere a tutti i servizi, ma un catalogo rinnovato e più in linea con le attuali tendenze tecnologiche; dunque, un OPAC di nuova generazione aperto alla collaborazione degli utenti e integrato graficamente nel sito web della biblioteca.

Sono stati creati diversi percorsi di ricerca ('catalogo', 'percorsi', 'biblioteca digitale' e 'Web') corrispondenti ai principali strumenti messi a disposizione della biblioteca: il catalogo, le bibliografie pubbliche create dai lettori e dai bibliotecari, gli oggetti digitalizzati, una selezione di risorse web. Sono state però conservate le potenti funzionalità della ricerca avanzata, dedicate agli utenti più esperti.

\section{Accesso all'OPAC /autenticazione}

Dalla pagina dedicata aicataloghi / si per i servizi bibliotecari

Possibilità di visualizzare l'interfaccia in più lingue

No

Altre preferenze/personalizzazioni

Sì, tramite autenticazione nel proprio spazio personale dove è possibile gestire profilo, situazione lettore,suggerimenti di acquisto, ricerche salvate, liste di documenti

Risorse incluse nell'OPAC

Dati e Database

Tutte le risorse già presenti nel catalogo tradizionale

Formato dei dati

n.d.

Maschera di ricerca principale

\section{Ricerca}

Monobox di ricerca google like con possibilità di filtro per tipologia di materiale

Tipologie di ricerca previste (searching)

Ricerca su tutti i campi, ricerca avanzata

Tipologie di ricerca previste (browsing)

Vetrina delle novità, top ten (libri più prestati degli ultimi due mesi); interfaccia visuale per la visualizzazione degli scaffali, browsing e ricerca per liste

Aiuti nella ricerca

Autocompletamento in fase di digitazione; video 'Impara a cercare' (vari videotutorial disponibili) dalla home page del catalogo; chiedi al bibliotecario presente in tutte le schermate

Acquisizione passiva di informazioni (tramite modalità push, durante il processo di ricerca)

Vetrina della biblioteca digitale: propone oggetti random estratti dalla biblioteca digitale 


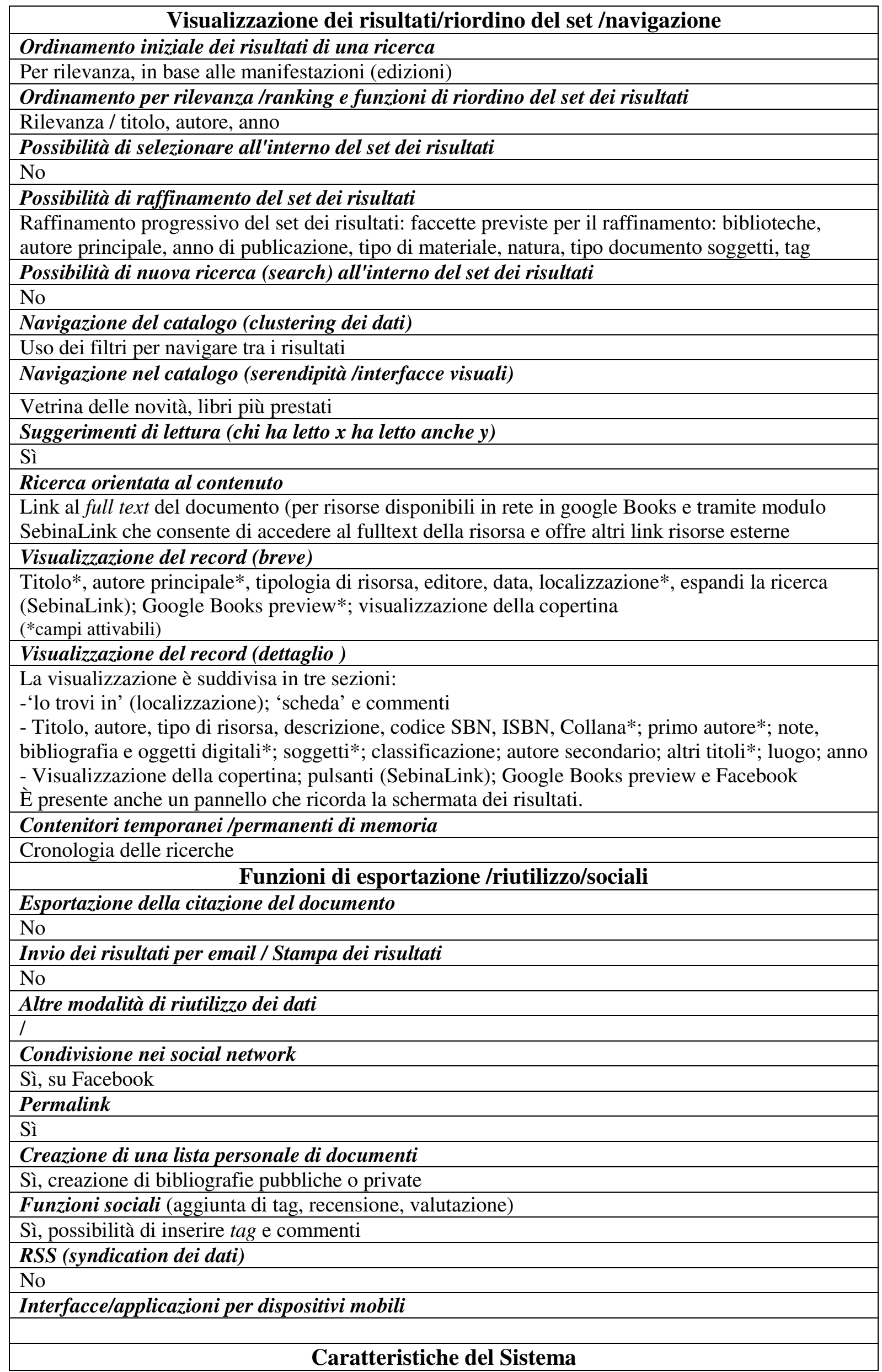




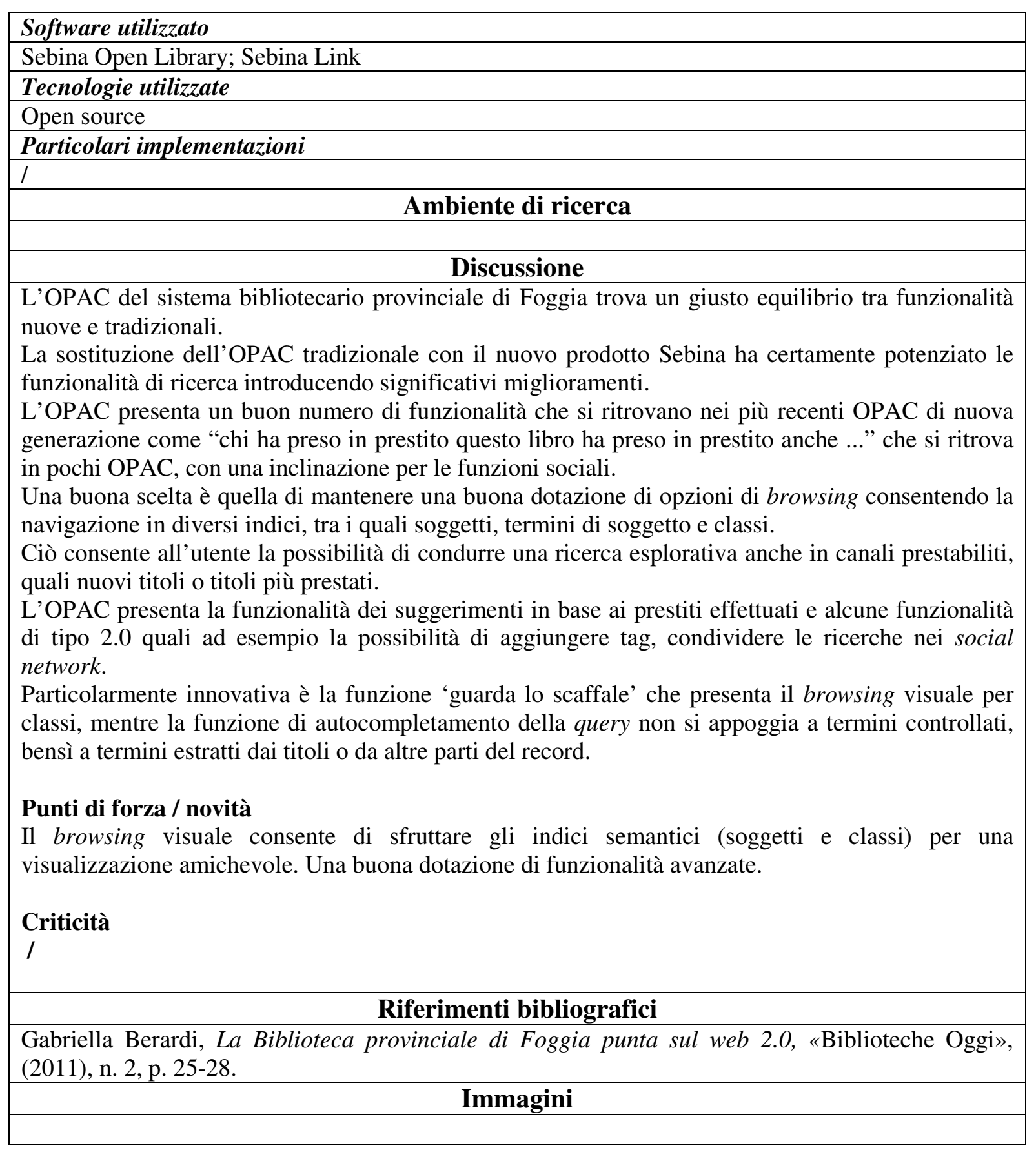




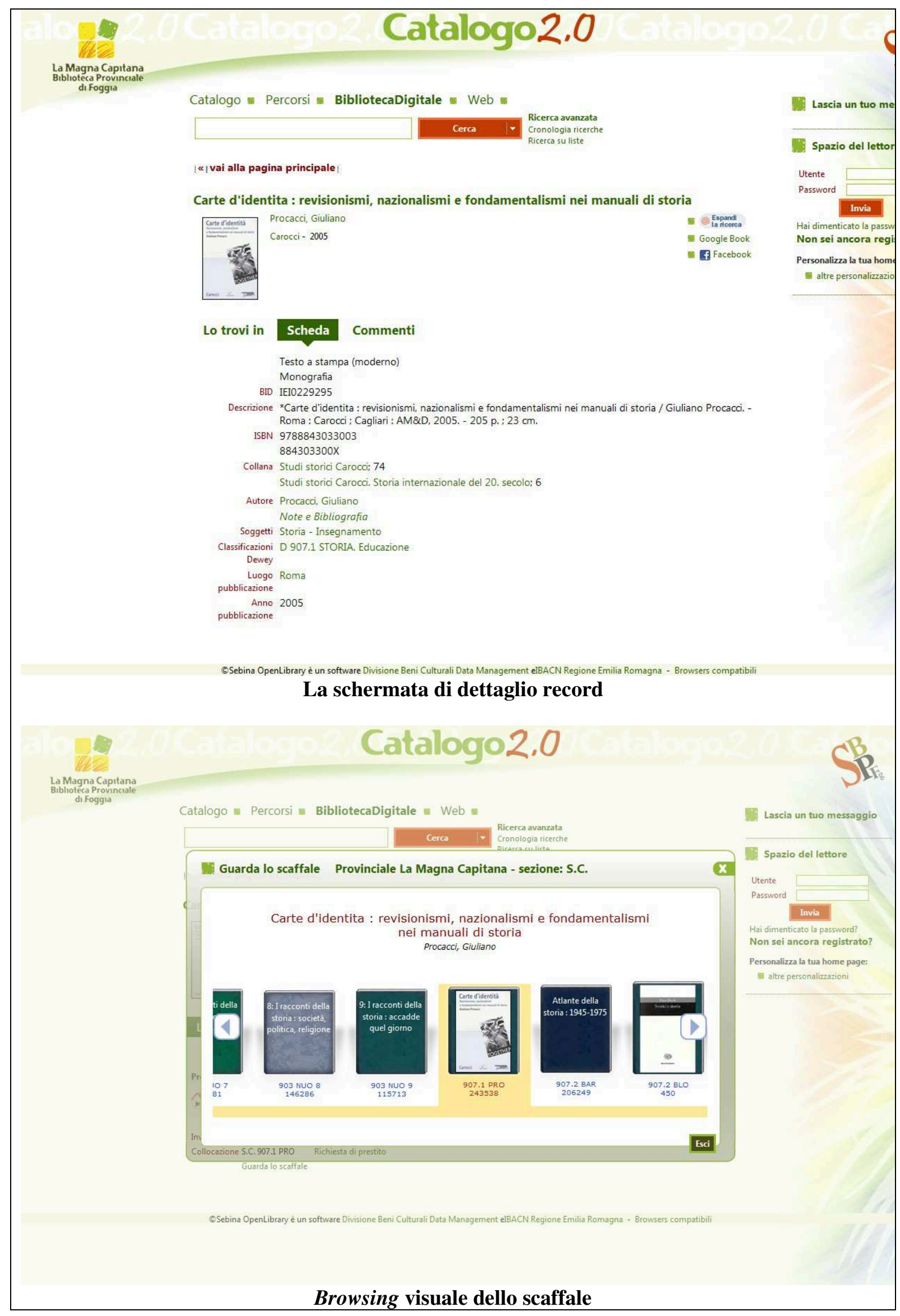




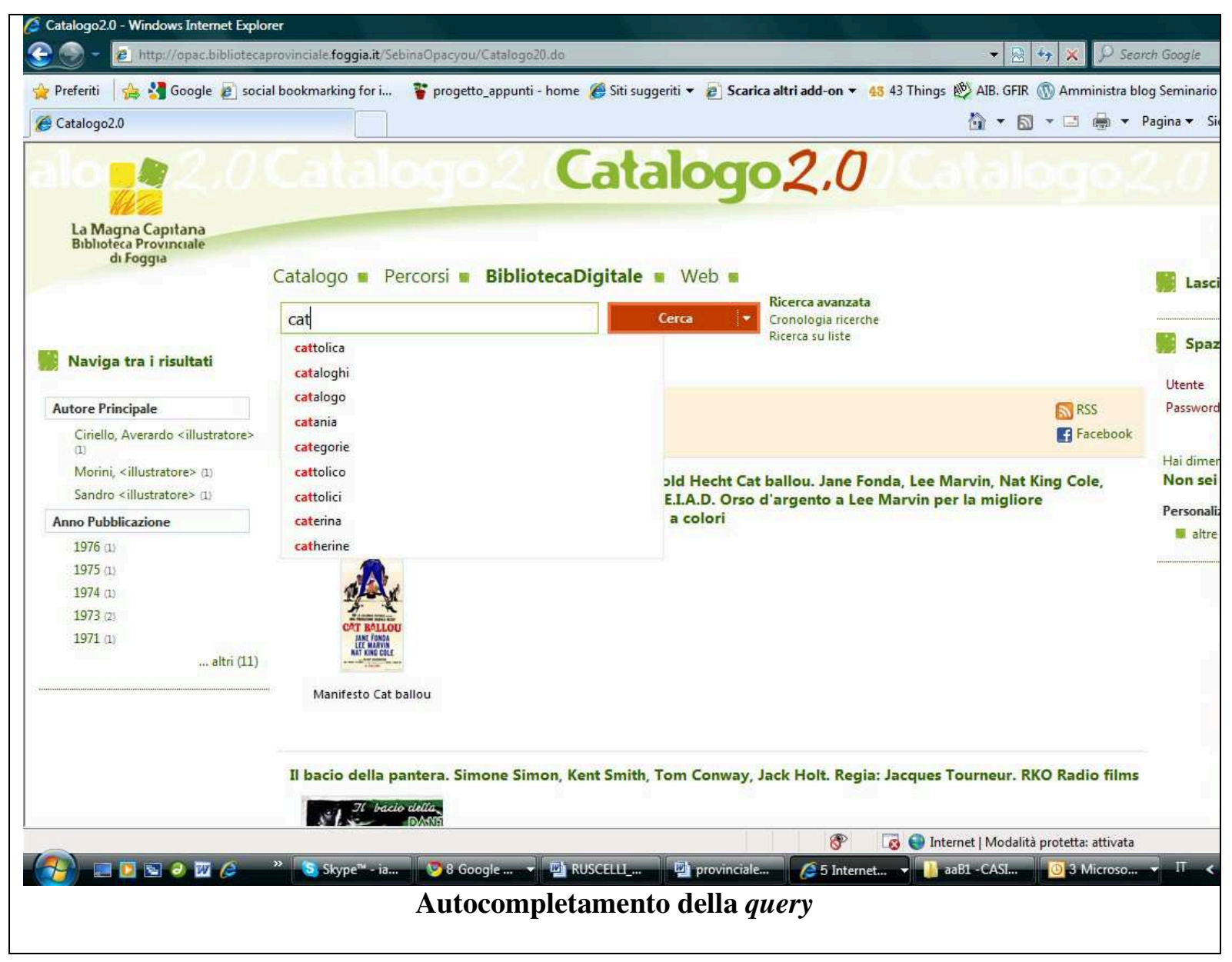




\section{Università degli Studi di Palermo}

URL: <http://unipa.summon.serialssolutions.com >

L'implementazione di Summon all'Università di Palermo costituisce uno dei primi casi italiani di sostituzione dell'OPAC con un discovery system.

Summon viene implementato nel sistema bibliotecario dell'Università di Palermo a partire dal 2011 per offrire un accesso unico alle risorse della Biblioteca digitale e la possibilità di ampliare i risultati delle ricerche grazie alle risorse che sono contenute nel suo indice centralizzato.

Prima di Summon l'università offriva in un portale una lista delle risorse possedute senza punti di accesso aggregati (es. Metalib o link resolver), rendendo difficoltoso l'accesso alle banche dati di ateneo.

Dopo un' analisi dei prodotti presenti sul mercato, il sistema bibliotecario ha scelto Summon per una serie di motivazioni, dai costi, alla ricchezza dei contenuti presenti nell'indice (oltre 630.000.000 di records), e per la presenza nell'indice di numerosi metadati relativi a risorse digitali presenti nella collezione (Elsevier) dell'ateneo. L'accesso a Summon viene integrato nella home page della biblioteca digitale. Attraverso Summon è possibile interrogare in un indice unico e in un'unica piattaforma le risorse della collezione digitale, le risorse interne a Summon e l'OPAC (Summon è compatibile con Marc e Marc 21) evitando la ricerca su piattaforme multiple e la ricerca federata.

Tre le caratteristiche giudicate importanti dall'istituzione: l'amichevolezza e semplicità di ricerca (maschera Google like), la ricchezza dei contenuti proposti (più di 630.000.000 di record per oltre 80 diversi tipi di pubblicazioni mappate con uno schema di metadati comune per ottimizzare la ricerca; oltre 6.800 editori internazionali tra cui Elsevier, Springer, Wiley, IEEE; oltre 130.000 periodici indicizzati a livello di articolo per quasi 140.000 .000 di articoli; e svariati milioni di quotidiani, monografie, articoli, brevetti); tempi rapidi di risposta grazie ai metadati raccolti e indicizzati preventivamente.

\section{Accesso all'OPAC /autenticazione \\ A tutti gli utenti del web, dalla pagina dell'OPAC \\ Possibilità di visualizzare l'interfaccia in più lingue}

Sì, inglese Altre preferenze/personalizzazioni

No

Risorse incluse nell'OPAC

Dati e Database

Risorse dell'OPAC di ateneo, della biblioteca digitale e le risorse presenti nell'indice centralizzato di Summon.

Formato dei dati

MARC21

Maschera di ricerca principale

Ricerca

Ricerca avanzata, con possibilità di passaggio alla ricerca monobox

Tipologie di ricerca previste (searching)

Ricerca monobox google like, ricerca avanzata

Tipologie di ricerca previste (browsing)

No

Aiuti nella ricerca

Suggerimento forse cercavi

Acquisizione passiva di informazioni (tramite modalità push, durante il processo di ricerca)

Visualizzazione dei risultati/riordino del set /navigazione

Ordinamento iniziale dei risultati di una ricerca

Per rilevanza, in base alle manifestazioni (edizioni) 
Ordinamento per rilevanza /ranking e funzioni di riordino del set dei risultati

Riordino del set dei risultati, per 'pertinenza', data (ascendente), data (discendente)

Possibilità di selezionare all'interno del set dei risultati

Tra i documenti di una specifica biblioteca, tra i documenti in fulltext, solo articoli peer review, escluso articoli di quotidiani, ricerca nel Catalogo Unico delle Biblioteche, estendi la ricerca alle risorse sterne all'ateneo.

Possibilità di raffinamento del set dei risultati

Raffinamento progressivo del set dei risultati

Faccette previste per il raffinamento: tipo di contenuto, biblioteca, termini di soggetto, data di

pubblicazione (slider), lingua

Possibilità di nuova ricerca (search) all'interno del set dei risultati

No

Navigazione del catalogo (clustering dei dati)

Uso dei filtri per navigare tra i risultati

Navigazione nel catalogo (serendipità /interfacce visuali)

No

Suggerimenti di lettura (chi ha letto $x$ ha letto anche $y$ )

No

Ricerca orientata al contenuto

Link al full text del documento (per le risorse disponibili in rete)

Visualizzazione del record (breve)

Comprende: titolo, autore, data, tipo di risorsa, link al record (dettaglio), link al full text (se

presente), visualizzazione della copertina, verifica disponibilità (dettaglio), salva articolo,

visualizzazione in anteprima del record

Visualizzazione del record (dettaglio )

La visualizzazione standard per le risorse librarie rimanda al record dell'OPAC tradizionale della biblioteca.

Contenitori temporanei /permanenti di memoria

Possibilità di salvare in memoria singoli item nello 'scaffale elettronico' dal dettaglio record

\section{Funzioni di esportazione /riutilizzo/sociali}

Esportazione della citazione del documento

No

Invio dei risultati per email / Stampa dei risultati

$\mathrm{Si}$, dal dettaglio record (con possibilità di salvare sul server) /No

Altre modalità di riutilizzo dei dati

No

Condivisione nei social network

No

Permalink

No

Creazione di una lista personale di documenti

$\mathrm{Si}$, nello scaffale elettronico personale tramite autenticazione

Funzioni sociali (aggiunta di tag, recensione, valutazione)

No

RSS (syndication dei dati)

No

Interfacce/applicazioni per dispositivi mobili

Sì

Software utilizzato

Caratteristiche del Sistema

Summon, Serial Solution

Tecnologie utilizzate

Proprietaria 


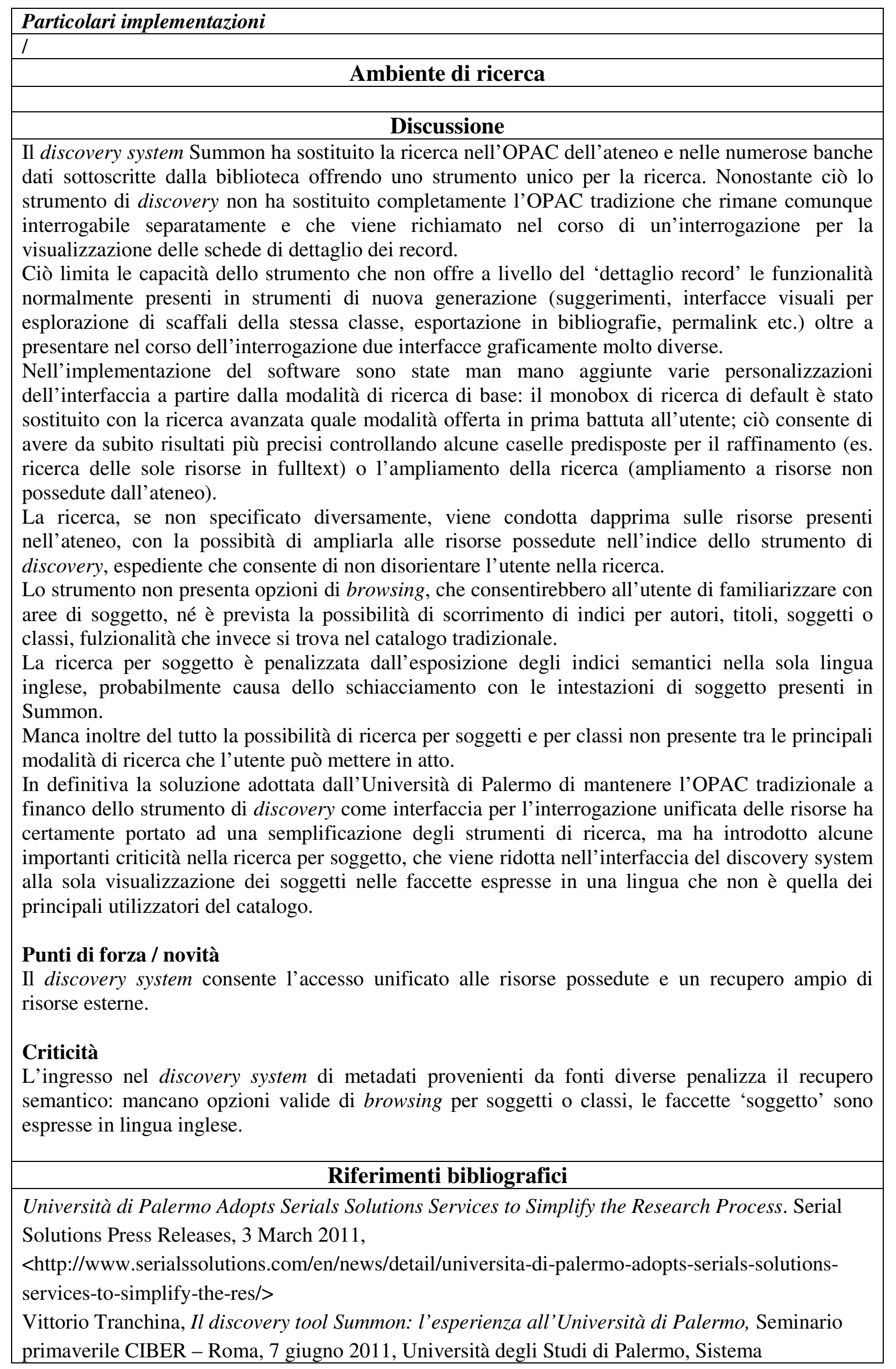


bibliotecario - Settore Biblioteca digitale, [slides],

<http://bib03.caspur.it/ocs/index.php/ciber/pr2011/paper/view/68/77>

\title{
Immagini
}

Aiuto | Info | Commenta | Italiano $\square$

\author{
(3) UNIVERSITÀ DEGLI STUDI DI PALERMO \\ Scopri facilmente il mondo di contenuti della biblioteca \\ Ricerca avanzata
}

La schermata iniziale 'Google like' 


\section{(4) UNIVERSITÀ DEGLI STUDI DI PALERMO}

\section{Scopri facilmente il mondo di contenuti della biblioteca}

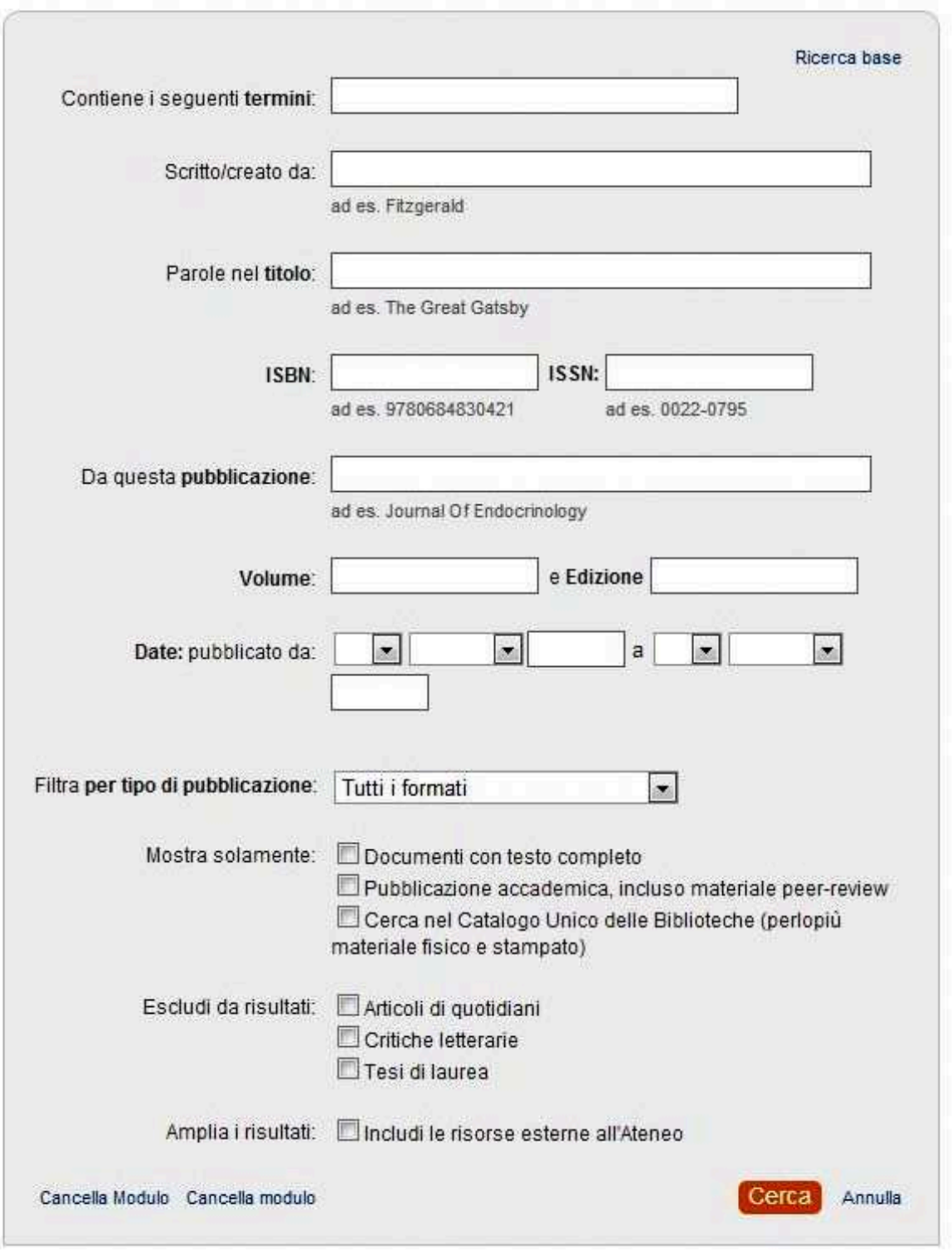

\section{Le opzioni di ricerca avanzata}




\section{Università degli Studi di Verona}

URL: <http://universe.univr.it/primo_library/libweb/action/search.do?vid=univr>

UniVerSe è uno dei primi esempi di implementazione del discovery system Primo nel nostro Paese. L'Università degli Studi di Verona, fondata nel 1982 e organizzata in otto facoltà e quindici dipartimenti, con circa 23 mila studenti è al terzo posto tra i migliori atenei italiani per didattica, ricerca scientifica e le strutture. Il Sistema Bibliotecario d'Ateneo è costituito da due biblioteche centralizzate, la Biblioteca centrale E. Meneghetti del polo medico-scientifico-tecnologico e la Biblioteca centralizzata A. Frinzi del Polo umanistico-economico-giuridico, alle quali fanno riferimento numerose biblioteche dipartimentali. Il Servizio di Automazione Biblioteche d'Ateneo sviluppa, coordina e armonizza le procedure gestionali automatizzate delle biblioteche e dei centri di documentazione dell'Ateneo. Implementato nel portale del Sistema bibliotecario di ateneo a partire dal maggio 2012, UniVerSe è stato presentato come un innovativo sistema integrato per la ricerca bibliografica, per la possibilità di interrogare tramite un'unica interfaccia web contemporaneamente OPAC, banche dati, periodici elettronici, e-book, risorse online gratuite e di ottenere l'accesso diretto al testo completo attraverso meccanismi di linking, tramite dal link resolver $\mathrm{SFX}{ }^{\circledR}$. L'interrogazione è arricchita dall' indice Primo Central e si avvale dei suggerimenti del servizio $B X \AA$, che indirizza l'utente ad altri articoli o materiali di interesse. Il testo completo di un articolo o la versione elettronica di un libro sono accessibili dalla rete di Ateneo in virtù degli abbonamenti sottoscritti o in modalità open access. Una delle particolarità di Primo è costituita dalle modalità di autenticazione: chi si identifica nell'accedere a UniVerSe è automaticamente identificato anche per i servizi personalizzati dell'OPAC di Ateneo dunque potrà visualizzare i record bibliografici dell'OPAC e usufruire dei servizi di prenotazione e rinnovo.

\section{Accesso all'OPAC /autenticazione}

Accesso a tutti gli utenti / autenticazione integrata con l'OPAC di ateneo

Possibilità di visualizzare l'interfaccia in più lingue

Sì, inglese

Altre preferenze/personalizzazioni

Risorse incluse nell'OPAC

Dati e Database

OPAC, banche dati, periodici elettronici, e-book, risorse online gratuite e altre risprse dell'indice centralizzato Primo Central

\section{Formato dei dati}

Unimarc

\section{Maschera di ricerca principale}

Monobox di ricerca google like con tre modalità di ricerca. 1. Catalogo di Ateneo + risorse online (default); Catalogo di Ateneo; Risorse online e la possibilità di ineserire i seguenti filtri: libri/ riviste/ articoli/ tutti ; che contiene i i termini della mia strategia di ricerca/con la mia frase esatta/inizia con; ovunque nel record/nel titolo/come autore-creatore/nel soggetto

\section{Tipologie di ricerca previste (searching)}

Ricerca monobox, ricerca avanzata (nel box monocampo è possibile inserire anche: ricerca per frase esatta, ricerca per parole libere o frasi, ricerca con caratteri jolly si possono anche escludere parole o frasi raggruppare termini in una stringa di ricerca, selezionare dove fare la ricerca.

Tipologie di ricerca previste (browsing)

Browsing dei tag di Universe/tag più popolari

Aiuti nella ricerca

No

Acquisizione passiva di informazioni (tramite modalità push, durante il processo di ricerca) 


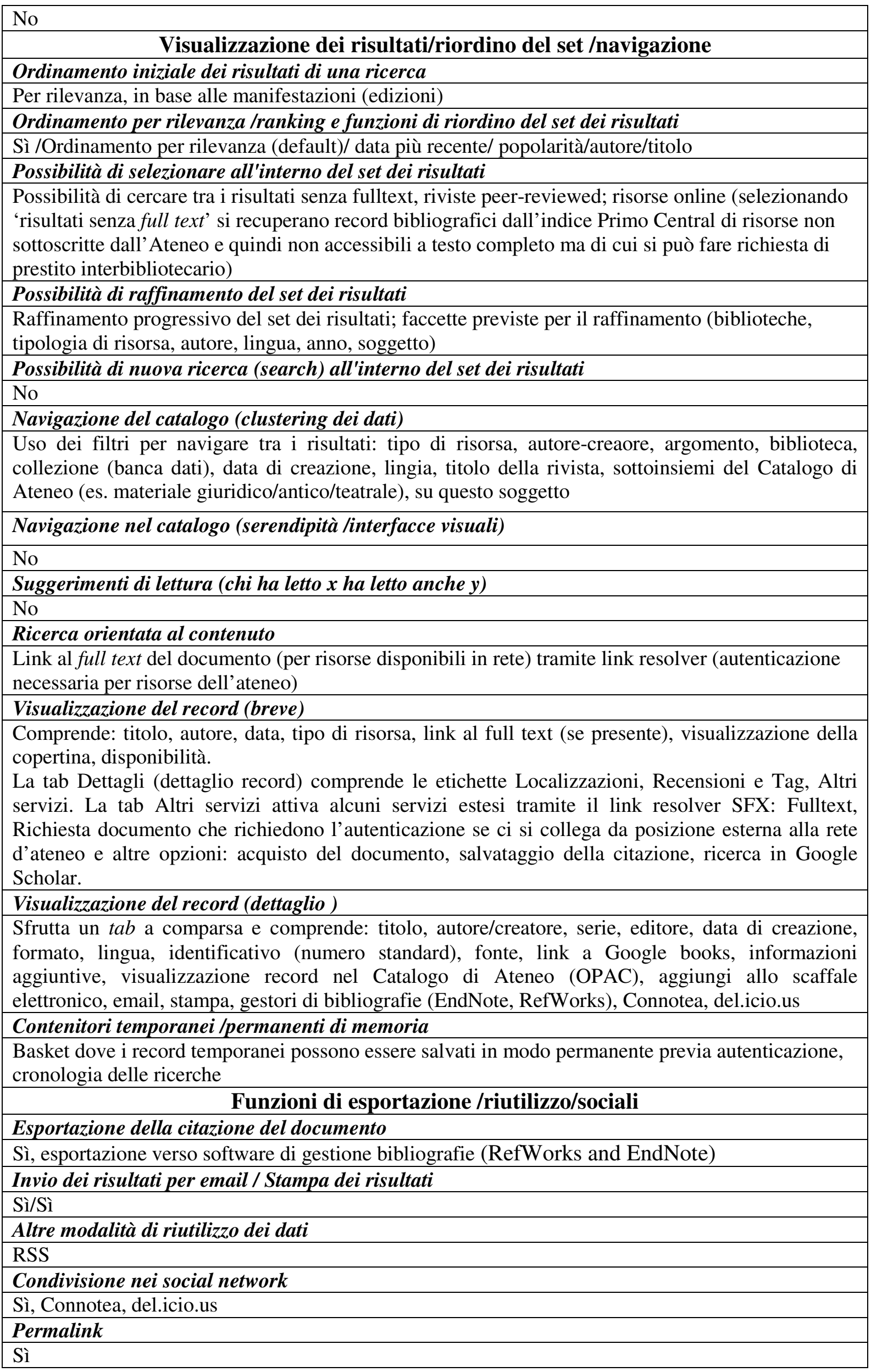




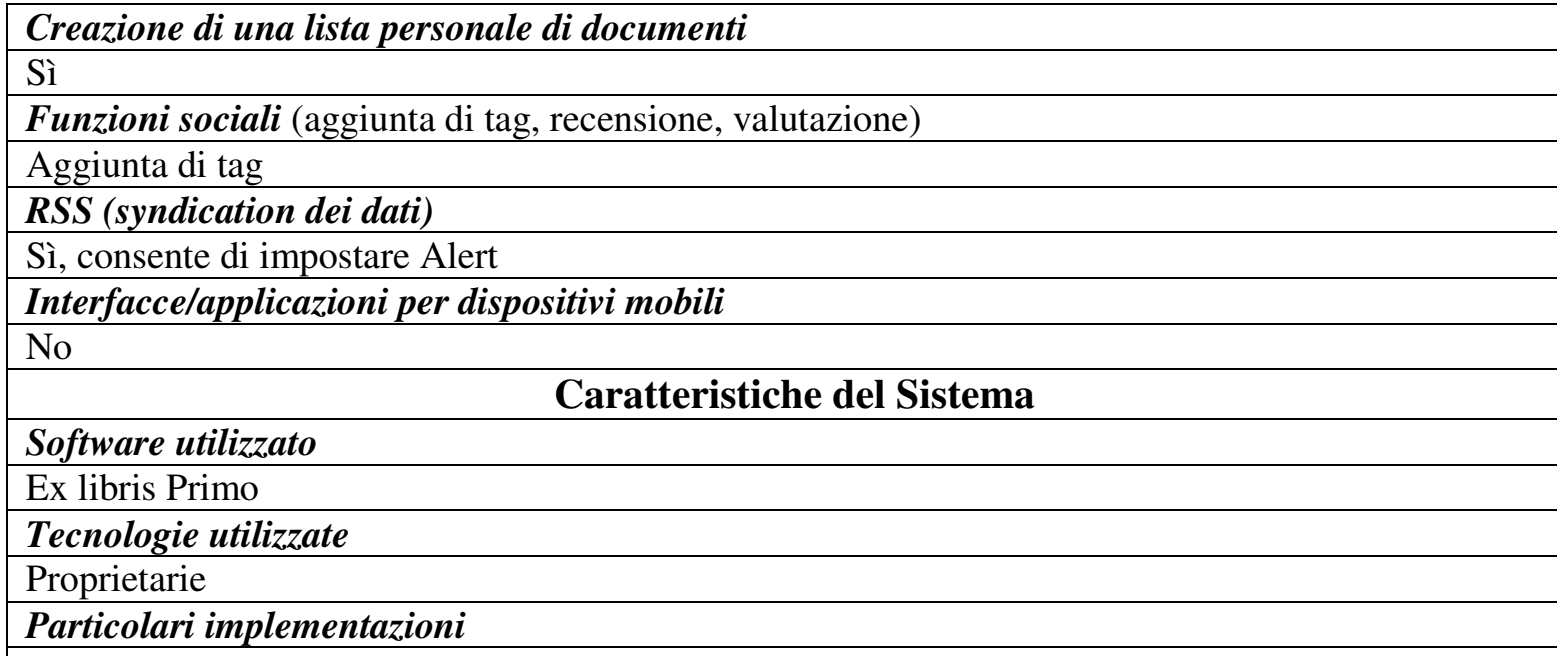

Possibilità di personalizzazione delle faccette per particolari tipologie di documenti (es. volumi antichi).

\section{Ambiente di ricerca}

Il discovey system ha sostituito l'accesso a diverse fonti, quali l'OPAC d'Ateneo (Aleph), le numerose banche dati e le riviste che erano precedentemente organizzate in una lista 'di tipo A-Z'. Nonostante la compatibilità quasi piena di queste liste con le riviste presenti nell'Indice di Primo, viene data la possibilità di cercare queste riviste fuori dallo strumento di discovery per la

\section{\begin{tabular}{l} 
percentuale non coperta dall'indice. \\
\hline \multicolumn{1}{l}{ Discussione }
\end{tabular}}

L'Università degli Studi di Verona è il primo ente accademico in Italia ad adottare Primo allineandosi a prestigiosi esempi internazionali quali la British Library, la Oxford University e la Freie Universität di Berlino.

La scelta di un sistema di discovery è stata motivata dall'esigenza manifestata dagli utenti di interrogare da un unico strumento l'OPAC, le riviste online e le varie banche dati offerte dalla biblioteca. Tra le funzionalità apprezzate, la possibilità di personalizzare i criteri di rilevanza e di ordinare i risultati per classi e sottoinsiemi di varia tipologia reati dalla biblioteca (es. libri antichi, materiale giuridico, materiale teatrale) che si propongono come ulteriori percorsi di navigazione e la possibilità di interrogazione tramite un'autenticazione unica (valida anche per accedere ai servizi dell'OPAC) e di usufruire di una reportistica sull'uso delle risorse elettroniche che la biblioteca possiede. Il discovery system consente di interrogare tramite l'interfaccia di Primo anche le sole risorse del catalogo offrendo agli utenti un'interfaccia di interrogazione user friendly, ma con tutti i limiti che gli strumenti per la scoperta presentano, tra i quali, ad esempio, le limitazioni che affliggono gli accessi semantici.

L'accesso all'OPAC tradizionale (Aleph), al momento, è stato mantenuto come possibilità per l'utente di effettuare ricerche più mirate (per soggetti e per classi), che non sono possibili nell'interfaccia del discovery system a causa della diversa omogeneità delle fonti presenti nell'indice di Primo.

Oltre a fornire una ricca funzione di aiuto in linea, a partire dall'attivazione del software la biblioteca "A. Frinzi" organizza, a cura del personale, corsi di formazione all'uso del catalogo collettivo d'Ateneo (OPAC) e del portale UniVerSe, rivolti a tutti gli utenti.

\section{Punti di forza/novità}

L'autenticazione integrata, la possibilità di cercare dentro lo strumento di discovery tra le sole risorse dell'OPAC.

\section{Criticità}

Il diverso trattamento degli accessi semantici negli strumenti per la scoperta non consentono di ottenere raggruppamenti utili, ciò si concretizza nella perdita di questi accessi e nella proposta di accessi alternativi per il browsing (es. i tag). 


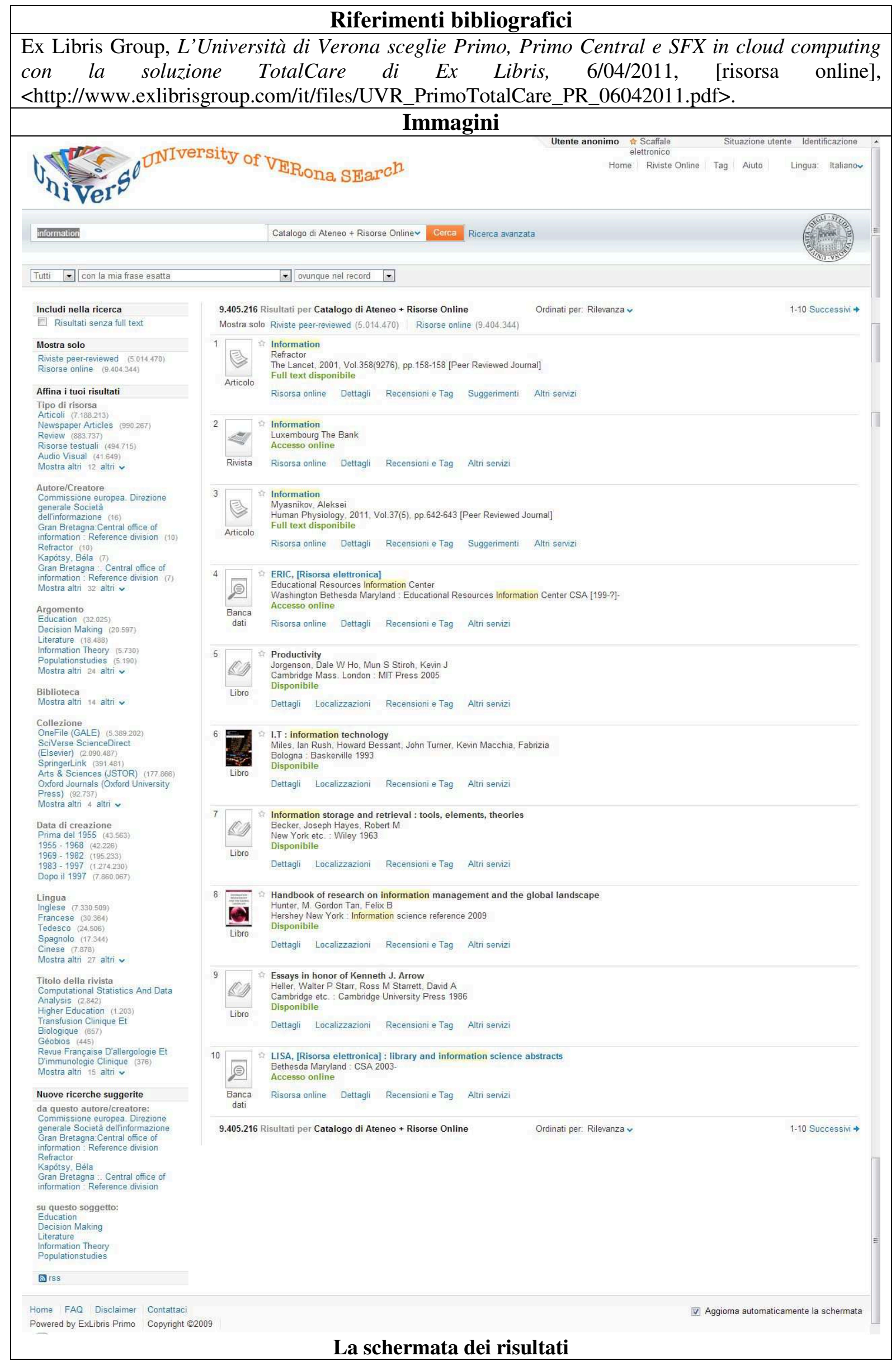




\section{Ann Arbor district library. SOPAC}

URL: <http://www.aadl.org/catalog>

SOPAC è un software open source creato da John Blyberg per le biblioteche pubbliche di Ann Arbor (US, Michigan) e implementato a partire dal 2007.

L'intenzione del progetto era quella di creare un nuovo OPAC fortemente integrato nel sito web della biblioteca e quindi fornire all'utente un unico indirizzo web sia per il sito della biblioteca sia per il catalogo. Per far ciò è stato scelto di utilizzare un CMS (content management system), cioè una piattaforma che viene solitamente usata per i blog, e di integrarvi il catalogo, costruito sopra l'OPAC tradizionale in forma di wrapper. La principale caratteristica è quella di orientarsi verso le funzioni sociali; non solo l'OPAC è costruito all'interno di un blog, ma offre numerose funzioni sociali, come la possibilità di aggiungere tag e recensioni e valutare le risorse. Questi dati sono indicizzati dal sistema e possono essere utilizzati come chiavi di ricerca. Ad esempio è possibile ottenere $\mathrm{i}$ dati ordinati in base alle valutazioni degli utenti; una funzione che si rivela utile per alcune tipologie di materiale, come i film e cd musicali.

\section{Accesso all'OPAC /autenticazione}

Dalla home page, autenticazione per i servizi (prestiti, prenotazioni) disponibile nella lista dei risultati e dal menù principale alla voce 'my account'.

\section{Possibilità di visualizzare l'interfaccia in più lingue}

Sì, (arabo, spagnolo, giapponese, coerano, cinese, russo). La traduzione si appoggia al servizio di traduzione di Google, dunque è possibile tradurre in contenuto dell'OPAC in tutte le lingue supportate da Google translate.

\section{Altre preferenze/personalizzazioni}

No

Risorse incluse nell'OPAC

Dati e Database

Risorse tradizionali (libri, DVD, periodici, mappe, risorse internet etc.)

\section{Formato dei dati}

UNIMARC

\section{Maschera di ricerca principale}

Accessibile dalla pagina dell'OPAC. Si contraddistingue per la doppia modalità di accesso ai contenuti del catalogo: search the catalogue (ricerca per campi di tipo google like) e browse the catalog che consente il browsing di categorie (New items, hot items, by format)

La maschera di ricerca è inserita nel contesto del blog della biblioteca. Sono dunque visibili nella schermata principale, i più recenti post, le ultime liste pubbliche create dagli utenti, la nuvola dei tag più popolari, le recensioni più recenti, un link ai più recenti post del blog.

\section{Tipologie di ricerca previste (searching)}

Ricerca in un box unico (google like) dalla pagina principale provvisto di riconoscimento vocale (con la possibilità di ricerca in tutti i campi (default) oppure nei campi titolo, autore, collane, tag, recensioni, soggetti, classi e possibilità di filtrare la ricerca per tipologia di materiale tutti (default)/libri, video/musica/audiolibri/riviste e in ogni singola biblioteca.

Ricerca avanzata: prevede la possibilità di cercare un termine in un campo, di scegliere la modalità di ranking e di applicare vari tipi di filtri (formati, biblioteche, destinazione, editore, intervallo di date)

Tipologie di ricerca previste (browsing)

È possibile effettuare il browsing nei nuovi arrivi, negli item più richiesti, per formato

Aiuti nella ricerca

No

Acquisizione passiva di informazioni (tramite modalità push, durante il processo di ricerca) No 


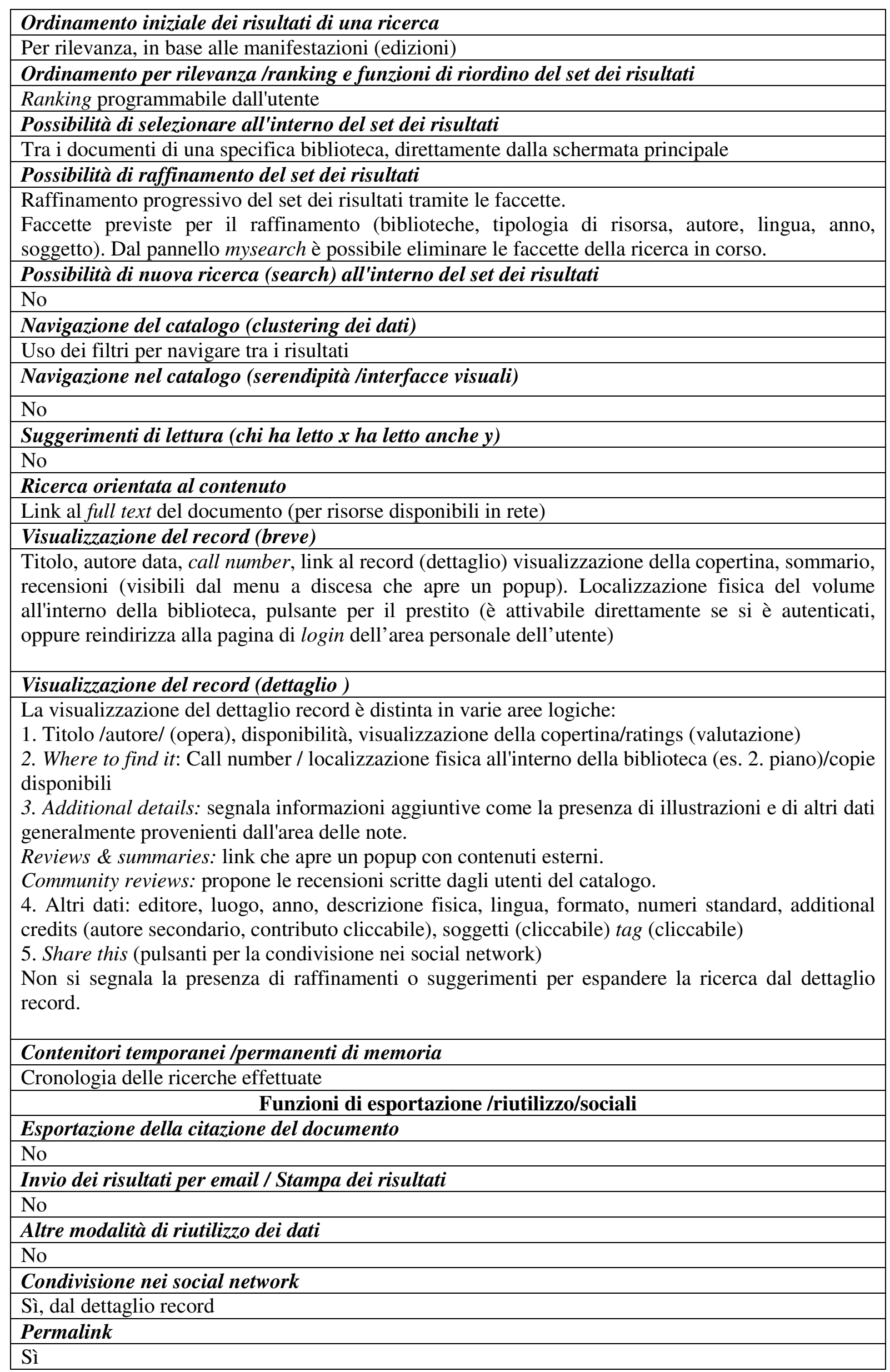




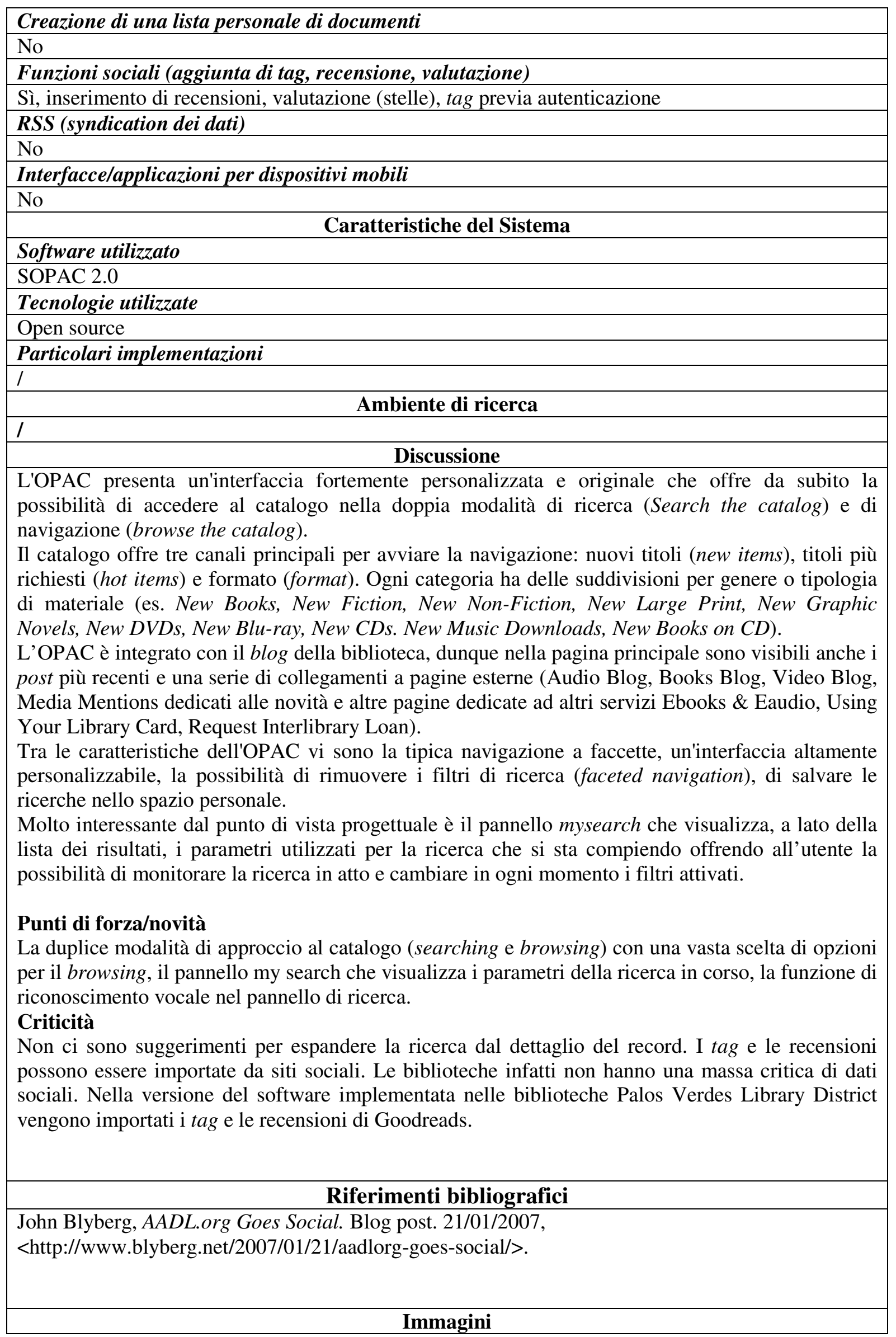




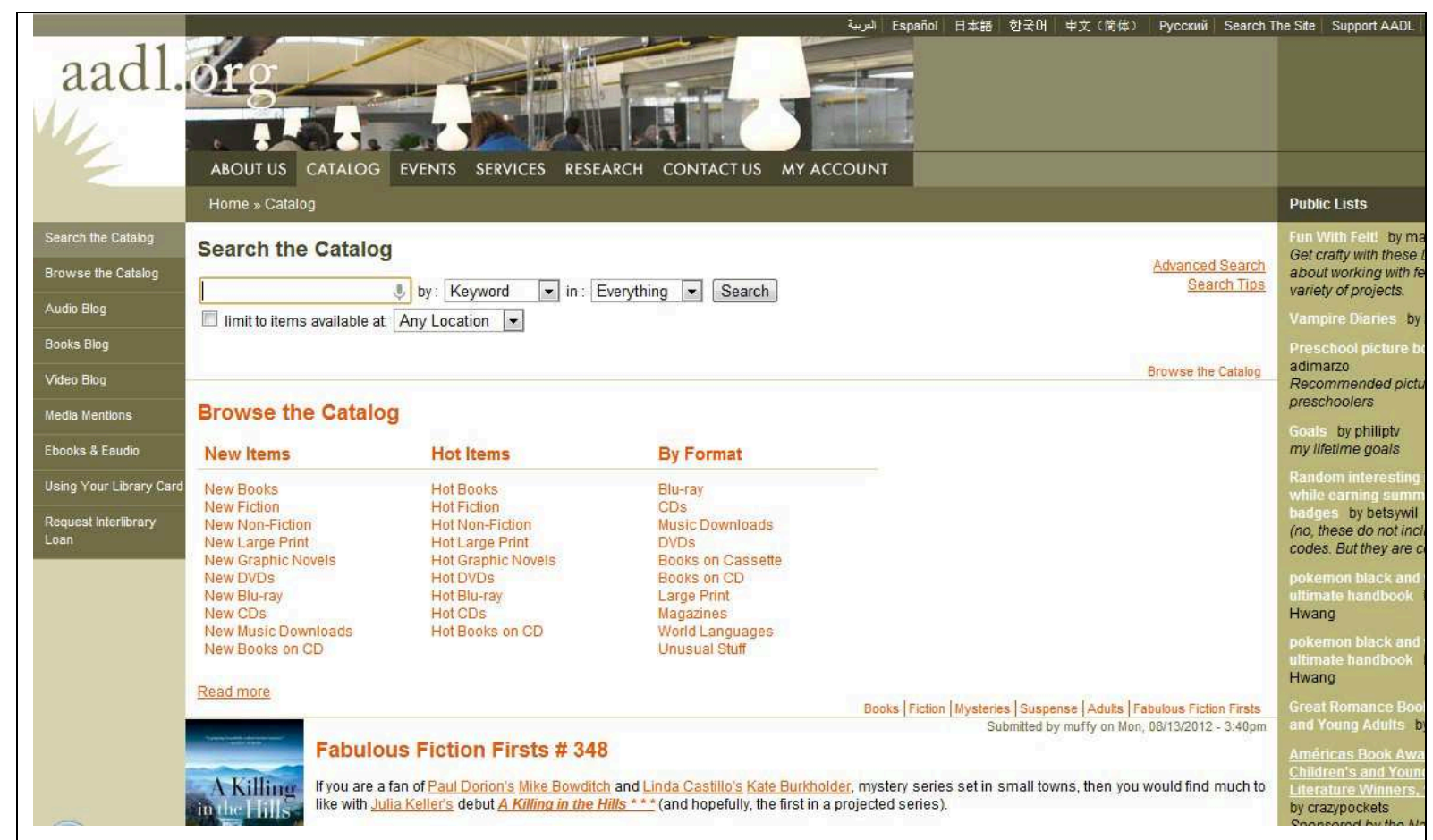

SOPAC. La schermata principale con le opzioni di searching e browsing. 


\section{Århus Statsbiblioteket. Summa}

URL: <http://www.statsbiblioteket.dk/>

Summa, il discovery system progettato alla Århus Statsbiblioteket (State and University Library) in Danimarca, è frutto di un progetto avviato nel $2006^{6}$.

Lo strumento viene presentato come un sistema integrato per la ricerca nelle diverse risorse che la biblioteca normalmente mette a disposizione dei suoi utenti ed è stato realizzato dai bibliotecari in cooperazione con sviluppatori di information technologies ed esperti di usabilità dei sistemi. Lo strumento non sostituisce il sistema integrato della biblioteca (ILS), ma si sovrappone ad esso come strumento per la ricerca.

Attualmente sono ricercabili attraverso Summa circa 2.6 milioni di record in formato danMARC2 provenienti dal software Horizon, 0.5 milioni di pubblicazioni catturate nel formato OAI Dublin Core e circa 100.000 record in Aleph nel formato danMARC2 provenienti dalle biblioteche dell'area scientifica dell'Università di Århus. I record sono sottoposti ad un'indicizzazione full-text e sono visualizzati ordinati per ranking in base a un algoritmo progettato ad hoc. L'OPAC consente l'integrazione di dati esterni provenienti da servizi web tramite un'interfaccia in $\mathrm{AJAX}^{7}$ che consente di implementare nell'OPAC le seguenti funzionalità:

* Raccomandazioni basate sui dati di prestito

* Suggerimenti nella forma "Forse cercavi ..."

* Recensioni e copertine provenienti da Amazon.com

* Schede degli autori ricavate da Litteratursiden (sito autorevole danese di letteratura)

* Musica e copertine CD da Bibliotekernes Netmusik (sito danese sulla musica gestito da biblioteche)

* OPenURL

* Altri suggerimenti basati sulle interazioni degli utenti

L'architettura di Summa consiste in una serie di moduli sviluppati in Java e J2EE che comunicano tra di loro tramite vari protocolli (tra i quali as JINI, RMI and SOAP web services) ${ }^{8}$. Summa è progettato per gestire quantità molto grandi di dati e in fase di indicizzazione si può provvedere a funzioni di normalizzazione e arricchimento dei metadati. L'indicizzazione può essere personalizzata tramite XSLT, tramite il formato XSL impostando una serie di parametri quali definizioni di campi, parametri di ranking, raggruppamenti, lingua per lo stemming ${ }^{9}$.

In Summa sono state impostate alcune caratteristiche come la visualizzazione delle opere correlate, un indice per la correzione dello spelling, la classificazione automatica (estrazione e

raggruppamento dei concetti). La versione 'beta' è stata lanciata tra il 2005 e il 2006.

Accesso all'OPAC /autenticazione

Dalla home page / Sì, per i servizi online

Possibilità di visualizzare l'interfaccia in più lingue

Sì, inglese

Altre preferenzelpersonalizzazioni

No

Dati e Database

Risorse incluse nell'OPAC

Tutte le risorse possedute dalla biblioteca; no ricerca federata (articoli)

Formato dei dati

DanMARC2

\section{Ricerca}

\footnotetext{
${ }^{6}$ Una prima versione di test con il nome Beta venne rilasciata nell'autunno 2005/2006.

${ }^{7}$ Asynchronous javascript XML

${ }^{8}$ Tutti i moduli possono essere amministrati tramite JSR-31(JMX). Summa può essre amministrato con software come IBM-Tivoli (cfr. Lund - Lauridsen -Hansen 2006)

${ }^{9}$ Hans Lund, Hans Lauridsen, Jens Hofman Hansen, Summa integrated search, State and University Library,

Denmark, 2006 [risorsa online] <http://www.statsbiblioteket.dk/summa/summaenglish.pdf>, p. 4-5.
} 


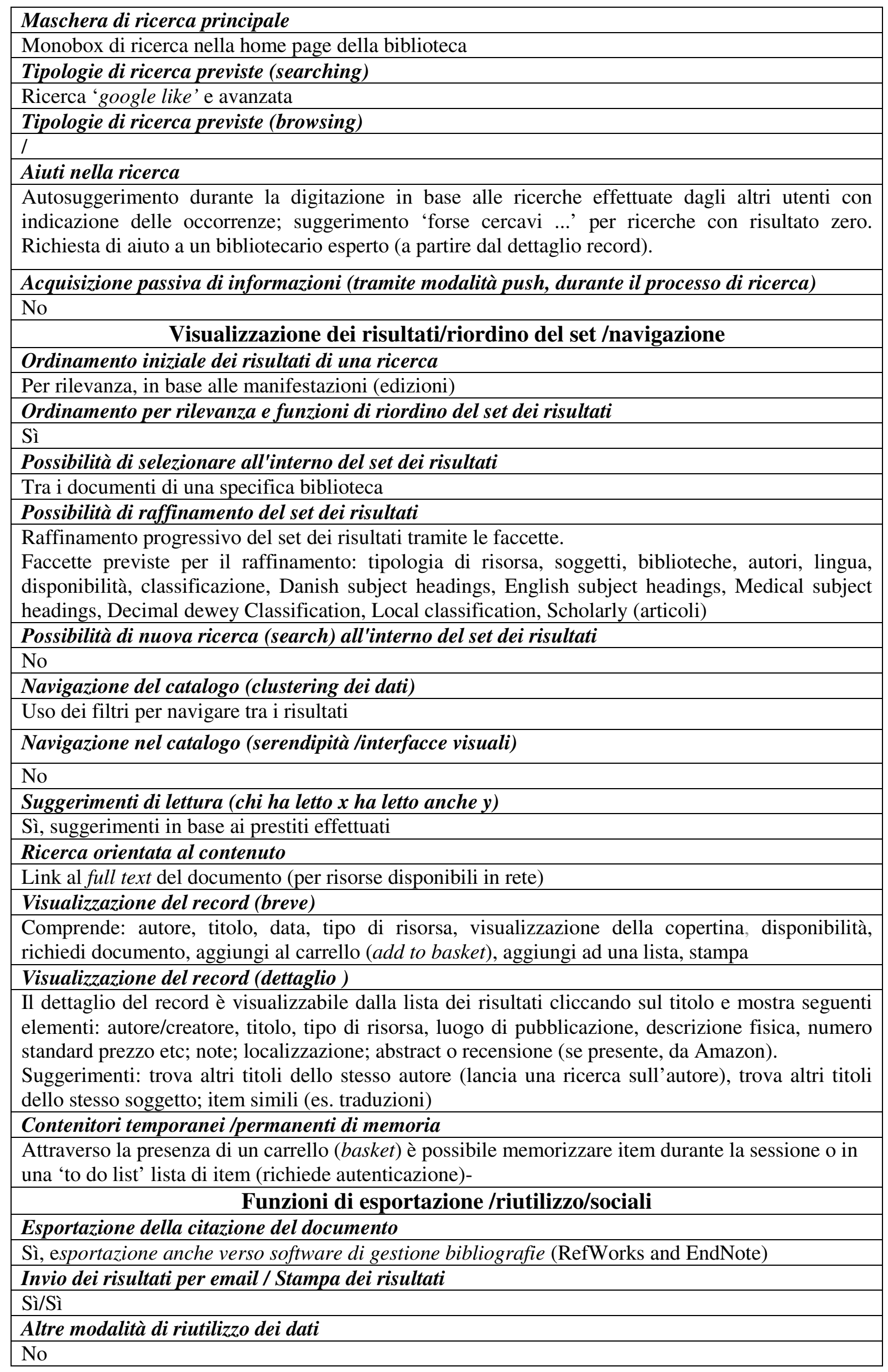




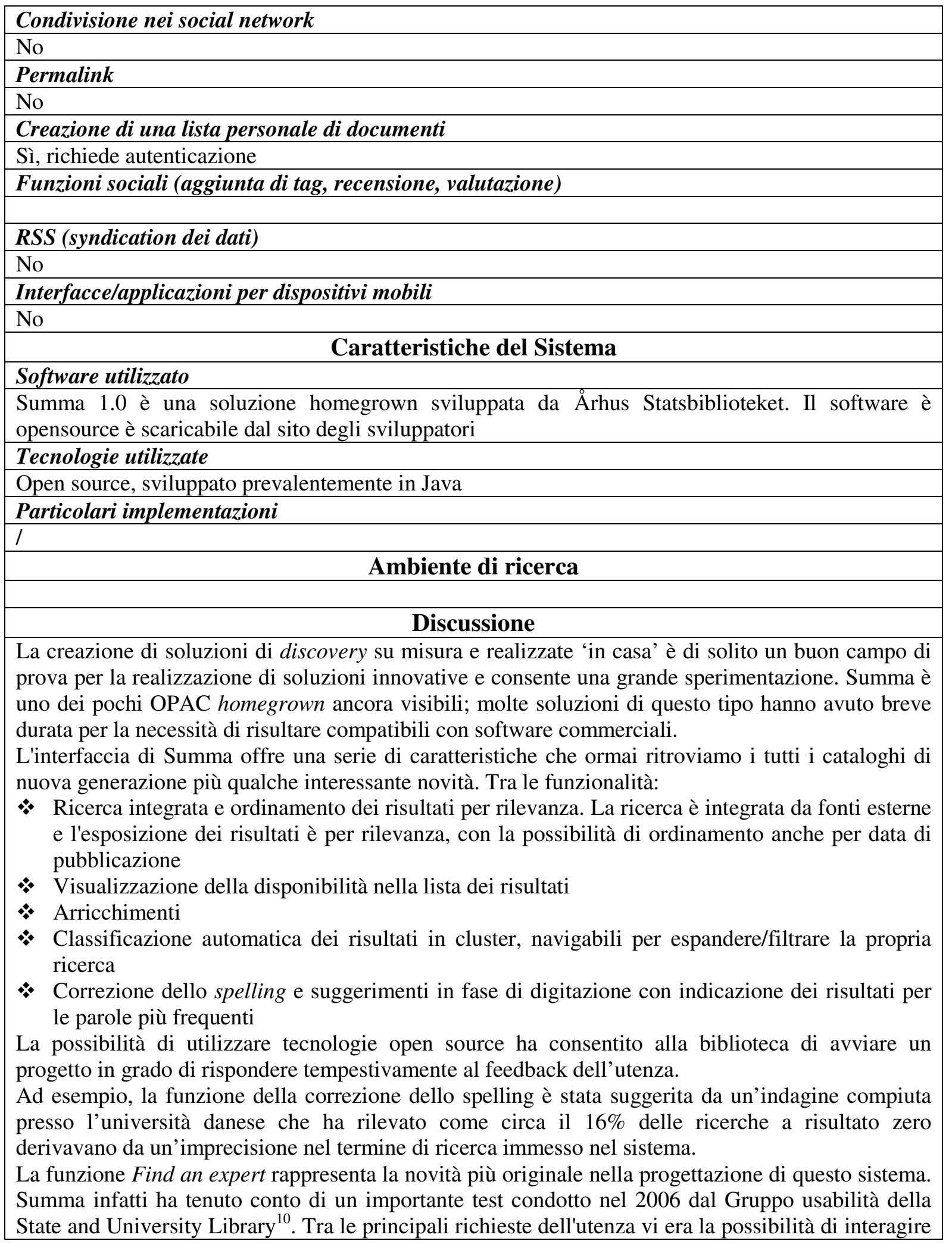

${ }^{10}$ Jeppe Lomholt AKSELBO - Lise ARNFRED, Sten BARFORT [et al.], The hybrid library: from the users' perspective cit. L'indagine ha avuto l'obiettivo quello di stabilire quale fosse la percezione degli utenti della biblioteca ibrida. Lo scopo dell'indagine era di raccogliere informazioni su sulla soddisfazione delle esigenze informative degli utenti attraverso l'uso delle risorse fisiche, digitali, attraverso l'aiuto dei bibliotecari in una biblioteca fisica. Le prospettive di miglioramento dell'OPAC sono descritte al par. 4 (p. 30).

Secondo il rapporto gli utenti hanno espresso un parere positivo sulla la possibilità di interagire con bibliotecari di reference specializzati all'interno dei risultati di una ricerca; sulla presenza abstract e sommari 
con bibliotecari di reference specializzati durante una ricerca. Summa fornisce nel risultato della una ricerca l'indicazione del bibliotecario specializzato nell'area di interesse. In un primo momento era possibile attivare una chat; successivamente la funzione chat è stata sostituita con il contatto mail del bibliotecario.

L'ingresso della funzione di reference all'interno dell'OPAC risponde alla volontà di offrire una mediazione nel corso del processo di ricerca, ove solitamente l'utente interagisce in solitudine con il sistema. A differenza delle altre esperienze, ove di solito si dà la possibilità di richiedere aiuto ad un bibliotecario generico, in questo caso la ricerca di aiuto è contestualizzata nella schermata dei risultati (il bibliotecario specializzato compare nella lista dei risultati) nel caso di corrispondenza tra l'interrogazione e l'ambito disciplinare di specializzazione del bibliotecario.

\section{Punti di forza/novità}

La possibità di chiedere aiuto ad un bibliotecario specializzato nell'area di interesse è una novità originale del catalogo e risponde alla necessità di una mediazione durante il processo di ricerca.

\section{Criticità}

È un peccato che la funzione di chat con il bibliotecario specializzato sia stata in breve tempo sostituita dalla possibilità di contattarlo via email.

\section{Riferimenti bibliografici}

Jeppe Lomholt Akselbo - Lise Arnfred, Sten Barfort [et al.], The hybrid library: from the users' perspective. A report for the DEFF project "The loaners' expectations and demands for the hybrid library", 2006, <http://www.statsbiblioteket.dk/summa/fieldstudies.pdf/view>.

Hans Lund - Hans Lauridsen - Jens Hofman Hansen, Summa integrated search, State and University Library, Denmark, 2006, <http://www.statsbiblioteket.dk/summa/summaenglish.pdf>.

Si vedano anche: sito web del progetto <http://www.statsbiblioteket.dk/summa/> e caratteristiche del software <http://www.statsbiblioteket.dk/summa/features-text-in-english $>$.

\begin{tabular}{|l|l|}
\hline & Immagini \\
\hline gol & Start search \\
\hline Igolan (81 maults) \\
gogh van (262 rosults) \\
gorilla (135 rosults) \\
gordiske knude (3 wesults) \\
gorm den gamle (43 rosults) \\
gondol (15 results) \\
godsk (100 results)
\end{tabular}

La funzione di autocompletamento della query è basata sulle interrogazioni formulate in precedenza dagli altri utenti.

nei risultati e sulla presenza di suggerimenti in base ai prestiti degli altri utenti. Hanno invece espresso parere neutrale sulle le recensioni di altri utenti, la presenza in OPAC delle tesi di laurea. 


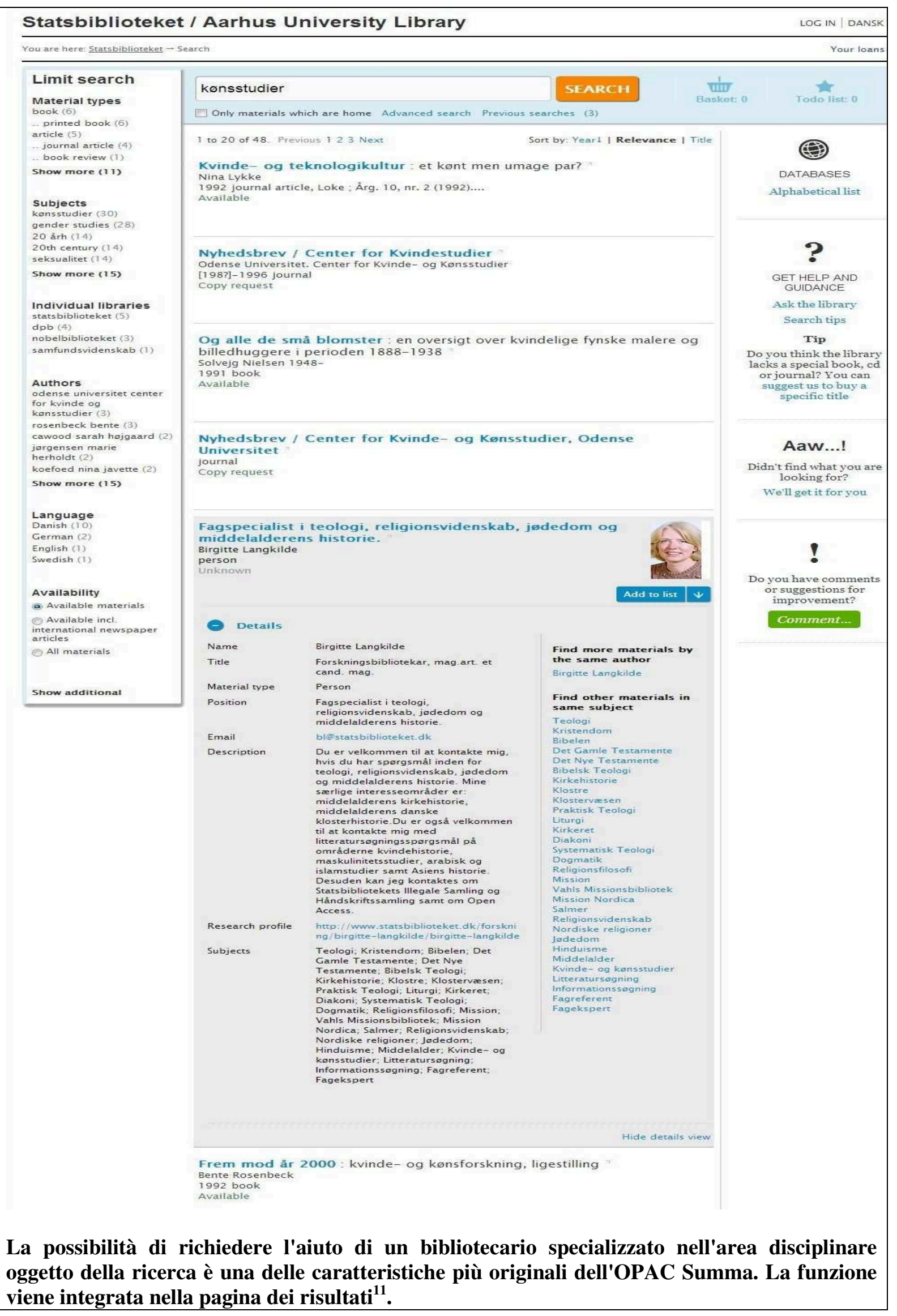

11 Hans Lund- Hans Lauridsen - Jens Hofman Hansen, Summa integrated search, State and University Library, Denmark, 2006, <http://www.statsbiblioteket.dk/summa/summaenglish.pdf>- 


\section{Université d'Artois. Visual Catalog}

URL: <http://visualcatalog.univ-artois.fr/vc2/>

Si tratta di un OPAC sperimentale progettato nel 2006 per le biblioteche universitarie di Paris $8^{12} \mathrm{e}$ di Artois (Arras, Lens, Béthune, Lievin-Angres, Douai) ed esteso nel 2008 alla biblioteca universitaria Lens.

Visual Catalog è un sistema di interrogazione ed esplorazione dei dati bibliografici. È un sistema sperimentale che mette a disposizione per la ricerca 145000 record bibliografici sfruttando nell'esposizione dei contenuti la ricchezza degli accessi semantici della Classificazione decimale Dewey e del soggettario RAMEAU..

La progettazione del sistema si fonda sull'ipotesi che un migliore sfruttamento delle collezioni si basa sull'esposizione dell'organizzazione intellettuale dei fondi documentari per mezzo della classificazione e di una descrizione concettuale e semantica di ogni notizia bibliografica attraverso il soggettario RAMEAU. L'OPAC è stato specificamente progettato per offeire un accesso per soggetto più potente alle raccolte, senza curarsi dell'integrazione con i servizi Lo strumento rappresenta dunque un'interfaccia di navigazione aggiuntiva per il catalogo classico al quale rimanda per le funzionalità di gestione dell'area personale dell'utente.

\begin{tabular}{|l|}
\hline Accesso all'OPAC /autenticazione \\
\hline Dalla home page / No \\
\hline Possibilità di visualizzare l'interfaccia in più lingue \\
\hline No \\
\hline Altre preferenze/personalizzazioni \\
\hline No \\
\hline \\
\hline Risorse incluse nell'OPAC \\
\hline 145.000 record bibliografici di monografie e riviste. \\
\hline Formato dei dati \\
\hline n.s. \\
\hline Maschera di ricerca principale \\
\hline Monobox di ricerca, per titolo \\
\hline Tipologie di ricerca previste (searching) \\
\hline Titolo, autore, soggetto RAMEAU, CDD (equivalente verbale) \\
\hline Tipologie di ricerca previste (browsing) \\
\hline Browsing gerarchico delle classi e dei soggetti a partire da ogni singolo record della lista risultati \\
\hline Aiuti nella ricerca \\
\hline No \\
\hline Acquisizione passiva di informazioni (tramite modalità push, durante il processo di ricerca) \\
\hline Sì, tramite l'esposizione visuale dei soggetti e delle classi collegate. \\
\hline \multicolumn{1}{|c|}{ Visualizzazione dei risultati/riordino del set /navigazione } \\
\hline Ordinamento iniziale dei risultati di una ricerca \\
\hline Per data (più recenti), in base alle manifestazioni \\
\hline Ordinamento per rilevanza /ranking e funzioni di riordino del set dei risultati \\
\hline
\end{tabular}

\footnotetext{
${ }^{12}$ Il progetto è di Fabrice Papy e di Sophie Chauvin (Lab. Document numérique \& Usages" http://doc.univparis8.fr). Sulle visualizzazioni dei dati bibliografici si veda anche: Visualisations heuristiques pour la recherche et l'exploration de données dynamiques : l'art informationnel en tant que révélateur de sens, Thèse de doctorat en SIC de Sophie Chauvin, Université Paris 8, 2005. L'implementazione della biblioteca dell'Università di Paris $8<$ http://visualcatalog.univ-paris8.fr/> non è più attiva.
} 


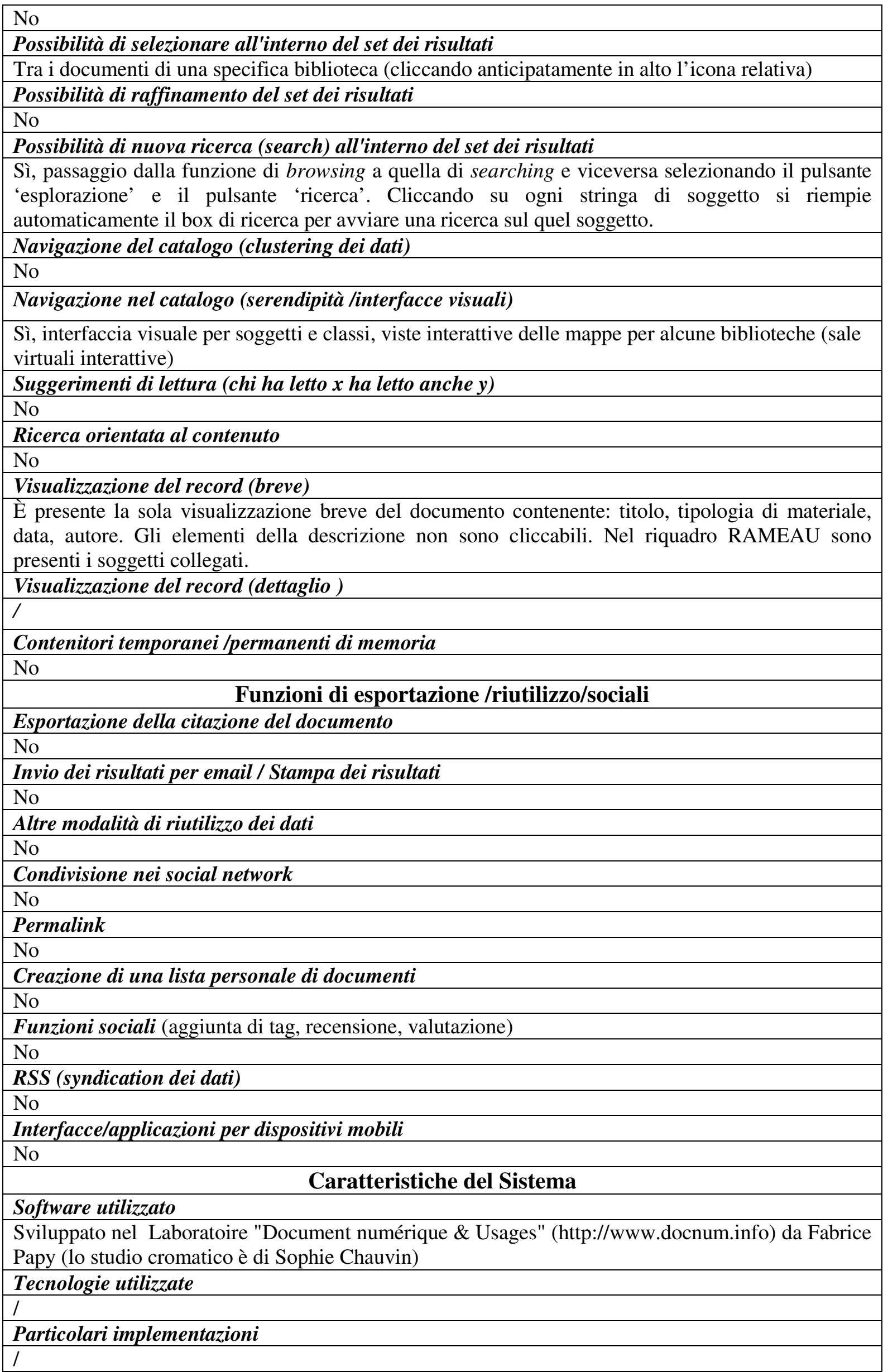




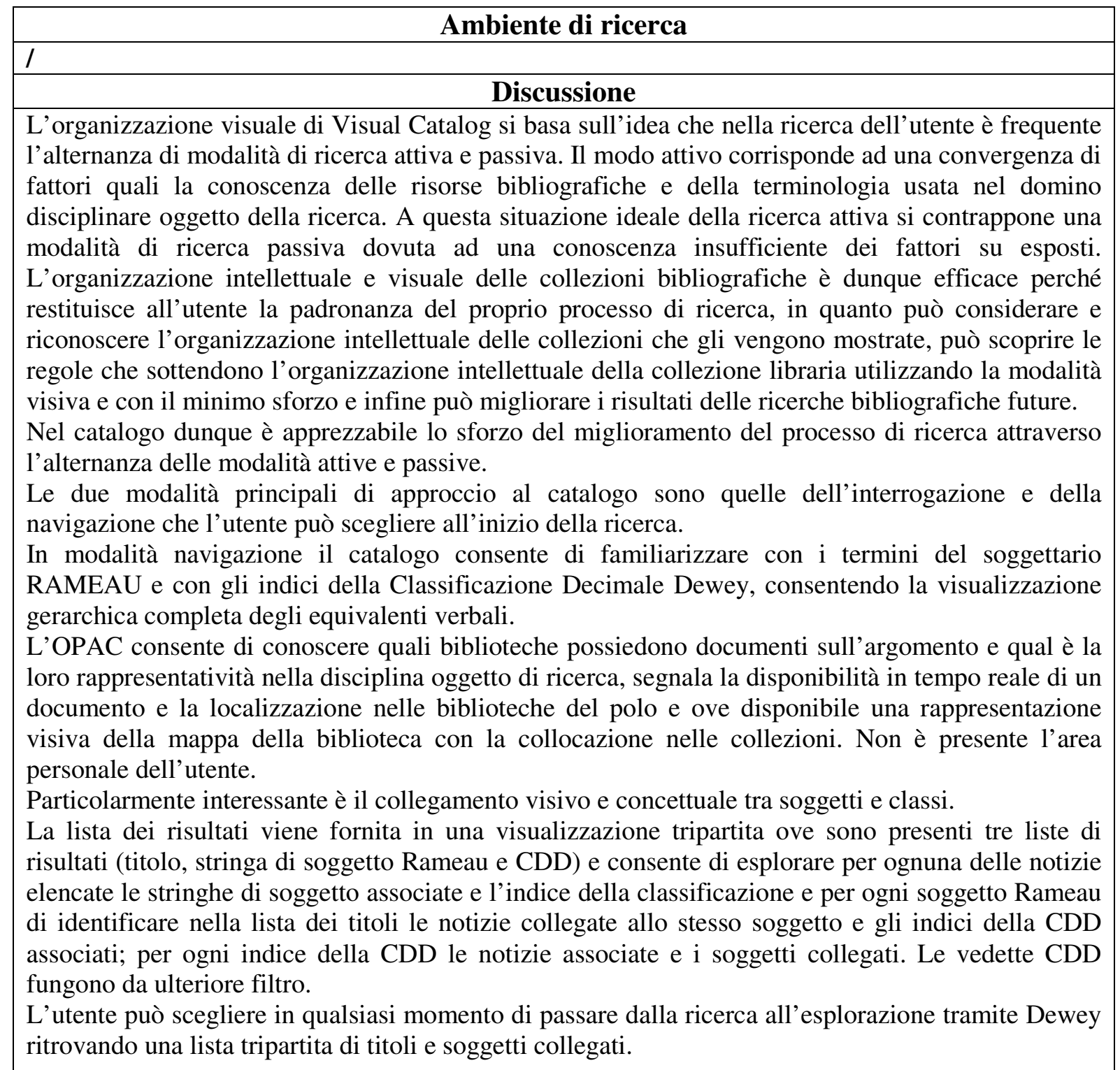

\section{Punti di forza/novità}

L'organizzazione visuale dell'interfaccia che consente di sfruttare gli accessi semantici per fornire un recupero di qualità e l'alternanza tra due le modalità di ricerca: interrogazione e navigazione.

\section{Criticità}

Trattandosi di un sistema sperimentale non si ritrovano funzionalità più avanzate di personalizzazione, gestionali, di arricchimento, sociali e di riutilizzo dei dati.

Il sistema sfrutta maggiormente la navigabilità della Classificazione Decimale Dewey rispetto ai soggetti: dalla visualizzazione del singolo record i soggetti collegati non sono navigabili, né cliccabili per formulare nuove interrogazioni che invece sono disponibili dalla schermata tripartita principale. Di ogni record viene fornita esclusivamente una visualizzazione breve: potrebbe essere fornito il collegamento alla scheda completa presente nell'OPAC tradizionale.

La grafica essenziale e un po' retrò penalizza questo potente strumento di ricerca.

\section{Riferimenti ibbliografici}

Sophie Chauvin - Fabrice Papy, Au-delà de la transfiguration du catalogue. Le Visual...Catalog, «BBF Paris», 50 (2005), n 4, p. 5-12 (<http://bbf.enssib.fr>).

Sophie Chauvin - Fabrice Papy, Peut-on déranger le bibliothécaire à la banque d'accueil ?ou comment rapprocher la communauté des usagers de celle des professionnels de la bibliothèque : l'expérience Visual...Catalog", CAIS/ACSI conference, London, Ontario, Canada, 2-4 giugno 2005, 
$<$ http://www.cais-acsi.ca/>.

Sophie Chauvin - Fabrice Papy, Pour une approche visuelle et ergonomique dans la recherche et l'exploration d'informations au sein d'un OPAC de SCD. L'exemple du Visual...Catalog, "Traité IC2, Management et gestion des STICS», Hermès Sciences Publications, marzo 2005.

Fabrice Papy, C. Leblond, L'interface de RI du Visual...Catalog : un outil innovant à «double détente», «Documentaliste-SI», 2007, n. 10.

Fabrice Papy, Le Visual...Catalog des universités de Paris 8 et d'Artois : un dispositif de recherche documentaire au service de la médiation sociale, [slides], 2008.

$<\mathrm{http}$ ///eduscol.education.fr/cdi/anim/reunion-des-interlocuteurs-academiques/reunions/reunion2008/politique-d-acq/visualcatalog $>$.

\section{Immagini}

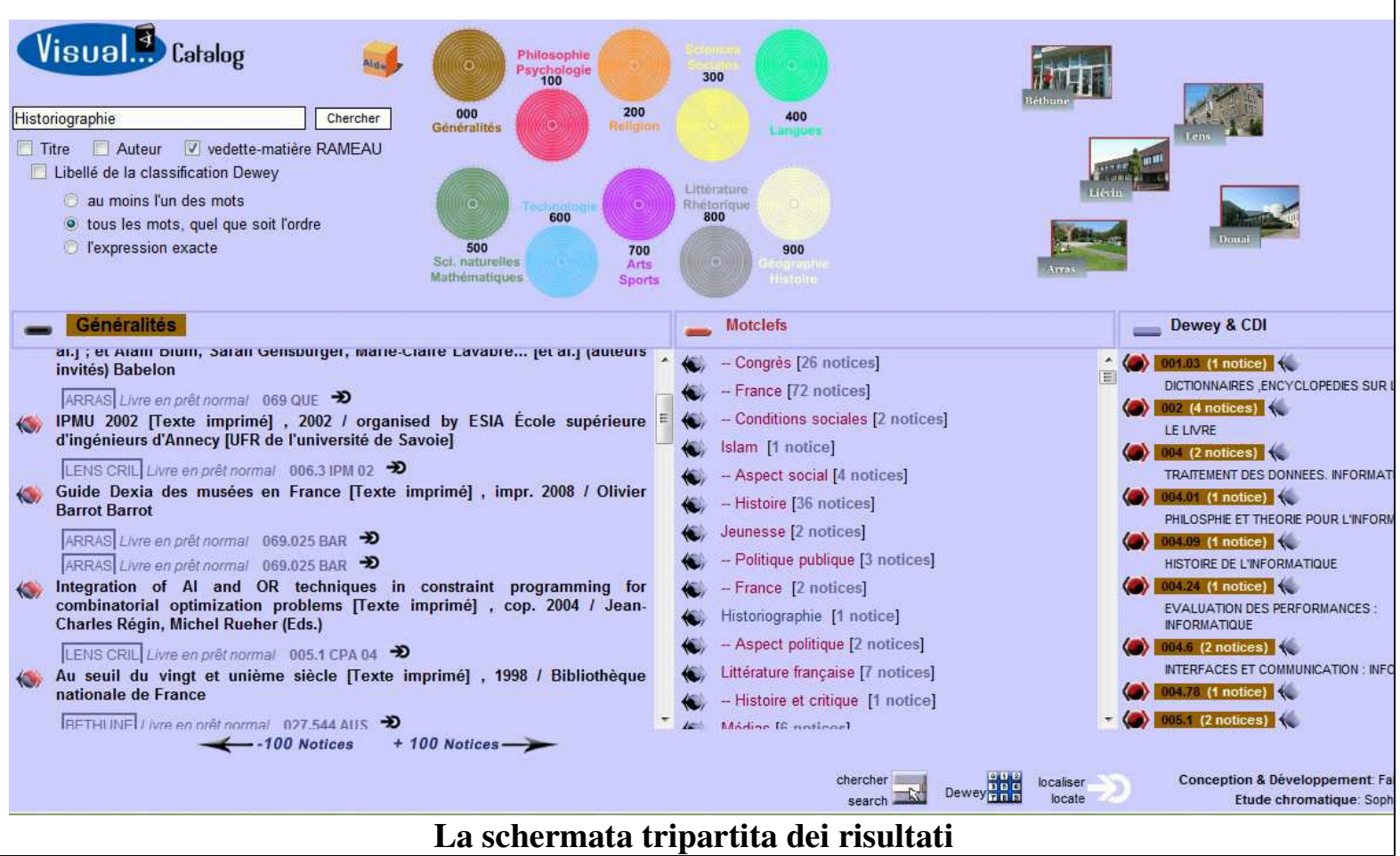




\begin{tabular}{|c|c|}
\hline \multirow{9}{*}{ 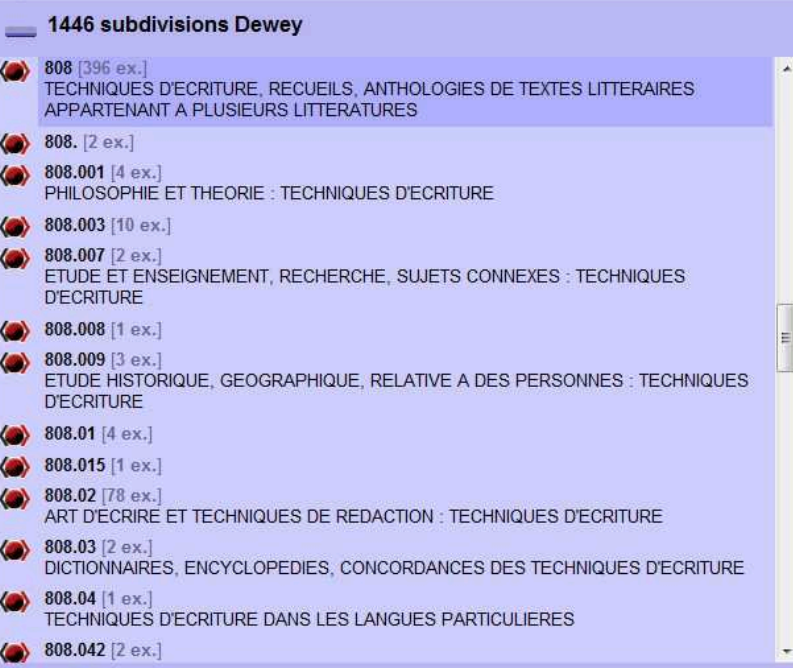 } & \multirow{8}{*}{ 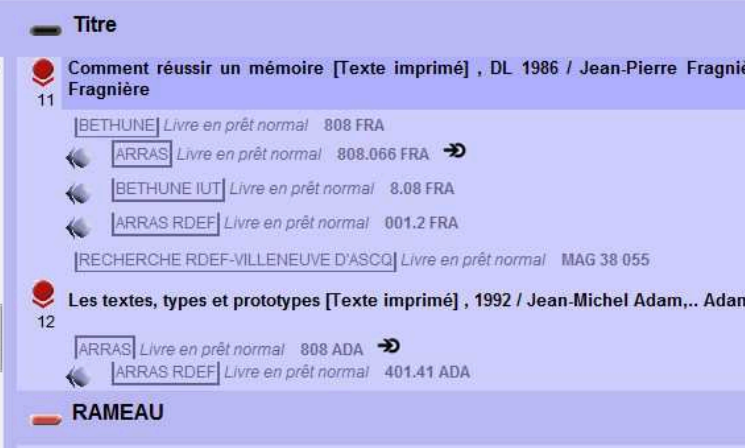 } \\
\hline & \\
\hline & \\
\hline & \\
\hline & \\
\hline & \\
\hline & \\
\hline & \\
\hline & \multirow{2}{*}{ 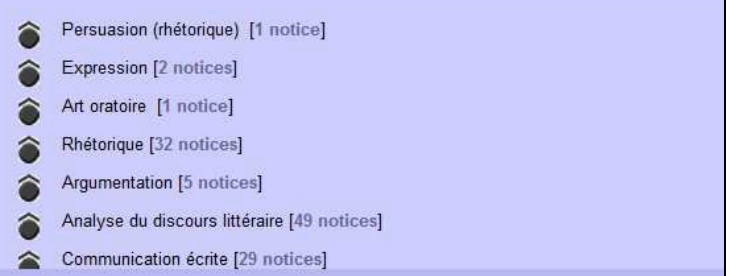 } \\
\hline$\leftarrow-100$ subdivisions $+100 \longrightarrow$ & \\
\hline & 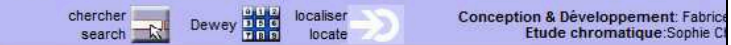 \\
\hline \multicolumn{2}{|c|}{ La modalità navigazione sfrutta il collegamento tra soggetti e classi } \\
\hline \multirow[t]{4}{*}{ BU d'Arras Etage } & 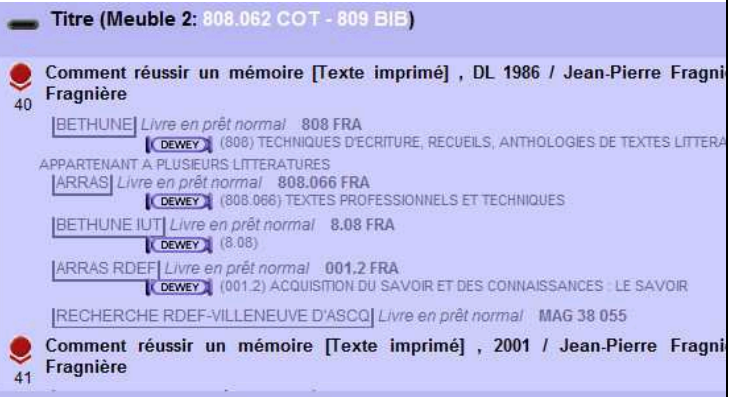 \\
\hline & - RAMEAU \\
\hline & $\begin{array}{l}\text { [0 notice] } \\
\text { Français (langue) [34 notices] } \\
\text { - Rédaction [31 notices] } \\
\text { Résumé de texte [14 notices] } \\
\text { Synthèse de textes [13 notices] } \\
\text { - Guides, manuels, etc. [35 notices] } \\
\text { Dissertation littérare [3 notices] } \\
\text { Analyse de contenu (communication) [2 notices] }\end{array}$ \\
\hline & 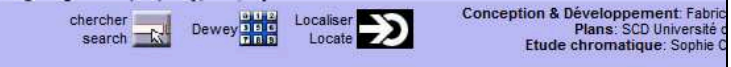 \\
\hline
\end{tabular}




\section{Svenska forskningsbibliotekens gemensamma katalog. OPAC Libris}

URL: <http://libris.kb.se/>

Realizzato con software open source ${ }^{13}$ e gestito dalla Royal library, l'OPAC LIBRIS raccoglie il posseduto di circa 200 biblioteche, musei, archivi svedesi e offre la maggior parte dei dati esposti sotto forma dei linked data.

L'OPAC contiene circa 6 milioni di record bibliografici e 20 milioni di localizzazioni.

Il nuovo OPAC comprende il posseduto delle 175 biblioteche facenti parte dello Swedish Union Catalogue, che utilizzavano lo stesso sistema di gestione (ILS) e il formato MARC21 per i record bibliografici e di autorità.

Progettare un catalogo elettronico considerando il contesto del Web è stato l'obiettivo degli sviluppatori. L'OPAC svedese è dunque il risultato di un processo di trasformazione dei dati che si è articolato in vari passaggi: l'esportazione dei dati dall'ILS (sistema di gestione proprietario) nel formato MARCXML, l'assegnazione di identificatori univoci alle risorse (URI), la trasformazione dei dati in triple RDF usando le ontologie definite in SKOS, Dublin Core, Bibliontology e DbPedia. Ciò ha significato la volontà di creare un catalogo aperto, in cui i dati (e non le pagine, o i record) possono entrare a far parte del Web Semantico, essere collegati ad altri dati e riutilizzati nel Web.

Venendo ai dettagli tecnici è stato costruito un RDF server wrapper $^{14}$ capace di fornire su richiesta $\mathrm{i}$ record bibliografici e di autorità nel formato RDF. Seguendo le regole dei linked data i dati vengono forniti di un URI persistente che possa essere accessibile via HTTP $^{15}$. Per i record bibliografici gli URI prendono la forma http://libris.kb.se/resource/bib/<number $>$ e http://libris.kb.se/resource/auth/<number> per i record di autorità.

I dati convertiti in triple possono essere interrogati usando SPARQL, uno strumento che richiede e analizza i dati e che è in grado di generare interrogazioni più complesse rispetto, ad esempio, ad SQL.

La volontà di rendere pienamente accessibile alla comunità del web i dati bibliografici ha spinto i progettisti ad adottare schemi di metadati conosciuti come Dublin Core per i record bibliografici e FOAF per le voci di autorità e SKOS per i vocabolari controllati. RDF Server Wrapper supporta Bibliontology, MODS, MarcOnt e altri standard.

Particolarmente interessante è la previsione di applicazione di FRBR seppur limitata al solo raggruppamento dei dati relativi all'opera. Ad ogni record vengono assegnate una o più chiaviFRBR consistenti nella coppia autore-titolo originale (uniforme) che vengono inclusi nel file RDF.

Accesso all'OPAC /autenticazione

A tutti gli utenti del web, autenticazione per i servizi (prestiti, prenotazioni, liste personali, selezione biblioteca preferita).

Possibilità di visualizzare l'interfaccia in più lingue

Sì, dal menu preferenze

Altre preferenzelpersonalizzazioni

Sì, dal menu preferenze: interfaccia (contrasto), visualizzazione di default del record per

13 Al vaglio dei progettisti è la possibilità di distribuire il software realizzato, vedi $<$ http://libris.kb.se/semweb> (in svedese).

${ }^{14}$ Il wrapper comunica con l' ILS sottostante usanto SQL e fornisce i record di un identificatore unico. Poi converte i record ISO2709 in una rappresentazione XML di MARC21; infine i record in formato XML vengono trasformati utilizzanto XSLT, che è il formato di output dei files.

15 Tramite il processo denominato HTTP content negotiation con cui un URI viene "dereferenziato" e reso accessibile in HTML: «Using HTTP content negotiation, the correct format can be delivered depending on the clients capabilities. This method uses the HTTP Accept header to tell the server what media types the client can handle and prefers. For example, the accept header text/html tells the server to deliver an HTML page suitable for a human user. An accept header containing, for example, text/rdf $+n 3$ or application/rdf $+x m l$ tells the server that the client is able to handle RDF. The server can either deliver the data in RDF directly or send an HTTP 302 or 303 response indicating that the information can be found at a different URL». 


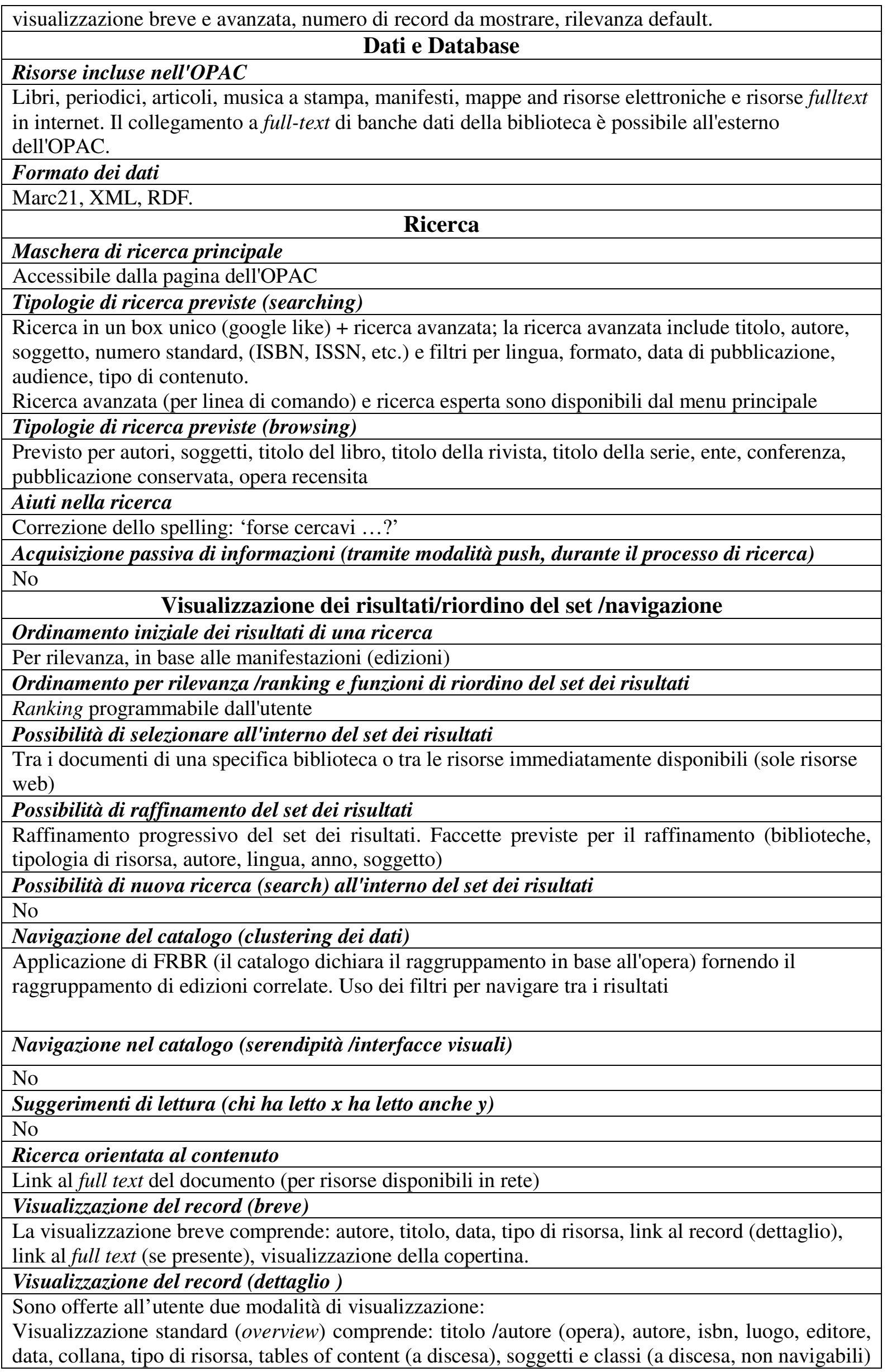


funzioni di esportazione: cita, salva, email, rss (solo nel caso di una ricerca per soggetto) / prestito /richiesta del documento, visualizzazione della copertina, elenco delle localizzazioni, altre edizioni Visualizzazione dettagliata (detail) contiene in forma estesa gli stessi dati della visualizzazione standard più le note di contenuto e il dettaglio del record MARC, link a LIBRIS Formathandbok (Information om MARC21).

Sono presenti varie possibilità di raffinamento/espansione della ricerca dalla visualizzazione del dettaglio: Altri titoli di (autori principali); Altri titoli su (soggetti - ritorna all'elenco dei record e lancia la ricerca sul soggetto); Cerca all'esterno (ricerca della manifestazione in Google, Google Book search, Google Scholar, Scirus, Librarything); link esterno all'autore su Wikipedia.

\section{Contenitori temporanei /permanenti di memoria}

Cronologia ricerche

\section{Funzioni di esportazione /riutilizzo/sociali}

\section{Esportazione della citazione del documento}

Sì, esportazione anche verso software di gestione bibliografie (RefWorks, EndNote, Zotero)

Invio dei risultati per email / Stampa dei risultati

Sì

Altre modalità di riutilizzo dei dati

No

Condivisione nei social network

Sì

\section{Permalink}

Sì, tramite URI

Creazione di una lista personale di documenti

No

Funzioni sociali (aggiunta di tag, recensione, valutazione)

No

RSS (syndication dei dati)

$\mathrm{Si}$

Interfacce/applicazioni per dispositivi mobili

No

\section{Caratteristiche del Sistema}

Software utilizzato

Homegrown, sviluppato ad hoc

Tecnologie utilizzate

Open source

Particolari implementazioni

Sono state realizzate dal team di sviluppo una serie di API per riutilizzare dati dal catalogo, $<$ http://api.libris.kb.se/>.

Ambiente di ricerca

I

Discussione

I cataloghi elettronici attuali, anche quelli definiti 'di nuova generazione', e gli strumenti per la scoperta utilizzano sovente tecnologie non adatte all'apertura dei dati e all'interoperabilità. I dati bibliografici vengono scambiati solo tra biblioteche tramite formati proprietari o non adatti all'interoperabilità con gli altri dati del web. Una grande opportunità di cambiamento viene oggi offerta dal Web Semantico e dalla tecnologia dei linked data che consente la produzione di dati aperti, interoperabili e riutilizzabili nel web. I dati prodotti dalle biblioteche possono così essere liberamente fruibili nel web con enormi vantaggi nella creazione, nella ricerca e nell'utilizzo dei dati bibliografici da parte degli utenti del catalogo. I linked open data offrono un enorme potenziale per collegare tra loro le risorse delle istituzioni del patrimonio culturale come biblioteche, archivi, musei. I dati bibliografici possono collegati a dati prodotti museali o archivistici accrescendo il loro valore e collaborando alla creazione di un grafo globale in grado di collegare tutte le risorse culturali tra loro alle altre risorse del web.

L'OPAC Libris, rappresenta un passo importante in questa direzione. L'OPAC è stato realizzato con 
la volontà di misurarsi con le innovazioni del Web Semantico e di verificarne le potenzialità. L'apertura dei dati bibliografici al web rappresenta per i progettisti un modo di creare interesse verso i dati bibliografici e le biblioteche anche da parte di comunità che non praticano formati e protocolli strettamente bibliotecari. Accanto ai formati tradizionali di 'uscita' (MARCXML, DC, JSON, MODS) l'esposizione dei dati in RDF consente di utilizzare i dati catalografici senza dover creare apposite API e di renderli disponibili sul web. Ciò consente non solo di utilizzare i dati bibliografici, ma anche di sfruttare i link di collegamento tra $i$ dati stessi. L'esposizione dei dati in RDF è stata compiuta sfruttando ontologie conosciute come SKOS, Bibliontology e DBPedia e per le voci di autorità FOAF. Gli elementi di Dublin Core sono stati utilizzati per collegare soggetti e autori e i concetti e le proprietà di SKOS per collegare concetti e stabilire relazioni tesaurali. Tra i vocabolari utilizzati vi è LCSH.info per i soggetti. L'OPAC Libris offre all'utente una navigazione semplificata e migliorata grazie al collegamento tra i dati bibliografici, ma soprattutto si prepara a diventare un punto di partenza per servizi innovativi.

Il consorzio svedese Libris da circa quarant'anni è oggetto di ispirazione per le attività e i servizi proposti; quest'anno in occasione del $40^{\circ}$ anno di attività cooperativa il tema delle giornate Ispirazione Libris ${ }^{16}$ è stato «dati aperti e libertà di informazione». In questo campo la biblioteca nazionale svedese ha infatti aperto nuove prospettive per lo sviluppo dei cataloghi elettronici e per la loro integrazione nel Web Semantico.

\section{Punti di forza/novità}

Le funzionalità di nuova generazione e l'esposizione dei dati sotto forma di linked data consentiranno di creare intorno ai dati del catalogo servizi avanzati.

\section{Criticità}

I

\section{Riferimenti bibliografici}

Martin Malmsten, LIBRIS available as Linked Data, 312 2008. Blog post. «Librisbloggen.kb.se», LIBRIS nationella bibliotekssystem. <http://librisbloggen.kb.se/2008/12/03/libris-available-aslinked-data/>.

Martin Malmsten Making a library catalogue part of the Semantic Web. In Greenberg J. and Klas W. (Eds) Metadata for semantic and social applications. Proceedings of the International Conference on Dublin Core and Metadata Applications. Berlin, 22-26 September 2008, p. 146-150. Singapore and Universitätsverlag Göttingen, Dublin Core Metadata Initiative, 2008 <http://webdoc.sub.gwdg.de/univerlag/2008/DC_proceedings.pdf>.

Martin Malmsten, Exposing Library Data as Linked Data, relazione tenuta al Satellite Meetings IFLA 2009, Emerging trends in technology: libraries between Web 2.0, semantic web and search technology, $\quad$ Florence, 19-20 Agosto 2009, $<$ http://www.ifla2009satelliteflorence.it/meeting3/program/assets/MartinMalmsten.pdf>.

Henrik Lindström - Martin Malmsten, User-centred design and the next generation OPAC - a perfect match?, <http://library.wur.nl/elag2008/presentations/Lindstrom_Malmsten.pdf>.

Anders Söderbäck,-- Martin Malmsten, LIBRIS. Linked Library Data, «Nodalities Magazine», 5(2008), p. 20-21, <http://www.talis.com/nodalities/pdf/nodalities_issue5.pdf>.

Martin Malmsten, Cataloguing in the open - the disintegration and distribution of the record, «LLIS.it», 4 (2013), n. 1, p. 417-424, <http://leo.cilea.it/index.php/jlis/article/view/5512>.

\footnotetext{
16 'Libris-inspirationsdagar' ovvero Inspirazione libris si è tenuto il 26 e 27 marzo 2012 presso la biblioteca nazionale svedese, <http://www.kb.se/aktuellt/utbildningar/2012/Nationell-samverkan/Librisinspirationsdagar/>.
} 


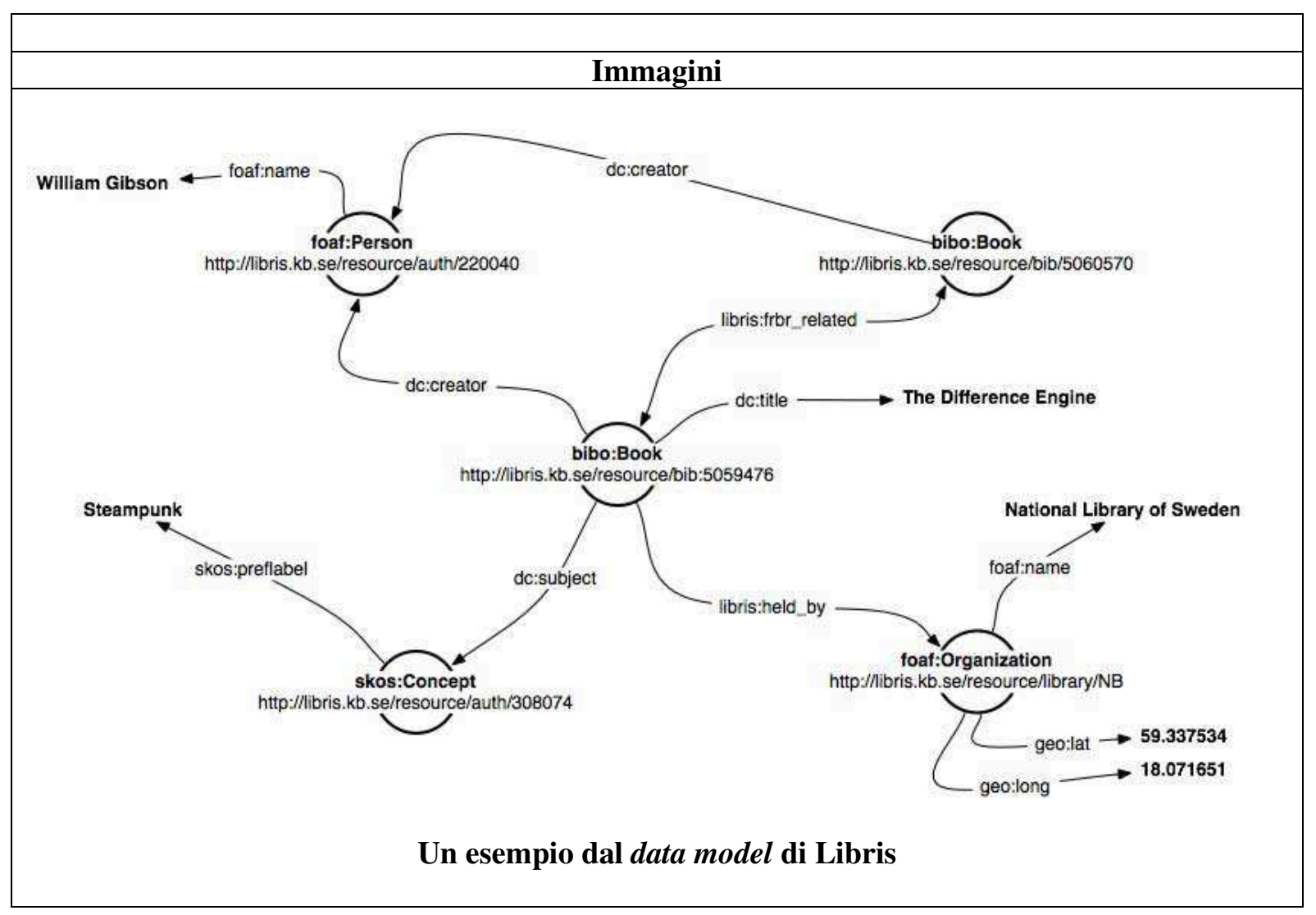




\section{New York Public Library}

URL: <http://nypl.bibliocommons.com/>

La New York Public Library (NYPL) creata nel 1895 è la terza più grande biblioteca del nord America e una delle più importanti e prestigiose biblioteche degli Stati Uniti dopo la Library of Congress, la Boston Public Library e le biblioteche universitarie di Harvard e Yale. Attualmente la biblioteca comprende quattro centri di ricerca e 90 filiali tra le quali spiccano quelle di Brooklyn e Queens. Il patrimonio conta più di 65 milioni di documenti e oltre 20 milioni di volumi e serve circa 18 milioni di utenti nazionali e 25 milioni di utenti di tutto il mondo che accedono alle collezioni e ai servizi online della biblioteca.

Il nuovo catalogo, attualmente realizzato con il software Bibliocommons, implementato nel settembre 2011, contiene circa 8 milioni di record dei quali 6 milioni di titoli catalogati dopo del 1972 e 2 milioni di titoli catalogati in precedenza. I record rappresentano collezioni in più di 370 lingue e dialetti e in una molteplicità di formati: libri, musica a stampa e registrazioni audio, micriforme, documenti d'archivio manoscritti, video e film, fotografie, stampe materiale cartografico, software e databases, slides e altro.

Il catalogo tradizionale WebPac Pro viene tuttora mantenuto per offrire una ricerca mirata e, per i volumi più antichi è presente un catalogo retrospettivo per opere catalogate prima del 1972.

Il catalogo mostra le più comuni caratteristiche degli OPAC di nuova generazione: navigazione a faccette e risultati ordinati per rilevanza consentono la navigazione nel catalogo.

Particolarmente ricche sono le funzioni sociali e i contributi che l'utente può apportare alle risorse catalografiche accedendo alla propria area personale che offre la possibilità di gestire la propria situazione utente e aggiungere contenuti alla risorsa, votare, aggiungere un commento, dei tag, un video (es. un'intervista con l'autore, un filmato relativo alla risorsa), un parere in merito ai destinatari (age suitability), notizie sul contenuto, citazioni, titoli simili, sommari, note private, escludere un titolo della propria lista dalla visualizzazione, segnalare alla biblioteca un contenuto inappropriato, ricevere riconoscimenti dalla community del catalogo (i riconoscimenti rimangono privati).

\section{Accesso all'OPAC /autenticazione}

Dal portale della biblioteca / area personale myNYPL per la gestione utente ed altri servizi. L'utente autenticato può contribuire direttamente al catalogo durante la navigazione.

\section{Possibilità di visualizzare l'interfaccia in più lingue}

No

Altre preferenze/personalizzazioni

Area personale dell'utente, dove è possibile visualizzare e gestire la propria situazione utente, salvare item in liste (my shelves) di documenti (da leggere, in lettura, per dopo), creare nuove liste, gestire la propria comunità di utenti ('my community' ove è possibile mettersi in contatto con altri utenti e condividere risorse, ricevere messaggi). Le liste create possono essere condivise su Facebook e Twitter

\begin{tabular}{|l|}
\hline \multicolumn{1}{|c|}{ Dati e Database } \\
\hline Risorse incluse nell'OPAC \\
\hline $\begin{array}{l}\text { Il catalogo contiene circa } 8 \text { milioni di record relativi a libri, musica a stampa e registrazioni audio, } \\
\text { microforme, documenti d'archivi, manoscritti, video e film, fotografie, stampe materiale } \\
\text { cartografico, software e databases, slides e altro. }\end{array}$ \\
\hline Formato dei dati \\
\hline MARC \\
\hline \multicolumn{1}{|c|}{ Ricerca } \\
\hline $\begin{array}{l}\text { Maschera di ricerca principale } \\
\text { autore, collana, soggetto, tag, lista, utente. }\end{array}$ \\
\hline Tipologie di ricerca previste (searching) \\
\hline Ricerca 'google like' e avanzata \\
\hline
\end{tabular}




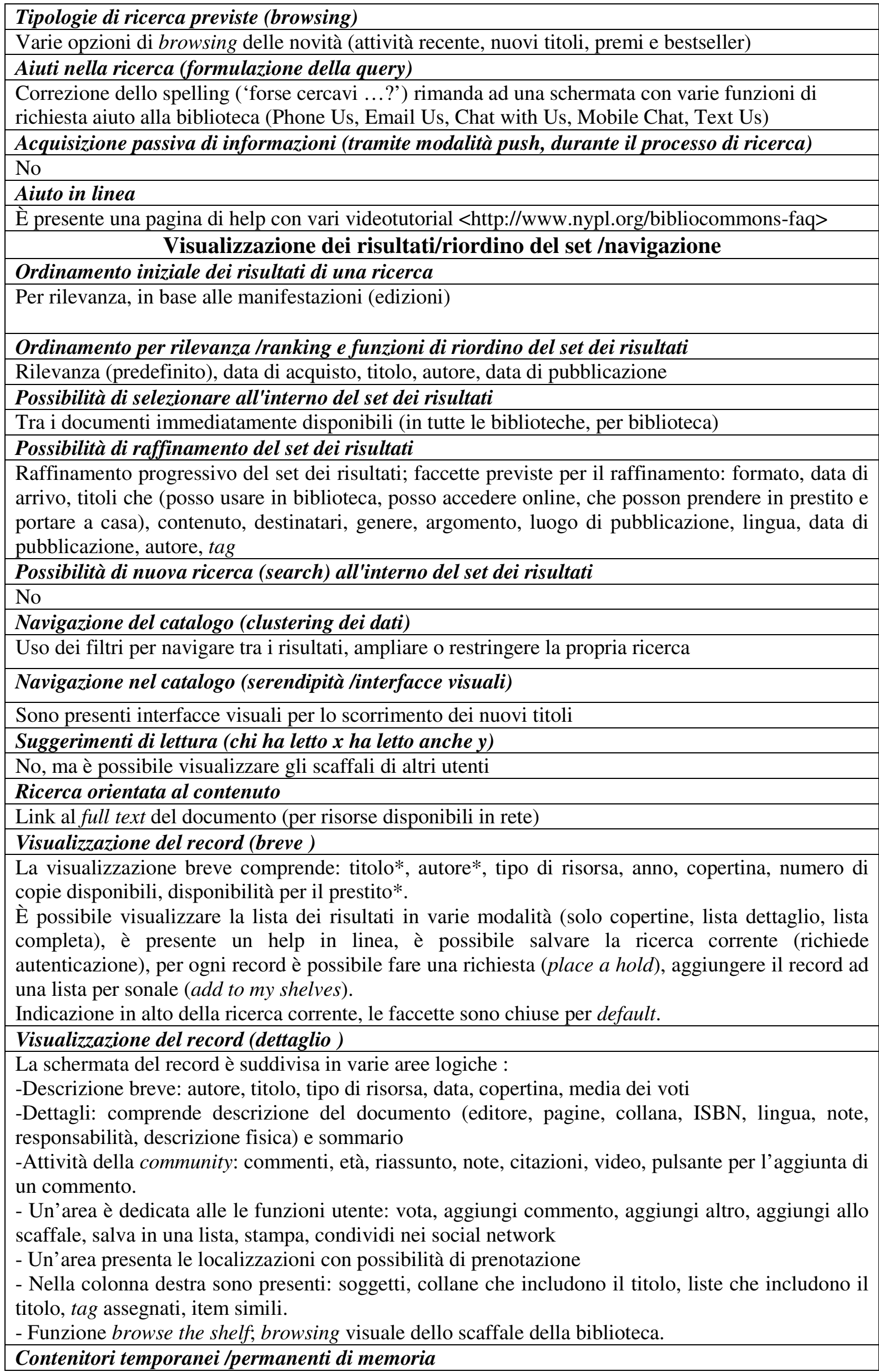




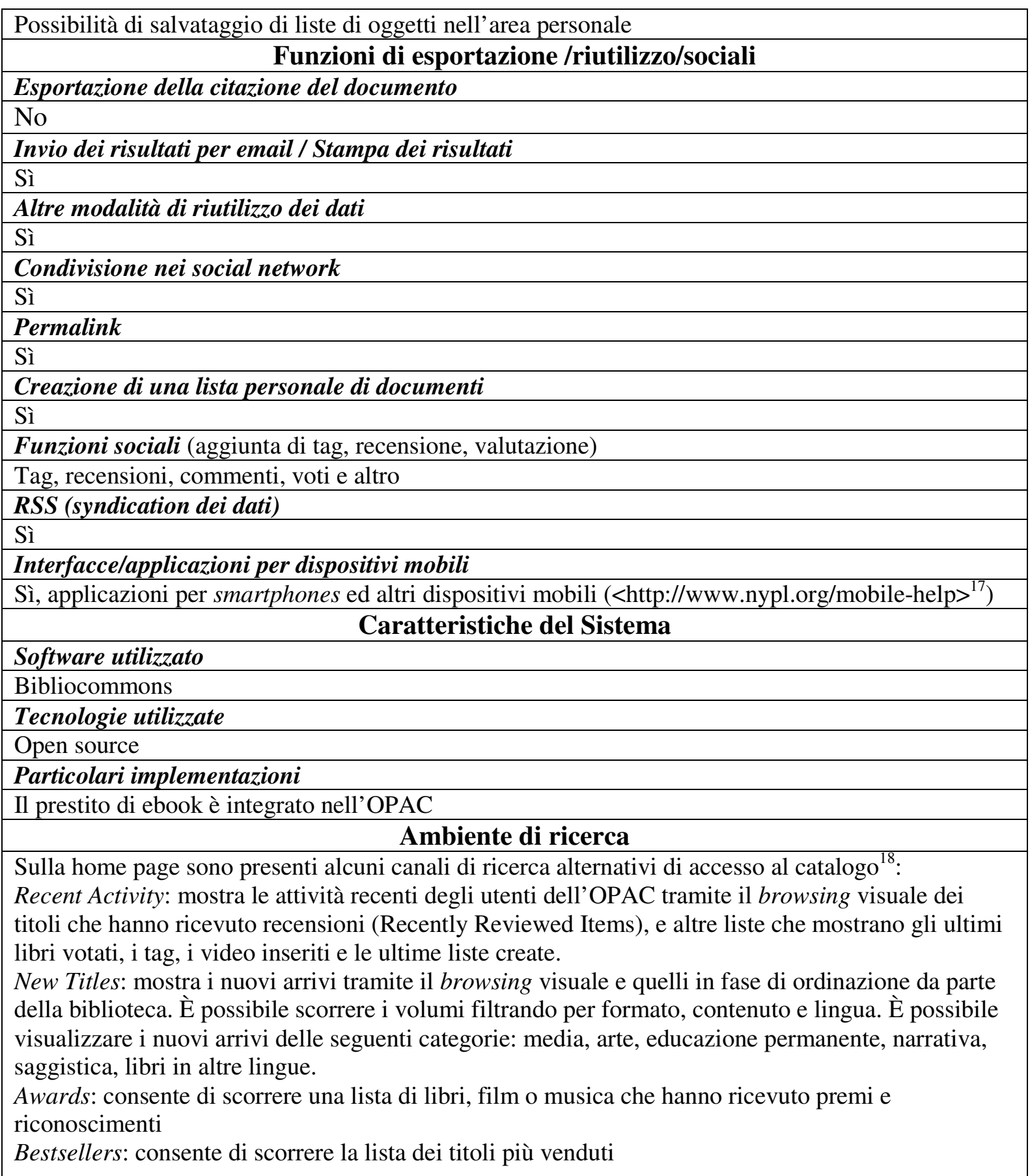

\section{Discussione}

L'OPAC della New York public library, come vari OPAC di biblioteche pubbliche, presenta numerose funzionalità che ne fanno un OPAC sociale di nuova generazione.

La scelta del software Bibliocommons, ricco di funzioni sociali, rispecchia l'esigenza di offrire un'interfaccia semplificata per la ricerca e di creare un punto di interrogazione per i numerosi materiali che la biblioteca offre (tra i quali le collezioni digitali), ma soprattutto di creare intorno al catalogo e alle sue risorse una rete di contenuti generati dagli utenti allo scopo di rafforzare la loro presenza e fidelizzali ai servizi della biblioteca.

Nella presentazione della notizia ampio spazio è dedicato alle funzionalità di interazione con le risorse catalografiche. L'utente autenticandosi può immettere non solo commenti, voti o recensioni,

\footnotetext{
${ }^{17}$ Molte applicazioni per dispositivi mobili create da biblioteche che utilizzano il software sono visibili anche in <http://www.youtube.com/user/bibliocommons>

${ }^{18}$ Queste sono create tramite una funzionalità del software che consente di creare guide per soggetto dagli oggetti presenti nel catalogo.
} 
ma anche riportare le citazioni che lo hanno più colpito, dare un parere sui potenziali destinatari della risorsa e organizzare in liste pubbliche e private i propri libri preferiti. All'interno della propria area personale l'utente può ricevere messaggi e mettersi in contatto con altri utenti del catalogo.

L'OPAC offerto agli utenti è dunque uno strumento sociale, ispirato alle applicazioni del Web 2.0, come le reti sociali, le librerie online e i siti di social cataloguing per favorire il più possibile la partecipazione attiva dell'utente. Nell'ottica della partecipazione il principale arricchimento del catalogo è quello della conversazione e dialogo con l'utente. L'OPAC offre all'utente la possibilità di arricchire le informazioni bibliografiche relative ai libri con i commenti, le recensioni, le opinioni o i voti e, accogliendo le folksonomie, assegnare per classificare le risorse.

È un peccato che questo strumento 'di nuova generazione' nell'accoglimento di una nuova semantica 'dal basso' finisca per trascurare gli accessi semantici tradizionali. Ciò si riflette nelle scelte compiute per la navigazione: le faccette di soggetto sono tra le meno evidenti e manca totalmente la faccetta della classificazione. Anche per questo motivo l'OPAC tradizionale, che consente ricerche mirate specie per soggetti e classi, non è stato abbandonato, ma completa l'offerta dei servizi della biblioteca, sebbene nella maggior parte dei casi l'OPAC di nuova generazione sostituisca il tradizionale catalogo.

\section{Punti di forza / novità}

La ricchezza degli strumenti sociali a disposizione degli utenti consente di creare attorno al catalogo una comunità di utenti e rafforza l'uso del catalogo.

\section{Criticità}

Il catalogo non sfrutta adeguatamente le opportunità di accesso per soggetto; manca anche la funzionalità di esportazione della citazione.

\section{Riferimenti bibliografici}

Marshall Breeding, New York Public Library Partners with BiblioCommons, Smart Libraries Newsletter, $31 \quad$ (2011) $\quad$ n. $\quad 9$, p. 2-4, <http://www.librarytechnology.org/ltgdisplaytext.pl?RC $=16143>$.

\section{Immagini}




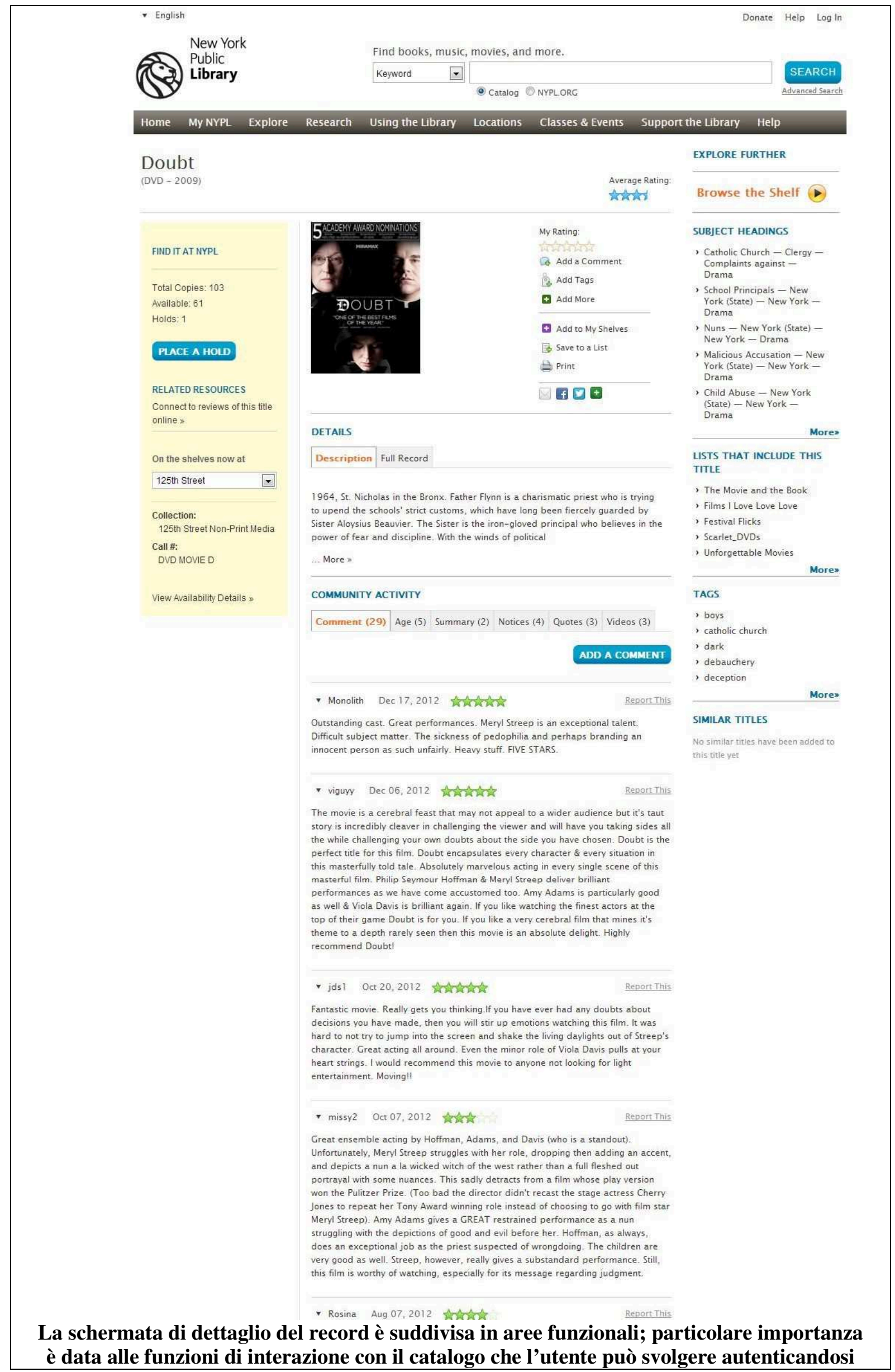




\section{North Carolina State University. Endeca e Summon}

URL: <http://www.lib.ncsu.edu/catalog/>

Endeca, implementato dalle biblioteche della North Carolina State University (NCSU) a partire dal 12 gennaio 2006, è il più noto OPAC di nuova generazione.

Il catalogo è conosciuto per l'introduzione della cosiddettà 'ricerca a faccette' affiancata dalla presentazione dei risultati ordinata per ranking (rilevanza) come modalità di navigazione del catalogo e di una serie di altre caratteristiche innovative che hanno segnato profondamente la progettazione delle interfacce per i cataloghi elettronici ${ }^{19}$.

Le biblioteche di NCSU acquistarono il software di Endeca Information Access Platform nel maggio 2005; fu così creata una commissione di studio affidata ad Emily Lynema che nel biennio 2005-2007 studiò l'implementazione del software con il preesistente OPAC Web2 dell'ILS Unicorn prodotto da Sirsi-Dynix. Già dal principio vi erano alcuni obiettivi che il team di sviluppo voleva ottenere dall'esperienza di uso del nuovo catalogo: semplicità nell'interfaccia, interattività nella fase di gestione dei risultati, elasticità nella ricerca (correzione degli errori in fase di digitazione), ordinamento per rilevanza dei risultati ottenuti alla maniera di Google, utilizzo dei web service (tecnologia del web 2.0 che permette il riutilizzo dei dati e mashup con il web) ${ }^{20}$. All'inizio il gruppo di studio lavorò su alcune caratteristiche principali rimandando funzionalità più avanzate alle versioni successive; tra le prime funzionalità introdotte vi erano la navigazione a faccette (faceted browse o navigation), la possibilità di ottenere informazioni in tempo reale sulla disponibilità dei documenti, link ai dati dell'OPAC Web2 in tempo reale, navigazione 'a briciole di pane', correzione dello spelling, il browsing gerarchico delle classi basato sulla LCC, il browsing dei nuovi titoli, visualizzazione semplificata e la possibilità di gestire risorse online (con varie forme di redirect a Journal finder / web2). Altre caratteristiche vennero aggiunte alla progettazione in seguito, quando Endeca venne adottato anche per il catalogo collettivo delle cosiddette 'biblioteche del Triangolo' le TRLN libraries (Duke, NCCU, NCSU, UNC-CH) ${ }^{21}$.

Al momento del lancio sul web dell'OPAC, nel 2006, Endeca coesisteva con l'ILS Unicorn di SirsiDynix e l'OPAC Web2; Endeca gestiva la ricerca per parola chiave mentre Web2 (il catalogo online preesistente) gestiva l'authority search (ricerca per campi) e la visualizzazione del dettaglio della pagina del record; i record MARC che venivano re-indicizzati in Endeca venivano poi esportati durante la notte da Unicorn; in sostanza, Endeca si configurava come la parte del'ILS dedicata alla ricerca e alla 'scoperta'.

Alcune funzionalità non supportate, ma sulle quali il team contava di lavorare in seguito, erano: l'aggregazione dei record per opera (attraverso l'implementazione del modello FRBR); la possibilità di personalizzazione del catalogo; l'aggiunta di folksonomies; l'aggiunta di caratteristiche di raccomandazione; le funzionalità 'carrello della spesa'.

Il 7 marzo del 2008, in coincidenza con l'avvio del catalogo unico, NCSU ha rilasciato Endeca 1.2, la nuova versione migliorata del catalogo. Nello stesso anno si resero disponibili i risultati di una prima indagine sull'uso dell'OPAC nel biennio 2006-2008 ${ }^{22}$. Probabilmente queste prime

\footnotetext{
${ }^{19}$ La navigazione guidata di Endeca, società che si occupa di strumenti di ricerca per aziende (tra i clienti vi sono grandi catene commerciali, imprese della finanza, della produzione enti governativi, editori) è stata progettata per rispondere a problemi del sovraccarico informativo (information overload) nella ricerca. Non si tratta quindi di una soluzione specificamente creata per le biblioteche, ma studiata per organizzare l'informazione e gestire la ricerca nei grandi siti web commerciali.

${ }^{20}$ Cfr. Charley Pennell, NCSU Endeca 2 1/2 years on: from NextGen to Normalcy, ALA Annual Meeting, Anaheim, CA, June, 2008 <www.lib.ncsu.edu/endeca/presentations/200806-ala-pennell.ppt>.

${ }^{21}$ Queste istituzioni dal 1985 al 1992 avevano mantenuto un catalogo collettivo; negli anni successivi si erano poi separate e avevano scelto per i propri OPAC soluzioni diverse, dal 2003 NCSU \& NCCU avevano adottato Unicorn; Duke aveva adottato Aleph; UNC-CH aveva scelto Millenium. Dopo la fusione di Sirsi e Dynix e l'acquisto di Endeca da parte di NCSU nel 2007 il TRLN approvò Endeca come unica interfaccia per il catalogo. Nel 2008 venne varato il nuovo TRLN Endeca.

${ }^{22}$ Da una prima indagine condotta allo scopo di valutare l'uso del nuovo catalogo nel biennio 2006-2008 e riportata da Charley Pennell (2008) risulta che il 73\% delle richieste fatte al catalogo è una ricerca per parola
} 
valutazioni hanno spinto i progettisti ad apportare alcuni correttivi come, ad esempio, eliminare la ricerca per classi, che prima si trovava in testa alla schermata principale dei risultati, come metodo principale di browsing del catalogo e localizzarla tra le faccette di raffinamento. La navigazione per classi come punto di partenza per l'esplorazione del catalogo è stata delegata a un'applicazione esterna al catalogo, la navigazione per classe è entrata a far parte delle faccette di raffinamento è diventata attivabile a partire dalla singola scheda (funzione browse the shelf), mentre nell'ultima versione del software è stata introdotta una funzione di scorrimento dello scaffale di tipo visuale.

Il record bibliografico di Endeca, ricavato dal modulo OPAC Web2 viene arricchito dalle copertine e dai sommari e dal catalogo è possibile in ogni momento espandere la ricerca all'esterno. È possibile anche attivare una visualizzazione del record MARC. Il catalogo delle biblioteche NCSU mostra solo ciò che è stato acquistato per le collezioni della biblioteca, per la maggior parte libri, risorse elettroniche e materiale in altri formati. I periodici vengono mostrati come titoli, mentre per la ricerca degli articoli in fulltext si fa riferimento a Summon.

Per quanto riguarda le funzioni di ricerca nel catalogo, nel 2006, anno del lancio della prima versione dell'OPAC, il catalogo veniva visualizzato nella pagina principale della biblioteca e presentava un box di ricerca attivabile con tre diverse modalità: search (portava ad una schermata con due diverse possibilità di ricerca: per parola chiave (gestita da Endeca) e per liste (gestita dal modulo OPAC Web2), browse (lanciava il browsing delle classi LC) e ricerca avanzata (apriva la schermata della ricerca avanzata). L'accesso diretto al catalogo dalla pagina principale della biblioteca è stato poi sostituito, a partire dal 2008, da uno strumento unificato di ricerca, QuickSearch, che permette di cercare in tutte le risorse della biblioteca (opzione di default) inviando ad una pagina apposita che mostra i primi risultati dal discovery tool Summon (articoli), dal

chiave; seguono nell'ordine le richieste per titolo (12\%), autore (7\%), ISBN (4\%), soggetto(3\%), titolo/autore, parola chiave/autore, autore/titolo, parola chiave/soggetto. La navigazione nelle singole faccette avviene per il $29 \%$ nelle faccette di soggetto (subject:topic), per il $21 \%$ nelle faccette format, call number, library, author, subject:genre (suddivisione formale del soggetto), subject:region (suddivisione geografica del soggetto), language, subject:era (suddivisione cronologica del soggetto), disponibilità, nuovi titoli. Rispetto al 2006, cioè al momento in cui la navigazione a faccette fece i suoi esordi nel primo OPAC di NCSU la navigazione per soggetto, prima utilizzata più o meno con la stessa frequenza della navigazione per classe $(18 \%$ e $19 \%)$, è cresciuta principalmente a discapito di quella per classi e la navigazione per formato è passata dal $10 \%$ al $21 \%$.

${ }^{23}$ L'algoritmo di ranking è stato modificato rispetto a quello fornito con il software acquistato da Endeca e si basa su alcuni fattori e sui dati provenienti da diversi moduli del sistema (ILS): confronto della interrogazione così come è inserita dall'utente; corrispondenza della frase per ricerche con più termini ; field match, cioè corrispondenza per campi dove ad ogni campo viene dato un diverso peso nell'algoritmo ad esempio la corrispondenza della parola nel campo titolo viene considerato più rilevante di una corrispondenza nel campo delle note; numero dei campi che hanno restituito un risultato; Weighted frequency (TF/IDF) o frequenza ponderata; data di pubblicazione, dati provenienti dalla circolazione (popolarità del documento).

${ }^{24}$ L'OPAC dialoga con altre applicazioni (es. Quick Search) attraverso la web API CatalogWS, sviluppata da Tito Sierra e Emily Lynema per richiedere informazioni in modo dinamico dal catalogo Endeca. Essa supporta un elenco ampio di applicazioni che permettono di sfruttare i dati del catalogo fuori dall'interfaccia OPAC. CatalogWS consente il colloquio con le seguenti applicazioni esterne, progettate dal team di NCSU libraries:

* New Books RSS: applicazione che permette di riceve i nuovi titoli del catalogo via RSS

* Catalog Results QS Module: modulo che integra i risultati dal catalogo nell'applicazione Library Quick Search

* MobiLIB catalog: applicazione che consente l'interrogazione del catalogo da un dispositivo mobile

* New Books Bookwall: interfaccia visuale che mostra una bacheca di copertine delle nuove acquisizioni <http://www.lib.ncsu.edu/display/bookwall/eboard.php>

* New Faculty Books: interfaccia visuale che mostra una bacheca di copertine dei nuovi libri editi dalla Facoltà <http://www.lib.ncsu.edu/display/facultybooks/eboard.php>

* FacetBrowser: una nuova interfaccia avanzata di browsing del catalogo

* Catalog Google Gadget: il gadget da incorporare nella pagina IGoogle che permette di fare una ricerca nel catalogo dell'ateneo

OpenSearch interface: risultati del catalogo in xml con OpenSearch (tecnologie per l'aggregazione e la trasmissione via RSS dei dati). 
catalogo, dal sito web della biblioteca e da altre fonti (es. Google Scholar, repository istituzionale); in questa schermata sono presenti la ricerca articoli (che reindirizza a Summon); la ricerca "libri e altro" che indirizza al catalogo NSCU. Oltre alla sostituzione dell'accesso diretto al catalogo con una preliminare pagina-filtro (Quick search) la ricerca a catalogo viene indicata con l'etichetta "books \&more".

Le principali funzionalità di ricerca nel catalogo searching e browsing (scorrimento), sono completate dalla chat con il bibliotecario. La funzione di ricerca (searching) è rappresentata dal box di ricerca nei campi che ha come caratteristica aggiuntiva (aggiunta nella seconda versione del software) la possibilità di cercare nuovamente all'interno di un primo set di risultati, ottenendo una utile integrazione delle funzioni di searching e browsing). Caratterizzano inoltre le funzioni di ricerca la correzione dello spelling (forse cercavi) stemming (aggiunta di prefissi e suffissi per evitare il risultato zero), la possibilità di accedere alla chat dal catalogo e la possibilità di intervenire sul set dei record per restringere solo ai volumi disponibili, a ciò che è disponibile online, o solo ai nuovi titoli (visualizza i titoli acquisiti nell'ultimo mese).

La visualizzazione dei risultati fornita da Endeca è rappresentata da un elenco di risultati ordinati per rilevanza ${ }^{23}$

La navigazione è l'ormai famosa faceted navigation o navigazione a faccette o guidata. L'intera collezione, ancora prima che l'utente formuli una interrogazione, o qualora il box di ricerca sia lasciato vuoto e venga premuto il pulsante 'cerca', è presentata suddivisa in faccette con l'esposizione delle occorrenze. Il raffinamento 'per faccette' prevede la possibilità di selezionare / deselezionare le faccette cioè di ampliare/ restringere la ricerca o tornare indietro.

La progettazione della pagina permette di fare ricerca e browsing in una singola schermata e di riordinare il set dei risultati; vi è, infine, la possibilità di espandere la ricerca tramite il pulsante Expand Your Search alle biblioteche del Triangle e alle altre biblioteche che aderiscono al catalogo collettivo Worldcat. Il catalogo, durante una ricerca, da già conto delle risorse presenti nelle altre risorse esterne indicando il numero di occorrenze corrispondenti all'interrogazione immessa.

Per quanto riguarda le funzionalità attivabili dall'utente, partendo da un record Endeca vi sono varie possibilità di riutilizzo dei dati ottenuti. È possible richiedere il documento (anche al domicilio dell'utente), mandarlo in formato testo a un telefonino, inviarlo via email, esportarlo in vari 'gestori di bibliografie', salvarlo nel 'carrello'. Nello spazio virtuale dell'utente è possibile effettuare varie operazioni sul record (esportazione, richiesta di prestito, abbonarsi ad una ricerca e riceverne periodicamente gli aggiornamenti via RSS, cioè direttamente da un browser o da un aggregatore di Feed RSS e senza visitare il catalogo).

Infine per quanto riguarda le caratteristiche di sistema, senza entrare nei dettagli estremamente tecnici in merito all'integrazione dell'OPAC Endeca con l'ILS di SirsiDynix, la ricerca per parola chiave e la navigazione guidata sono completamente eseguiti da Endeca. I record Marc vengono esportati dal sistema Unicorn, modificati, e ri-processati dal software Endeca. La visualizzazione dettagliata del record è affidata al catalogo Web2 di Sirsi Dynix. La ricerca dei record di autorità (la ricerca "inizia con") viene ancora svolta da Web2 e Unicorn.

Il catalogo implementa alcune tecnologie come il permalink (link permanente a un record o una risorsa) e le API (Application Programming Interface) per comunicare con altre applicazioni ${ }^{24}$.

Accesso all'OPAC /autenticazione

Accesso dal box di ricerca Quick search integrato nella home page della biblioteca, accesso anche dalla home page dell'OPAC.

Possibilità di visualizzare l'interfaccia in più lingue

No

Altre preferenze/personalizzazioni

No

Risorse incluse nell'OPAC

Sono inclusi libri, ebook, riviste, fil,m musica, documenti governativi e altre risorse tradizionali

Formato dei dati

MARC21

Ricerca 


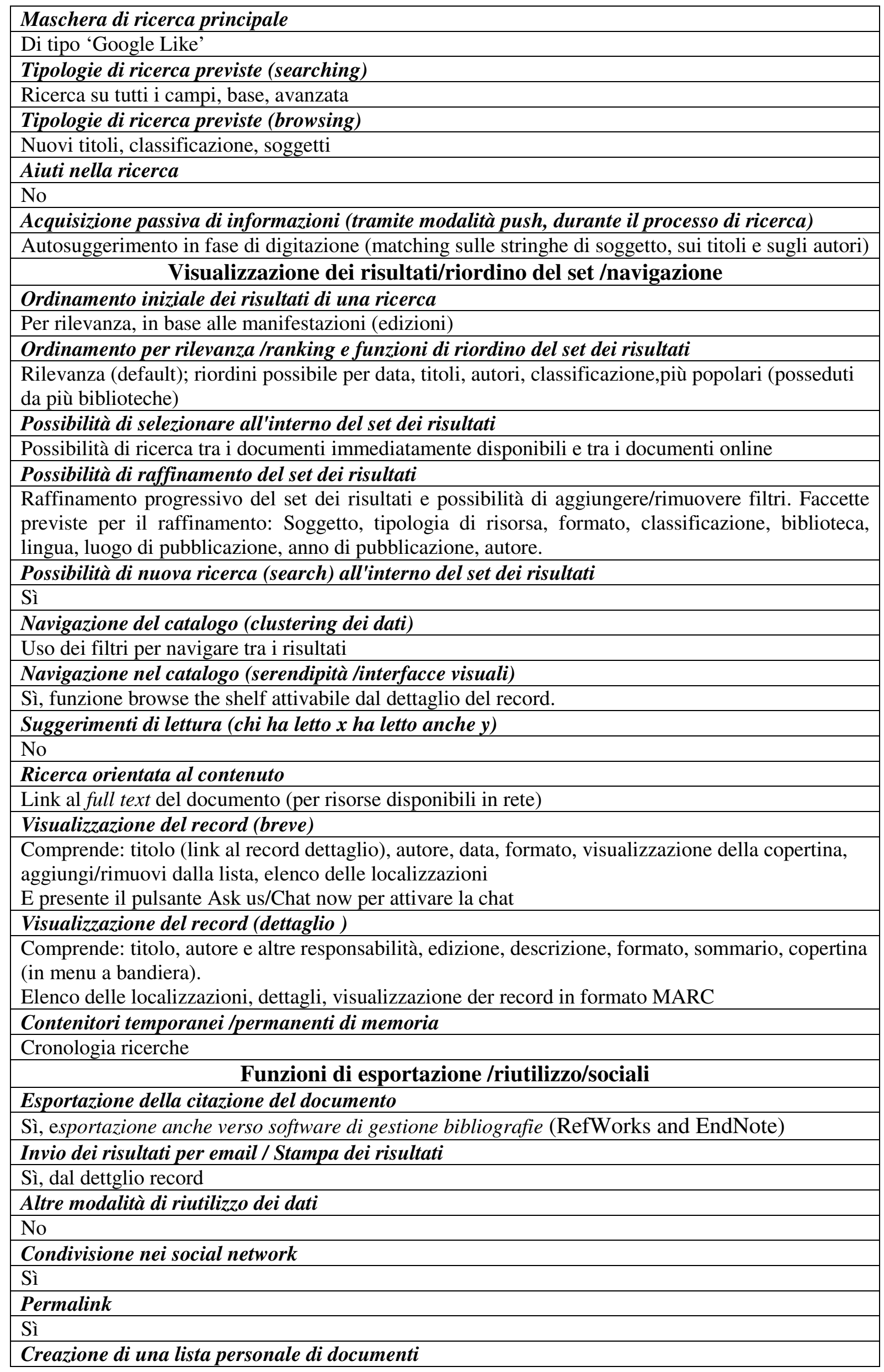




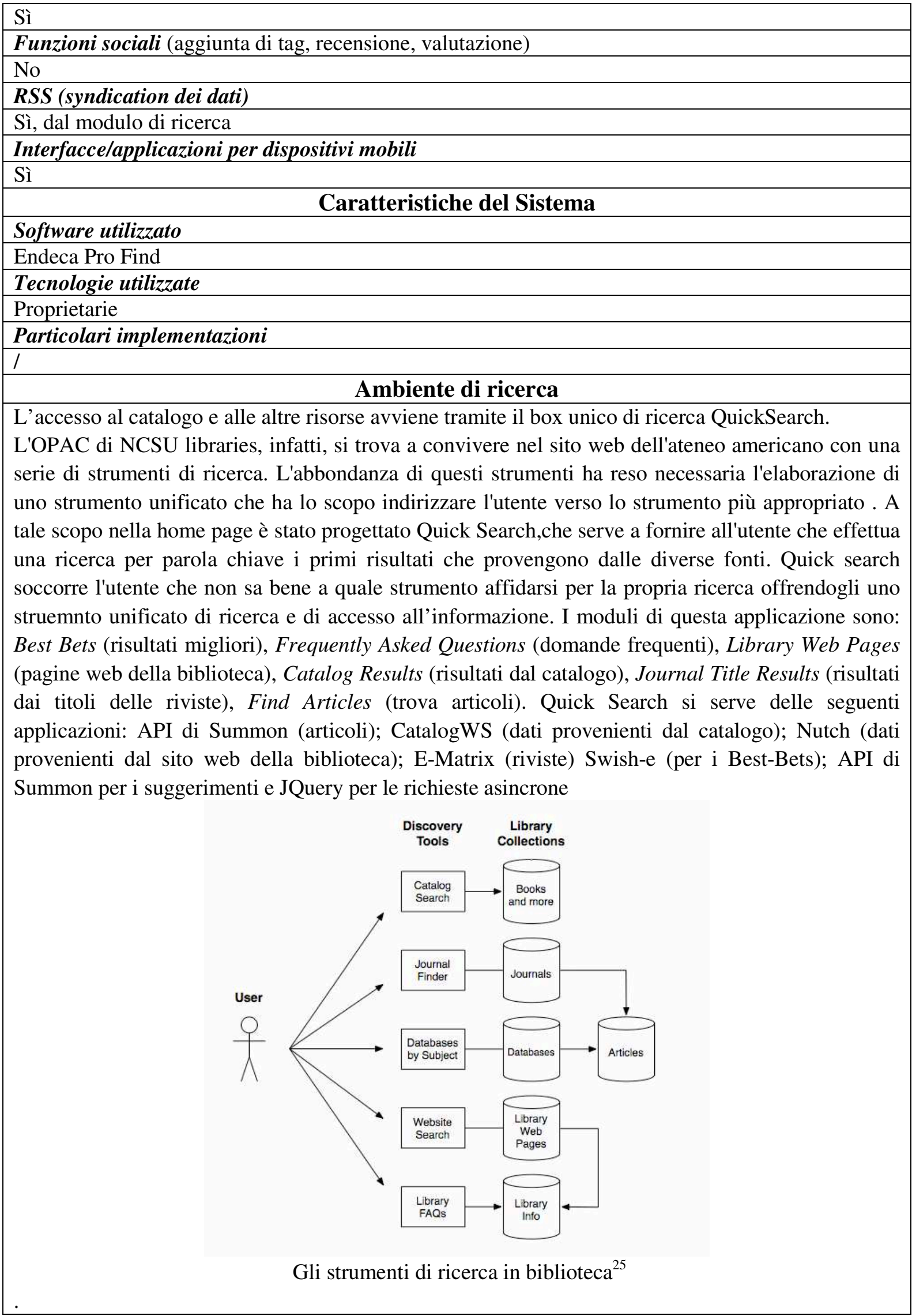

${ }^{25}$ Tito Sierra, A single search box interface to the NCSU libraries, two years later, Digital Library Federation Spring Forum 2007, Pasadena, California, April 24, 2007, $<$ http://www.lib.ncsu.edu/dli/projects/quicksearch/>. 


\begin{abstract}
Il discovery tool Summon
Il catalogo delle biblioteche NCSU mostra solo ciò che è stato acquistato per le collezioni della biblioteca, per la maggior parte libri, risorse elettroniche, e materiale in altri formati. Per la ricerca 'a livello di articolo' nell'aprile 2010 NCSU ha affiancato all'OPAClo strumento per la scoperta Summon (<http://www.serialssolutions.com/summon/ $>$ ).

Il discovery tools di Serial solutions, disponibile in commercio dal luglio 2009, viene definito nel sito web «Un servizio per la scoperta nel web che permette di cercare velocemente, trovare e accedere a contenut affidabili e attendibili. È uno strumento che va oltre la ricerca federata, oltre i cataloghi di nuova generazione per creare un servizio completamente nuovo per le biblioteche. Attraverso una semplice ricerca, il servizio Summon da accesso a una vastissima serie di contenuti autorevoli - digitali e a stampa, audio e video, singoli articoli e interi e journals, in qualsiasi formato». Non c'è dunque bisogno di trasmettere la ricerca ad altri database; il software fornisce una singola casella di ricerca in cui l'utente può introdurre qualsiasi termine e ricevere velocemente una serie di risultati in una lista ordinata per rilevanza. I contenuti indicizzati in Summon sono di svariati tipi: record del catalogo della biblioteca, articoli di riviste elettroniche, banche dati, articoli di giornale, ebook, tesi di laurea, depositi istituzionali, atti di convegni, letteratura grigia, riferimenti citati, relazioni, documenti provenienti da collezioni e altro $^{26}$.

Summon ha dato modo al catalogo NCSU di risolvere il problema legato alla ricerca degli articoli pubblicati sulle riviste; una volta trovato l'articolo Summon attiva un resolver per offrire all'utente abilitato la possibilità di autenticarsi e scaricare il full text.

L'accesso al catalogo non è intuitivo. L'utente viene infatti indirizzato al catalogo attraverso l'interfaccia di ricerc fornita da Quicksearch selezionando Books\&more. Più opportuno sarebbe fornire all'utente un'etichetta più esplicativa (es. Catalog).
\end{abstract}

\title{
Discussione
}

Endeca è uno dei più noti OPAC di nuova generazione e forse il più documentato insieme a WorldCat.

Le biblioteche della NCSU, che lo hanno adottato a partire dal 2006, forniscono un buon esempio di ambiente complesso, dove vengono sfruttate le nuove tecnologie per fornire l'accesso a fonti diverse. L'OPAC di nuova generazione Endeca, il discovery tool Summon e gli altri strumenti predisposti dalle biblioteche NCSU per esplorare le collezioni documentarie ci mostrano quanto sia complesso progettare ambienti di ricerca efficaci.

La soluzione scelta da Endeca per progettare la ricerca nell'OPAC ha preferito puntare su caratteristiche molto richieste e familiari all'utente (ranking, box di ricerca unico, faccette) e le caratteristiche dapprima anticipate di implementazione di FRBR per il raggruppamento in base all'opera, ad ormai sette anni dall'avvio del nuovo catalogo non sono state ancora implementate.

Il punto di forza di Endeca è quello di aver progettato una ricerca 'a faccette' dove il browsing permette una ricerca guidata e senza sforzo per l'utente. Mentre la ricerca (searching) richiede di formulare una interrogazione e di descrivere in anticipo le nostre esigenze informative al contrario il browsing mostra direttamente ciò che è disponibile, mostra il vocabolario usato e ci ricorda di cosa potremmo avere bisogno.

La possibilità di usare le API per richiamare i dati del catalogo da fonti diverse ha permesso di costruire applicazioni esterne che consentono di esplorare il catalogo in vari modi; ad esempio si può navigare il catalogo partendo dalle classi o dai soggetti o dalle altre faccette (negli strumenti Facetbrowser e New books bookwall).

Poche le caratteristiche di tipo 2.0 implementate; il catalogo non ha giocato la carta dell'innovazione sulle caratteristiche sociali (ad esempio le folksonomies, che nel 2006 costituivano una novità e lo stesso Endeca aveva in programma di aggiungere al catalogo non sono poi state implementate) ma sulla novità nell'interfaccia e sul ranking dei risultati.

${ }^{26}$ Cfr. infra, Appendice B. Software per OPAC di nuova generazione e servizi di Web scale discovery. Summon. 
L'intreccio di strumenti per la ricerca che le biblioteche della North Carolina State University mettono a disposizione dell'utente ci da senz'altro una prima idea dell'articolato ambiente informativo che le biblioteche sono oggi chiamate a gestire.

\section{Punti di forza / novità}

L'interfaccia innovativa di Endeca, le funzioni di browsing del catalogo, la costruzione intorno al catalogo di un sistema di ricerca ricco che integra i vari strumenti di ricerca presenti in biblioteca.

\section{Criticità}

L'interfaccia 'esplorativa' di Endeca basata sulla coppia relevance ranking/navigazione a facccette estratte dai metadati ha segnato la nascita di un modello di successo nello sviluppo delle interfacce per OPAC penalizzando la possibilità di esplorare in maniera significativa l'universo bibliografico rappresentato nel catalogo.

\section{Riferimenti bibliografici}

Per la descrizione del progetto, la bibliografia, presentazioni, demo del software e avanzamento del progetto (compresa una bibliografia sull'information retrieval) si veda: $<$ http://www.lib.ncsu.edu/endeca>; Una demo è presente in: $<$ http://www.lib.ncsu.edu/endeca/presentations/endeca-demo.wmv>.

L'usabilità del catalogo è stata oggetto di due report interni che a distanza di due anni hanno verificato l'effettiva rispondenza delle scelte catalografiche sull'uso del catalogo (Littletree 2008, Lynema 2006). Una webcast presentazione di Pace si trova sul sito della Library of Congress webcasts all'indirizzo: http://www.loc.gov/today/cyberlc/feature_wdesc.php?rec=4107 NCSU's Faceted Catalog: 18 Months Later.

Kristin Antelman, - Emily Lynema - Andrew K. Pace, Toward a Twenty-first Century Library Catalog, «Information Technology \& Libraries», 25 (2006), n. 3 p. 128-139 oppure: $<$ http://eprints.rclis.org/archive/00007332/01/antelman_lynema_pace.pdf>

Maria Collins - Jacquie Samples - Charley Pennell - David GOLDSMITH, Magnifying the ILS with Endeca, «The Serials Librarian», 51 (2010), n. 3/4, p. 75-100.

Sandy Littletree, Endeca catalog usability test: findings report, (internal report), February 2008. $<$ http://www.lib.ncsu.edu/endeca/publications.html>.

Emily Lynema, Endeca catalog usability test: findings report (internal report), July 2006. $<$ http://www.lib.ncsu.edu/endeca/publications.html $>$.

Cory Lown, A Transaction Log Analysis of NCSU's Faceted Navigation OPAC. A Master's Paper for the M.S. in L.S degree,. April, 2008, <http://ils.unc.edu/MSpapers/3387.pdf>.

Charley Pennell, NCSU Endeca 2 1/2 years on: from NextGen to Normalcy, ALA Annual Meeting, Anaheim, CA, June, 2008 <www.lib.ncsu.edu/endeca/presentations/200806-ala-pennell.ppt>.

Tito Sierra, Improving the catalogue interface using Endeca, Capitalize on access October 2006. $<$ http://www.lib.ncsu.edu/endeca/presentations.html>.

Tito Sierra, A single search box interface to the NCSU libraries, two years later, Digital Library Federation Spring Forum 2007, Pasadena, California, April 24, 2007. $<$ http://www.lib.ncsu.edu/dli/projects/quicksearch/>.

\section{Immagini}




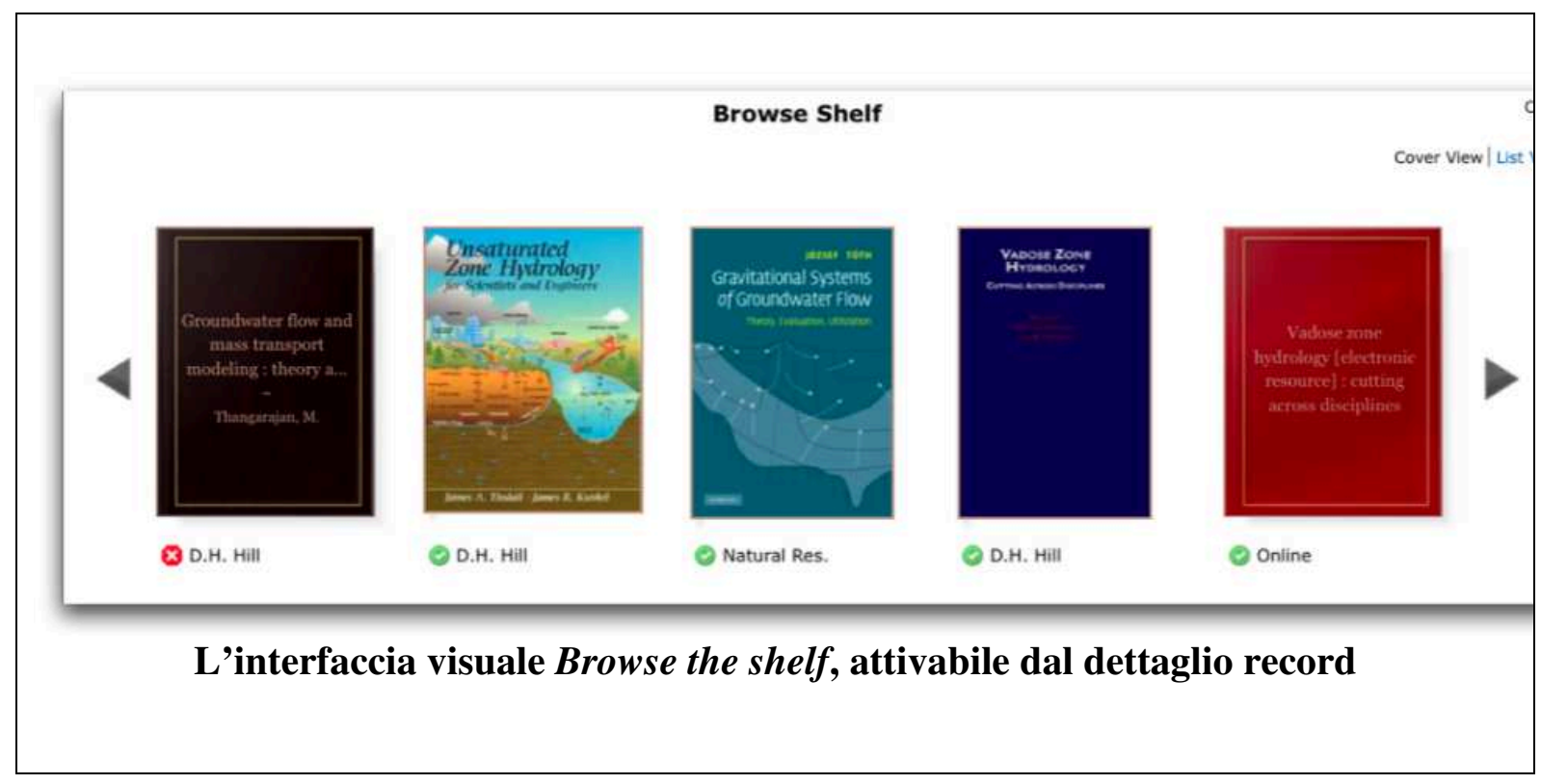




\section{OCLC. WorldCat}

URL: <www.worldcat.org>

WorldCat è il catalogo del consorzio di biblioteche OCLC $^{27}$, nonché il più grande catalogo collettivo del mondo comprendente 275 milioni di record bibliografici e più di un miliardo e mezzo di risorse possedute dalle biblioteche partecipanti che lo incrementano collettivamente. Il catalogo include record bibliografici di varie tipologie di risorse; oltre ai libri, video, periodici il database include articoli, musica, ebooks, genealogie, oggetti d'arte, oggetti digitali, siti web, include anche libri rari e oggetti unici. In Worldcat sono indicizzati anche contenuti provenienti da OAIster, JSTOR Archive, Elsevier, Medline, ERIC, British Library Inside Serials, ArticleFirst. Nel catalogo sono catalogate risorse in più di 470 lingue provenienti da 112 diversi paesi del mondo con record prevalentemente in lingua inglese, ma anche in Arabo, Bengali, Cinese, Cyrillic, Greco, Ebraico, Giapponese, Coreano, Latino, Tamil and Thailandese. Tra le istituzioni rappresentate vi sono i cataloghi di 44 biblioteche nazionali, tra le quali Australia, Polonia e Nuova Zelanda. Con l'ingresso nel consorzio delle biblioteche italiane del Catalogo bibliografico Trentino l'interfaccia è stata tradotta ed è oggi disponibile anche in italiano.

Le registrazioni bibliografiche vengono mantenute e incrementate cooperativamente dai bibliotecari di tutto il mondo secondo standard internazionali e verificate da OCLC.

I record vengono arricchiti tramite copertine, sommari, note sugli autori, recensioni, estratti e altri collegamenti esterni e in fase di ricerca i risultati vengono esposti per rilevanza, e raggruppati in base al modello FRBR tramite l'apposito algoritmo sviluppato da OCLC.

La visualizzazione raggruppa le diverse espressioni facilitando la visualizzazione di opere presenti in formati diversi. Particolarmente interessante è anche la recente visualizzazione dell'interfaccia nella lingua di provenienza dell'utente.

Nell'agosto 2012 WorldCat ha annunciato l'esposizione dei dati bibliografici sottoforma di linked data utilizzando Schema.org.

Accesso all'OPAC /autenticazione

Accesso web, autenticazione per alcuni servizi

Possibilità di visualizzare l'interfaccia in più lingue

L'interfaccia si adatta automaticamente alla lingua di provenienza dell'utente

Altre preferenze/personalizzazioni

Possibilità di personalizzazione dal proprio account

\section{Dati e Database}

Risorse incluse nell'OPAC

Libri, video, periodici, articoli in full text di riviste, musica, ebooks, genealogie, oggetti d'arte, oggetti digitali, siti web, risorse online, archivi fotografici, visuali e multimediali. Indicizza anche i contenuti provenienti da OAIster, JSTOR Archive, Elsevier, Medline, ERIC, British Library Inside Serials, ArticleFirst.

Formato dei dati

MARC 21, MARCXML, RDF (tramite Schema.org)

Maschera di ricerca principale

\section{Ricerca}

Di tipo google like con la possibilità di selezionare il canale (tutto, libri, DVD, CD, Articoli)

Tipologie di ricerca previste (searching)

${ }^{27}$ Fondata nel 1967, OCLC è un'organizzazione che fornisce servizi alle biblioteche con lo scopo di ridurre I costi e massimizzare l'accesso all'informazione bibliografica. Aderiscono al network più di 72.000 biblioteche di tutto il mondo localizzate in 179 paesi diversi che utilizzano i servizi di OCLC per acquisire, catalogare, prestare e preservare le risorse bibliografiche. OCLC mantiene il catalogo collettivo WorldCat che al momento è il più grande database del mondo per la scoperta e l'accesso all'informazione bibliografica. 


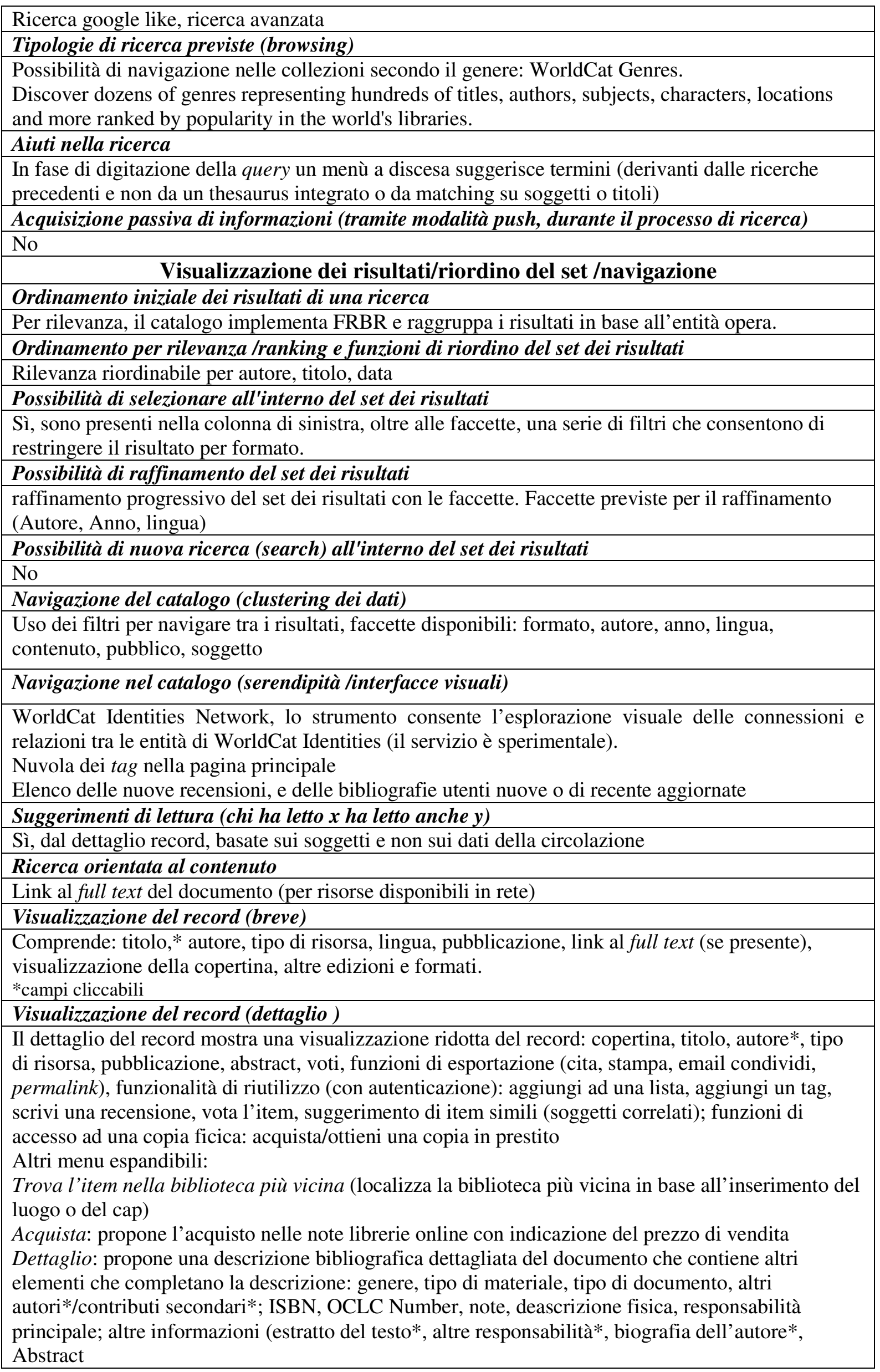


Recensioni: mostra le recensioni degli utenti del social network Goodreads

Tag: mostra i tag inseriti dagli utenti registrati in WorldCat

Soggetti correlati: mostra i soggetti correlati*

Linked data: mostra i linked data presenti nel documenti in base allo schema fornito da Schema.org.

*Campi cliccabili

Contenitori temporanei /permanenti di memoria

Possibilità di salvataggio di record in liste personalizzate (richiede autenticazione). Nello spazio personale sono presenti liste già pronte (le cose che raccomando, le cose che ho, cose da prendere in prestito) ed è possibile crearne di nuove.

Funzioni di esportazione /riutilizzo/sociali

\section{Esportazione della citazione del documento}

si, è possibile ottenere le citazioni del materiale librario in cinque stili comuni, ed esportarli in una varietà di formati, tra cui EndNote, Gestione Reference e RefWorks.

Invio dei risultati per email / stampa dei risultati

Sì /Sì

Altre modalità di riutilizzo dei dati

Varie possibilità di riutilizzo dei dati tramite Widgets appositamente creati, API e Apps

Condivisione nei social network

Sì,

Permalink

Sì

Creazione di una lista personale di documenti

Sì, tramite autenticazione

Funzioni sociali (aggiunta di tag, recensione, valutazione)

Sì

RSS (syndication dei dati)

Sì

Interfacce/applicazioni per dispositivi mobili

Sì, disponibile per IOS apple a Android

Caratteristiche del Sistema

Software utilizzato

Proprietario, sviluppato da OCLC

Tecnologie utilizzate

Proprietarie

Particolari implementazioni

I

\section{Ambiente di ricerca}

WorldCat.org è inserito in un ambiente di ricerca ricco sviluppato da OCLC. L'utente ha la possibilità di esplorare il catalogo anche utilizzando strumenti esterni:

WorldCat Genres. Modellato sul popolare FictionFinder, offre la possibilità di esplorare le collezioni di narrativa (titoli, soggetti, personaggi, luoghi) ordinate per popolarità attraverso i principali canali, costituiti dai generi letterari.

WorldCat Identities Network. Offre la possibilità di navigare tra i collegamenti delle voci di autorità utilizzando lo strumento WorldCat Identities Network, un'interfaccia visuale che consente di esplorare i collegamenti tra le entità che vengono disposte in forma di grafo e di nuvola (tag cloud) che mostra le personalità più influenti ovvero che possiedono il maggio rumero di collegamenti ('Top 100 Identities').

\section{Discussione}

Worldcat è il più grande catalogo al mondo ed il primo OPAC di nuova generazione in cui è stato applicato il modello FRBR per la visualizzazione dei dati bibliografici. Tra le caratteristiche più importanti, oltre alla creazione di un algoritmo per la visualizzazione delle notizie bibliografiche in base all'entità opera di FRBR, sono da segnalare le numerose caratteristiche sociali, un ampliamento delle funzioni di riutilizzo che si è indirizzata tra le prime allo sviluppo di applicazioni per la tecnologia mobile (applicazioni e interfacce per cellulari e smartphones). 
WorldCat è frutto della grande esperienza di OCLC che ha avviato vari progetti interessanti come FAST e Fictionfinder e WorldCat Identities, indirizzati a creare interfacce amichevoli e arricchite di esplorazione per le opere di narrativa e per le voci di autorità.

Uno dei primi impegni che OCLC ha assunto nella progettazione del catalogo WorldCat è stato quello dello studio delle possibilità di implementazione di FRBR, avviando un gruppo di studio che ha prodotto una ricca bibliografia di articoli e contributi sull'argomento ${ }^{28}$. L'obiettivo è stato raggiunto tramite la creazione di un sofisticato algoritmo (OCLC Work-Set Algorithm) che consente il raggruppamento automatico dei record in base all'opera; l'implementazione di FRBR è dunque ancora parziale anche se con questo espediente è possibile ottenere una visualizzazione migliorata del set dei risultati.

WorldCat si distingue per una serie di caratteristiche comuni a tutti gli OPAC di nuova generazione: nella home page è presente un'interfaccia di ricerca semplificata 'Google like' con la possibilità di ricerca in diversi canali (libri, DVD, CD, articoli) o nell'intero catalogo. È possibile compiere anche una ricerca in modalità avanzata ricercando nei metadati bibliografici MARC. Nella schermata principale è possibile autenticarsi nella propria area personale, che darà la possibilità, oltre all'interazione con la propria situazione utente e le funzionalità di gestione di prestiti, rinnovi, di interagire con le risorse visualizzate nel catalogo per salvare ricerche, creare liste, bibliografie, inserire tag, commenti, voti o recensioni alle risorse presenti nel catalogo. Nella home page del catalogo vengono presentati gli altri strumenti di interrogazione o di interazione con il catalogo (es. le numerose API, i widgets, etc.), vengono forniti video esplicativi (video tutorial) sulla creazione di bibliografie, vengono visualizzati i tag e le biblioteche più popolari e le più recenti interazioni degli utenti con il catalogo (ultime liste create e le recensioni aggiunte dagli utenti).

La ricerca manifesta le caratteristiche consuete di esposizione degli item per ranking (l'ordinamento predefinito mostra gli item più recenti), con la possibilità di filtrare e navigare utilizzando le faccette, una lista di risultati che viene notevolmente abbreviata tramite il raggruppamento in base all'opera. Scelto un singolo item è possibile visualizzare tutte le edizioni e i formati (le entità espressione e manifestazione).

La strategia intrapresa negli ultimi anni da OCLC con WorldCat è quella di ampliare la visibilità dei dati bibliografici sul web. Questa si è dapprima manifestata nell'indicizzazione dei record del catalogo WorldCat nei principali motori di ricerca e in Google e negli strumenti Google books e Google Scholar, ove sono stati resi disponibili dei link 'find in a library' collegati alle biblioteche del catalogo.

Successivamente OCLC ha lanciato al sua 'Web-scale strategy' per portare la ricerca a livello globale presentando il primo sistema per la scoperta WorldCat Local (WCL) come alternativa ad altri prodotti come Aquabrowser, Primo Discovery and delivery, Encore. Il progetto pilota fu avviato da University of Washington libraries a partire dal 2007. Il prodotto consente di sfruttare le funzionalità di ricerca e il database di WorldCat adattandolo alle esigenze di ogni biblioteca, che così può disporre si un'interfaccia personalizzata di Worldcat e delle numerose funzionalità del programma. WCL consente di mostrare un'interfaccia WorldCat per recuperare dapprima le risorse presenti nella singola biblioteca, per poi estendere la ricerca a tutte le biblioteche del network e a livello globale. L'adesione a WCL comprende la possibilità di accedere tramite il programma alle basi dati fulltext per recuperare gli articoli delle riviste sottoscritte dalla biblioteca tramite il servizio NetLibrary/EJournals, offrendo un solo punto di interrogazione per banche dati fulltext e senza dover consultare interfacce diverse. WorldCat Link Manager è un servizio Open URL che consente di espandere la ricerca a documenti remoti liberamente accessibili su internet oppure tra quelli posseduti in licenza dalla biblioteca.

Particolare importanza riveste la recente decisione di WorldCat di rendere disponibili i dati catalografici in forma di linked data aderendo alle specifiche di Schema.org. In tal modo le pagine dei record bibliografici vengono accompagnate da tag semantici che consentono di integrare i dati di WorldCat con gli altri dati presenti nel Web Semantico. Ciò prevede l'aggiunta nel dettaglio del record della possibilità per l'utente di visualizzare i dati semantici relativi agli item, che vengono inseriti in base all'ontologia proposta da Schema.org (http://schema.org/Book).

Questa volontà di apertura dei dati bibliografici per rispondere alla tendenza oggi in atto di apertura dei dati verso il Web Semantico ha avuto i primi positivi riscontri nella politica catalografica di

\footnotetext{
${ }^{28}$ L'attività è documentata all'URL: <http://www.oclc.org/research/activities/frbr.html>
} 
OCLC che ha recentemente rivisto le pratiche per il riutilizzo dei dati bibliografici contenuti nell'OPAC WorldCat (<http://www.oclc.org/worldcat/recorduse/datalicensing/default.htm $>$ ) passando da licenze restrittive a modalità di utilizzo più aperte ${ }^{29}$.

In definitiva le funzionalità di nuova generazione, l'applicazione seppur automatizzata del modello FRBR, l'apertura dei dati attraverso Schema.org, pongono al momento il catalogo WorldCat tra i più avanzati sistemi di ricerca presenti in rete.

\section{Punti di forza / novità}

Completezza delle funzioni di riutilizzo dei dati, applicazione del modello FRBR seppure relativa al raggruppamento in base all'opera, utilizzo dei linked data per esporre i record bibliografici nel Web, altri canali di esplorazione del catalogo, la presenza di applicazioni per dispositivi mobili. La traduzione dell'interfaccia in un gran numero di lingue.

\section{Criticità}

L'implementazione di FRBR potrebbe essere migliorata estendendola ai gruppi 2 e 3 del modello.

\section{Riferimenti bibliografici}

Stefano Crivellaro, WorldCat.org. Caratteristiche di un OPAC di nuova generazione, «Biblioteche oggi», 28 (2010), n. 8, p. 13-22

Per l'implementazione del modello FRBR in WordlCat si rimanda a OCLC, FRBR bibliography, version 13.3, 2008-11-15, <http://infoserv.inist.fr/wwsympa.fcgi/d_read/frbr/FRBR_bibliography.rtf>.

Sull' applicazione dei linked data in Worldcat si veda:

OCLC, OCLC provides downloadable linked data file for the 1 million most widely held works in WorldCat, $\quad$ www.oclc.org, $14 \quad$ August 2012 , $<$ http://www.oclc.org/us/en/news/releases/2012/201252.htm>.

Altri dettagli sull'OPAC sono presenti in: <http://www.oclc.org/worldcat/web/default.htm>.

\section{Immagini}

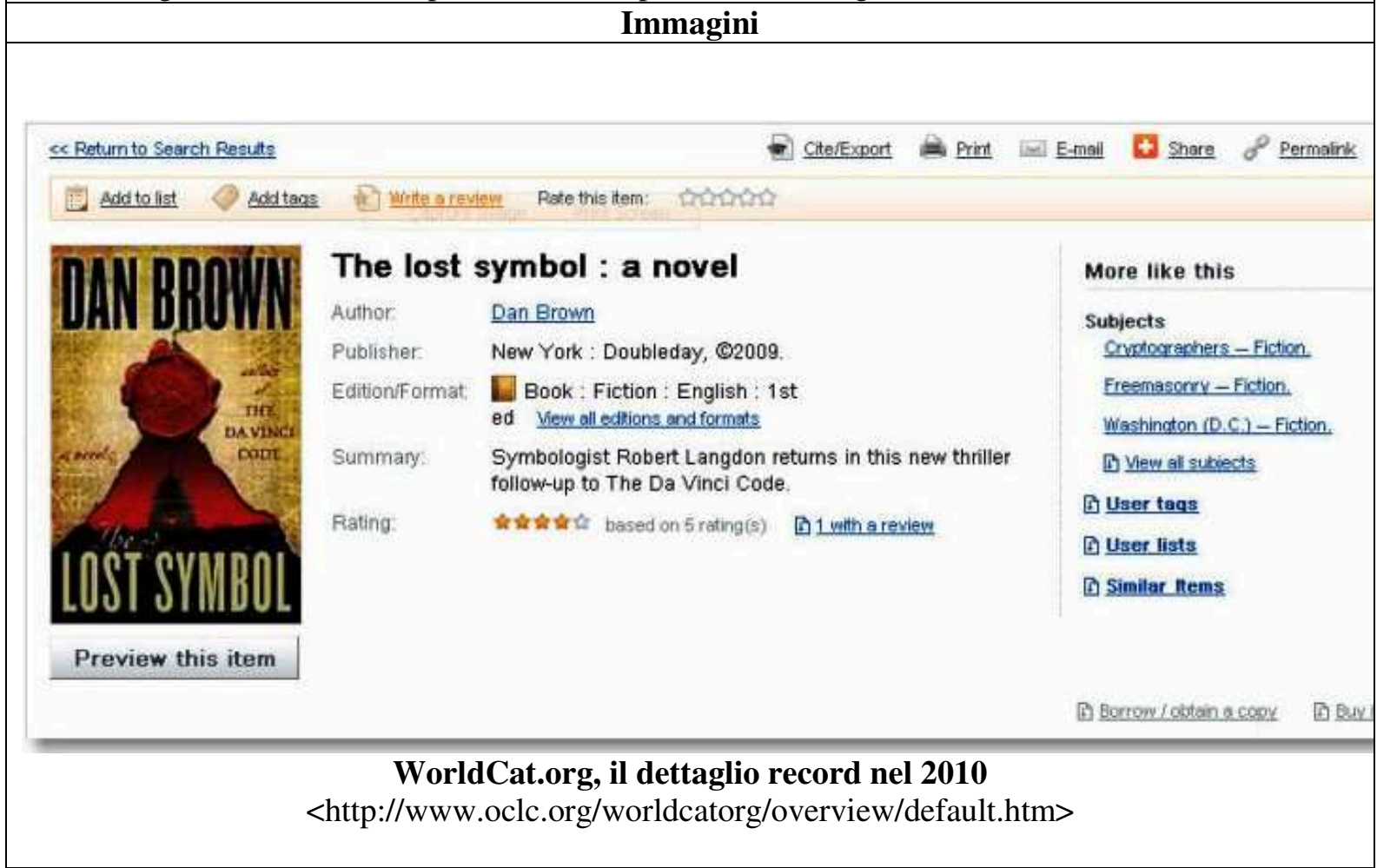

\footnotetext{
${ }^{29}$ La licenza utilizzata è ODC-BY <http://opendatacommons.org/licenses/by/1.0/>.
} 


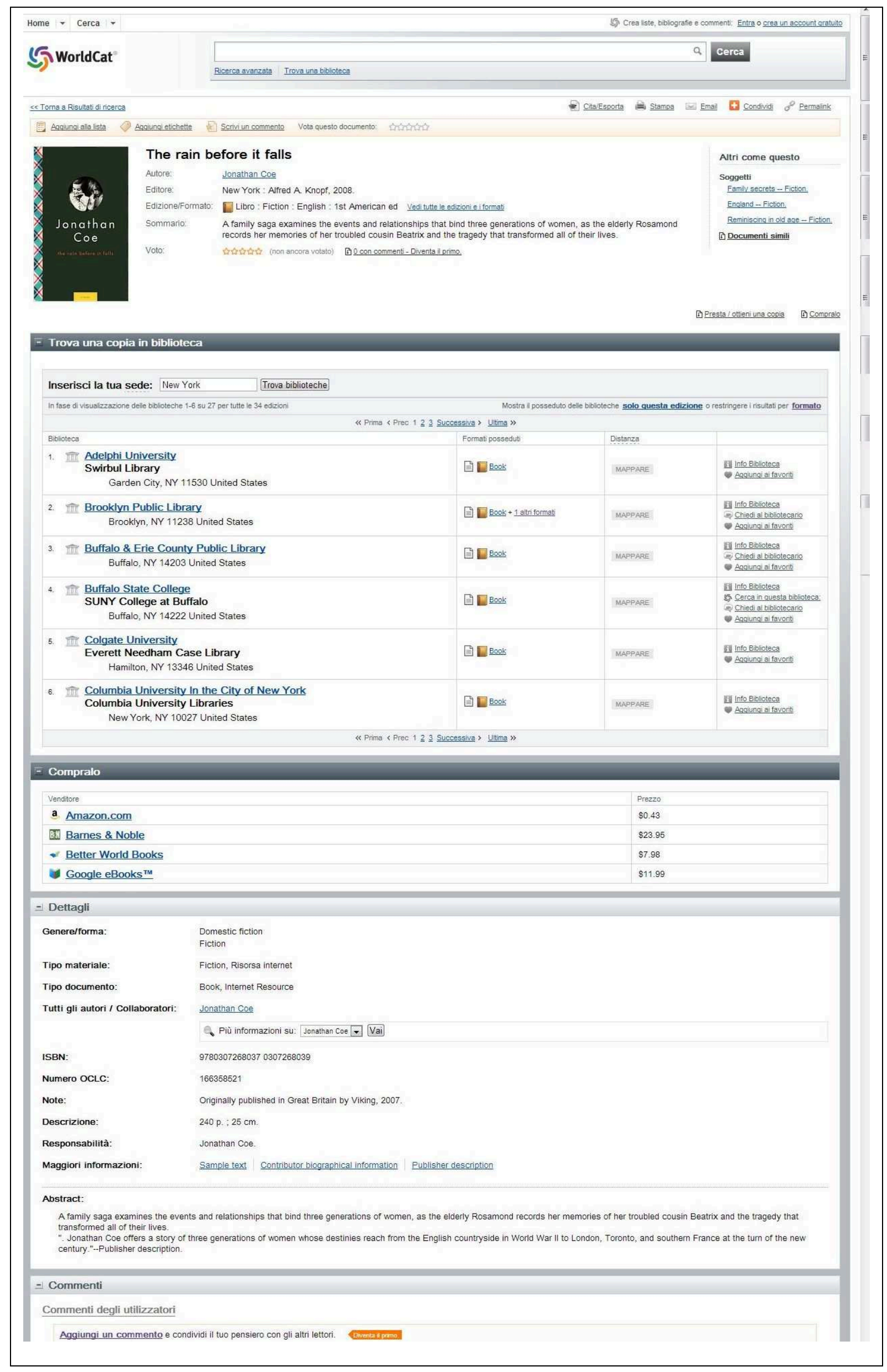




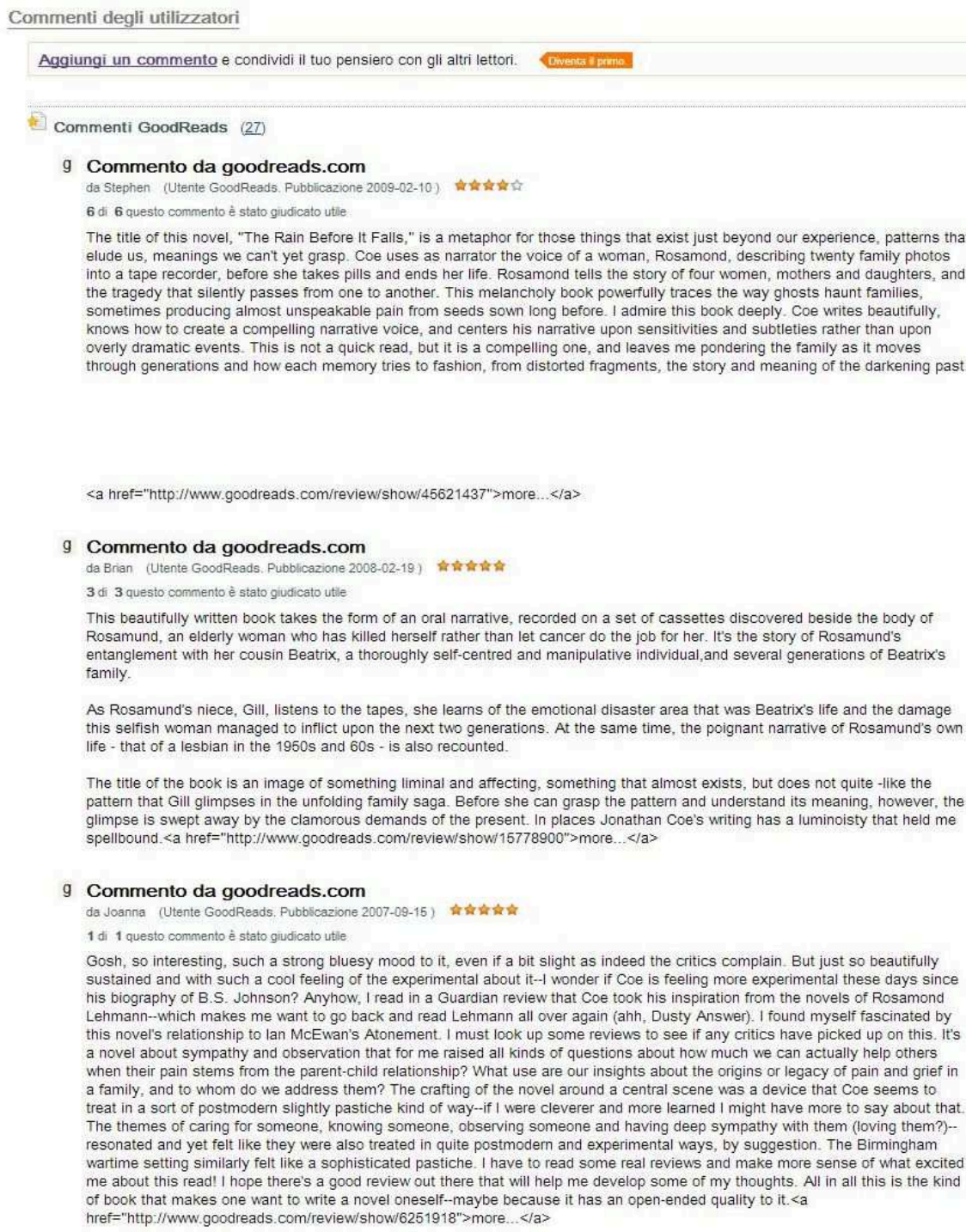

g Commento da goodreads.com

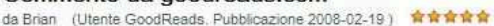

3 di 3 questo commento è stato givicicato utile

This beautifully witten book takes the form of an oral narrative, recorded on a set of cassettes discovered beside the body of Rosamund, an elderly woman who has killed herself rather than let cancer do the job for her. It's the story of Rosamund's entanglement with her cousin Beatrix, a thoroughly self-centred and manipulative individual, and several generations of Beatrix's family

As Rosamund's niece, Gill, listens to the tapes, she learns of the emotional disaster area that was Beatrix's life and the damage this selfish woman managed to inflict upon the next two generations. At the same time, the poignant narrative of Rosamund's own life - that of a lesbian in the $1950 \mathrm{~s}$ and $60 \mathrm{~s}$ - is also recounted

The title of the book is an image of something liminal and affecting, something that almost exists, but does not quite -like the pattern that Gill glimpses in the unfolding family saga. Before she can grasp the pattern and understand its meaning, however, the spellbound <a href="http///wmw goodreads com/review/show/15778900">more _</a $>$

g Commento da goodreads.com

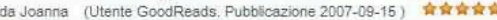

1 di 1 questo commento è stato giudicato utile

Gosh, so interesting, such a strong bluesy mood to it, even if a bit slight as indeed the critics complain. But just so beautifully sustained and with such a cool feeling of the experimental about it--I wonder if $C o e$ is feeling more experimental these days since (ent this novel's relationship to lan McEwan's Atonement I must look up some reviews to see if any critics have picked up on this. It's a novel about sympathy and observation that for me raised all kinds of questions about how much we can actually help others when their pain stems from the parent-child relationship? What use are our insights about the origins or legacy of pain and grief in a family, and to whom do we address them? The crafting of the novel around a central scene was a device that Coe seems to treat in a sort of postmodern slightly pastiche kind of way-if I were cleverer and more learned I might have more to say about that The themes of caring for someone, knowing someone, observing someone and having deep sympathy with them (loving them?)-resonated and yet felt ilke they were also treated in quite postmodern and experimental ways, by suggestion. The Birmingham wartime setting similarly felt like a sophisticated pastiche. I have to read some real reviews and make more sense of what excited me about this read! I hope there's a good review out there that will help me develop some of my thoughts. All in all this is the kind of book that makes one want to write a novel oneself--maybe because it has an open-ended quality to it:< href="http://www. goodreads. com/review/show/6251918">more...</a

Leggi più commenti di utenti GoodReads so

\section{- Etichette}

Aggiungi etichette: per "The rain before it falls"

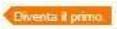

- Documenti simili

Soggetti correltai: (3)

Family secrets - Fiction.

England - Fiction.

Reminiscing in old age - Fiction.

\pm Dati collegati

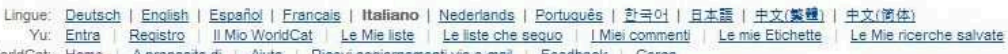

Worldcat. Home Aproposito di Aiuto Riceviagiomamentivia e-mal Feedback Cerca

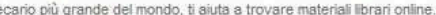

La lunga schermata del dettaglio record in WorldCat nel 2013 si distingue per alcune caratteristiche avanzate: la doppia presentazione del record (con una scheda per i dettagli), commenti da siti sociali, link alle librerie online, etichette, voti e suggerimenti. 


\section{Harvard University. OPAC Hollis}

URL: <http://hollis.harvard.edu>

La biblioteca universitaria di Harvard fondata nel 1638 da un nucleo di 400 volumi appartenenti a John Harvard è la più antica biblioteca degli Stati Uniti. Con più di 70 biblioteche, la maggior parte situate a Cambridge e a Boston nel Massachussets, il sistema bibliotecario di Harvard possiede attualmente più di 17 milioni di volumi e una ricca collezione di risorse digitali. Ogni biblioteca di facoltà è specializzata in un particolare settore disciplinare. Attraverso questi materiali le biblioteche supportano le esigenze di studio e ricerca degli studenti e della comunità internazionale di studiosi. Tra le discipline rappresentate oltre alla letteratura e alla storia americana e di altri paesi l'antropologia, l'architettura, la tecnica bancaria, l'economia, la medicina, la botanica, la zoologia, l'archeologia e la linguistica, la storia dell'arte, la storia delle donne e dell'educazione.

L'OPAC Hollis, cui aderiscono tutte le biblioteche universitarie dell' ateneo, comprende non solo i materiali a stampa delle biblioteche ma anche archivi e collezioni speciali che raccolgono materiali di varia tipologia che vanno dai documenti più antichi a quelli digital born.

Le collezioni speciali occupano infatti una parte importante del posseduto delle biblioteche dell'ateneo e comprendono un ricco patrimonio di materiali rari e unici che si presentano in una varietà di formati che vanno dai papiri ai manoscritti alle fotografie, mappe pitture e sculture, documenti sonori e audiovisivi. Collezioni più recenti includono anche siti web, blog e altro materiale esclusivamente digitale.

Il sistema bibliotecario dal 2002 adotta il gestionale Aleph500 e il modulo OPAC denominato Hollis ; il 7 aprile del 2009 è stato lanciato in versione beta il nuovo catalogo Hollis che utilizza il software Aquabrowser ${ }^{30}$ e il precedente OPAC Aleph è stato denominato Hollis Classic.

Quasi tutto il contenuto delle collezioni è descritto nel nuovo OPAC Hollis che ingloba il contenuto proveniente dall'OPAC Aleph (circa 11 milioni di record) e di alcuni repositories di oggetti digitali, archivistici e museali; alcuni materiali provenienti da collezioni digitali sono descritti in singoli archivi.

Il Discovery Advisory Group (DAG), cui è stato affidato lo sviluppo del nuovo sistema di discovery, ha previsto alcune priorità nella personalizzazione di Aquabrowser quali l'esportazione verso gestori di bibliografie, l'aggiunta di opzioni di ricerca avanzata, l'implementazione di caratteristiche 2.0 come i feed RSS e il tagging, il raggruppamento in base all'opera, l'espansione dei record di autorità per i soggetti.

Attualmente le biblioteche di Harvard forniscono due principali accessi per la ricerca:

Hollis Classic è l'interfaccia OPAC di Aleph che contiene records di libri, manoscritti, documenti governativi, mappe, microforme, documenti musicali, registrazioni audio, materiale visivo e risorse elettroniche. Oltre alla localizzazione del materiale HOLLIS Classic fornisce i servizi all'utenza (prestiti, rinnovi, prenotazioni, salvataggio di ricerche)

L'OPAC Hollis raccoglie, oltre al contenuto di Hollis Classic, anche i materiali presenti in due importanti collezioni: VIA (Visual Information Access) e OASIS (Online Archival Search Information System).

Per quanto riguarda le riviste è possibile visualizzarle a livello titolo, in quanto l'OPAC non è integrato con la ricerca articoli. I record possono essere salvati per tutto il corso della sessione o in via permanente tramite il modulo My discoveries che, previa autenticazione, gestisce l'area dei servizi all'utente.

Accesso all'OPAC /autenticazione

Pagina dedicata, cui si accede dalla home page della biblioteca selezionando Search\&find /autenticazione per l'aggiunta di tag e la creazione di liste di documenti (tramite il modulo My discoveries)

\section{Possibilità di visualizzare l'interfaccia in più lingue}

No

\footnotetext{
${ }^{30}$ L'annuncio in <http://hul.harvard.edu/ois/news/2009/html/2009-03-31_0918_aleph.html>
} 


\begin{tabular}{|c|}
\hline Altre preferenze/personalizzazioni \\
\hline 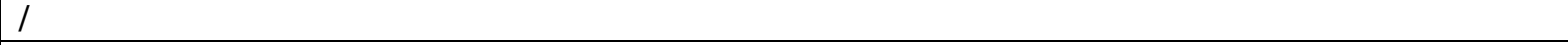 \\
\hline Dati e Database \\
\hline Risorse incluse nell'OPAC \\
\hline $\begin{array}{l}\text { Libri, periodici, manoscritti, documenti governativi, mappe, microforme, documenti musicali, } \\
\text { registrazioni audio, materiale visivo e risorse elettroniche, documenti d'archivio e oggetti digitali } \\
\text { presenti in VIA (Visual Information Access) e OASIS (Online Archival Search Information } \\
\text { System) }\end{array}$ \\
\hline Formato dei dati \\
\hline 1 \\
\hline Ricerca \\
\hline Maschera di ricerca principale \\
\hline Monobox di ricerca 'google like' \\
\hline Tipologie di ricerca previste (searching) \\
\hline Ricerca si tutti i campi e avanzata (per campi) \\
\hline Tipologie di ricerca previste (browsing) \\
\hline 1 \\
\hline Aiuti nella ricerca \\
\hline Correzione dello spelling 'forse cercavi ... ?' \\
\hline Acquisizione passiva di informazioni (tramite modalità push, durante il processo di ricerca) \\
\hline No \\
\hline Visualizzazione dei risultati/riordino del set/navigazione \\
\hline Ordinamento iniziale dei risultati di una ricerca \\
\hline Per rilevanza, in base alle manifestazioni (edizioni) \\
\hline Ordinamento per rilevanza /ranking e funzioni di riordino del set dei risultati \\
\hline Si / Possibilità di riordino del set \\
\hline Possibilità di selezionare all'interno del set dei risultati \\
\hline Tra i documenti di una specifica biblioteca \\
\hline Possibilità di raffinamento del set dei risultati \\
\hline $\begin{array}{l}\text { Raffinamento progressivo del set dei risultati } \\
\text { Faccette previste per il raffinamento (biblioteche, tipologia di risorsa, autore, lingua, anno, soggetto) }\end{array}$ \\
\hline Possibilità di nuova ricerca (search) all'interno del set dei risultati \\
\hline No \\
\hline Navigazione del catalogo (clustering dei dati) \\
\hline Uso dei filtri per navigare tra i risultati \\
\hline Navigazione nel catalogo (serendipità /interfacce visuali) \\
\hline No \\
\hline Suggerimenti di lettura (chi ha letto $x$ ha letto anche y) \\
\hline No \\
\hline Ricerca orientata al contenuto \\
\hline Link al full text del documento (per risorse disponibili in rete) \\
\hline Visualizzazione del record (breve) \\
\hline Comprende: titolo, autore, pubblicazione, tipo di risorsa \\
\hline Visualizzazione del record (dettaglio ) \\
\hline $\begin{array}{l}\text { Comprende: autore, titolo, pubblicazione, descrizione, soggetti, numero standard, Hollis number, } \\
\text { permalink, localizzazione con stato, link ai servisi di ILL e al servizio Borrow Direct, icona } \\
\text { materiale, preview risorsa in Google Book Search, 'save or tag' (accesso a My discoveries trial); } \\
\text { opzioni di esportazione (stampa, mail, facebook, twitter, invia sms, aggiungi record alla lista, } \\
\text { esporta in Endnote, Refworks. }\end{array}$ \\
\hline Contenitori temporanei /permanenti di memoria \\
\hline $\begin{array}{l}\text { Salvataggio delle ricerche tramite il modulo My Discoveries (al momento è in prova e richiede } \\
\text { autenticazione) }\end{array}$ \\
\hline Funzioni di esportazione /riutilizzo/sociali \\
\hline Esportazione della cita \\
\hline
\end{tabular}




\begin{tabular}{|l|}
\hline Sì, esportazione anche verso software di gestione bibliografie (RefWorks and EndNote) \\
\hline Invio dei risultati per email / Stampa dei risultati \\
\hline Sì/ Sì \\
\hline Altre modalità di riutilizzo dei dati \\
\hline / \\
\hline Condivisione nei social network \\
\hline Sì \\
\hline Permalink \\
\hline Sì, dal dettaglio record \\
\hline Creazione di una lista personale di documenti \\
\hline Sì, \\
\hline Funzioni sociali (aggiunta di tag, recensione, valutazione) \\
\hline $\begin{array}{l}\text { Sì, possibilità di aggiunta di tag alle risorse tramite il modulo My discoveries (richiede } \\
\text { autenticazione) }\end{array}$ \\
\hline RSS (syndication dei dati) \\
\hline No \\
\hline Interfacce/applicazioni per dispositivi mobili \\
\hline Sì, applicazione per Iphone \\
\hline Software utilizzato \\
\hline Serials Solutions Aquabrowser Library \\
\hline Tecnologie utilizzate \\
\hline Proprietarie \\
\hline Particolari implementazioni \\
\hline Non statistiche del Sistema \\
\hline
\end{tabular}

Non è stata implementata la caratteristica interfaccia visiva di Aquabrowser che suggerisce termini sinonimi o collegati

\section{Ambiente di ricerca}

Il sito web delle biblioteche di Harvard offre numerosi punti di accesso alle risorse. Oltre agli OPAC Hollis e Hollis Classic è presente l'accesso diretto alle collezioni digitali tramite vari cataloghi speciali che le biblioteche mettono a disposizione per l'utenza.

OASIS cerca in una vasta collezione di collezioni di manoscritti e materiale archivistico presente ad Harvard, tra cui lettere, diari, fotografie e oggetti.

VIA è un catalogo per risorse visive come pitture, sculture, fotografia, disegni e stampe conservate nelle biblioteche, negli archivi e nei musei di Harvard.

WAX è un catalogo speciale di copie di siti web dei dipartimenti di Harvard e di copie di siti esterni all'istituzione collezionati dalla biblioteca di Harvard.

IQSS Dataverse è un database di scienze sociali di collezioni presenti nel mondo, comprendenti dati provenienti da ICPRS, Roper, NARA, Census Bureau e altri organismi di ricerca.

DASH è il repository centrale e ad accesso aperto dei prodotti della ricerca dell'ateneo, nel quale è possibile cercare articoli, documenti e tesi e dissertazioni.

HGL è una collezione universale di dati geografici, mappe storiche digitalizzate e relative informazioni che possono essere visualizzate e scaricate per l'uso in software GIS (Geographic Information System, ovvero un software per visualizzare dati geo-referenziati).

Le biblioteche mettono a disposizione per la ricerca altri canali: i titoli di riviste possono essere ricercati tramite Citation Linker o un'apposita lista di riviste possedute. I singoli articoli di riviste possono essere ricercate tramite autenticazione in Academic Search Premier, attraverso Metalib+ (che cerca nell'indice Primo Central), in JSTOR, in PubMed, in Web of Science, o senza autenticazione nel repository DASH, in Google Scholar e in Citation Linker. Le risorse elettroniche possono essere recuperate tramite liste A-Z (per titoli e soggetti). Per la ricerca di notizie e avvenimenti storici la biblioteca offre tramite autenticazione i servizi LexisNexis, Factiva e Historical Newspapers, infine per le tesi oltre ai cataloghi e al repository istituzionale è a disposizione previa autenticazione la banca dati universale ProQuest Dissertations.

Per l'utilizzo delle risorse l'utente deve autenticarsi; l'ateneo gestisce varie tipologie di autenticazione a seconda dei servizi da utilizzare: HOLLIS / HOLLIS Classic, Borrow Direct, Scan 
\& Deliver, ILL, Special Collections, E-Research, Metalib+, RefWorks.

\section{Discussione}

Il sistema bibliotecario di Harvard ha adottato il sistema per la scoperta Aquabrowser per offrire l'accesso unificato alle risorse presenti nel catalogo e nelle collezioni documentarie messe a disposizione dalle biblioteche d'ateneo in interfacce interrogabili separatamente.

In particolare la scelta di un software come Aquabrowser risponde alla necessità di rendere interrogabili e visibili le collezioni digitali che le biblioteche possiedono.

Nel nuovo catalogo Hollis Aquabrowser consente una ricerca facilitata di fonti eterogenee tramite singolo box di ricerca, dunque l'uso di Hollis è appropriato per una ricerca semplificata. I risultati sono ordinati per ranking e tramite le faccette l'utente può raffinare i risultati utilizzando dei filtri. Hollis soccorre l'utente in fase di interrogazione con la funzione di correzione dello spelling, mostrando le varianti ortografiche, propone i sommari del contenuto delle pubblicazioni, restituisce descrizioni di immagini presenti nel database VIA e gli aiuti alla ricerca (di OASIS).

La ricerca nel catalogo tradizionale Hollis Classic viene attualmente proposta all'utente per una ricerca più mirata. Attraverso il catalogo classico è possibile sfruttare funzionalità di browsing per scorrere liste navigabili di autori, titoli e soggetti e avvalersi di funzionalità di ricerca avanzate ad esempio cercando la frase esatta o combinando gli operatori booleani; inoltre Hollis Classic consente di ottenere descrizioni, localizzazioni e informazioni sul prestito aggiornate in tempo reale, di utilizzare la ricerca per classi e di ricercare caratteri non latini (es. lingua cinese o araba).

Nessuno dei due cataloghi supporta la ricerca degli articoli di riviste, che vengono presentare a livello titolo. Per trovare le descrizioni di articoli sono previsti due strumenti esterni al catalogo: Citation linker consente di trovare articoli per titolo e citazione e E-research consente di trovare risorse digitali ed e-journals sottoscritti dalla biblioteca per soggetto o titolo ${ }^{31}$.

L'implementazione di Aquabrowser è stata personalizzata, ad esempio, è stato scelto di non mostrare la tipica word cloud dei termini varianti prevista dal software Aquabrowser ${ }^{32}$.

La compresenza dell'OPAC tradizionale da affiancare ad un sistema per la scoperta come Aquabrowser si rivela una scelta appropriata per offrire all'utente una ricerca semplificata delle risorse offerte dalle biblioteche senza sacrificare la possibilità di compiere una ricerca mirata e di sfruttare la navigazione tra gli indici semantici.

L'utente viene informato tramite la guida in linea della copertura e delle caratteristiche dei due strumenti e delle differenze nella ricerca e nella precisione dei risultati ottenibili.

\section{Punti di forza / novità}

La presenza di più punti di accesso alle collezioni della biblioteca viene bene esplicitata nella home page evitando di disorientare l'utente. La pagina di accesso alle risorse è sufficientemente chiara nell'indirizzare l'utente allo strumento più appropriato. La compresenza dell'OPAC tradizionale e dello strumento di discovery consentono all'utente di compiere ricerche a gradi diversi di completezza e precisione.

\section{Criticità}

La ricerca articoli è affidata a strumenti separati che vanno interrogati al di fuori dei cataloghi.

\section{Riferimenti bibliografici}

Medialab Solutions, AquaBrowser Library chosen by Harvard University, March 9, 2009, «Library

technology guides», < http://www.librarytechnology.org/ltg-displaytext.pl?RC=13856>.

\footnotetext{
31 Rispettivamente <http://hul.harvard.edu/lib/sfxmini.html> e <http://eresearch.lib.harvard.edu/V> (il servizio è gestito tramite il software Metalib)

${ }^{32}$ Caratteristica distintiva del software Aquabrowser Library è la funzione di navigazione delle informazioni attraverso un grafo (word-cloud) costituito da termini relazionati in vario modo al termine ricercato. Ponendo al centro del grafo il termine (o i termini) ricercato, il sistema recupera e presenta, in forma di suggerimenti di esplorazione, una serie di termini associati al termine di ricerca (i termini piu' utilizzati nei record in associazione a quello ricercato), per traduzioni (le traduzioni linguistiche del termine ricercato), sinonimi, varianti ortografiche, altri temini della gerarchia thesaurale.
} 


\section{Immagini}

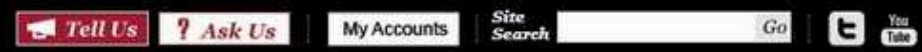

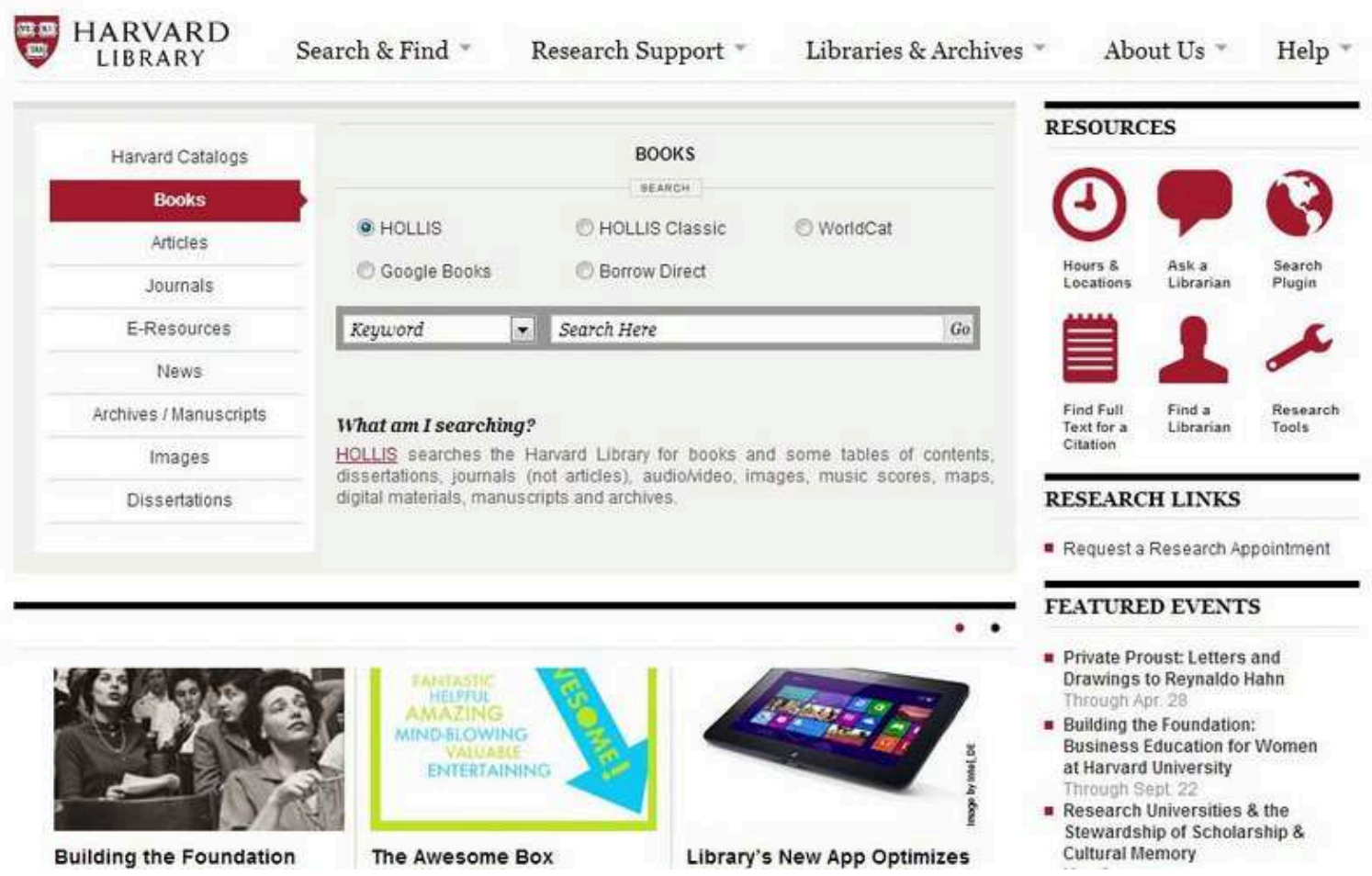

Gli accessi alle risorse della biblioteca

mARVARD LIBRARY

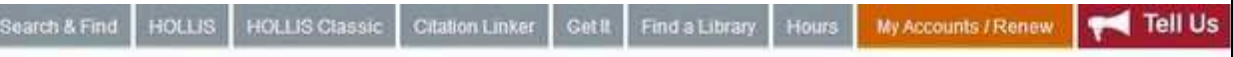

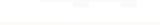

Text-Oaly / Accessible Version

My Discoveries [TRIAL]

Help

Ask a Librarian / Requests

My HOLLIS ACcount/ Renew

\section{HOLLIS}

Results 1.25 of 77,850 for water, sorted by: relevance

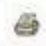

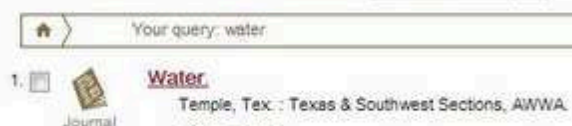

Joumal Tempie, Tex:
iserial

2. III Waterielectronicresourcel.

Base, Switzerland: MDFi Pub.

Serial sove ortag

3. I. Water I

1 Canibndge, Mass : MIT Press; 02009

, Saveor tag.

4. 11) Water the final resource : how the potitics of water will impact the world! Houston, Ba, 1928.

Petersted, Hangshire: Herrman House, 2008

$$
\text { , Save or tag. }
$$

5. III Water for health, fochealing, for life: voute not sick, voure thirstyl Batmangheid, $F$ New York : Warner Books, c2003 a seve or tisg

6. WI Water the fate of our mast precious resource: De Viliers, Marc

Boston, MA: Houghton Mttin, 2000.

s Save or tas -

7. II Water a scurce of conflict or cooperation? Enfeld, N.H. : Science Publishers, c2007.

回四四

Showing results from:

Allocatons

Refine

7 Online

, Onine (16,615)

\section{$\checkmark$ Publictition Dite}

, In the last 3 years $(2,239)$

$\rightarrow$ in the last 10 yesrs $(13,932)$

2. Al years by decade (75,463).

7 Author/ Creator

, Goological Survey (U.S) (1,537)

, Christe, Marean 3 Woods 77698

> Feininger, Lyonel, 1871-1968 (606)

, Unted States. Congress. Senate (505)

85,642 move.

DForinat

> Book $(63,170)$

, image (6.844)

> Journal/Serial (2.545)

Map $(1,706)$

6 more.

\section{Tlunsuage}

> Engish (68, 280)

> French 22,710

\section{La schermata dei risultati}




\section{The Hong Kong University of Science and Technology library. SmartCat con Scriblio}

URL: <http://catalog.ust.hk/catalog/smartcat.php>

SmartCat è una delle poche implementazione oggi visibili di Scriblio, l'OPAC sperimentale basato sulla piattaforma CMS Wordpress e creato da un team di esperti bibliotecari e programmatori con il supporto di Andrew Mellon Foundation ${ }^{33}$.

Basandosi interamente sulla nota piattaforma Wordpress, tra le più comuni per la creazione di blog, l'OPAC può considerarsi come un wrapper, ovvero un dispositivo che cattura i dati presenti in un catalogo senza la creazione di indici centralizzati.

Tra le novità recenti introdotte in fase di sviluppo dall'Università il supporto per i dispositivi mobili (novembre 2011) per sistemi operativi Apple e Android e una ottimizzazione del sito per la visione sui browser di tali dispositivi (visibile in <http://catalog.ust.hk>).

Altre recenti innovazioni sono:

1. la visualizzazione del numero corrente di una rivista per quelle riviste che forniscono i feed RSS per i propri numeri questi feed possono essere visualizzati dall'utente e salvati ${ }^{34}$;

2. la possibilità di visualizzare il contenuto con Google Book Preview ${ }^{35}$;

3. la possibilità di esportare la citazione in vari stili (APA, Chicago, Harvard, MLA and Turabian) e in software per la gestione di bibliografie ${ }^{36}$;

4. la possibilità di esportare il record in RDF in forma di linked data e di visualizzarlo nell'apposito lettore OpenLink Data Explorer ${ }^{37}$ Un esempio si trova in: $<$ http://linkeddata.uriburner.com/ode/?uri=http $\% 3 \mathrm{~A} \% 2 \mathrm{~F} \% 2 \mathrm{Fcatalog}$.ust.hk $\% 2 \mathrm{Fcatalog} \% 2 \mathrm{Fbib} \% 2 \mathrm{~F}$ b847551\%2Frdf>).

La principale novità, seppur in fase di testo, è data dalla funzionalità di visualizzazione dei dati contenuti nel record in forma di linked data tramite un apposito visualizzatore.

\section{Accesso all'OPAC /autenticazione}

a tutti gli utenti del web, dalla pagina dell'OPAC/ autenticazione per i servizi

Possibilità di visualizzare l'interfaccia in più lingue

No

Altre preferenze/personalizzazioni

No

Risorse incluse nell'OPAC

Libri, risorse elettroniche, riviste, video, microforme, manoscritti, mappe, audio

Formato dei dati

MARC

Maschera di ricerca principale

La maschera di ricerca principale propone una scelta tra ricerca a catalogo e ricerca articoli (offerta tramite software Ex Libris). Ricerca multipla per campi con possibilità di scelta dell'ordinamento e filtri per lingua, formato, tipologia di materiale (collezione dei libri, periodici, risorse online).

Tipologie di ricerca previste (searching)

\footnotetext{
${ }^{34}$ Un esempio in <http://catalog.ust.hk/catalog/bib/b847551>.

${ }^{35}$ Esempio in $<$ http://catalog.ust.hk/catalog/bib/b1131455>.

${ }^{36}$ Esempio in <http://catalog.ust.hk/catalog/bib/b1140479>.

${ }^{37}$ The OpenLink Data Explorer è un'estensione per browser (attualmente disponibile per Firefox, Safari e Google Chrome) che aggiunge una nuova opzione alle funzionalità di Web User Agent creando nel menu contestuale un nuovo collegamento che mostra le sorgenti dei dati associati a una pagina web. Lo strumento consente dunque di esplorare i dati grezzi (raw data) e le relazioni tra i dati di una pagina web e consente di visualizzare sia i link ipertestuali che e i link semantici tra dati del web.
} 


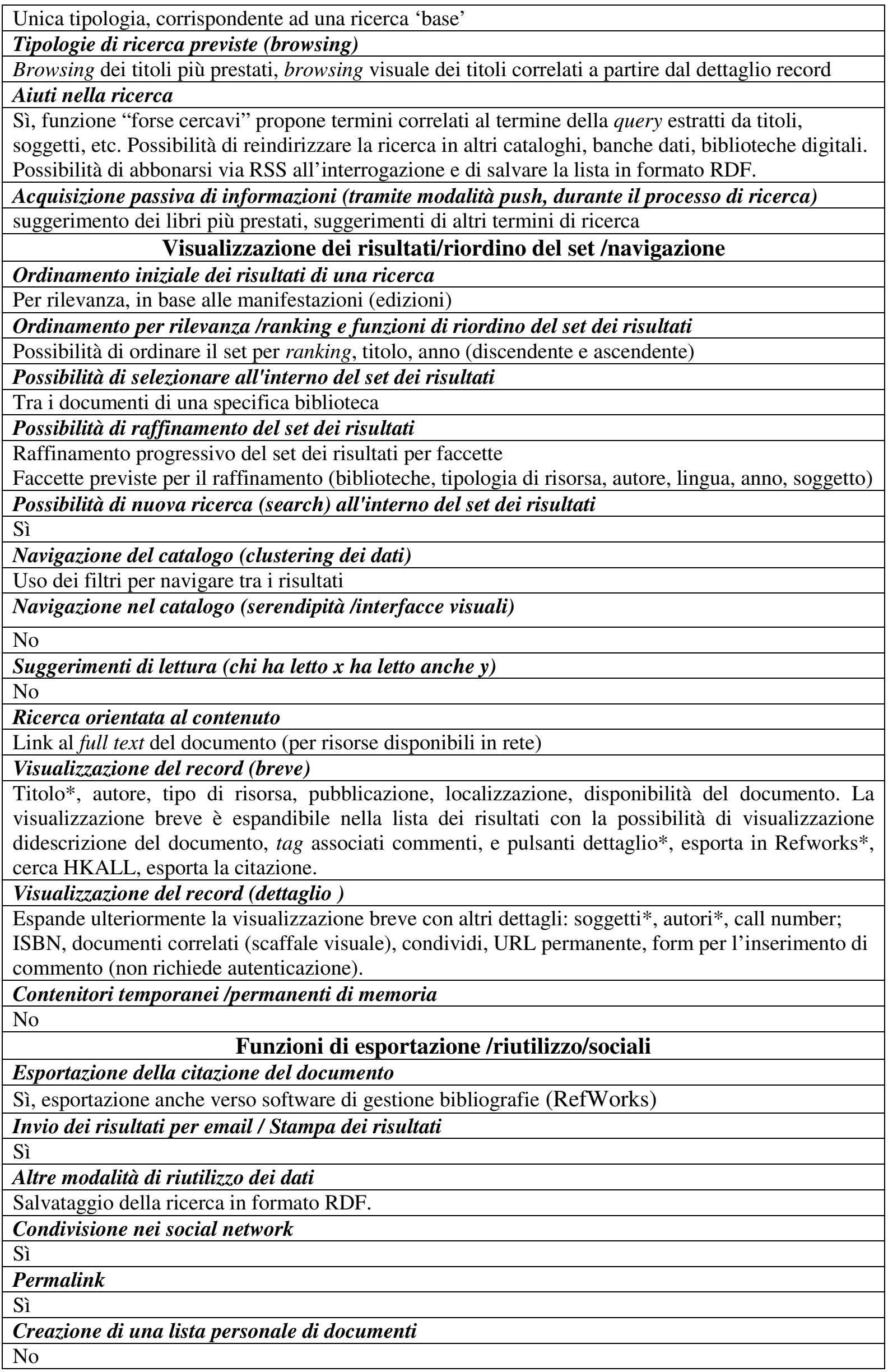


Funzioni sociali (aggiunta di tag, recensione, valutazione)

Sì, aggiunta di tag e commenti (non richiede autenticazione)

RSS (syndication dei dati)

Possibilità di abbonarsi alla ricerca effettuata via RSS

Interfacce/applicazioni per dispositivi mobili

Sì, applicazioni mobili (apps) per Apple OS e Androis OS

Caratteristiche del Sistema

Software utilizzato

Scriblio ver 2.7

Tecnologie utilizzate

Open source, basata su piattaforma Wordpress

Particolari implementazioni

I

Ambiente di ricerca

I

Discussione

Smart Cat implementa il sofware Scriblio basato su CMS Wordpress e rappresenta una delle prime soluzioni per OPAC di nuova generazione ad aver aggiunto al catalogo arricchimenti e funzionalità di riutilizzo dell'informazione. Si tratta in questo caso di un OPAC wrapper, ovvero un'intefaccia aggiuntiva al catalogo tradizionale che non comporta la presenza di indice centrale. Ciò comporta una limitazione nelle funzionalità rispetto ad altri software commerciali. Tuttavia l'OPAC dispone di alcune funzionalità interessanti come ad esempio l'esportazione del record sottoforma di linked data nel formato RDF.

\section{Punti di forza / novità}

L'uso di uno strumento open source e l'esposizione dei dati sottoforma di linked data consente di salvare i dati della ricerca nel formato RDF.

\section{Criticità}

I

\section{Riferimenti bibliografici}

Si veda il sito del software Scriblio <http://scriblio.net/>. 


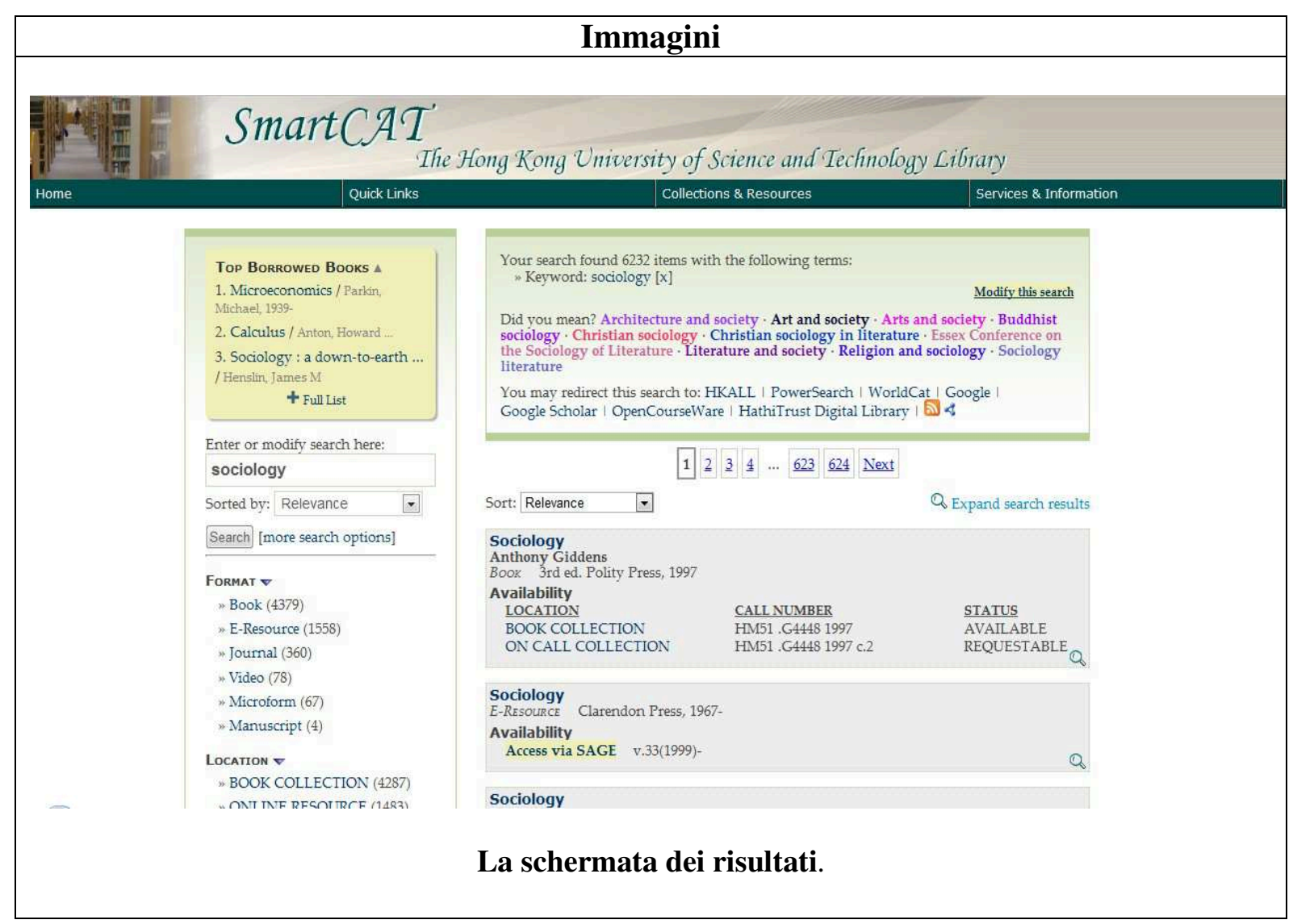




\section{Universität Hamburg. Beluga}

URL: <http://beluga.sub.uni-hamburg.de/>

Beluga è un'interfaccia per un catalogo di nuova generazione sviluppata in casa (homegrown) alla State and University Library di Amburgo per l'Università e per altre cinque biblioteche universitarie dell'area di Amburgo. In Beluga sono registrati circa 7 milioni di titoli provenienti da sei biblioteche di Amburgo: State and University Library di Amburgo 5 biblioteche specialistiche dell'Università di Amburgo (UHH) (Staatsbibliothek Hamburg (Stabi) und Fachbibliotheken der Universität Hamburg (UHH); Università Tecnica di Amburgo-Harburg (TU) (Technische Universität Hamburg-Harburg); Università di Scienze Applicate di Amburgo (HAW) (Hochschule für Angewandte Wissenschaften Hamburg (HAW); Scuola di Musica e Teatro (HfMT) Hochschule für Musik und Theater (HfMT); Biblioteca dei docenti di Amburgo Hamburger (Lehrerbibliothek); HafenCity Universität (HCU) ${ }^{38}$. Il progetto è stato parzialmente finanziato da un consorzio locale di e-learning con lo scopo di creare un catalogo unico per tutte le biblioteche dell'area di Amburgo e per il collegamento di questo strumento ai course management system usato nelle 5 università pubbliche (OLAT, Blackboard, StudIP, Moodle, and CommSy).

Dopo il lancio in rete di un terzo prototipo nell'aprile 2009 il progetto Beluga è ufficialmente terminato nel novembre 2010 e Anne Christensen, creatrice del progetto, ha passato la direzione a Frederik Jan Maas che è diventato responsabile di ogni implementazione e sviluppo della piattaforma dal 2011/01/01 e Beluga è stato pubblicato online.

A partire dall'agosto 2012 la necessità di interfacciarsi con un sistema di discovery ha condotto ad una revisione radicale; oggi Beluga è un'implementazione personalizzata dello strumento per la scoperta open source VuFind.

\section{Accesso all'OPAC /autenticazione}

A tutti gli utenti del web, autenticazione per i servizi

Possibilità di visualizzare l'interfaccia in più lingue

No

Altre preferenze/personalizzazioni

I

Risorse incluse nell'OPAC

Dati e Database

Libri, periodici, articoli, risorse elettroniche in full text

Formato dei dati

Marc 21

Maschera di ricerca principale

Accessibile dalla pagina dell'OPAC, google like

Tipologie di ricerca previste (searching)

Ricerca in un box unico (google like) (possibilità di cercare fin da subito in una singola biblioteca)

Tipologie di ricerca previste (browsing)

Previsto per autori, soggetti, titolo del libro, titolo della rivista, titolo della serie, ente, conferenza, pubblicazione conservata, opera recensita

Browsing dei nuovi titoli nella pagina dell'OPAC

\section{Aiuti nella ricerca}

Autosuggerimento nella fase di digitazione della query tramite un menù a discesa

se la ricerca dà risultato zero, in caso di errore in fase di digitazione vengono forniti aiuti (forse cercavi ...)

Acquisizione passiva di informazioni (tramite modalità push, durante il processo di ricerca)

\footnotetext{
${ }^{38}$ Dati aggiornati a febbraio 2013.
} 


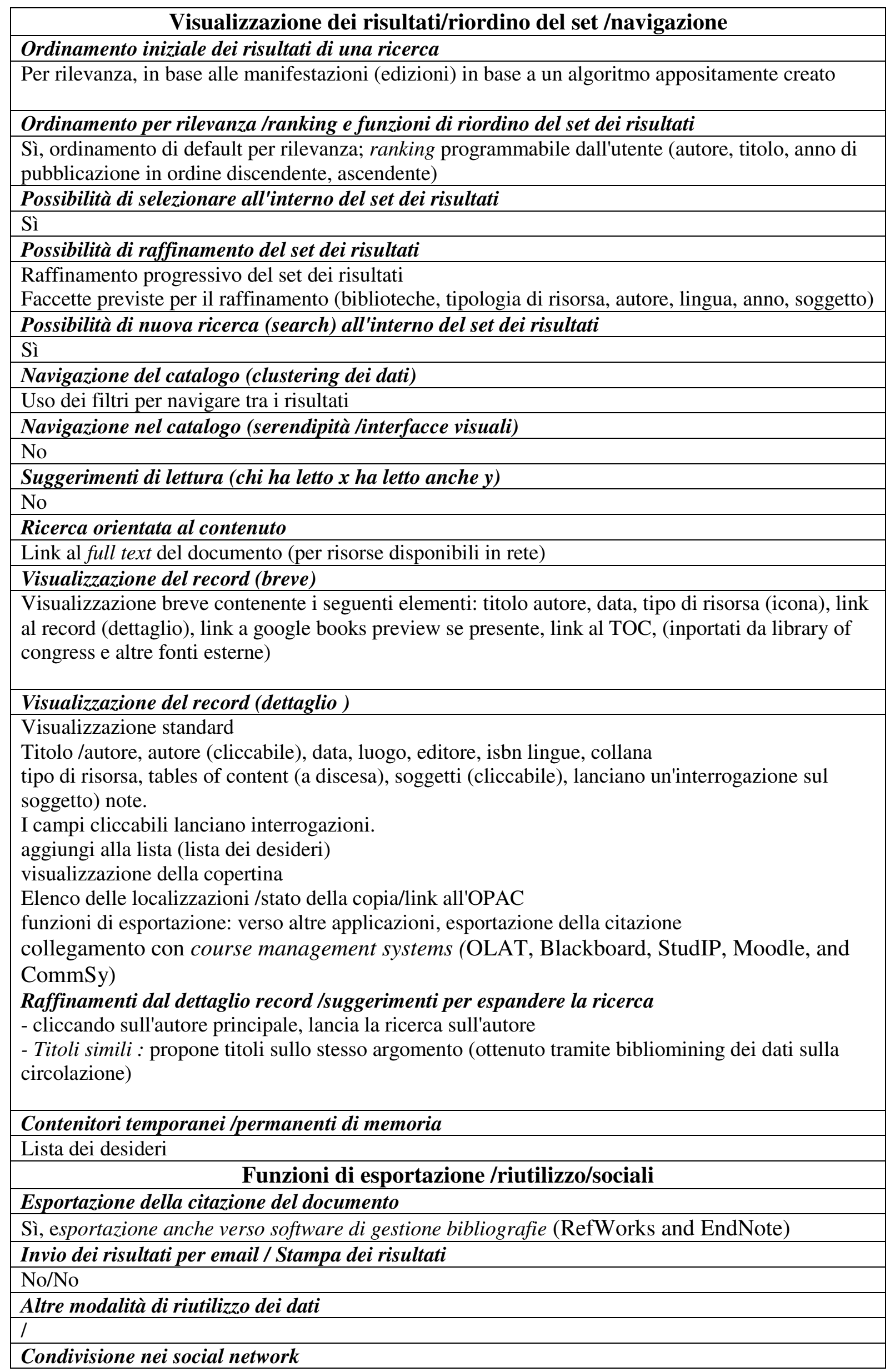




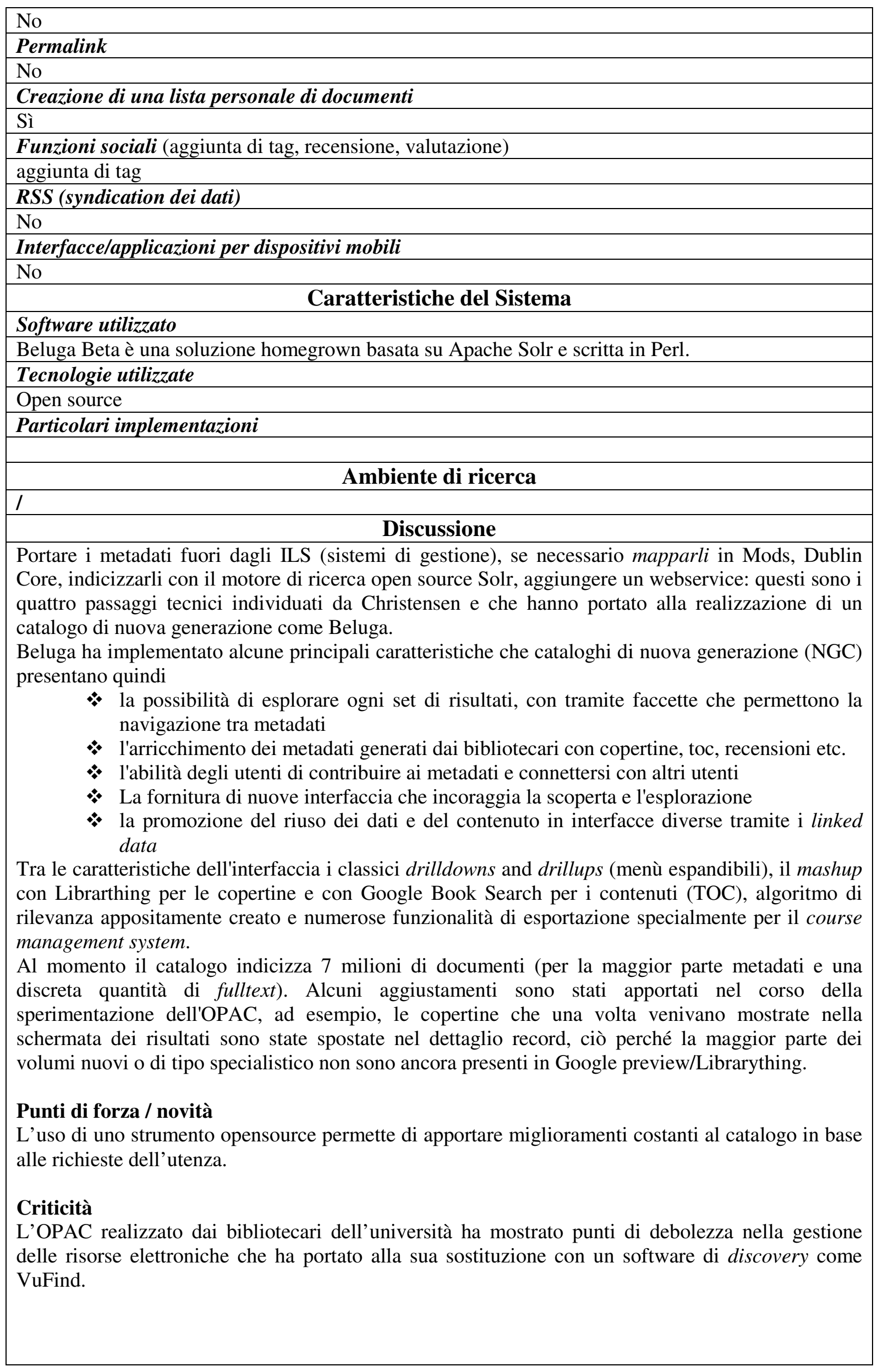




\section{Riferimenti bibliografici}

Ann Christensen, Next generation catalogs: What do users think? Conclusions from the beluga project. Convegno IFLA 75 pre-satellite Event. Emerging trends in technology: libraries between Web. 2.0 semantic web and search technology. Firenze 19-20 agosto 2009 ,

<http://www.ifla2009satelliteflorence.it/meeting3/program/assets/AnnChristensen.pdf>.

Beluga, 23 May $2009<$ http://beluga.sub.uni-hamburg.de/>.

Henrik Lindström - Martin Malmsten, User-centred design and agile development: rebuilding the Swedish National Union Catalogue, «Code4Lib 5 .2008-12-15. 22 May 2009, <http://journal.code4lib.org/articles/561>.

Beluga geht an den Start!, 3. Dezember 2012, «Beluga blog», <http://beluga-blog.sub.unihamburg.de/blog/>.

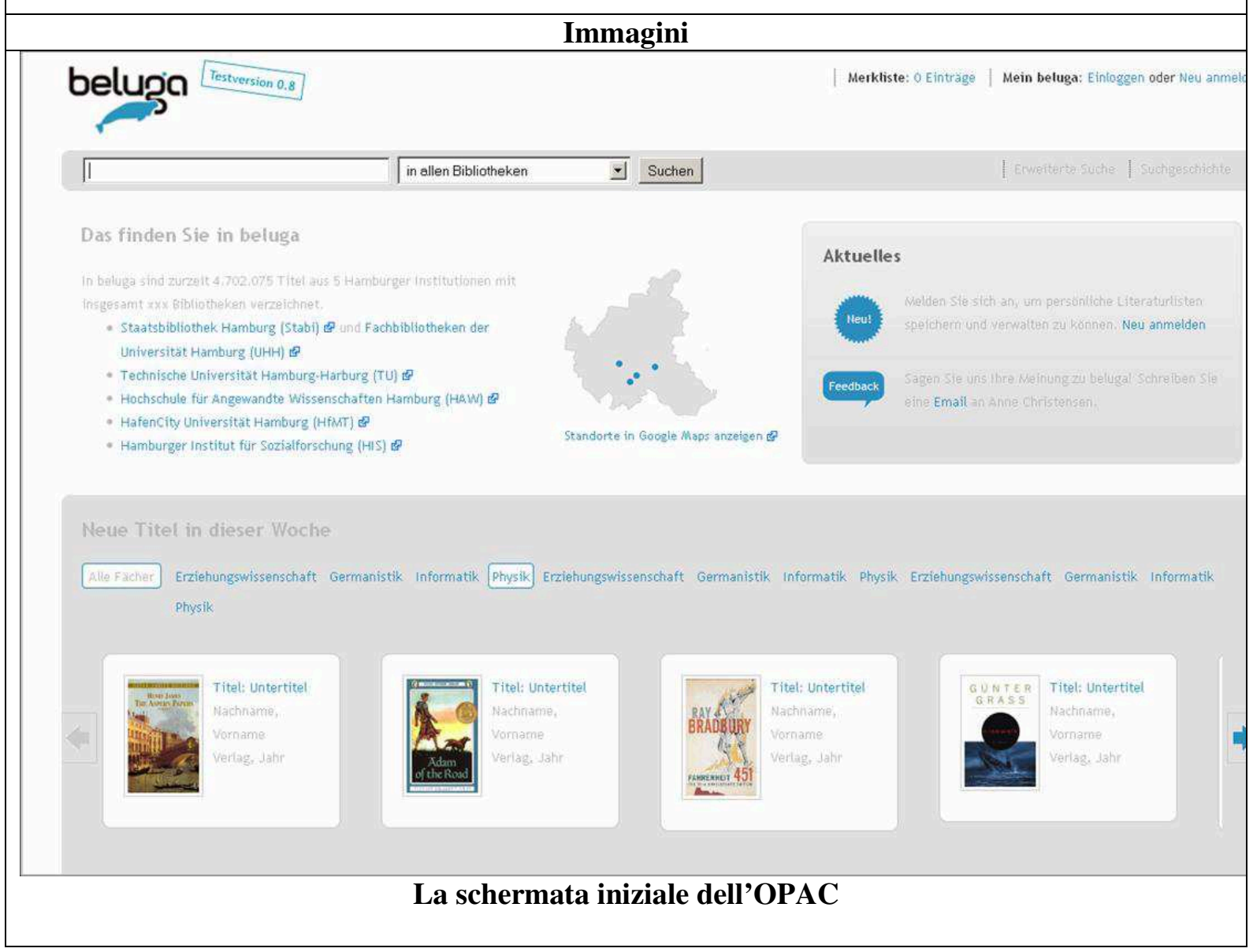




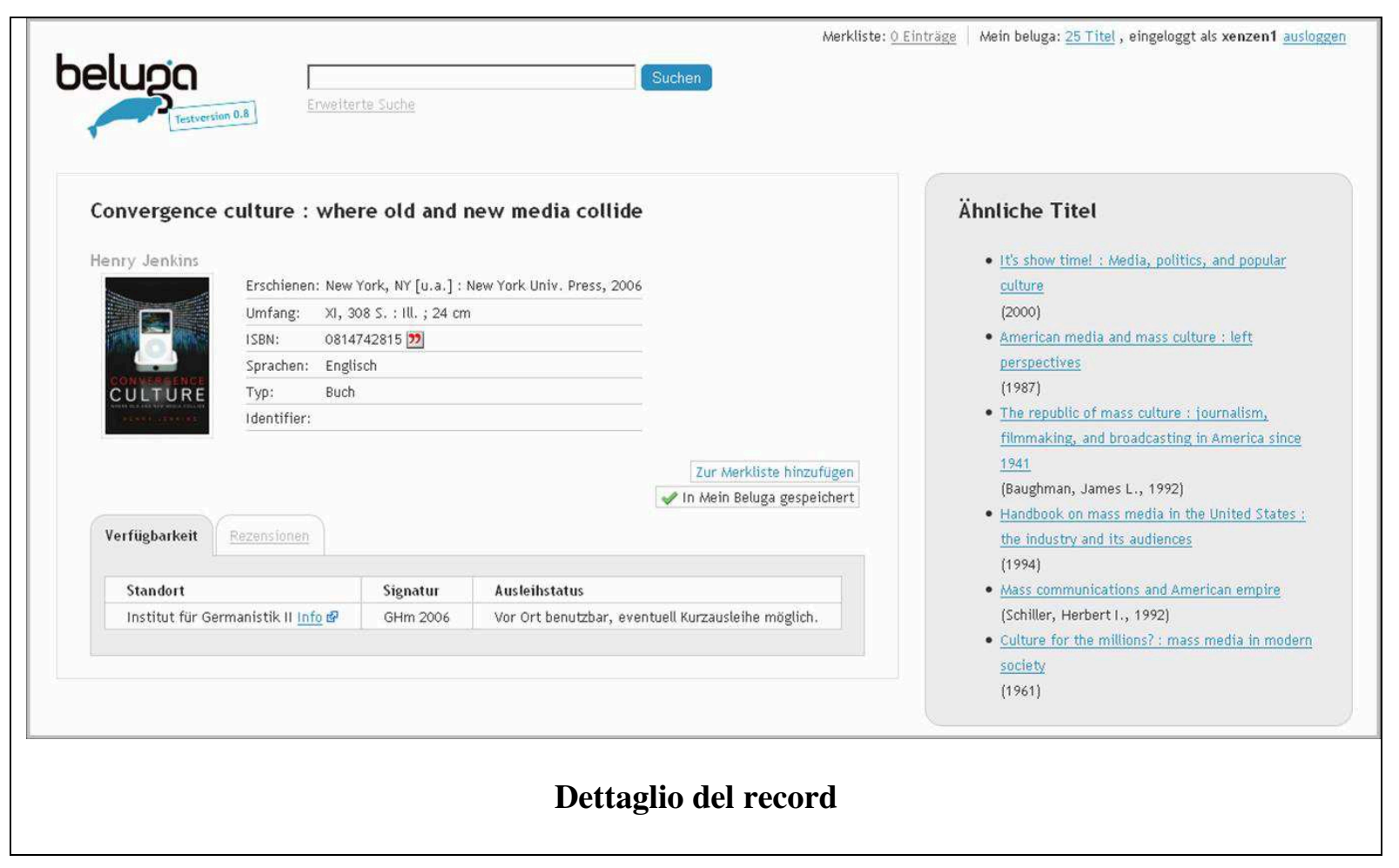




\section{University of California Libraries. Melvyl}

URL: <http://melvyl.worldcat.org/>

Il progetto Melvyl@UCLA di evoluzione del catalogo elettronico delle biblioteche dell'università californiana è un seguito del progetto Melvyl che aveva condotto allo studio dell'interfaccia Revyl dove venivano introdotti i suggerimenti di lettura.

L'Università della California preparò nel 2005 il report Rethinking how we provide bibliographic services for the University of California, dove analizzava le nuove modalità di fornire i servizi bibliografici e i nuovi progetti per il catalogo online e apriva la strada ad un ripensamento delle funzionalità del catalogo nella direzione dei next generation catalogs. Nel report di Bibliographic Services Task Force (BSTF) del 2006, bibliotecari ed esperti affermarono la necessità di una cambiamento del sistema e cominciarono ad indagare opportunità di partnership con OCLC .

California Digital library, il gruppo di studio per le biblioteche digitali dell'UC (SOPAG) ha pubblicato nel 2006 The Melvyl Recommender Project. Nel progetto venivano delineate una serie di modifiche urgenti da apportare al catalogo; il rapporto definiva i concetti chiave della strategia di rinnovamento del catalogo in quattro essenziali aree di intervento

1. miglioramento della ricerca e del recupero dell'informazione

2. architettura dell'OPAC

3. Adozione di nuove prassi catalografiche

4. supporto continuo all'innovazione

I bibliotecari dell'Università decisero successivamente di indagare l'opportunità di istituire una partnership con OCLC per esplorare la possibilità di costruire il nuovo catalogo Melvyl basandosi su una versione prototipale di Worlcat.org.

Il nuovo catalogo denominato Next generation Melvyl è frutto del lavoro del Discovery and delivery group $(<\mathrm{http} / / / \mathrm{www} . c d l i b . o r g /$ services/d2d/>), gruppo di ricerca sulle nuove soluzioni per la scoperta.

Il nuovo catalogo apporta quale principale novità l'uso di Worldcat local, primo e più importante sistema di discovery che offre la possibilità di usare l'interfaccia di ricerca innovativa di WorldCat per cercare nel contenuto delle biblioteche dell'ateneo $\mathrm{e}$ in quello delle biblioteche di WorldCat.org ${ }^{39}$.

Il 27 maggio 2008 viene lanciato il progetto pilota denominato Next-Generation Melvyl Pilot che contiene i record delle biblioteche dei 10 campus collegati, e gli utenti vengono incoraggiati a testare il catalogo e ad offrire il proprio feedback. Dall'OPAC si può cercare oltre che nelle biblioteche di UCL anche quelli delle altre biblioteche facenti parte della rete OCLC, negli articoli indicizzati in ERIC e MEDLINE, in pubblicazioni governative e articoli di argomento generale recuperati in ArticleFirst. UC-eLink (attiva il resolver) piò essere utilizzato per recuperare i full tet posseduti dall'Università della California.

Nel luglio del 2009 è stato pubblicato in rete il nuovo OPAC ${ }^{40}$. Il nuovo progetto di appoggia al software WorldCat Local, allo scopo di migliorare l'accesso a tutte le risorse dell'ateneo e di integrare la coperta di risorse appartenenti ad altre biblioteche. Il catalogo negli ultimi tre anni è stato oggetto di vari aggiustamenti tra i quali quelli rivolti ad integrare il conteuto in fulltext delle banche dati: la ricerca prevede il recupero dei risultati dalle banche dati dell'ateneo con possibilità di loggarsi subito per visualizzare e accedere al contenuti in fulltext riservati. Anche la ricerca

\footnotetext{
${ }^{39}<$ http://libraries.universityofcalifornia.edu/about/uc_oclc.html $>$ e <http://www.library.ucla.edu/facultynews/11811.cfm>

${ }^{40}$ Per le differenze tra il sistema precedente e il nuovo sistema denominato Next-Generation Melvyl si veda: $<$ http://melvyl.cdlib.org/F/?func=file\&file_name=help-ngm-learnmore $>$ ).
} 
avanzata consente è stata modificata per consentire la ricerca in specifiche banche dati.

\section{Accesso all'OPAC /autenticazione}

A tutti gli utenti del web; autenticazione per i servizi. L'autenticazione viene richiesta anche al momento della visualizzazione dei risultati, ma è data all'utente la possibilità di accedere al catalogo senza autenticazione (come ospite)

Possibilità di visualizzare l'interfaccia in più lingue

$\mathrm{Si}$, interfaccia in inglese, tedesco, spagnolo, francese, cinese, olandese, portoghese

Altre preferenze/personalizzazioni

I

Risorse incluse nell'OPAC

\section{Dati e Database}

Tutte le risorse dell'ateneo e delle biblioteche che presenti in WorldCat. Le ricerche recuperano articoli provenienti da WorldCat e attraverso la ricerca federata anche records di articoli fulltext provenienti da GPO Monthly Catalog, ArticleFirst, Medline, ERIC e British Library Inside Serials. È prevista anche l'inclusione di metadati di oggetti digitali. I database rappresentati sono ACM Digital Library, Academic Search Complete, Anthropology Plus, e altri 25 databases minori.

Formato dei dati

MARC

Maschera di ricerca principale

\section{Ricerca}

Di tipo Google like, affiancato da menu a discesa per delimitare la ricerca alle biblioteche UCLA o alle biblioteche presenti in WorldCat.org

Tipologie di ricerca previste (searching)

Google like, ricerca a linea di comando e ricerca esperta

Tipologie di ricerca previste (browsing)

I

Aiuti nella ricerca

Menù a discesa con suggerimento delle stringhe di ricerca, in base a ricerche già effettuate

Acquisizione passiva di informazioni (tramite modalità push, durante il processo di ricerca)

\section{Visualizzazione dei risultati/riordino del set /navigazione}

Ordinamento iniziale dei risultati di una ricerca

Per rilevanza, based on OCLC algorithm, che mostra i risultati raggruppati per opera con la possibilità di vedere 'edition and formats'.

Ordinamento per rilevanza /ranking e funzioni di riordino del set dei risultati

Sì, ordinamento per sola rilevanza, per luogo e rilevanza e in base ad altri parametri (author, title, date: oldest or newest first).

Possibilità di selezionare all'interno del set dei risultati

Tra i documenti di una specifica biblioteca

Possibilità di raffinamento del set dei risultati

Raffinamento progressivo del set dei risultati

Faccette previste per il raffinamento: (formato, tipologia di risorsa, autore, data, lingua, contenuto, audience, soggetto, più altre faccette espandibili)

La faccetta database consente di espandere /limitare la ricerca al contenuti delle banche dati (Worldcat. org, MEDLINE, Article First, Eric, etc)

Possibilità di nuova ricerca (search) all'interno del set dei risultati

No

Navigazione del catalogo (clustering dei dati)

Uso delle faccette per la navigazione

Navigazione nel catalogo (serendipità /interfacce visuali)

No

Suggerimenti di lettura (chi ha letto $x$ ha letto anche $y$ ) 


\begin{tabular}{|c|c|}
\hline $\mathrm{Nc}$ & \\
\hline & icerca orientata al contenuto \\
\hline & ink al full text del documento (per risorse disponibili in rete) \\
\hline & isualizzazione del record (breve) \\
\hline & $\begin{array}{l}\text { a citazione breve include: titolo, autore, editore, luogo/data di pubblicazione, lingua, formato, } \\
\text { BN/ISSN, soggetti, audience, copertina, il campo database è stato aggiunto successivamente. }\end{array}$ \\
\hline & isualizzazione del record (dettaglio ) \\
\hline & $\begin{array}{l}\text { ella visualizzazione del dettaglio record vengono fornite informazioni aggiuntive: titolo della } \\
\text { rie, edizioni, descrizione fisica, note, riassunto e abstract, recensioni e localizzazioni nelle } \\
\text { blioteche. Sono aggiunte, ove possibile, le copertine da Google Book Search. La visualizzazione } \\
\text { ettagliata del record contiene anche: localizzazione nelle biblioteche del campus, e nelle altre } \\
\text { blioteche di WorldCat; 'Buy it': acquisto del volume; dettagli del volume con collegamento a } \\
\text { ografie degli autori e abstract; recensioni degli utenti, tag popolari e vista della tag cloud, soggetti } \\
\text { prrelati (i link lanciano una nuova ricerca per il soggetto); utenti che hanno il libro in liste } \\
\text { ubbliche. }\end{array}$ \\
\hline & ontenitori temporanei /permanenti di memoria \\
\hline Sì & \\
\hline & Funzioni di esportazione /riutilizzo/sociali \\
\hline & sportazione della citazione del documento \\
\hline & $\begin{array}{l}\text { possibile esportare singoli record o liste di record nei più conosciuti formati citazionali. È prevista } \\
\text { che l'esportazione verso software per la gestione di bibliografie e in vari formati (HTML, RTF, } \\
\text { IS, RefWorks, and EndNote). }\end{array}$ \\
\hline & ivio dei risultati per email / Stampa dei risultati \\
\hline Sì & \\
\hline & ltre modalità di riutilizzo dei dati \\
\hline $\mathrm{Nc}$ & \\
\hline & ondivisione nei social network \\
\hline & , (tramite il servizio Addthis) \\
\hline & ermalink \\
\hline Sì & \\
\hline & reazione di una lista personale di documenti \\
\hline & $\begin{array}{l}\text { possibile creare liste personali di documenti previa autenticazione e tenere in memoria oggetti } \\
\text { ella sessione di ricerca }\end{array}$ \\
\hline & unzioni sociali (aggiunta di tag, recensione, valutazione) \\
\hline & , tramite autenticazione \\
\hline & SS (syndication dei dati) \\
\hline $\mathrm{N}$ & \\
\hline & iterfacce/applicazioni per dispositivi mobili \\
\hline $\mathrm{N}$ & \\
\hline & Caratteristiche del Sistema \\
\hline & oftware utilizzato \\
\hline & JorldCat Local \\
\hline & ecnologie utilizzate \\
\hline & roprietaria \\
\hline & articolari implementazioni/caratteristiche \\
\hline & hat con il bibliotecario nella schermata dei risultati e nel dettaglio record \\
\hline & $\begin{array}{ll}\text { Ambiente di ricerca } \\
\end{array}$ \\
\hline I & \\
\hline & Discussione \\
\hline & $\begin{array}{l}\text { OPAC Melvyl è il frutto di numerosi studi che da tempo l'ateneo dedica al proprio catalogo. La } \\
\text { ecisione di avvalersi di WordlCat Local come software sul quale lavorare per il miglioramento del } \\
\text { atalogo è stata l'occasione per accelerare il processo di rinnovamento, che avviato nel } 2005 \text { si è } \\
\text { oncluso nel } 2009 \text { con il lancio del nuovo catalogo online. Le modifiche che l'ateneo ha apportato a } \\
\text { JorldCat negli ultimi tre anni sono state indirizzate al miglioramento del recupero all'interno }\end{array}$ \\
\hline
\end{tabular}


dell'OPAC di tutte le banche dati sottoscritte dall'ateneo.

A tale necessità si riconducono alcune scelte strategiche, quali la possibilità di loggarsi fin da subito, che viene richiesta dal sistema.

\section{Punti di forza / novità}

La chat, attivabile dalla schermata di ricerca avanzata, nella schermata dei risultati e nel dettaglio del record è posizionata dunque nei luoghi in cui è maggiormente necessaria.

\section{Criticità}

I

\section{Riferimenti bibliografici}

Colleen Whitney - Lisa R. Schiff, The Melvyl Recommender Project: Developing Library Recommendation Services. UC Office of the President: California Digital Library, 2006, $<\mathrm{http}: / /$ escholarship.org/uc/item/94d0r3cx>

California Digital Library, The Melvyl Recommender Project Web site, . September 2005.

$<$ http://cdlib.org/inside/projects/melvyl_recommender/>.

University of California Libraries, UC/OCLC Pilot Implementation.

$<$ http://libraries.universityofcalifornia.edu/about/uc_oclc.html $>$.

California Digital Library, The Melvyl Recommender Project final report. 2006, $<$ http://www.cdlib.org/inside/projects/melvyl_recommender/report_docs/Mellon_final.pdf/>.

\section{Immagini}

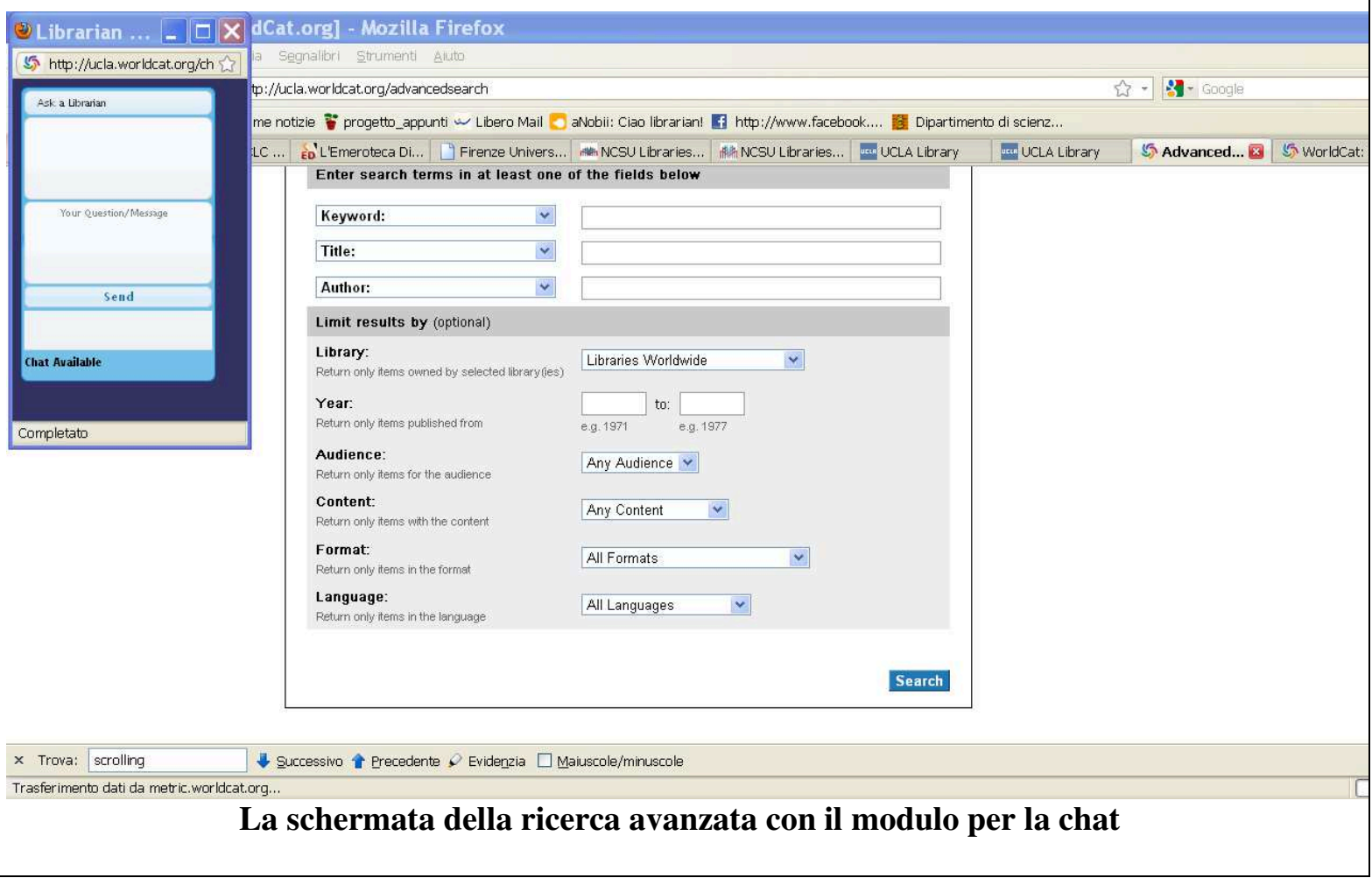




\section{University of Virginia Library. Blacklite}

URL: <http://virgobeta.lib.virginia.edu/>

Blacklite $^{41}$ è un progetto open source sviluppato all'Università della Virginia ${ }^{42}$ a partire dal 2008. Nato con l'obiettivo di utilizzare la tecnologia open source per i cataloghi di biblioteca e sperimentare le nuove possibilità del mashup, l'interfaccia di ricerca Virgobeta, punto di arrivo del progetto, viene definita un OPAC Mashup ${ }^{43}$.

Blacklite è un software offerto gratuitamente su licenza Apache. Il progetto Blacklite prende avvio dal progetto Collex sviluppato nell'ateneo da alcuni studiosi come strumento open source per visualizzare cataloghi contenenti informazioni di vario tipo. Con l'utilizzo di Solr e Ruby on Rails, Collex permetteva indicizzare da una gran varietà di fonti esterne le risorse full-text che potevano essere cercate, provviste di tag e ricombinate con la tecnica del mashup in vari modi. Successivamente il gruppo di Collex e la biblioteca di ateneo unirono gli sforzi per far indicizzare nel programma anche record in formato MARC e farne un catalogo che potesse sostituire l'OPAC tradizionale allora in uso. Il cuore dell'infrastruttura tecnologica è Lucene, motore di ricerca flessibile e opensource e Solr, un web service wrapper per Lucene, scelto come piattaforma ideale per sperimentare le nuove tecniche del mashup. Il nuovo Virgo è stato lanciato in versione beta il 15 luglio 2010 ed è stato continuamente migliorato dallo staff; l'accesso al catalogo tradizionale, rinominato Virgo Classic, è stato mantenuto come opzione per una ricerca più mirata e costituisce ancora oggi uno strumento che si affianca al catalogo di nuova generazione Virgo.

\section{Accesso all'OPAC /autenticazione}

Dalla pagina dell'OPAC e integrato anche nella home page $<$ http://research.lib.virginia.edu/ $>$

Possibilità di visualizzare l'interfaccia in più lingue

No

Altre preferenze/personalizzazioni

No

Risorse incluse nell'OPAC

Catalogo ( «Find books, print journals, maps, videos, digitized materials, and more from the Library's collection»); articoli («Find articles from the Library's collection of subscription online journals. Most articles have full-text available»). Le ricerche in musica e video hanno apposite interfacce di ricerca per questi materiali.

\section{Formato dei dati}

Tradizionale, MARC

\section{Maschera di ricerca principale}

Box di ricerca Google like; ricerca avanzata catalogo; ricerca avanzata articoli.

con possibilità di (switch) selezionare i risultati da catalogo, articoli, musica, video. La ricerca di default è impostata su catalogo + articoli.

Autocompletamento nel box principale in base alle ricerche svolte da altri utenti

Tipologie di ricerca previste (searching)

Ricerca google like; ricerca avanzata.

Vi sono due tipologie di ricerca avanzata: per il catalogo e per le riviste.

\section{Tipologie di ricerca previste (browsing)}

${ }^{41}$ Per i dettagli del progetto si veda: <http://projectblacklight.org/>

${ }^{42}$ L'Università della Virginia (chiamata anche U.Va., UVA, Mr. Jefferson's University o The University ha sede a Charlottesville, in Virginia, ed è stata fondata da Thomas Jefferson e aperta al pubblico nel 1819. È tra le più grandi biblioteche statunitensi con 5.1 milioni di libri, 18.7 milioni di manoscritti e 551.000 ebooks.

${ }^{43}$ Cfr. Elisabeth (Bess) Sadler, Joseph Gilbert, Matt Mitchell, Library catalog mashup: using Blacklight to expose collections in Nicole C. Engard, Library Mashups: Exploring New Ways to Deliver Library Data, Medford, Information Today, Inc, 2009, p. 115-127. 


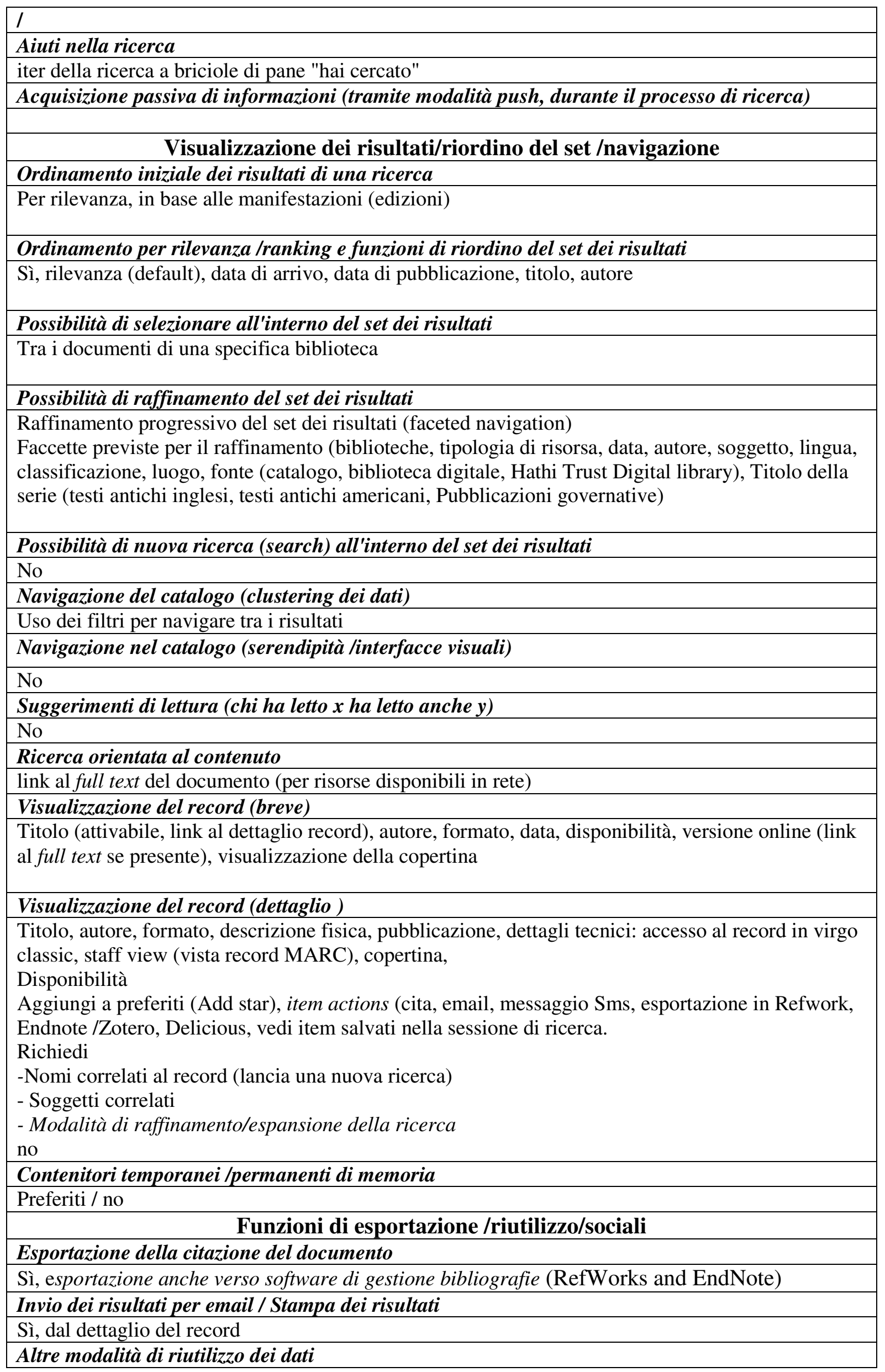




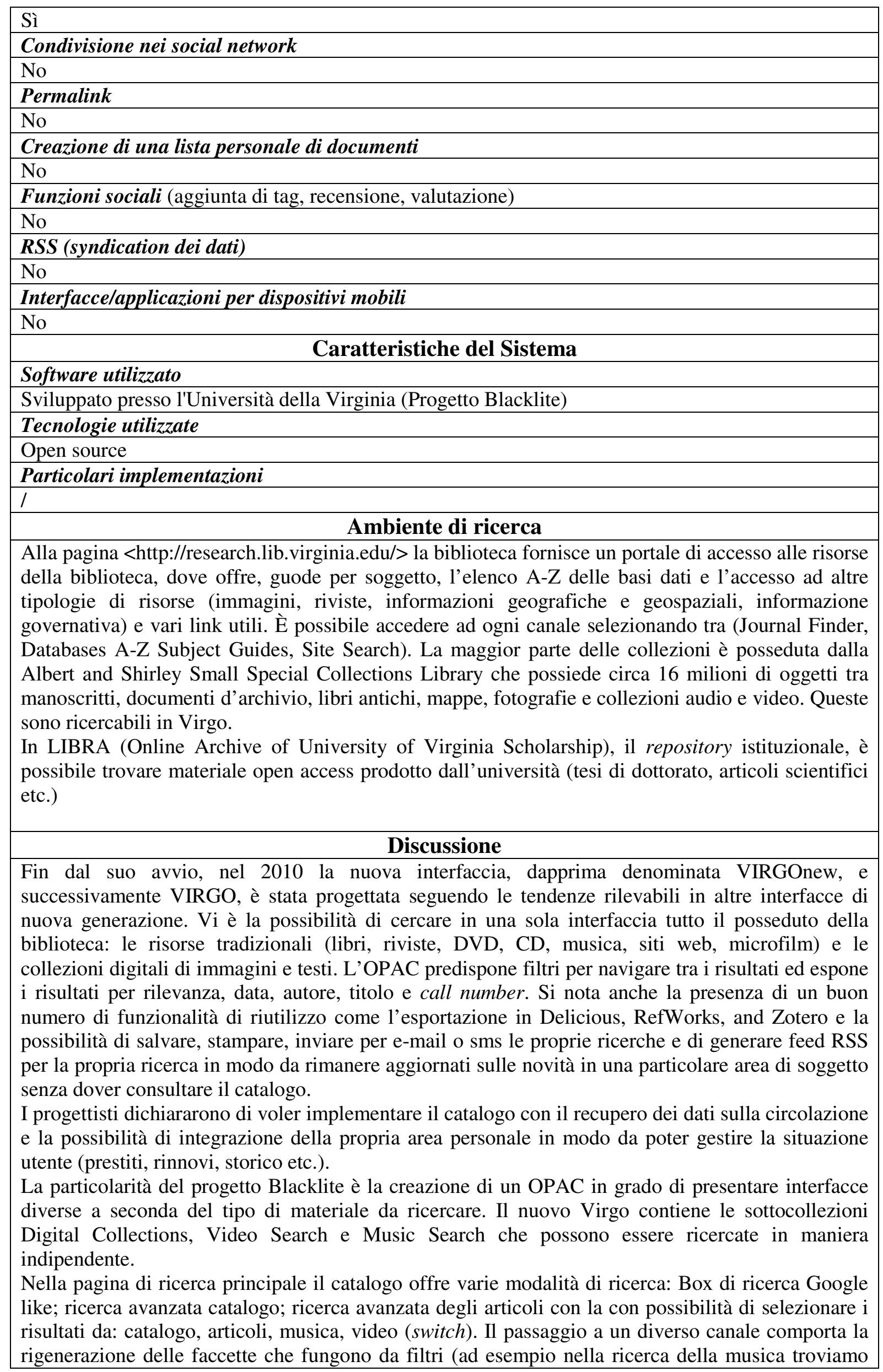


per il raffinamento le faccette 'strumento' e 'data di composizione' che non sono presenti in altri canali). L'opzione 'catalog + articles search' mostra i risultati in due colonne affiancate, è sempre possibile passare da un canale di ricerca all'altro tramite l'opzione switch presente in una barra di scelta dei canali. Molto interessante è la navigazione 'a briciole di pane' che consente di visualizzare il percorso di ricerca svolto e di eliminare / aggiungere i filtri per ampliare/restringere la propria ricerca.

\section{Punti di forza / novità}

L'OPAC presenta nell'interfaccia una chiara suddivisione dei diversi canali di ricerca che l'utente può utilizzare e una buona interfaccia di navigazione che mostra filtri specifici per diverse tipologie di risorse. La ricerca avanzata mostra suggerimenti accanto ai campi di ricerca.

\section{Criticità}

La mancanza di opzioni di browsing in base agli accessi semantici o altri canali.

\section{Riferimenti bibliografici}

Elizabeth (Bess) Sadler, Project Blacklight: a next generation library catalog at a first generation university, «Library Hi Tech», 27 (2009), n.1, p. 57-67.

Elisabeth (Bess) Sadler, Joseph Gilbert, Matt Mitchell, Library catalog mashup: using Blacklight to expose collections in Nicole C. Engard, Library Mashups: Exploring New Ways to Deliver Library Data, Medford, Information Today, Inc, 2009, p. 115-127.

\section{Immagini}




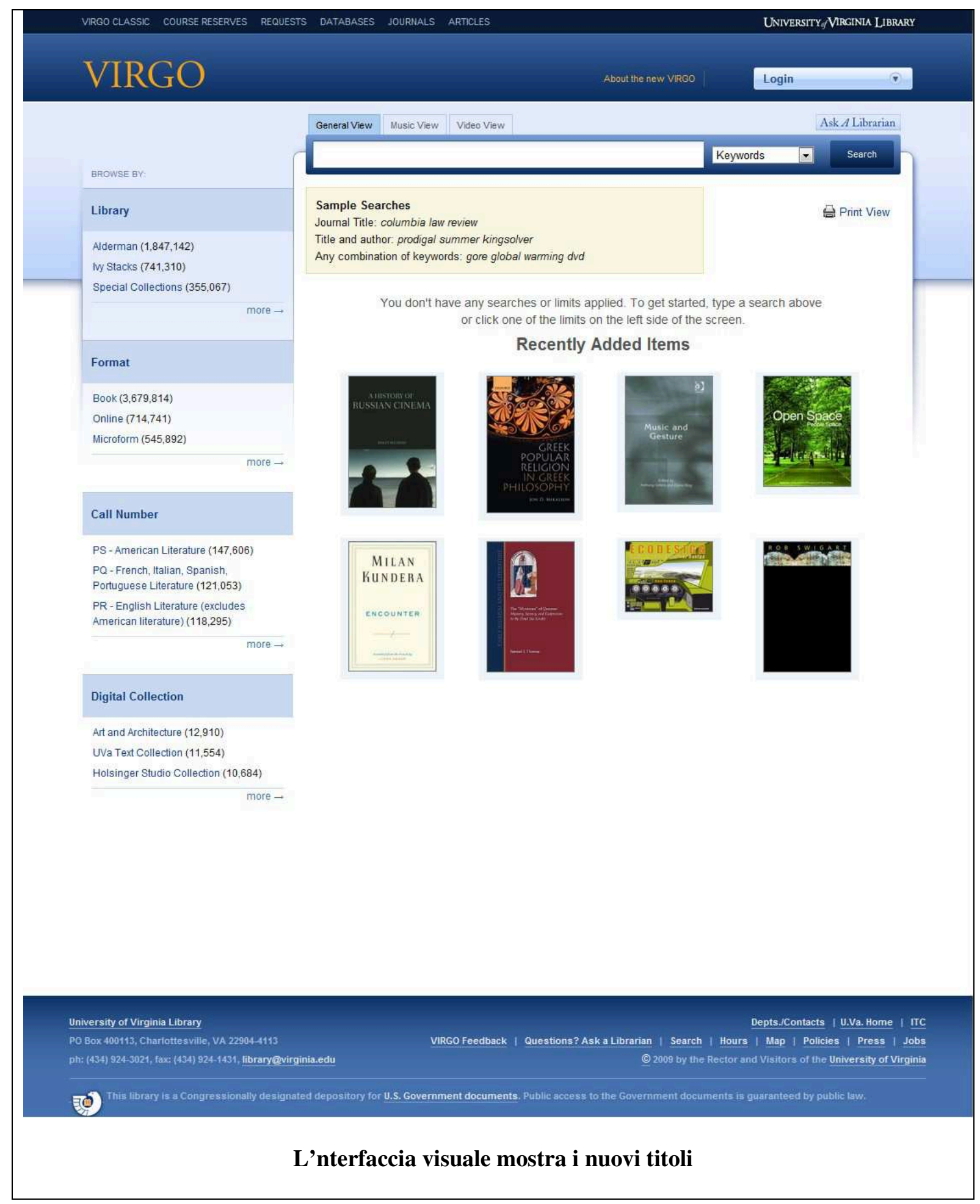




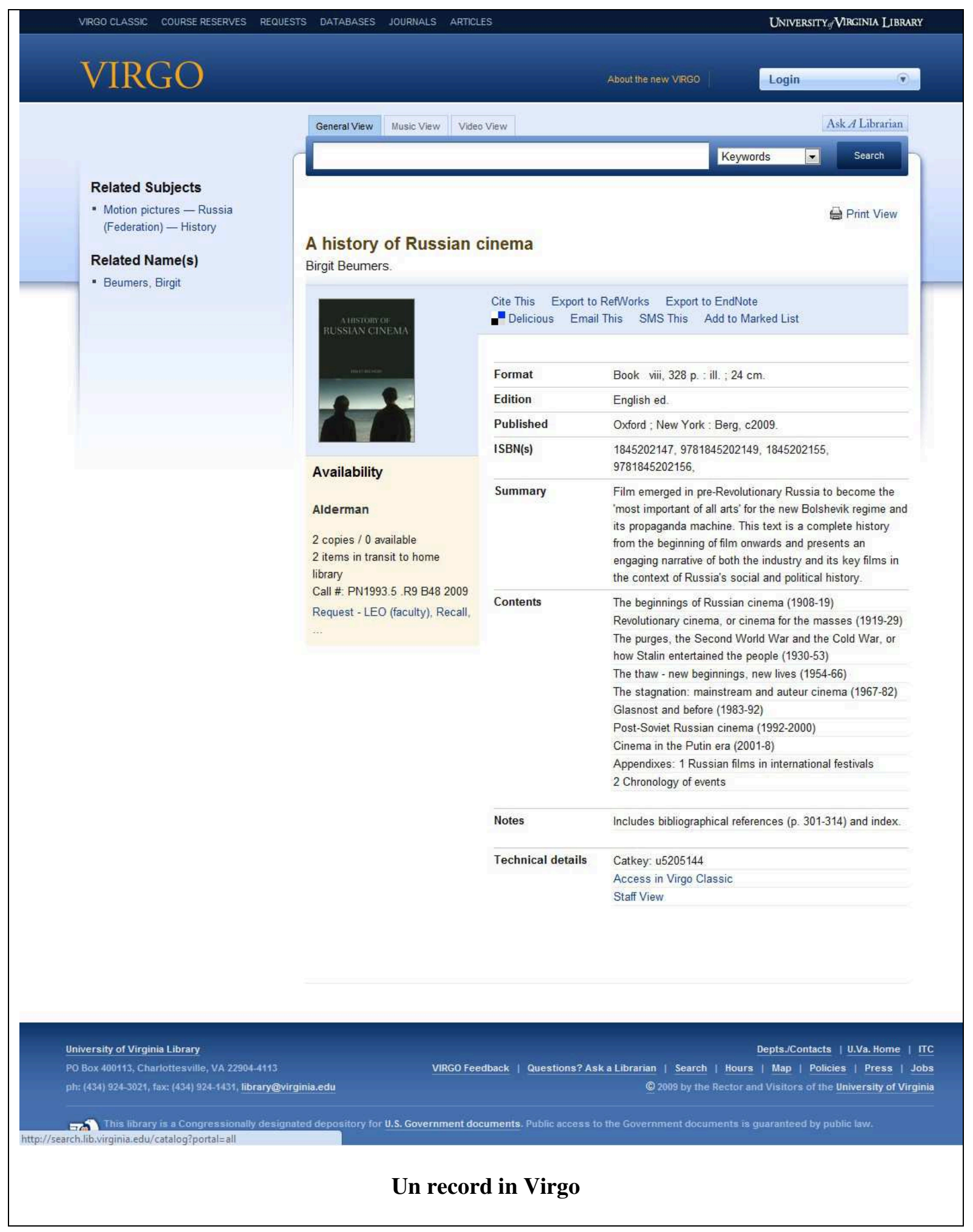




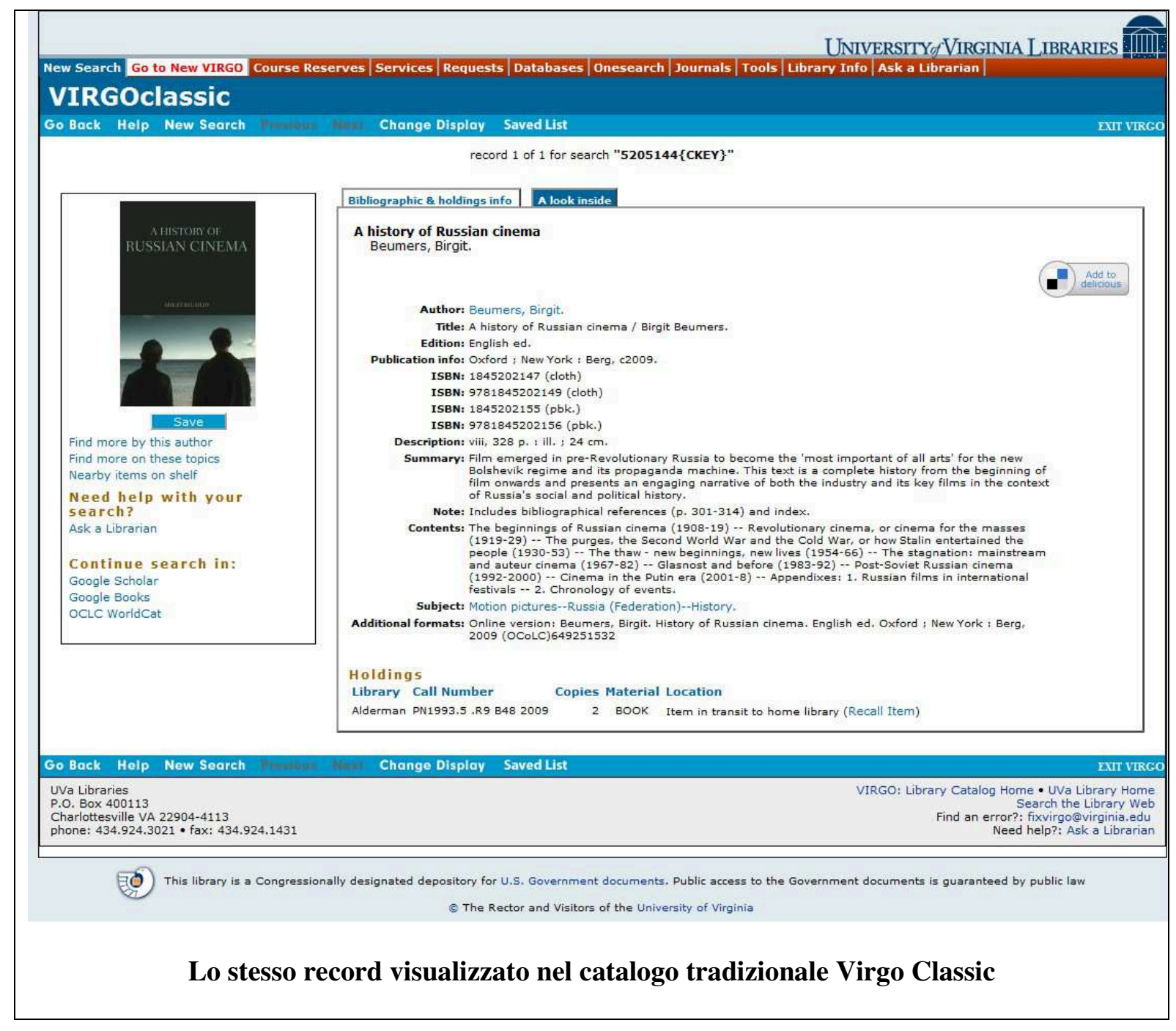




\section{Villanova University}

URL: <https://library.villanova.edu/Find>

VuFind è un software open source che si definisce 'interfaccia per la scoperta' creato e mantenuto dalla Villanova University, Falvey memorial library. L'implementazione e il rilascio del nuovo OPAC risalgono all'Agosto 2008. Tra le tante funzionalità del software che si ritrovano nell'implementazione alla Villanova sono da citare: suggerimenti di risorse simili; feed RSS del catalogo; export delle citazioni in formati standard; URL persistenti, che facilitano la possibilità di segnalare record per bibliografie o inviarli per posta elettronica e l'indicizzazione da parte dei motori di ricerca generalisti; interfaccia disponibile in diverse lingue; possibilità di creare indici per scorrimenti personalizzati; semplice personalizzazione grafica dell'interfaccia; apertura dei dati: predisposizione OpenSearch, OAI-PMH ecc. con la possibilità di impostare semplici collegamenti da cataloghi esterni; utilizzo di contenuti esterni (es. copertine e recensioni, da GoogleBooks, OpenLibrary, Amazon e altri fornitori); uso di API per l'integrazione con servizi remoti (es. il servizio XISBN di OCLC, per il reperimento di altre edizioni del documento visualizzato disponibili nel catalogo). VuFind applica il modello FRBR segnalando altre edizioni dal dettaglio record.

\section{Accesso all'OPAC /autenticazione}

Dalla home page / autenticazione per alcuni servizi (prestito aggiunta di contenuti sociali)

Possibilità di visualizzare l'interfaccia in più lingue

Disponibile, non implementata

Altre preferenzelpersonalizzazioni

Sì

Risorse incluse nell'OPAC

\section{Dati e Database}

Libri, riviste, risorse audio e video, risorse elettroniche

Formato dei dati

MARC, export dei dati in OpenSearch, OAI-PMH

Maschera di ricerca principale

Ricerca

Box di ricerca google like, menù a discesa per ricerca per campi

Tipologie di ricerca previste (searching)

Google like, ricerca avanzata per campi

Tipologie di ricerca previste (browsing)

Browsing di autori, titoli, soggetti, classi a partire da un termine di ricerca; (browsing visuale a faccette)

Aiuti nella ricerca

suggerimento ricerche alternative, alla presentazione della schermata dei risultati.

Acquisizione passiva di informazioni (tramite modalità push, durante il processo di ricerca) No

Ordinamento iniziale dei risultati di una ricerca

Per rilevanza, in base alle manifestazioni (edizioni).

Ordinamento per rilevanza /ranking e funzioni di riordino del set dei risultati

Riordino del set, per data, call number, autore, titolo

Possibilità di selezionare all'interno del set dei risultati

No

Possibilità di raffinamento del set dei risultati

Raffinamento progressivo del set dei risultati

Faccette previste per il raffinamento (biblioteche, tipologia di risorsa, autore, lingua, anno, soggetto)

Possibilità di nuova ricerca (search) all'interno del set dei risultati 
No

Navigazione del catalogo (clustering dei dati)

Uso dei filtri per navigare tra i risultati,

raffinamento per collezione, formato, data (con slide), classe, argomento, autore, lingua

\section{Navigazione nel catalogo (serendipità /interfacce visuali)}

Interfaccia visuale a faccette (tipo Flamenco) delle risorse bibliografiche disponibile. Si accede dalla home page del catalogo, dalla tag "Browse" <https://library.villanova.edu/Find/Browse/Home>

Suggerimenti di lettura (chi ha letto $x$ ha letto anche y)

No

Ricerca orientata al contenuto

Link al full text del documento (per risorse disponibili in rete)

Visualizzazione del record (breve)

Titolo*, autore*, titolo, data, call number, collocazione nell'edificio, disponibilità, tipo di risorsa, visualizzazione della copertina, aggiungi ai preferiti* (richiede autenticazione), trova nella mappa* (lancia un'applicazione che consente di visualizzare la posizione dello scaffale nella mappa dell'edificio).

(*ampi cliccabili)

Visualizzazione del record (dettaglio )

Titolo /autore (opera),

autore*, altri autori*, formato, lingua, pubblicazione, collana*, soggetti*, tag* (pulsante aggiungi tag - richiede autenticazione).

In alto sono presenti le funzioni di esportazione: cita, invia testo come sms, email, esporta in software di gestione bibliografie, aggiungi ai preferiti.

In basso varie tabs: holdings, description, table of contents, comments, review, excerpt, staff view.

Localizzazioni (richiede autenticazione), Descrizione (descrizione fisica, ISBN), toc, commenti, recensioni visualizzazioni di estratti vengono visualizzate se disponibili.

Sulla destra vengono suggerite altre letture (scelte tra gli scritti dell'autore principale) e altre

edizioni. Un dettaglio di un record è visibile in https://library.villanova.edu/Find/Record/59045)

(*campi cliccabili)

Contenitori temporanei /permanenti di memoria

History ricerche effettuate (Search history)

Funzioni di esportazione/riutilizzo/sociali

Esportazione della citazione del documento

Sì, esportazione anche verso software di gestione bibliografie (RefWorks and EndNote)

Invio dei risultati per email / Stampa dei risultati

Sì

Altre modalità di riutilizzo dei dati

Sì

Condivisione nei social network

No

Permalink

Sì

Creazione di una lista personale di documenti

Sì

Funzioni sociali (aggiunta di tag, recensione, valutazione)

Sì,

RSS (syndication dei dati)

Non implementata, ma supportata dal software

Interfacce/applicazioni per dispositivi mobili

No

Software utilizzato

Caratteristiche del Sistema 


\begin{tabular}{|c|}
\hline Vu Find \\
\hline Tecnologie utilizzate \\
\hline Open source, basato motore di ricerca Apache Lucene/Solr \\
\hline Particolari implementazioni \\
\hline 1 \\
\hline Ambiente di ricerca \\
\hline $\begin{array}{l}\text { L'interfaccia del catalogo tradizionale VuCat è ancora visibile e interrogabile all'URL } \\
\text { <http://voyager.villanova.edu/cgi-bin/Pwebrecon.cgi?DB=local\&PAGE=First>. } \\
\text { Il catalogo è inserito in un ambiente di ricerca particolarmente ricco dove agli utenti vengono forniti } \\
\text { vari canali di accesso alle risorse della biblioteca; tra gli strumenti a disposizione segnaliamo le } \\
\text { guide di soggetto (subject guides), un elenco A-Z dei database, Journal finder per la ricerca delle } \\
\text { riviste, E-Reference per risorse di reference a stampa e online. }\end{array}$ \\
\hline Discussione \\
\hline 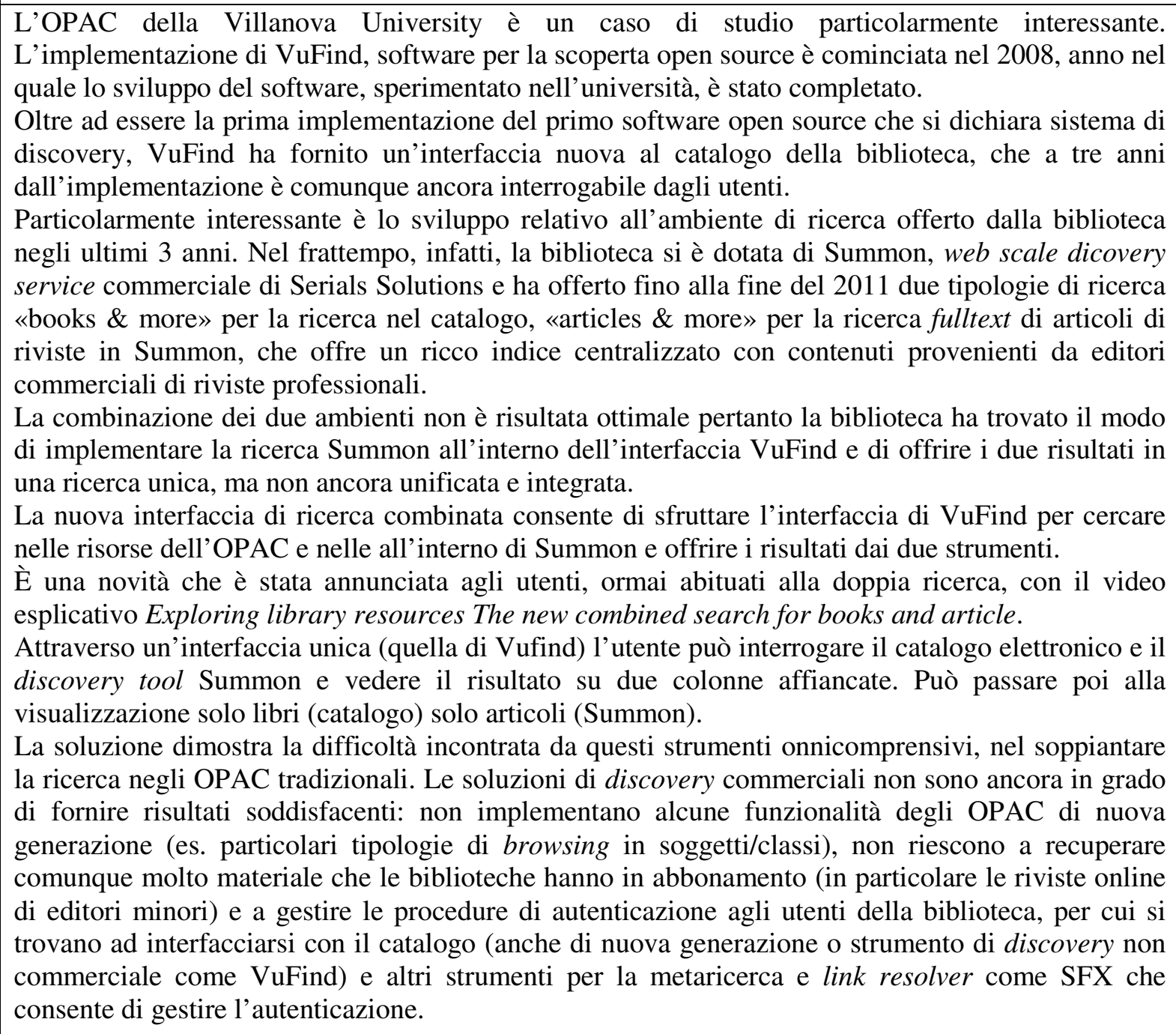 \\
\hline $\begin{array}{l}\text { Punti di forza / novità } \\
\text { VuFind fornisce alla biblioteca un catalogo di nuova generazione completo di tutte le funzionalità } \\
\text { necessarie, compresi gli utili suggerimenti. }\end{array}$ \\
\hline $\begin{array}{l}\text { Criticità } \\
\text { La difficoltà di integrare nel software VuFind la ricerca di articoli ha portato alla sperimentazione } \\
\text { originale di integrare il discovery system Summon dentro l'interfaccia di VuFind ottenendo però una } \\
\text { lista di risultati non integrata. }\end{array}$ \\
\hline
\end{tabular}




\section{Bibliografia}

John Houser, The VuFind implementation at Villanova University, «Library Hi Tech»,27 (2009), no.1, p. 93-105, <http://dx.doi.org/10.1108/07378830910942955>.

\section{Immagini}

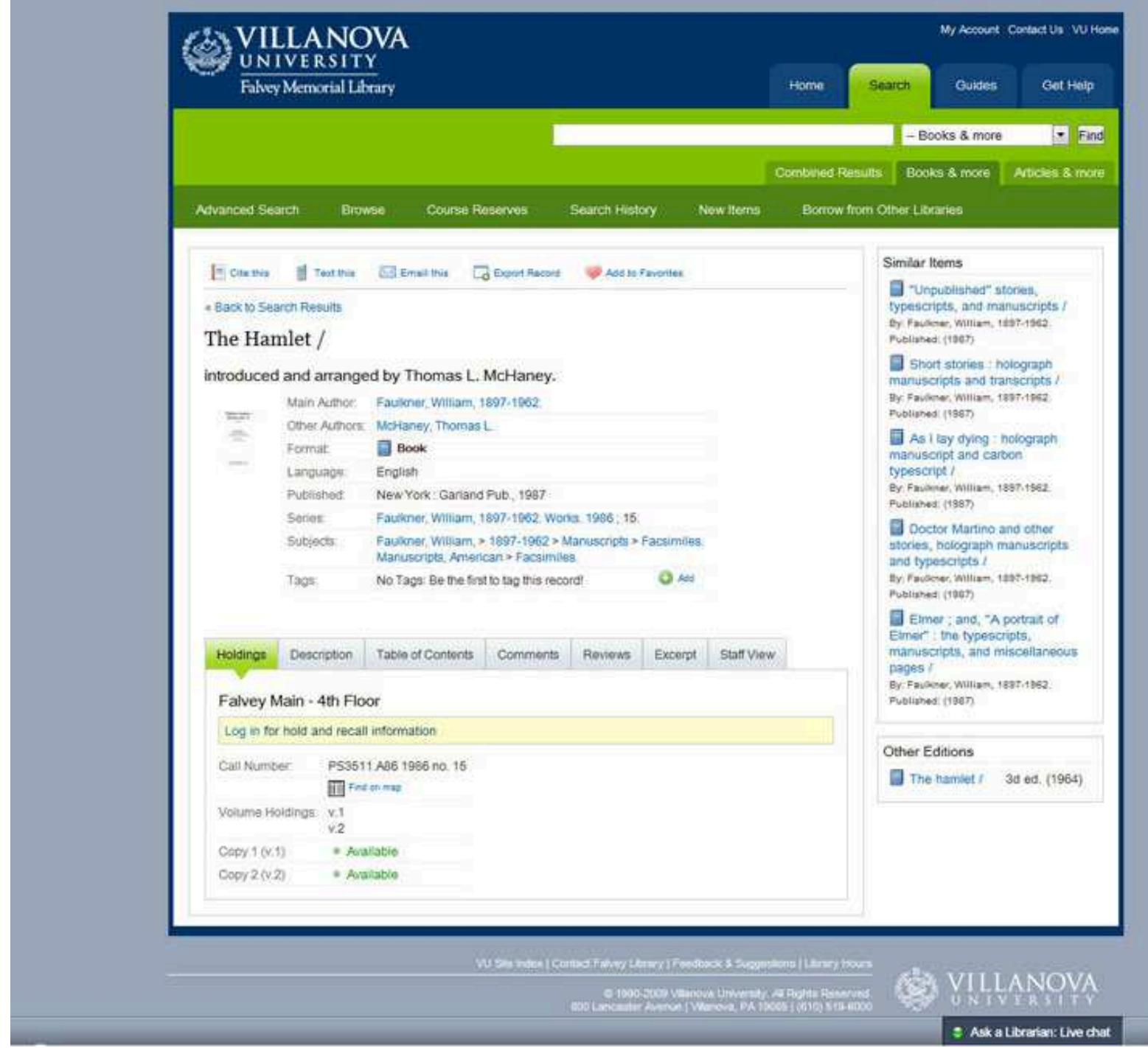

Dettaglio del record

(OPAC Villanova University- Falvey memorial library) 


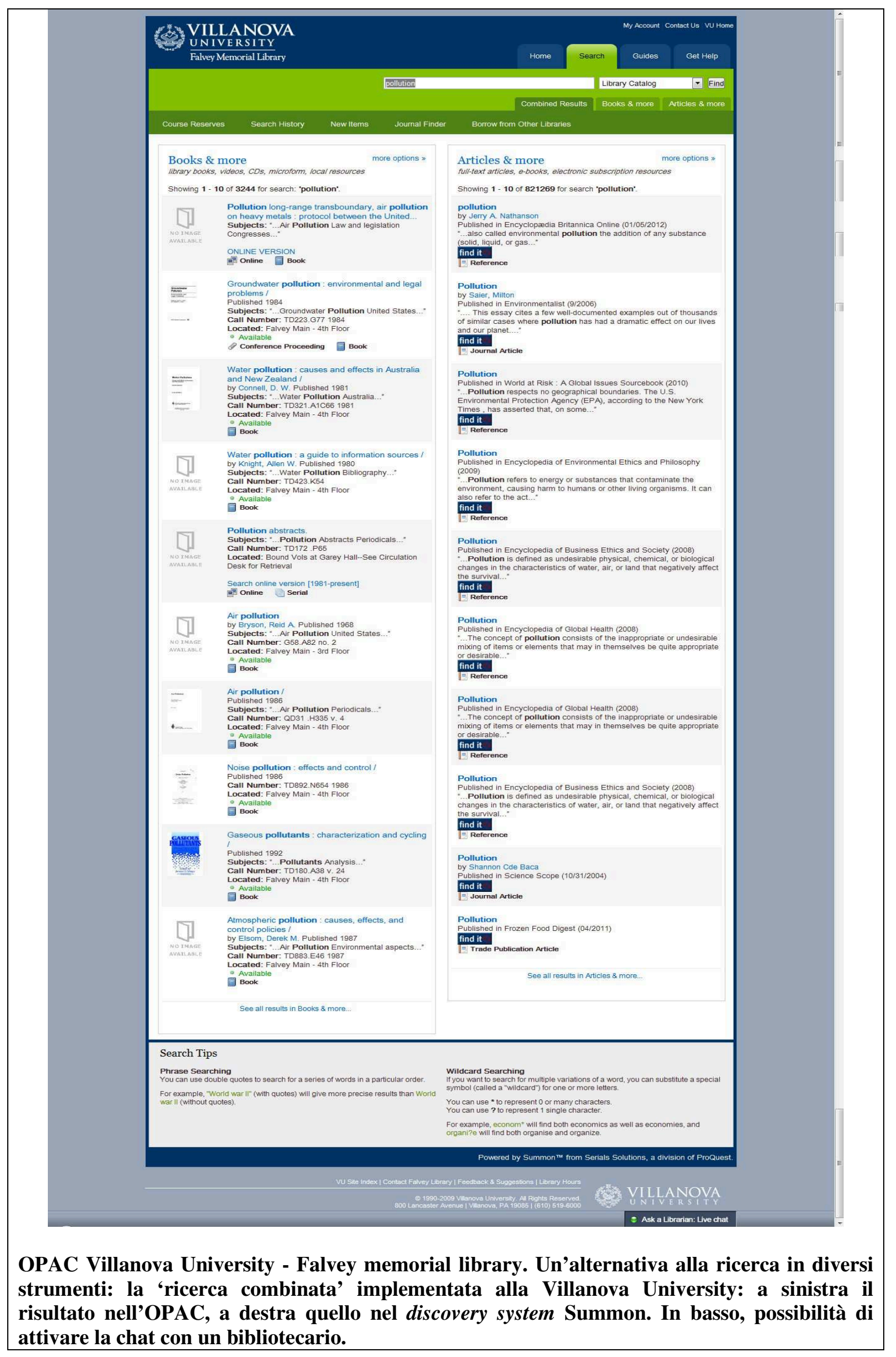





\section{APPENDICE B. SOFTWARE PER OPAC DI NUOVA GENERAZIONE E SERVIZI DI WEB SCALE DISCOVERY ${ }^{1}$}

\section{AQUABROWSER}

AquaBrowser è un un'interfaccia per la ricerca e la scoperta. Il software è costituito da un motore di ricerca affiancato da una applicazione visuale che consente il recupero di informazioni dal catalogo di una biblioteca o anche da più cataloghi di provenienza eterogenea. La sua funzionalità più popolare, anche se non necessariamente la più importante, è la possibilità di recupero per rilevanza tramite una 'nuvola semantica' che ricorda certe applicazioni sociali.

Creato e sviluppato dalla azienda olandese Medialab, è stato acquisito successivamente da Serial Solutions, ditta del gruppo ProQuest con sede a Seattle, a sua volta parte del Cambridge Information Group, di cui fa parte anche l'editore Bowker. Questo intreccio societario consente l'integrazione di numerosi prodotti e Web-scale Management Solutions che l'azienda produce, compresa LibraryThing for Libraries (prodotta da Bowker in integrazione con LibraryThing), grazie anche al modulo MyDiscoveries, che introduce le caratteristiche del Web 2.0. Con MyDiscoveries, AquaBrowser combina le collezioni delle biblioteche con i contributi, le opinioni e i suggerimenti della comunità degli utenti. My Discoveries si presenta come una community sociale di libri realizzata all'interno di AquaBrowser.

Le principali funzionalità del prodotto sono quelle di 'Search, Discover e Refine' che è anche il motto con cui Aquabrowser riassume le sue caratteristiche essenziali: ricerca semplificata con ordinamento per rilevanza; scoperta di altre risorse tramite la nuvola semantica; raffinamento tramite la navigazione a faccette.

\begin{tabular}{|l|l|}
\hline Sviluppatore/i & Serials Solutions (Distributore per l'Italia: @ CULT) \\
\hline Sito web & $\begin{array}{l}<\text { http://www.serialsolutions.com> } \\
(<\text { http://www.atcult.it>) }\end{array}$ \\
\hline Licenza/costi & Proprietario / costi n.d. \\
\hline Implementazione & Piattaforma Windows \\
\hline \multicolumn{2}{|c|}{ Dati, database, architettura del software } \\
\hline ILS supportati & $\begin{array}{l}\text { Tutti i maggiori ILS. Quando utilizzato come } \\
\text { interfaccia di ricerca unica (es. consorzio) è in grado } \\
\text { di supportare più ILS diversi. }\end{array}$ \\
\hline Workflow dei dati & Harvesting dall'ILS e dal altri repositories \\
\hline Accesso a collezioni digitali /e-journals & $\begin{array}{l}\text { L'applicazione può importare collezioni digitali in } \\
\text { XML e altri linguaggi strutturati. Accesso a } e- \\
\text { journals tramite affiancamento di applicativi per la } \\
\text { ricerca federata forniti da Serial Solutions }\end{array}$ \\
\hline $\begin{array}{l}\text { Integrazione con strumenti di ricerca } \\
\text { federata }\end{array}$ & $\begin{array}{l}\text { No, opzionale con affiancamento di applicazioni per } \\
\text { la ricerca federata. }\end{array}$ \\
\hline \multicolumn{1}{|c|}{ Ricerca nel catalogo / Visualizzazione dei record } \\
\hline Caratteristiche del motore di ricerca & $\begin{array}{l}\text { Proprietario. Il motore di ricerca è Igor sviluppato da } \\
\text { SS in circa dieci anni e con caratteristiche di ricerca } \\
\text { fuzzy, stemming. }\end{array}$ \\
\hline Aiuti nella ricerca & $\begin{array}{l}\text { Suggerimento: Forse cercavi; varianti del termine } \\
\text { dalla cloud dinamica }\end{array}$ \\
\hline
\end{tabular}

${ }^{1}$ La presente appendice fornisce una rassegna dei più noti software per OPAC di nuova generazione e strumenti di discovery. Salvo diversa indicazione tutti i dati si intendono aggiornati al 20.04.2013. I dati delle installazioni sono ricavati dal sito web Library technology guides, <http://www.librarytechnology.org>. 


\begin{tabular}{|c|c|}
\hline Tipologie di ricerca & Ricerca semplice e ricerca 'esatta' o avanzata \\
\hline Tipologie di navigazione & A faccette e visuale tramite la tag cloud dinamica \\
\hline Visualizzazione risultati & Ordinamento per rilevanza \\
\hline Arricchimenti & $\begin{array}{l}\text { Disponibili tramite servizi a sottoscrizione: } \\
\text { copertine, sommari, abstract. }\end{array}$ \\
\hline Raccomandazioni & No \\
\hline Tag clouds & Sì \\
\hline Community & No \\
\hline Interfacce visuali & $\begin{array}{l}\text { Sì, interfaccia visuale cloud dinamica che mostra i } \\
\text { termini affini e correlati. Richiede l'istallazione del } \\
\text { plugin Adobe Flash }\end{array}$ \\
\hline Implementazione del modello FRBR & No \\
\hline \multicolumn{2}{|c|}{ Altre caratteristiche } \\
\hline $\begin{array}{l}\text { Tagging/commenti/ } \\
\text { recensioni/voti }\end{array}$ & $\begin{array}{l}\text { Attraverso il componente MyDiscoveries gli utenti } \\
\text { possono inserire tag e recensioni, creare liste } \\
\text { pubbliche e private, votare gli item. } \\
\mathrm{E} \text { possibile anche includere copertine da } \\
\text { Librarything. }\end{array}$ \\
\hline Esportazione citazione & Sì, è possibile creare liste di documenti. \\
\hline Altre caratteristiche & $\begin{array}{l}\text { Interfaccia per dispositivi mobili (palmari e } \\
\text { smartphone) }\end{array}$ \\
\hline \multicolumn{2}{|c|}{ Installazioni } \\
\hline \multicolumn{2}{|c|}{$\begin{array}{l}1505 \text { biblioteche in tutto il mondo. Tra le biblioteche pubbliche si segnalano: Queens Borough } \\
\text { Public Library, Denver public library; tra le accademiche: Harvard University (catalogo Hollis), } \\
\text { University of Chicago, Oklahoma State University, University of Pittsburgh. } \\
\text { In questi anni AquaBrowser ha rafforzato la propria presenza in Italia. Fra i clienti importanti } \\
\text { segnaliamo la Soprintendenza speciale per i beni archeologici di Napoli e Pompei, la biblioteca del } \\
\text { museo Galileo, COBIRE (coordinamento delle biblioteche e delle strutture documentarie della } \\
\text { Regione Toscana). }\end{array}$} \\
\hline \multicolumn{2}{|c|}{ Riferimenti bibliografici /sitografia } \\
\hline \multicolumn{2}{|c|}{$\begin{array}{l}\text { Rapporto sulle biblioteche italiane } 2009-2010 \text { a cura di Vittorio Ponzani, direzione scientifica di } \\
\text { Giovanni Solimine, Roma, Associazione Italiana Biblioteche, 2010, p. 158-170. }\end{array}$} \\
\hline
\end{tabular}




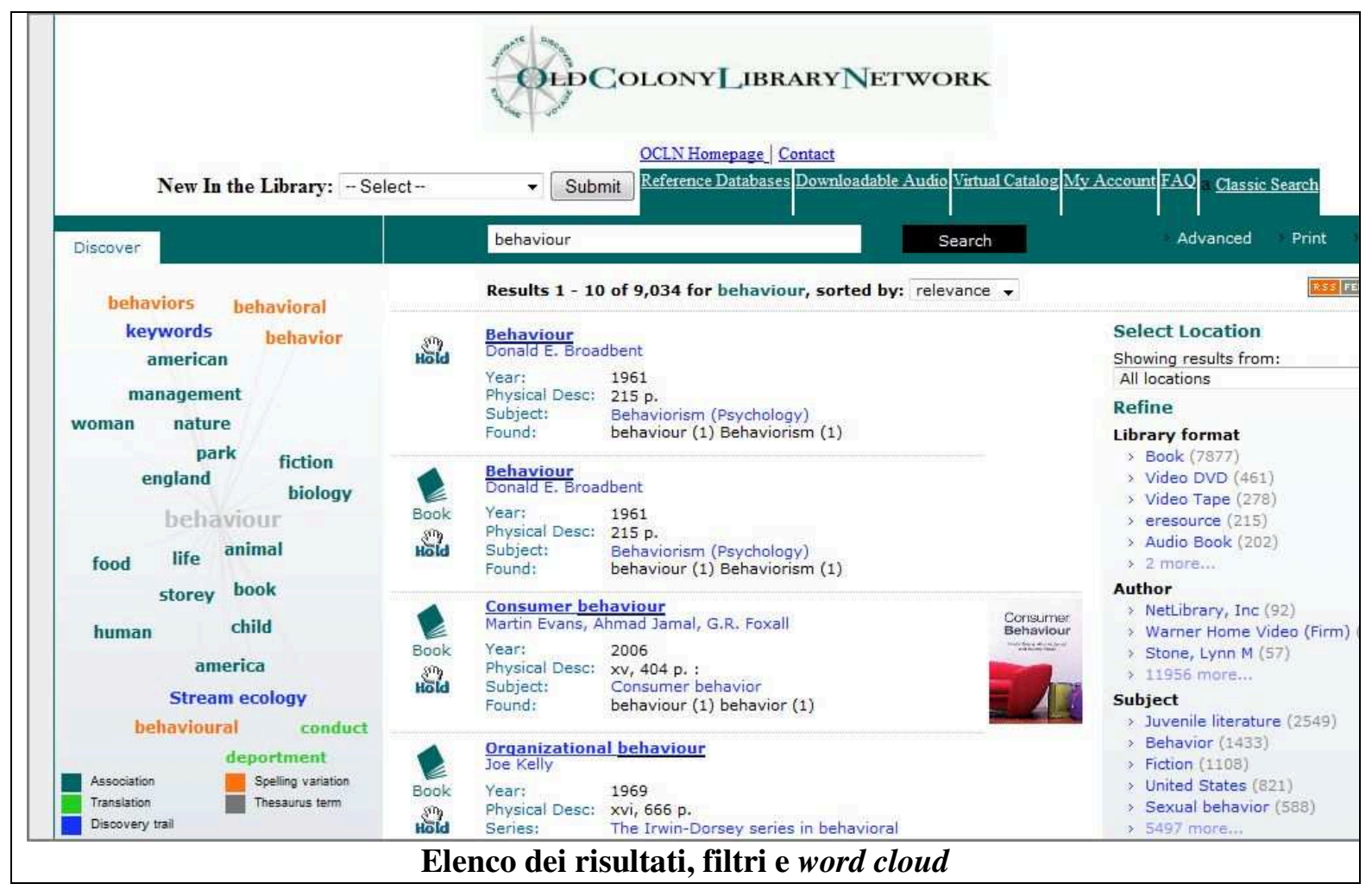




\section{SUMMON}

«Your library, with a single starting point» è il motto di Summon, il primo Web scale discovery di Syndetic Solutions presentato per la prima volta al convegno annuale dell'ALA nel 2009. L'azienda produttrice lo definisce un vero Web-Scale Discovery service dove è indicizzabile e recuperabile un vastissimo elenco di contenuti, dai libri ai video alle risorse digitali come gli articoli di riviste, oltre alle collezioni possedute dalla biblioteca: «It goes beyond federated search, beyond next-generation catalogs to create an all-new service for libraries». In Italia il prodotto viene pubblicizzato con il motto «SUMMON = Google... for libraries».

Tra le caratteristiche del prodotto sono da citare:

1)Unico box di ricerca per tutti i documenti e sostituzione degli strumenti di ricerca federata: attraverso un semplice campo di ricerca monobox ed un indice unico dei contenuti, Summon fornisce l'accesso a tutte le collezioni della biblioteca. Offrendo un'unica piattaforma di ricerca, Summon sostituisce la ricerca su piattaforme multiple e la ricerca federata che offrono risultati parziali, spesso incompleti e richiede tempi lunghi di risposta. Attualmente l'indice di Summon comprende oltre 1 miliardo di record, provenienti dai database commerciali, da fonti open access, editoriali, dal catalogo della biblioteca (indipendentemente dal sistema di automazione) e dalle collezioni locali. I contenuti dell'indice sono in aumento grazie alla collaborazione con più di 9000 editori internazionali (tra i quali ProQuest, Elsevier, IEEE, Springer, Wiley, ISI, Nature, EIU) Summon offre contenuti in lingua italiana grazie agli accordi con Casalini Libri, Franco Angeli Editore, Chiriotti Editore, Società Italiana di Biologia Marina, Vita e Pensiero Università Cattolica del Sacro Cuore, Unicredit Banca di Roma, FAO. I titoli presenti nella piattaforma sono elencati in: $<\mathrm{http} / / / \mathrm{www}$.serialssolutions.com/assets/publications/Summon-represented-titles.pdf>

2)Suggerimenti, esportazione delle citazioni, integrazione con link resolver, supporto dei dispositivi mobili citazioni, integrazione con link resolver, supporto dei dispositivi mobili.

\begin{tabular}{l|l} 
Sviluppatore/i & Serials Solutions (distributore per l'italia Cenfor International)
\end{tabular}

\begin{tabular}{l|l}
\hline Sito web & $<$ http://www.serialssolutions.com/en/services/summon/>
\end{tabular}

\begin{tabular}{l|l} 
Licenza/costi & Software proprietario \\
\hline
\end{tabular}

\begin{tabular}{|l|l|}
\hline Implementazione & Soluzione in hosting: nessun investimento hardware né risorse
\end{tabular} umane

Dati, database, architettura del software

\begin{tabular}{l|l} 
ILS supportati & Tutti i principali ILS. Summon è compatibile con i formati
\end{tabular} Unimarc e MARC21.

\begin{tabular}{ll|l} 
Workflow dei dati & I metadati, ottenuti direttamente dagli editori grazie agli accordi
\end{tabular} diretti di Serials Solutions, vengono pre-raccolti, normalizzati, deduplicati, fusi tra loro per ottenere un unico record il più completo possibile.

I metadati sono poi arricchiti dall'indice dei fulltext, dalla classificazione "Peer reviewed" tratta da Ulrich's, dalla funzionalità di conteggio delle citazioni tratta da ISI Web of Science e dal CrossRef DOI, prima di essere resi disponibili agli utenti Summon. L'indice si basa su Metadata Object Description Schema (MODS).

Viene implementato dai tecnici di Serials Solutions un processo automatico di scarico dei record dal sistema di automazione originario e importazione automatica nell'indice di Summon, per assicurare la perfetta sincronizzazione dei due sistemi. Una volta rilevato in Summon un record di interesse tratto dall'OPAC della Biblioteca, l'utente tramite un link verificherà la disponibilità del testo direttamente sull'interfaccia originaria (es. Aleph, Sebina OPAC, ecc). 


\begin{tabular}{|c|c|}
\hline $\begin{array}{l}\text { Accesso a collezioni } \\
\text { digitali /e-journals }\end{array}$ & $\begin{array}{l}\text { Accesso a oltre } 1 \text { milione di record bibliografici provenienti da } \\
\text { oltre } 7000 \text { editori internazionali. Collezioni di ebook, video, } \\
\text { periodici, tesi di dottorato, atti di convegni, letteratura grigia e } \\
\text { reports. }\end{array}$ \\
\hline $\begin{array}{l}\text { Integrazione con strumenti } \\
\text { di ricerca federata }\end{array}$ & $\begin{array}{l}\text { Summon si sostituisce alla ricerca federata presentando un indice } \\
\text { unificato con metadati provenienti da una vasta serie di } \\
\text { contenuti. } \\
\text { è garantita una facile integrazione con link resolver e piattaforme } \\
\text { di e-learning. } \\
\text { L'accesso al full-text degli articoli in formato elettronico o degli } \\
\text { eBook è immediato attraverso il Link Resolver (Summon è } \\
\text { compatibile con tutti i Link Resolver in commercio). La ricerca } \\
\text { viene effettuata dapprima all'interno delle risorse della } \\
\text { Biblioteca, ma può essere allargata a tutto il contenuto di } \\
\text { Summon (oltre } 900 \text { milioni di record). }\end{array}$ \\
\hline \multicolumn{2}{|c|}{ Ricerca nel catalogo /Visualizzazione dei record } \\
\hline $\begin{array}{l}\text { Caratteristiche del motore } \\
\text { di ricerca }\end{array}$ & Proprietario, sviluppato da Syndetic Solutions \\
\hline Aiuti nella ricerca & $\begin{array}{l}\text { Suggerimenti 'did you mean ...' in caso di errata digitazione o } \\
\text { scarsi risultati uso di Database recommender per risorse non } \\
\text { indicizzate. }\end{array}$ \\
\hline Tipologie di ricerca & 'Google like' \\
\hline Tipologie di navigazione & navigazione a faccette \\
\hline Visualizzazione risultati & $\begin{array}{l}\text { Risultati presentati in ordine di rilevanza. Sono disponibili varie } \\
\text { opzioni per raffinare l'elenco dei risultati, attraverso filtri, uso di } \\
\text { faccette, per organizzare facilmente i risultati per tipo di } \\
\text { contenuto, soggetto, biblioteca, lingua ed altro. L'elenco dei } \\
\text { risultati include anche l'abstract, la collocazione, la disponibilità } \\
\text { di full-text online. }\end{array}$ \\
\hline Arricchimenti & Abstract \\
\hline Raccomandazioni & Sì \\
\hline Tag clouds & No \\
\hline Community & No \\
\hline Interfacce visuali & No \\
\hline $\begin{array}{l}\text { Implementazione del } \\
\text { modello FRBR }\end{array}$ & No \\
\hline \multicolumn{2}{|r|}{ Altre caratteristiche } \\
\hline $\begin{array}{l}\text { Tagging/commenti/ } \\
\text { recensioni/voti }\end{array}$ & No \\
\hline Esportazione citazione & $\begin{array}{l}\text { Formattazione ed esportazione delle citazioni nei principali } \\
\text { gestori di bibliografie }\end{array}$ \\
\hline \multirow[t]{3}{*}{ Altre caratteristiche } & $\begin{array}{l}\text { Ricerca disciplinare: lo strumento Discipline Scoped Searching } \\
\text { consente di effettuare ricerche selezionando una specifica area } \\
\text { disciplinare e ottenere la massima precisione nei risultati. }\end{array}$ \\
\hline & $\begin{array}{l}\text { Supporto per devices mobili: il sistema riconosce } \\
\text { automaticamente l'accesso effettuato tramite smartphone e si } \\
\text { adatta per offrire una modalità di visualizzazione ottimizzata per } \\
\text { lo schermo di cellulari e dispositivi mobili. }\end{array}$ \\
\hline & $\begin{array}{l}\text { Filtro per full-text e testi peer-reviewed. Possibilità di restringere } \\
\text { i risultati della ricerca a contenuti full text e/o a materiale } \\
\text { accademico. }\end{array}$ \\
\hline \multicolumn{2}{|r|}{ Installazioni } \\
\hline
\end{tabular}




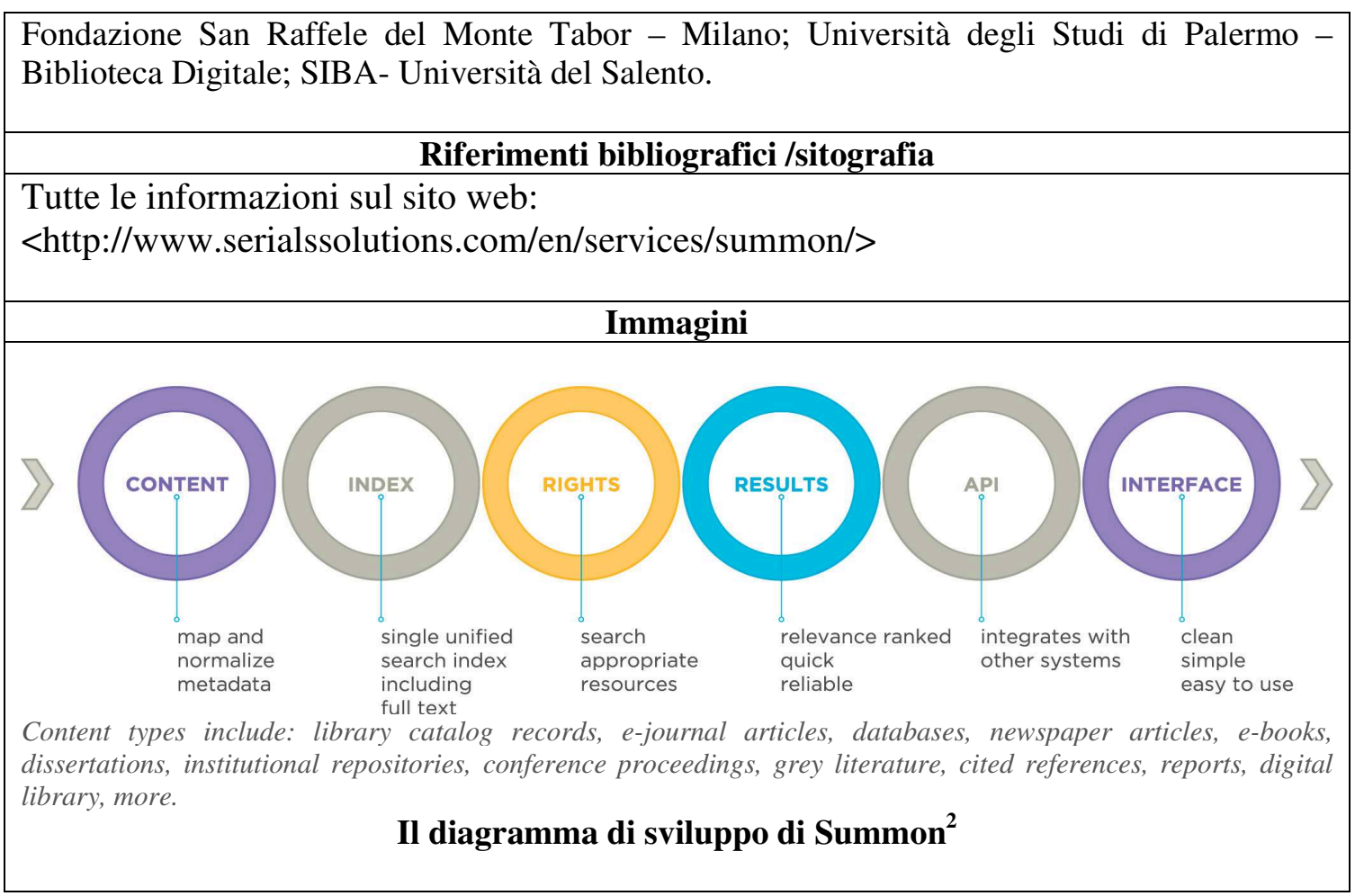

\footnotetext{
${ }^{2}$ Serial Solutions $<$ http://www.serialssolutions.com/en/services/summon>.
} 


\section{EBSCO DISCOVERY SERVICE}

«EBSCO Discovery Service Takes Discovery to the Next Level». Ebsco discovery service (EDS) lanciato nel 2011 è il software dell'azienda americana Ebsco che promette quattro principali funzionalità: accesso semplice e veloce ai contenuti a stampa ed elettronici della biblioteca, la più completa lista di partners (editori); metadati di alta qualità e indicizzazione di alto livello, indicizzazione per soggetto ricavata da Indici di soggetto Ebsco (e non tramite la ricerca federata), interazione in tempo reale con i dati dell'OPAC.

EDS Base Index, l'indice aggregato, rappresenta i contenuti provenienti da circa 20,000 fornitori e metadati provenienti da 70,000 editori comprendendo ad oggi più di 64,000 riviste e giornali, con 320 milioni di articoli, 400 atti di congressi, circa 6 milioni di libri, 825,000 tra CD e DVD e centinaia di migliaia di altre risorse.

Gli utenti che sottoscrivono EDS possono costruire la propria collezione importando nel database i dati delle loro collezioni, inclusi i dati provenienti dall'OPAC, dai repositories istituzionali, da database, collezioni archivistiche e altro.

Uno dei servizi principali forniti da EDS è quello di consentire il caricamento nella piattaforma dell'intero catalogo della biblioteca, in modo tale che i contenuti siano ricercabili insieme agli altri in un'interfaccia di ricerca unificata.

Tra le caratteristiche che EDS prevede nell'interfacciamento con il catalogo sono da menzionare le seguenti funzionalità: 1) facile accesso a tutti i contenuti del catalogo; 2) aggiornamento dei risultati con disponibilità in tempo reale e aggiornamenti quotidiani, possibilità di cercare per biblioteca (es. in un catalogo collettivo). 3) arricchimento: i contenuti del catalogo possono essere arricchiti con copertine, soggetti, disponibilità, premi, citazioni e recensioni; 4) ricerca full-text per i libri contenuti in EBSCO's eBook collections 5) box di ricerca personalizzabile dalla biblioteca 6) ricerca One-stop 7) collegamento ai contenuti fulltext sottoscritti dalla biblioteca con EBSCO A-to-Z e LinkSource.

\begin{tabular}{|c|c|}
\hline Sviluppatore/i & Ebsco Publishing \\
\hline Sito web & <http://www.ebscohost.com/> \\
\hline Licenza/costi & Proprietario \\
\hline Implementazione & \\
\hline \multicolumn{2}{|c|}{ Dati, database, architettura del software } \\
\hline ILS supportati & Tutti i principali ILS sono supportati \\
\hline Workflow dei dati & $\begin{array}{l}\text { Harvesting dei metadati dall'OPAC e da altri } \\
\text { repositories }\end{array}$ \\
\hline Accesso a collezioni digitali /e-journals & Tramite EDS Base Index \\
\hline $\begin{array}{l}\text { Integrazione con strumenti di ricerca } \\
\text { federata }\end{array}$ & $\begin{array}{l}\text { Il software sostituisce la ricerca federata con } \\
\text { l'indicizzazione in un indice centralizzato }\end{array}$ \\
\hline \multicolumn{2}{|c|}{ Ricerca nel catalogo / Visualizzazione dei record } \\
\hline \multicolumn{2}{|l|}{ Caratteristiche del motore di ricerca } \\
\hline \multicolumn{2}{|l|}{ Aiuti nella ricerca } \\
\hline Tipologie di ricerca & 'Google like', avanzata \\
\hline Tipologie di navigazione & A faccette \\
\hline Visualizzazione risultati & $\begin{array}{l}\text { Risultati ordinati per ranking. } \\
\text { personalizzabile e } \\
\text { istallazione }^{3}\end{array}$ \\
\hline Arricchimenti & $\begin{array}{l}\text { I contenuti del catalogo possono essere arrichiti } \\
\text { con copertine, soggetti, disponibilità, premi, } \\
\text { citazioni e recensioni, preview del libro su } \\
\text { Google Libri }\end{array}$ \\
\hline
\end{tabular}

\footnotetext{
${ }^{3}$ Il ranking è ampiamente configurabile: <http://www.ebscohost.com/discovery/technology/relevancyranking>
} 


\begin{tabular}{|c|c|}
\hline Raccomandazioni & Sì \\
\hline Tag clouds & No \\
\hline Community & No \\
\hline Interfacce visuali & No \\
\hline Implementazione del modello FRBR & No \\
\hline \multicolumn{2}{|c|}{$\begin{aligned} \text { Altre caratteristiche } \\
\end{aligned}$} \\
\hline $\begin{array}{l}\text { Tagging/commenti/ } \\
\text { recensioni/voti }\end{array}$ & Commenti e recensioni \\
\hline Esportazione citazione & Sì \\
\hline Disponibilità in tempo reale & Sì \\
\hline \multicolumn{2}{|c|}{ Installazioni } \\
\hline \multicolumn{2}{|c|}{395 istallazioni, in Italia l' Università degli Studi di Napoli Federico II } \\
\hline \multicolumn{2}{|c|}{ Riferimenti bibliografici /sitografia } \\
\hline \multicolumn{2}{|c|}{$\begin{array}{l}\text { Roberta Barbieri, EBSCO Discovery Service: esperienze a confronto, «Biblioteche oggi», } 31 \\
\text { (2013), n. } 2 \text {, p. } 80 \text {. } \\
\text { <http://www.ebscohost.com/> }\end{array}$} \\
\hline
\end{tabular}




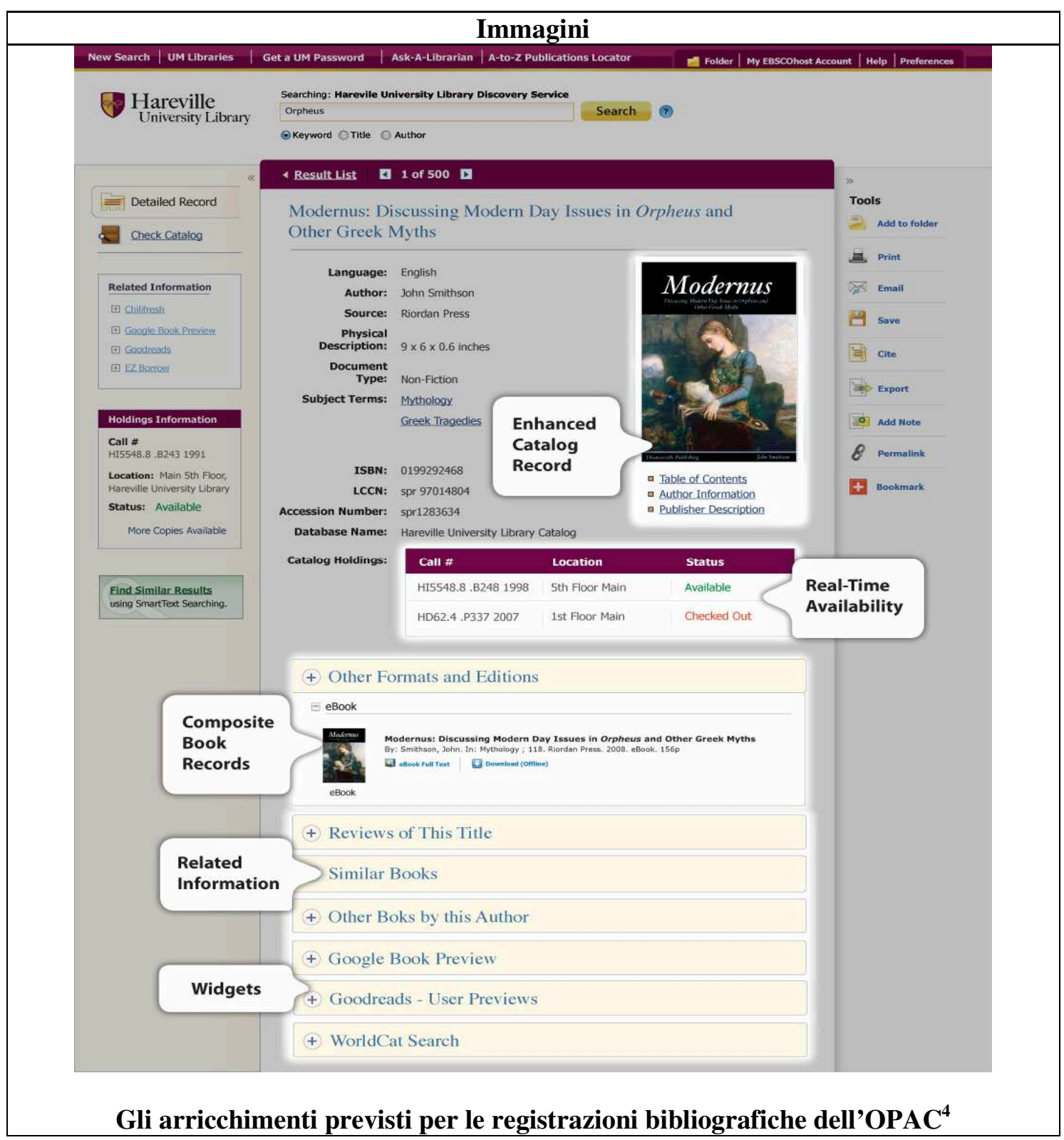

${ }^{4}$ Ebsco host $<$ http://www.ebscohost.com/discovery/catalog/catalog-customization $>$. 


\section{BIBLIOCOMMONS}

«A far better online catalog is just the beginning» è il motto di BiblioCommons, servizio fornito dalla omonima azienda canadese, che si configura come un'interfaccia di nuova generazione per il catalogo. Oltre alle più comuni caratteristiche offerte dai next generation catalogs (visualizzazioni arricchite, navigazione a faccette e relevance ranking) il prodotto ha puntato dapprima su arricchimenti sociali come tag e commenti, mentre oggi si concentra sull'integrazione con una community di utenti.

Il 'Commons' del nome dell'azienda fa, appunto, riferimento alla ricchezza di funzioni sociali e di community che consentono all'utente di interagire con l'OPAC per aggiungere commenti, tag e classificazioni, e condividere i propri elementi preferiti su Facebook o Twitter. Gli utenti possono creare elenchi che possono mantenere privati o pubblici ovvero utilizzabili da altri utenti con gusti o esigenze simili. La prima implementazione si registra biblioteca pubblica di Oakville in Ontario, Canada nel 2007. Fino a qualche tempo fa l'unico ILS supportato era Horizon. Nel 2009 il software è entrato in una nuova fase di sviluppo Attualmente il software BiblioCommons è in fase di sviluppo e offrirà a breve una suite completa di prodotti. Al momento il prodotto centrale della suite BiblioCore,si propone di sostituire tutte le funzionalità dell'OPAC tradizionale, mentre Bibliomobile consente di interrogare l'OPAC sugli smartphone. Particolarità della piattaforma sono le caratteristiche sociali integrate nel software, un'architettura SaaS basata su tecnologie open source, la possibilità di esportare i dati e riutilizzarli tramite apposite API. Il target è principalmente quello delle biblioteche di pubblica lettura. Tra le biblioteche che hanno implementato il sistema vi è anche la New York public library.

\begin{tabular}{|l|l|}
\hline Sviluppatore/i & BiblioCommons \\
\hline Sito web & <www.bibliocommons.com> \\
\hline Licenza/costi & Proprietario, sottoscrizione annuale \\
\hline Implementazione & $\begin{array}{l}\text { Servizio fornito come SAS e basato su } \\
\text { tecnologie open-source come Ruby on Rails, } \\
\text { Solr/Lucene, and PostgreSQL. }\end{array}$ \\
\hline ILS supportati & $\begin{array}{l}\text { Hati, database, architettura del software } \\
\text { Millennium, Evergreen }\end{array}$ \\
\hline Workflow dei dati & $\begin{array}{l}\text { Prevede l'istallazione di un connettore che } \\
\text { consente l'estrazione dei dati dall'ILS tramite } \\
\text { harvesting e la sincronizzazione con i server }\end{array}$ \\
\hline Accesso a collezioni digitali /e-journals & RiblioCore. \\
\hline $\begin{array}{l}\text { Integrazione con strumenti di ricerca } \\
\text { federata }\end{array}$ & $\begin{array}{l}\text { Si, integrazione con strumenti per la ricerca } \\
\text { federata e con altri strumenti per importare post } \\
\text { da blog, wiki ed eventi all'interno } \\
\text { dell'interfaccia }\end{array}$ \\
\hline Raratteristiche del motore di ricerca & $\begin{array}{l}\text { Supporto dello stemming. Natural Language } \\
\text { detection interpreta le ricerche popolari ad } \\
\text { esempio una query come 'libri per bambini in } \\
\text { cinese' viene interpretata come 'language: } \\
\text { Chinese AND format: book AND audience: } \\
\text { children'. Supporto Unicode nativo per la ricerca } \\
\text { e visualizzazione in lingue e scritture diverse. }\end{array}$ \\
\hline Aiuti nella ricerca & $\begin{array}{l}\text { Suggerimenti in fase di digitazione ricavati dai } \\
\text { record di autorità. Autosuggerimenti in fase di }\end{array}$ \\
\hline
\end{tabular}




\begin{tabular}{|c|c|}
\hline & $\begin{array}{l}\text { ricerca e browsing di termini autorizzati: autori e } \\
\text { soggetti. Funzionalità di correzione dello } \\
\text { spelling (forse cercavi) in caso di risultato zero } \\
\text { ed errori di digitazione. }\end{array}$ \\
\hline Tipologie di ricerca & $\begin{array}{l}\text { Google like, ricerca avanzata intelligente con } \\
\text { possibilità di generare stringhe da connettere con } \\
\text { operatori booleani e numerosi campi per la } \\
\text { ricerca avanzata precisa. Browsing dei soggetti e } \\
\text { delle classi con l'opzione browse the shelf a } \\
\text { partire dal record. }\end{array}$ \\
\hline Tipologie di navigazione & $\begin{array}{l}\text { Ricerca a faccette che include alcuni valori come } \\
\text { destinatari, fiction/non fiction, disponibilità. Le } \\
\text { classificazioni destinatari e genere possono } \\
\text { essere specificati con apposite regole } \\
\text { personalizzabili. }\end{array}$ \\
\hline Visualizzazione risultati & $\begin{array}{l}\text { Relevance ranking. L'algoritmo si adatta } \\
\text { automaticamente ai dati sulla circolazione per } \\
\text { determinare i risultati più pertinenti. }\end{array}$ \\
\hline Arricchimenti & $\begin{array}{l}\text { Record bibliografico arricchito con copertine etc. } \\
\text { BiblioCommons also provides full integration of } \\
\text { a broad range of data types from third-party data } \\
\text { enhancement suppliers including Content Café } \\
\text { and Syndetics. }\end{array}$ \\
\hline Raccomandazioni & Sì \\
\hline Tag clouds & No \\
\hline Community & $\begin{array}{l}\text { Si, è possibile anche basare } \mathrm{i} \text { suggerimenti sulle } \\
\text { attività recenti della community per scoprire } \\
\text { nuovi item. }\end{array}$ \\
\hline Interfacce visuali & $\begin{array}{l}\text { Possibilità di vedere separatamente gli articoli } \\
\text { 'Just Arrived' and 'On Order' che possono } \\
\text { essere suddivisi per categorie e filtrati per by } \\
\text { formato, lingua, destinatari, e in base al } \\
\text { contenuto (fiction/non-fiction). } \\
\text { Interfaccia visuale "a carosello" delle copertine } \\
\text { per ogni tipologia di vista. Utilizzando lo } \\
\text { strumento di ricerca avanzata di BiblioCommons } \\
\text { è possibile per l'amministatore personalizzare le } \\
\text { categorie nella schermata nuovi titoli. } \\
\text { Aggiornamento automatico dei titoli in ogni } \\
\text { "vista" configurata. }\end{array}$ \\
\hline Implementazione del modello FRBR & No \\
\hline \multicolumn{2}{|c|}{ Altre caratteristiche } \\
\hline $\begin{array}{l}\text { Tagging/commenti/ } \\
\text { recensioni/voti }\end{array}$ & Sì \\
\hline \multirow[t]{4}{*}{ Esportazione citazione } & Sì \\
\hline & $\begin{array}{l}\text { Possibilità di impostare avvisi (alerts) per } \\
\text { ripetere queries ed importare dati aggiornati } \\
\text { dall'OPAC tramite URL permanenti. }\end{array}$ \\
\hline & Nuovi titoli aggiornati automaticamente \\
\hline & $\begin{array}{l}\text { Database per premi letterari e bestseller. } \\
\text { 'Staff picks', un'interfaccia che consente di } \\
\text { creare un riquadro che mostra consigli dei libri } \\
\text { scelti dai bibliotecari. }\end{array}$ \\
\hline \multicolumn{2}{|r|}{ Installazioni } \\
\hline
\end{tabular}




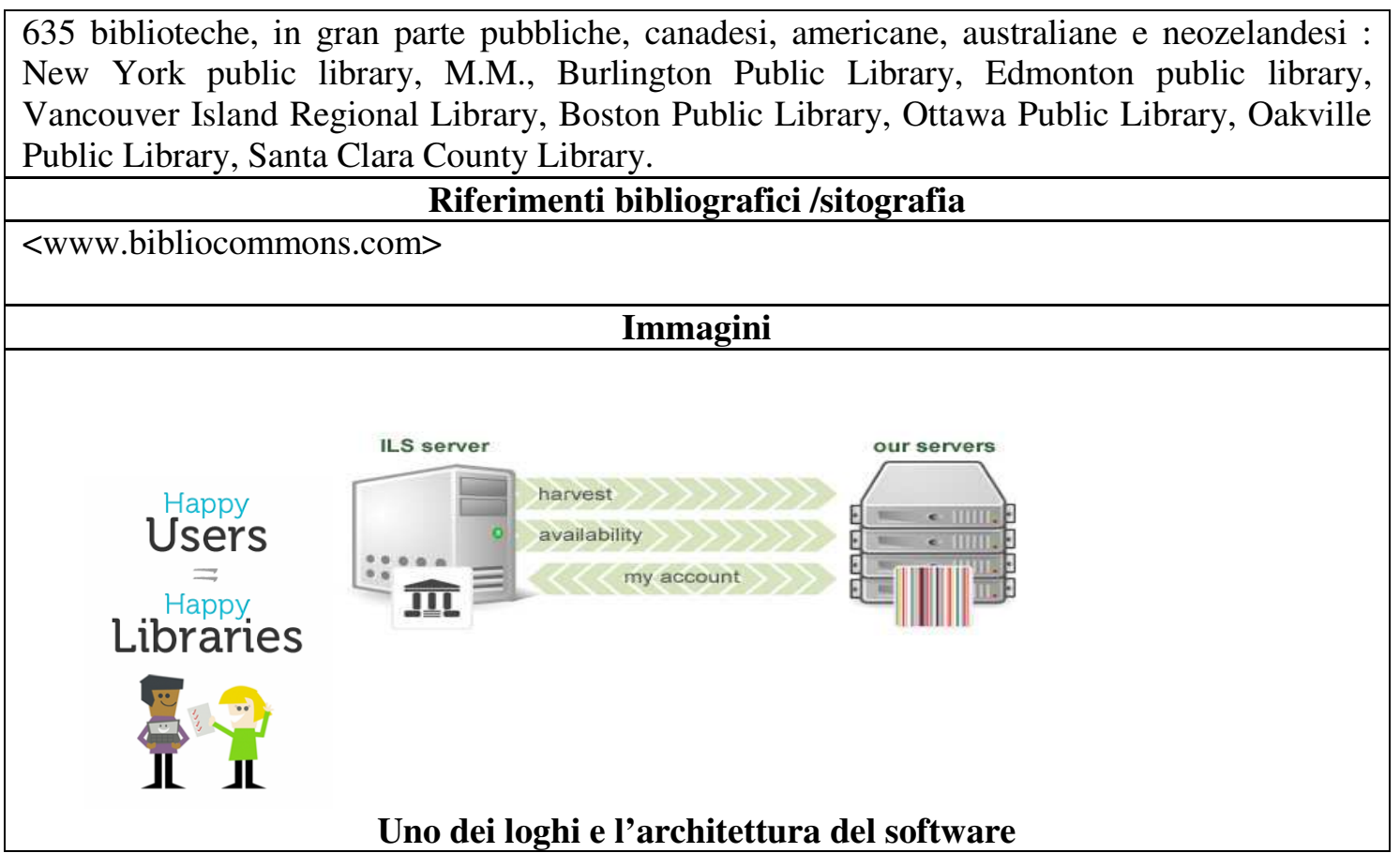




\section{ENCORE}

Encore è il software creato da Innovative Interfaces, già sviluppatore dell'ILS Millennium. Tra le caratteristiche del software vi sono la ricerca da unico punto di più risorse, risultati ordinati per rilevanza secondo l'algoritmo proprietario RightResult ${ }^{\mathrm{TM}}$, navigazione a faccette, una word cloud per correlare i tag, e il browsing dello scaffale virtuale.

Il software si integra con strumenti per la ricerca federata e la ricerca di contenuto arricchito può avvenire anche tramite harvesting dei metadati. Offre inoltre varie funzionalità 'sociali' come la possibilità di creare una community tramite il tagging delle risorse, le recensioni, le valutazioni e la possibilità di interagire con noti social network come Facebook.

Se usato in combinazione con gli altri strumenti di Innovative interfaces il software garantisce il recupero dei dati bibliografici in tempo reale da strumenti di gestione delle risorse elettroniche.

\begin{tabular}{|c|c|}
\hline Sviluppatore/i & Innovative interfaces \\
\hline Sito web & $\begin{array}{l}<\text { <http://www.iii.com/products/encore.shtml>, } \\
<\text { http://encoreforlibraries.com/> }\end{array}$ \\
\hline Licenza/costi & Proprietario \\
\hline Implementazione & Versione in hosting o istallazione locale \\
\hline \multicolumn{2}{|r|}{ Dati, database, architettura del software } \\
\hline ILS supportati & Compatibile con la maggior parte degli ILS \\
\hline Workflow dei dati & Harvesting dei metadati dall'ILS e da altri repositories \\
\hline $\begin{array}{l}\text { Accesso a collezioni digitali } \\
\text { le-journals }\end{array}$ & Tramite OAI-PMH \\
\hline $\begin{array}{l}\text { Integrazione con strumenti } \\
\text { di ricerca federata }\end{array}$ & $\begin{array}{l}\text { Compatibile e integrabile con altri strumenti di ricerca federata } \\
\text { sviluppati da Innovative interfaces }\end{array}$ \\
\hline \multicolumn{2}{|c|}{ Ricerca nel catalogo / Visualizzazione dei record } \\
\hline $\begin{array}{l}\text { Caratteristiche del motore di } \\
\text { ricerca }\end{array}$ & Proprietaro \\
\hline Aiuti nella ricerca & / \\
\hline Tipologie di ricerca & 'Google like' \\
\hline Tipologie di navigazione & Navigazione a faccette \\
\hline Visualizzazione risultati & Ordinamento per ranking \\
\hline Arricchimenti & Tutti gli arricchimenti di Syndetic solutions \\
\hline Raccomandazioni & Sì \\
\hline Tag clouds & Sì \\
\hline Community & No \\
\hline Interfacce visuali & Browsing visuale dello scaffale \\
\hline $\begin{array}{l}\text { Implementazione del } \\
\text { modello FRBR }\end{array}$ & No \\
\hline \multicolumn{2}{|r|}{ Altre caratteristiche } \\
\hline $\begin{array}{l}\text { Tagging/commenti/ } \\
\text { recensioni/voti }\end{array}$ & Sì \\
\hline Esportazione citazione & Sì \\
\hline \multicolumn{2}{|r|}{ Installazioni } \\
\hline \multicolumn{2}{|l|}{1231 istallazioni } \\
\hline \multicolumn{2}{|r|}{ Riferimenti bibliografici /sitografia } \\
\hline$<$ http://encoreforlibraries.c & $m>$ \\
\hline
\end{tabular}




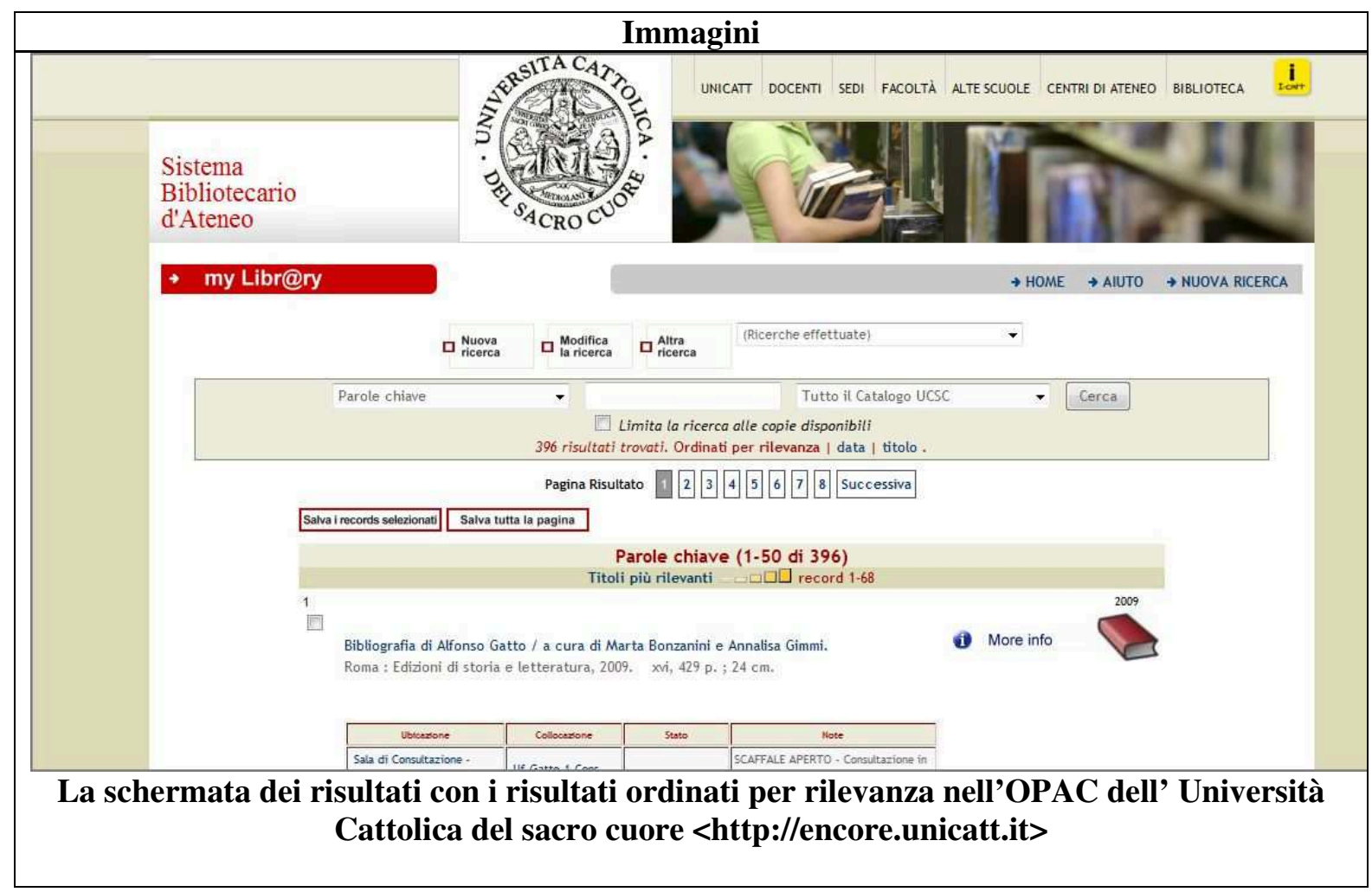




\section{PRIMO DISCOVERY AND DELIVERY}

Primo è la soluzione di discovery e delivery di ExLibris, azienda software leader del settore e nota per i gestionali Voyager e Aleph500.

Il software, sviluppato a partire dal 2006 consente attraverso la sua architettura modulare di a) esportare i dati dal catalogo, dall'archivio digitale, da ambienti locali di e-learning e pubblicarli sulla piattaforma di publishing di Primo, arricchendo ogni record con link a fonti esterne per il recupero di ToC, abstract, copertine ed altro. È possibile deduplicare i record, e raggruppare diverse manifestazioni di una stessa opera secondo tecniche di FRBR. Tutti i record che passano attraverso il processo di publishing di Primo vanno a far parte di un indice locale omogeneo;

b) Consentire agli utenti di fare ricerca simultaneamente sia sull'indice locale (che contiene le informazioni provenienti dagli applicativi locali come il catalogo, l'archivio digitale o altri repositories) sia sull'indice centrale di Primo Central creato e mantenuto da Ex Libris.

Primo Central è un 'mega-aggregato di informazioni bibliografiche', abstract e, in molti casi, di testi integrali di articoli provenienti dai database di editori e da aggregatori a livello mondiale. L'azienda ha previsto che Primo Central conterrà oltre 500 milioni di record entro la fine del 2010 e che tali dimensioni saranno destinate ad aumentare in modo esponenziale negli anni successivi.

Il processo di ricerca, la qualità e la visualizzazione dei record sono omogenee e i tempi di risposta sono molto rapidi. Primo Central limita dunque la necessità di ricorrere alle singole interfacce native delle varie risorse o quella di adottare sistemi di meta-ricerca che, come è noto, pongono problemi non trascurabili sia da un punto di vista della scarsa rapidità con la quale vengono visualizzati i risultati sia per la ovvia necessità che i sistemi di meta ricerca hanno di appiattire ad un minimo comune denominatore la qualità dei metodi di ricerca.

Primo e Primo Central rappresentano insieme un ambiente di discovery integrato ed omogeneo attraverso il quale l'utente finale può scoprire i materiali di interesse attraverso un processo che l'azienda definisce coinvolgente e appagante oltre che certificato da un punto di vista della qualità dei contenuti.

La ricerca simultanea sui due indici - quello relativo ai dati provenienti dal catalogo che, per dimensioni, non può certo competere con quello relativo ai dati raccolti in Primo Central e, appunto, Primo Central - potrebbe far supporre che i dati provenienti dal catalogo vengano "annegati" nell'enorme quantità di informazioni disponibili e, quindi non presentati fra i primi risultati in ordine di rilevanza. In realtà, grazie a sofisticati algoritmi di relevance ranking e a tecniche di "boosting" dei dati provenienti dalle risorse locali, a parità di importanza rispetto ai criteri di ranking, Primo è in grado di presentare fra i primi risultati i record relativi ai materiali disponibili localmente.

Tra le innovazioni recenti del prodotto è allo studio la possibilità di introdurre funzionalità di browsing che ancora mancano nell'interfaccia del prodotto.

Nel dicembre 2012 Ex Libris ${ }^{\circledR}$ Group ha infine annunciato la sua adesione al W3C Schema Bib Extend Community Group che raggruppa esponenti di diverse comunità che hanno interesse ad estendere i propri schemi di metadati per favorire la presenza di dati bibliografici compatibili con il web. Il gruppo opera all'interno del progetto Schema.org ${ }^{5}$ che consente alle pagine web di condividere uno schema strutturato che consente ai motori di ricerca di riconoscere il contenuto semantico presente nelle pagine web.

\begin{tabular}{|l|l|}
\hline Sviluppatore/i & Exlibris \\
\hline Sito web & $<$ http://www.exlibrisgroup.com/category/PrimoOverview $>$ \\
\hline
\end{tabular}

\footnotetext{
5 Ex Libris Promotes Linked Data Techniques to Make Library Data More Open and Accessible, [comunicato stampa], 5 dicembre 2012, Exlibrisgroup.com, $<$ http://www.exlibrisgroup.com/default.asp?catid=\{916AFF5B-CA4A-48FD-AD54-

9AD2ADADEB88 $\} \&$ details_type $=1 \&$ itemid $=\{$ 97C7DCE3-2AF6-442A-88C0-9E7C8299EA9B $\}>$
} 


\begin{tabular}{|c|c|}
\hline Licenza/costi & Proprietario \\
\hline Implementazione & $\begin{array}{l}\text { Installabile localmente, oppure è possibile scegliere l'opzione SaaS } \\
\text { (Software as a Service) di ExLibris. In questo caso l'applicazione sarà } \\
\text { ospitata in Cloud Computing e non sarà necessario mettere a } \\
\text { disposizione hardware e personale per la gestione sistemistica } \\
\text { localmente. Nell'opzione SaaS è inoltre possibile richiedere il servizio } \\
\text { Total Care di Ex Libris attraverso il quale l'Istituzione è sollevata da } \\
\text { ogni attività di implementazione iniziale e di gestione nel tempo } \\
\text { dell'applicativo. }\end{array}$ \\
\hline & Dati, database, architettura del software \\
\hline ILS supportati & Compatibile con tutti i principali ILS in commercio. \\
\hline Workflow dei dati & $\begin{array}{l}\text { Harvesting dei metadati dall'ILS, attraverso il protocollo OAI-PMH, o } \\
\text { export in MARCXML; importazione dei dati sulla circolazione e delle } \\
\text { funzioni utente }\end{array}$ \\
\hline $\begin{array}{l}\text { Accesso a collezioni } \\
\text { digitali /e-journals }\end{array}$ & $\begin{array}{l}\text { Primo recupera: 1) le informazioni provenienti dai repository locali } \\
\text { (cataloghi, archivi digitali, ambienti di e-learning), dell'Istituzione e/o } \\
\text { delle istituzioni collegate; 2)Primo Central. Indice contenente } \\
\text { centinaia di milioni di record e in costante crescita, contiene risorse in } \\
\text { licenza d'uso e ad accesso libero, e-books, e-journals, banche dati } \\
\text { bibliografiche; 3) la knowledge base di MetaLib: } 2115 \text { risorse remote } \\
\text { di cui } 658 \text { ad accesso gratuito; 4) la knowledge base di SFX: milioni di } \\
\text { e-journals (oltre } 25.000 \text { ad accesso gratuito) ed e-books (migliaia dei } \\
\text { quali ad accesso gratuito). }\end{array}$ \\
\hline $\begin{array}{l}\text { Integrazione } r \\
\text { strumenti di ricerca } \\
\text { federata }\end{array}$ & Integrazione con gli indici di SFX e Metalib. \\
\hline \multicolumn{2}{|c|}{$\begin{array}{ll}\text { Ricerca nel catalogo / Visualizzazione dei record } \\
\end{array}$} \\
\hline $\begin{array}{ll}\text { Caratteristiche } & \text { del } \\
\text { motore di ricerca } & \end{array}$ & I \\
\hline Aiuti nella ricerca & Did You Mean: per la correzione di termini digitati in modo errato \\
\hline Tipologie di ricerca & Di tipo google like (default) e avanzata \\
\hline $\begin{array}{l}\text { Tipologie di } \\
\text { navigazione }\end{array}$ & Navigazione a faccette \\
\hline $\begin{array}{l}\text { Visualizzazione } \\
\text { risultati }\end{array}$ & $\begin{array}{l}\text { Possibilità di ordinamento dei risultati di ricerca secondo vari criteri } \\
\text { che vanno dalla rilevanza, all'ordine alfabetico per autore o per titolo, } \\
\text { all'ordine per data di pubblicazione, al grado di popolarità all'interno } \\
\text { della comunità di utenti }\end{array}$ \\
\hline Arricchimenti & Sì \\
\hline Raccomandazioni & $\begin{array}{l}\text { Sì, tramite percorsi di ricerca che suggeriscono ricerche alternative } \\
\text { rilevanti rispetto alla ricerca impostata dall'utente. Primo offre servizi } \\
\text { di 'raccomandazioni' sia per gli item dal catalogo (generalmente libri) } \\
\text { grazie a sistemi di terze parti come Library Thing e BibTip, sia per i } \\
\text { record che si riferiscono ad articoli da riviste elettroniche. I questo } \\
\text { caso specifico il servizio di raccomandazioni presente in Primo e bX. } \\
\text { bX nasce da un progetto di ricerca condotto da Herber Van De Sompel } \\
\text { (l'inventore di SFX e dello standard OpenURL) e si basa sull'analisi } \\
\text { dei dati di utilizzo di utenti da tutto il mondo che, attraverso i link di } \\
\text { SFX in ogni sessione di lavoro "visitano" una serie di articoli correlati } \\
\text { gli uni agli altri. Tutte queste transazioni vengono tracciate nei file di } \\
\text { log dei server SFX e raccolte centralmente nel database di bX } \\
\text { mantenuto da Ex Libris. Le relazioni fra i vari articoli oggetto delle } \\
\text { transazioni effettuate vengono analizzate e attraverso meccanismi } \\
\text { molto sofisticati vengono messe in evidenza le relazioni più stringenti. } \\
\text { BX è un servizio opzionale. }\end{array}$ \\
\hline
\end{tabular}




\begin{tabular}{|c|c|}
\hline Tag clouds & No \\
\hline Community & Sì \\
\hline Interfacce visuali & No \\
\hline $\begin{array}{l}\text { Implementazione del } \\
\text { modello FRBR }\end{array}$ & $\begin{array}{l}\text { Primo dichiara di implementare il modello FRBR, per deduplicare i } \\
\text { dati contenuti in diverse fonti. }\end{array}$ \\
\hline \multicolumn{2}{|r|}{ Altre caratteristiche } \\
\hline $\begin{array}{l}\text { Tagging/commenti/ } \\
\text { recensioni/voti }\end{array}$ & Commenti, tagging \\
\hline Esportazione citazione & $\begin{array}{l}\text { È possibile esportare il riferimento URL dal proprio spazio personale } \\
\text { Primo dove conservare e organizzare i materiali di interesse oppure } \\
\text { inviarli ad altri spazi del web (connotea, de.li.cio.us, refworks, } \\
\text { endnote,etc...) }\end{array}$ \\
\hline Area personale & $\begin{array}{l}\text { Primo offre ad ogni utente che si autentichi sul sistema un'area } \\
\text { personale che contiene uno scaffale elettronico Inoltre, nell'area } \\
\text { personale l'utente può vedere l'elenco delle ricerche eseguite nella } \\
\text { sessione di lavoro attiva e lo storico delle ricerche eseguite nel } \\
\text { passato. Ogni ricerca eseguita può essere trasformata in alert o in } R S S \\
\text { così che il sistema fornisca automaticamente all'utente aggiornamenti } \\
\text { relativi a nuove informazioni aggiuntesi ai record recuperabili } \\
\text { attraverso quella determinata strategia di ricerca. }\end{array}$ \\
\hline Widgets & $\begin{array}{l}\text { Possibilità di Impostare Primo come search box nel proprio browser o } \\
\text { come motore di ricerca preferito. }\end{array}$ \\
\hline Chat & $\begin{array}{l}\text { h) Usare una chat per dialogare con lo staff della biblioteca } \\
\text { i) Usare strumenti come "Ask a Librarian" o un Help Desk virtuale } \\
\text { all'interno dell'ambiente di Primo }\end{array}$ \\
\hline Dispositivi mobili & Gli utenti possono utilizzare Primo attraverso palmari, IPhone e IPad \\
\hline \multicolumn{2}{|c|}{ Installazioni } \\
\hline \multicolumn{2}{|c|}{$\begin{array}{l}425 \text { istallazioni (ultimo aggiornamento } 13 \text { gennaio 2013) Tra gli altri si segnalano: Primo Ver.3: } \\
\text { Yonsey University; Vanderbilt University; Iowa University; Primo Ver.2: British Library; NLB } \\
\text { Singapore; Oxford University. In Italia: Università Ca' Foscari Venezia, Università di Verona, } \\
\text { Università di Camerino, Università di Macerata, Università di Torino e Biblioteca Europea di } \\
\text { Informazione e Cultura (BEIC). }\end{array}$} \\
\hline \multicolumn{2}{|r|}{ Riferimenti bibliografici /sitografia } \\
\hline \multicolumn{2}{|c|}{ 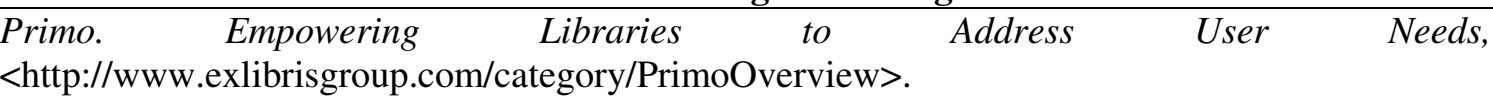 } \\
\hline & Immagini \\
\hline
\end{tabular}




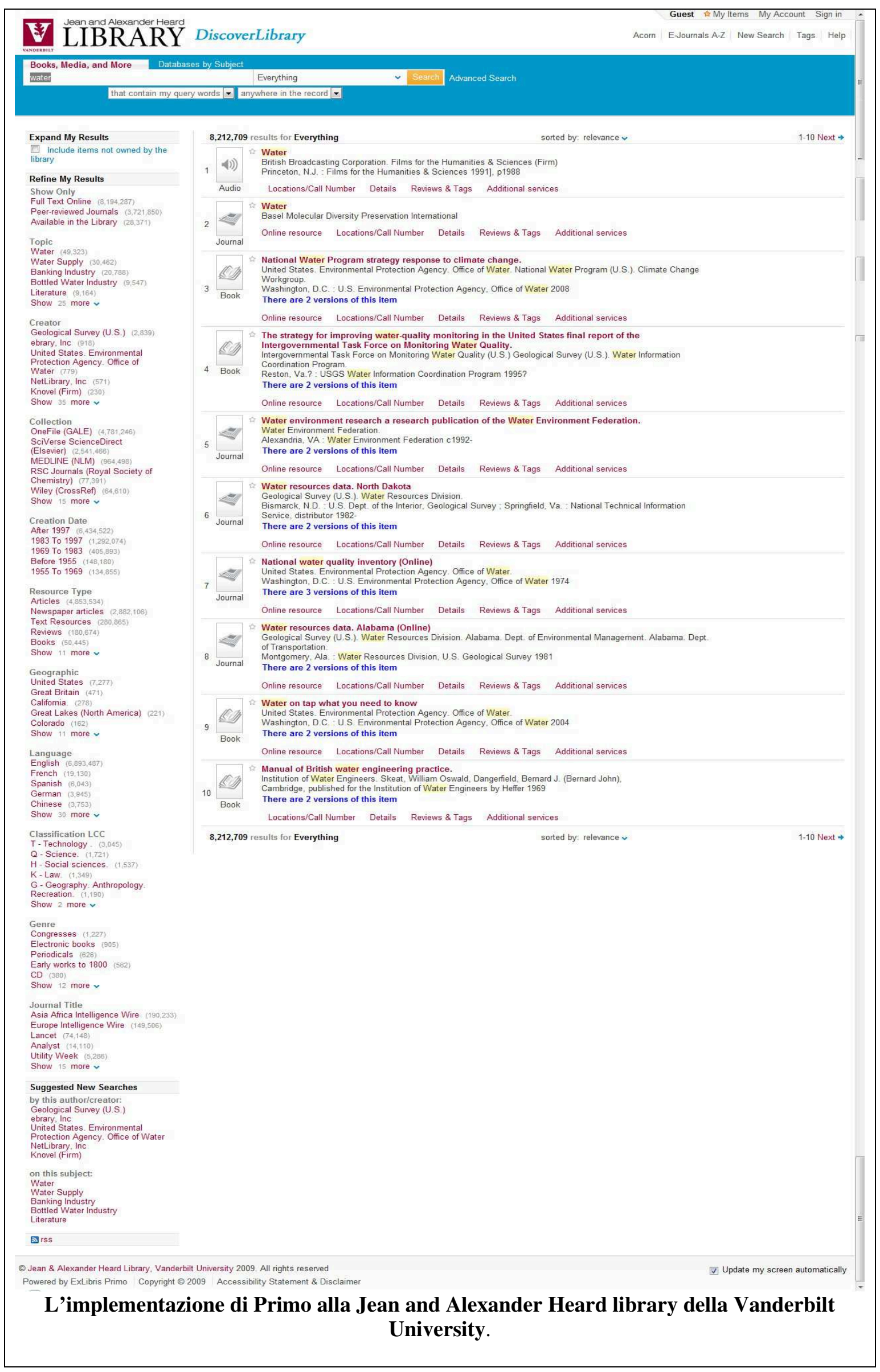




\section{DISCOVERY NG}

Discovery NG è un 'OPAC di nuova generazione' sviluppato dalla azienda italiana Comperio Basato sul sistema per la gestione di contenuti (Content Management System) open source SilverStripe per il quale Comperio ha sviluppato un modulo chiamato Liquens Discovery NG si presenta come un OPAC con integrato un engine di social network per aggregare gli utenti in una community web. Il prodotto permette di integrare in un unico portale OPAC e sito della biblioteca.

Il CMS facilita la pubblicazione e strutturazione dei contenuti sul web, senza la necessità di conoscere il linguaggio HTML o altri linguaggi di programmazione.

Il software può gestire il portale di più biblioteche: l'architettura di Discovery permette di gestire in maniera efficace la comunicazione sia a livello di rete che per ciascuna biblioteca. È possibile gestire eventi e news, pagine di presentazione dello staff e dell'orario, così come la pubblicazione di bibliografie grazie all'uso di appositi widget.

Il software si distingue per l'integrazione di funzioni sociali: gli utenti del portale hanno la possibilità di prenotare il materiale da casa, votare e recensire il catalogo, salvare ricerche $\mathrm{e}$ creare bibliografie personalizzate. Grazie alla presenza dei forum di discussione, utenti e bibliotecari possono contribuire alla costruzione della community online.

Il team prevede di sviluppare nei prossimi mesi all'interno dell'OPAC un 'plugin sociale' per la condivisione dei contenuti sui principali social network ${ }^{6}$.

\begin{tabular}{|l|l|}
\hline Sviluppatore/i & Comperio \\
\hline Sito web & $<$ http://www.comperio.it/> \\
\hline Licenza/costi & $\begin{array}{l}\text { DiscoveryNG è distribuito come software libero secondo la } \\
\text { GNU Affero General Public License version 3. }\end{array}$ \\
\hline Implementazione & $\begin{array}{l}\text { Basato su software open source (Silverstripe, Apache } \\
\text { Solr,YAML, jQuery, jQueryUI; LESS, Subtlepatterns, Fugue } \\
\text { icons per la grafica) }\end{array}$ \\
\hline
\end{tabular}

\begin{tabular}{|c|c|}
\hline \multicolumn{2}{|c|}{ Dati, database, architettura del software } \\
\hline ILS supportati & Supporto SBN \\
\hline Workflow dei dati & \\
\hline $\begin{array}{l}\text { Accesso a collezioni } \\
\text { digitali /e-journals }\end{array}$ & No \\
\hline $\begin{array}{l}\text { Integrazione con strumenti } \\
\text { di ricerca federata }\end{array}$ & No \\
\hline
\end{tabular}

Caratteristiche del motore / /

di ricerca

\begin{tabular}{|l|l|}
\hline Aiuti nella ricerca & Sì, autocompletamento \\
\hline Tipologie di ricerca & Box unico google like \\
\hline Tipologie di navigazione & A faccette \\
\hline Visualizzazione risultati & Ranking \\
\hline Arricchimenti & Copertine, sommario, allegati, dettagli, commenti, voti \\
\hline Raccomandazioni & No \\
\hline Tag clouds & Sì \\
\hline Community & Sì \\
\hline Interfacce visuali & Sì \\
\hline $\begin{array}{l}\text { Implementazione del } \\
\text { modello FRBR }\end{array}$ & No
\end{tabular}

\begin{tabular}{|l|l|} 
modello FRBR & \\
\hline & Altre caratteristiche
\end{tabular}

\begin{tabular}{l|l} 
Tagging/commenti/ & Tagging, commenti, recensioni, voti
\end{tabular}

${ }^{6}$ L'annuncio si trova nel blog di Comperio: <http://www.comperio.it/comperio-blog/condividereautomaticamente-le-proprie-recensioni-di-discoveryng-su-facebook-o-twitter-ecc/>. 


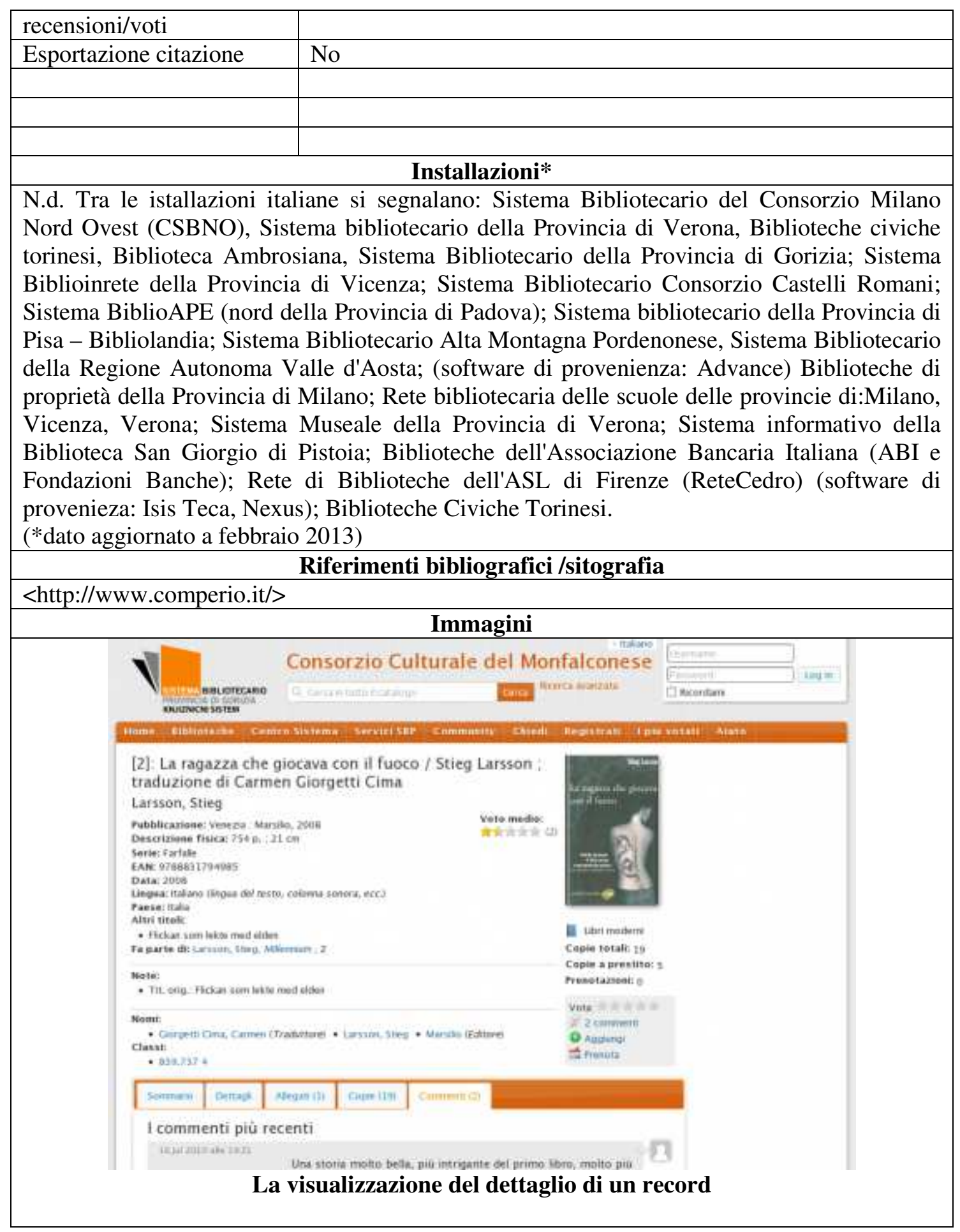




\section{VUFIND}

Il software VuFind è un software per la scoperta ('library resource portal') open source progettato per fornire un accesso unificato 'one stop shop' alle risorse delle biblioteche.

Scopo del progetto, sviluppato dalla Villanova University's Falvey Memorial Library e rilasciato in versione 1.0 nel luglio 2010, è la progettazione di un software in grado di sostituire l'OPAC tradizionale fornendo accesso ai record catalografici, collezioni digitali, repositories istituzionali, bibliografie ed altre risorse bibliotecarie.

VuFind è sviluppato in una serie di elementi modulari e dunque ampiamente flessibile e personalizzabile; essendo progettato con software open source consente di modificare o aggiungere componenti al sistema ed è facilmente integrabile in un sito web.

Il software è basato sul motore di ricerca Apache SOLR che consente la cosiddetta ricerca 'a faccette' e l'esposizione dei risultati per ranking; tra le altre caratteristiche: stato dei record e informazioni sulla disponibilità in tempo reale, i suggerimenti di lettura che vengono mostrati nel dettaglio del record, la possibilità di salvare e organizzare i risultati in liste personalizzate, le possibilità di browsing delle risorse.

I record sono arricchiti di biografie degli autori dinamicamente recuperate da Wikipedia e di altre informazioni contestuali e dotati di URL persistenti che li rendono utilizzabili e accessibili dalle pagine web.

Vi è la possibilità di creazione di liste e bibliografie da parte degli utenti ed esportare le citazioni in formati standard o in Zotero e altre applicazioni simili per la gestione di bibliografie grazie alla compatibilità con il protocollo CoinS per la gestione delle citazioni bibliografiche.

L'interfaccia può essere personalizzata in varie lingue (tra le quali Italiano, Inglese, Francese, Spagnolo, Portoghese Brasiliano, Cinese, Tedesco, Inglese, Francese, Olandese, Giapponese) con la possibilità di personalizzazione in nuove lingue e la possibilità di modificare le parole dell'interfaccia.

È possibile accedere ai dati di Vu Find attraverso Open Search, OAI, Solr. Vi è la possibilità di creare indici per scorrimenti personalizzati e di semplice personalizzazione grafica dell'interfaccia.

$\mathrm{Vu}$ Find può utilizzare contenuti esterni (es. copertine e recensioni, da GoogleBooks, OpenLibrary, Amazon e altri fornitori) e utilizzare API per l'integrazione con servizi remoti (es. con il servizio xISBN di OCLC per il reperimento di altre edizioni del documento visualizzato disponibili nel catalogo).

VuFind dispone di varie API per interagire con i moduli di ricerca e per estrarre i dati. È possibile operare la syndication dei dati (feed RSS) e utilizzare OpenSearch, OAI-PMH per impostare semplici collegamenti da cataloghi esterni, utilizzare gli algoritmi del software con OpenSearch o avere un accesso completo ai dati da indicizzare e gestire personalizzando il motore di ricerca Solr che gestisce la ricerca e l'indicizzazione dei dati.

Infine Vu Find si interfaccia con soluzioni commerciali per la scoperta come Summon, Ebsco discovery, Wordlcat.

L'ultima versione (VuFind 1.3) rilasciata il 30 gennaio 2012 ha introdotto varie novità: un rinnovato "book bag" (carrello della spesa) con varie funzionalità (save, email, export multiple records) e nuovi pug-ins opzionali (timeline visuale, integrazione con Google Maps ed Europeana), un miglioramento delle funzioni di syndication dei dati che consente di condividere i risultati di VuFind con servizi commerciali esterni e piattaforme come SciVerse di Elsevier e Syndetics.

\begin{tabular}{|l|l|}
\hline Sviluppatore/i & Villanova University Falvey Memorial Library. \\
\hline Sito web & $<$ http://vufind.org/> \\
\hline Licenza/costi & $\begin{array}{l}\text { Open source con licenza GNU General Public } \\
\text { License / liberamente scaricabile dal sito web dello } \\
\text { sviluppatore }\end{array}$ \\
\hline
\end{tabular}




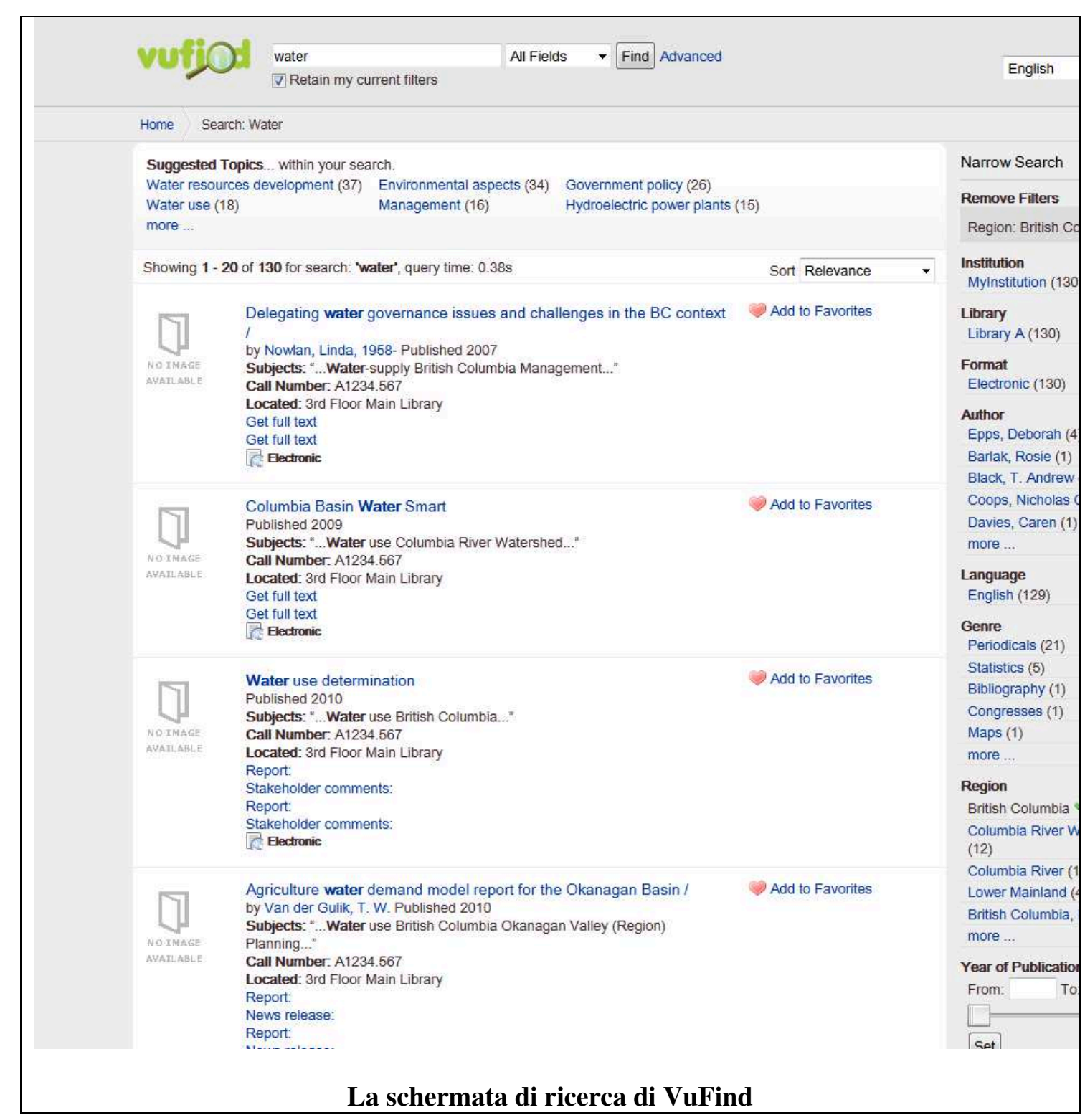




\section{WORLDCAT LOCAL}

WorldCat Local è stato il primo sistema di discovery creato allo scopo di sostituire il catalogo locale offrendo al contempo l'accesso ad una straordinaria quantità di contenuti esterni. Con WordlCat local le biblioteche possono offrire all'utente ò' accesso da una singola interfaccia a tutti i materiali della biblioteca e ai servizi correlati. Tra le risorse incluse vi sono quelle dei partners Google Books, HathiTrust, JSTOR e OAIster. Attraverso l'interfaccia di WorldCat Local gli utenti visualizzeranno prima il posseduto della singola biblioteca, per poi ampliare la ricerca all'intero catalogo globale WorldCat, con la possibilità di visualizzare il posseduto delle biblioteche che aderiscono al catalogo collettivo.

Tramite WorldCat Local è possibile collegarsi rapidamente i materiali che interessano e grazie all'integrazione con i servizi di fornitura locali, visualizzare cosa è immediatamente disponibile per l'accesso, accedere alla copia elettronica e attivare servizi di prestito e prenotazioni.

Grazie alla collaborazione con partner commerciali, la visualizzazione del dettaglio record si presenta particolarmente ricca (copertine, toc, recensioni etc). E poi possibile sfruttare la visualizzazione di WorldCat Identities per visualizzare la pagina relativa ad un autore, scoprendone i contributi ad una disciplina o ad un genere letterario, la fortuna editoriale etc grazie alla ricca pagina di informazioni del record di autorità. Attraverso WordlCat local le biblioteche possono disporre delle interfacce per dispositivi mobili e delle funzionalità di condivisione nei social network più diffusi.

L'abbonamento a WorldCat Local comprende l'accesso a circa 1,9 miliardi di record bibliografici relativi a libri, banche dati, articolo, ebooks, record di depositi istituzionali, tesi, dissertazioni, audio video, risorse web, materiali archivistici, spartiti etc.

\begin{tabular}{|l|l|}
\hline Sviluppatore/i & $\begin{array}{l}\text { Online Computer Library Center (OCLC), distributore per l'Italia } \\
\text { Ifnet srl. }\end{array}$ \\
\hline Sito web & <http://www.oclc.org/worldcatlocal/default.htm> \\
\hline Licenza/costi & A pagamento \\
\hline Implementazione & $\begin{array}{l}\text { Accesso tramite Web browser, collegamento all'ILS in tempo reale } \\
\text { tramite appositi connettori }\end{array}$ \\
\hline \multicolumn{2}{|l|}{ Dati, database, architettura del software } \\
\hline ILS supportati & Tutti i principali ILS \\
\hline Workflow dei dati & Collegamento all'ILS per i dati della circolazione \\
\hline $\begin{array}{l}\text { Accesso a collezioni } \\
\text { digitali /e-journals }\end{array}$ & $\begin{array}{l}\text { Le collezioni digitali possono essere importate in WCL tramite } \\
\text { harvesting dal catalogo collettivo WordCat.org. Gli e-journals } \\
\text { vengono importati tramite WordCat.org. Sono presenti 91,495 riviste } \\
\text { rappresentate, provenienti da varie fonti (ArticleFirst, MEDLINE, } \\
\text { ERIC, British Library Inside Serials, PapersFirst, ProceedingsFirst, } \\
\text { JSTOR, OAIster and Elsevier). } \\
\text { Circa 15 milioni di record provengono da repositoeires full text ad } \\
\text { accesso aperto (Google Books, OAIster, JSTOR, NDLTD: Networked } \\
\text { Digital Library of Theses and Dissertations). }\end{array}$ \\
\hline $\begin{array}{l}\text { Integrazione con } \\
\text { strumenti di ricerca } \\
\text { federata }\end{array}$ & Sì \\
\hline \multicolumn{2}{|l|}{ Ricerca nel catalogo / Visualizzazione dei record } \\
\hline $\begin{array}{l}\text { Caratteristiche del motore } \\
\text { di ricerca }\end{array}$ & Proprietario \\
\hline Aiuti nella ricerca & Auto suggerimenti, interfaccia multilingue \\
\hline Tipologie di ricerca & Google like, avanzata \\
\hline Tipologie di navigazione & A faccette \\
\hline Visualizzazione risultati & Ordinati per ranking \\
\hline Arricchimenti & Sì \\
\hline
\end{tabular}




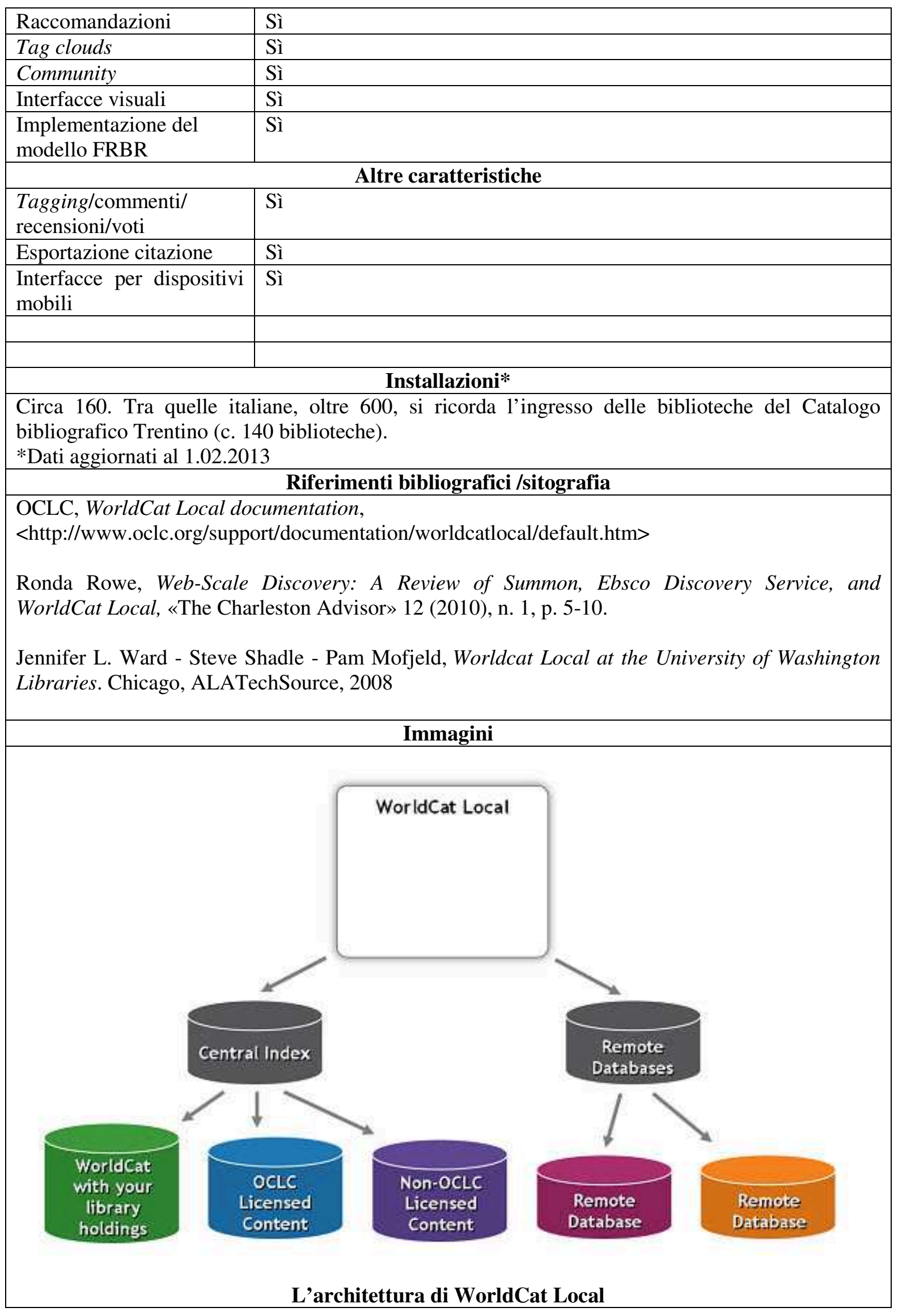





\section{APPENDICE C. PROGETTI DI IMPLEMENTAZIONE DI FRBR NEGLI OPAC. SISTEMI COMPLETI, PROTOTIPI, SOFTWARE}

La presente appendice fornisce una breve rassegna delle principali implementazioni di FRBR in OPAC e biblioteche digitali ${ }^{1}$.

\section{1) Sistemi completi}

\section{UCLA FILM AND TELEVISION ARCHIVE}

Sviluppatore: University of California at Los Angeles (UCLA)

URL: <www.cinema.ucla.edu>

«Documenting the 20th Century and beyond» si legge nella home page dell'OPAC di UCLA Film and Television Archive, uno dei più grandi repositories di film e produzioni per la televisione. The UCLA Film \& Television Archive è dopo la the Library of Congress, la più grande collezione di materiali multimediali negli Stati Uniti, con più di 220,000 titoli di film e produzioni per la televisione e 27 milioni di metri di cinegiornali. L'obiettivo è di conservare e rendere disponibile ogni sorta di materiale filmico e televisivo. Il sistema è pienamente operativo, è basato su FRBR e utilizza il software ILS Voyager. Il catalogo tratta i record di autorità come record per le opere, i record bibliografici come espressioni e i record relativi alle localizzazioni come record delle manifestazioni, implementando le entità del primo gruppo del modello.

\section{WORLDCAT.ORG}

Sviluppatore: Online Computer Library Center (OCLC)

URL: <http://worldcat.org>

WorldCat.org è il catalogo delle biblioteche appartenenti affiliate al consorzio OCLC. Con più di

1 L'elenco riprende la lista di implementazioni fornita da Zhang e Salaba (Yin Zhang - Athena Salaba, Implementing FRBR in libraries: key issues and future directions, New York, Neal-Schuman Publishers, 2009) ampliandola e aggiornandola per quanto riguarda i nuovi sviluppi e fornendo i principali riferimenti bibliografici. Un'altra utile lista delle implementazioni è fornita in <http://www.frbr.org/categories/implementations>. Per le implementazioni già descritte nell'appendice A. Casi di Studio si riporta in questa sede solo una breve descrizione. 
268 milioni di record e il contenuto di 10.000 biblioteche, è il più grande catalogo collettivo al mondo, contenente risorse di diversa tipologia («books, music, videos, digital audiobooks, article citations, documents and photos of historical note, and digital versions of rare items»). Il catalogo si basa su OCLC WorldCat ed è open access. Oggi WorldCat offre una serie di funzionalità avanzate 'di nuova generazione': navigazione a faccette, creazione e condivisione di bibliografie personalizzate, esportazione delle citazioni, tagging e altre funzioni sociali, applicazioni per dispositivi mobili, API, widgets, etc. Il catalogo utilizza OCLC Work-Set Algorithm per raggruppare automaticamente i record bibliografici in base all'entità opera del modello FRBR; per ogni opera è dunque possibile vedere un secondo livello delle manifestazioni collegate. WorldCat oggi si presenta come il più grande network di contenuti e servizi alle biblioteche.

Riferimenti bibliografici

Rick Bennett - Brian F. Lavoie, - Edward T. O'Neill, The Concept of a Work in WorldCat: An Application of FRBR. Library Collections, Acquisitions and Technical Services 27 (2003) n. 1, $<$ http://www.oclc.org/research/publications/archive/2003/lavoie_frbr.pdf>.

Edward T. O'Neill, FRBR: Application of the entity-relationship model to Humphry Clinker. «Library Resources and Technical Services», 46 (2002), n. 4, $<$ <ttp://www.oclc.org/research/publications/archive/2002/oneill_frbr22.pdf>

Thomas B. Hickey - Edward T. O'Neill - Jenny Toves, Experiments with the IFLA Functional Requirements for Bibliographic Records (FRBR), «D-Lib Magazine», 8, (2002) n. 9.

\section{2) Sistemi sperimentali e progetti}

\begin{tabular}{|c|}
\hline AUSLIT \\
\hline Sviluppatore: National Library of Australia \\
\hline URL: <www.austlit.edu.au> \\
\hline \multirow{5}{*}{$\begin{array}{l}\text { AusLit è un consorzio australiano di studiosi e bibliotecari nato per sviluppare progetti correlati alla } \\
\text { letteratura australiana. Il suo omonimo database ha implementato il modello FRBR per la } \\
\text { descrizione delle edizioni a stampa della letteratura australiana. Il software implementa le entità del } \\
\text { primo gruppo previste da FRBR compresa l'espressione (in Austlit denominata 'versione') per } \\
\text { organizzare una vastissima collezione di letteratura di autori australiani o su argomenti legati alla } \\
\text { cultura e alla storia australiana. L'entità item è rappresentata dalle localizzazioni delle biblioteche. }\end{array}$} \\
\hline \\
\hline \\
\hline \\
\hline \\
\hline Tra le funzionalità del catalogo la possibilità di visualizzare a partire da una ricerca per autore, le \\
\hline opere di cui è autore e le opere di cui è soggetto (works of e works by), la possibilità di selezionare \\
\hline il genere delle opere, con i riferimenti alle espressioni e manifestazioni. \\
\hline AustLit non è un catalogo 'pubblico' ed è accessibile previa sottoscrizione, infatti, si serve dei suoi \\
\hline
\end{tabular}


apporti per continuare ad aggiornare il database con nuove pubblicazioni.

AusLit ha arricchito il modello FRBR con l'entità eventi tratta dai modelli INDECS e ABC Harmony.

Riferimenti bibliografici

Kerry Kilner, The AustLit Gateway and scholarly bibliography: a specialist implementation of the FRBR in Functional Requirements for Bibliographic Records (FRBR): Hype, or Cure-All?edited by Patrick Le Boeuf, Binghamton, the Haworth Press, 2005. Pubblicato anche in «Cataloging \& Classification Quarterly», 39 (2005), n. 3-4. Abstract disponibile in $<\mathrm{http}: / /$ catalogingandclassificationquarterly.com/ccq39nr1-2.html>.

\section{FICTIONFINDER}

Sviluppatore: Online Computer Library Center (OCLC)

URL: <http://fictionfinder.oclc.org>

FictionFinder è un sistema sperimentale per le opere di narrativa che fornisce accesso a circa 2.9 milioni di record bibliografici relativi a libri, e-books e materiale audio provenienti dal database di OCLC WorldCat. Per raggruppare i documenti a livello dell'opera, FictionFinder si serve di OCLC Work-Set Algorithm. Caratteristica del catalogo è la possibilità di cercare oltre che tramite i punti di accesso tradizionali (autore, titolo, soggetto) anche per protagonisti, premi letterari, e sommari. I risultati sono ordinati in base all'opera e per ogni opera viene visualizzato un 'livello' che risulta una combinazione delle entità espressione e manifestazione. Filtri di raffinamento consentono di riordinare il set di risultati per lingua e per formato o in base ad altri attributi. Al momento ${ }^{2}$ l'interfaccia di Fiction Finder non è utilizzabile e si fa riferimento alla nuova interfaccia WorldCat Genres $^{3}$, che consente di ricercare i documenti per genere letterario.

\section{Riferimenti bibliografici}

Diane Vizine-Goetz, FictionFinder: a FRBR works-based prototype. In Back to the future, ALCTS Preconference, ALA Annual Meeting, Orlando, June 25, 2004. [S. 1.], ALA, 2004, $<$ http://www.ala.org/ala/alcts/alctsconted/presentations/VizineGoetz.pdf>.

Diane Vizine-Goetz, FictionFinder: Imagining What a "Next Generation" Catalog Might Look Like. Presentation given at the OCLC Members Council Research and New Technologies Interest Group meeting, 6 February 2007, Québec, QC (Canada) e Dublin, Ohio (USA), 2007,

\footnotetext{
${ }^{2}$ Ultima consultazione il 30 aprile 2012.

${ }^{3}<$ http://www.worldcat.org/genres/>. Le principali categorie ricercabili sono: Adventure, Horror, Occult, Spy Stories, Fantasy, Legends, Love \& Romance, War Stories, Historical Mystery, Science Fiction, Westerns.
} 
<http://www.oclc.org/research/memberscouncil/2007-02/vizine-goetz.pdf>.

\section{LIBRARY LABS OF LIBRARIES AUSTRALIA}

Sviluppatore: National Library of Australia, National Australian Bibliographic Database

URL: <http://1101.nla.gov.au>

Libraries Australia FRBR prototype è un sistema sperimentale definito dagli sviluppatori come sistema 'FRBR-like'. Il sistema si basa sulla possibilità di cercare tra i record bibliografici in formamo MARC usando Lucene per il recupero e l'archiviazione dei dati. La base dati di riferimento è Australian National Bibliographic Database (ANBD) che contiene circa 16 milioni di record bibliografici posseduti dalle biblioteche australiane. Il sistema (denominato Demostrator) estrae le entità e le relazioni dai record che vengono recuperati tramite una ricerca full-text e li restituisce ordinati per rilevanza, raggruppati per data, soggetto (in forma gerarchica o a faccette), forma, genere, autore, Dewey o Classificazione LC. Il sistema è anche in grado di proporre suggerimenti, che derivano dal matching con gli accessi per soggetto, e di offrire suggerimenti dello spelling (che dal 2008 è stato sostituito da Yahoo). I record correlati vengono mostrati in una struttura «FRBR-like» e accresciuti con record provenienti da OCLC (usando OCLC's XISBN service) arricchiti dalle ratings e dalle recensioni di Amazon, mostrando i link agli OPAC delle biblioteche collegate per le localizzazioni. È possibile selezionare da subito tra le risorse online ${ }^{4}$. Gli items sono raggruppati prima per tipologia (i.e. book, musical score, film, etc.), poi per lingua, per anno di pubblicazione ed editore. Una caratteristica interessante e originale è il raggruppamento in base all'entità super-opera.

Il sistema si propone ancora oggi come interfaccia sperimentale dunque non supporta l'integrazione con un gestionale (ILS).

Riferimenti bibliografici

National Library of Australia, Lucene NBD Home Prototype, [Canberra], National Library of Australia, 2006, <http://1101.nla.gov.au/index.jsp>.

\begin{tabular}{|l|}
\hline \\
\hline \\
\hline PERSEUS DIGITAL LIBRARY \\
\hline Sviluppatore: Tufts University \\
\hline URL: <www.perseus.tufts.edu/hopper $>$ \\
\hline
\end{tabular}

\footnotetext{
${ }^{4}$ Funzione che gli sviluppatori hanno giudicato particolarmente importante.
} 
Progettato a partire dal 1985 Perseus Digital Library, oggi giunto alla versione 4.0, nota anche come Perseus Hopper, è una biblioteca interamente digitale di testi classici ordinati gerarchicamente in base alle entità FRBR del primo gruppo. Attualmente contiene varie collezioni digitali (ricercabili anche separatamente: Greek and Roman Materials, Art \& Archaeology Artifact, Arabic Materials, Germanic Materials, 19th-Century American, Renaissance Materials, Richmond Times Dispatch, Humanist and Renaissance Italian Poetry in Latin) e descrizioni di una vastissima collezione di oggetti digitali (monete, vasi, sculture, gemme, monumenti). I numerosi testi classici e gli oggetti vengono esposti in FRBR secondo le entità opera, espressione e manifestazione. Alcuni record relativi alle collezioni contengono opere diverse, dunque il catalogo pone l'accento sullo sviluppo delle relazioni 'intero-parte' tra opere, espressioni e manifestazioni. I progettisti di Perseus hanno sottolineato la necessità di identificatori univoci per le entità di FRBR es. le opere. L'interfaccia è interrogabile online dal 2005.

Riferimenti bibliografici

Alison Babeu, Building a "FRBR-Inspired" Catalog: The Perseus Digital Library Experience, [S. 1.], Perseus Digital 1/31/2008, $<$ http://www.perseus.tufts.edu/ ababeu/PerseusFRBRExperiment.pdf>.

David Mimno - Gregory Crane - Alison Jones, Hierarchical catalog records: implementing a $\begin{array}{llllll}\text { FRBR catalog. } & \text { «D-Lib }\end{array}$ $<$ http://www.dlib.org/dlib/october05/crane/10crane.html>.

\section{KENT STATE UNIVERSITY FRBR PROJECT}

Sviluppatore: Kent State University School of Library and Information Science

URL: <http://frbr.slis.kent.edu> (non più raggiungibile)

The Kent State University FRBR Project è un progetto dell'omonima università nato per sviluppare un catalogo open source e basato sul modello FRBR.

Il progetto "FRBR-Based Systems to Effectively Support User Tasks and Facilitate Information Seeking” è stato condotto dal 2006 al 2010 da Yin Zhang e Athena Salaba, professori alla School of Library and Information Science at Kent State University.

Scopo del progetto è stato quello di valutare i sistemi esistenti che implementavano FRBR alla luce delle funzioni utente e della ricerca dell'informazione e di costruire un catalogo basato su FRBR basato su un'indagine sull'utenza, uno studio Delphi che è stato riassunto nel contributo Zhang Salaba (2009). La collezione usata per progettare il sistema contiene record bibliografici e di autorità provenienti dai record della Library of Congress records presenti in OCLC WorldCat (circa 13 milioni di record bibliografici e oltre 7 milioni di record di autorità) e utilizza il software open source Koha. 
Riferimenti bibliografici

Yin Zhang - Athena Salaba, What is Next for FRBR? A Delphi Study, «The Library Quarterly», vol. 79 (2009), n. 2, p. 233-255.

\section{VARIATIONS [POI ] SCHERZO (VARIATIONS/FRBR TEST CATALOGUE)}

Sviluppatore: Indiana University

URL: <http://variations2.indiana.edu> [poi] <http://www.dlib.indiana.edu/projects/vfrbr/ projectDoc/admin/news.shtml>

Il software Variations, in uso dal 1996 all'Indiana University è un sistema open source progettato su un modello entità-relazione, che si ispira al modello FRBR. Nel 2005 fu pubblicata la seconda versione (Variations 2) e nel 2009 la terza versione (Variations 3) ${ }^{5}$.

La base per i test è una collezione di audio video, registrazioni, anche in forma elettronica provenienti dall'Indiana University Cook Music Library ad uso dell'università di Bloomington.

Nel 2008 è stato finanziato un nuovo progetto triennale di evoluzione del software ${ }^{6}$ (IMLS-funded Variations/FRBR project) che ha avuto come principale obiettivo l'adeguamento al modello FRBR e che ha condotto alla nuova interfaccia Scherzo pubblicata nel settembre del 2010 accessibile all'indirizzo <http://vfrbr.info/search>. L'obiettivo è progettare e mostrare come potrebbe presentarsi un catalogo aderente al modello concettuale FRBR. I record contenuti da Indiana University Digital Music Library sono più di 80,000 costituiti da registrazioni sonore provenienti dalla William and Gayle Cook Music Library in the Jacobs School of Music dell'Indiana University.

Nel novembre 2010 è stata rilasciata la versione in XML Schema per i dati $\mathrm{FRBR}^{7}$ e dall'agosto 2011 Variations/FRBR project espone i suoi dati sotto forma di linked data, ha rilasciato il codice sorgente e condotto test di usabilità per introdurre miglioramenti alle funzioni di ricerca.

\footnotetext{
${ }^{5}$ Questa terza versione è stata pubblicata in open source in <http://variations.sourceforge.net/>

${ }^{6}$ Gli obiettivi del progetto Variations/FRBR:

«- Convert the production Variations digital music library system deployed at Indiana University to use a FRBR-compliant data model;

- Create and release publicly a FRBRization algorithm for MARC records for musical scores and recordings, focusing particularly on appropriate handling of multi-Work Manifestations;

- FRBRize existing MARC records for all score and recording holdings in the IU William and Gayle Cook Music Library (approximately 80,000 bibliographic records for audio recordings and 105,000 records for scores), and load them into the Variations system;

- Make FRBRized records available for community use via OAI-PMH, SRU, and batch download;

- Design and implement a new, openly-accessible search interface for discovering FRBRized data;

- Design and implement a new cataloging system for FRBRized data that takes advantage of the distinction between the FRBR entities yet supports efficient data entry, and;

- Perform usability testing on the new end-user and cataloger interfaces to evaluate their effectiveness».
} 
Riferimenti bibliografici

Jenn Riley, Variations as a Testbed for the FRBR Conceptual Model, «D-Lib Magazine», 14 (2008), n. 11/12, <http://www.dlib.org/dlib/november08/11inbrief.html\#RILEY>.

Juliet L. Hardesty, Steven Harris, Anna Coogan, and Mark Notess, Scherzo Usability Test Report: Testing a FRBR Search Interface for Music. Digital Library Program Indiana University, January 3 ,

$<$ http://www.dlib.indiana.edu/projects/vfrbr/projectDoc/usability/usabilityTest/ScherzoUTestRepo rt.pdf>.

\section{PARADIGMA}

Sviluppatore: National Library of Norway

URL: (non più disponibile)

Il progetto Paradigma è stato applicato ad una collezione di documenti digitali provenienti dal deposito legale Norwegian Legal Deposit Act. Lo scopo del software era di costituire una piattaforma basata su FRBR applicata ai documenti internet. In particolare il progetto ha lavorato sull'adattamento delle entità FRBR manifestazione e item per renderle compatibili con i documenti web, che presentano particolari problematiche dovute alla volatilità di questi documenti.

Riferimenti bibliografici

Ketil Albertsen - Carol Van Nuys, Paradigma: FRBR and Digital Documents, «Cataloging \& Classification Quarterly», 39 (2005), n. 3/4, p. 125-49.

Carol Van Nuys- Ketil Albertsen - Linda Pedersen- Asborg Stenstad. Paradigma Project: il deposito legale delle risorse remote nell'esperienza norvegese. Una ricerca di soluzioni per metadati e servizi agli utenti, «Biblioteche oggi», 2005 n. 1, p. 17-28.

\section{ABC HARMONY}

Sviluppatore: Cornell Digital Library Research Group, USA; Distributed Systems Technology Centre, Australia; Institute for Learning and Research Technology UK

URL: <www.ilrt.bris.ac.uk/discovery/harmony>

The Harmony Project è un progetto internazionale finanziato da DSTC, JISC, and NSF per indagare le problematiche relative alla descrizione di documenti multimediali nelle biblioteche

\footnotetext{
${ }^{7}$ Versione 1.1 di XML Schema progettata per la rappresentazione dei dat i FRBR in XML. L'annuncio su: $<$ http://vfrbr.info $>$
} 
digitali. Le relazioni presenti nelle collezioni di testi, immagini, audio e video comprendono elementi spaziali, temporali, strutturali e semantici di cui si deve dar conto. Il progetto mira alla collaborazione tra comunità che producono metadati ${ }^{8}$, come archivi e musei, per sviluppare un modello concettuale che supporti l'interoperabilità tra i diversi domini e comunità, utilizzando anche gli strumenti del Web Semantico. Altri riferimenti su $<$ www.metadata.net/harmony/ABCV2.htm>

Riferimenti bibliografici

Bearman D., G. Rust, et. al. A Common Model to Support Interoperable Metadata, «D-lib Magazine», January 1999.

\section{3) Software}

\section{VISUALCAT}

Sviluppatore: Portia

URL: <www.portia.dk/websites/productgallery.htm>

VisualCat è un software per la catalogazione basato su RDF e FRBR in grado di gestire i dati bibliografici in una visualizzazione basata sul modello FRBR.

Il software viene descritto come un modulo OPAC in grado di integrarsi con altri applicativi ILS e particolarmente progettato per cataloghi collettivi. Dotato di flessibilità, gestisce differenti formati e linguaggi (differenti formati MARC tramite mappatura MARC/XML, collegamento a risorse RDF, ricerca e recupero tramite Z39.50 e supporto Microsoft.net per agire sul Web, supporto Unicode. Il software è stato sviluppato a partire dal 2002 intorno all'idea di implementare RDF e FRBR servendosi degli strumenti allora disponibili. I formati MARC vengono gestiti tramite schemi XML, la validazione è affidata a schemi XML e XSL(danMARC2 XML Schema) I record di autorità sono gestiti tramite RDF Schemas (<http://www.w3.org/TR/rdf-schema/>) Vari metadati (come Dublin Core e FRBR) vengono combinati in un authority file in RDF. Al progetto parteciparono British library e la biblioteca nazionale di Roma. Lo sviluppo del software si è fermato al 2005.

Riferimenti bibliografici

Poul Henrik Jørgensen, RDF and FRBR applications in Semantic Web and Libraries, 26 Library

\footnotetext{
${ }^{8}$ Il progettoHarmony prende a riferimento per il suo sviluppo una serie di iniziative e progetti where Harmony research

Dublin Core Metadata Initiative, MPEG-7, Open Archives Initiative, RDF, RSS.
} 
Systems Seminar, Rome, 17-19 April 2002 / European Library Automation Group (ELAG). Roma, $\begin{array}{lllll}\text { Biblioteca nazionale } & \text { centrale } & \text { 2002], }\end{array}$ $<$ http://www.portia.dk/pubs/elag/RdfAndFrbrApplications.pdf>.

Poul Henrik Jørgensen, RDF, IFLA FRBR and XML in VisualCat client, 2000, $<$ http://www.portia.dk/pubs/AccessY2K/VisualCatRdf/VisualCatRdf.htm>.

\section{THE EXTENSIBLE CATALOG(XC)}

Sviluppatore: University of Rochester Libraries

URL: <www.extensiblecatalog.org>

Tra i progetti di questa rassegna, The eXtensible Catalog (XC) è il più recente. Sviluppato dalle biblioteche dell'University of Rochester River Campus e finanziato da Andrew W. Mellon Foundation, XC fornisce una piattaforma per metadati "estendibile" (da qui il nome EXtensible Catalog) e in grado di supportare più schemi che possono essere cercati simultaneamente e supporta le funzionalità per una navigazione 'FRBR-like'.

Definito un software di nuova generazione, open source e user-centered, permette di trasformare i dati MARC in linked data e visualizzarli in un sistema per la scoperta a faccette che implementa FRBR e RDA per le sole entità del primo gruppo.

Sviluppato per gestire il materiale tradizionale e digitale, il software si integra con altri strumenti bibliotecari per la metaricerca ed è strutturato in diversi moduli che possono essere implementati anche separatamente (Drupal Toolkit XC discovery user interface gestisce l'interfaccia del software, Metadata Services Toolkit Tools per elaborare i metadati, più altri due strumenti che consentono la connettività con l'ILS sottostante: OAI Toolkit sincronizza i metadati MARC provenienti dagli ILS con XC, NCIP Toolkit importa i dati gestionali dell'ILS).

Particolarmente importante nello sviluppo del sono stati i test di usabilità svolti durante la progettazione per garantire un software progettato in base alle necessità degli utenti.

\section{Riferimenti bibliografici}

Nancy Fried Foster - Katie Clark - Kornelia Tancheva - Rebekah Kilzer, Scholarly Practice, Participatory Design and the eXtensible Catalog, Association of College and Research Libraries. Division of the American Library Association, Chicago, 2011.

Nancy Fried Foster, Ryan RANDALL, Designing the Academic Library Catalog: A Review of Relevant Literature and Projects, 2007 <http://hdl.handle.net/1802/8409>. 


\section{LIBDB}

Sviluppatore: Morbus (pseudonimo di Hemenway, Kevin)

URL: <http://sourceforge.net/projects/libdb>

LibDB è un software open source per biblioteche ispirato ad FRBR e RDF che supporta la catalogazione di varie tipologie di risorse. Il software, sviluppato a partire dal 2004, è ancora registrato sulla piattaforma Sourceforge.net, ma il progetto non è più attivo dal 2007.

\section{REDLIGHTGREEN}

Sviluppatore: Research Libraries Group (now part of OCLC)

URL: non più disponibile

Oggi non più attivo, RedLightGreen è stato il primo sistema basato su FRBR. Il software creato dal Research Libraries Group (RLG) ${ }^{9}$ era nato per testare l'applicazione di FRBR ai record catalografici, ma permetteva anche di creare citazioni dalle pubblicazioni recuperate.

Dal 1 luglio del 2006 RLG è stato acquisito da OCLC ed è stato annunciato che il software sarebbe stato rimpiazzato da WorldCat (Worldcat.org). RedLightGreen implementava le entità del gruppo 1 ma solo parzialmente. Opere ed espressioni erano fuse in un solo livello per creare insiemi titolo.

Riferimenti bibliografici

Merrilee Proffitt, RedLightGreen: FRBR between a rock and a hard place in Back to the future, ALCTS Preconference, ALA Annual Meeting, Orlando, June 25, 2004, [S. 1.]: ALA, 2004 (non più disponibile online)

\footnotetext{
${ }^{9}$ The Research Libraries Group (RLG) è un consorzio di biblioteche statunitensi che ha sviluppato progetti di un certo rilevo come Eureka interlibrary search engine, RedLightGreen, database bibliografico e ArchiveGrid, un database di descrizioni archivistiche. Fondato da un gruppo di biblioteche di ricerca (Columbia, Harvard and Yale and the New York Public Library) nel 1974, due anni dopo la nascita di OCLC come alternativa e in opposizione alla gestione dei record da parte di OCLC, RLG aveva creato il catalogo unico RLIN (Research Libraries Information Network). Nel giugno 2006 RLG si è fusa con OCLC e il suo catalogo è stato inglobato in OCLC WorldCat. (Per l'annuncio si deda: $<$ http://www.oclc.org/news/releases/200618.htm>)I progetti di ricerca sono oggi gestiti dal nuovo gruppo OCLC Programs and Research.
} 


\section{VIRTUA INTEGRATED LIBRARY SYSTEM}

Sviluppatore: VTLS (Visionary Technology in Library Solutions)

URL: <www.vtls.com/products/virtua>

Virtua è un sistema di gestione per biblioteche che consente l'integrazione di record basati su FRBR con i record tradizionali. A seconda del record cui accede, il sistema può mostrare una visualizzazione gerarchica delle entità di FRBR. Il software offre anche un'opzione Software as a Service (SaaS) per la visualizzazione FBRR che può essere linkata dal catalogo verso un server VTLS. Una lista di presentazioni del software è disponibile in: $<$ http://www.vtls.com/Corporate/FRBR.shtml>.

Riferimenti bibliografici

VTLS Inc. VTLS announces first production use of FRBR, Blacksburg, VA: VTLS Inc., January 07, 2004, <http://www.vtls.com/Corporate/Releases/2004/6.shtml>.

\section{4) Algoritmi e strumenti per data FRBR-zation}

\section{BIBSYS}

Sviluppatore: Norwegian National Library, Norwegian University of Science and Technology, Library of Norway

URL: <www.bibsys.no>

BIBSYS è un sistema sperimentale progettato dall'agenzia norvegese Bibsys. Scopo del progetto di ricerca è stato indagare i metodi per applicare FRBR ai dati MARC. Esito del progetto è stato un tool per estrarre gli elementi FRBR dai record MARC.

Riferimenti bibliografici

Trond Aalberg- Ole Husby - Frank Berg Haugen, A tool for converting from MARC to FRBR. in Julio Gonzalo, Costantino Thanos, M. Felisa Verdejo and Rafael C. Carrasco Research and Advanced Technology for Digital Libraries. 10th European Conference, ECDL 2006, Alicante, Spain, September 17-22, 2006. Proceedings, «Lecture Notes in Computer Science», 4172 (2006), p. 453-456.

Trond Aalberg, From MARC to FRBR: a case study in the use of the FRBR model on the BIBSYS database in Bibliotheca Universalis: How to organize chaos? 11-12 August 2005 [S. 1.]: Finnish Library Association, 2005 (non più disponibile). 


\section{IFPA (ISIS FRBR PROTOTYPE APPLICATION)}

Sviluppatore: Roberto Sturman, Università di Trieste

URL: <http://pclib3.ts.infn.it/frbr/wwwisis/FRB2.01/FORM.HTM>

IFPA (ISIS FRBR Prototype Application) è un tool FRBR frutto di un esperimento condotto presso

il Dipartimento di Fisica dell' Università di Trieste e coordinato da Roberto Sturman.

Scopo del software è stata l'implementazione del modello FRBR per l'ambiente software CDS/ISIS usato dalla biblioteca. L'applicativo è in grado di gestire le entità e le relazioni e la più recente versione del software IFPA2 rilasciata nel luglio 2008 serve come applicativo per il software UNESCO ISIS ISIS. Una dimostrazione è visibile nella pagina del progetto. I moduli per la gestione di FRBR sono distribuiti come open source sotto licenza GNU GPL.

Riferimenti bibliografici

[Roberto Sturman], IFPA (ISIS FRBR Prototype Application) home page [online]. [Trieste, Italy]: [Physics Department Library, University of Trieste], ultimo aggiornamento 23.11.2004, $<$ http://pclib3.ts.infn.it/frbr/FRBR.htm>.

Roberto Sturman, Implementing the FRBR conceptual approach in the ISIS software environment: IFPA (ISIS FRBR Prototype Application). In Patrick LE BCUF, Functional Requirements for Bibliographic Records (FRBR): Hype, or Cure-All?, Binghamton, The Haworth Press, 2005. Pubblicato anche in «Cataloging \& Classification Quarterly», 39 (2005), n . 3-4. Abstract in $<$ http://catalogingandclassificationquarterly.com/ccq39nr1-2.html $>$ Full text in $<$ http://pclib3.ts.infn.it/frbr/CCQ-IFPA.pdf>.

\section{FRBR DISPLAY TOOL}

Sviluppatore: Network Development and MARC Standards Office -Library of Congress

URL: <www.loc.gov/marc/marc-functional-analysis/tool.html>

FRBR Display Tool è un'applicazione creata dal Network Development and MARC Standards Office Della Library of Congress che consente di creare visualizzazioni basate su FRBR in base ai dati estratti dai record MARC. Il progetto prende avvio dalla pubblicazione nel 2001 da parte del Network Development and MARC Standards Office del documento Displays for Multiple Versions from MARC 21 and FRBR", che indicava come FRBR potesse essere utilizzato per visualizzare i record bibliografici, e forniva alcuni esempi pratici. 
FRBR Display Tool si basa su quell'analisi ed è un programma in XSLT che trasforma i dati bibliografici dei record MARC in visualizzazioni significative in base alle entità del gruppo 1 di FRBR. The FRBR Display Tool ordina e combina set di record bibliografici usando il modello FRBR e generando visualizzazioni gerarchiche di opere. Gli sviluppatori ritengono lo strumento flessibile e adattabile alle necessità di ogni tipologia di biblioteche, in output si possono avere più o meno informazioni modificando il foglio di stile esterno in XSL che controlla la visualizzazione senza modificare il programma. Così il programma può essere adottato dalle biblioteche per sperimentare una visualizzazione del catalogo secondo FRBR, raggruppando i record secondo le entità di FRBR senza dover apportare modifiche ai record catalografici. Il programma è giunto alla versione 2.0. Una lista di discussione (MARCFRBR electronic discussion list) è stata creata allo scopo di fornire uno spazio di discussione sugli esperimenti e le implementazioni dell'applicazione.

\section{Riferimenti bibliografici}

Miao Jin -Nashaat I. Sayed, FRBR-izing library collections in Mississippi Library Association [2004] Conference Poster Session Abstracts, Jackson, MLA, 2004, $<\mathrm{http} / / /$ www.misslib.org/activities/conf/poster.php>.

Sally McCallum, The FRBR Tool of the Library of Congress, in Bibliotheca Universalis: How to organize chaos? 11-12 August 2005, [S. 1.], Finnish Library Association, 2005, $<$ http://www.fla.fi/frbr05/McCallumHelsinki_frbrtool.pdf $>$.

Jackie RADEBAUGH -Corey KEITH, FRBR Display Tool in Patrick LE BEUF, Functional Requirements for Bibliographic Records (FRBR): Hype, or Cure-All?, Binghamton, The Haworth Press, 2005. Pubblicato anche in Cataloging \& Classification Quarterly, 39 (2005), n. 3-4. ISSN $<$ http://catalogingandclassificationquarterly.com/ccq39nr1-2.html>.

\section{OCLC WORK-SET ALGORITHM}

\section{Sviluppatore: Online Computer Library Center (OCLC)}

URL: <www.oclc.org/research/software/frbr/default.htm>

OCLC Work-Set Algorithm fa parte di un insieme di progetti che OCLC Research ha sviluppato per l'implementazione di FRBR nel catalogo $\operatorname{WorldCat}^{10} \mathrm{e}$ viene presentato come un algoritmo in grado di convertire i dati bibliografici di database bibliografici MARC21 secondo il modello FRBR. In particolare il software è stato sviluppato per esplorare la possibilità di un processo automatico di data FRBRzation come base per implementare su larga scala sistemi basati su FRBR. L'algoritmo utilizza sia i record di autorità sia i record bibliografici per raggruppare i record catalografici a

\footnotetext{
${ }^{10}$ FictionFinder, xISBN e progetti di indagine su casi di studio: Case Study: The FRBRization of Humphry Clinker; Extending the Case of Clinker, Work Records in WorldCat.
} 
livello dell'opera. Per far ciò ci si è serviti di un caso di studio, un'opera particolarmente diffusa e complessa, allo scopo di comprendere le relazioni tra i record bibliografici e gli oggetti bibliografici che essi rappresentano e per valutare se l'informazione disponibile nei record bibliografici sia sufficiente ad identificare tutte le entità previste dal modello.

L'attività sviluppo del software è ormai cessata, ma rimane consultabile la pagina del progetto all'indirizzo <www.oclc.org/research/software/frbr/default.htm>.

Riferimenti bibliografici

OCLC, OCLC Work-Set Algorithm <www.oclc.org/research/software/frbr/default.htm>.

\begin{tabular}{|l|}
\hline \multicolumn{1}{|c|}{ FRBR FLOATER } \\
\hline Sviluppatore: Monte Sano Associates \\
\hline URL: <www.montesanoassociates.com/apps-msafrbr.htm> \\
\hline $\begin{array}{l}\text { FRBR Floater è un servizio accessibile alle biblioteche tramite sottoscrizione. Il software offre la } \\
\text { possibilità agli utenti di vedere in fase di navigazione in una finestra pop-up le diverse edizioni di } \\
\text { un'opera, e di fare browsing in una lista strutturata in base alle opere e manifestazioni. }\end{array}$ \\
\hline
\end{tabular}

\section{OPENFRBR}

Sviluppatore: William Denton

URL: <www.openfrbr.org>

OpenFRBR è un software open source che consente una parziale implementazione del modello FRBR.

\section{TOOL FOR CONVERTING BIBLIOGRAPHIC RECORDS}

Sviluppatore: Norwegian National Library, Norwegian University of Science and Technology, Library of Norway

URL: <www.ercim.org/publication/Ercim_News/enw66/aalberg.html>

Lo sviluppo di questo strumento di conversione è collegato al progetto BIBSYS, avviato dalla biblioteca nazionale norvegese implementare il modello FRBR al proprio catalogo. Lo strumento effettua una conversione completa dei record MARC per elencare entità, attributi e relazioni dei record bibliografici. 


\section{BIBLIOGRAFIA ${ }^{1}$}

Jeppe Lomholt AKSELBO - Lise ARNFRED, Sten BARFORT [et al.], The hybrid library: from the users' perspective: a report for the DEFF Project "The loaners" expectations and demands for the hybrid library. February 2006, National Library and Copenhagen University Library, Aarhus School of Business Library, State and University Library, and University Library of Southern Denmark, 21 September 2006, $<$ http://www.statsbiblioteket.dk/summa/fieldstudies.pdf/view>.

Vilma ALBERANI - Elisabetta POLTRONIERI, Documentazione e scienza dell'informazione: interazioni con la biblioteconomia, «Bollettino AIB», 43 (2003), n. 2, p. 189-211.

Dean ALLEMANG - James HENDLER, Semantic web for the working ontologist, Morgan Kaufmann Publishers - Elsevier, 2008.

AMERICAN LIBRARY ASSOCIATION - CANADIAN LIBRARY ASSOCIATION, CHARTERED INSTITUTE OF LIBRARY AND INFORMATION PROFESSIONALS (GREAT BRITAIN)- JOINT STEERING COMMITTEE FOR DEVELOPMENT OF RDA, RDA: resource description \& access, Chicago, American Library Association, 2010.

Chris ANDERSON, The long tail, «Wired», 12 (2004) n. 10, $<$ http://www.wired.com/wired/archive/12.10/tail.html>.

Chris ANDERSON, La coda lunga. Da un mercato di massa a una massa di mercati, trad. it. di Susanna Bourlot, Torino, Codice, 2007.

Paul ANDERSON, What is Web 2.0? Ideas, technologies and implications for education, JISC tech watch report (Joint Information Systems Committee-UK), february 2007. $<$ http://www.jisc.ac.uk/media/documents/techwatch/tsw0701b.pdf>.

\footnotetext{
${ }^{1}$ Tutte le pagine web sono state controllate l'ultima volta il 30 aprile 2013.
} 
Kristin ANTELMAN, - Emily LYNEMA - Andrew K. PACE, Toward a twenty-first century library catalog, «Information Technology \& Libraries», 25 (2006), n. 3 p. 128-139 oppure: <http://eprints.rclis.org/archive/00007332/01/antelman_lynema_pace.pdf>

ASSOCIAZIONE ITALIANA BIBLIOTECHE (AIB). Sezione Toscana, Seminario su FRBR (Functional requirements for bibliographic records) - Firenze, 27-28 gennaio 2000 [online], [Firenze], Associazione italiana biblioteche, Sezione Toscana. $<$ http://www.aib.it/aib/sezioni/toscana/conf/cfrbr.htm>. Disponibile anche a stampa:

Seminario FRBR : Functional requirements for bibliographic records = Requisiti funzionali per record bibliografici, Firenze, 27-28 gennaio 2000 : atti a cura di Mauro Guerrini $=$ FRBR Seminar $:$ Functional requirements for bibliographic records $=$ Requisiti funzionali per record bibliografici, Florence, 27th-28th January 2000 : proceedings / edited by Mauro Guerrini, Roma, AIB, 2000.

ASSOCIAZIONE ITALIANA PER I DIRITTI DI RIPRODUZIONE DELLE OPERE DELL'INGEGNO (AIDRO), Osservatorio permanente sui contenuti digitali. Indagine 2009, Nielsen, $2009<w w w . o s s e r v a t o r i o c o n t e n u t i d i g i t a l i . i t>$.

Thomas BAKER, Designing data for the open world of the Web, «JLIS.it», 4 (2013), n. 1, p. 63-66, <http://leo.cilea.it/index.php/jlis/article/view/6308/7865>.

Terry BALLARD- Anna BLAINE, User search-limiting behavior in online catalogs, «New Library World», 112, (2011) n. 5-6, p. 261-273.

Laura BALLESTRA, Servizi on line personalizzati: luci e ombre, in La biblioteca su misura. Verso la personalizzazione del servizio, atti del convegno di "Biblioteche oggi", Milano, 9-10 marzo 2006, a cura di Claudio Gamba - Maria Laura Trapletti, Milano, Editrice Bibliografica, 2007, p. 243-267.

Albert BANDURA, Self efficacy: towards a unifying theory of behavioural change, «Psychological Review», 84 (1977), p. 191-215.

Gitte BARLACH "My library": un accesso integrato alle risorse della biblioteca basato sui servizi web, in La biblioteca su misura. Verso la personalizzazione del servizio, atti del 
convegno di "Biblioteche oggi", Milano, 9-10 marzo 2006, a cura di Claudio Gamba Maria Laura Trapletti, Milano, Editrice Bibliografica, 2007, p. 119-128.

Marcia J. BATES, Information search tactics, «Journal of the American Society for Information Science», 30 (1979), p. 205-214.

Marcia J. BATES, Subject access in online catalogs: a design model, «Journal of the American Society for Information Science», 37 (1986), n. 6, p. 357-376.

Marcia J. BATES, The design of browsing and berrypicking techniques for the online search interface, «Online Review», 13 (1989) n. 5, p. 407-424.

Marcia J. BATES, Where should the person stop and the information search interface start? «Information Processing \& Management», 26, (1990), p. 575-591.

Marcia J. BATES, Toward an integrated model of information seeking and searching, Keynote Address, Fourth international Conference on Information Needs, Seeking and Use in Different Contexts, Lisbon, Portugal, September 11, 2002, <http://www.gseis.ucla.edu/faculty/bates/articles/info_SeekSearch-i-030329.html>.

Marcia J. BATES, Task Force Recommendation 2.3 Research and design review, improving user access to library catalog and portal information, final report, Version 2.3, 2003, <http://www.loc.gov/catdir/bibcontrol/2.3BatesReport6-03.doc.pdf>.

Marcia J. BATES, Berrypicking in Theories of information behaviour, edited by Karen E. Fisher - Sanda Erdelez - Lynne Mckechnie, Medford, published for the American Society for Information Science and Technology by Information Today, 2005, p. 58-62.

Marcia J. BATES, An introduction to metatheories, theories and models in Theories of information behaviour, edited by Karen E. Fisher - Sanda Erdelez - Lynne Mckechnie, Medford, published for the American Society for Information Science and Technology by Information Today, 2005, p. 1-24. 
Marcia J. BATES, What is browsing—really? A model drawing from behavioural science research, «Information Research», 12 (2007), n. 4 p. 330, anche in $<$ http://InformationR.net/ir/12-4/paper330.html>.

Marcia J. BATES, Information, in Encyclopedia of Library and Information sciences, 3 ed., edited by Marcia J. Bates and Mary Niles Maack, New York, CRC Press, 2010 p. 2347-2360, anche in <http://pages.gseis.ucla.edu/faculty/bates/articles/information.html>.

Marcia J. BATES, Information behavior in Encyclopedia of Library and Information sciences, 3 ed., edited by Marcia J. Bates and Mary Niles Maack, New York, CRC Press, 2010 p. 2381-2391, anche in $<$ http://www.gseis.ucla.edu/faculty/bates/articles/information-behavior.html>.

Gregory BATESON, Verso un'ecologia della mente, trad. it. di Giuseppe Longo, Milano, Adelphi, 1976.

Zygmunt BAUMAN, Homo consumens: lo sciame inquieto dei consumatori e la miseria degli esclusi, Trento, Erickson, 2007.

Zygmunt BAUMAN, La società sotto assedio, trad. it. di Sergio Minucci, Roma [etc.], Laterza, 2007.

Zygmunt BAUMAN, Modernità e olocausto, trad. it. di Massimo Baldini, Bologna, Il mulino, 2010.

Zygmunt BAUMAN, Modernita liquida, trad. it. di Sergio Minucci, Roma-Bari, Laterza, 2010.

David BAWDEN, Information and digital literacies; a review of concepts, «Journal of Documentation», 57 (2001), n. 2, p. 218-259.

David BAWDEN, Smoother pebbles and the shoulders of giants: the developing foundations of information science, «Journal of Information Science», 34 (2008), n 4, p. 415-426. 
David BAWDEN - Lyn ROBINSON, The dark side of information: overload, anxiety and other paradoxes and pathologies, «Journal of Information Science», 35 (2009) n. 2, p. 180191 ,

<http://intranet.welch.jhmi.edu/intranet/journalclub/reference/journalclub32010dblr.pdf>.

Dave BECKETT - Brian MCBRIDE, RDF/XML Syntax Specification (Revised)W3C Recommendation 10 February 2004,<http://www.w3.org/TR/REC-rdf-syntax/>.

Nicholas J. BELKIN, Anomalous states of knowledge as a basis for information retrieval, «Canadian Journal of Information Science», 5, (1980), p. 133-143.

Nicholas J. BELKIN - Colleen COOL - Adelheit STEIN -Ulrich THIEL, Cases, scripts and information seeking strategies: on the design of interactive information retrieval systems, «Expert Systems with Applications», 9, (1995) p. 379-395.

Nicholas J. BELKIN - R. N. ODDY - H. M. BROOKS, ASK for Information Retrieval: Part II. Results of a Design Study, «Journal of Documentation», 38 (1982), n. 3, p. 145164.

François BELLEAU Marc-Alexandre NOLIN -Nicole TOURIGNY- Philippe RIGAULTJean MORISSETTE, Bio2RDF: towards a mashup to build bioinformatics knowledge systems, « Journal of Biomedical Informatics », 41 (2008) n.5, p. 706-716.

Richard BENNET - Christel HENGEL - Thomas B. HICKEY- Edward T. O'NEILL Barbara B. TILLETT, Virtual International authority file [Presentazione ad ALA Annual Conference, New Orleans, LA, June 22-28, 2006], $<$ http://www.oclc.org/research/projects/viaf/ala2006c.ppt>.

Sue BENNETT - Karl MARTON - Lisa KERVIN, The "digital native” debate: a critical review of the evidence, «British journal of educational technology», 39 (2008), n. 5, p. 775786. 
Nicola BENVENUTI, Dal MARC ai metadati: comincia una nuova stagione per le biblioteche, «Bibelot Blog», blog post, 21 Maggio 2007, $<\mathrm{http}: / /$ www.bibelot.toscana.it/?p=130>.

Nicola BENVENUTI, Social tagging e biblioteche: implicazioni e suggestioni di una "classificazione generata dagli utenti che emerge attraverso un consenso dal basso", «Biblioteche oggi», 25, (2007), n. 3, p. 35-42.

Giovanni BERGAMIN, OPAC: migliorare l'esperienza degli utenti. in Atti del seminario Il catalogo oggi: le norme catalografiche fra consolidamento e fluidità, Modena, 13 $\begin{array}{lllllll}\text { dicembre 2007. } & \text { «Bibliotime», n.s. } 11 & \text { (2008), } & \text { n. } & 1 \text {, }\end{array}$ $<$ http://didattica.spbo.unibo.it/bibliotime/num-xi-1/bergamin.htm>.

Giovanni BERGAMIN - Anna LUCARELLI, The Nuovo soggettario as a service for the linked data world, «JLIS.it», 4 (2013), n. 1, p. 214-226, $<$ http://leo.cilea.it/index.php/jlis/article/view/5474/7903>.

Tim BERNERS-LEE, Linked data - Design issues, 2006, $<$ http://www.w3.org/DesignIssues/LinkedData.html>.

Tim BERNERS-LEE [et al.] Uniform Resource Identifier (URI): generic syntax. Request for Comments: 3986, 2005, <http://tools.ietf.org/html/rfc3986>.

Tim BERNERS-LEE - James HENDLER - Ora LASSILA, The Semantic Web, «Scientific American», 284, 5, (2001) p. 34-43.

Tim BERNERS-LEE, Nigel SHADBOLT e Wendy HALL, The Semantic Web revisited, $\begin{array}{lllllll}\text { «Intelligent } & \text { systems», } & 21 & \text { (2006), } & \text { n. } & 3, & \text { p. }\end{array}$ <http://eprints.soton.ac.uk/262614/1/real/OLD_Semantic_Web_Revisted.pdf>.

Maria Teresa BIAGETTI, Nuovi modelli catalografici, «Bibliotheca», 2 (2002), p. 38-59. 
Maria Teresa BIAGETTI, Indici semantici e ricerca scientifica, in L'organizzazione del sapere. Studi in onore di Alfredo Serrai, a cura di Maria Teresa Biagetti, Milano, Sylvestre Bonnard, 2004, p. 15-29.

Maria Teresa BIAGETTI, Le ontologie come strumenti per l'organizzazione della conoscenza in rete in Numero speciale monografico su le ontologie a cura di Maria Teresa Biagetti, «AIDA informazioni», $28 \quad$ (2010) n. 1-2, p. 9-31, $<$ http://www.aidainformazioni.it/2010/122010.html>.

Maria Teresa BIAGETTI, Nuove funzionalità degli OPAC e relevance ranking. «Bollettino AIB», vol. 50 (2010) n. 4, p. 339-356.

Maria Teresa BIAGETTI, Pertinence perspective and OPAC enhancement, in Paradigms and conceptual systems in knowledge organization. Proceedings of the Eleventh International ISKO Conference 23-26 February 2010, Roma. Edited by Claudio Gnoli and Fulvio Mazzocchi. Würzburg, Ergon Verlag, 2010, p. 334-340.

Carlo BIANCHINI, FRBR prima di FRBR. Il numero di libro nella Colon Classification, «JLIS.it», 1 (2010), n. 1, p. 11-39, <http://leo.cilea.it/index.php/jlis/article/view/31>.

Carlo BIANCHINI, Library Linked Data e il futuro delle biblioteche, unpublished - draft 6 marzo 2012, <http://eprints.rclis.org/16886/>.

Carlo BIANCHINI - Mauro GUERRINI, Quis custodiet ipsos custodes? : osservazioni sulle relazioni fra FRBR, ICP, ISBD e RDA, «Il bibliotecario», $3^{\mathrm{a}}$ serie (2009), n. 1, p. 5985 .

Bonaria BIANCU, Opac 2.0: BNCF. «The Geek Librarian», blog post, 28 febbraio 2006. $<$ http://bonariabiancu.wordpress.com/2006/02/28/opac-20-bncf>.

Bonaria BIANCU, Aggregare le informazioni sul web: esperienze a confronto in Biblioteche come conversazioni: 2.0 due punto zero intorno e oltre l'architettura: Giornata di studio del CNBA 2007, Roma, 18 maggio 2007 / a cura di Serena Sangiorgi e Sandra 
Astrella. - [S.1.] : Coordinamento nazionale biblioteche di architettura, c2008, anche in $<$ http://digital.casalini.it/17240611>.

\section{BIBLIOGRAPHIC SERVICES TASK FORCE. UNIVERSITY OF CALIFORNIA} LIBRARIES, Rethinking how we provide bibliographic services for the University of California. Final report: December 2005, $<$ http://libraries.universityofcalifornia.edu/sopag/BSTF/Final.pdf>.

BIBLIOTECA NAZIONALE CENTRALE DI FIRENZE, Nuovo soggettario: guida al sistema italiano di indicizzazione per soggetto. Prototipo del Thesaurus, Milano, Bibliografica, @2006.

La biblioteca su misura. Verso la personalizzazione del servizio, atti del convegno di "Biblioteche oggi", Milano, 9-10 marzo 2006, a cura di Claudio Gamba e Maria Laura Trapletti. Milano, Editrice Bibliografica, 2007.

Chris BIZER - Richard CYGANIAK - Tom HEATH, How to publish Linked data on the web, <http://sites.wiwiss.fu-berlin.de/suhl/bizer/pub/LinkedDataTutorial/20070727/>.

Christian BIZER- Tom HEATH, - Tim BERNERS-LEE, Linked data: principles and sttate of the art, [relazione presentata a] 17th International World Wide Web Conference, W3C Track @ WWW2008, Beijing, China, 23-24 April 2008, <http://www.w3.org/2008/Talks/WWW2008-W3CTrack-LOD.pdf>.

Christian BIZER- Tom HEATH - Tim BERNERS-LEE, Linked data - The story so far, «IJSWIS», 5 (2009) n. 3, p. 1-22.

Pia BORLUND, The concept of relevance in IR, «Journal of the American Society for Information Science and Technology», 54 (2003), n. 10, p. 913-925.

Christine L. BORGMAN, Why are online catalogs still hard to use?, «Journal of the American Society for Information Science», 47 (1996), n. 7, p. 493-503. 
Jennifer BOWEN, Metadata to support next-generation library resource discovery: Lessons from the eXtensible Catalog, Phase 1., «Information Technology and Libraries», 27 (2008), n. 2, p. 6-19, <https://urresearch.rochester.edu/handle/1802/5757>.

Jennifer BOWEN, Moving library metadata toward linked data: opportunities provided by the eXtensible Catalog in Diane I. Hillmann- Michael Lauruhm, Making metadata work harder: celebrating 15 years of Dublin Core. Proceedings of the International Conference on Dublin Core and Metadata Applications, Pittsburgh 20-22 October 2010, Dublin Core $\begin{array}{llll}\text { Metadata } & \text { Initiative, 2010, 44-59, }\end{array}$ $<$ http://dcpapers.dublincore.org/ojs/pubs/article/view/1010/979>.

Danah BOYD, Taken out of context: american teen sociality in networked publics. $\mathrm{PhD}$ Dissertation. University of California-Berkeley, School of Information, 2008.

Oriana BOZZARELLI - Maria CASSELLA Gli identificativi persistenti. A Torino un seminario di presentazione. «Biblioteche Oggi», 29 (2011) n. 8 p. 66-70, anche in $<$ http://eprints.rclis.org/handle/10760/16344>.

Marshall BREEDING, Next-Gen Library Catalogs, New York, Neal-Schuman Publishers, 2010 .

Marshall BREEDING, State of the Art in Library Discovery 2010, «Computers in libraries», 30 (2010), n. 1 p. 31-35, anche in <http://www.librarytechnology.org/ltgdisplaytext.pl?RC=14574>.

Sergey BRIN - Lawrence PAGE, The anatomy of a large-scale hypertextual web search engine, Seventh International World-Wide Web Conference, Seventh International WorldWide Web Conference (WWW 1998), April 14-18, 1998 Brisbane, Australia, $<$ http://infolab.stanford.edu/ backrub/google.html>.

Bertram C. BROOKES, The foundations of information science. Part 1: Philosophical aspects. «Journal of Information Science», 2 (1980), p. 125-133. 
Cinzia BUCCHIONI, SFX e OpenURL: gli esperimenti del team di Van de Sompel. «Bibliotime», V (2002), n. 2, <http://didattica.spbo.unibo.it/bibliotime/num-v2/bucchion.htm>.

Cinzia BUCCHIONI - Serafina SPINELLI, Teoria e pratica del linking citazionale «Biblioteche Oggi», $25 \quad$ (2007), $\quad$ n. $\quad 8, \quad$ p. $\quad 70-86$, <http://www.bibliotecheoggi.it/2007/20070807001.pdf>.

Pino BUIZZA, Dai principi di Parigi a FRBR, «Bibliotime», V, (2002) n. 1, $<$ http://www.spbo.unibo.it/bibliotime/num-v-1/buizza.htm>.

Pino BUIZZA, Gli opac: funzionalità e limiti nel mondo del web, «Bibliotime», XI (2008), n.1<http://didattica.spbo.unibo.it/bibliotime/num-xi-1/buizza.htm>.

Timothy BURKE, Burn the catalog, 20 January 2004, [Blog post], <http://www.swarthmore.edu/SocSci/tburke1/perma12004.html>.

John D. jr. BYRUM, Recommendations for urgently needed improvement of OPAC and the role of the National Bibliographic Agency in achieving it, World Library and Information Congress: 71th IFLA General Conference and Council. Libraries - A voyage of discovery. August 14th - 18th 2005, Oslo. <http://www.ifla.org/IV/ifla71/papers/124eByrum.pdf> ; trad. italiana: Byrum, John D. jr, Raccomandazioni per miglioramenti urgenti dell'OPAC. Il ruolo delle agenzie bibliografiche nazionali, «Biblioteche Oggi», 23 (2005), n. 10, p. 5-14.

Karen CALHOUN, The changing nature of the catalog and its integration with other discovery tools. Final report prepared for the Library of Congress, 17 March 2006, $<$ http://www.loc.gov/catdir/calhoun-report-final.pdf>.

CALIFORNIA DIGITAL LIBRARY, The Melvyl Recommender Project final report, 2006 ,

$<$ http://www.cdlib.org/services/publishing/tools/xtf/melvyl_recommender/report_docs/Me llon_final.pdf>. 
Rafael CAPURRO - Birger HJØRLAND, The concept of information, «Annual Review of Information Science and Technology», 37 (2003), n. 1, p. 343-411 (Draft version in $<$ http://www.capurro.de/infoconcept.html>).

Allyson CARLYLE, Ordering author and work records: an evaluation of collocation in online catalog displays, «Journal of the American Society for Information Science», 47 (1996), n.7, p. 538-554.

Allyson CARLYLE - Lisa M. FUSCO, Functional requirements for bibliographic records: Understanding FRBR as a conceptual model: FRBR and the bibliographic universe, «Bulletin of the American Society for Information Science and Technology», 33 (2007), n. 6, p. 12-16.

Nicholas G. CARR, Internet ci rende stupidi?: come la rete sta cambiando il nostro cervello, trad. it. di Stefania Garassini, Milano, Raffaello Cortina, 2011.

Donald Owen CASE, Looking for information : a survey of research on information seeking, needs, and behavior, 2. ed, Bingley, Emerald, c2008.

Maria CASSELLA, Le risorse elettroniche nei portali di biblioteche, «Biblioteche Oggi», 25 (2007), n. 10, p. 5-15.

Maria CASSELLA- Paola GARGIULO, Il workflow delle risorse elettroniche remote, «Biblioteche oggi», $24 \quad$ (2006), $\quad$ n. $\quad 6, \quad$ p. $46-58$, $<$ http://www.bibliotecheoggi.it/2006/20060604601.pdf>.

Manuel CASTELLS, La nascita della società in rete, Milano, EGEA-Università Bocconi, 2002.

Mario CEROTI, Rassegna critica della letteratura scientifica italiana sugli OPAC, «Biblioteche Oggi», 30 (2012), n. 11, p. 15-27. 
Ken CHAD - Paul MILLER, Do libraries matter? The rise of Library 2.0. A white paper. Version 1.0 November 2005 , $<$ http://www.talis.com/applications/downloads/white_papers/DoLibrariesMatter.pdf>.

Elfreda CHATMAN, The impoverished life world of outsiders, «Journal of the American society of Information science», 47 (1996), p. 193-206.

Ann CHRISTENSEN, Next generation catalogs: What do users think? Conclusions from the Beluga project. Convegno IFLA 75 pre-satellite Event. Emerging trends in technology: libraries between Web. 2.0 semantic web and search technology. Firenze 19-20 agosto 2009 ,

$<$ http://www.ifla2009satelliteflorence.it/meeting3/program/assets/AnnChristensen.pdf>.

La conoscenza come bene comune: dalla teoria alla pratica, a cura di Charlotte Hess Elinor Ostrom, ed. italiana a cura di Paolo Ferri, premessa di Fiorello Cortiana, Milano, Mondadori, 2009.

Erica COSIJN - Peter INGWERSEN, Dimensions of relevance, «Information Processing and Management: an International Journal archive», 36 (2000), n. 4, p. 533-550.

Karen COYLE, The library catalog in a 2.0 world, «Journal of Academic Librarianship», 33 (2007), n. 2, p. 289-291, anche in <http://www.kcoyle.net/jal_33_2.html>.

Karen COYLE, Murdering MARC, «Coyle's InFormation», September 2006, $<$ http://kcoyle.blogspot.com/2006/09/murdering-marc.html>.

Karen COYLE, Understanding the Semantic Web: bibliographic data and metadata, «Library Technology Reports», 46 (2010) n. 1 (numero monografico).

Karen COYLE, RDA vocabularies for a twenty-first-century data environment, «Library Technology Reports», 46 (2010) n. 2 (numero monografico).

Karen COYLE, Linked Data: an Evolution, «JLIS.it», 4 (2013), n. 1, p. 53-62, $<$ http://leo.cilea.it/index.php/jlis/article/view/5443/7889>. 
Karen COYLE - Diane HILLMANN, Resource Description and Access (RDA). Cataloging rules for the 20th century, «D-Lib Magazine», 13 (2007), n. 1/2, $<$ http://www.dlib.org/dlib/january07/coyle/01coyle.html>

Walt CRAWFORD, Library 2.0 and "Library 2.0", «Cities \& Insights», 6 (2006), n. 2. $<$ http://citesandinsights.info/civ6i2.pdf>

Luigi CROCETTI - Rossella DINI, ISBD (M). Introduzione ed esercizi, 3. ed., Milano, Editrice Bibliografica, 1995.

Gianfranco CRUPI, Beyond the Pillars of Hercules: Linked data and Cultural heritage, $\begin{array}{llllll}\text { «LIS.it», } & 4 & \text { (2013), } & \text { n. } & 1, & \text { p. }\end{array}$ $<$ http://leo.cilea.it/index.php/jlis/article/view/8587/7887>.

Carlos CUADRA, Experimental studies of relevance judgments: final report. Vol. 1: Project summary, Santa Monica, System Development Corp., 1967.

Charles Ammi CUTTER, Rules for a printed dictionary catalogue, in Department of Interior, Bureau of Education, Public libraries in the United States of America. Their history, condition, and management.Special report. Washington, Government Printing Office, 1876.

Alan DANSKIN, Linked and open data: RDA and bibliographic control, «JLIS.it», 4 (2013), n. 1, p. 147-159, <http://leo.cilea.it/index.php/jlis/article/view/5463/7897>.

The Dark side of Google, luci e ombre di Google, <http://eprints.rclis.org/bitstream/10760/12843/1/thedarksideofgoogle.pdf>

Ian DAVIS, Talis, Web 2.0 and all that, «Internet Alchemy blog», blog post, 4. 7. 2005, $<$ http://iandavis.com/blog/2005/07/talis-web-20-and-all-that $>$.

Ian DAVIS, - Richard NEWMAN, Expression of core FRBR concepts in RDF, , [S. 1.]: I. Davis, c2005, <http://vocab.org/frbr/core >. 
Shelda DEBOWSKI, Wrong way: Go back! An exploration of novice search behaviours while conducting an information search, «The Electronic Library», 19 (2001), p. 371-382.

Alison DELLIT - Tony BOSTON, Relevance ranking of results from MARC-based catalogues: from guidelines to implementation exploiting structured metadata. National $\begin{array}{llll}\text { Library Of Australia } & \text { 2009, }\end{array}$ <http://www.nla.gov.au/openpublish/index.php/nlasp/article/view/1052/1321>.

Tom DELSEY, Functional analysis of the MARC 21 bibliographic and holdings formats, Washington, Library of Congress, January 4 2002, <http://www.loc.gov/marc/marcfunctional-analysis/home.html>.

Lorcan DEMPSEY, The sound of words: Amazoogle and Googlezon, January 29, 2005, blog post <http://orweblog.oclc.org/archives/000562.html>.

Lorcan DEMPSEY, The (digital) library environment: ten years after, «Ariadne», 46 (2006) <http://www.ariadne.ac.uk/issue46/dempsey/intro.html>.

Lorcan DEMPSEY, Outside in and inside out, «Lorcan Dempsey's Weblog», blog post, 11. 01. 2010, <http://orweblog.oclc.org/archives/002047.html>.

Lorcan DEMPSEY, Thirteen ways of looking at libraries, discovery, and the catalog: scale, workflow, attention, «EDUCAUSE Review Online», 10. 12. 2012, $<$ http://www.educause.edu/ero/article/thirteen-ways-looking-libraries-discovery-andcatalog-scale-workflow-attention>.

Carmel DENHOLM - Leto KAULER - Jan LAVELLE - Lloyd SOKVITNE, Making the new OPAC seamless: dealing with the transition from "finding" to "getting" "Library Hi Tech», 27 (2009), n.1, p. 13-29.

William DENTON, FRBR and the history of cataloging in Understanding FRBR: what it is and how it will affect our retrieval tools, edited by Arlene Taylor, Westport, Libraries Unlimited, 2007, p. 35-57. 
Antonella De ROBBIO, My library. Note informative e tecniche stilate sulla base della documentazione disponibile e sulla base di una prima sperimentazione tecnica del software. A cura di Antonella De Robbio. Gruppo di Lavoro per Integrazione delle Risorse Elettroniche IRE, Sistema Bibliotecario di Ateneo. Università degli Studi di Padova, Unpublished report, 28.02.2002, ultimo aggiornamento 30.03.2002, $<$ http://eprints.rclis.org/4031/>.

Antonella DE ROBBIO, Servizi bibliotecari personalizzati basati su rss feeds : $i$ diversi volti di un nuovo canale di comunicazione, in La biblioteca su misura. Verso la personalizzazione del servizio, atti del convegno di "Biblioteche oggi", Milano, 9-10 marzo 2006, a cura di Claudio Gamba e Maria Laura Trapletti, Milano, Editrice Bibliografica, 2007, p. 184-206.

Antonella DE ROBBIO, Forme e gradi di apertura dei dati. I nuovi alfabeti dell'Open Biblio tra scienza e società, «Biblioteche Oggi», 30 (2012), n. 6, p. 11-24.

Antonella DE ROBBIO - Silvia GIACOMAZZI, Dati aperti con LODe, «Bibliotime», anno XIV (2011), n. 2, <http://didattica.spbo.unibo.it/bibliotime/num-xiv-2/derobbio.htm>.

Brenda DERVIN, From the mind's eye of the user: the sense-making qualitativequantitative methodology. In Jack D. Glazier-Ronald R. Powell, Qualitative research in information management, Englewood, Libraries Unlimited Inc., 1992.

Brenda DERVIN, Chaos, order, and Sense-Making: a proposed theory for information design, in Robert Jacobson, Information design, Cambridge, MIT Press, 1999, p. 35-57.

DIGITAL LIBRARY FEDERATION. ILS DISCOVERY INTERFACE TASK GROUP (ILS-DI), Technical Recommendation. An API for effective interoperation between integrated library systems and external discovery applications. Revision 1.1. December 8, 2008 ,

$<$ http://www.diglib.org/architectures/ilsdi/DLF_ILS_Discovery_1.1.pdf>. 
Leigh DODDS - Ian DAVIS, Linked data patterns. A pattern catalogue for modelling, publishing, and consuming Linked data, 2011, [ebook], $<$ http://patterns.dataincubator.org/book/>.

Ákos DOMANOVSZKY, Functions and objects of author and title cataloguing, München, Verlag Dokumentation, 1975.

Phillip M. EDWARDS, Taylor Question-Negotiation in Theories of information behaviour, edited by Karen E. Fisher - Sanda Erdelez - Lynne Mckechnie, Medford, published for the American Society for Information Science and Technology by Information Today, 2005, p. 358-362.

David ELLIS, A behavioural approach to information retrieval system design «Journal of Documentation», 45 (1989), p. 171-212.

Nicole C. ENGARD, Library Mashups: Exploring New Ways to Deliver Library Data, Medford, Information Today, Inc, 2009.

Zorana ERCEGOVAC, Toward a global access to bibliographic information: converging patterns, new paradigms: presented as a keynote presentation at the COBISS/SICRIS User Conference in Maribor, Slovenia, 29 November 2000, [Los Angeles]: [University of California], [s. d.], <http://www.cs.ucla.edu/Leap/zer/maribor.htm>. Disponibile anche in lingua slovena: «Obvestila», 5 (2000), n. 4, p. 4-28.

Sanda ERDELEZ, Information encountering: a conceptual framework for accidental information discovery in Pertti Vakkari-Reijo Savolainen-Brenda Dervin, Information seeking in context: proceedings of international conference on research in information needs, seeking and use in different context, London, Taylor Graham, 1997, p. 412-421.

Elena ESCOLANO RODRİGUEZ, ISBD adaptation to semantic web of bibliographic data in linked data, «JLIS.it», $4 \quad$ (2013), $\quad$ n. $\quad 1, \quad$ p. 119-138, $<$ http://leo.cilea.it/index.php/jlis/article/view/5484/7895>. 
Giampaolo FABRIS, Societing: il marketing nella società postmoderna, Milano, Egea, 2008.

Karl V. FAST-D. Grant CAMPBELL, "I Still Like Google": University Student Perceptions of Searching OPACs and the Web in Proceedings of the ASIS Annual Meeting, Medford, Information Today, 2004, p. 138-146, anche online in: $<$ http://www.asis.org/Conferences/AM04/abstracts/137.html>.

Rahmatollah FATTAHI, The relevance of cataloguing principles to the online environment: an historical and analytical study: a thesis submitted in fulfillment of the requirements for the degree of doctor of philosophy in the School of information, library and archive studies, University of South Wales [Sidney, Australia], 1997, anche online in $<$ http://wilma.silas.nsw.edu.au/students/rfattahi/ chappref.htm>.

Rahmatollah FATTAHI, From information to knowledge: superworks and the challenges in the organization and representation of the bibliographic universe $=$ Dall'informazione alla conoscenza: le super-opere e le sfide dell'organizzazione e rappresentazione dell'universo bibliografico : Lectio magistralis in Biblioteconomia, Firenze, Università degli Studi di Firenze, 2010, Firenze, Casalini Libri, 2010.

Nicky FERGUSON - Seb SCHMOLLER - Neil SMITH, Personalisation in presentation services. A report commissioned by JISC (Joint Information Systems Committee-UK), 15 July 2004, <http://www.therightplace.plus.com/jp/jp-study-15.pdf>.

Paolo FERRI, Nativi digitali, Milano, Mondadori 2011.

Luciano FLORIDI, Semantic Conceptions of Information, First published 5. 10. 2005, substantive revision 28. 1. 2011, <http://plato.stanford.edu/entries/information-semantic/>.

Luciano FLORIDI, La rivoluzione dell'informazione, trad. it. di Massimo Durante; prefazione di Juan Carlos De Martin, Torino, Codice, 2012.

Susan FOLKMAN, Personal control and stress and coping processes: a theoretical analysis. «Journal of Personality and Social Psychology», 46 (1984), p. 839-852. 
Kevin FORD, LC Classification as linked data, «JLIS.it», 4 (2013), n. 1, p. 161-176, $<$ http://leo.cilea.it/index.php/jlis/article/view/5467/7899>.

Giovanni FORNERO, Postmoderno e filosofia in Nicola Abbagnano, Storia della filosofia, vol 4. La filosofia contemporanea, Milano, Tea, 1996, p. 3- 48.

Giovanna FRIGIMELICA, OPAC arricchiti: alcuni esempi italiani, «AIB Notizie», 19 (2007) n. 5, <http://www.aib.it/aib/editoria/n19/0507.htm3>.

Raffaella GADDONI - Maria Laura VIGNOCCHI, Nella selva oscura degli Opac, «Bibliotime», IX (2006), n.3 <http://www2.spbo.unibo.it/bibliotime/num-ix-3/gadvign.htm>.

Anna GALLUZZI, Biblioteche per la città, Roma, Carocci, 2009.

Carlo GHILLI - Mauro GUERRINI, Introduzione a FRBR: Functional requirements for bibliographic records $=$ Requisiti funzionali per record bibliografici, Milano, Bibliografica, 2001.

William GIBSON, Neuromante, trad. it. di Giampaolo Cossato e Sandro Sandrelli,Milano, Mondadori, 2003.

Claudio GNOLI, Blopac semantici, «Bibliotime», XI (2008), n.1, $<$ http://didattica.spbo.unibo.it/bibliotime/num-xi-1/gnoli.htm>.

Claudio GNOLI - Vittorio MARINO - Luca ROSATI, Organizzare la conoscenza. Dalle biblioteche all'architettura delle informazioni per il Web, Milano, Hops Tecniche nuove, 2006.

Natalia GODBOLD, Beyond information seeking: towards a general model of information behaviour, «Information Research», 11 (2006) n. 4, p. $269<$ http://InformationR.net/ir/114/paper269.html>. 
Michael GORMAN, Metadati o catalogazione? Una falsa alternativa, traduzione a cura di Stefano Gambari e Mauro Guerrini, «Biblioteche oggi», 19 (2001), n. 6, p. 8-18.

Giuseppe GRANIERI, Umanità accresciuta. Come la tecnologia ci sta cambiando. RomaBari, Laterza, 2009.

Patrick GRIFFIS - Cyrus FORD, Enhancing OPAC records for discovery, «Information technology and libraries», 28 (2009), n. 4, p. 191-193.

Teresa GRIMALDI, L'oggetto della descrizione bibliografica. Seminario su FRBR (Functional requirements for bibliographic records), Firenze, 27-28 gennaio 2000, online, <http://www.aib.it/aib/sezioni/toscana/conf/frbr/grimaldi.htm>.

Thomas Robert GRUBER, A translation approach to portable ontology specifications, «Knowledge Acquisition», 5 (1993) n. 2, p. 199-220.

Mauro GUERRINI, Le funzioni del catalogo dall'ICCP a FRBR, AIB. SEZIONE TOSCANA, <http://www.aib.it/aib/sezioni/toscana/> [2000], $<$ http://www.aib.it/aib/sezioni/toscana/conf/frbr/guerrini.htm>.

Mauro GUERRINI, Catalogazione, Roma, AIB, 2005.

Mauro GUERRINI, Catalogazione in Biblioteconomia: principi e questioni, a cura di Giovanni Solimine e Paul Gabriele Weston, Roma, Carocci, c2007, p. 185-220.

Mauro GUERRINI, I Principi internazionali di catalogazione (ICP). Universo bibliografico e teoria catalografica all'inizio del XXI secolo, postfazione a cura di Attilio Mauro Caproni, Milano, Editrice Bibliografica, 2012.

Mauro GUERRINI - Tiziana POSSEMATO, Linked data: a new alphabet for the semantic $\begin{array}{llllll}\text { web, } & \text { (2013), } & \text { n. } & 1, & \text { p. } & \text { 67-90, }\end{array}$ $<$ http://leo.cilea.it/index.php/jlis/article/view/6305>. 
Barrie GUNTHER - Ian ROWLANDS - David NICHOLAS. The Google generation: are ICT innovations changing information seeking behaviour?, Oxford, Chandos publishing, 2009.

Jutta HAIDER and David BAWDEN, Conceptions of 'information poverty' in library and information science: a discourse analysis, «Journal of Documentation» 63, (2007), n. 4, p. $534-557$.

Jan HANNEMANN - Jürgen KETT, Linked data for libraries, relazione presentata World Library and Information Congress: 76th IFLA General Conference and Assembly Open access to knowledge - promoting sustainable progress, Gothenburg, 10-15 Agosto 2010, [online] <http://www.ifla.org/files/hq/papers/ifla76/149-hannemann-en.pdf>.

Corey A. HARPER - Barbara B. TILLETT, Library of Congress controlled vocabularies and their application to the Semantic Web, «Cataloging \& Classification Quarterly», 43 (2007), n. 3-4, p. 47-68.

Christopher G. HARRIS, Catalog Manifesto, «Infomancy», 14.6.2007, blog post, $<$ http://schoolof.info/infomancy/?p=388>.

Marti A. HEARST, Clustering versus faceted categories for information exploration, «Communication of the ACM», 4 (2006), n. 4, p. 59-61, oppure $<$ http://people.ischool.berkeley.edu/ hearst/papers/cacm06.pdf>.

Tom HEATH, - Christian BIZER, Linked data: evolving the Web into a global data space, «Synthesis Lectures on the Semantic Web: Theory and Technology», 1 (2011) n. 1, 1-136. $<$ http://linkeddatabook.com/editions/1.0/>.

Thomas B. HICKEY, The Virtual International Authority File: expanding the concept of universal bibliographic control, «NextSpace», $13 \quad$ (2009), $<$ https://www.oclc.org/content/dam/oclc/publications/newsletters/nextspace/nextspace_013 $. p d f>$. 
Charles HILDRETH, Pursuing the ideal: generations of online catalogs, in Brian AveneyBrett Butler, Online catalogs, Online Reference, Converging Trends, Chicago, American Library Association, 1984, p. 31-56.

Charles HILDRETH, Beyond boolean: designing the next generation of online catalogs, «Library Trends,» 35 (1987), n. 4, p. 647-667.

Charles R. HILDRETH, Advancing toward the E3 OPAC: the imperative path, in Noelle Van Pulis, Think Tank on the Present and Future of the Online Catalog: Proceedings, American Library Association, Chicago, 1991, p. 39-48.

Charles R. HILDRETH, Online catalog design models: are we moving in the right direction? A report submitted to the Council on library resources, August, 1995, [risorsa online]. $\quad<$ http://eprints.rclis.org/archive/00000198/> oppure $<$ http://myweb.cwpost.liu.edu/childret/clr-opac.html>.

Charles R. HILDRETH, The GUI OPAC: approach with caution, «The public-access computer systems review», 6 (1995), n. $5 . \quad$ p $1-6$ oppure $<$ http://epress.lib.uh.edu/pr/v6/n5/hild6n5.html>.

Diane HILLMANN - Karen COYLE - Jon PHIPPS - Gordon DUNSIRE, RDA Vocabularies. «D-Lib Magazine», $16 \quad$ (2010), $\quad$ n. <http://www.dlib.org/dlib/january10/hillmann/01hillmann.html>

Birong HO - Keith KELLEY - Scott GARRISON, Implementing VuFind as an alternative to Voyager's WebVoyage interface: one library's experience, «Library Hi Tech», 27 (2009), n. 1, p. $82-92$.

The hybrid library: from the users' perspective. A report for the DEFF project "The loaners' expectations and demands for the hybrid library", 2006, $<$ http://www.statsbiblioteket.dk/summa/fieldstudies.pdf/view>.

Bernadette HYLAND - Boris VILLAZÓN-TERRAZAS, Cookbook for Open Government Linked Data, <http://www.w3.org/2011/gld/wiki/Linked_Data_Cookbook>. 
ICCU - Istituto Centrale per il Catalogo Unico delle biblioteche italiane e per le informazioni bibliografiche. Commissione RICA, L'applicazione del modello FRBR ai cataloghi: problemi generali e di impiego normativo. 22 ottobre 2001, [risorsa online,

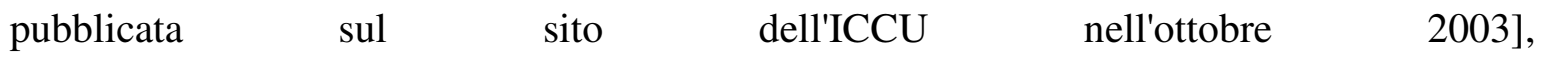
$<$ http://www.iccu.sbn.it/upload/documenti/rica-frbr.pdf>.

ICCU - Istituto Centrale per il Catalogo Unico delle biblioteche italiane e per le informazioni bibliografiche, Regole italiane di catalogazione: REICAT, Roma, ICCU, 2009.

IFLA, Guidelines for online public access catalogue (OPAC) displays : final report, May 2005 / recommended by the task force on Guidelines for OPAC Displays, approved by a the Standing Committee of the IFLA Cataloguing Section, München, K. G. Saur, 2005.

IFLA, International Standard Bibliographic Description (ISBD), Preliminary consolided edition, recommended by the ISBD Review Group, approved by the Standing Committee of the IFLA Cataloguing Section, München, Saur, 2007.

IFLA, IFLA Cataloguing Principles: Statement of International Cataloguing Principles (ICP) and its glossary edited by Barbara Tillett and Ana Lupe Cristán, München, K.G. Saur, $2009<$ http://www.ifla.org/VII/s13/icc/imeicc-statement_of_principles-2008.pdf> [trad. it. Dichiarazione di principi internazionali di catalogazione, Roma, ICCU, 2009, $<$ http://www.iccu.sbn.it/genera.jsp?id=94>, oppure $<$ http://www.ifla.org/files/cataloguing/icp/icp_2009-it.pdf>.

IFLA, International Standard Bibliographic Description (ISBD), edizione consolidata preliminare raccomandata dall'ISBD Review Group; approvata dallo Standard Committee dell'IFLA Cataloguing Section. Edizione italiana / a cura dell'Istituto centrale per il catalogo unico delle biblioteche italiane e per le informazioni bibliografiche (ICCU), Roma, ICCU, 2010.

IFLA. ISBD Review Group e International Federation of Library Associations and Institutions. Cataloguing Section. Standing Committee, ISBD International Standard Bibliographic Description, Berlin, De Gruyter Saur, 2011. 
IFLA. STUDY GROUP ON THE FUNCTIONAL REQUIREMENTS FOR BIBLIOGRAPHIC RECORDS, Functional requirements for bibliographic records: final report, Münich, K. G. Saur, 1998. Disponibile anche online all'URL: <http://www.ifla.org/VII/s13/frbr/frbr.pdf>, o <http://www.ifla.org/VII/s13/frbr/frbr.htm>. Trad. italiana: Requisiti funzionali per record bibliografici, ed. italiana a cura dell'Istituto centrale per il catalogo unico delle biblioteche italiane e per le informazioni bibliografiche, Roma, ICCU, 2000.

IFLA STUDY GROUP ON THE FUNCTIONAL REQUIREMENTS FOR BIBLIOGRAPHIC RECORDS. Functional requirements for bibliographic records: final report. Approved by the Standing Committee of the IFLA Section on Cataloguing. September 1997. As amended and corrected through February 2009, <http://www.ifla.org/VII/s13/frbr/frbr_2008.pdf>.

IFLA. UBCIM, Working Group on Functional Requirements and Numbering of Authority Records (FRANAR), Functional requirements for authority records: a conceptual model, Draft 2005-06-15 <http://www.cidoc-crm.org/docs/frbr_oo/frbr_docs/FRANARConceptual-M-Draft-e.pdf>

IFLA. WORKING GROUP ON FUNCTIONAL REQUIREMENTS FOR SUBJECT AUTHORITY RECORDS (FRSAR), Functional Requirements for Subject Authority Data, A Conceptual Model (FRSAD), Berlin -München, De Gruyter Saur, 2011, anche online: IFLA. Functional Requirements for Subject Authority Data (FRSAD) Final Report Approved by the Standing Committee of the Classification and Indexing Section, June 2010, <http://www.ifla.org/files/classification-and-indexing/functional-requirements-forsubject-authority-data/frsad-final-report.pdf>.

Peter INGWERSEN, Information retrieval interaction, London, Taylor Graham, 1992.

Peter INGWERSEN, Cognitive perspectives of information retrieval interaction, «Journal of Documentation», 52 (1996), n. 1, p. 3-50. 
Peter INGWERSEN, Interactive Framework for information Seeking and interactive Information Retrieval in Theories of information behaviour, edited by Karen E. Fisher Sanda Erdelez - Lynne Mckechnie, Medford, published for the American Society for Information Science and Technology by Information Today, 2005, p. 215-220.

Peter INGWERSEN - Kalervo JÄRVELIN, The turn: integration of information seeking and retrieval in context, Heidelberg, Springer, 2005.

Interactive information seeking, behaviour and retrieval, edited by Ian Ruthven and Diane Kelly, London, Facet, 2011.

INTERNATIONAL CONFERENCE ON SCIENTIFIC INFORMATION, Proceedings of the International Conference on Scientific Information. Washington, National Academy of Sciences, 1959.

Henry JENKINS, Cultura convergente, trad. it. di Vincenzo Susca, Maddalena Pepacchioli, Virginio B. Sala, Milano, Apogeo, 2010.

Jesper KALLEHAUGE, Stage-driven information seeking process: value and uncertainty of work tasks from initiation to resolution, «Journal of Information Science», 36 (2010), n. 2, p 242-262.

Axel KASCHTE, Linked open data on its way into next generation library management and discovery solutions, «JLIS.it», 4 (2013), n. 1, p. 305-312, $<$ http://leo.cilea.it/index.php/jlis/article/view/5492/7913>.

George KELLY, A theory of personality: the psychology of personal constructs, New York, W. W. Norton \& Co, 1963.

James KRIKELAS, Information-seeking behavior: patterns and concepts, «Drexel library quarterly», 19 (1984), p. 5-20.

Carol Collier KUHLTHAU, Seeking meaning: a process approach to library and information services, Westport, Libraries Unlimited, 2004. 
Kathryn LA BARRE, Faceted navigation and browsing features in new OPACs: A more robust solution to problems of information seekers? In Joseph T. Tennis, Proceedings North American Symposium on Knowledge Organization 2007 1, p. 100-104, Toronto, 2007. Abstract in:

<http://arizona.openrepository.com/arizona/bitstream/10150/106157/1/71_La_Barre_(exte nded)2.pdf $/>$.

David LANKES, Fighting for the soul of libraries, June 12th, 2009, <http://quartz.syr.edu/rdlankes/Presentations/2009/NYSLAA.pdf>.

David LANKES, Joanne SILVERSTEIN, Scott NICHOLSON, Participatory networks: the library as conversation, «Information Research», 12 (2007), n. 4, $<$ http://informationr.net/ir/12-4/colis/colis05.html>. La traduzione italiana si trova in: <http://www.aib.it/aib/cg/gbdigd07.htm3>.

Richard LAZARUS - Susan FOLKMAN, Stress, appraisal, and coping, New York, Springer Pub. Co, 1984.

Eric LEASE MORGAN, Next generation library catalog, «Infomotions.com», 02-062006, <http://infomotions.com/musings/ngc/>.

Eric LEASE MORGAN, A 'Next-Generation' library catalog - Executive summary (Part \#1 of 5), July 7, 2006, «LITA Blog: Library Information Technology Association», $<$ http://litablog.org/2006/07/07/a-next-generation-library-catalog-executive-summary-part$1-$ of- $5 />$.

Claudio LEOMBRONI, Appunti per un'ontologia delle biblioteche digitali: considerazioni sulla Biblioteca digitale italiana, «Bollettino AIB», 44 (2004), n. 2, p. 115-131.

Rick LEVINE - Cristopher LOCKE - Doc SEARLE - David WEINBERGER, The Cluetrain Manifesto. The end of business as usual, Roma, Fazi, 2001. 
Dirk LEWANDOWSKI, Web searching, search engines and information retrieval, «Information Service and Use» 25 (2005), n. 3/4 p. 137-147.

\section{LIBRARY OF CONGRESS. NETWORK DEVELOPMENT AND MARC STANDARDS}

OFFICE, Functional analysis of the MARC 21 bibliographic and holdings formats, updated and revised, Library of Congress, April 6, 2006, <http://www.loc.gov/marc/marcfunctional-analysis/functional-analysis.html>.

LIBRARY OF CONGRESS. WORKING GROUP ON THE FUTURE OF BIBLIOGRAPHIC CONTROL, On the record: report of the Library of Congress Working Group on the future of bibliographic control, January 9, 2008, $<$ http://www.loc.gov/bibliographic-future/news/lcwg-ontherecord-jan08-final.pdf>.

LIBRARY LINKED DATA INCUBATOR GROUP, Library linked data incubator group final report, unpublished draft 6. 3. 2012, <http://www.w3.org/2005/Incubator/lld/XGRlld-20111025/>.

LIBRARY LINKED DATA INCUBATOR GROUP, Library linked data incubator group: use cases, W3C Incubator Group report, Daniel Vila Suero editor, 25 October 2011. <http://www.w3.org/2005/Incubator/lld/XGR-lld-usecase-20111025/>. Ultima versione in $<$ http://www.w3.org/2005/Incubator/lld/XGR-lld-usecase/>.

David LINDAHL, EXtensibleCatalog's 2010, $<$ http://www.screencast.com/users/eXtensibleCatalog>.

David LINDAHL, EXtensible Catalog: transforming legacy metadata towards FRBR, RDA, and Linked Data. ALA Midwinter 2011, $<$ http://www.extensiblecatalog.org/learnmore/publications/ala-midwinter-2011-lita-nextgeneration-catalog-ig-extensible-catalog-transfo>.

David LINDAHL, EXtensible Catalog: software portfolio, presentazione tenuta a ALA Midwinter 2011 - LITA Next Generation Catalog IG, 9 gennaio 2011 $<$ http://docushare.lib.rochester.edu/docushare/dsweb/Get/Document-47024>. 
Linking Government Data, David Wood editor, New York, Springer, 2011.

Cory LOWN, A transaction log analysis of NCSU's faceted navigation OPAC. A master's paper for the M.S. in L.S degree. April, 2008,< http://ils.unc.edu/MSpapers/3387.pdf>.

Geert LOVINK, Zero comments. Teoria critica di Internet, trad. it. di Alessandro Delfanti, Milano, Mondadori, 2008.

Jean-François LYOTARD, La condizione postmoderna. Rapporto sul sapere, 2. ed. Milano, Feltrinelli, 1981.

Martin MALMSTEN, LIBRIS available as Linked data, 312 2008. Blog post. LIBRIS nationella bibliotekssystem, «Librisbloggen.kb.se» <http://librisbloggen.kb.se/2008/12/03/libris-available-as-linked-data/>.

Martin MALMSTEN, Making a library catalogue part of the Semantic Web. In J. Greenberg - W. Klas, Metadata for semantic and social applications. Proceedings of the International Conference on Dublin Core and Metadata Applications. Berlin, 22-26 September 2008, pp. 146-150. Singapore and Universitätsverlag Göttingen, Dublin Core Metadata Initiative, 2008 <http://webdoc.sub.gwdg.de/univerlag/2008/DC_proceedings.pdf>.

Martin MALMSTEN, Exposing library data as Linked data, [relazione tenuta al Satellite Meetings IFLA 2009, Emerging trends in technology: libraries between Web 2.0, semantic web and search technology, Florence, 19-20 Agosto 2009], <http://www.ifla2009satelliteflorence.it/meeting3/program/assets/MartinMalmsten.pdf>.

Martin MALMSTEN, Cataloguing in the open - the disintegration and distribution of the record, «JLIS.it», $4 \quad$ (2013), $\quad$ n. $\quad 1, \quad$ p. $417-424$, $<$ http://leo.cilea.it/index.php/jlis/article/view/5512>.

Diego MALTESE, La biblioteca come linguaggio e come sistema, Milano, Editrice Bibliografica, 1985. 
Thomas MANN, Is precoordination unnecessary in LCSH? are Web sites more important to catalog than books? A reference librarian's thoughts on the future of bibliographic control, Library of Congress, Washington D.C., 15-17 November 2000. $<$ http://www.loc.gov/catdir/bibcontrol/mann_paper.pdf>.

Thomas MANN, Will Google's keyword searching eliminate the need for LC cataloging and classification? August 15 2005, <http://www.guild2910.org/searching.htm>.

Thomas MANN, Survey of library user studies, October 2005, <http://www.guild2910.org/google.htm>.

Thomas MANN, The changing nature of the catalog and its integration with other discovery tools. Final report, March 17, 2006, prepared for the Library of Congress by Karen Calhoun. A critical review, 3 April 2006. <http://guild2910.org/AFSCMECalhounReviewREV.pdf> ; trad. italiana: Thoman Mann, Il catalogo e gli altri strumenti di ricerca: un punto di vista dalla Library of Congress, «BollettinoAIB», 46 (2006), 3, p. 186-205.

Thomas MANN, What is going on at the Library of Congress?. Prepared for AFSCME 2910 by Thomas Mann, reference librarian in the Library of Congress Main reading room, June 19, 2006, <http://www.guild2910.org/AFSCMEWhatIsGoingOn.pdf>.

Thomas MANN, More on what is going on at the Library of Congress. Prepared for AFSCME 2910, 2007, <http://www.guild2910.org/AFSCMEMoreOnWhatIsGoing.pdf>.

Thomas MANN, The Peloponnesian war and the future of reference, cataloging, and scholarship in research libraries. Prepared for AFSCME 2910 The Library of Congress Professional Guild representing over 1,600 professional employees www.guild2910.org. Library of Congress, Washington D.C., Library of Congress, 2007, <http://guild2910.org/Pelopponesian\%20War\%20June\%2013\%202007.pdf>.

Thomas MANN, "On the record" but off the track: a review of the report by The Library of Congress Working Group on The Future of Bibliographic Control, with a further 
examination of Library of Congress cataloging tendencies, 2008. <http://www.guild2910.org/WorkingGrpResponse2008.pdf>.

Christopher D. MANNING - Prabhakar RAGHAVAN -Hinrich SCHÜTZE, Introduction to Information Retrieval, Cambridge University Press, 2008. Anche online all'URL: $<$ http://nlp.stanford.edu/IR-book/>.

Gary MARCHIONINI, An invitation to browse: designing full-text systems for novice users, «The Canadian Journal of Information Science», 12 (1987), n. 3/4, p. 69-79.

Gary MARCHIONINI, Information seeking in electronic environments, Cambridge, Cambridge University Press, 1995.

Gary MARCHIONINI - Ryen WHITE, Find what you need, understand what you find, «International Journal of Human-Computer Interaction», 23 (2007) n. 3, p. 205-237. <http://www.tandfonline.com/doi/abs/10.1080/10447310701702352\#preview>.

Andrea MARCHITELLI, OPAC, bloOPAC e social networking: gestione della conoscenza 2.0?. slides presentate al 3' Incontro ISKO Italia - UniMIB, Milano, 2007. <http://eprints.rclis.org/archive/00010667/01/opac-blopac-e-social-networking1722.pdf>.

Andrea MARCHITELLI, La biblioteca nella percezione degli utenti: $i$ risultati di tre indagini di OCLC, «AIB Notizie», 20 (2008), n. 4, p. 13-14 oppure <http://www.aib.it/aib/editoria/n20/0413.htm3>.

Andrea MARCHITELLI, Il buono, il brutto, il cattivo: esistono davvero gli OPAC 2.0?, slides presentate all'incontro CILEA “Oltre l'OPAC”. Jesi, Biblioteca Planettiana, 9 giugno 2008 ,

<http://eprints.rclis.org/archive/00013795/01/Il_buono\%2C_il_brutto\%2C_il_cattivo.pdf>.

Andrea MARCHITELLI, E pur si muove? Servizi integrati e di nuova generazione per la ricerca informativa, slides presentate al Seminario CENFOR "Le Biblioteche del 2011 scommettono sul futuro? Nuove tecnologie per l'accesso alle collezioni e servizi di telefonia mobile per gli utenti”, Milano, 4 Marzo 2011. 
Andrea MARCHITELLI, Giovanna FRIGIMELICA, OPAC, Roma, AIB, 2012.

Andrea MARCHITELLI, - Tessa PIAZZINI, OPAC, SOPAC e Social networking: cataloghi di biblioteca 2.0? «Biblioteche Oggi», 26 (2008), n. 2, p. 82-92, anche in $<$ http://www.bibliotecheoggi.it/content/20080208201.pdf>.

Deanna MARCUM, The future of cataloguing. Address to the Ebsco leadership seminar. Boston, Massachusetts, January 16, 2005, $<$ http://www.loc.gov/library/reports/CatalogingSpeech.pdf $>$.

Deanna MARCUM, A bibliographic framework for the digital age, 31. 10. 2011, <http://www.loc.gov/marc/transition/pdf/bibframework-10312011.pdf>.

Karen MARKEY DRABENSTOTT, Do non-domain experts enlist the strategies of domain experts? «Journal of the American Society for Information Science and Technology», 54 (2003), n. 9, p. 836-854, anche online in $<$ http://deepblue.lib.umich.edu/bitstream/handle/2027.42/35291/10281_ftp.pdf?sequence= $1>$.

Karen MARKEY, The online library catalog. Paradise lost and paradise regained?, «DLib Magazine», 13 (2007), n. 1/2.

Karen MARKEY, Twenty-five years of end-user searching, Part 1: fesearch findings, «Journal of the American Society for Information Science and Technology», 58 (2007) n. 8, p. 1071-1081, <http://hdl.handle.net/2027.42/56093>.

Karen MARKEY, Twenty-five years of end-user searching, Part 2: future research directions. «Journal of the American Society for Information Science and Technology», 58 (2007) n. 8, 1123-1130, <http://hdl.handle.net/2027.42/56094>.

Melvin E. MARON, - John L. KUHNS, On relevance, probabilistic indexing and information retrieval. «Journal of the ACM», 7 (1960) n, 3, p. 216-244. 
Gabriele MEßMER, Linking library metadata to the Web: the German experiences, $\begin{array}{llllll}\text { «LIS.it», } \quad 4 & \text { (2013), } & \text { n. } & 1, & \text { p. }\end{array}$ $<$ http://leo.cilea.it/index.php/jlis/article/view/5507/7878>.

Fabio METITIERI, Il grande inganno del web 2.0, Roma-Bari, Laterza, 2009.

Fabio METITIERI, L'OPAC collaborativo tra socialità e folksonomia. «Biblioteche Oggi», 27 (2009), n.2, p. 7-14.

Alistair MILES - Sean BECHHOFER, SKOS Simple Knowledge Organization System eXtension for Labels (SKOS-XL) Namespace Document - HTML variant 18th August 2009 recommendation edition, <http://www.w3.org/TR/skos-reference/skos-xl.html>.

Steven J. MILLER, Metadata and cataloging online resources: selected reference documents, web sites and articles. Compiled by Steven J. Miller, University of WisconsinMilwaukee School of Information Studies, Updated June 1, 2011, $<$ http://www.uwm.edu/ mll/resource.html>.

Steven J. MILLER, Metadata for digital collections. A how-to-do-it manual, New York, Neal-Schuman Publishers, 2011, Sito web con esempi: <http://www.nealschuman.com/metadata-digital-collections>.

Joan S. MITCHELL - Michael PANZER, Dewey linked data: Making connections with old friends and new acquaintances, «JLIS.it», 4 (2013), n. 1, p. 177-200, $<$ http://leo.cilea.it/index.php/jlis/article/view/5467>.

Stefano MIZZARO, Relevance: the whole history. «Journal of the American Society for Information Science», 48 (1997) n. 9, p. 810-832.

Stefano MIZZARO, How many relevances in information retrieval? «Interacting with Computers», 10 (1998), n. 3, 303-320.

Rossana MORRIELLO, La coda lunga e le biblioteche, «Bollettino AIB », 49 (2009) 1, p. 69-82. 
Peter MORVILLE, Authority, «Semantic studios», [blog post], 11. 10. 2005, $<$ http://semanticstudios.com/publications/semantics/000057.php>.

Peter MORVILLE - Louis ROSENFELD, Information architecture for the World Wide Web. Sebastopol, CA, O'Reilly, 2007.

Peter MORVILLE - Jeffery Callender, Search patterns, Sebastopol, CA, O'Reilly Media, 2010.

Andrew NAGY, Analyzing the Next-Generation catalog, «Library Technology Reports», ALA TechSource, 2011.

Jacob NIELSEN, Mental models for search are getting firmer, «Jakob Nielsen's Alertbox», May 9 2005, <http://www.useit.com/alertbox/20050509.html>.

NISO, Standards and best practices for library discovery services based on indexed search: a proposed NISO work item voting member ballot, September 2 - October 2, 2011, Work item proposal: $<$ http://www.niso.org/apps/group_public/document.php?document_id=7175>.

Jan NOLIN, "Relevance" as a boundary concept: Reconsidering early information retrieval. «Journal of Documentation», 65 (2009), n. 5, 745-767.

Donald A. NORMAN- Stephen W. DRAPER, User centered system design: new perspectives on human-computer interaction, Hillsdale, Lawrence Erlbaum Associates, 1986.

OAI PMH Guidelines, <http://www.openarchives.org/OAI/2.0/guidelines.htm>.

Edward T. O'NEILL, FRBR: application of the entity-relationship model to Humphry Clinker, «Library Resources and Technical Services», 46 (2002) n. 4, p. 150-159. 
ONLINE COMPUTER LIBRARY CENTER (OCLC), OCLC White paper on the information habits of college students, published electronically by the OCLC Online Computer Library Center, Inc., June 2002. $<$ http://www5.oclc.org/downloads/community/informationhabits.pdf>.

ONLINE COMPUTER LIBRARY CENTER (OCLC), The 2003 OCLC environmental scan: pattern recognition. A report to the OCLC membership. Dublin, Online Computer Library Center, 2003, <http://www.oclc.org/reports/escan/>.

ONLINE COMPUTER LIBRARY CENTER (OCLC), Perceptions of libraries and information resources. A report to the OCLC membership. Dublin, Online Computer Library Center, $2005<$ http://www.oclc.org/reports/pdfs/Percept_all.pdf>.

ONLINE COMPUTER LIBRARY CENTER (OCLC), Sharing, privacy and trust in our networked world. A report to the OCLC membership. Dublin, Online Computer Library Center, $2007<$ http://www.oclc.org/reports/pdfs/sharing.pdf >

ONLINE COMPUTER LIBRARY CENTER (OCLC), Online catalogs. What users and librarians want : an OCLC report, [a cura di] Calhoun, Karen - Diane Cellentani, Dublin Ohio, OCLC, 2009 <http://www.oclc.org/reports/onlinecatalogs/fullreport.pdf>.

ONLINE COMPUTER LIBRARY CENTER (OCLC), Perceptions of libraries, 2010: context and community, [a cura di] Cathy De Rosa et al. OCLC, 2011, <http://www.oclc.org/reports/2010perceptions.htm>.

ONLINE COMPUTER LIBRARY CENTER (OCLC), OCLC adds linked data to WorldCat.org, $20 \quad$ Giugno 2012, <http://www.oclc.org/uk/en/news/releases/2012/201238.htm>.

Tim O'REILLY, What is Web 2.0 Design patterns and business models for the next generation of software, «O'Rellynet.com», $30 \quad$ september 2005. $<$ http://oreilly.com/web2/archive/what-is-web-20.html>- 
Tim O'REILLY, Web 2.0: compact definition?, «O'Reilly Radar», blog post, October 1 2005. <http://radar.oreilly.com/archives/2005/10/web_20_compact_definition.html>.

Tim O'REILLY, Web 2.0 compact definition: trying again «O'Reilly Radar», blog post, december 10, 2006, <http://radar.oreilly.com/archives/2006/12/web_20_compact.html>.

George ORWELL, 1984, traduzione di Stefano Manferlotti, Milano, Mondadori, 2004.

John PALFREY - Urs GASSER, Nati con la rete. La prima generazione cresciuta su internet. Istruzioni per l'uso, Milano, Rizzoli, 2009.

Michael PANZER - Marcia Lei ZENG, Modeling classification systems in SKOS: some challenges and best-practice recommendations, in Semantic interoperability of linked data : Proceedings of the international Conference in Dublin Core and Metadata Applications, Seoul, October 12-16, 2009, edited by S. Oh. Sugimoto - S. A. Sutton, Seoul, Dublin Core Metadata Initiative and Nation Library of Korea, 2009, p. 3-14. Disponibile anche all'URL: <http://dcpapers.dublincore.org/index.php/pubs/article/view/974/944>.

Ioannis PAPADAKIS - Konstantinos KYPRIANOS - Rosa MAVROPODI - Michalis STEFANIDAKIS, Subject-based information retrieval within digital libraries employing

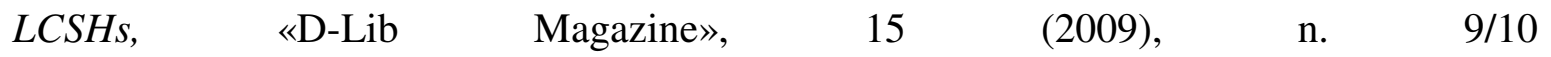
$<$ http://www.dlib.org/dlib/september09/papadakis/09papadakis.html>.

Glenn E. PATTON - IFLA Working Group on Functional Requirements and Numbering of Authority Records (FRANAR) - International Federation of Library Associations and Institutions, Functional requirements for authority data: a conceptual model, München, K.G. Saur, 2009.

Alberto PETRUCCIANI, Ragioni e principi della revisione delle RICA: per un nuovo codice italiano di catalogazione, «BollettinoAIB», 45 (2005), n. 2, p. 149-184.

Alberto PETRUCCIANI, La catalogazione, il mercato e la fiera dei luoghi comuni, «BollettinoAIB», 43 (2006) n. 3 p. 177-206. 
Alberto PETRUCCIANI, Le nuove RICA nel contesto dell'evoluzione delle normative catalografiche, $\quad$ «Bibliotime», XI $\quad$ (2008), $\quad$ n. $<$ http://didattica.spbo.unibo.it/bibliotime/num-xi-/petrucci.htm>.

Alberto PETRUCCIANI, Ancora su Google e la giungla digitale: altri misteri, novità e tendenze, «AIB Studi», 52 (2012) n. 2, p. 197-204, $<$ http://aibstudi.aib.it/article/view/6326/7213>.

Alison Jane PICKARD, La ricerca in biblioteca. Come migliorare i servizi attraverso gli studi sull'utenza, introduzione, traduzione e cura di Elena Corradini; prefazione di Anna Maria Tammaro, Milano, Editrice Bibliografica, 2010.

William Gray POTTER, Expanding the online catalog, «Information Technology and Libraries», 8 (1989), n. 2 p. 99-104.

Marc PRENSKY, Digital natives, digital immigrants, «On the Horizon», 9 (2001), n. 5, p. 1-6, <http://www.scribd.com/doc/9799/Prensky-Digital-Natives-Digital-ImmigrantsPart1>.

Marc PRENSKY, Digital natives, digital immigrants, part 2: Do they really think differently «On the Horizon», 9 (2001), n. 6, p. 1-6.

Emanuele QUINTARELLI, Folksonomies: power to the people. [Relazione presentata al convegno ISKO Italia - UniMIB, Milano, 24 giugno 2005, $<$ http://www.iskoi.org/doc/folksonomies/>.

RDA Relationships for Works, Expressions, Manifestations, Items, $<$ http://metadataregistry.org/schema/show/id/13.html>.

RDA Toolkit: Resource Description \& Access, [2010], $<$ http://access.rdatoolkit.org/index.php>.

Jef RASKIN, Interfacce a misura d'uomo, Milano, Apogeo, 2003. 
Edie RASMUSSEN, Access models in Interactive information retrieval, edited by Ian Ruthven and Diane Kelly, London, Facet, 2010, p. 95-111.

Alan M. REES - Douglas O. SCHULTZ, A field experiment approach to the study of relevance assessments in relation to document searching: Final report to the National Science Foundation, Cleveland, Case Western Reserve University. Center for Documentation and Communication Research, 1967.

Jeremy RIFKIN, L'era dell'accesso. La rivoluzione della new economy, trad. di Paolo Canton, Milano, Mondadori, 2000.

Art RHYNO, The end of ILS, «Inside OLITA», 5 (2001).

Riccardo RIDI, La Biblioteca come ipertesto. Verso l'integrazione dei servizi e dei documenti, Milano, Editrice Bibliografica, 2007.

Pat RIVA, FRBR Review Group initiatives and the world of linked data, «JLIS.it», 4 (2013), n. 1, p. 105-118, <http://leo.cilea.it/index.php/jlis/article/view/5482/7893>.

Stephen E. ROBERTSON, The probability ranking principle in $I R$, «Journal of Documentation», 33 (1977) p. 294-304.

Stephen E. ROBERTSON - Micheline HANCOCK-BEAULIEU, On the evaluation of IR systems, «Information Processing \& Management», 28 (1992) n. 4, p. 457-466.

Stephen E. ROBERTSON - Karen SPÄRCK JONES, Relevance weighting of search terms, «Journal of the American Society for Information Science», 27 (1976), n. 3, p. 129146.

Joseph J. Jr. ROCCHIO, Relevance feedback in information retrieval, in Gerard SALTON, The SMART retrieval system: experiments in automatic document processing, Prentice Hall, 1971, p. 313-323. 
Jonathan ROCHKIND "FRBRization" is not FRBRization «Bibliographic Wilderness», Blog post. 31/07/2008 <http://bibwild.wordpress.com/2008/07/31/frbrization-is-notfrbrization/>.

Gino RONCAGLIA, Blogosfera e feed rss : una palestra per il semantic web?, « Networks», 2003, n. 2, <http://www.merzweb.com/testi/saggi/blogosfera.pdf> oppure $<\mathrm{http}: / /$ eprints.rclis.org/5742/>.

Luca ROSATI, Un modello integrato di interazione uomo-informazione, Trovabile, 2007, $<$ http://trovabile.org/articoli/un-modello-integrato-di-interazione-uomo-informazione> .

Luca ROSATI, Per un accesso multidimensionale all'informazione o della classificazione a faccette, «The Information Architecture Institute Italia», 2003 $<$ http://iainstitute.org/it/articoli/000204.html>.

Luca ROSATI, Architettura dell'informazione: trovabilità: dagli oggetti quotidiani al Web, Milano, Apogeo, 2007.

Elizabeth SADLER, Project Blacklight: a next generation library catalog at a first generation university, «Library Hi Tech», 27 (2009), n.1, p. 57-67.

Athena SALABA - Yin ZHANG, From a conceptual model to application and system development, «Bulletin of the American Society for Information Science and Technology», 33 (2007), n. 6, p. 17-23. Disponibile anche online all'URL: $<$ http://www.asist.org/Bulletin/Aug-07/Bulletin_AugSep07.pdf>.

Alberto SALARELLI, Biblioteca e identità: per una filosofia della biblioteconomia, Milano, Ed. Bibliografica, 2008.

Alberto SALARELLI, Biblioteconomia e information science: diversi linguaggi per due naturali alleati? Relazione presentata al convegno I nuovi alfabeti della biblioteca, Milano, Fondazione Stelline, 15-16 marzo 2012. 
Alberto SALARELLI, Introduzione alla scienza dell'informazione, Milano, Bibliografica, 2012.

Gerard SALTON, Automatic text processing: the transformation, analysis, and retrieval of information by computer, Reading, Addison-Wesley, 1988.

Michele SANTORO, La disarmonia prestabilita: per un approccio ibrido alla conoscenza e ai suoi supporti, «Biblioteche oggi», 20 (2002), n.5, p. 46-57.

Michele SANTORO, Questa sera si cataloga a soggetto. Breve analisi delle folksonomies in prospettiva bibliotecaria, «Bibliotime», $\mathrm{X} \quad$ (2007), $\quad \mathrm{n} . \quad 2$ $<$ http://www2.spbo.unibo.it/bibliotime/num-x-2/santoro.htm>.

Tefko SARACEVIC, Relevance: a review of and a framework for the thinking on the notion in information science, "Journal of the American Society for Information Science», 26 (1975) n. 6, p. 321-343.

Tefko SARACEVIC, Relevance reconsidered, in Peter Ingwersen - Niels Ole Pors, Proceedings Colis 2: Second international conference on conceptions of Library and information science: integration in perspective, October 13-16, 1996 Copenhagen, Royal School of Librarianship, 1996, p. 201-218.

Tefko SARACEVIC, The stratified model of information retrieval interaction: Extension and applications. «Proceedings of the American Society for Information Science», 34 (1997), p. 313-327.

Tefko SARACEVIC, Information science, «Journal of the American Society for Information Science», 50 (1999), n. 12, p. 1051-1063.

Tefko SARACEVIC Relevance: a review of the literature and a framework for thinking on the notion in information science. Part II: nature and manifestations of relevance, «Journal of the American Society for Information Science and Technology», 58 (2007), n. 3, p. 1915-33. 
Tefko SARACEVIC, "Relevance: a review of the literature and a framework for thinking on the notion in information science. Part III: Behavior and effects of relevance", «Journal of the American Society for Information Science and Technology», 58 (2007) n. 13, pp. 2126-44.

Tefko SARACEVIC, Information Science in Encyclopedia of Library and Information sciences, 3 ed., a cura di Marcia J. Bates e Mary Niles Maack, New York, CRC Press, 2010, p. 2381-2391.

Tefko SARACEVIC - Paul B. KANTOR, Studying the value of library and information services. I. Establishing a theoretical framework. «Journal of the American Society for Information Science», 48 (1997), n. 6, p. 527-542.

Reijo SAVOLAINEN- Jarkko KARI, Facing and bridging gaps in Web searching. «Information Processing Management», 42 (2006), n. 2, p. 519-537.

Graham SEAMAN, Adapting VuFind as a front-end to a commercial discovery system, «Ariadne», 68 (2012), <http://www.ariadne.ac.uk/issue68/seaman>.

Andrea SEMPRINI, La società di flusso: senso e identità nelle società contemporanee, Milano, Franco Angeli, 2003.

Karen G. SCHNEIDER, How OPACs suck, Part 1: relevance rank (or the lack of it), 13. 3. 2006, [blog post], <http://www.techsource.ala.org/blog/2006/03/how-opacs-suck-part-1relevance-rank-or-the-lack-of-it.html>.

Toby SEGARAN - Colin EVANS - Jamie TAYLOR, Programming the Semantic Web. Build flexible applications with graph data, Beijing, O'Reilly, 2009.

Alfredo SERRAI, Biblioteconomia come scienza: introduzione ai problemi e alla metodologia, Firenze, Olschki, 1973.

Alfredo SERRAI, Indici, logica e linguaggio : problemi di catalogazione semantica, Roma, Consiglio nazionale delle ricerche, Laboratorio di studi sulla ricerca e sulla documentazione scientifica, 1974. 
Alfredo SERRAI, La biblioteca di fronte alla rivoluzione concettuale e tecnologica della scienza moderna, in I congressi 1965-1975 dell'Associazione Italiana Biblioteche, a cura di Diana La Gioia, Roma: AIB, 1977, p. 105-122.

Alfredo SERRAI, Del catalogo alfabetico per soggetti: semantica del rapporto indicale, Roma, Bulzoni, 1979.

Alfredo SERRAI, Guida alla biblioteconomia, Firenze, Sansoni, 1981.

Alfredo SERRAI, Biblioteche e cataloghi, Firenze, Sansoni, 1983.

Alfredo SERRAI, Ricerche di biblioteconomia e bibliografia, Firenze, Giunta regionale toscana, La Nuova Italia, 1983.

Alfredo SERRAI, Dalla informazione alla bibliografia: la professione bibliotecaria. Milano, Bibliografica, 1984.

Alfredo SERRAI, Il cimento della bibliografia, Milano, Sylvestre Bonnard, 2001.

Alfredo SERRAI - Marco MENATO, Biblioteche e bibliografia : vademecum disciplinare e professionale, Roma, Bulzoni, 1994.

Robert B. SETTLE - Pamela L. ALRECK, Reducing buyers' sense of risk. «Marketing Communications», 14 (1989) p. 34-40.

Claude Elwood SHANNON, A mathematical theory of communication, «Bell System Technical Journal», 27, (1948), p. 379-423 e 623-656.

Claude Elwood SHANNON - Warren WEAVER, The Mathematical Theory of Communication, Urbana, University of Illinois Press, 1949. 
Jesse Hauk SHERA, Knowing books and men; knowing computers too, Littleton, Libraries Unlimited, 1973.

Clay SHIRKY, Here comes everybody. The power of organizing without organizations, New York: Penguin Press, c2008.

Tito SIERRA, Improving the catalogue interface using Endeca, Capitalize on access, 2006, <http://www.lib.ncsu.edu/endeca/presentations.html>.

Tito SIERRA, A single search box interface to the NCSU libraries, two years later, Digital Library Federation Spring Forum 2007, Pasadena, California, April 24, 2007. $<$ http://www.lib.ncsu.edu/dli/projects/quicksearch/>.

Aida SLAVIĆ, Interface to classification: some objectives and options. «Extension \& Corrections to the UDC», 28 (2006), <http://dlist.sir.arizona.edu/1621/>.

Lloyd SOKVITNE, Redesigning the OPAC: moving outside of the ILMS, «Australian Academic \& Research Libraries», 37 (2006), n. 4, p 246-259. Oppure $<$ http://www.nla.gov.au/lis/stndrds/grps/acoc/documents/Sokvitne.doc>.

Giovanni SOLIMINE, La biblioteca e la rete nella società dell'apprendimento, in L'organizzazione del sapere: studi in onore di Alfredo Serrai / a cura di Maria Teresa Biagetti, Milano, Sylvestre Bonnard, 2004.

Iryna SOLODOVNIK, Comunicazione e ricerca semantica di contenuti informativi tra metadati, Linked Open Data e ontologie, «Bibliotime», XIV (2011), n. 2 $<$ http://didattica.spbo.unibo.it/bibliotime/num-xiv-2/solodovnik.htm>.

Amanda SPINK, Multiple search sessions model of end-user behavior: An exploratory study, «Journal of the American Society for Information Science», 47 (1996), p. 603-609.

Amanda SPINK, Study of interactive feedback during mediated information retrieval. «Journal of the American Society for Information Science», 48 (1997) n. 5, p. 382-394. 
Amanda SPINK - Robert M. Losee, Feedback in information retrieval, «Annual Review of Information Science and Technology», 31 (1996), p. 33-78.

Tom STEELE, The new cooperative cataloging, «Library Hi Tech», 27 (2009), n.1, p. 6877.

Robi STURMAN, Il software open source per la gestione integrata delle biblioteche: una nuova risorsa?, «Bollettino AIB», 44 (2004), n. 3 p. 257-270.

James SUROWIECKI, The wisdom of crowds. Why the many are smarter than the few and how collective wisdom shapes business, economies, societies, and nations, New York: Doubleday, 2004. In traduzione italiana: ID., La saggezza della folla, Milano, Internazionale, 2007.

Elaine SVENONIUS, Unanswered questions in the design of controlled vocabularies, «Journal of the American Society for Information Science», 37 (1986) n. 5, p. 331-340.

Elaine SVENONIUS, The intellectual foundation of information organization. Digital libraries and electronic publishing, Cambridge, MIT Press, 2000. In traduzione italiana:, Elaine SVENONIUS, Il fondamento intellettuale dell'organizzazione dell'informazione, traduzione di Maria Letizia Fabbrini; introduzione di Mauro Guerrini, Firenze, Le lettere, 2008.

Don R. SWANSON, Subjective versus objective relevance in bibliographic retrieval systems, «The Library Quarterly», 56 (1986), n. 4, p. 389-398.

Winnie TAM - Andrew M. COX - Andy BUSSEY, Student user preferences for features of next-generation OPACs: A case study of University of Sheffield international students. «Program: Electronic Library \& Information Systems», 43 (2009), n. 4, p. 349-374.

Arthur TAYLOR - Xiangmin ZHANG - William J. AMADIO, Examination of relevance criteria choices and the information search process, «Journal of Documentation», 65 (2009), n. 5, p. $719-744$. 
Arthur TAYLOR, User relevance criteria choices and the information search process, «Information Processing and Management», 48 (2012), n. 1, p. 136-153.

Robert S. TAYLOR, Question-negotiation and information seeking in libraries, «College and research libraries», 29 (1968), p. 178-194.

Roy TENNANT, Cross-database search: one-stop shopping, «Library Journal»,126 (2001), n 17, p. 29-30.

Roy TENNANT, MARC must die, «Library Journal», 127 (2002), n. 17, p. 26-27 <http://www.libraryjournal.com/article/CA250046.html>.

Roy TENNANT, MARC exit strategies, «Library Journal», 127 (2002) n. 19, p. 27-28. $<$ http://www.libraryjournal.com/article/CA256611.html?q=tennant+exit>.

Roy TENNANT, Lipstick on a pig, «Library Journal» 130 (2005) n. 7, p. 34. <http://www.libraryjournal.com/article/CA516027.html >.

Roy TENNANT, Demise of the local catalog, «Library Journal», 132 (2007), n. 12, p.28. $<$ http://www.libraryjournal.com/article/CA6457238.html>.

Theories of information behaviour, edited by Karen E. Fisher - Sanda Erdelez - Lynne Mckechnie Medford, published for the American Society for Information Science and Technology by Information Today, 2005.

Barbara B. TILLET, What is FRBR? a conceptual model for the bibliographic universe, revised february 2004, Library of Congress Cataloging Distribution Service, <http://www.loc.gov/cds/downloads/FRBR.PDF>, pubblicato anche in «Technicalities», 25 (2003), n. 5.

Barbara B. TILLET, RDA (Resource Description and Access): lo sviluppo di un nuovo codice di catalogazione internazionale, «Bibliotime», XI (2008), $\quad$ n. 1 $<$ http://didattica.spbo.unibo.it/bibliotime/num-xi-1/tillett.htm>. 
Barbara TILLETT, Keeping libraries relevant in the Semantic Web with RDA: Resource Description and Access, «JLIS.it», 2(2011) n. 2, p. 1-19, $<$ http://leo.cilea.it/index.php/jlis/article/view/4760>.

Barbara B. TILLETT, RDA and the Semantic Web, Linked data environment, «JLIS.it», 4 (2013), n. 1, p. 139-146, <http://leo.cilea.it/index.php/jlis/article/view/6303/7876>.

Alain TOURAINE, La società post-industriale, trad. it di Rolando Bussi, Bologna, Il Mulino, 1970.

Alain TOURAINE, Critica della modernità, trad. it. di Francesco Sircana, Milano, Il Saggiatore, 1997.

Jennifer TRANT, Studying social tagging and folksonomy: a review and framework, «Journal of Digital Information» 10 (2009) n.1, anche in $<$ http://dlist.sir.arizona.edu/2595/>.

Giovanni TUMMARELLO, Richard CYGANIAK, Michele CATASTA, Szymon DANIELCZYK, Renaud DELBRU, Stefan DECKER, Sig.ma: Live views on the Web of Data, «Journal of Web Semantics: Science, Services and Agents on the World Wide Web», 8 (2010) n. 4, p. 355-364.

Understanding FRBR: what it is and how it will affect our retrieval tools, edited by Arlene Taylor, Westport, Libraries Unlimited, 2007.

UNIVERSITY COLLEGE LONDON - BRITISH LIBRARY - JOINT INFORMATION SYSTEMS COMMITTEE, Information behaviour of the researcher of the future, [London], UCL, 2008 , $<$ http://www.jisc.ac.uk/media/documents/programmes/reppres/gg_final_keynote_1101200 8.pdf>.

Pertti VAKKARI, A theory of the task-based information retrieval process: A summary and generalization of a longitudinal study, «Journal of Documentation», 57 (2001), n.1, p. 44-60. 
Jason VAUGHAN, Investigations into library web scale discovery services, «Information Technology and Libraries», [preprint] <http://www.ala.org/ala/mgrps/divs/lita/ital/prepub/vaughan2011.pdf>.

Jason VAUGHAN, Web scale discovery services, Chicago, ALA TechSource, 2011.

Roberto VENTURA, User satisfaction e personalizzazione: quale rapporto, in La biblioteca su misura. Verso la personalizzazione del servizio, atti del convegno di "Biblioteche oggi", Milano, 9-10 marzo 2006, a cura di Claudio Gamba e Maria Laura Trapletti, Milano, Editrice Bibliografica, 2007, pubblicato anche in «Biblioteche Oggi», 24 (2006), n. 6, p. 29-44.

Roberto VENTURA, Il senso della biblioteca. Tra biblioteconomia, filosofia e sociologia, Milano, Bibliografica, 2011.

Brian VICKERY, The structure of information retrieval systems. In Proceedings of the International Conference on Scientific Information, vol. 2, Washington, National Academy of Sciences, 1959, p. 1275-1290.

Brian C. VICKERY, On retrieval system theory, London, Butterworths, 1965.

Brian C. VICKERY- Alina VICKERY, Information science in theory and practice, London, Bowker-Saur, 1992.

Boris VILLAZON TERRAZAS - Oscar CORCHO, Methodological guidelines fot publishing linked data, <http://delicias.dia.fi.upm.es/wiki/images/7/7a/07_MGLD.pdf>.

Diane VIZINE-GOETZ, FictionFinder: a FRBR works-based prototype, in Back to the future, ALCTS Preconference, ALA Annual Meeting, Orlando, June 25, 2004 [on line], [S. 1.], ALA, 2004, <http://www.ala.org/ala/alcts/alctsconted/presentations/VizineGoetz.pdf>.

Diane VIZINE-GOETZ, FictionFinder: imagining what a "Next generation" catalog might look like. Relazione presentata a OCLC Members Council Research and New 
Technologies Interest Group meeting, 6 February 2007, Québec, QC (Canada) and Dublin, Ohio (USA), 2007, <http://www.oclc.org/research/memberscouncil/2007-02/vizinegoetz.pdf>.

Max WEBER, Economia e società. 1. Teoria delle categorie sociologiche, trad. it. di Tullio Bagiotti, Franco Casabianca, Pietro Rossi, Edizioni di Comunità, Torino 1999.

Romain WENZ, Linked open data for new library services: the example of data.bnf.fr, «JLIS.it», 4 (2013), 4 n. $\quad 1, \quad$ p. 403-416, $<$ http://leo.cilea.it/index.php/jlis/article/view/5509/7919>.

Gernot WERSIG, The problematic situation as basic concept of information science in the framework of the social sciences. In Theoretical problems for Informatics: new trends in informatics and its terminology, Moscow, International Federation for Documentation, 1979, p. 48-57.

Paul Gabriele WESTON, FRBR e utente: considerazioni sulla ricerca, AIB. Sezione Toscana, [relazione presentata a Seminario su FRBR (Functional requirements for bibliographic records), Firenze, 27-28 gennaio 2000], $<$ http://www.aib.it/aib/sezioni/toscana/conf/frbr/weston.htm>.

Paul Gabriele WESTON, Tra controllo bibliografico e controllo intellettuale: qualche riflessione sui sistemi bibliografici elettronici, [relazione presentata all'International Conference Electronic Resources, Roma, 2001], $<$ http://eprints.rclis.org/4121/1/weston_ita.pdf/>.

Paul Gabriele WESTON, Il catalogo elettronico. Dalla biblioteca cartacea alla biblioteca digitale, Roma, Carocci, 2002.

Paul Gabriele WESTON, Dal controllo bibliografico alle reti documentarie. Il catalogo elettronico nella prospettiva dell'interoperabilità tra sistemi eterogenei, «Biblioteche Oggi», 20 (2002), n. 7, p. 44-56. 
Paul Gabriele WESTON, Gli strumenti della cooperazione in rete : dal catalogo elettronico ai sistemi della ricerca interdisciplinare, Napoli, ClioPress, 2003, anche in $<$ http://www.storia.unina.it/cliopress/weston.html>.

Paul Gabriele WESTON, La gestione elettronica delle biblioteche in Biblioteconomia: principi e questioni, a cura di Giovanni Solimine e Paul Gabriele Weston. Roma, Carocci, 2007, p. 221-256.

Paul Gabriele WESTON, Caratteristiche degli opac e strategie delle biblioteche, «Bibliotime», XI (2008), n.1, <http://didattica.spbo.unibo.it/bibliotime/num-xi1/weston.htm>.

Paul Gabriele WESTON - Salvatore VASSALLO ... “E il navigar m'è dolce in questo mare”. Linee di sviluppo e personalizzazione dei cataloghi, in Claudio GAMBA - Maria Laura TRAPLETTI, La biblioteca su misura. Verso la personalizzazione del servizio, atti del convegno di "Biblioteche oggi", Milano, 9-10 marzo 2006, a cura di C. Gamba e M. L. Trapletti. Milano, Editrice Bibliografica, 2007, p. 130-167.

Jezmynne WESTCOTT-Alexandra CHAPPELL- Lebel CANDACE, LibraryThing for libraries at Claremont, «Library Hi Tech», 27 (2009), n.1, p. 78-81.

Ryen WHITE, Interactive tecniques in Interactive information seeking, behaviour and retrieval edited by Ian Ruthven and Diane Kelly, London, Facet, 2011, p. 171-188.

Thomas D. WILSON, On user studies and information needs, «Journal of Documentation», 37 (1981), n. 1, p. 3-15, ripubblicato in «Journal of Documentation», 62 (2006), n. 6, pp. 658-670, <http://informationr.net/tdw/publ/papers/1981infoneeds.html>.

Thomas D. WILSON, Exploring models of information behaviour: the 'Uncertainty' Project, «Information Processing and Management», 35(1999), p. 839-849.

Thomas D. WILSON, Models in information behaviour research, «Journal of Documentation», 55 (1999), n. 3, p. 249-270, anche in <http://informationr.net/tdw/publ/papers/1999JDoc.html>. 
Thomas D. WILSON - Christina WALSH, Information behaviour: an interdisciplinary perspective, Sheffield, University of Sheffield, Department of Information Studies, 1996, $<$ http://informationr.net/tdw/publ/infbehav/cont.html>.

Iris XIE, Interactive information retrieval in digital environments, New York, IGI Global, 2008.

Yunjie (Calvin) XU-Zhiwei Chen, Relevance judgment: what do information users consider beyond topicality?, «Journal of the American Society for Information Science and Technology», 57 (2006), n. 7, p. 961-973.

Fugang XU, A search engine that answers questions, «Crienglish.com», 10. 10. 2005 <http://english.cri.cn/2238/2005-10-10/33@275908.htm>.

Sharon Q. YANG - Kurt WAGNER, Evaluating and comparing discovery tools: How close are we towards next generation catalog? «Library Hi Tech», 28, (2010), n. 4, p. 690709.

Sharon Q. YANG - Melissa A. HOFMANN, Next generation or current generation?: A study of the OPACs of 260 academic libraries in the USA and Canada, «Library Hi Tech», 29 (2011), n. 2, p. 266-300.

Martha M. YEE, System design and cataloging meet the user: user interfaces to online public access catalogs, «Journal of American Society for Information Science», 42 (1991), n. 2, p. 78-98. < http://escholarship.ucop.edu/uc/item/2rp099x6 >.

Martha M. YEE, FRBRization: a method for turning online public finding lists into online public catalogs, «Information Technology and Libraries», 24 (2005), n. 3, pag. 77-95, anche in $<\mathrm{http}: / /$ repositories.cdlib.org/postprints/715>.

Martha M. YEE Applying FRBR to library catalogues : a review of existing FRBRization projects. [Relazione presentata al seminario Beyond the OPAC : future directions for Web- 
based catalogues. Perth, Western Australia, settembre 2006] <http://www.nla.gov.au/lis/stndrds/grps/acoc/papers2006.html>.

Martha M. YEE, Beyond the Opac: future directions for Web-based catalogues, [Relazione presentata al seminario Beyond the OPAC : future directions for Web-based catalogues. Perth, Western Australia, settembre 2006] <http://www.nla.gov.au/lis/stndrds/grps/acoc/papers2006.html>.

Martha M. Yee, FRBR and moving image materials: content (Work and Expression) versus carrier (Manifestation) in Understanding FRBR: what it is and how it will affect our retrieval tools, edited by Arlene Taylor, Westport, Libraries Unlimited, 2007, p. 117-130.

Martha M. YEE, Can bibliographic data be put directly onto the Semantic Web?, «Information Technology and Libraries», 28 (2009), n. 2, p. 55-80, $<$ http://escholarship.org/uc/item/91b1830k>.

Martha M. YEE, The single shared catalog revisited, in Conversations with catalogers in the 21st century edited by Elaine Sanchez Santa Barbara, Libraries Unlimited, 2011, p. 121-136.

Holly YU-Margo YOUNG, The impact of Web search engines on subject searching in OPAC, «Information Technology And Libraries», 23 (2004), n. 4, p. 168-180, anche in <http://www.ala.org/ala/lita/litapublications/ital/volume23a/number4a/yu.pdf>.

Yin ZHANG - Athena SALABA, Implementing FRBR in libraries: key issues and future directions, New York, Neal-Schuman Publishers, 2009.

Chaim ZINS, Models for classifying Internet resources, «Knowledge organization», 29 (2002), p. 20- 28.

George Kingsley ZIPF, Human behaviour and the principle of least-effort: an introduction to human ecology, Cambridge, Addison-Wesley, 1949. 
Maja ŽUMER - Marcia Lei ZENG, Modelling Knowledge Organization Systems and Structures: A discussion in the context of conceptual and data models. Slides presentate a: ISKO UK Biennial Conference, London, 4-5 luglio 2011 $<$ http://www.iskouk.org/conf2011/ppt/zumer.pdf>. 